\title{
2019 Scientific Session of the Society of American Gastrointestinal and Endoscopic Surgeons (SAGES), Baltimore, Maryland, USA, 3-6 April 2019: 2019 Posters
}

(C) Springer Science+Business Media, LLC, part of Springer Nature 2019

P001

Delay in Emergency Hernia Surgery is Associated with Worse Outcomes

Ira L Leeds, MD, MBA, ScM, Christian D Jones, MD, MS, Elliott R Haut, MD, PhD, Joseph V Sakran, MD, MPA, MPH, Sandra R Dibrito, MD, PhD, Ryan Fransman, MD, Alistair J Kent, MD, MPH; Johns Hopkins University School of Medicine

Introduction: The purpose of this study was to determine if variation in the practice of deferring surgery for preoperative optimization or surgeon availability impacts surgical outcomes.

Methods: The national NSQIP databases from 2011 to 2016 were queried for emergent surgeries for abdominal hernia resulting in obstruction or gangrene by primary post-op diagnosis. Diaphragmatic hernias, elective cases and cases without the specific designation emergent were also excluded. Patients were grouped by time in days from date of admission to date of surgery: Same day, next day, and second day or longer. These groups were then examined for differences in NSQIP defined major morbidities and mortality. We used a multinomial propensity score weighting for covariates' clustering across the timing groups then performed weight-adjusted multivariate logistic regression.

Results: The effective population size was 79,145 ; selected unadjusted characteristics are shown (Table). After adjusted analysis, the odds of major complication were increased by $25 \%(\mathrm{aOR}=1.25$, $95 \%$ CI $1.07-1.47, \mathrm{p}=0.005$ ) for delays to the next day, and $50 \%$ for delays to the second day or longer $(\mathrm{aOR}=1.50,95 \%$ CI $1.22-1.86$, $\mathrm{p}<0.001$ ). There was no difference of risk of 30-day mortality when adjusting for other factors for next day surgery (aOR 1.37, CI $0.52-$ $3.60, \mathrm{p}=0.51$ ) or surgery deferred $\geq 2$ days (aOR 1.79 , CI 0.56 $5.74, \mathrm{p}=0.32$ ).
Conclusions: Delay in surgery for emergent hernias substantially and progressively increased odds of major morbidity but not mortality in a propensity adjusted analysis of NSQIP data, though limitations exist based on NSQIP structure and reporting mechanisms. Patients presenting with hernia and indications for urgent surgical intervention be taken to the operating room as soon as physiologically feasible. Delays for resuscitation and optimization beyond physiologic tolerance of the procedure may increase the risk of major morbidity.

\begin{tabular}{lrrrc}
\hline $\begin{array}{l}\text { Variable }(\%) \\
\mathrm{n}=79,145\end{array}$ & Same-day & Next-day & Further delayed & $\mathrm{p}$ \\
\hline Preoperative sepsis & 21.3 & 21.6 & 22.1 & 0.6 \\
Diabetes & 16.9 & 17.1 & 19.2 & 0.08 \\
Hypertension & 51.6 & 51.6 & 57.5 & $<0.001$ \\
Dyspnea & 7.0 & 7.1 & 8.2 & 0.2 \\
COPD & 7.9 & 7.7 & 9.9 & 0.07 \\
Active smoker & 20.8 & 20.5 & 18.4 & 0.06 \\
\hline
\end{tabular}


P002

\section{Retroperitoneal Duodenal Foreign Body Perforation - A Novel Laparoscopic Approach}

Nicholette Goh, MBBS, Sunder Balasubramaniam, Wee Ming Tay, Mui Heng Goh; Tan Tock Seng Hospital

Introduction: Gastrointestinal perforation from foreign bodies can lead to life threatening sepsis, and pose a significant challenge given the need to drain the septic source as well as safely extract the offending object.

Case Description: A 71-year-old Chinese lady presented with abdominal pain and constitutional symptoms without peritonitis or fever. A computed tomographic (CT) scan of the abdomen revealed two retroperitoneal abscesses with a $4 \mathrm{~cm}$ fishbone within (Image 1). The cavities were $(5.8$ by $2.4 \mathrm{~cm})$ and $(10$ by $7 \mathrm{~cm}$ ) in size, one of which was surrounding the right external iliac artery. There was a visible connection from the third portion of the duodenum to the superior abscess cavity.

She underwent radiology-guided drainage of the collections, followed by a gastrograffin swallow which did not demonstrate an ongoing leak. Retrieval of the fishbone was first attempted endoscopically. This was done by placing a $5 \mathrm{~mm}$ laparoscopic port into the cavity to insufflate it with gas, followed by introduction of a flexible choledochoscope. Unfortunately, the bone could not be visualised, and the procedure was hence converted to open retroperitoneal approach via a $4 \mathrm{~cm}$ incision. The bone was successfully retrieved (Image 2), and a repeat CT done post-operatively showed near resolution of the abscess and complete removal of the fishbone. The patient was fit for discharge on the 4th postoperative day.

Discussion: Intestinal perforation from the ingestion of a foreign body most commonly affects the colon and terminal ileum. If the perforation is intra-peritoneal, peritonitis usually results; however retroperitoneal perforations may seal off and hence present in a more insidious fashion.

To our knowledge, this is the first such report of retroperitoneal abscess due to a foreign body managed by minimally invasive techniques. Fluoroscopic extraction by the radiologist was considered in this case but deemed unsuitable due to the risk of fragmentation and having pieces left behind. The retroperitoneal approach combined with interventional radiology allowed us to avoid the morbidity of laparotomy and speed the patient's recovery. As expertise develops further, it is hoped that minimally invasive techniques like those used in retroperitoneoscopic adrenalectomy or pancreatic debridements can be used in such cases in the future. These can also be considered for trauma patients with projectile injuries who face a similar problem.

Conclusion: Multidisciplinary collaboration allowed for a novel method of extraction of a fishbone, and hence faster recovery than would be expected with traditional laparotomy and extraction.

Image 1

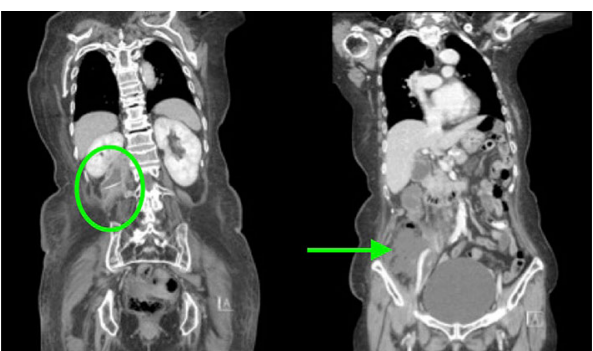

Image 2

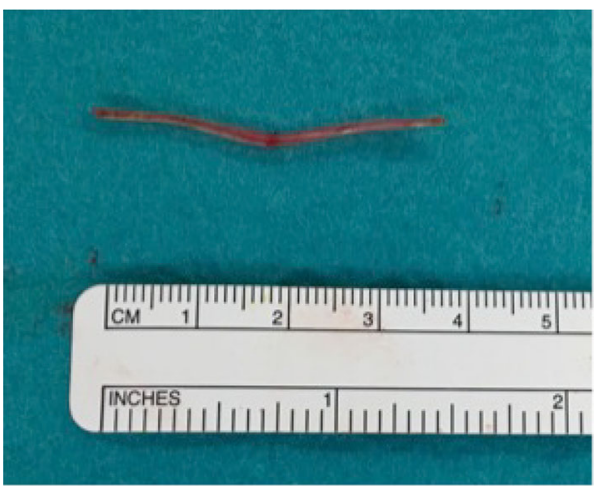

P003

Perforated Appendicitis Wash or Dry Abdominal Cavity: Myth or Reality

Carlos A Rosero, MDFACS, Andres G Moreno, MDFACS; Hospital Carlos Andrade Marín, Quito, Ecuador

Introduction: Seeking the best treatment of the peritoneal cavity in case of secondary peritonitis is a surgeons' permanent concern and it is essential for the adequate management of the patient.

Objective: To compare the results of thoroughly washing the peritoneal cavity vs simply just drying the peritoneal cavity in cases of secondary peritonitis due to acute perforated appendicitis.

Materials and methods: A retrospective observational study was carried out in the General Surgery Department at Carlos Andrade Marín Specialties Hospital, from January 2016 to December 2017. The study main end-point was assessing the postoperative complication between peritoneal lavage versus cavity drying.

Results: The records of 301 patients, 212 (70.4\%) who underwent peritoneal lavage and 89 $(29.6 \%)$ with just drying the peritoneum. The frequency of complications between both groups did not reach statistical significance. The only significant variable was the operative time (OR 1.01, $\mathrm{p}<0.005$ )

Conclusions: No statistically significant differences were found with the type of cavity management in patients with peritonitis secondary to perforated appendicitis (lavage versus cavity drying).

Keywords: Peritoneal lavage, Peritoneal cavity drying, Perforated appendicitis, Peritoneal cavity.

\section{P004}

\section{Guidelines for Acute Care Surgery Implementation are Necessary}

Holly B Cunningham, Joshua J Weis, Luis R Taveras, Steven Boll, Tarik D Madni, Jonathan B Imran, Meaghan Colletti, Maryanne L Pickett, Joseph P Minei, Michael W Cripps; University of Texas Southwestern

Introduction: The creation of an acute care surgery (ACS) service has been associated with improved hospital efficiency and clinical outcomes; however, specific guidelines for implementing this model have not been developed. As a result, there has been significant variation in how individual institutions apply the principals of an ACS model to their practice. We sought to evaluate the impact of an ACS model at a private, non-trauma hospital with no in-house, overnight attending.

Methods: A retrospective review of all general surgery (GS)/ACS admissions and consults was conducted at an academic, private, non-trauma hospital from July 2015 to July 2018 . An ACS service was established in July 2016. Clinical, demographic, and financial variables were queried from the electronic medical record. Parametric and non-parametric analyses were used when appropriate. Prior to July 2016, the GS service was staffed during weekdays by a single surgeon who was responsible for managing consults as well as maintaining an elective GS practice. Weekend and night coverage was home-call shared between this surgeon and other surgical subspecialists. Within the ACS model, weekday elective cases and clinics were maintained by the GS staff while emergency GS consults were assigned to a separate, dedicated ACS surgeon. Overnight and weekend home-call was divided amongst ACS staff, many of whom were critical care boarded.

Results: In total, 3164 hospital account records (HARs) were reviewed. There were 1016 HARs in the pre-ACS group and 2148 HARs in the post-ACS group. During the pre-ACS time period, there were 505 admissions and 511 consults. Post-ACS implementation, there were 1135 admissions and 1013 consults. Groups were similar in age $(p=.88)$, gender (preACS $57.6 \%$ male, post-ACS $58.8 \%$ male), and race (70\% white). Total hospital charges between groups were statistically similar $(\mathrm{p}=.51)$. Net revenue and contribution margin between groups were also similar ( $\mathrm{p}=.44, \mathrm{p}=.07$, respectively). Analysis of total length of stay (LOS) and intensive care unit LOS revealed no difference $(\mathrm{p}=96, \mathrm{p}=94$ respectively). In subset analyses, no differences were seen in total LOS for cholecystectomies ( 4.25 vs 4.14 days, $\mathrm{p}=.84$ ) or bowel obstructions ( 5.30 vs 4.95 days, $\mathrm{p}=.71$ ). Conclusions: The creation of an ACS service in a private, non-trauma hospital without concurrent institutional culture change does not result in the financial benefits or clinical outcome improvements previously reported. Guidelines for optimal implementation should be established. Further studies will aim to define appropriate measures of successful ACS implementation as well as predictors of success in various hospital settings. 


\section{P005}

\section{The Great Imitator: Acute Gangrenous Cholecystitis}

Allyne Topaz, MD, Lauren Poliakin, MD, Luca Milone, MD; The Brooklyn Hospital Center

Acute gangrenous cholecystitis mimicking acute cardiovascular diseases may lead to a delay in surgical management of cholecystitis. Common symptoms associated with ischemic heart disease, such as chest pain and shortness of breath, may also be associated with noncardiac conditions, like cholecystitis. Acute cholecystitis can causes transient or dynamic ECG changes, likely secondary to gallbladder distension. We have reported 2 cases in a period of 2 months, where the patients presented with cardiac-like symptoms. Both patients were admitted for management of acute cardiovascular conditions, and eventually diagnosed with acute cholecystitis on abdominal ultrasound. The patients were female and obese. Minimally invasive cholecystectomy was performed for both patients, which subsequently led to improvement of symptoms postoperatively.

Case 1: An 81 year old obese female presented to the emergency department with tachycardia and complaints of chest pain and right upper quadrant pain. Her ECG showed atrial fibrillation. Immediate focus and workup surrounded the atrial fibrillation; including imaging to rule out mesenteric ischemia. Abdominal ultrasound showed multiple gallstones with features of acute cholecystitis. After resuscitation, the patient underwent a laparoscopic subtotal cholecystectomy for acute gangrenous cholecystitis. The degree of inflammation necessitated stapled removal of the gallbladder with placement of a JP drain. The patient recovered well postoperatively.

Case 2: A 59 year old obese female with a long standing cardiac history presented to the emergency department with complaints of chest pain and epigastric pain. The patient was admitted for further work-up and management of acute coronary syndrome. After life threatening cardiac conditions were ruled out, an abdominal ultrasound showed multiple gallstones with evidence of acute cholecystitis. Robotic assisted subtotal cholecystectomy was performed. The patient had a left sided gallbladder. Given the degree of inflammation, a partial cholecystectomy including lysis of adhesions, and needle decompression was performed. A postoperative ERCP was later performed revealing a small bile leak necessitating sphincterotomy. The patient did well postoperatively and was discharged uneventfully. Acute gangrenous cholecystitis may manifest with cardiac-like symptoms postponing the patient's work-up and treatment of cholecystitis. Cholecystitis is a common surgical condition that requires prompt recognition and diagnosis. Timely recognition of gangrenous cholecystitis provides proper treatment and can prevent unnecessary interventions.

\section{P006}

\section{Small Bowel Obstructions Following the Use of Barbed Sutures: A Systematic Review}

Benjamin Clapp, MD, Loyd Christensen, BS, Robert Jones, Carlos Lodeiro, BS, Ellen Wicker, DO, William Klingsporn, MD, Alan Tyroch, MD; Texas Tech HSC Paul Foster School of Medicine

Introduction: Barbed sutures were introduced in 2004. Their adoption and use has been widespread across all surgical specialties. One of the infrequent complications seen with the use of barbed sutures are small bowel obstructions. In this study, we perform a systematic review of the literature to characterize small bowel obstructions (SBO) after the use of barbed sutures in a variety of operative procedures.

Methods: A review of the literature was performed by searching PubMed and Ovid. We used the search terms: "barbed" "suture" "bowel" and "obstructions." For each case report we examined the initial surgical procedure, type of barbed suture used, the type of complication, the time to complication, the presentation, and the type of operative interventions required for said complication.

Results: Our review of the literature revealed 18 different cases of small bowel obstruction (SBO) secondary to the use of barbed sutures. The two most index common procedures, with a total of 4 cases each were myomectomy and hysterectomy. The next two most common procedures were TAPP hernia repair and sacral colpopexy. Different types of sutures were reported with the V-Loc barbed suture the most common type, involved in 14 of the 18 cases. The average time of presentation to SBO was found to be 18.3 days post-op (1-49 days). A total of 15 patients $(83.3 \%$ ) presented with abdominal pain. Other common complaints included vomiting $(50 \%)$, abdominal distension $(27.8 \%)$, inability to tolerate food $(22.2 \%)$ and constipation $(16.7 \%)$. Most patients had a CT done, with the most common finding being a transition point in the small bowel. A total of 5 cases were also found to have a possible volvulus on $\mathrm{CT}$, and only 1 case was reported to have strangulation.

Of these patients, 14 underwent a diagnostic laparoscopy, 3 of them had a diagnostic laparotomy and one of them had a hepatojejunostomy revision. In most cases, the removal of the redundant barbed suture was enough to release the small bowel and clear the obstruction

Conclusions: Surgeons should have a high index of suspicion for SBO if a patient presents with obstructive symptoms after a surgery that used barbed sutures. This will often present as a mesenteric volvulus on computed tomography. These particular SBOs require operative exploration, with laparoscopy being successful in the majority of cases.

\section{P008}

Use of Departmental Morbidity and Mortality Conference as a Real Time Barometer of Outcomes and Quality in Pediatric Appendectomy

Julie A Disano, MD, Mary C Santos, MD, Vamsi V Alli, MD, Morgan K Moroi, BS, Robert E Cilley, MD; Penn State Hershey Medical Center

Introduction: Weekly morbidity and mortality conferences (M\&M) are educational staples, playing an important role in quality improvement. After noticing an increase frequency of pediatric appendectomy complications during $\mathrm{M} \& \mathrm{M}$, we explored the accuracy of this finding.

Methods: M\&M listings were reviewed for complications following appendectomy. Data was collected from January 2013 through December 2016 and analyzed in semiannual intervals (CY1: January-June; CY2: July-December). The total number of appendectomies performed was determined from billing data. Complication rates were compared to institutional Pediatric NSQIP data.

Results: 771 appendectomies were performed during the study period with 63 reported complications $(8.17 \%)$. M\&M data indicated an increase in postoperative complications during the CY2-2015 period to $13.58 \%$, from $11.59 \%$ in CY1-2015. This was similarly seen in NSQIP data, with a rise in complications from $2.20 \%$ to $8.20 \%$ between CY1 and CY2 of 2015.

Conclusions: Observed trends in institutional M\&M data recognized an increase in reported postoperative complications in CY2-2015, a finding confirmed by pediatric NSQIP data. Using external national benchmarks we validated a cheap and reliable method of QI and outcomes measures based on regularly collected M\&M data. Regular review of M\&M data provides a cost effective and accurate means of assessing quality in addition to reinforcing the adoption of practice-based learning and systems-based practice. Additionally, this method is a useful barometer for QI at non-NSQIP participating institutions. 


\section{P009}

Solitary Ileal Lymphangioma, A Rare Cause of Obscure Intestinal Bleeding Requiring Transfusion Successfully Treated with Laparoscopic Resection

Justin D Sargent, DO ${ }^{1}$, Hassan Masoudpoor, MD ${ }^{1}$, Jeffrey J Kraft, $\mathrm{MD}^{2}$, John Davis, MD ${ }^{1}$; ${ }^{1}$ Hackensack Meridian Health Palisades, ${ }^{2}$ Hackensack University Medical Center

Introduction: Small bowel lymphangioma is a rare benign vascular system tumor that mostly presents in children as head, neck or axillary region masses. Generally they are asymptomatic and don't require treatment, unless complications arise, such as bleeding or obstruction. Small bowel lymphangiomas are very rare in adults and intestinal bleeding secondary to a solitary ileal lymphangioma presenting with severe anemia requiring transfusion are rarely reported. Case Description: 54 year old male presented to ED with sudden onset of lower abdominal pain without remarkable past medical history. A small urinary tract stone with hydronephrosis was identified. Incidentally on CT an area of mural thickening of a short segment of small bowel in the left lower quadrant was noted. Mild asymptomatic anemia was found, and patient was recommended outpatient endoscopic follow-up with analgesics for the renal calculi.

The patient was lost to follow up due to insurance reasons and returned to our hospital after three months with recurrent and severe anemia requiring blood transfusion and intravenous iron treatment. He denied any episodes of melena, hematochezia, chest pain, nausea and vomiting. The patient only complained of the mild exertional SOB, but was compensating by decreasing activity at home. Clinical examination revealed marked conjunctival pallor. No masses were palpated on rectal or abdominal examination. On admission a microcytic anemia with hemoglobin of $4.3 \mathrm{~g} / \mathrm{dL}$ was noted. CT was performed showing segmental circumferencial thickening of a loop of ileum without perienteric stranding and lymphadenopathies. Remainder of the large and small bowel including terminal ileum was identified normal. The patient received a transfusion prior to upper and lower endoscopy.

Esophagogastroduodenoscopy was normal except mild gastritis. Colonsocopy didn't reveal active bleeding or abnormalities in the colon. No bleeding was noted in the terminal ileum. Subsequently, he underwent technetium labeled red blood cell scan and no focus of active bleeding identified.

Given the CT findings and endoscopic workup, the patient underwent diagnostic laparoscopy and intraoperatively an $8 \mathrm{~cm}$ section of the mid-ileum was found distended distinctly from the surrounding bowel and it was resected with the feeding mesentery with a $5 \mathrm{~cm}$ margin. Histologic examination showed multiple endothelium lined cystic spaces and associated smooth muscle fibers consistent with a lymphangiomatous lesion.

Discussion: Lymphangiomas in the jejunum or ileum are extremely rare and surgery can be necessary when bleeding is not controlled by gastroenteroscopy. To avoid recurrence, laparoscopic segmental bowel resection is an optimal approach.
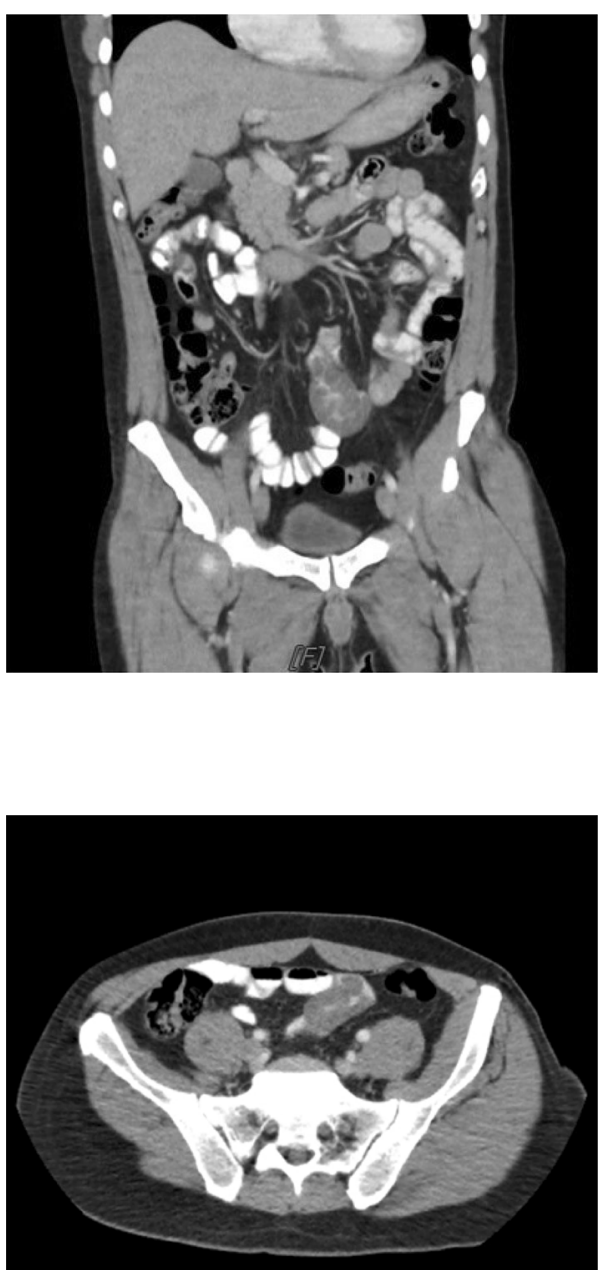
P010

\section{Weekday or Weekend Hospital Admission: Does it Matter for Acute Care Surgery?}

Ibrahim Albabtain ${ }^{1}$, Roaa Alsuhaibani ${ }^{1}$, Sami Almalki ${ }^{1}$, Nada Alhassan ${ }^{2}$, Hassan Arishi ${ }^{1}$, Hatim Alsulaim ${ }^{1}$; ${ }^{1}$ King Abdulaziz Medical City, ${ }^{2}$ King Saud bin Abdulaziz University for Health Sciences

Introduction: During weekends, hospitals usually reduce their staffing levels and services. This might result in decreased quality of healthcare or so-called the weekend effect. In this study, we aimed to determine the impact weekends on two common emergency general surgeries and their outcomes, in terms of day of operation and admission.

Methods and Procedures: This retrospective cohort study was conducted at a tertiary care hospital between January and December 2016. Surgical procedures included were cholecystectomy and appendectomy. Patients' demographic, co-morbidities, admission date, surgery date, complications, readmission and follow-up details were collected from the electronic medical records. Postoperative outcomes associated with weekend admission were identified by multivariable analysis using univariable and multivariable logistic regression models controlling for potential confounders. Results: A total of 539 patients were included. Median age for weekday admissions was 31 years (IQR: 22, 45), and 32 years (IQR: 23, 49.75) for weekend admissions. The majority of patients were admitted during weekdays $(\mathrm{n}=391)$. No significant difference was found in the type of surgery performed between weekday and weekend admissions ( $p$ value 0.384 ). Surgeries tend to be delayed by a median of one day for weekend admissions compared to weekdays with similar overall length of stay for both groups. Weekend admissions were associated with higher complication rates compared with weekday admissions $(12.2 \% \mathrm{vs}$. $6.1 \%$ ). Regarding the day of surgery, 444 surgeries were performed during weekdays while 86 surgeries were performed during weekends. Patients who were operated on weekends were younger in age compared with weekdays ( 32 vs. 30 years old, p-value 0.019 ). The percentage of female patients undergoing surgery during weekends was lower compared with weekdays $(40.7 \%$ vs. $53.8 \%)$. Both groups had similar complication rates (7.7 in weekdays vs. $9.3 \%$ in weekends, p-value .605). During weekends, appendectomies were performed more $(77.9 \%$ vs. $45.9 \%$ ), and less cholecystectomies were performed ( $22.1 \%$ vs. $54.1 \%$, p-value 0.000$)$.

Conclusions: Patients admitted on weekends tended to have their surgeries delayed by one day, with more complication rates. Patient operated on over weekends were younger and less likely to be females. Appendectomy was the most common performed surgery over the weekend.

\section{P011}

\section{Massive Splenic Infarction from Hypoperfusion}

Nancy Panko, MD, Seeyuen Lee, MD, Melissa Boyle, MD, Phillip Leggett, MD; Houston Northwest Medical Center

Introduction: Massive splenic infarction (MSI) due to hypoperfusion is rare, with only one source documenting MSI from hypoperfusion in a patient with septic shock. We report a case of MSI managed with laparoscopic splenectomy three weeks after an exploratory laparotomy performed for control of a bleeding duodenal ulcer.

Case: Patient is a 28 year old man who presented with rectal bleeding. He underwent imaging but then sustained cardiac arrest due to hypovolemia. He responded to resuscitation and vasopressors, and then surgery was consulted for evaluation. We attempted endoscopic management of a bleeding duodenal ulcer with arterial hemorrhage. The ulcer was injected and clipped without success. The patient was then taken emergently for exploratory laparotomy with duodenotomy and ligation of the gastroduodenal artery. A drain was left adjacent to the duodenotomy and feeding jejunostomy was placed. The patient was extubated after definitive abdominal closure on post-operative day two. He received over 30 units of blood products in the first 24 hours. Post-operatively, the patient had shock liver, acute renal failure requiring hemodialysis, and VTE. He developed fever 10 days postoperatively, and work up was initiated with no significant source. CT of the abdomen and pelvis was performed (Figure 2). The spleen was noted be significantly enlarged without evidence of perfusion. No other abnormalities were noted. The patient was counseled that splenic infarction was likely the source of his fever. He was initially asymptomatic, but then developed abdominal pain and anorexia. This did not improve with conservative measures and he agreed to surgery.

Patient underwent uneventful laparoscopic splenectomy on postoperative day 24. Pathology showed marked splenic necrosis.

He recovered uneventfully, and was seen in clinic with no additional complaints.

Discussion: MSI is a rare occurrence, with less than twenty documented cases. The majority of these cases occurred secondary to blood disorders or coagulopathies, malignancy, infection, and organ transplant. Only one other case has been reported linking MSI to hypoperfusion and shock. This was attributed to both hypoperfusion as a result of septic shock as well as hypercoaguability related to the inflammatory response.

Splenic preservation is preferred in most cases of splenic injury, to preserve immune function. Splenectomy is reserved for patients with refractory abdominal pain, hemorrhage, abscess, or pseudocyst. In our case, the patient had significant abdominal pain, and requested operative management after a trial of conservative therapy. We were able to perform his splenectomy laparoscopically, which further sped his recovery.

\section{P012}

Laparoscopic Management of Large Ruptured Subcapsular Liver Hematoma After Laparoscopic Cholecystectomy

Justin D Sargent, DO, Steven P Shikiar, MD; Hackensack Meridian Health Palisades

Introduction: Laparoscopic cholecystectomy has become the standard of care for symptomatic biliary disease due to its minimally invasive nature and low complication rate. Here we present a rare complication of a large ruptured subcapsular liver hematoma and its successful management with laparoscopic control of the hepatic hemorrhage.

Case Report: An 18-year-old female presented with a 9 day history of epigastric and RUQ abdominal pain with daily episodes of emesis. Pain was initially colicky but became constant. Patient was initially evaluated at an outside hospital with abdominopelvic ultrasound, HIDA and EGD. U/S showed acute cholecystitis with gallstones, wall thickening, and pericholecystic fluid. HIDA showed normal filling with decreased ejection fraction. Endoscopy showed a grossly normal stomach and duodenum without signs of gastritis/ ulcers. Patient refused cholecystectomy at that time but returned to our hospital and was taken to the operating room for diagnostic laparoscopy.

Intraoperatively her liver and bowel appeared normal. Her gallbladder was grossly distended. A cholecystectomy was performed in the standard fashion without difficulty. Hemostasis of the GB fossa was noted at the end of the case.

On POD\#1 she had a vasovagal syncopal episode when standing. HGB dropped from 16 to 10 and a leukocytosis of $39 \mathrm{k}$ was noted. Abdominal U/S was performed and showed significant simple RUQ fluid. Three hours later hemoglobin of 7.5 was noted. She remained HD stable. Tachycardia continued to worsen and she was taken to the OR for diagnostic laparoscopy. Intraoperatively a large subcapsular liver hematoma over the superior surface of the right dome of the liver was noted with a free rupture of Glisson's capsule at the anterior margin of the liver. 1.4 L of hemoperitoneum was evacuated. The liver and abdomen were thoroughly inspected and the majority of the liver capsule was intact. A stable clot was noted under the capsule and left in place. Several small areas of oozing were noted and the free edge of the capsule was packed with surgicel and floseal. No active bleeding was noted at the end of the case. Intraoperatively she received $3 \mathrm{u}$ pRBC and FFP.

She had an uneventful post-operative course. After discharge she received a CT scan revealing no sequelae from her surgeries.

Discussion: Exploration of the postoperative patient displaying signs and symptoms of hemorrhage is always indicated. Minimally invasive methods to evaluate and control hemorrhage can be safely used in select cases with a low threshold to convert to open technique.

\section{P013}

Case Series of Seventeen Patients with Acute Appendicitis in Pregnancy: Management Algorithm and Laparoscopic Approach

Georges Abizeid, Hager Aref, Obada Alhallak; International Medical Center

Introduction: Acute appendicitis (AA) is the most common general surgical problem encountered during pregnancy, affecting 1 in 500 to 1 in 2000 pregnancies. Emergency Department, General Surgery, and Obstetric physicians will face this challenging situation of abdominal pain in a pregnant woman suspected of AA especially in diagnosis, management, and surgical approach.

Objectives: First, to report our experience of operated pregnant patients for AA; Second, to propose an algorithm to facilitate the optimal management. Third, to suggest strongly, after analysis of the results of this series and review of literature that Laparoscopic appendectomy (LA) is considered as the "new standard of care".

Methods: Between November 2013 and January 2018, seventeen patients diagnosed with AA, who underwent LA $(\mathrm{N}=17)$ were included in this study. Data collection and details were reviewed retrospectively based on medical records.

Results: Retrospective analysis of 17 consecutive cases of suspected AA during pregnancy was carried out. (Nov. 2013 - January 2018). Patients were in their first (35\%), second (53\%) and third trimesters (12\%). The average gestational age was 17.5 weeks [5-27]. All patients had preoperative Ultrasound with (54\%) accuracy, non-conclusive in 7 cases. MRI done for six of these seven patients confirmed the diagnosis of AA in 5 patients (84\%). All patients were operated laparoscopically, no conversion done. Negative appendectomy rate was (6\%). Postoperative complications were $(6 \%)$ with paralytic ileus treated conservatively. No obstetrical or fetal complications found. No differences were observed between trimesters in terms of clinical presentation and outcome except for operative technical modifications. Conclusion: Acute appendicitis in pregnancy is a clinically challenging situation that often cannot be faced without the aid of radiological investigations. Ultrasound and Magnetic Resonance Imaging may help to avoid late diagnosis and contribute to decreasing the rate of negative appendectomy. Operating with no delay will reduce the appendicular perforation and the feto-maternal complications. Laparoscopic appendectomy in pregnancy is the standard of care, regardless of the trimester. 
P014

\section{Multi-Modal Minimally Invasive Approach to Boerhaave's Syndrome: A Case Report}

Kalyan Gorantla, MD, MBA, Seeyuen Lee, MD, MPH; University of Texas at Houston/Houston Northwest Medical Center

Background: Boerhaave's syndrome, or effort rupture of the esophagus, is a life-threatening emergency and fatal if left untreated. It is associated with high morbidity and mortality often due to a delay in diagnosis. Given the critical nature of the disease, prompt surgical management is recommended. The management traditionally involved controlling sepsis with laparotomy, thoracotomy, or both to repair the site of esophageal perforation and mediastinal debridement/drainage. Since esophageal perforations, and specifically Boerhaave's syndrome, are uncommon, ideal management guidelines have not been standardized. The authors present a case where a multi-modal minimally invasive approach was used to address the problem of Boerhaave's syndrome.

Methods: Initial experience of managing Boerhaave's syndrome in a multidisciplinary manner using laparoscopic, thoracoscopic (VATS), and endoscopic techniques is reported. Results: Emergent laparoscopic Nissen fundoplication with transabdominal drainage of mediastinum and feeding jejunostomy was utilized to initially treat a patient with Boerhaave's syndrome. VATS was subsequently utilized to treat a left-sided pleural effusion and empyema. A persistent leak was then managed endoscopically with an esophageal stent. The patient recovered, the stent was removed, and the patient was discharged by hospital day 24 . At 8 weeks follow-up, the patient developed symptoms of dysphagia due to an esophageal stricture. The patient underwent endoscopic balloon dilation of the stricture. At 3 month follow-up, the patient was tolerating a diet and gaining weight with no signs of dysphagia. Conclusion: A multi-modal minimally invasive approach to treating Boerhaave's syndrome is feasible. Using a combination of laparoscopic, thoracoscopic, and endoscopic approaches can potentially mitigate the morbidity and mortality of open esophageal surgery. Further studies need to be conducted to evaluate and potentially standardize approaches to treating Boerhaave's syndrome incorporating minimally invasive techniques.

\section{P015}

\section{Intraoperative Findings and the Diagnosis of Complicated Appendicitis. It is not What You Think}

Luis R Taveras, MD, Omar Harirah, BS, Dang-Huy Do, BA, Folarin M Adeyemi, BS, Maryanne L Pickett, MD, Holly B Cunningham, MD, Jonathan B Imran, MD, Tarik D Madni, MD, MBA, Meaghan M Colletti, John Kubasiak, MD, Michael W Cripps, MD, MSCS University of Texas Southwestern

Introduction: The objective of this study is to evaluate the relationship between intraoperative (IO) findings and the diagnosis of complicated appendicitis (CA). Postoperative management of these patients is highly dependent on the operative findings. Furthermore, IO assessment is a strong prognostic predictor of clinical outcomes.

Methods: A multicenter cross-sectional study surveyed all surgeons performing emergent laparoscopic appendectomies from September 2017 to September 2018. The survey collected information regarding disease severity, case complexity, and IO findings. General demographics, preoperative parameters, operative details, and histopathological (HP) descriptions were collected for all cases. Descriptive statistics were compared using Chi square and t-test where appropriate. Significance was set at $\mathrm{p}<0.05$ with a two-tailed distribution. Predictors of a diagnosis of CA were evaluated using a multivariable regression model.

Results: Twelve surgeons completed surveys for 263 patients, of which $57.9 \%$ were male and median age was 33 years old (IQR 24.5-43). Respondents rated case difficulty, on a Likert-type scale, with a median score of 2 out of 5 (IQR 1-3). Conversion to open rate was $0.004 \%$. The most common IO finding was hyperemia (45.63\%). Median OR time was 49 (IQR 39-64.5) minutes. An IO diagnosis of CA was made in $12.55 \%$ of cases, while $4.56 \%$ were reported as complicated by HP. IO diagnosis has a sensitivity of $75 \%$ (CI 95\% 50.9\%, $91.34 \%$ ), specificity of $81.59 \%$ (CI 95\% $76.08 \%, 86.29 \%$ ) and accuracy of $81.08 \%$ (CI 95\% $75.77,85.66)$.

Cohorts were divided by HP diagnosis. Those with CA had higher creatinine $(0.86$ vs 0.77 , $\mathrm{p}=0.031)$, higher heart rates (96.76 vs 85.32, $\mathrm{p}=0.007)$, increased rate of appendicolith on imaging $(55.56 \%$ vs $31.14 \%, \mathrm{p}=0.034)$ and larger appendix diameter (millimeters) on imaging ( 14.11 vs $11.38, \mathrm{p}<0.001$ )

Operative findings predictive of IO diagnosis were abscess $(33.312,2.270-488.879$, $\mathrm{p}=0.011)$, necrotic appendiceal wall (OR 13.71,95\% CI 2.148-87.497, p = 0.006) and purulent fluid (OR 7.703, 1.902-31.204, $\mathrm{p}=0.004)$. While the findings that correlated with a HP diagnosis were gross perforation (OR 14.381, 95\% CI 3.232-63.992, p < 0.001) and areas of necrosis (OR 5.36, 95\% CI 1.249-23.039, $\mathrm{p}=0.02$ ), neither presence of purulent fluid or abscess was predictive for HP diagnosis.

Conclusion: Intraoperative findings of abscess and purulent fluid have a significant weight in the IO diagnosis of CA but do not correlate with a HP diagnosis. These findings start to explain the dichotomy between IO and HP diagnosis.

\section{P016}

\section{The Impact of a Dedicated Emergency Surgery and Trauma Team on Appendicitis Outcomes}

Sabrina Cheok Hx, Dr, Serene Goh Sn, Woan Wui Lim, Anil Rao Dinkar, Kok Yang Tan, Jerry Goo Tt; Department of General Surgery, Khoo Teck Puat Hospital

Introduction: The Emergency Surgery and Trauma (ESAT) is a consultant led service to streamline emergency and elective workloads. As appendicectomy is one of the commonest emergency surgeries performed, we aim to compare outcomes of patients with appendicitis in the ESAT model as compared to the traditional on-call model.

Methods: We conducted a retrospective review of patients admitted to KTPH between two 6-month time periods: May-October 2014 (6 months pre-ESAT) vs January-June 2018 (post-ESAT) Patient demographics, diagnoses, and operations were compared. Efficiency, clinical outcomes and hospital bill savings were evaluated.

Results: There were 179 patients in the pre-ESAT period and 167 patients in the post-ESAT period. Patient demographics and comorbidities were comparable: mean age was 38.6 ? 15.8 , majority were males $(63.9 \%)$ and mean Charlson's comorbidity index was 0.08 . In the post-ESAT period patients had longer duration of symptoms 2.0 ? 1.8 days vs 1.8 ? 1.6 days $(\mathrm{p}=0.08)$. There were more perforated appendixes in the post-ESAT period $25.8 \%$ vs pre-ESAT period $20.1 \%(\mathrm{p}=0.221)$. Majority underwent laparoscopic appendicectomy $85 \%$ (pre-ESAT) vs $89.3 \%$. More patients in pre-ESAT period had conversion to open or open appendicectomy $(\mathrm{p}=0.05)$. The remainder had antibiotics or percutaneous drainage for appendiceal abscesses. There were lower surgical complications $7.8 \%$ vs $9.5 \%$ in post-ESAT period $(\mathrm{p}=0.02)$, shorter length of stay $2.5 ? 2.0$ days vs $2.7 ? 2.9(\mathrm{p}=0.07)$ and higher supervision rates during surgery in the post-ESAT period $(\mathrm{p}=0.01)$. The hospital bill sizes were comparable $(\mathrm{p}=0.475)$.

Conclusion: The ESAT service has improved outcomes of patients with appendicitis without increased costs.

\section{P017}

Does Peritoneal Irrigation Reduce Postoperative Intra-abdominal Abscess Rates After Laparoscopic Appendectomy?

Kevin Bain, $\mathrm{DO}^{1}$, Derek $\mathrm{Lim}^{1}$, Andrew Lelchuk, $\mathrm{DO}^{2}$, Vadim Meytes, $\mathrm{DO}^{3} ;{ }^{1} \mathrm{~N} Y U$ Langone Hospital - Brooklyn, ${ }^{2}$ Nova Southeastern University College of Osteopathic Medicine, ${ }^{3}$ Vassar Brothers Medical Center

Introduction: Intra-abdominal abscess (IAA) after laparoscopic appendectomy (LA) is a major cause of morbidity, can prolong hospitalization, and can increase hospital costs. Risk factors include diabetes mellitus, obesity, age, and perforated appendicitis.

Case Report: 15 year old female with 3 days of right-sided abdominal pain. A CT scan demonstrated perforated appendicitis, and the patient was taken for diagnostic laparoscopy About 500 milliliters of pus was evacuated from the peritoneal cavity. A perforated appendix was visualized and removed in the normal fashion. Irrigation with $3 \mathrm{~L}$ of normal saline (NS) was performed.

Postoperatively, the patient continued intravenous antibiotics. She persisted to have an uprising leukocytosis to $13.0 \mathrm{~K} / \mathrm{uL}$ on postoperative day 5. Further imaging demonstrated multiple intra-abdominal abscesses. Interventional radiology performed transgluteal drainage, with placement of a pigtail catheter. The patient was discharged home on antibiotics, with the catheter in place.

Discussion: Postoperative IAA after LA remains a debated topic. Studies have shown as high as a $20 \%$ abscess formation rate status post appendectomy for perforated appendicitis. Debate still remains between irrigation versus suction alone. The theory behind irrigation is dilution of bacterial concentration. Others believe irrigation actually spreads contamination, and dilutes immune system mediators.

In 2008, Hussain et al. described a prospective study of 283 patients with acute appendicitis. Their technique to reduce postoperative IAA included irrigating all four quadrants of the peritoneal cavity with $3 \mathrm{~L}$ of NS. No patients developed postoperative IAA, and they concluded that copious irrigation and drainage significantly decreased the rate of postoperative IAA.

In 2015, Cho et al. performed a retrospective cohort study to examine risk factors for postoperative IAA after LA, and concluded the exact opposite. They analyzed 1,817 LA and the only difference was that "peritoneal irrigation was performed significantly more often in the IAA group than in the non-IAA group $(\mathrm{p}<0.001)$." They concluded that peritoneal irrigation increased the incidence of postoperative IAA

More recently, in 2016 Snow et al. published the first prospective, randomized controlled trial (RCT) in adults comparing irrigation versus suction alone during LA. In 81 patients, they found "the rate of IAA was equivalent between groups treated with peritoneal irrigation and suction alone." This conclusion coincides with the only other prospectively conducted RCT on this topic, which was conducted in children.

Conclusion: There remains to be limited high-quality prospective studies to compare peritoneal irrigation to suction alone for preventing IAA after LA. 
P019

\section{Case Report: An Unusual Case of Acute Appendicitis - Cecal Endometriosis}

Romina Deldar, $\mathrm{MD}^{1}$, Chaitanya Vadlamudi, MD ${ }^{1}$, Gao L Chen, $\mathrm{MD}^{2} ;{ }^{1}$ Georgetown University Hospital, ${ }^{2}$ Kaiser Permanente, MidAtlantic States

Introduction: Acute appendicitis can arise from many etiologies. We present a case of acute appendicitis that was radiographically suspicious for appendiceal mucocele. However, final pathology revealed luminal obstruction secondary to cecal endometriosis. Cecal endometriosis is exceedingly rare, and only a few case reports describe this as a cause of appendicitis.

Methods and Procedure: A healthy 33-year-old woman with no prior gynecologic history presented on day five of her menstrual cycle with 48-hours of right lower quadrant abdominal pain, nausea, emesis, and leukocytosis of $13,900 / \mathrm{mm}^{3}$. Computed tomography (CT) of the abdomen revealed a 10-centimeter, distended and fluid-filled appendix, suspicious for mucocele (Figure 1). As clinical and radiographic evidence was consistent with acute appendicitis, the patient was taken for laparoscopic appendectomy. Intraoperatively, the appendix was noted to be massively dilated and associated with a firm cecal mass just beyond the appendiceal base. These findings raised suspicion for appendiceal mucinous neoplasm, therefore the operation was converted to open. A partial cecectomy was performed in addition to appendectomy. The appendix, cecal base, and mesoappendix were removed en bloc with no spillage. No mucinous deposits or other abnormalities were identified. The patient tolerated the procedure well and was discharged home on post-operative day one.

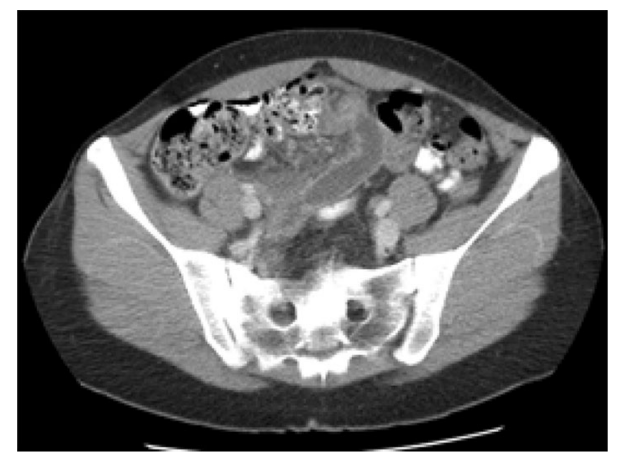

Figure 1. CT axial view showing dilated fluid-filled appendix

Results: Three independent pathologists reviewed the specimens and found no evidence of an appendiceal mucinous neoplasm. Fluid within the appendiceal lumen did not stain positive for mucin. The specimen was consistent with acute appendicitis without evidence of malignancy. Interestingly, the cecum at the appendiceal base was noted to contain endometrial glands with surrounding stromal tissue in the muscularis propria, consistent with endometriosis (Figure 2). During menses, increased inflammation and tissue edema of the endometrial tissue positioned at the appendiceal base may have led to luminal obstruction and subsequent acute appendicitis. The patient had no other symptoms of endometriosis.

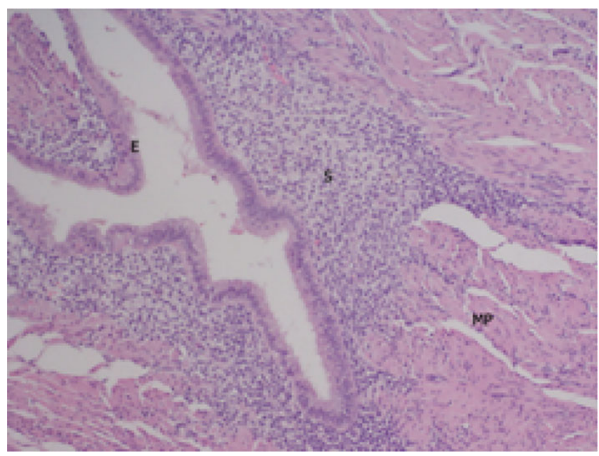

Figure 2. Focus of endometriosis in the muscularis propria (MP) of the cecum. Endometrial gland (E) and surrounding stromal tissue (S). Hematoxylin and eosin stain, $\times 100$. Conclusion: Cystic dilatation of the appendix can result from luminal obstruction secondary to a wide spectrum of benign or neoplastic causes. Clinically suspicious appendiceal mucocele should be resected intact to prevent mucinous deposits from seeding the abdomen and causing pseudomyxoma peritonei. Pathologic examination can reveal an unexpected diagnosis. This case simultaneously highlights an unusual presentation of endometriosis and an extraordinarily rare etiology of acute appendicitis.

\section{P020}

Case of Intussusception after Roux-en-Y gastric Bypass

Ahmed Abdelhady, MD, FACS, MRCSeng, MRCSglas, MSC, Mohamed Elkady, FRCS, Ire, Ghaleb Aboalsamh, MBBS, SBGS, MSC; National Guard Health Affairs Saudi Arabia

The patient was a 46 year-old female who presented to the emergency room with a sudden severe abdominal pain as well as nausea and vomiting that began the day before after a meal. She is 3 years post retrocolic RYGBP. On examination, the patient was well nourished, afebrile, and with normal vital signs. She was in moderate distress with pain out of proportion to the physical examination. Her abdomen was soft, and there were no signs of peritoneal irritation or distension. Laboratory data were unremarkable. Computed tomography scan revealed a long segment obstructive retrograde small bowel intussusception adjacent to the jejuno-jejunal anastamosis and associated with complete proximal small bowel obestruction. The patient was immediately taken to the operating room.

\section{P021}

Is The Risk of Venous Thromboembolic Event (VTE) Higher In Emergency General Surgery? Results From The National Surgical Quality Improvement Program (NSQIP)

Samuel W Ross, MD, MPH ${ }^{1}$, John C Kubasiak, $\mathrm{MD}^{2}$, Lindsey $\mathrm{P}$ Mossler, $\mathrm{MD}^{3}$, Luis R Taveras, $\mathrm{MD}^{2}$, Thomas H Shoultz, $\mathrm{MD}^{2}$, Herbert A Phelan, $\mathrm{MD}^{2}$, Michael W Cripps, $\mathrm{MD}^{2}$; ${ }^{1}$ Carolinas Medical Center, ${ }^{2}$ University of Texas Southwestern Medical Center, ${ }^{3}$ University of Indiana School of Medicine

Introduction: Trauma patients have an increased risk of VTE partly due to increased inflammation, and, therefore, are recommended to have increased VTE chemoprophylaxis dosing. A similar inflammatory physiology occurs in the patients with Emergency General Surgery (EGS) operative pathology, however, it is not well studied if this process leads to an increased VTE risk in this population. We hypothesized that EGS cases have a higher risk of VTE than their elective counterparts.

Methods: The American College of Surgeons NSQIP database was queried from 2005-2016 for all open and laparoscopic cholecystectomies (OC and LC), ventral hernia repairs (OVHR and LVHR), and partial colectomies (OPC and LPC) to give a sample of commonly encountered EGS procedures that have elective counterparts. Elective surgeries were then compared to emergent ones using univariate statistics with VTE at 30 days being the primary outcome. A multivariate analysis controlling for age, gender, BMI, cancer, bleeding disorders, pregnancy, surgery type, and open status was then performed. Results: There were 604,537 surgeries over 12 years: 285,847 cholecystectomies $(12.7 \%$ OC vs. $87.3 \%$ LC); 158,500 VHR (79.8\% OVHR vs. $20.2 \%$ LVHR); and 160,190 partial colectomies (61.3\% OPC and $38.7 \%$ LPC). There were $4.607(0.8 \%)$ patients with DVT and $2,648(0.4 \%)$ with PE, and a total 6,624 (1.1\%) patients with VTE. Patients with emergent surgery were more likely to be younger, male, lower BMI, and have higher white blood counts and lower albumin $(\mathrm{p}<0.001$ for all). Patient outcomes by emergent status are displayed in Table 1 . When VTE risk was examined by open versus laparoscopic surgery, as expected, VTE risk increased with invasiveness $(2.0 \mathrm{vs} 0.3 \%$ for all; $2.3 \mathrm{vs}$. $0.3 \%$ for cholecystectomy, 1.0 vs. $0.4 \%$ for VHR, and 3.2 vs. $1.2 \%$ for partial colectomy; $\mathrm{p}<0.001$ for all). On multivariate analysis, emergent surgery was an independent predictor of VTE (OR 1.8; 95\% CI 1.4-2.2), with almost twice the odds of VTE as elective surgery. As was open surgery $(2.7,2.0-3.5)$ with almost three times the risk of VTE when controlling for emergent status. Additionally, more extensive surgeries had higher VTE odds when compared to cholecystectomy; VHR $(1.8,1.4-2.4)$ and partial colectomy $(3.1,2.4-4.0)$.

Conclusion: Emergent surgery was an independent predictor of VTE when compared to their elective counterparts. Given this increased risk, higher dosing and earlier VTE chemoprophylaxis should be considered in emergent and more extensive operations, especially when performed open, to reduce the risk of potentially lethal VTE.

\begin{tabular}{|c|c|c|c|}
\hline & $\begin{array}{c}\text { Emergent } \\
n=183,099(\%)^{*}\end{array}$ & $\begin{array}{c}\text { Elective } \\
n=421,438(\%)^{*}\end{array}$ & p value \\
\hline Laparoscopic & $98,369(53.7)$ & $247,862(58.8)$ & \\
\hline Operative time (minutes) & $108.5 \pm 77.3$ & $113.6 \pm 96.6$ & $<0.0001$ \\
\hline $\begin{array}{l}\text { Hospital Length of Stay } \\
\text { (days) }\end{array}$ & $8.1 \pm 10.4$ & $2.9 \pm 5.8$ & $<0.0001$ \\
\hline Wound Complications & $9,758(5.3)$ & $15,121(3.6)$ & $<0.0001$ \\
\hline Minor Complications & $37,068(40.3)$ & $39,960(20.7)$ & $<0.0001$ \\
\hline Major Complications & $25,812(14.1)$ & $15,517(3.7)$ & $<0.0001$ \\
\hline DVT & $2,562(1.4)$ & $2,045(0.5)$ & $<0.0001$ \\
\hline PE & $1190(0.7)$ & $1458(0.4)$ & $<0.0001$ \\
\hline VTE & 3,443 (1.9) & $3,181(0.8)$ & $<0.0001$ \\
\hline Days to DVT from surgery & $10.7 \pm 7.7$ & $12.1 \pm 7.8$ & $<0.0001$ \\
\hline Days to PE from surgery & $10.4 \pm 7.9$ & $10.2 \pm 7.8$ & 0.427 \\
\hline 30-day Mortality & $6,601(3.6)$ & $1,842(0.4)$ & $<0.0001$ \\
\hline
\end{tabular}




\section{P022}

Peritoneal Adhesions Seen During Laparoscopic Appendicectomy Post Prior Pelvic Floor Surgery

Pravish Rai Sookha, MD, MS, PhD; Welkin hospital, Clinic Muller, Clinic du Nord

During the last 226 adult female appendicectomies, 55 were found to have underwent prior open pelvis floor surgeries. The most common surgeries included LSCS, ovarian cystectomy, salpingo-oophrectomy and endometriosis. Due to prior open surgery scars, all the surgeries were started with the Hasson's method. Out of the 55 cases peritoneal adhesions affecting the surgery were seen in 28 cases, while in the rest either very minimal or no adhesions were seen. The pelvic surgeries were further divided in 2 classes - LSCS (20 cases) - The rest 35 cases. LSCS had 6 cases of adhesions of out 20 surgeries whereas in the other group 22 cases had adhesions out of 35 surgeries.

Results: In our cohort $51 \%$ of cases after open pelvic surgery presented with adhesions. All cases of laparoscopic appendicectomy were completed laparoscopically after careful dissection of adhesions. Conclusions: Adhesions due to prior surgery are not a contraindication for laparoscopic surgery. Adhesions after pelvic surgeries are more common after emergency surgeries than after LSCS.

\section{P023}

\section{Laparoscopic Repair with ERAS Pathway for Perforated} Duodenal Ulcer

Yoshihito Shinohara, MD; Teinekeijinkai Medical Center

Background: Several studies have shown that, when compared with a conventional perioperative care, Enhanced recovery after surgery (ERAS) pathway was associated with a reduction in the length of hospital stay and rates of complication. Whether such a program is a feasible and beneficial in the setting of emergency ulcer surgery remains unknown.

Methods: This single-center, retrospective case-series study. From January 2005 to April 2017, 98 patients with Perforated Duodenal Ulcer (PDU) underwent laparoscopic repair with simple closure with omentopexy or Graham patch repair. Patients who underwent open surgical repair or standard care were excluded. The Primary outcomes were the length of hospital stay (LOS), morbidity and mortality within 30 days after surgery. The secondary outcomes measured included various functional recovery parameter as individual components of the ERAS protocol. Data were collected retrospectively to assess the efficacy of the ERAS protocol and included all patients undergoing LR. All data analysis.

Results: A total of 110 patients were diagnosed with PDU during the study period. 98 patients were initially performed laparoscopic approach. 88 patients $(90 \%)$ performed laparoscopic repair (LR). LR included laparoscopic simple suture repair with omentopexy $(n=73)$ and Graham omental patch $(\mathrm{n}=15) .82$ patients were adapted ERAS pathway. The reasons for non-adaptation to 6 patients $(6 \%)$, the required vasopressor for intraoperative shock $(n=1)$, the required ventilator for respiratory failure $(n=1)$, elderly patient with large perforation $(n=1)$, delirium immediately after operation $(n=2)$, and ileus $(n=1)$. Median of LOS was 4 days (quartile range: $4-5$ ). There was no 30-d mortality and readmission. There was one case above grade 3 of the Clavien-Dindo classification because of leakage with percutaneous drainage. There was no reoperation. Median of solid diet was 2 days (quartile range: 2 -2). The overall compliance of ERAS protocol was $61 \%$ (50 cases). The variances show in Table 4 which of the prolong of NPO $(n=15: N G$ tube $n=5$, pain and abdominal distension $n=5$, suspect of leakage $n=4$ ), prolong of LOS $(n=13$ : delayed diet $n=4$, SSI $n=1$, patients hope $n=1$, no known reasons $n=7)$, and delayed drain removing $(n=2$ : suspect of abscess $\mathrm{n}=2$ ).

Conclusions: In conclusion, ERAS pathways seem safe and feasible for patients undergoing LGPR for PDU. In addition, ERSA pathway with LGPR for PDU make it possible to improve the early oral intake and discharge. It may be possible to early return to society for patients and to reduce health care costs. 


\section{P024}

A Review of Management of Hernias Containing the Vermiform Appendix

Kevin Bain, $\mathrm{DO}^{1}$, Nicholas Morin, $\mathrm{DO}^{1}$, Vadim Meytes, $\mathrm{DO}^{2}$, Michael Nicoara, DO ${ }^{1}$, Galina Glinik, MD ${ }^{1} ;{ }^{1} \mathrm{NYU}$ Langone Hospital - Brooklyn, ${ }^{2}$ Vassar Brothers Medical Center

Introduction: Hernias involving the appendix are unusual and are often found during surgical exploration. Only approximately $1 \%$ of all hernias contain a portion of the vermiform appendix. The rarity of these hernias makes for a difficult discussion on standard of management.

Case report: 33 year old male who presented to the ED with right lower quadrant abdominal pain for ten days, and a CT scan demonstrated acute perforated appendicitis.

During diagnostic laparoscopy, the appendix was visualized herniating through a previously undescribed orifice in the lateral right iliac fossa. The orifice was lateral to the femoral triangle, in the so called "triangle of pain," which is a "V" shaped area bounded by the iliopubic tract, testicular vessels, and peritoneal fold.

Using laparoscopic technique, the appendix was reduced, and an endoscopic stapler was used to remove the appendix. The hernia was primarily repaired, and surgery was completed in the usual manner. Discussion: The most commonly described hernia which contains the appendix is the Amyand hernia. This occurs when the appendix becomes trapped within an inguinal hernia sac. The incidence ranges from $0.19 \%$ to $1.7 \%$. An Amyand hernia is three times more likely to be seen in childhood due to a persistently patent processus vaginalis. The next most commonly described is De Garengeot's hernia. This occurs when the appendix is trapped within a femoral hernia sac, and occurs in approximately $1 \%$ of all femoral hernia cases. It differs from the Amyand hernia in that it is more commonly found in females, and follows a bimodal age distribution.

The mechanism by which appendicitis develops within hernias is not fully understood, and is extremely rare with rates ranging from $0.07-0.13 \%$. In these instances, perforation only occurs in approximately $0.1 \%$ of the cases. When perforation does occur, there is increased mortality due to the spread of severe peritoneal sepsis.

Therapeutic strategies for these hernias depend on the condition of the appendix. The Losanoff and Basson classification is a management strategy for dealing with Amyand hernias. The Rikki modification adds to this in dealing with incisional hernias. The general principle shared between the two is that for a non-inflamed appendix, the patient should have hernia repair without appendectomy. When an acutely inflamed appendix is encountered, appendectomy should be performed and the hernia should be primarily repaired. These principles should be applied to all hernias containing the appendix in order to avoid postoperative complications.

\section{P025}

Does Time of Day Matter for Acute Cholecystectomy in an Acute Care Surgery Model?

Ilya Rakitin, MD, Cletus Stanton, MD, Michael Dix, BA, Nathan Ziegler, BA, Jerry Stassinopoulos, MD, MBA, Jeffery Johnson, MD, Nadia Obeid, MD, Nathan Schmoekel, DO; Henry Ford Hospital

Introduction: Prompt cholecystectomy for acute cholecystitis is well accepted; however, whether this warrants urgent nocturnal surgery remains a matter of debate. Differences in available resources, multitasking, and sleep hygiene may affect performance. This could impact conversion rates to open surgery, length of operation, hospital length of stay, and complications such as bile duct injury, blood loss, and major organ injury. Some evidence supports improved outcomes in patients who can avoid after-hour cholecystectomy. We aimed to determine if outcomes changed based on time of day for laparoscopic cholecystectomy. Our null hypothesis is that night time cholecystectomy is associated with worse outcomes.

Methods and Procedures: Retrospective analysis of 486 patients aged $\geq 18$ years who underwent cholecystectomy in a large, urban level 1 tertiary care center with a longstanding acute care surgery model. Demographics, procedural information, time of day of surgery, and postoperative outcomes were collected and stratified over a 4-year period from 2013-2017. Sixty five percent of the patients were female with an average age of 49.6 years and an average BMI of $32.7 \mathrm{~kg} / \mathrm{m}^{2}$. A total of $230(47 \%)$ patients underwent laparoscopic cholecystectomy for acute cholecystitis. Almost half (45\%) underwent nighttime operation defined as procedure start time between the hours of 19:00 to 07:00.

Results: Analysis of the 230 patients with acute cholecystitis revealed an overall conversion rate to open of $14 \%(\mathrm{n}=32)$, bile leak (duct of Luschka or cystic) $3.5 \%(n=8), 30$-day readmission rate of $7.4 \%$ $(n=17)$, and length of stay of 3.9 days. Subgroup analysis of patients having nighttime cholecystectomy for cholecystitis $(\mathrm{N}=103)$ revealed $6.1 \%$ conversion to open, $0.9 \%(n=2)$ bile leak, $2.6 \%$ $(n=6) 30$-day readmission rate, and 3.43 days length of stay. Other outcomes including mortality, cardiac events, pneumonia, stroke, PE, blood loss requiring blood transfusion were not statistically significant. One patient had a common bile duct injury in the daytime group that required conversion to open and hepaticojejunostomy.

Conclusions: Despite prior evidence that favors delaying cholecystectomy until daytime to avoid complications, our study did not support this. We found no difference in outcomes between daytime and nighttime cholecystectomy and conclude that cholecystectomy can safely be performed during any time of the day with similar complication rates. This may reflect the volume of experience in after-hours acute care surgery at this regional referral center. 


\section{P026}

Is There a "weekend effect" in Patients with Necrotizing Fasciitis: A Nationwide Analysis

Heather Peluso, $\mathrm{DO}^{1}$, Wesley B Jones, MD ${ }^{1}$, Marwan S Abougergi, $\mathrm{MD}^{2}$; ${ }^{1}$ Greenville Health System, ${ }^{2}$ Catalyst Medical Consulting, LLC

Introduction: We sought to determine the impact of day of admission on treatment outcomes and resource utilization among patients with necrotizing fasciitis in the United States.

Methods: This is a retrospective cohort study using the 2014 National Inpatient Sample. Discharges were included if they had a principal diagnosis of necrotizing fasciitis. Patients $<18$ years and non-urgent admissions were excluded. The primary outcome was inpatient mortality. Secondary outcomes were morbidity (sepsis and prolonged mechanical ventilation (PMV)), treatment metrics (time to surgery) and resource utilization (length of stay (LOS) and total hospitalization costs and charges). The following confounders were adjusted for using multivariate regression analysis: patient's sex, age, race, Charlson comorbidity index, median income in patient's zip code, hospital urban location, region, teaching status and bedsize.

Results: 3,590 patients were included in the study, $24 \%$ of whom were admitted on weekends. The mean age was 53 (52 - 54) years and $42 \%$ were Female. Weekend admission was not a predictor of mortality (adjusted odds ratio (aOR):1.29 (0.38-4.33), $\mathrm{p}=0.68)$. Rates of septic shock (aOR: $1.03(0.51-2.08), \mathrm{p}=0.93)$ and PMV (aOR: 0.87, $(0.39-1.95), p=0.74)$ were similar for both groups. Time to surgery (adjusted mean difference (amDiff):1.15 (0.77-1.71) days, $\mathrm{p}=0.50$ ) was similar for patients admitted on weekdays and weekends. Furthermore, LOS (amDiff:-1.77 (-4.04-0.50) days, $\mathrm{p}=0.13)$, total hospitalization costs $(\$-4,788 \quad(\$-11,833-\$ 2,256), \mathrm{p}=0.18)$, and charges (amDiff:\$-15,276 $(\$-44,906-\$ 14,353), \mathrm{p}=0.31)$ were also similar for both groups.

Conclusion: In-hospital mortality, morbidity, treatment metrics, and resource utilization were not different between patients admitted on weekends and on weekdays. Therefore, day of admission does not affect treatment outcomes or resource utilization among patients with necrotizing fasciitis.

Table 1: Primary and Secondary Outcomes.

\begin{tabular}{|c|c|c|}
\hline & $\begin{array}{l}\text { Adjusted Odds ratio } \\
\text { ( } 95 \% \text { confidence interval) }\end{array}$ & $\begin{array}{l}P \\
\text { value }\end{array}$ \\
\hline In-hospital mortality & $1.29(0.38-4.33)$ & 0.65 \\
\hline \multicolumn{3}{|l|}{ Morbidity } \\
\hline Septic Shock & $1.03(0.51-2.08)$ & 0.93 \\
\hline \multirow[t]{2}{*}{ PMV } & $0.87(0.39-1.95)$ & 0.74 \\
\hline & $\begin{array}{l}\text { Adjusted Mean Difference * } \\
\text { (95\% confidence interval) }\end{array}$ & \\
\hline \multicolumn{3}{|l|}{ Treatment metrics } \\
\hline $\begin{array}{l}\text { Time from admission } \\
\text { to surgery }\end{array}$ & 1.15 (0.77-1.71) days & 0.50 \\
\hline \multicolumn{3}{|l|}{ Resource Utilization } \\
\hline LOS & $-1.77(-4.04-0.50)$ days & 0.13 \\
\hline Total costs & $\$-4,788(\$-11,833-\$ 2,256)$ & 0.18 \\
\hline Total charges & $\$-15,276(\$-44,906-\$ 14,353)$ & 0.31 \\
\hline
\end{tabular}




\section{P027}

Small Bowel Obstruction and Perforation from Ingested Fish Bone Treated with Laparoscopic Bowel Resection

Justin D Sargent, DO, Steven P Shikiar, MD, John Davis, MD; Hackensack Meridian Health Palisades

Introduction: There is a wide range of variability in presentation for patients whom ingest foreign bodies (IFB). Most commonly patients are asymptomatic as the foreign body passes through the gastrointestinal tract without any complications. Patients may also present with obstruction, hemorrhage or perforation and subsequent sepsis of any part of the alimentary tract from esophagus to anus. When operative intervention is required traditionally exploratory laparotomy is performed. Here we will present a case of perforating and obstructing IFB treated successfully with laparoscopic bowel resection with intracorporeal anastomosis.

Case Description: A 67 year-old male, without previous abdominal complaints, presented to the ER with acute onset severe 10/10 sharp, constant, diffuse abdominal pain with associated nausea that began a few hours prior to arrival. Patient had never had any similar symtpoms. No past abdominal surgeries. Palpation of the abdomen showed diffuse tenderness with rebound tenderness in the lower abdomen. Workup was significant for a leukocytosis of 18,300 and CT imaging showing a focal area of small bowel perforation in the RLQ associated with a linear, radiopaque object in the lumen of the bowel at this level with associated bowel obstruction proximally. Further questioning revealed patient had eaten fish 2 days prior without any recollection of swallowing any bones. NG tube was placed and the patient was placed on empirical IV antibiotics and taken to the operating room.

Diagnostic laparoscopy was performed. A loop of small bowel was identified with a small perforation and associated induration of adjacent mesentery. A thin linear object was identified to be protruding from the lumen. Two healthy areas of adjacent bowel were identified. The mesentery to the bowel was divided. The proximal and distal areas of resection were divided with an endo GIA stapler. An intracorporeal bowel anastomosis was performed with the common channel created with an endo GIA stapler. The common enterotomy was closed with 3-0 vicryl in a running fashion. The specimen was removed in through a Pfannenstiel incision. The specimen was then opened on the back table and visualization of the bowel reveled a small linear fish bone with associated ulceration of the mucosa. Post-operatively the patient had return of bowel function on POD\#2, diet was advanced discharged on POD\#3.

Conclusion: Laparoscopic bowel resection with intracorporeal anastomosis can be a viable option in patients requiring small bowel resection for perforating ingested foreign bodies.
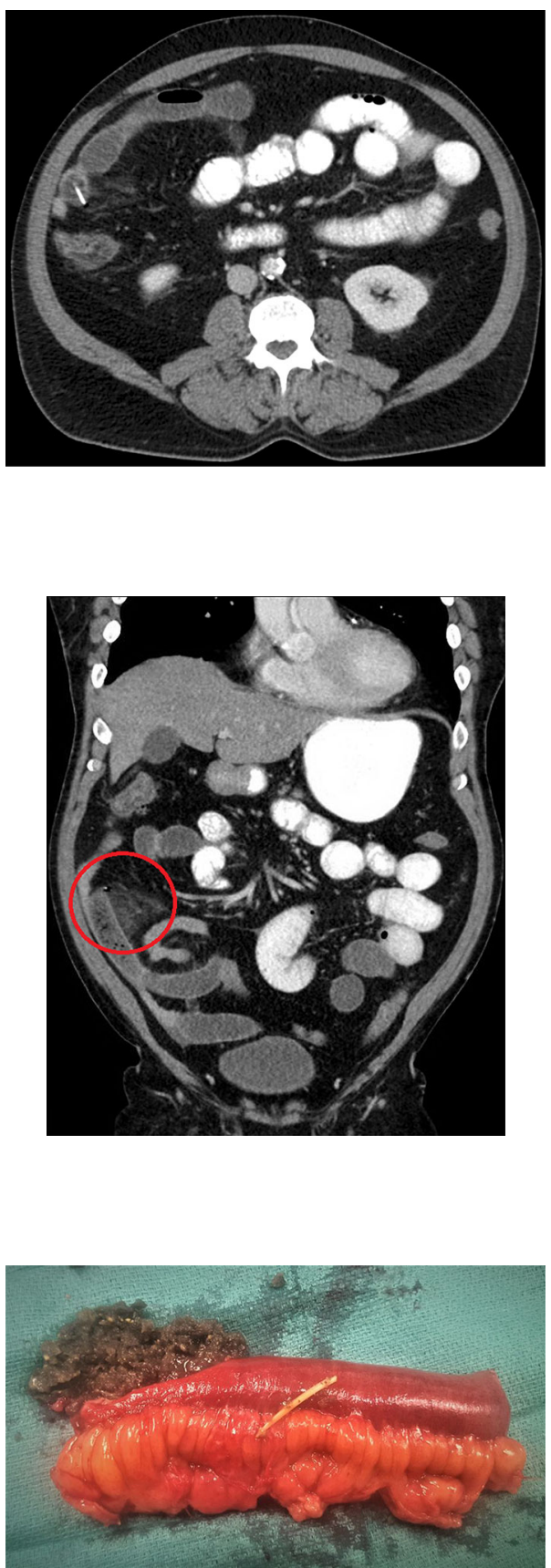


\section{P028}

Appendiceal Intussusception due to a Mucinous Neoplasm: a Crossroads of Two Rare Conditions

Alyssa Mowrer, MD, Clayton Theleman, MD, Thomas Rossi, MD, FACS; University of Illinois College of Medicine at Peoria

Introduction: Neoplasms of the appendix occur in less than $1 \%$ of all appendectomy specimens. Mucoceles of the appendix are mucin filled cystic lesions that are classified as either benign, a cystadenoma, or malignant, a cystadenocarcinoma. Rupture of mucoceles of the appendix is associated with peritoneal tumor implantation and mucinous ascites; a condition known as pseudomyxoma peritonei that carries a median 10-year survival rate of only $63 \%$. Although the medical approach for mucinous neoplasms has been somewhat controversial, the standard of care remains prompt surgical resection.

Case Description: A 49-year-old female presented with 3 days of periumbilical abdominal pain. She described nausea with emesis, decreased bowel function, and an acute weight loss of $10 \mathrm{lb}$ since onset of symptoms. A CT scan demonstrated appendiceal intussusception.

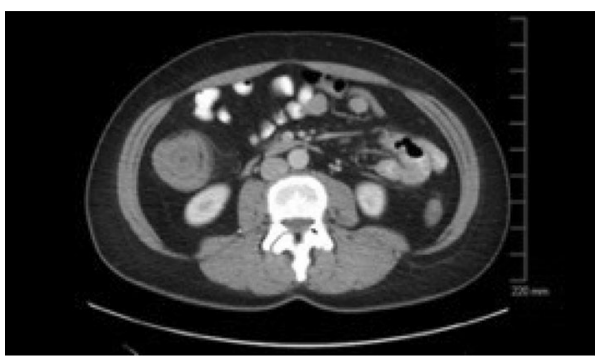

Figure 1: Dilated, fluid filled, blind ending structure measuring $4 \mathrm{~cm}$ in diameter consistent with the appendix.

The patient was then taken to the operating room for resection. Due to the high clinical suspicion for an appendiceal tumor and significant risk of pseudomyxoma peritonei with rupture of an appendiceal mucocele, an open approach to a right hemicolectomy was planned. A firm mass palpated within the cecum at the base of the appendix was found to be inspissated mucin from the tumor. The specimen was removed intact.

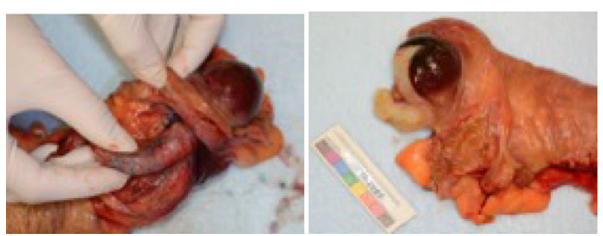

Figure 2: Surgical specimen including appendix and right colon with intussusception of appendiceal base into cecum.

Final pathology was consistent with a low grade appendiceal mucinous neoplasm that extended through the muscularis propria and into peri-appendicular tissue.

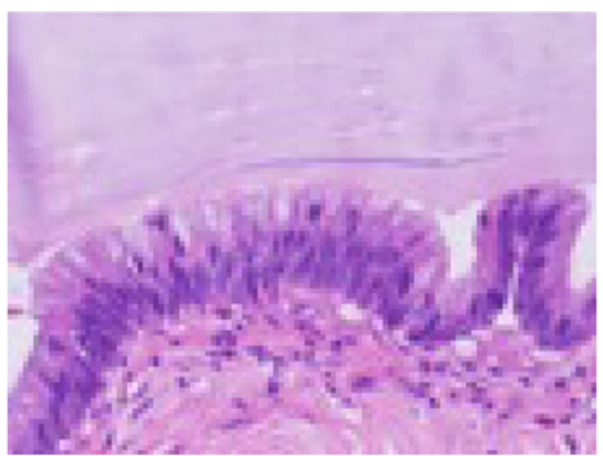

Figure 3: Tumor with mild cytologic atypia. Cellular mucin goblets extruding into appendiceal lumen. Histologic grade 1 (well differentiated) and stage T3NOMO.

Discussion: This case of a suspected mucocele of the appendix was complicated by the presentation of appendiceal intussusception. With a devastating possible complication of rupture of the appendiceal mucocele seeding the peritoneum and progressing to pseudomyxoma peritonei, we elected to perform an open approach. Although there have been case reports discussing endoscopic reduction of an appendiceal intussusception, this patient presented with partial obstructive symptoms which contributed to the decision for first line intervention with operative management.

At this point, there is limited information in the literature involving the extremely rare combination of an appendiceal mucocele with the clinical presentation of an obstruction at an intussuscepted appendix. We hope to contribute to the growing body of literature to provide the general surgeon with recommended guidelines when presented with this unusual clinical scenario. 
P029

Gastrointestinal Stromal Tumor of the Appendix with associated Mucocele Mimicking Acute Perforated Appendicitis Treated with Laparoscopic Appendectomy

Elizabeth A Verrico, DO ${ }^{1}$, Justin Sargent, $\mathrm{DO}^{1}$, Lindsay Tse, $\mathrm{DO}^{2}$, German Costa, $\mathrm{MD}^{1}$, Steven Shikiar, $\mathrm{MD}^{1}$; ${ }^{1}$ Hackensack Meridian Health Palisades Medical Center, ${ }^{2}$ Houston Methodist Hospital

Gastrointestinal stromal tumors (GIST) are the most common mesenchymal neoplasms of the gastrointestinal tract and most often present in the stomach and small bowel although they may also present in the large bowel or esophagus. GIST of the vermiform appendix, however, is extremely rare, and represents $<1 \%$ of all GIST

This is a case of a 54-year-old male who presented with acute right lower quadrant abdominal pain with associated nausea. His medical history was only significant for hypertension and his surgical history included a laparoscopic right inguinal hernia repair. On physical exam he was tender in the right lower quadrant and suprapubic region with mild localized guarding without generalized peritoneal signs. Laboratory results were only significant for a mildly elevated white blood cell count. CT was performed and demonstrated an inflamed appendix with periappendiceal inflammatory change and a $2.3 \times 4 \mathrm{~cm}$ rim enhancing fluid collection consistent with perforated appendicitis. The patient was given a course of intravenous antibiotics and taken to the operating room for laparoscopic appendectomy Significan inflammation was noted in the right lowe inflammation was Pathology revealed focal spindle cell proliferation at the distal portion of the appendix positive for CD 117 and CD 34, consistent with GIST, and focal mild luminal distention with luminal mucoid material and extravasation into the wall/periappendiceal tissue consistent with mucocele.

GIST of the vermiform appendix is infrequently reported in the literature with varying presentations from incidental diagnosis during surgery for another pathology or at autopsy, to signs and symptoms similar to acute appendicitis. Due to the variability of presentation and the uncommon pathological finding of GIST of the appendix, it is very difficult to diagnose in the preoperative period. Although rare, obstruction of the appendiceal lumen can lead to secondary inflammation and thus acute appendicitis-like symptoms. appendiceal lumen can lead to secondary inflammation and thus acute appendicitis-like symptoms.
Therefore, appendiceal GIST should be considered in patients presenting with acute right lower quadrant abdominal pain, especially if imaging findings are concerning for possible mass of the appendix. Appendectomy should be performed in order to obtain appropriate pathological examination including immunohistochemical staining and evaluation of malignant potential so that treatment can be tailored on an individual basis.

\section{P031}

The Strengths and Limitations of Image-Based Anatomic Severity for Acute Appendicitis in the Japanese Healthcare System

Masakazu Fujii ${ }^{1}$, Yusuke Watanabe, $\mathrm{MD}^{2}$, Chisato Ichimaru, $\mathrm{MD}^{1}$, Shintaro Takeuchi, MD ${ }^{1}$, Kiyotaka Imamura, MD $^{1}$, Kentaro Katou, $\mathrm{MD}^{1}$, Yoshihiro Kinoshita, MD ${ }^{1}$, Minoru Takada, $\mathrm{MD}^{1}$, Yoshiyasu Anbo, MD ${ }^{1}$, Fumitaka Nakamura, MD ${ }^{1} ;{ }^{1}$ Department of Surgery, Teine Keijinkai Medical Center, ${ }^{2}$ Department of Gastroenterological Surgery II, Hokkaido University Faculty of Medicine

Introduction: Validity evidence of the American Association for the Surgery of Trauma (AAST) grading system for appendicitis has been reported in the US and South America. We evaluated whether the preoperative AAST severity scores correlate with outcomes of appendicitis in the Japanese healthcare system and correspond with intraoperative findings in order to forecast case difficulties.

Methods and Procedures: Patients ( $\geq 18$ years) with a preoperative diagnosis of appendicitis during 2013-2017 in a major teaching hospital were reviewed. The image-based AAST (iAAST) grades were assigned based on CT findings (range 1-5, grade $\geq 3$ were defined as perforated appendicitis). Demographics, operative findings, and procedure types were collected. Outcomes including 30-day mortality, complications based on Clavien-Dindo categories, length of stay, and institutional costs were reviewed. Descriptive and univariate analyses were performed to compare iAAST grades with operative findings and clinical outcomes. The correlation of iAAST with intraoperative AAST grades was calculated using a kappa statistic. Malignant cases were excluded from data analysis.

Results: A total of 406 consecutivepatients with a median [IQR] age of 45 [32-63] (range 18-92) were analyzed (46\% female). The iAAST grade is as follows: Grade I (115, 28\%), Grade ? (46, $11 \%)$, Grade ?(144, 36\%), Grade ? $(58,14 \%)$,and Grade ? $(43,11 \%)$. Management included appendectomy $(\mathrm{n}=295,76 \%)$, interval appendectomy $(\mathrm{n}=36,9.0 \%)$, and conservative management with antibiotics $(n=63,15 \%)$. Operative management consisted of laparoscopic appendectomy $(L A ; n=319,93 \%)$, LA with partial cecectomy $(\mathrm{n}=13,3.8 \%)$, laparoscopic ileocecal resection $(\mathrm{n}=3,0.9 \%)$, and open surgery $(\mathrm{n}=8,2.3 \%)$.Among all patients, increased iAAST grade correlated with length of stay $(\mathrm{rs}=0.44$ $\mathrm{p}<0.01)$ and institutional cost $(\mathrm{rs}=0.30, \mathrm{p}<0.01)$ regardless of management pathways. Of 295 patients with initial operative management performed by surgeons at varying levels of experience [24 surgical trainees $(72 \%), 8$ attending surgeons $(28 \%)]$, the iAAST grade associated with operative time $(r s=0.33$ $\mathrm{p}<0.01$ ) but didn't correlate with procedure types. No 30-day mortality was observed, and overall complication rate was $10 \%$ (Clavien-Dindo $\geq 2$ ). From operative findings, $29 \%$ of the patients with iAAST grade $\leq 2(\mathrm{n}=121)$ were diagnosed with perforated appendicitis, and $48 \%$ with iAAST grade $3-5(\mathrm{n}=174)$ were diagnosed with non-perforated appendicitis (kappa coefficient $=0.22, \mathrm{p}<0.01$ ). Perforated appendicitis significantly increased operation time $(65[50-85]$ vs. $102[72-127], \mathrm{p}<0.01)$ and complication rate $(\mathrm{p}<0.01)$, regardless of level experience.

Conclusions: Although the iAAST grade predicts clinical outcomes in a Japanese population, the preoperative grading has some limitations to forecast intraoperative findings. Better estimations of intraoperative findings may help predict case difficulty to optimize the efficacy of surgical care in teaching hospitals.

\section{P032}

Sarcopenia Predicts Postoperative Mortality in Emergency Abdominal Surgery. The Effect is More Significant in Emergency Surgery Compared to Elective Surgery: Meta-analysis and Comparative Cohort Study of Literature

Sheik Rehman, Mr, Shahab Hajibandeh, Mr; Royal Bolton Hospital

Objectives: To investigate the effect of sarcopenia on postoperative mortality in patients undergoing emergency abdominal surgery and to compare postoperative mortality in patients with sarcopenia undergoing emergency abdominal surgery with those undergoing elective abdominal surgery.

Methods: A search of electronic information sources was conducted to identify all observational studies comparing sarcopenia with no sarcopenia in a) emergency abdominal surgery and b) elective abdominal surgery. We also identified the available cohort of patients in the literature with sarcopenia undergoing abdominal surgery and divided the entire cohort into two groups based on exposure to emergency surgery o elective surgery. The primary outcome measure of this study was postoperative 30-day mortality.

Results: Overall, 4 studies, enrolling a total of 734 patients, were eligible for the comparison in emergency setting and 16 studies, enrolling a total of 4590 patients, were eligible for the comparison in elective setting. Sarcopenia is associated with significantly higher risk of 30-day mortality (RR: $2.15, \mathrm{P}<0.0001$ ), 1-year mortality (RR: $1.97, \mathrm{P}<0.0001$ ), total complications (RR: $2.07, \mathrm{P}=0.0008$ ), and need for ICU admission mortality (RR: $1.97, \mathrm{P}<0.0001$ ), total complications (RR: $2.07, \mathrm{P}=0.0008$ ), and need for $\mathrm{ICU}$ admission
(RR: $1.38, \mathrm{P}=0.003$ ) and significantly longer length of ICU stay (MD: $2.26, \mathrm{P}=0.006$ ) and length of hospital stay (MD: 2.46, $\mathrm{P}<0.00001$ ) compared to no sarcopenia in patients undergoing emergency abdominal surgeries. Sarcopenia was also associated with significantly higher risk of 30-day mortality in patients undergoing elective abdominal surgery $(R R: 2.15, P=0.002)$. Emergency abdominal surgery in patients with sarcopenia was associated with significantly higher risk of 30-day mortality compared to elective surgery (OR: 12.00, $\mathrm{P}<0.00001)$

Conclusions: Sarcopenia is an independent predictor of postoperative mortality in emergency abdominal surgery. 
P033

Conversion from Laparoscopy to Laparotomy in Patients Undergoing Surgery for Small Bowel Obstruction: Does Prior History of SBO or Previous Abdominal Surgery Matter?

Remealle A How, MD ${ }^{1}$, Valerie G Sams, MD ${ }^{1}$, Christopher Corkins, MD ${ }^{1}$, John C Graybill, MD ${ }^{1}$, James Aden ${ }^{1}$, Martin D Zielinski, MD², Daniel C Cullinane, $\mathrm{MD}^{3}$, Kenji Inaba, $\mathrm{MD}^{4}$, Daniel D Yeh, $\mathrm{MD}^{5}$, Salina Wydo, MD 6 , David S Turay, $\mathrm{MD}^{7}$, Andrea Pakula, MD, $\mathrm{MPH}^{8}$, Therese M Duane, MD, MPH ${ }^{9}$, Jill Watras, MD ${ }^{10}$, Kenneth A Widom, $\mathrm{MD}^{11}$, John Cull, MD ${ }^{12}$, Carlos J Rodriguez, DO, MBA ${ }^{13}$, Eric A Toschlog, MD ${ }^{14}$, Mohamed D Ray-Zack, $\mathrm{MD}^{2}$, Matthew C Hernandez, MD ${ }^{2}$, Asad Choudhry, $\mathrm{MD}^{2}$, Richard Lesperance, $\mathrm{MD}^{1} ;{ }^{1}$ San Antonio Uniformed Services Health Education Consortium (SAMMC), ${ }^{2}$ Mayo Clinic, ${ }^{3}$ Marshfield Clinic, ${ }^{4}$ University of Southern California, ${ }^{5}$ University of Miami, ${ }^{6}$ Cooper University Hospital, ${ }^{7}$ Loma Linda University, ${ }^{8}$ Kern Medical Center, ${ }^{9}$ John Peter Smith Hospital, ${ }^{10}$ Inova Fairfax Hospital, ${ }^{11}$ Geisinger Medical Center, ${ }^{12}$ Greenville Memorial Hospital, ${ }^{13}$ Walter Reed National Military Medical Center, ${ }^{14}$ East Carolina University

Introduction: Laparoscopic adhesiolysis can be used for small bowel obstruction (SBO); however, conversion to laparotomy is frequent. In this study, we aimed to determine whether patient factors such as prior SBO or abdominal surgery were associated with an increased likelihood of conversion from laparoscopy (LS) to laparotomy (LT) in patients with SBO.

Methods and Procedures: We performed a post hoc analysis of the EAST SBO database and included patients who initially underwent a laparoscopic approach. Patient history, admission physiology, laboratory data, and operative details were reviewed and compared between patients whose operations remained LS and those whose operations converted to LT. Descriptive statistics were calculated, and comparisons between groups were performed using Chi squared test, Fisher's exact test, and t test.

Results: Of the SBO patients $(\mathrm{n}=1322), 464$ patients required surgery $(35 \%)$. LS was initially attempted in 100 cases $(21 \%)$. Of those, $56 \%$ required LT. Between groups, there were no differences in admission physiology or laboratory values. The rates of prior SBO admission or abdominal surgery were not significantly different between groups ( $\mathrm{p}$-values $>0.05$; Fig. 1). More LT patients required small bowel resections (59\% vs. $14 \%, \mathrm{p}<0.001)$, anastomoses $(54 \%$ vs. $14 \%, \mathrm{p}<0.001)$, and had operative findings of perforation $(9 \%$ vs. $0 \%, p=0.014)$ compared to LS patients. There were more nontherapeutic explorations in the LS group (p-value $=0.04$; Fig. 2 ).

Conclusion: More than half of patients undergoing laparoscopy for SBO require conversion to laparotomy. No pre-operative patient factors, including prior hospitalization for SBO or previous abdominal surgery, were predictive of increased likelihood of conversion. Approach to a successful therapeutic laparoscopic intervention for small bowel adhesiolysis may not depend on the patient's pre-operative history but more on intraoperative findings.
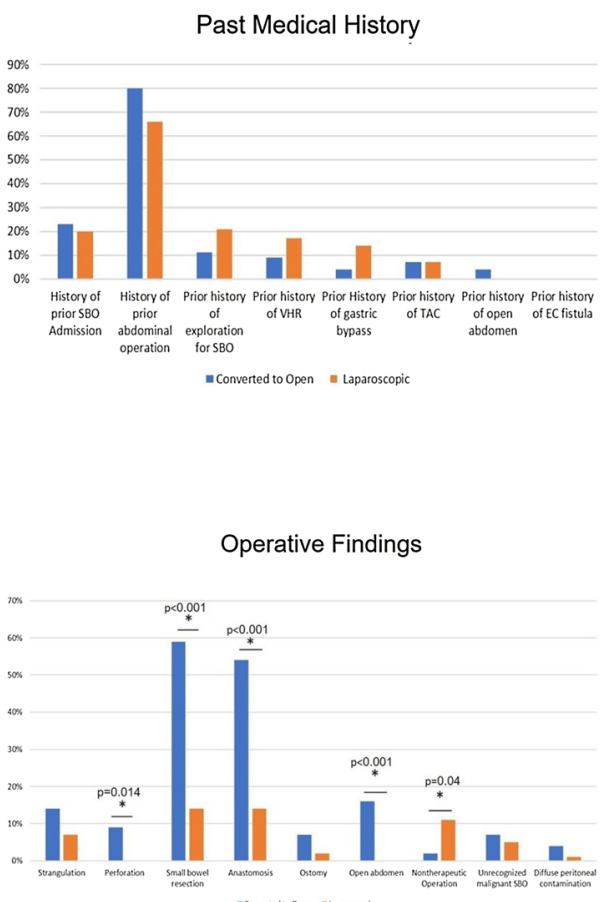


\section{P034}

'Rare Case of Mesentrico Axial Gastric Volvulus - A Series of Three Cases'

\section{Sumita Jain, Professor; SMS Hospital}

Gastric Volvulus was first described in 1896 by Berti. It is a rotation of stomach around a fixed axis which is greater than 180 degrees. The incidence if this in males and females are equal. It is mainly observed in infants and young adults and rarely seen in the elderly (>50 yrs). A dreaded complication of gastric volvulus is gastric strangulation (observed in $28 \%$ cases). It's classification is proposed by Singleton as (i) Organo-axial type (59\%), (ii) Mesentero-axial type (29\%) and (iii) Mixed (12\%).

We have experience of treating three cases of 'mesentrico axial gastric volvulus'. All patients presented in acute stage having upper abdominal pain, distention and recurrent vomiting. All patients underwent upper GI endoscopy and barium meal to confirm the diagnosis. These were managed through laproscopic surgery. All our patients had uneventful surgeries and are doing well in follow-up. Conclusion: From our experience with this rare presentation of mesentrico axial gastric volvulus, we conclude that this disease can be managed by minimal invasive surgery with no morbidity and mortality. The component of surgery should include reduction of volvulus, excision of hernial sac, repair of diaphragmatic defect and anti reflux procedure (if required), and gastrostomy tube placement. The excision of hernial sac and gastrostomy tube placement prevents recurrence and further complications. Hence, our procedure is superior to other procedures like endoscopic reduction and gastrostomy or alpha-loop or J-type technique. There are many other variations of gastropexy available like tanner's slide, oozler's operation, gastrojejunostomy with gastro-colic discontinuation, but our experience has shown that a simple pexy by doing 2 point fixation - suturing the fundus to the diaphragm and gastorstomy in the distal stomach helps to prevent recurrence.

\section{P035}

The Presence of Air Fluid Levels in the Mesenteric Veins as an Indicator of Early Bowel Ischemia

Vinay Bajaj, MD, Anthony Dippolito, MD; Easton Hospital

Our patient is a 67 year old male with significant history of insulin dependent diabetes, end stage renal disease on hemodialysis, cerebrovascular accident, and ischemic cardiomyopathy with EF 25\% who was initially admitted for worsening dyspnea on exertion. $\mathrm{He}$ was found to be in flash pulmonary edema secondary to cardiogenic shock, and was being treated for the same. During his recovery patient started complaining of new onset 10/10 abdominal pain after his morning dialysis session, and was sent for a CT scan of his abdomen. On examining the patient he complained of no tenderness to palpation, no rebound or guarding, but returned to fetal position as soon as the exam was completed. Lab findings revealed a type II MI, with Troponin level of 1.66 and a lactic acidosis of 2.9. CT showed massive portovenous gas (PVG) with an impressive air fluid level in the mesenteric veins as shown below. At this point the patient was rushed to the operating room for an exploratory laparotomy that showed patchy dusky jejunal loop with a palpable and dopplerable superior mesenteric artery. At this point a decision to place an abthera vac was made with plans for a second look surgery within 24-48 h. Discussion: The presence of PVG on radiologic testing is usually considered a sign of impending doom. Factors that can cause this sign historically include, most commonly, bowel necrosis, ulcerative colitis intra-abdominal sepsis, small bowel obstruction, gastric ulcers, chronic obstructive pulmonary disease and even chronic steroid use in adults. The mortality rates vary from $56-90 \%$ based on the underlying etiology. Bowel ischemia, especially in adults, is especially morbid with rates from $75-90 \%$ cited in literature. The extent of bowel ischemia in the bowel wall is divided into three stages: Stage I: reversible ischemic enteritis limited to the mucosa; Stage II: necrosis of the mucosal and submucosal tissues, which may lead to fibrotic strictures; Stage III: the entire wall is affected by ischemia. PVG when associated with ischemia is usually related to Stage III and carries the highest mortality. Although CT scans are neither sensitive nor specific for the detection of bowel ischemia, the presence of PVG should raise concerns. Detection of air fluid levels in the pre-portal venous system as seen here could be an early indicator of the same, requiring emergent intervention to operate and concurrently to attempt to reverse the underlying etiology.

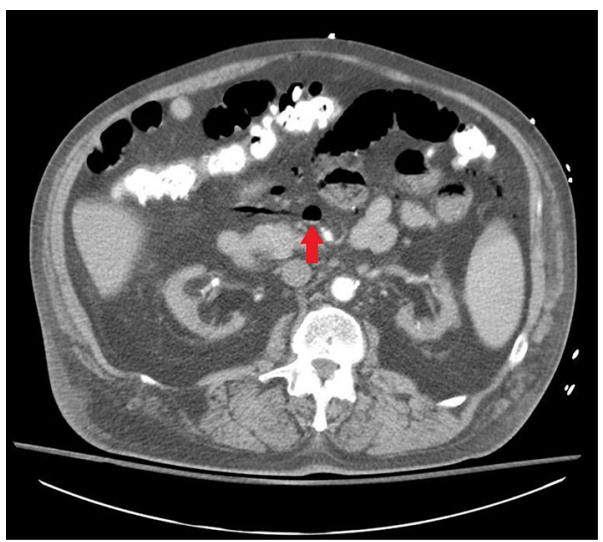


P036

\section{Posterior Gastric Perforation with Laparoscopic Omental Patch} Repair

Derek Lim, DO ${ }^{1}$, Shinban Liu, DO ${ }^{1}$, Nicholas Morin, DO $^{1}$, Vadim Meytes, $\mathrm{DO}^{2} ;{ }^{\mathrm{T}} \mathrm{NYU}$ Langone Medical Center - Brooklyn, ${ }^{2}$ Vassar Brothers Medical Center

Case Presentation: A 64-year-old female with a past medical history of chronic back pain with daily NSAID use had complaints of 5 days of abdominal pain, malaise, nausea, and vomiting. She was tachycardic in the emergency room with generalized peritonitis on physical exam. A CT scan of the abdomen demonstrated extraluminal air and oral contrast extravasation in the lesser sac, suggestive of a gastric perforation.

A diagnostic laparoscopy was performed and upon initial inspection, the lesser omental sac was noted to be full of succus and air. The greater curvature was dissected and a $3 \mathrm{~cm}$ pre-pyloric perforated ulcer was identified on the posterior surface of the stomach. An omental patch was secured with seromuscular 2-0 silk sutures across the ulcer. An esophagogastroduodenoscopy visualized the repaired non-bleeding ulcer and a post-pyloric NGT was placed intraoperative. The remainder of her hospital course was uncomplicated and her diet was advanced after a negative gastrograffin study.

Discussion: Posterior gastric ulcer perforations are an uncommon surgical emergency. Less than $1 \%$ of peptic ulcer perforations occur in the posterior stomach. Symptoms vary depending on the location of the perforation. Posterior pre-pyloric perforation causes leakage of stomach contents within the lesser sac. Generalized peritonitis occurs with contamination through the Foramen of Winslow into the peritoneal cavity. Posterior post-pyloric perforation contaminates the retroperitoneal space and may present as back pain or retroperitoneal abscess formation. CT imaging is the modality of choice to identify the location and extent of the perforation. Gastric perforations may be repaired with an omental patch, wedge resection, or gastric reconstruction depending on the size of the perforation. In rare instances, perforations may be contained on imaging and can be managed nonoperatively through careful observation, gastric decompression, bowel rest, and empiric antibiotics. However, extreme vigilance with a low threshold for operation is warranted with conservative management.

Conclusion: posterior gastric ulcer perforations are rare and insidious, requiring prompt diagnosis and treatment. The clinical presentation may be atypical and a CT scan is the gold standard for diagnosis. Laparoscopic and open approaches are both acceptable methods of repair depending on operator skill and clinical status of the patient.

\section{P037}

Clinical Course of Patients Presenting to the Emergency Department with Small Bowel Obstruction

Maria S Altieri, $\mathrm{MD}^{1}$, Lisa A Bevilacqua, $\mathrm{MD}^{2}$, Jie $\mathrm{Yang}, \mathrm{PhD}^{1}$, Chencan Zhu, MS ${ }^{1}$, Konstantinos Spaniolas, MD ${ }^{1}$, Mark Talamini, MD, FACS, MBA ${ }^{1}$, Aurora D Pryor, MD, FACS ${ }^{1}$; ${ }^{1}$ Stony Brook University School of Medicine, ${ }^{2}$ Thomas Jefferson University Hospitals

Introduction: Small Bowel Obstruction (SBO) is a common pathology requiring surgical workup and treatment; it is also often a consequence of prior abdominal surgery. Admissions for SBO and abdominopelvic adhesions have been estimated as high as 350,000 per year in the US alone. However, little is known regarding the anticipated clinical course of patients presenting to an emergency department (ED) with SBO. This includes rates of admission, need for surgery, or transfer to a tertiary care facility. This study aims to identify all patients who presented to the ED in New York State with SBO and follow their clinical course, with a focus on predictors of transfer.

Methods and Procedures: The New York SPARCS administrative database was used to identify all patients who presented to an ED with the diagnosis of SBO between 2012-2014. Patients were followed to identify discharges from the ED, admissions, operations, and transfers. To evaluate predictors of transfer, Chi square tests with exact p-values based on Monte Carlo simulation were utilized to examine the marginal association between categorical variables and transfers. Factors that were significantly associated with transfer $(\mathrm{p}<0.05)$ were further evaluated as possible risk factors for transfer in a multivariable logistic regression model.

Results: Between 2012-2014, there were 43,567 ED visits for SBO from 35,646 patients. Figure 1 shows the percentage discharged, admitted, transferred, and deceased. Of those requiring surgery, 94.2\% underwent an operation at the initial presenting institution while $5.8 \%$ had surgery after transfer. The mean time-to-surgery was 0.7 days $(\mathrm{SD}=0.83)$ and 2.47 days $(\mathrm{SD}=4.82)$ at presenting and transfer institutions, respectively. Independent predictors for transfer included age over 18-29 ( $\mathrm{p}=0.03)$, white non-Hispanic race/ethnicity $(\mathrm{p}<0.0001)$, and location in Western NY $(\mathrm{p}<0.0001)$. There was no effect of insurance status on transfer $(p=0.38)$. Interestingly, presence of any comorbidity resulted in a lower likelihood of transfer while any complication during initial visit significantly increased the likelihood of transfer.

Conclusions: To our knowledge, this is the first study that examines what happens to patients presenting with SBO in the ED using a large state-wide database. We found an in-hospital mortality rate of $1.9 \%$, a surgery rate of $17.6 \%$ during initial visit, and a transfer rate of $4.5 \%$. Further research is needed to determine demographic and clinical predictors of these outcomes.

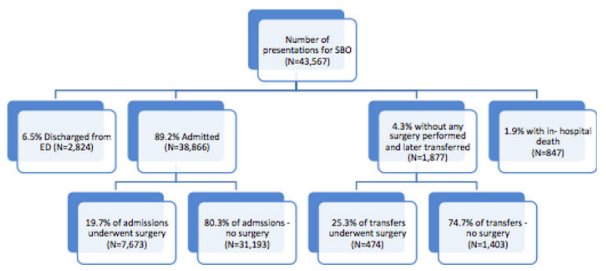


P038

\section{The Incidence and Risk Factors OF Venous Thromboembolism After Operative Management of Patients with Complicated Diverticulitis}

Mustafa Al-Jubouri, MD, MRCSI, Mohanad Baldawi, MD, Mustafa Baldawi, MD, Munier Nazzal, MD, FACS, Francis Burnicardi, MD, FACS; University of Toledo

Introduction: The prevalence of colonic diverticular disease increases with age and reaches over $70 \%$ in octogenarians. Dietary modification and the use of antibiotics are the main form of management of uncomplicated acute diverticulitis. But for complicated cases, surgical intervention is required.

Major surgery is a significant risk factor for venous thromboembolism. The incidence and risk factors of venous thromboembolism in patients undergoing colonic surgery for complicated diverticulitis has not been studied.

This study aims to determine the risk factors and the incidence of postoperative venous thromboembolism (VTE) within a 30 day postoperative period, in patients with complicated diverticulitis requiring surgical intervention.

Methods: Retrospective review from the American College of Surgeons National Surgical Quality Improvement Program (ACS NSQIP). All patients who underwent colonic resection for complicated acute diverticulitis from the years 2012-2016 were included in our analysis. Data regarding patient demographics, comorbidities, type of operative intervention and occurrence of postoperative VTE was recorded.

Results: A total of 47,890 patients underwent colorectal resection for complicated acute diverticulitis during the years 2012-2016. The incidence of VTE was $1.6 \%(\mathrm{n}=774)$ within 30 days postoperatively.

Univariate analysis showed an increased risk of VTE with increasing age, African-American race, diabetes mellitus, congestive heart failure, COPD, hypertension, chronic steroid use, emergent surgery, open surgery, preoperative sepsis, preoperative blood transfusion, impaired renal function, and intraoperative blood transfusion. Multivariate analysis identified 9 independent predictors of postoperative VTE: age $>70$ years, chronic steroid use, emergent surgery, open surgery, preoperative PRBC transfusion, preoperative sepsis, BUN $>20 \mathrm{mg} / \mathrm{dl}$, hematocrit $<36$ and intraoperative blood transfusion.

Conclusion: Multiple risk factors are associated with higher risk VTE among patients with complicated acute diverticulitis undergoing colorectal resection. Awareness of these factors can help the colorectal or general surgeons predict patients at high risk of developing VTE and therefore, modify and be more vigilant in their prophylaxis measures.

\section{P039}

\section{Laparoscopic Management of Small Bowel Obstruction}

\section{Prof Subhash Khanna, MS, FICS, FIAGES, FALS; Swagat Super} Speciality Surgical Institute

Background: Acute abdomen is one of the commonest presenting symptom to an emergency physician and surgeon In the day to day clinical practice.

Acute abdominal may be caused by various causes but small bowel obstruction is one of the common causes of those patients particularly having history of previous surgery Although considered a relative contraindication diagnostic laparoscopy is increasingly being used in small bowel obstruction not only for diagnosis but also as a therapeutic tool. We had been using diagnostic laparoscopy in all our cases of bowel obstruction presenting to us particularly those patients who did not have massive distension and who were not in shock

With growing experience small bowel obstruction is no more a contraindication to diagnostic laparoscopy rather most of these cases can be better managed with laparoscopy compared to convention surgery.

Methods: We searched medical records of medical records of all patients of acute abdomen undergoing diagnostic laparoscopy in our both centers during the period from august 2000 to june 2018 and reviewed the medical records of all the patients were reviewed to obtain data on feasibility, intra operative findings and also the total number of cases who needed a small incision for segmental resection or enterotomy.

We shall be presenting our data of all cases of bowel obstruction presenting to us and shall also share the technique of release of obstruction with video clippings of internal hernias, band obstruction, obstruction due to Meckel's and many such rare conditions including trichobezoar.

Conclusion: Diagnostic laparoscopy should always remain a preferred option in patients presenting with small bowel obstruction particularly those without massive abdominal distension as many of these patients can be better managed by laparoscopy much better way compared to unnecessary laparotomy causing morbidity. We shall present our series and some of the rare cases presented to us and who underwent therapeutic laparoscopic management successfully.

\section{P040}

\section{The Surgical Management of Ingested Sharp Foreign Objects in the Small Bowel (Adults): A Case Series and Review of the Literature}

Nicholas Morin, DO, Shinban Liu, DO, Erika King, George Ferzli, MD; NYU langone Brooklyn

Introduction: The surgical management of arrested sharp foreign bodies (FB) in the small bowel is often managed with a concurrent approach that balances endoscopy, laparoscopy, and laparotomy for their removal. Controversy exists as to the timing of surgical intervention and the management of asymptomatic patients. Here we discuss the management and present a case series with a review of the current literature.

Cases Series: Patient one underwent endoscopic retrieval of a wire bristle embedded in the duodenum. Patient two underwent diagnostic laparoscopy converted to open small bowe resection of chronically retained FB after it eroded through the bowel wall of the mid jejunum. Patient three underwent laparoscopic removal via enterotomy and primary repair of a chicken bone embedded in the terminal ileum.

Discussion: Any arrested sharp foreign body should be surgically removed in a timely fashion. The literature shows that longer delays to the operating room, and asymptomatic patients with retained small FBs, increases the risk of significant morbidity; such as migration of the FB through the bowel wall, enterocolic fistula, aortic - duodenal fistula, and frank acute perforation. Any known or suspected ingestion of a sharp FB should be staged with imaging if it is detectable. If progression fails, then surgery is indicated. If it has arrested in the stomach or duodenum endoscopic retrieval is appropriate. A skilled endoscopist can attempt balloon endoscopy for proximal jejunal FB retrieval, but retrieval should not be delayed. Laparoscopic retrieval is preferable when endoscopic attempts have failed. This can be performed via an enterotomy and primary repair for small FBs or laparoscopic small bowel resection with primary anastomosis. If there is frank perforation (or a skilled laparoscopic surgeon is not available) then a laparotomy and washout with resection of affected segment may be indicated.

Conclusion: Retrieval after ingestion of a sharp FB should be performed in a timely manner. A review of the literature shows that chronically retained FBs, and traditional lons delays to monitor for transit of the FB increase morbidity and mortality.

\section{P042}

\section{Laparoscopic Spleen Preserving Distal Pancreatectomy for Grade} IV Pancreatic Injury

Srikanth Gadiyaram, Dr, Gaurav Singh, Dr, Yashas H Ramegowda, Dr; Sahasra Hospitals, Center of Excellence for Gastroenterology

Background: Laparoscopic distal pancreatectomy with spleen preservation is one of the standardized procedures in selected cases with distal pancreatic tumors but is technically demanding in the setting of pancreatic trauma when distal pancreatic resection is required. We herein present a video with operative steps

Case Report: A 15 year old girl presented $24 \mathrm{~h}$ after trauma to upper abdomen while playing Kabaddi. She was hemodynamically stable and and had guarding and tenderness in her upper abdomen. MDCT of the abdomen revealed minimal hemoperitoneum, no pneumoperitoneum and transected pancreas opposite the vertebral body (grade IV pancreatic injury). She was resuscitated and taken up for emergency laparoscopic exploration. At laparoscopy there was $500 \mathrm{ml}$ of blood in the peritoneal cavity. Lesser sac was opened by dividing gastrocolic omentum with harmonic shears. A large hematoma was seen occupying the space between the transected head and body of the pancreas with extension of the hematoma into the mesocolon and mesentery. There was no obvious duodenal injury. A laparoscopic spleen preserving distal Pancreatectomy was performed in the following steps.

Step 1 - Division of gastrocolic omentum and placement of a gastric traction suture to provide wide exposure of lesser sac.

Step 2 - Evacuation of the hematoma by gentle suctioning.

Step 3 - Careful dissection behind the transected body of pancreas to identify the splenoportal venous confluence.

Step 4 - Progressive division of branches of the splenic vein and artery to the body and tail of pancreas using harmonic shears.

Step 5 - Bagging the specimen and extraction through the $12 \mathrm{~mm}$ port site in an endobag. Step 6 - Fibrin glue injection at the proximal transected surface and drain placement. The operative procedure took $2 \mathrm{~h}$ and 45 min.Her post operative period was uneventful. The abdomen drain was removed on the 4th POD. She was discharged on a normal diet on postoperative day 7 .

Conclusion: Laparoscopic distal panreatectomy with spleen preservation is feasible and safe in an emergent situation in a hemodynamically stable patient 
P043

\section{Systemic Review on Surgical Management of Colonic Injuries in Trauma: To Divert or to Anastomose?}

\section{Man Hon Tang, Daniel Lee; Khoo Teck Puat Hospital}

Introduction: There has been more evidence to support the role of primary repair or anastomosis for colonic injuries in trauma. However, there are no randomized trials especially on blunt trauma or in cases of damage control (DC) setting.

Aims: To perform a systemic review of studies comparing outcomes of fecal diversion (FD) versus primary repair/anastomoses $(\mathrm{PR} / \mathrm{A})$ in traumatic colonic injuries

Methods: A systematic review was performed as per PRISMA guidelines utilizing three electronic databases: Pubmed, EMBASE, and Cochrane Library resources. Primary and secondary outcomes are overall mortality and anastomotic leak rates respectively.

Results: There were 13 studies identified, including 10 retrospective, 2 prospective cohort and 1 randomised studies. Data from 1890 patients were abstracted including need for damage control surgery, surgical interventions, mortalities and complications. Overall, $71.3 \%$ of the patients underwent a PR/A, with a lesser proportion (64.5\%) being performed in the DC group. The overall mortality is $3.59 \%$ ( $2.89 \%$ in PR/A vs $5.30 \%$ in FD). The overall anastomotic leak rate is $5.64 \%$, and it is significantly higher in the DC group $(16.7 \%)$. Most papers identified significant blood transfusion, severe abdominal contamination and physiological instability as risk factors for poor outcomes.

Conclusion: It is safe to perform PR/A in stable patients with traumatic colonic injuries. FD should be recommend in unstable patients especially in a DC setting or in those at higher risk for complications

\section{P045}

Pyloroplasty as a Rescue Procedure for Morbidly Obese Patients with Refractory Gastroparesis after Sleeve Gastrectomy

Keneth Hall, MD, FACS, FASMBS, Raelina S Howell, MD, Harika Boinpally, MD, Patricia Cherasard, PAC, Patrizio Petrone, MD, Collin E Brathwaite, MD, FACS, FASMBS; NYU Winthrop Hospital

Introduction: Patients with morbid obesity and gastroparesis can be treated with sleeve gastrectomy (SG), which has been shown to increase gastric emptying, decrease transit time, and increase glucagon-like peptide levels. Historically, in the setting of refractory gastroparesis following SG, conversion to Roux-en-Y gastric bypass (RNY) was used as a salvage procedure. However, there are limited surgical options for patients with refractory gastroparesis who are poor RNY candidates (i.e. high risk for anastomotic breakdown such as in Crohn's disease, high-dose steroids, immunosuppressed) or who are unwilling to undergo RNY. This case series describes the unique surgical management technique of rescue pyloroplasty with sleeve gastrectomy (SG) for patients with morbid obesity (body mass index $[\mathrm{BMI}] \geq 35 \mathrm{~kg} / \mathrm{m}^{2}$ ) and refractory gastroparesis.

Methods: A retrospective chart review was performed for patients with morbid obesity and gastroparesis who underwent SG and simultaneous or subsequent pyloroplasty by a single surgeon at a Metabolic and Bariatric Surgery Accreditation and Quality Improvement Program Center of Excellence from August 2016 through July 2018. Patient workup, surgical techniques, and outcomes were assessed.

Results: Three patients underwent SG and simultaneous pyloroplasty $(\mathrm{n}=2)$ or subsequen pyloroplasty $(\mathrm{n}=1)$ and were included in this case series. The first patient was a 70-year-old female with a BMI of 36 who had undergone placement and removal of two prior adjustable gastric bands. She underwent laparoscopic SG, but complained of bloating and dysphagia during postoperative visits and was found to have gastroparesis on a gastric emptying study. She then underwent robotic revision SG and pyloroplasty with subsequent symptom resolution. The second patient was a 46-year-old male with diabetic gastroparesis (hemoglobin A1c 6.8) and a BMI of 40 who was offered RNY, but elected for SG with pyloroplasty and also had postoperative resolution of symptoms. The third case was a 34-year-old female with a BMI 37.8 and idiopathic, refractory gastroparesis who underwent laparoscopic SG and pyloroplasty. She was readmitted on postoperative day seven for liquid intolerance that resolved with conservative, non-operative management and she was discharged home the same day with continued symptom resolution during subsequent follow-up.

Conclusion: We have demonstrated good results with the use of rescue pyloroplasty following SG in patients with morbid obesity and refractory gastroparesis. In patients with gastroparesis who have already undergone sleeve gastrectomy, surgeons should consider pyloroplasty as a salvage maneuver prior to conversion to RNY, keeping in mind that conversion is still an option if symptoms persist.
P044

\section{CT Scan Measurement of Gastric Volumes Pre and Post Laparoscopic Sleeve Gastrectomy. Does the Resected Volume Correlates to Weight Loss Results?}

Fernandez Ananin Sonia, PhD, Rodriguez-Otero Carlos, Balague Ponz Carmen, Gonzalo Prats Berta, Pernas Canadell Juan Carlos, Garay Solá Meritxell, Targarona Soler Eduardo; Hospital de la Santa Creu i Sant Pau

Laparoscopic sleeve gastrectomy (LSG) has enormously grown in popularity as a stand-alone procedure for the treatment of morbid obesity. The aim of this study was to assess if the resected and the remnant gastric volumes (measured by multi-detector CT scans) were predictors for effectiveness of weight loss at 1 year after surgery.

Materials and methods: Sixty-four patients with BMI $>40 \mathrm{~kg} / \mathrm{m}^{2}$ or $>35 \mathrm{~kg} / \mathrm{m}^{2}$ and medical comorbidities underwent LSG between January 2012 and October 2016 included into a RCT (NCT02144545) study comparing different bougie sizes (33 Fr vs 42 Fr). Multi-slice CT scans were performed preoperatively, 2 months, and 1 year after surgery in those patients, to evaluate the gastric volume with a dedicated examination protocol. Parameters were compared to percentage of excess weight loss (\%EWL) at 1 year.

Results: Females accounted for $68.7 \%$ of patients. Mean age was 50.2 years. Mean preoperative BMI was $44.5 \mathrm{~kg} / \mathrm{m}^{2}$, and mean preoperative gastric volume measured by CT volumetry was $686.8 \mathrm{ml}$. \%EWL at 1 year was $60.1 \pm 19.3 \%$. Mean remnant gastric volume was $103.5 \mathrm{ml}$ at 2 months and $178.5 \mathrm{ml}$ at 1 year, this increase was statistically significant $(\mathrm{p}<0.001)$. No differences were found between the gastric volume increase after surgery ( 2 months volumen/ 1 year months volume months) with weight loss results at 1 year $(\mathrm{r}=0.016, \mathrm{p}=0.92)$. No significant differences were found in \%EWL (61\% vs 59\%) with the different bougie sizes $(p=0.327)$. A significant correlation was found between the percentage of resected stomach (preoperative volume $/ 2$ months volume) and $\% \mathrm{EWL}(\mathrm{r}=0.322, \mathrm{p}=0.043)$. We also found correlation between the resected volume (preoperative volume/2 months volume) with weight loss results at 1 year $(r=0.384, p=0.014)$

Conclusion: LSG is an effective treatment for inducing weight loss, and it seems that the bougie size should be tailored in each patient to resect a suitable gastric volume. Gastric dilatation does not seem to have an impact in short-term results.

\section{P046}

\section{LSG: One Minute to Fire is Better than 20 Secs?}

Mirto Foletto, MD, Pasquale Auricchio, MD, Mostafa Altowerqi, MD, Alice Albanese, MD; University of Padua

Introduction: Laparoscopic Sleeve Gastrectomy (LSG) is one of the most popular bariatric procedure, with good outcomes in terms of weight loss and comorbidities resolution in the mid- and long-term.

Staple line bleeding is one of the major related complications, occurring in $1-4 \%$ of cases Adequate tissue compression is considered of utmost importance to create a good staple line Objectives: To evaluate the impact of the time of stapler closure before firing on staple line bleeding.

Methods: From January to August 2018, 116 patients (mean age 43 years old, F 69, M 47, mean BMI $40 \mathrm{~kg} / \mathrm{m}^{2}$ ) underwent LSG at our Institution. In 57 patients stapler was kept closed for $60 \mathrm{~s}$ (group 1) before firing, while in the remaining 59 the time of compression was $20 \mathrm{~s}$ (group 2) Mean operative time, Haemoglobin loss (DHb) and drainage output (ml) in 1st and 2nd postoperative day (POD) were collected and compared. $\mathrm{P}<0,005$ was considered significative. Results: DHb (pre-op vs POD1) resulted higher in group $1(10.9 \pm 0.07$ vs $8.01 \pm 0.7$ $\mathrm{P}=0,0001)$. The same was observed for drainage output in POD 1 and $2(81.3 \pm 48.37$ vs. $57.5 \pm 9.37, \mathrm{p}=0.003 ; 70.9 \pm 51.7$ vs $67.88 \pm 9.38 \mathrm{ml}, \mathrm{p}=0.001)$

Mean operative time was shorter in group $1(63.2 \pm 13.9 \mathrm{~min}$ vs $68.7 \pm 9.3 \mathrm{~min}, \mathrm{p}=0.001)$. Conclusions: According to our experience, the longer time of tissue compression before stapler firing reduce intra-operative staple line bleeding during and mean operative time, but doesn't affect $\mathrm{Hb}$ changes in the early post-operative period (POD1 and 2). 


\section{P047}

\section{Rates of Reoperation and Intervention Within 30 Days} of Bariatric Surgery

Farah Ladak, MD, MPH, Jerry Dang, MD, PhDc, Noah Switzer, MD, MPH, FRCSC, Valentin Mocanu, MD, Daniel W Birch, MSc, MD, FRCSC, FACS, Shazeer Karmali, MD, MPH, FRCSC, FACS; University of Alberta

Introduction: The objective of this study was to identify early complications that result in intervention or reoperation following sleeve gastrectomy (SG) and Roux-en-Y gastric bypass (RYBG), the timeframe within which to expect them, and factors that influence the likelihood of intervention and reoperation. Complications arising from RYGB and SG are not insignificant and can necessitate additional invasive interventions or reoperation. This study provides a granular assessment of the morbidity associated with these procedures.

Methods and Procedures: Data for this study were obtained from the Metabolic and Bariatric Surgery Accreditation and Quality Improvement Program's participant use files for 2015 and 2016. Statistical analysis was performed using STATA 15 (StataCorp, College Station, TX). Between group differences were assessed via univariate analysis where chi2 was used for categoric data and independent t-test was used for continuous data. Predictors for reoperation and intervention were identified using multivariable logistic regression analysis. A combination of forward and purposeful selection was used in the creation of the final model. A goodness of fit test was conducted to estimate the predictive utility of the model.

Results: In 2015 and 2016, 243,747 underwent RYGB or SG, of which 3,014 had a reoperation and 1,536 had an intervention. Complications occurred in $5.48 \%$ of RYGB patients and $2.28 \%$ of SG patients, the most common of which was bleeding. Anastomotic/ staple line leaks were rare, affecting only $0.08 \%$ and $0.16 \%$ of SG and RYGB patients, respectively. SG was associated with far fewer interventions $(0.85 \%$ vs. $2.2 \%)$. and re-operations than RYGB $(0.67 \%$ vs. $2.5 \%$ ) and was associated with a lower risk of re-intervention and re-operation (OR: $0.45, \mathrm{p}<0.001$ ). Renal insufficiency, including dialysis dependency, was an important predictor of reoperations among bariatric surgery patients. This was also true of interventions however, history of pulmonary embolism and use of therapeutic anticoagulation were marginally stronger predictors.

Conclusions: This study provides a representative assessment of perioperative morbidity in two of the most commonly performed bariatric procedures: SG and RYGB. Complications were predominantly bleeding and soft tissue infections with a very low incidence of anastomotic or staple line leaks. Reoperation and intervention were relatively infrequent but more common among RYGB patients, corroborating what has been observed in smaller studies. These findings, in conjunction with new efficacy data demonstrating comparable long-term weight loss between RYGB and SG, provide further support for the safety, effectiveness and cost efficiency of SG.

\section{P048}

Does Bougie Size and Distance from the Pylorus affect Rates of Dehydration Following Laparoscopic Sleeve Gastrectomy?: An MBSAQIP Analysis

Ivy N Haskins, MD, Ada Graham, MD, Sheena W Chen, MD, Andrew D Sparks, MS, Paul P Lin, MD, FACS, Hope T Jackson, MD, Khashayar Vaziri, MD, FACS; George Washington University

Introduction: Laparoscopic sleeve gastrectomy (SG) is the most commonly performed bariatric procedure in the United States. Despite its prevalence, there are many technical factors related to this procedure that are not standardized. Specifically, the size of the bougie used and the distance from the pylorus used to create the gastric sleeve varies both by surgeon and across institutions. The purpose of this study was to determine if there was an association between either bougie size or distance from the pylorus on the rate of dehydration following laparoscopic SG.

Methods: All patients undergoing first-time, elective laparoscopic SGs from 2015-2016 were identified within the American College of Surgeons Metabolic and Bariatric Surgery Quality Program (ACSMBSAQIP) database. The association of bougie size and distance from the pylorus on the rate of dehydration following laparoscopic SG within the first 30-days postoperatively was investigated using multivariate logistic regression analysis.

Results: A total of 170,751 patients met inclusion criteria. The most commonly used bougie size was 36 French (Fr) and the most common distance from the pylorus was $5 \mathrm{~cm}(\mathrm{~cm})$. The patients were divided into four different groups based on bougie size and distance from the pylorus. Group 1 included bougie size $<36 \mathrm{Fr}$ and pylorus distance $<4 \mathrm{~cm}$, Group 2 included bougie size $>36 \mathrm{Fr}$ and pylorus distance $<4 \mathrm{~cm}$, Group 3 included bougie size $>36 \mathrm{Fr}$ and pylorus distance $>4 \mathrm{~cm}$, and Group 4 included bougie size $<36 \mathrm{Fr}$ and pylorus distance $>4 \mathrm{~cm}$. Patients in Group 4 were significantly less likely than patients in Group 1 to be treated for dehydration within the first 30 days postoperatively $(p=0.04)$. There were no other statistically significant differences between the four groups.

Conclusion: Distance from the pylorus, but not bougie size, is significantly associated with dehydration requiring treatment following laparoscopic SG. Specifically, a longer distance from the pylorus is significantly associated with a decreased risk of being treated for dehydration within the first 30-days following laparoscopic SG (OR: 0.82, CI: $0.67-0.99, p=0.04)$. Consideration should be made for standardizing distance from the pylorus during laparoscopic SG. 
P049

The Impact of Severe Obesity on Electrolyte Imbalance and its Potential Effects on the Sodium/Potassium-ATPase Pump Regulation

Cristian Milla Matute, MD, Maria C Fonseca, MD, Carlos Rivera, MD, David Romero Funes, MD, Emanuele Lo Menzo, MD, PhD, FACS, FASMBS, Samuel Szomstein, MD, FACS, FASMBS, Raul Rosenthal; Cleveland Clinic Florida

Introduction: Sodium/Potassium-ATPase ( $\mathrm{Na}+/ \mathrm{K}+$-ATPase) is a membrane protein responsible for the active transport of sodium and potassium ions across the plasma membranes on eukaryotes. Recent studies have shown that obesity, among other complications, induces an aberrant activity of $\mathrm{Na}+/ \mathrm{K}+-$ ATPase, causing extracellular and intracellular ions imbalance. Hyperinsulinemia, leptin resistance, and hyperandrogenism are factors that play a key role in $\mathrm{Na}+/ \mathrm{K}+-$ ATPase expression and regulation. The aim of this study is to analyze our bariatric patient population with electrolyte disturbances before and after surgery.

Methods: After IRB approval, we retrospectively reviewed the electronic records of all patients that underwent bariatric surgery at Cleveland Clinic Florida between the years 2003 and 2017. Inclusion criteria were BMI $>35$, serum sodium and potassium measurements pre-operatively and post-operatively at 3 months follow up. Patients with comorbidities under pharmacological treatment and/or hormonal replacement therapy were excluded from this study. SPSS software was used to apply a t-test for means.

Results: From a total of 5,373 patients reviewed, 22.5\% $(n=1,558)$ patients met the inclusion criteria. This patient population was predominantly female $77.8 \%$ (N: 1,212), the most prevalent procedure was RNYGB $61 \%(\mathrm{~N}: 950)$, the mean age was $54 \mathrm{y} \pm 13$. The mean BMI was $44 \pm 8 \mathrm{~kg} / \mathrm{m}^{2}$ at the time of the procedure and $32 \pm 9 \mathrm{~kg} /$ $\mathrm{m}^{2}$ at 3 months follow up. We identified $37 \%(\mathrm{n}=589)$ of patients with serum electrolyte imbalance preoperatively versus $3.1 \%(n=47)$ postoperatively at three months follow up. Before surgery $18 \%$ $(\mathrm{n}=106)$ patients had hypokalemia, $17.6(\mathrm{n}=104)$ hyperkalemia, $56.8 \%(\mathrm{n}=335)$ patients had hyponatremia and $6.6 \%(\mathrm{n}=39)$ had hypernatremia. At 3 months follow up after surgery a total of $21.2 \%$ $(\mathrm{n}=10)$ had hypokalemia, 34\% $(\mathrm{n}=16)$ hyperkalemia, 17\% $(\mathrm{n}=8)$ patients had hyponatremia and $27.6 \%(n=13)$ had hypernatremia. Conclusions: Electrolytes disturbances are just one of the multiple manifestations of $\mathrm{Na}+/ \mathrm{K}+$-ATPase pump impaired activity in patients with severe obesity. The prevalence of obese electrolytes disturbance is higher than in the general population. Rapid weight loss after bariatric interventions showed a significant improvement of serum electrolytes levels. However multiple factors might be related to these changes. Further studies with the inclusion of more variables should be performed in order to better understand these findings.
P050

Bleeding Dieulafoy Ulcer After Gastric Sleeve: A Case Report and Examination of the Need for Preoperative Esophagogastroduodenoscopy Before Bariatric Surgery

Michael Nicoara, DO, Nicholas Morin, DO, Shinban Liu, DO, Corneliu Vulpe, MD, George Ferzli, MD; NYU Langone Brooklyn

Introduction: There is still controversy regarding whether or not a preoperative esophagogastroduodenoscopy (EGD) should be done before bariatric surgery; does it change surgical course, and does it prevent postoperative complications? Here we present a relevant case and review the current literature relating to preoperative EGDs in bariatric surgery.

Case Presentation: A 51 year female presents for preoperative workup prior to undergoing a restrictive bariatric procedure. A standard institutional preoperative workup was performed without an EGD. Patient returned postoperative day number two with hematemesis. Emergent EGD showed clotted blood in the stomach and a Dieulafoy ulcer that was successfully clipped by the endoscopist.

Methods: A literature review was performed using Pubmed. Search term "EGD bariatric surgery" was used.

Results: PubMed search returned 50 papers, and 13 were selected for review due to their relevance; 4 against EGD, 5 in favor of EGD, and 4 in favor if patient has reflux symptoms.

Discussion: A Dieulafoy ulcer is rare, accounting for 1-2\% of upper gastrointestinal bleeding presentations. Our patient presented with classic findings of this lesion: larger diameter vessel on the lesser curve of the stomach, about $6 \mathrm{~cm}$ from the gastroesophageal junction, with vessel protruding through a mucosal defect with active arterial bleeding. It is uncertain if a preoperative EGD would have located this lesions and/or provided a means for intervention before presentation. Our literature review on the subject shows that preoperative EGD rarely changes surgical management. They do change medical management in a significant number of cases. With the majority of papers in favor of preoperative EGD (albeit 4 only if patient has symptoms of GERD) there is evidence in favor of performing a preoperative EGD before bariatric surgery.

Conclusion: The current literature is equivocal regarding a preoperative EGD as it rarely changes the surgical management, but often changes the medical management. Even though the yield is small, we recommend preoperative EGD before bariatric procedures for medical optimization; in order to avoid the potentially devastating consequences associated with a missed lesions such as the one presented.

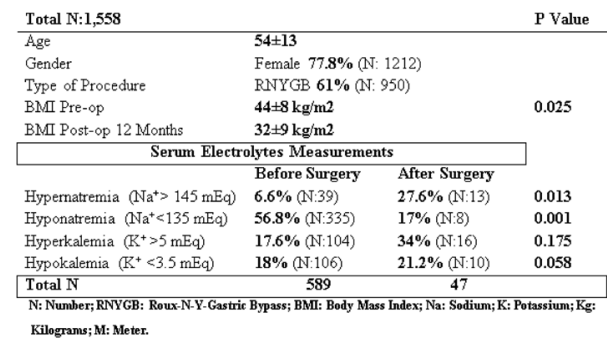




\section{P051}

Gastric Pseudocyst associated with a prolapsed Laparoscopic Adjustable Gastric Band

Tianming Liu, MD ${ }^{1}$, Ziyad Nasrawi, $\mathrm{MD}^{1}$, George John, $\mathrm{MS}^{2}$, Darren Kong, BS ${ }^{2}$, Piotr Gorecki, MD, FACS ${ }^{1}$; ${ }^{1}$ New York Presbetyrian Brooklyn Methodist Hospital, ${ }^{2}$ Rowan School of Osteopathic Medicine

Introduction: Due to long term failures and complications, the number of laparoscopic adjustable gastric bands (LAGB) being performed in the United States each year is decreasing. We report a case of the prolapsed (slipped) gastric band associated with perigastric pseudocyst and discuss the pathophysiology.

Case report: A 42-year-old woman with a history of morbidobesity and LAGB placed 8 years ago presented emergently with abdominal pain, dehydration, vomiting and severe gastroesophageal reflux (GERD). Emergent plain radiogram revealed prolapsed LAGB. Postoperatively the patient experienced an excellent weight reduction with 94\% Excess Weight Loss and a BMI of $23.5 \mathrm{~kg} / \mathrm{m}^{2}$. At emergent laparoscopy, a $6 \mathrm{~cm}$ cystic mass was found in association with the prolapsed LAGB. The perigastric cyst was dissected and excised. Intraoperative endoscopy did not reveal communication to the gastric lumen. The LAGB was explanted without evidence of gastric erosion. Pathologic evaluation revealed a smooth thin walled pseudocyst with inflammatory and mesothelial cells. The patient recovered well and was discharged the following day.

Discussion: Perigastric pseudocyst associated with LAGB has been reported in the literature. It's pathogenesis may be connected to foreign body reaction. We propose collagen and granulomatous tissue deposits around the LAGB and the gastro-gastric plication area contributed to this rare entity. The fibrotic tissue blocks lymphatic channels which may result in an accumulation of lymphatic fluid. This condition may be revealed during emergent exploration or during the preoperative advanced imaging such as the CT imaging. Pathological findings consistent with fibrotic and inflammatory tissue confirm the diagnosis and rules out malignancy and true gastric cyst. Lack of epithelial lining confirms the diagnosis of pseudocyst.

Conclusion: Pseudocysts may form as a mechanism of foreign body reaction. Slipped LAGB could be a contributing factor as in this case. Intraoperative photographs and endoscopic images will be presented. Additional observational studies and reports are needed to further understand the pathophysiology of this entity and to determine the causal association with a band and particularly a prolapsed LAGB.

Figure 1: B marks the normal location and position of the LAGB. * marks the flow of contrast which is normal through the GE junction and stomach.

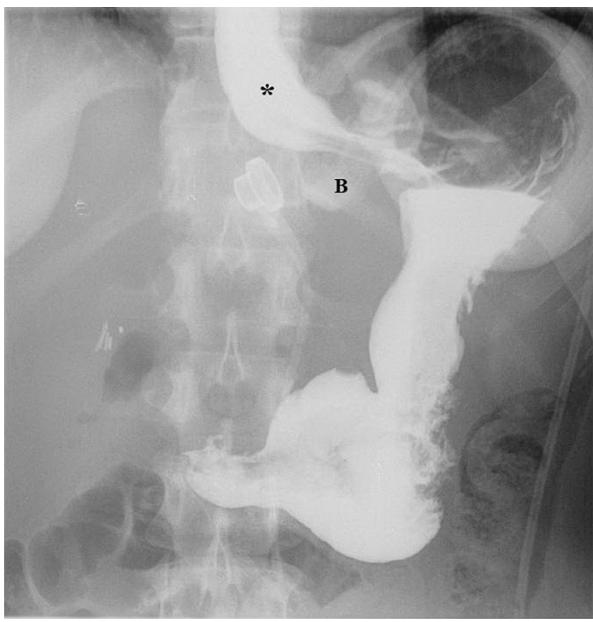

Figure 2: B, marks the slipped lap band. * marks dilation of the of the proximal stomach and esophagus.

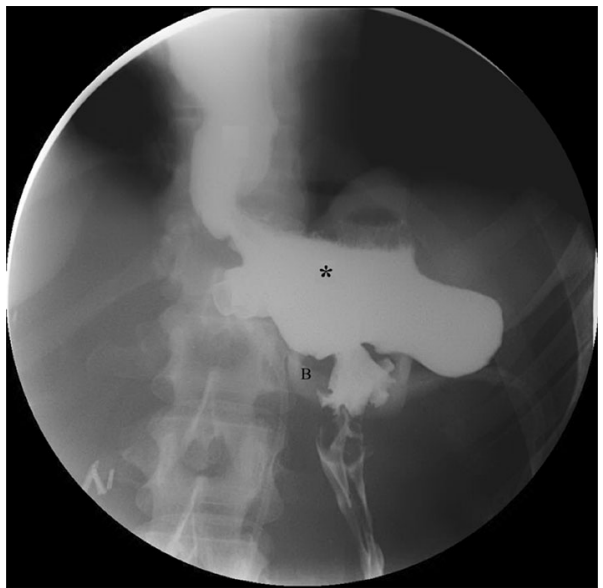

Figure 3: $6 \mathrm{~cm}$ cystic mass associated with gastric band.

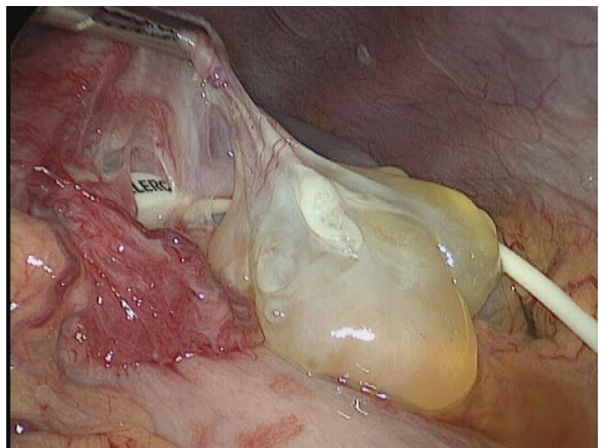

Figure 4: Gastric psuedocyst wall, consistent with fibrous cyst wall, focal mild hemorrhage, and organizing chronic inflammation/fibrosis.

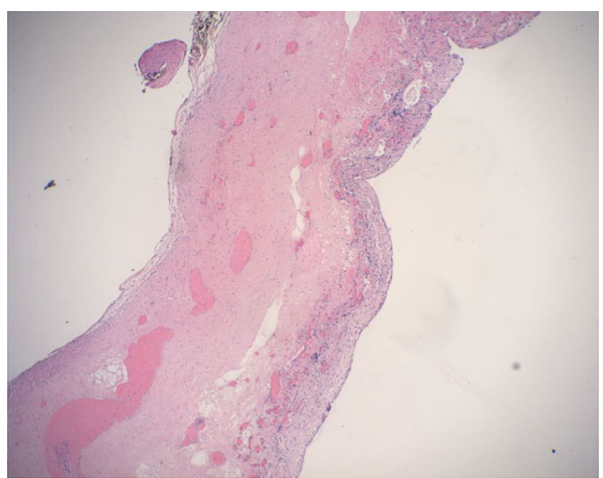


P052

\section{Use of Laparoscopic Witzel Gastrostomy without Gastropexy} in Bariatric and General Surgery

Joshua Davies, MD, Luise Pernar, MD, Brian Carmine, MD, Donald Hess, MD, Cullen Carter, MD; Boston Medical Center

Introduction: Gastrostomy placement is the preferred means of long term enteral feeding for patients who cannot eat by mouth. During laparoscopic gastrostomy, it is standard to perform gastropexy, apposing visceral and parietal peritoneum. However, in some settings, such as the presence of an antecolic roux limb from prior gastric bypass, gastropexy is not possible. This study reports a series of cases where gastrostomy was performed via a witzeled approach without gastropexy.

Methods and Procedures: A retrospective chart review was performed of all patients at a tertiary academic medical center who underwent witzeled gastrostomy without gastropexy over a three year period. In each case, an 18 French feeding tube was brought through the abdominal wall and placed into the fundus of the stomach and secured with a pursestring suture. A $5 \mathrm{~cm}$ serosalized witzel tunnel was created around the tube using running silk suture. No gastropexy was performed, and no drain was placed in any case.

Results: Between September 1, 2015 and August 1, 2018, 6 patients underwent 7 witzeled gastrostomy procedures. The patients ranged in age from 39 to 86 . In three cases, patients had undergone prior major upper abdominal surgery where adhesive disease prevented gastropexy. In the other four cases, the patients had undergone prior gastric bypass with antecolic antegastric position of the roux limb. Indications for procedure were dysphagia in two cases, critical illness in one, chronic nausea and malnutrition after gastric bypass in three cases, and perforated marginal ulcer in one case. No patient suffered leakage of gastric contents into the peritoneum, and there were no postoperative complications or mortality related to the gastrostomy procedure.

Conclusion: In cases where enteral access is necessary, and where the stomach cannot reach the anterior abdominal wall for gastropexy due to prior surgeries, a witzeled gastrostomy without gastropexy is a safe option, and in this small series resulted in no morbidity or mortality.

\section{P054}

Pre-existing Cardiac Disease is Associated with Increased Risk of Peri-Operative Mortality Among Patients Undergoing Laparoscopic Sleeve Gastrectomy and Roux-en-Y Gastric Bypass

Victoria M Gershuni, MD, MSGM, Michael W Foster, MD, Colleen M Tewksbury, PhD, MPH, RD, Eduardo Rame, MD, Kristoffel R Dumon, MD, Noel N Williams, MD; Hospital of the University of Pennsylvania

Introduction: Severe obesity and its related complications, including hyperlipidemia, hypertension, insulin resistance, and obstructive sleep apnea (OSA), confer increased risk of developing cardiac disease. Paradoxically, patients with overweight and obesity have lower cardiac-related mortality than their normal-weight, cardiovascular risk-adjusted counterparts, which suggests that obesity may exert a slightly protective effect in this population. It is unclear, however, whether cardiac disease portends worse surgical outcomes following bariatric surgery.

Methods: Using the MBSAQIP Participant Use Data File, a retrospective analysis of patients undergoing laparoscopic and laparoscopic-assisted Roux-en-Y Gastric Bypass (LRYGB) or sleeve gastrectomy (LSG) between 2016-2017 was performed. Patients were classified as having cardiac disease by history of prior myocardial infarction (MI), percutaneous coronary intervention (PCI), or cardiac surgery. Patients were assessed for 30-day mortality and incidence of peri-operative cardiac arrest/need for CPR.

Results: Of patients undergoing bariatric surgery $(n=325,653), 4,405$ have history of MI, 7,097 have had PCI, and 3,774 have had prior cardiac surgery. Compared to the rest of the cohort, cardiac patients $(3.5 \%)$ had a greater number of comorbidities as demonstrated by increased prevalence of hypertension $(88.9 \%$ vs. $47.7 \%, \mathrm{p}<0.0001)$, hyperlipidemia $(71.2 \%$ vs. $23.2 \%, \mathrm{p}<0.0001)$, diabetes $(54.6 \%$ vs. $25.8 \%, \mathrm{p}<0.0001)$, and renal insufficiency $(4.1 \%$ vs. $0.54 \%, \mathrm{p}<0.0001)$. Prior MI, PCI, and cardiac surgery were each associated with significantly increased risk of intra-operative cardiac arrest requiring CPR (RR: 5.65; 95\% CI 3.1-10.4, RR 4.88, 95\% CI: 2.9-8.3, RR: 7.26; 95\% CI 4.0-13.1, respectively) and 30-day mortality (RR: $4.29 ; 95 \%$ CI 2.7-6.8, RR 3.85, 95\% CI: 2.6-5.7, RR $4.46,95 \%$ CI: 2.7-7.3, respectively).

Conclusion: Patients with significant pre-existing cardiac disease as indicated by prior cardiac intervention were at greatly increased risk for perioperative mortality, either at time of surgery or in the initial 30 days following the operation. Severe obesity is a known risk factor for cardiac disease suggesting benefit for bariatric surgery in this population; however, cardiac patients undergoing bariatric surgery may require additional preoperative treatment, perioperative interventions, and postoperative monitoring.

\section{P055}

\section{Impact of Sleeve Gastrectomy on Gastric Emptying Scintigraphy and Weight Loss in an Ethnically Diverse Bariatric Patient Cohort}

Matthew Knouse, $\mathrm{MD}^{1}$, Perry Orthey, $\mathrm{BS}^{1}$, Henry Parkman, $\mathrm{MD}^{2}$, Michael A Edwards, MD, FACS, FASMBS ${ }^{2} ;{ }^{1}$ Temple University Hospital, ${ }^{2}$ lewis Katz School of Medicine at Temple University

Background: Sleeve gastrectomy (SG) is the most common bariatric operation performed Its impact on gastric emptying remains controversial. In this study, we evaluated the impact of SG on gastric emptying scintigraphy (GES) and weight loss.

Methods: We performed a retrospective analysis of SG patients who had both a preoperative and postoperative GES between 2012 and 2017. Postoperative gastric retention (GR) and weight loss (TBWL) were compared among ethnic groups. All analysis was performed with IBM SPSS Statistics 24 . A p value $<0.05$ was considered significant.

Results: Of 435 SG cases, 17 patients with a preoperative and postoperative GES were analyzed. Retention was higher in white patients $(\mathrm{p}=0.014)$. Preoperatively, $35.3 \%(2 \mathrm{hr}$ GES) and $29.4 \%$ (4-hr GES) had gastroparesis (GP). Gastroparesis decreased after SG on both 2 -hr $(42.4 \%$ vs $11.1 \%, \mathrm{p}<0.0001)$ and $4-\mathrm{hr}(8.9 \%$ vs $2.2 \%, \mathrm{p}=0.01)$ GES. TBWL was less in Hispanics at $12(\mathrm{p}=0.036)$ and $18(\mathrm{p}=0.058)$ months. All patients had normal postoperative GES at 2-hr and 4-hr scintigraphy. No correlation could be made between postoperative gastroparesis and TBWL. On average, SG was initiated $4.93 \mathrm{~cm}$ proximal the the pylorus. There was no correlation between TBWL and distance from the pylorus.

Conclusion: SG significantly improves gastric retention in this ethnically diverse patient cohort with severe obesity. There was no significant difference in postoperative gastric retention and weight loss among ethnic groups. Further research and a larger sample size are needed to further validate these findings and better understand the impact of sleeve gastrectomy on gastric emptying scintigraphy and postoperative weight loss in ethnically diverse patients with severe obesity.

\section{Does Resected Stomach Weight Predict Weight Loss after Laparoscopic Sleeve Gastrectomy?}

Avian Chang, Sarang Kashyap, Manthan Makadia, Vinay Singhal; Easton Hospital

Background: Laparoscopic sleeve gastrectomy (LSG) has gain popularity as a standalone bariatric procedure and is being performed with increasing frequency. Limited data exists on predictors of post-operative weight loss. The aim of this study is to determine if resected stomach weight could predict post operative weight loss following a LSG.

Method: This is a retrospective review of patients who underwent a standard LSG for morbid obesity over a $40 \mathrm{~F}$ bougie. Stomach weight was recorded and data was recorded for post-operative weight loss. Mean values were compared using t-tests and analysis of variance. Pearson's coefficient was used for correlation.

Results: Patients are still being followed however 107 patients underwent a LSG during $39.43 \pm 15.74,49.01 \pm 20.05$ and $52.40 \pm 23.46 \%$ at 6 weeks, 3,6 and 12 months respectively. Mean weight of stomach removed was $114.39 \pm 48.85 \mathrm{gm}$. No significant difference was seen between amount of stomach removed and mean \%EBWL. Positive had a larger stomach compared to females.

Conclusion: Mean \%EBWL after LSG is significant although weight of stomach removed weight had a larger amount of resected stomach. this time. Mean percent excess body weight loss (\%EBWL) was $26.49 \pm 14.75$, correlation was seen between pre-operative weight and amount of stomach removed. Males did not affect post-operative weight loss. Males and patients with a higher pre-operative 
P056

Use of Transversus Abdominis Plane Block with Liposomal Bupivacaine in Patients Undergoing Bariatric Surgery: A CaseControl Study

Allison M Barrett, MD, FACS ${ }^{1}$, John A Afthinos, MD, FACS ${ }^{1}$, Christopher Funfgeld, PAC, MPAS ${ }^{1}$, Vaughn E Nossaman, MD ${ }^{2}$, Kevin K Kresofsky, $\mathrm{MD}^{2}$; ${ }^{1}$ Long Island Jewish Forest Hills Hospital, ${ }^{2}$ Nassau University Medical Center

Introduction: The overuse of postoperative narcotics has gained attention due to the epidemic of opiate abuse in the United States. Multimodal postoperative pain management strategies can address this through the addition of non-opiate analgesia and are incorporated within enhanced recovery after surgery (ERAS) pathways. We hypothesize that the use of an intraoperative transversus abdominis plane (TAP) block with liposomal bupivacaine will decrease the use of narcotic pain medications in patients undergoing bariatric surgery.

Methods: This is a retrospective review of in-hospital pain medication use for patients who underwent bariatric surgery from 2016-2018 at a single institution. From 2016 - 2017 patients received injection of bupivacaine locally at incision sites. From 2017 - 2018, patients received a laparoscopic-guided TAP block with a mixture of $20 \mathrm{~mL}$ of liposomal bupivacaine, $30 \mathrm{~mL}$ of $0.5 \%$ bupivacaine, and $100 \mathrm{~mL}$ of normal saline at the beginning of the procedure. This was injected by the surgeon bilaterally at the level of the anterior axillary line, with extension into the subcostal space. Injections were performed with a 20G or $22 \mathrm{G}$ spinal needle and were placed with laparoscopic visualization. Postoperatively, all patients received standing intravenous ketorolac and acetaminophen, unless contraindicated. Patients were excluded from the study if they required a concomitant procedure, returned to the OR, had alterations in surgical technique, or were on narcotics or benzodiazepines preoperatively. In-hospital narcotic use was converted to morphine equivalent units, according to previously published equianalgesic tables.

Results: A total of 80 patients underwent bariatric surgery during the time period and 15 patients were excluded. TAP block was performed in 39 patients, compared to 24 who did not have a TAP block. Mean age was 41 , and pre-operative BMI was not different between the groups. Intraoperative dosing of morphine equivalent units, acetaminophen, and ketorolac were also not statistically different between the groups. Use of narcotics on postoperative day 0 was significantly less for the TAP-block group (5.97 vs $10.14 \mathrm{mg}$ morphine, $\mathrm{p}=0.016$ ). Pain scores in the PACU and up to $24 \mathrm{~h}$ postoperatively were not different, nor was the use of acetaminophen and ketorolac postoperatively or length of stay. There were no medication-related adverse events.

Conclusion: Performance of an intraoperative TAP block decreases the use of postoperative narcotic medication use, but does not affect length of stay. As a part of an ERAS protocol, a TAP block is a useful approach for minimizing postoperative opiate use.

\section{P057}

\section{The Preoperative Weight Loss Program and Predictive Marker of Postoperative Weight Loss in Sleeve Gastrectomy}

Hideya Kashihara, Mitsuo Shimada, Kozo Yoshikawa, Jun Higashijima, Tomohiko Miyatani, Takuya Tokunaga, Masaaki Nishi, Chie Takasu; Department of surgery, Tokushima university

Background: Our department perform preoperative weight loss program for the purpose of decreasing the perioperative complication in sleeve gastrectomy (SG). The aim of this study is to show the surgical outcome of SG and predictive marker of postoperative weight loss. Patients and Methods:

1. 13 obese patients who underwent SG were enrolled in the preoperative weight loss program (Duration; 37.2 days). Preoperative weight loss program contains calorie restriction $(1200 \mathrm{kcal} / \mathrm{day})$ and exercise by the nutrition support team.

2. The relationship between \% EWL in postoperative 1 year and preoperative neutrophil/lymphocyte ratio (NLR) was examined in the cases with less postoperative weight loss after SG

Results:

1. The preoperative weight loss program showed decrease of body weight $(-12.1 \mathrm{~kg}$, $9.1 \%)$, visceral fat mass $(230.9 \rightarrow 192.9)$, improvement of transaminase and maintenance of skeletal muscle. There was no perioperative complication. \%EWL in 3, 6 months and 1 year were $46.3,50.8$ and $47.4 \%$. In postoperation, SG improved NASH (AST/ALT, FIB4 index, liver to spleen ratio in CT value). Obesity related disease were improved (diabetes; $75 \%$, hypertension; $64 \%$, hyperlipidemia; $71 \%$, SAS; 90\%). NLR in postoperative 3 months showed decrease $(2.68 \rightarrow 1.86)$. So, immune function was improved.

2. There was negative relationship between preoperative NLR and \%EWL in postoperative 1 year $(\mathrm{R} 2=0.52)$. Comparing preoperative NLR in $\% \mathrm{EWL}<50 \%$ and $950 \%$ in postoperative 1 year, $<50 \%$ was 2.97 and $? 50 \%$ was 2.01 (cut-off 2.25 ). Preoperative NLR predicts postoperative weight loss in SG.

Conclusion: Preoperative weight loss program is effective in SG. However, more strong preoperative intervention or bypass surgery may be needed in the cases with NLR?2.25.

\section{P058}

Impact of smoking on elective bariatric surgery postoperative complications: analysis of the MBSAQIP database

Jingliang Yan, MD, PhD, Brandon Williams, MD, Matthew Spann, MD, Chetan Aher, MD, Wayne English, MD; Vanderbilt University Medical Center

Introduction: Bariatric surgery is an effective means of achieving weight loss and improvement of metabolic co-morbidities. Enhancing quality and safety profile is imperative as the number of bariatric procedures increases worldwide. Smoking has been associated with increased postoperative complications after many surgical procedures. We aim to study how preoperative smoking status affects postoperative outcomes in bariatric patients using the Metabolic and Bariatric Surgery Accreditation and Quality Improvement Program (MBSAQIP) database.

Methods and Procedures: Patients in the MBSAQIP 2016 data registry undergoing elective bariatric procedures were identified and included. Odds ratio (OR) and 95\% confidence interval (CI) of postoperative complications in recent smokers compared to nonsmokers were calculated.

Results: A total of 184,749 patients undergoing elective bariatric surgery in 2016 were identified. Of these patients, 16,084 (8.6\%) reported smoking cigarettes within 1 year prior to the bariatric operations. Length of stay was slightly longer in patients with a smoking history ( 1.8 vs 1.7 days). Several postoperative complication rates were increased with smoking. Unplanned re-intubation $(\mathrm{OR}=1.70$, CI 1.23-2.34), Clostridium difficile infection $(\mathrm{OR}=1.64, \mathrm{CI} 1.15-2.33)$, postoperative incisional hernia $(\mathrm{OR}=1.62$, CI 1.02-2.55), and 30-day mortality related to the operation $(\mathrm{OR}=1.80, \mathrm{CI} 1.02-3.18)$ were associated with an odds ratio of at least 1.5. In addition, intraoperative or postoperative transfusion requirement $(\mathrm{OR}=1.20$, CI 1.00-1.44), unplanned $\mathrm{ICU}$ admission $(\mathrm{OR}=1.34$, CI 1.12 $1.58), 30$-day reoperation $(\mathrm{OR}=1.23, \mathrm{CI} 1.08-1.39)$, 30-day readmission $(\mathrm{OR}=1.16, \mathrm{CI}$ 1.07-1.25), and need for intervention within 30 days $(\mathrm{OR}=1.25$, CI $1.10-1.40)$ were associated with an odds ratio of greater than 1 .

Conclusion: Prior smoking history within a year of bariatric surgery is associated with an increase in multiple postoperative complications as well as mortality. Smoking cessation therefore may improve outcomes among bariatric patients. 


\section{P059}

\section{Computational Tools for the Reliability Assessment and the Engineering Design of Procedures and Devices in Bariatric Surgery}

Chiara Giulia Fontanella, PhD, Mirto Foletto, MD, C Salmaso, I Toniolo, Alice Albanese, MD, Pasquale Auricchio, MD, Lino Polese, Professor, Mostafa Altowerqi, MD, Emanuele Luigi Carniel, Professor; University of Padua

Background: Sleeve Gastrectomy (SG) is one of the most performed bariatric procedure worldwide. Unsatisfactory weight loss and de novo gastroesophageal reflux (GERD) are the major long-term concerns, which can be related to the gastric pouch conformation.

Aims: A novel approach is required to better assess SG in the long run. Rational criteria are advocated to provide engineering tools for the forecast of SG efficacy and the identification of the optimal postsurgical stomach configuration.

Methods: The methods of computational and experimental biomechanics allow investigating interactions between bolus and stomach tissues. As bolus interacts with stomach lining, stretching forces develop and their magnitudes increase with food intake. Such mechanical stimuli act on gastric receptors, initiating neural signals, which convey information to the brain. The brain responds by releasing neuro-transmitters that elicit the feeling of satiety, depending on intensity of stomach wall mechanical stimulation A computational model of the stomach was developed by means of histo-morphometric investigations and mechanical tests. Computational analyses were performed to evaluate stomach functionality in both pre- and post-surgical conformation, as the stomach pressurevolume behaviour and the mechanical stimulation of gastric receptors. The stomach computational model was exploited to investigate an endoscopic approach to sleeve gastrectomy. Different clips conformations were investigated, as anchors, spiders, screws and spirals, considering both the endoscopic applicability and the capability to support intraluminal loads.

The results showed the potentiality of computational methods to investigate stomach functionality and to optimize procedures and techniques in bariatric surgery.
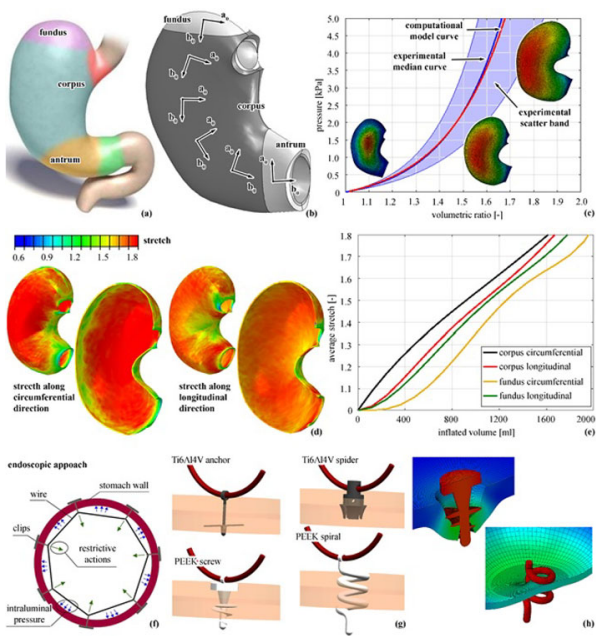

Biomechanical approach to stomach functionality. Schematic representation of stomach conformation and regions (a). Virtual solid models of stomach regions and layers with indication of local material directions (b). Model validation by the analysis of stomach inflation behavior: comparison of experimental data and model results (c). Results from computational analyses of stomach functionality: distribution of stretch components along circumferential and longitudinal directions within the muscular layer at $1200 \mathrm{ml}$ inflated volume (a); average values of stretch components in fundus and corpus regions depending on inflated volume (b). Endoscopic approach to bariatric surgery: schematic representation of intraluminal fixation devices (f); clips design for endoscopic fixation, as anchor, spider, screw and spiral; analysis of interaction phenomena between clips and stomach tissues (h). 


\section{P060}

\section{Can Bariatric Surgery Prevent the Development of Metabolic} Syndrome?

Salman Alsabah, MBBS, MBA, FRCSC ${ }^{1}$, Eliana Al Haddad ${ }^{2}$, Aliaa Al-Mutawa ${ }^{1}$, Mohammad Al-Mutawa ${ }^{1}$, Jonathon D Vaz ${ }^{1}{ }^{1}$ Kuwait University, ${ }^{2}$ Columbia University Medical Center

Introduction: Obesity has been directly correlated with the development of metabolic syndrome, a life-threatening disorder. With the constant rise of obesity in the world, this correlation has become of major significance.

Methods: A retrospective analysis of LSG patients' medical records was performed between October 2008-2017 at a single institute in Kuwait. 1818 patients that have not been previously diagnosed as diabetic and/or dyslipidemic were included.

Results: Patients were followed-up for a 5-year period. Pre-operative weight and body mass index(BMI) were $123.8 \mathrm{~kg}$ and $46.1 \mathrm{~kg} / \mathrm{m}^{2}$. The greatest weight loss was achieved at 1.5 years post-operatively, correlating to a \%excess weight-loss(EWL) of $73.8 \%$ and change in BMI of $14.62 \mathrm{~kg} / \mathrm{m}^{2}$. Pre-operatively, only $45.1 \%$ of the patients exhibited normal FBS levels. This number dropped significantly throughout the follow-up period, corresponding to a normal FBS level in $77.8 \%$ at 12 months post-op. Only $20 \%$ of the patients had normal $\mathrm{HbA1c}$ levels pre-operatively, and this number increased to a maximum of $50.4 \%$ at 1 -year. When it came to looking at the effect of LSG on lipid status, it was seen that it had little effect on total cholesterol and LDL levels, while our patients demonstrated significantly favorable results on HDL, VLDL and TG numbers

Conclusion: Our study was able to prove substantial positive results with regards to metabolic syndrome post-LSG, with a significant improvement in the glycemic and lipid profile of patients from numbers that are considered dangerous, to those that are in the normal range post-operatively. This improvement is not just due to the weight loss associated with the surgery, but also due to the nature of this procedure.

\section{P061}

Banded Versus Non-banded Roux-en-Y Gastric Bypass for Morbid Obesity: A Systematic Review and Meta-analysis of Randomized Controlled Trials

Saeed Shoar, MD ${ }^{1}$, Zhamak Khorgami ${ }^{2}$, Essa Aleassa, MD ${ }^{1}$, Stacy A Brethauer ${ }^{1}$, Ali Aminian ${ }^{1}$; ${ }^{1}$ Bariatric and Metabolic Institute, Department of General Surgery, Cleveland Clinic, Cleveland, $\mathrm{OH},{ }^{2}$ Department of Surgery, University of Oklahoma, College of Medicine, Tulsa, OK

Introduction: Despite remarkable effects of bariatric surgery in achieving weight loss and improvement of obesity-related comorbidities, efforts still continue to improve the long-term outcomes of bariatric surgery. Banded Roux-en-Y gastric bypass (RYGB) has been continuously scrutinized in comparison to standard (non-banded) RYGB in terms of durability of benefits and band-related complications.

Methods: In an attempt to conduct a meta-analysis of high-quality studies comparing the banded versus non-banded RYGB, we systematically searched the PubMed/Medline and Cochrane Database until September 2018 for the published randomized controlled trials (RCTs) comparing these two procedures. Meta-analysis was performed to pool the data on excess weight loss, food tolerability (including the incidence of postoperative dysphagia and emesis), and postoperative complications.

Results: Three RCTs were eligible to be included into this metaanalysis comprising a total of 494 patients ( 247 in each group). Two RCTs provided 2-year postoperative data and one study reported 5-year data. Age ranged between 21 and 49.8 years and the initial BMI was between 42 and $64.8 \mathrm{~kg} / \mathrm{m}^{2}$. Pooled results on the postoperative outcomes were compared between the two groups (Figure). Excess weight loss was significantly greater in the banded RYGB than in non-banded RYGB (mean difference $5.63,95 \%$ confidence interval (CI) 3.26-8.00). Postoperative food intolerance, emesis, and dysphagia were more commonly seen after the banded RYGB (odds ratio $3.76,95 \%$ CI 2.27-6.24). Nevertheless, major postoperative complications did not significantly differ between the two groups (Figure 1). Conclusion: Findings of this meta-analysis of RCTs indicate that excess weight loss by banded RYGB would be only $5 \%$ greater than that of non-banded RYGB (about 1 point difference in BMI) at the expense of more food intolerance and postoperative vomiting; however, the frequency of postoperative complications is not significantly different. Larger RCTs with longer follow-up time would be necessary to delineate the safety and durability of banded RYGB.

Figure 1. Comparison of excess weight loss outcome (above), food intolerance (middle), and postoperative complications (below) between the banded and non-banded RYGB.

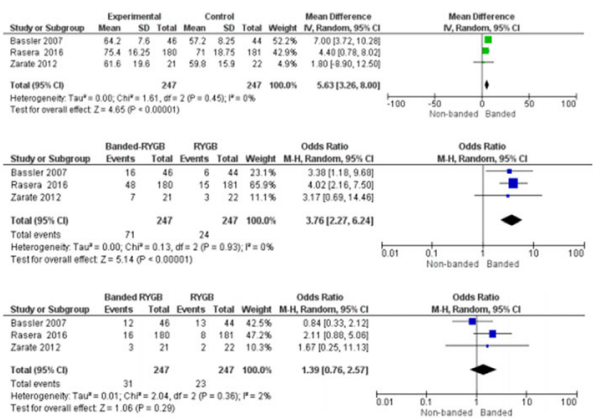


P062

\section{Weight Loss by Obesity Stage: Dual Intra-Gastric Balloon Outcomes}

A. Dorsey, MD, S. Ahmed, MD, J. M Morton, MD, MPH, FACS, FASMBS; Stanford

Introduction: The Reshape ${ }^{\circledR}$ intra-gastric balloon is a saline-filled dual chamber intra-gastric balloon approved by the FDA for weight loss in patients with BMI of $30-40 \mathrm{mg} / \mathrm{kg}^{2}$ who otherwise failed to lose weight with diet and exercise. The intra-gastric balloon promotes weight loss by reducing gastric capacity and enhancing satiety. The aim of this paper is to determine the weight loss attained at 3,6, and 12 months with the intra-gastric balloon among BMI ranges.

Methods: At a single academic center, preoperative, 3 month, 6 month, and 12 month data were prospectively collected from individuals undergoing ReShape ${ }^{\circledR}$ Intra-gastric Balloon placement. Patients were categorized into BMI 30-34.9 (Stage 1), 35-39.9 (Stage 2 ), and $>40$ (Stage 3). Data were analyzed with Student T tests, ANOVAs and Chi square tests.

Results: Forty six patients underwent placement and subsequent removal of the intra-gastric balloon from 2015 - 2018. Sixty five percent of patients were women. Mean age at placement was $48 \mathrm{yrs}$, mean weight and BMI at placement were $344 \mathrm{lbs}$ and $38 \mathrm{~kg} / \mathrm{m}^{2}$. Seventy eight percent of patients were on pre-operative weight loss medications, which included Lomaira, Contrave, Saxenda, and Metformin. There were no differences in percentage of patients with preoperative diabetes (Stage 1-12\% vs Stage 2- 6\% vs Stage 3-9\%, $\mathrm{p}=0.8)$, hyperlipidemia $(17 \%$ vs $12 \%$ vs $45 \%, \mathrm{p}=0.09)$ or OSA ( $18 \%$ vs $29 \%$ vs $36 \%, p=0.5$ ). Mean duration of balloon use was 6.5 months. All stages of obesity had significant weight loss: Stage 1 BMI pre-op, 3, 6, 12 mo: 32.4, 29.2, 28.6, 28.7 ( $\mathrm{p}<0.01$ ); Stage 2, BMI pre-op, 3, 6, 12 mo: 37.5, 33.5, 32.2, 31.5 ( $\mathrm{p}<0.01$ ); Stage 3 BMI pre-op, 3, 6 mo: 48.1, 43.1, 41.3, 40.3 (p <0.01). Stage 1 had the greatest \% excess weight loss (\%EWL) at 3 and 6 months ( 3 mo: Stage $1-61 \%$ vs Stage-2 39\% vs Stage 3-24\%, p < 0.01; 6 mo: $71 \%$ vs $51 \%$ vs $33 \%, \mathrm{p}<0.01)$. No difference in $\%$ EWL at $12 \mathrm{mo}(60 \%$ vs $58 \%$ vs $40 \%(\mathrm{p}=0.5)$. All groups had similar percent decrease in BMI (3 mo: Stage 1- $10 \%$ vs Stage 2- $11 \%$ vs Stage $3-10 \%, p=0.7 ; 6$ mo: $11 \%$ vs $14 \%$ vs $14 \%, \mathrm{p}=0.5 ; 12$ mo: $10 \%$ vs $16 \%$ vs $16 \%$, $\mathrm{p}=0.2)$.

Conclusions: Our study shows significant weight loss following intragastric balloon placement among all stages. Weight loss was sustained even after balloon removal.

\section{P063}

\section{Analysis of Bariatric Surgical Procedures in Adolescents from National Inpatient Sample}

Fereshteh Salimi Jazi, MD ${ }^{1}$, Tamta Chkhikvadze, MD² Junxin Shi, MD, $\mathrm{PhD}^{3} ;{ }^{1}$ University of Texas Medical Branch, ${ }^{2} \mathrm{NYU}$ Langone HospitalBrooklyn, Department of Medicine, NYU School of Medicine, Brooklyn, NY, USA, ${ }^{3}$ Ohio State University, The Research Institute at Nationwide Children's Hospital, Columbus, OH, USA

Purpose: To identify if advancement of minimally invasive surgery and increasing number of trained minimally invasive surgeons had impact on utilization of bariatric surgical procedures in children and adolescents in the era of obesity epidemics.

Methods: We analyzed 10 years of National Inpatient Sample (NIS) throughout 2005-2014yy. We extracted discharges of patients $<20 y$, with associated ICD-9 diagnosis and procedure codes of morbid obesity (278.01, V85.4), open gastric bypass (OGB- 44.31, 44.39, 43.89), laparoscopic gastric bypass (LGB- 44.38), sleeve gastrectomy (SG43.82), laparoscopic gastric banding (LAGB- 44.95) and laparoscopic gastroplasty (LG-44.68). Technical and systemic surgical complications were identified using according ICD-9 codes. All extracted numbers were weighted to national estimates. Trends and available socio-demographics were reviewed. Mean length of stay (LOS) and mean charges (MC) adjusted by inflation index were analyzed.

Results: Throughout the analyzed years total number of major bariatric procedures remained relatively unchanged (Table 1), with substitution of most procedures with SG since 2011 (Figure 1). LAGB and LG procedures have been essentially abandoned. OGB constituted only minor portion $(2.3 \%)$ of total bariatric procedures by $2014.98 \%$ of all bariatric procedures were performed in adolescents $13-19 y y$, and $2 \%$ in children $<12 y$. By 2014 two of the most commonly performed bariatric procedures in adolescents were SG (64\%) and LGB (29\%). Sustained significant female $(76 \%)$ vs. male $(24 \%)$ predominance, with majority of patients being white $(56 \%)$, and privately insured $(65 \%)$, as well as increasing access to treatment for least wealthy patients $(21 \%$ - > $30 \%)$ and those with public insurance $(13.5 \%->30 \%)$ was noted. While procedures were equally distributed between teaching and non-teaching urban hospitals before 2011, majority of cases have shifted to teaching institutions since ( $80 \%$ vs. $20 \%$ ). Only non-reportable number of inpatient deaths and overall $\sim 2-4 \%$ of complication rate was identified, most common being hemorrhage and respiratory complications. No cardiac complications have been reported since 2011, but increased incidents of acute kidney failure were observed. MC have increased $(\$ 46,300$ - $>\$ 54,049)$ and LOS has marginally decreased over the years(2.67- > 2.39).

Conclusion: Despite above described favorable shifts in between procedure types and socioeconomics, our study did not demonstrate increase in utilization of weight loss surgery in adolescents. Bariatric procedures are underutilized but continuously performed in this population, following adult curves. SG has demonstrated to be safe, effective and has been extensively performed since 2011 , constituting $64 \%$ of all adolescent bariatric procedures in 2014. Multidisciplinary efforts should be made to increase identification and referral of eligible candidates for surgery.

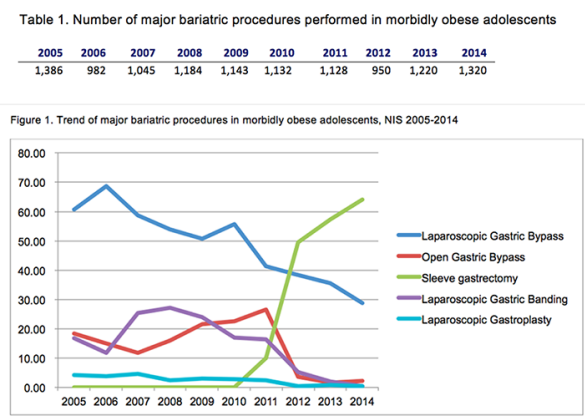




\section{P065}

\section{Newly Reported Complication After Gastric Bypass: Vertical Band Through the Gastrojejunostomy}

\section{Hossam S Alslaim, MD, Renee Hilton, MD; Augusta University}

Roux-en-Y gastric bypass (RYGB) is one of the most common procedures currently performed for surgical treatment of morbid obesity in the United States. Dysphagia and chronic abdominal pain after RYGB for morbid obesity are common and often these complications have multiple etiologies. Determining the exact etiology can be challenging but necessary to provide adequate symptom relief. We present a 39-year-old female who underwent RYGB 10 years ago that was complicated by ulceration, perforation and surgical revision. She presented with progressive dysphagia and abdominal pain. Upper digestive endoscopy revealed a vertical tissue band at the gastrojejunal anastomosis. After complete work up to rule out other causes of abdominal pain and dysphagia, she underwent an interventional endoscopy with resection of the band. At follow up she has had complete symptom resolution. Post-operative GI symptoms after RYGB are common. The differential diagnosis for these presentations is broad and the symptoms alone are poor predictors of the endoscopic pathology. Along with our specific case report we discuss treating dysphagia following RYGB based on literature review. With the increasing number of patients undergoing bariatric procedures, not only bariatric surgeons, but also primary providers, general surgeons, and gastrointestinal physicians must understand the treatment of the variable complications. Endoscopy provides a minimally invasive approach to treating complications after surgery, and many times can avoid the need for additional invasive surgery.
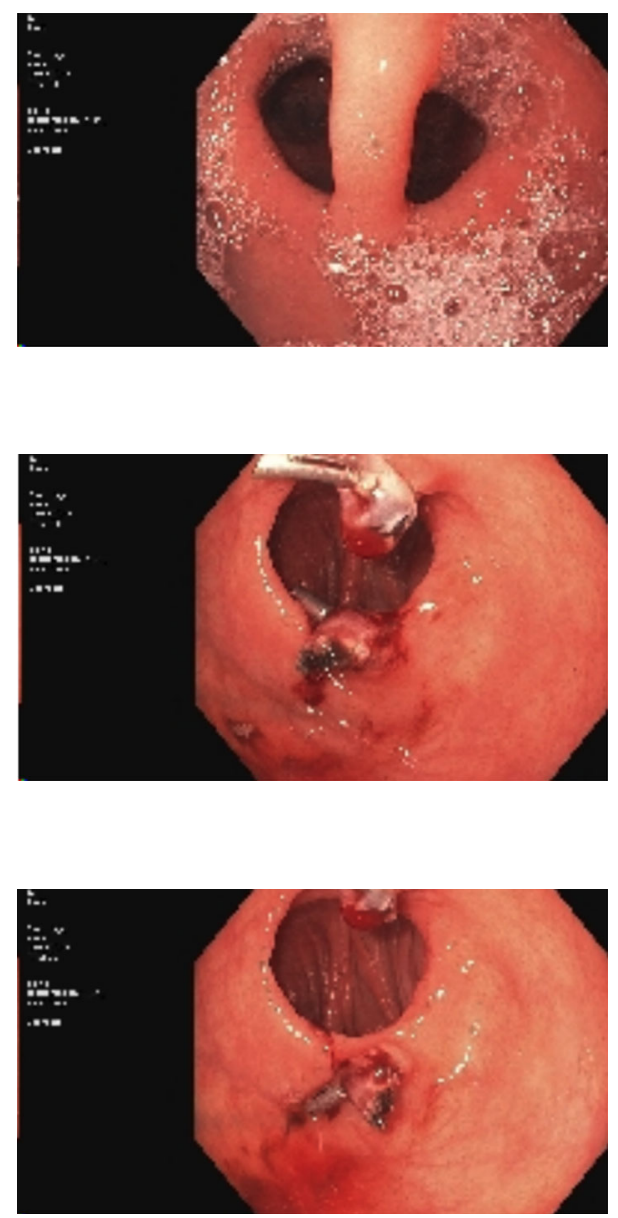

\section{P066}

Ricotta Cheese Disease: Lactobezoar After Roux-en-Y Gastric Bypass

John Mayo, MD, Robert Conrad, MD, Bridget Colgan, MD, William Harjes, MD, Robert Lim, MD; Tripler Army Medical Center

Background: Gastric bezoar is a rare but known complication following Roux-en-Y gastric bypass (RYGB). These bezoars are typically composed of indigestible plant matter and are usually associated with an underlying stricture. Lactobezoars, on the other hand, are composed of compacted milk proteins and gastric secretions that are reported in the literature to occur almost exclusively in neonates. We present the first case to our knowledge of a lactobezoar occurring after bariatric surgery, specifically without any underlying anastomotic stricture. This also appears to be the first case report of a lactobezoar occurring in an adult patient not taking high-density tube feedings and not critically ill.

Case Presentation: 49-year-old female three weeks status post RYGB for class III obesity presented with symptoms of gastric outlet obstruction after having added ricotta cheese several times daily to her post surgery diet of Greek yogurt and protein shakes. The patient underwent upper endoscopy and was found to have a lactobezoar that was successfully removed endoscopically. There was no underlying anastomotic stricture. The patient then tolerated a full liquid diet and was discharged later that same day.

Conclusion: Lactobezoar is a rare diagnosis in adults, reported to occur in those who are critically ill and on high-density gastric tube feedings. This report shows it can occur in the gastric pouch of a RYGB in patients who consume milk-based protein shakes and in this case ricotta cheese. The treatment is via endoscopy and dietary guidance with nutritionist consultation. 


\section{P067}

Role of Preoperative Body Mass Index on Estimated Weight Loss, Total Weight Loss, and Remission of Comorbidities After Bariatric Surgery

Cristian Milla Matute, MD, Maria C Fonseca Mora, MD, Mauricio Sarmiento Cobos, MD, Joel S Frieder, MD, Emanuele Lo Menzo, MD, PhD, FACS, Samuel Szomstein, MD, FACS, FASMBS, Raul J Rosenthal, MD, FACS, FASMBS; Cleveland Clinic Florida

Introduction: Currently, bariatric surgery (BS) is the only long-term treatment for obesity. Demographic, medical and social variables have been thoroughly evaluated as influential on BS outcomes. We found scarcity of literature in regards to the impact of preoperative BMI (Body Mass Index) on BS total weight loss and remission of comorbidities. The aim of this study is to understand the impact of preoperative BMI on BS outcomes.

Methods: After IRB approval, a retrospective review of all patients who underwent bariatric surgery from 2004-2017 was performed. Demographics and comorbidities were analyzed. Patients were assigned into three BMI groups; 30-39.9, 40-49.9, > 50. Total weight loss, excess body mass index loss, and comorbidities were analyzed at a 12-month postoperative follow-up period. Student's T-test and ANOVA was used for means and Chi Square for categorical values, all tests were two-tailed and performed at a significant level of $<0.05$.

Results: A total of 1,389 patients were analyzed. This population was predominantly female $70 \%(\mathrm{n}=972)$, the most common procedure performed was LSG (Longitudinal Sleeve Gastrectomy) $65.3 \%$ $(\mathrm{n}=903)$ and had a mean age of $54 \pm 11$ years. From this total population $42 \%(\mathrm{n}=583)$ were between $30-39.9 \mathrm{~kg} / \mathrm{m}^{2}$ BMI, $43 \%$ $(\mathrm{n}=595)$ in $40-49.9$ BMI and $15 \%(\mathrm{n}=211)$ was above 50 BMI. At 12 months follow-up the category 30-39.9BMI had a 66\% EBMIL (Percentage of Excess Body Mass Index Loss), the category 40-49.9 BMI had 59\% EBMIL and the category above 50BMI had 55\% EBMIL ( $\mathrm{p}=0.02$ ). Regarding Total Weight Loss, category 30-39.9 BMI lost $21.9 \pm 14 \mathrm{~kg}$, category 40-49.9 BMI lost $32.3 \pm 17 \mathrm{~kg}$ and category above 50 BMI lost $49.3 \pm 22 \mathrm{~kg}(\mathrm{p}=0.001)$. When analyzing remission of comorbidities, we observed equal remission rates of Diabetes, High Blood Pressure, and Dyslipidemia amongst all three BMI categories and all were found to have statistical significance.

Conclusions: Bariatric patients with BMI ranging from 30-39.9 km/ $\mathrm{m}^{2}$ had higher \% EBMIL, yet patients with BMI above 50 had greater total weight loss. Remission of comorbidities rates were similar among all categories and equally statistically significant. Although many other variables contribute to the weight loss results, it seems that proportionally the initial BMI has an influence the final weight loss outcome.

\section{P068}

\section{Resolution of Sleep Apnea After Bariatric Surgery}

Babak Katiraee, $\mathrm{MD}^{1}$, Peter Powles, MD, FRACP, FRCPC, $\mathrm{ABSM}^{2}$, Dennis Hong, MD, MSc, FRCSC, FACS ${ }^{1}$, Scott B Gmora, MD, FRCSC, FACS ${ }^{1}$, Mehran Anvari, MB, BS, PhD, FRCSC,

FACS ${ }^{1}$; ${ }^{1}$ Centre for Minimal Access Surgery (CMAS), Hamilton, $\mathrm{ON}, \mathrm{CA},{ }^{2}$ St. Joseph's Healthcare Hamilton

Background: Obesity is one of the risk factors for obstructive sleep apnea (OSA), increasing its risk by tenfold. Positive Airway Pressure (PAP) is a proven method of treating obstructive sleep apnea, but compliance is reported to be less than $50 \%$. Although patients achieve important weight loss after bariatric surgery, literature is conflicting regarding improvement of obstructive sleep apnea symptoms.

Purpose: This study aims to assess the effects of bariatric surgery on sleep apnea symptoms, apnea/hypopnea index (AHI) scores, overnight oximetry and use of PAP treatment.

Methodology: A retrospective chart review was completed of patients who underwent bariatric surgery at our institution between March 2013 and September 2014. We included all patients who had completed a preoperative overnight polysomnography, had diagnoses of OSA, and were on PAP prior to surgery. Patients that did not complete a postoperative polysomnography were excluded.

Results: In total 57 patients were recruited (42 RNYGB and 15 VSG), of which 47 were woman. We observed a decrease in body weight (Kg.) of $24 \%$, and a decrease in BMI of $23 \%$ in accordance with their post bariatric state. With respect to their sleep apnea, there was a decrease in AHI of 59\% and a decrease in their Arousal Index by $34 \%$. The lowest recorded $\mathrm{Sa} 02$ levels increased by $11 \%$, while Hypoxia Time decreased by $59 \%$. This lead to a decrease in the requirement of PAP use by $65 \%$. Of the patients remaining on PAP, the pressures required were reduced by $25 \%$. The average interval between surgery and follow-up polysomnography was 22 months.

Conclusions: Bariatric surgery significantly improved OSA scores and decreased PAP use in relation to decreases in BMI and body weight. It also improved oxygen saturation levels and decreased Arousal Index scores leading to better sleep quality. We consider that OSA follow up should be done routinely after bariatric surgery for patients previously on PAP.

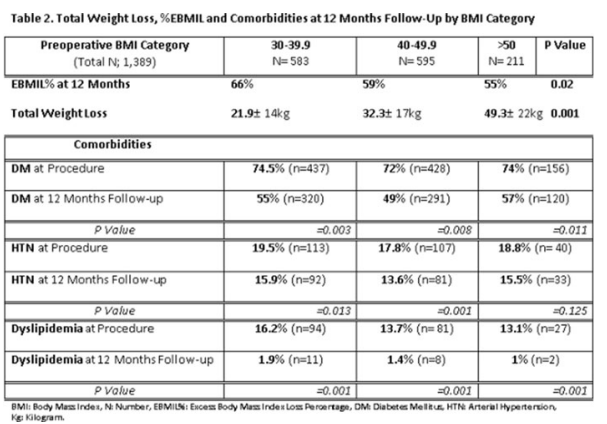




\section{P069}

\section{'Gastric Bypass With or Without Cholecystectomy?' A 10 Year} Perspective'

Robert M Cunningham, MD, Jason E Kuhn, DO, Marcus Fluck, James T Dove, Katherine T Jones, Ryan D Horsley, MD, Jon D Gabrielsen, MD, Anthony T Petrick, MD, David M Parker, MD; Geisinger

Introduction: The aim of the study was to evaluate outcomes of selective concurrent cholecystectomy and long term biliary outcomes after Roux-en-Y Gastric Bypass (RYGB). Methods: We performed a retrospective analysis of patients who underwent Laparoscopic RYGB (LRYGB) between 2008 and 2018. Chi square, Fisher's exact, or Wilcoxon ranksum tests were used to compare outcomes.

Results: 3004 patients underwent a RYGB (LRYGB $\mathrm{n}=2453$, open RYGB $\mathrm{n}=533$ ). At time of review, $54.7 \%(n=1643)$ of patients had undergone a cholecystectomy. $31.1 \%$ of patients $(n=933)$ had a cholecystectomy prior to RYGB, $16.9 \%(n=509)$ had a concurrent cholecystectomy and $51.9 \%(\mathrm{n}=1562)$ did not have a cholecystectomy. After LRYGB, 12.9\% $(\mathrm{n}=202)$ underwent an interval cholecystectomy. Of those who underwent LRYGB, $29.9 \%(\mathrm{n}=735)$ had a prior cholecystectomy. Those who underwent concurrent cholecystectomy/LRYGB $(\mathrm{n}=328)$ were compared with LRYGB alone $(\mathrm{n}=1231)$. The concurrent cholecystectomy group were significantly older, higher percentage of females, higher preoperative BMI, higher Charlson Comorbidity index and a higher medication count. The two groups demonstrated no significant difference in BMI nadir, length of stay, complications or mortality.

Conclusions: The study supports that a higher percentage of bariatric patients will undergo cholecystectomy. Long-term follow-up demonstrates a higher rate of interval cholecystectomy than previously reported. Concurrent cholecystectomy can be performed safely without an increase in length of stay, complications or 30-day mortality.

Table 1

Laparoscopic RYGB versus combined RYGB/cholecystectomy

\begin{tabular}{llll}
\hline & $\begin{array}{l}\text { LRYGB } \\
\text { only }\end{array}$ & $\begin{array}{l}\text { Concurrent } \\
\text { cholecystectomy }\end{array}$ & \\
\hline & $\mathrm{n}=1231(\%)$ & $\mathrm{n}=328(\%)$ & $p$ value \\
Female & $910(73.9)$ & $266(81.1)$ & 0.008 \\
Age,years & $43.9(11.5)$ & $45.9(11.2)$ & 0.008 \\
BMI,initial & $47.0(7.7)$ & $48.1(8.3)$ & 0.032 \\
BMI,nadir & $31.2(7.3)$ & $31.3(7.6)$ & 0.948 \\
\%BMI Change, nadir & -33.3 & -34.6 & 0.117 \\
Comorbidity Score & $4.4(2.2)$ & $4.8(2.4)$ & 0.025 \\
Medications & $5.4(3.5)$ & $5.9(3.6)$ & 0.0279 \\
Length of stay, days & $1.7(1.2)$ & $1.8(1.1)$ & 0.116 \\
Readmission, 30 Day & $126(10.2)$ & $43(13.1)$ & 0.1368 \\
Any reintervention & $40(3.3)$ & $14(4.3)$ & 0.370 \\
Minor Complication & $89(7.2)$ & $31(9.5)$ & 0.180 \\
Major Complication & $26(2.1)$ & $12(3.7)$ & 0.107 \\
Mortality, 30 Day & $1(0.1)$ & $1(0.3)$ & 0.377 \\
\hline
\end{tabular}

\section{P070}

\section{Single Institution Outcomes of ASMBS' Employing New Enhanced Recovery Goals in Bariatric Surgery (ENERGY) Initiative}

Sara Monfared, MD, Marisa Embry, RN, Ambar Banerjee, MD, Dimitrios Stefanidis, PhD, MD; Indiana University

Introduction: Enhanced recovery programs have proven effective after various operation but their value after minimally invasive bariatric surgery is less clear and potentially harder to demonstrate given already speedy recovery. Employing New Enhanced Recovery Goals in Bariatric Surgery (ENERGY) is a new program of the American Society for Metabolic and Bariatric Surgery that aims to improve postoperative outcomes. Our objective was to assess the impact of ENERGY implementation on postoperative outcomes after bariatric surgery and identify strategies that have the highest opioid-sparing effect.

Methods: Prospectively collected data on the Metabolic and Bariatric Surgery Accreditation and Quality Improvement Program (MBSAQIP) database were reviewed. Patien outcomes after laparoscopic roux-en-Y gastric bypass (RYGB) and laparoscopic sleeve gastrectomy (SG) during the year before and the year after implementation of ENERGY (July 2017) were compared. Data analyzed included age, gender, procedure type, length of stay (LOS), 30-day complications, readmissions, and reoperations. Multilinear regression analysis was performed to control for age, gender and procedure type as possible confounders. Average morphine equivalent dosage used during their hospital stay was compared between those who received regional anesthetic block and those who did not. Results: Outcomes from 531 patients were analyzed, 206 before and 325 after ENERGY implementation. Mean LOS decreased by 0.2 days after ENERGY $(\mathrm{p}=0.085)$. The percentage of patients with LOS $\geq 4$ days also decreased from $9.2 \%$ to $6.5 \%(p=0.24)$. Postoperative complication, readmission and reoperation rates were similar between the two groups (table 1) even after controlling for age, gender and procedure type as possible confounders. Patients who received a local anesthetic regional block used an average of $30.5 \mathrm{mg}$ of morphine less during their hospital stay compared with those who did not $(\mathrm{p}=0.052)$.

Table 1 Comparison between control and ENERGY group
\begin{tabular}{|l|l|l|l|}
\hline Variables & Control group & ENERGY group & P value \\
\hline N & 206 & 325 & \\
\hline Age, mean \pm SD & $46.0 \pm 11.8$ & $45.1 \pm 11.4$ & 0.354 \\
\hline Female (\%) & $156(76 \%)$ & $271(83 \%)$ & 0.030 \\
\hline Procedure type & & & \\
\hline RYGB (\%) & $146(71 \%)$ & $200(61 \%)$ & 0.028 \\
\hline SG (\%) & $60(29 \%)$ & $125(39 \%)$ & \\
\hline Postoperative Outcomes & & & \\
\hline LOS, days, mean \pm SD & $1.9 \pm 1.7$ & $1.7 \pm 1.1$ & 0.085 \\
\hline Patients' LOS $\geq 4$ days (\%) & $19(9.2 \%)$ & $21(6.5 \%)$ & 0.240 \\
\hline Complication rate (\%) & $17(8.3 \%)$ & $25(7.7 \%)$ & 0.816 \\
\hline Readmission rate $(\%)$ & $16(7.8 \%)$ & $27(8.3 \%)$ & 0.824 \\
\hline Reoperation rate (\%) & $5(2.4 \%)$ & $5(1.5 \%)$ & $0.521 *$ \\
\hline *Fisher's Exact Test Used & & & \\
\hline
\end{tabular}

Conclusions: Promising downward trends in patient LOS, complication, readmission and reoperation rates were observed after the implementation of ENERGY in our bariatric program that warrant further study and analysis within a larger sample. Regional anesthesia limits opioid use after surgery and should be considered. 


\section{P071}

\section{Use of Intraoperative Provocative Leak Testing in Elective} Laparoscopic Bariatric Procedures

Bhavani Pokala, MD, Priscila R Armijo, MD, Corrigan L Mcbride, MD, Dmitry Oleynikov, MD; University of Nebraska Medical Center

Introduction: Intraoperative leak testing (IOLT), surgical drains, and swallow studies are commonly employed for early detection of gastrointestinal leaks following bariatric surgery, however, their utility remains widely debated. Our aim was to examine the frequency and outcomes of IOLT with laparoscopic sleeve gastrectomy (LSG) and laparoscopic roux-en-y gastric bypass (LRYGB).

Methods: The 2016 Metabolic and Bariatric Surgery Accreditation and Quality Improvement Project (MBSAQIP) database was analyzed for adults who underwent primary elective LSG or LRYGB. Emergent cases, those unable to be followed for 30 days, and patients with previous foregut or revisional surgery were excluded. Cases with IOLT and without IOLT (NIOLT) were compared within each procedure group. Statistical analysis was performed using IBM SPSS $25.0, \alpha=0.05$.

Results: 164,567 LSG patients $($ IOLT $=126,291$; NIOLT $=38,276)$ and 70,148 LRYGB patients (IOLT $=65,007$; NIOLT $=5,141$ ) were included in the study. LSG with IOLT had higher rates of staple line reinforcement $(69.2 \%$ vs $61.3 \%$; $p<.001)$, oversewing $(22.5 \%$ vs $21.6 \% ; \mathrm{p}<.001)$, and surgical drain placement $(21.3 \%$ vs $9.5 \%$; $\mathrm{p}<.001)$ than LSG NIOLT. For LSG, both bariatric and general surgeons performed IOLT in majority of cases $(77.4 \%$ and $66.9 \%$, respectively). Mean operative time for LSG with IOLT was $77.2 \pm 37.1 \mathrm{~min}$ vs $64.7 \pm 30.7$ min without. For LRYGB, cases with IOLT also had a higher rate of surgical drain placement $32.8 \%$ vs $21.8 \% ; \mathrm{p}<.001)$ than NIOLT. IOLT was performed routinely during LRYGB by bariatric $(92.6 \%)$ and general surgeons $(97.5 \%$; $\mathrm{p}<.001)$. Mean operative time for LRYGB with IOLT was $116.8 \pm 51.7 \mathrm{~min}$ vs $106.0 \pm 47.8 \mathrm{~min}$ NIOLT. For both procedures, leak rates were higher with IOLT (LSG: $0.4 \%$ vs $0.3 \% ; \mathrm{p}<.001$, LRYGB: $1.0 \%$ vs $0.4 \%, \mathrm{p}<.001$ ), however, logistic regression revealed that IOLT was not independently associated with post-operative leak.

Conclusions: IOLT is used in the vast majority of LSG and LRYGB cases. Our results show a statistically significant increase in operative time without significant decrease in post-operative leak rates in cases when IOLT was performed. Therefore, we suggest that IOLT may be unnecessary in elective LSG and LRYGB cases. In the future, MBSAQIP should consider capturing results of IOLT and discriminating between recurrent or previously undiagnosed leak in the postoperative setting.

\section{P072}

Single Anastomosis (Mini) Gastric Bypass versus Sleeve Gastrectomy: Meta-analysis of Randomized Clinical Trials

Zhamak Khorgami ${ }^{1}$, Saeed Shoar, MD ${ }^{2}$, Philip R Schauer, MD $^{2}$, Stacy A Brethauer, $\mathrm{MD}^{2}$, Ali Aminain, $\mathrm{MD}^{2} ;{ }^{1}$ University of Oklahoma, College of Medicine, Tulsa, OK, ${ }^{2}$ Bariatric and Metabolic Institute, Department of General Surgery, Cleveland Clinic, Cleveland, $\mathrm{OH}$

Introduction: While sleeve gastrectomy (SG) is the most common bariatric procedure worldwide, single anastomosis (mini) gastric bypass (SAGB) is rapidly gaining acceptance. As mid- to long-term effects of SAGB on weight and obesity-related comorbidities become available, a systematic review of the literature to summarize the available high quality data is required to direct future investigations and clinical practice.

Methods: A systematic literature review was conducted in PubMed/ Medline and Cochrane Database through September 2018 to identify RCTs which compared weight loss outcome, diabetes mellitus (DM) and hypertension (HTN) remission, and postoperative complications between SAGB and SG.

Results: 3 RCTs encompassing 595 patients (297 SAGB patients and 298 LSG patients) were eligible to be enrolled into this meta-analysis. The longest follow-up was 5 years for 2 RCTs and 3 years for 1 RCT. Weight loss outcome was pooled based on percentage of total weight loss in 2 studies (significant in favor of SAGB) and percent excess weight loss in 2 studies (not significantly different). Moreover, while SAGB had a mild superiority in DM remission (effect size $=0.16$, $\mathrm{p}=0.02$ ), the difference was not significant for HTN resolution (effect size $=0.17, p=0.06)$. Postoperative adverse events were comparable between the 2 procedures (odds ratio $=0.80, \mathrm{p}=0.42$ ). Conclusion: Findings of this meta-analysis suggest that SAGB is associated with more weight loss compared with SG. Furthermore, while SG has remarkable effects on DM and HTN, SAGB is associated with about $15 \%$ higher chance of remission for both comorbidities. Both procedures are comparably safe.

Figure. Comparison of weight loss outcome (above: \%weight loss and \% excess weight loss), resolution of comorbid diabetes mellitus and hypertension (middle), and postoperative adverse events (below)

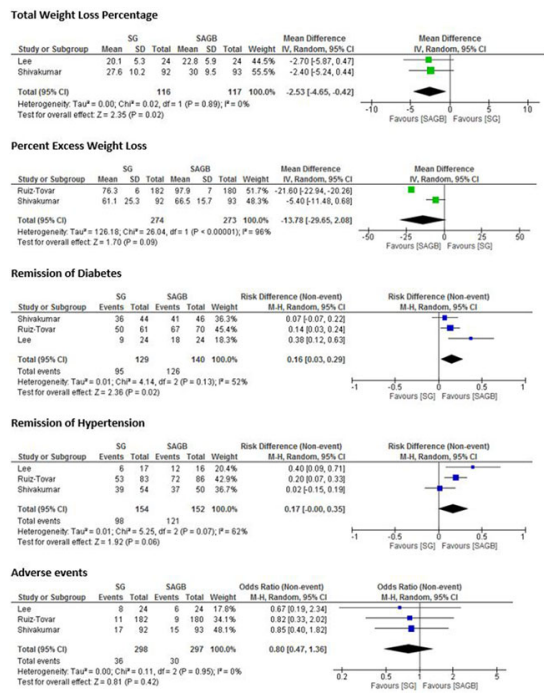




\section{P073}

The Impact of Impulsivity on Weight Loss after Bariatric Surgery - A Systematic Review

Charleen Yeo, Danson Yeo, Rachel Chen, Gabriel Low, Jiazheng Yeo, Myint Oo Aung, Jaideepraj Rao, Aaryan Koura, Sanghvi Kaushal, Adrian Toh; Tan Tock Seng Hospital, Singapore

Background: Psychopathology has been associated with poorer outcomes after bariatric surgery. Impulsivity has been shown to be associated with obesity through links to pathological eating behavior such as binge eating. Recent literature suggests that impulsivity is linked to poorer outcomes post-bariatric surgery. The aim of this systematic review is to synthesize the current evidence on the impact of impulsivity on post-bariatric surgery weight loss.

Methods: A literature review was performed in August 2018. Original studies investigating the relationship between impulsivity and weight loss post-bariatric surgery were evaluated. Results: Six studies with a total of 911 patients were analyzed. There were two casecontrol, two prospective observational and two retrospective observational studies. The post-operative follow-up ranged from 0.5 to 12 years. The most common measure of trait impulsivity was the Barratt Impulsivity Score (BIS), which was used in five studies. Only one study demonstrated a direct association between BIS scores and post-operative weight loss. Two studies reported an indirect effect of impulsivity on weight loss, mediated via pathological eating behavior. Scores specific to state impulsivity showed a significant impact on post-surgical weight loss.

Conclusion: Impulsivity may adversely affect post-operative outcomes after bariatric surgery. However, this may be specific to state impulsivity rather than trait impulsivity. Patients with a higher state impulsivity may benefit from closer follow-up post-bariatric surgery.

\section{P074}

\section{Liver Abscesses without leaks in Revisional Bariatric Surgeries: Management and Outcomes}

A Zaw, MD, G Khair, MD, FACS, K Singh, MD, FACS, FASMBS, MBA; St Agnes

Introduction: Obesity is a global epidemic and has become a public health crisis in the United States. Bariatric surgery is considered the most effective way to achieve long term weight loss. Roux-n-Y gastric bypass (RYGB) and sleeve gastrectomy are two of the most commonly performed bariatric procedures. Most significant complications are staple line bleeding, stricture, and staple line leak. Formation of liver abscess without intra-abdominal infectious process is a rare complication after bariatric surgery.

Methods and Procedures: Two cases of liver abscesses after revisional bariatric surgery. Results: We report two cases of pyogenic liver abscesses after laparoscopic gastric bypass revision and sleeve gastrectomy. These were managed by percutaneous drainage and antibiotics.

Conclusion: Liver abscess is a rare complication of revisional bariatric surgery and could be due to multiple factors; it could be due to a clinical leak, subclinical leak, pyelophlebitis of the porto-mesenteric veins or from the combination of parenchymal liver injury and bacterial seeding from staple line when leak is not evident. Most liver abscesses respond to percutaneous drainage and antibiotic administration.

\section{P076}

Diabetes Improvement and Resolution Following Laparoscopic Sleeve Gastrectomy Versus Sleeve Gastrectomy with Loop Bipartition

Vladimir Grubnik, MD, Professor, Vadim Ilyashenko, $\mathrm{PhD}$, Viktor Grubnyk, Stanislav Usenok, PhD; Odessa National Medical University

Introduction: The prevalence of type 2 diabetes is growing internationally. Obesity is an independent risk factor for development of type 2 diabetes mellitus (DM) and other metabolic disorders. The aim of this study was to compare the effectiveness of laparoscopic sleeve gastrectomy (LSG) with new surgical procedure: sleeve gastrectomy with loop bipartition (LSG + LB).

Methods: 28 obese patients with DM type 2 were divided in two groups: I group (15 patients) consisted from 9 women and 6 men age 36 to 64 years, mean body mass index (BMI) was $49.7 \pm 8.6 \mathrm{~kg} / \mathrm{m}^{2}$. LSG was performed in all patients of the I group. II group (13 patients) consisted from 7 women and 6 men, age 39-69 years, mean BMY was $51.8 \pm 7.4 \mathrm{~kg} / \mathrm{m}^{2}$. LSG + LB was performed in all patients of the II group. These was no statistically significant difference between two groups in demographic, BMY, and comorbidities. Excess weight loss percentage (\%EWL), the total weight loss percentage (\% TWL) and diabetes improvement were analyzed.

Results: There were no serious complications and mortality in the both groups. After 18 months the patients of the II group lost more \% TWL and \%EWL and the difference was statistically significant $(\mathrm{p}<0.05) . \% \mathrm{EWL}$ in the I group was $69.7 \pm 9.2 \%$, in the patients of the II group $-80.7 \pm 13.4 \%(\mathrm{p}<0.05)$. Resolution of DM type 2 was in $7(47 \%)$ patients from the I group and in $10(77 \%)$ from the II group $(\mathrm{p}<0.01)$.

Conclusion: Sleeve gastrectomy with loop bipartition is more effective than LSG in the treatment of DM type 2 associated with obesity.

\section{P077}

\section{Lipid Profiles Following Sleeve Gastrectomy Vs Roux-En-Y Gastric Bypass - A Meta-Analysis}

Caleb J Ba Mendoza ${ }^{1}$, Aisha Tabba ${ }^{1}$, Osamuyi Idubor ${ }^{1}$, Joel Miller ${ }^{1}$, Aliu Sanni, MD, FACS ${ }^{2}$; ${ }^{1}$ Philadelphia College of Osteopathic Medicine, ${ }^{2}$ Eastside Bariatric and General Surgery LLC

Introduction: Morbid obesity is associated with increased mortality secondary to cardiovascular risk factors including diabetes, hypertension and dyslipidemia. The most effective intervention for morbid obesity and associated risk factors is bariatric surgery. The aim of this study is to evaluate changes in lipid profiles following Laparoscopic Gastric Sleeve (LSG) or Laparoscopic Roux-en-Y Gastric Bypass (LRYGBP) one year after surgery.

Methods and Procedures: A systematic review of studies between 2005 and 2017 was conducted through Pubmed to identify studies with comparative data on lipid levels between Sleeve Gastrectomy and Roux-en-Y Gastric bypass postoperatively at 1 year. Outcomes analyzed were total cholesterol, LDL cholesterol, HDL cholesterol, triglycerides, and BMI. The results are expressed as standard difference in means with standard error. Statistical analysis was done using fixed-effects meta-analysis to compare the mean value of the two groups. (Comprehensive Meta-Analysis Version 3.3.070 software; Biostat Inc., Englewood, NJ).

Results: Four out of 101 studies were quantitatively assessed and included in this metaanalysis. Among the studies, 1405 patients underwent Laparoscopic Roux-en-Y Gastric Bypass and 198 patients underwent Laparoscopic Sleeve Gastrectomy. There was significantly lower cholesterol $(-0.667 \pm 0.102 ; \mathrm{p}<0.05)$ and LDL levels $(-0.714 \pm 0.103$, $\mathrm{p}<0.05$ ) for those patients who underwent LRGYBP as compared to LSG. HDL levels ($0.107 \pm 0.001, \mathrm{p}=0.287)$, Triglyceride levels $(-0.149 \pm 0.101, \mathrm{p}=0.140)$, and BMI $(0.119 \pm 0.163, \mathrm{p}=0.463)$ at 12 months postoperatively were similar in both groups. Conclusion: Lipid profiles are significantly reduced following LRYGBP when compared to LSG in bariatric patients. 
P078

\section{Bariatric Surgery Outcomes as a Function Of Patient Characteristics and Subjective Experience}

Jamil S Samaan, BS, Evan T Alicuben, MD, Elaine Qian, BS, Kulmeet Sandhu, MD, Adrian Dobrowolsky, MD, Kamran Samakar, MD; University of Southern California

Introduction: Primary outcomes of bariatric surgery are often defined as excess weight loss and comorbidity resolution. Sparse literature exists on the relationship between psychosocial characteristics and patient outcomes. Similarly, little is known about the relationship between patient satisfaction after surgery and psychosocial characteristics. We sought to examine subjective measures of satisfaction as a function of personal and behavioral characteristics.

Methods and Procedures: A retrospective chart review was conducted on patients who underwent laparoscopic sleeve gastrectomy (LSG) and roux-en-y gastric bypass (RYGB) from August 2002 to November 2017 at a single institution, multi-surgeon, tertiary care academic hospital. Preoperative surveys were reviewed and a telephone questionnaire was performed for gathering demographic and personal data. The telephone survey consisted of a standardized satisfaction survey using a leikert scale to assess response to the following statements: bariatric surgery improved my quality of life (Q), I am satisfied with my surgical outcomes (S), I would undergo bariatric surgery again (A). Inclusion criteria was patients who completed a telephone interview. Exclusion criteria was those who did not complete the interview. Fishers exact test was used for statistical analysis.

Results: A total of 514 patients were included. $76 \%$ were female, $71 \%$ underwent RYGB and $29 \%$ underwent LSG. Median age at time of surgery was 47.0 (95\% $\mathrm{Cl} 45.6-48.8)$. Average follow up was 6.99 years $(\mathrm{SD}=4.30)$.

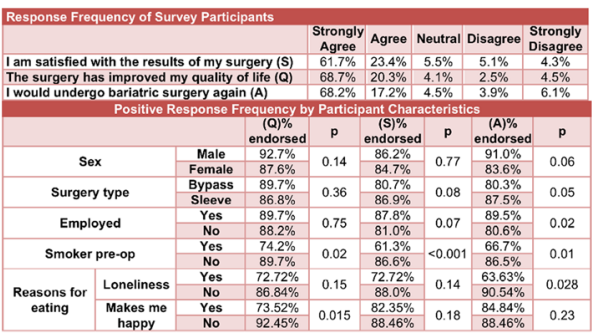

Conclusion: Bariatric surgery patients are overwhelmingly satisfied largely independent of psychosocial characteristics. There were some notably interesting findings that predicted lower satisfaction scores including preoperative smoking status and the reasons for overeating.

\section{P079}

\section{Retrograde Jeujunojeujnal Intussusception Post Roux En Y Gastric Bypass}

Ahmed Abdelhady, MD, FACS, MRCS, Ghaleb Aboalsamh, MBBS, SBGS, Msc; National Guard Health Affairs

The patient was a 46 year-old female who presented to the emergency room with a sudden severe abdominal pain as well as nausea and vomiting that began the day before after a meal. She is 3 years post retrocolic RYGBP. On examination, the patient was well nourished, afebrile, and with normal vital signs. She was in moderate distress with pain out of proportion to the physical examination. Her abdomen was soft, and there were no signs of peritoneal irritation or distension. Laboratory data were unremarkable. Computed tomography scan revealed a long segment obstructive retrograde small bowel intussusception adjacent to the jejuno-jejunal anastamosis and associated with complete proximal small bowel obestruction. The patient was immediately taken to the operating room.

\section{P080}

\section{Comparison of Long-Term Weight Loss After Laparoscopic Sleeve Gastrectomy and Laparoscopic Roux-En-Y Gastric Bypass from a Single Center in Thailand}

Pattharasai Kachornvitaya, MD, Suthep Udomsawaengsup, MD, Chadin Tharavej, MD, Suppaut Pungpapong, MD, Krit Kitisin, MD, Patpong Navicharern, MD; Chula Minimally Invasive Surgery Center, Department of Surgery, Faculty of Medicine, Chulalongkorn University, Bangkok, Thailand

Introduction: A sleeve gastrectomy (LSG) and laparoscopic Roux-en-Y gastric bypass (LRYGB) are two of the most widely used bariatric procedures in Thailand but comparison of long-term outcomes are still limited. The aim of this study is to compare the degree of long-term weight loss after the laparoscopic Roux-en-Y gastric bypass (LRYGB) and sleeve gastrectomy (LSG).

Materials and Methods: A retrospective analysis was performed for all patients who underwent LSG or LRYGB between April 2005 and May 2018 at a single institution with a least 1 year to 5 years of follow up. Demographic data comprised age, sex, preoperative body weight, excess body weight, body mass index (BMI) and co-morbidities. The primary outcomes were percentage total weight loss and percentage excess weight loss. The secondary outcomes was postoperative complications.

Results: Of the total 368 patients, 163 underwent primary LSG and 205 patients underwent LRYGB between April 2005 and May 2018. Preoperative BMI in the LSG group was significantly more than the LRYGB group $\left(53.7 \pm 12.3 \mathrm{~kg} / \mathrm{m}^{2}\right.$ vs $48.4 \pm 8.3 \mathrm{~kg} / \mathrm{m}^{2}$, $\mathrm{p}<0.001$ ). Co-morbidities between LSG and LRYGB were similar. Mean percentage of excess weight loss (\%EWL) at 5 years was $36.7 \pm 51.0 \%$ in the LSG group vs $62.3 \pm 25.3 \%$ in the LRYGB group ( $p=0.04)$. Mean percentage of total weight loss (\%TWL) at 5 years was $19.2 \pm 23.0 \%$ in the LSG group vs. $27.9 \pm 11.8 \%$ in the LRYGB group $(\mathrm{p}=0.252)$. At 5 years, no significant difference was found in the percentage of patients who had $>50 \%$ EWL between groups. Overall complications were $4.3 \%$ in the LSG group vs. $11.7 \%$ in the LRYGB group $(\mathrm{p}=0.011)$. The mortality rate was $0 \%$. Conclusion: Our center revealed LRYGB had better results than LSG in terms of percentage excess weight loss at 5 years. On the other hand, LSG had lower overal complications than LRYGB.

\section{P081}

\section{Early Signs of Postoperative Hemorrhage in Bariatric Surgery}

Michael Farrell, MD, MS, Zugui Zhang, PhD, Bayo Gbadebo, MBA, Caitlin Halbert, DO, MS; Christiana Care Health System

Introduction: The aim of this study is to identify potential risk factors or early indicators, specifically related to perioperative blood pressure, and its association with perioperative hemorrhage in the bariatric population. Laparoscopic bariatric surgery in the United States has been steadily increasing over the past several years. Between 2011 and 2015, the annual number of cases has increased by $24 \%$. Although rare, hemorrhagic complications (HC) occur at a rate of $1-5 \%$ and can lead to significant morbidity and mortality. By identifying factors which may place a patient at a higher chance of $\mathrm{HC}$, surgeons can potentially mitigate those risks. These modifications could reduce morbidity and limit the requirement of transfusions or reoperations.

Methods and Procedures: A retrospective case-control series was performed to include all patients who underwent either laparoscopic sleeve gastrectomy (SG) or laparoscopic Roux-en-Y gastric bypass (GB) between 2015 and 2018 at a single bariatric center of excellence. Propensity matching was completed for age, gender, procedure, body mass index, and comorbidities including diabetes, hypertension, heart disease, anticoagulation/ antiplatelet use and smoking status. Maximum and minimum systolic blood pressures (SBP), diastolic blood pressures (DBP), and mean arterial pressures (MAP) were compared between groups at time of admission, intraoperative, and during the remainder of initial hospital stay. Chi square and odds ratio estimates were used to compare groups.

Result: A total of 1488 procedures were performed with 1203 de novo SG, and 285 de novo GB. Revisional and robotic bariatric cases were excluded from the study. HC occurred in 11 $(0.74 \%)$ total patients, $8 \mathrm{GS}$ and $3 \mathrm{~GB}$. Three patients required transfusion transfusion of one unit packed red blood cells (pRBCs). Eight patients required at least 2 units pRBCs and all required operative treatment. There was no difference between the procedure performed and risk of developing $\mathrm{HC}$ (p- $=0.96$ ). Additionally, there was no difference between groups for preoperative or intraoperative blood pressure findings or postoperative DBP or MAP ( $\mathrm{p}=0.3-0.95)$. After accounting for baseline hypertension, mean postoperative maximum (102 vs $146 \mathrm{mmHg}$ ) and minimum SBP (102 vs $112 \mathrm{mmHg}$ ) were significantly lower in patients who developed $\mathrm{HC}(\mathrm{p}<0.001)$

Conclusion: Relative decreased SBP in the immediate post-operative period may be an early sign of $\mathrm{HC}$ in bariatric patients. There was no associated increased risk of developing $\mathrm{HC}$ with preoperative or intraoperative blood pressure changes 


\section{P082}

\section{Challenges Associated with the Bariatric Surgery in Poland}

Tomasz Stefura ${ }^{1}$, Jakub Dros ${ }^{1}$, Artur Kacprzyk ${ }^{1}$, Katarzyna Chlopas ${ }^{1}$, Oksana Skomarovska $^{1}$, Marta Krzysztofik ${ }^{1}$, Katarzyna Major ${ }^{2}$, Mateusz Rubinkiewicz, $\mathrm{MD}^{3}$, Mateusz Wierdak, $\mathrm{MD}^{3}$, Michal Wysocki, $\mathrm{MD}^{3}$, Magdalena Pisarska, $\mathrm{MD}^{3}$, Piotr Malczak, $\mathrm{MD}^{3}$, Michal Pedziwiatr, $\mathrm{MD}, \mathrm{PhD}^{3}$, Andrzej Budzynski, $\mathrm{MD}, \mathrm{PhD}^{3}$, Piotr Major, $\mathrm{MD}, \mathrm{PhD}^{3}$; ${ }^{1}$ Students' Scientific Group at 2nd Department of General Surgery, Jagiellonian University Medical College, Krakow, Poland, ${ }^{2}$ Faculty of Health Sciences, Jagiellonian University Medical College, Krakow, Poland, ${ }^{3}$ 2nd Department of General Surgery, Jagiellonian University Medical College, Krakow, Poland

Introduction: Due to constantly growing demand for surgical treatment of obesity in Poland, there is a need for creating new bariatric centers and further improving presently active ones. Therefore, we aimed to identify which stages conducting peri-operative care and organizing modern bariatric center pose the greatest challenge currently in Poland.

Materials and Methods: An anonymous survey was designed and distributed to Polish bariatric surgeons. Our questionnaire was divided into three parts: demographic characteristics, difficulties assessed on a scale 1-5 associated with peri-operative care for bariatric patients and organization and running of bariatric centers in which participants are currently working.

Results: Overall, 70 surgeons and surgical residents from 17 Polish surgical centers participated in our survey. The most difficult element of the pre-operative care was compliance with recommendation to cease smoking $(3.47 \pm 1.28)$. The most difficult obstacle during postoperative care period was implementation of the ERAS protocol $(2.27 \pm 1.31)$. Funding for the bariatric treatment was obtained exclusively from National Health Fund by 60 respondents $(85.7 \%)$ working in 15 different bariatric centers $(88.2 \%)$. Among elements of bariatric infrastructure access to operating theater equipment sized for morbidly obese patients was reported to be the most difficult $(3.8 \pm 1.68)$.

Conclusion: Pre-operative recommendations including smoking, physical activity or weight-loss are difficult to execute. Introducing ERAS protocol based peri-operative care in bariatric departments remains to be difficult in polish reality. The development of specialized bariatric centers included in the centralized register and equipped with specialized infrastructure for obese patients seems to be the next step to improve post-operative results.

\section{P083}

\section{Impact of Preoperative Opioid Use on Surgical Outcomes Following Bariatric Surgery}

Chenchen Tian, $\mathrm{BHSc}^{1}$, Azusa Maeda, $\mathrm{PhD}^{2}$, Allan Okrainec, MDCM, MHPE, FACS, FRCSC ${ }^{2}$, Timothy Jackson, MD, MPH, FRCSC, FACS ${ }^{2} ;{ }^{1}$ University of Toronto, Faculty

of Medicine, ${ }^{2}$ University Health Network, Division of General Surgery

Introduction: With rising opioid-related morbidity and mortality, it is critical to understand the implications chronic opioid use on surgical outcomes. Preoperative opioid use before elective abdominal and emergency general surgery has been associated with increased length of stay, costs of care, and readmission rates. Thus, preoperative opioid use represents a potentially modifiable risk factor and a novel target to improve surgical quality of care. The objective of this study was to explore the implications of preoperative opioid use in patients undergoing bariatric surgery on surgical outcomes.

Methods: A single-center retrospective cohort study was performed using the Metabolic and Bariatric Surgery Accreditation and Quality Improvement Program (MBSAQIP) and Ontario Bariatric Network (OBN) databases. Patients were selected if they underwent any bariatric procedure between January 1, 2010 and March 31, 2018. Preoperative opioid use, coded as a binary exposure variable, was retrospectively identified from the home medication list in the preoperative evaluation. The primary outcome was the rate of postoperative complications within 30-days of surgery. Secondary outcomes included hospital length of stay, intraoperative complications, and operative time. Continuous variables were compared using Student t-test or Wilcoxon rank-sum test, as appropriate. Categorical variables were compared using Chi squared tests.

Results: Overall, 2479 patients met inclusion criteria. Among those, $291(11.7 \%)$ patients used opioids preoperatively. The majority case mix consisted of Roux-en-Y (88\%) and sleeve gastrectomy (11.9\%). Opioid users (compared to opioid-naïve) had longer operative time $(151 \mathrm{~m}$ vs $144 \mathrm{~m}, \mathrm{p}<.05)$ and higher rates of intraoperative complications $(3.8 \%$ vs $2.0 \%, \mathrm{p}<.05)$.

In a subset of 1266 patients evaluated postoperatively, $123(9.7 \%)$ patients used opioids preoperatively. The majority case mix consisted of Roux-en-Y (85.8\%) and sleeve gastrectomy (14.1\%). In this cohort, opioid users (compared to opioid-naïve) had higher but not statistically significant rates of postoperative complications $(9.0 \%$ vs 6.3 ) and statistically significantly longer hospital length of stay (2.6 vs 2.1 days, $\mathrm{p}<.001$ ).

Conclusions: Based on MBSAQIP and OBN registry data, opioid users (compared to opioid-naïve) had prolonged operative time, higher rates of intraoperative complications, and prolonged hospital length of stay after bariatric surgery. Preoperative opioid use may complicate perioperative and postoperative management in bariatric procedures. Further investigation is needed to evaluate preoperative opioid use as a potentially modifiable risk factor to improve bariatric quality of care and postoperative outcomes. 


\section{P084}

Risks of Sleeve Gastrectomy Versus Gastric Bypass Among Patients with Kidney Disease

John R Montgomery, MD ${ }^{1}$, Seth A Waits, MD ${ }^{1}$, Justin B Dimick, MD, $\mathrm{MPH}^{2}$, Dana A Telem ${ }^{2}$, ${ }^{1}$ University of Michigan, Dept Transplant Surgery, ${ }^{2}$ University of Michigan, Center for Healthcare Outcomes \& Policy

Objective: Roux-en-Y gastric bypass (RYGB) and laparoscopic sleeve gastrectomy (LSG) have similar long-term weight-loss and comorbidity-improvement among obese patients with chronic kidney disease (CKD) or end-stage renal disease (ESRD). As such, the decision to proceed with RYGB versus LSG can be controversial and is often left to surgeon and/or patient preference. No published, generalizable data exists about the perioperative risks of these operations. To inform operative decision-making, we performed an analysis of perioperative safety of RYGB versus LSG in obese patients with CKD or ESRD using a national registry capturing $>95 \%$ of bariatric operations.

Methods: Patients with CKD (creatinine $\geq 2 \mathrm{mg} / \mathrm{dL}$, but not on dialysis) or dialysis-dependent ESRD who underwent primary, laparoscopic-RYGB or LSG between 2015-2016 were identified from the Metabolic and Bariatric Surgery Accreditation and Quality Improvement Program participant use file. The primary outcome was a composite variable of death and severe, life-threatening complications within 30-days of operation. Logistic regression was used to compare adverse outcomes between patients who underwent RYGB versus LSG. Subgroup analyses were then performed among CKD and ESRD patients.

Results: During the study period, 2,357 primary, laparoscopic bariatric operations were performed on obese patients with CKD $(\mathrm{n}=1521,64.5 \%)$ or ESRD $(\mathrm{n}=836,35.5 \%)$; of these, 1,704 $(72.3 \%)$ were LSG and $653(27.7 \%)$ were RYGB. After adjusting for patient age, smoking status, hypertension, diabetes, and functional status, there was a trend towards RYGB association with the primary outcome of death or severe, life-threatening complications (6.7vs4.9\%), but this was not statistically significant (aOR 1.41[0.962.07], $\mathrm{p}=0.078$ ). Major contributors to the composite primary outcome between RYGB and LSG patients were reoperation (4.0vs3.1\%, $\mathrm{p}=0.3)$, major infection $(1.8 \mathrm{vs} 0.6 \%, \mathrm{p}=0.005)$, transfusion $\geq 3$ units $(1.2 \mathrm{vs} 0.5 \%, \mathrm{p}=0.046)$, and leak $(0.9 \mathrm{vs} 0.4 \%, \mathrm{p}=0.084)$. In the CKD subgroup, RYGB was associated with progression to renal failure requiring dialysis $(2.8 \mathrm{vs} 0.8 \%, \mathrm{p}=0.002)$, and major infection (2.0vs0.5\%, p = 0.006). In the ESRD subgroup, RYGB was associated with myocardial infarction $(0.7 \mathrm{vs} 0.0 \%, \mathrm{p}=0.033)$.

Conclusion: In a contemporary cohort of bariatric surgeries, nearly one-third of obese CKD and ESRD patients undergo RYGB. Our analysis shows increased risk of major infection and transfusion $\geq 3$ units among RYGB patients when compared to LSG. Furthermore, CKD patients undergoing RYGB are three times more likely to experience progressive renal failure requiring dialysis. Given the clinical similarity of LSG and RYGB in terms of weight-loss and comorbidity-improvement among CKD and ESRD populations, we strongly recommend LSG as the preferred bariatric surgery unless otherwise contraindicated.

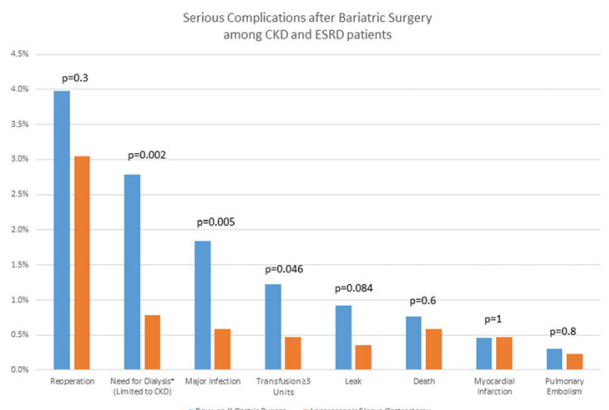




\section{P085}

The Use of Bariatric Surgery for Treatment of Nonalcoholic Fatty Liver Disease: A Systematic Review and Meta-Analysis

Yung Lee, BHSc ${ }^{1}$, Aristithes Doumouras, MD, MPH, FRCSC ${ }^{1}$, James $\mathrm{Yu}_{\mathrm{BHSc}}{ }^{1}$, Karanbir Brar, BHSc ${ }^{2}$, Laura Banfield, MLIS, MHSc ${ }^{1}$, Scott Gmora, MD, FRCSC, FACS ${ }^{1}$, Mehran Anvari, MD, PhD, FRCSC, FACS ${ }^{1}$, Dennis Hong, MD, MSc, FRCSC,

FACS ${ }^{1} ;{ }^{1}$ McMaster University, ${ }^{2}$ University of Toronto

Introduction: We aim to conduct a systematic review and metaanalysis to critically evaluate the benefits and harms of bariatric surgery on Nonalcoholic fatty liver disease in patients with obesity. NAFLD has become one of the most common chronic liver diseases in the world, affecting 20-30\% of the general population in Western countries and costing the US healthcare system $\$ 32$ billion annually. There is a rapidly growing body of evidence demonstrating the complete resolution of NAFLD following the sustained weight loss induced by bariatric surgery.

Methods: MEDLINE, EMBASE, CENTRAL, and Web of Science were searched up to May 2018. Studies were eligible for inclusion if a study compared biopsy results of NAFLD before and after bariatric surgery in patients with obesity. Exclusion criteria were studies with $<10$ patients or cirrhosis. Primary outcomes were biopsy-confirmed resolution of NAFLD (steatosis, inflammation, ballooning degeneration, fibrosis) and NAFLD activity score (NAS); secondary outcome was worsening of NAFLD (liver side effects) and change in liver volume after surgery. Grading of Recommendations, Assessment, Development, and Evaluation (GRADE) to assess quality of evidence. Pooled proportions and mean differences (MD) were calculated using the random effects meta-analysis and Freeman-Tukey double arsine transformation. Heterogeneity was quantified using the $\mathrm{I}^{2}$ statistic and publication bias was assessed using a funnel plot.

Results: Among 1,695 studies identified, 32 cohort studies involving a total of 3,093 biopsies met the inclusion criteria. Bariatric surgery resulted in a biopsy-confirmed resolution of steatosis in $66 \%(95 \%$ Confidence Interval (CI), 56-75\%), inflammation in 50\% (95\% CI, $35-64 \%$ ), ballooning degeneration in $76 \%$ (95\% CI, 64-86\%), and fibrosis in $40 \%$ of patients (95\% CI, $29-51 \%$ ). NAS was significantly reduced after bariatric surgery (Mean Difference (MD) 2.39; 95\% CI, 1.58-3.20; $\mathrm{p}<0.001)$. Liver volume by magnetic resonance imaging and showed significant reductions in liver volume 6 months after bariatric surgery (MD $469.35 \mathrm{~cm}^{3}$; 95\% CI, 297.02-641.68, $\mathrm{p}<0.001)$. However, bariatric surgery resulted in new or worsening features of NAFLD such as fibrosis in $12 \%$ of patients $(95 \% \mathrm{CI}$, 5-20\%). The overall GRADE quality of evidence was very low.

Conclusions: Bariatric surgery performed on obese patients with NAFLD leads to complete resolution of NAFLD in a substantial proportion of patients. However, there is a minor chance of patients developing new or worsening histological features of NAFLD. High quality randomized controlled trials are needed to confirm the therapeutic benefits of bariatric surgery for NAFLD.

\section{P086}

The Role of Serum Ferritin as Marker of Chronic Inflammation in Patients Undergoing Bariatric Surgery

Cristian Milla Matute, MD, Rene Aleman, MD, Maria C Fonseca Mora, MD, Francisco Ferri Abreu, MD, Emanuele Lo Menzo, MD, PhD, FACS, FASMBS, Samuel Szomstein, MD, FACS, FASMBS, Raul J Rosenthal, MD, FACS, FASMBS; Cleveland Clinic Florida

Introduction: Ferritin is a cellular protein for iron storage, and it has been recently recognized as an acute phase reactant and marker of acute and chronic inflammation. Along with transferrin and the transferrin receptor, ferritin is a member of the protein family that orchestrates cellular defense against oxidative stress and processes. Obesity is a well-known chronic inflammatory state. The aim of this study is to analyze the variations of serum ferritin as an alternate potential marker of inflammation in severely obese subjects that undergo bariatric surgery.

Methods: After IRB approval, we retrospectively reviewed the electronic charts of all patients who underwent bariatric surgery at Cleveland Clinic Florida from 2005 to 2016 . We included all patients with serum ferritin measurements at the time of the surgery and a control measurement at 12 and 24 months after bariatric surgery. Demographics, type of procedure and comorbidities were reviewed. High values were determined $>120 \mathrm{ng} / \mathrm{dl}$ as a mean for both genders. Patients with hematologic pathology, cancer, and autoimmune diseases were excluded from this analysis. SPSS software was used to apply a t-test for means.

Results: From the of 1,260 patients analyzed with serum ferritin measurements before, surgery and at 12 and 24 months after surgery at follow up, a total of $24.7 \%(n=312)$ patients had abnormally high ferritin measurements before surgery. This population had a mean age of 57 years, a mean BMI of $42 \pm 7$ before bariatric surgery, and Female gender was predominant with $62 \%(\mathrm{n}=192)$. Regarding the type of procedure laparoscopic sleeve gastrectomy (LSG) was performed in 58\% $(\mathrm{n}=183)$, Roux-N-Y Gastric Bypass $(\mathrm{RNYGB})$ in $33 \%(\mathrm{n}=103)$ and Laparoscopic Gastric Banding (LGB) in $8 \%$ $(\mathrm{n}=25)$. At the time of bariatric surgery, the serum measurements of ferritin had a mean value of $153.7 \pm 27$ and after surgery a mean value of $137 \pm 23(\mathrm{p}=0.32)$.

Conclusions: Our study shows no statistically significant difference in serum ferritin level before and after surgery. Although many different indicators of chronic inflammation vary after bariatric surgery, Ferritin by itself does not seem to be helpful as surrogate for chronic inflammation measurements in this patient population. Further studies with a higher number of patients should be performed on an in order to better understanding the role of ferritin as an inflammatory marker.

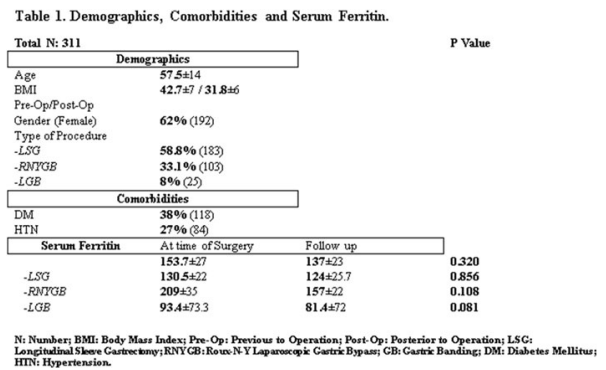


P087

\section{Early Outcomes Following Bariatric Surgery: Primary Vs Revisional Surgery}

Connal Robertson-More, $\mathrm{MD}^{1}$, Aryan Modasi, MD, $\mathrm{MSc}^{2}$, Jerry T Dang, MD ${ }^{2}$, Noah Switzer, MD, $\mathrm{MPH}^{3}$, Daniel W Birch, MD, $\mathrm{MSc}^{2}$, Shahzeer Karmali, MD, MPH ${ }^{2} ;{ }^{1}$ University Hospitals Coventry and Warwickshire NHS Trust, ${ }^{2}$ University of Alberta, ${ }^{3}$ Ohio State University

Introduction: Secondary to insufficient weight loss, weight regain or complications related to primary bariatric surgery, a growing proportion of individuals are seeking revisional bariatric surgery. Despite this, limited knowledge regarding the complication profile of revisional compared to primary bariatric procedures exists.

Methods and Procedures: The MBSAQIP datasets from 2015 and 2016 were combined in a single database and individuals undergoing primary and revisional laparoscopic Roux-en-Y gastric bypass (LRYGB) or laparoscopic sleeve gastrectomy (LSG) were identified based on CPT codes. Standard demographic information and complications within 30 days of surgery were analyzed for differences between those undergoing primary versus revisional procedures.

Results: Within the combined 2015/2016 MBSAQIP database, 278,869 and 20,159 individuals underwent primary and revisional procedures, respectively. Proportionally, LSG represented $71.7 \%$ and $60.1 \%$ of the primary and revisional procedures, respectively. Individuals undergoing revisional procedures had a greater mean age (44.7 vs. 47.8 yrs), a lower pre-operative BMI (45.5 vs. $42.3 \mathrm{~kg} / \mathrm{m}^{2}$ ), represented a greater proportion of females, and displayed similar preoperative comorbidity profiles outside of a lower proportion of individuals with diabetes, and sleep apnea. Additionally, a greater proportion of individuals with gastroesophageal reflux disease was observed in the revisional groups.

With respect to early peri-operative complications, revisional LRYGB was associated with a significantly higher mortality rate ( 0.015 vs. $0.26 \%, p=0.025)$. Both revisional groups had a greater serious complication rate $(2.7$ vs. $4.4 \%, \mathrm{p}<0.001$ and 6.4 vs. $10.4 \%$, $\mathrm{p}<0.001$, pLSG vs rLSG and pLRYGB and rLRYGB), in particular, a greater post-operative bleed, leak and 30-day readmission, reoperation and reintervention rates. Multivariable logistic regression analysis determined that revisional LRYGB procedures were independently predictive of serious complications (OR $1.52,95 \%$ CI 1.40 to $1.65, \mathrm{p}<0.001$ ), leaks (OR $2.40,95 \%$ CI 1.97 to $2.92, \mathrm{p}<0.001$ ) and bleeds (OR 1.44, 95\% CI 1.23 to $1.69, \mathrm{p}<0.001$ ). Whereas revisional LSG procedures were independently predictive of serious complications (OR 1.46, 95\% CI 1.33 to $1.61, \mathrm{p}<0.001$ ) and leaks (OR 2.14, 95\% CI 1.77 to $2.59, \mathrm{p}<0.001$ ).

Conclusions: Utilizing the MBSAQIP database differences in demographic and comorbidity profiles were found between individuals undergoing primary vs revisional bariatric surgery. Further, revisional LRYGB procedures were observed to have a higher perioperative mortality and to be independently associated with the risk of serious complications, bleeds and leaks. On the other hand, revisional LSG was independently associated with the risk of serious complications and leaks.

\section{P088}

Utility of Inflammatory Markers in Detection of Perioperative Morbidity After Bariatric Procedures - Multicenter Study

Piotr Major, MD, $\mathrm{PhD}^{1}$, Michal Wysocki, MD ${ }^{1}$, Tomasz Stefura ${ }^{1}$, Jakub Dros ${ }^{1}$, Artur Kacprzyk ${ }^{1}$, Katarzyna Chlopas ${ }^{1}$, Katarzyna Major $^{2}$, Piotr Malczak ${ }^{1}$, Magdalena Pisarska ${ }^{1}$, Michal Pedziwiatr ${ }^{1}$, Andrzej Budzynski, Professor ${ }^{1} ;{ }^{1}$ 2nd Department of General Surgery, Jagiellonian University Medical College, ${ }^{2}$ Faculty of Health Sciences, Jagiellonian University Medical College, Krakow, Poland

Introduction: We aimed to determine if there are correlations between of C-reactive protein, procalcitonin and white blood count measured in first, second and third postoperative day after bariatric surgeries and postoperative morbidity (1-month and 12-months), length of hospital stay (LOS) and hospital readmissions.

Methods and Procedures: Retrospective cohort study of patients who underwent laparoscopic sleeve gastrectomy (SG), Roux-en-Y gastric bypass (GB) or mini-gastric bypass (MGB) for morbid obesity in seven referral bariatric centers. Patients were divided into two groups: complicated group - patients with postoperative complications (in 1 month or 12 months postoperative period) and noncomplicated group - patients without postoperative morbidity. Primary endpoint: to determine utility of routinely tested inflammatory markers in prognosis of perioperative (30-days) and postoperative (12-months excluding first 30-days) morbidity rates. Secondary endpoints: utility of inflammatory markers to determine risk of LOS after bariatric surgeries and readmissions.

Results: 2125 patients, 1448 females (68\%) and 677 males (32\%) in median age of 43 (35-52) years were included. Postoperative morbidity was 155 patients (7.29\%), of who 112 cases occurred in first postoperative month and 43 cases after first postoperative month during 12-months follow-up. Sex, age, BMI and co-morbidities did not differ complicated and non-complicated cases. In complicated group LOS was significantly longer as well as readmission rate. CRP (OR: 1.02, 95\%CI: 1.01-1.03), WBC (1.23, 1.15-1.32), PCT (17.18, 3.09-95.46) on POD1 used as continuous variables were significantly increasing OR for perioperative morbidity. ROC analysis indicated suggested cut-off point of CRP on POD1 indicating for risk of perioperative morbidity for $23.8 \mathrm{mg} / \mathrm{L}$, WBC for $10 \times 103 / \mathrm{mm}^{3}$, and PCT for $0.22 \mathrm{ng} / \mathrm{mL}$. In case of postoperative morbidity inflammatory parameters on POD1 were not significantly related to ORs (p-values respectively: $0.713,0.189,0.889$ ). CRP (OR: 1.02 , 95\%CI: 1.01 1.03), WBC (1.10, 1.02-1.20), PCT (4.7, 1.12-19.64) were increasing OR for prolonged LOS. ROC analysis indicated suggested cut-off point of CRP on POD1 indicating for risk of prolonged LOS for $35 \mathrm{mg} / \mathrm{L}$, WBC for $8.9 \times 103 / \mathrm{mm}^{3}$, PCT for $0.05 \mathrm{ng} / \mathrm{mL}$. CRP (OR: 1.01, 95\%CI: $1.00-1.03)$, WBC $(1.21,1.09-1.33)$ on POD1 were significantly increasing OR for readmissions, while PCT was not (6.67, 0.97-45.80). ROC analysis indicated suggested cut-off point of CRP on POD1 indicating for risk of readmissions for $94 \mathrm{mg} / \mathrm{L}$, WBC for $14.5 \times 103 / \mathrm{mm}^{3}$.

Conclusion: Even moderate increase in inflammatory parameters on POD1 should alert surgeon for possibility of increased chance for perioperative morbidity, prolonged LOS and hospital readmissions. 
P089

\section{Internal Hernia Following Roux-En-Y Gastric Bypass: Our} Institution's 5 Year Experience

Victoria Needham, MD ${ }^{1}$, Jazmin Juarez ${ }^{2}$, Diego Camacho, $\mathrm{MD}^{1} ;{ }^{1}$ Montefiore Medical Center, ${ }^{2}$ Albert Einstein College of Medicine

Introduction: We investigated the incidence of internal hernia (IH) in patients undergoing abdominal exploration at any interval following Roux-en-Y gastric bypass. Of patients found to have IH, we evaluated their clinical and radiographic presentations leading up to operative re-intervention, as well as the technical aspects of their initial bypass, in efforts to elucidate predictors of this bariatric morbidity.

Methods: We used a single-institution database from 2013-2017 to conduct a review of 213 cases of abdominal exploration (diagnostic laparoscopy or exploratory laparotomy) in patients with a history of bariatric surgery.

Results: 110 patients had a history of Roux-en-Y gastric bypass (11 open, 99 laparoscopic). In this group, upon operative re-exploration, 29 patients $(26.1 \%)$ were found to have $\mathrm{IH}$ via one of the defects created by the prior bypass surgery. Of these, 15 underwent antecolic configuration of the roux limb and 11 underwent retrocolic configuration ( 3 unknown) at their index operation. All patients presented with abdominal pain, while physical exams ranged from mild tenderness to peritonitis. The mean white blood cell count at presentation was 9.4 (SD 3.4). 69\% of patients had a CT scan with at least one finding concerning for internal hernia: swirl sign (58.6\%), mesenteric edema (41.4\%), free fluid $(20.1 \%)$ and jejunojejunostomy (JJ) to right of midline (17.2\%). 5 patients $(17.2 \%)$ had a definitive small bowel obstruction diagnosed on preoperative CT scan, defined as oral contrast cutoff at a transition point in the small bowel. Of patients with an internal hernia with available operative records, 1 patient herniated through a JJ mesenteric defect not closed at initial operation, 6 via a closed JJ defect, 8 via an unclosed Petersen's defect, 9 via a closed Petersen's defect, and 3 via a transverse mesocolic defect. All defect closure that was performed during initial bypass surgery was noted to be done using nonabsorbable polyester sutures.

Conclusions: In our population, IH was found in over a quarter of patients who underwent abdominal exploration following Roux-en-Y gastric bypass. Abdominal pain and CT findings concerning for internal hernia appear to be valuable factors in predicting the presence of IH. However physical exam, leukocytosis and complete bowel obstruction on CT appear to be less reliable as grounds for suspicion. Closure of mesenteric defects did not appear to prevent IH, however further investigation is needed to determine the role of surgical technique in the risk of developing eventual $\mathrm{IH}$.

\section{P090}

\section{Effects of Social And Behavioral Risk Factors on Bariatric Surgery Success}

Jamil S Samaan, BS, Evan T Alicuben, MD, Elaine Qian, BS, Yousaf Malik, BS, Stephanie Chang, BS, Kamran Samakar, MD; University of Southern California

Introduction: Bariatric patients are a unique population whose personal characteristics, behavioral patterns and social risk factors are poorly understood. A greater understanding of this patient population may lead to improved whole person care. We aim to examine the biographical, social, and medical profile of our bariatric patient population and determine if any of these factors correlate with weight loss success after surgery.

Methods and Procedures: A retrospective chart review was conducted on patients who underwent laparoscopic sleeve gastrectomy (LSG) and roux-en-y gastric bypass (RYGB) from August 2002 to November 2017 at a single institution, multi-surgeon, tertiary care academic hospital. Preoperative surveys were reviewed for gathering demographic and personal data. Inclusion criteria was patients who completed preoperative surveys. Exclusion criteria was those who did not complete preoperative surveys. Fisher's exact test was used for statistical analysis.

Results: 513 patients were included in the study. $72.7 \%$ were female, the average age at the time of surgery was $47(\mathrm{SD}=11.98)$, average BMI at the time of surgery was $44.7(\mathrm{SD}=8.61)$ and the average follow up was 5.5 years $(\mathrm{SD}=4.09)$. Results are reported as percentage of patients achieving greater than $50 \%$ excess weight loss.

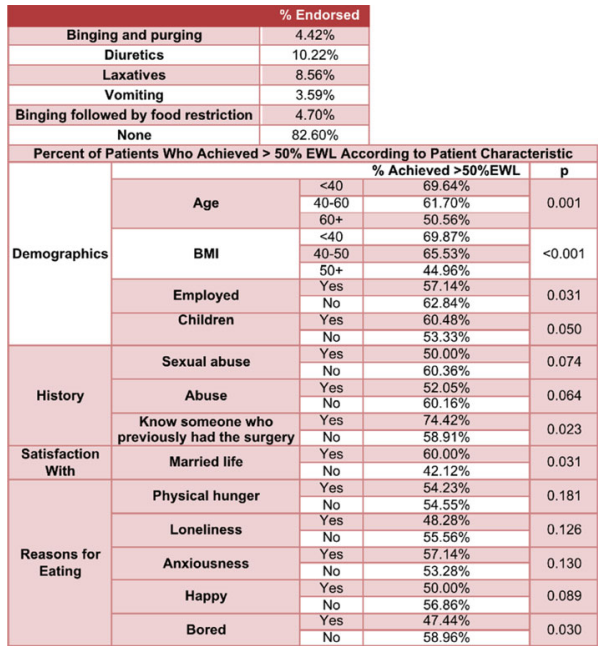

Conclusion: Demographic data for our patient population is similar to published reports. Weight loss surgery success was improved in younger patients, those who had known someone to previously undergo bariatric surgery, and in patients with lower starting BMI. Boredom as the reason for eating was associated with decreased rates of surgical success. 
P091

\section{What Makes Bariatric Operation Difficult - Result of National Survey}

Tomasz Stefura ${ }^{1}$, Mateusz Rubinkiewicz, MD ${ }^{2}$, Mateusz Wierdak, $\mathrm{MD}^{2}$, Jakub Dros ${ }^{1}$, Artur Kacprzyk ${ }^{1}$, Oksana Skomarovska ${ }^{1}$, Marta Krzysztofik $^{1}$, Katarzyna Chlopas ${ }^{1}$, Michal Wysocki, MD ${ }^{2}$, Magdalena Pisarska, $\mathrm{MD}^{2}$, Piotr Malczak, $\mathrm{MD}^{2}$, Michal Pedziwiatr, $\mathrm{MD}, \mathrm{PhD}^{2}$, Andrzej Budzynski, MD, $\mathrm{PhD}^{2}$, Piotr Major, $\mathrm{MD}, \mathrm{PhD}^{2}$; ' ${ }^{1}$ Students'

Scientific Group at 2nd Department of General Surgery, Jagiellonian University Medical College, Krakow, Poland, ${ }^{2}$ 2nd Department of General Surgery, Jagiellonian University Medical College, Krakow, Poland

Introduction: The most commonly performed bariatric procedures include laparoscopic sleeve gastrectomy (LSG), laparoscopic Rouxen-Y gastric bypass (LRYGB) and mini gastric bypass, also known as one anastomosis gastric bypass (MGB-OAGB). A study comparing the degree of difficulty of those procedures could serve as a guide for decision making in bariatric surgery. Moreover, it would help to further improve training programs and curriculums for general surgery trainees.

Materials and Methods: An anonymous internet-based survey was designed to evaluate subjective opinions of surgeons and surgical residents in training in Poland. It covered baseline characteristics of the participant, incidence of technical difficulties during bariatric operations in groups of patients based on the Body Mass Index (BMI), difficulty of LSG, MGB-OAGB, LRYGB and particular stages of each operation assessed on a scale 1-5.

Results: Overall, 70 surgeons and residents from 16 Polish surgical centers participated in our survey. Surgeons reported the highest incidence of technical difficulties during bariatric operation in patients with BMI above 60 . The incidence of difficulties seems to correlate with increasing BMI. Mean difficulty degree of LSG was $2.34 \pm 0.89$. The reinforcing staple line with sutures was considered most difficult stage of this operation $(3.17 \pm 1.19)$. The LRYGB operation had an average difficulty level of $3.87 \pm 1.04$. Creation of the gastrojejunostomy was considered the most difficult stage of LRYGB with mean difficulty level $(3.68 \pm 1.16)$. Responders to our survey assessed mean degree of difficulty of MGB-OAGB's as $2.34 \pm 0.97$. According to participating surgeons creating the gastrojejunostomy is the most difficult phase of this operation $(3.68 \pm 1.16)$

Conclusion: The LSG is perceived by surgeons as relatively easy operation and good to start with. The LRYGB was considered to be the most technically challenging procedure among operations included in our survey. Operative stages, which require intra-abdominal suturing with laparoscopic instruments seem to be the most difficult phases of any operation.

\section{P092}

\section{Bariatric Surgery in Patients with BMI Greater Than 70 and/} or Age Greater Than 70: Outcomes Analysis

David M Pechman, MD ${ }^{1}$, Ruben Salas, $\mathrm{MD}^{1}$, Corin M Kinkhabwala, $\mathrm{BA}^{2}$, David Weithorn ${ }^{1}$, MD, Diego R Camacho, MD ${ }^{1}$; ${ }^{1}$ Montefiore Medical Center, ${ }^{2}$ Albert Einstein College of Medicine

Introduction: Bariatric surgery offers patients tremendous benefits to their short and long-term health and their quality of life. Careful patient selection and consideration of risk is especially critical in elective surgical procedures. Risk aversion is important, however "high risk" patients may have the greatest potential to benefit from weight-loss surgery and improvement of comorbid disease. Evidencebased medicine is instrumental in the assessment of risk versus benefit, however data is lacking for several high risk patient populations, including those at the extremes of BMI or age. This study assessed morbidity and mortality data for patients with BMI greater than $70 \mathrm{~kg} / \mathrm{m}^{2}$ or age greater than 70 years.

Methods: Patients were selected from operative logs at Montefiore Medical Center from 2014-2017. Patients were included if they underwent primary sleeve gastrectomy (SG) or Roux-en-Y gastric bypass (GB). Patients with preoperative BMI greater than $70 \mathrm{~kg} / \mathrm{m}^{2}$ were assigned to the BMI $70+$ cohort. Patients age 70 and above were assigned to the AGE $70+$ cohort. All other patients were assigned to the control cohort. Length of stay and 30-day morbidity and mortality were assessed.

Results: 3451 patients underwent non-revisional bariatric surgery, including $2300(66.6 \%)$ SG and 1151 (33.3\%) GB. The BMI $70+$ cohort included $34(1.0 \%)$ patients, 30 SG and 4 GB. The AGE $70+$ cohort included $11(0.3 \%)$ patients, too few for comparison. The BMI $70+$ patients were younger $(36.2$ vs 41.6 years, $\mathrm{p}=0.009$ ) and more frequently male $(35.3 \%$ vs $16 \%, \mathrm{p}=0.002)$ than control patients, but demographics were otherwise similar. Rates of comorbid conditions were similar as well. Operative time was similar for BMI70 + undergoing SG (61.5 vs $67.5 \mathrm{~min}, \mathrm{p}=0.248)$ and GB (112 vs $107 \mathrm{~min}, \mathrm{p}=0.778$ ) relative to control. BMI70 + was associated with increased incidence of pulmonary embolism $(2.9 \%$ vs $0.1 \%$, $\mathrm{p}=0.029)$ and readmission $(8.9 \%$ vs $3.2 \%, \mathrm{p}=0.061)$. BMI70 + and control patients had similar lengths of stay (2.1 vs 1.8 days, $\mathrm{p}=0.097)$ and comparably low rates of surgical site infection (0 vs $0.9 \%, \mathrm{p}=0.942)$, acute coronary syndromes $(0 \%$ vs $0.06 \%$, $\mathrm{p}=0.98)$, pneumonia ( 0 vs $0.03 \%, \mathrm{p}=0.990)$, renal failure (none), and mortality (0\% vs $0.09 \%, \mathrm{p}=0.971)$.

Conclusion: BMI70 + patients undergoing bariatric surgery had increased rates of pulmonary embolism and readmission, however overall major morbidity and mortality were similar to control patients. Further study is warranted to help guide patient selection and operative planning for patients at the extremes of BMI and age. We next plan to assess preoperative factors and outcomes using national MBSAQIP and NSQIP databases. 


\section{P093}

Development of Consensus-Derived Quality Indicators for Laparoscopic Sleeve Gastrectomy

Shannon Stogryn, MSc, MBBS, FRCSC, Alistair Sharples, MBChB, MSc, FRCS, Krista Hardy, MSc, MD, FRCSC, FACS, Ashley Vergis, MMEd, MD, FRCSC, FACS; University of Manitoba

Introduction: Synoptic operative reporting has gained popularity due to the poor overall quality of dictated narrative reports. Bariatric surgery is a rapidly expanding field and sleeve gastrectomy is the most common bariatric procedure. The objective was to systematically develop a list of operative report quality indicators for a laparoscopic sleeve gastrectomy (LSG) to generate validated items to include in a synoptic operative report for LSG.

Methods: A Delphi protocol was used to determine quality indicators for a LSG report. Bariatric surgeons across Canada were recruited with physician key stakeholders to participate via a secure web-based platform. We aimed for one representative bariatric surgeon from all Royal College of Physicians and Surgeons Regions in Canada. Participants initially submitted quality indicators for a LSG. Suggested quality indicators were assessed and grouped by theme. Items were then rated on a 5-point Likert scale in subsequent rounds. For consensus, a score of $70 \%$ (mean 3.5/5) or greater indicated inclusion of an item and $30 \%$ (mean 1.5/5) or less denoted exclusion. Elements scoring $30-70 \%$ consensus were re-circulated in subsequent rounds to generate the final list of quality indicators.

Results: Seven bariatric surgeons were invited. We one representative from all regions in Canada. The 3 multidisciplinary invitees were comprised of: 1 academic minimally invasive/acute care surgeon, 1 tertiary abdominal radiologist, and gastroenterologist with expertise in endoscopic management of bariatric complications. The overall survey response rate was $90.0 \%(9 / 10)$ and identified 61 potential quality indicators for consideration. In the second-round survey 53 items reached inclusion consensus (see Table 1).

\section{TABLE 1 - QUALITY INDICATORS FOR LSG}

\begin{tabular}{lrl}
\hline Headings & \# items & Example \\
\hline Team demographics & \multicolumn{1}{c}{11} & Surgeon \\
Patient demographics & 5 & BMI \\
Pre-operative events & 1 & Weight lost on pre-op diet \\
Intra-op details & 14 & Leak test \\
Sleeve formation & 11 & \# stapler firings \\
Closure details & 10 & Port closure \\
Post-op details & 1 & Post-operative condition
\end{tabular}

Conclusion: This study established consensus-derived multidisciplinary quality indicators for LSG operative reports. This will allow further assessment of the quality of existing documentation and will afford the development of a synoptic report that may improve this documentation.

\section{P094}

Laparoscopic Sleeve Gastrectomy as a Proposed Treatment for Gastroparesis - A Case Series

Katelin A Mirkin, MD, Ann M Rogers, MD; Penn State Health Milton S. Hershey Medical Center

Introduction: In patients with severe obesity and gastroparesis, sleeve gastrectomy can improve symptoms and may be a reasonable treatment option. We report a case series of four patients with gastroparesis documented by preoperative nuclear gastric emptying study, who experienced improvement in symptoms after laparoscopic sleeve gastrectomy.

Methods and Procedures: A prospectively maintained database of all patients who underwent sleeve gastrectomy at our institution from 2008-2017 was reviewed, searching for patients with preoperative clinical diagnosis of gastroparesis and gastric emptying studies. Outcomes assessed were clinical resolution of symptoms with followup of 1 year.

Results: Four patients met inclusion criteria, all females, ages 34 to 58 , with BMIs ranging from 41-44. Preoperatively, the patients had symptoms of nausea and vomiting that failed to improve with medical management. Two of the patients had insulin-dependent diabetes. All four patients underwent laparoscopic sleeve gastrectomy; one also underwent anterior cruraplasty for a small hiatal hernia, without complications. Two of the patients were discharged on post-operative day one and the others on post-operative day two, all tolerating a liquid diet. At 1 year follow up, BMI had decreased by an average of 5.9 points. At 1 year follow-up, all four patients reported complete resolution of symptoms.

Conclusions: To date there are only 13 reported cases in the literature of improvement of gastroparesis after sleeve gastrectomy. In bypass patients, gastroparesis was previously treated with an adjuvant gastrostomy tube in the remnant stomach to prevent nausea or distention. This is likely unnecessary. Sleeve gastrectomy led to resolution of gastroparesis in all patients in our database, and may be the bariatric procedure of choice for patients with this diagnosis. 


\section{P095}

Factors Associated with Open Conversion During Laparoscopic Roux-En-Y Gastric Bypass: A National Database Analysis

Eric S Wise, MD, Adam Sheka, MD, Keith Wirth, MD, Sayeed Ikramuddin, MD, Daniel Leslie, MD; University of Minnesota

Introduction: Open conversion (OC) is an infrequent, highly undesirable necessity during elective minimally invasive bariatric surgery. Characterization of those patients at greatest risk for OC is poor. In this study, we seek to determine those preoperative variables that may portend an increased risk of OC. We also aim to characterize the association of $\mathrm{OC}$ on postoperative morbidity and mortality.

Methods and Procedures: Using the robust Metabolic and Bariatric Surgery Accreditation and Quality Improvement Program 2016 dataset, 38,907 patients ( $\geq 18$ years of age, body-mass index $[\mathrm{BMI}] \geq 35 \mathrm{~kg} / \mathrm{m}^{2}$ ) who underwent elective, multi-port laparoscopic Roux-en-Y gastric bypass (LRYGB) were included. Preoperative patient and surgeon factors were tested for associations with open conversion using bivariate and subsequent multivariate nominal logistic regression analysis. Bivariate analysis was subsequently used to characterize the association between OC and a panel of 30-day postoperative morbidities. The Mann-Whitney U test and Chi squared test were used for continuous and categorical data comparisons, respectively. All patient variables and outcomes were as defined by the Participant Use Data File User Guide. A criterion of $\mathrm{P} \leq .05$ was taken to denote statistical significance.

Results: Among the 38,907 patients, there were 79 (0.2\%) OCs. On bivariate analysis, variables associated with $\mathrm{OC}$ were advanced age, African American race, greater BMI, gastroesophageal reflux disease, chronic obstructive pulmonary disease, hypertension requiring 3 +medications, therapeutic anticoagulation use, prior obesity/foregut surgery, chronic steroid use and non-independent functional status $(\mathrm{P} \leq .05)$. The multivariate nominal logistic regression model considering patient variables with sufficient prevalence ( $n \geq 5$ among OC patients) generated an area under the receiver-operating characteristic curve of $0.75\left(\mathrm{n}=35,008, \mathrm{r}^{2}=.06\right.$, Chi squared $=66.3, \mathrm{P}<.001$ ). Independent risk factors for $\mathrm{OC}$ included advanced age $(\mathrm{P}<.001$, odds ratio $1.04,95 \%$ confidence interval [1.02-1.06]), higher BMI $(\mathrm{P}<.001,1.06$ [1.04-1.08]), and previous foregut/obesity surgery $(\mathrm{P}<.001,3.9$ [2.3-6.5])(Table 1). OC patients had longer lengths of stay (median 4 vs. 2 days, $\mathrm{P}<.001$ ) and operative times (median 238 vs. $110 \mathrm{~min}, \mathrm{P}<.001$ ), as well as greater rates of perioperative transfusion $(\mathrm{P}=.03)$, unplanned ICU admission $(\mathrm{P}<.001)$, 30-day mortality $(\mathrm{P}<.001)$, 30-day reoperation $(\mathrm{P}=.02)$ and 30 -day readmission $(\mathrm{P}<.001)$ (Table 2).

Conclusions: During elective LRYGB, OC is rare and associated with increased 30-day postoperative morbidity and mortality. We have identified several critical risk factors independently associated with OC. Derived from a robust national database, these data may guide intraoperative and postoperative expectations for the patient and bariatric surgical team.

\section{P096}

Roux-en-Y Gastric Bypass vs. Vertical Sleeve Gastrectomy for Diabetic Patients: 5 Year Outcomes

Vanessa Boudreau, MD, Karen Barlow, Hons, BSc, Scott Gmora, MD, Dennis Hong, MD, Mehran Anvari, MD, PhD; Center for Minimal Access Surgery, McMaster University, Ontario, Canada

Introduction: Bariatric surgery is a known effective treatment for diabetes in obese patients.

Methods: Analysis of the Ontario Bariatric Registry data was performed to compare long term outcomes in diabetic patients who underwent Roux-en-Y Gastric Bypass (RYGB) or Vertical Sleeve Gastrectomy (VSG) between 2010-2018. Intention-to-treat analysis was performed. Results include conversions and revisions.

Results: Of the 5,101 diabetic patients that underwent bariatric surgery, 4,244 (83\%) had RYGB (BMI 48.2, age 49.1; 75\% female) and $847(16.6 \%)$ had VSG (BMI 53.3, age $52.5 ; 69.1 \%$ female). There were 786 patients with RYGB available for follow up at 3 years and 198 patients at 5 years. Among the VSG patients, 92 were available for follow-up at 3 years and 17 at 5 years. There were 38 VSG patients that required a conversion due to weight regain/ineffective weight loss compared to 2 RYGB patients. Data below includes converted patients using intention to treat analysis.

\begin{tabular}{|c|c|c|c|c|}
\hline & & RYGB & VSG & p-value \\
\hline \multirow[t]{2}{*}{ Conversion Rate } & 3 years & $0.2 \%$ & $32.6 \%$ & $<0.05$ \\
\hline & 5 years & $1.0 \%$ & $47.1 \%$ & $<0.05$ \\
\hline \multicolumn{5}{|l|}{ Weight Loss } \\
\hline \multirow[t]{2}{*}{$\%$ EWL } & 3 years & $63.7 \%$ & $43.6 \%$ & $<0.05$ \\
\hline & 5 years & $60.3 \%$ & $52.9 \%$ & NS \\
\hline \multicolumn{5}{|l|}{ Diabetes Control } \\
\hline \multirow[t]{2}{*}{ No Diabetes Medication } & 3 years & $69.8 \%$ & $66.7 \%$ & NS \\
\hline & 5 years & $65.2 \%$ & $56.2 \%$ & NS \\
\hline \multirow[t]{2}{*}{$\mathrm{HbA1c}$} & 3 years & $6.1 \%$ & $6.3 \%$ & $<0.05$ \\
\hline & 5 years & $6.3 \%$ & $6.5 \%$ & NS \\
\hline \multirow[t]{2}{*}{ Remission $(\mathrm{HbA} 1 \mathrm{c}<6 \%)$} & 3 years & $51.3 \%$ & $45.5 \%$ & NS \\
\hline & 5 years & $47.3 \%$ & $50 \%$ & NS \\
\hline \multirow{2}{*}{$\begin{array}{l}\text { Partial Remission } \\
\qquad(\mathrm{HbA} 1 \mathrm{c}<6.5 \%)\end{array}$} & 3 years & $15.5 \%$ & $15.6 \%$ & NS \\
\hline & 5 years & $16.3 \%$ & $7.1 \%$ & NS \\
\hline
\end{tabular}

Conclusion : Both RYGB and VSG are effective with favorable weight loss and diabetes control. RYGB resulted in lower diabetes medication use and lower $\mathrm{HbA} 1 \mathrm{c}$ at each follow-up.
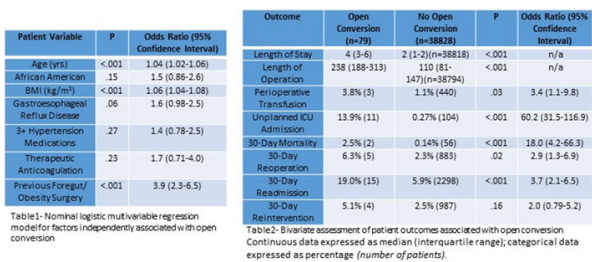


\section{P097}

The Impact of Bariatric Surgery on Hypothyroidism: A Two Year Follow Up

Michael Zhou, BA, Jordan Grubbs, MD, David Berler, MD, Benjamin E Schneider, MD, Sara A Hennessy, MD; University of Texas Southwestern Medical Center

Introduction: Thyroid dysfunction and obesity are intricately connected with a positive correlation between BMI and TSH. Bariatric surgery is one of the most effective long-term treatments of obesity and its co-morbidities. Likewise, changes in thyroid function, correlating decrease of medication dosage, and even resolution of hypothyroidism have been reported in post-operative patients. However, the literature is sparse on the long-term effects of bariatric surgery on hypothyroidism. In this study, we aimed to review the effects of bariatric surgery on hypothyroidism over 2 years.

Methods and Procedures: A retrospective review was conducted of patients undergoing sleeve gastrectomy (SG) and Roux-en-Y gastric bypass (RNYGB) procedures at a university-affiliated academic institution from 2014 to 2016. Patients with hypothyroidism were evaluated for changes in thyroid stimulating hormone (TSH) and dose of hypothyroid medication over follow up periods of 6,12 , and 24 months were evaluated. Patients were compared by comorbidities, type of surgery, BMI change and outcomes.

Results: Of the 401 bariatric surgery patients, 63 patients had preoperative diagnosis of hypothyroidism. Of the patients with hypothyroidism, 24 patients had adequate TSH follow up. Preoperative mean TSH was 2.15 with a BMI of 43.6. At 24 months there was a decrease to a TSH of 1.33 and a BMI of 33.2 (Table 1). Of these patients, $4(16.7 \%)$ had a dose decrease in their thyroid replacement medication postoperatively with an average of reduction in dose by $31.2 \%$.

When comparing SG and RNYGB in hypothyroid patients, there was a significant difference in BMI change of 7.97 and 13.5, respectively $(\mathrm{p}=0.03)$, and TSH decreased by 0.21 and 0.64 , respectively $(\mathrm{p}=0.04)$.

\begin{tabular}{ccccc} 
& Pre-Operative $(n=24)$ & $\mathbf{6}$ months $(n=24)$ & $\mathbf{1 2}$ months $(n=18)$ & $\mathbf{2 4}$ months $(n=12)$ \\
\hline TSH & 2.15 & 1.46 & 1.49 & 1.33 \\
BMI & 43.6 & $35.5^{*}$ & 32.62 & 33.18
\end{tabular}

Table 1. TSH and BMI changes over follow up periods. *: Statistical significant decrease in BMI occurred post-operatively $(p<0.01)$. There was no statistically significant decline in TSH and no correlation between BMI and TSH changes.

Conclusion: Bariatric patients with well-controlled preoperative hypothyroidism are safe to undergo either sleeve gastrectomy or Roux-en-Y gastric bypass surgery. They can continue to achieve adequate control of their hypothyroidism without a negative impact on absorption of hypothyroid medications. There is a trend towards improvement in hypothyroidism with decreases in thyroid medication doses. Roux-en-Y gastric bypass is potentially superior to gastric sleeves in treatment of hypothyroidism, with significant improvement in thyroid function. A prospective study is necessary to understand the true impact of bariatric surgery on hypothyroidism.

\section{P098}

\section{Outcomes After Laparoscopic Sleeve Gastrectomy at a High} Volume Center

Chetna Bakshi, MD, Sara Siskind, MD, Christopher Taglia, MD, Larry Gellman, MD, Dominick Gadaleta, MD; Zucker School of Medicine at Hofstra/Northwell

Background: Laparoscopic sleeve gastrectomy has gained interest in the United States due to its efficacy and relative safety ${ }^{1}$. Many studies have been done comparing sleeve gastrectomies with other bariatric procedures. High volume centers have developed early recovery protocols to decrease hospital length of stay without increasing morbidity or post-operative re-admissions ${ }^{2}$. The aim of this study was to demonstrate superior outcomes and lower complication rates at a high volume center for sleeve gastrectomies, using a similar technique.

Methods: A retrospective chart review was undertaken, looking at outcomes after sleeve gastrectomies performed between 2012 and 2017 by three surgeons who utilize a similar technique at a single Bariatric Center of Excellence. All patients who had additional bariatric procedures listed, such as gastric band removal, were excluded. The primary end points were post-operative complications including ICU stay, mortality, and readmission rates.

Results: 812 patients were included in the study with a mean BMI of $45.59 \pm 7.36$. Average hospital length of stay was $1.15 \pm 1.19$ days for the index hospitalization. None of the patients experienced any intra-operative complications. Only four patients $(0.49 \%)$ had an ICU admission within 30 days, and there was only one mortality $(0.12 \%)$. Four patients $(0.49 \%)$ required discharge to a rehabilitation facility, and four patients $(0.49 \%)$ experienced dehydration requiring treatment. Twenty patients $(2.46 \%)$ presented to the ED but only 16 $(1.97 \%)$ required re-admission, and only 5 of these $(0.62 \%)$ required an intervention.

Conclusions: The overall complication rate in this study is lower than that described in the literature. The re-admission rate was $1.97 \%$, significantly lower than the $3.8 \%$ quoted in previous studies ${ }^{3}$. Readmission complications in this study included venous thromboembolic events, wound infections, and dehydration. Notably, no leaks were seen. This study effectively demonstrates that high volume bariatric centers with standardized techniques and protocols can achieve lower complications rates, thus minimizing patient injury and preserving valuable healthcare resources. 
P099

\section{Surgical Complications Following Roux-En-Y Gastric Bypass and Sleeve Gastrectomy in Patients Aged ? 65 Years from 2006- 2016}

Rebekah Wood, BS ${ }^{1}$, Mukund Srinivas, BS ${ }^{1}$, Mike Bottomley, $\mathrm{MS}^{2}$, Priti Parikh, $\mathrm{PhD}^{1}$, Joon K Shim, MD, MPH, FACS ${ }^{1}$; Wright State University Boonshoft School of Medicine, ${ }^{2}$ Wright State University

Introduction: With the obesity epidemic in America, this study aimed to evaluate the outcomes of obese patients over the age of sixty-five after either laparoscopic/robotic Roux-En-Y or laparoscopic/robotic sleeve gastrectomy in one single tertiary institution. There are an increase number of patients over the age of sixty-five with indications for bariatric surgery. Studies have shown efficacy and safety of bariatric surgery in this age group.

Methods: We retrospectively reviewed 70 laparoscopic/robotic Roux-En-Y gastric bypass and 74 laparoscopic/robotic sleeve gastrectomy that were performed on patients aged $\geq 65$ years at a tertiary center from January 2006 to December 2016. Revisional surgeries were excluded. A logistical regression was run with 'yes/no' to 'patient experienced a complication' as the dependent variable. Demographic characteristics and perioperative data were analyzed including type of procedure, gender, smoking status, and BMI as independent variables. SAS version 9.4 (SAS Institute, Inc., Cary, NC) was used for analysis.

Results: Tukey's multiple comparison procedure adjusts the P-values to control for the number of comparisons made so that the experiment-wise type I error rate is held constant at alpha $=0.05$. The odds ratio estimate for laparoscopic/robotic Roux-En-Y gastric bypass is 3.183 . The adjusted $95 \%$ confidence interval for the true odds ratio is $(1.082,9.366)$. Based on the adjusted P-value of 0.03 , there is strong evidence to suggest there is a significant difference in the odds of having a complication for patients who underwent laparoscopic/ robotic Roux-En-Y gastric bypass versus patients who underwent laparoscopic/robotic gastric sleeve. There were a total of 32 patients who experienced a complication after laparoscopic/robotic Roux-En$\mathrm{Y}$ gastric bypass. Eight of these 32 patients experienced more than 1 complication. There were a total of 17 patients who experienced a complication after laparoscopic gastric sleeve. No patients experienced multiple complications. Ulcer, perforation, surgical site infection, respiratory failure, acute kidney injury and bleeding were observed more frequently in laparoscopic/robotic Roux-En-Y gastric bypass. These complications were further categorized as short and long term complications.

Conclusion: For obese patients over the age of sixty-five, the odds of experiencing a complication postoperatively for patients undergoing laparoscopic/robotic Roux-En-Y gastric bypass were 3.183 times the odds of experiencing a complication postoperatively for patients undergoing a laparoscopic/robotic sleeve gastrectomy at our center. However, the $95 \%$ confidence interval indicates that the true odds ratio could be as low as 1.082 times more likely or as high as 9.366 times more likely in patients undergoing laparoscopic Roux-En-Y gastric bypass.

\section{$\mathbf{P 1 0 0}$}

The Safety and Efficacy Of Bariatric Surgery in the Elderly

Brian J Shea, MD ${ }^{1}$, William Boyan, MD ${ }^{1}$, Jonathan Decker, MD ${ }^{1}$, Ethan Paulin, $\mathrm{MD}^{1}$, Vincent Almagno, BS ${ }^{2}$, Wiley Abbott, $\mathrm{BS}^{2}$, Joseph Bahgat, BS ${ }^{2}$, Gurdeep Matharoo, MD, FACS, FASMBS ${ }^{1}$, Steven Binenbaum, MD, FACS, FASMBS ${ }^{1}$, Frank

Borao ${ }^{1} ;{ }^{1}$ Monmouth Medical Center, ${ }^{2}$ St. George's University School of Medicine

Introduction: Bariatric surgery has been demonstrated to improve obesity related comorbidities and be superior to medical therapy alone. Many of these trials demonstrating this benefit studied patient populations at average risk for surgery, which typically included younger patients. This study seeks to examine the safety of bariatric surgery in the over 60 population, and to determine if these patients share the same the benefits in terms of weight as their younger counterparts.

Methods and Procedures: The records of all patients undergoing bariatric surgery at a bariatric surgery center of excellence were examined between January 2012 and December 2016. Patients were separated into two groups; those over the age of 60 and those under age 60. Data for perioperative factors were then recorded, including length of stay, complications, thirty day readmission and reoperation. Follow up data was then recorded, including patient's excess body weight loss and percentage change in body mass index. Weight loss was followed over four time intervals: one week post-operative, short term (1-3 months) intermediate term (4-8 months) and long term (9 months to last available follow up). Student's T Test and Chi square analysis was used to determine if differences in the above characteristics were statistically significant.

Results: There were 90 patients in the over 60 group, and 453 in the under 60 group. Average age in these groups were 64.3 years and 42.9 years respectively. Average ASA classification was significantly higher in the over 60 group (3.01 vs. 2.76 ; $p<0.001)$. There was no significant difference in length of hospital stay between groups ( $2.3 \mathrm{vs}$ $2.1 ; \mathrm{p}=0.15)$. Rates of complications were similar between groups $(7.7 \%$ vs $5.1 \% ; p=0.5)$. There was also no statistically significant difference in readmission $(2.2 \%$ vs $3.5 \% ; \mathrm{p}=0.5)$ or reoperation ( $11 \%$ vs. $9 \% ; p=0.55)$. The mean follow up time for patients in the over 60 group was 14.2 months, and 12.1 months in the under 60 group. In terms of weight loss, with longer term follow up younger patients experienced more excess body weight loss $(54.7 \%$ vs $46.2 \%$; $\mathrm{p}=0.008$ ).

Conclusion: The results of this series demonstrate that bariatric surgery in elderly patients is not only safe, but effective. Although younger patients tended to lose more weight, elderly patients still lost a clinically significant amount of weight. Age alone should not preclude a patient from undergoing bariatric evaluation. 


\section{P101}

Obesity and Non-alcoholic Fatty Liver Disease: Cause and Effect?

Khaleel Mohammad, MD ${ }^{1}$, Marcoandrea Giorgi, MD ${ }^{1}$, Jawad Ali, $\mathrm{MD}^{1}$, Rouzbeh Moustaedi, $\mathrm{MD}^{2}$, Aaron Carr, MD ${ }^{1}$, Mohamed Ali, $\mathrm{MD}^{1}$; ${ }^{1} \mathrm{UC}$ Davis Medical Center, ${ }^{2}$ The Permanente Medical Group

Introduction: Obesity has been commonly cited to be a major risk factor for the occurrence and progression of non-alcoholic fatty liver disease (NAFLD). In particular, bariatric surgery patients are hypothesized to be at highest risk for NAFLD and its consequences. In this study, we sought to characterize the extent of NAFLD, the prevalence of progression to NASH and fibrosis, and identify patient characteristics that correlate with fatty liver disease in this high-risk population.

Methods: Core liver biopsy was obtained on 529 consecutive patients undergoing bariatric surgery and assessed for the degree of steatosis, portal inflammation, and hepatic fibrosis. Demographic, anthropometric, laboratory, and co-morbidity data were collected and analyzed to identify relationships between the clinical condition of the patient and NAFLD.

Results: The study included 529 patients with an average body mass index (BMI) and age of $45.3 \pm 7.5 \mathrm{~kg} / \mathrm{m}^{2}$ and $45.3 \pm 11.2$ years, respectively. Metabolic syndrome was present in $71.8 \%$ of patients. Hepatic steatosis, NASH and fibrosis were present in 69.6, 44.2 and $18.1 \%$ of patients, respectively. There was a significant direct correlation between degree of steatosis and preoperative BMI $(\mathrm{p}<0.05)$, serum triglycerides $(\mathrm{p}<0.0001)$, and hemoglobin A1C (HbA1C) $(\mathrm{p}<0.0001)$, while HDL showed an inverse correlation with the degree of hepatic steatosis $(\mathrm{p}<0.001)$. Patients with metabolic syndrome had more steatosis $(p<0.0001)$ and steatohepatitis $(\mathrm{p}<0.005)$ than patients without metabolic syndrome.

Conclusions: Factors such as elevated BMI, triglycerides, HbA1C and presence of metabolic syndrome correlated with the degree of hepatic steatosis. Although the majority of patients in this study exhibited hepatic steatosis, $30.4 \%$ of patients did not have steatosis despite having the high risk traditionally ascribed to advanced obesity. These findings suggest that the mere presence of obesity may not be enough to promote the progression of NAFLD to NASH and fibrosis and indicate that metabolic dysregulation may play a more vital role.

\section{P102}

Despite Risk Factors, the Incidence of C. Difficile Infection in Bariatric Patients Remains Low and Laparoscopic Sleeve Gastrectomy Confers the Lowest Risk

Luke Putnam, MD, Sahil Gambhir, MD, Gabrielle Stretz, BSc, Areg Grigorian, MD, Megan T Smith, PhD, Brian R Smith, MD, Ninh T Nguyen, MD, Shaun Daly, MD; University of California Irvine Medical Center

Introduction/Objectives: Clostridium Difficile is a significant source of morbidity in hospitalized patients. Obesity and major gastrointestinal operations are known risk factors for clostridium difficile infection (CDI). Currently, there is limited data suggesting laparoscopic roux-en-Y gastric bypass (LRYGB) is also associated with an increased incidence of CDI compared to laparoscopic sleeve gastrectomy (LSG). We hypothesized the rate of CDI in the bariatric population is elevated given inherent risk factors of this patient population and that LSG may confer less risk than LRYGB.

Methods: The Metabolic and Bariatric Surgery Accreditation and Quality Improvement Program database was queried to identify patients who underwent LSG and LRYGB between 2015 and 2016. Data was collected from each patient's index admission. As a surrogate for the true incidence of CDI, treatment of CDI was used. Descriptive statistics and multivariable logistic regression analyses were performed to obtain odds ratios (OR) and 95\% confidence intervals (CI).

Results: During the study period, 167,563 (71\%) patients underwent LSG and 67,525 (29\%) underwent LRYGB. The overall incidence of CDI in our study population was $0.07 \%$. The incidence of CDI in index hospital admission in patients undergoing LSG was $0.05 \%$ compared to $0.09 \%$ in patients undergoing LYRGB. The risk of CDI during the index hospital admission in LSG patients compared to LRYGB patients was 0.64 (95\% CI 0.47-0.88; $\mathrm{p}=0.006)$.

Conclusion: While literature reports obesity remains a risk factor for development of CDI, the overall incidence of $0.07 \%$ remains low in postoperative bariatric patients. CDI does not represent a significant source of postoperative morbidity during index admission. In bariatric patients, those undergoing LSG have a significantly decreased risk of developing CDI compared to LRYGB patients. 


\section{$\mathbf{P 1 0 3}$}

Opioid Use Reduction Secondary to Remission of Osteoarthritis Associated Pain in Severely Obese Subjects Undergoing Bariatric Surgery

Maria C Fonseca, MD, Cristian Milla Matute, MD, Camila Ortiz Gomez, MD, Francisco Ferri, MD, Emanuele Lo Menzo, MD, PhD, FACS, FASMBS, Samuel Szomstein, MD, FACS, FASMBS, Raul J Rosenthal, MD, FACS, FASMBS; Cleveland Clinic Florida

Background: Unbearable pain associated with symptomatic Osteoarthritis(OA) is among the causes of work loss worldwide. Schedule II/III opioids are frequently used for this purpose, however is highly associated to abuse. The aim of this study is to describe OA in our population and the opioid reduction and cessation after undergoing Bariatric Surgery.

Methods: After IRB approval, we performed a retrospective analysis of all severely obese patients with a BMI above $35 \mathrm{~kg} / \mathrm{m}^{2}$ and Osteoarthritis who underwent bariatric surgery from January 2004 to February 2018 at our institution. OA remission was assessed by the decrease in the dose of opioid medication for pain management or cessation during 6 and 12 months. Patients were matched for demographics. We excluded patients who received opioids due to Polymyalgia rheumatic and fibromyalgia.

Results: From 4226 patients, $11 \%(\mathrm{~N}=483)$ met the inclusion criteria. Female gender was predominant $(\mathrm{N}=335 ; 69.4 \%)$. Average BMI was $43.93 \pm 8.8 \mathrm{~kg} / \mathrm{m}^{2}$. Performed surgeries were as follows: LSG in $43.8 \%(\mathrm{~N}=213)$ patients, RYGB in $39.9 \%(\mathrm{~N}=194)$ patients and $\mathrm{AGB}$ in $16.1 \%(\mathrm{~N}=78)$ patients (table 1). Remission was achieved in $95 \%(\mathrm{~N}=495)$ and $97 \%(\mathrm{~N}=471)$ of the patients at 6 and 12 months respectively. Overall statistical significance was attributed to Opioid dose reduction at 6 and 12 months was found in $54.2 \%(\mathrm{~N}=13)$ and $75 \%(\mathrm{~N}=9)(\mathrm{p}=0.02$ and $\mathrm{P}=0.06)$ respectively, when compared to preoperatively. Oxycodone was predominantly prescribed at 6 months in $54.2 \%(\mathrm{~N}=13)$ and 12 months in $41.7 \%(\mathrm{~N}=5)$ of the patients, with no statistical difference among procedures ( $\mathrm{p}=0.15$ in both terms) (Table 2).

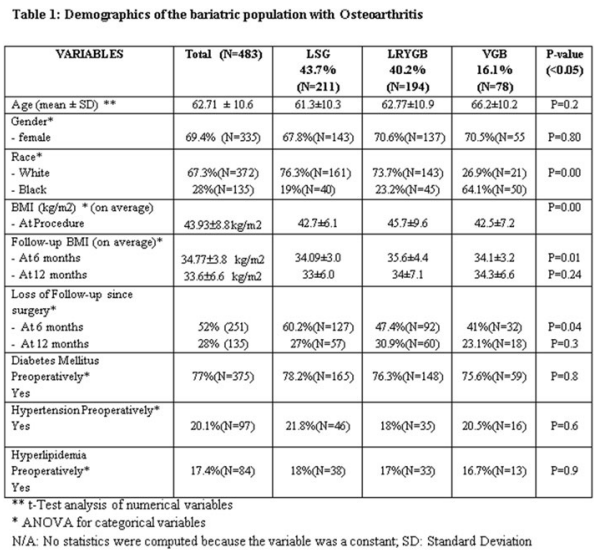

Laparoscopic Sleeve Gastrectomy (LSG), Laparoscopic Rouz-en-Y Gastric Bypass (LRYGB), Laparoscopic Adjustable Gastric Banding (LAGB).
Table 2: Bariatric patients with Osteoarthritis remission rate at 6 and 12 months

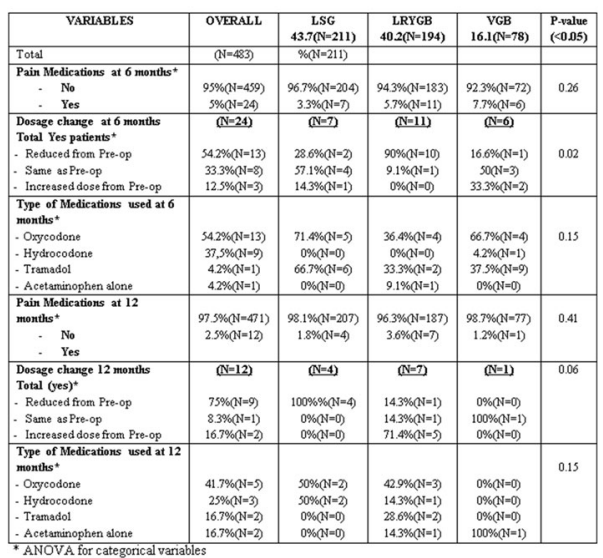

N/A: No statistics were computed because the variable was a constant, SD: Standard Deviation

Laparoscopic Sleeve Gastrectomy (LSG), Laparoscopic Roux-en-Y Gastric Bypass (LRYGB),
Laparoscopic Adjustable Gastric Banding (LAGB)

Conclusion: In spite of the discriminated consumption of opioids for pain control, Bariatric Surgery seems to reduce substantially their use, by achieving OA remission in $97 \%$ of our patients at 12 months. Further studies will need to be done to confirm these findings. 


\section{P104}

Laparoscopic Selective Intra-abdominal Denervation Associated to a Duodenum Ileal Interposition and Sleeve Gastrectomy for the Treatment of Type 2 Diabetes (SID-DISG)

Luciana J El-Kadre, MD, PHD, FACS ${ }^{1}$, Aureo L De Paula, MD, $\mathrm{PHD}^{2}$, Augusto C Tinoco, MD, PHD, FACS ${ }^{1}$, Surendra Ugale, MD ${ }^{3}$, Jose Ribamar De Azevedo, MD, PHD, FACS ${ }^{4}$, Carolina De Paula, $\mathrm{MD}^{2}$, Leonardo Ferraz, MD ${ }^{5}$ ' ${ }^{1}$ Sao Jose do Avai Hospital, ${ }^{2}$ Hospital de Especialidades, ${ }^{3}$ Kirloskar Hospital, ${ }^{4}$ Americas

Hospital, ${ }^{5}$ Bonsucesso Federal Hospital

No single medication can approach all the pathophysiologic disturbances that characterize type 2 diabetes (T2D). Current medical therapy involves the combined use of multiple drugs to control the disease. With this concept in mind, the laparoscopic duodenum ileal interposition and sleeve gastrectomy (DISG) is an operation that intends to address the different components of the pathophysiology of T2D. The objective of this study is to evaluate the early results of this operation combined with a selective intra-abdominal denervation in diabetic patients with BMI below 35.The procedure was performed in 33 patients. 29 were men and 4 women. Mean age was 56.3 years (3471). Mean BMI was $32.2 \mathrm{~kg} / \mathrm{m}^{2}$ (26.7-34.9). All patients had evidence of stable treatment with oral hypoglycemic agents and or insulin for at least 12 months. Insulin therapy was been used by $36 \%$ of the patients. Mean duration of T2DM was 11.4 years (4-32). Mean A1c was $9 \%$. Coronary heart disease was diagnosed in 27.3 , hypertension in 72.7 , dyslipidemia in 94 , retinopathy in 24.2 , nephropathy in 24.2 and cholelithiasis in $18.2 \%$ of the patients.Mean post-operative follow-up was 22 months (9-33). Mean postoperative BMI was $23.3 \mathrm{~kg} / \mathrm{m}^{2}$ (19.6-27). Mean A1c was 5.8\%, ranging 4.6 to 8 . Overall, $87.9 \%$ of the patients achieved an adequate glycemic control (A1c $\leq 6)$ without anti-diabetic medication and $83.3 \%$ had fasting glucose $<126 \mathrm{mg} / \mathrm{dl}$. Arterial hypertension was controlled in $95.8 \%$ of the patients, hypercholesterolemia in $96.8 \%$, and hypertrygliceridemia in $93.1 \%$. The results of this short-term study demonstrated that the combination of a selective intra-abdominal denervation to the laparoscopic DISG improved the glycemic, hypertensive and dyslipidemic remission rates of T2D patients with BMI below 35. The scalpel seems to be an effective tool in controlling diabetes using the concept of an endocrine-based, pathophysiological, understanding of the disease.

\section{P105}

The Role of Laparoscopic Bariatric Procedures in the Improvement of Pre-operative Uncontrolled/Resistant Hypertension and its Impact on the 10-Year Risk of Cardiovascular Disease

David Romero Funes, MD, David Gutierrez Blanco, MD, Camila Ortiz Gomez, MD, Joel S Frieder, MD, Cristian Milla Matute, MD, Emanuele Lo Menzo, MD, Samuel Szomstein, MD, Raul Rosenthal, MD; Cleveland Clinic Florida

Introduction: Although several studies have addressed the improvement of hypertension (HTN) after bariatric surgery, only a few of them specifically addressed the effects of the procedures on uncontrolled/resistant HTN. The aim of our study is to assess the remission of uncontrolled/resistant hypertension and its impact in the risk of cardiovascular disease (CVD) of morbidly obese patients following laparoscopic bariatric procedures.

Methods: We retrospectively reviewed all patients who underwent Laparoscopic Bariatric surgery at our institution from 2010-2014. We used the guidelines of the American College of Cardiology (ACC) to define HTN and uncontrolled/resistant hypertension. Only patients who met the criteria for the ACC and the calculation of the Framingham-BMI 10-year risk score pre-operatively at 3 months and at 12 months follow-up were included.

Results: Of the 1,200 patients reviewed, 203(16.91\%) met the required criteria of uncontrolled/resistant HTN according to the ACC and had the required variables for the risk assessment of CVD using the FraminghamBMI 10-year risk score. The most prevalent procedure was Laparoscopic sleeve gastrectomy LSG $\mathrm{n}=133$ (65.51\%). The most significant improvement found was an amelioration of $15 \mathrm{mmhg}$ and $13 \mathrm{mmgh}$ when comparing of systolic blood pressure pre-operatively, at 3 and 12 months follow-up respectively $(\mathrm{P}=0.0001)$. The improvement in the risk of CVD is shown by an absolute risk reduction(ARR) of $14 \%$ at 3 months and $15 \%$ at 12 months follow-up, both evidencing statistically significant $(\mathrm{P}=0.0001)$ In the patients with resistant hypertension the mean heart age was $80.81 \pm 9.11$. With regard to the Heart age, we observe an improvement of 6.76 and 7.33 years when comparing heart age pre-operatively, at 3 months and at 12 months follow-up. Both showing statistical significance $(p=0.0001)$ Conclusions: Our results suggest that laparoscopic bariatric procedures have a positive impact on patients with uncontrolled/resistant hypertension. This results also translates into significant cardiovascular disease risk reduction. Furthermore, the Cardiovascular disease risk reduction also demonstrates a most dramatical improvement in patients with resistant hypertension.

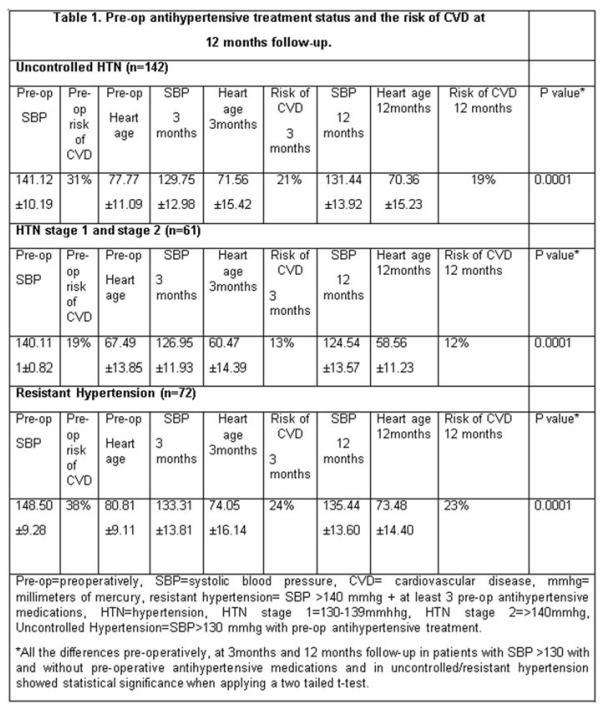




\section{P106}

\section{Gastric Cancer After Roux-en-Y Gastric Bypass: Case Report}

Diego L Lima, MD ${ }^{1}$, Victor M Diniz, MD ${ }^{1}$, Camila Maria N Firme, $\mathrm{MD}^{1}$, Jose Julio F Arruda, $\mathrm{MD}^{1}$, Raquel N Cordeiro, Medical Student $^{2}$, Lucas M Aires Camara, MD ${ }^{1}$, Lucas M Vasconcelos De Albuquerque, $\mathrm{MD}^{1}$, Marconi Roberto L Meira, $\mathrm{MD}, \mathrm{MsC}^{1}$, Geraldo Jose P Wanderley, $\mathrm{MD}^{1} ;{ }^{1}$ State Servers Hospital, ${ }^{2}$ Pernambuco Health Faculty

Introduction: The incidence of gastric adenocarcinoma in patients undergoing Roux-en-Y gastric bypass (BPGYR) is rare. Over the past 10 years, just over 30 cases have been reported, most of which were in the excluded stomach. The pathogenesis is still unknown. However, it is believed that chronic reflux, $\mathrm{H}$. pylori infection and gastric stasis of undigested food would be associated with the genesis of cancer. Symptomatology usually includes nonspecific symptoms, the most common being abdominal pain. The most accurate diagnostic method is upper GI. However, the difficulty in accessing the remaining stomach in patients submitted to bypass, ends up delaying the diagnosis in these patients.

Case Report: A 68-year-old woman who had been submitted to BPGYR for morbid obesity 15 years ago presented with epigastric pain and melena, and a mass was identified by computed tomography of the abdomen and Upper GI. In view of persistent bleeding and acute anemia, laparotomy with total gastrectomy and esophageal-jejunal anastomosis was performed. Histology showed a well differentiated adenocarcinoma. Surgery had no major bleeding and no further complications. Patient was discharged and had adjuvant chemotherapy.

Conclusion: Gastric adenocarcinoma's symptomatology is unspecific in most of the patients. Its diagnosis is also postponed because it is very difficult to analyze of the excluded stomach. When these patients present general and unspecific symptoms, it is recommended to perform a CT scan or an Upper GI in a center of excellence.

\section{P108}

\section{Concurrent Paraesophageal Hernia Repair and Sleeve Gastrectomy is Safe and Well Tolerated}

Eugene Wang, MD, Chaitanya Vadlamudi, MD, Elizabeth Zubowicz, MD; Medstar Georgetown-Washington Hospital Center

Introduction: The aim of this study is to evaluate short term morbidity, if any, of concomitant paraesophageal hernia repair (PHR) in patients undergoing laparoscopic sleeve gastrectomy (LSG). LSG is now the most widely used surgical intervention for morbid obesity, however significant concern exists about exacerbating or creating de novo gastroesophageal reflux disease (GERD) in these patients. Undiagnosed or untreated paraesophageal hernias may contribute to symptomatic GERD in these patients. They are present in $15 \%$ of patients with body mass index (BMI) over $35 \mathrm{~kg} / \mathrm{m}^{2}$. Intra-operative diagnosis of hiatal hernias is estimated to occur in $1 / 3$ of LSG performed.

Methods and Procedures: Retrospective, single center case control study (2016-2018) using data from Metabolic and Bariatric Surgery Accreditation and Quality Improvemen Program. Inclusion criteria: patients who underwent concurrent LSG and PHR. Primary endpoints: operative time (minutes), length of stay (LOS, days), BMI difference, emergency department (ED) visits, and mortality. Independent t-test was used for continuous data. Pearson's Chi squared test was used for categorical data, reported as percentages.

Results: Nine patients were identified with concurrent surgery by LSG and PHR, with 30 day follow-up. 562 control patients during this time period underwent LSG only. There were no statistically significant differences between the two groups' co-morbidities in terms of diabetes, obstructive sleep apnea, GERD and hypertension. The LSG and PHR group was approached robotically $77.7 \%$ of the time, which was more frequent than the LSG only group which was approached robotically $40.5 \%$ of the time, $p=0.024$. The operative time for the LSG and PHR group was on average 134.2 $\pm 69.7 \mathrm{~min}$, which was longer than the LSG only group, which averaged $101.4 \pm 45.4 \mathrm{~min}, \mathrm{p}=0.062$. There were no statistically significant differences between the two groups in terms of LOS $1.1 \pm 0.3$ vs $1.4 \pm 1.9$ days $(\mathrm{p}=0.472)$, BMI difference post-surgery $3.0 \pm 0.6$ vs $3.3 \pm 2.6$ $(\mathrm{p}=0.242)$, ED visits $11.1 \%$ vs $10.1 \%(\mathrm{p}=0.924)$, and mortality $0 \%$ vs $0 \%(\mathrm{p}=0.858)$. Conclusion: Concurrent surgery with LSG and PHR is safe to perform during the same operation, with no statistical difference in LOS, weight loss, morbidity or mortality, and only had minimally longer operative times. Given the high incidence of reflux symptoms after LSG, future studies should investigate improvement with LSG and PHR as opposed to LSG alone in those with a concurrent paraesophageal hernia.

\section{P109}

\section{Does Adding a Concurrent Surgery to a Laparoscopic Roux en Y Gastric Bypass Lead to Increased Morbidity?}

Haley Leesley, MD, Mia Shapiro, MD, Todd Stafford, MD, Beth Ryder, MD, Dean Roye, Sivamainthan Vithiananthan, MD; Warren Alpert Medical School of Brown University/Miriam Hospital, Providence, Rhode Island, USA

Introduction: Morbidly obese patients being evaluated for gastric bypass will often present with concurrent surgical pathology. The aim of this study is to determine if surgical risk is greater in patients undergoing an additional procedure at the time of laparoscopic roux-en-y gastric bypass (LRYGB) as compared to patients undergoing LRYGB alone.

Methods: A retrospective review was performed on all patients who underwent LRYGB or LRYGB plus a concurrent procedure between 2013-2017 at our institution using the Metabolic and Bariatric Surgery Accreditation and Quality Improvement Program (MBSAQIP) database. The most commonly performed concurrent procedures we included were paraesophageal hernia repair, ventral/umbilical hernia repair and cholecystectomy for symptomatic cholelithiasis. Our primary outcome was 30-day morbidity as defined by MSBAQIP. Our secondary outcomes included length of surgery, hospital length of stay, thirty-day readmission and major morbidity.

Results: We identified 620 total patients who underwent either a LRYGB or a LRYGB with one of the concurrent procedures listed above. 564 patients (91\%) underwent LRYGB alone and $56(9 \%)$ underwent LRYGB with a concurrent procedure. Patients who underwent LRYGB with concurrent procedures were significantly older (median age: 47.0 vs 43.0 , $\mathrm{p}=0.02)$, however had a lower preoperative BMI $\left(45 \pm 6.7 \mathrm{vs} 47.8 \pm 7.5 \mathrm{~kg} / \mathrm{m}^{2}, \mathrm{p}=0.004\right)$. There were no significant differences in other medical comorbidities, including smoking, COPD, sleep apnea or hypertension. The average length of surgery was higher for patients undergoing a concurrent procedure $(163 \mathrm{~min} \pm 47.7$ vs. $146.7 \min \pm 47.5, \mathrm{p}=0.005)$. In regards to our primary outcome, we found that patients who underwent concurrent procedures were more likely to suffer a post-op morbidity (OR 4.11, 95\% CI 1.74-9.72, $\mathrm{p}=0.005$ ) when compared to those who had a LRYGB alone. They were also significantly more likely to suffer a major morbidity (OR 2.97, 95\% CI 1.06-8.34) and the odds of a 30-day post-operative readmission was over 6 times higher $(\mathrm{OR} 6.05,95 \% \mathrm{CI} 1.96-18.8, \mathrm{p}=0.005)$.

Conclusions: We found that adding a concurrent procedure on to a LRYGB was associated with increased length of surgery, increased rates of 30-day readmissions, and increased overall 30-day morbidity along with major morbidity. 


\section{P110}

\section{The Impact of Low Preoperative Albumin on Short-term} Outcomes After Bariatric Surgery

Amlish B Gondal, MD, Matthew E Mobily, MD, MPH, Iman Ghaderi, MD, MSc, MHPE; University of Arizona

Introduction: Obesity and malnutrition often coexist; malnutrition in turn is an established risk factor for adverse outcomes after surgery. Preoperative albumin is routinely used as surrogate parameter for nutritional status prior to surgery. The aim of this study was to assess the effect of low preoperative albumin on outcomes within 30 days after bariatric surgery.

Methods: An observational study of patients undergoing primary sleeve gastrectomy (SG) and gastric bypass (GB) from the MBSAQIP ${ }^{\circledR}$ PUF 2016 was performed. Chi square and two tailed t-test analyses were used for categorical and continuous outcomes, respectively, with $\mathrm{p}<0.05$ denoting statistical significance. Logistic regression analysis was performed and odds ratio (OR)along with $95 \%$ confidence intervals (CI) were calculated for significant risk factors for Clavien category IV (CD-IV), category V (CD-V) and were calculated for significant risk factors for Clavien category IV (CD-IV), category V (CD-V) and
composite infection rate (CIR) (defined as occurrence of any of the following: pneumonia, superficial SSI, composite infection rate (CIR) (defined as occurrence of any of the following: pneumonia, super
deep SSI, UTI, sepsis, septic shock or clostridium difficile infection) after bariatric surgery.

Results: A totla of 106526 patients were included, $79.7 \%$ were female, $73.8 \%$ were white, and mean preoperative BMI was $44.3 \pm 13.7 \mathrm{~kg} / \mathrm{m}^{2}$. SG was performed in $72 \%$ in the patients and $28 \%$ patients underwent GB. Ten percent patients $(\mathrm{n}=11559)$ had low preoperative albumin $(<3.5 \mathrm{~g} / \mathrm{dL})$ where the rest had normal albumin. The group with low and normal albumin had comparable baseline demographics and distribution of comorbidities.

When compared to patients with normal albumin, patients with low albumin experienced higher rates of Clavien category V complication $(0.2 \%$ vs $0.08 \%, \mathrm{p}<0.001)$, Clavien category IV complications $(1.3 \%$ vs $0.8 \%, \mathrm{p}<0.001)$, composite infection rate $(2 \%$ vs $1.4 \%, \mathrm{p}<0.001)$ and 30 -day readmission rate $(5.3 \%$, $3.8 \%, \mathrm{p}<0.01)$. Those with low preoperative albumin also had a longer length of hospital stay $(1.9 \pm 1.7$ vs. $1.6 \pm 1.2$ days). On logistic regression, low preoperative albumin was associated with higher odds of Clavien category V complications $(\mathrm{OR}=22, \mathrm{CI}=1.4-3.5, \mathrm{p}<0.001)$, Clavien category IV complication $(\mathrm{OR}=1.4, \mathrm{CI}=1.1-1.6, \mathrm{p}<0.001), \mathrm{and}, \mathrm{ny}-\mathrm{cuse}$ ifection $(\mathrm{OR}=1.3, \mathrm{CI}=1.1-1.6)$. The regesion

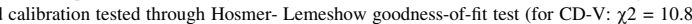
$\mathrm{p}=0.21$; for CD-IV: $\chi^{2}=6.8, \mathrm{p}=.55$; for CIR: $\chi^{2}=3.5, \mathrm{p}=0.89$ ). Figure 1 demonstrates the discriminatory capacity of regression models for CD-V, CD-IV and CIR

Conclusion: Preoperative albumin levels below $3.5 \mathrm{~g} / \mathrm{dL}$ are a significant risk factor for serious short termmorbidity after primary bariatric surgery. Further research should focus on nutritional risk stratification and rehabilitation of patients prior to bariatric surgery.

\section{P113}

Risk Factors of Intraoperative Difficulties During Laparoscopic Sleeve Gastrectomy

Artur Kacprzyk $^{1}$, Magdalena Pisarska, $\mathrm{MD}^{2}$, Tomasz Stefura ${ }^{1}$, Piotr Malczak, MD ${ }^{2}$, Marcin Dembinski, MD, PhD ${ }^{2}$, Jakub Dros ${ }^{1}$, Katarzyna Chlopas ${ }^{1}$, Michal Wysocki, MD ${ }^{2}$, Michal Pedziwiatr, MD, $\mathrm{PhD}^{2}$, Andrzej Budzynski, MD, $\mathrm{PhD}^{2}$, Piotr Major, MD, $\mathrm{PhD}^{2}$; ${ }^{1}$ Students' Scientific Group at 2nd Department of General Surgery, Jagiellonian University Medical College, Krakow, Poland, ${ }^{2}$ 2nd Department of General Surgery, Jagiellonian University Medical College, Krakow, Poland

Introduction: Laparoscopic Sleeve Gastrectomy (LSG) is one of the most frequently performed bariatric procedures worldwide. Preoperative knowledge concerning risk factors of potential intraoperative diffculties may help to predict outcomes and influence the operative approach.

Aim of the study: Our purpose was to identify potential risk factors of intraoperative difficulties during LSG.

Materials and methods: The analysis included consecutive patients who underwent LSG betwee December 2009 and April 2017. Patients with intraoperative difficulties were submitted to Group 1, patient without intraoperative difficulties to Group 2. Demographic parameters were assessed for potential risk factors of intraoperative difficulties. Length of stay (LOS) and complication rate were also analysed.

Results: Group 1 consisted of $37(11.71 \%)$ and Group 2 of $279(88.29 \%)$ patients. Besides rates of diabetes, pulmonary disease and sleep apnea, which were higher in group 1, there were no statistical differences between the groups based on demographic parameters. Univariate logistic regression found that risk factor affecting intraoperative difficulties included BMI $>45 \mathrm{~kg} / \mathrm{m}^{2}(\mathrm{OR} 2.15,95 \%$ CI $1.05-4.39, \mathrm{p}=0.0362)$ experience of operating surgeon (OR $9.22,95 \% \mathrm{CI} 4.31-19.72, \mathrm{p}=0.0058)$, incidence of diabetes (OR 2.44 $95 \%$ CI $1.19-4.98, \mathrm{p}=0.0146)$ or pulmonary disease (OR 12.22, 95\% CI 1.97-75.75, $\mathrm{p}<0.0001)$. In the multivariate logistic regression model only experience of operating surgeon (OR 8.61, 95\% CI 3.75-19.72, $p<0.0001)$ remained significant factor affecting intraoperative difficulties.

Conclusions: The only significant factor contributing to the incidence of intraoperative difficulties is the experience of the surgeon.

Keywords: sleeve gastrectomy, intraoperative difficulties, risk factors, postoperative complications
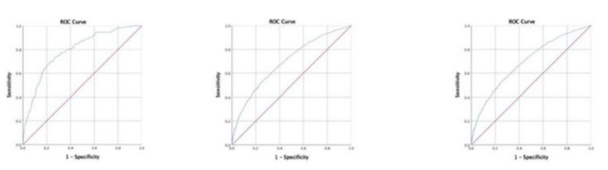

\section{P111} The Utility of Routine Preoperative Upper Gastrointestinal Series
for Laparoscopic Sleeve Gastrectomy

Warren Sun, MD ${ }^{1}$, Jerry T Dang, MD ${ }^{1}$, Noah J Switzer, MD, MPH, FRCSC $^{2}$, Daniel Birch, MD, MSc, FRCSC ${ }^{1}$, Shahzeer Karmali, MD, MPH, FRCSC ${ }^{1} ;{ }^{1}$ University of Alberta, ${ }^{2}$ Ohio State University Wexner Medical Center

Introduction: We aim to study the diagnostic utility of routine UGI series for preoperative evaluation of LSG in our center. Laparoscopic sleeve gastrectomy (LSG) has become the most commonly performed primary bariatric procedure for the treatment of severe obesity in North America. However, there is no consensus for the preoperative diagnostic evaluation for patients undergoing LSG. The role of preoperative upper gastrointestinal (UGI) series to evaluate candidates for LSG is debatable.

Methods and Procedures: A retrospective chart review for patients planning to undergo LSG with one surgeon at our hospital from May 2015 to April 2017 was completed. Primary outcomes included UGI findings and consequential changes in clinical management. Secondary outcomes included preoperative findings and consequential changes in clinical
symptomology and postoperative complications.

Results: 36 patients were identified from billing records of a single surgeon and were originally scheduled to undergo LSG. Four patients were male and $32(88.9 \%)$ were female. The average age was $43.2 \pm 2.1$ years and average preoperative BMI was $47.3 \pm 1.2 \mathrm{~kg} / \mathrm{m}^{2} .22(61.1 \%)$ patients underwent a preoperative UG series, of which, $8(36.4 \%)$ patients had hiatal hernias, $9(40.9 \%)$ had gastroesophageal reflux, and $2(9.1 \%)$ had dysmotility. Additionally, four ( $18.2 \%$ ) of the 22 patients had a change from LSG to laparoscopic Rouxen-Y gastric bypass (LRYGB) due to significant reflux or dysmotility found on UGI. Patients who did not sons. $18(50.0 \%)$ patients underwent a preoperative EGD, of which $4(22.2 \%)$ had esophagitis and $5(27.8 \%)$ had gastritis. One additional patient required a change of surgical management to LRYGB due to reflux found on EGD. Overall, $31(86.1 \%)$ patients had LSG and $5(13.9 \%)$ patients had LRYGB. The average postoperative BMI was $38.7 \pm 1.4 \mathrm{~kg} / \mathrm{m}^{2}$, after an average follow-up period of $8.3 \pm 0.8$ months. Four (11.1\%) patients had postoperative complications, including two LSG requiring revision to LRYGB for reflux esophagitis, one LSG with dysphagia, and one LRYGB with a marginal ulcer.

Conclusion: Our cohort demonstrated that preoperative UGI has the potential to screen for pathology that may affect outcomes after LSG, and changed clinical management in almost one-fifth of patients undergoing UGI. Overall, UGI is a relatively simple and inexpensive test for the preoperative evaluation of patients before LSG.

\section{P114}

Endoluminal Revision of the Dilated Gastrojejunostomy: The State-of-the-art Cure of Dumping Syndrome After Gastric Bypass

\section{Hamzeh Halawani, MD; Farah Medical Complex}

Dumping syndrome is an underdiagnosed consequence after Roux en Y Gastric bypass. High osmolar foo that passes rapidly due to accelerated pouch emptying and a dilated Gastro-Jejunal Anastomosis (GJA) results in osmotic overload and classical symptoms of hypoglycemia. New data links the presences of a dilated GJA and dumping syndrome to weight regain after gastric bypass. For those patients who suffer from intractable dumping syndrome, dietary changes, somatostatin analogues and surgical approaches are all well dercibed. Endeluminl reduction of the GJA using a full thichess endoscopic suturing device is an descibed. Endolo dilated $>10 \mathrm{~mm}$ GJA has been shown to be a major and an independent predictor or weight regain. Endoluminal solutions for patients who underwent a bariatric procedure is an exciting field for both the surgeon and the gastroenterologist. We present a case after a complete cure of intractable dumping syndrome using an endoscopic full-thickness suturing device. 


\section{P115}

\section{Hormonal Response of Sleeve Gastrectomy. Does the Size} of the Bougie Have an Influence?

Berta Gonzalo, Carlos Rodríguez-Otero, Meritxell Garay, Mercedes Camacho, Sonia Fernández, Inka Miñambres, Irene Gómez, Eulàlia Ballester, Carmen Balagué; Hospital Sant Pau

Background amb Aims: Laparoscopic vertical gastrectomy (LSG) is a growing technique in recent decades for the treatment of obesity. There are different theories about the influence of LSG on the hormones involved in glycolipid metabolism. The aim of this study is to analyze the influence of this technique on the levels of GIP, GLP-1, glucagon, ghrelin, insulin, leptin and PYY and the presence of differences according to the two bougie sizes used for gastrectomy.

Materials and methods: Prospective randomized study (Protocol IIBSP-RES-2012-178, EAES Research Project) that included patients undergoing vertical gastrectomy randomized into 4 groups according to distance to the pylorus at the beginning of gastrectomy ( $2 \mathrm{vs}$ $5 \mathrm{~cm}$ ) and size of the probe used for the gastrectomy (bougie size, $33 \mathrm{vs} 42 \mathrm{Fr}$ ). A total of 33 patients ( 24 women and 9 men) who had completed the complete follow-up one year after the intervention were analyzed. The blood values ??of GIP, GLP-1, glucagon, ghrelin, insulin, leptin and PYY were determined preoperatively, postoperatively immediately ( $48 \mathrm{~h}$ post IQ), 2 months and 1 year postoperatively.

Results: The average decrease of the hormones studied, at in the immediate postoperative period, at 2 months and a year after sleeve gastrectomy were (pg/ml): GIP $48.65 \pm 10.09$ / $33.15 \pm 11.97 / 37.84 \pm 10.45$, GLP-1 $6.29 \pm 6.85(\mathrm{p}=0.36) / 13.01 \pm 5.07 / 17.71 \pm 8.03$, glucagon $-13.73 \pm 7.28(\mathrm{p}=0.06) / 15.06 \pm 6.14 / 21.78 \pm 5.68$, ghrelin $33.55 \pm 6.73 /$ $30.36 \pm 7.02 / 32.49 \pm 6.76$, Insulin $209.56 \pm 108.98 \quad(\mathrm{p}=0.06) / 704.13 \pm 124.32$ $788.91 \pm 97.76$, Leptin $\quad-6964.24 \pm 4757.32 \quad(\mathrm{p}=0.15) / 24143.03 \pm 4744.26 /$ $24192.67 \pm 5368.06$ and PYY $14.51 \pm 20.08 \quad(\mathrm{p}=0.47) / 8.19 \pm 12.79 \quad(\mathrm{p}=0.53) /$ $1.29 \pm 9.65(\mathrm{p}=0.89)$. All results were statistically significant excepte those specified with the value of $\mathrm{p}$.

Conclusion: Laparoscopic vertical gastrectomy has significant effects on the hormones involved in obesity, mainly at two months and one year after surgery. The size of the bougie is not an influential factor in the observed hormonal response.

\section{P116}

\section{Clinical Outcomes of Laparoscopic Greater Curvature Plication and Laparoscopic Sleeve Gastrectomy}

Caleb J Ba Mendoza ${ }^{1}$, Osamuyi Idubor ${ }^{1}$, Aisha Tabba ${ }^{1}$, Joel Miller ${ }^{1}$, Aliu Sanni, MD, FACS ${ }^{2}$; ${ }^{1}$ Philadelphia College of Osteopathic Medicine, ${ }^{2}$ Eastside Bariatric and General Surgery LLC

Introduction: Laparoscopic greater curvature plication (LGCP) is an emerging restrictive bariatric procedure that successfully reduces the gastric volume by folding of the greater curvature of the stomach. Laparoscopic Sleeve Gastrectomy (LSG) and LGCP have been both successfully performed in recent years. The aim of this study is to compare the clinical outcomes of LSG vs. LGCP.

Methods and Procedures: A systematic review was conducted through PubMed to identify pertinent studies from 2009-2016 with comparative data on patients who underwent LGCP and LSG. The outcomes analyzed were \%EWL at 12 months, operative times and length of hospital stay. The results are expressed as standard difference in means with standard error. Statistical analysis was done using fixed-effects meta-analysis to compare the mean value of the two groups. (Comprehensive Meta-Analysis Version 3.3.070 software; Biostat Inc., Englewood, NJ).

Results: Five out of 215 studies were quantitatively assessed and included for meta-analysis. A total of 460 subjects underwent bariatric surgery; 221 had LGCP and 239 had LSG. The \% EWL $(-0.541 \pm 0.095, \mathrm{p}<0.0001)$ was higher in the patients who underwent LSG when compared to LGCP. There was no difference in the mean operative times $(0.073 \pm 0.095, \mathrm{p}=0.441)$ or hospital length of stay $(-0.021 \pm 0.094, \mathrm{p}=0.825)$ between both group of patients.

Conclusion: LSG results in higher weight loss at 12 months when compared to LGCP.

\section{P117}

Outcomes Following Robotic-Assisted Compared to Conventional Laparoscopic Primary Bariatric Surgery: A Review of the MBSAQIP Database

Edwin Acevedo, $\mathrm{MD}^{1}$, Huaqing Zhao, $\mathrm{PhD}, \mathrm{MS}^{2}$, Xiaoning Lu, $\mathrm{MS}^{2}$, Rohit Soans, $\mathrm{MD}^{2}$, Michael A Edwards, MD, FACS,

FASMBS ${ }^{2} ;{ }^{1}$ Temple University Hospital, ${ }^{2}$ Lewis Katz School of Medicine at Temple University

Introduction: Robotic-assisted bariatric surgery (RBS) is increasingly performed. The overall benefit of RBS compared to conventional laparoscopic bariatric surgery (LBS) remain controversial. In this study, we compare outcomes between RBS and LBS using a large risk-stratified clinical database. We hypothesize that LBS is safer and more cost-effective than RBS.

Methods: A retrospective analysis of the 2015 and 2016 Metabolic and Bariatric Surgery Accreditation and Quality Improvement Program (MBSAQIP) database was performed. Primary RBS and LBS were analyzed. 1:1 case-controlled matching was performed. Cases and controls were match by demographics (age, gender, race, $\mathrm{BMI}$ ) and preoperative comorbidities. Outcomes measures included operative length $(\mathrm{OL})$, length of stay (HLOS), 30-day outcomes and complications.

Results: 278,761 primary bariatric operations were identified (93\% LBS, 7\% RBS). $79 \%$ were female and $64.7 \%$ white. Mean age and BMI were 44.6 years and $45.5 \mathrm{~kg} / \mathrm{m}^{2} .16,291$ matched RBS and LBS cases and controls were identified, and outcomes compared (Table 1). Conversion rate was higher in RBS $(0.53 \%$ vs $0.1 \%, \mathrm{p}<0.0001)$. RBS was associated with longer OL (118 min vs $85 \mathrm{~min}, \mathrm{p}<0.0001)$ and HLOS ( 1.83 days vs 1.7 days, $\mathrm{p}<0.0001$ ). Readmission, reintervention, reoperation and leak rates were higher in RBS. Bleeding, cardiac arrest and 30-day mortality rates were higher in LBS. There was no difference in mortality related to bariatric surgery ( $\mathrm{p} 0.74$ ).

Conclusion: Robotic-assisted bariatric surgery is associated with longer OL, HLOS and higher complication rates, likely contributing to less cost-effectiveness. Both approaches remain safe, but LBS is associated with a higher all-cause 30 -day mortality.

Table 1. Matched cohort outcome

\begin{tabular}{|c|c|c|c|}
\hline \multicolumn{4}{|l|}{ Approach } \\
\hline Outcomes, N (\%) & $\begin{array}{l}\text { LBS } \\
(N=16,291)\end{array}$ & $\begin{array}{l}\text { RBS } \\
(N=16,291)\end{array}$ & p-value \\
\hline 30-Day Readmission & $604(3.71)$ & 715 (4.39) & 0.0018 \\
\hline 30-Day Intervention & $229(1.41)$ & $278(1.71)$ & 0.0028 \\
\hline 30-Day Reoperation & 194 (1.19) & $232(1.42)$ & 0.064 \\
\hline 30-Day Mortality & $18(0.11)$ & $8(0.05)$ & 0.05 \\
\hline CPR & $12(0.07)$ & $3(0.02)$ & 0.02 \\
\hline Transfusion & $96(0.59)$ & $66(0.41)$ & 0.018 \\
\hline Aggregate Bleeding & $68(0.42)$ & $48(0.29)$ & 0.063 \\
\hline Organ Space SSI & $35(0.21)$ & $62(0.38)$ & 0.006 \\
\hline 30-Day Drain Present & $27(0.17)$ & $70(0.43)$ & $<0.0001$ \\
\hline Agregate Leak & $36(0.22)$ & $66(0.41)$ & 0.0029 \\
\hline
\end{tabular}




\section{P118}

Incidence of Perioperative Hypoglycemia in Bariatric Patients with Diabetes Mellitus Type II: A Single Institution Experience

Cristian Milla Matute, MD, Maria C Fonseca, MD, Camila Ortiz Gomez, MD, Francisco Ferri Abreu, MD, Emanuele Lo Menzo, MD, PhD, FACS, FASMBS, Raul Rosenthal; Cleveland Clinic Florida

Introduction: Bariatric surgery (BS) has shown to be an effective method to permanently reduce weight and improve comorbidities associated with obesity. The most common cause of hypoglycemia is medications used to treat diabetes mellitus such as insulin and sulfonylureas. The incidence of hypoglycemia after BS has been reported ranging from $0-4.5 \%$, although most of the studies consist of small case series. The aim of this study is to analyze the incidence of hypoglycemia in bariatric patients with Diabetes type II at our institution.

Methods: After IRB approval, a retrospective chart review of all patients who underwent bariatric surgery at our institution between 2006 and 2017 was performed. Patients with Diabetes type II and older than 18 years of age that underwent BS were included in the analysis. Fasting hypoglycemia was defined as a measurement of blood glucose level of $<75 \mathrm{mg} / \mathrm{ml}$, with or without symptoms. The perioperative period was defined as 30 days before and 30 days after the BS date. SPSS software was used to perform descriptive statistics and Chi Square for categorical values. A P value $<0.05$ was considered significant.

Results: A total of 4,098 patient met the inclusion criteria. The predominant gender was female $67.7 \%(\mathrm{n}=2,818)$, the mean age was $55.2 \pm 7$, mean BMI $40.6 \pm 8$ and the most common ethnicity represented was Caucasian $73.9 \%(n=3,032)$. Regarding the type of surgery, 60\% $(\mathrm{n}=2,465)$ of the patients had LSG (Longitudinal Sleeve Gastrectomy), 29\% $(\mathrm{n}=1,186)$ had RNYGB (Roux-en-YGastric Bypass), $10 \%(\mathrm{n}=423)$ Gastric Banding (LAGB), and $0.5 \%$ (24) had revisions. The incidence of hypoglycemia pre-operatively was $2.9 \%$ (86), and $4.04 \%$ (56) in the post-operative period. When analyzing the impact demographics and each procedure on the incidence of hypoglycemia in both groups, none of the variables resulted in statistically significant differences (table 1).

Conclusions: This is the first study to analyze perioperative hypoglycemia in bariatric patients with Diabetes Mellitus type II. Our reported incidence of hypoglycemia is $3.4 \%(n=142)$ in the perioperative period of Bariatric Surgery. There was no statistical significant associated with hypoglycemia and type of bariatric procedure.

\begin{tabular}{|c|c|c|c|}
\hline Demographics & $\begin{array}{l}\text { Total Population } \\
4,098 \\
\text { Total Inciden ce (3.4\%) }\end{array}$ & $\begin{array}{l}\text { Hypoglycemia PreOp } \\
86 / 2,962 \\
(2.9 \%) \\
\end{array}$ & $\begin{array}{l}\text { Hypoglycemia PostOp } \\
56 / 1,383 \\
(4.04 \%)\end{array}$ \\
\hline Age & $55.2 \pm 7$ & $67 \pm 9.8$ & $47.2 \pm 4.3$ \\
\hline BMl at Procedure & $40.6 \pm 8$ & $39.1+6.6$ & $44+5.1$ \\
\hline Ethnicity(Caucasian) & $73.9 \%(3,032)$ & $62.7 \%$ & $67.8 \%$ \\
\hline Gender (Female) & $68.76 \%(2,818)$ & $80 \%$ & $71.4 \%$ \\
\hline $\begin{array}{l}\text { Type of Procedure } \\
\text { LSG } \\
\text { RNYGB } \\
\text { LAGB } \\
\text { REVISION } \\
\end{array}$ & $\begin{array}{l}60.1 \%(2,465) \\
28.9 \%(1,186) \\
10.3 \%(423) \\
0.53 \%(24)\end{array}$ & $\begin{array}{l}54 \% \\
33 \% \\
9 \% \\
4 \% \\
\end{array}$ & $\begin{array}{l}57 \% \\
37 \% \\
6 \% \\
0 \%\end{array}$ \\
\hline $\begin{array}{l}\text { Procedure } \\
\text { Total } 142\end{array}$ & $\begin{array}{l}\text { Pre-Op } \\
86\end{array}$ & $\begin{array}{l}\text { Post-Op } \\
56\end{array}$ & PValue \\
\hline LSG & 45 & 32 & 2.33 \\
\hline RNYGB & 28 & 21 & 1.12 \\
\hline LGB & 8 & 3 & 3.02 \\
\hline REVISION & 3 & 0 & 0.23 \\
\hline Total Incidence & $2.9 \%$ & $4.04 \%$ & \\
\hline
\end{tabular}

\section{P119}

Measure Twice, Cut Once: Anatomy-Based Sleeve Gastrectomy Associated with Dramatic Benefits Versus Suction Bougie in Sleeve Gastrectomy Pouch Creation

Jonathan R Thompson, MD ${ }^{1}$, Vikrom K Dhar, $\mathrm{MD}^{1}$, Dennis J Hanseman, $\mathrm{PhD}^{2}$, Brad M Watkins, MD ${ }^{1}$, Tayyab S Diwan, $\mathrm{MD}^{1}$, Thomas Inge, $\mathrm{MD}, \mathrm{PhD}^{3}$, John M Morton, $\mathrm{MD}, \mathrm{MPH}^{4}$; ${ }^{1}$ University of Cincinnati College of Medicine, ${ }^{2}$ SET Data

Collaborative, ${ }^{3}$ Children's Hospital Colorado and University of Colorado, Denver, ${ }^{4}$ Stanford School of Medicine

Introduction: Anatomic imperfections in the sleeve gastrectomy pouch have been implicated in increased gastroesophageal reflux disease (GERD) and food intolerance following LSG. Anatomybased sleeve gastrectomy (ABS) has been developed to improve the shape, volume and anatomic consistency of the laparoscopic sleeve gastrectomy (LSG) pouch. We report our one-year results using an ABS technique.

Methods: A quality improvement data collaborative was initiated with custom fields added to the Metabolic and Bariatric Surgery Accreditation and Quality Improvement Program (MBSAQIP) database. Determination of "not human subjects research" was obtained from our IRB. ABS was implemented beginning in 6/2016. ABS consists of planning a sleeve gastrectomy pouch by marking the stomach $1 \mathrm{~cm}$ from the gastroesophageal junction, $3 \mathrm{~cm}$ from the IA, and $6 \mathrm{~cm}$ from the pylorus. The marks are lined up beside a $25 \mathrm{~cm}$ clamp and a $60 \mathrm{~mm}$ endocutter is used to resect the stomach. 641 ABS cases were compared to 737 cases where a $40 \mathrm{~F}$ suction bougie was used for pouch creation (1/2014-5/2016). No programmatic changes were made during this time. 30-day safety, 6-month and 1-year GERD and weight loss outcomes were compared. Students $t$ test and Chi square tests were used as appropriate.

Results: No significant differences in gender, preoperative BMI, or operative time were identified between groups (all $\mathrm{p}>0.05$ ). Average age was 45 in the bougie group and 43.5 in ABS $(p=0.02)$. ABS was associated with shorter length of stay (1.2 vs $1.7, \mathrm{p}<0.01), 42 \%$ fewer readmissions $(3.3 \%$ vs $5.7 \%, \mathrm{p}=0.03), 61 \%$ fewer readmissions due to nausea/vomiting ( $1.1 \%$ vs $2.9 \%, \mathrm{p}=0.02)$. ABS patients had a $48 \%$ lower 6 -month GERD rate $(21 \%$ vs $40 \%$, p $<0.01), 86 \%$ higher 6-month GERD resolution rate (54\% vs $29 \%$, p < $<.01$ ), $49 \%$ lower 1-year GERD rate (20\% vs 39\%, p < 0.01$)$, $106 \%$ higher 1 -year GERD resolution rate $(72 \%$ vs $35 \%, \mathrm{p}<0.01)$. Both groups had similar 1-year \% total weight loss $(26.3 \%$ vs $27.3 \%, \mathrm{p}=0.41)$. Conclusion: Anatomy-based sleeve gastrectomy offers benefits over sleeve gastrectomy with suction bougie with regard to 30-day safety and dramatic improvement of GERD rates at 6 months and 1 year. 


\section{P120}

Rogue Staples: An Alarming Trend Observed Over the Past Two Years

Allan E Stolarski, MD, MS, Olga Beresneva, MD, Cullen Carter, MD, Luise Pernar, MD, Brian Carmine, MD, Donald Hess, MD; Boston University Medical Center

Introduction: Our objective is to raise awareness of complications arising from loose or improperly folded staples ("rogue staples") that originated from a staple closure device that was appropriately fired. Linear staplers are frequently used instruments across all disciplines of surgery and are highly relied upon tools to effectively complete operations in a safe, efficient, and reliable manner.

Methods and Procedures: At a busy urban tertiary care center from August 1st. 2016 until August 31st 2018 we observed seven cases of rogue staples causing bowel obstruction after several gastric bypasses and one appendectomy performed by various surgeons. Demographic data collected include age, procedure, and identification of the involved staple loads. Outcomes of interest include the number of days between the index surgery and presentation with obstruction, length of stay after representation, and the morbidity of observed patients.

Results: Over a two-year period we have identified seven cases related to a rogue staple protruding beyond the staple line after correct closure technique was applied resulting in adhesion (3 patients; $43 \%$ ) or hooking of nearby bowel (4 patients; $47 \%$ ). All of these patients required return to the operating room, during which time six patients $(86 \%)$ were found to have a small bowel obstruction, and one patient (14\%) suffered from internal hernia. The average time to representation was 8.57 days (range: 2-17 days). The average length of stay after representation was 4.29 days (range: 1-9 days), indicating and increased use of hospital resources.

Conclusion: Over two years, we present a series of seven cases where an improperly formed staple from a linear stapler caused direct mechanical obstruction or adhesion formation. These cases resulted in bowel obstruction or internal hernia in the early postoperative period requiring reoperation. To our knowledge, this is the largest case series in the literature describing this early postoperative complication. Due to our recent experiences following gastric bypass or appendectomy, general and bariatric surgeons should be aware of these potentially significant consequences of linear staplers.

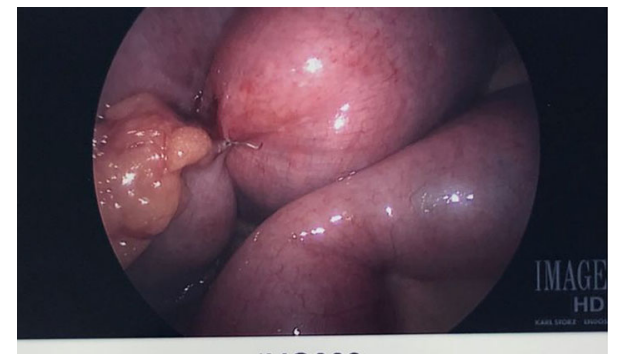
IMG002

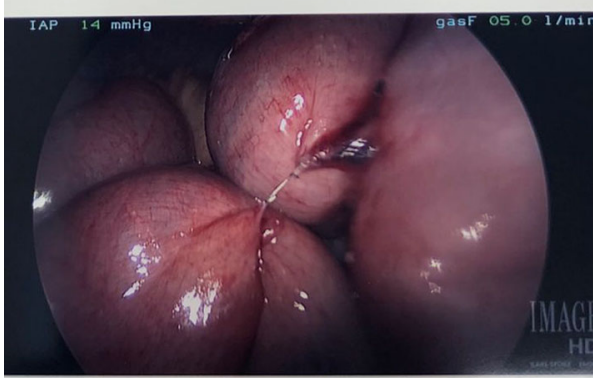

P121

Reversal of RYGB and Revision to Single Anastomosis Duodenal Ileal Bypass with Sleeve Gastrectomy (SADI-S) Using a Two Stage Approach. Safety, and 30 Day Outcomes for Patients with Failed RYGB

Ryan Fairley, DO ${ }^{1}$, Moataz Bashah, $\mathrm{MD}^{2}$, Danial Cottam, $\mathrm{MD}^{3}$, Helmuth T Billy ${ }^{\mathrm{T}}{ }^{1}$ Community Memorial Hospital, Ventura California, ${ }^{2}$ Hamad Medical Center, Doha Qatar, ${ }^{3}$ Bariatric Medical Institute, Salt Lake City, Utah

Background: Failed Roux-Y Gastric Bypass (RYGB) is a difficult problem with few options for treatment. We report a series of ten consecutive morbidly obese patients with weight regain following RYGB who were converted to SADI-S using a 2 stage approach.

Methods: 10 patients with a history of laparoscopic RYGB and weight regain $(B M I>35)$ were evaluated for surgical revision. Each patient completed medical weight management consisting of monthly appointments with a dietician, psychological evaluation and follow up with the physician team. All patients failed to achieve any significant weight loss. Each patient underwent laparoscopic reversal of the RYGB as a first stage followed by revision to laparoscopic SADI-S as a second stage.

Results: 10 morbidly obese patients underwent revision following weight regain after RYGB. Average pre-operative BMI was 44.3 with a range of 37.6 to 54.1. All patients underwent EGD without evidence of abnormal pathology. EGD revealed an average gastric pouch length between 5 and $6 \mathrm{~cm}$. There was no evidence of pouch dilation, fistula, anastomotic dilation or other abnormality that might have contributed to weight regain. Presenting weight ranged from $210.5 \mathrm{lb}$ to $362.4 \mathrm{lb}$. Each patient underwent laparoscopic reversal of their gastric bypass to normal anatomy. The average time from primary RYGB to reversal of gastric bypass was 8 years. Average operative time to perform the reversal RYGB to normal anatomy was 170.9 min. Average length of stay was 2.5 days.

Time between reversal of RYGB to laparoscopic SADI-S ranged from 3 to 6 months. Preoperative weight at the time of SADI-S ranged from $215.5 \mathrm{lb}$ to $353.8 \mathrm{lb}$. Average operating time to perform the laparoscopic SADI-S was $198.3 \mathrm{~min}$. Average length of stay was 2.7 days. 30 day post operative weight ranged from 196.6 to $316.6 \mathrm{lb}$ and the average weight lost per patient in the first 30 days was $19.85 \mathrm{lb}$.

In the 30 days following SADI-S, 2 patients were seen in the emergency department for reflux, both treated with proton pump inhibitors not requiring admission. There were no reoperations, there were no deaths and there were no readmissions

Conclusions: Our series of 10 patients undergoing a two stage approach to revise failed RYGB to SADI-S appears to be a promising and safe approach to the challenge of weight regain following RYGB. Further long term follow up and a larger series will be needed to demonstrate safety and efficacy, 
$\mathbf{P 1 2 2}$

\section{P124}

\section{Preoperative Use of Arepitant for Laparoscopic Sleeve Gastrectomy and its Impact on Hospital Length of Stay in a Community Hospital Setting}

Kristin Mccoy, MD, Meaghan Broderick, MD, James Bonheur, MD, Phillip Bilderback, MD; Stamford Hospital

Introduction: Laparoscopic sleeve gastrectomy was first performed in 2000 as a component of the biliopancreatic diversion with duodenal switch. Today, it is gaining popularity in the bariatric field as a stand-alone procedure for weight loss. However, patients continue to complain of postoperative nausea, vomiting and dry heaving due to the new anatomical constriction. Nausea and vomiting can be harmful to the newly formed staple line. Several anti-emetics are utilized in the postoperative patients. We aim to investigate the use of arepitant which is a neurokinin-1 inhibitor as a prophylactic anti-emetic in laparoscopic sleeve gastrectomy patients.

Methods: 36 patients from a multicenter bariatric practice were studied prospectively between October 2017- Septmeber 2018. Patient data collected included age, gender, BMI, post-operative use of anti-emetic medicine, episodes of emesis, and length of stay. Statistical analysis was performed on post-operative episodes of emesis, and administered doses of antiemetic medicine.

Results: There were 18 patients in each of the cohorts. The population was predominantly female with only one male in each of the cohorts. The average age in the arepitant group was 41.6 vs. 43.2 in the control group. Average starting body mass index (BMI) in the arepitant group was 41.9 vs. 41.0 in the control group. There was no statistical difference in BMI or age. The arepitant group required less post-operative emetics with $7.6 \mathrm{mg}$ of Zofran administered in the arepitant group and $9.6 \mathrm{mg}$ in the control group. The length of stay was also shorter in the arepitant group as compared to the control group (1.55 days vs. 1.73 days). There was no significant difference in number of episodes of emesis between the arepitant and control arms.

Conclusion: The preoperative use of arepitant aids in reducing the postoperative nausea and vomiting patients experience. Our results show promise in decreasing the length of stay in laparoscopic sleeve gastrectomy patients, as well as the amount of post-operative antiemetics required. Arepitant should be considered in the use of bariatric elective procedures.

\section{Validation of the Modified Frailty Index as a Predictor of Outcomes After Bariatric Surgery}

$\underline{\text { Jakub Dros }^{1}}{ }^{1}$, Tomasz Stefura ${ }^{1}$, Artur Kacprzyk ${ }^{1}$, Katarzyna Chlopas ${ }^{1}$, Magdalena Pisarska, $\mathrm{MD}^{2}$, Michal Wysocki, MD², Michal Pedziwiatr, MD, $\mathrm{PhD}^{2}$, Andrzej Budzynski, MD, PhD, Professor ${ }^{2}$, Piotr Major, $\mathrm{MD}, \mathrm{PhD}^{2}$; ${ }^{1}$ Students' Scientific Group at 2nd Department of General Surgery, Jagiellonian University Medical College, Krakow, Poland, ${ }^{2}$ 2nd Department of General Surgery, Jagiellonian University Medical College, Krakow, Poland

Introduction: Obesity and aging are associated with declines in organ functions and constitute a marker of frailty. A modified frailty index (MFI) is a confirmed tool predicting postoperative morbidity and mortality, but its usefulness in the field of bariatric surgery has not been sufficiently investigated. As the population ages and more elderly patients seek bariatric procedures, it is essential to evaluate the method which may potentially improve quality and efficacy of the treatment.

Objectives: To assess the usefulness of MFI as a predictor of short- and long-term outcomes of bariatric surgery.

Methods: The retrospective analysis was conducted among 731 patients who underwent laparoscopic bariatric surgery during eight-year period. MFI was calculated using 11 variables defined in the National Surgical Quality Improvement Program of the American College of Surgeons. Primary endpoints of the study include intra- and postoperative parameters, secondary endpoints - long-term effects of bariatric treatment after one-year follow-up. The correlations between MFI and surgical outcomes were assessed. Additionally, a cutoff MFI score of 0.18 and above classified patients as "frail".

Results: Increasing MFI was not significantly associated with longer operative time ( $p=0.41$ for sleeve gastrectomy, $\mathrm{p}=0.88$ for Roux-and- $Y$ gastric bypass), higher rates of intraoperative adverse events $(\mathrm{p}=0.36)$, postoperative complications $(\mathrm{p}=0.08)$, reoperations $(\mathrm{p}=0.16)$, readmissions $(p=0.21)$ and longer hospital stay $(p=0.25)$. Multivariate logistic regression models adjusted for relevant intergroup baseline differences did not show increased risks of negative intra- and postoperative outcomes in "frail" patients. Increasing MFI was negatively correlated with effects of bariatric treatment in terms of percentage of weight loss $(p=0.02)$ and percentage of excessive weight loss $(p<0.01)$, but not percentage of excessive body-mass index loss $(p=0.09)$. Conclusion: Estimation of MFI in bariatric patients does not constitute a method predicting intra- and postoperative outcomes of the surgery. However, high MFI is associated with inferior long-term effects of bariatric treatment in terms of weight loss.

Keywords: bariatric surgery, modified frailty index, postoperative complications

\section{$\mathbf{P 1 2 5}$}

\section{Outcome of Laparoscopic Sleeve Gastrectomy in Morbidly Obese Thai Patients}

Pakkavuth Chanswangphuvana $^{1}$, Ajjana Techagumpuch ${ }^{1}$, Karikarn Auksornchart ${ }^{2}$, Sanit Wichansawakun ${ }^{3}$; ${ }^{1}$ Division of Gastrointestinal and Endoscopic Surgery, Department of Surgery, Thammasat University, Pathum Thani, Thailand, ${ }^{2}$ Division of Trauma and Critical Care, Department of Surgery, Thammasat University, Pathum Thani, Thailand, ${ }^{3}$ Division of Clinical Nutrition, Department of Medicine, Thammasat University, Pathum Thani, Thailand

Background: Because of technical simplicity and the relatively good outcome, Laparoscopic Sleeve Gastrectomy (LSG) as a standalone bariatric procedure has rapidly gained popularity worldwide. LSG was also the most common bariatric procedure in Thailand. In our hospital, obesity clinic was established in June 2015 and the first bariatric procedure was LSG that was performed in November 2015. The objective of this study was to evaluate the outcomes for LSG in morbidly obese Thai patients.

Methods: There were 64 morbidly obese Thai patients underwent bariatric procedure that 52 procedures $(81.3 \%)$ were LSG as a standalone procedure. The mean age was 37.6 years (19-58 years). The mean preoperative body weight and body mass index (BMI) were $140.7 \mathrm{~kg}(85-195 \mathrm{~kg})$ and $51.2 \mathrm{~kg} / \mathrm{m}^{2}(33.2-71.1 \mathrm{~kg} / \mathrm{m} 2)$ respectively. Twenty four super morbidly obese patients $(46.2 \%)$ that BMI was more than $50 \mathrm{~kg} / \mathrm{m}^{2}$ were included. All patients were evaluated and managed under a strict multidisciplinary team approach.

Results: There were neither morbidity nor mortality. The mean BMI declined to $36.5 \pm 9.1 \mathrm{~kg} / \mathrm{m}^{2}$ at 1 year and $31.3 \pm 7.3 \mathrm{~kg} / \mathrm{m}^{2}$ at 2 years $(\mathrm{p}<0.001)$. The mean percent total body weight loss (\%TWL) and mean percent excess body weight loss was $32.0 \pm 13.3 \%$ and $62.1 \pm 25.1 \%$ at 1 year and $39.0 \pm 13.2 \%$ and $78.5 \pm 23.8 \%$ at 2 years. Complete remission of type 2 diabetes was achieved in 8 patients (36.4\%). Super morbidly obese patients had a tendency to achieve less weight loss. Revision of LSG were required in eight patients $(15.4 \%)$ due to insufficient weight loss.

Conclusion: LSG for Thai morbidly obese patients is effective with good short-term outcomes. For super morbidly obese patients, other surgical options may be required. The revision of LSG is possible and mainly for insufficient weight loss.

\section{Evaluating the Safety of Bariatric Surgery for Weight Loss in Class I Obesity: A Propensity-Matched Analysis of North American data}

Gary G Gamme, $\mathrm{MD}^{1}$, Jerry Dang, $\mathrm{MD}^{2}$, Noah Switzer, $\mathrm{MD}^{3}$, Richdeep Gill, $\mathrm{MD}^{4}$, Daniel Birch, $\mathrm{MD}^{2}$, Shahzeer Karmali, $\mathrm{MD}^{2} ;{ }^{1}$ University of Ottawa, ${ }^{2}$ University of Alberta, ${ }^{3}$ Ohio State, ${ }^{4}$ University of Calgary

Background: Bariatric surgery is a safe and effective treatment for severe obesity. However, there has been an evolving role for bariatric surgery as a primary treatment in the management of Class I obesity. Objectives: We aimed to assess safety of surgery by comparing the surgical safety of LSG and LRYGB in Class I obesity to those with Class 2 obesity and higher with an analysis of a large-scale matched patient cohort analysis. Setting International database, USA and Canada.

Methods: We performed a retrospective analysis using the MBSAQIP database, which collects patient information from over 790 bariatric surgery centers in North America. Patients included in our analysis underwent surgery in the years 2015 and 2016 and had either LRYGB or LSG for weight loss. A propensity matched analysis was performed between patients with Class I obesity and Class II obesity and higher for factors predictive of major complications and mortality.

Results: Initial analysis revealed 274,091 patients. Propensity matching resulted in 9,104 patients for analysis in each arm. The overall major complication rate between the two matched groups was $3.9 \%$ for Class I and 3.5\% for Class 2 and higher $(p=0.09)$. We did not find that Class I obesity was associated with an increased risk of 30-day complication or death.

Conclusions: In our analysis of propensity-matched patients undergoing LSG and LRYGB for weight loss, Class I obesity did not have statistically higher risk of postoperative complication rates compared to Class II and higher. 
P126

Feasibility of Laparoscopic Sleeve Gastrectomy for Morbidly Obese Patients with Cardiomyopathy

Fahad Ba Mehriz, Consultant Laparoscopic and Bariatric ${ }^{1}$, Hassan Arishi, General Surgery Resident ${ }^{2}$, Mohammed Alali, General Surgery Resident ${ }^{1} ;{ }^{1}$ King Khalid university hospital, ${ }^{2}$ King Abdulaziz Medical City, National guard health affair

Background: Morbidly obese patients with associated co-morbidities have high perioperative morbidity and mortality. Cardiomyopathy found to be a predictor for mortality in those undergoing bariatric surgery. Laparoscopic sleeve gastrectomy (LSG) with its low complications rate could be safe option for those patients.

Methods: A retrospective study of morbidly obese patients with cardiomyopathy underwent LSG (2016-2018). Length of stay, 30-day morbidity, mortality, and emergency department visits were assessed. The aim is to assess the feasibility and safety of LSG in morbidly obese patients with cardiomyopathy.

Results: Elven patients ( 7 men) with mean age $41.27 \pm 5.16$ years, preoperative body mass index (BMI) $52.78 \pm 4 \mathrm{~kg} / \mathrm{m}^{2}$ (39-88), and mean ejection fraction $38.73 \%$ (20.3-73.9). Six patients $(54.5 \%)$ were having American Society of Anesthesiologists (ASA) class III. The mean length of stay was 5.6 days. There was no 30 -day mortality. One patient was readmitted due to sever dehydration and acute kidney injury.

Conclusion: Our study showed that LSG is feasible and safe for morbidly obese patients with cardiomyopathy. Further researches are recommended to assess postoperative ventricular function and quality of life.

\section{P128}

\author{
Non-internal Hernia Bowel Obstructions After Roux-en-y Gastric \\ Bypass
}

Brandon W Vanderwel, MD, Kevin M Reavis, MD, Jan C Jay, MD, Valerie J Halpin, MD; Legacy Good Samaritan Medical Center

Background: Roux-en-y gastric bypass is a proven therapeutic treatment for metabolic diseases and morbid obesity. Internal hernias as a cause of bowel obstruction after roux-eny have been extensively studied and surgical techniques have been developed to reduce their incidence. There is a paucity of data, however, about the incidence and etiology of non-internal hernia bowel obstructions (NIHBO).

Methods: A retrospective review of our institutional MBSAQIP database from 01/01/2015 - 08/01/2018 was used to identify NIHBO after roux-en-y gastric bypass. PHI was deidentified and data about patient characteristics, comorbidities, and clinical course were collected from the medical record.

Results: Twenty patients were identified as meeting the eligibility criteria for NIHBO after roux-en-y gastric bypass. Each patient that experienced a NIHBO was categorized by etiology: intraluminal hemorrhage $(10 \%)$, technical $(20 \%)$, unclear etiology $(20 \%)$, food bezoar $(20 \%)$, and adhesive disease $(30 \%)$. Intraluminal hemorrhage as a source of obstruction presented within 1-2 days after surgery and were operatively managed to resolve the hemorrhage. All technical obstructions presented within 5 days of discharge (average post operative day 4) and were successfully treated with an operation, where a clear technical issue was identified and corrected. Patients with unclear etiology of obstruction presented within 2-7 days after surgery (average post operative day 4) and all were successfully managed with supportive care. All food bezoar obstructions presented within 30 days of discharge (average post operative day 8 ) and all were successfully treated with nonoperative management. Adhesive obstructions presented within a range of 3-75 days after discharge (average post operative day 26) and all were treated with an operation. There were no mortalities.

Conclusion: There are a variety of causes of post operative bowel obstruction after roux-eny gastric bypass. Technical, bleeding, and adhesive disorders are successfully managed with surgery. Dietary causes can be managed nonoperatively.

\section{P129}

\section{Chronic Prescription Opioid Use Does Not Impact Outcomes after Bariatric Surgery}

Nicole Shockcor, Sakib Adnan, Ariel Siegel, Sami Tannouri, Mark Kligman; University of Maryland

Introduction: Over the last decade, United States healthcare providers dispensed over 200 million opioid prescriptions annually for chronic pain. Here we aimed to determine the effect of prescription opioid use on weight loss post laparoscopic Roux-en-Y gastric bypass (LRYGB) as well as laparoscopic sleeve gastrectomy (LSG).

Methods: We completed a retrospective review of chronic prescription opioid use in 1177 consecutive patients undergoing primary bariatric surgery at a single institution. Patients were grouped into chronic prescription opioid users (OU), defined as ongoing opioid use for $>3$ months at the time of surgery, and non-users (NU), defined as no opioid use prior to surgery. Patients undergoing remedial operations (conversions or revisions), and those lost to follow up were excluded.

Results: In the 1177 patients included in this analysis, $133(11.3 \%)$ were identified as chronic prescription opioid users. 465 (39.5\%) underwent LSG and $713(60.6 \%)$ underwen LRYGB with similar rates in both groups. At 2 months OU patients lost on average $54.88 \mathrm{lb}$, similar to NU patients $56.8 \mathrm{lb}$ (p-value 0.402 ). Similar outcomes persisted longterm at 1 year follow up with average weight loss in the OU group $93.46 \mathrm{lb}$ vs NU $97.91 \mathrm{lb}$ (p-value 0.458). Postoperative complications and rates of follow up were studied and similar between groups.

Conclusions: A significant proportion (11.3\%) of bariatric patients have active narcotic prescriptions, and given the ongoing opioid epidemic, understanding the short and longterm effects on surgical patients is key. Initial analysis here supports similar weight loss outcomes in prescription opioid users as compared to non-users. 
P130

Reducing Readmission Following Bariatric Surgery: Is There an App for This?

Jordan Heuser, $\mathrm{MD}^{1}$, Azusa Maeda, $\mathrm{PhD}^{2}$, Caterina Masino, $\mathrm{MA}^{2}$, Timothy Jackson, MD, MPH ${ }^{1}$, Allan Okrainec, MD,

MHPE $^{1}$; ${ }^{1}$ Department of Surgery, Faculty of Medicine, University of Toronto, ${ }^{2}$ Division of General Surgery, University Health Network

Introduction: Despite efforts to improve recovery through the enhanced recovery after surgery (ERAS) pathway, visits to the emergency department (ED) and readmissions remain a critical issue in bariatric surgery patients. The purpose of this study is to determine the impact of a patient education mobile app on healthcare utilization and outcomes of patients undergoing bariatric surgery.

Methods: Bariatric surgery patients were recruited prospectively to use a mobile app that provides evidence-based surgical and dietary education, allows tracking of postoperative symptoms for 30 days, and alerts patients at risk of ED visits or readmission when necessary. The cohort was compared to the group of patients who did not enroll in the app for the same study period of September 2017 to April 2018. Data on 30-day readmissions, ED visits, and postoperative occurrences were collected from the Metabolic and Bariatric Surgery Accreditation and Quality Improvement Program (MBSAQIP) database to compare the healthcare utilization between the two groups. Data from the mobile app was additionally analyzed to evaluate user satisfaction and usage rates.

Results: There were 338 bariatric surgery patients included during the study period, with 104 patients who enrolled in the mobile application. There was an equal distribution based on age, gender, BMI and type of bariatric procedure performed between patients with and without the app. There was no difference in ED visits $(7 / 104,6.7 \%$ vs. $14 / 234,6.0 \%)$ or readmissions $(6 / 104,5.8 \%$ vs. $7 / 234,3.0 \%)$ between patients with and without the app, respectively. The mobile app surveys indicated that the overall ratings were high $(37 / 50,74 \%)$ and the usage rates were high $(86 \%$ of patients used the app at least once and usage was over $50 \%$ for post-operative days 1 to 7 ). In addition, although the MBSAQIP data revealed no difference in ED visits, the survey from the mobile app showed that $12.6 \%$ of patients perceived that they had avoided at least one ED visit.

Conclusions: Overall, patient satisfaction and use of the mobile app were high, although a reduction in ED visits and readmissions was not found despite a significant percentage of patients that reported avoided ED visits because of the mobile app. Further investigation is warranted to evaluate reduction of other healthcare resources such as nurse educators, as well as to increase the proportion of patients that enroll in the mobile app and allow for transition to a paperless patient information pamphlet.

\section{P131}

\section{Text Mining Techniques for Bariatric Patient Personal} Statements

Kristen M Saad, MS ${ }^{1}$, Paige L Martinez, $\mathrm{MS}^{2}$, Larissa A Mcgarrity, $\mathrm{PhD}^{3}$, Ellen H Morrow, $\mathrm{MD}^{2}$, Eric T Volckmann, $\mathrm{MD}^{2}$, Juliana $\mathrm{S}$ Simonetti, $\mathrm{MD}^{4}$, Anna R Ibele, MD ${ }^{2}$; ${ }^{1}$ University of Utah School of Medicine, ${ }^{2}$ University of Utah Department of Surgery, ${ }^{3}$ University Hospital Rehabilitation Psychology, ${ }^{4}$ University of Utah Department of Internal Medicine

Introduction: Bariatric surgery is one of the best-evidenced treatments for obesity, a complex medical condition with both physiologic and psychosocial contributors. As such, it's become standard of care at some institutions to elicit pre-surgical written personal statements regarding patient motivation for seeking bariatric surgery. While these statements are often used as a "jumping-off point" for clinical discourse, they also represent a rich data source for text analytics.

This pilot study $(\mathrm{n}=50)$ presents novel interdisciplinary methodological approaches and best practices for bariatric personal statement text mining to uncover and elucidate lexical and psychosocial patterns in presurgical patients.

Methods and Procedures: A proof-of-concept dataset of pre-surgical personal statements and associated demographic factors was constructed using an IRB-approved database, and subsequently analyzed with R. The dataset was cleansed, normalized, and descriptive statistical techniques applied to better understand the population of statements. The statements were then analyzed for word frequency, topic affiliations, and sentiment to better understand underlying patterns in patient motivation and psychological state.

Results: Word clouds, comparative graphs, and sentiment charts are used to effectively communicate the results of text mining studies using the pilot dataset. Methods, lessons learned, and best practices for database design, data cleansing, descriptive analytics, advanced computational techniques, and result integration and operationalization will be presented.

Conclusions: By combining a wide range of computational methods including word counts, lexical diversity, collaborative filtering, and sentiment analysis, underlying text patterns can be detected and analyzed for clinical use as well as potential association with postsurgical and long-term outcomes. While bariatric survey statements have been evaluated and subjectively scored by teams of researchers, this is the first known investigation utilizing computational text analytics to evaluate statements elicited as standard-of-care. This project represents a step forward in the field as it leverages a novel application of interdisciplinary techniques to enable clinicians to understand patient motivations and population patterns using patients' own words.

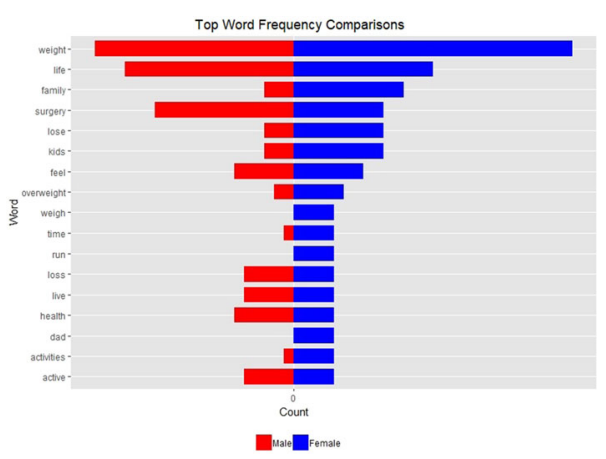


$\mathbf{P 1 3 2}$

Impact of Bariatric Surgery on Male Sex Hormones and Sperm Quality: A Systematic Review and Meta-Analysis

Yung Lee, BHSc ${ }^{1}$, Jerry Dang, $\mathrm{MD}^{2}$, James $\mathrm{Yu}, \mathrm{BHSc}^{1}$, Chunhong Tian, $\mathrm{PhD}^{2}$, Noah Switzer, MD, $\mathrm{MPH}^{2}$, Daniel W Birch, MD, $\mathrm{MSc}^{2}$, Shahzeer Karmali, MD, MPH ${ }^{2},{ }^{1} \mathrm{McMaster}$ University, ${ }^{2}$ University of Alberta

Background: This systematic review and meta-analysis aims to establish the effects of bariatric surgery on male sex hormones, sperm parameters, and sexual function. Men with obesity experience lower testosterone levels, lower sexual satisfaction, and reduced fertility. The literature on the effect of bariatric surgery on male sex hormones and sperm quality is considerable and has not been comprehensively reviewed and meta-analyzed.

Methods: We searched the following databases covering the period from database inception through June 2018: MEDLINE, EMBASE, Web of Science, and Scopus. Articles were eligible for inclusion if the studies examined the effect of bariatric surgery on male sex hormones and sperm parameters in patients with obesity. Primary outcomes of interest were: (1) sex hormones (luteinizing hormone ( $\mathrm{LH}$ ), follicle stimulating hormone (FSH), total estradiol, free estradiol, total testosterone, free testosterone, dehydroepiandrosterone (DHEA), androstenedione, sex hormone binding globulin (SHBG), prolactin, inhibin B) and (2) sperm quality (sperm volume, sperm concentration, \% total motility, \% normal morphology, \% progressive motility. Secondary outcome was sexual function (International Index of Erectile Function (IIEF) score). Pooled estimates were calculated using random effects meta-analyses and heterogeneity was quantified using the inconsistency (I2) statistic.

Results: A total of 28 cohort studies with 1,022 patients were identified from 3,896 potentially relevant citations. Both free and calculated testosterone levels were significantly increased after bariatric surgery (Mean Difference (MD) $-7.47 \mathrm{nM}, 95 \% \mathrm{CI}-8.62$ to $6.31, \mathrm{p}<0.001$ and $\mathrm{MD}-0.05 \mathrm{nM}, 95 \% \mathrm{CI}-0.07$ to $-0.02, \mathrm{p}<0.001$ respectively). Consistent with the increase in testosterone levels, $\mathrm{LH}$, FSH, and SHBG levels were also significantly increased after surgery. In contrast, free and total estradiol and prolactin levels were significantly decreased after bariatric surgery. From only five studies that reported the IIEF score, bariatric surgery led to a small, but statistically significant increase in erectile function after surgery (MD -0.46 , $95 \% \mathrm{CI}-0.89$ to $-0.02, \mathrm{p}=0.04)$. However, bariatric surgery did not affect any of the sperm parameters, DHEA, androstenedione, and inhibin B levels. Most of the sex hormone meta-analyses results had considerable heterogeneity (I2>50\%).

Conclusions: Sustained weight-loss induced by bariatric surgery had an effect on male sex hormones and decreased female sex hormones in male patients with obesity. However, sperm quality and function were not improved after surgery. Long-term comparative studies or adequately powered randomized controlled trials are warranted to examine the impact of bariatric surgery on male sex hormones and sperm quality.

\section{P133}

The Impact of Protein Malnourishment on Bariatric Surgery Outcomes: An Mbsaqip Analysis

Michael Mazzei, MD ${ }^{1}$, Jeremy Van De Rijn ${ }^{2}$, Dominic Recco ${ }^{2}$, Rajiv Raghavan, MD ${ }^{1}$, Matthew Knouse, $\mathrm{MD}^{1}$, Michael Edwards, $\mathrm{MD}^{1}$, Eric Velazquez, $\mathrm{MD}^{1} ;{ }^{1}$ Temple University Hospital, ${ }^{2}$ Temple University Lewis Katz School of Medicine

Introduction: While morbidly obese patients have an excess of stored calories, this is not necessarily commensurate with adequate nourishment. Nutritional deficiencies are commonly identified in the bariatric population; in particular, hypoalbuminemia has been identified in up to $15 \%$ of patients prior to bariatric surgery. Protein malnutrition has been implicated as a risk factor for poor outcomes in a number of surgical populations, and may represent a potentially modifiable preoperative risk factor in the bariatric patient. In this study, we evaluate the effects of hypoalbuminemia on 30-day bariatric outcomes.

Methods: From the American College of Surgeons Metabolic and Bariatric Surgery Accreditation Quality Improvement Program (MBSAQIP) database, we identified patients with albumin levels recorded within 30 days prior to weight loss surgery in 2015-2016. An unmatched cohort analysis as well as a 1:1 propensity-matched cohort analysis was performed to assess the relationship between preoperative albumin levels and thirty-day postoperative outcomes and complication rates.

Results: Of the 195,407 patients with albumin levels recorded in the thirty days prior to weight loss surgery, $12,124(6.2 \%)$ had protein malnutrition (albumin $<3.5 \mathrm{~g} / \mathrm{dl}$ ). At baseline, hypoalbuminemic patients had higher BMI (48.9 vs. $45.1, \mathrm{p}<0.001)$ with significantly increased rates of comorbid conditions, including heart disease, diabetes, and COPD. These patients had longer postoperative length of stay ( 2.1 vs 1.7 days, $\mathrm{p}<0.001)$, and higher rates of readmission (6.1\% vs. $4.3 \%, \mathrm{RR}=1.41, \mathrm{p}=0.001)$, unplanned ICU admission $(1.4 \%$ vs. $0.8 \%, \mathrm{RR}=1.77, \mathrm{p}<0.001)$, re-intervention $(2.5 \%$ vs. $1.5 \%, \mathrm{RR}=1.61, \mathrm{p}<0.001)$, and wound infections $(1.4 \%$ vs. $0.8 \%$, $\mathrm{RR}=1.59, \mathrm{p}<0.001)$. On $1: 1$ propensity-matched analysis of 24,064 patients controlling for age, BMI, operation type, and major comorbidities, the findings of poorer outcomes among patients with preoperative protein malnutrition were preserved, including higher rates of readmission $(6.1 \%$ vs. $5.1 \%, \mathrm{RR}=1.18, \mathrm{p}=0.004)$, re-intervention $(2.4 \%$ vs. $1.8 \%, \mathrm{RR}=1.33, \mathrm{p}=0.002)$, and wound infections ( $1.3 \%$ vs. $1.0 \%, \mathrm{RR}=1.33, \mathrm{p}=0.019)$.

Conclusions: In this large database analysis, protein malnutrition independently increases the risk of 30-day adverse outcomes following primary bariatric surgery. This is especially true in the context of a higher prevalence of comorbid conditions in this patient population, which further increases the risk. Because hypoalbuminemia may be viewed as a modifiable comorbid condition, weight loss surgery should be deferred for patients with protein malnutrition until this condition is corrected preoperatively. 


\section{P134}

\section{Resolution of Co-morbidities in Pediatric Patients Undergoing Bariatric Surgery}

Aryan Meknat, MD, Gustavo Fernandez-Ranvier, MD, PhD, Vivienne Cabreza, MD, Kaitlyn E Billington, DNP, AGPCNPBC, Daniela E Guevara, MD, Daniel M Herron, MD, Matthew L Dong, MD, Abiba Salahou; Mount Sinai Hospital

Introduction: Adolescent obesity is currently an epidemic in the western world. With increased prevalence of obesity in the pediatric population, we are seeing a significantly younger onset of metabolic syndrome (1). This can potentially lead to a greater rise in premature morbidity and mortality from cardiovascular disease, as a result of prolonged exposure to modifiable risk factors $(1,2)$. The objective of this study was to evaluate the effect of bariatric surgery on obese pediatric patients who had obesity-related comorbidities.

Methods: We retrospectively reviewed our prospectively maintained database and included in the analysis pediatric patients (range; 15 to 21 years) who underwent a laparoscopic sleeve gastrectomy (LSG) or a laparoscopic roux-en-y gastric bypass (LRYGB) between 2011-2016, and those who subsequently followed up at one and two years. Attention was placed on those who had obesity-related co-morbidities (e.g. type 2 diabetes mellitus, dyslipidemia, and/or obstructive sleep apnea) and whether or not they were resolved after the bariatric procedure.

Results: The review yielded 70 patients; $12(17.14 \%)$ had sleep apnea (necessitating the use of CPAP), $4(4.28 \%)$ had type 2 DM, and $2(2.85 \%)$ had dyslipidemia. Sixty eight percent of all co-morbidities were resolved at one-year follow-up with no recurrence at two years. These left three patients with sleep apnea, who all stated their need for CPAP was decreasing and one patient with type 2 DM after having undergone a LRYGB. There were fifty-two other pediatric patients who underwent either LSG or LRYGB that did not have an associated co-morbidity, and they did not develop one when seen on follow-up. Conclusion: Our study showed $68 \%$ of patients had complete resolution of their preexisting co-morbidities at one-year follow up. Evaluation at long-term follow up is necessary to determine if the rate of resolution improves or if there are recurrences. More studies are showing that obesity in the pediatric population is associated with mortality secondary to the associated co-morbidities. These same studies are showing safe and effective results of pediatric bariatric procedures with respect to weight loss and resolution of co-morbidities analogous to the adult population (1-3)

\section{P135}

\section{Gastrojejunostomy Stricture after Roux-en-Y Gastric Bypass, A 17 Year Experience}

Brittany Nowak, MD, George Fielding, MD, Marina Kurian, MD, Bradley Schwack, MD, Andrea Bedrosian, MD, Christine RenFielding, MD; New York University Langone Medical Center

Introduction: The gastrojejunostomy (GJ) during Roux-en-Y gastric bypass (RYGB) can be performed by stapled or hand-sewn techniques, and is at risk for anastomotic stricture, reported in the literature at rates from 0 to $33 \%$. This study reviews a single center's experience with anastomotic stricture and intervention required.

Methods and Procedures: A retrospective chart review was performed of 904 patients who underwent RYGB as primary or revisional surgery at a single institution from October 2000 through September 2017. There were 182 patients excluded for follow up duration of less than 1 year, 5 for an esophagojejunostomy rather than GJ, and 1 for gastroparesis as the surgical indication rather than morbid obesity. This left 716 patients to be included in the study. Demographic and operative data were collected including technique for GJ, postoperative follow up, and complications, with a focus on GJ stricture and subsequent interventions.

Results: Gastrojejunostomy (GJ) was performed with a 25 CEEA stapler in $674(94.1 \%)$ patients, with a linear stapler in $25(3.5 \%)$, was hand-sewn in $7(1.3 \%)$, and the technique was unknown in the remaining $8(1.1 \%)$. Roux-en-Y gastric bypass was performed as a primary surgery in $522(72.9 \%)$ patients and as a revisional surgery in $194(27.1 \%)$.

Stricture of the GJ was diagnosed in $29(4.1 \%)$ patients. The average time to diagnosis of early strictures occurring prior to 3 months was 40.3 days, and for late strictures was 871 days. By technique, stricture was diagnosed in 26 (3.9\%) patients in the 25 CEEA group, 1 $(4 \%)$ in the linear stapler group, and $2(22.2 \%)$ in the hand-sewn anastomosis group. In primary RYGB patients stricture was diagnosed in 20 (3.8\%) patients, and in revisional RYGB in $9(4.6 \%)$ patients $(\mathrm{p}=0.626)$. Esophagogastroduodenoscopy (EGD) with dilation was performed at least once (1-9 times) in 26 patients, 2 with concomitant stenting, 2 required operative intervention, and 1 patient awaits operative intervention. Both patients who required surgery also had marginal ulcers, and possible gastro-gastric fistula at time of surgery. Conclusion: The results of this study show that the 25 CEEA circular stapler is a reasonable technique for performance of the GJ anastomosis in RYGB, with a stricture rate of $3.9 \%$. There is also a slightly increased stricture rate in revisional surgical patients, though not statistically significant.

\section{P136}

\section{A Systematic Review on the Use of Biochemical Markers as a Predictive Tool for Post-operative Complications After Bariatric Surgery}

Siddharth Shinde, Gabriel Marcil, Juan Arminan, Artan Reso, Estifanos Debru, Neal Church, Richdeep Gill, Philip Mitchell; University of Calgary

Introduction: The aim of this study was to assess the use of acute phase reactants, specifically C-Reactive Protein (CRP), as a marker which may precede the onset of clinical manifestations of post-operative complications after bariatric surgery. Presently there are no systematic reviews/meta-analysis on validated biochemical markers for early prediction or identification of patients who are prone to post-operative complications. While early discharge under the Enhanced Recovery After Surgery (ERAS) protocol after bariatric surgery has its advantages, it can often mask post-operative complications until after the patient has been discharged. The current best diagnostic method for complications remains clinical suspicion, which can guide further biochemical and radiographic investigations.

Methods and procedures: A comprehensive literature search was conducted through Medline, Embase, Scopus, Web of Science, Dare, Cochrane library, and HTA database from January 2008 through October 2017. All human prospective or retrospective studies, non-randomized comparison studies, and case series involving more than ten patients were considered for inclusion. The target patients were adults ( $\geq 18$ years) who have under gone surgical management of obesity. The primary outcome of interest was level of acute phase reactants in patients who develop complications following bariatric surgery. Secondary outcomes aimed to determine the association of acute phase reactants with complication stratified by the bariatric surgical procedure performed and type of complication.

Results: Thirteen studies were included in our analysis ( $\mathrm{n}=20,639$ patients). The average patient age was $42.14 \pm 2.62$ years. The average pre-operative BMI was $45.49 \pm 2.96 \mathrm{~kg} /$ $\mathrm{m}^{2} .79 .0 \%$ of patients were female. 1403 patients had at least one post-operative complication of any type (leak, bleed, or abscess). The overall post-operative complication rate was $6.8 \%$. From a limited analysis due to heterogeneity of our studies the mean Postoperative Day (POD) 2 CRP in patients with any complication was $197.04 \pm 53.94 \mathrm{mg} / \mathrm{L}$, while the mean POD $2 \mathrm{CRP}$ in patients with no complication was $62.45 \pm 33.36 \mathrm{mg} / \mathrm{L}$ Conclusions: Timely diagnosis of post-operative complications after bariatric surgery remains challenging. Preliminary analysis demonstrates higher POD 2 CRP in patients who develop post-operative complications after bariatric surgery. CRP could provide a costeffective option for screening for post-operative complications in conjunction with clinical suspicion. However, there is no clear consensus on timing of biomarker measurement and cut-off values that indicate the need for re-operation. Next steps in this study will be to stratify CRP for leaks, abscesses, and bleeds based on type of procedure performed. 
P137

Initial Outcomes of One Anastomosis Gastric Bypass at a Single Institution

Mohammad Zagzoog, MD ${ }^{1}$, Wisam Jamal, MD $^{2}$, Salma Sait ${ }^{3}$, Ashraf Maghrabi, $\mathrm{MD}^{3} ;{ }^{1}$ King Abdulaziz Medical city, ${ }^{2}$ University of Jeddah, ${ }^{3}$ King Abdullaziz University

Introduction: One anastomosis gastric bypass (OAGB) is an emerging bariatric procedure, which has been reported to be safe and effective. This study aims to evaluate the short-term outcome of OAGB and its mid-term effects on weight loss and remission of Type 2 Diabetes Mellitus (T2DM). Methods: A retrospective review of patients who had undergone OAGB between January 2013 and January 2016 in King Abdulaziz University Hospital, Jeddah, Saudi Arabia is presented here. Patients; perioperative characteristics, biochemical profile (HbAlc and iron) and details on subsequent weight loss in terms of Body Mass Index (BMI) and Excess Weight Loss percentage (\%EWL) along with early and late postoperative complications were evaluated.

Results: Out of the forty-three patients who underwent OAGB, 38 were included in this study and completed the 2-years follow up. Average operative time was $107.5 \pm 21.8 \mathrm{~min}$ and average length of hospital stay was $2.5 \pm 0.64$ day. Mean preoperative BMI was $48.6 \pm 9 \mathrm{~kg} / \mathrm{m}^{2}$ and at 1 and 2 years of follow up was $30.6 \pm 8.7$ and $27.5 \pm 6.3$, respectively. No mortality, anastomotic leak or bleeding were reported. Most common mid-term complication was iron deficiency anemia $(\mathrm{n}=7 / 38)$. Remission of T2DM at 6 month was $81.8 \%$. Patients with preoperative T2DM for less than 10 years showed better remission $(\mathrm{p}=0.024)$.

Conclusion: Our analysis suggests that OAGB is a safe and effective weight loss procedure that carries low perioperative risk and acceptable nutritional complications in the midterm, with a notable remission of T2DM. Preoperative duration of T2DM plays a major role in achieving remission after OAGB.

\section{P138}

Short and Mid term Outcomes Comparing Morbidity and Weight Loss After Laparoscopic Sleeve Gastrectomy vs. Roux-En-Y Gastric Bypass in patients over 65 years of age and older

Joel S Frieder, MD, Camila Ortiz Gomez, MD, Rene Aleman, MD, Maria C Fonseca, MD, David Romero Funes, MD, Emanuele Lo Menzo, MD, $\mathrm{PhD}$, FACS, FASMBS, Samuel Szomstein, MD, FACS, FASMBS, Raul J Rosenthal, MD, FACS, FASMBS; Cleveland Clinic Florida

Background: Bariatric surgery in the elderly population has been reported as feasible and safe Laparoscopic Sleeve Gastrectomy (LSG) seems to have fewer complications than Laparoscopic Roux-En-Y Gastric Bypass (RYGB), even in the over 65 age group. We analyzed the difference in weight loss between LSG and RYGB in patients $\geq 65$ years of age.

Methods: After IRB approval we retrospectively reviewed 2,486 patients, who underwent either LSG or RYGB between 2005 and 2018 at our institution. Basic demographics, preoperative BMI and comorbidities were described. We identified all patients $\geq 65$ years old and subsequently divided them in two groups depending on the type of bariatric procedure performed. Analysis and comparison of outcomes between these groups were done. Post-operative BMI was reviewed at 6 , 12 and 24 months and \%EBMIL was calculated accordingly. T-test and $\mathrm{Chi}^{2}$ analysis were performed for nominal and categorical variables, respectively.

Results: From 2,486 patients reviewed, $22.73 \%(\mathrm{n}=565)$ were $\geq 65$ years old. From these, $43.19 \%(\mathrm{n}=244)$ underwent LSG and $56.81 \%(\mathrm{n}=321)$ RYGB (Table 1). Caucasians and females were predominant in both groups. Mean age was similar for both populations (LSG:71.10 \pm 3.96 , RYGB:71.67 \pm 4.54$)$. Pre-procedure mean BMI was similar in both groups ( $40.46 \pm 5.48$ for LSG vs. $43.68 \pm 7.22$ for RYGB). Postoperative follow-up rates were similar in both groups at 12 and 24 months (LSG:51.23\% and 31.56\%; RYGB:48.29\% and 34.27\%; $p=0.4883$ and $p=0.4976$ ). The \%EBMIL at 6,12 and 24 months was higher for the RYGB group vs. the LSG group $(59.27 \pm 27.91, \quad 72.10 \pm 29.51, \quad 77.35 \pm 26.11 \quad$ vs. $\quad 50.16 \pm 21.85, \quad 55.21 \pm 25.58$ $43.85 \pm 32.23 ; p=0.0006, p<0.0001$ and $p<0.0001$; respectively). Complication rates were significantly higher in RYGB vs. LSG $(27.73 \%$ vs $9.43 \% ; p<0.0001)$. We observed significantly higher anastomotic ulcer and stricture rates for RYGB vs. LSG $(7.17 \%$ and $5.92 \%$ vs. $0 \%$ and $0 \% ; p<0.0001$ and $p=0.0015$, respectively). RYGB had a higher rate for GI obstruction requiring intervention ( $2.18 \%$ vs. $0.41 \% ; p=0.0776$ ). A similar De Novo GERD rate was noted in both procedures ( $3.74 \%$ vs. $3.69 \% ; p=0.9753$ ). No leaks were reported in either group (Table 2 ). Conclusions: Both LSG and RYGB are effective weight loss procedures for patients $\geq 65$ years of age. RYGB seems to have higher \%EBMIL at 1 and 2 years, however it has almost three times higher complication rate than LSG.
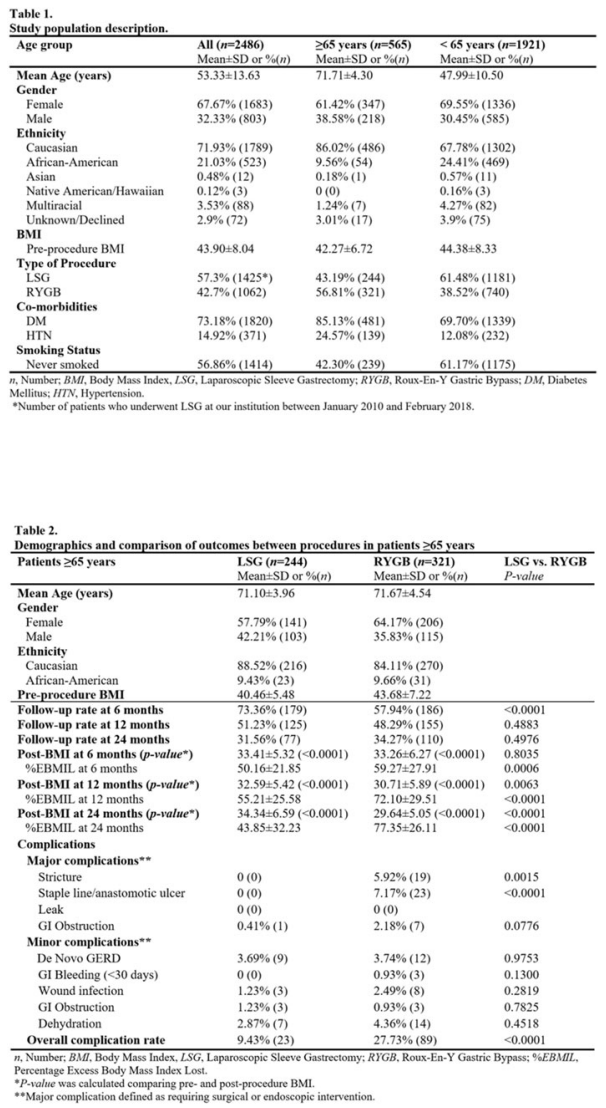


\section{P139}

\section{Outcomes of Bariatric Surgery in Super Super Morbidly Obese} Patients

Raelina S Howell, MD, Harika Boinpally, MD, Elizabeth Carruthers, MSN, RN, Keneth Hall, MD, FACS, FASMBS, Jun Levine, MD, Armando Castro, MD, Patrizio Petrone, MD, Collin Em Brathwaite, MD, FACS, FASMBS; NYU Winthrop Hospital

Introduction: Increased body mass index (BMI) is associated with poor bariatric surgical outcomes. The risks of bariatric surgery in the super super morbidly obese (BMI $\geq 60 \mathrm{mg}$ / $\mathrm{kg} 2$ ) merit further investigation. This study examines short-term outcomes of bariatric surgical procedures in this population.

Methods: Our prospectively-maintained database was retrospectively reviewed for patients with BMI $\geq 60 \mathrm{mg} / \mathrm{kg} 2$ who underwent bariatric surgery at our Center of Excellence over a 13-year period ending June 2018. Demographic data was summarized using descriptive statistics for quantitative variables, and frequencies and percentages for categorical variables. Statistical analyses were made using Chi Square.

Results: Two hundred fourteen procedures were performed on 207 patients over the 13-year period. Excluded were four aborted procedures, one internal hernia repair, and one lap band removal. Four laparoscopic sleeve gastrectomies (LSG) were aborted due to extensive adhesions $(n=3)$, and respiratory distress prior to incision $(n=1)$. Two hundred eight cases were eligible for inclusion. One hundred thirty-six patients were female $(65.4 \%)$. Mean age was 43 years (range 17-68), BMI $65.9 \mathrm{mg} / \mathrm{kg} 2$ (60-95), and weight $411 \mathrm{lb}$ (range 265-639). Co-morbidities included obstructive sleep apnea $(\mathrm{n}=154 ; 74 \%)$, hypertension $(\mathrm{n}=125 ; 60 \%)$, gastroesophageal reflux disease $(\mathrm{n}=94 ; 45 \%)$, osteoarthritis $(\mathrm{n}=91 ; 44 \%)$, and diabetes mellitus $(\mathrm{n}=65 ; 31.3 \%)$. There were 97 roux-en-y gastric bypasses (46\%), 88 LSG (42\%) and 23 adjustable gastric bands (11\%). These included primary $(n=181 ; 87 \%)$, conversion $(n=20 ; 9.6 \%)$, and revision $(n=7 ; 3.4 \%)$ procedures. Technique was primarily minimally-invasive $(75 \%$ laparoscopic, $24 \%$ robotic, and $1 \%$ open). Complications were graded according to the Clavien-Dindo classification system: 1 grade I, 1 grade II, 3 grade IIIa, 3 grade IIIb, and 3 grade IVa. Thirty-day events included: 11 complications (5.3\%; including 1 leak [0.5\%] and 1 deep vein thrombosis [0.5\%]), six readmissions (3\%), and four reoperations $(1.9 \%)$, which involved repair of staple-line leak $(\mathrm{n}=1)$, repair of incisional hernias $(\mathrm{n}=1)$, uterine dilation and curettage for vaginal bleeding $(n=1)$, and cholecystectomy for biliary colic $(n=1)$. There were no mortalities. Complications occurred in $14.8 \%$ of conversion/revision cases and $3.9 \%$ in primary cases $(\mathrm{p}=0.0395)$ with no difference in complications between laparoscopic $(4.5 \%)$ and robotic $(6.1 \%)$ modalities $(\mathrm{p}=0.7051)$.

Conclusion: Super super morbidly obese patients may undergo bariatric surgery safely, with no mortalities using minimally-invasive techniques. Revision procedures may increase the risk.

\section{P140}

\section{One Hundred Most Frequently Cited Studies on Sleeve Gastrectomy}

Tomasz Stefura $^{1}$, Katarzyna Chlopas ${ }^{1}$, Jakub Dros ${ }^{1}$, Artur Kacprzyk ${ }^{1}$, Michal Wysocki, MD², Magdalena Pisarska, MD², Michal Pedziwiatr, $\mathrm{MD}, \mathrm{PhD}^{2}$, Andrzej Budzynski, $\mathrm{MD}, \mathrm{PhD}^{2}$, Piotr Major, $\mathrm{MD}, \mathrm{PhD}^{2} ;{ }^{1}$ Students' Scientific Group at 2nd Department of General Surgery, Jagiellonian University Medical College, Krakow,

Poland, ${ }^{2}$ 2nd Department of General Surgery, Jagiellonian University Medical College, Krakow, Poland

Introduction: Sleeve gastrectomy (SG) is currently one of the most popular bariatric operations and one of the most frequently and thoroughly studied areas in bariatric surgery. Multiple previous publications have focused on the 100 most frequently cited paper investigating various topics in surgery to underline which authors, centers, countries, or journals have most strongly influenced particular area. The aim of this study was to analyze and summarize the characteristics of the most frequently cited studies focusing on SG.

Materials and Methods: We used the Web of Science database (Thomson Reuters, Philadelphia, PA, USA) to identify all studies focused on SG published from 1945 to 2018 The term "sleeve gastrectomy" was used to reveal 100 most cited records.

Results: The most frequently cited publication had 471 citations. The highest mean number of citations per year was 65.2. Studies were frequently published in the year 2010 and 2013. Overall, 61 among included publications were observational studies. Articles were most commonly published in bariatric surgery oriented journals. The most frequent country of origin was United States of America. Usually articles were written by authors from academic departments, working in a surgical institution. Most of the studies focused on the short- and long-term clinical outcomes of SG.

Conclusion: Our study indicates an increase in medical researchers' interest in the subject of SG and underlines the need to perform studies with a higher level of evidence, preferably randomized clinical trials, to further analyze the outcomes and basic science behind SG.

\section{P141}

An Observational Study to Evaluate Metabolic Effects of Bariatric Surgery on Morbidly Obese Patients

Richa Jaiswal, Dr, Sumit Talwar, Dr, Bariatric Surgeon; Manipal Hospitals, Bengaluru

Introduction and Objective: Metabolic syndrome is a known association of obesity and is also associated with diabetes and increased cardiovascular risk. In this study we evaluated the metabolic effects of bariatric surgery on morbidly obese patients.

Materials \& Methods: We recruited 40 morbidly obese patients; 26 underwent Laparoscopic sleeve gastrectomy (LSG) and 14 underwent Roux en Y gastric bypass (LYRGB) of which seven and nine were diabetic respectively. All patients were evaluated for weight, BMI, FBS, PPBS, HbA1c, C-Peptide, Lipid parameters, Mean Arterial Pressure at baseline and at two weeks and at six months post surgery.

Results: We achieved significant reduction in weight $(\mathrm{p}<0.0001)$ by paired Friedman test was noted at and six months (mean $=94.3 \mathrm{~kg}$ ) postoperative as compared to baseline $($ mean $=129.7 \mathrm{~kg})$. A mean weight loss of $52 \%$ for \%Excess weight loss and \%Excess BMI loss. Fasting Blood Glucose was significantly reduced at two weeks (mean $=132.2$ $\mathrm{mg} / \mathrm{dl} ; \mathrm{p}=0.04$ ) and six months (mean $=96.7 \mathrm{mg} / \mathrm{dl} ; \mathrm{p}=0.01$ ) postoperatively. A total of $56.3 \%$ diabetic patients achieved an $\mathrm{HbAlc}$ levels $<5.5$ at six months post surgery with or without the use of antidiabetic medications. Majority of diabetic patients $(93.8 \%, 15 / 16$ patients) were able to achieve an $\mathrm{HbAlc}=6.5$ at 6 months post surgery. C-Peptide levels showed significant difference as compared to baseline (mean $=6.3)$ to those at 6 month (mean $=3.8 ; p=0.03)$ and also there was a significant difference in reduction of use of antidiabetic medications at six months postsurgery (mean $=0.3 ; \mathrm{p}<0.0001$ ) when compared to baseline (mean $=1.4)$. Difference in improvement in deranged lipid parameter $(\mathrm{p}<0.0001)$ and Mean Arterial Pressure $(\mathrm{p}<0.0001)$ became quite significant at six months post surgery.

Conclusion: Bariatric surgery thus helps in reducing weight and also helps in treatment of diabetes, correction of deranged lipid profile and reduces Mean arterial blood pressure. Keywords: BMI, Weight loss, FBS, HbA1c, Lipid parameters, Laparoscopic sleeve gastrectomy, Laparoscopic Roux en Y Gastric Bypass. 
P142

Bariatric Surgery in Patients with Body Mass Index $<35$ : a Systematic Review and Meta Analysis

Chris G Smith, Dr ${ }^{1}$, Fatima Haggar, Dr ${ }^{2}$, Joe Mamazza, Dr ${ }^{2}$, Bryan Curtis, $\mathrm{Dr}^{1}$, Michael Hogan, Dr ${ }^{1}$, Dave Pace, $\mathrm{Dr}^{1}$, Darrell Boone, $\mathrm{Dr}^{1}$, Lisa Bacque, $\mathrm{Dr}^{1}$, Dimitry Terterov, $\mathrm{Dr}^{1}$, Priscille Cyr, $\mathrm{Dr}^{1}$, Aryan Modasi, Dr ${ }^{1}$, Erin Mayo, $\mathrm{Dr}^{1}$, Vanessa Falk, $\mathrm{Dr}^{1}$, Hensley Mariathas, $\mathrm{Dr}^{1}$; ${ }^{1}$ Memorial University of Newfoundland, ${ }^{2}$ University of Ottawa

Background: Bariatric surgery has been shown to be safe and effective for the treatment of morbid obesity and related comorbidities. The goal of the current study is to explore the role of surgery in patients with moderate obesity or body mass index $(\mathrm{BMI})<35$.

Methods: Systematic review and Meta analysis was performed focusing solely on patients with BMI $<35$ who underwent laparoscopic roux en y gastric bypass (REYGB), sleeve gastrectomy (LSG), or adjustable gastric banding (AGB). Data were limited to randomized controlled trials and prospective cohort studies. Primary outcome measure was fasting plasma glucose (FPG). Secondary outcome measures included hemoglobin Alc (HbAlc), and other obesity related comorbidities.

Results: 13 studies were included in the analysis. Surgery was associated with significantly improved FPG compared to medical therapy (WMD -3.24, 95\% CI -4.45; -2.02). Surgery was also associated with improved HbAlc, body weight, BMI loss, waist circumference, and resolution of hypertension and dyslipidemia. Improvements were also seen with respect to obstructive sleep apnea, osteoarthritis, gastroesophageal reflux, infertility, urinary stress incontinence, and venous stasis. These results were consistent across each surgical procedure. 2 randomized controlled trials compared REYGB to LSG and subgroup analysis was performed which revealed no difference with respect to glucose metabolism however REYGB was associated with greater BMI loss and decreased waist circumference. Perioperative complications were comparable to morbidly obese subjects.

Conclusion: REYGB, LSG and AGB appear to be safe and effective in the treatment of obesity and related comorbidities in patients with BMI $<35$. REYGB and LSG have similar effects on FPG and HbAlc however REYGB appears to have improved results with respect to waist circumference and BMI

\section{P143}

\section{Hiatal Dissection in Conjunction with Sleeve Gastrectomy is Associated with Increased Rates of Readmission and Reoperation}

Anna R Ibele, MD, Paige L Martinez, MS, Chelsea M Allen, PhD, Mark A Taylor, MD, Matthew Kingsbury, BS, Ellen H Morrow, MD, Robert E Glasgow, MD, Eric T Volckmann, MD; University of Utah

Introduction: Hiatal hernia repair at the time of bariatric surgery adds additional operative time and technical complexity to the operation. While early postoperative complications of conventional hiatal hernia repair and paraesophageal hernia repair performed with fundoplication are well described, the incidence of perioperative complications in patients undergoing hiatal dissection and closure in conjunction with sleeve gastrectomy has not been established. We wished to determine whether performing a hiatal hernia repair in conjunction with sleeve gastrectomy was associated with increased risk of adverse perioperative outcomes.

Methods and Procedures: Registry data from the American College of Surgeons' MBSAQIP database from January 2015 to December 2016 was reviewed to assess for the presence of a hiatal or paraesophageal hernia repair performed in conjunction with a sleeve gastrectomy. Patients were grouped into two cohorts depending on the presence or absence of hiatal dissection at the time of sleeve gastrectomy. Regression models were constructed to assess for incidence of postoperative nausea and vomiting with nutritional depletion, sepsis, stricture, anastomotic leak, need for therapeutic endoscopy, 30 day readmission and 30 day reoperation rates. Depending on the type of postoperative outcome variable, logistic regression (binary response) or Poisson regression (count response) was used, controlling for demographic covariates.

Results: In the two year period, 44,291 patients underwent sleeve gastrectomy with hiatal hernia repair and 155,477 underwent sleeve gastrectomy without hiatal dissection. The addition of a hiatal repair to a sleeve gastrectomy was associated with a $14 \%$ increase in the odds of diagnosis of postoperative nausea and vomiting with nutritional depletion (OR: 1.14; $95 \%$ CI: $1.03,1.26 / \mathrm{p}=0.010)$, a $10 \%$ increase in the odds of 30 day readmission $(\mathrm{OR} 1.10$; $95 \% \mathrm{CI}: 1.03,1.17 / \mathrm{p}=0.003)$ and a $17 \%$ increased odds of 30 day reoperation $(\mathrm{OR} 1.17 ; 95 \%$ CI: $1.04,1.31 / \mathrm{p}=0.010)$. There was no significant difference in rates of sepsis, stricture, anastomotic leak or need for therapeutic endoscopy.

Conclusion: Hiatal hernia repair performed in conjunction with sleeve gastrectomy imparts an increased risk of nausea and vomiting with nutritional depletion, 30 day readmission, and 30 day reoperation compared to patients having sleeve gastrectomy performed without hiatal hernia repair. In patients with a hiatal hernia, the decision to perform sleeve gastrectomy with hiatal repair should be undertaken with caution because of these increased perioperative risks.

\section{P144}

Roux-en-Y Gastric Bypass vs. Vertical Sleeve Gastrectomy: Long Term Surgical Outcomes

Vanessa Boudreau, MD, Karen Barlow, HonsBSc, Scott Gmora, MD, Dennis Hong, MD, Mehran Anvari, MD, PhD; Center for Minimal Access Surgery, McMaster University, Ontario, Canada

Introduction: Vertical Sleeve Gastrectomy (VSG) is becoming an increasingly popular surgical treatment for morbid obesity compared to Roux-en-Y Gastric Bypass (RYGB) in North America.

Methods: Data collected in Ontario Bariatric Registry between 2010-2018 was used to compare long term outcomes of patients undergoing VSG or RYGB (currently the gold standard). Intention to treat analysis was performed. Results include conversions and revisions.

Results: Of the 18431 patients that underwent surgical treatment, $15,379(81.4 \%)$ had RYGB (baseline BMI 48.2; age 44.3 years; $84.3 \%$ female) and 2572 (13.6\%) had VSG (baseline BMI 53.3, age 48.2 years; $75.4 \%$ female). Surgical complications were reported in $5.6 \%$ of RYGB patients and in $2.8 \%$ of VSG patients. There were $50 \mathrm{VSG}$ patients that required a conversion due to weight regain/ineffective weight loss compared to 4 RYGB patients. The follow-up data were available for 2384 RUGB at 3 years and 569 at 5 years. For VSG, 236 patients had 3 year follow-up and 40 patients had 5 year follow-up.

\begin{tabular}{lllll}
\hline & & RYGB & VSG & p-value \\
\hline Conversion & 3 year & $0.2 \%$ & $36.0 \%$ & $<0.05$ \\
& 5 year & $1.5 \%$ & $47.5 \%$ & $<0.05$ \\
\%EWL & 3 year & $68.8 \%$ & $49.3 \%$ & $<0.05$ \\
& 5 year & $62.3 \%$ & $46.4 \%$ & $<0.05$ \\
Improvement in GERD & 3 year & $46.9 \%$ & $-3.9 \%$ & $<0.05$ \\
& 5 year & $45.7 \%$ & $-6.0 \%$ & $<0.05$ \\
Improvement in Diabetes & 3 year & $56.0 \%$ & $39.7 \%$ & NS \\
Improvement in OSA & 5 year & $41.5 \%$ & $40.9 \%$ & NS \\
& 3 year & $27.3 \%$ & $6.6 \%$ & $<0.05$ \\
Improvement in musculoskeletal pain & 5 year & $48.9 \%$ & $27.1 \%$ & $<0.05$ \\
& 3 year & $38.0 \%$ & $22.3 \%$ & $<0.05$ \\
& 5 year & $34.3 \%$ & $7.0 \%$ & $<0.05$ \\
\hline
\end{tabular}

Conclusion: RYGB results in more favorable weight loss and reduced weight regain as well as better resolution of GERD, musculoskeletal pain and OSA symptoms and has a significantly less likelihood for conversion due to weight regain or side effects at 5 years. 


\section{P145}

\section{Early Experience with Endoscopic Gastrogastric Fistula Closure After Gastric Bypass}

Matthew Tufts, Thadeus Trus, MD, Stuart R Gordon, MD; Dartmouth-Hitchock Medical Center

Background: Gastrogasrtic fistula (GGF) is a rare but serious complication of gastric bypass surgery. Overall incidence has been reported to be from $1.2 \%$ to as high as $12 \%$. Pathogenesis is due to incomplete division of the stomach, staple line breakdown, or a leak/marginal ulcer leading to subsequent fistula formation. Symptoms included epigastric pain, reflux, lack of early satiety and weight gain Traditionally, fistula closure is achieved by open or laparoscopic revisional surgery. Recently, endoscopic closure has been reported with variable levels of success.

Methods: All Apollo Overstitch ${ }^{\mathrm{TM}}$ GGF closures at our institution were followed prospectively. Inclusion criteria were, history of gastric bypass surgery and endoscopic documentation of GGF. All patients underwent mucosal cauterization using argon plasma coagulation of the fistula tract prior to closure. In some cases closure was augmented by large over the scope clip placement. We examined, time from index operation to fistula diagnosis, fistula size, technique of bypass (open versus laparoscopic), symptoms of GGF, mechanism of fistula formation, number of endoscopic interventions, length of follow up and degree of fistula closure.

Results: 6 patients underwent endoscopic GGF closure. 5 patients had open gastric bypass, 1 had laparoscopic bypass. GGF diagnosis was made 13.3 years after index operation (range 8-20 years). GGF fistula was secondary to staple line breakdown in 4 patients, 2 were due to marginal ulceration/perforation. Average fistula size was $2 \mathrm{~cm}$, (range .5$8 \mathrm{~cm}$ ). The most common symptoms of fistula were weight gain and reflux Average follow up was 20.8 months, (range 1-51 months) An average of 2 endoscopic closure attempts were performed(range 2-5). 2 patients also underwent over the scope clip placement. 2 patients experienced weight loss after endoscopic closure. 4 patients have endoscopic documentation of persistent fistula, one was lost to follow up and 1 has yet to be endoscopically re-evaluated. 1 patient underwent operative revision after closure failure. Discussion: GGF is a rare complication of gastric bypass. GGF should be suspected in bypass patients that have reflux or weight gain after bypass surgery.. These data suggest GGF fistula endoscopic closure has a high failure rate, particularly after staple line breakdown. Operative revision remains the optimal treatment. More studies are needed to elucidate which patients may respond to endoscopic fistula closer.

\section{P146}

\section{Longer Operative Times is an Independent Risk Factor for Increased Risk of Pulmonary Embolism in Laparoscopic Gastric Bypass Compared to Laparoscopic Sleeve Gastrectomy}

Sahil Gambhir, MD, Reza F Alizadeh, MD, Colette S Inaba, MD, Megan T Smith, PhD, Jeffry Nahmias, MD, Brian R Smith, MD, Ninh T Nguyen, MD, Shaun Daly, MD; University of California Irvine Medical Center

Introduction/Objectives: Postoperative venous thromboembolism (VTE) is a leading cause of morbidity and mortality in bariatric surgery. There is limited data comparing VTE disease between various types of bariatric surgery. Operative time has been found to be an independent predictor of postoperative VTE. We hypothesized the incidence of VTE would be higher in Laparoscopic Gastric Bypass (LRYGB) compared to Laparoscopic Sleeve Gastrectomy (LSG) and operative time could be an independent predictor.

Methods: The Metabolic and Bariatric Surgery Accreditation and Quality Improvement Program database was queried to identify patients who underwent LSG or LRYGB between 2015- 2016. Perioperative data were compared using univariate analysis including t-tests and Fischer's test. Adjusted odds ratios (AOR) for the risk of deep venous thrombosis and/ or pulmonary embolus (PE) were determined using multivariable logistic regression analysis.

Results: During the study period 167,563 (71\%) patients underwent LSG and 67,525 (29\%) underwent LRYGB. Demographics were similar between both groups, including age, sex, body mass index, history of DVT (1.37\% versus $1.78 \%)$, history of PE (1.02\% vs. $1.18 \%)$ and history of smoking $(8.91 \%$ vs. $8.72 \%)$ respectively. Patients diagnosed with perioperative PE have longer operative times in both LSG ( $86 \mathrm{~min}$ vs. $65 \mathrm{~min}$ ) and LRYGB (135 min vs. $108 \mathrm{~min}$ ) than patients without diagnosis of PE. LRYGB patients experience longer length of stay (2.06 days vs. 1.62 days). There was no significant difference in risk of postoperative DVT between LSG and LRYGB (OR 0.968, CI 0.767-1.220, p >0.05). Compared to LRGYB, LSG was associated with decreased risk of pulmonary embolism (PE) (AOR 0.614, CI $0.462-0.816 \mathrm{p}=0.004)$. LRGYB patients with PEs had a statistically longer operative time compared to LSG patients with PEs ( $135 \mathrm{~min}$ vs. $86 \mathrm{~min} ; \mathrm{p}=0.001$ ). Conclusion: Patients undergoing either LSG or LRYGB and have longer operative times, have a higher incidence of PE. However, LSG confers a lower risk of pulmonary embolism compared to LRYGB. After multivariate analysis, operative time is an independent risk factor for higher PE rates in LRYGB patients compared to LSG patients.

\section{P147}

Our Initial experiance of Performing Laparoscopic Bariatric Surgery in a Public Teaching Hospital in a Middle Ecomic Country Like Pakistan

Danish Ali, Muhammad Adeel Kaiser, Muhammad Farooq Afzal; Lahore General hospital, Lahore

Introduction and Purpose: After establishing foothold in the west, pandemic of obesity now threatens the developing countries of Asia like Pakistan. Pakistan has witnessed unprecedented growth of obese individuals which is causing serious public health concern. Bariatric surgery is now established as the first line treatment for weight loss for morbidly obese patients. The treatment however is costly and carries its own risks and benefits. Although bariatric surgery has been practiced in Pakistan for more than 10 years now but its use is still only limited to private hospitals as a treatment option for the very few who can afford it. But things are changing now as we have started the first Bariatric Surgery Program for the public sector in Lahore. We would like to share our initial experience. Methodology: In a period of 18 months from January 2017 to May 2018 a total of 18 cases have been performed. Demographics, gender, comorbidities, preop weight and BMI of al patients were recorded. Informed consent had been taken from all the patients. All the equipment and gadgets had been arranged and managed by hospital and department of surgery unit-I at LGH. Type of procedure and procedure related complications were recorded. All the patents had been planned to have follow up at 1st, 3rd, 6th, 12th and 24th months. Post op weight, excess weight loss and resolution of comorbidities were also recorded and analyzed in SPSS.

Results: Total 18 patients were included. Median age was 34 years and median BMI was 41. 16 sleeve gastrectomies and 2 minibypass was performed. Weight reduction of $11 \mathrm{~kg}$ at 1 montg, $27.8 \mathrm{~kg}$ at 3 months, $52.8 \mathrm{~kg}$ at 6 months and $72 \mathrm{~kg}$ at 12 months. There was complete resolution of symptoms in patients.

Conclusion: Morbid obesity is serious health concern in developing countries like Pakistan. Bariatric surgery has proven itself the best modality to not just only treat the morbid obesity but also in significantly decreasing the obesity related comorbidities. The treatment is however costly and had never been practiced in a public sector hospital. According to results of our study, Bariatric surgery can be safely practiced in a Public Sector Teaching hospital in a developing countries like Pakistan Bariatric surgery in a public sector hospital in a lower middle income country is as safe and effective as in any private setup. We hope the government acknowledges our efforts and extends this program to other teaching hospitals across the country. 
P148

\section{Liraglutide in a Tertiary Bariatric Clinic: A Case Series}

Daniel Ta, BSc, Jerry T Dang, MD, Arya M Sharma, MD, PhD, Shahzeer Karmali, MD, MPH, Renuca Modi, MD; University of Alberta

Introduction: The objective of this study is to demonstrate the use of liraglutide in various patient scenarios in a tertiary bariatric clinic. Liraglutide is a glucagon-like peptide type 1 (GLP-1) analogue. Its efficacy for weight management has been demonstrated in various clinical trials, indicating its potential as a pharmacological option for patients with obesity.

Case Descriptions:

Case 1: A 26-year old female started liraglutide treatment to induce weight loss preoperatively. She sufficiently loss weight and successfully underwent laparoscopic sleeve gastrectomy.

Case 2: A 39-year old female was assessed for obesity. To become eligible for surgery, the patien needed to lose a substantial amount of weight. She began liraglutide treatment and was successfully bridged to laparoscopic Roux-en-Y gastric bypass (RYGB).

Case 3: A 31-year old male started liraglutide with plans to undergo bariatric surgery. However, after successful weight loss, the patient opted to cancel the procedure and remain on long-term liraglutide due to its flexibility in lifestyle and behavior.

Case 4: A 29-year old female started liraglutide and successfully lost weight. However, the patient was paying for liraglutide out of pocket, and was forced to discontinue. As a result, she experienced weight recidivism. The patient elected to go back on liraglutide on a lower dose to decrease cost and was subsequently able to lose weight. She successfully underwent laparoscopic RYGB.

Case 5: A 51-year old female underwent successfully laparoscopic RYGB. However, over the years, she began to experience weight recidivism. When she began liraglutide therapy, her weight recidivism reversed, and she lost weight

Case 6: A 39-year old female had polycystic ovarian syndrome (PCOS) and infertility. The patient wished to pursue in vitro fertilization (IVF) but was advised to reduce her BMI prior to treatment. She was referred to a bariatric clinic and began liraglutide therapy. She was able to reduce her BMI and successfully underwent IVF shortly after.

Conclusion: This case series provides evidence that liraglutide may potentially be a useful pharmacological option in a bariatric clinic. Patients in our cases tolerated liraglutide well, however, liraglutide has been associated with nausea and vomiting, gastroparesis and esophageal dysmotility. Liraglutide is a useful adjunct in a multispecialty bariatric clinic for weight loss. Liraglutide can be used in various patient scenarios: as a bridge to bariatric surgery, for long-term weight loss or to pursue fertility treatment. Further research on liraglutide should be performed, especially in these various patient scenarios to determine its efficacy.

\section{P149}

\section{Management of Gastroesophageal Reflux in Patients with Laparsocopic Sleeve Gastrectomy: Hill Modified Technique}

Ricardo Nassar, MD, Surgeon, Gastrointestinal Surgery, Juan David Hernandez, MD, Surgeon, FACS, Felipe Giron, MD, MSc, Alberto Ricaurte, MD, Surgeon, Juan David Linares, MD, Surgeon, Natan Zundel, MD, Surgeon; Fundación Santa fe de Bogota

Introduction: Obesity has become a worldwide public health problem, affecting rich and poor countries alike. Bariatric surgery is still the best treatment to achieve significant and long lasting weight loss and control of comorbidities. Laparoscopic Sleeve Gastrectomy (LSG) has become the most frequently practiced operation, even more than laparoscopic Roux-en $-\mathrm{Y}$ gastric bypass. GERD is a common disease among obese patients with prevalence between $39 \%$ and $61 \%$ before surgery. There is controversy in choosing the best approach to manage GERD whether if it was present before, worsens, or appears de novo in relation with bariatric surgery. It has been suggested that GERD can be either treated or prevented using a technique inspired in Hill's posterior gastropexy. We present our experience with this procedure.

Objective: To describe both surgical technique and results of a treatment for GERD based in Hill technique, which can be carried out simultaneously in patients undergoing LSG, or in patients who already have been operated in the past.

Methods: Retrospective observational study based on a prospectively recorded database of patients with GERD who underwent a "Hill modified technique" either concomitantly with a LSG or who presented with GERD after a previous LSG. Patients were followed-up for at least 3 years since 2014, both for obesity control and GERD, this last based on the presence or absence of symptoms. All patients treated were included, and all of them had preoperative studies showing pathologic reflux. Surgical technique is based in obtaining an intra-abdominal esophageal length of minimum $3 \mathrm{~cm}$, posterior closure of the hiatus, and posterior fixation of gastroesophageal junction to the crus, with at least two non-absorbable stitches.

Results: A total of 18 consecutive patients underwent closure of the hiatus and posterior gastropexy. 5 patients had the procedure alone to treat post-LSG symptomatic GERD. 13 had the procedure simultaneously with LSG. There were no complications associated to the procedure and none of the patients needed reintervention or medication out of the standard protocol. Postoperative controls were at 1, 3, 6, 12,18, 24 and 36 months. All patients have shown satisfactory results in the control and management of both obesity and GERD, remaining asymptomatic during the study period.

Conclusion: "Hill modified technique" has shown adequate control of GERD symptoms in patients with LSG with no complications. Longer series and comparison with other strategies will allow to determine protocols for GERD treatment in patients undergoing bariatric surgery.

\section{P150}

\section{Longer Biliopancreatic Limb Length in Roux-en-Y Gastric Bypass Does Not Promote Greater Weight Loss}

Chetna Bakshi, $\mathrm{MD}^{1}$, Julio Teixeira, $\mathrm{MD}^{2}$; ${ }^{1}$ Zucker School of Medicine at Hofstra/Northwell, ${ }^{2}$ Lenox Hill Hospital, Northwell Health

Background: Roux-en-y gastric bypass promotes weight loss by restrictive and malabsorptive means. The malabsorptive effects are due to bypassing a portion of jejunum as well as hormonal pathways that are not fully understood ${ }^{1}$. There is variation in the lengths of the biliopancreatic limb (BPL) and alimentary limb (AL) lengths among different centers. Whereas some studies have shown increased weight loss with increased BPL and $\mathrm{AL}$ lengths ${ }^{2}$, others have shown no difference $^{3}$. The aim of our study was to compare weight loss with a BPL length of $100 \mathrm{~cm}$ versus $150 \mathrm{~cm}$, and a stable AL $(100 \mathrm{~cm})$

Methods: Single center retrospective review of 50 patients, 25 patients with a BPL length of $100 \mathrm{~cm}$ (group 1) versus 25 with a BPL length of $150 \mathrm{~cm}$ (group 2), between 2015-2016 at our Bariatric Center of Excellence. Weights were measured preoperatively and at varying times of follow up from 20 days to over 2 years.

Results: We calculated and compared the average decrease in weight change from surgery to follow-up weigh-in between the two groups. There was no significant difference in average weigh decrease between the groups; group 1 vs $2[27.69 \mathrm{~kg} \mathrm{~s} \pm 14.79 \mathrm{~kg}$ vs $28.87 \mathrm{~kg} \pm 13.77 \mathrm{~kg}$, $\mathrm{p}=0.77]$. There was also no significant difference in average days to follow up weigh-in between group 1 vs group 2 [285.28 days vs 281.32 days, $p=0.93]$.

Conclusions: It is theorized that a longer BPL length provides more malabsorption, and therefore should provide greater weight loss. However, in our study, no weight loss difference was seen in patients with a BPL length of $100 \mathrm{~cm}$ versus $150 \mathrm{~cm}$, with a stable AL of $100 \mathrm{~cm}$. Even after controlling for differences in surgical technique between surgeons and different centers, no added advantage to lengthening the BPL was seen in terms of weight loss. Further research is needed to determine other effects of lengthening the BPL, such as effects on comorbidities, hormonal changes, and deleterious effects.

\section{P151}

\section{A Comparison of the Effects Of Roux-en-Y Gastric Bypass and Sleeve Gastrectomy on Body Mass Composition as Measured by Air Displacement Plethysmography}

Rhys Kavanagh, MD, Smith Jessica, MD, Debra Allan, RN, MSN, Peter Nau, MD, MS; University of Iowa Hospitals and Clinics

Introduction: The roux-en-y gastric bypass (RYGB) and sleeve gastrectomy (SG) are the mos commonly performed bariatric surgeries worldwide. Evidence suggests that the malabsorptive component of the RYGB leads to disparate changes in fat and lean mass when compared to that which is seen with restrictive-centric efforts. This can impart deleterious effects on the patient such as an inability to complete activities of daily living, decreased bone mineral density and basa metabolic rate. The SG omits the intestinal bypass and is thought to lack the malabsorptive qualities seen with the RYGB. Little evidence exists on the effects of SG on body composition. This study seeks to compare patients who received a RYGB to SG with regards to change in excess body weight and body composition using Air Displacement Plethysmography (BodPodTM). It is hypothesized that patients who undergo RYGB will experience more profound loss in lean body mass than with $\mathrm{SG}$.

Methods: Patients were selected according to NIH guidelines for bariatric surgery and completed a full multidisciplinary evaluation and monitored weight loss preoperatively. Patients underwent SG or RYGB based on surgeon recommendation, patient preference and medical comorbidities. Body composition was calculated using whole body densitometery (BodPodTM, Cosmed Chicago, IL). Patients underwent testing pre-operatively, six months and 12 months post-operatively. Measurements of change in total body mass, fat mass and lean mass as well as calculation of change in percent excess body weight were performed. Statistical analysis was completed using SPSS Results: 63 patients were enrolled. 33 patients underwent SG and 30 had a RYGB. Mean \%EBW loss for SG and RYGB was $47.2 \%$ and $53.4 \%$ respectively (P value $=0.1795 \% \mathrm{CI}-14.8$ to 2.6 ). Mean \% change in fat mass for the SG and RYGB groups was $9.2 \%$ and $10.51 \%$ respectively (P value $=0.2595 \% \mathrm{CI}-0.86$ to 3.22 ). The mean $\%$ change in lean mass for the SG group and RYGB group was $9.4 \%$ and $10.49 \%$ respectively $(\mathrm{P}$ value $=0.3895 \% \mathrm{CI}-2.8$ to 1.13 )

Conclusions: Both the SG and RYGB provide equivalent, clinically significant loss in excess body weight. Despite the malabsorptive component of the RYGB, a greater degree of lean body mas loss was not observed when compared to the SG. This data can be used in patient counselling and patient selection when discussing the pros and cons of each procedure. 


\section{$\mathbf{P 1 5 2}$}

\section{Predictors and Outcomes of Bleeding After Sleeve Gastrectomy:} An Analysis of the MBSAQIP Data Registry

Valentin Mocanu ${ }^{1}$, Jerry Dang ${ }^{1}$, Daniel Skubleny ${ }^{1}$, Noah Switzer ${ }^{2}$, Daniel Birch ${ }^{1}$, Shahzeer Karmali ${ }^{1} ;{ }^{1}$ University of Alberta, ${ }^{2}$ The Ohio State University

Background: The purpose of this study is to examine predictors of and outcomes associated with postoperative bleeding in patients undergoing laparoscopic sleeve gastrectomy (SG) using the Metabolic and Bariatric Surgery Accreditation and Quality Improvement (MBSAQIP) data registry.

Bleeding following SG is a common complication associated with significant morbidity and a drastic increase in healthcare resources. Multiple strategies have been developed to minimize bleeding including varying bougie size, staple line reinforcement, and intraoperative tranexamic acid. These techniques, however, have been implemented without a clear understanding of the pre-, intra-, and post-operative predictors of bleeding in patients undergoing sleeve gastrectomy.

Methods and Procedures: We identified all MBSAQIP patients who underwent SG in 2015 and 2016. Primary outcomes of interest include identifying the prevalence, impact, and predictors of bleeding in SG patients. Our secondary outcomes of interest include characterizing overall complication rates in SG patients. Univariate analysis of pre-, intra-, and post-operative variables was performed using Chi squared tests for categorical data and independent sample t-test for continuous data. A non-parsimonious multivariable logistic regression model was then developed to determine predictive factors for development of postoperative bleed.

Results: A total of 175353 patients underwent laparoscopic SG from 2015 to 2016 . The majority of patients were female (79.0\%), with a mean age of 44.4 (SD 12.0) years and a mean BMI of $45.2 \mathrm{~kg} / \mathrm{m}^{2}\left(\mathrm{SD} 7.9 \mathrm{~kg} / \mathrm{m}^{2}\right)$. A total of $4366(2.5 \%)$ patients had a postoperative bleed associated with a mortality of $1.0 \%$. The mean operative time was $74.0 \mathrm{~min}$ (SD $36.6 \mathrm{~min}$ ) with a mean bougie size of $36.9 \mathrm{~F}$ (SD 2.9F), and a mean pylorus distance of $4.80 \mathrm{~cm}(\mathrm{SD} 1.1 \mathrm{~cm})$. Staple line reinforcement was used in $67.8 \%$ of patients while $22.4 \%$ were oversewn. Bleeds were associated with a statistically significant increase in all complication rates. Multivariable logistic regression analysis revealed the following independent predictors of leak: bougie size, BMI, female, chronic steroids, dialysis, prior history MI, ASA, GERD, prior cardiac surgery, hypertension, prior DVT, renal insufficiency, therapeutic anticoagulation, diabetes, functional status, COPD, sleep apnea, and operating time.

Conclusion: Overall bleed rate following SG was $2.5 \%$ with bleed significantly increasing all other complications, readmission and reoperation rates and mortality at 30 days. Despite adoption of novel operative techniques to minimize bleed rates, none were protective after adjusting for confounders. Preoperative optimization of patient comorbidities prior to surgery may therefore have the greatest role in reduce bleeding after SG.

\section{P153}

Geriatric Robotic-assisted Bariatric Surgery: How do Sleeve Gastrectomy and Roux-en-Y Gastric Bypass Compare in Terms of Short-term Morbidity

Amlish B Gondal, MD, Matthew E Mobily, MD, MPH, Iman Ghaderi, MD, MSc, MHPE; University of Arizona

Introduction: In the United States, more than $10 \%$ of bariatric procedures in academic centers are performed in elderly patients. Higher morbidity and mortality rates have been described in patients above 65 years old. However, there is limited data about the relative safety of robotic assisted sleeve gastrectomy (RA-SG) and Roux-en-Y gastric bypass (RARYGB) in this subset of patients. We aimed to compare 30-day safety of these procedures in geriatric patients.

Methods: Using current procedural terminology codes, RA-SG and robotic assisted RARYGB procedures performed on patients greater than 65 years of age were identified from the Metabolic and Bariatric Surgery Accreditation and Quality Improvement Program (MBSAQIP) participant use file 2016. Demographic data, baseline comorbidities, perioperative data and 30-day outcomes were examined. Outcomes of interest were summarized with descriptive statistics and two tailed analyses were performed using Chi squares for categorical variables (reported as proportions) and t-tests for continuous variables (reported as mean $\pm \mathrm{SD}$ ).

Results: A total of 698 patients were identified; $61.9 \%$ patients underwent RA-SG whereas $38.1 \%$ underwent RA-RYGB. Mean age was $68.4 \pm 2.83$, mean body mass index was $42.7 \pm 7.44,71.8 \%$ were female, $85 \%$ were white, and $82.5 \%$ patients were classified as ASA class III.

Both the RA-SG group and RA-RYGB group were similar in baseline characteristics except patients who underwent were RA-RYGB had higher prevalence of gastroesophageal reflux disease $(54 \%$ vs. $43 \%, p=0.002)$ and patients who underwent RA-SG had higher prevalence of hypertension $(21 \%$ vs. $14 \%, \mathrm{p}=.02)$.

Patients who underwent RA-RYGB had a longer length of hospital stay, operative time, reoperation and readmission rates within 30 days after surgery (Table 1). Major organ system morbidity was similar in both groups. There were no deaths in either group. Conclusion: Robotic assisted bariatric surgery in patients 65 years and older appears safe However, robotic assisted gastric bypass is associated with higher rates of reoperation and readmission. Prospective data is needed to evaluate long-term efficacy of these procedures in the elderly.

\begin{tabular}{|c|c|c|c|}
\hline 30-day outcome & RA.SG (n= 432) & RA-RYGB $(n=266)$ & |p \\
\hline LOS (Mean $\pm S D)$ & $1.83 \pm 1.3$ & $2.23 \pm 2.54$ & .006 \\
\hline Operative time & $102.02 \pm 42.8$ & $164.18 \pm 73.3$ & .001 \\
\hline Readmission & $4.1 \%$ & $8.2 \%$ & 0.029 \\
\hline Reoperation & $0.4 \%$ & $3 \%$ & 0.008 \\
\hline $\begin{array}{l}\text { Emergency } \\
\text { Department visit }\end{array}$ & $5.7 \%$ & $4.5 \%$ & 0.491 \\
\hline $\begin{array}{l}\text { At least one Re- } \\
\text { intervention }\end{array}$ & $1.6 \%$ & $3 \%$ & 0.283 \\
\hline $\begin{array}{l}\text { Transfusion within } 72 \\
\text { hours of the } \\
\text { operation }\end{array}$ & $0.2 \%$ & $0.7 \%$ & 0.157 \\
\hline $\begin{array}{l}\text { Clostridium difficile } \\
\text { colitis }\end{array}$ & 0 & $0.7 \%$ & 0.145 \\
\hline $\begin{array}{l}\text { Treatment for } \\
\text { dehydration }\end{array}$ & $1.8 \%$ & $3.3 \%$ & 0.215 \\
\hline $\begin{array}{l}\text { Organ/space surgical } \\
\text { site infection }\end{array}$ & 0 & 1.12 & 0.05 \\
\hline Leak & $0.2 \%$ & $0.3 \%$ & 1.00 \\
\hline $\begin{array}{l}\text { Unplanned } \\
\text { intubation }\end{array}$ & $0.4 \%$ & 0.3 & 1.00 \\
\hline
\end{tabular}

castric bypass 
P154

\section{Predictors and Outcomes of Leak After RYGB : An Analysis of the MBSAQIP Data Registry}

Valentin Mocanu ${ }^{1}$, Jerry Dang ${ }^{1}$, Farah Ladak $^{1}$, Noah Switzer ${ }^{2}$, Daniel Birch $^{1}$, Shahzeer Karmali ${ }^{1}$; ${ }^{1}$ University of Alberta, ${ }^{2}$ The Ohio State University

Background: The purpose of this study is to examine gastrointestinal leak in patients undergoing Roux-en-Y gastric bypass (RYGB) using the Metabolic and Bariatric Surgery Accreditation and Quality Improvement (MBSAQIP) data registry. Gastrointestinal leak is one of the most severe postoperative complications following RYGB, occurring in up to $2 \%$ of all patients. This has led to adoption of simpler procedures, such as sleeve gastrectomy (SG), which have improved safety profiles but potentially less effective long-term metabolic outcomes. Yet, in contrast to SG, a paucity of modern literature exists regarding predictors of leak for RYGB.

Methods And Procedures: We identified all MBSAQIP patients who underwent RYGB in 2015 and 2016. Primary outcomes of interest include identifying the prevalence, impact, and predictors of leak in RYGB patients. Our secondary outcomes of interest include characterizing overall complication rates in RYGB patients. Univariate analysis of pre-, intra-, and post-operative variables was performed using Chi squared tests for categorical data and independent sample t-test for continuous data. A non-parsimonious multivariable logistic regression model was then developed to determine predictive factors for development of leak.

Results: A total of 77596 patients underwent RYGB from 2015 to 2016. The majority of patients were female $(79.8 \%)$, white $(75.9 \%)$, and underwent laparoscopic RYGB $(89.7 \%)$. The mean age of patients was 45.2 years (SD 11.9) with a mean BMI of $46.3 \mathrm{~kg} / \mathrm{m}^{2}$ (SD 8.17). Complication rates for RYGB were low with a mortality of $0.2 \%$ and a total complication rate of $7.5 \%$. A total of 476 leaks were identified with an overall leak rate of $0.6 \%$ and a mortality of $1.5 \%$. Leak was associated with a statistically significant increase in all complication rates. Multivariable logistic regression analysis revealed the following statistically significant independent predictors of leak: BMI, age, ASA score $>3$, prior PE, and partially dependent functional status. Albumin and increased operative time were the only independent protective variables after adjusting for confounders and interactions. Conclusion: Using the robust MBSAQIP database, we found RYGB to be a safe procedure with low morbidity and mortality. The overall leak rate was $0.6 \%$ with leak significantly increasing all other complications, readmission and reoperation rates at 30 days. Logistic regression identified prior PE and partially dependent functional status as the two largest predictors of leak while increased albumin was the only protective factor. Optimizing pre-operative nutrition and strength in these patients through structured multidisciplinary programs may therefore have a role in the ongoing improvement of outcomes following RYGB.

\section{P155} Incidence of Gastroesophageal Reflux in Morbidly Obese Patients
Taken to Laparoscopic Sleeve Gastrectomy: 2 Years of follow-up

Carlos Luna, MD, Ruben Luna, MD, Luis F Cabrera, General Surgeon, Laura Quintero; Bosque University

Introduction: Gastroesophageal reflux disease are one of the most common disorders in morbidly obese patients. Despite the positive effect of laparoscopic sleeve gastrectomy regarding weight loss and improvement in obesity co-morbidities, there are concerns about the development of de novo gastroesophageal reflux disease. Several published follow-up studies report an increased rate of gastroesophageal reflux after a laparoscopic sleeve gastrectomy. However, the literature on this topic is ambivalent.

Objetives: Identify the incidence of gastroesophageal reflux in morbidly obese patients brought to laparoscopic sleeve gastrectomy in 2 years of follow-up.

Materials and Methods: Prospective observational study. Laparoscopic sleeve gastrectomy patients were studied before and at 2 years follow-up. Demographics, anthropometrics, status of comorbidities, perioperative data, gastroesophageal refluxsymptoms with de GerdQ score were evaluated.

Results: 129 morbidly obese patients without gastroesophageal refluxwere treated with laparoscopic sleeve gastrectomy and follow for 2 years with the GerdQ score every 6 months, with a incidence of de novo gastroesophageal reflux, with a GerdQ score of 8 or more was the $12 \%$.

Conclusions: The incidence of de novo gastroesophageal reflux in laparoscopic sleeve gastrectomy patients can be reduced by performing a standardized and highquality technique by a group of experienced and certified bariatric surgery.

\section{P156}

Medical Complications in 166,601 Surgical Patients with Morbid
Obesity Varying Directly with Increasing Age Independent of Bmi

Luke Perry, DO, Kevin Engledow, DO, Gus Slotman, MD; Inspira Medical Center Vineland New Jersey

Introduction: In today's overweight society, every surgeon must operate on medically high-risk morbidly obese patients. In this milieu of toxic obesity, every clinical insight helps. Although we have reported increased obesity co-morbidities in Medicare patients, some of whom are younger on disability, the specific risks of weight-related peri-operative problems by age are unknown. Objective: To identify the incidence of obesity co-morbidities by decades of age in pre-operative bariatric surgery patients.

Methods: Pre-operative data on 166,601 patients from the Surgical Review Corporation's BOLD database was analyzed by age: $<30 \quad(\mathrm{n}=18,119), 30-40$ $(\mathrm{n}=41,879), 40-50 \quad(\mathrm{n}=46,911), 50-60 \quad(\mathrm{n}=40,788), 60-70 \quad(\mathrm{n}=17,475,>70$ $(\mathrm{n}=1,429)$ years. Data included demographics, BMI, and \% incidence of 33 obesity co-morbid conditions. Statistics: ANOVA for continuous variables; Dichotomous variables by general linear models modified for binomial distribution. Results: BMI varied inversely by age $(48+-8<30$ to $44+-8>70)$, as did female/male percent $(84 / 16<30$ to $64 / 36>70)(\mathrm{p}<0.0001)$. African-American/ Caucasian/Hispanic race percent varied from 12.4/67.5/12.6 in the $<30$ group to $7.0 / 86.6 / 2.2$ among patients $>70$ years of age $(\mathrm{p}<0.0001)$. Variations by age of obesity co-morbidities are displayed in the Table. The incidence of hernia, abdominal panniculitis, angina, cholelithiasis, CHF, DVT/PE, fibromyalgia, impaired function, GERD, diabetes, gout, hypertension, ischemic heart disease, dyslipidemia, leg edema, back pain, musculoskeletal pain, obesity hypoventilation, PVD, pulmonary hypertension, stress incontinence, and unemployment increased directly with increasing age, peaking in the $>70$ group (12) and the 60-70 years cohort $(10)(\mathrm{p}<0.0001)$. Asthma, depression, psychological impairment, and liver disease were highest in the 40-60 decades, but lower $<30$ and $>70(\mathrm{p}<0.0001)$. Alcohol/tobacco/substance use, PCOS, mental health diagnosis, and pseudotumor cerebri (n-6) were inversely proportional to increasing age $<30$ to $60-70,>70$ $(\mathrm{p}<0.0001)$.

Conclusion: Among adult surgical patients with obesity, the incidences of weightrelated medical conditions vary by age. Younger patients are heavier, more frequently female, African-American or Hispanic, and have more psychological/ behavioral issues. The major cardiopulmonary, abdominal/hepatobiliary, endocrine/ metabolic, and weight-induced somatic issues increase in prevalence directly with increasing age. This severe age variation in morbid obesity suggests exaggerated adverse effects the longer one has obesity. Although BOLD did not capture the length of time each patient was morbidly obese, these result suggest the concept of obesity years, with entrenched co-morbidities accumulating the longer patients carry excess weight. These results may not be perfectly representative of all obese surgical patients. Nevertheless, applying this advance knowledge clinically may facilitate presumptive management of obese surgical patients, reduced peri-operative adverse events, and improving outcomes.

\begin{tabular}{|c|c|c|c|c|c|c|}
\hline \multicolumn{7}{|c|}{ Table: Variation of Obesity Co-Marbidities by Age in Adult Surgical Patients } \\
\hline \multirow[b]{2}{*}{$\begin{array}{l}\text { CO-MORBiorities (\% of } \\
\text { each age eroup } \\
\text { affected) }\end{array}$} & \multicolumn{6}{|c|}{ Age } \\
\hline & $<30$ & 30-40 & 40-50 & 50.60 & 60.70 & $>70$ \\
\hline & 5.82 & 6.09 & 6.19 & 7.31 & 7.67 & \\
\hline Angine & 1.45 & 1.74 & 2.34 & & & \\
\hline & 17.16 & 16.22 & & & 18.21 & \\
\hline Bsck Pain & 43.28 & 46.33 & 47.3 & & \$1.51 & 50.73 \\
\hline $\begin{array}{cl}\text { Cholelithisasis } \\
\text { CHE }\end{array}$ & ${ }_{0.42}^{12.1}$ & 16.88 & 19.55 & 22.71 & $\begin{array}{c}25.53 \\
5.57\end{array}$ & 24.28 \\
\hline$\frac{\text { CHFI }}{\text { DVT/PE }}$ & $\begin{array}{l}0.42 \\
1.26\end{array}$ & $\begin{array}{l}0.74 \\
1.86\end{array}$ & $\begin{array}{l}1.33 \\
2.66\end{array}$ & $\begin{array}{l}3.01 \\
3.72\end{array}$ & $\begin{array}{l}5.57 \\
4.59\end{array}$ & $\begin{array}{l}6.58 \\
5.18\end{array}$ \\
\hline Depression & 28.48 & 32.43 & 34.91 & 38.04 & 34.88 & 27.5 \\
\hline Diabetes & 14.16 & 22.62 & 33.72 & 46.28 & 55.69 & 57.66 \\
\hline Drue Use & 0.65 & 0.43 & 0.43 & 0.36 & 0.17 & 0.28 \\
\hline Dysalipidemis & 17.8 & 27.9 & 41.59 & 55.28 & 64.17 & \\
\hline EtOH Use & 32.71 & 32.97 & 31.48 & 28.63 & 25.83 & 24.14 \\
\hline $\begin{array}{l}\text { Fibromyaleis } \\
\text { GERO }\end{array}$ & $\begin{array}{l}0.84 .3 \\
37.3\end{array}$ & $\begin{array}{r}1.98 \\
43.96\end{array}-10$ & $\begin{array}{r}3.36 \\
47.57\end{array}$ & $\begin{array}{l}4.68 \\
49.9\end{array}$ & $\begin{array}{l}4.79 \\
48.5\end{array}$ & 3.29 \\
\hline Gout & $\begin{array}{l}37.3 \\
1.18 \\
\end{array}$ & ${ }_{2.22}^{439}$ & 3.1 & $\begin{array}{r}49.9 \\
4.7\end{array}$ & 6.64. & $\begin{array}{l}44.44 \\
8.19\end{array}$ \\
\hline Hernis & 1.58 & 3.35 & 5.18 & 6.82 & & 7.42 \\
\hline Hypertension & 28.77 & 42.62 & 59.32 & 74.41 & 82.23 & 84.81 \\
\hline Impaired function & 0.63 & 1.04 & 2.35 & 4.46 & 7.61 & 9.8 \\
\hline Ischemic Heart Disease & 0.61 & 1.26 & 3.01 & 6.41 & 12.3 & 18.33 \\
\hline Lee Edems & $\begin{array}{r}15.88 \\
465\end{array}$ & $\begin{array}{c}22.67 \\
5.38\end{array}$ & $\begin{aligned} 27.6 \\
6.35\end{aligned}$ & 32.4 & 36.24 & 35.62 \\
\hline $\begin{array}{l}\text { Liver Disecese } \\
\text { Menstrual Irreqularity }\end{array}$ & $\begin{array}{r}4.66 \\
19.56\end{array}$ & $\begin{array}{r}5.38 \\
19.17 \\
\end{array}$ & $\begin{array}{r}6.35 \\
2257\end{array}$ & $\begin{array}{r}6.85 \\
2465\end{array}$ & $\begin{array}{r}5.89 \\
24.56\end{array}$ & $\begin{array}{r}3.99 \\
23.65\end{array}$ \\
\hline 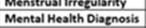 & $\begin{array}{l}1.96 \\
10.12\end{array}$ & $\begin{array}{l}19.17 \\
11.04\end{array}$ & $\begin{array}{l}22.57 \\
10.77\end{array}$ & & $\begin{array}{r}24.56 \\
8.01\end{array}$ & $\begin{array}{l}{ }^{23.65} \\
0.45\end{array}$ \\
\hline MSK Pain & 29.88 & 36.32 & 42.82 & 49.99 & 53.34 & 53.88 \\
\hline Obesity Hypoventilation & 0.97 & 1.22 & & 2.21 & 3.39 & \\
\hline Obstructive Sleep Apnes & 28.73 & 38.37 & 46.69 & 51.08 & 52.13 & 48.29 \\
\hline PVD & 0.26 & $\begin{array}{r}0.39 \\
8.87\end{array}$ & 0.87 & 1.67 & $\begin{array}{c}2.81 \\
0.68\end{array}$ & 3.36 \\
\hline & 12.19 & 8.87 & 3.76 & 1.41 & 0.68 & 0.35 \\
\hline 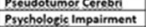 & $\begin{array}{c}2.25 \\
14.58\end{array}$ & $\begin{array}{l}2.24 \\
16.1\end{array}$ & $\begin{array}{l}1.182 \\
16.6\end{array}$ & $\begin{array}{l}1.26 \\
16.94\end{array}$ & $\begin{array}{r}0.8 \\
14.71\end{array}$ & $\begin{array}{r}0.49 \\
11.41\end{array}$ \\
\hline Pulm, HTN & 3.44 & 3.93 & 4.29 & 5.03 & 5.71 & 5.53 \\
\hline Stressis Incontinence & 12.59 & 19.26 & 23.86 & 26.82 & 28.59 & 30.65 \\
\hline Tobacteo Use & 9.2 & & & 5.15 & 3.43 & \\
\hline
\end{tabular}




\section{P157}

\section{Metabolic Outcomes After Bariatric Surgery for Indigenous Patients in Ontario}

Olivia Lovrics, BSc, MScA ${ }^{1}$, Aristithes G Doumouras, MD, $\mathrm{MPH}^{1}$, Scott Gmora, $\mathrm{MD}^{2}$, Mehran Anvari, MB, BS, PhD, MD², Dennis Hong, MSc, MD ${ }^{2}$; ${ }^{1}$ St Joseph's Healthcare, Hamilton, Ontario, Canada, ${ }^{2}$ McMaster University, Hamilton, Ontario, Canada

Introduction: In 2013, over $18 \%$ of Canada's general adult population was considered obese $\left(\mathrm{BMI}>30 \mathrm{~kg} / \mathrm{m}^{2}\right.$ ), compared to $25.7 \%$ of Canada's First Nations, Inuit, and Metis (collectively, Indigenous) peoples. Obesity-related comorbidities affect indigenous Canadians more than non-indigenous, contributing to a lower lifeexpectancy in indigenous Canadians than non-indigenous. Bariatric surgery has been demonstrated to be an effective treatment for obesity, but this treatment has not been studied in Canadian indigenous populations. Accordingly, the purpose of this study is to determine the effect of bariatric surgery on metabolic outcomes for indigenous populations.

Methods and Procedures: Prospectively collected data from the Ontario Bariatric Registry was used in this study. All individuals who underwent bariatric surgery between March 2010 and February 2018 were included in initial analysis. Postoperative outcomes in the database include diabetes, hypertension, GERD, and medication requirements. Demographics, baseline characteristics, and univariate outcomes were assessed using either the Pearson Chi Squared test or t-test. A multivariable regression model for BMI change at 6 months and 1 year was utilized with both a complete case analysis and multiple imputation.

Results: Overall, 16,629 patients were identified of which 338 self-identified as indigenous, 13,502 as non-indigenous, and 2,789 did not enter a designation and were excluded. Overall follow-up rates were $67.5 \%$ at 6 -months and $52.0 \%$ at 1 -year. Baseline demographics were not statistically different between indigenous and nonindigenous patients; however, rates of hypertension $(\mathrm{p}=0.03)$ and diabetes $(<0.001)$ were higher in indigenous populations. Indigenous patients utilized presurgical medical and allied healthcare specialists, investigations and procedures at rates similar to non-Indigenous patients, except in the cases of physiotherapists, psychologists, nurse practitioners and diabetes nurses, who were more likely to be seen by indigenous patients. In univariable analysis, at 1 year, change in BMI was similar between groups (Indigenous: $15.8 \pm 6.0 \mathrm{~kg} / \mathrm{m}^{2}$; Non-indigenous: $16.1 \pm 5.6 \mathrm{~kg} / \mathrm{m}^{2}, \mathrm{p}=0.362$ ). After adjustment, BMI change for indigenous patients, compared to non-indigenous, was not different at 6-months (Effect Size $=0.07,95 \% \mathrm{CI}-0.45$ to $0.58, \mathrm{p}=0.803$ ) and 1 -year $($ Effect Size $=-0.24$, $95 \%$ CI -0.93 to $0.45, p=0.489)$, respectively. Rates of diabetes, hypertension, GERD, and medication use were similar at 1-year between the two populations despite differences at baseline.

Conclusions: Indigenous Ontarians appear to respond as well as non-indigenous Ontarians to bariatric surgery in terms of weight loss and resolution of relevant comorbidities. Accordingly, this treatment, and the standard outcomes associated with it, should be considered for all indigenous patients who qualify.

\section{P158}

Learning Curve for Robotic Sleeve Gastrectomy, Roux-en-Y \& One-Anastomosis Gastric Bypass

Jackly M Juprasert, $\mathrm{MD}^{1}$, Lauren Tufts ${ }^{1}$, Katherine D Gray, $\mathrm{MD}^{1}$, Omar Bellorin, $\mathrm{MD}^{2}$, Gregory Dakin, $\mathrm{MD}^{1}$, Alfons Pomp, $\mathrm{MD}^{1}$, Cheguevara Afaneh, MD ${ }^{1}$, Francesca M Dimou, MD ${ }^{1}$; ${ }^{1}$ NewYorkPresbyterian Hospital/Weill Cornell Medicine, ${ }^{2}$ The Valley Hospital/ Valley Health System

Introduction: The safety and efficacy of robotic bariatric surgery has been established. However, the learning curve has been variably documented and assessed. In this study, we describe our experience with the learning curve in robotic sleeve gastrectomy (RSG), robotic one-anastomosis bypass (ROAB), and robotic Roux-enY gastric bypass (RRYGB).

Methods: Consecutive patients undergoing primary robotic bariatric surgery from October 2015 to July 2018 by a minimally invasive fellowship (MIF)-trained surgeon (Surgeon 1) during his first three years of attending practice were included. Demographic and perioperative data were collected via retrospective chart review. The primary outcome was the learning curve in RSG, RRYGB, and ROAB, represented as the change in operative time over the course of this study and calculated by linear regression fit lines over the number of procedures performed. Secondary outcomes were estimated blood loss (EBL), length of stay (LOS), 90-day readmission, and morbidity. To externally validate our single-surgeon results, we compared our data to Surgeon 2 who trained under Surgeon 1. We report on Surgeon 2's learning curve and outcomes during his first year in practice in 2017. Resident and fellow participation did not change significantly throughout the study period.

Results: A total of 241 patients undergoing RSG $(n=162)$, RRYGB $(n=53)$, and $\operatorname{ROAB}(\mathrm{n}=26)$ by Surgeon 1 were included. Median age was $42 \pm 12.5$ years (range 18-72). $75 \%$ were female. $67 \%$ of patients had ASA scores $\geq 3$. Mean preoperative BMI was $45.9 \pm 8.9$ (23.3-92.5). Mean operative time for RSG for 2015-2016, 2016-2017, and 2017-2018 were $110 \pm 26$ (73-185), $98.3 \pm 25$ (60$211)$, and $90.8 \pm 24$ (54-211) respectively. Mean operative time for RRYGB for 2015-2016, 2016-2017, and 2017-2018 were $200 \pm 36$ (141-268), $178 \pm 44$ (117$278)$, and $142 \pm 37$ (87-278) respectively. Mean operative time for ROAB for 2016-2017 and 2017-2018 were $104 \pm 21$ (72-142) and $98.2 \pm 19$ (71-142) respectively. Operative time decreased over time for all three procedures (Figure 1). There were no conversions to open. Mean EBL was $33.2 \pm 37 \mathrm{~mL}(0-250)$. Mean LOS was $2.18 \pm 1.83$ days (1-26). 90-day readmission rate was $6 \%$. Overall morbidity rate was $5.8 \%$. Mortality was zero. The RSG learning curve was shorter for Surgeon 2 with a comparable complication rate of $4 \%$ (Figure 1); Surgeon 2 did not perform enough RRYGB or ROAB cases to construct learning curves.

Conclusions: RSG, RRYGB, and ROAB operative time consistently and rapidly decreased while morbidity and readmission rates remained low, suggesting that the learning curve for robotic bariatric surgery is quick without compromising patient safety or increased morbidity.

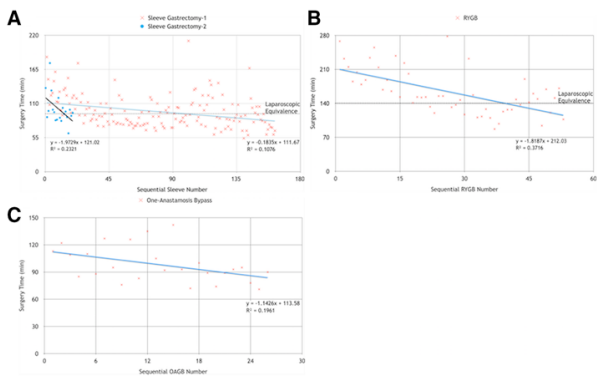


P159

Critical Role of the Postoperative Visits to a Hospital Following Laparoscopic Adjustable Gastric Banding (LAGB)

Minyoung Cho, $\mathrm{MD}, \mathrm{PhD}^{1}$, Jung-Eun Kim, $\mathrm{MD}, \mathrm{PhD}^{1}$, Bodri Son, $\mathrm{MD}, \mathrm{PhD}^{1}$, Gyu-Hee Chae, MD, PhD ${ }^{1}$, Jae-Yong So, MD, $\mathrm{PhD}^{1}$, SunHo Lee, MD, $\mathrm{PhD}^{2}$, Ha-Jin Kim, MD, $\mathrm{PhD}^{3}$, Kyungnam Eoh, $\mathrm{MD}^{1}$, Yun-Chan Park, $\mathrm{MD}^{4}$, Nam-Chul Kim, MD, $\mathrm{PhD}^{4} ;{ }^{1} 365 \mathrm{mc}$ Obesity Clinic LAMS Center, ${ }^{2}$ Global $365 \mathrm{mc}$ Hospital, ${ }^{3}$ Seoul $365 \mathrm{mc}$

Hospital, ${ }^{4}$ Busan $365 \mathrm{mc}$ Hospital

Background: Chronic illness including hypertension and diabetes means treatment is necessary throughout life. Obesity has declared a chronic disease. Many patients believe that the bariatric surgery is not a start to weight loss but a last resort. Patients tend to ignore the importance of visiting hospitals when they reach appropriate adjustment levels of the laparoscopic gastric banding (LAGB). This study evaluated what happens in that case.

Methods: The data were recorded by patients' hospital visits who undertook the same day LAGB using LAP ${ }^{\circledR}$ APs for 8 years. The loss to follow-up (LTFU) is defined as the case where no visit has been made to a hospital for more than six months since LAGB.

Results: Only 497 out of 1,086 patients were followed up during the study (45.8\%). Mean preoperative BMI was $35 \mathrm{~kg} / \mathrm{m}^{2}$ and age was 32 years-old. $90.9 \%$ of the patients showed more than one LTFU $(\mathrm{n}=452, \mathrm{p}<0.0001)$. The first LTFU started at $14.7(0.2-52.8)$ months after LAGB. $78.7 \%$ of the LTFU patients occurred more than 1-year LTFU duration. \%EBMIL at the first LTFU was 54.3\% ($61.3 \sim+253)$. The gap time from the first LTFU to hospital revisit was 20.1 months $(3 \sim 72.8)$. \%EBMIL at the revisit was $50.1 \%(-381.9 \sim+306.3)$. $51 \%$ of the LTFU patients showed weight gain $(+7.3 \mathrm{~kg})$ and the rest was lost $(-$ $8.8 \mathrm{~kg}) .80 .5 \%$ of the LTFU patients complained and developed adverse symptoms and signs such as solid food intolerance $(40.3 \%)$, esophageal dilatation (16.2\%), esophageal barium stasis $(12.8 \%)$, liquid intolerance $(5.1 \%)$, pouch dilatation $(3.5 \%)$, slippage $(2.4 \%)$, and erosion $(0.2 \%)(\mathrm{p}<0.0001)$. \%EBMIL of the LTFU patients vs. Non-LTFU patients was $21.9 \%$ vs. $25.9 \%, 60.9 \%$ vs. $70.8,59.3 \%$ vs. $76.5 \%, 60 \%$ vs. $95.6 \%, 49.6 \%$ vs. $88.3 \%, 45.6 \%$ vs. $106.3 \%$ at post-LAGB $1,12,24$, 36,48 , and 60 months, respectively $(\mathrm{p}<0.05)$. Pseudoachalasia was more frequently developed in the LTFU $(27.7 \%$ vs. $11.1 \%, \mathrm{p}<0.05)$. Among the study participants, 39.2 percent $(n=195)$ of the bands were removed and there was no difference between the two groups. Up to this series, the total explantation rate was $18 \%$.

Conclusion: It is usually difficult to follow up regularly after bariatric surgery because patients mistake obesity as a treatment for weight loss through simple restrictive dietary control. To ensure a safe and good weight loss through the LAGB, proper diet and lifestyle changes must be made through regular hospital visits.

\section{P160}

The Role of Statins and Post Bariatric Surgery Pericardial Fat Reduction in Preventing Coronary Artery Disease

Mauricio Sarmiento-Cobos, MD, Lisandro Montorfano, MD, Carlos Rivera, MD, Camila Ortiz Gomez, Elliot Wasser, MD, Emanuele Lo Menzo, MD, PhD, FACS, FASMBS, Samuel Szomstein, MD, FACS, FASMBS, Raul Rosenthal, MD, FACS, FASMBS; Cleveland Clinic Florida

Background: Pericardial fat is a type of visceral adipose tissue (VAT) with multiple endocrine and inflammatory functions, which may play an important role in the pathogenesis of coronary artery disease. Due to its anatomical location, it has been associated with vulnerability of the atherosclerotic plaque. Lipid-lowering therapy has been shown to halt the progression of atherosclerotic plaques and to induce regression of cardiac adipose tissue. The aim of this study is to report the statin effect on pericardial fat after bariatric surgery.

Methods: A linear measurement of the pericardial fat thickness ( $\mathrm{PFt}$ ) was recorded before and after Bariatric Surgery (BS), from the chest and abdominal CT scans. We divide the patients into two groups; patients using statins (Group 1), and patients not using statins (Group 2). PFt was measured in both groups at the right ventricular wall, perpendicular to the myocardium, at the level of the sternum. We compared the measurements before and after BS, and the risk of developing CAD by Framingham heart study. Common demographics and comorbidities were collected along with lipid profile preoperative and postoperative and were analyzed between the groups. Results: From our bariatric population, 82 patients met inclusion criteria. We identified 31 patients in Group 1 and 51 patients in Group 2. In group one 41.93\% $(\mathrm{n}=13)$ and in group $270.58 \%(\mathrm{n}=36)$ were females. The average age for group one was $61.35+11.14$ years and $57.27+9.001$ years for group two $(\mathrm{p}=0.091)$. The percentage of estimated BMI loss (EBMIL) \% at 12 months in group one was $57.32+31.08(\mathrm{~N}=28)$ versus $73.30+31.16(\mathrm{~N}=42)$ in group two $(\mathrm{p}=0.04)$. Pericardial fat thickness loss was $17.07+10.86 \%$ in group one versus $11.78+6.35 \%$ in group 2 . The risk of CAD one year after BS was $12.22+6.57$ $(\mathrm{n}=9)$, and in group two was $6.78+2.38(\mathrm{n}=9) \mathrm{p}=0.04$.

Conclusion: Obese patients have higher VAT deposits. Pericardial adipose tissue is highly associated with the risk of developing CAD. Pericardial fat can be a target by pharmaceutical agents like statins. In our population, the use of statins combined with bariatric surgery is linked to a significant decrease in pericardial fat thickness and to a lower risk of CAD. Further studies may be needed to better assess these findings.

Table 1. Variables and Demographics.

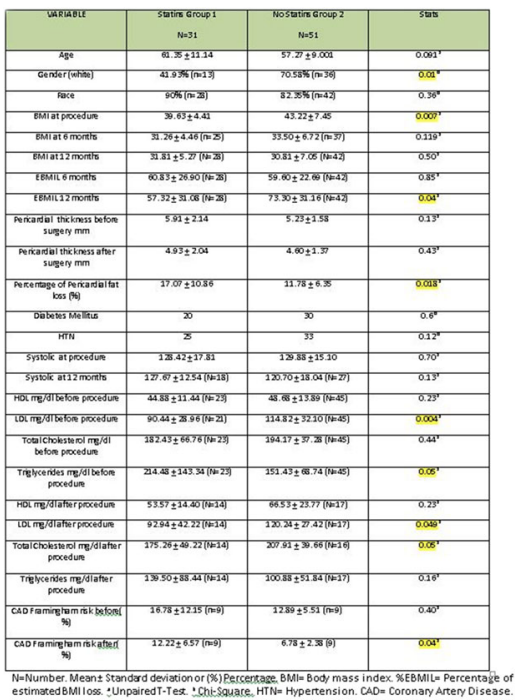




\section{P161}

\section{Weight Loss After Bariatric Surgery Predicts an Improvement} in the Non-Alcoholic Fatty Liver Disease (NAFLD) Fibrosis Score

\section{Charleen Yeo, Marc Ong, Anton Cheng, Chun Hai Tan; Khoo Teck} Puat Hospital, Singapore

Introduction: Non-alcoholic fatty liver disease (NAFLD) is one of the most common liver diseases worldwide, and has been shown to be present in up to $90 \%$ of bariatric surgery patients. Bariatric surgery has grown to be an internationally accepted option for the treatment of obesity and type II diabetes mellitus. Previous literature has demonstrated improvement in liver steatosis, inflammation and fibrosis in patients undergoing bariatric surgery. Our study aims to investigate if bariatric surgery results in an improvement in NAFLD fibrosis scores, and if there is a correlation with weight loss.

Materials and Methods: A retrospective study was conducted on all patients who underwent bariatric surgery from 2010 to 2016 in our institution, using a prospectively collected bariatric surgery database. Patients who had a redo bariatric surgery, or other concomitant gastric pathologies requiring surgical intervention were excluded. Indications for bariatric surgery followed the guidelines of the Asia-Pacific Metabolic and Bariatric Surgery Society. Patient demographics, clinical characteristics, operative details, and surgical outcomes were collected. All patients were followed up for at least one year postoperatively. Statistical analysis was performed using SPSS Version 21, with p-value of less than 0.05 considered statistically significant.

Results: There were a total of 304 patients in this study, of which the majority were females $(\mathrm{n}=178,59 \%)$. Data are represented in mean \pm 2 standard deviation unless stated otherwise. Mean age was $40 \pm 8$ years old. Pre-operative mean weight and body mass index (BMI) was $116 \pm 24$ kilograms, and $42 \pm 3.6 \mathrm{~kg} / \mathrm{m}^{2}$ respectively. The mean preoperative NAFLD fibrosis score was - 1.42. 1-year and 2-year follow up post bariatric surgery demonstrated a decrease in mean weight from 116 to 86 to 82 kilograms, and a decrease in in mean BMI from 42 to 31 to $30 \mathrm{~kg} / \mathrm{m}^{2}$. There was also an improvement in mean NAFLD scores from -1.42 to -1.99 to -1.84 . The correlation between weight loss and the improvement in NAFLD fibrosis scores was statistically significant at both the 1 st and 2nd post-operative year, with a r-coefficient of 0.36 and 0.463 respectively, and p-values of $<0.001$

Conclusion: Bariatric surgery results in weight reduction as well as an improvement in the NAFLD fibrosis score. The degree of weight loss predicts the extent of improvement in NAFLD fibrosis scores. More long term studies need to be conducted to assess if this effect is permanent.

\section{P162}

\section{Implementation of an ERAS Pathway for Gastric Bypass Results in Reduced Costs and Length of Stay}

Anne P Ehlers, MD, MPH, Judy Y Chen-Meekin, MD, Andrew S Wright, Saurabh Khandelwal, MD; University of Washington

Background: Enhanced Recovery After Surgery (ERAS) pathways are well studied in colorectal surgery patients and are increasingly applied to other surgical populations. The effectiveness of ERAS pathways on outcomes and costs for patients undergoing Roux-en-Y gastric bypass (RYGB) remains to be determined.

Methods: Single-institution retrospective study of prospectively collected administrative data for patients undergoing RYGB from July 2015-August 2018. The ERAS pathway was implemented in June 2016 and consisted of standardized pre-operative teaching; multimodal analgesia and anti-emetics in the perioperative period; early oral intake; and standardized discharge instructions. We compared outcomes for patients treated prior to ERAS implementation (July 2015-May 2016) to those treated after ERAS implementation (July 2016-August 2018). The primary outcomes were length of stay (LOS), mean direct hospital costs, and 30-day readmission rates.

Results: Of the 311 patients undergoing RYGB, 116 had surgery before ERAS implementation and 195 did after. Patients treated after ERAS implementation had a 0.8 day shorter hospital LOS (1.9 versus 2.7 days) and direct hospital costs that were \$1,096 lower $(\$ 10,796$ versus $\$ 11,892)$. The 30 -day readmission rate was similar $(6.2 \%$ versus $6.0 \%)$. Conclusion: Implementation of an ERAS pathway was associated with hospital LOS that was nearly one day shorter, and cost savings of more than $\$ 1000$ per patient. This resulted in 156 fewer inpatient days and more than $\$ 200,000$ in direct cost savings. Despite this, there was no appreciable change in the rate of 30-day readmission. This suggests that for patients undergoing elective RYGB, ERAS pathways can increase efficiency without negatively impacting patient outcomes. In an era when patient outcomes are increasingly scrutinized while reimbursement is declining, an ERAS pathway may help streamline patient care and maximize hospital resources.

\section{P163}

Survey of Perceptions and Educational Needs of Primary Care Providers in Ontario, Canada Regarding Management of Patients with Morbid Obesity

Boris Zevin, MD, PhD, FRCSC, FACS ${ }^{1}$, Nancy Dalgarno, $\mathrm{PhD}^{2}$, Mary Martin, $\mathrm{MSc}^{3}$, Colleen Grady, $\mathrm{DB}^{3}$, Linda Chan, $\mathrm{MPH}^{2}$, Robyn Houlden, MD, FRCPC ${ }^{4}$, Richard Birtwhistle, MD, MSc, FCFP $^{3}$, Karen Smith, MD, FRCPC ${ }^{5}$, Rachel Morkem, MSc ${ }^{3}$, David Barber, MD, CCFP ${ }^{3} ;{ }^{1}$ Department of Surgery, Queen's University, ${ }^{2}$ Office of Professional Development and Educational Scholarship, Queen's University, ${ }^{3}$ Department of Family Medicine, Queen's University, ${ }^{4}$ Department of Medicine, Queen's University, ${ }^{5}$ Department of Physical Medicine and Rehabilitation, Queen's University

Introduction: Five percent of the Canadian population has morbid obesity; however, access to surgical and medical weight loss interventions for these patients remains limited. This study explored the knowledge, experience, perceptions, and educational needs of Primary Care Providers (PCP) in managing patients with morbid obesity and obesity-related comorbidities in primary care settings.

Methods: We surveyed practicing PCPs, including primary care physicians and nurse practitioners, in the South East Local Health Integration Network in Southeast Ontario, Canada using a 24-question survey. The survey was circulated via mail, fax, email or inperson at events to 591 PCPs. Data was collected between October 2017 and June 2018, and analyzed with SPSS using descriptive statistics.

Results: One hundred and three participants responded to the survey (17.4\%). The majority were primary care physicians $(91.8 \%)$, followed by nurse practitioners $(6.2 \%)$ and other primary health care providers $(2.0 \%)$. Overall, PCPs reported that, on average, $11.6 \%$ of their patient population qualifies for bariatric surgery, though the majority (77.0\%) reported that they refer fewer than $20 \%$ of these patients for weight loss interventions. In terms of satisfaction with the outcome of recommending treatments for patients with morbid obesity, bariatric surgery had the highest reported level of satisfaction $(71.9 \%$ were satisfied/very satisfied), while the outcome of recommending weight loss diets was among the lowes (18.7\% were satisfied/very satisfied). Twenty-two percent of PCPs agreed/strongly agreed that they are hesitant to refer patients for bariatric surgery, and $43.5 \%$ and $53.5 \%$ agreed/ strongly agreed that they were concerned with the risks associated with bariatric surgery and with postoperative surgical complications, respectively. Nearly half (43.4\%) strongly agreed/agreed that future treatment of patients with morbid obesity must be based primarily on bariatric surgery with appropriate behavioral and dietary modifications. Nearly all respondents $(88.5 \%)$ believe there is a need for education of PCPs on bariatric surgery. Conclusions: Despite an overall perception that bariatric surgery is successful in the treatment of patients with morbid obesity, PCPs continue to under-refer their patients for surgical weight loss interventions. This appears to be due to a lack of knowledge about and experience with bariatric surgery among PCPs. Our results can inform the development of continuing professional development programs that support PCP education in management of patients with morbid obesity. 
P164

\section{A Comparison of Surgical Outcomes Before and After Implementation of a Bariatric Surgery Program}

Elizabeth M Carr ${ }^{1}$, Samaad Malik, BSc, MSc, MD, FRCSC ${ }^{2} ;{ }^{1}$ University of Victoria, ${ }^{2}$ Unit of British Columbia, Island Medical Program Victoria General Hospital

Introduction: Laparoscopic sleeve gastrectomy has become the most common procedure for patients with morbid obesity worldwide. Dedicated bariatric surgery programs have become the central intake for patients; however, there is limited evidence to show improved outcomes with this approach. This study aims to evaluate surgical outcomes following laparoscopic sleeve gastrectomy (LSG) procedures pre- and post- implementation of a formal bariatric surgery program (BSP).

Methods and Procedures: A retrospective analysis of surgical outcomes for patients undergoing LSG pre-BSP implementation from December 20, 2011 to December 31, 2014 to post-BSP implementation January 01, 2015 to May 14, 2018 was conducted. Data was analyzed from the Plexia EMR system from patients that had undergone LSGs performed by a same single surgeon in Victoria, BC, CANADA. Patients who were considered for revisional bariatric surgery were excluded from analysis. Patient demographics, operative time, complications, and length of postoperative stay (LOS) were statistically analyzed utilizing an unpaired two sample t-test.

Results: A total of 381 patients were included in the study, $309(81.1 \%)$ patients were female and $72(18.9 \%)$ patients were male. Mean age was $47.1 \pm 10.4$ years, with a mean pre-operative BMI of $44.9 \pm 6.1 \mathrm{~kg} / \mathrm{m}^{2}$. Pre-BSP mean OR time was $55.8 \pm 10.0 \mathrm{~min}$, and the post-BSP mean OP time was $57.3 \pm 27.8 \mathrm{~min}$. There were no sleeve leaks nor deaths in our study. Three $(0.9 \%)$ patients returned to hospital postoperatively, for dehydration and pain. The average length of postoperative stay was reduced from $2.3 \pm 0.75$ days preinstitution to $1.3 \pm 0.65$ days post-institution $(\mathrm{P}<0.001)$

Conclusion(s): Implementation of a formal bariatric institution may correlate with reduced postoperative length of stay for LSG procedures. More studies are required to ensure BSPs are improving surgical and patient outcomes.

\section{P165}

\section{Efficacy of Sleeve Gastrectomy vs. Roux-en-y Gastric Bypass Amongst Obese Pediatric Patients}

Aryan Meknat, MD, Gustavo Fernandez-Ranvier, MD, PhD, Vivienne Cabreza, MD, Daniela E Guevara, MD, Kaitlyn E Billington, DNP, AGPCNPBC, Daniel M Herron, MD, Matthew L Dong, MD, Abiba Salahou; Mount Sinai Hospital

Background: Despite the fact that childhood obesity has become an epidemic in the western world with lifestyle changes having a limited success in sustaining weight loss; algorithms that incorporate bariatric surgery for adolescent weight management programs are lacking (1). Data comparing the efficacy of different bariatric procedures among pediatric patients is sparse. The safety and efficacy of the laparoscopic sleeve gastrectomy (LSG) and the laparoscopic roux-en-y gastric bypass (LRYGB), in the pediatric population, have been independently demonstrated (2). When comparing LSG with LRYGB in the adult population, there is no significant difference in short or mid-term weight loss (3). The objective of this study was to determine whether one bariatric surgery was more effective at lowering short-term (at one follow-up) BMI than another.

Methods: We retrospectively analyzed our series of pediatric patients (range; 15 to 21 years) who underwent either a LSG or LRYGB between 2011-2016, and those who subsequently followed up in one year.

Results: A total of 63 patients followed up at one year after having one of the two bariatric procedures done. 56 underwent LSG, while 7 underwent LRYGB. The mean pre- and postoperative BMIs for those who underwent LSG were 46.212 \pm 6.29 and $33.192 \pm 6.066$, respectively. The mean pre- and post-operative BMIs for those who underwent LRYGB were $46.124+4.413$ and $32.052+6.955$, respectively. A t-test was used to measure the difference between the post-operative BMIs and a p-value of 0.646 was discovered. Conclusion: Much like the adult population, there was no clinical or statistically significan difference in post-operative BMIs when comparing LSG vs. LRYGB at one year follow up. LRYGB, amongst the adults, produced better long-term weight loss (3). Therefore, the long-term efficacy and morbidity between these two bariatric procedures still needs to be determined.

\section{P166}

\section{Incidentaloma of Duodenal Pancreatic Heterotopia in Laparoscopic Sleeve Gastrectomy : A Case Report and Literature Review}

Mohammed N Alali, MD ${ }^{1}$, Fahad Bamehriz, MD ${ }^{1}$, Hassan Arishi, $\mathrm{MD}^{2}$, Waad Y Yaseen, $\mathrm{MD}^{3}$; ${ }^{1}$ King Khalid University Hospital,college of medicine,KSU, Riyadh,KSA, ${ }^{2}$ King Abdulaziz Medical City, National Guard health affair, Riyadh,KSA, ${ }^{3}$ AlNoor hospital, Ministry of health, Makkah

Introduction: Pancreatic heterotopia or Heterotopic pancreas (HP) is the presence of pancreatic tissue outside the normal anatomical boundaries. It is usually found in the upper gastrointestinal tract - specifically, the stomach, duodenum, and proximal jejunum. The incidence of HP is $0.25-1.2 \%$.

$\mathrm{HP}$ is usually asymptomatic and diagnosed during histopathological examinations with extremely rare malignant transformation.

We are reporting the first case report of incidentaloma of duodenal HP in obese patient during laparoscopic sleeve gastrectomy (LSG).

Case Report: A 15 year-old male obese patient (body mass index: $39.49 \mathrm{~kg} / \mathrm{m}^{2}$ ), otherwise medically and surgically free, admitted electively for LSG. Intraoperatively, after a standard LSG was done a 2-3 cm doudenal nodule was noted incidentally to be attached to the mesenteric surface of the first part of duodenum. The decision was to do a wedge resection of the mass followed by closing the opening using 3 interrupted stitches. Post-operative course was uneventful and patient was discharged after less than $24 \mathrm{~h}$. The histopathological examination of the duodenal nodule showed HP tissue.

Later during follow up, patient was doing well and tolerating oral intake.

Conclusion: Incidental pathology during bariatric surgery found to be around $2 \%$, most commonly gastrointestinal stromal tumors (GISTs). We are reporting first case of incidental duodenal HP which was removed safely during LSG, further researches are recommended to look for the safety of excision of such lesion at the time of LSG

\section{P167}

\section{The Use Of Non-steroidal Anti-inflammatory Drugs After Sleeve Gastrectomy}

Alan Begian, BS, Jamil Samaan, BS, Evan Alicuben, MD, Angelica Hernandez, MD, Kulmeet Sandhu, MD, Adrian Dobrowolsky, MD, John Lipham, MD, Kamran Samakar, MD; University of Southern California

Introduction: Non-steroidal anti-inflammatory drugs (NSAID) are one of the most commonly used drugs for symptomatic relief of pain. More than 30 million Americans use NSAIDs daily and studies have demonstrated that those in higher BMI categories are more likely to be regular NSAID users compared to those in the lowest BMI categories. Nonetheless, NSAID use in the bariatric surgery population is strongly discouraged with some centers adopting a zero-use policy. The recommendations against NSAID use are based on low level evidence (Grade C) as published in the Clinical Practice Guidelines for the Perioperative Nutritional, Metabolic, and Nonsurgical Support of the Bariatric Surgery Patient. These recommendations were primarily aimed at preventing complications of NSAID use in gastric bypass patients but have been adopted to patients undergoing sleeve gastrectomy. The effects of NSAID use on sleeve gastrectomy patients has not been adequately studied to determine brest practice guidelines.

Methods and Procedures: A retrospective review of patients who underwent laparoscopic sleeve gastrectomy (LSG) at a single institution, multi-surgeon, academic tertiary hospital between January 1, 2014 and November 1, 2017 was performed. A phone interview and chart review were completed on the identified patient population.

Results: 423 LSG patients were identified for inclusion in the study. $76.7 \%$ of patients were female, average age was 48.5 years old, average preoperative BMI was 44.9 , and average follow up was 28.7 months. There were zero identified cases of sleeve complications secondary to NSAID use including gastritis, bleeding, ulceration, perforation, or leak. 116 phone surveys were completed with $62.9 \%(\mathrm{n}=73)$ of respondents reporting some NSAID use after LSG. Of the respondents who reported using NSAIDs after surgery, $41.1 \%$ reported that they used them often $>$ once/week or daily), $28.8 \%$ reported occasional use $(>1 /$ month but $<1 /$ week), and $30.1 \%$ reported rare use $(<1 /$ month $)$. Nearly $27 \%$ of respondents reported regular NSAID use after LSG.

Conclusion: NSAID use in our bariatric surgery population is high despite an institutional policy to prohibit their use among all bariatric patients. Despite the high incidence of NSAID use in our study population, we could not identify a single case of NSAID induced gastrointestinal complications in our retrospective review. NSAID use after LSG may be a safe and viable pain management strategy that needs further evaluation. 


\section{P168}

\section{Influence of HbA1c on Postoperative Outcomes of Bariatric} Surgery

Michal Wysocki, MD ${ }^{1}$, Piotr Major, MD, $\mathrm{PhD}^{1}$, Tomasz Stefura ${ }^{1}$, Jakub Dros ${ }^{1}$, Artur Kacprzyk ${ }^{1}$, Katarzyna Chlopas ${ }^{1}$, Katarzyna Major $^{2}$, Piotr Malczak ${ }^{1}$, Magdalena Pisarska ${ }^{1}$, Michal Pedziwiatr ${ }^{1}$, Andrzej Budzynski ${ }^{1} ;{ }^{1}$ 2nd Department of General Surgery, Jagiellonian University Medical College, ${ }^{2}$ Faculty of Health Sciences, Jagiellonian University Medical College, Krakow, Poland

Introduction: The aim of this study was to investigate the influence of baseline glycated hemoglobin level (HbAlc) level in bariatric patients on postoperative outcomes. Studies investigating the association of $\mathrm{HbA1c}$ with surgical outcomes have shown conflicting results. We found scarce of clinical data regarding influence of baseline $\mathrm{HbA} 1 \mathrm{c}$ on bariatric surgeries postoperative morbidity and readmission what was inspiration to conduct this multicenter retrospective study.

Methods and Procedures: Retrospective cohort study analyzed patients who underwent laparoscopic sleeve gastrectomy (SG), Rouxen-Y gastric bypass (RYGB) or mini-gastric bypass (MGB) for morbid obesity in seven referral bariatric centers. Patients were divided into groups depending on preoperative HbAlc: HbAlc $<$ $5.7 \%$; $5.7-6.4 \%$ and $\geq 6.5 \%$. Primary endpoints: influence of $\mathrm{HbA} 1 \mathrm{c}$ level on perioperative (30-days) and postoperative (12-months) morbidity rates, operation time, length of hospital stay (LOS) and readmission rate.

Results: (32\%). Median age was 43 (35-52) years. Median HbA1c was 5.7 (5.3-6.1). HbA1c $<5.7 \%$ was present in 1044 patients $(49 \%)$, $\mathrm{HbA1c5.7-6.4 \%}$ in 734 (35\%), and $\mathrm{HbAlc} \geq 6.5 \%$ in $347(16 \%)$. Percentage of male patients increased in groups from $26 \%$ in $\mathrm{HbA} 1 \mathrm{C}<5.7 \%$ to $47 \%$ in $\mathrm{HbA} 1 \mathrm{C} \geq 6.5 \%$ significantly. Same tendency through groups we observed in case of BMI and age. Uncontrolled diabetes $(\mathrm{HbA} 1 \mathrm{C} \geq 6.5 \%)$ was present in $185(8.7 \%)$ patients, while $162(7.62 \%)$ patients were not on antidiabetic medications despite having $\mathrm{HbA} 1 \mathrm{C} \geq 6.5 \%$. Median operative time in patients with $\mathrm{HbA} 1 \mathrm{C} \geq 6.5 \%$ was significantly longer than in $\mathrm{HbA} 1 \mathrm{C}<5.7 \%$ and $\mathrm{HbA} 1 \mathrm{C}$ 5.7-6.4\%. 30-days morbidity rate was $5.27 \%$ (112 patients) and did not differ groups significantly, as 12 -months morbidity rate (excl. 30-days) of $2.02 \%$ (43 patients). LOS did not differ groups significantly. 44 patients were readmitted in observation period $(2.07 \%)$ in general. Patients having $\mathrm{HbA} 1 \mathrm{C}$ in range of $5.7-6.4 \%$ and with $\mathrm{HbA} 1 \mathrm{C} \geq 6.5 \%$ did not have significantly increased odds for perioperative morbidity, 12-months postoperative morbidity as compared with those with $\mathrm{HbA1C}<5.7 \%$. Patients with $\mathrm{HbA} 1 \mathrm{c} \geq 6.5 \%$ had increased OR for prolonged LOS as compared to those with $\mathrm{HbA} 1 \mathrm{C}<5.7 \%$ (OR: 1.45; 95\%CI: 1.07-1.97). HbA1c did not influence OR for readmissions. Patients with baseline HbA1c $\geq 8 \%$ had significantly increased chances for hospital readmission (OR 3.53, 95\%CI: 1.35-9.21).

Conclusion: Baseline level of glycated hemoglobin did not influence chance for perioperative morbidity, 12-months postoperative morbidity and prolonged LOS. Patients with $\mathrm{HbA} 1 \mathrm{c} \geq 8 \%$ have increased chance for hospital readmissions.

\section{P169}

\section{Bariatric Surgery in Elderly Patients: 3-Year Outcomes}

Vanessa Boudreau, MD, Scott Gmora, MD, Dennis Hong, MD, Mehran Anvari, MD, PhD, Karen Barlow, Hons, BSc, Olivia Lovrics, MsC; Center for Minimal Access Surgery, McMaster University, Ontario, Canada

Introduction: Only a few studies have studied bariatric surgery in the elderly population.

Methods: Data from the Ontario Bariatric Registry between 2010-2015 was used for this retrospective study to determine outcomes of bariatric surgery in the elderly population, compared with the population under 65 years old, with 3-year follow-up.

Results: 6420 patients underwent RYGB or SG, and completed at least 1-year follow-up. Of these, 140 patients $(2 \%)$ were in the elderly cohort (BMI 48.4, age 66 years old, 70\% female), with 101 patients undergoing RYGB (72.1\%) and 39 undergoing SG (27.9\%). At baseline, the elderly group had more hypertension ( $80.0 \%$ vs $44.9 \%)$, diabetes $(86.0 \%$ vs $31.1 \%)$ and hyperlipidemia ( $86.6 \%$ vs $32.7 \%)$ than the younger group (BMI 49.5, age 45 years old, $84 \%$ female). Outcomes at 3-year follow-up are as follows:

\begin{tabular}{|c|c|c|c|}
\hline & Under 65 & 65 and over & p-value \\
\hline \multicolumn{4}{|l|}{ Decrease in BMI } \\
\hline 1 year & $16.4(5.6)$ & $17.1(5.7)$ & NS \\
\hline 3 years & $16.1(5.8)$ & $16.5(6.1)$ & NS \\
\hline \multicolumn{4}{|l|}{ Complications n (\%) } \\
\hline Mortality 30-day & 0 & 0 & NS \\
\hline Overall complications & $523(8.3)$ & $11(7.9)$ & NS \\
\hline Leak & $4(0.1)$ & $0(0)$ & NS \\
\hline DVT/PE & $3(0.1)$ & $1(0.7)$ & $<0.05$ \\
\hline Hospitalization & $199(3.2)$ & $4(2.9)$ & NS \\
\hline Revisional Surgery & $25(0.4)$ & 0 & NS \\
\hline \multicolumn{4}{|c|}{ Comorbidities improvement ( $\%$, from baseline) } \\
\hline \multicolumn{4}{|l|}{ Diabetes } \\
\hline 1 year & $40.8 \%$ & $53.6 \%$ & $<0.05$ \\
\hline 3 years & $38.9 \%$ & $55.9 \%$ & $<0.05$ \\
\hline \multicolumn{4}{|l|}{ Hypertension } \\
\hline 1 year & $40.9 \%$ & $58.9 \%$ & $<0.05$ \\
\hline 3 years & $40.9 \%$ & $54.8 \%$ & $<0.05$ \\
\hline \multicolumn{4}{|l|}{ Hyperlipidemia } \\
\hline 1 year & $35.8 \%$ & $39.6 \%$ & NS \\
\hline 3 years & $40.4 \%$ & $37.9 \%$ & NS \\
\hline
\end{tabular}

Conclusions: Gastric bypass and sleeve gastrectomy have similar overall mortality and morbidity rates for elderly patients, when compared to the younger patients. The elderly group may have an increased thromboembolic risk. Further study is needed to evaluate this finding. 
P170

Repair of Paraesophageal Hernia at the Time of Bariatric Surgery: A Propensity Matched Analysis of the Mbsaqip Database

Joshua Hefler, MD ${ }^{1}$, Jerry Dang, MD ${ }^{1}$, Noah Switzer, MD, MPH ${ }^{2}$, Valentin Mocanu, MD ${ }^{1}$, Daniel W Birch, MD, MSc, FRCSC ${ }^{1}$, Shazheer Karmali, MD, MPH, FRCSC ${ }^{1} ;{ }^{1}$ University of Alberta, ${ }^{2}$ Ohio State University

Introduction: The purpose of this study is to examine short-term outcomes of patients undergoing bariatric surgery with concurrent paraesophageal hernia (PEH) repair versus bariatric surgery alone. PEHs are relatively common amongst obese patients. They must be repaired either before or concurrently with bariatric surgery. However, there remains debate on whether this is best done prior, in a separate operation, or at the time of bariatric surgery. Methods \& Procedures: The Metabolic and Bariatric Surgery Accreditation and Quality Improvement Program (MBSAQIP) data registry was used to identify patients who had undergone bariatric surgery with concurrent PEH repair. Patients undergoing primary bariatric surgery at an accredited institution between 2015 and 2016 were included. Bariatric surgery procedures included laparoscopic Roux-en-Y gastric bypass (LRYGB) or laparoscopic sleeve gastrectomy (LSG). A propensity-score matching algorithm, accounting for patient age, sex, body mass index (BMI) and comorbidities, was used to match these patients to a cohort of patients who underwent bariatric surgery alone. Overall 30-day incidence of major complications was the primary outcome. Secondary outcomes included mortality, length of operation, length of stay, readmission and reoperation.

Results: The MBSAQIP database identified 222,320 bariatric procedures without PEH and 42,732 procedures with concurrent PEH repair ( $16.12 \%$ of the total). Baseline age, BMI, and comorbidities were significantly different between cohorts. With one-to-one propensity score matching, 42,379 pairs were selected. Background characteristics, including age, sex, preoperative BMI and preoperative comorbidities did not differ statistically between matched cohorts. There was no statistically significant difference in 30-day major complications (PEH $3.48 \%$ vs no PEH $3.36 \%$, p = 0.317) or mortality (PEH $0.06 \%$ vs no PEH $0.08 \%, \mathrm{p}=0.189$ ) after surgery. Reoperation within 30 days (PEH $1.24 \%$ vs no PEH $1.11 \%, \mathrm{p}=0.08$ ) were also similar. Rates of readmission were higher with concurrent PEH repair (3.99 vs 3.59\%, p = 0.002) and length of stay was longer without PEH repair (1.74 vs 1.63 days, $\mathrm{p}<0.001)$. The cohort including PEH repair did have slightly longer operative times $(87.09$ vs. $81.19 \mathrm{~min}, \mathrm{p}<0.001)$.

Conclusions: With a total of 42,732 concurrent PEH repair procedures, this is the largest retrospective study to date. Our analysis indicates that the incidence of major complications for bariatric surgery with concurrent PEH repair is similar to bariatric surgery alone. However, rates of readmission and operative time are higher with concurrent PEH repair. Overall, this study demonstrates the safety of concurrent bariatric surgery and PEH repair.

\section{P171}

ROUX-en-Y Gastric by Pass Impact on Obese Patients Erosive Esophagitis and Barret's Esophagous

Franco J Signorini, $\mathrm{MD}^{1}$, German R Viscido, $\mathrm{MD}^{1}$, Veronica Gorodner, MD ${ }^{2}$, Lucio R Obeide ${ }^{1}$, Federico Moser ${ }^{1}$; ${ }^{1}$ Hospital Privado Universitario de Córdoba, ${ }^{2}$ Unidades Bariatricas, Buenos Aires

Introduction: The incidence of erosive esophagitis (EE) and Barret's esophagus (BE) is as high as $26 \%$ and $6 \%$ respectively in bariatric patients. Although the benefits of Roux-en-Y gastric by pass (RYGB) regarding gastroesophageal reflux disease (GERD) have been well documented, the reports of the effects of this procedure among EE and BE are scarce. The aim of this study is to evaluate the postoperative evolution of EE and BE after RYGB.

Methods and Procedures: A retrospective study from a prospective database was performed. Patients who had EE or BE and RYGB in our institution between 2013 and 2017 were included. Demographics, BMI and \%EWL evolution were analysed. Upper endoscopy pre and post operative and their biopsies results were assesed. Post operative data was collected at 24 months after surgery. EE was classified following the Los Angeles criteria Results: 64 patients were included, 55 had EE and 9 BE. Mean age, BMI and \%EWL were 46.9 years, $44.29 \mathrm{~kg} / \mathrm{m}^{2} \pm 3.5$ and $78.5 \pm 5.8 \%$ respectively. Patients with EE were distributed as follows: A: $54.5 \%(\mathrm{n}=30), \mathrm{B}: 34.5 \%(\mathrm{n}=19), \mathrm{C}: 10 \%(\mathrm{n}=5)$ and $\mathrm{D}: 2 \% .87 \%$ and $84 \%$ of patients with grade $\mathrm{A}$ and $\mathrm{B}$ resolved their condition respectively $(\mathrm{p}<0.001$ for both cases). $20 \%$ of patients with grade $\mathrm{C}$ resolved while $80 \%$ improved to grade $\mathrm{A}$. The only patient with grade $\mathrm{D}$ resolved his esophagitis.

Nine patients had histological diagnosis of BE preoperatively. $4(45 \%)$ were short segment Barret's esophagus (SSBE) and 5 (55\%) were long (LSBE). 75\% of SSBE $(\mathrm{p}=0.1429)$ and $40 \%$ of LSBE $(\mathrm{p}=0.1667)$ had endoscopic and histopathological resolution. One patien (25\%) with SSBE and 2 patients $(40 \%)$ with LSBE remained the same. 1 patient $(20 \%)$ with LSBE improved to SSBE.

Conclusion: In our experience, RYGB promoted the improvement and even resolution of $\mathrm{EE}$ and $\mathrm{BE}$ in a significant number of patients, without observing disease progression in any case. Long term studies should be perform in order to establish EE and BE definite behaviour after RYGB.

\section{P172}

\section{Intragastric Balloon Outcomes in Mexican Population of the General Hospital "Dr. Manuel Gea González" in the Last 5 Years}

Luis A Topete, MD, Luz S Romero, MD, Rodrigo Aceves, MD, Martin E Rojano, Roberto Delano, MD, José De Jesus Herrera, MD; Hospital General "Dr. Manuel Gea González"

Introduction: In 1985, Garren-Edwards introduce the intragastric balloon. It was removed from market after several complications such as deflation and migration of the balloon. Outcomes in the usage of intragastric balloon published in other countries show a percentage of excess weight loss of $60 \%$, and a weight regain of $35 \%$ after retrieval of balloon. Intragastric balloon therapy has been use as first option therapy for patient with obesity type I and also as bridge therapy in patients with morbid obesity, Nonetheless, there are none clinical trials publish in Mexico of the outcomes of usage of intragastric balloon.

Objective: Main purpose of the study is to report the percentage of excess weight loss and weight regain after retrieval of intragastric balloon in the General Hospital "Dr. Manuel Gea González" in the last 5 years.

Materials and methods: We conducted a research from database at the Clinic of Obesity of the General Hospital "Dr. Manuel Gea González" in search of patients who underwent an endoscopic intragastric balloon placement as therapy of weight loss from January 2012 to November 2017. We analyze demographics characteristics of patients, weight before intragastric balloon therapy, type of intragastric balloon, time of intragastric balloon therapy, symptoms after intragastric balloon placement. Follow up of patient with weight was recorded at 1, 3, 6, 9, 12 and 18 months. Additionally, it was specifying if the patient underwent a bariatric surgery after retrieval of intragastric balloon.

Outcomes: From January 2012 to November 2017, we found 22 patients who underwent intragastric balloon therapy. The average age of patient was 41.6 years, average Body Mass Index was $41.4 \mathrm{~kg} / \mathrm{m}^{2}, 6$ patients were male and 16 were women. Percentage of excess weight loss was $34.9 \%$ with a weight regain after retrieval of intragastric balloon at 1 year of follow up. We had the same outcomes in terms of percentage of excess weight loss with the two different types of intragastric balloon. We included patients undergoing interval therapy and also as first option therapy. Five patients underwent bariatric surgery after balloon retrieval reaching a percentage of excess weight loss of $45.6 \%$ six months after removal. In terms of symptoms, none of the patients underwent early removal of the balloon and only 7 patients suffer from mild symptoms after placement of the balloon.

Conclusions: Our study shows similar outcomes as previous reports regarding weight regain and percentage of excess weight loss. 
$\mathbf{P 1 7 3}$

Comparing Mini-gastric Bypass and ROUX-en-Y Gastric Bypass for Bariatric Surgery Using the Metabolic and Bariatric Surgery Accreditaion and Quality Improvement (MBSAQIP) Database

Conrad Moher, MD, Daniel Skubleny, MD, Jerry T Dang, MD, Daniel W Birch, MD, FRCSC, Shahzeer Karmali, MD, FRCSC; University of Alberta

Introduction: The aim of this study is to determine whether MGB may be a reasonable alternative to RYGB by comparing post-operative complication rates. Mini-gastric bypass (MGB) is continuing to gain evidence as a viable bariatric procedure. Compared to the twoanastomosis laparoscopic Roux-en-Y gastric bypass (RYGB), the MGB is performed with a single gastrojejunal anastomosis and is arguably a simpler and faster procedure in experienced hands. Significant criticism surrounds the MGB, including complications related to bile-acid reflux and the potential risk of future gastro-esophageal cancer.

Systematic reviews from Europe and Asia have established MGB as a safe and effective procedure. North American comparisons of MGB and RYGB are currently limited. This is the first study using the MBSAQIP database to compare MGB and RYGB.

Methods and Procedures: This is a retrospective study using the MBSAQIP database. Univariate and multivariable analyses on multiple outcome measures for all primary MGB and RYGB procedures was performed. Revisional procedures were excluded. Data analyzed included demographic information, operative time, perioperative outcomes, and complication rates. Measured 30-day outcomes included: death, venous thromboembolism (VTE), bleed, leak, re-operation, re-admission, length of stay, and re-intervention. Results: From 2015-2016, a total of 112 MGB and 78,883 RYGB were performed. There was no difference in patient demographics except: mean body mass index (BMI) $(\mathrm{MGB}=44.0, \mathrm{RYGB}=46.3 ; \mathrm{p}=0.059)$, dialysis dependence $(\mathrm{MGB}=0.9 \%, \mathrm{RYGB}=$ $0.2 \% ; \mathrm{p}=0.064)$, history of DVT $(\mathrm{MGB}=4.5 \%, \mathrm{RYGB}=1.9 \% ; \mathrm{p}=0.040)$, and therapeutic anticoagulant use $(\mathrm{MGB}=8.9 \%, \mathrm{RYGB}=2.6 \% ; \mathrm{p}=0.000)$. Mean operative time was longer for MGB (MGB = $135.6 \mathrm{~min}$, RYGB $=119.6 \mathrm{~min}$; $\mathrm{p}=0.002$ ). 30-day complication rates showed no significant difference in mortality, leak, bleed, re-operation and re-intervention. Significant differences were found in rates of VTE (MGB $=1.8 \%$, RYGB $=0.4 \%, p=0.025)$, readmission $(\mathrm{MGB}=9.8 \%, \mathrm{RYGB}=6.1 \% ; \mathrm{p}=0.099)$, length of stay lasting greater than 7 days $(\mathrm{MGB}=3.6 \%, \mathrm{RYGB}=1.0 \% ; \mathrm{p}=0.007)$, and acute renal failure $(\mathrm{MGB}=0.9 \%$, RYGB $=0.1 \% ; \mathrm{p}=0.026$ )

Conclusions: Our analysis of the MBSAQIP database found that MGB had longer operative times and increased complications in several categories. Significant discrepancy in data volume between MGB and RYGB within the MBSAQIP database limits the ability to establish robust conclusions. Surgical volume and differences in geographical procedural preferences likely contributes to the increased complications found for MGB. The need for additional MGB data within the MBSAQIP database must be balanced with the notion that surgeons should continue to perform procedures in which they maintain large volumes in order to mitigate procedural complications.

\section{P174}

\section{Re-emergence of Diabetes After Sleeve Gastrectomy in Patients} with Long Term Follow-up

Franco J Signorini, $\mathrm{MD}^{1}$, German R Viscido, $\mathrm{MD}^{1}$, Veronica Gorodner, $\mathrm{MD}^{2}$, Luciano Arocena, $\mathrm{MD}^{1}$, Lucio R Obeide, $\mathrm{MD}^{1}$, Federico Moser, $\mathrm{MD}^{1} ;{ }^{1}$ Hospital Privado Universitario de Córdoba, ${ }^{2}$ Unidades Bariatricas, Buenos Aires

Introduction: Diabetes (DBT) improvement or remission associated to bariatric surgery have been shown by several studies. Data about the recurrence of DBT after surgery remains scarce and very little information can be collected regarding sleeve gastrectomy (SG). The main goal of our study was to determine the incidence of durable remission of DBT after sleeve gastrectomy and factors associated with DBT recurrence.

Methods and Procedures: A retrospective study of all patients $(n=47)$ with DBT who had undergone primary LSG at a single institution and had more than eight years of followup with complete laboratory data available was perfomed. Patients with inadequate weight loss were compared with patients with adequate weight loss. Patients with long time of diabetes evolution were also compared with those with less than 10 years of evolution. Finally, patients that needed insulin for glycemic control were analyzed separately.

Results: After SG, all patients had rapid improvement of their DBT. 83\% achieved complete resolution and $17 \%$ registered an improvement in their metabolic control within the first year. The average follow-up time was 8.8 years. At this point, the mean post-operative $\%$ EWL was $51 \pm 19.39 \%$, HbAlc $6.45 \pm 1.28 \%$ and FG $118.7 \pm 27.75 \mathrm{mg} / \mathrm{dl} .31 \%$ of the patients with initial resolution after surgery experienced recurrence. 6 out of the $8(75 \%)$ patients who registered an initial improvement worsened their metabolic control. Those who recurred or worsened had an inadequate mean $\%$ EWL (46\%) and $67 \%$ needed more than one drug preoperatively for their DBT control. The results of a comparison of patients with adequate weight loss $(\mathrm{n}=25)$ versus inadequate weight loss $(\mathrm{n}=22)$ reported a $\%$ EWL of $66,44 \%( \pm 11.38)$ and $34,04 \%( \pm 11.25)$ respectively (p?0.001). Statistical difference was obtained when the number of patients with remission or improvement was compared with those with recurrence or worsening among this groups ( $\mathrm{p}$ ?0.0255). Patients with long time DBT evolution $(\mathrm{n}=14)$ were compared with those with short time DBT evolution $(n=33): 50 \%$ vs $37 \%$ of patients recurred respectively (p?0.704). All the patients who required insulin $(n=5)$, had initial remission or improvement. $60 \%$ recurrence or worsening was registered in the long term, none of them required insulin and their DBT could be managed with drugs and even without medication.

Conclusion: Sleeve gastrectomy significantly improves diabetes control and appear to alter the trajectory of the disease without resulting in a permanent cure. The result seems to be assosiated to weight loss and insulin requierements.

\section{P175}

\section{Laparoscopic Sleeve Gastrectomy with Hiatal Hernia Repair in a Morbidly Obese Patient with Gastroesophageal Reflux Disease}

Krista Angeli P Delos Santos-Belen, MD, Miguel C Mendoza, MD, Judy Carissa M Atazan, MD; Asian Hospital and Medical Center

Introduction: Laparoscopic sleeve gastrectomy (LSG) has increasingly become one of the most commonly performed bariatric procedures in the past decade, with proven excellen outcomes in achieving excess weight loss, resolution of comorbidities, and low perioperative morbidity. Preoperative gastroesophageal reflux disease (GERD) in obesity is considered a relative contraindication due to the possibility of having worse reflux symptoms postoperatively. In general, developing GERD symptoms is also recognized as a late complication after LSG. For those with known preoperative GERD secondary to a hiatal hernia, newer techniques have been recently implemented such as performing a concomitant hiatal hernia repair, for GERD treatment and prevention as well. This case report aims to show the key steps in performing LSG with hiatal hernia repair (LSG + HHR) as a single stage procedure.

Methods: This is a case of a 30 year-old male with morbid obesity (BMI $=37.2 \mathrm{~kg} / \mathrm{m}^{2}$ ), Type 2 Diabetes Mellitus, Obstructive Sleep Apnea, and GERD. Preoperative gastroscopy, esophagogram, and abdominal CT showed a hiatal hernia type I. Patient was referred to the following services for multidisciplinary perioperative management and clearance prior to surgery: Gastroenterology, Endocrinology, Cardiology, Pulmonology, Psychiatry, and Nutrition. The patient underwent LSG + HHR as a single stage procedure.

Results: On the 1st postoperative day, an esophagogram was done showing smooth flow and no leak of contrast ingested. Patient was able to tolerate general liquids and was discharged on 2nd day after surgery. Short term outcomes evaluated included postoperative bleeding, anastomotic leakage, surgical site infection, and reflux symptoms - none of which were reported in the immediate postoperative period and on followup consults on 1st and 4th weeks after discharge.

Conclusions: LSG + HHR is an effective and feasible option in morbidly obese patients with GERD. Further studies are recommended to evaluate the outcomes as compared to conventional two-staged or bypass procedures. 
P176

Neural Mechanisms of Taste Preference Changes following Rouxen Y Gastric Bypass and Vertical Sleeve Gastrectomy

Kimberly Smith, $\mathrm{PhD}^{1}$, Afroditi Papantoni, $\mathrm{BS}^{1}$, Marga Veldhuizen, $\mathrm{PhD}^{2}$, Timothy Moran, $\mathrm{PhD}^{1}$, Susan Carnell, $\mathrm{PhD}^{1}$, Kimberley E Steele, $\mathrm{MD}, \mathrm{PhD}^{1} ;{ }^{1}$ The Johns Hopkins University School of Medicine, ${ }^{2}$ Yale University, The John B Pierce Laboratory, Inc

Background: Changes in taste preferences have been reported following bariatric surgery. To investigate neural mechanisms for such changes and differentiation by surgery type, we measured behavioral and neural responsiveness to varying concentrations of sugar and fat-containing tastants in patients before and 2 weeks after vertical sleeve gastrectomy (VSG) or Roux-en Y gastric bypass (RYGB).

Methods: Prior to surgery, patients were presented with 12 taste stimuli varying in sugar and fat content and asked each patient to rate each stimulus on a visual analog scale and select the stimulus they preferred most. Patients then underwent a functional magnetic resonance imaging (fMRI) brain scan in which they were orally presented with the following stimuli in a randomized order across multiple trials: high fat (cream, 0\% added sugar), high sugar (skim milk, 20\% added sugar), the patient's most preferred tastant, and a tasteless solution. Two weeks following bariatric surgery, patients repeated the taste preference test and fMRI.

Results: A total of 34 patients (VSG; $N=17$ and RYGB; $N=17$ ) participated prior to and two weeks following bariatric surgery. The mean pre-surgical BMI for VSG (42.36, SD 4.43) and RYGB (43.52, SD 3.68); 2 weeks mean post surgery BMI VSG (39.77, SD 3.89) and RYGB (40.64, SD 3.90). Initial whole-brain analyses indicate increased blood-oxygen-level dependent (BOLD) responses in the insula and amygdala to the high fat and high sugar solutions, respectively, in RYGB patients relative to VSG patients 2 weeks following surgery. Greater post-surgical decreases in BOLD responses in the insula and striatum to the patients' pre-surgical most preferred solution were seen in RYGB patients relative to VSG patients.

Conclusion: Our preliminary data suggest that bariatric surgery may decrease preferences for high energy-dense foods by modulating brain reward responses to combined tastes of sugar and fat, and that the extent of changes in taste-responsive neural activation may differ by surgery type (supported by 1 K23DK100559)

\section{P178}

Outcomes from Explantation of Laparoscopic Adjustable Gastric Band: an Institutional Analysis from a Canadian Bariatric Centre of Excellence

Shannon E Stogryn, MBBS, MSc, FRCSC ${ }^{1}$, Azusa Maeda, $\mathrm{PhD}^{1}$, Steve J Maclellan, MD, FRCSC ${ }^{1}$, Ashley Vergis, MMEd, MD, FRCSC, FACS ${ }^{2}$, Allan Okrainec, MD, MHPE, FRCSC ${ }^{1}$, Timothy Jackson, MD, MPH, FRCSC, FACS ${ }^{1} ;{ }^{1}$ University of Toronto, ${ }^{2}$ University of Manitoba

Introduction: Laparoscopic Adjustable Gastric Banding (LAGB) is a common bariatric procedure that has experienced a significant decline. This is primarily due to poor sustained weight loss and high revision rates compared to its contemporary bariatric procedures. Explantation of LAGB is commonly performed at our institution and often concurrently converted to other bariatric procedures. Previously reported adverse event rates for LAGB removal alone was $6.8 \%$. The objective of this study is to evaluate the outcomes after LAGB removals at our institution including conversions to other bariatric procedures.

Methods: Patients were identified using the Metabolic and Bariatric Surgery Accreditation and Quality Improvement Program (MBSAQIP) database from the Toronto Western Hospital site, filtered by LAGB removal based on the principal procedure code and concurrent procedural terminology (CPT) codes $(43773,43774)$. Patients undergoing simultaneous conversion to other bariatric procedures were included. Outcomes were evaluated for 30-day morbidity, mortality, and readmission.

Results: Between 2011-2018, 93 patients met our inclusion criteria. The majority of patients were female $(84.95 \%)$ with a mean body mass index (BMI) of 42.31 ( \pm SD 9.48) and mean age of 49.12 years $( \pm$ SD 10.77 years). All LAGB removals were performed laparoscopically with only 1 conversion to open $(1.08 \%)$. The majority were elective procedures $(96.77 \%)$ with only 3 emergency cases $(3.23 \%)$. Mean length of stay was 2.19 days ( \pm SD 2.29 days). Post-operative complications at 30 days occurred in $11.83 \%$ of patients with a $4.30 \%$ readmission rate. There were no deaths. Surgical site infections (SSI) accounted for $81.82 \%$ of complications (54.55\% superficial SSI, $27.27 \%$ deep SSI). Thirty-day adverse event rate for LAGB removal alone was $15.00 \%$. Emergent LAGB explantation had a $33.33 \%$ complication rate. LAGB was converted to another bariatric procedure in $56.99 \%$ of cases (48.39\% Laparoscopic Roux-en-Y Gastric Bypass (LRYGB), 8.6\% Sleeve Gastrectomy (LSG)). Conversion to LSG was associated with the highes 30-day post-operative complication rate (37.50\%) compared to conversion to LRYGB at $2.22 \%$ $(\mathrm{p}=0.375)$.

Conclusion: Thirty-day complication rates for removal of LAGB and conversion to other bariatric procedures is significant and may be higher than rates previously reported for LAGB removal alone. This case series suggests that conversion to sleeve gastrectomy may have the highest postoperative complication rate.

\section{P177}

\section{A Novel Gastrointestinal Sealant for Use on Gastric Staple Lines: A Pilot Study}

Tovah Z Moss, MD ${ }^{1}$, Nathan Gasek ${ }^{2}$, Weiss Dan ${ }^{1}$, Patrick Forgione, $\mathrm{MD}^{1} ;{ }^{1} \mathrm{UVM},{ }^{2}$ University of Connecticut

Introduction: Leakage of gastric/intestinal contents from disrupted gastrointestinal staple or suture lines is a significant cause of morbidity and mortality for surgical patients undergoing many gastrointestinal surgeries. For example, the incidence of gastric leak after sleeve gastrectomy from staple line disruption is approximately $2.2 \%$ and thus affects 2,329 patients annually (Parikh et al., 2013). We have developed multiple materials that show promising qualities for use as GI sealants. These materials are biological polymers that can be tailored and functionalized to harness qualities such as tissue-adhesiveness and increased tensile strength. This pilot study aimed to systematically assess one promising compound, methacrylated gelatin (GELMA) in ex vivo rat stomach models. Methods and Procedures: Rat stomachs were harvested, the esophagus was cannulated using 18 -gauge cannulas and sealed with 0 silk or Ethilon suture. The stomachs were then lavaged to remove excess stomach contents before stapling across the body of the stomach, excluding the pylorus, with vascular staple loads (Medtronic Endo GIA Gray Articulating Reload $30 \mathrm{~mm}$ Extra Thin/Vascular). The stomach was then tested using our burst pressure device until an air leak was detected. The max pressure was then recorded. $25 \%$ (w/v) GELMA sealant $(200 \mathrm{uL})$ was then applied to the previously-tested bare staple line (for paired analysis) and re-tested for burst pressure once again. These results were then compared to an equal number $(n=5)$ of rat stomachs that were stapled with buttressed staple loads (Medtronic Endo GIA Reinfored Medium/Thick staple loads). Results: The mean burst pressure was higher in the GELMA 25\% group compared to the bare staple lines, despite these stomachs being tested a second time $(202.251 \mathrm{cmH} 2 \mathrm{O}$ vs 483.306 $\mathrm{cmH} 2 \mathrm{O}, \mathrm{p}=0.134)$. The GELMA-enforced staples had significantly higher mean burst pressures than the commercially-available buttressed staple group $(483.306 \mathrm{cmH} 2 \mathrm{O}$ vs $165.844 \mathrm{cmH} 2 \mathrm{O}$, $\mathrm{p}=0.036$ ).

Conclusions: These are encouraging preliminary results in evaluating this novel material for use as a gastric staple line reinforcement in bariatric surgery. These results show that GELMA may provide a safe, effective, and practical tool as a gastrointestinal sealant and staple/suture line reinforcement.

Resources: PARIKH, M., ISSA, R., MCCRILLIS, A., SAUNDERS, J.K., UDE-WELCOME, A. \& GAGNER, M. 2013. Surgical strategies that may decrease leak after laparoscopic sleeve gastrectomy: a systematic review and meta-analysis of 9991 cases. Ann Surg, 257, 231-7. 


\section{P179}

\section{Mental Health and Bariatric Surgery}

Jamil S Samaan, BS, Evan T Alicuben, MD, Elaine Qian, BS, Kulmeet Sandhu, MD, Adrian Dobrowolsky, MD, Kamran Samakar, MD; University of Southern California

Introduction: The relationship between mental health and bariatric surgery has not been well established. We aim to investigate how subjective measurement of depression and anxiety responded to bariatric surgery in our patient population and examine changes in mental health as a function of bariatric surgery outcomes.

Methods and Procedures: A retrospective chart review was conducted on patients who underwent laparoscopic sleeve gastrectomy (LSG) and roux-en-y gastric bypass (RYGB) from August 2002 to November 2017 at a single institution, multi-surgeon, academic hospital. Preoperative surveys and a telephone questionnaire were utilized for gathering data regarding personal characteristics and mental health. The telephone survey utilized a leikert scale to assess patient postoperative satisfaction and quality of life. Inclusion criteria included patients who completed a telephone interview. Exclusion criteria included patients who did not complete the telephone interview or preoperative questionnaires. Fisher's exact test was used for statistical analysis.

Results: There were a total of 514 patients, $76 \%$ female, $71 \%$ underwent RYGB and $29 \%$ LSG with a median age of $47.0(95 \% \mathrm{Cl} 45.6-48.8)$. Median preoperative BMI was 42.6, range 29.95-80.24 (95\% Cl 41.8-43.2). Median postoperative BMI points lost was 10.6 (95\% CI 10.0-11.0). Average follow up was 6.99 years $(\mathrm{SD}=4.30)$. Surgery success was defined as excess weight loss $(\mathrm{EWL})$ of $>50 \%$.

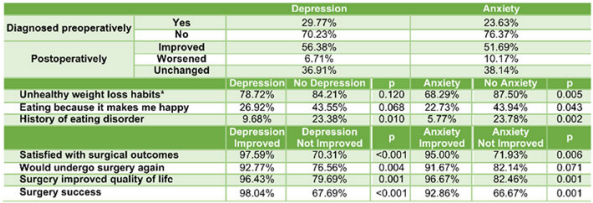

* Use of diuretics and laxatives, binging and purging, vomiting, binging followed by food restriction

Conclusion: Depression and anxiety symptoms improved significantly after bariatric surgery in our study. Those who improved also demonstrated higher improvement in quality of life and postoperative satisfaction. Interestingly, surgery success was statistically correlated with improvement in symptoms. Interpretation of the relationship between symptom improvement and mental health is difficult to ascertain and objective prospective trials are needed to further understand these relationships.

\section{P180}

\section{Balloons Gone Wild: Spontaneous Hyperinflation of Orbera Intragastric Balloon Causing Gastric Outlet Obstruction}

Ransford Commey, MD, Ryan Lehmann, DO, FACS, FASMBS, Norbert Richardson, MD, FACS, FASMBS; St Alexius Hospital

Background: Several endoscopic bariatric therapies have been approved for use in the treatment of obesity and metabolic syndrome. The Orbera Intragastric balloon is one of the first devices to be approved in the United States. It is a single saline-filled balloon $(400-700 \mathrm{~mL})$ that is endoscopically inserted into the stomach for a period of 6 months. Rare complications of intragastric balloons include hyperinflation, deflation, gastric outlet obstruction, and pancreatitis. Spontaneous hyperinflation with resultant gastric outlet obstruction requires balloon removal.

Methods: We present the case of a 44-year-old female, BMI 37.6, who underwent placement of Orbera Intragastric balloon. She presented 2-1/2 months later with complaints of worsening nausea, vomiting and increasing fullness in the left upper quadrant. A computed tomography scan showed the balloon in position within the stomach but significantly enlarged at $15.6 \times 14.2 \times 12.4 \mathrm{~cm}$ with an air-fluid level. The spontaneously hyperinflated balloon was causing a gastric outlet obstruction. She was taken to the operating room and the balloon was endoscopically removed. Results: The patient lost approximately 40Lbs from time of balloon insertion to removal. During endoscopy, the balloon was visualized in the gastric antrum. It appeared maximally inflated and tense with an air-fluid level. Attempt at the normal aspiration procedure resulted in balloon rupture and spillage of the saline content which was then suctioned. The deflated balloon was removed endoscopically in its entirety. The patient was discharged home the same day.

Conclusion: Spontaneous hyperinflation of the Orbera Intragastric balloon is a possible complication of the procedure. Clinicians must maintain a high index of suspicion in patients who present with symptoms of increasing left upper quadrant fullness and gastric outlet obstruction following balloon insertion. Endoscopic treatment of this complication with balloon removal is feasible and should be carried out following clinical and radiographic diagnosis.

\section{P181}

\section{Attending Specialization and 30-Day Outcomes following Laparoscopic Bariatric Surgery}

Ivy N Haskins, MD, Sheena W Chen, MD, Ada Graham, MD, Andrew D Sparks, MS, Paul P Lin, MD, FACS, Hope T Jackson, MD, Khashayar Vaziri, MD; George Washington University

Introduction: Surgeon and hospital volume are two factors that have been shown to impact outcomes following bariatric surgery. However, there is a paucity of literature investigating surgeon training on bariatric surgery outcomes. The purpose of this study was to determine if bariatric specialty training leads to improved short-term outcomes following laparoscopic bariatric surgery. Methods: All patients undergoing first-time, elective, laparoscopic bariatric surgery from 2015-2016 were identified within the Metabolic and Bariatric Surgery Accreditation and Quality Improvement Program (MBSAQIP) database. These patients were divided into two groups based on surgeon training, including general surgery training only or metabolic and bariatric surgery training. Thirty-day outcomes following laparoscopic bariatric surgery were compared between the two groups using multivariate logistical regression analysis.

Results: A total of 140,340 patients met inclusion criteria; 4.598 (3.3\%) patients underwent bariatric surgery by a general surgeon. These patients were lower risk as evidenced by their overal functional status and American Society of Anesthesia (ASA) classification. Despite being lowe risk, patients who underwent surgery by a general surgeon were significantly more likely to be discharged to a facility (OR: 3.6, CI: 2.56-5.13, p < 0.0001) while patients who underwent surgery by a metabolic and bariatric surgeon were significantly less likely to be readmitted to the hospital within 30-days of their index bariatric surgery (OR: 0.81, CI: 0.67-0.97, p = 0.02).

Conclusion: Patients who undergo bariatric surgery by a general surgeon are more likely to be discharged to a facility and to be readmitted within 30-days of surgery. These differences in 30-day outcomes could be related to differences in postoperative support provided by the surgeon types and the institutions where they perform bariatric surgery. Additional information is needed with regards to the type of postoperative support provided following bariatric surgery and to determine if general surgeons are more or less likely to perform bariatric procedures at designated centers of excellence.

\section{$\mathbf{P 1 8 2}$}

Pre-operative Risk Factors for 30-Day Mortality After Primary Laparoscopic Bariatric Procedures

Samantha Warwar ${ }^{1}$, Noah J Switzer ${ }^{1}$, Jerry Dang ${ }^{2}$, Megan Delisle ${ }^{3}$, Carla Holcomb $^{1}$, Shahzeer Karmali ${ }^{2}$, Susan Maurer ${ }^{1}$, Bradley Needleman ${ }^{1}$, Sabrena Noria ${ }^{1} ;{ }^{1}$ The Ohio State University, ${ }^{2}$ University of Alberta, ${ }^{3}$ University of Manitoba

Introduction: The purpose of this study was to identify predictors of 30-day mortality after bariatric surgery using the Metabolic and Bariatric Surgery Accreditation and Quality Improvement Program (MBSAQIP) database.. With improvements in perioperative care, 30-day mortality rates after bariatric surgery are $<0.1 \%$; a rate similar to elective laparoscopic cholecystectomy. With such low mortality rates, identifying patients at increased risk becomes difficult. Given the emphasis on shared decision-making and informed consent, predictive tools have become increasingly useful to guide physician-patient discussions.

Methods and Procedures: This is a retrospective review of prospectively collected data from the MBSAQIP database for the years 2015 and 2016. Patients $>18$ yo, who underwent a primary laparoscopic gastric bypass (RYGB) or sleeve gastrectomy (LSG) were included in the analysis. The outcome of interest was 30-day mortality. Multivariable logistic regression analysis selected by univariable screen was performed. A derivation was created using the 2015 dataset and validated using the 2016 data. Variables with $\mathrm{p}$ value $<0.05$ in univariate analysis were included in the predictive model. A forward selection algorithm with an entry $p$ value of $<0.01$ was used to build a logistic regression model predicting probability of death within 30 days using the derivation data set. The area under the receiver operating characteristic curve was calculated for the derivation and validation dataset. The accuracy of the predictions was assessed with the Brier score

Results: A total of 248,286 patients were included in the analysis. Mean age was $44.64+11.93$ years, with the majority of subjects being female ( $80 \%$ ). Overall 30-day mortality rate was $0.1 \%$, with 249 reported events. In the multivariable model, age (OR-1.04,95\% CI 1.02-1.05), gender(OR-0.56, 95\% C 0.42-0.73), BMI(OR-1.05, 95\% CI 1.04-1.06), COPD (OR-1.82, 95\% CI 1.09-3.04), operation length (OR-1.29, 95\% CI 1.13-1.49), and therapeutic anticoagulation (OR-2.51, 95\% CI 1.67-3.77) were all risk factors for 30 day mortality. The following prediction model was created. Risk of Death $=\left(13^{*}\right.$ therapeutic_anticoagulation $)+(0.5 * \mathrm{BMI})+(0.5 *$ Age $)+\left(3^{*}\right.$ Operationlength $)+(-10 *$ Female $)+(8 *$ Blackrace $)+(3 *$ Diabetes $)$

Subjects were stratified into high $(>5 \%)$, medium $(1-5 \%)$, and low risk groups $(<1 \%)$ for mortality at 30-days, based on points received from the model. The discrimination of the model was 0.80 Conclusion: While death following bariatric surgery is rare, approximately $0.1 \%$, a cohort of patients exist who are at increased risk. Identifying these patients before surgery may allow for proper informed consent and preoperative optimization. 


\section{P183}

\section{Cannabis Use May Affect Early Outcome Weight Loss Post Bariatric Surgery}

Nicole Shockcor, Sakib Adnan, Ariel Siegel, Eric Wise, Nabeel Zafar, Mark Kligman; University of Maryland

Introduction: With the decriminalization of marijuana, it is imperative that we understand how cannabis use can affect surgical outcomes. Previous studies in transplantation have indicated no difference in morbidity or mortality after receiving a kidney allograft, but the affect on bariatric patient outcomes remains to be uncovered. Here we aimed to determine the effect of marijuana use on weight loss post laparoscopic Roux-en-Y gastric bypass (LRYGB) as well as laparoscopic sleeve gastrectomy (LSG)

Methods: 1177 patients who underwent bariatric surgery from 2012-2017 at a single institution were identified. Patients who underwent LRYGB revisions, conversions to LRYGB, laparoscopic band removal with conversion, and lost to follow up were excluded. Patients were followed two years post procedure. SPSS software was utilized to determine covariate effects.

Results: In the 1177 patients included in this retrospective analysis, $73(6.2 \%)$ patients were identified as cannabis users. This was defined by a positive toxicology screen and/or reported use. LSG was performed in 465 patients $(39.5 \%)$ while LRYGB was performed in $712(60.5 \%)$. Postoperative complications were no different between groups, including all cause complications, infection, venothrombolic events, and reoperation. Early 2 month average weight loss was greater in cannabis users $(68.02 \mathrm{vs} .55 .83 \mathrm{lb})$ than non-users, with the cannabis group having lost a larger percentage of excess body weight. Long-term BMI results showed no difference in excess BMI between cannabis users and non-users at 6 months $\left(10.55 \mathrm{vs} 10.07 \mathrm{~kg} / \mathrm{m}^{2}\right)$ and 1 year $(6.48 \mathrm{vs}$ $6.91 \mathrm{~kg} / \mathrm{m}^{2}$ ) post procedure.

Conclusions: With nationwide changes in cannabis use and regulation, it is important to note that its use may have no effect on morbidity or mortality after bariatric surgery. With this preliminary retrospective analysis, a trend was seen towards greater initial excess weight loss in cannabis users; however, further investigation is warranted to fully characterize attributing factors.

\section{P184}

\section{One Anastomosis Gastric Bypass (OAGB) and Esophageal Reflux. A Cross Sectional Study}

Luciano J Deluca, MD, Patrcio J Cal, MD, Tomas C Jakob, MD, Martin Pruss, MD, Ezequiel O Fernandez, MD; CMPFA Churruca Visca

Introduction: Obesity is associated with multiple metabolic disorders and is a risk factor for several upper gastrointestinal tract diseases. GERD's prevalence can rise to $49.6 \%$ in the obese population.

Controversy has arisen regarding the severity of esophageal reflux after the one anastomosis gastric bypass (OAGB)

The objective of this study was to evaluate GERD following SAGB.

Methods: Between April 2015 and March 2018, 66 OAGBs were performed either laparoscopically or with robotic assistance.

Patients with at least 1 year follow up were evaluated via a standardized reflux questionnaire (GERDq), upper GI endoscopy and $24 \mathrm{~h}$ pH-impedanciometry performed and interpreted by surgical team to assess acid and non-acid esophageal reflux.

Results were shown as means \pm standard deviations or as percentage of the total.

Results: A total of 51 patients achieved 1 year follow-up. Mean age was $45.4 \pm 8.1$ years, BMI

$46.7 \pm 7 \mathrm{~kg} / \mathrm{m}^{2} ; 59,1 \%$ were women. GERD q was obtained in 33 patients, with a mean score of $1.43 \pm 2.8$. Only one patient showed a result higher than 8 .

Upper GI endoscopy was performed on 31. Compared to preoperative study, 1 patient showed resolution of esophagitis, and de novo esophagitis was assesed in 2 . No other erosive changes were recorded.

19 patients underwent ph-impedanciometry. Average Demeester score was $6.7 \pm 1.8$. 5 patients presented a score between 14.7 and 18,7. All of them showed a GERDq of 0 and non erosive upper GI endoscopy.

Average total time with acid reflux was $1.84 \%$, all patients were below $6 \%$. Four patients showed higher than normal number of weakly alkaline episodes, although percentage of total time in alkaline reflux was normal in all studies $(0,36 \% \pm 0,9)$. All of them presented a GERDq of 0 and a normal upper GI endoscopy. Only one patient showed a positive symptomatic index (SI). Conclusion: OAGB seems to result in a low incidence of GERD. Even though 6,45\% showed to have de novo esophagitis, $\mathrm{pH}$-impedanciometry and questionnaires showed promising results.

\section{P185}

Outcomes Following Revision and Conversion Robotic-Assisted Compared to Conventional Laparoscopic Bariatric Surgery: A Review of the 2015 and 2016 MBSAQIP Participant User Files Database

Edwin Acevedo $\mathrm{Jr}, \mathrm{MD}^{1}$, Huaqing Zhao, $\mathrm{PhD}, \mathrm{MS}^{2}$, Xiaoning Lu, $\mathrm{MS}^{2}$, Michael A Edwards, MD, FACS, FASMBS ${ }^{2} ;{ }^{1}$ Temple University Hospital, ${ }^{2}$ Lewis Katz School of Medicine at Temple University

Introduction: Revisional bariatric surgery is being increasingly performed and is associated with higher operative risks. Optimal technique to minimize complications remains controversial. Here, we report a retrospective review of the Metabolic and Bariatric Surgery Accreditation and Quality Improvement Program (MBSAQIP) Participant User Files (PUF) database, comparing outcomes between for revision/conversion bariatric surgery using conventional laparoscopic (LBS) and robotic-assisted (RBS) approaches.

Methods: Revisional/conversion cases were identified in the 2015 and 2016 MBSAQIP PUF database. Selected cases were characterized into two groups (RBS; LBS). Case-controlled matching (1:1) was performed of the RBS and LBS cohorts. Cases and controls were match by demographics (age, gender, race, BMI) and preoperative comorbidities. Outcomes measures included operative length (OL), length of stay (HLOS), 30-day outcomes, and complications.

Results: 26,404 revision/conversion cases were identified (93.3\% LBS, 6.7\% RBS). $85.6 \%$ were female and $67 \%$ white. Mean age and BMI were 48 years and $40.9 \mathrm{~kg} / \mathrm{m}^{2} .1,144$ matched RBS and LBS cases and controls were identified and outcomes compared (Table 1). 30-day follow-up was less for LBS $(93.2 \%$ vs $95.5 \%, p=0.024)$. RBS was associated with significantly longer operative times $(\mathrm{p}<0.0001)$ and hospital length of stay $(\mathrm{p}=0.0002)$. Unplanned ICU admission $(1.31 \%$ vs $0.52 \%, p=0.049)$, aggregate bleeding $(p=0.069)$ and aggregate leak $(p=0.087)$ were higher in RBS. 30-day mortality, reoperation, readmission, intervention and all other complications were similar.

Conclusion: In this matched cohort analysis of revision/conversion bariatric surgery, roboticassisted and conventional laparoscopic approaches seem to be equally safe, but RBS is likely less cost-effective due to longer operative times and hospital length of stay.

\begin{tabular}{llll}
\hline Outcomes in case-controlled matched cohorts & & \\
\hline Surgical Approach & & & \\
\hline & $\begin{array}{l}\text { Conventional laparoscopic } \\
{[\mathrm{n}=1,144]}\end{array}$ & $\begin{array}{l}\text { Robotic-assisted } \\
{[\mathrm{n}=1,144]}\end{array}$ & $\mathrm{p}$ value \\
\hline $\begin{array}{l}\text { OL_Minutes } \\
\quad \text { (mean } \pm \text { SD) }\end{array}$ & $121.71 \pm 67.45$ & $177.42 \pm 79.40$ & $<0.0001^{*}$ \\
$\begin{array}{l}\text { HOS_Days } \\
\quad \text { mean } \pm \text { SD) }\end{array}$ & $2.19 \pm 3.09$ & $2.38 \pm 3.07$ & $0.0002^{*}$ \\
$\begin{array}{l}\text { Outcome, no. } \%) \\
\text { Conversion rate }\end{array}$ & $11(0.96)$ & & \\
$\begin{array}{l}\text { 30-day unplanned ICU } \\
\text { admission }\end{array}$ & $6(0.52)$ & $13(1.14)$ & 0.68 \\
30-day reoperation & $32(2.8)$ & $15(1.31)$ & $0.049^{*}$ \\
30-day mortality & $2(0.17)$ & $42(3.67)$ & 0.24 \\
Death related & $2(0.17)$ & $3(0.26$ & 0.65 \\
Aggregate leak & $7(0.61)$ & $2(0.17)$ & 0.36 \\
Aggregate bleeding & $4(0.35)$ & $15(1.31)$ & 0.087 \\
\hline
\end{tabular}




\section{P186}

\section{Google Trends and Bariatric Surgery; A Google Search Data Analysis from 2004 to 2018}

Aimal Khan, $\mathrm{MD}^{1}$, Thaer Obeid, $\mathrm{MD}^{2}$, Renee Tholey, $\mathrm{MD}^{2}$, Alfred Trang, $\mathrm{MD}^{2}$, Ramsey Dallal, $\mathrm{MD}^{2} ;{ }^{1}$ University of Alabama at Birmingham, ${ }^{2}$ Einstein Healthcare Network

Introduction: Despite being a reliable and effective long-term tool in combatting obesity, the percentage of people undergoing bariatric surgery has remained stagnant over the last decade. Public interest in bariatric surgery is one of the main drivers of its utilization, however measuring it using traditional methods is challenging.

Materials and Methods: Google Trends is a useful internet tool that can be used for quantifying public's interest in a particular topic. It reports internet searches about a particular term as search volume indexes (SVIs). SVIs are normalized values based on total searches during a specified period for a given region. We queried Google trends for "bariatric surgery", "gastric bypass", "gastric sleeve", and "lap band" from January 2004April 2018 and analyzed their results

Results: The results of this study show that the public's interest in bariatric surgery has been steadily increasing since 2004 after falling to its lowest point in December 2010. We also show that the interest in lap band and gastric bypass has been gradually declining, with sleeve gastrectomy (SG) taking over the most searched bariatric procedure in 2018. State and city wise analysis show Michigan and New York City to have the highest proportion of Google searches for "bariatric surgery" respectively.

Discussion: Our study provides insight into the interest levels of US population in bariatric surgery. These findings can aid in the development of awareness campaigns via Internet, aimed at surgeons and the general public by helping better understand the US population's interest and means of accessing information regarding bariatric surgery.

\section{P187}

Risk Factors and Outcomes for Bleeding Following Bariatric Surgery: Results from the Metabolic and Bariatric Surgery Accreditation and Quality Improvement Program (MBSAQIP)

Aimal Khan, MD, Katey Feng, MPH, Richard D Stahl, MD, Abhishek D Parmar, MD, MS, Jayleen M Grams, MD, PhD; University of Alabama at Birmingham

Introduction: Bleeding following bariatric surgery can be a life-threatening complication. Here, we aimed to evaluate risk factors and outcomes of patients with postoperative bleeding using a national database from accredited bariatric centers.

Methods: The Metabolic and Bariatric Surgery Accreditation and Quality Improvemen Program (MBSAQIP) databases for 2015-2016 were used to identify patients with postoperative bleeding following laparoscopic sleeve gastrectomy (SG) or Roux-en-Y gastric bypass (RYGB). Patients undergoing emergency or revisional surgery or had their approach converted intraoperatively were excluded. Multivariate logistic regression was used to identify the risk factors for postoperative bleeding.

Results: A total of 326,841 patients underwent either SG (70\%) or RYGB (30\%). Of these, $1,592(0.5 \%)$ had postsurgical bleeding. RYGB were more likely to have a bleeding complication than SG (AOR 2.7, 95\% CI $=2.375-2.982$, p $<0001$ ). Other factors associated with increased risk of postoperative bleeding included preoperative therapeutic anticoagulation (AOR 3.6, 95\% CI $=3.017-4.338, \mathrm{p}<0.0001$ ), history of pulmonary thromboembolism (AOR 1.4, 95\% CI $=1.047-1.849, \mathrm{p}=0.0227$ ) or deep venous thrombosis $(A O R 1.3,95 \% \mathrm{CI}=1.002-1.670, \mathrm{p}=0.0479)$, renal insufficiency (AOR 2.8, 95\% $\mathrm{CI}=2.030-3.801, \quad \mathrm{p}<0.0001), \quad$ hypertension $\quad(\mathrm{AOR} \quad 1.2, \quad 95 \% \quad \mathrm{CI}=1.028-1.287$, $\mathrm{p}=0.0147)$, diabetes $(\mathrm{AOR} 1.2,95 \% \mathrm{CI}=1.086-1.355, \mathrm{p}=0.0006)$, gastroesophageal reflux disease (AOR 1.1, 95\% CI $=1.028-1.265, \mathrm{p}=0.0130$ ), obstructive sleep apnea (AOR 1.3, 95\% CI $=1.028-1.272, \mathrm{p}=0.0132)$, and male $\operatorname{sex}(\mathrm{AOR} 1.6,95 \% \mathrm{CI}=1.429$ $1.789, \mathrm{p}<0.0001)$. ASA class, anastomotic or staple line provocative testing, and placement of a surgical drain were not associated with postoperative bleeding. On subgroup analysis of SG patients, use of staple line reinforcement material (AOR 0.722, 95\% CI = $0.612-0.852, \mathrm{p}=0.0001$ ), over-sewing of the staple line (AOR $0.791,95 \% \mathrm{CI}=0.646$ $0.969, \mathrm{p}=0.0234$ ), and increased distance of the staple line from the pylorus (AOR 0.933 , $95 \% \mathrm{CI}=0.878-0.991, \mathrm{p}=0.0232)$ were all associated with decreased risk of postoperative bleeding. Larger sleeve bougie sizes were associated with increased risk of postoperative bleeding (AOR 1.031, 95\% CI $=1.010-1.052, \mathrm{p}=0.0037$ ). Patients with postoperative bleeding had significantly higher rates of unplanned admission to the ICU $(25.25 \%$ vs $0.82 \%, \mathrm{p}<0.0001)$, increased length of stay (4.15 days vs 1.82 days, $\mathrm{p}<0.0001)$ and mortality $(1.82 \%$ vs $0.11 \%, \mathrm{p}<0.0001)$

Conclusions: The overall rate of bleeding following index bariatric surgery is lower than previously thought. Numerous patient- and operation-specific risk factors for postoperative bleeding are identified and may be useful for improving risk stratification, patient counseling, and development of processes to reduce the risk of postoperative bleeding.

\section{P188}

"Intraoperative Leak Test (ILT) is Not Associated with Improvement in 30-day Outcomes After Primary Laparoscopic Sleeve Gastrectomy (LSG): MBSAQIP PUF, 2015-2016."

Ghaith Khair, MD, FACS, Anne Sill, MSHS, Andrew Averbach, MD, FACS, FASMBS; Saint Agnes Healthcare

Background: According to ASMBS estimate for 2017, LSG constituted over $60 \%$ of al bariatric procedures. Between 2011 and 2017 the leak rate has decreased from 2\% and currently stabilized at $0.3 \%$. A variety of ILT is used, in order to prevent the most serious complications of this procedure. Metabolic and Bariatric Surgery Accreditation and Quality Improvement Program (MBSAQIP) data indicate that ILT is performed in three quarters of LSG cases. However, at present the effectiveness of ILT in improving outcomes is still unclear.

Methods: MBSAQIP 2015-2016 Participant Use File (PUF) data were used to select only primary LSG cases. Two study groups were based on the use of ILT, and multiple primary outcomes were compared using Chi square tests and T-tests. Leak rate was calculated based on several reported outcomes. Data set reflected only the fact that ILT was performed without definition of the particular variant

Results: A total number of 185,867 primary LSG were performed; 143,400 patients (77.2\%) had ILT and 42,467 patients $(22.8 \%)$ did not. The post-operative leak rate was similar $(0.3 \%$ - with ILT and $0.2 \%$ - with no ILT; P $=0.367)$. There were no statistica differences between study groups in rates of mortality, overall morbidity, re-operation, reintervention and hospital readmission. In cases with no ILT, bougie size of Fr 40 or higher were used more frequently with average size of $37.1+8.1 \mathrm{Fr}$ compared to $36.8+8.4 \mathrm{Fr}$ in ILT group. OR time was significantly increased in ILT group $(76.7+32.7 \mathrm{~min}$ vs $68.6+32$ min; $\mathrm{P}<0.001$ )

Table 1: Analysis of MBSAQIP 2015-2016 PUF, primary LSG

\begin{tabular}{llll}
\hline Variable & No ILT & ILT performed & P value \\
\hline Number of patients & $42,467(22.8 \%)$ & $143,400(77.2 \%)$ & \\
Bougie size < 40 Fr & $67.5 \%$ & $72.3 \%$ & $<0.001$ \\
Bougie size > 40 Fr & $32.5 \%$ & $27.7 \%$ & \\
Average bougie size Fr & $37.1+8.1$ & $36.8+8.4$ & $<0.001$ \\
OR time min. & $68.6+32$ & $76.7+32.7$ & $<0.001$ \\
Hospital LOS days & $1.62+1.4$ & $1.64+1.5$ & 0.001 \\
Mortality & $0.1 \%$ & $0.1 \%$ & 0.850 \\
Overall morbidity & $4.6 \%$ & $4.6 \%$ & 0.467 \\
Leak postop & $0.2 \%$ & $0.3 \%$ & 0.367 \\
Reoperation & $0.9 \%$ & $0.8 \%$ & 0.126 \\
Reintervention & $0.9 \%$ & $1.0 \%$ & 0.047 \\
Readmission & $3.3 \%$ & $3.2 \%$ & 0.357 \\
\hline
\end{tabular}

Conclusion: The routine use of ILT in LSG is not associated with reduced leak rates or with improved 30-day outcomes. 
P189

Dual Academic Institutional Experience of Secondary Operations after Sleeve Gastrectomy

Victoria Lyo, $\mathrm{MD}^{1}$, Ryan Macht ${ }^{2}$, Yalini Vigneswaran ${ }^{1}$, Andrew Posselt $^{2}$, Jonathan Carter $^{2}$, Stanley Rogers ${ }^{2}$, Andrea Stroud ${ }^{1}$, Stephanie Wood ${ }^{1}$, Farah Husain ${ }^{1} ;{ }^{1}$ OHSU, ${ }^{2}$ UCSF

Introduction: Although laparoscopic sleeve gastrectomy (LSG) is the most common bariatric operation performed, patients can experience severe GERD, strictures, insufficient weight loss, and leaks. The incidence, indications, and patient outcomes of a secondary operation or conversion to bypass are not well understood.

Methods and Procedures: We reviewed institutional MBSAQIP data from two academic, tertiary referral centers for bariatric operations between 2014-2018. We identified patients who underwent secondary operations after LSG and evaluated their demographics and early postoperative outcomes.

Results: Of the 1713 bariatric operations performed, 316 (18.4\%) were secondary after prior bariatric surgery: 165 after gastric banding, 82 after bypass, and 55 after LSG. We identified 49 operations after LSG in 42 patients, excluding six port-site hernia repairs. Seven of the 49 cases were washouts or tube placements in duplicate patients. Most LSGs $(32 / 49,65.3 \%)$ were performed outside of our institutions. Mean time to revisions was 2.7 years (range: $0.23-9.1$ years).

Among the 42 reoperative LSG patients, 32 (76.2\%) had conversions from sleeve to bypass Of these conversions, three had concurrent HH repairs and one had a concurrent Heller myotomy. The remaining 10 patients had: four HH repairs, two gastroplasties, one incisural seromyotomy, one sleeve fundal revision, one conversion to esophago-jejunostomy for a chronic GC fistula, and one gastrobronchial fistula takedown.

Most patients had multiple indications for reoperation. Grouped by primary indication, the most frequent reason for surgery after LSG was GERD in 17 patients, four of whom had esophagitis, four HHs, and three inadequate weight loss. Of all patients with GERD (26 patients), $84.6 \%$ had their symptoms resolve with reoperation. Two of the four without resolution of GERD had a sleeve revision or HH repair alone.

Of the nine patients who had incisural strictures, six had resolution of oral intolerance after revision; three patients had persistent nausea with no strictures on EGD. All eight patients undergoing conversions for inadequate weight loss (baseline BMI $56.7 \mathrm{~kg} / \mathrm{m} 2$ to post-LSG BMI 48.8, EBWL 21.1\%) had successful weight loss to an average BMI of 44.7 and EBWL to $40.2 \%$. One of seven reoperations for fistulas or leaks failed leak closure. Among all reoperative 42 patients, 14 patients $(33.3 \%$ ) had complications requiring further procedures. Conclusion: Symptoms and complications after LSG can persist and these patients may need secondary operations. At our tertiary bariatric centers, secondary operations successfully treated the primary indications for reoperation and should be offered, but can be challenging with higher complication rates.

\section{P190}

Patient Therapeutic Education: Smartphone-based Applications for the Bariatric Surgery Patient

Rene Aleman, MD, Cristian Milla Matute, MD, Maria Fonseca Mora, MD, Joel S Frieder, MD, Emanuele Lo Menzo, MD, PhD, FACS, FASMBS, Samuel Szomstein, MD, FACS, FASMBS, Raul J Rosenthal, MD, FACS, FASMBS; Cleveland Clinic Florida

Background: Patient therapeutic education (PTE) encourages patient involvement and it has demonstrated efficacy in treating various chronic conditions. With the ever-growing smartphone ownership in the U.S., packaged software applications <'apps'>, have become widely popular for the access of health-related information, including bariatric surgery. The aim of this study is to review currently available apps in terms of PTE for bariatric surgery. Methods: The search for smartphone apps was conducted using three U.S. web stores: Apple's App Store, Google Play (Android) and Blackberry World. The keyword 'bariatric surgery' was used in order to retrieve the available related apps. The content quality and quantity of the apps was determined in accordance to PTE definition by the World Health Organization (WHO) as: "helping the patient and his family to acquire knowledge and competencies about the disease and its treatment, in order to better collaborate with the caregivers, and to improve his quality of life". Data was collected and posteriorly analyzed from the app overview description provided by the developer.

Results: Overall, a total of 306 apps were identified in the web stores used for the search $($ Google Play $=249$, Apple App Store $=41$, and BlackBerry World $=16)$. Of the entire query, $14.71 \%(\mathrm{~N}=45)$ of the apps classified as PTE apps, $4.25 \%(\mathrm{~N}=13)$ were targeted for surgeons, $1.31 \%(\mathrm{~N}=4)$ targeted sales related to bariatric surgery, and $1.63 \%(\mathrm{~N}=5)$ were literature reference apps. None of the apps reviewed required payment for download. Seven percent $(\mathrm{N}=22)$ of the apps had customer review with an overall average of 4.01/5. Nineteen apps were duplicated in the Google Play and the Apple App stores. Furthermore, $77.77 \%$ of the PTE apps had some sort of medical professional involvement.

Conclusions: There are currently 45 apps available for patients specifically directed to PTE on bariatric surgery. With the current trend on smartphone/app patient education, further studies are needed to determine the impact of mobile-based information in current bariatric clinical practice. Additionally, although apps are a way to educate potential bariatric surgery candidates, expert professional follow up should be emphasized to guarantee satisfactory outcomes.

\begin{tabular}{|l|l|l|l|l|l}
\hline Web store & $\begin{array}{l}\text { arget } \\
\text { audience }\end{array}$ & $\begin{array}{l}\text { Price } \\
\text { range } \\
(\$)\end{array}$ & $\begin{array}{l}\text { Apps with } \\
\text { customer } \\
\text { reviews }\end{array}$ & $\begin{array}{l}\text { Average } \\
\text { customer } \\
\text { rating from 15 }\end{array}$ & $\begin{array}{l}\text { Apps with medical } \\
\text { professional } \\
\text { involvement }\end{array}$ \\
\hline $\begin{array}{l}\text { Google Play } \\
\text { Store }\end{array}$ & Patients & 0 & 15 & 4.22 & 17 \\
\hline $\begin{array}{l}\text { ADpie ApD } \\
\text { Store }\end{array}$ & Patients & 0 & 7 & 3.81 & 18 \\
\hline $\begin{array}{l}\text { BlackBerry } \\
\text { Wordd }\end{array}$ & Patients & N/A & N/A & N/A & N/A \\
\hline
\end{tabular}

\section{P191}

Incidence of Gallstone Formation After Bariatric Surgery. A Prospective Study and Follow up

Juan E Contreras, Professor, Jorge Bravo, MD, Ismael Court, MD; Clinica Santa Maria

Background: Chilean prevalence of cholelithiasis is $13.1 \%$ in men and $36.7 \%$ women and $70 \%$ of population has overweight or obesity.

Objective: The aim is to evaluate the incidence of cholelithiasis after bariatric surgery. Methods: It is a prospective one year study of 444 patient operated by obesity, of which $86 \%$ Sleeve Gastrectomy and 14\% Gastric Bypass.

Follow up until 5 years. Average age 38 years, weight preop average $100 \mathrm{Kgs}$ and average BMI 36.27

A total of 345 cases were included after exclusion criterion

(previous and concomitant chlolecisthectomy and revision surgery)

All patients have ultrasound study at third month after de bariatric surgery. 163 patients los the follow up and were discarded.Total included was 182 (155 sleeve and 27 Bypass)

Results: 86 patients developed Cholelithiasis (47\%) after bariatric procedure. Women than men ratio of 2,2: 1 .

Presentation with $73 \%$ with asyntomatic cholelitiasis and $21 \%$ with acute cholecistitis or pain

75 cases of 155 (48\%) in Sleeve Gastrectomy and Bypass with 11 cases of 27(40\%).

$65 \%$ of patients under 40 years developed cholelithiasis, and older than 40 years it was $35 \%$.

In $65 \%$ Cholelithiasis were detected at first year of F.U. Obesity grade I and II it was associated with higher prevalence of cholelithiasis compared to III-IV. $86 \%$ and $14 \%$ respectively.

Fast and effective EWL were associated with increased incidence of cholelithiasis.

Conclusion: patients undergoing bariatric surgery suffer a significant incidence of cholelithiasis during the first year. 


\section{P192}

The Impact of ADHD on Outcomes Following Bariatric Surgery: A Systematic Review and Meta-analysis

Valentin Mocanu ${ }^{1}$, Iran Tavakoli ${ }^{1}$, Jerry Dang ${ }^{1}$, Noah Switzer ${ }^{2}$, Daniel Birch ${ }^{1}$, Shahzeer Karmali ; ${ }^{1}$ University of Alberta, ${ }^{2}$ The Ohio State University

Introduction: The objective our study was to carry out a systematic review and meta-analysis to examine the impact of attention deficit and hyperactivity disorder (ADHD) on bariatric surgery outcomes. Despite the effectiveness of bariatric surgery, about 10 to $20 \%$ of patients continue to regain weight after the procedure. New evidence supports that ADHD may be directly associated with obesity and may affect outcomes following bariatric surgery. However, certain psychiatric illnesses, such as ADHD are rarely screened for, leading to a continued lack of data on the interaction between ADHD and bariatric surgery.

Methods and Procedures: A comprehensive literature search for both published and unpublished studies of ADHD and bariatric surgery from 1946 to August 2018 was performed. The search was conducted using the Medline, EMBASE, Scopus, the Cochrane Library, and Web of Science databases as well as conference abstracts. Our search strategy terms included "(ADHD OR attention deficit hyperactivity disorder) AND (bariatrics OR obesity surgery OR gastric bypass OR gastric sleeve OR Roux-en-Y OR RYGB OR sleeve gastrectomy)" and was limited to human studies in the English language. Preliminary database search of the literature yielded 104 articles after 70 duplicates were removed

Results: A total of five studies with 492 patients were included. The overall ADHD rate was $20.9 \%$ with reported rates ranging from $7 \%$ to $38 \%$. The weighted mean age was $44.0 \pm 10.2$ years, the weighted sex was $83.6 \%$ female, and the weighted mean follow-up was 22.2 months. Preoperative weighted mean BMI was 43.7 versus a postoperative weighted mean BMI of 34.7. No statistical significance was observed for mean BMI difference between non-ADHD vs. ADHD patients undergoing bariatric surgery (Figure 1) (3 studies; MD -2.66; CI -7.54 to $2.13 ; p=0.28$ ). Statistical significance was, however, observed for postoperative follow-up between patients with ADHD vs. non-ADHD subjects ( 3 studies; MD $-7.28 ;-13.83$ to -0.73 ; $\mathrm{p}=0.03$ ).

Conclusion: Patients with ADHD do not have a statistically significant mean BMI difference following bariatric surgery but have a statistically significant reduction in postoperative follow-up versus non-ADHD patients. Targeted strategies aimed at improving clinic attendance for this at-risk ADHD population may improve bariatric outcomes and minimize recidivism rates.

\section{P193}

Gastric Plication as a Component in a Comprehensive Weight Loss Program in Egyptian Patients

Ashraf A Bakr, Professor, Dr, Mohamed Y Selim, Professor, Dr; Faculty of Medicine, Cairo University

Introduction: Obesity in Egypt soared to a high 40\%. Consequently, the rate of bariatric surgery increased fourfold, including laparoscopic greater curvature plication (LGCP). Illiteracy and poor patient compliance affect the outcome of surgical procedures, including bariatric procedures.

The aim of this study was to evaluate the impact of intensive patient education and strict postoperative care and monitoring on the outcome of LGCP.

Patients and Methods: This study included 53 patients, presenting with morbid obesity with Body Mass Index $(\mathrm{BMI})<40 \mathrm{~kg} / \mathrm{m}^{2}$. The study was conducted from June 2016 till February 2018. All patients were enrolled in a comprehensive program. The complements of the program included preoperative orientation about

- Obesity

- Nutrition (pre and postoperative)

- Weight loss surgery

- How to maintain weight loss after surgery

- Changes expected in co-morbidities

The patients then underwent LGCP by a standardized technique. The patients were followed postoperatively using a strict program that included clinic visits, phone calls and social media communication. The main components of the follow up were

- Morbidity

- Weight loss

- Changes in co-morbid disease

- Patient satisfaction

Results: The participants were mostly females (42/53), with mean BMI $39.1 \pm 0.19 \mathrm{~kg} / \mathrm{m}^{2}$, mean age $33.7 .4 \pm 1.29$ years. Postoperative follow up was complete in 45 patients and partial in 8 patients. Seven patients suffered from transient nausea and vomiting that lasted for one week and managed conservatively. Six patients complained from hair loss. The mean total body weight loss was $25 \% \pm 4.5$ after 6 month. No revisions and no re-operations were needed in this series. Patient satisfaction was in the range of $94 \%$. Improvement in the management of co morbidities occurred in 7 patients (14.8\%).

Disucssion: LGCP is a bariatric procedure that requires no resection or bypass of the gastrointestinal tract. No staplers are needed, therefore the cost of the procdrure is significantly reduced. The procedure takes a longer time to perfom and needs special skills, yet for BMI belwow $4 \mathrm{o} \mathrm{kg} / \mathrm{m}^{2}$ the excess body weight loss postoperatiely is satisfactory. Patients do not require extensive dietary supplements postoperatively. The down side is that the changes in comorbidities are not as high as other bariatric procedures.

Conclusion: LGCP is good option for morbidly obese patients with BMI below 40 with close postoperative monitoring. The low cost is an added advantage, especially in low-income countries. 


\section{P194}

\section{Surgical Technique for Sleeve Gastrectomy: Comparison Between Medial Versus Lateral Approach at 3 Years}

Vanessa Boudreau, MD, Scott Gmora, MD, Dennis Hong, MD, Mehran Anvari, MD, PhD, Karen Barlow, Hons, BSc, Olivia Lovrics, MSc; Center for Minimal Access Surgery, McMaster University, Ontario, Canada

Introduction: No consensus exists in the literature for the best surgical technique when performing a sleeve gastrectomy. Medial and lateral approaches have been described, but no study could identify if one technique is better in terms of clinical outcomes. This study aims to compare the medial and lateral approach for sleeve gastrectomy (SG) in terms of intraoperative and postoperative complications, and weight loss, and to determine if both techniques are equivalent.

Methods: Data from the Ontario Bariatric Registry was used for this retrospective study to compare the effectiveness and safety of sleeve gastrectomy using the medial approach versus the lateral approach, during a 3-year follow-up after SG.

Results: Between January 2010 and June 2015, 564 patients underwent a sleeve gastrectomy surgery at St Joseph's Healthcare Hamilton. 229 patients underwent a medial approach (72.9\% female, age 46.9, BMI 54.4) and 335 underwent a lateral approach (73.1\% female, age 48.8, BMI 56.3). Both groups were comparable in terms of baseline comorbidities. Weight loss and rate of complications at 3-year follow-up are as follows:

\begin{tabular}{llll}
\hline & Medial approach & Lateral approach & p-value \\
\hline Weight loss & & & \\
Decrease in BMI (SD) & & & \\
1 year & $15.9(7.0)$ & $15.0(7.6)$ & NS \\
3 years & $14.5(6.7)$ & $13.7(8.3)$ & NS \\
OR time minutes (SD) & $70.4(15.8)$ & $95.3(31.3)$ & $<0.05$ \\
Complications n (\%) & & & \\
Mortality 30 days & 0 & 0 & NS \\
Overall complications & $10(4.4)$ & $11(3.3)$ & NS \\
Wound infection & $1(0.4)$ & $2(0.6)$ & NS \\
Stenosis and stricture & $2(0.9)$ & $1(0.3)$ & NS \\
Hospitalization & $4(1.7)$ & $6(1.8)$ & NS \\
Revisional surgery & $3(1.3)$ & $4(1.2)$ & NS \\
\hline
\end{tabular}

Conclusions: Both the lateral and the medial approach are effective and safe for performing the sleeve gastrectomy. No significant differences were seen in weight loss and rate of complications at 3 years. The medial approach may decrease operative time. Further study is needed to evaluate this finding.

\section{P195}

Trends in Drain Utilization in Bariatric Surgery: An Analysis of the MBSAQIP Database 2015-2016

Robert Cullen, BS, Ira L Swinney, MS, Alan Tyroch, MD, Ellen Wicker, DO, Ginger Coleman, MD, Calvin Mcnelly, BS, Babak Sadri, BS, Carlos Lodeiro, BS, Chris Dodoo, MS; Texas Tech HSC Paul Foster School of Medicine

Introduction: Laparoscopic gastric bypasses (GB) and sleeve gastrectomies (SG) are the two most common bariatric operations. There have been changes in surgical technique and surgical norms over time. The main advance has been moving towards laparoscopic surgery. With the implementation of enhanced recovery protocols, surgeons tend to use less invasive methods of perioperative management. This includes decrease in foley catheterization, the use of nasogastric tubes and drains. There is still controversy about the use of drains and indications for drain placement. As practice patterns change and enhanced recovery protocols are adopted, the use of drains should decrease.

Methods: The MBSAQIP database was queried for the years 2015 and 2016. Our inclusion criteria included all patients undergoing a primary GB or SG. Revisions were excluded. We examined demographics, operative characteristics, the use of drains, and post operative complications. Continuous variables were summarized using mean and standard deviations (SD). Categorical variables were summarized using frequencies and proportions. Students T- test (Wilcoxon sum rank test in the case of skewed data) and Chi squared analysis was used to assess the baseline differences in drain utilization. To assess the factors that predict drainage usage, the generalized linear models with family Poisson and link log was used obtain the relative risk measures. $\mathrm{P}$ values $<5 \%$ were considered statistically significant. All analyses were carried out using STATA V15.

Results: In 2015-2016 there were 240,462 bariatric cases performed without drains and 70,292 with drains. $31.4 \%$ of GB patients had a drain placed but only $18.5 \%$ of SG patients. The percentage of GB that had a drain dropped from $33.1 \%$ to $29.7 \%$ during the study period and SG dropped from $20.3 \%$ to $17.0 \%$. For GB and SG, there was no difference in provocative testing, swallow studies, operative length, reoperations, readmissions or death Patients that had drains placed were more likely to have a provocative test at the time of surgery (relative risk (RR) 1.9) and to have a postoperative swallow study (RR 2.67) Conclusions: Drains are still commonly used in bariatric patients. Over the study period, there was a slight decrease in the use of drains in both bypass and sleeve patients. Patients with a drain were more likely to have had a provocative test and a swallow study. Drain placement was not associated with higher complications.

\section{P196}

Revisional Surgery Results After Sleeve Gastrectomy. A Retrospective Cohort Study Surveying 2nd Stage BPD/DS

Zvi H Perry, MD, PhD, Mohammed Al Abri, MD, Olivier Court, MD, Amin Andalib, MD, MPH, Sebastian Demyttenaere, MD; Center for Bariatric Surgery, Division of General Surgery, Department of Surgery, McGill University, Montreal, Quebec

Introduction: Sleeve gastrectomy is becoming the most popular bariatric procedure in the world. However, there is ongoing debate as to the appropriate surgical procedure after failed sleeve gastrectomy. In this study, we aimed to evaluate patients who underwent prior sleeve surgery who subsequently underwent biliopancreatic diversion with duodenal switch (BPD/DS).

Methods: A retrospective analysis of a prospectively maintained database of laparoscopic sleeve gastrectomies (LSG) was performed between January 2006 and March 2018. Data analyzed included age, weight loss, postoperative complications and long-term outcomes. Results: 173 patients were enlisted in our database as having lap BPD/DS. Of these patients, 91 subsequently had revisional surgery to (BPD/DS). Of the 91 patients 56 $(61.5 \%)$ were female. Mean follow up time was $5.5( \pm 2.7)$ years. Mean time between the sleeve and the revisional surgery was $21.5( \pm 16)$ months. The mean age upon the sleeve procedure was $41.8( \pm 10.5)$, while the mean age upon the revisional duodenal switch was $43.7( \pm 10.6)$. Pre-sleeve mean body mass index (BMI) was $63.5( \pm 9.7) \mathrm{kg} / \mathrm{m}^{2}$. Pre-BPD mean BMI was $46.3( \pm 7.7) \mathrm{kg} / \mathrm{m}^{2}$ and at the end of the follow up period the mean BMI was $39.5( \pm 7.8) \mathrm{kg} / \mathrm{m}^{2}$. Differences between pre-op BMI and pre-revision, pre-revision and end of follow-up BMI, as well as between pre-op BMI and end of the follow-up were all significant $(\mathrm{p}<0.001) .20$ patients suffered from a complication $(22 \%)$, with the mos common being leak ( 9 patients, $9.9 \%$ ), and infection (6 patients, $6.6 \%$ ). No death occurred. Discussion and Conclusions: Controversy exists regarding technical aspects of revisional LSG. In our study, we have seen that post-sleeve duodenal switch had substantial weight loss, with a satisfactory complication profile. Longer follow up is needed to determine if this improvement in weight loss remains in the long term. 


\section{P197}

\section{Risks of Bariatric Surgery Among Patients with End-Stage Renal Disease}

John R Montgomery, MD ${ }^{1}$, Seth A Waits, MD ${ }^{1}$, Justin B Dimick, $\mathrm{MD}, \mathrm{MPH}^{2}$, Dana A Telem, MD, $\mathrm{MPH}^{2} ;{ }^{1}$ University of Michigan, Dept Transplant Surgery, ${ }^{2}$ University of Michigan, Center for Healthcare Outcomes \& Policy

Objective: The association between bariatric surgery and long-term weight loss and comorbidity improvement among obese patients with end-stage renal disease (ESRD) is well established. Moreover, for patients who subsequently undergo kidney transplantation, it is associated with improved patient and graft survival. However, the perioperative risks of bariatric surgery among obese ESRD patients are poorly characterized as data are limited to single-center studies that lack generalizability and may not detect rare events. In this context, we performed an analysis of perioperative safety of bariatric surgery in obese patients with ESRD using a national registry capturing $>95 \%$ of bariatric operations.

Methods: Patients who underwent primary, laparoscopic sleeve gastrectomy or gastric bypass between 2015-2016 were identified from the Metabolic and Bariatric Surgery Accreditation and Quality Improvement Program participant use file. The primary outcome was a composite variable of death and severe, life-threatening complications within 30 days of operation. Logistic regression was used to compare adverse outcomes between ESRD patients (defined as dialysis-dependent) and those with normal renal function.

Results: During the study period, 299,373 bariatric operations were performed; of these, $836(0.3 \%)$ patients had ESRD. ESRD patients were more likely to be older $(48.6 \pm 10.4 \mathrm{vs}$ $44.8 \pm 11.9$ years, $\mathrm{p}<0.001$ ), male ( 43.7 vs $20.2 \%, \mathrm{p}<0.001)$, hypertensive ( 88.4 vs $72.2 \%, \mathrm{p}<0.001)$, diabetic $(55.0$ vs $25.7 \%, \mathrm{p}<0.001)$, and have poorer functional status (5.9 vs $1.0 \%$ partially-or-fully dependent, $\mathrm{p}<0.001$ ). After adjusting for patient age, smoking status, hypertension, diabetes, and functional status, ESRD was associated with increased chance of death or severe, life-threatening complications $(4.8$ vs $1.9 \%$, aOR 2.61 [1.93-3.52], $\mathrm{p}<0.001)$. This difference was primarily driven by higher incidence of reoperation ( 3.6 vs $1.3 \%, \mathrm{p}<0.001)$, transfusion $\geq 3$ units $(0.8$ vs $0.2 \%, \mathrm{p}<0.001)$, and death $(1.0$ vs $0.1 \%, \mathrm{p}<0.001)$ among ESRD patients.

Conclusion: ESRD is associated with increased risk of death and severe, life-threatening complications after bariatric surgery when compared to patients with normal renal function. However, the absolute risk is low and largely driven by increased reoperation rates; it should not be prohibitive for operative consideration. Given the established long-term benefits of bariatric surgery in obese ESRD patients and improved post-transplantation patient and graft survival, this supports consideration of CMS-mandated referral for comprehensive weight-loss management with availability of surgical options in all obese ESRD patients, and especially among patients being considered for renal transplantation.

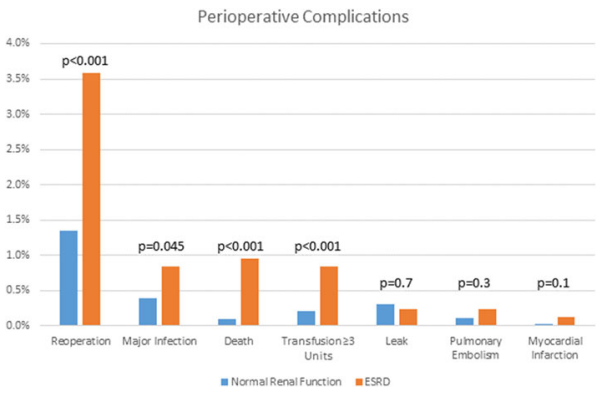

\section{P198}

The Role of Sleeve Gastrectomy in Glycemic Control in Patients with Morbid Obesity and Type 2 Diabetes

Taryel Omerov, $\mathrm{PhD}^{1}$, Gulay Mamedova, $\mathrm{Md}^{2}$, Nadir Zeynalov, $\mathrm{PhD}^{1}$, Nuru Bayramov, Professor ${ }^{1} ;{ }^{1}$ Azerbaijan Medical University, ${ }^{2}$ Modern hospital

Key words: Obesity, sleeve gastrectomy, type II diabetes, bariatric surgery. Aim of the study: Study of the results of sleeve gastrectomy in patients with type II diabetes with excessive obesity in the postoperative period.

Materials and Methods: 84 obese patients have undergone a sleeve gastrectomy procedure in Azerbaijan Medical University and Modern Hospital clinic 2012 through 2018 years. A $59(70,2 \%)$ of patients were females, $25(29,8 \%)$ were males, mean age was $37.9(15-59)$ years. Preoperative weight of patients ranged from 102 to $220 \mathrm{~kg}$, BMI $35.7-80.5 \mathrm{~kg} / \mathrm{m}^{2}$. The current trials have shown the growing burden of obesity incidence among population. Above all, the proportion of the patients with type 2 diabetes among morbid obese patients is significantly high. Taking in accounts above mentioned, we decided to investigate the correlation between glycemic control and rate of weight decrease among patients with morbid obesity, underwent stomach bariatric surgery. We followed the 84 patients with morbid obesity and prediabetes and diabetes type 2 , who underwent bariatric surgery and observed their glycemic level. The 30 patients (35/7\%) had the history of diabetes type 2 for 2 year, in 27 patients $(32.1 \%$ ) the history of diabetes was for 10 years, whereas 12 patients (14.3\%) had diabetes for more than 10 years. The 15 patients $(17.9 \%)$ had prediabetes. Results: The dynamic of anthropometric parameters showed significant decrease of weight in comparison with initial level (38\%), the BMI decreased from $46.8 \mathrm{~kg} / \mathrm{m}^{2}(42.3-54.3)$ to $27.6 \mathrm{~kg} / \mathrm{m}^{2}(25.4-30.9),(\mathrm{p}<0.001)$. In two years following operation weight parameters were decreased in comparison with initial level for $42 \%$, the BMI was decreased up to $26.8 \mathrm{~kg} / \mathrm{m}^{2}(23.1-28.4)$. The remission of type 2 diabetes was observed in $98.6 \%$ patients $(\mathrm{n}=82)$ within three years of following up ( $\mathrm{Hb} 1 \mathrm{Ac}<0.6 \%$, fasting glucose level $<5.6$ $\mathrm{mmol} / \mathrm{l})$

Conclusion: Thus, the procedure sleeve gastrectomy of patients with Type II diabetes mellitus was not only effective in reducing body mass index and correction of metabolic syndrome. First of all, it has been proven to be a satisfactory control of stable glycemic control as well as carbohydrate metabolism in the postoperative period.

\section{P199}

\section{SILS (Single Incision Laparoscopic Surgery)}

Lakshman Agarwal, Mbbs, MS; Swai Man Singh Medical College, Jaipur, Rajasthan, India

Gall stones (cholelithiasis) are common condition. Advent of ultra sonography has increased its detection in a large way. The exact number of cholecystectomy performed in India is difficult to know as there is no standard national registery for it. We are practising SILS since last 4 years and the number exceed 600 .

Langenbuch in 1882 did first open cholecystectomy, Conventional four port laparoscopic cholecystectomy (Philippe Mouret 1987), Single incision laparoscopic cholecystectomy (Navarra 1997) and NOTES (Natural orifice transluminal endoscopic surgery)

Materials and Methods: This study comprises of 600 cases of SILC (87\% female and $13 \%$ male)

A four fascial incision approach was used with conventional staright and reticulating instrument.

Results: This study comprises of 600 cases of SILC ( $87 \%$ female and $13 \%$ male) with AGE ranging from age 9-90 (mean 39.91)

USG Findings in our study were Cholelithiasis with chronic cholecystitis (553 patients), Cholelithiasis with acute cholecystitis (36 patients), Acalculouschlecystitis (7 patients) And Gall bladder polyp (4 patients)

Previous Abdominal Surgery- Lap tubectomy 3\%, hysterectomy 2\%, L.S.C.S. 3\%, Appendicectomy $2 \%$, Exploratory Laparotomy $2 \%$.

Intra-Op Findings were - Adhesion 20\%, distended gall bladder $12 \%$, Contracted gall bladder $9 \%$, normal gall bladder $59 \%$

Operative time range from 26.3 to $75 \mathrm{~min}$ (mean $46.96 \mathrm{~min}$ )

Intra-Op Complications were - bleeding 5\%, content leak from gallbladder $9 \%$ and spillage of stones in $5 \%$ patients

Conversion rate was $3 \%$. Post-Operative Hospital stay was $1.24+0.46$ days

Post-Operative Complications (wound infections) was $2 \%$

Scar length was $2.07 \mathrm{~cm}$ (range $1.6-2.7 \mathrm{~cm}$ )

Conclusion: SILC is feasible, safe and reproducible with shorter hospital stay and better cosmesis, but has larger learning curve and longer operative time.

It does not add to cost if conventional instruments are used as we did in our study. 
P200

\section{Direct Laparoscopic Transverse Abdominis Plane (TAP) Block:} New Approach

\section{Ali Hasan, MD; Saudi German Hospital, UAE}

The transverse abdominis plane (TAP) block is a peripheral nerve block designed to anesthetize the nerves supplying the anterior abdominal wall (T6 to L1). It was first described in 2001 by Rafi as a traditional blind landmark technique using the lumbar triangle of Petit. The initial technique described the lumbar triangle of Petit as the landmark used to access the TAP in order to facilitate the deposition of local anaesthetic solution in the neurovascular plane. Other techniques include ultrasound-guided access to the neurovascular plane via the mid-axillary line between the iliac crest and the costal margin, Open transversus abdominis plane block,and a subcostal access termed the 'oblique subcostal' access.

Objectives: New technique review aiming to describe the technique of direct laparoscopic technique in transverse abdominal plan blockade for different surgical interventions

Methods: Detailed review of TAP blockad starting from relevant anatomy, brief description of old TAP blockade techniques. Details of Direct Laparoscopic TAP blockade, with description of complications, technical difficulties, results and expectations

Conclusion: Direct laparoscopic transverse abdominal plan blockades is simple procedure, with high quality efficacy, can be used routinely in most of laparoscopic surgery procedures of the abdomen and pelvis

I describe here a new laparoscopic technique for safe, effective transverse abdominis plane (TAP) block which is handable and accessible for all laparoscopic surgeon

This paper shows the feasibility of alternative approach for transverse abdominis plane (TAP) block in laparoscopic cholecystectomy

\section{P201}

\section{Our Retraction Methods for Securing the Surgical Field that Facilitates Laparoscopic Pancreaticoduodenectomy}

Nagakawa Yuichi, MD, FACS, Yatsuka Sahara, Chie Takishita, Yousuke Hijikata; Department of Gastrointestinal and Pediatric Surgery, Tokyo Medical University

Laparoscopic pancreaticoduodenectomy (LPD) comprises various procedures including reconstruction and requires an advanced level of laparoscopic skill. Therefore, techniques to reduce the difficulty of LPD are needed. The visual field for LPD is different from that of open surgery. Even experts of laparoscopic surgery may increase the difficulty if the surgical field is not secured, increase surgical time and blood loss, and cause vascular injury. The establishment of effective retraction methods is important at each site to achieve an effective three-way retraction. Although the SMA first approach is widely used in open pancreaticoduodenectomy, it is difficult to laparoscopically expose the origin of the IPDA from left side of the SMA. We introduce the proximal dorsal jejunal vein (PDJV) preisolation method which facilitate approaching the SMA from the right side of the SMA under good surgical field. Although the mean operation time from the initial cases to 47 cases was 551.6 (374-646) minutes, operation time from 60 to 85 cases was significantly reduced from the initial cases was significantly decrease Although laparoscopic pancreaticoduodenectomy (LPD) is considered as minimally invasive surgery, an advanced level of laparoscopic skill is still required. LPD comprises various procedures including reconstruction. Recently, laparoscopic pancreaticoduodenectomy (LPD) has been used as a nove minimally invasive surgery. However, advanced laparoscopic skills are required. Therefore, techniques to reduce the difficulty of LPD are needed. The visual field for LPD is different from that of open surgery. Even experts of laparoscopic surgery may increase the difficulty if the surgical field is not secured, increase surgical time and blood loss, and cause vascular injury. The establishment of effective retraction methods is important at each site to achieve an effective three-way retraction. Although the artery-first approach is widely used in open pancreaticoduodenectomy, it is difficult to laparoscopically expose the origin of the IPDA from left side of the SMA. We introduce the proximal dorsal jejunal vein (PDJV) preisolation method which facilitate approaching the SMA from the right side of the SMA under good surgical field. Although the mean operation time from the initial cases to 47 cases was 551.6 (374-646) minutes, operation time from 61 to 85 cases was significantly reduced from the initial cases was significantly decrease ( $405.1 \mathrm{~min}$; $370-480$ ) by the above attempt. We introduce our approaches to reduce surgical difficulty of LPD.by the above attempt. We introduce our approaches to reduce surgical difficulty of LPD.

\section{P202}

Gangrenous Cholecystitis: Delays in Surgical Evaluation And Outcomes in the Era of Minimally Invasive Surgery

Rebecca C Gologorsky, $\mathrm{MD}^{1}$, Justin Tse, $\mathrm{MD}^{2}$, Dylan Wolman, $\mathrm{MD}^{2}$, Aya Kamaya, $\mathrm{MD}^{2} ;{ }^{1}$ University of California San Francisco-East Bay, ${ }^{2}$ Stanford University

Introduction: Gangrenous cholecystitis (GC) is a highly morbid complication of acute cholecystitis (AC). Risk factors for gangrenous progression are poorly described, as are preoperative clinical features and post-operative outcomes in the era of minimally invasive surgery.

Methods and Procedures: Patients who underwent cholecystectomy for AC from January 2014-May 2018 at a single academic medical center were retrospectively reviewed, with pathologic confirmation of GC vs. uncomplicated acute cholecystitis (UAC) in all included patients.

Pertinent clinical features, demographics, and laboratory values were recorded. Two-tailed t-tests and Fisher's exact tests were used to determine statistical significance for continuous and categorical variables, respectively.

Results: Among the 101 patients reviewed, $48(48 \%)$ had GC and $53(52 \%)$ had UAC Patients with GC were older $(62 \pm 17$ vs. $48 \pm 18 ; \mathrm{p}=0.0001)$ and predominantly male $(65 \%$ vs $38 \%$; $=0.005)$. Delays in diagnosis or treatment were significantly more common among patients with GC ( $46 \%$ vs $17 \%, \mathrm{p}=0.0025)$, defined as a failure to recognize or treat acute cholecystitis at initial emergency department or urgent care visit up to 14 days prior to surgical admission (Table 1). Among patients with delayed care, none were surgically evaluated until subsequent admission. Mean time from imaging to surgical consult did not differ between groups ( $4.3 \mathrm{~h}$ vs. $4.6 \mathrm{~h}, \mathrm{p}=0.9167$ ). Following surgical consultation, there was no difference in mean time to surgery $(24.6 \mathrm{~h}$ vs. $21.0 \mathrm{~h}, \mathrm{p}=0.4128)$. Among patients with GC, 17 (35\%) underwent open cholecystectomy, of which $13(27 \%)$ were converted from a laparoscopic approach. Among patients with UAC, only 2 (3.7\%; $\mathrm{p}=0.0001$ ) underwent open cholecystectomy, both converted from a laparoscopic approach. Postoperatively, $5(10 \%)$ patients with GC were admitted to the ICU versus 1 $(2 \%)$ UAC patient $(\mathrm{p}=0.0994)$, and total length of hospitalization was greater among GC patients $(5.0 \mathrm{~d}$ vs. $2.7 \mathrm{~d} ; \mathrm{p}=0.0022)$. Complication rates were higher among GC patients $(17 \%$ vs. $3.8 \% ; p=0.0441)$, with greater complication severity, including one death $(2 \%)$ Conclusion: GC is a clinically under-recognized surgical urgency. Delays in diagnosis of $\mathrm{AC}$ and subsequent surgical consultation contribute to its prevalence. A need for improved diagnostic recognition of $\mathrm{AC}$ is necessary to reduce risk of progression to $\mathrm{GC}$, and to rapidly treat GC on presentation.

\begin{tabular}{lll} 
& $\begin{array}{l}\text { Gangrenous } \\
\text { Cholecystitis ( } \mathrm{n}=22)\end{array}$ & $\begin{array}{l}\text { Uncomplicated Acute } \\
\text { Cholecystitis ( } \mathrm{n}=9)\end{array}$ \\
\cline { 2 - 4 } Initial ED/urgent care & & \\
diagnosis & & 4 \\
Symptomatic cholelithiasis & 3 & 1 \\
Constipation/GI upset & 3 & 3 \\
GERD & 2 & 1 \\
Patient refused care & 2 & 0 \\
Musculoskeletal & 2 & 0 \\
Other & 5 &
\end{tabular}




\section{P203}

\section{Epidemiological Profile and Evaluation of Health Care in Patients with Biliary Atresia: A Descriptive Study}

Caline S Medeiros, Medical Student ${ }^{1}$, Eduardo V Holanda, Medical Student ${ }^{1}$, Rodrigo M Gallindo, MD, $\mathrm{phD}^{1}$, Raquel N Cordeiro, Medical Student ${ }^{1}$, Diego L Lima, MD ${ }^{2}$; ${ }^{1}$ Pernambuco Health College, ${ }^{2}$ State Servers Hospital

Introduction: Biliary atresia (BA) is a rare pediatric pathology, but it is the main cause of liver transplantation in children when not treated with the Kasai procedure. There is a tendency for the surgical procedure to be delayed throughout Brazil and an investigation of its cause is necessary.

Objective: to determine the epidemiological profile of the patient with BA and to evaluate the health care focused on this diagnosis.

Methods: a descriptive, retrospective, cross-sectional study was performed with the medical records of patients diagnosed with BA and followed between 1996 and 2015.

Results: We analyzed the medical records of 72 patients but only 52 patients had complete records available. Twenty-six patients were included in the study: the mean age at admission was 87,9 days. 24 cases were diagnosed with ultrasonography and 10 needed to repeat the exam for confirmation. The Kasai surgery were performed in $50 \%$ of the patients, $38.4 \%$ of them in the appropriate time. Of the patients who were operated late, $25 \%$ were transplanted and $50 \%$ died. In the patients who had the procedure early, only one was transplanted and there were no deaths.

Conclusion: The diagnostic difficulties: failure in suspecting of BA in primary or tertiary care, and the high rate of false-negative results in USG delayed the surgical treatment, contributing to the worst outcome for patients who were operated late.

\section{P205}

Laparoscopic Management of Gastro Intestinal Stromal Tumours Ajay H Bhandarwar, MS, FMAS, FIAGES, FAIS, FICS, FBMS ${ }^{1}$, Jalbaji P More, MS ${ }^{1}$, Amol N Wagh, MS, FMAS, FIAGES, FAIS, FICS, FBMS ${ }^{1}$, Shekhar A Jadhav, MS, FMAS ${ }^{1}$, Amarjeet E Tandur, $\mathrm{MS}^{1}$, Priyanka Saha, MBBS ${ }^{1}$, Khushboo Kadakia, MBBS ${ }^{1}$, Soumya Chatnalkar, MBBS ${ }^{1}$, Ruchira Bhattacharya, MBBS $^{1}$, Shraddha R Gajbhiye, MBBS, MD Associate Professor ${ }^{2}$; ${ }^{1}$ Grant Government Medical College \& Sir J.J. Group of Hospitals, Mumbai, India, ${ }^{2}$ Govt. Medical College, Nagpur,Maharashtra,India

\section{Abstract}

Background: Gall bladder perforation is a rare clinical entity but life-threatening complication of cholecystitis with or without stones and associated with increased rate of mortality and morbidity due to late presentation and diagnosis.Niemeier classified gallbladder perforation into three types.

Objectives: To discuss the laparoscopic management of gall bladder perforation in emergency patients at tertiary care centre in government setup.

Method: Patients diagnosed as gall bladder perforation type $1 \& 2$ were included in the study.

Results: 13 patients ( 5 males, 8 females) with an average age of $58 \pm 2$ years had Gall bladder perforation. Patients with Gall bladder perforation type 1,2 were 4 and 9 . All patients were subjected to surgery via laparoscopic approach with conversion to open in 1 case. Gallbladder perforation was located on the fundus in 8 cases and body in 4 cases and 1 at hartman's pouch. The average duration of stay in patients with laparoscopic surgery was 4 days while it was $8-10$ days in case of open surgery. The overall mortality rate was $7.7 \%$ ( 1 cases). Complications included wound infection in 4 cases

Conclusion:The laparoscopic approach can be a safe and feasible method in order to treat both the cause and the complication in this situation. Early diagnosis and appropriate minimally invasive approach are the key to manage this condition.

\section{P204}

\section{An Unusual Presentation of Acute Cholecystitis: Gallbladder Volvulus}

Elizabeth E Price, DO, Luciano Dimarco, DO; UPMC Pinnacle Community Osteopathic Hospital

Gallbladder volvulus is an uncommon disease caused by torsion of the gallbladder around its mesentery along the axis of the cystic duct and artery, ultimately leading to gallbladder ischemia and obstruction of biliary drainage. The etiology of the condition is unknown; however, hypothesized contributory factors include anatomical abnormalities including a long gallbladder mesentery allowing it to float freely from the liver bed and easily twist upon itself. Volvulus can be seen more commonly in older females and is likely due to loss of visceral fat with aging. This diagnosis was first discovered in 1898 by an American surgeon Wendel, who referred to the condition as "floating gallbladder." Gallbladder volvulus only accounts for 1 out of 365,000 cases of gallbladder disease. Thus, although rare, laparoscopic cholecystectomies are one of the most common surgical procedures performed in the United States and it is important for surgeons to familiarize themselves with the condition due to its severe complications.

Here, we present a case of a 77-year-old female who presented for right upper quadrant pain, weight loss and nausea associated with eating. CT scan and ultrasound demonstrated a distended hydropic-appearing gallbladder with pericholecystic fluid and wall thickening without evidence of gallstones. Upon laparoscopic inspection, the gallbladder was necrotic, gangrenous and mostly detached from the liver bed as a result of twisting upon its mesenteric stalk. The mesentery was unable to be detorsed because the gallbladder was enlarged and necrotic in appearance with only its neck clearly identifiable. A laparoscopic subtotal reconstituting cholecystectomy was able to be performed without postoperative complication.

In conclusion, gallbladder volvulus is a rare presentation of acute right upper quadrant abdominal pain. The patient's clinical presentation and imaging can mimic those seen in patients with biliary colic or acute cholecystitis. Pre-operative diagnosis is rare; however, a high suspicion should prompt urgent surgical intervention in order to prevent necrosis and possible perforation. Ideally, the surgeon should attempt to return the gallbladder to its anatomic position. However, due to the severe distension and the necrotic presentation usually seen with this disease, obtaining the critical view of safety can present as an intraoperative challenge. Intraoperative decision making plays a crucial role in this condition in order to proceed with the procedure safely and without increasing morbidity and mortality. 


\section{P206}

Trans-nasobiliary Cholangiography Guided Laparoscopic Cholecystectomy After ERCP

Alaa Sewefy, Dr; Minia university

Introduction: Endoscopic retrograde cholangiopancreatography (ERCP) and laparoscopic cholecystectomy (LC), up till now, is the most common management of gallstone combined with CBD stones. In difficult LC, intraoperative cholangiography (IOC) is recommended to identify the anatomy of the biliary tree. This study is a randomized clinical trial aimed to evaluate the routine insertion of nasobiliary (NB) catheter during ERCP to be used as a method for IOC during LC in difficult cases.

Methods and procedure: One hundred sixty patients had combined gallstone \& CBD stone underwent ERCP followed by LC in the same session. They divided into equal 2 groups: NB group, in which NB catheter was inserted after CBD clearance, Control group in which only CBD clearance was done. In NB group: tans-nasobiliary IOC was done during LC. At the end of the procedure trans-nasobiliay methylene blue test was done to detect any hidden biliary injury.

Result: Eighty seven patients (54.4\%) were male and 73 (45.6\%) were female. Median age was 56 . The average operative time in NB group was $120 \mathrm{~min}$. VS. $129 \mathrm{~min}$. in Control group $(\mathrm{P}=0.002)$. No conversion in NB group (0\%) VS. 6 cases $(7.5 \%)$ in Control group $(\mathrm{P}=0.013)$. One case of biliary leak $(1.3 \%)$ occurred in NB group, which discovered by intraoperative methylene blue test and was managed conservatively by leaving the MB tube in place till the postoperative trans-NB cholangiography revealed no leak, VS. 2 cases $(2.5 \%)$ in Control group $(\mathrm{P}=0.560)$, the 2 cases were discovered postoperatively and managed by hepaticojejenostomy. The average postoperative hospital stay was $1 \pm 0.2$ days in NB group VS. $1.6 \pm 3.3$ days in Control group $(\mathrm{P}=0.1)$.

Conclusion: Insertion of NB catheter during ERCP is simple and dynamic method for IOC. It decreases the conversion rate. It doesn't decrease the incidence of BDI but it can diagnose, minimize the severity and treat BDI with shorter operative time

Figure 1: Endoscopic picture of $N B$ tube

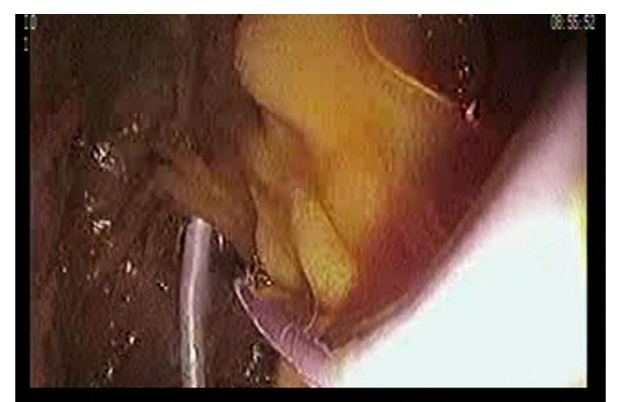

Figure 2: Trans- NB IOC with the supposed cystic duct is closed with empty clipper to make sure that it was not the CBD

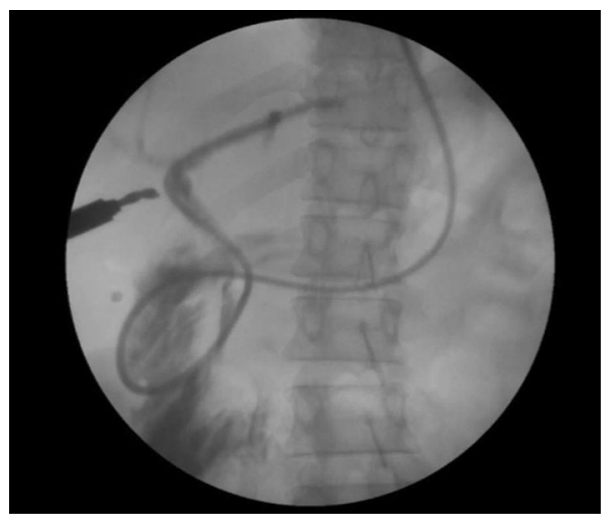

Figure 3: Intraoperative Trans-NB Methylene blue test with postive leak which was not discovered before the test

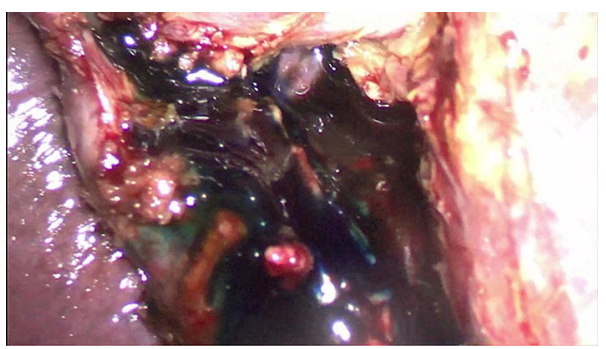




\section{Over Usage of ERCP Following Negative MRCP in Patients with Suspected Choledocholithiasis}

Damian Korsich, Swati Patel, Paula Veldhuis, Sebastian De La Fuente; Florida Hospital Orlando

Introduction: The ideal algorithm for evaluation of the biliary system remains contentious. Historically, patients with suspected choledocholithiasis were subject to intraoperative cholangiograms at the time of cholecystectomy but with the introduction of less invasive, albeit more expensive imaging studies, this approach has changed in recent years. Not uncommonly, patients with suspected choledocholithiasis undergo Magnetic Resonance Cholangiopancreatography (MRCP) to determine the presence of stones in the bile duct system. If choledocholithiasis is indeed found on MRCP, Endoscopic Retrograde Cholangiopancreatography (ERCP) is often recommended to clear the bile duct system preoperatively. In this study, we aim to determine the number of patients with suspected choledocholithiasis that despite a negative MRCP, were still subject to ERCP.

Methods: A retrospective review of medical records and administrative databases was used to include all consecutive patients suspected of gallstones, biliary obstructions and common bile duct filling defects between August 2010 to August 2017. Inclusion criteria included all patients who underwent MRCP for these indications. Subgroup analysis then included those that were subjected to ERCP.

Results: A total of 7,488 patients underwent MRCP for suspected common bile duct stones during the study period. Of these, 5,126 patients were found to have no stones on MRCP. Of the patients that did have stones on MRCP, 806 were also found subsequently to have stones on ERCP. There were 209 patients with stones on MRCP but were found not to have lithiasis on ERCP. Interestingly, a total number of 1178 patients underwent an ERCP despite a MRCP that had shown no evidence of choledocholithiasis.

Conclusion: A significant number of patients with suspected choledocholithiasis undergo MRCP at a single institution, in spite of evidence that shows that simple lab testing can often be sufficient. There are also a substantial number of patients with no radiologic evidence of choledocholithiasis that are subject to unnecessary invasive procedures.

\section{P208}

\section{The Outcome of Laparoscopic Cholecystectomy in Situs Inversus Totalis: Technique and Anatomical Variation. Case Report}

Omar A Ibrahim, MD, Surgical Resident, Tahir I Yunus, General Surgery and Bariatric Consultant, Ahmed M Mai, MD, Surgical Resident; International Medical Center

Situs inversus totalis (SIT) is an rare medical condition where thoracic and abdominal organs are in the mirror-image reversal of their natural position. Many difficulties faces surgeons to diagnose and manage chronic cholecystitis via laparoscopic cholecystectomy in patient with SIT. This is because the changes in the anatomical positions of organs influences the location of signs and symptoms of the diseased organ in addition to the special demands on the diagnostic and surgical skills of the surgeons. The current case report illustrates one of these cases in term of diagnosis and management.

Case presentation: A 32 years old female came to the clinic with chronic cholecystitis and multiple gallstones, in addition to dextrocardia on chest radiograph. The CT scan demonstrated SIT, cholecystitis and cholelithiasis. Laparoscopic cholecystectomy was conducted using mirror image port placement of conventional laparoscopic cholecystectomy. A right-handed surgeon conducted the operation, with reoriented visual images and the surgical steps were performed in clock wise rotation manner.

Conclusion: Chronic cholecystitis in SIT patient is considered to be a rare condition. Several precautions should be taken for proper diagnosis and adequate laparoscopic cholecystectomy procedures. Mirror image techniques, using right-handed surgeon, reoriented visual images and performing in a clock wise rotation were essential steps for the surgery success.

\section{Single Incision Laparoscopic Cholecystectomy (SILC) for Emergency and Elective Cases: Eight-year Experience in More Than 250 Patients}

Fernando Arias, MD, FACS ${ }^{1}$, Gabriel Herrera, MD ${ }^{1}$, Camilo Cetares, $\mathrm{MD}^{2}$, Manuel Arrieta, $\mathrm{MD}^{3} ;{ }^{1}$ University Hospital Fundación Santa Fe de Bogota - University of the Andes, Bogotá Colombia, ${ }^{2}$ University Hospital Fundacion Santa Fe de Bogota, ${ }^{3}$ University of Sabana, Bogota, Colombia

Introduction: Single incision laparoscopic cholecystectomy has gained increasing attention ove conventional technique due to the potential benefits such as less postoperative pain, less length of hospital stay and more patient satisfaction regarding post-operative scar and cosmesis. However, it is a technically challenging procedure with an apparent increased risk of bile duct injury. Methods: We review medical records from July 2008 to December 2016 of patients who underwent single incision laparoscopic cholecystectomy performed by our group. Our team used SILS port, GelPort, Triport and hybrid port (Alexis + glove) to perform the procedure. The following data was collected: patient's demographics, indications for surgery, single port devised used, operative time, conversions, postoperative complications, length of hospital stay and mortality. Results: A total of 273 patients underwent SILC from the registry. The median age was 44.2 years with a mean BMI of 24.1. The mean surgical time was $72 \mathrm{~min}$; we exclude 3 patients that additionally underwent to exploration of the common bile duct. There was no mortality in hospital and no cases of 30-day-mortality. One procedure was converted to multi-port and none to open surgery. A total of 7 postoperative minor complications with no bile duct injuries were reported. The mean length of hospital stay was $14.86 \mathrm{~h}$. Finally, there were $133(48.7 \%)$ emergent and 140 (51.3\%) elective cases.

Conclusions: SILC is a safe and a feasible alternative to standard laparoscopic cholecystectomy even in emergency cases. Our series show similar outcomes and complication rates compared to cases reported in literature about standard multiport cholecystectomy. Finally, experience of the group is important to obtain appropriate results.

\section{Gallbladder Perforation with Hemoperitoneum Secondary to Ulceration from Densely Packed Gallstones in a Minimally Symptomatic Patient}

Elizabeth A Verrico, DO ${ }^{1}$, Lindsay Tse, $\mathrm{DO}^{2}$, Justin Sargent, $\mathrm{DO}^{1}$, Fred Wolodiger, $\mathrm{MD}^{3}$, Steven Shikiar, $\mathrm{MD}^{1}$; ${ }^{1}$ Hackensack Meridian Health Palisades Medical Center, ${ }^{2}$ Houston Methodist Hospital, ${ }^{3}$ Englewood Hospital and Medical Center

Acute cholecystitis is one of the most common general surgery pathologies. Perforated cholecystitis is a rare potentially life threatening complication of acute cholecystitis. It is often described as acute perforation with acute peritonitis, subacute perforation with abscess and localized peritonitis, or chronic perforation with cholecystoenteric fistulation. Rare cases of spontaneous perforation have been reported but almost all cases are associated with acute inflammation.

This is a case of a 51 year old female who presented with mild non-radiating right upper quadrant and epigastric abdominal pain without associated nausea, vomiting, or fever. She denied similar symptoms in the past and her past medical history was only significant for hypertension and migraine headaches. She had no prior history of abdominal surgery. Physical exam revealed tenderness in the right upper quadrant without peritoneal signs. Labs were unremarkable except for mild leukocytosis. Ultrasound revealed a gallbladder filled with stones without pericholecystic fluid or gallbladder wall thickening. Due to persistence of symptoms the patient was taken to the operating room for laparoscopic cholecystectomy. Upon entry into the peritoneal cavity, hemoperitoneum was noted, particularly in the right paracolic gutter and perihepatic spaces. Upon further inspection and dissection, a gall bladder ulceration and perforation was noted with bleeding of the ulcerated edges and multiple stones visible. Successful laparoscopic cholecystectomy was performed with evacuation of the hemoperitoneum and collection of loose gallstones. Post-operative course was uneventful. Pathology revealed a $2.3 \mathrm{~cm}$ jagged hemorrhagic ulceratation/ perforation of the gall bladder with multiple stones causing indentations in the mucosa with moderate acute and chronic cholecystitis.

Perforated cholecystitis is uncommon. Presentation varies and can be indistinguishable from uncomplicated acute cholecystitis or can present with peritonitis, sepsis, and hemodynamic instability. This case demonstrates a very unique presentation of gall bladder perforation. The patient was minimally symptomatic without definite signs or symptoms of acute cholecystitis There was minimal to no bile leakage from the gall bladder but there was hemoperitoneum and hemorrhage from the ulcerated edges of the perforation. Consideration of perforated cholecystitis is important on the differential diagnosis when treating patients presenting with right upper quadrant abdominal pain. There is relatively high morbidity and mortality from delay in diagnosis and high index of suspicion is required. Treatment varies depending on presentation. 


\section{P211}

\section{Recurrent Right Upper Quadrant Pain and Complications of Progression}

Zachary Walker, Kejal Shah, MD, Michael P Meara, MD, MBA, FACS; The Ohio State University Wexner Medical Center

Background: Complicated Cholelithiasis can result in episodes of cholangitis and Mirizzi syndrome, extrinsic compression of the common hepatic duct by a stone in the cystic duct or gallbladder. Endoscopic retrograde cholangiopancreatography (ERCP) can be used for diagnosis and stent placement to temporarily treat Mirizzi syndrome. Cholecystectomy remains the definitive treatment for Mirizzi syndrome. Chronic inflammation in Mirrizi syndrome can result in fibrosis of surrounding tissues complicating resection and increasing risk of iatrogenic bile duct injury and intraperitoneal bleeding. The subject of this case study presented with extensive fibrosis extending O.R time.

Case Presentation: A 35-year-old Caucasian female presented to the emergency department jaundiced with right upper quadrant pain and emesis for three days. Her vital signs were within normal limits. Her history was significant for the previous diagnosis of cholelithiasis with subsequent common bile duct (CBD) stent placement two years prior. Ultrasound lead to the diagnosis of choledocholithiasis with common bile duct dilation to $11 \mathrm{~mm}$ on date of admission. An ERCP was performed on the day of admission with the removal and replacement of the previous stent. ERCP lead to suspicion of Mirizzi syndrome due to apparent hepatic duct filling defect which was confirmed on MRI. Three weeks later a robotic cholecystectomy was performed with an operative time of two hours and fifteen minutes. One week postoperative symptoms resolved with no complications.

Conclusions: Mirizzi syndrome can cause repeated inflammation of the cystic walls and subsequent fibrosis may cause adherence to nearby structures $(1,7)$, which complicated this procedure. Avoiding extensive fibrosis and inflammation with appropriately timed surgery in calculous cholecystitis may decrease complications.

Keywords: Mirizzi Syndrome, Choledocholithiasis, robotic cholecystectomy

\section{$\mathbf{P 2 1 4}$}

Primary Closure of Common Bile Duct with Biliary Stents

Vladimir Grubnik, MD, Professor ${ }^{1}$, Viktor Grubnyk ${ }^{1}$, Vadim Ilyashenko, $\mathrm{PhD}^{1}$, Alexander Kovalchuk, MD, Professor ${ }^{2} ;{ }^{1}$ Odessa National Medical University, ${ }^{2}$ Ternopil State Medical University

Introduction: Laparoscopic common bile duct exploration (LCBDE) for choledocholithiasis is a popular option in many surgical institutes. Decompression of biliary system via T-tube post supraduodenal choledochotomy has been the traditional surgical practice. Primary closure of common bile duct (CBD) has been shown to reduce hospital stay but bears a risk of bile leak. We conducted a prospective randomized trial to compare complications and length of stay in patients undergoing biliary stent insertion versus T-tube drainage following LCBDE via choledochotomy.

Methods and Procedures: The study involves 65 patients with choledocholithiasis who underwen LCBDE and decompression of the biliary system by either antegrade biliary stent or T-tube insertion. A 7 French biliary stent (9-10 cm long) have been placed in 33 patients (group I), T-tube insertion has been used for 32 patients (group II). The length of hospital stay and complications were recorded. All transcystic explorations were excluded.

Results: There were no signi?cant differences between groups with respect to age, sex, comor bidities, number and size of CBD stones. Postoperative complications have been observed in 4 patients $(12.5 \%)$ in the T-tube group (two patients needed reoperation: 1 patient for dislocation of T-tube, another one for biliary peritonitis due to extraction of T-tube 6 weeks after operation). And 1 patient $(3.0 \%)$ in the biliary stent group $(\mathrm{p}<0.05)$. The mean postoperative hospital stay was $3.4 \pm 1.3$ days for group I, and $6.5 \pm 1.6$ days for group II $(\mathrm{p}<0.05)$

Conclusions: Our results showed a reduction of length of hospital stay and morbidity following stent insertion compared to T-tube drainage. Also, the use of biliary stent after LCBDE can reduce costs and increase patient satisfaction.
P212

\section{Cholelithiasis in Sickle Cell Disease}

Prachi Mahajan, MS, FRCS, FMAS, FIAGES; Mahajan ortho and surgical hospital

Vidarbha region of Central India hosts a large population suffering from sickle cell disease (SCD). Patients may be divided into 2 categories depending upon whether they harbour the sickle cell trai ' SA' pattern or 'SS' haemoglobinopathy. $25 \%$ of people suffering from either condition have gallstones due to haemolysis owing to polymerization of the $\mathrm{HbS}$ within the Red Blood Cells and sequestration of RBC's in the spleen. This leads to the formation of pigment stones in the gall bladder.

Most of the patients have symptoms such as chronic right upper quadrant with intermittent pain acute exacerbations. Patients may be jaundiced due to the chronic haemolysis and also have bouts of obstructive jaundice from calculi that may intermittently slip into the common bile duct unless treated.

Materials and Methods: 70 patients with cholelithiasis were studied over 5 years. 42 patients had sickle cell trait while 28 had 'SS' disease. The age range was between $15-40$ years, with a mean age of 24 years. All the patients had symptoms of chronic cholecystitis. Mean bilirubin was $4.8 \mathrm{mg} / \mathrm{DL}$ Laparoscopy revealed characteristic features of chronic cholecystitis in all patients. The gall bladders were small, shrunken, thick walled and contained multiple pigmented calculi.

The technical difficulties were as follows:

1) Very small size of gall bladder in all (100\%) patients.

2) Chronic adhesions in the Calot's triangle in $75 \%$ of patients.

3) Thickened short contracted cystic duct in $90 \%$ of patients.

4) Impacted stones at the neck, causing difficulty in dissection (20\%)

5) Intrahepatic gall bladder (25\%) Results: Subtotal cholecystectomy had to be performed in 3 cases due to severe adhesions in the Calot's triangle.

Post operative complications: Sickle cell crisis- acute chest syndrome, joint painsin 3 cases Extended hospital stay of over 5 days in 15 patients due to SCD related complications. Port infection in 4 patients. Mortality in 1 patient due to sickle cell crisis.

Conclusion:

1) Laparoscopic cholecystectomy is technically difficult in patients with sickle cell conditions due to chronic inflammatory changes in the area.

2) Meticulous pre and post operative care is mandatory with adequate fluid infusions, good oxygenation, maintenance of OR temperature above 25 degrees Celsius, pre operative folic acid, sodium bicarbonate, hydroxyurea to prevent hypoxaemia, dehydration and hypothermia.

\section{$\mathbf{P 2 1 5}$}

Results in the Endoscopic Management of Giant Common Bile Duct Stones: Stent and Pneumatic Dilatation

Carlos Leal, MD, General Surgery, Gastroenterology ${ }^{1}$, Andres Mendoza, MD, General Surgery ${ }^{1}$, Luis Cabrera, MD, General Surgery $^{1}$, Mauricio Pedraza ${ }^{1}$, Sebastian Sanchez ${ }^{1}$, Jean Pulido ${ }^{1}$, Daniel Gomez, MD, FACS ${ }^{2} ;{ }^{1}$ Bosque University, ${ }^{2} \mathrm{CPO}$

Background: The endoscopic management of choledocholithiasis remains a challenge for the general surgeon due to the complications that can arise from it. Traditional management has been surgical, but in recent years endoscopic management has had great relevance due to the high rate of effectiveness and for demonstrating lower morbidity and mortality rates.

Materials and Methods: We describe our experience at a third level surgical center of patients taken to Endoscopic Retrograde Cholangiopancreatography (ERCP), endoscopic balloon dilation and placement of biliary prosthesis (stent), during January 2016 to July 2017, for the management of giant common bile duct stones.

Results: A total of 11 cases completed the inclusion criteria. Eight (72.7\%) patients corresponded to the female gender. Patient age averaged 62.3 years. A resolution frequency of giant common bile duct stones was found with this endoscopic procedure in $90 \%$, without any reported complications. A decrease in the size of the stones, with a range of 2-3 procedures per patient.

Conclusion: The endoscopic procedure with ERCP dilatations of the bile duct and stent placement proves to be effective in the management of giant common bile duct stones with low complication rates.

Key Words: Common bile duct stones, stent, pneumatic dilatation 
P216

\section{Endoscopic Management of the Difficult Hepatic Biliary Duct} Stone with Digital Direct Cholangiography

Renzo Pinto, Efrain J Isaacs, Laura Quintero, Luis F Cabrera, General Surgeon, Daniel Gomez, MD, FACS, Mauricio Pedraza, Jean Pulido; Bosque University

Introduction:Digital direct cholangiography or Spyglass (Boston Scientific Corp., Natick, MA, USA) is a modern and simplified technique, which allows a single endoscopist a better visualization of the biliary tract and equipment maneuverability. Using this unique technique, it is possible to fragment and extract bile stones using a mechanical lithotripter or a pulsed dye laser, with a successful rate greater than $90 \%$

Objective: To describe the utility of the direct cholangioscopy and laser lithotripsy in the management of the difficult common bile duct stones, in two patients with biliary stenosis Materials and Methods: 2 patients were treated in the Bogota, Colombia, between the years of 2015 and 2018 in a fourth level hospital, who presented with distal biliary stenosis and choledocolitiasis. This patients also had difficult bile duct stones criteria, which is the reason why direct cholangioscopy and laser lithotripsy was indicated, with $100 \%$ success and no complications.

Results: The 2 cases had complete resolution of hepatolithiasis, being able to overcome the distal stenosis and preventing new episodes of cholangitis.

Conclusions: Digital direct cholangiography or Spyglass, is an endoscopic technique which allows direct vision of the biliary tract and the bile ducts. Using this technique, it is possible to fragmentate all the bile stones and overcome bile duct stenosis, prevent cholangitis and spare the patient of a possible hepatectomy

\section{P218}

Intraoperative Fluoroscopic-guided Balloon Choledocholithotomy and Laparoscopic Cholecystectomy : A New Technique for the Treatment of Choledocholithiasis

Yasunobu Kobayashi, $\mathrm{MD}^{1}$, Hironori Ohdaira, $\mathrm{MDPhD}^{1}$, Mutsumi ${ }_{\text {Kaji, MD }}^{1}$, Norihiko Suzuki, MD ${ }^{1}$, Satoshi Narihiro, MD ${ }^{1}$, Taigo Hata, $\mathrm{MD}^{1}$, Soujun Hoshimoto, MDPhD ${ }^{1}$, Masashi Yoshida, $\mathrm{MDPhD}^{1}$, Jun Horiguchi, MDPhD ${ }^{1}$, Eigoro Yamanouchi, MDPhD ${ }^{2}$, Masaki Kitajima, MDPhD ${ }^{1}$, Yutaka Suzuki, MDPhD ${ }^{1} ;{ }^{1}$ Dept of Surgery, International University of Health and Welfare, ${ }^{2}$ Dept of Radiology, International University of Health and Welfare

Background: Patients with choledocholithiasis often are undergone preoperative endoscopic retrograde cholangiography (ERCP) following laparoscopic cholecystectomy (LC) However, ERCP has risks of acute pancreatitis, bleeding, and perforation. Consequently, there might be unexpected interval between ERCP and LC.

Objective: We report on a single-stage operation (LC + fluoroscopic-guided balloon choledocholithotomy) as a new technique of treatment for choledocholithiasis.

Patients: From April 2015 to August 2018, 15 patients with choledocholithiasis (male: female $=6: 9$, median 75 (range, 62 - 91) years old) ) were enrolled.

Methods: Calot's triangle were dissected and exposed with the same procedure as conventional LC. Cystic duct was incised, the sheath of angiography catheter percutaneously was placed through right upper quadrant, and cannulated to cystic duct. Guide wire was inserted with fluoroscopy, and cannulated the duodenum beyond the Vater papilla. Existence of choledocholithiasis was confirmed, Vater papilla was dilated with balloon Subsequently, the balloon was expanded upstream of the choledocholithiasis and stones ware removed by the extrusion method. Common bile duct was imaged again to confirm that there was no remaining stone. Finally temporally plastic stent was placed.

Results: Operation was completed in all 15 patients, and in all cases stones could be removed. Median operative time was $139 \mathrm{~min}$ (range, 87-341 $\mathrm{min}$ ) and median fluoroscopic time of stone removal was $45 \mathrm{~min}$ (range, 7-120 min). Median postoperative hospital stay was 3 days (range, 2-11 days). No complications related to surgery were observed in all cases, and it was confirmed that there was no residual stone in the biliary tract by MRCP one month after the operation.

Conclusion: $\mathrm{LC}+$ fluoroscopic stone removal technique is safe and reliable and can be one of new treatments for choledocholithiasis.

\section{P219}

\section{Clinical Differences of Young Population Underwent Laparoscopic Cholecystectomy}

Yoo Shin Choi, Suk Won Suh, MD, Seung Eun Lee; Chung-Ang University

Introduction: Laparoscopic cholecystectomy (LC) in young population is relatively uncommon, despite being one of the most common surgical procedures in adults. Although clinical characteristic of adult patients with gallbladder (GB) disease is well established, scanty information have been for youth. In the present study, we aimed to comprehensively review the young population underwent LC compared to older population.

Methods: A total 2,115 patients who received LC for GB stones were retrospectively analyzed. The patients were categorized into two clinical groups according to the age of patients: (young $(<24)$ group and the elder group). We compared two groups according to its clinical characteristics.

Results: In univariate analysis, significant factors between two groups were found in the concomitant of choledocholithiasis and American Society of Anesthesiologists score I/II. By multivariate analysis, the concomitant of choledocholithiasis (OR 1.152, 95\% CI, 0.663 $-2.001, p<0.001$ ) were independent factors between young group and the elder group. Conclusions: In our study, young population with gallstone disease had more prevalence of choledocholithiasis. Therefore, young patients with gallstone disease require special attention for choledocholithiasis. 
P220

\section{Hyperkinetic Gallbladder: An Indication for Cholecystectomy?}

Baongoc Nasri, MD, $\mathrm{PhD}^{1}$, Brian Gilchrist, $\mathrm{MD}^{2}$, Timothy Glass, $\mathrm{MD}^{1}$, Jonathan Saxe, $\mathrm{MD}^{1} ;{ }^{1}$ St. Vincent Hospital, ${ }^{2} \mathrm{New}$ York University Winthrop Hospital

Background: The main indications for laparoscopic cholecystectomy are stone related diseases in adults. With a normal abdominal ultrasound (US), a hepatobiliary iminodiacetic acid (HIDA) scan with ejection fraction (EF) is recommended to evaluate gallbladder function. Biliary dyskinesia or low gallbladder ejection fraction $(\mathrm{GBEF}<35 \%)$ is a recognized indication for cholecystectomy in children. However, not only do patients have low ejection fractions, but a significant number have hyperactive ejection fractions with GBEF $>80 \%$. There are multiple articles showing long term resolution of symptoms in children with hyperkinetic ejection fractions on HIDA scan. The purpose of this study is to evaluate whether hyperkinetic gallbladder (GBEF $>80 \%$ ) could be an indication for cholecystectomy in adult population.

Methods: Data were consecutively collected from all patient underwent laparoscopic cholecystectomies between June 2012 and June 2017 at a single institution. Patients less than 18 year of age or missing data were excluded. Patients with a negative US (no stone, no sludge, no gallbladder wall thickening) and GBEF greater than $80 \%$ were included in this study.

Results: Over a five-year period from June 2012 until June 2017, 36 patients were identified to fit the inclusion criteria for this study. There were 33 women and 3 men with a mean age of $52 \pm 13.87$ years, all had an GBEF greater than $80 \%$ with a mean of $89 \pm 4.45 \%$. Mean BMI was $30 \mathrm{~kg} / \mathrm{m} 2$ (range, 14- 48.8) 3 patients $(8.3 \%)$ had daily bouts of nausea or emesis. All patients had biliary colicky pain for more than one month. Patients had undergone several additional studies: endoscopy (23), colonoscopy (6), gastric emptying study (6), CT scan (9). Pathology reports were reviewed: only 3 $(8.3 \%)$ patients had a normal gallbladder, $27(75 \%)$ had chronic cholecystitis, and $6(16.7 \%)$ had chronic cholecystitis with cholesterolosis. All patients were seen in follow up 4 to 6 weeks postoperatively. 33 had complete resolution of symptoms, two had partial resolution and one had no change. There was a complete resolution rate of $91.7 \%$ and an improvement rate of $97.2 \%$.

Conclusions: Long term resolution of symptoms in children with hyperkinetic ejection fractions on HIDA scan have been documented. In this series of adult patients who presented with biliary symptoms, negative ultrasound and elevated GBEF on HIDA scan (EF $>80 \%$ ), laparoscopic cholecystectomy led to significant rate of symptomatic relief. This disease process requires further analysis, but this could represent a new indication for laparoscopic cholecystectomy in the adult population.

\section{P221}

Laparoscopic Cholecystectomy: A Review of Operative Timing and Complications

Chetna Bakshi, MD, Gainosuke Sugiyama, MD, FACS, Charles Choy, MD, FACS, Gene Coppa, MD, FACS, Antonio Alfonso, MD, FACS, Paul Chung, MD; Zucker School of Medicine at Hofstra/ Northwell

Background: Laparoscopic cholecystectomy is one of the most common operations performed in general surgery in the United States. It has been suggested that increased operative time (OT) is correlated with increased risk of complications in laparoscopic surgeries across various surgical fields ${ }^{1,2,3}$.

Objective: To determine if increased operative time is associated with increased risk of complications in laparoscopic cholecystectomy. Methods: Using ACS NSQIP from 2006-2015, we identified all adult ( $\geq 18$ years) patients that underwent an emergent laparoscopic cholecystectomy with a postoperative diagnosis of cholecystitis performed within 3 days of admission, by a general surgeon with a wound classification of clean/contaminated or contaminated. We excluded cases with preoperative SIRS/sepsis, ASA class IV or V, and cases that had additional procedures listed. We also limited our analysis to cases with OT $\geq 15 \mathrm{~min}$ and $\leq 360 \mathrm{~min}$. Risk variables included age, sex, race, morbid obesity (BMI $\geq 40 \mathrm{~kg} / \mathrm{m} 2$ ), functional status, ASA class, and operative time. Outcome variables included postoperative superficial surgical site infection (SSI), deep SSI, organ-space SSI, dehiscence, pneumonia, reintubation, failure to wean from ventilator, pulmonary embolism, renal failure, urinary tract infection, cardiac arrest, myocardial infarct, bleeding, deep vein thrombosis, sepsis, septic shock, return to the operating room, and death. Multivariable logistic regression was performed adjusting for all risk variables. Postoperative length of stay (LOS) was analyzed using negative binomial regression adjusting for all risk variables.

Results: A total of 7,031 cases met inclusion criteria, of which the majority were women $(71.5 \%)$, Caucasian $(80.0 \%)$, with a mean age of 46.1 years. Median OT was $63 \mathrm{~min}$, first quartile was $46 \mathrm{~min}$ and third quartile was $87 \mathrm{~min}$. Logistic regression analysis showed that increased OT (third vs first quartile) was an independent risk factor for superficial SSI (OR 1.75, 95\% CI 1.36-2.25, p < 0.0001), organspace SSI (OR 1.77, 95\% CI 1.33-2.35, $\mathrm{p}<0.0001$ ), dehiscence (OR $2.03,95 \%$ CI 1.01-4.07, $\mathrm{p}=0.0470)$, septic shock (OR 1.81, 95\% CI $1.06-3.09, \mathrm{p}=0.0286$ ). Increased OT was independently associated with increased LOS (fourth vs 1st quartile: IRR 1.53, p $<0.0001$; third vs 1st quartile: IRR $1.29, \mathrm{p}<0.0001 ; 2$ nd vs 1 st quartile: IRR $1.16, \mathrm{p}<0.0001$ ).

Conclusion: In this large observational study we found that increased OT is independently associated with morbidity and increased postoperative LOS following laparoscopic cholecystectomy for cholecystitis. Prospective studies are warranted to determine whether increased OT from resident training is a contributing factor. 


\section{P222}

Laparoscopic Completion Cholecystectomy for Left out Gall Bladder Remnant and Stump: Technique and Results of a Series of 52 Cases

\section{Subhash Khanna, PROF; Swagat Super Speciality Surgical Institute}

Background: Laparoscopic cholecystectomy is presently the standard of care for most cases of calculus cholecystitis. Although it is practiced and being routinely done in both acute and chronically inflammed gall bladders, but many a times a surgeon knowingly or inadvertently ends up leaving a large stump of gall bladder and cystic duct that may harbor a calculus.

Such large stump may be the cause of persistent pain and may necessitate removal of the stump or may at times be a formal completion cholecystectomy.

In a span of nearly 20 years we encountered 52 such cases of left out stump and post partial cholecystectomy gall bladder remnant.

We are presenting the technique and our results of laparoscopic excision in these cases.

The Technique: Of the 52 cases, 35 were post conventional cholecystectomy having a scar (sub costal in all 34 except one right paramedian) and rest 17 were after laparoscopic cholecystectomy. A modified open technique was used to create pneumoperitoneum. In most of the post open cholecystectomy cases gross omental adhesions were seen to the scar and the falciform ligament.

The second port was made in the left midclavicular line to the left of umbilicus. The first step was to remove adherent falciform and omental adhesions from the previous laparotomy scar, followed by separating the hepatic adhesions from diaphragm. At this stage the third and mid clavicular port was made followed by right anterior axillary port. Finally with gentle blunt dissection the omental adhesions were lysed from the gall bladder fossa and the stump exposed. The stump varied in size from $1 \mathrm{~cm}$ to $5 \mathrm{~cm}$ and many a times clips were seen on the wall of the left out gall bladder. Complete removal of gall bladder was successful in 51 out of 52 cases with no major morbidity.

Discussion: Although a simple technique Laparoscopic cholecystectomy at times ends up with unwanted complications. One of the rare causes of post laparoscopic cholecystectomy pain is the left out stone in the cystic duct remnant or the gall bladder remnant. We present our series of 52 cases of excision of left out gall bladder and stump in a span of last 20 years. All such cases can be done laparoscopically. Usually the cystic duct and artery can be dissected in most of these cases. Laparoscopy should be the standard technique for all such cases of incomplete removal of gall bladder.

\section{$\mathbf{P 2 2 3}$}

\section{Determinant of Quality of Life After Laparoscopic Cholecystectomy}

Heba A Elghalban, MD ${ }^{1}$, Nagwa Nashat, MD ${ }^{1}$, Rami R Mustafa, $\mathrm{MD}^{2}$, Hatem Soltan, $\mathrm{MD}^{3}$, Hala Shaheen, $\mathrm{MD}^{1}$, Taghreed Farahat, $\mathrm{MD}^{1}$; ${ }^{1}$ Family Medicine Department, Faculty of Medicine, Menoufia University, ${ }^{2}$ Department of surgery, Cleveland Medical Center, University Hosptals, ${ }^{3}$ Department of surgery, Faculty of medicne, Menoufia University

Introduction: cholecystitis is a striking public health issue with high socioeconomic impact. About $10 \%-15 \%$ of the general population have gallstone, with a high risk for morbidity or even mortality. Risk factor for cholecystitis include increasing age, female sex, certain ethnic groups, obesity or rapid weight loss, certain diet, drugs and pregnancy. Gallstone disease is the second most costly digestive disease and the single most common indication for abdominal surgery; as more than $75 \%$ of patients who suffer from cholelithiasis combined with chronic cholecystitis undergo laparoscopic cholecystectomy (LC) surgery. The measurement of quality of life (QOL) issues make it possible to obtain standardized information about a patient perception of the specific disease and its impact on his or her life. Quality of life (QOL) is a critical consideration when evaluating treatment options for cholelithiasis. Therefore, understanding the postoperative physical, psychological, and social outcomes associated with cholecystectomy is essential. few studies have assessed the role of socioeconomic characteristics on outcomes after a cholecystectomy.

Methods: a cohort study that was conducted through an entire year (December 2016-december 2017); in Menoufia University hospital as all patient prepared for laparoscopic cholecystectomy was offered to join the study, with exclusion of those who refuse to participate, having malignancy, patient with sever organic or psychiatric illness and contraindication for anesthesia. Preoperatively patients were interviewed and promoted to complete a pre-designed questionnaire that include full patient history (age, sex, history of present illness with its onset, duration and any associated gastroenterological symptoms. Past medical history of presence of comorbid conditions, previous operations and hospitalization. Patients also subjected to detailed general and local physical examination. Revision patient's investigation include U/S and some lab correlation when needed. Complete operative details collected. postoperatively all patients were interviewed for gastrointestinal quality-of-life index (GIQLI) after a month of the operation day; as part of the follow up process for the patients.

Results: among 200 patients enrolled in the study, $(76 \%$ were females), mean age $(43 \pm 2.65)$, with prevalence of $(64 \%)$ of low socioeconomic level. Although more female patients were enrolled in the study; male patients had lower score of (GQOLI). Compared to younger groups, patients older than 65 years old were the lowest in the (GQOLI) scores.

Conclusion: age and sex could be used as a predictor of quality of life after laparoscopic cholecystectomy.

Keywords: cholecystitis, gallstone, quality of life . 
$\mathbf{P 2 2 4}$

Incidence of Concomitant Vascular Injuries in Postcholecystectomy Benign Biliary Strictures and Impact on Longterm Outcomes

Saurabh Galodha, MSMCh ${ }^{1}$, Rajan Saxena, MS ${ }^{2}$, Rajneesh K Singh, $\mathrm{MSMCh}^{2}$, Ashok K Gupta, $\mathrm{MCh}^{2}$, Anu Behari, $\mathrm{MS}^{2}$, Vinay K Kapoor, $\mathrm{MS}^{2} ;{ }^{1}$ Indira Gandhi Medical College, Shimla, ${ }^{2}$ SGPGIMS, Lucknow

Objective: The impact of concomitant vascular injury on the longterm outcome of post- cholecystectomy benign biliary stricture (BBS) repair is controversial. In this study we tried to find out the incidence of a concomitant vascular biliary injury (CVBI) and their impact on the long-term outcomes.

Methods: All consecutive patients with BBS from December 2010 to May 2012 were included. Magnetic resonance angiography (MRA) with MRCP was done prior to repair. Long-term outcomes were analysed on basis of McDonald grading.

Results: 36 patients of BBS were included. Median age was 36 (15$70)$ years and $28(78 \%)$ were females. 10 patients $(28 \%)$ had prior failed repair. CVBI was present in $22(61 \%)$ and all had right hepatic artery (RHA) injury. Laparoscopic cholecystectomy was primary surgery in 18 patients with VBI (82\%) and in 5 patients without CVBI $(\mathrm{p}=0.016)$. Right portal vein injury was present in one patient. CVBI was noted in 9 patients with prior failed repair $(\mathrm{p}=0.027) .23$ (64\%)patients had high strictures (Bismuth Type $\geq 3$ ); 19 had CVBI $(\mathrm{p}<0.001) .34$ patients underwent Roux en Y hepaticojejunostomy (RYHJ). Mean operative time was $3.2 \pm 1.1 \mathrm{~h}$ and mean blood loss was $300 \pm 150 \mathrm{ml}$. Post-operative complications were present in 13 $(36 \%)$ patients but there was no perioperative mortality. At median follow up of 42 months $(24-60)$, there were 8 failures in CVBI group (Success $=64 \%$ ). Of these, 1 patient underwent right hepatectomy, 3 had percutaneous dilatation and 4 underwent revision RYHJ. In patients without CVBI only 1 patient had failure $(\mathrm{p}=0.07)$.

Conclusion: Concomitant vascular injuries have considerable impact on long-term outcomes of post-cholecystectomy BBS repair and are significantly associated with failed repairs. MR angiography is a good adjunct in the diagnostic armamentarium of BBS and can help in appropriate management of these patients.

\section{P226}

Endoscopic Transpapillary Stenting for the Management of Acute Cholecystitis

Danielle Hayes, MD, Gary Lucas, MD, Bryce French, MD, Andrew Discolo, MD, Rajnish Mishra, MD, Sean Wells, MD; Swedish Medical Center - First Hill

Introduction: Cholecystectomy is the gold standard treatment of acute cholecystitis. Patients with multiple comorbidities who are considered high-risk surgical candidates are commonly recommended to undergo percutaneous cholecystostomy tube placement; however, long-term external drainage is undesirable for many patients. Endoscopic transpapillary stent placement (ETSP) has been described as an alternative method for decompression of the gallbladder. The purpose of our study is to assess which patients would benefit from this treatment compared to traditional treatments of cholecystitis.

Methods: We performed a retrospective chart review of patients with cholecystitis who underwent ETSP at our institution between January 2015 and July 2018. This study was performed to identify indication, comorbidities, length of stay, labs, outcomes, additional procedures, and whether cholecystectomy was eventually performed.

Results: During the study period, 12 patients underwent ETSP. The mean age was 68.2 years ( \pm SD 12.4) with an average ASA class of 3.2. The Charlson Comorbidity Index was greater than seven in $75 \%$ of patients, indicating a zero percent 10-year survival. The NSQUIP surgical risk calculator estimated an average mortality risk for laparoscopic cholecystectomy of $4.8 \%( \pm 3.3,95 \% \mathrm{CI})$ in our study population; the estimated risk in the general population is $0.1 \%$. Resolution of symptoms with endoscopic drainage was achieved in 11 of 12 patients $(91.7 \%)$; one patient experienced no symptom resolution with endoscopic drainage nor subsequent percutaneous cholecystostomy tube placement. Six of 12 patients underwent interval cholecystectomy.

Adverse events occurred in four cases which consisted solely of postERCP stent migration or occlusion. Estimated time to stent occlusion or migration ranged from 20-400 days. Two patients died in the time of the study, one from sepsis in the setting of metastatic pancreatic cancer and the second from biliary sepsis after stent migration.

Conclusion: Endoscopic transpapillary stent placement is an effective and safe method for the temporary management of acute cholecystitis in high risk surgical patients. We recommend attempting ETSP as a temporizing measure for acute cholecystitis in high risk surgical patients who are undergoing ERCP for other diagnostic or therapeutic purposes and patients with anatomy that would make percutaneous cholecystostomy tube placement challenging. Randomized studies would be helpful to further investigate the utility and safety of ETSP in the management of acute cholecystitis.

Keywords: Endoscopic Transpapillary Stenting; Cholecystitis; Gallbladder Drainage; Percutaneous Cholecystostomy Tube; Cholecystectomy 


\section{P227}

\section{Sinistroposition of the Gallbladder}

David L Rhoiney, DO, Raymond Laird, DO; Beaumont HealthTrenton

Introduction: Sinistroposition of the gallbladder is a rare anomaly and has been reported to occur in approximately $0.3 \%$ of patients surgically treated for their gallbladder disease. Knowledge of this variation is paramount as the presentation of sinistroposition is not unlike traditional right-sided gallbladders and is often identified at the time of the operation.

Case Presentation: A 43-year-old woman with a history of gallstones and symptoms suspicious for acute cholecystitis was taken to the operating room for a laparoscopic cholecystectomy. Upon entrance into the abdomen and inspection of the liver, the gallbladder was observed to be present to the left of the falciform ligament and likely arising from segment III of the liver. An uneventful laparoscopic cholecystectomy was completed with some modification of technique and the patient did very well postoperatively.

Sinistroposition of the gallbladder is a rare anatomic variation, which is typically diagnosed upon intraoperative inspection of the liver bed during laparoscopic cholecystectomy. There are two anatomical variations of the presentation and the surgeon must be familiar with both in order to perform a laparoscopic cholecystectomy in a safe-manner.

Conclusion: Laparoscopic cholecystectomy can be performed safely without modification of technique provided the surgeon is aware of the anatomical variations which commonly occur with sinistroposition of the gallbladder.

\section{P228}

Efficacy of Pancreatic Enzyme Replacement Therapy in the Treatment of Pancreatic Exocrine Insufficiency Following Pancreatic Surgery, A Systematic Review

Alysha R Keehn, MD, MSc, candidate ${ }^{1}$, Stephen N Quigley, MD, FRCSC $^{2}$, Elijah Dixon, MD, MSc, FRCSC ${ }^{1}$; ${ }^{1}$ University of Calgary, Department of Surgery, ${ }^{2}$ Memorial University, Department of Surgery

Background: Mortality from pancreatic surgery has decreased over the years, however morbidity remains high. $45 \%$ of patients demonstrate pancreatic exocrine insufficiency prior to surgery with post-operative incidences ranging form $56-98 \%$. This contributes to poor quality of life, readmission to hospital and reduced survival.

Objectives: Few studies have reported on the efficacy of pancreatic enzyme replacement therapy following pancreatic surgery. The aim of this review is to systematically summarize the evidence to guide and inform practice.

Methods: A systematic review was conducted using Medline, Embase, Cochrane Central Register of Controlled Trials, CINAHL \& Google Scholar. Bibliographies of all included publications were cross-referenced. PRISMA guidelines were used to structure the review and evidence was appraised using the GRADE system.

Results: Eleven studies were included (4 prospective observational and 7 RCTs). Pancreatic enzyme replacement therapy was found to significantly reduce fecal fat excretion $(p<0.001)$ in 3 RCTs and significantly reduce stool frequency $(\mathrm{p}<0.001)$ in $1 \mathrm{RCT}$. It was found to significantly improve both the coefficient of fat absorption and of nitrogen absorption $(p<0.001,0.05$ respectively) in 2 RCT's. There is mixed evidence for whether PERT improves mean body weight and BMI. 3 RCT's showed an improvement in clinical symptomatology with PERT while 1 found no difference.

Conclusion: There is grade A evidence to support that PERT reduces fecal fat excretion and improves stool frequency, and fat/nitrogen absorption. There is mixed evidence (Grade C) that it has an effect on mean body weight and BMI. There is Grade A evidence to support a reduction in clinical symptoms. The data from this systematic review will be used in a subsequent meta-analysis. Key Words: Pancreatic exocrine insufficiency, pancreatic enzyme replacement therapy, pancrealipase, CREON $^{\circledR}$, pancreatic surgery, pancreaticoduodenectomy

\section{P229}

Feasibility of Two-port Laparoscopic Cholecystectomycompared to Standard Four-port Laparoscopic Cholecystectomy: Randomized Control Study

Ibrahim A Salama, MDPhD; Department of Surgery,National Liver Institutee, Menoufia University

Introduction: Two-port laparoscopic Cholecystectomy (LC) has been proposed as a safe and feasible technique. However, there are limited Studies to evaluate the effectiveness of the procedure. This is a prospective randomized control study to Compare the standard four-port LC with two-port L.C.

Materials and Methods: A total of 316 Consecutive patients undergoing LC were randomized to four-port (Group A)/two-port mini LC (Group B) from January 2013 to January 2018 In two-port LC, a 10-mm umbilical and a 5-mm left hypochondruim port were used with polyprolene thread was used for lateral -cephalic retraction of the fundus and another thread used for traction of the Hartman pouch if needed. Outcomes measured were duration and difficulty of Operation, post-operative pain, analgesia requirements, Post-operative stay, complications and cosmetic score at 30 days.

Results: Out of 316 patients, the ratio of $\mathrm{M}$ : F was 1:4, with mean age $40.79 \pm 12.6$ years.. The mean Operative time were similar $(\mathrm{P}=0.727)$. Addition 3rd port was used in seven cases. Post-operative pain was significantly low in the two-port group at up to $24 \mathrm{~h}(\mathrm{P}=0.023)$. The overall analgesia requirements $(\mathrm{P}=0.003)$ and return to daily activity $(\mathrm{P}=0.00)$ were Significantly lower in two-port group. The cosmesis score of the two-port group was better than four-port group $(\mathrm{P}=0.00)$. However, the length of hospital stay $(\mathrm{P}=0.760)$ and complications $(\mathrm{P}=0.247)$ were similar between the two groups.

Conclusion: Two -port LC result in reduced pain, need for analgesia, and improved cosmesis without increasing the operative time and complication rates compared to that in four-port LC. Thus, it can be recommended in selected patients 
P230

\section{Endoscopic Biliary Intervention Following Orthotopic Liver Transplantation}

Krishnaraj Mahendraraj, MD, Guillermo Medrano Del Rosal, MD, Michelle Kruse, BS; Lincoln Medical Center

Introduction: Biliary tract complications that require endoscopic decompression represent a greater challenge after liver transplantation than in non transplanted patients. Immunosuppressive therapy predisposes patients to a greater risk of infections and poor healing process. This comparative study analyzes risk factors and outcomes in liver transplant patients with biliary pathology that requires endoscopic intervention.

Methods: Information obtained from National Inpatient Sample database over a 15-year period (2001-2011). This included a total 10,252 patients who underwent liver transplantation, $581(6 \%)$ if these patients experienced biliary system complications. Univariate and multivariate was obtained via Statistical Package for the Social Sciences (SPSS). Statistical significance was accepted for p-value $<0.05$.

Results: A total of 581 patients underwent endoscopic retrograde cholangiopancreatography (ERCP) after hepatic transplantation, $80 \%$ of them had sphincterotomy and stent placement. These were mostly seen in men $(64.5 \%)$, caucasians $(69.8 \%)$ with a mean age of 52 years, however these results were similar to the no intervention group. These patients were diabetic with chronic complications $(4.9 \%$ vs $2.9 \%$ ) and had congestive heart failure (3.8\% vs $2.4 \%)$. Choledochoenterostomy was a protective factor for having biliary pathology requiring endoscopic intervention. Biliary complications that required intervention were; choledocholithiasis (18.8\% vs $9.5 \%)$, cholangitis ( $36.7 \%$ vs $7.5 \%$ ), biliary stricture ( $36.7 \%$ vs $3.8 \%$ ), biliary leak $(0.7 \%$ vs $0.1 \%)$. Length of stay $>1$ week was greater in the biliary intervention group $(98.3 \%$ vs $77.6 \%)$. Significant complications seen in patients requiring intervention were; sepsis $(23.2 \%$ vs $11.8 \%$ ), urinary tract infections $(12.6 \%$ vs $8.5 \%)$, acute renal failure $(39.9 \%$ vs $31.1 \%)$, peritonitis or intra abdominal abscess $(11.4 \%$ vs $6.0 \%$ ), wound infection $(11.7 \%$ vs $6.0 \%$ ) and acute rejection $(46.1 \%$ vs $18.4 \%$ ). In-hospital mortality was significantly higher for patients with biliary pathology requiring endoscopic intervention $(7.2 \%$ vs $5.3 \%)$.

Conclusion: Biliary system pathologies requiring endoscopic intervention had life threatening complications and thus mortality rate on the same admission. Interestingly, choledochoenterostomy decreased the risk of biliary tract pathology that required instrumentation.

\section{P231}

Less Invasive Single Port Laparoscopic Cholecystectomy with 2 Fine Needle Forceps

Junko Takita, MD, Norihiro Haga, MD, Norihiro Masuda, MD, Yuta Shibasaki, MD; NHO Utsunomiya National Hospital

Introduction: Single port surgery (SPS) has been reported to reduce the abdominal wall damages. To reduce the length of umbilical scar and to keep the triangulation, we use 2 additional fine needle forceps for laparoscopic cholecystectomy (LC).

Patients and Methods: From 2007 to May 2018, 597 consecutive LC patients were retrospectively investigated. There were 345 male and 252 female. Severe cholecystitis was observed in $30 \%$ of the cases. We use two $5 \mathrm{~mm}$ ports ( 1 for the scope and 1 for the operator's right hand forceps) through an umbilical multi-channel port and 2 additional $2.4 \mathrm{~mm}$ fine needle instruments are pierced. One of the forceps is placed on the right side of the lower end of sternum and the other is on the right side of abdomen. A $5 \mathrm{~mm}$ flexible scope allowed us to keep the triangular formation easily. We performed cholecystectomy by this plus two punctures SPS (PTP-SPS) for 464 patients. The rest of patients were operated by one $11 \mathrm{~mm}$ port, one $5 \mathrm{~mm}$ port and two fine needle forceps as conventional reduced port surgery (RP). We employed the RP method until 2011, however from 2012 to now, we perform cholecystectomy by PTP-SPS. We studied the safety and usefulness of PTP-SPS from the viewpoints of operation time and the complications.

Results: Median operation time of PTP-SPS (464 cases) was 83 (28227) minutes, while RP (133 cases) was 84 (26-191) minutes. In PTPSPS, 66 cases $(14.2 \%)$ needed 1 or 2 additional $5 \mathrm{~mm}$ ports and 7 $(1.5 \%)$ were converted to open surgery. Postoperative complications were conservatively treated 2 bile leakage $(0.4 \%)$ and 3 incisional hernia $(0.6 \%)$. There was no severe wound infection in our series. In RP, 2 cases were converted to open surgery (1.5\%). Severe postoperative complication was 1 incisional hernia $(0.75 \%)$ that needed surgical repair. Umbilical scars and the pierced needle instrument scars became gradually invisible within 1 or 2 months. There was no learning curve with operators changing over from RP to PTP-SPS method.

Conclusions: There were no differences between PTP-SPS and RP in operation time and complications. Operative scar of PTP-SPS is smaller than RP because of using the fine needle forceps instead of $5 \mathrm{~mm}$ port. Therefore, the patients appreciate PTP-SPS by better cosmesis than the conventional LC or RP. PTP-SPS may become the standard approach as less invasive laparoscopic cholecystectomy. 


\section{P232}

Surgical Response in Patients with Normokinetic Biliary Colic

Robert C Wright, MD, FACS, Rachel Robles, Haley Peffer, Nina Thach, Robert J Wright; Meridian Surgery Center

Background: Clinical response of patients with symptomatic biliary colic but with atypical findings of gallbladder polyps, hyper-dynamic gallbladder and otherwise negative biliary work up are underrepresented in the literature.

Methods: A clinical outcome study with a retrospective design compared the pre-operative pain to the post-operative pain reported by patients with pre-operatively diagnosed biliary dyskinesia to all other patients with biliary colic and atypical pre-operative diagnoses. A visual analog scale was used. All patients underwent surgery at Meridian Surgery Center from the years 2010 to 2017. 600 patients were reviewed for biliary dyskinesia, gallbladder polyps, hyper-dynamic gallbladder and negative work up. Patients with cholelithiasis or sludge were excluded from the study. Post-operative pathology reports were reviewed.

Results: Responses were received from 17 biliary dyskinesia patients, 10 hyper-dynamic patients, 10 polyps patients, 3 adenomyosis patients and 9 negative work up patients. Based on a 10 point pain scale, we found a mean drop in pain scale among biliary dyskinesia patients (-5.6) and among all other atypical findings (-5.7), with -6.1 for hyper-dynamic patients, -5.7 for adenomyosis patients, -6.0 for polyps patients and -5.0 for negative work up patients. There is no significant difference between pain recovery of patients with biliary dyskinesia and those with another atypical diagnosis. From the surgical pathology reports, all 17 of the of the biliary dyskinesia patients had chronic cholecystitis. Similarly, 9 hyper-dynamic patients, 10 polyps patients, 9 negative work up patients and 3 adenomyosis patients had chronic cholecystitis. Only 2 of the polyp patients' pathology reports showed polyps. Gallstones were reported in 2 biliary dyskinesia patients, 1 hyper-dynamic patient and 2 of the 3 adenomyosis patients. One patient had a completely normal report was in the hyper-dynamic group. Our poor response rates were disappointing.

Conclusion: These results allow us to conclude that there is a comparable surgical pain relief between biliary colic patients with gallbladder polyps, hyper-dynamic gallbladder, negative work up and those with conventional biliary dyskinesia.

\section{P233}

Comparison of Long-term Outcomes Between Laparoscopic and Open Liver Resection in Combined Hepatocellular Carcinoma and Intrahepatic Cholangiocarcinoma

So Hyun Kang, Woohyung Lee, Youngrok Choi, Ho-Seong Han, Yoo-Seok Yoon, Jai Young Cho, Kilhwan Kim, In-Gun Hyun, Sunjong Han; Seoul National University Bundang Hospital

Introduction: Combined hepatocellular carcinoma and cholagiocarcinoma (HCC-CCC) is a rare primary hepatic neoplasm with incidence reporting up to $3.5 \%$. Liver resection is still the preferred method for curative treatment. With the advancement of minimally invasive techniques, laparoscopic liver resection (LLR) is now a possible option for patients with resectable HCC-CCC, yet there are no published studies that analyze the feasibility of LLR in HCC-CCC alone. This study aims to compare the long-term survival and postoperative complications of LLR with open liver resection (OLR) in HCC-CCC.

Methods: Patients who underwent liver resection for HCC-CCC from August 2004 to June 2015 were enrolled. Those who received palliative surgery, and those who underwent open conversion after laparoscopic surgery were excluded. Medical records of these patients were retrospectively reviewed. Primary endpoint was 3-year diseasefree survival (DFS) and 3-year overall survival (OS), and secondary enpoints were 3-year disease-specific survival, 1-year OS, 1-year DFS, operative outcome, and postoperative complications. KaplanMeier survival analysis was performed to compare survival.

Results: After exclusion, 13 patients were enrolled in the OLR group and 13 patients in the LLR group. There was no difference in age, gender, type of liver surgery (major or minor hepatectomy), tumor location (favorable or unfavorable), liver function, size of largest tumor, and pathologic stanging. Mean operation time for OLR was $314.2 \pm 135.1 \mathrm{~min}$ and for LLR it was $337.3 \pm 155.9 \mathrm{~min}$ with no statistical significance $(\mathrm{p}=0.690)$. There was also no statistical difference in estimated blood loss $(1173.1 \pm 1459.7$ vs $453.8 \pm 271.9$, $\mathrm{p}=0.105$ ). The 3-year disease free survival for the OLR group was $38.5 \%$ while the LLR group was slightly higher with $53.8 \%$, but there was no statistical difference $(\mathrm{p}=0.828)$. The 3 -year overall survival was the same in both groups $(69.2 \%)$. The 3 -year disease-specific survival was also analyzed, and the OLR group was higher with $76.9 \%$ to $73.1 \%$ for the LLR group, but there was also no statistical significance $(\mathrm{p}=0.591)$.

Conclusion: Laparoscopic liver resection for HCC-CCC is technically feasible showing similar long-term outcome with open liver resection. However, a large scale cohort study is still needed to provide better evidence, and until then, careful patient selection must be taken place. 


\section{$\mathbf{P 2 3 4}$}

\section{Laparoscopic Management of Type II (Rare Type) Choledochal Cyst}

\section{Sumita A Jain, Senior Professor; SMS Hospital and Medical College}

Choledochal cysts (CCs) are rare medical conditions with an incidence in the western population of 1 in 100 000-150 000 live births. $50 \%-80 \%$ are type I, $2 \%$ type II, $1.4 \%-4.5 \%$ type III, $15 \%-35 \%$ type IV $20 \%$ type V.

The etiology of CCs is still unclear. Babbitt's theory of cysts caused by an abnormal pancreaticobiliary duct junction (APBDJ) such that the pancreatic duct and the common bile duct meet outside the ampulla of Vater, thus forming a long common channel, has gained much popularity.

$\mathrm{CC}$ is a premalignant state. The overall risk of cancer has been reported to be $10 \%-15 \%$, and increases with age.

MRCP is currently the most accurate preoperative imaging study to assess cyst anatomy and classify the disease according to standard Todani classification

The treatment of choice for choledochal cysts is complete excision with construction of a biliary-enteric anastomosis to restore continuity with the gastrointestinal tract.

Type I: is complete excision of the involved portion of the extrahepatic bile duct; \& Roux-en-Y hepaticojejunostomy

Type II: Complete excision of the dilated diverticulum comprising a type II choledochal cyst; the resultant defect in the common bile duct is closed over a T-tube

Type III (choledochocele): $3 \mathrm{~cm}$ or less can be treated with endoscopic sphincterotomy, $>3 \mathrm{~cm}$ are excised surgically via a transduodenal approach.

Type IV: Complete excision of dilated duct followed by a Roux-en-Y hepaticojejunostomy.

Type V (Caroli disease): One lobe-Hepatic Lobectomy, Bilobar-Liver transplantation.

We present a 17 year old girl who had type 2 (saccular) choledochal cyst which is very rare and that's why Very few cases of laparoscopic management of type 2 choledochal cyst has been reported in the literature. We managed this case by laparoscopic excision of choledchal cyst in its entirety and closure of defect.

\section{P235}

The Impact of Clostridium Difficile Infection on Treatment Outcomes Among Patients with Acute Pancreatitis: A Nationwide Analysis

Heather Peluso, $\mathrm{DO}^{1}$, Wesley B Jones, MD ${ }^{1}$, Marwan S Abougergi, $\mathrm{MD}^{2}$; ${ }^{1}$ Greenville Health System, ${ }^{2}$ Catalyst Medical Consulting, LLC

Introduction: Antibiotics are frequently used to treat acute pancreatitis before the presence of infected necrosis is established, which predisposes patients to Clostridium Difficile Infection (CDI). We sought to determine the impact of CDI on mortality, 30-day readmission, morbidity and resource utilization among patients with acute pancreatitis in the United States.

Methods: Retrospective cohort study using the 2014 National Readmission Database, the largest publicly available national readmission database. Patients were included if they had a principal diagnosis of acute pancreatitis. Exclusion criteria were age $<18$ years and December admission. Readmission was defined as the first admission to any hospital for any non-trauma diagnosis within 30 days of the index admission. The primary outcome was in-hospital mortality rate. Secondary outcomes were 30-day readmission, morbidity (intensive care unit (ICU) admission, systemic inflammatory response syndrome with organ failure (SIRS/OF), total parenteral nutrition (TPN), shock) and resource utilization (length of stay (LOS), total hospitalization costs and charges). The following confounders were accounted for using multivariate regression analysis: age, sex, median income in patient's zipcode, Charlson comorbidity score, hospital urban location, bedsize, teaching status.

Results: 236,066 patients were included, 2,362 of whom developed CDI. The mean age was 52.3 years and $47 \%$ were female. After adjusting for confounders, CDI was associated with $>2$-fold increase in mortality (adjusted odds ratio (aOR: $2.64(1.70-4.12), \mathrm{p}<0.01)$. In addition, patients with CDI were twice as likely to be readmitted within 30 days (aOR: $1.97(1.68-2.30), \mathrm{p}<0.01)$. CDI was associated with a substantial increase in morbidity: ICU admission (aOR: 9.07 (6.88-11.96), p < 0.01), SIRS/OF (aOR: 3.26 (1.72-6.17),p < 0.01), TPN (aOR: 5.68 (4.55-7.08),p < 0.01), and shock (aOR: 6.50 (4.51$9.36), \mathrm{p}<0.01)$. Finally, CDI was associated with significantly higher resource utilization: LOS (adjusted mean difference (aMD): 7.07 (6.33-7.81) days,p < 0.01), total hospitalization costs (aMD: \$16,278 $(\$ 13,820-\$ 18,736), \mathrm{p}<0.01)$ and charges $(\mathrm{aMD}: \$ 60,758(\$ 49,896-$ $\$ 71,620), \mathrm{p}<0.01)$.

Discussion: The development of CDI among patients with acute pancreatitis has a detrimental effect on treatment outcomes. It is associated with substantially higher mortality, 30-day readmission, morbidity and resource utilization. Therefore, judicious antibiotics use in this setting is of crucial importance and should be advocated for at the national level. 


\section{P236}

\section{Laparoscopy Versus Transmural Endoscopic Drainage} of a Pancreatic Pseudocyst

Ionut Bogdan I Diaconescu, MD, PhD, Matei Razvan Bratu, Madalina Ilie, Gabriel Constantinescu, Mircea Beuran; Carol Davila University of Medicine and Pharmacy

Introduction: Laparoscopic cystogastrostomy is an suitable procedure for the drainage of pancreatic pseudocysts if the cysts is present in the lesser sac (retrogastric)

Methods: We present a series of 7 consecutive cases. First option was endoscopic transmural drainage like an ideal procedure. It was tried, but for two cases it failed, because the cyst wall was thick and the cyst had completely compressed the stomach, making endoscopic manipulation difficult inside the stomach.

Results: For 5 cases Endoscopic drainage was the only procedures. Laparoscopic drainage was a simple solutions to the problems in managing a huge pancreatic pseudocyst of $17 \mathrm{~cm} \times 18 \mathrm{~cm}$ in a 57 years old male with a history of acute pancreatitis. Ultrasound and computed tomography revealed showed a walled off cystic collection that pushed the stomach superiorly. The cyst was exposed by dissecting the lesser omentum and found to have no adhesion to the surrounding tissues. Anastomosis was performed using an endoscopic linear stapler via small cystotomy and gastrotomy openings on the lesser curvature, which were then sutured laparoscopically. The postoperative course was uneventful.

Conclusion: Laparoscopic surgery is recommended as a safe, reliable, and minimally invasive treatment for managing pancreatic pseudocyst. Both techniques are suitable but with different indications that should be adapted to the case.

\section{P237}

\section{The Endoscopic Correction of Anastomotic Strictures After Liver Transplantation}

Bolatbek Baimakhanov, Prof, PhD, MD, Yerlan Abdirashev, MD, Maksat Doskhanov, MD, Nurken Abdiyev, MD; JSC "National Scientific center of surgery named after A.N. Syzganov's"

Background/Aims: The purpose is a retrospective analysis of endoscopic treatment (ET) of anastomotic strictures (AS) after LDLT and DDLT.

Methods: In the period from December 2015 to August 2018, we analyzed 107 patients after LT. LDLT was performed in $89(83.1 \%)$ patients and DDLT in 18 $(16.9 \%)$ patients. The whole liver in $18(16.9 \%)$ patients, the right lobe in 81 $(75.7 \%)$ patients, the left lobe in $7(6.5 \%)$ patients, right posterior section in 1 (0.9\%) patient. In $107(100 \%)$ cases after LT in $25(23.3 \%)$ patients BC of bile ducts were occurred. The early strictures $(<90$ days) of duct to duct anastomosis developed in $11(44 \%)$ patients and $15(54 \%)$ recipients developed late strictures (> 720 days). The ET was performed to 20 (80\%) patients. Other BC of $5(20 \%)$ patients was solved by PTBD and open surgery.

Results: The retrograde ET was successful in $14(70 \%)$ patients. In $11(55 \%)$ cases we deployed plastic stent. To $3(15 \%)$ patients only balloon dilation of AS was done. The $6(30 \%)$ cases of ET were not effective. The main reasons were late diagnostic and treatment, severe stenosis of AS and unsufficient certain necessary equipments (guidewire, stents etc.). Also it was due to a small experience in this pathology. However, by growing of experience, the amount of successful ET increased.

Conclusions: Thus, ET on time is good solution to solve BC after LT. These category patients require careful and timely diagnosis of early BC (MRCP etc.), using single-use and sufficient quantity accessories, advanced experience of ERCP.

\section{P238}

Adenomyomatous Hyperplasia of Distal Common Bile Duct: A Case Report and Review of the Literature

Paul S Chandler, MD, Jonathan Harris, Danny Sherwinter, MD; Maimonides Medical Center

Adenomyomatous hyperplasia $(\mathrm{AH})$ is commonly found in the gallbladder and is considered a tumor-like inflammatory lesion arising from RokitanskyAschoff sinus. It is extremely rare in the extrahepatic bile duct and only 15 cases have been reported to date. We describe a 63-year-old male patient who presented with cholangitis, underwent an extensive diagnostic workup, and ultimately had a Whipple procedure. Final pathology showed a $2.0 \times 1.5 \times 0.5 \mathrm{~cm}^{3}$ granular lesion in the distal common bile duct. There was prominent biliary epithelial proliferation with tubular-papillary architecture and minimal nuclear atypia in association with chronic inflammation, stroma reaction and smooth muscle proliferation. $\mathrm{AH}$ of the extrahepatic bile duct is a benign process but often requires a major operation to definitively diagnose.

\section{P239}

Total Laparoscopic Cholecystectomy in Moderate to Severe Acute Cholecystitis: Still a Safe Procedure

Juan D Hernandez, MD, FACS ${ }^{1}$, Gabriela L Larios, MD, FACS ${ }^{2}$, Diana C Quintero $^{2}$, Gabriel Herrera, MD, FACS ${ }^{1}$, Roosevelt Fajardo, MD, FACS ${ }^{1}$, Felipe Perdomo, $\mathrm{MD}^{3}$, Francisco $\mathrm{J}$ Diaz, $\mathrm{MD}^{1}$, Ricardo $\mathrm{M}$ Nassar, $\mathrm{MD}^{3} ;{ }^{1}$ Hospital Universitario Fundacion Santa Fe de Bogota, Universidad de los Andes School of Medicine, ${ }^{2}$ Universidad de los Andes School of Medicine, ${ }^{3}$ Hospital Universitario Fundacion Santa Fe de Bogota

Introduction: Cholecystectomy is one of the most common procedures in general surgery and was responsible for the dissemination of laparoscopy among surgeons from the late 1980's. Due to the catastrophic implications of bile duct injury, major efforts such as SAGES safe cholecystectomy program have been put forth to reduce this risk in laparoscopic cholecystectomy (LC), especially in severe cases. Also, bleeding and infection are major concerns. However, it is feared that an excess of precaution may lead to unnecessary conversions or partial cholecystectomies, procedures that can carry their own complications. A case series of LCs in complex cholecystitis and the incidence of intraoperative and postoperative complications is presented.

Methods: A prospectively maintained database was queried for patients who underwent LC at a tertiary care university hospital between January 2017 and June 2018, all conducted by surgeons with laparoscopic expertise. Inclusion criteria were patients with moderate or severe cholecystitis according to Tokyo guidelines 2018 (TG18). Exclusion criteria are age under 17 years, cholecystectomy as a secondary procedure, open cholecystectomy, mild cholecystitis according to TG18, pregnancy and incomplete records. The primary outcomes were three main complications: bile duct injury, hemorrhage or bleeding and infection. The secondary outcomes were conversion and mortality.

Results: Of 764 consecutive patients, 149 were identified as having moderate cholecystitis (19.5\%), and 5 as severe cholecystitis $(0.65 \%)$, and comprised the study group. The frequency of main complications reported in our study was 1 infection and 1 major bleeding (1.29\% total); there were no bile duct injuries. There were two conversions, one due to technical difficulties and associated medical conditions of the patient. The other one was due to difficult bleeding control, also requiring subtotal cholecystectomy. There was only one other subtotal cholecystectomy, performed because Calot triangle was unidentifiable. It was completed laparoscopically. Only one mortality was found and it was not associated with the procedure (leukemia).

Discussion: This series of patients with moderate to severe cholecystitis who underwent total LC shows good outcomes with low incidence of complications and no bile duct injuries. This incidence was not superior to that in international literature. Conversion to open or subtotal was decided only in truly severe disease or when the anatomy was impossible to discern. TG18 frequently did not correlate to severity or difficulty. A consensus on the indications or findings to decide conversion or subtotal cholecystectomy should be achieved to prevent overuse and the complications associated to them. 
P240

\section{Laparoscopic Bile Duct Injury.Effect of Surgical Repair}

Shahidur Rahman, Professor; Bangobandhu Sheikh Mujib Medical University

Introduction: The aim of this study was to analyze the surgical treatment results of major bile duct injuries.

A single institution prospective analysis of 30 patients with bile duct injuries, underwent surgical repair.

Methods: From January 2004 to september 2018, a prospective records of all patients with a BDI following LC was The bile duct injuries were classified using the Stewart-Way classifion. Class II injuries consisted of lateral damage to the hepatic duct with a resultant stenosis and/or fistula. Class III injuries, the most common involved transection and excision of a variable length of the duct, which always included the cystic duct-common duct junction. The surgeon transected the common duct (deliberately, thinking it was the cystic duct) early in the dissection and transected the common hepatic duct. Class III injuries were subdivided based on the proximal extent of the injury as follows: in class IIIa injuries, a remnant of the common bile duct or common hepatic duct remained; in class IIIb injuries, the proximal transaction was at the bifurcation of the common hepatic duct; in class IIIc injuries, the bifurcation of the common hepatic duct had been excised, and in class IIId injuries, the proximal line of resection was above the first bifurcation of the lobar ducts (into segmental ducts).

Results: Over 14 years, 30 patients were treated for a major BDI following LC. Patient demographics were notable for women with a mean age of 45.5 years (median 44 years). All patients sustained their BDI at outside hospital. The mean interval from the time of BDI to referral was 22 weeks (median 3 weeks). Thirty patients underwent definitive biliary reconstruction hepaticojejunostomY. Five patients sustained at least 1 postoperative complication. The most common complications were wound infection, cholangitis, and intraabdominal abscess/biloma The mean postoperative length of stay was 9.5 days (median 9 days). Later repairs might have been more successful than earlier ones. Multivariate analysis, however, showed that the timing of the repair was unimportant. Instead, success correlated with: eradication of intra-abdominal infection; complete preoperative cholangiography; use of correct surgical technique, and repair by a biliary surgeon.

Conclusions: In conclusion, this study demonstrates that the most important factors associated with the success of biliary reconstruction include the complete eradication of intra-abdominal infection (drainage of all bile and fluid collections), complete characterization of the injury with cholangiography, use of the correct surgical technique, and repair performed by an experienced biliary surgeon.

\section{P241}

The Application of Laparoscopy in Staged and Redo Surgery in Children with Choledochal Cysts

Mei Diao, Professor, Long Li, Professor; Department of Pediatric Surgery, Capital Institute of Pediatrics

Purpose: Conventionally, staged and redo surgeries are thought to be contra-indications for laparoscopy in children with choledochal cysts (CDC) because of adhesions, deranged anatomy, and demanding techniques. The current study is to assess the efficacy of laparoscopic staged and redo surgeries in CDC children.

Methods: Between January 2006 and September 2018, 178 patients were referred to our hospital for the second stage or redo surgeries. Of them, 163 patients successfully underwent laparoscopic definitive surgeries, including 1$)$ staged surgery $(n=79)$, i.e. external biliary drainages before cyst excision and RYHJ for i) CDC perforation with general peritonitis $(n=60)$, and ii) severe inflammation or neonates with huge CDCs $(n=19)$; 2$)$ redo surgeries $(n=84)$ : i) revision of choledocho- or cholecysto-jejunostomies $(n=4)$, ii) postoperative bile leak repairs ( $n=16,3$ of them caused by aberrant hepatic ducts which located in middle portion of cystic duct), iii) postoperative biliary obstructions $(n=64)$. A series of transabdominal retraction sutures were placed through 1) serosa of gallbladder fundus or gallbladder fossa in staged and redo surgeries respectively, 2) proximal common hepatic duct to facilitate dissection and anastomosis, 3) proximal $\rightarrow$ distal $\rightarrow$ posterior wall of CDC in staged surgery, 4) hepatic lobe to facilitate anastomosis of aberrant hepatic duct to jejunum.

Results: Conversion rate was $8.4 \%$ (15 out of 178 patients), including 1) unclear anatomical structure caused by dense adhesions $(n=5), 2)$ uncontrolled oozing $(\mathrm{n}=4), 3)$ stenotic segments extended to the intrahepatic bile ducts, which required extensive dissections $(n=4)$, 4) anastomosis of aberrant hepatic duct to jejunum in early practice $(n=2)$. Of remaining 163 patients, mean age at surgery was 3.5 years. Average operative time was $4.5 \mathrm{~h}$. Mean postoperative hospital stay, resumption of full diet, and duration of drainage were 6.5 days, 3.1 days, and 4.2 days respectively. Median follow-up period was 45 months. None of patients had biliary re-obstruction, intrahepatic stone formation, cholangitis, pancreatic fluid leak, pancreatitis, wound infection/dehiscence, or accidental injury of viscera which were directly adherent to the abdominal scar of primary surgery. Two (1.2\%) patients with perforated CDCs who underwent staged surgeries developed bile leaks. The bile leaks were caused by unrecognized aberrant hepatic ducts and repaired laparoscopically. Liver function tests were normalized within 1 year.

Conclusions: In experienced hands, laparoscopic staged and redo surgeries is safe and effective in selected CDC children. 


\section{P242}

\section{Clinical, Radiological and Pathological Presentation of Intramural Intraductal Papillary Mucinous Neoplasm of the Gallbladder}

Indraneil Mukherjee, Aleksandr Demin, DO, Ian Provancha, Jocelyn Villanueva, Anupma Agarwal; Staten Island

Introduction: The clinicopathologic characteristics of epithelial neoplasms of the gallbladder is limited, due to its rarity and because of the variability in terminology. The World Health Organization classification of 2010 added Mucinous cystic neoplasms as a separate entity. The Category of Intracystic papillary neoplasm was created to encompass a vast spectrum of lesions, ranging from innocuous cyst lined by benign epithelium without atypia to extensive invasive carcinomas of mucinous type. We present a case of an incidentally found Intra mural Intraductal Papillary Mucinous Neoplasm of the Gallbladder.

Clinical Details: A 67-year-old lady with a BMI of 27 presented with postprandial Right Upper Quadrant Pain. Past Medical History of H.Pylori Gastritis which had been eradicated and $3.3 \mathrm{~cm}$ stable Liver Hemangioma. She also had a remote 20 Packyears of smoking.

Imaging: Sonogram for suspected Gallstones, did not show any gallstones. MRI showed a focal form of adenomyomatosis of the gallbladder fundus and small hemangioma and cysts. Hepatobiliary Scintigraphy with HIDA (99mTc-hepatic iminodiacetic acid) and CCK (cholecystokinin)-stimulated cholescintigraphy demonstrated a gallbladder ejection fraction of $8 \%$.

Management: She underwent an uneventful Laparoscopic Cholecystectomy for Biliary Dyskinesia.

Pathology: Grossly gallbladder measured $9 \times 3 \times 2 \mathrm{~cm}$, with smooth and glistening serosa. The gallbladder contained thin green bile with no stones. The wall measuring $0.1 \mathrm{~cm}$ in thickness and the mucosa was unremarkable. A thin-walled cyst measuring $2.3 \times 1.8 \times 1.2 \mathrm{~cm}$ was seen at the fundus. On cut sections, the cyst contained clear fluid and had a firm, white, $0.1 \mathrm{~cm}$ thick wall. It was deemed to be adenomyoma with low grade mucinous dysplastic process or "mural IPMN" pattern is at the fundus of the gallbladder away from the uninvolved cystic duct margin. It was deemed safe to assume that this was clinically inconsequential and the patient was not offered any further invasive treatment other than follow up.

Conclusion: Adenomyomatous (AM) is seen in $<7 \%$ of cholecystectomies. It does not have the association of being neoplastic itself, but rather appears to be a developmental/malformative process, possibly a version of a duplication/diverticulum. Thus, adenomyomatous nodule may be a more accurate name. Mucinous change with papillary units ("mural IPMN" pattern) occurs in 7\%. High-grade dysplasia is seen in $3 \%$ and invasive carcinoma, often small, arises in $2 \%$. Overall prognosis is good, but depends on degree of dysplasia or invasion.

\section{P243}

Muscle Splitting Cholecystectomy: A Prospective Study

Ashish Prasad Rajbhandari, MS, Surgery; Nepal Medical College Teaching Hospital

Laparoscopic cholecystectomy is now regarded as the gold standard treatment for symptomatic cholelithiasis. Muscle splitting mini cholecystectomy is a good alternate option for those who are not fit for the laparoscopic procedure and in those institutes where the laparoscopic facilities are not available. It is the muscle division which is supposed to be responsible for postoperative pain and the resultant local and systemic effects.

All cases of symptomatic cholelithiasis were advised laparoscopic cholecystectomy and only those cases which opted for open cholecystectomy or were not fit for the laparoscopic procedure were included in the study. 16 cases of consecutive open cholecystectomies underwent muscle splitting procedure from June 2016 to May 2018 at Stupa community hospital. 13 were female and age ranged from $19-$ 82 years. I case was of morbid obesity with a weight of $99.6 \mathrm{~kg}$ and BMI of 44.2 cases were of preoperative diagnosis of empyema gallbladder and 1 case was of perforated gall bladder. The subcutaneous fat thickness ranged from 1.5 to 4 inches. All cases had a transverse incision in the RUQ around the tip of the 9th coastal cartilage; the length was usually around $5 \mathrm{~cm}$ (mini cholecystectomy)except for the complicated cases which extended to $7 \mathrm{~cm}$. Drain was kept in the 3 complicated cases. The duration of surgery varied from 35- $85 \mathrm{~min}$.

Post operative pain scale was taken in all cases, on 1st post operative day pain scale ranged from 4-6 and on 2nd post operative day 2-5. 13 cases (except the complicated cases) were discharged on the 2 nd post operative day. All cases with drain had an uneventful period with no leakage of bile and were removed on the 3rd post operative day and discharged on the 5 th post operative day after completion of a course of antibiotics.

Muscle splitting cholecystectomy is a good alternative to traditional rectus muscle dividing open cholecystectomy and maybe comparable to laparoscopic cholecystectomy as it offers less post operative hospital stay, post operative pain and discomfort with no increased incidence of intra or post operative complications and can be safe and effectively performed where and when needed. 
P244

Single-Incision Laparoscopic Cholecystectomy in Patients with Previous Upper Abdominal Surgery

Junpei Suzuki, Nobumi Tagaya, Horoshi Kusaba, Yuichi Obana, Yuhei Nakano, Masako Mizoguchi, Yukino Yoshimura, Takashi Arai, Yako Hasegawa, Junichi Suzuki, Tomoki Kido, Koji Matsushita, Nobuhisa Teranishi, Toshifumi Arai, Tetsuya Kurosaki, Masayuki Hatanaka; Itabashi Chuo General Hospital

Introduction: Single-incision laparoscopic cholecystectomy (SILC) has become a treatment of choice for symptom gallbladder diseases. However, its indication is still limited to uncomplicated cases. We investigated the feasibility and safety of SILC for the patients with previous upper abdominal surgery.

Patients and Methods: Recent five years we performed SILC in 307 patients with various gallbladder diseases. The patients were classified into 2 groups: Group1, patients with a prior history of upper abdominal surgery $(n=14)$; Group 2 , patients without any risk factors including acute cholecystitis or morbid obesity $(n=247)$. The data were analyzed in age, conversion rate, operation time, blood loss, postoperative complication, and postoperative hospital stay.

Results: There were no significant differences between PUAS and UC in gender (M:F) $(10: 4$ and $116: 131, \mathrm{p}=0.075), \mathrm{BMI}\left(\mathrm{kg} / \mathrm{m}^{2}\right)$ (24.4: 23.9, $\mathrm{p}=0.717)$, blood loss $(\mathrm{ml})(8.1: 7.7, \mathrm{p}=0.954)$ and postoperative hospital stay (day) $(3.5: 3.2, \mathrm{p}=0.282)$. Mean age (years) (66.0 and 56.5: $\mathrm{p}=0.004)$ and operation time (min) of PUAS and UC (115.0 and 84.8; $\mathrm{p}=0.003)$ were significant factors. There was no conversion to open laparotomy in both groups. Additional ports were inserted one case $(7.1 \%)$ in PUAS and 11 cases $(4.5 \%)$ in UC ( $\mathrm{p}=0.642)$. Postoperative bile leakage was seen one case in UC, and it showed spontaneous remission.

Conclusion: Previous upper abdominal surgery is not a contraindication to perform a safe SILC, however, previous upper abdominal surgery is associated with a prolonged operation time.

\section{P245}

Possible Variables to Develop a Scoring System to Predict Preoperative Diagnosis of Acute Cholecystitis

Theophilus Pham, MBA ${ }^{1}$, Daron Jacob, BS ${ }^{1}$, Chanaka Kahathuduwa, $\mathrm{PhD}^{2}$, Adel Alhaj Saleh, MD, MRCS ${ }^{1}$, Amir H Aryaie, MD,

FACS ${ }^{1} ;{ }^{1}$ Texas Tech University Health Sciences Center, ${ }^{2}$ Texas Tech University

Introduction: Cholecystitis and symptomatic cholelithiasis are two diseases that can present with very similar general symptoms making it difficult to distinguish between them based solely on clinical findings. Abdominal ultrasounds (US), and hepatobiliary iminodiacetic acid (HIDA) scans, are used to confirm diagnosis.

Objective: to determine predictive factors that can help the clinician more precisely diagnose acute cholecystitis for patients who were diagnosed with symptomatic cholelithiasis based on ultrasound.

Method: A retrospective review of all patients diagnoses with symptomatic cholelithiasis for last five years based on US. Data collection included demographics, admission, presence of nausea, presence of pain, presence of postprandial pain, positive Murphy's sign, serum alkaline phosphatase (ALP), serum bilirubin, total White blood cell count, and the presence of a left shift in neutrophils.

Results: 287 patients with preoperative diagnosis of symptomatic cholelithiasis were included in the study, $226(78.7 \%)$ of them had intra-operative findings of either acute or chronic cholecystitis. Pathology confirmed inflammation of the gall bladder in $282(98.3 \%)$ of the patients. Presence of macroscopic features of cholecystitis during surgery was best predicted by the combination of sex, bilirubin level and the presence of left shift of neutrophils. The odds of a male patient having macroscopic features of cholecystitis was 1.12 times greater than the odds of a female patient having features of cholecystitis during surgery $(p=0.03)$. Similarly, the odds of a surgeon encountering features of acute or chronic cholecystitis was 1.17 time higher for patients with left shifted neutrophils $(p=0.003)$. Each $1 \mathrm{mg}$ increase in serum bilirubin decreased the odds of having macroscopic features of cholecystitis by approximately $4 \%$ (OR = $0.96, \mathrm{p}=0.037)$. (Table \#1)

Conclusion: Our data showed that only male sex, the presence of leftshifted neutrophils, and bilirubin were statistically significant to predict the intraoperative finding of cholecystitis. Additional studies could be used to expand the data pool in order to create a comprehensive preoperative scoring system to further differentiate between symptomatic cholelithasis and acute cholecystitis.

\begin{tabular}{|l|l|l|l|l}
\hline Predictor & $\begin{array}{l}\text { Coefficient (log- } \\
\text { transformed } \\
\text { odds ratio) }\end{array}$ & $\begin{array}{l}\text { Standard error } \\
\text { coefficient }\end{array}$ & t-statistic & p-value \\
\hline Male sex & 0.114 & 0.053 & 2.155 & 0.032 \\
\hline $\begin{array}{l}\text { Left shifted } \\
\text { neutrophils }\end{array}$ & 0.160 & 0.053 & 3.022 & 0.003 \\
\hline Bilirubin (mg/dl) & -0.042 & 0.020 & -2.083 & 0.037 \\
\hline
\end{tabular}

Table 1: Results of the best fitting logistic regression model predicting the presence of macroscopic features of acute or chronic cholecystitis during surgery 


\section{P246}

\section{Impact of On-Table ERCP in the Acute Setting on Length of Stay} at a Tertiary Care Center

Samantha R Witte, MD, Katelin Mirkin, MD, Jerome Lyn-Sue, MD; Penn State Health

Introduction: The management of acute complicated cholecystitis with choledocholithiasis diagnosed either during the initial workup or in the operating room by cholangiogram can be a complex and challenging problem to manage. We compared outcomes between patients who underwent preoperative ERCP followed by delayed cholecystectomy during the same hospital admission with patients who underwent a combined procedure with intraoperative ERCP. We hypothesize that on-table ERCP will decrease length of stay and associated hospital cost, without increasing the rate of procedural complication.

Methods and Procedures: We retrospectively reviewed the 2017-2018 database of an academic hospital for patients with acute complicated cholecystitis or choledocholithiasis who underwent laparoscopic cholecystectomy and ERCP. The study population was then divided into two cohorts: those who underwent preoperative ERCP followed by laparoscopic cholecystectomy, and those who underwent combined interventions. Primary outcomes evaluated were hospital length of stay, discharge destination, and days remaining in hospital after operation.

Results: A total of 29 patients were included in this study. One patient was excluded for oncologic pathology, and one patient was excluded for pregnancy. 16 underwent preoperative ERCP and 11 underwent combined interventions. There was no difference in patient age between the two groups ( $p$-value 0.77 ). All patients presented with a diagnosis of acute cholecystitis with or without pancreatitis. Patients who were confirmed or suspected to have choledocholithiasis underwent ERCP either pre-operatively or as a combined procedure. The average length of stay was compared between the two groups. Patients who underwent two separate procedures had an average length of stay of 6 days while patients who underwent a combined procedure with a single anesthetic exposure had an average length of stay of 3.45 days, which was a statistically significant difference with a p-value of 0.05 .

Conclusions: This data suggests that there is a benefit both for patient outcomes as well as from the standpoint of cost to performing combined procedures when feasible, even in an urgent setting.

\section{P247}

\section{Acute Intestinal Obstruction Due to Gallstone Ileus: Case Report}

Gessica P Vasconcelos, MD ${ }^{1}$, Diego L Lima, $\mathrm{MD}^{2}$, Raquel N Cordeiro, Medical Student ${ }^{3}$, Italo C Moreira, MD , Adalberto G Araujo, MD ${ }^{1}$; ${ }^{1}$ Getulio Vargas Hospital, ${ }^{2}$ State Servers Hospital, ${ }^{3}$ Pernambuco Health College

Introduction: Gallstone ileus (GI) have an incidence of $0.3 \%$ to $0.5 \%$ of the patients with cholelithiasis. It is the cause of intestinal obstruction in 1 to $4 \%$ of the cases. Typically, GI starts with chronic cholelithiasis, which evolves with the formation of a cholecystoduodenal fistula, with the passing of a large calculus to the gastrointestinal tract, resulting in obstruction. Approximately $50 \%$ of patients with the disease have a history of gallstones, but only 0.3 to $1.5 \%$ of patients with gallstones present GI.

Case Report: A 64 years-old male, with history of no bowel movements, nausea, vomits and abdominal pain for 3 days. A nasogastric tube presented fecal fluid in high quantity $(500 \mathrm{ml}$ in $12 \mathrm{~h})$. CT scan of the abdomen showed an image that suggested a gallstone ileus. Patient was submitted to an emergency surgical procedure with the following findings: distension of jejunal loops in its proximal third by a stone of $4.0 \times 3.0 \mathrm{~cm}$, stuck $160 \mathrm{~cm}$ of the ileocecal valve. Besides, an intense blockage involving duodenum, epiploon and gallbladder was noted (a cholecystoduodenal fistula). The surgical team opted to perform an enterolithotomy. The surgical team opted to not approach the blockage area which could cause an iatrogenic lesion of the biliary tract. Patient had no further complications, with good oral diet and he was discharged 6 days after the surgery.

\section{P248}

Live QA, Live Image Sharing and Grading to Evaluate the CVS in Cholecystitis

Kathryn S Sobba, MD, Adolfo Fernandez, MD, Stephen Mcnatt, MD, Myron Powell, MD, Andrew Nunn, MD, Amy Hildreth, MD, Clancy Clark, MD, Barbara Yoza, PhD, Lauren Mccormack, MD, Amit Saha, $\mathrm{PhD}$, Jessica Gross, MD, Preston Miller, MD, Carl Westcott, MD, Matthew Tufts, MD; Wake Forest Baptist Medical Center

Objective: The Critical View of safety is a technique widely believed to decrease the risk of bile duct injury. The general quality of CVS in cholecystectomy has been studied and those evaluations generally questioned the consistency and accuracy of the CVS in practice. The aim of this study is to use an already established SMS image sharing and grading system to evaluate if and how cholecystitis changes surgeons ability to attain a CVS.

Methods and Procedures: Live QA is an image sharing and grading system where a standard doublet CVS image set can be sent by sms picture/text via hand held smart phones to a group of experts. No PHI is put at risk. A grade for the two images is immediately provided and returned to the operating team. 38 urgent laparoscopic cholecystectomies done for cholecystitis and 117 done for gallstones by sixteen surgeons between 8/2017 and 9/2018 were included. CVS doublets were attained prior to clipping and sent out to the group. The images were graded remotely and returned. The ability to attain good grades and the consistency of scores amongst the graders were compared between the two groups.

Results: There were no bile duct injuries or leaks. There was 1 post operative hematoma in the inflamed group that required operative evacuation. The average time to first response was less than $2 \mathrm{~min}$ and the average number of responses was 3.6. Average CVS scores were lower for cholecystitis patients (anterior average 5.41 vs 5.45 in cholecystitis (p value 0.836 ), posterior average 5.16 vs 4.95 in cholecystitis ( $p$ value 0.375 )), however a sufficient CVS grade (5 or better in at least one view) was attained in 36 of 38. The consistency of CVS scores for cholecystitis patients was $91 \%$ for anterior images and $86 \%$ for posterior images ( $\mathrm{p}$ value 0.69 ).

Conclusions: Adequate CVS is almost always attainable in cholecystitis, however pericystic inflammation decreases CVS quality and grading agreement. The posterior view suffers and varies the most. Larger sample sizes would be needed to study the CVS and the effect of this image grading system on adverse events associated with cholecystectomy. 
P249

Safety and Feasibility of Day Care Laparoscopic Cholecystectomy in a Tertiary Care University Hospital - A Randomized Controlled Study

Anubhav Vindal, MS, DNB, MRCSEd, FRCSGlas, FACS, FAIS, Saurav Kumar, MBBS, Pawanindra Lal, MS, DNB, FRCSEd, FRCSGlas, FACS, FAIS; Division of Minimal Access Surgery, Department of Surgery, Maulana Azad Medical College, New Delhi, India

Background: Day care laparoscopic cholecystectomy (DCLC) is increasingly replacing inpatient cholecystectomy in the developed nations. Early mobilization and return to normal activities has many positive implications for the patient, the hospital, and society. However, the safety and efficacy of DCLC is yet to be tested in developing nations especially in centres without dedicated units and well-established protocols for day care surgery.

We present the results of our prospective randomized double blind study to evaluate feasibility and safety of DCLC in unselected patients in a tertiary care university hospital of a developing country without a separate facility for day care surgery.

Methods and Procedures: Hundred patients of symptomatic gall stone disease planned for elective LC were included in the study. The only selection criteria that were used to screen the patients were residence within $1 \mathrm{~h}$ of travel time to the hospital, ability to comprehend written, verbal and telephonic instructions and a responsible adult company at home.

The patients were randomized at the conclusion of the surgery into two groups of 50 patients each (DCLC and in-patient LC). The surgeon and the observer were blinded to the groups of the patients.

Post-operative pain (at 6, 12, $24 \mathrm{~h}$ and 1 week) (using VAS), time of return to activities and patient satisfaction (using Capuzzo score) were recorded and compared between the two groups. Discharge and readmission rates were also recorded in the DCLC group.

Results: The mean VAS at 6, 12, $24 \mathrm{~h}$ and 1 week was more or less similar in the two groups $(\mathrm{p}=0.637,0.612,0.582$ and 0.536 respectively). However the mean time to return to activities was significantly lower $(\mathrm{p}<0.0001)$ and mean Capuzzo score (patient satisfaction) higher $(\mathrm{p}<0.05)$ in DCLC group compared to in-patient LC group. The discharge rate for the DCLC group was $98 \%$ (49/50 patients discharged as planned). There were no readmissions in this group (0\%).

Conclusions: DCLC is safe and feasible in unselected patients even in centres without dedicated day care units. Proper patient counselling and adherence to standard discharge protocols may help in reducing readmission rates and increasing patient satisfaction and acceptability of this pathway. Developing countries have a lot to gain from day care approach, in the form of decreased health care costs, higher turnover of hospital beds, and decreased loss of work days.
$\mathbf{P 2 5 0}$

Outsized Approaches in the Diagnosis of Biliary Pancreatitis

I. Fedoriv, MD, V. Myesoyedova, MD; Dpt. of Surgery. IvanoFrankivsk national medical university. Ukraine

The purpose of the study was to determine the differences in the dynamics of glycemia in the first few minutes after glucose loading, because in that time the diagnostically valuable indicators that would show a statistically proven correlation with the severity of pancreatitis were supposed to be detected.

The main criteria for the selection of patients for examination were: the presence of gallstone disease, an attack of cholecystitis during the hospitalization or in the recent medical history and the presence of the pancreatic lesion syndromes.

Methods of the Research: general-clinical to clarify the diagnosis and monitor the dynamics of the disease; biochemical - determination of the level of cholecystokinin, alpha-amylase and gamma-glutamyl transpeptidase of serum; instrumental methods, such as ultrasound examination of the liver bile ducts and pancreas were used.

Results of the research and their discussion. 120 patients were examined, which were divided into 4 groups.

The results of examinations of 30 volunteers without pathology of biliary tract and pancreas were used as a control.

The age of the patients ranged from 20 to 80 years. The average age of patients with gallstone disease, complicated by acute pancreatitis was 49,6 years. Among them, females were predominant - 94 (78,3\%). The largest number of patients was in the age group of 40-49 and $50-59$ years old $(20,8 \%$ and $18,3 \%$ respectively). There were 26 men $(21,7 \%)$, the highest number of men ( 8 men) was in the age group of $50-59$, which was $6,7 \%$.

\section{Conclusions:}

1. An exacerbation of biliary pancreatitis in gallstone disease does not lead to complete insular insufficiency. $\beta$-cells respond adequately to the amount of glucose in the blood.

2. In acute biliary pancreatitis the violation of the dynamics of the insulin response to intravenous glucose stimulation has been confirmed. There was a certain delay in the reaction of islet cells, which was from 1 to $10 \mathrm{~min}$.

3. The insulin response over 3 min shows an incretory pancreatic insufficiency in the early stages of pancreatitis.

4. Based on the findings of the study, we have proposed the use of an intravenous glucose tolerance test in the diagnosis of acute biliary pancreatitis, since its advantage is the ability to avoid the effect of the rate of absorption of glucose in the digestive tract on the result of investigation. Thus, this test reflects the adequacy of the reaction of the insulin apparatus of the pancreas to the administration of glucose. 


\section{P251}

\section{Usefulness of the Laparoscopic Approach for Liver Resection} of Multi-site Lesions

Masayasu Aikawa, Kenichiro Takase, Yukihiro Watanabe, Katsuya Okada, Kojun Okamoto, Shinichi Sakuramoto, Shigeki Yamaguchi, Isamu Koyama; Saitama medical university, international medical center

Introduction: The laparoscopic liver resection (LLR) has become widespread because it is less invasive. However, the evidence of its less invasiveness has not been reported in multiple resections for hepatomas. We investigated the less invasiveness and safety of LLR in multiple resections in comparison with those of the laparoscopic and open approaches.

Methods: Between 2008 and 2018, of 340 patients who underwent LLR for whole hepatoma in our facility, 36 underwent LLR for dualsite hepatoma. Before definitive induction of the LLR, 30 patients underwent open liver resection (OLR) for dual-site hepatoma. The patients' background, characteristics, and perioperative outcomes were compared between LLR and OLR for dual-site hepatoma.

Results: No significant differences in the patients' background were found between the two groups except for the type of hepatoma. The operative durations were prolonged in the LLR group as compared with those in the OLR group. The blood loss volumes, pain score, and hospital stay in the LLR group were lower than those in the OLR group. A postoperative complication higher than grade III according to the Clavien-Dindo classification did not occur in any of the patients in the LLR group but occurred in 3 patients in the OLR group. Conclusion: OLR requires an extended wound for resection of multisite lesions in hepatoma. In contrast, LLR makes it possible to resect multi-site lesions by the addition of a few laparoscopic ports. LLR may be useful, particularly for resection of multi-site lesions.

\section{$\mathbf{P 2 5 2}$}

Biliary Hyperkinesa: Is It a True Indication for Cholecystectomy?

Ratnakishore Pallapothu, MD, FACS, FASMBS ${ }^{1}$, Matthew Protas, MSIII $^{2}$, Todd Prier, MD, FACS ${ }^{3}$, Jerome Brustein, MD $^{3}$, Nirupama Anne, MD, FACS ${ }^{1}$; ${ }^{1}$ Our Lady of Lourdes Memorial Hospital, Binghamton, NY. SUNY Upstate Medical University, Binghamton Clinical Campus, Clinical Assistant Professor of Surgery., ${ }^{2}$ SUNY Upstate Medical University Syracuse NY, ${ }^{3}$ Our Lady of Lourdes Memorial Hospital, Binghamton, NY

Background: Biliary hyperkinesia is poorly understood and the diagnosis is questioned. The literature regarding surgical intervention for biliary hyperkinesia is limited. We present a case series of 41 patients who underwent laparoscopic cholecystectomy for biliary hyperkinesia and had symptomatic relief.

Methods: Patients presenting with symptoms of biliary colic, with a negative ultrasound for gallstones, underwent HIDA scan with CCK. Patients with ejection fraction (EF) greater than $80 \%$ were considered to have biliary hyperkinesia based on radiology literature. An IRB approval was obtained for a prospective study for the team comprising of surgeons and a radiologist. The study time was between March 2011- May 2018. 41 patients with ongoing refractory symptoms consistent with biliary colic, affecting quality of life, with negative upper GI work up (endoscopy), were considered for laparoscopic cholecystectomy. All the 41 patients were followed subsequently with postoperative follow up visits.

Results: 41 patients underwent laparoscopic cholecystectomy. 33 patients were women and 8 patients were men. 14 patients had EF between $80-90 \%$ and 27 patients had EF between $90-100 \%$. The chief complaint in all these patients was abdominal pain in epigastrium or right upper quadrant. The mean duration of symptoms is at least 6 months. The final histopathology showed cholecystitis in 32 patients; chronic cholecystitis $(\mathrm{n}=31)$ and one patient had acute cholecystitis $(n=1)$. Cholesterolosis in 11 patients and 8 patients had no pathologic findings. 40 patients had symptomatic relief and improved quality of life after surgical intervention. One patient had no change in her symptomatology. None of these patients had any postoperative complications.

Conclusion: There is enough evidence regarding the surgical intervention for symptomatic biliary hypo/dyskinesia $(\mathrm{EF}<35 \%)$. The literature regarding the diagnosis of biliary hyperkinesia which is defined as EF $>80 \%$ is limited and most commonly misinterpreted as normal gall bladder function. Hence the diagnosis is missed and patients continue to have ongoing symptoms typical for biliary colic affecting the quality of life with multiple office and emergency room visits. Even though our number is limited, as demonstrated in our case series the entity of biliary hyperkinesia is true, as cholecystitis is noted in most pathology specimens (approximately 80\%) and symptomatic response was noted in $98 \%$ of the patients with no postoperative complications. Hence cholecystectomy should be considered as an option for symptomatic patients with $\mathrm{EF}>80 \%$ when conservative measures such as life style and diet changes have failed and the symptoms become refractory. 


\section{P253}

\section{The Use of Indocyanine Green (ICG) for Image-Guided Laparoscopic Cholecystectomy. Comparison of Two Methods of ICG Administration}

Nikolaos Antoniou, Orestis Ioannidis, Ioannis Mantzoros, Stathis Kotidis, Loukiani Kitsikosta, Dimitris Konstantaras, Konstantinos Tsalis; Aristotle University

Laparoscopic cholecystectomy is one of the most commonly performed operations worldwide. Bile duct injury (BDI) is a rare but very serious complication of of the procedure, with a significant impact on quality of life and overall survival. The high frequency of BDI with laparoscopic cholecystectomy was first considered to be a consequence of the initial learning curve of the surgeon, but it later became clear that the primary cause of BDI is misinterpretation of biliary anatomy. Intraoperative cholangiography (IOC) has been advised by many authors as the technique reduces the risk of BDI. However, the procedure has inherent limitations and is therefore reserved for select cases.Fluorescent cholangiography using indocyanine green(ICG) is a novel approach, which offers real-time intraoperative imaging of the biliary anatomy. A comparative study was contacted by administering ICG intravenously or intrabiliary during the operation.

Forty patients scheduled to undergo an elective lap. cholecystectomy were randomly divided in two groups:

- In Group A ICG was administered in a dose $2.5 \mathrm{mg}$ in $2 \mathrm{~mL}$ solution intravenously $1 \mathrm{~h}$ before surgery.

- In Group B ICG was injected intrabiliary in a $0.025 \mathrm{mg} / \mathrm{mL}$ solution mixed with the patient's bile.

Results

- Group A. Intravenous ICG was administered in 20 patients. There was no any reaction and the extrahepatic biliary anatomy was identified well. There was no BDI or any complication related to the procedure.

- Group B. ICG was injected intrabiliary in 20 patients during the laparoscopic procedure. In all but one patient the extrahepatic biliary tree was delineated very well. In one patient part of ICG solution was injected into the gallbladder wall and this resulted in a partially confusing image. There was no BDI and no postoperative complication

Conclusions: Fluorescence cholangiography can be used during laparoscopic cholecystectomy to obtain fluorescence images of the bile ducts following intrabiliary injection during the operation or intravenous injection $1 \mathrm{~h}$ before the procedure. The later technique is more easy to perform and does not require catheterization of the biliary tree.

\section{$\mathbf{P 2 5 4}$}

Simplified Laparoscopic Bile Duct Reconstruction Using a Double-Omega: An 18 Patient Case Series Introducing a Novel Surgical Approach

Daniel Gomez, MD, FACS ${ }^{1}$, Luis F Cabrera, $\mathrm{MD}^{2}$, Ricardo Villarreal $^{2}$, Andres Urrutia ${ }^{3}$, Eric Vinck ${ }^{2}$, Mauricio Pedraza ${ }^{2}$, Sebastian Sanchez ${ }^{2}$, Jean Pulido ${ }^{2} ;{ }^{1}$ Military University, ${ }^{2}$ Bosque University, ${ }^{3}$ Universidad Pedagógica y Tecnológica de Tunja

Introduction: The management of Laparoscopic from the benign bile duct pathologies has become a mainstay approach because of the advantages offered to patients. Even though patients recover from the use of laparoscopic approach swiftness, bile duct trauma secondary to laparoscopic manipulation still remains a significant complication with an incidence of $0.6 \%$ in laparoscopic gallbladder surgeries.

Methods: We describe a case series use of 18 patients taken to a novel modified and simplified laparoscopic technique for a hepatic duct jejunostomy with a Roux in $\mathrm{Y}$ reconstruction reducing the surgical complexity and the duration of surgery of bile duct reconstruction secondary to benign bile duct pathologies.

Results: The surgery was allocated an average of $139 \mathrm{~min}$, an average of $15 \mathrm{cc}$ for intraoperative bleeding, no re-intervention was required, there were no mortalities, and the average period of hospitalization was 5 days. No patients required ICU admission, no patients had postoperative bile leaks, none were converted to open surgery and all patients had oral feeding and adequate tolerance at POP 2. Long-term term follow-up showed no bile stenosis at 12 months following surgery.

Conclusion: This simplified laparoscopic approach to bile duct reconstruction long with Roux in $\mathrm{Y}$ anastomosis seems to be an effective, seemingly safe alternative to bile duct surgery offering patients a quicker surgery time with fewer complications. Key words: Laparoscopic; approach; bile duct; reconstruction; duct surgery.

\section{P256}

\section{A Case Report of Double Gallbladder and Choledochal Cyst in a Single Patient}

Abdulaziz A Arishi, MD, Amin M Ahmad, MD, Wesley C Judy, David Heidt, MD; University of Toledo Medical Center

Introduction: Double gallbladder is an uncommon condition. The incidence of Double gallbladder alone is about 1 in 3000-4000 cases where the incidence of choledochal cyst is ranging from 1 in 13000 persons to 1 in 200000 persons. There is no known reported case of both anomalies in a single patient.

Presentation of case: This is a 26-year-old male who presents with intermittent right upper quadrant abdominal pain for one month associated with nausea and no vomiting. Ultrasound of abdomen revealed duplications of gallbladder. MRCP shows duplication of gallbladder, and Type I choledochal cyst. No family history of cancer. He underwent an exploratory laparotomy, cholecystectomy, common bile duct resection, and hepaticojejunostomy.

Discussion: Double gallbladder and choledochal cyst are well described rare clinica entities. Classification system of abnormal anatomic variation of gallbladder was developed by Harlaftis and Boyden. Proposed theories of embryologic development suggest that it develops either as a bifurcation of the cystic primordia or duplication of it. Gallbladder duplication can be classified as a type-I anomaly a split primordia gallbladder, where gallbladder can be partially, incomplete or completely split. Type-II anomaly, which is the most common, where two separate gallbladders, each with their own cystic duct, or a rare type-III anomaly, where triple gallbladders draining by $1-3$ separate cystic ducts. In other hand choledochal cyst was classified by Todani into five types based on location and the degree of biliary tract dilation. Choledochal cyst are associated with high risk of cholangitis, pancreatitis, and cancer development. Surgery is indicated to prevent those complications.

Conclusion: The important point of this case is the unusual presentation of two relatively rare congenital conditions. A literature search showed an absence of similar cases ever being reported. Knowledge of anatomy and high level of alertness for congenital abnormalities is key for safe surgical operation. Failure to recognize those anomalies are associated with increased operative difficulty and complications. 


\section{P257}

Laparoscopic Common Bile Duct Decompression with Primary Closure for CBD stones following failed ERCP: A Case Series

Daniel Gomez, MD, FACS ${ }^{1}$, Luis F Cabrera, General

Surgeon ${ }^{2}$, Andres Mendoza ${ }^{2}$, Ricardo Villarreal ${ }^{2}$, Mauricio Pedraza $^{2}$, Jean Pulido ${ }^{2}$, Eric Vinck ${ }^{2}$, Sebastian Sanchez ${ }^{2} ;{ }^{1} \mathrm{CPO},{ }^{2}$ Bosque University

Background: Despite studies showing the effectiveness of common bile duct surgery (CBD) using laparoscopy in the international literature, In Colombia there are no series detailing the benefits and advantages of this approach as an alternative to open surgery in CBD stone obstruction. Here we present a case series on our experience with this technique on complex common bile duct stone obstruction using decompressive laparoscopy following failed ERCP.

Methods: During a time frame of 4 years from June 2014 to April 2018, 182 patients were taken to laparoscopic CBD decompression surgery due to complex stone obstruction. Records were reviewed and analyzed for demographic data, mean operative time, blood loss, intra or postoperative complications and hospital stay.

Results: All procedures were carried out successfully with no conversions registered, $100 \%$ of these procedures were CBD explorations with choledocotomy and primary duct closure, and in those patients with gallbladders, cholecystectomy was also performed (78\%); all procedures using a laparoscopic approach. Surgery duration averaged $85 \mathrm{~min}$, intraoperative bleeding averaged $15 \mathrm{cc}$, no patients required additional surgery, there were no mortalities, none required ICU admission and average hospital stay was 5 days.

Conclusions: A laparoscopic approach to complex CBD stone obstruction with primary closure after failed ERCP is safe and effective, and in experienced hands offers advantages over open surgery and endoscopic techniques.

Key words: Laparoscopic; approach; bile duct; reconstruction; duct surgery

\section{P258}

Minilaparoscopic Cholecystectomy: The Lessons Learned After 18 Years of Experience and More Than 2,530 Cases

Diego L Lima ${ }^{1}$, Raquel N Cordeiro ${ }^{2}$, Gustavo Henrique B De Goes ${ }^{3}$, Gustavo L Carvalho, MD, $\mathrm{phD}^{3}$; ${ }^{1}$ State Servers Hospital, ${ }^{2}$ Faculdade Pernambucana de Saude, ${ }^{3}$ University of Pernambuco

Objective: To show the results after 18 years of experience of a single surgical team in the Brazil performing minilaparoscopic cholecystectomy by clipless technique.

Method: A total of 2,536 consecutive patients who underwent MLC were analyzed, from January 2000 to January 2018. Of total, 1973 $(77,8 \%)$ were women whose age ranged from 6 to 99 years old. Surgical technique: after performing the pneumoperitoneum at the umbilical site, four trocars were inserted; three of $3.5 \mathrm{~mm}$, and one of $11 \mathrm{~mm}$ in diameter, through which a laparoscope was inserted. Neither the 3-mm laparoscope, nor clips, nor manufactured endobags were used. The cystic artery was safely sealed by electrocautery near the gallbladder neck, and the cystic duct was sealed with surgical knots. Removal of the gallbladder was carried out, in an adapted bag, through the 11-mm umbilical site.

Results: The operation video mean time was $24 \mathrm{~min}$. There was conversion to LC in 32 cases, which represents $1.261 \%$ of total. There was no conversion to open surgery, no bleeding, no bile ducts injury, no bowel lesion, no reoperation or mortality, no important complications in intra and postoperative time. There were just 11 cases $(0.433 \%)$ of umbilical hernia, which occurred in the first 1,000 surgeries, and 23 cases $(0.906 \%)$ of minor umbilical infection that was treated with antibiotics. There was great satisfaction of patients regarding postoperative pain and reduced hospital stay.

Conclusion: The clipless MLC shows to be a safe and effective technique, with a lower learning curve when compared to other techniques. 


\section{P259}

\section{Rare Case of Extra-hepatic Biliary Duplication}

Nima Fatehi, Praneetha Narahari, MD, Sean Tower, MD; Saint Agnes Medical Center

Introduction: Aberrant right hepatic duct entering cystic duct is fairly common $(18 \%)$. Main hepatic duct flowing into cystic duct is extremely rare. We report an exceptionally rare case of duplicated right hepatic duct, arising from anterior segments 5 and 8 and draining into the cystic duct.

Case Summary: A 66-year-old male with diabetes, hypertension and CAD, presented with acute cholecystitis. He was obese with RUQ tenderness. LFT's were normal, with WBC of $12 \mathrm{k}$ hyperglycemia of 330, lactic acid of 2.5 . US and CT were suggestive of cholelithiasis, CBD of $7 \mathrm{~mm}$, normal pancreas. Proceeded with Lap Chole/IOC. Dissection initiated at thegallbladder/cystic duct junction. The cystic duct appeared thick and could not be dissected easily. Hence the infundibulum was dissected exposing the base of the gallbladder (cholecysto hepatic plate) and cystic duct partially transected close to the gallbladder and cholangiogram obtained. Abnormal anatomy detected, with duplicated right biliary system. The cystic duct was very short proximal to the insertion of the dilated, anomalous right hepatic duct. There was no filling defect, with good flow into duodenum. No arterial anomaly noted. MRCP was performed post lap chole to delineate the segmental anatomy and to identify reason for anomalous ductal dilatation. MRCP did not show any filling defects, and confirmed the presence of 2 right hepatic ducts. The anterior segments 5 and 8 joined to form the anomalous right hepatic and drained into the cystic duct. The segment 8 duct was dilated, possibly from the spiraling cystic duct, with inadequate emptying. The right hepatic from the posterior segments reached the confluence in a normal fashion and continued to the common hepatic duct.

Conclusion: The anomalous right biliary duct duplication, with the anterior right hepatic duct draining to cystic duct is extremely rare, and underscores the importance of identifying the biliary anatomy to prevent a bile duct injury. Bile duct injury imposes severe consequences to the patient, the surgeon, is burdensome to the medical system, and leads to loss of productivity to the society. Although the incidence of congenital anomalies is low, it is prudent to define the anatomy and prevent complications. We propose routine use of cholangiogram and substantiate the benefits of such a practice, in not only identifying anomalies, and detecting CBD stones, but also limiting the extent of ductal injury by early recognition.

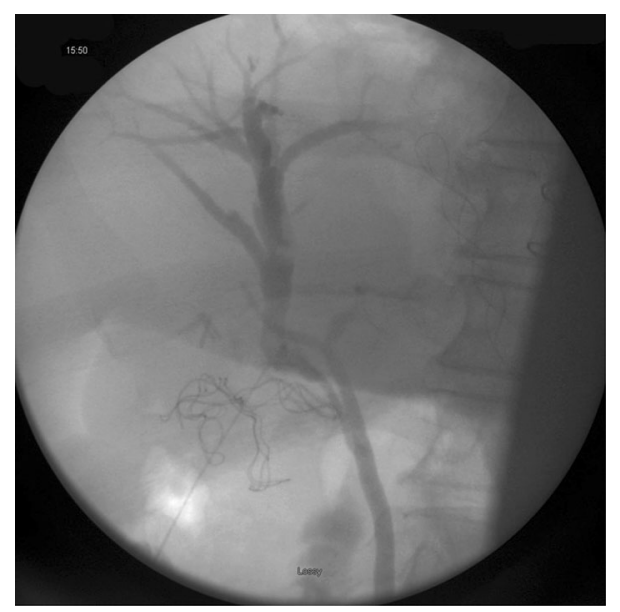

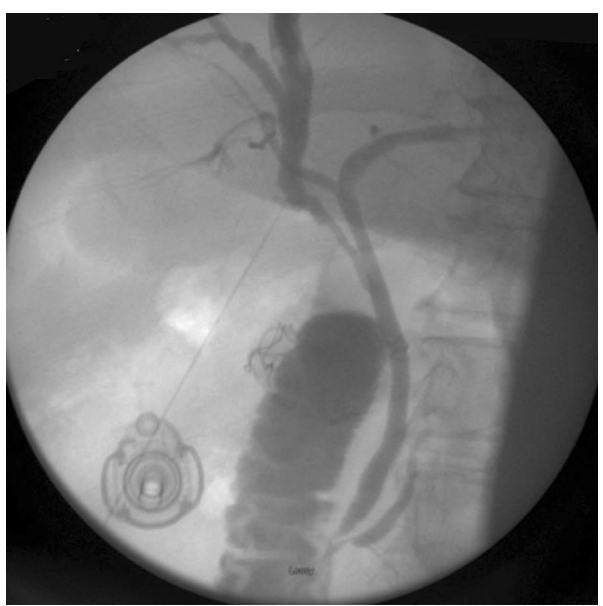
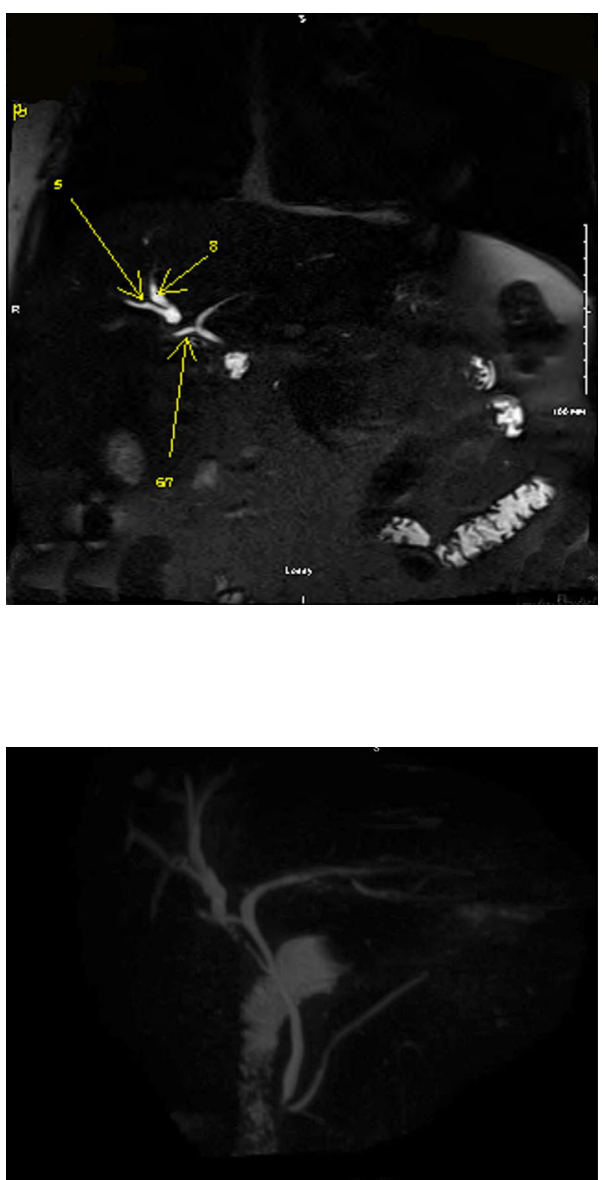
P260

Pancreatic Necrosectomy: Predicting the Morbidity and Mortality of Pancreatic Debridment

Noah J Swann, MD, Nobel Letendre, BS, Brian Cox, MD, James Recabaren, MD, FACS; Huntington Hospital

Introduction: Debridement of pancreatic necrosis is traditionally considered a morbid procedure. Previous multi-center studies using 2007 data have demonstrated 6.8\% 30-day mortality for pancreatic necrosectomy; Laparoscopic and percutaneous management of pancreatic and peri-pancreatic necrosis have become increasingly prevalent, but haven't fully supplanted the traditional open pancreatic Necrosectomy.

Objective: To evaluate the outcomes of pancreatic necrosectomy. Patient demographics, Ranson criteria, patient morbidity and mortality (both observed and expected) were analyzed. A multivariate stepwise regression was performed to analyze the clinical and laboratory parameters predictive of mortality. Patient demographics and comorbidities were analyzed. Post-operative complications were also evaluated.

Participants: The NSQIP national database was searched for all patients who had pancreatic or peri-pancreatic debridement using the Current Procedural Technology (CPT) code 48015 from January 1st, 2007 to December 31st 2015. After exclusion,1590 patients were identified during a 9 year interval.

Results: The patient population was predominantly male (71.2\%) and Caucasian $(69.8 \%)$. The mean age was 53.2 years; mean BMI was 29.7. Smokers comprised $24.1 \%$ of the cohort, with $33.9 \%$ of the cohort manifesting diabetes. The in hospital mortality (30 day) was 6.8\%. Patient BMI (OR 1.02-1.07), Age (1.01-1.03), BUN (1.02-1.03), INR (1.48-4.46), albumin (0.36-0.54) and total bilirubin (1.16-1.31) were independent predictors of mortality. All of these factors reached statistical significance with p-values $<0.05$ and significant odds ratios.

Conclusions: Our data demonstrates that pancreatic and peri-pancreatic debridement continues to be a morbid procedure, with 30 day in-hospital mortality (6.8\%) and overall morbidity similar to previous multi-center studies. We reaffirmed that BUN and Age were independent risk factors for mortality. BMI, INR, total bilirubin and albumin have also been demonstrated to be independent markers for mortality.

\section{P261}

Comparison Between Transpapillary Vs. Transduodenal Biliary Decompression for Biliary Obstruction: A meta-analysis

Aaron Pinnola, DO, Yuan Du, Paula Veldhuis, Sebastian De La Fuente; Institute for Surgical Advancement, Florida Hospital Orlando

Introduction: The effectiveness of transpapillary endoscopic retrograde cholangiopancreatography (ERCP) with stenting for palliative relief of biliary obstruction is well established. Recently, technical advances have led to the development of transduodenal endoscopic ultrasound guided biliary drainage (EUS-BD) for use in cases where the native papilla cannot be accessed. Traditionally, this technique has been mainly restricted to settings of a previously failed ERCP, but primary EUS-BD has now garnered interest to avoid ERCP procedure-related complications. In this study, we performed a metaanalysis comparing efficacy and complications of both approaches.

Methods: Articles written in English were searched in Pubmed. Studies with less than 10 patients, studies with no complications reported, when no comparison between techniques was done or studies with pediatric patients were excluded. Primary outcomes were defined as technical success and procedural complications. Secondary outcomes were clinical success and subsequent stent malfunction. Patient demographics and etiology of obstruction were collected. The pooled proportions were computed using either fixed-effects or random-effects models depending on homogeneity or heterogeneity. The Cochran Q test and I2 were used to evaluate for heterogeneity. The $95 \%$ CIs were calculated for all points estimates. P-value $<0.05$ was considered as statistically significant. Statistical analysis was performed using the $\mathrm{R}$ software. For binary outcome data, the relative risks (RR) were estimated.

Results: Initial database search yielded 245 studies from which 50 were selected for review, and 7 were chosen based on inclusion criteria for final analysis. There was no statistically different technical success rate found when comparing primary EUS-BD to ERCP RR: 1.04 (95\% CI $(0.96-1.13)), \mathrm{p}=0.33$. Likewise, primary EUS-BD compared to ERCP had no statistically different procedural complication rate 1.39 (95\% CI $(0.47-4.06)), \mathrm{p}=0.55$. Based on the Egger regression test and symmetric distribution, there was no obvious publication bias detected.

Conclusions: Primary EUS-BD for biliary obstruction was not associated with either an increased risk of procedural complications or technical success. This technique, however should be limited to palliative procedures to avoid biliary leakage and dissemination of malignant cells in patients with resectable disease. Further studies will be needed to assess the procedure's effect on long term patient outcomes and strict indications for use over primary ERCP. 
P262

\section{Scoring System for Preoperative Diagnosis of Gangrenous Cholecystitis}

Rebecca C Gologorsky, $\mathrm{MD}^{1}$, Justin R Tse, $\mathrm{MD}^{2}$, Aya Kamaya, $\mathrm{MD}^{2} ;{ }^{1}$ University of California San Francisco-East Bay, ${ }^{2}$ Stanford University

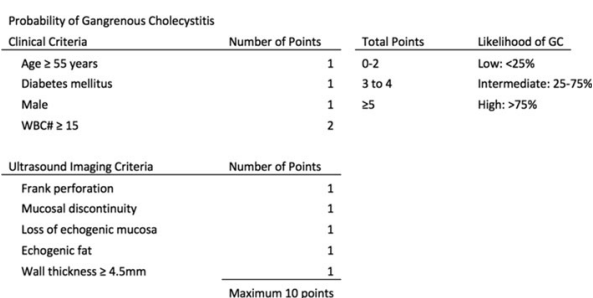

Gangrenous cholecystitis is a severe complication of acute cholecystitis and is associated with high morbidity and mortality. There are few characteristic clinical indicators of gangrenous cholecystitis, making preoperative diagnosis difficult. Previously published algorithms involve extensive mathematics and therefore have limited application. We identify sonographic and clinical features of gangrenous cholecystitis and propose a scoring system to facilitate preoperative diagnosis.

We retrospectively reviewed all adult patients from 1/2014-5/2018 who underwent abdominal ultrasound within 48 of cholecystectomy for acute cholecystitis. A diagnosis of gangrenous cholecystitis (GC) versus uncomplicated acute cholecystitis (UAC) was confirmed by review of pathology reports. Pertinent clinical features, demographics, and laboratory values were recorded. Ultrasonographic imaging features were reviewed by a blinded abdominal radiologist. Two-tailed t-tests and Fisher's exact tests were used to determine statistical significance for continuous and categorical variables, respectively. A multivariate analysis was performed using all statistically variables to create a model to predict a diagnosis of gangrenous cholecystitis.

One-hundred one patients met inclusion criteria. Forty-eight (48\%) had GC and $53(52 \%)$ had UAC. Points were assigned to each associated clinical or ultrasonographic feature as detailed in table 1. A total score of 0-2 points correlated with low probability $(<25 \%), 3-4$ points with intermediate probability $(25-75 \%)$, and 5 or more points high probability $(>75 \%)$ of GC. Of our patient cohort $45 \%$ (45/101) fell into the low probability, 29\% (29/101) fell into the intermediate probability, and $27 \%$ (27/101) fell into the high probability category. There were no UAC patients who were categorized as high risk. We propose a simple scoring system based on clinical and imaging features to facilitate preoperative diagnosis of GC. Prompt identification of GC may improve treatment planning and clinical outcomes. Evaluation of the impact of diagnosis timing versus surgical intervention and outcomes should be studied, as well as clinical factors that lead to late-stage diagnosis of cholecystitis.

\section{$\mathbf{P 2 6 4}$}

NSQIP Database Analysis does not Support Preoperative Antibiotic use for Outpatient Laparoscopic Cholecystectomy

Sora Ely, MD ${ }^{1}$, Rebecca C Gologorsky, $\mathrm{MD}^{1}$, Michelle R Huyser, $\mathrm{MD}^{1}$, Genna Beattie, MD ${ }^{1}$, C K Chang, MD ${ }^{2}$; UCSF East Bay Surgery, ${ }^{2}$ Kaiser Permanente Oakland Medical Center

Introduction: Debate continues regarding utility and safety of routine antibiotic prophylaxis for elective laparoscopic cholecystectomy. Several small, randomized controlled trials have failed to demonstrate benefit, while a handful of larger meta-analyses have reported statistically significant reductions in surgical site infections. However, most of these studies come from clinical settings outside the United States. We used a national database to examine factors associated with postoperative infection in outpatient laparoscopic cholecystectomy.

Methods: We retrospectively reviewed outpatient laparoscopic cholecystectomy cases from the 2015-2016 American College of Surgeons National Surgical Quality Improvement Program (NSQIP) database. Chi square test and ANOVA were used to identify pre- and intra-operative NSQIP variables associated with postoperative surgical infection, defined as any surgical site, deep wound, or organ space infection.

Results: Postoperative surgical infection occurred in 347 of 41,665 cases $(0.8 \%)$. Notably, incidence of Clostridium difficile infection in this population was also low $(0.2 \%)$, but not insignificant by comparison. Furthermore, the majority $(91.9 \%)$ of C.difficile infections occurred in the absence of a surgical infection.

Factors associated with higher infection rates included: age $>89$ years, hypertension, congestive heart failure, acute renal failure, dialysis, and chronic steroid use $(\mathrm{p}<0.05)$. Strongest predictors of postoperative infection included higher wound classification $(\mathrm{p}<0.001)$ and longer operative time $(>80 \mathrm{~min}$, $\mathrm{p}<0.001)$. Infection rates were low in wound classes $1-3$, with a marked increase only in class $4(0.7 \%, 0.7 \%, 1.2 \%, 3.2 \%$ respectively).

Using these two clinical variables, we developed a simple scoring system to predict infection risk. Figure 1 shows postoperative infection rates stratified by this score: operating room minutes $\leq 80$ (0) or $>80$ (1) plus wound class $1-3$ (0) or 4 (1).

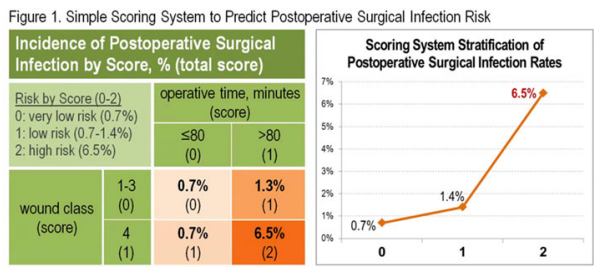

Conclusions: Risk of postoperative surgical infection after outpatient laparoscopic cholecystectomy is low, and the incidence of C. difficile infection is non-trivial. Although antibiotics may be associated with lower rates of postoperative infection, their marginal clinical benefit in the setting of low absolute risk is tempered by the risk of $\mathrm{C}$. difficile infection, antibiotic resistance, and other adverse effects of antibiotic use.

We recommend against routine antibiotic prophylaxis in low-risk patients undergoing elective, outpatient laparoscopic cholecystectomy. Antibiotics should be considered in cases for which the wound class is 4 and/or the operative time exceeds $80 \mathrm{~min}$. Surgeons may wish to utilize this scoring system intra-operatively to inform their decision to administer antibiotics during the case once operative time can be reasonably estimated. A randomized controlled trial is needed to validate this recommendation. 


\section{P265}

The Benefits of Our Glove Method for Single Port Laparoscopic Cholecystectomy

Noriaki Kameyama, MD, PhD, Yuki Mae, MD, Masashi Yahagi, MD, Kenta Inomata, MD, Tomoko Takesue, MD, Yoshinobu Akiyama, MD, PhD; KKR Tachikawa Hospital

Background: We introduced single port laparoscopic surgery at our hospitals in May 2009 and our experiences of single port laparoscopic surgery now number more than 1000 as of March 2018. Single port laparoscopic surgery has been gaining attention because of its effectiveness in reducing postoperative wound pain and superior cosmetic results with minimal scarring. In our hospitals, $80 \%$ of single port laparoscopic surgery cases were benign diseases such as cholecystectomy, appendectomy, and inguinal hernioplasty (TAPP and TEP). The most common procedure was cholecystectomy (43\%). We performed single port laparoscopic cholecystectomy via an $1.0 \mathrm{~cm}$ transumbilical incision (the minimum size for inserting the wound retractor) with the glove method. We examined the cost benefits of this method compared to other platforms for single port laparoscopic surgery and conventional laparoscopic cholecystectomy. Methods: An $1.0 \mathrm{~cm}$ incision was made at the bottom of the umbilicus. After small laparotomy, the Alexis wound retractor (size XXS) was inserted. After inserting the wound retractor, the surgical glove is attached and two low-profile laparoscopic $5 \mathrm{~mm}$ ports are inserted through the holes of the surgical glove with cut fingertips. The original pre-bending forceps inserted directly through the hole of the cut fingertip. We usually use $5 \mathrm{~mm}$ flexible laparoscopes by Olympus. The data from 484 cases of cholecystectomy performed by this technique were analyzed.

Results: A supplemental miniport or $5 \mathrm{~mm}$ port including conversion to conventional laparoscopic cholecystectomy, was required for $6.4 \%$, and the conversion to open surgery in only two cases $(0.4 \%)$. The rate of complications is only $2.9 \%$ with only one case of incisional hernia. Our follow-up outcome is continued until one year after surgery. Average hospital stay was 3.5 days, and operation time was $70 \mathrm{~min}$ overall. (63 min for expert surgeon) The average BMI in our patients was $24.6 \mathrm{~kg} / \mathrm{m}^{2}$

The cost of our glove method was $\$ 140.40$ compared to $\$ 224.50$ for "EZ-access", the most popular platform for single port laparoscopic cholecystectomy in Japan and \$163.60 for conventional laparoscopic cholecystectomy.

Conclusions: Single port laparoscopic cholecystectomy can be successfully performed via a minimal $1.0 \mathrm{~cm}$ transumbilical incision with our glove method. Our method for single port cholecystectomy is not only minimally invasive but also relatively safe and has cost benefits.

\section{P266}

Outcome of Laparoscopic Common Bile Duct Exploration After Failed Ercp. A Comparative Study

Davide Di Mauro $^{1}$, Edoardo Ricciardi ${ }^{1}$, Leandro Siragusa ${ }^{2}$, Antonio Manzelli ; ${ }^{1}$ Royal Devon and Exeter NHS Foundation Trust, Exeter, UK, ${ }^{2}$ Tor Vergata University Hospital, Rome, Italy

Introduction: Common bile duct stones (CBDS) are treated with endoscopic retrograde cholangiopancreatography (ERCP) followed by laparoscopic cholecystectomy (LC) or with the single-stage laparoscopic common bile duct exploration (LCBDE) and LC. Multiple ERCP attempts and failure increase the risk of post-procedural complications. In such circumstances surgery is advocated.

The aim of the study is to compare the outcome of LCBDE and LC in patients who had never had an ERCP, to that of patients who underwent previously failed ERCP.

Methods and Procedures: A retrospective analysis of 54 patients undergoing LCBDE and LC between 2010 and 2017, was performed. Patients were divided in 2 groups: primary surgery (group 1), surgery after failed ERCP (group 2). Demographics and preoperative investigations results were collected. Comparative outcomes were CBD clearance rate, operative time, conversion to open rate, postoperative morbidity, mortality, hospital stay. Student's t-test was used for nonparametric data; two-tailed Chi square or Fisher's tests were used to compare differences in frequencies. Results were considered as statistically significant when $\mathrm{p}<0.05$.

Results: Results are shown in table 1 below:

\begin{tabular}{|l|l|l|l}
\hline Parameter & Group 1 (21) & Group 2 (33) & P \\
\hline Agc mcan \pm SD & $49.23 \pm 16.3$ & $61.73 \pm 18.9$ & $<0.05$ \\
\hline CBD diameter (mm) mean \pm SD & $7.51 \mathrm{~mm} \pm 2.62$ & $11.78 \pm 4.08$ & 0.0001 \\
\hline CBD clearance (\%) & $19(90.5)$ & $30(90.9)$ & NS \\
\hline Local dense fibrosis (\%) & $4(19)$ & $16(48.4)$ & $<0.05$ \\
\hline Conversion to open (\%) & $2(9.5)$ & $4(12.1)$ & NS \\
\hline $\begin{array}{l}\text { CBDE approachn. } \\
\text { Transcystic }\end{array}$ & 14 (66.7) & $4(12.1)$ & 0.0001 \\
Choledochal & $7(33.3)$ & $29(87.9)$ & \\
\hline T-tube insertion (\%) & $1(4.8)$ & $5(15.2)$ & NS \\
\hline Intraoperative time (min) mean \pm SD & $130.3 \pm 83.72$ & $178.73 \pm 57.22$ & $<0.05$ \\
\hline Complicationsn (\%) & $2(9.5)$ & $5(15.2)$ & $\mathrm{NS}$ \\
\hline & & & \\
\hline
\end{tabular}

No postoperative mortality was recorded.

Conclusions: LCBDE and LC is safe and effective in patients who had previous failed ERCP. If ERCP failure is anticipated and/or the risk of post-ERCP complications is high, surgery should be considered as the first line treatment of CBDS. Longer intraoperative time and hospital stay are expected. 


\section{P267}

Intracorporeal Versus Extracorporeal Anastomosis in Minimally Invasive Right Hemicolectomy: A Single Institution Case Series Comparison

Peyman Lavi, MD, Vikram Attaluri, MD, Elisabeth C Mclemore, MD; Los Angeles Medical Center, Southern California Permanente Medical Group

Intracorporieal Anastomosis (ICA) vs. Extracorporeal Anastomosis (ECA)

\begin{tabular}{|c|c|c|}
\hline & ICA & ECA \\
\hline Number of Patients & 20 & 21 \\
\hline $\begin{array}{l}\text { Number of Laparoscopic Cases } \\
\text { (\# Robotic Cases) }\end{array}$ & $17(3)$ & $20(1)$ \\
\hline Median Age (Range) & 65 Years old $(38-87)$ & $\begin{array}{l}73 \text { years old (54- } \\
87)\end{array}$ \\
\hline Female Sex & 10 Female & 10 Female \\
\hline Median BMI (Range) & $26(19-43)$ & $24(18-36)$ \\
\hline Colon Cancer Diagnosis & 16 & 20 \\
\hline $\begin{array}{l}\text { Median OR Time in Minutes } \\
\text { (Range) }\end{array}$ & $168 \min (83-227)$ & $\begin{array}{l}130 \text { Minutes (76- } \\
\text { 320) }\end{array}$ \\
\hline $\begin{array}{l}\text { Median Length of Stay in Days } \\
\text { (Range) }\end{array}$ & 2 days (1-17) & 3 days $(1-14)$ \\
\hline \multicolumn{3}{|l|}{ COMPLICATIONS } \\
\hline Wound: Surgical Site Infection & 1 & 0 \\
\hline Wound: Seroma & 1 & 0 \\
\hline Thromboembolic Event & $\begin{array}{l}1 \text { Mesenteric Vein } \\
\text { Thrombosis }\end{array}$ & $\begin{array}{r}1 \text { Pulmonary } \\
\text { Embolism }\end{array}$ \\
\hline Urinary Tract Infection (UTI) & 1 & 1 \\
\hline Ileus & 1 & 1 \\
\hline Bacteremia & 0 & 1 \\
\hline Anastomotic Bleeding & 0 & 1 \\
\hline
\end{tabular}

Background: Minimally invasive right hemicolectomy with intra-corporeal anastomosis (ICA) has been associated with reduced ileus and shorter length of stay when compared to extra-corporeal anastomosis (ECA). The ICA technique was adopted by colorectal surgery in June of 2015. The short-term outcomes before and after ICA adoption are reviewed

Methods: An IRB approved retrospective case series review was performed. All laparoscopic and robotic right hemicolectomy cases performed by colorectal surgery at a single teaching institution before and after adoption of ICA into clinical practice were included.

Results: 41 patients underwent right hemicolectomy for colon cancer in the majority of patients from 10/2014 - 6/2018. ECA was performed in 21 cases. The median operative time was longer in the ICA group (168 vs. $130 \mathrm{~min}$ ). The median length of stay (LOS) was shorter in the ICA group ( 2 days vs. 3 days). The complication rate was similar with the exception of anastomotic bleeding requiring blood transfusion in the ECA group only.

Conclusions: Right hemicolectomy with ICA has similar outcomes compared to ECA. ICA is associated with a shorter LOS and lower anastomotic bleeding related complications when compared to ECA in the early adoptive phase.

\section{P268}

Is Laparoscopic Colectomy Suitable for Right Colon Cancer More Than 6 Centimeter? A Case-Matched Study with ShortTerm and Long-Term Outcomes

Weisheng Chen, Jiang Wei, Kai Li, Jun Yan, Xiaoyu Dong, Aorong Ouyang, Dexin Chen, Shaopeng Zhou, Huixin Xu, Pu Luo;

Department of General Surgery, Nanfang Hospital, Southern Medical University

Background: In China, the indication of laparoscopic right colectomy is tumor less than $6 \mathrm{~cm}$. The purpose of this study is to investigate whether laparoscopic right colectomy is suitable for right colon cancer more than $6 \mathrm{~cm}$.

Methods: A case-matched study was conducted to compare short-term and long-term outcomes between laparoscopic right colectomy and open right colectomy using propensity scores from patients with right colon cancer more than $6 \mathrm{~cm}$ between January 2006 and December 2015 .

Results: In our institution, 224 eligible patients with right colon cancer more than $6 \mathrm{~cm}$ underwent laparoscopic or open right colectomy by same surgical team. 150 patients were selected by 1:1 propensity score matching, with 75 patients in the laparoscopic group and 75 in the open group. Mean operating time, hospital costs, postoperative complication rate were similar between two groups. Length of incision, estimated blood loss, time to first flatus, time to first liquid intake, hospital stay of laparoscopic group were significantly shorter than those of open group. Harvest lymph node of laparoscopic group was significantly more than that of open group. Conversion was performed in $6.67 \%$ (5 out of 75) cases. The significant differences of overall survival and disease-free survival between two groups were not observed $(p=0.843 \& p=0.765$, respectively).

Conclusions: Laparoscopic right colectomy is suitable for patients with right colon cancer more than $6 \mathrm{~cm}$ in centers with expertise in minimally invasive surgery.

Comparisons between laparoscopic colectomy $(\mathrm{LC})$ group and open colectomy (OC) group in overall survival and disease-free survival

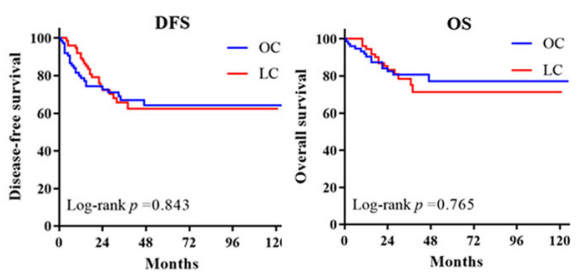


P269

\section{Comparison of Preoperative Versus Postoperative Transversus Abdominis Plane Block in Patients Undergoing Robotic-Assisted Colorectal Surgery}

Julia Xia, BA ${ }^{1}$, Terrah J Paul Olson, MD ${ }^{1}$, Yuan Liu, SPH ${ }^{1}$, Shawn Tritt, $\mathrm{MD}^{2}$, Seth A Rosen, $\mathrm{MD}^{1}$; ${ }^{1}$ Emory University School of Medicine, ${ }^{2}$ Emory Johns Creek Hospital

Introduction: Using transversus abdominis plane block (TAP) decreases pain scores and narcotic use post-operatively after colorectal surgery (CRS). It is unclear if TAP effectiveness varies if performed before versus after surgery. Methods: All eligible patients undergoing CRS are offered TAP. In 2017, we transitioned from pre-operative to post-operative TAP to improve efficiency. Using a prospectively maintained database of robotic-assisted surgery, we compared 115 patients who underwent pre-operative TAP with 53 patients who underwent post-operative TAP. Primary endpoints were pain scores and morphine equivalent (ME) use post-operatively. Secondary endpoints included perioperative factors contributing to pain scores and post-operative ME use. Summary statistics and univariate analysis by non-parametric tests were utilized.

Results: From April 2015 through May 2018, 168 patients received TAP before or after CRS. The cohort included $79(47.0 \%)$ women, average age $59.11( \pm 12.32)$ years, and mean body mass index (BMI) $28.32( \pm 5.82) \mathrm{kg} /$ $\mathrm{m}^{2}$. Surgery indication was cancer in $66(39.3 \%)$, polyp in $43(25.6 \%)$, and diverticulitis in $43(25.6 \%)$ patients. Right colectomy was performed in 61 $(36.3 \%)$, low anterior resection (LAR) in $46(27.4 \%)$ and sigmoid colectomy in $40(23.8 \%)$ patients. Demographics of the two groups were equivalent. Comparison of post-operative pain scores and ME use revealed no significant differences (See Table and Graph). Average pain scores and ME use were lower in patients who were older, underwent right colectomy, or had intracorporeal anastomosis.

Conclusions: In our cohort, no differences were seen in pain scores or ME use for patients receiving TAP pre-operatively versus post-operatively.
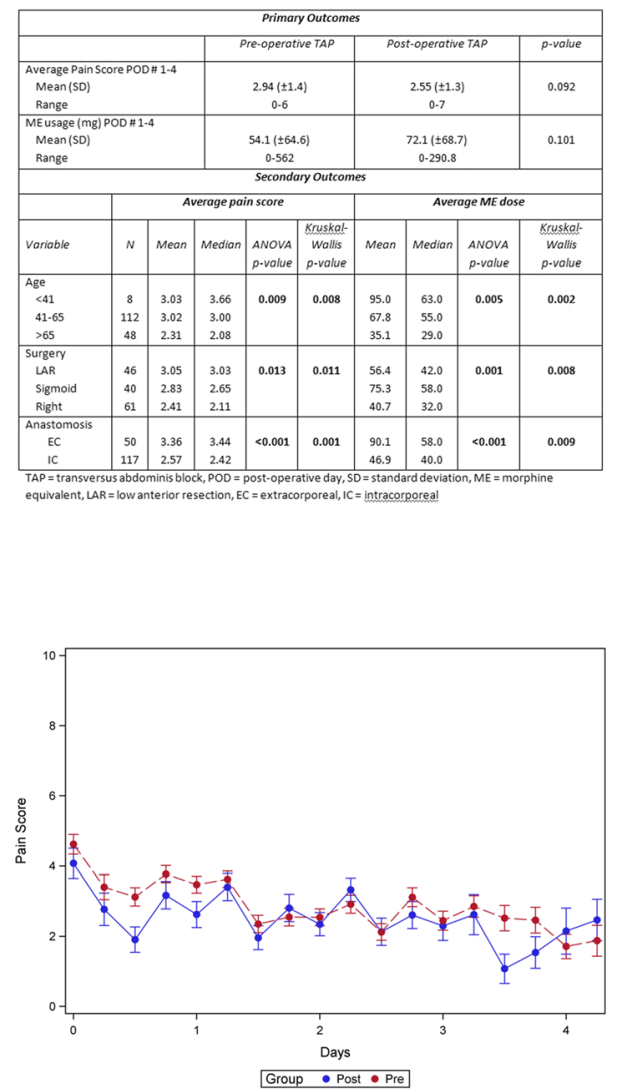

P270

A North American Single-Blinded Pilot Randomized Controlled Trial for Outpatient Non-antibiotic Management of Acute Uncomplicated Diverticulitis (Mud Trial): Feasibility and Lessons Learned

Safiya Al-Mansouri, MD, MSc ${ }^{1}$, Ebram Salama, MD ${ }^{1}$, Sebastien Lachance, MD, FRCSC ${ }^{1}$, Sarah Faris-Sabboobeh, MSc ${ }^{2}$, Julie Savard, $\mathrm{RN}^{2}$, Nancy Morin, MD, FRCSC ${ }^{2}$, Carol-Ann Vasilevsky, MD, FRCSC $^{2}$, Gabriela Ghitulescu, MD, FRCSC ${ }^{2}$, Julio Faria, MD, FRCSC $^{2}$, Marylise Boutros, MD, FRCSC ${ }^{2} ;{ }^{1}$ McGill University, ${ }^{2}$ Jewish General hospital

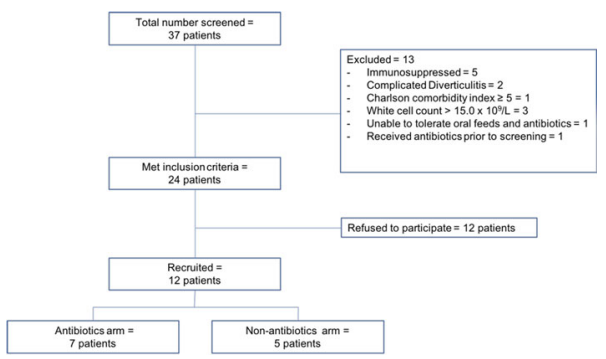

Introduction: Antibiotics remain the mainstay of treatment of uncomplicated diverticulitis in North America despite recent European literature challenging their utility. This pilot study assesses the feasibility of the first North American randomized controlled trial for outpatient non-antibiotic management of uncomplicated diverticulitis (clinicaltrials.gov identifier-NCT03146091).

Methods and Procedures: After institutional review board approval, adults presenting to the Emergency Department from April to September 2018 with CT-proven acute uncomplicated left-sided diverticulitis were screened for eligibility, and those meeting inclusion/exclusion criteria offered enrollment (Figure-1). Participants were randomized to receive either oral antibiotics or analgesia alone. Follow-up included daily phone calls for 7 days by a blinded nurse and clinic visits at 14-, 30-, and 60-days. Our primary endpoint was recruitment rate. Secondary outcomes included median visual analogue pain scores at 7 days and treatment failures (defined as persistent diverticulitis at 60-days, progression to complicated diverticulitis, hospital admission, and mortality). We determined a sample size of 40 patients would allow for an estimation of the recruitment rate to within a 14\% margin of error with $95 \%$ confidence. Reporting of the pilot complied with the 2010-CONSORT extension for Pilot/Feasibility Trials.

Results: Of the 37 patients screened, 24 met inclusion/exclusion criteria and 12 consented to participation. This represented a recruitment rate of $32 \%$. Patients refusing participation $(\mathrm{n}=12)$ cited inability to attend clinic follow-ups, preconceived expectations of receiving antibiotics, and belief that antibiotics would expedite recovery. Seven patients were randomized to the antibiotic arm and 5 to the non-antibiotic arm. Median age of participants was 54 (30-70) years with an even gender distribution. Eleven patients (92\%) completed the 7-day phone follow-up and expressed that they were satisfied and reassured by the phone calls. Ninety percent completed at least 30 days of follow-up, but only $44 \%$ adhered to all scheduled visits. Median follow-up was 45 days. One patient $(8 \%)$ was lost to follow-up. There were no treatment failures in either group. Median pain scores at 7 days were 0 for both arms (0-2 for controls, $0-4$ for experimental).

Conclusions: A large-scale RCT is feasible assuming a $32 \%$ recruitment rate and $92 \%$ retention rate. Recruitment and retention rates are likely to improve with (1) a more rigorous electronic platform to facilitate screening and appropriate implementation of inclusion/exclusion criteria, and (2) less stringent follow-up (i.e. a single visit at 30-days and phone follow-ups over the study year). Further investigation into non-antibiotic management of acute uncomplicated diverticulitis is warranted. 
P271

\section{All That is Right Lower Quadrant Pain is Not Appendicitis}

Rebekah S Wood, BS ${ }^{1}$, Michael D Sarap, MD, FACS ${ }^{2}$; ${ }^{1}$ Wright State Boonshoft School of Medicine, ${ }^{2}$ South Eastern Ohio Regional Medical Center

Introduction: Three rare surgical cases in a rural setting over the course of three weeks of right lower quadrant abdominal pain. Resulting diagnoses of infarcted cecal diverticulum, cecal perforation due to actinomycosis infection, and low grade appendiceal mucinous neoplasm

Case Presentation, Management, and Outcome: 55-year-old male presented to emergency department with sudden onset right lower quadrant abdominal pain and nausea without fever or vomiting. CT scan with contrast showed no intestinal obstruction or inflammation, normal appendix, no abnormal fluid or gas collection, present diverticulosis without associated inflammation, and no kidney stones. He was admitted and given pain medicine and IV hydration without any improvement of symptoms. Decision was made to take patient to the operating room due to concerns for an ongoing acute process from no improvement of symptoms, peritoneal signs on exam, and white count of 16,000. Patient underwent laparoscopy and was found to have an infarcted cecal diverticulum. Pathology reported transmural necrosis and acute suppurative inflammation of cecal diverticulum. On post operative day 1 he was eating, walking and feeling well.

14-year-old male presented for intermittent right lower quadrant abdominal pain for 5 months after a negative appendicitis workup. Upon presentation, CT and ultrasound showed large phlegmon in right lower quadrant. Patient was taken to surgery for laparoscopy, conversion to laparotomy with ileocecectomy and primary small bowel-colon anastomosis. Pathology returned as perforation at base of cecum as primary event leading to abscess formation with possible cause being an Actinomycoses infection due to presence of sulfur granules. Pathology specimen sent to Children's infectious disease also indicated Actinomycoses as cause of perforation.

59-year-old male with mild right lower quadrant pain was referred after colonoscopy showed inverted large appendix and benign biopsies of the base of the appendix. Patient underwent outpatient laparoscopic appendectomy with removal of cuff of cecum due to concern for malignant etiology. Pathologic diagnosis of low-grade appendiceal mucinous neoplasm. Patient followed-up in office with complete resolution of symptoms.

Discussion: Appendicitis is a common etiology of right lower quadrant pain. However, there are many causes of right lower quadrant pain that mimic appendicitis. These other causes may differ from an appendicitis presentation in only one aspect. Full appendicitis workup if possible or exploratory laparoscopy with surgical abdomen while keeping in mind other etiologies of right lower quadrant pain, allowed for tremendous improvement post operatively for all three cases.

\section{P272}

\section{Laparoscopic Versus Open Emergent Sigmoid Resection for Perforated Diverticulitis}

Yongjin F Lee, MD, June Hsu, MD, Michael Battaglia, MS, Robert K Cleary, MD; St. Joseph Mercy Ann Arbor

Introduction: The role of the laparoscopic approach to emergent resection for diverticulitis is unclear. Previous studies have demonstrated its safety and feasibility. There is a need for a large riskadjusted database analysis of emergent operations for Hinchey III or IV diverticulitis that focuses on the role for minimally invasive options. The objective of this study is to compare outcomes between the laparoscopic and open approach to emergent sigmoidectomy for perforated diverticulitis.

Methods and Procedures: The national American College of Surgeons-National Surgical Quality Program (ACS-NSQIP) database was queried for cases of laparoscopic and open emergent sigmoid resection for perforated diverticulitis from January 1, 2012 through December 31, 2016. Propensity scores were converted to weights and weighted generalized linear models created. 30-day outcomes were compared between emergent laparoscopic and open sigmoidectomy. Two subgroup analyses were performed comparing 1) laparoscopic to open Hartmann's procedure, and 2) laparoscopic to open sigmoidectomy with primary anastomosis and diverting stoma.

Results: 2,845 patients were included in the study - 2,581 open, and 264 laparoscopic sigmoidectomy. Compared to the laparoscopic approach, open sigmoidectomy had a higher rate of complications overall ( $\mathrm{p}<0.001)$, and a longer hospital length of stay $(\mathrm{p}<0.001)$. Specifically, the open approach had higher rates of ileus $(\mathrm{p}=0.003)$, acute renal failure $(\mathrm{p}<0.001)$, unplanned intubation $(\mathrm{p}<0.001)$, superficial SSI $(p<0.001))$, re-operation within 30 days $(p=0.04)$, and discharge destination other than home $(\mathrm{p}<0.001)$. Mortality rate favored the laparoscopic to open approach (2.99\% vs. $6.97 \%$, respectively), although this difference was not statistically significant $(p=0.089)$. Subgroup analyses showed similar findings. 2,007 patients underwent open and 152 patients underwent laparoscopic Hartmann's procedure. Compared to laparoscopic, open Hartmann's had an overall higher rate of complications $(\mathrm{p}<0.001)$, including a higher rate of acute renal failure $(p<0.001)$, pneumonia $(p=0.02)$, unplanned intubation $(\mathrm{p}<0.001)$ and superficial SSI $(\mathrm{p}<0.001)$. Mortality was higher but not statistically significant following open compared to laparoscopic Hartmann's (7.35\% vs. $2.09 \%$, p = 0.07). Subgroup analysis comparing laparoscopic and open sigmoidectomy with primary anastomosis and diverting stoma favored the laparoscopic approach, although it was limited by sample size $(\mathrm{n}=10$ and 85 , respectively).

Conclusion: The laparoscopic approach has better outcomes than the traditional open approach in emergent sigmoid resection for perforated diverticulitis. Additional studies are needed to confirm this finding in a randomized controlled setting. 


\section{P273}

Open Colectomies Associated with Increased Retrieval of Twelve or More Lymph Nodes Compared to Laparoscopic Colectomies in Colon Cancer Surgery

Yana Puckett, MD, MPH, MBA, MSc, Diana Mitchell, MBA, RN, Theophilus Pham, MBA, Amir Aryaie, MD; Texas Tech University Health Sciences Center

Background: Studies have suggested that excising 12 or more lymph nodes during colectomy in patients with colon cancer is associated with improved survival. To date, no study has investigated whether minimally invasive surgery affects ability to retrieve $12+$ lymph nodes in elective colon cancer surgery. We elected to determine whether a difference exits on ability to retrieve $12+$ nodes in elective colon cancer colectomies performed open vs. laparoscopic.

Methods: The National Surgical Quality Improvement Program (NSQIP) database was analyzed for the year 2014, 2015. Inclusion criteria was Colon Cancer (ICD-9 Code 153.9), age greater than 18 . Exclusion criteria was missing data. Data abstracted included number of lymph nodes retrieved and type of operation performed. Data was compared between patients that underwent laparoscopic colectomy compared to open colectomy. Binary logistic regression was used to identify confounding variables in retrieval of $12+$ lymph nodes.

Results: After accounting for missing cases, a total of 18,792 patients with a diagnosis of colon cancer were analyzed. Greater than 12 lymph nodes were retrieved in $88 \%(16,538)$ of patients. Overall mean lymph nodes retrieved were the same between two groups 20 (SD 11) $(\mathrm{p}=0.43)$. However, open operative approach compared to laparoscopic was associated with $15 \%$ greater odds of retrieval of 12 + lymph nodes (OR 1.148; 95\%CI (1.035-1.272); P = 0.009).

Conclusion: Majority of colectomies, whether done open or laparoscopically, retrieve 12 or more lymph nodes. However, there may be increased odds of retrieving more than 12 lymph nodes with open approach compared to laparoscopic.

\section{P274}

\section{The Relaparoscopy Threshold in Colorectal Surgery}

Mariana Matzner Perfumo, MD, José Piatti, MD, Mariano Laporte, MD, Maximiliano Bun, MD, Nicolás Rotholtz, MD; Hospital Aleman

Introduction: In the last few years there was an increased use of laparoscopic approach for colorectal surgery as well as the use of this approach to treat postoperative complications (re laparoscopy). Like in other procedures, re-laparoscopy in colorectal surgery requires a learning curve. The aim of this study was to identify variables that determines the acquisition of the learning curve.

Methods and Procedures: A consecutive cohort of patients who underwent re-laparoscopy after colorectal surgery was analyzed from a prospectively collected database during the period 2002-2017. Patients were divided into two groups according to surgeon's experience: G1: less than five years of attending; G2: more than five years of attending. Demographics data, intraoperative complications, and postoperative outcomes were analyzed.

Results: From a total of 1580 patients who underwent laparoscopic colorectal resection, $146(9,3 \%)$ required a re-operation. One hundred of them underwent a re-laparoscopy. The median age was 60.5 (2792) years. There were no differences in demographic data and conversion rate between the groups. In G2 CT scan was used as diagnostic tool before re-laparoscopy with lower frequency $\{\mathrm{G} 1$ : $37(74 \%)$ vs. G2 $26(52 \%) ; \mathrm{p}<0.05)\}$. There was a trend towards shorter time to decide the re-laparoscopy as well as shorter operative time in $\mathrm{G} 2(\mathrm{p}<0,08)$. There were no differences in length of hospital stay, post-operative morbidity and mortality rate.

Conclusion: Experienced colorectal surgeons showed earlier time to decide the re-laparosopy and shorter surgical time as compare to their younger counterparts. Time to re-laparoscopy and use of imaging studies to diagnose postoperative complications might be good indicators of reaching the learning curve in colorectal surgery.

\begin{tabular}{|l|c|c|c|c|c|}
\hline \multicolumn{1}{|c|}{ Variables } & $\begin{array}{c}\text { <12 Nodes } \\
\text { Retrieved } \\
(\mathbf{n}=2,254)\end{array}$ & $\begin{array}{c}>12 \text { Nodes } \\
\text { Retriered } \\
(\mathbf{n}=16,538)\end{array}$ & $\begin{array}{c}\text { Odds } \\
\text { Ratio }\end{array}$ & $\begin{array}{c}95 \% \text { Confidence } \\
\text { Interval }\end{array}$ & P-Value \\
\hline $\begin{array}{l}\text { Laparoscopic } \\
\text { Colectomy } \\
(\mathrm{n}=2,960)\end{array}$ & $15.00 \%(444)$ & $85.00 \%(2,516)$ & 1.122 & $0.985-1.279$ & 0.084 \\
\hline $\begin{array}{l}\text { Oopen } \\
\text { Colectomy } \\
(\mathrm{n}=5,852)\end{array}$ & $9.71 \%(568)$ & $90.29 \%(5,284)$ & 1.148 & $1.035-1.272$ & 0.009 \\
\hline
\end{tabular}


P275

Does Enhanced Recovery After Surgery Affect Time to Delivery of Adjuvant Systemic Therapy in Patients with Stage III Colon Cancer?

Tiffany Paradis, Maude Trepanier, Pepa Kaneva, A. Sender Liberman, Patrick Charlebois, Barry L Stein, Liane S Feldman, Lawrence Lee; McGill University Health Center

Introduction: Timely delivery of adjuvant systemic therapy is important for oncologic outcomes in stage III colon adenocarcinoma. However, many patients do not receive or have delays in adjuvant treatment secondary to poor recovery or postoperative complications. Enhanced recovery pathways(ERP) decrease complications and improve recovery, but their effect on delivery of adjuvant treatment is unknown. Therefore, the objective of this study is to determine the effect of ERPs on the time to delivery of adjuvant systemic therapy and overall survival(OS) in patients with resected stage III adenocarcinoma.

Methods: All patients with stage III adenocarcinoma undergoing elective surgery at a single colorectal referral centre from 01/2005-12/2013 were reviewed. Patients were divided into two groups: ERP and conventional car$\mathrm{e}(\mathrm{CC})$. The ERP was implemented fully in 2010. The main outcome measured was time to initiation of adjuvant systemic therapy (none, $\leq 8$ weeks, and $>$ 8 weeks) and 5-year overall survival(OS). 30-day postoperative complications were grouped using the Clavien-Dindo classification into none, mild (ClavienDindo 1-2) and severe (Clavien-Dindo $\geq 3$ ). Kaplan-Meier method was used to estimate OS. Multinominal logistic regression was performed to identify predictors of time to initiation of adjuvant chemotherapy (reference group: $\leq 8$ weeks). A Cox proportional hazard model was used to determine predictors of 5-year OS.

Results: A total of 209 patients were included(112 ERP, 97 CC). Patients were well balanced between groups. ERP was associated with shorter hospitalization (4 days[IQR3-7] vs. CC 6 days[IQR 5-10], $\mathrm{p}<0.001$ ) and fewer complications $(39.1 \%$ vs. $57.6 \%, \mathrm{p}=0.028)$. Median time to initiation of adjuvant therapy (ERAS 67 days[IQR57-80] vs. CC 65 days[IQR56-80], $\mathrm{p}=0.415$ ) and delays in initiation $(63.1 \%$ vs. $63.5 \%, \mathrm{p}=0.288)$ were similar (Figure 1A). Five-year OS was also similar between the two groups (ERAS $56.6 \%$ vs. CC $45.0 \%$, logrank $\mathrm{p}=0.319)$. The multinomial model for time to initiation of adjuvant treatment reported that only older age (OR1.05 per year, 95\%CI1.011.11) and severe complications (OR9.92, 95\%CI1.02-30.04) independently predicted no receipt. Only male gender (OR2.38, 95\%CI1.01-5.56) predicted delay $>8$ weeks. In the adjusted survival analysis, no adjuvant treatment (HR2.56, 95\%CI1.17-5.60), stage IIIC (HR3.36, 95\%CI1.40-8.05), and severe complications (HR2.78, 95\%CI1.06-7.35) were associated with poorer 5-year OS. Perioperative care was not independently associated with delivery of adjuvant treatment or 5-year OS.

Conclusion: Perioperative management did not affect delivery of adjuvant systemic therapy or long-term survival for stage III colon cancer. The occurrence of severe complications appears to have the greatest effect on both adjuvant treatment and overall survival.

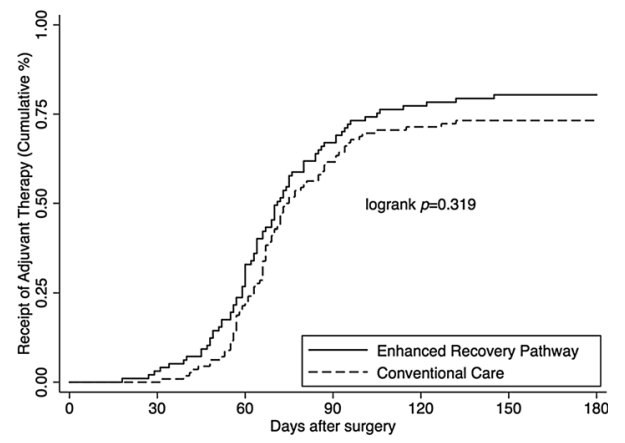

P276

A Unique Case of Metastatic Cervix Squamous Cell Carcinoma Presenting as a Large Bowel Obstruction

Andrew Lelchuk, $\mathrm{DO}^{1}$, Nicholas Morin, $\mathrm{DO}^{2}$, Vadim Meytes, $\mathrm{DO}^{3}$, Michael Nicoara, $\mathrm{DO}^{2}$, Kevin Bain, $\mathrm{DO}^{2} ;{ }^{1}$ Nova Southeastern University College of Osteopathic Medicine, ${ }^{2} \mathrm{NYU}$ Langone Hospital - Brooklyn, ${ }^{3}$ Vassar Brothers Medical Center

Introduction: Cervical cancer is the third most common cancer in women. Histologically, squamous cell carcinoma (SCC) accounts for approximately $70 \%$ of all cervical cancers. Disease progression through metastasis or recurrence usually occurs within the first two years of treatment. Metastasis to the gastrointestinal tract is extremely uncommon.

Case report: A 47 year-old female presented to the ED with abdominal pain and bloody stool for two days. Past history was significant for hysterectomy for uterine leiomyomas. Pathology revealed high grade dysplasia with squamous cell carcinoma in situ, and negative margins.

In the ED a CT scan was obtained which demonstrated dilated loops of bowel, with a transition point at the rectosigmoid junction secondary to a soft tissue density.

The patient was taken to the operating theatre for non resolving high grade colonic obstruction. A densely adherent mass was discovered in the pelvis. En bloc resection was performed of the rectosigmoid colon, bilateral ovaries, and portion of the bladder wall. Surgical pathology demonstrated poorly differentiated squamous cell carcinoma of cervical origin.

Discussion: Squamous cell carcinoma (SCC) of the colon is a rare entity. As a primary tumor its pathogenesis is unclear, and as a secondary tumor it arises through metastasis. It is important to differentiate between the two, as metastatic SCC to the colon carries a significantly worse prognosis. In either case, early detection and prompt intervention improve overall survival and disease free survival rates.

Colonic metastasis is often reported from primary sites such as the breast, kidney, ovary, and melanomas. It is rare that metastatic disease to the colon arises from the cervix. In advanced SCC of the cervix, distant metastasis occurs in $9-27 \%$ of patients. The most common sites include lung and para-aortic lymph nodes.

In general, sigmoid colon metastasis carries a poor prognosis due to its late presentation. The treatment for colonic metastatic tumor arising from cervical SCC remains controversial, as not enough cases have been reported to compare treatment outcomes. Surgery and debulking are the primary treatment modalities, while the role for radiotherapy and chemotherapy remain somewhat ambiguous.

Conclusion: In this report, we describe the case of colonic metastasis presenting as a large bowel obstruction. Surgery is the primary mode of therapy. Radiotherapy is indicated for unresectable masses, while the role of chemotherapy remains controversial. Ultimately, resection of metastatic lesions may help in palliative measures, and prevent recurrent obstructions. 


\section{P277}

Transanal Minimally Invasive Surgery for the Treatment of T1/ T2 Rectal Cancer in Elderly Patients

Yu Sato, MD, PhD, Takashi Oshiro, MD, PhD, Tomoaki Kitahara, MD, Yutaka Yoshida, MD, Tasuku Urita, MD, Ryuichi Takagi, MD, Kentaro Kawamitsu, MD, Kengo Kadoya, MD, Taiki Nabekura, MD, Shin-Ichi Okazumi, MD, PhD; Department of Surgery, Toho

University Sakura Medical Center

Introduction: In elderly patients with cancer, radical surgery is sometimes not selected because of their vulnerabilities. For cases of rectal cancer, total mesorectal excision has been regarded as a standard therapy. However, it can cause some lethal complications and impair the quality of life. Local excision (LE) for low to mid rectal cancer in elderly patients with T1/T2 lesions is an attractive therapeutic method. This study revealed the outcomes of our conventional transanal LE and evaluated the benefit of transanal minimally invasive surgery (TAMIS) for the treatment of $\mathrm{T} 1 / \mathrm{T} 2$ rectal cancer in elderly patients.

Methods and Procedures: Fifteen patients with low rectal tumors underwent conventional non-endoscopic transanal LE at our institution between January 2012 and December 2016. After this period, TAMIS was applied to the LE in elderly patients with T1/T2 rectal cancer, and chemoradiation (45 Gy plus $1650 \mathrm{mg} / \mathrm{m}^{2}$ of capecitabine per day) was administered to those who were pathologically diagnosed as possessing high-risk factors for lymph node metastasis. We presented 3 cases of this treatment.

Results: In the cohort of conventional transanal LE, the range of tumor location was 1 to $5 \mathrm{~cm}$ from the anal verge. Piecemeal resection of the tumor was performed in 2 patients. Postoperative bleeding occurred in 1 patient. Six patients $(40 \%)$ had positive or unevaluable margins, and 3 patients (20\%) had recurrences. Among the TAMIS group, en bloc full-thickness resection with clear margins was possible in all patients. No intraoperative or postoperative complications occurred, and anal function after treatment was retained as that before surgery. One patient had a pT2 tumor with lymphatic invasion and received adjuvant radiotherapy, but he developed distant recurrences 3 months after surgery. On the other hand, 2 patients had pT1 cancer and were followed-up observationally without any additional treatment. Both patients did not show evidence of recurrence.

Conclusions: TAMIS followed by chemoradiotherapy was demonstrated to be safe for elderly patients with $\mathrm{T} 1 / \mathrm{T} 2$ rectal cancer. While long-term oncological outcomes are limited, this treatment might be less invasive for vulnerable patients.

\section{P278}

The Feasibility and Application Value of D3 Lymph Node Dissection around the Superior Mesenteric Artery with Preservation of Autonomic Nerves for the Treatment of Right Hemi-colon Cancer

Xiaojiang Yi, Dechang Diao; Department of Gastrointestinal (Tumor) Surgery, Guangdong Province Hospital of Chinese Medicine, The Second Affiliated Hospital of Guangzhou University of Chinese Medicine

Objective: To explore the feasibility and application value of the preservation of plant nerve function in the radical resection for right hemicolon cancer.

Methods: Clinical data of 55 cases of right hemicolon cancer undergoing laparoscopic D3 + CME radical resection, including 29 cases of partial resection of superior mesenteric plexus (nerve partial resection group, NPRG) and 26 cases of completely preserved of mesenteric plexus (nerve preserved group, NPG), from January 2017 to July 2018 in the department of Gastrointestinal Surgery of Guangdong Province Hospital of Traditional Chinese Medicine were retrospectively analyzed. Intra-operative and postoperative complications were compared between the two groups.

Results: The baseline information was not significantly different between the two groups (all $\mathrm{P}>0.05$ ). The operation time in the NPG and the NPRG group was $(164.0+19.8)$ and $(176.0+19.7) \mathrm{min}$ respectively, which has a significant difference $(\mathrm{P}<0.05)$. The number of harvested lymph nodes in the two groups were $28.5+7.8$ and $27.6+6.47$ respectively $(\mathrm{P}>0.05)$, which was not significantly different. One case (3.8\%) of Chylous leakage in the NPG group, which was significantly lower than that of the NPRG group (11 cases, $37.9 \%)(\mathrm{P}<0.05)$, meanwhile, the postoperative abdominal pain occurred in 4 cases $(15.4 \%)$ and 12 cases $(41.4 \%)$ in the two groups, which was significantly different $(\mathrm{P}<0.05)$. Compared with the NPRG group, there was no significant difference in the operative blood loss, postoperative time to flatus and postoperative hospitalization time in the NPG group $(\mathrm{P}>0.05)$. All patients were followed up for more than 6 months, and the rates of 30-day re-hospitalization and mortality were 0 .

Conclusion: It is safe and feasible to preserve the plant nerve in the operation of right hemicolon cancer and prevents the postoperative gastrointestinal dysfunction caused by nerve injury. 


\section{P279}

Recent Advances and Comparison of Different Fistula-in-ano Classifications: Has the Problem Been Solved?

Pankaj Garg, Dr ${ }^{1}$, Sachi Singhal, MBBS $^{2}$, Sachin B Jamma, MBBS, $\mathrm{MS}^{3}$, Suresh Vasistha, MBBS, $\mathrm{MS}^{4} ;{ }^{1}$ Garg Fistula Research Institute, ${ }^{2}$ Dayanand Medical College, Ludhiana, Punjab, India, ${ }^{3}$ Ashwini Rural Medical College, Kumbhari, Solapur, Maharashtra, India, ${ }^{4}$ Mangalam Hospital, Gurgaon, India

Objective: Classification is done to grade the disease according to severity and guide regarding its management. There are four classifications published for fistula-in-ano- $\operatorname{Parks}^{1}(1976)$, St James university hospital(SJUH) ${ }^{2}(2000)$ and Standard Practice Task Force $(\mathrm{SPTF})^{3}(2005)$ classification and a recent $\mathrm{Garg}^{4}(2017)$ classification. Considering there is lot of confusion regarding management of fistulain-ano, the objective of the study was to ascertain as which classification is most relevant and useful to the operating surgeon.

Method: The basis, methodology, utility(correlation with disease severity and guidance regarding disease management) and strong points of each classification were analyzed.

Results: All the classifications are compared in Figure-1.

Parks and SPTF were based on clinical experience, SJUH was MRI based and Garg was based on both clinical experience and MRI. Parks and SJUH did not correlate with disease severity whereas Garg and SPTF correlated well with disease severity. Whereas Parks, SJUH and SPTF were not validated by patient data, Garg classification was validated by patient data (440 patients). Whereas Parks, SJUH and SPTF had no role in the disease management, Garg classification guided the operating surgeon regarding the treatment of all types of anal fistulas. Garg grade I-II are simple fistulas and can be safely managed by fistulotomy without any risk to continence whereas Garg grade III-V are complex fistulas and fistulotomy should not be even attempted in these fistulas. The latter may be dealt with sphincter saving procedures like advancement flap, Anal Fistula Plug, LIFT or VAAFT. Thus this classification guides a general surgeon regarding the fistulas (grade I-II) which can be easily managed and the fistulas (grade III-V) that need to be referred to an expert(Figure-2).

Conclusion: Garg classification is a significant advancement over existing classification (Parks, SJUH \& SPTF) with regard to accuracy and utility to the operating surgeon. It classifies the fistulas accurately as per disease severity as well as guides the operating surgeon regarding the disease management. Therefore, Garg classification should be used by radiologists and the surgeons.
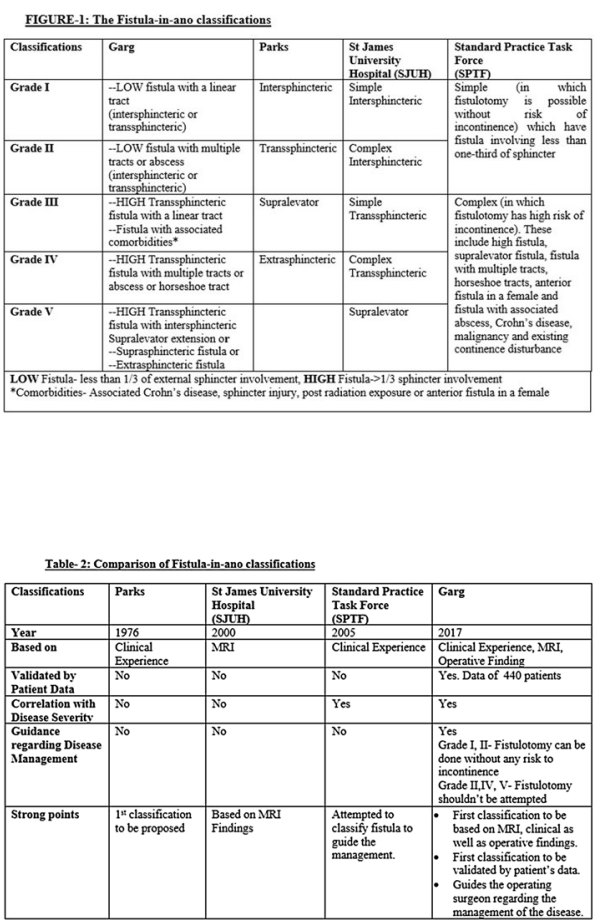

References

(1) Parks A. G.et al.(1976) A classification of fistula-in-ano. Br J Surg 63:1-12

(2) Morris J.et al.(2000) MR imaging classification of perianal fistulas. Radiographics 20:623-635

(3) Whiteford M. H.et al.(2005) Practice parameters for treatment of fistula-in-ano (revised). Dis Colon Rectum 48:1337-1342

(4) Garg P(2017) Comparing existing classifications of fistula-in-ano in 440 operated patients: Is it time for a new classification? Int J Surg 42:34-40 
P280

\section{Malignant Mixed Mullerian Tumor (MMMT) Presents as a Retro-rectal Mass: A Case Report}

Anthony Dakwar, MD, Melissa S Zoumberos, MD, Alexandria Glenn, MD, Sowsan Rasheid, MD; University of South Florida, Tampa General Hospital

Malignant Mixed Mullerian Tumors (MMMT) are a rare and aggressively malignant cancer of the female genital tract, usually arising from the uterus, cervix, and ovaries. MMMT are considered a carcinosarcoma, due their histological characteristics of both epithelial cells as well as connective tissues. Prognosis is mainly poor, even after appropriate surgery and adjuvant therapy treatments. We report a case of a MMMT having an unusual presentation as a retro-rectal tumor.

MMMT are mostly consistent with a history of vague abdominal pain and associated vaginal bleeding. These tumors can grow to a substantial size and encompass the entirety of the abdominal compartment, as an intra-peritoneal mass. The patient we are presenting complained of vague perineal pain along with a radiculopathy to her right leg. Physical exam revealed an enlarged right buttock, with mass effect along with erythema and skin-dimpling (Figure 1). The abdominal and pelvic exams were normal. Vaginal ultrasound and computed tomography (CT) did not illustrate the origin of this tumor, only confirming it location in the pre-sacral space tracking into the her pelvic organs. Magnetic resonance imaging (MRI) was obtained to further characterize the mass. It showed a $16 \times 11 \times 12 \mathrm{~cm}$ heterogenous mass with cystic components, hyper-intense on T2 weighted image (Figure 2). Tumor markers and other lab work were unremarkable.

The patient underwent a retro-rectal tumor excision via Kraske approach. The tumor appeared to be involving the uterus and base of the cervix at the time of surgery, requiring a trans-abdominal approach to adequately remove it and all its components. Patient recovered well and discharged home. Pathology revealed that the tumor was indeed a MMMT, arising from polypoid endometriosis.

In conclusion, we report a case of a Malignant Mixed Mullerian Tumor that presented in the pre-sacral space. Tumors of gynecological origin are known to occur in the retro-rectal space, however such a rare pathology as MMMT usually is limited to the peritoneal cavity. A high index of suspicion must be maintained during the evaluation of giant retro-rectal tumors that are found to be heterogenous with multiple cystic components on pre-operative imaging. The extent of disease in this case and its unusual location is indicative of the potential for aggressive endometrial transformation and migration.

Figure 1. Physical exam findings of right buttock.

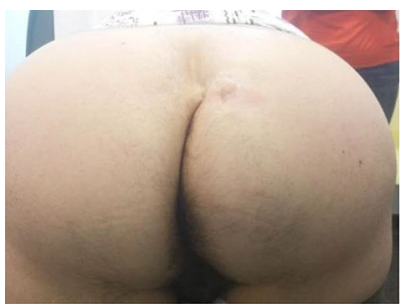

Figure 2.

Magnetic Resonance Image (MRI) of retro-rectal tumor.

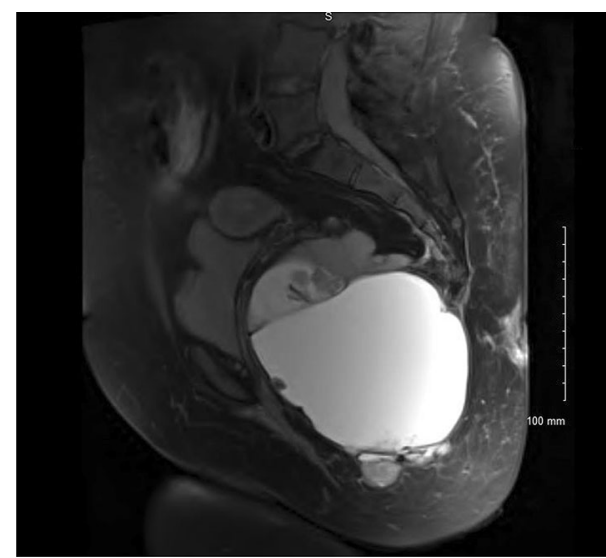

\section{P281}

\section{Combined Laparoscopic Right Hemicolectomy and Sigmoid} Colectomy for Simultaneous Cecal and Sigmoid Volvulus

\section{Hugo Bonatti, Kiran Khosha; Meritus Health, Hagerstown MD}

Introduction: Cecal (CV) and sigmoid volvulus (SV) are acute surgical disorders frequently requiring emergent surgery. Colonoscopic decompression may temporize the condition; however; CV and SV may recur requiring colectomy. Only few cases of combined $\mathrm{CV}$ and $\mathrm{SV}$ have been reported and open surgery has remained the more common approach.

Case Report: A 40-year-old African-American female with cerebral palsy came to the ER with acute abdominal distention and pain. CT-scan revealed acute CV Multiple episodes of SV had been treated nonoperatively in the past. The proximal bowel was decompressed through her PEG tube and the large bowel by colonoscopy. Laparoscopic (partial) colectomy with ostomy was planned. The patient was placed supine, trocars were placed in the left upper (U) and left lower quadrant (LQ) and into an old upper midline incision from a fundoplication. Transverse colon adhesions at the PEG tube site were lysed, the right $(\mathrm{R})$ colon was completely mobilized together with $25 \mathrm{~cm}$ terminal ileum involved in the torque; an extra corporeal ileotransverse anastomosis was created using a $4 \mathrm{~cm}$ periumbilical incision, which was closed. Trocars were switched to the RU and RLQ. The elongated, twisted sigmoid colon was mobilized out of the pelvis, the mesentery was divided and the rectum was stapled. The specimen was removed through the colostomy site in the LLQ. The postoperative course was uneventful.

Conclusion: We report the first laparoscopic right hemicolectomy plus sigmoid colectomy preserving the transverse/descending colon for combined $\mathrm{CV}$ and SV. Colonoscopic decompression temporized the disorder allowing for subsequent minimally invasive surgery. 


\section{P282}

Outcomes Following Colorectal Surgery: A Comparative Study of CR -POSSUM and ACS - NSQIP Risk Calculator

Nitin Vashistha, MS, FACS ${ }^{1}$, Arati Verma, MBBS, DHHM, PGDMLS $^{2}$, Dinesh Singhl, MS, FACS, DNB, Surgical, Gastroenterology ${ }^{1}$; ${ }^{1}$ Department of Surgical Gastroenterology, Max Super Speciality Hospital, Delhi, India, ${ }^{2}$ Department of Medical Quality, Max Super Speciality Hospital, Delhi, India

Background: Several studies have reported outcomes of colorectal surgery (CRS) based either on CR - POSSUM or ACS - NSQIP risk calculator. To the best of our knowledge, no study to date has evaluated/compared outcomes of the two risk calculators on same patient cohort .

We report outcomes of elective and emergency CRS by CR - POSSUM and ACS - NSQIP risk calculator.

Patients and Methods: This is a single center observational study of 86 consecutive adult ( $\geq 18$ years) patients who underwent elective or emergency, resective \&/or reconstructive CRS from March 2013 to March 2018. Procedures such as appendicectomy, diverting colostomy, laparoscopic rectopexy were excluded from analysis.

Data was accessed from institutional electronic health record system. The CR - POSSUM and ACS - NSQIP scores for each patient was calculated. Actual outcomes were then compared with those predicted by risk calculators.

Results: Of the 86 patients (60 Indians and 26 International) there were 56 males and 30 females with a mean age of 57 (range 18-93) years. Of these $59(68.6 \%)$ underwent elective whereas $27(31.4 \%)$ had emergency CRS. Sixty two (72\%) and 24 (38\%) patients underwent open and minimally invasive procedures (laparoscopic -21 and robotic -3 ) respectively. The median length of stay was 08 (range 02-40) days. The mortality following elective and emergency operations was $1 / 59(1.6 \%)$ and $8 / 27(29.6 \%)$ respectively.

By CR- POSSUM, ratio of observed to expected mortality was 1.29 with no significant difference between the two values.

Objective comparison between overall actual outcomes and those predicted by ACS - NSQIP risk calculator revealed no significant difference in any of the parameters studied (Figure 1).

A comparison of actual and predicted ACS - NSQIP outcomes in elective and emergency CRS is provided in Table 1. The score significantly underestimated SSI and over predicted the length of stay following emergency CRS.

Conclusions: Both CR - POSSUM and ACS - NSQIP risk calculator accurately predicted mortality in our cohort of CRS patients. CR POSSUM provides information on single domain of mortality only and requires operative findings to calculate the score and is therefore more useful for surgical audits. In comparison ACS - NSQIP risk calculator provides comprehensive information prior to surgical intervention that may be useful for both individual prognostication as well as surgical audits.
Figure 1: Actual versus predicted outcomes by ACS-NSQIP risk calculator

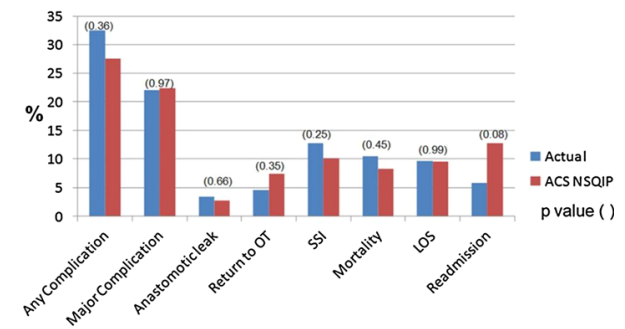

Table 1: Outcomes of Emergency and Elective CRS by ACS-NSQIP risk calculator

\begin{tabular}{|c|c|c|c|c|c|c|}
\hline & \multicolumn{6}{|c|}{ Table 1. Outcomes of CRS: AC } \\
\hline \multirow[t]{2}{*}{ Parameter } & \multicolumn{3}{|c|}{ Emergency $(n=27)$} & \multicolumn{3}{|c|}{ Elective (n=59) } \\
\hline & Observed & \begin{tabular}{|l} 
Predicted \\
\end{tabular} & Pvalue & Observed & Predicted & $\begin{array}{l}\text { Pvalue } \\
\end{array}$ \\
\hline $\operatorname{Los}($ Mean \pm SD) & $12.26 \pm 7.34$ & $17.87 \pm 6.32$ & 0.01 & $8.36 \pm 3.48$ & $5.78 \pm 1.71$ & 0.00 \\
\hline Return to OT (\%) & $1(3.7)$ & $3.37(12.46)$ & 0.20 & $3(5.08)$ & $2.97(5.04)$ & 0.99 \\
\hline $\begin{array}{l}\text { Anastomotic } \\
\text { Leak }(\%)\end{array}$ & 0 & $0.86(3.17)$ & 0.36 & $3(5.08)$ & $1.48(2.51)$ & 0.21 \\
\hline sSI (\%) & $7(25.9)$ & $2.85(10.54)$ & 0.01 & $5(8.47)$ & $5.75(9.75)$ & 0.75 \\
\hline $\begin{array}{l}\text { Any } \\
\text { Complicastion (\%) }\end{array}$ & $18(66.6)$ & $\begin{array}{l}12.91 \\
\text { (49.82) }\end{array}$ & 0.16 & $10(16.94)$ & $10.68(18.1)$ & 0.84 \\
\hline $\begin{array}{l}\text { Major } \\
\text { complication (\%) }\end{array}$ & $13(48.1)$ & $\begin{array}{l}10.79 \\
(39.94)\end{array}$ & 0.50 & $6(10.16)$ & $8.36(14.17)$ & 0.41 \\
\hline Readmission (\%) & $1(3.7)$ & $4.73(17.5)$ & 0.09 & $4(6.77)$ & $6.14(10.40)$ & 0.39 \\
\hline Mortality (\%) & $8(29.6)$ & $6.51(24.11)$ & 0.56 & $1(1.69)$ & $0.49(0.83)$ & 0.47 \\
\hline
\end{tabular}




\section{P283}

\section{Initial Experience Using Transanal Approach to Low Rectal} cancer

\section{Adrian Maghiar, George Dejeu, Octavian Maghiar; Spital Pelican} Oradea

In our institution we have a vast experience with rectal cancer patients, with a number of over 50 cases per year that get resection with anastomosis. In the recent years we have started studying and attending courses to using TA-TME. We have a 8 years experience of using transanal devices for both benign and malignant disease, but we only started doing TA-TME in our Clinic this year.

We present our experience after 5 cases of low rectal cancer patients using the transanal approach for the rectal dissection, using the conventional laparoscopic approach for the vascular dissection and the left colon mobilisation (we always mobilise the splenic flexure).

We operated on 4 men and 1 female. All patient were between the ages of 60 and 75, with no major comorbidities ( 3 male and 1 female with hypertension and 2 male with diabetes). The mean operating time was $230 \mathrm{~min}$, with the first case lasting $320 \mathrm{~min}$ and last one 195 min (skin to skin). All cases were R0 resection, with an average lymph node harvest of 16 nodes (13 to 25), and an average height of the anastomosis of $3 \mathrm{~cm}(1,5$ to $4 \mathrm{~cm})$. The average length of stay was 7 days (5 to 14). All patients had a ileostomy done prior to neoadjuvant therapy. 2 patients needed readmission before 30 days after surgery (one for constipation and dehydration, and one for vomiting). None patients presented with fistula, and all patients had their ileostomy reversed after 6 weeks with normal bowel function at 3 and 6 months (3 cases have more than 6 months, one has less than 3 months after surgery). We conclude that TA-TME is feasible in a center with a reasonably high volume of rectal cancer patients and a big experience with advanced oncologic laparoscopic surgery. The duration of surgery goes down with experience, but the visualisation during dissection is much superior than that of the normal laparoscopic approach. Needs further study to see the benefits compared to the robotic approach (we have no experience with the robot).

\section{$\mathbf{P 2 8 4}$}

Trends and Clinical Outcomes in Young Patients with Colorectal Cancer: A Comparative Review of Emergency vs Elective Surgical Cases

Edith Loo, $\mathrm{Ms}^{1}$, Serene Goh, $\mathrm{Dr}^{2}$, Daniel Lee, $\mathrm{Dr}^{2} ;{ }^{1}$ Lee Kong Chian School of Medicine, ${ }^{2}$ Khoo Teck Puat Hospital

Introduction: This study strives to determine trends and clinical outcomes for patients diagnosed with CRC under age 50. This study also aims to evaluate differences in outcome between elective and emergency young CRC cases, as it is stipulated that younger patients who present in acute settings and undergo emergency surgery may have a poorer prognosis. To date, the role of young age of CRC diagnosis as an adverse prognostic factor remains controversial.

Methods: A retrospective study was conducted on 99 patients under 50 years of age presenting to Khoo Teck Puat Hospital (KTPH) with primary CRC from 2010-2017. Patients were stratified into elective or emergency surgery subgroups. Chi square test was used for comparison of the two subgroups, Cox's proportional hazard regression was used to multivariately assess predictors of outcome and survival analysis was undertaken using Kaplan-Meier survival curves.

Results: In our cohort, $54.5 \%$ presented with Stage 3 CRC and above. $68.4 \%$ underwent elective surgery, while $31.6 \%$ underwent emergency surgery, of which $66.6 \%$ presented with obstruction and $33.3 \%$ with perforation. $62.1 \%$ underwent open surgery, while $37.9 \%$ underwent laparoscopic surgery. On univariate analysis, factors demonstrating a significant association with poor prognosis were high CEA levels, advanced overall stage, $\mathrm{T}$ stage, presence of signet ring histology, vascular invasion, presence of metastasis and emergency surgery. On multivariate analysis, only advanced stage (stage III and IV) and presence of signet ring histology were independent predictors of poor outcome. Survival outcome was significantly poorer for patients who underwent emergency surgery compared to elective surgery.

Conclusion: Young CRC does not have a poorer prognosis. Advanced stage and signet ring cell histology are both indicators of poor outcome. Emergency presentation exhibits poorer survival, where patients present at later stages with higher rates of metastasis. This raises concerns regarding public knowledge of red-flag symptoms and awareness among clinicians during evaluation of younger patients, to avoid severe ramifications of delayed diagnosis. 
P285

A New Minimally Invasive Sphincter-saving Procedure to Treat Highly Complex Anal Fistulas : Transanal Opening of Intersphincteric Space (TROPIS) Procedure in 238 High Complex Anal Fistulas

Pankaj Garg, MBBS, MS, FASCRS ${ }^{1}$, Sachi Singhal, MBBS ${ }^{2}$, Sachin B Jamma, MS ${ }^{3}$; ${ }^{1}$ Garg Fistula Research Institute, Panchkula, Haryana, India, ${ }^{2}$ Dayanand Medical College and Hospital, Ludhiana, Punjab, India, ${ }^{3}$ Ashwini Rural Medical College, Kumbhari, Solapur, Maharashtra, India

Aims: In complex fistulas with significant intersphincteric component(high transsphincteric, horseshoe and supralevator fistulas), the intersphincteric component acts like an abscess. Simply draining the intersphincteric sepsis once as is done in LIFT procedure would lead to recurrence in many cases (up to 50\%). In such fistulas, laying open the intersphincteric part of the fistula through the transanal route(deroofing the abscess) ensures healing in most cases. This is the basis of TROPIS(transanal laying open of intersphincteric space) procedure. TROPIS was done and evaluated in high complex fistulas. Methods: All consecutive operated patients of complex high fistulain-ano included. All fistula were high (involving more than one-thirds of sphincter complex). Simple fistula in which fistulotomy was possible were excluded. Preoperative MRI scan was done in all the patients. The main outcome measures were healing rate, hospital stay, objective incontinence scores

Operative Procedure: A curved artery forceps was inserted through the internal(primary) opening into the intersphincteric part of the fistula tract. The mucosa and the internal sphincter over the artery forceps were laid open inside the rectum with electrocautery. The incision, starting from the internal opening, was usually curvilinear but could also be oblique, depending upon the direction of the intersphincteric tract. In case of horseshoe fistula, the incision extended on both sides of the midline posterior internal opening. In case of supralevator extension/tract, the incision was extended from the midline posterior internal opening up to the supralevator rectal opening(Figure-1).

Results/Outcome: 238 patients with high complex fistula-in-ano were operated over 4 years with a follow-up of 4-45 months (median15 months). 29 patients were excluded due to short follow-up. Male/ Female: $189 / 20$, age-39.06 \pm 9.3 years. $78 \%$ (163/209) were recurrent, $89 \%$ (186) had multiple tracts, $36.4 \%$ (76) had horseshoe tract, $27.8 \%(58)$ had supralevator extension and $37.3 \%(78)$ had associated abscess. Fistula healed completely in $83.3 \%(174 / 209)$ and didn't heal in $16.7 \%(35 / 209) .27 / 35$ of these were reoperated with the same procedure and fistula healed in 21 patients. Thus overall healing rate was $93.3 \%$ (195/209). There was no significant change in incontinence scores.

Conclusions: The success rate of TROPIS $(>93 \%)$ in high complex fistula(all were high and majority were recurrent fistula with multiple tracts, horseshoe tracts and supralevator extension) is quite impressive. The external sphincter is not cut or damaged due to which the risk to continence is minimized. The technique is simple, minimally invasive, easy to reproduce, associated with little pain and early resumption of normal activities.
P286

Colorectal Cancer in Patients Under Age 50: Trends in Staging and Mortality

$\underline{\text { Jaclyn Malat, } \mathrm{DO}^{1}}$, Sarah Martin, $\mathrm{BS}^{1}$, Adrian Ong, $\mathrm{MD}^{2}$, Michael Brown, MD, MBA, FACS ${ }^{2}$; ${ }^{1}$ Philadelphia College of Osteopathic Medicine, ${ }^{2}$ Reading Hospital, Tower Health

Objective: The aim of this study is to compare stage and mortality in colorectal cancer (CRC) patients above and below age 50 . We hypothesize that the stage at diagnosis and mortality will both be higher in patients under age 50. Recent national reports show an increase in CRC diagnoses in younger patients. This correlates with the recent change by the American Cancer Society to beging screening colonoscopies at age 45 , instead of age 50. Data on this younger patient population, including stage of diagnosis and mortality however, are limited and require further investigation.

Methods: A 20-year retrospective review of electronic medical records was completed at the McGlinn Cancer Institute from 1997 to 2017 of all patients diagnosed with CRC. This data was then aggregated by age, gender, tumor location, stage and mortality over the past 20 years. Univariate analysis of variables was performed where appropriate. Statistical significance was indicated by a $p$ value of 0.05 .

Results: Of 3041 patients diagnosed with CRC over the last 20 years, $231(7.6 \%)$ patients were below age 50 . These younger patients were diagnosed at higher stages compared to patients age 50 and above; $71.4 \%(165 / 231)$ of younger patients compared to $56.5 \%(1589 / 2810)$ of those above age 50 were diagnosed at stage 2 or higher $(\mathrm{p}<0.0001)$. Despite this, the mortality rate over 20 years in younger patients was lower compared to those above age 50 (35.5\% vs $59.3 \%$, $\mathrm{p}=0.0001$ ). There was no statistically significant difference in gender or location of tumor between these two age groups.

Conclusion: Although patients under age 50 were more likely to be diagnosed at higher stages of CRC, they demonstrated a lower mortality rate when compared to patients above age 50 . Based on our data, initiating screening colonoscopies at age 45 would likely downgrade the stage at diagnosis however, its effect on overall survival has yet to be determined. 


\section{P287}

Intracorporeal Versus Extracorporeal Anastomosis in MinimallyInvasive Rectosigmoid Resection

Kasim Mirza, MD, Carey Wickham, MD, Andreas M Kaiser, MD, FACS, FASCRS; USC Colorectal Surgery

Introduction: Intracorporeal anastomosis (ICA) during minimally invasive colorectal resections (robotic/laparoscopic) has been thought to have advantages over extracorporeal anastomosis (ECA). It avoids exteriorization of the colon with room air exposure, traction, and manipulation in the resection and anastomosis phase and for placement of an EEA stapler anvil. We previously described the technique of a robotic single-dock intracorporeal anastomosis for rectosigmoid resections. The purpose of this study was to compare outcomes between robotic and laparoscopic ICA and ECA for sigmoid and low anterior rectal resections (LAR). We hypothesized that ICA was associated with decreased rates of ileus and LOS.

Methods: Patients who between 2015 and 2018 underwent a laparoscopic or robotic sigmoidectomy or LAR performed by a single surgeon at a tertiary referral center were retrospectively analyzed. Included were any minimally-invasive sigmoidectomy or LAR for benign or malignant disease with primary stoma-free anastomosis. ICA was defined as laparoscopic or single-dock robotic resection with intracorporeal colon resection and anvil placement, whereas ECA was defined as exteriorization of more than the specimen for resection, anvil placement, or anastomosis. Exclusion criteria included conversion from MIS to open surgery, creation of a stoma, or hand-sewn coloanal anastomosis.

The patients were grouped into ICA versus ECA. Data analyzed and compared included patient demographics, operative time, peri- and postoperative morbidity, and length of stay. Statistical analysis was performed on SAS software using 2-tailed t-test for continuous variables, and Chi squared or fisher exact test for categorical data.

Results: Of 64 patients identified, 40 met the criteria with 20 ICA (10 LARs and 10 sigmoidectomies) and 20 ECA (17 LARs and 3 sigmoidectomies). There were no significant differences between the groups for age, gender, ASA, or BMI. Average LOS was shorter in ICA (3.5 vs 5.3 days) without reaching statistical significance $(\mathrm{p}=0.088)$. While the rate of prolonged postoperative ileus showed no difference, overall complications were significantly higher in ECA $(8 / 20$ vs $2 / 20,=p=0.029)$. Responsible for this difference was a significantly lower complication rate after ICA for LARs when compared to ECA $(\mathrm{p}=0.026)$. Without reaching statistical significance, secondary outcomes such as operative time (254 vs $231 \mathrm{~min}, \mathrm{p}=0.24)$ and leak rate $(2 / 20,0 / 20, \mathrm{p}=0.49)$ favored ICA.

Conclusion: Minimally-invasive ICA was associated with favorable outcomes compared to ECA with significantly decreased overall postoperative complications and a trend to shorter LOS without an increase in operative time. ICA for rectosigmoid resections appears to offer an advantage and should be further evaluated

\section{P288}

Transanal Extraction for Rectal Cancer After TME: Always, Never or Sometimes?

Jonathan Josse, MD, Brigitte Anderson, BS, Henry Schoonyoung, MD, John Marks, MD; Lankenau Medical Center

Introduction: TaTME has emerged as a new popular approach for rectal cancer surgery. An incision through the anus or rectum provides a natural orifice route to deliver the specimen and avoid an abdominal extraction site. Concerns regarding the applicability of this approach in patients with large tumors, or high BMI and possible oncologic risks of this approach have often limited the adoption of Transanal extraction (TAE). We examine our experience with the routine use of TAE for TaTME and TATA over a 10 year period, to identify its impact on TME grade, local recurrence (LR) and morbidity.

Methods: From a prospectively maintained laparoscopic and rectal cancer database, all patients with primary adenocarcinoma of the rectum, treated with neoadjuvant therapy and a TaTME/TATA between 2008-2018 were identified. Retrospective review of the operative notes determined the route of specimen extraction in these patients as either abdominal (abd) or Transanal (TAE). The TAE group was analyzed to determine overall TME completeness, LR and morbidity. Subclass analysis of these outcomes was carried out based on $\mathrm{BMI} \geq 30$, size, $\geq 5 \mathrm{~cm}$, and circumferential versus non-circumferential cancers.

Results: Of the 128 eligible patients, $99.2 \%$ of specimens were extracted transanally, and comprise these results $(\mathrm{N}=127)$. The mean age of our patients was 59 years old, with $28 \%$ being female. TME grade on final pathology was incomplete- $2 \%$, near complete$3 \%$, and Complete- $95 \%$. Local recurrence was $1.6 \%$. There was one case of carcinomatosis. Overall 60-day morbidity was $32.3 \%$ with $63.4 \%$ having a Clavien-Dindo score of $\leq 2$. There was no mortality. The effect of BMI and size of the cancer, on TME, LR and morbidity are shown in Table 1.

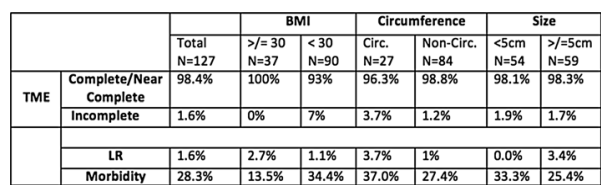

Conclusion: This study establishes that the Transanal extraction of the specimen can be routinely used for TaTME, without impairing the quality of the TME, increasing LR rates, carcinomtosis or morbidity. Transanal extraction is shown as safe for morbidly obese patients with large cancers as well. The primary concerns of Transanal extraction of the rectal cancer specimen regarding the compromise of the TME specimens and/or increased morbidity appears unfounded. 
P289

\section{Stoma Reversal and Postoperative Complications After} Laparoscopic Intersphinteric Resection for Rectal Cancer

Nao Obara, Shigeki Yakaguchi, Yasumitsu Hirano, Toshimasa Ishii, Hiroka Kondo, Kiyoka Hara, Shintaro Ishikawa, Takuhisa Okada, Liming Wong; Saitama medical university International medical Center

Introduction: Recently, the opportunity to perform laparoscopic intersphincteric resection (ISR) has been increased to preserve the anus. Temporary feacal diversion reduces the consequences of anastomotic leakage, and resulted in the increasing numbers of patients with loop ostomy after laparoscopic ISR. The aim of this study was to clarify the rate of stoma reversal and the postoperative complications after laparoscopic ISR for rectal cancer.

Patients and Methods: We retrospectively examined 139 patients who have underwent laparoscopic ISR for rectal cancer with defunctioning ostomy in our hospital between 2010 and 2017 with a follow-up period of at least one year. Results: The median age at the time of creation of the stoma was 65-years old, and median BMI was $22.8 \mathrm{~kg} / \mathrm{m}^{2}$. Of these, 102 were male and 37 were female. The preoperative comorbidities were 34 hypertensions, 25 diabetes, 11 respiratory diseases, 9 ischemic heart diseases, 8 cerebrovascular diseases, 5 arrhythmias and 4 hepatitis. The treatment performed before laparoscopic ISR included 18 chemo-radio therapies, 13 endoscopic tumor resections, 2 transanal tumor resections and one chemotherapy. The positions of defunctioning ostomy were 131 ileums and 8 transverse colons. Postoperative complications after laparoscopic ISR were 18 anastomotic leakages, 9 anastomotic strictures, 8 stoma outlet obstructions, 6 rectal prolapses, 5 bowel obstructions, 3 dysuria and one peri-stomal fistula. One hundred and twenty three patients (88\%) had stoma reversal, and median time to reversal was 4.2 months. In 16 patients $(12 \%)$, stoma reversal was not performed. The reasons were 10 tumor metastasis or recurrence of rectal cancer, 3 anastomotic leakage or stricture and 3 patients' reques. In 16 patients $(13 \%)$ after stoma reversal, reconstructions of ostomy were performed because of 6 anastomotic recurrences of cancer, 3 anastomotic strictures, 2 late anastomotic leakages, 2 poor anal function, one recto-seminal vesicle fistula, one perforation of colon and one perforation of small intestine. In 110 patients (79\%), stoma reversal was performed in the follow-up period of at least one year.

Conclusion: Twenty nine patients $(21 \%)$ undergone laparoscopic ISR with defuntioning ostomy did not have stoma reversal or had stoma recreation after reversal in the follow-up period. It is important to detect the patient who may not have stoma reversal preoperatively and should be considered for permanent stoma.

\section{$\mathbf{P 2 9 0}$}

The Current Status of TaTME-ISR and Robot-LAR for Rectal Cancer in Comparison with Lap-ISR and Lap-LAR

Takuya Miura, Yoshiyuki Sakamoto, Hajime Morohashi, Hayato Nagase, Tatsuya Yoshida, Kentaro Sato, Yutaro Hara, Kenichi Hakamada; Hirosaki university

Background/Aim: Laparoscopic-Intersphincteric resection (ISR) and Low anterior resection (LAR) for rectal cancer is widely accepted, but the usefulness and problems of TaTME and Robot remained unclear. By comparing with Lap, we will examine the current status of TaTME-ISR and Robot-LAR.

Methods: We compared 13 patients of Lap-ISR + diverting stoma (DS) with 15 patients of TaTME-ISR + DS and 19 patients of Lap-LAR + DS with 7 patients of robot-LAR + DS retrospectively. In our hospital, TaTME was only introduced to ISR. LLND cases were excluded in the comparison between Robot and Lap, because Robot-lateral lymph node dissection (LLND) was only performed in one patient. The patients who underwent a partial resection of adjacent organs, pelvic exenteration, and simultaneous resection other than pelvic organs were excluded.

\section{Results}

1. Lap vs TaTME-ISR. In the characteristics, distance from tumor margin to anal verge was statistically longer in TaTME $(3 \mathrm{~cm} / 5 \mathrm{~cm}$ ) but gender (male, $69 \% / 82 \%)$, BMI $(22.8 / 22.4)$, preoperative chemotherapy $(23 \% / 46 \%)$, tumor diameter $(3 \mathrm{~cm} / 4 \mathrm{~cm}), \mathrm{T} 3(15 \% / 21 \%)$, lymph node metastasis $(7 \% / 6 \%)$ and lateral lymph node dissection $(61 \% / 46 \%)$ were not obviously different. Postoperative complications in Lap were $15 \%$ of anastomotic leakage (Grade A 1, B 1), $15 \%$ of intracorporeal SSI, $15 \%$ of stoma related complications and $7 \%$ of urinary retention (Grade 2). TaTME had $20 \%$ of anastomotic leakage (GradeA 1, B 2), $53 \%$ of intracorporeal SSI, $46 \%$ of stoma related complications and $6 \%$ of urinary retention (Grade 1). The definitive stoma was created in $7 \%$ after Lap-ISR and 33\% after TaTME.

2. Lap-LAR vs Robot-LAR. In the characteristics, Lap group had more advanced tumor such as size $(15 \mathrm{~mm} / 35 \mathrm{~mm})$, T3 $(52 \% / 14 \%)$, and lymph node metastasis $(42 \% / 0 \%)$. Gender (male, $100 \% / 100 \%)$, BMI $(22.5 / 24.0)$, preoperative chemotherapy $(5 \% / 0 \%)$, and distance from tumor margin to anal verge $(9 \mathrm{~cm} / 7 \mathrm{~cm})$ were not obviously different. Operative time was longer in Robot (260 $\mathrm{min} / 404 \mathrm{~min}$ ) but blood loss was not different $(15 \mathrm{ml} / 20 \mathrm{ml})$. Postoperative complications in Lap-LAR were $31 \%$ of anastomotic leakage (Grade A 1, B 3, C 2), $21 \%$ of stoma related complications and $5 \%$ of urinary retention (Grade 2). Robot-LAR had $0 \%$ of anastomotic leakage, $28 \%$ of stoma related complications and $14 \%$ of urinary retention (Grade 1).

Conclusions: Compared to Lap-ISR, TaTME-ISR in the early stage of introduction had many intracorporeal SSI, stoma-related complications and definitive stoma. Robot-LAR can reduce anastomotic insufficiency as compared with Lap-LAR. 


\section{P291}

\section{Impact of Perioperative Variables According to the Start Time} of Laparoscopic Colorectal Surgery

Mariana Matzner Perfumo, MD, Maria Elena Peña, MD, Jose Piatti, MD, Maximiliano Bun, MD, Nicolás Rotholtz, MD; Hospital Aleman

Introduction: The operation start time might be one of the many factors that can contribute to the patient's outcomes. The aim of this study was to evaluate the relationship between operation start time and patient outcomes in laparoscopic colorectal surgery.

Methods and Procedures: A series of patients who underwent elective colorectal surgery was analyzed from a prospective data base since January 2005 from June 2018. Patients were divided in two groups according to the start time of surgery. G1: 8am-12am and G2: 12:01 am-20 pm. At the same time the procedures were categorized in: Simple procedures (segmental resections and anterior resection) and complex procedures (total colectomies, total proctocolectomy, low anterio resection, abdominoperineal resection). Demographic data, hospital stay, type of complication, number of reoperations morbidity and mortality were analyzed.

Results: A total of 1339 patients underwent laparoscopic elective colorectal surgery. $715(54 \%)$ Were included in G1 and $624(46 \%)$ in G2. The media age was 61 years. No differences between both groups were identified in demographic data. Time of surgery was lower in G2 with no statistically significant differences. Although simple procedures were performed more frequently in G2 $(460(73,7 \%)$ vs G1 $(491(68,7 \%)) \mathrm{p}<0,04)$ the rate of intraoperative complications was higher in this group $(\mathrm{G} 2(67(10.7 \%) / \mathrm{G} 1: 54(7,6 \%) \mathrm{p}<0.04)$. However there no differences in conversion rate. The length of hospital stay was less in G1 (G1: 4,6, G2:5,5 p <0.001). The most common complication was Clavien I in G1 (G1:135 (19\%), G2:92 (14,7\%), p < 0,04). Reoperations were more often in G2 (77(12,3\%/G1:63 (9\%)); $\mathrm{p}<0.03$. Mortality rates were higher in G2 $(15(2,4 \%) / \mathrm{G} 17(1 \%) \mathrm{p}<0.04)$

Conclusion: Start time of surgery after 12 am generates an impact on patient's outcomes in laparoscopic colorectal surgery as the increased morbidity and mortality.

\section{P292}

\section{Perioperative Nutrition-Focused Intervention in Enhanced Recovery After Surgery}

Zhigang Xue, MD, Jianchun Yu, MD, Weiming Kang, MD, Zhiqiang $\mathrm{Ma}, \mathrm{MD}, \mathrm{PhD}$, Xin Ye, MD, Yuguang Huang, MD, Lijian Pei, MD; Peking Union Medical College Hospital, Chinese Academy of Medical Sciences

Aim: Enhanced recovery after surgery (ERAS) program focusing on nutritional risk assessment and comprehensive perioperative nutritional intervention is limited. We aimed to study the safety and efficacy of ERAS program focusing on the integrative clinical nutrition concept.

Methods: Our study was a single-center, prospective cohort study. The ERAS group included special focus on preoperative nutritional assessment, nutritional supplements by oral or tube feeding, early postoperative enteral nutrition (EN), combined with other series of ERAS items including no routine mechanical bowel preparation, preoperative carbohydrate loading, NSAIDs as analgesic meditation, no routine of gastric tube, no routine or early removal of peritoneal drainage and so on. The control group was under traditional perioperative management including routine mechanical bowel preparation, preoperative fasting and fluids limitation for $6-8 \mathrm{~h}$ prior to induction of anesthesia, opioid analgesia, routine placement of gastric tube and peritoneal drainage, postoperative parenteral nutrition (PN) and so on. The primary outcome was postoperative length of hospital stay (LOS), and secondary outcomes included total hospitalization cost, postoperative complication rate, readmission rate within postoperative 60 days.

Results: From December 2015 to April 2018, we enrolled 204 patients with GI surgery in the Department of General Surgery, Peking Union Medical College Hospital, and Chinese Academy of Medical Sciences. 102 patients in the ERAS process group, and 102 patients in the traditional process group. The postoperative LOS in the ERAS group was significantly lower than that in the traditional group $(7.2 \pm 4.5$ days vs. $9.8 \pm 4.8$ days, $p<0.001)$. Total hospitalization cost in the ERAS group was significantly lower than that in the traditional process group $(41125 \pm 18593$ RMB vs. $51512 \pm 19453$ RMB, p $<0.001)$ as well. The postoperative EN start time in ERAS group was on $2.4 \pm 1.8$ days after surgery (POD), and the traditional group was on POD $5.1 \pm 1.2$ $(\mathrm{p}<0.001)$. There was no significant difference in the incidence of postoperative complication between the two groups (ERAS group: $9.8 \%$ vs, traditional group $13.7 \%, \mathrm{p}=0.646$ ), and readmission rate within postoperative 60 days were $2.9 \%$ and $2.0 \%$ respectively.

Conclusion: Perioperative Nutrition-focused Intervention including preoperative nutritional assessment and intervention and early postoperative EN as well as other ERAS programs are safe and effective in GI surgery. Nutrition-enhanced ERAS program helps to reduce postoperative hospitalization and total cost significantly, while showing no increase of postoperative complications and readmission rate within POD 60. Integrative clinical nutrition-focused program may be more reasonable and feasible when achieving enhanced recovery after GI surgery.

\section{P293}

Short Term Outcomes of Laparoscopic Ventral Mesh Rectopexy in the Management of Complete Rectal Prolapse

Anwar Zeb Khan, Muhammad Adeel Kaiser, Danish Ali, Muhammad Farooq Afzal; Lahore General hospital, Lahore

Purpose: To analyze the short outcome of laparoscopic mesh rectpexy in the management of rectal prolapse

Methods and Material: This is a case series of 6 patients with rectal. Prolapse who was admitted through Opd. They have been subjected to clinical examination and sigmoidoscopy. They have underwent laparoscopic ventral mesh rectpexy. Post of bowel functions and complications have been documented.

Results: Median age was 38.male into female ratio was 1:1. Median operation time was $130 \mathrm{~min}$ Their hospital stay was 3 days. They have been observed in follow up and no complication has been documented.

Conclusion: We suggest that laparoscopic ventral mesh rectpexy is a safe technique and nerve sparing method is good and result in less complication and less recurrence. 


\section{P294}

Laparoscopic Low Anterior Resection for Diverticular Disease on a Patient with Situs Inversus Totalis

Lucia Collar Yagas, $\mathrm{MD}^{1}$, Nicolas M Cordoba, $\mathrm{MD}^{2}$, Jan P Kamiski, MD, MBA ${ }^{1}$, Joaquin J Estrada, MD, FACS, FACRS ${ }^{1}$; ${ }^{1}$ Advocate Illinois Masonic Medical Center, ${ }^{2}$ Private Practice

Situs inversus totalis (SIT) is a rare condition with an approximate incidence of 1:10,000 live births, in which the position of the thoracic and abdominal organs is inverted presenting as a mirror image of the normal anatomic layout called situs solitus. Colonic diverticulosis is, on the other hand, a common clinical condition with a prevalence that increases with age, being 1 to $2 \%$ in patients under the age of 30 and over $40 \%$ after age 60 . Approximately a quarter of such patients will develop acute diverticulitis.

We present a case of SIT and recent history of complicated acute diverticulitis, and our experience performing a laparoscopic low anterior resection. To our knowledge, there have been no similar cases previously reported in the literature.

A 29-year-old male with SIT and polysplenia presented after an episode of acute sigmoid diverticulitis complicated by an intra-abdominal abscess. He had a previous episode of uncomplicated diverticulitis.

Due to the recurrent episodes of diverticulitis, the complicated course of the last one, and the patient's abnormal topography, a sigmoid colon resection was recommended, as well as an appendectomy in view of the atypical location of the appendix.

A laparoscopic low anterior resection (LAR) with primary anastomosis and an appendectomy were performed. Special consideration regarding port placement and position of the surgeon and assistant were taken. The operator was situated at the left side of the patient and the assistant at the right, which is opposite to a conventional laparoscopic LAR. Ports were placed in a mirrored fashion to their regular placement. The surgeon used his left hand as the main working hand for most of the procedure. Extra care was taken due to the presence of multiple accessory spleens. The surgery was completed safely with an operating time of $180 \mathrm{~min}$ and minimal blood loss.

Conclusion: In a patient with SIT and history of diverticulitis, surgery should be indicated to avoid future problems such as misdiagnosis, delay in treatment and the possible complications that these might bring. Similarly, a prophylactic appendectomy should be performed at the time of a laparoscopy for any other indication.

SIT represents a technical challenge for the surgeon. A laparoscopic approach is feasible and safe in the hands of an experienced surgeon. Understanding the aberrant anatomy and being able to adjust technical aspects of the surgery to this condition are key elements for the success of a laparoscopic LAR.
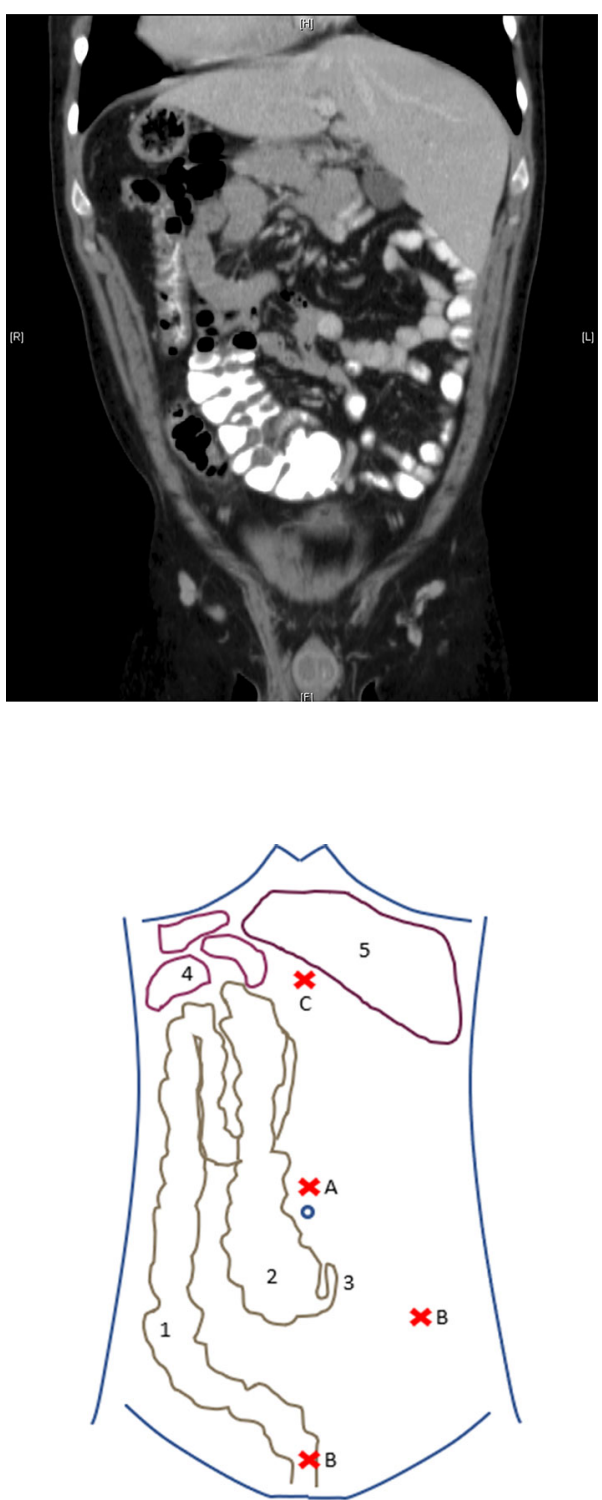

Anatomical layout and port placement.

1: Sigmoid colon, 2: Cecum, 3: Appendix, 4: Accessory Spleens, 5: Liver, A: Camera port, B: Surgeon's port, C: Assistant's port 


\section{P295}

Efficacy and Safety of Laparoscopic-Assisted Versus Traditional Open Surgery for Synchronous Multiple Primary Colorectal Cancers

Ziming Cui, Xiaoyu Dong, Weisheng Chen, Dexin Chen, Wei Jiang, Kai Li, Jun Yan; Department of General Surgery, Nanfang Hospital, Southern Medical University

Background: There is still no consensus on the treatment of synchronous multiple primary colorectal cancers (CRCs). In this study, we aimed to compare the efficacy and safety of laparoscopic-assisted versus traditional open surgery for multiple primary CRCs.

Methods: Between March 2006 and March 2018, 115 patients who suffered from synchronous multiple primary CRC with clinical stage among T1-4N02M0 were included. Efficacy and safety of laparoscopic-assisted group $(n=75)$ and traditional open group $(n=40)$ were compared. All patients were followed up every 6 months and were recorded the survival.

Results: Two patients were converted to open surgery in the laparoscopicassisted group. Both length of total and postoperative hospital stay were significantly shorter in the laparoscopic-assisted group than the traditional open group (median 16 vs 19 days, $\mathrm{p}=0.004$; median 10 vs 12 days, $\mathrm{p}=0.006$, respectively). The laparoscopic-assisted group yielded less blood loss (median 100 vs $125 \mathrm{~mL}, \mathrm{p}=0.003$ ), shorter postoperative exhaust time but longer operation time (median 230 vs $197 \mathrm{~min}, \mathrm{p}=0.031$ ) than the traditional open group. There was no significant difference on the overall survival and diseasefree survival between two groups.

Conclusion: Our results suggested that laparoscopic-assisted surgery is a more efficacious, relatively safer and minimally invasive treatment for synchronous multiple primary CRCs. Further randomized controlled trials with larger sample sizes are needed to strengthen the conclusion of this study.

\section{P296}

\section{Early Discharge with Transversus Abdominis Plane (TAP) Block as Component of Colon Resection Protocol}

Natalie Pozzi, Landon Guntman, MD, Logan Brady, Phillip Corvo; Saint Mary's Hospital

Background: Enhanced Recovery After Surgery (ERAS) protocols have been shown to optimize recovery and reduce hospital length of stay. Adverse effects from long-acting medications such as opioids, sedatives, and hypnotics slow recovery. This study aims to assess the impact of the addition of the Transversus Abdominis Plane (TAP) block as a component of non-opioid pain management for elective colorectal surgery patients at a community hospital. Method(s): A retrospective chart review was performed from October 2015 December 2016 for all adult patients undergoing general anesthesia and elective colon resection. Patients who received TAP blocks in addition to general anesthesia were compared to patients who received general anesthesia alone. Length of stay was calculated for each patient from time of admission to discharge from the hospital.

Results: Thirty-four patients who were treated with TAP block were compared to 32 patients who received conventional care. Length of stay was $25 \mathrm{~h}$ shorter $(\mathrm{P}=0.02)$ for patients who received general anesthesia with a TAP block compared to patients who received general anesthesia only. Additionally, compared with other surgeons at our institution, those following the established ERAS protocol with the addition of TAP block had an average length of stay shortened by $31 \mathrm{~h}(\mathrm{P}=0.07)$.

Conclusions: As part of the ERAS protocol, TAP blocks using liposomal bupivacaine significantly reduce hospital length of stay in patients undergoing elective colorectal surgery.

\section{P297}

Transanal Total Mesorectal Excision for Low Rectal Cancer: A Case-Matched Study Comparing TaTME Versus Standard Laparoscopic TME

Magdalena Mizera, Grzegorz Torbicz, Natalia Gajewska, Jan Witowski, Mateusz Rubinkiewicz, MD, PhD, Mateusz Wierdak, MD, Magdalena Pisarska, MD, Piotr Major, MD, PhD, Piotr Malczak, MD, Michal Pedziwiatr, MD, PhD; 2nd Department of General Surgery, Jagiellonian University Medical College, Cracow, Poland

Background: Transanal total mesorectal excision (TaTME) is emerging as a novel alternative to laparoscopic total mesorectal excision (LaTME). The aim of this study was to compare clinical and pathological results from these two techniques in patients undergoing rectal resections for low rectal cancer. Materials and Methods: Thirty-five patients undergoing TaTME were matched with 35 patients operated on using LaTME. Composite primary endpoint (complete TME, negative circumferential resection margin [pCRM], and distal resection margin [pDRM]) was used to assess pathological quality specimens. Secondary outcomes included operative and postoperative parameters (operative time, total blood loss, postoperative morbidity, length of stay, 30-day mortality).

Results: Composite primary endpoint was achieved by $85 \%$ of subjects in the TaTME group and $82 \%$ of subjects in the LaTME group $(\mathrm{p}=0.66)$. Mean pCRM was $1.1 \pm 1.29 \mathrm{~mm}$ vs. $0.99 \pm 0.78 \mathrm{~mm}(\mathrm{p}=0.25)$. Distal pDRM was $1.57 \pm 0.92 \mathrm{~cm}$ and $1.98 \pm 1.22 \mathrm{~cm}(\mathrm{p}=0.15)$. In the TaTME and LaTME groups, respectively. Complete mesorectal excision was achieved in $89 \%$ and $83 \%$ of subjects, while excision was nearly complete for the remaining $11 \%$ and $17 \%(p=0.23)$. Secondary outcomes were not significantly different. Conclusions: TaTME appears to be a non-inferior alternative to laparoscopic surgery. In comparison to LaTME, TaTME allows for similar quality of specimens with comparable clinical outcomes. 
P298

\section{Standarding Nursing Care to Ehnance Recovery After Colorectal Surgery}

Lauren Charles-Johnson, BS, RN, BSN, Miranda Aigeldinger, RN, BSN, PCCN; Lankenau Medical Center

Standardizing nursing care for colorectal surgical patients will enhance recovery by reducing physiologic and psychological stress, improve patient post op pain, expedite bowel function, and decrease cost by a shorter hospital stay and reduced post op complications.

The Enhanced Recovery after Surgery (ERAS) pathway involves continuous teaching and nursing interventions at all stages of care. When a patient comes to the hospital for pre-admission testing (PATs) the nurse discusses with them their plan of care including what to do the night before surgery, realistic postoperative pain expectations, the effects of early ambulation on the healing process, and the negative effects of opioid medication on bowel function. Instructions include drinking a carbohydrate load (12 oz) or clear fluids up to $2 \mathrm{~h}$ prior to surgery and taking Entereg the night before. Upon admission, Entereg is administered with a small sip of water if needed, along with Tylenol, Gabapentin, and Celebrex by the preoperative nurse. Teaching done in PATs is reiterated.

Once in the OR the CRNA uses a Clearsite to monitor fluid volumes, propofol to reduce postoperative nausea and vomiting, a ketamine or lidocaine drip are administered for the duration of the case, and a local nerve block using bupivacaine to the anterior abdominal wall is administered once the procedure is complete. The intraoperative goal is euvolemia, defined as having the total fluid volume less than $2 \mathrm{~L}$ above total losses. In the PACU the nurse assesses pain and administers pain medication as ordered or uses integrative therapy (Reiki, positioning, music, and guided imaginary). The PACU nurse assesses the IV fluid rate, questions the use of an NG tube or Foley catheter, and continues to educate the patient about pain management and the importance of day of surgery ambulation.

Upon arrival to the inpatient unit the nurse maintains the use of multimodel pain management converting to oral medications, uses aggressive antiemetic therapy (Dexamethasone 4-10 mg and Ondansetron $4 \mathrm{mg}$ ), removes the Foley or nasogastric tube, promotes chewing gum, and begins an ambulation schedule. The nurse ambulates the patient as soon as possible the day of surgery setting specific goals.

Since being implemented the average length of stay (5-7 days) is declining with a few patients leaving as early as post op day 2. Many patients have shown enhanced recovery which is a patient who is eating, drinking, and mobilizing well. The newly standardized ERAS pathway is largely the reason for these patients' success.
P299

Robotic Right Colectomy with Complete Mesocolic Excision and Suprapubic Approach: Clinical and Oncologic Results of a Consecutive Single-Centre Experience

Wanda Petz, MD, Emilio Bertani, MD, Simona Borin, MD, Alessandra Piccioli, Uberto Fumagalli Romario, Giuseppe Spinoglio, MD, FACS; European Institute of Oncology

Background: Single-center non randomized series have shown that D3 lymphadenectomy and complete mesocolic excision (CME) in the surgical treatment of colon cancer can provide better oncologic results in comparison with conventional technique in which integrity of mesocolon is not preserved and central lymph nodes are not removed. However, this technique is technically demanding, particularly with a laparoscopic approach.

Aim of this study is to describe a robotic suprapubic approach to right colectomy with CME and D3 lymphadenectomy and to report oncologic safety and short term outcomes.

Methods: Data from 57 consecutive patients who received a robotic right colectomy with suprapubic approach for right colon cancer are presented.

Surgery is realized with the $\mathrm{Da}$ Vinci $\mathrm{Xi}^{\circledR}$ system and all trocars are placed along a horizontal line 3-6 $\mathrm{cm}$ above the pubis. CME with fluorescence-guided D3 lymphadenectomy is performed.

After Indocyanine Green intravenous injection, bowel stumps perfusion is assessed, and an intracorporeal isoperistaltic side-to-side mechanical anastomosis with robotic linear stapler and manual closure of insertion holes is realized.

The specimen is extracted through a Pfannenstiel incision.

Results: Patients median age was 69 years, median body mass index was $27 \mathrm{~kg} / \mathrm{m}^{2}$. Median operative time was $228 \mathrm{~min}$, blood loss was negligible, no conversions to open or laparoscopic surgery occurred. Median hospital stay was six days; two postoperative grade IIIa Clavien-Dindo complications occurred, no 30-days postoperative death was registered. Resection margins were negative in all patients; median tumour diameter was $3.6 \mathrm{~cm}$, median specimen length was $40 \mathrm{~cm}$, median number of harvested lymph nodes was 37 .

Conclusions: Robotic right colectomy with cme using a suprapubic approach is a feasible and safe technique that allows for an extended lymphadenectomy and provides high quality surgical specimens. 


\section{P300}

\section{Current Status of taTME in China}

Liang Kang; the sixth affiliated hospital of Sun Yat-sen University

Methods: A questionnaire survey was conducted in 71 large medical centers in 23 provinces of China. The survey included the time to launch taTME, the number of taTME cases completed, the occurrence of complications and the difficulties encountered at present.

Results: A total of 71 units completed the questionnaires, of which $2 / 3$ units have begun to carry out taTME, a total of 915 taTME cases were counted, of which 211 completed the most cases, 10 units completed more than 30 cases $(14.1 \%)$. The total complication rate was $138(15 \%)$. Among them, anastomotic leakage was found in 75 cases $(8.2 \%)$, urinary retention in 21 cases $(2.3 \%)$, fecal incontinence and anastomotic stenosis in 8 cases $(0.9 \%)$ and urethral injury in 6 cases $(0.6 \%)$. Other complications included intestinal obstruction in 5 cases, anastomotic bleeding in 4 cases, diffuse peritonitis in 3 cases, vaginal injury in 2 cases, ureteral injury in 1 case. Surveys of technical difficulty showed that $50 \%$ of the surgeons thought lateral dissociation was more difficult, $43 \%$ thought anterior dissociation was more difficult, and 35\% thought purse suture was more difficult. Analysis of the factors affecting the development of taTME showed that $70.4 \%$ of the surgeons believed that there was a lack of professional equipment, $35 \%$ of the surgeons believed that the anatomical structure was unclear, $29 \%$ of the surgeons believed that the transanal operation was inexperienced and they were not confident to carry out it for the time being.

Conclusion: At present, the development of taTME in China is still in its infancy, and there are still many technical problems to be solved. The lack of professional instruments and equipment is the main problem for this operation.

\section{P301}

Postpolypectomy Electrocoagulation Syndrome: A Rare Imitator of Perforation after Colonoscopy

Shinban Liu, DO ${ }^{1}$, Nicholas Morin, $\mathrm{DO}^{1}$, Vadim Meytes, $\mathrm{DO}^{2} ;{ }^{1} \mathrm{NYU}$ Langone Medical Center, ${ }^{2}$ Vassar Brothers Medical Center

Case Description: A 78-year-old woman presents to the emergency department with complaints of abdominal pain. Earlier that day she had undergone a routine screening colonoscopy with polypectomy of 3 sessile polyps removed from the transverse colon. She was hemodynamically stable but febrile (101.3?). Laboratory values were significant for white blood cell count of $16.3 \mathrm{~K} / \mathrm{uL}$ but otherwise grossly within normal limits. A computed tomography of the abdomen and pelvis demonstrated no free intraperitoneal air and focal wall thickening of the mid transverse colon containing biopsy clips. Due to her clinical presentation with localized peritonitis, laboratory workup, and imaging studies the patient was brought urgently to the operating room for diagnostic laparoscopy. Upon insertion of the laparoscope, no perforation or gross spillage was identified after examination of the bowel. The prior polypectomy site in the mid transverse colon was located with identification of colonoscopic tattoo and noted to have surrounding hyperemia and edema consistent with transmural burn without perforation. The remainder of her hospital course was unremarkable and was ultimately discharged home with suspected postpolypectomy electrocoagulation syndrome.

Discussion: Postpolypectomy electrocoagulation syndrome (also known as polypectomy syndrome and transmural burn syndrome) is a rare condition with an incidence of $0.07 \%$ following colonoscopic polypectomy. Development of this syndrome is associated with large amounts of thermal energy over extended periods of time when performing extensive endoscopic submucosal dissection. The electrical current is theorized to extend past the targeted mucosa into the muscularis propria and serosa creating a transmural burn without associated perforation. Clinically this may manifest as localized peritonitis secondary to serosal irritation and subsequent inflammatory response. Patients may also present with symptoms of fever, leukocytosis, and imaging demonstrating inflammation with or without microperforation such as in this case- all of which are concerning for an acute surgical abdomen. This diagnosis should be suspected in patients following polypectomy with electrocautery but should be confirmed with imaging to evaluate for frank perforation and free air rather than focal wall thickening and inflammatory changes as seen in this syndrome. Treatment of this syndrome consists of conservative management with nothing by mouth, antibiotics, and intravenous fluids until symptoms improve with the majority of patients fully recovering. Recognition of this condition is important as unnecessary surgery can be avoided. 
P302

\section{Adoption of Laparoscopic Colorectal Surgery in the Elderly Population: Current State and Value Proposition}

Deborah S Keller, MS, MD, James M Kiely, MD, Daniel P Geisler, MD, Ravi P Kiran, MD; Columbia University Medical Center

Background: The economic and clinical benefits of laparoscopic colorectal surgery are proven, yet laparoscopy remains underutilized in appropriate cases in the United States. Use of laparoscopy in the elderly may remain specifically underutilized, despite reports of safety and feasibility. Since the elderly constitute the greatest surgical colorectal volume, our goal was to identify trends in utilization and outcomes for laparoscopic colorectal surgery in this cohort.

Methods: Review of the Premier Inpatient Database was performed for elective inpatient colorectal resections between $1 / 1 / 2010$ and $9 / 30 / 2015$. Patients were stratified into elderly ( $\geq 65$ years) and non-elderly cohorts ( $<65$ years), then grouped into open or laparoscopic procedures. The main outcome measures were the trends in utilization by approach and total costs for the episode of care (considering the inpatient and 30-day post-discharge periods), hospital length of stay (LOS), readmission, and complications by approach in the elderly. Multivariable regression models were used to control for differences across the platforms, adjusting patient demographic, comorbidities and hospital characteristics.

Results: Laparoscopic adoption was greater and steadily increased in the nonelderly, while in the elderly, laparoscopy rates increased until 2013, then declined, with increasing rates of open surgery. In the elderly, laparoscopy was associated with significantly lower mean total costs $(\$ 4,012$ less/case), complications and readmissions less likely ( $36 \%$ and $33 \%$ less, respectively), and shorter LOS (2.59 less days) than open cases (all p < 0.0001). When complications occurred, they were less severe and the readmission episodes were less costly with laparoscopy than open colorectal surgery.

Conclusion: The adoption of laparoscopic colorectal surgery in the elderly has lagged behind that of younger patients and has even declined slightly in recent years. Considering the reduced overall cost with improved clinical outcomes, there is a tremendous value proposition, in addition to the clinical benefits, for patients and hospitals with increased use of laparoscopic colorectal surgery in the elderly.

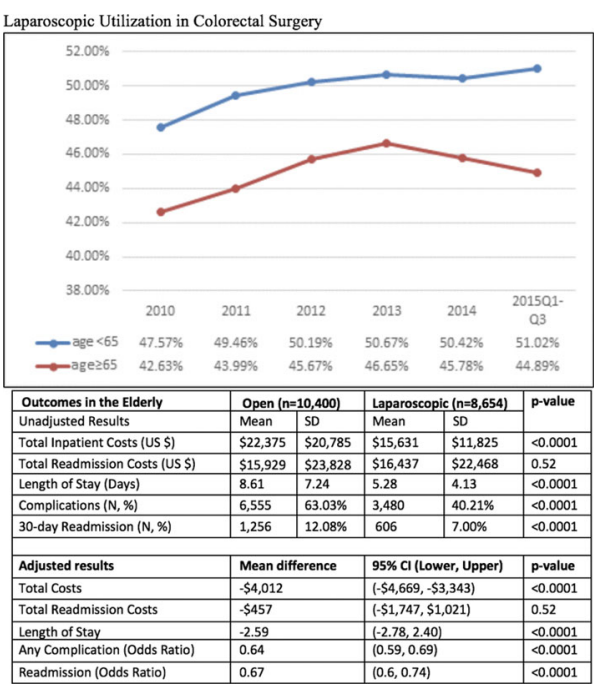

\section{P303}

\section{Incidence of Colon Resections is Increasing in the Younger Populations: Should an Early Initiation of Colon Cancer Screening be Implemented?}

Maria S Altieri, MD, MS ${ }^{1}$, Hannah Thompson, $\mathrm{MD}^{2}$, Aurora Pryor, $\mathrm{MD}^{1}$, Jie Yang, $\mathrm{MD}^{2}$, Siao Sun, $\mathrm{BS}^{2}$, Konstantinos Spaniolas, $\mathrm{MD}^{2}$, Mark Talamini, MD, $\mathrm{MBA}^{2}$, Jill Genua, $\mathrm{MD}^{2} ;{ }^{1}$ Washington University School of Medicine, ${ }^{2}$ Stony Brook University Hospital

Introduction: The American Cancer Society recently lowered the recommended age for screening of colorectal cancer (CRC) to age 45 due to recent data showing increased incidence of CRC in younger populations. The purpose of this study was to evaluate if younger patients have increased likelihood of resection for CRC (CRR) through the use of a statewide longitudinal database. Methods: The New York SPARCS administrative database was used to identify all patients with diagnosis of colon cancer from 2000-2016. Through the use of ICD-9 and ICD-10 procedure codes, patients undergoing colorectal resections were identified. Patients were divided into seven age groups: 21-30, 31-40, 41-50, 51-60, 61-70, 71-80 and > 80. Patients' characteristics, demographics, co-morbidities, and complications were evaluated. Duplicate records, missing information, and age $<21$ were excluded from analysis. For multiple procedures, only the first procedure was included in the analysis. Chi square test was used to compare patients' characteristics, comorbidities and complications among age groups. The linear trend of colon resection in different age groups over years was examined using log-linear Poisson regression models with year as an explanatory variable. Relative risks (RR) with $95 \%$ confidence interval were reported.

Results: Following inclusion and exclusion criteria, there were 73,697 colon resection surgeries extracted from 2000 to 2016. Trends are shown on Figure 1. Younger age was significantly associated with increased CRR over time. Patients age 21-70 had a significantly increasing trend over the years (age group 21-30: $\mathrm{RR}=1.06$, p-value $<0.0001$; age group 31-40: RR 1.04, $\mathrm{p}<0.0001$; age group 41-50: RR 1.04, $\mathrm{p}<0.0001$; age group 51-60: RR 1.02 $\mathrm{p}<0.0001$ ); age group 61-70: RR 1.01, $\mathrm{p}=0.0012)$. Patient age $>70$ was significantly associated with decreasing trend of CRR over the years (age group 71-80: RR0.98, p < 0.0001 an age group $>80$ : RR 0.99, p-value $<0.0001$ ). Conclusion: Over the years, younger patients have an increased trend of undergoing colorectal resections, with up to a $6 \%$ yearly increase over the studied period. New screening initiation guidelines should be considered and awareness among clinicians and the general public should be increased.

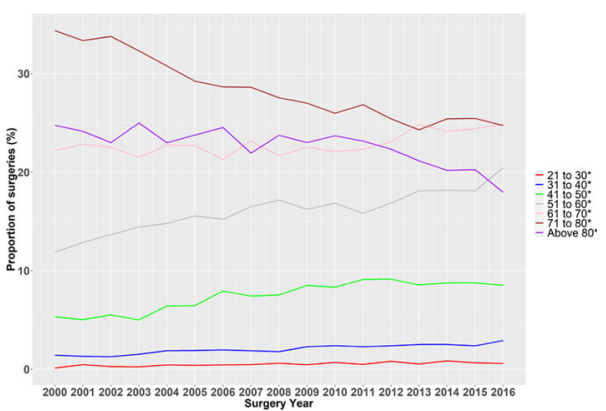




\section{P304}

\section{Laparoscopic Management Of A Rare Adult Patent Urachus} Presenting as a Colovesical Fistula

\section{Esther H Cha, MD, David Lisle, MD, P. Jeffrey Ferris, MD; Medstar} Health Baltimore

Introduction: Urachal ramnants result from a failure in the obliteration of the allantois at $4-5$ th week of gestation which connects the bladder to the umbilicus. This congenital anomaly occurrence is rare occurring about $1.6 \%$ in children and less than $1 \%$ in adults. We present a rare case of adult patent urachus presenting as a colovesical fistula complicated by chronic sigmoid diverticulitis managed by laparoscopy.

Case Presentation: A 63-year-old gentleman referred to a colorectal surgeon's office from his urologist for evaluation of symptoms consistent with pneumaturia. He described passing occasional gas during urination and feculent urine. However, he denied having any night sweats, fever, chills, or unintentional weight loss. As his preoperative work-up, patient obtained contrast-enhanced computed tomographic (CT) cystogram which revealed thickened bladder dome and adjacent sigmoid colon inflammation, and air in the bladder concerning for colovesical fistula. Subsequently, he underwent colonoscopy which had to be aborted due to severe inflammation at the proximal sigmoid colon due to diverticular disease. Then the patient was taken to the operating room for a laparoscopic resection. Once splenic flexure was mobilized laparascopically, it was noted that the thickened sigmoid colon was adherent to the lower mid-abdomen entering a triangular structure located superior to the dome of bladder. This patent urachus along with the segment of adherent sigmoid colon were resected and extracted with small midline incision. Final surgical pathology revealed chronic diverticulitis of the sigmoid colon and colo-urachus fistula without any malignancy. Patient had uncomplicated postoperative recovery course and was discharged home on postoperative day 7.

Discussion: The persistent urachal lumen has variety of clinical presentations, most commonly as periumbilical skin infection. Involution of this allantoic duct and cloaca becomes fibrotic cord known as the median umbilical ligament. There are four variants of urachal anomalies: cysts, sinus, diverticulum or a patent urachus. Urachal remnants progressing to malignancy are rare, less than $1 \%$, and most common malignancy type is urachal adenocarcinoma. Surgery remains as the primary treatment option. The traditional surgical approach to resection involves excision with low midline laparotomy or semicircular infraumbilical incision. Few reports include laparoscopic and robotic approaches with safe, successful outcomes.

Conclusion: Adult urachal remnant is rare and surgery is the treatment of choice as it prevents both recurrence of symptoms and malignant transformation. Laparoscopy is a useful and safe technique for the management of persistent or complicated urachus.

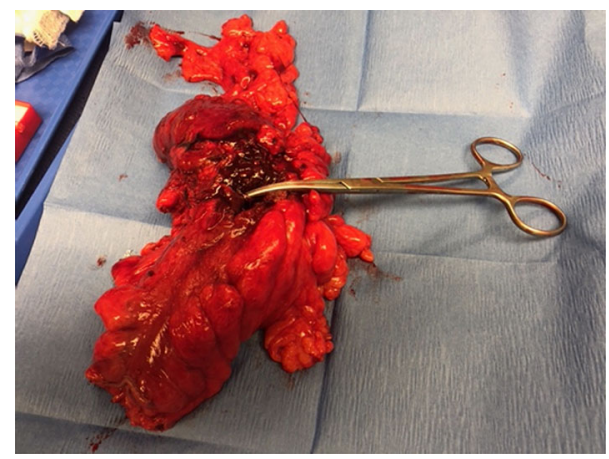

\section{P305}

Transanal Total Mesorectal Excision for Diffuse Cavernous Hemangioma of the Rectum

Ziwei Zeng, Shuangling Luo, Liang Kang; the Sixth Affiliated Hospital, Sun Yat-sen University

Aims: To evaluate the safety and feasibility of transanal total mesorectal excision (TaTME) for diffuse cavernous hemangioma of the rectum (DCHR).

Methods: All DCHR patients who underwent TaTME in our hospital between January 2014 and June 2018 were reviewed.

Results: A total of 7 patients with a diagnosis of DCHR underwent TaTME during the study period. Four patients $(57.1 \%)$ were male, with a mean age at surgery of ...years.. Recurrent painless rectal bleeding was the chief symptom in all patients. The mean age was 32 years old (range 21-54). The median duration of symptoms was 10 years (range 1 month-50 years). The level of hemoglobin at admission ranged from 59.0 to $148.0 \mathrm{~g} / \mathrm{L}$ (mean $106.6 \mathrm{~g} / \mathrm{L}$ ), and the level of MCV ranged from $75.1 \mathrm{fl}$ to $93.5 \mathrm{fl}$ (mean $83.7 \mathrm{fl}$ ). Colonoscopy, CT and MRI were important in the diagnosis of DCHR because of their high positive rates and accurate features. All of the lesions are between the anal tube and the descending colon. Two patients could be found enlarged serpentine vessel in other tissues and organs. After admission, all the patients underwent TaTME and four patients had simultaneous loop ileostomy. The mean operative time was $278 \mathrm{~min}$ (range 168-400 $\mathrm{min}$ ). The median amount of intraoperative blood loss was $50 \mathrm{ml}$ (range $10-300 \mathrm{ml}$ ). The mean distance from anal verge to anastomosis $2.2 \pm 0.2 \mathrm{~cm}$. The anastomosis was fashioned with a stapler in two patients $(28.6 \%)$. There were no intraoperative and postoperative complication happened. All the patients continued to recover well from the surgery, and nobody needed postoperative blood transfusion.

Conclusions: The definite diagnosis rate of DCHR is low. Preoperative MRI and CT examination can make a definitely diagnosis and determine the extent of the lesions. DCHR mostly restricts to the rectum, sigmoid colon, anal wall and mesorectum. The best treatment for DCHR is completely lesions resection. It is safety and feasibility to therapy DCHR by TaTME. Moreover, transanal procedure (TaTME) might have huge potential in other rectal diseases. 
P307

\section{Fitway Alabama Colorectal Cancer Prevention Program:} Analysis of Results

Greg S Thompson, MD, Lee West, MD, Mark A Parker, MD; Brookwood Baptist Health

Colorectal cancer is the 4 th most common cancer and 2 most common COD. In Alabama, it is among the top 6 in regards to mortality. There are several methods of screening for CRC but the most widely used are stool tests and colonoscopy. Stool tests include the FIT and FOBT which require samples with low sensitivity and will miss polyps. These are recommended as annual screening in conjunction with flexible sigmoidoscopy. Ultimately statistics have shown a $67 \%$ reduction in risk of death for CRC compared to no endoscopy.

The FITWAY program is a statewide program of the ADPH aimed at providing colorectal screening to AL residents. Patients receive a FIT test at their local clinic at age 50 and would be referred to have a colonoscopy if positive or recommended under current screening guidelines. Our study looked at the results a specific practice which is made up of 3 experienced colorectal surgeons who have participated in the program since May 2016. Data from 265 patients spanning 15 months was reviewed concentrating on age, gender, comorbidities, FIT results, prep adequacy and each of those associated ADR.

265 patients were examined and of the $19 \%$ who had a positive FIT, $45 \%$ had an adenoma found on endoscopy. Of the 26 pts w/negative FIT, 30\% had an adenoma detected on endoscopy. The average age of patients screened was 56 and this was the first colonoscopy for $85 \%$ of them. $2 / 3$ of patients screened were women. Overall patient prep was acceptable with $93 \%$ having adequate prep and only $3 \%$ having to be aborted. The ADR was equivalent to the national rate for endoscopists at $37 \%$ and well above the accepted rate of $>25 \%$. Of the comorbidities assessed for patients w/adenomas detected, 71\% had HTN and $56 \%$ had history of smoking. $35 \%$ of those with hypertension had an adenoma found and 55\% of smokers had an adenoma detected. 1 cancer was detected throughout all these.

We estimate 6 colorectal cancers prevented and 325 thousand dollars saved in the small study thus far. FITWAY has provided a cost effective screening and prevention service to the underserved population of Alabama. With adequate funding, a strong referral base of PCP's, and sufficient amount of gastroenterologists and colorectal surgeons we could begin to chip away at the national statistics and bring down Alabama's incidence rate of colorectal cancer.

\section{P308}

Accuracy of Preoperative Endoscopic Localization of Left-Sided Neoplasm by Gastroenterologist

Kayleigh Risser, RA, Reza Gamagami, MD; Silver Cross Hospital

Background: With increasing adoption of laparoscopy for the treatment of colorectal neoplasms, accurate preoperative localization is crucial as most tumors are invisible intraoperatively. There is usually no tactile feedback to assess the presence of a neoplasm. Furthermore, for rectal and rectosigmoid neoplasms, an accurate distance from anal verge will impact whether a patient will receive a colorectal anastomosis versus a coloanal anastomosis with a diversion. The aim of this study was to determine if there was a significant difference between the location of the left-sided colonic neoplasm described by the gastroenterologist as compared to the one performed by the operating surgeon prior to surgery.

Patients and Methods: A single surgeon retrospective analysis of patients referred for left-sided colonic neoplasm $(n=85)$ for laparoscopic surgery from May 2012 to August 2018 was evaluated. Assessed data included: rectal vs. colonic, presence or absence of endoscopic tattoo and/or clip, and description of anatomical location and distance from the anal verge. Findings were compared to the operating surgeon's preoperative endoscopic evaluation.

Results: We identified 85 patients, 7 with colonic and 68 patients with rectal and rectosigmoid neoplasms. The absolute difference between the gastroenterologist and the surgeon for anatomic location for rectal/rectosigmoid and colonic neoplasms was $24 \%$ and $45 \%$ respectively. For patients with rectal neoplasm, $16 \%$ of patients had no data for distance from anal verge by the gastroenterologist. The discrepancy between the gastroenterologist and the surgeon for distance from anal verge for rectal/rectosigmoid neoplasms was subcategorized to 2 to $<5 \mathrm{~cm}, 5-10 \mathrm{~cm}$ and $>10 \mathrm{~cm}$, with observed difference of 33,15 and $4 \%$ respectively.

Conclusion: There is a significant difference between the location of left-sided colorectal neoplasm reported by the gastroenterologist and that determined by the surgeon preoperatively. Patients undergoing left-sided colonic resection should undergo endoscopic evaluation by the operating surgeon before elective resection. Efforts should be made to improve endoscopic localization by gastroenterologist to accurately guide surgical resection. 


\section{P310}

FasciaOriented Laparoscopic Total MesorectalExcisionforRectal Cancer: Can It Better Protect the Nerve Function?

Dechang Diao, MD, Xinquan Lu, MD, Xiaojiang Yi, MD, Hongming Li, MD, Jin Wan, MD; Department of Gastrointestinal (Tumor) Surgery, Guangdong Province Hospital of Chinese Medicine, the Second Affiliated Hospital of Guangzhou University of Chinese Medicine

Background According to previous reports, Laparoscopictotal mesorectalexcision(LTME) for rectal cancer is considered as a mainstream surgery.However, due to the technical challenge and the risk of complications, most surgeons perform the dissection along the vessel or nerve of the rectum. Herein, we described a "fasciaoriented" approach for LTME forrectal cancer(fo-LTME).

Methods A total of 95 cases were collected from January 2017 to August 2018. 45 cases were included in the fo-LTMEgroup, which was compared with 50 cases with "spaceoriented" approach (soLTME). For fo-LTME group, our method was to incise the medial mesorectal membrane bridge (Tri-junction), enter the Toldt's space and free the mesorectum closing to the proper rectal fascia (PRF) and dorsal lobe of sigmoid mesoclon. The root of the inferior mesenteric artery and rectal mesentery were exposed and separated, and the No. 253 lymph nodes and the root of rectal mesentery were dissected en-bloc. The mesentery is removed along the fascia of the mesorectum to ensure that the fascia is intact. Resecting the mesentery at the end of the mesorectum and baring the rectum upwards, and the rectum was removed $2 \mathrm{~cm}$ from the distal edge of the tumor. For so-LTME group, the mesorectum was freebetween the layer of PRF and anterior vegetative membrane.

Results The mean operation time was without statistical significance between the fo-LTME and so-LTME groups (136.5 vs $137.1 \mathrm{~min}, \mathrm{p}>0.05$ ) and it was the same with the mean blood loss ( 35.0 vs $38.5 \mathrm{ml}, \mathrm{p}>0.05$ ). The mean number of harvested lymph nodes was 17.3in fo-LTMEgroup, compared with 19.2 in so-LTME group $(\mathrm{p}>0.05)$. For postoperative rectal quality assessment, foLTMEgroup (A/B grade; 42/3 cases) was without statistical significance compared with so-LTME group (A/B grade; 46/4 cases). Erectile functionassessment 2 weeks after surgery, dysfunctionwas occurred in 1 to 21 male patients in fo-LTMEgroup, compared with 6 to 26 cases in so-LTME group ( $p=0.08)$, There was no conversion in both two groups. Anastomotic leakage was occurred in 2 patientsfor fo-LTME group and 3 for so-LTME group ( $\mathrm{p}>0.05$ ), and no postoperative mortality was observed.

Conclusion The initial results suggest that the "fasciaoriented" approach for LTME may be a feasible and safe procedure. It may have some advantages in protecting bladder urodynamic and sexual nerves

\section{P311}

Demographics, Presentation and Short-Term Outcomes of Early Onset Rectal Cancer: A Contemporary Analysis from a Large Urban Hospital System

Anthony P D'andrea, MD, MPH ${ }^{1}$, Jani J Lee, $\mathrm{MD}^{2}$, Jordan M Cuevas, $\mathrm{BS}^{1}$, Deepika Bhasin, $\mathrm{MPH}^{1}$, Antoinette Bonaccorso, $\mathrm{MD}^{1}$, Marcher Thompson, $\mathrm{MD}^{3}$, Michael Buckstein, $\mathrm{MD}^{3}$, Randolph M Steinhagen, $\mathrm{MD}^{1}$, Patricia Sylla, $\mathrm{MD}^{1} ;{ }^{1}$ Division of Colorectal Surgery,

Department of Surgery, Icahn School of Medicine at Mount

Sinai, ${ }^{2}$ Department of Surgery, Mount Sinai St. Lukes-

West, ${ }^{3}$ Department of Radiation Oncology, Icahn School of Medicine at Mount Sinai

Background: While it has been well-documented recently that the incidence of early onset rectal cancer (EORC) is rising, there is a paucity of data on clinical presentation, treatment course, and specific outcomes.

Methods: A retrospective analysis was performed of a contemporary cohort of rectal cancer patients $<50$ years of age treated at a large urban tertiary hospital system between 2013 and 2018. Demographics, presentation, treatment and outcomes were evaluated.

Results: 60 patients with EORC were included with $58 \%$ males. Average age was 41.3 years (range $25-50$ ), with $17 \%$ of patients with $\mathrm{BMI} \geq 30$. Presenting symptoms included rectal bleeding $(72 \%)$, change in bowel habits (10\%), rectal (5\%) and abdominal pain $(5 \%)$, and median duration of symptoms was 16 weeks (range 2-250). A family history of colorectal cancer was elicited in $12 \%$, and $3 \%$ had documented microsatellite instability. Only $7 \%$ of EORC were detected on screening colonoscopy in otherwise asymptomatic patients. Cancer staging was completed within an average of 2 weeks (range 1-12). Tumors were staged I (25\%), II (22\%), III (42\%), IV (12\%). Median distance from the anal verge was $6 \mathrm{~cm}$ (range 0-13.5). Among $56(93 \%)$ patients staged by pelvic MRI, invasion into the mesorectal fascia was evident in $29 \%$ and involvement of the internal or external anal sphincters in 4 patients (7\%). Synchronous tumors were present in $11 \%$. Local excision including polypectomy was performed alone in $4(67 \%)$ patients with favorable T1 cancer. Fullcourse neoadjuvant treatment was administered to 42 patients $(70 \%)$ including 34 stage II/III, 2 stage I, and 6/7 stage IV patients following good tumor response with chemotherapy. Among 33 patients who underwent post-CRT restaging by MRI, $27.3 \%$ demonstrated downstaging. Ultimately, 38/42 (90\%) of stage I-IV patients treated with neoadjuvant therapy underwent TME with curative intent. Of the 48 patients who underwent TME, $43(86 \%)$ underwent sphincter preserving surgery and $39(81 \%)$ through a minimally invasive approach that included laparoscopic $(41 \%)$, robotic $(28 \%)$, and transanal total mesorectal excision (31\%). Conversion to open surgery occurred in 4 cases $(10 \%)$. Incomplete TME grading was noted in $5.5 \%$ and positive margins in $7 \%$. At a median follow-up of 15 months (range 1-63), local and distant recurrences were $11 \%$ and $28 \%$ respectively with overall survival of $92.5 \%$.

Conclusion: While this data is limited to a single large urban hospital system, this is the most contemporary series on the demographics, clinical presentation, treatment strategies and short-term outcomes in patients with EORC. 


\section{P312}

Laparoscopic Total Pelvic Exenteration Using a Linear Stapler for Urethral and Dorsal Vein Complex Transection

\author{
Akihiro Kondo $^{1}$, Yuji Nishizawa ${ }^{2}$, Hiroshi Taketani ${ }^{1}$, Naoki \\ Yamamoto ${ }^{1}$, Takahisa Fujita ${ }^{1} ;{ }^{1}$ Takamatsu Hospital, ${ }^{2}$ National \\ Cancer Center Hospital East
}

Introduction: Total pelvic exenteration (TPE) is an invasive operation for the management of advanced low rectal cancer. Laparoscopic surgery offers intraand postoperative benefits even in patients with advanced low rectal cancer requiring TPE. However, dorsal vein complex (DVC) and urethral transection during TPE are time-consuming and technically challenging procedures associated with a high rate of hemorrhage. This study aimed to show the use of a linear stapler for DVC and urethral transection during laparoscopic TPE. We have additionally assessed the surgical benefit of this method.

Methods and Procedures: An 85-year-old man with advanced low rectal cancer and suspected prostatic invasion underwent laparoscopic TPE. After medial-to-lateral retroperitoneal dissection and division of the inferior mesenteric artery, posterior dissection was performed to the level of the levator ani muscle. The bilateral ureters were mobilized and divided at the level of the ureterovesical junction. Anteriorly, the Retzius and the paravesical spaces were exposed to the level of the endopelvic fascia. Bilateral dissection was performed along the internal iliac vessels, and branches from these vessels were divided. After dissection of the endopelvic fascia, the levator ani muscle, and the ischiorectal fossa using a combined abdominal and perineal approach the DVC and urethra were completely exposed. Finally, en bloc transection of the DVC and urethra was performed using a linear stapler that was inserted from the perineal aspect. The specimen was extracted through the abdominal incision. After primary closure of the perineal wound, we constructed the ileal conduit and an endo-sigmoidostomy.

Results: The DVC and the urethra were easily transected without significant bleeding, and the operative procedure was relatively less time consuming. Total operating time was $513 \mathrm{~min}$ and estimated blood loss was $629 \mathrm{~mL}$. Postoperatively, he developed pelvic dead space infection; however, he responded to conservative treatment.

Conclusion: DVC and urethral transection using a linear stapler could be a simple and useful method associated with minimal bleeding during laparoscopic TPE performed for advanced low rectal cancer.

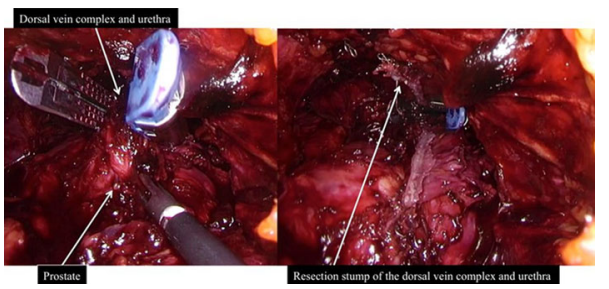

\section{P314}

Synchronous Colorectal Carcinoma Manifested as Three Separate Colon Masses: A Case Report and Review of Literature

Alyssa Imperatore, DO ${ }^{1}$, Linda Szczurek, DO, FACOS ${ }^{2}$, Alan J Shienbaum, $\mathrm{DO}^{2} ;{ }^{1} \mathrm{~J}$ Jefferson Health/Rowan University, ${ }^{2}$ Jefferson Health

Colorectal cancer (CRC) is the second leading cause of cancer-related deaths in the United States amongst cancers that affect both genders. A small entity of this common disease is synchronous colorectal carcinoma, defined as more than one primary detected on initial presentation. This accounts for $0.5-8 \%$ of all colorectal carcinomas. Prognostic implications appear to be similar to that of solitary CRC, however more investigation must be performed. Below is a case of synchronous colorectal carcinoma in which three separate colon masses were discovered on initial presentation.

This is a 78 year-old female who presented with shortness of breath on exertion for two weeks and intermittent black stools. Further workup revealed symptomatic anemia. The patient had never had a colonoscopy or EGD in the past. She was taken for endoscopy which demonstrated three polypoid lesions in the rectum and three separate colon masses: cecum, sigmoid colon and rectosigmoid. Preliminary pathology showed high grade dysplasia in the cecum and the patient was then taken for laparoscopic subtotal colectomy with rectal exam under anesthesia. Final pathology revealed moderately differentiated invasive adenocarcinoma arising from tubulovillous adenomas with lymphovascular and perineural invasion, five out of 12 lymph nodes involved. Patient had an uncomplicated post-operative course and was discharged home on post-op day four.

Synchronous colorectal cancer may be more prevalent than even documented secondary to not fully diagnosing or patients undergoing palliative surgery prior to complete diagnosis. This is a topic that could benefit from a large research experiment or meta-analysis to better answer the question as to how to effectively manage these patients.
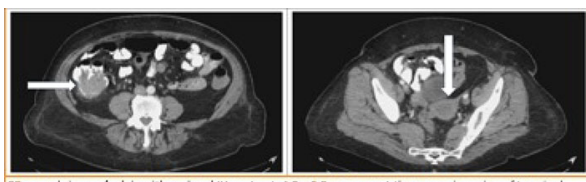

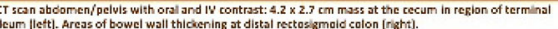

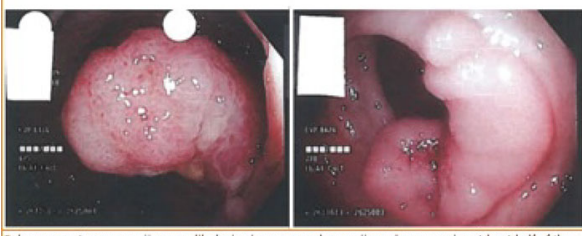

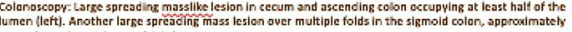

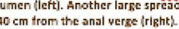

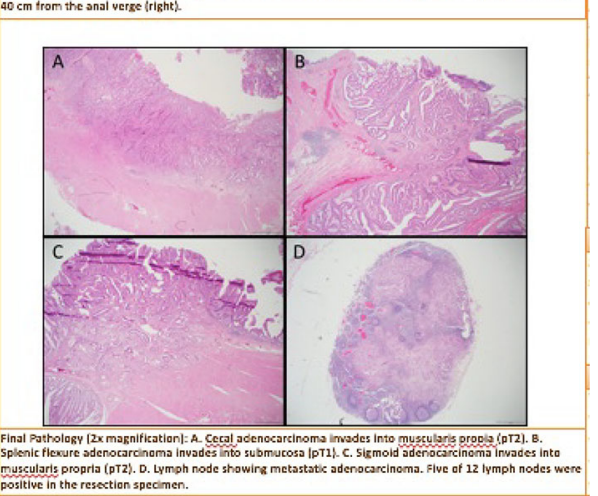




\section{P315}

\section{Natural Orifice Specimen Extraction in Laparoscopic Surgery} for Colonic Crohn's Disease - Transanal Approach

Leonardo C Duraes, MD, $\mathrm{PhD}^{1}$, Tracy L Hull ${ }^{2}$, Luca Stocchi, MD², Scott R Steele, MD, $\mathrm{PhD}^{2}$, Conor P Delaney, $\mathrm{MD}, \mathrm{PhD}^{2}$, Mariane Camargo $^{2}$, Emre Gorgun ${ }^{2}$, Hermann Kessler ${ }^{2}$; ${ }^{1}$ Johns Hopkins, ${ }^{2}$ Cleveland Clinic Foundation

Background: Although laparoscopic colorectal resections have been associated with lower risks of wound-related complications when compared with open resections, they still have a $10.3-22.7 \%$ rate of surgical site infection and a $6.0-10.8 \%$ incisional hernia risk. To date, one method to reduce wound complications is natural orifice specimen extraction (NOSE). Concerns associated with the NOSEtechnique include bacterial contamination of the peritoneal cavity, inflammatory response, and postoperative outcomes, including postoperative pain and the functional outcomes. In Crohn's disease, the use of the anus as an extraction site also avoids mini-laparotomy to remove the specimen after proctectomy. However, these patients are at higher risk for perineal complications. The implications of transanal specimen extraction during laparoscopic surgery for Crohn's disease have not been clearly delineated.

Methods: An institutional database was queried to identify patients undergoing laparoscopic total proctocolectomy for Crohn's disease between 1995 and 2013, in whom the specimen was extracted transanally. Perioperative outcomes were assessed.

Results: Thirty-four patients were identified (16 total proctocolectomies and 8 completion proctectomies). The mean age of patients was 42.6 years. Nineteen patients $(79 \%)$ had previous abdominal operations. Most patients were female (63\%). Four patients underwent robotic procedure (1 total proctocolectomy and 3 completion proctectomies), 3 patients underwent single-port procedure (3 completion proctectomies), and 1 patient underwent hand-assisted laparoscopic surgery (total proctocolectomy). Eight patients had perianal Crohn's disease. The median length of hospital stay was 6 days. Six patients were readmitted, and 4 patients were reoperated within 30 days ( 2 pelvic septic complications, 1 inadvertent enterotomy, and 1 perineal debridement). Two patients had perineal wound infections with delayed healing. No abdominal wound complications were observed.

Conclusion: Natural orifice specimen extraction during laparoscopic surgery for Crohn's colitis is safely feasible and may be considered despite higher risk for perineal challenges in patients with Crohn's disease.

\section{P316}

\section{Laparoscopic Repair of Cecal Bascule}

Shinban Liu, DO, Nicholas Morin, DO, Vadim Meytes, DO, George Ferzli, MD; NYU Langone Hospital - Brooklyn

Introduction: Cecal volvulus is a rare form of obstruction that occurs when there is torsion or rotation of mobile cecum or ascending colon. It accounts for only 1-3\% of all adult intestinal obstructions and is believed to have three main subtypes: clockwise axial rotation, loop torsion of the cecum with the terminal ileum, and anterior-superior folding without rotation-known as a cecal bascule. Cecal bascule presents less commonly, is less likely to create vascular compromise, and more frequently occurs in younger female patients. While abdominal plain film may reveal a characteristic "coffee bean" shaped cecum, computed tomography remains more specific in identifying cecal volvulus, differentiating between subtypes, and risk stratifying patients for operative management. We present the case of a 41 year old female with a cecal bascule treated with laparoscopic cecopexy and incidental appendectomy.

Case Presentation: 41 year old female with surgical history of abdominoplasty and caesarean section presents with 3 days of severe right lower quadrant abdominal pain with multiple episodes of nausea and vomiting. CT demonstrated cecal bascule formation without features of ischemic change or perforation. Patient was brought to the operating room and a redundant right colon with floppy cecum was identified. Multiple interrupted sutures were placed between the colonic tenia and lateral peritoneum to restrict the mobility of the cecum. Due to the retrocecal location of the appendix following cecopexy, an incidental appendectomy was performed. The patient had an uncomplicated postoperative course with unremarkable outpatient follow up.

Discussion: Cecal bascule is an uncommon form of cecal volvulus with anterior folding that can lead to obstructive symptoms. Initial diagnostic imaging may include abdominal plain film; however CT is more sensitive and assists with diagnostic specificity and operative planning. Nonsurgical treatment of cecal volvulus via endoscopy is limited, relatively ineffective, and associated with increased ischemia. Thus, surgical management should be the primary treatment modality. If nonviable bowel is encountered intraoperatively, resection is mandatory. With viable bowel, surgical management includes detorsion and fixation with cecopexy or hemicolectomy. Studies have shown increased risk of recurrence with cecopexy so many advocate for hemicolectomy as a definitive procedure. However, hemicolectomy is comparatively associated with increased mortality. Ultimately the choice of operation should be based on patient risk stratification, viability of the involved bowel segment, and operator experience. 


\section{P317}

Perineal Proctosigmoidectomy is an Acceptable and a Safe Procedure for Irreducible Rectal Prolapse

Amir Shaban, MD Gastrointestinal and Laparoscopic Surgery, Hossam Barakat Elbohoty, MD GIT Surgery, Hossam Ramadan Mousa, MD Surgical Oncology; Tanta University Hospitals

Irreducible or incarcerated rectal prolapse is a rare complication of rectal prolapse, the issue of management of rectal prolapse is controversial and the guidelines for the treatment of rectal prolapse recommends individual selection of the best proper surgical procedures based on each patient's overall condition. However, in cases of irreducible or incarcerated rectal prolapse the surgical procedures are very limited we had five cases of irreducibility complicated chronically progressive form of complete rectal prolapse. All cases were managed conservatively at first by trial of manual reduction but failed, application of sugar was done in three from the five cases but failed and hence emergency surgery through perineal proctosigmoidectomy was done. In the period from 2013 till end of 2017 we received five patients with irreducible incarcerated rectal prolapse in Tanta University hospital. Three of them were males and the reaming two were females the median age of these patients was 43 years. The duration of irreducibility at presentation varied from $16 \mathrm{~h}$ to 4 days trial of manual reduction was done in all five cases after injection of strong analgesia but failed. Two patients had patches of gangrene so no sugar application attempted. Two patients had medical morbidity one of them diabetic and the other was cardiac patient but with controlled cardiac functions. The mean length of the prolapsed rectum varied between 9-14 cm with a mean length of $11.2 \mathrm{~cm}$. Two cases had patches of ulceration and gangrene, two cases with ulceration and edema, and one case with only edema. All cases subjected to perineal proctosigmoidectomy (Altemier procedure) and a covering ileostomy, levatorplasty added in only one case. The mean operative time was $83 \mathrm{~min}$. Postoperative complications occurred in two cases; one of them wound infection and the other wound infection and partial anastomotic dehiscence, the two cases successfully managed conservatively. The timing of restoration of small bowel continuity ranged from 8-24 wks with a median value of $13 \mathrm{wks}$. The period of follow up ranged from 1-3 years in which all patients were doing well except one case suffered incontinence to fluid stool. No recurrence of prolapse occurred in any case.

\section{P318}

Can You Operate on a Mirror Image? Laparoscopic Sigmoid Colectomy in Kartagener syndrome

Hamda Al Zarooni, MD, Shadi Al Bahri, MD, Guido Mannaerts, MD, PhD; Tawam Hospital Johns Hopkins International

Introduction: Kartagener syndrome is a congenital, autosomal recessive disorder that affects ciliary motility. It presents as a triad of situs inversus, bronchiectasis and sinusitis, with a prevalence of 1 in 12,500 to 1 in 50,000. Situs inversus, is an anatomical abnormality with preserved organs physiological function, where the visceral organs are mirrored from their normal position.

Case Presentation: 64 year old male known to have Kartagener syndrome with situs inversus totalis variant. His comorbidities include atrial fibrillation, dyslipidemia, hypertension, metallic aortic valve replacement and coronary artery stenting. He presented with rectal bleeding and was diagnosed with sigmoid colon adenocarcinoma. Preoperative planning included colonoscopy with tattooing of the tumor site, CT angiography of the abdomen and pelvis to delineate vascular and anatomical variations. Patient was classified as ASA 3 with moderate cardiac risk. Important operative steps included placing the patient in lithotomy position, with the surgeon standing to the left and the assistant to the right of the patient. Four trocars were used; supra-umbilical $12 \mathrm{~mm}$ camera port, LLQ $12 \mathrm{~mm}$ and left flank $5 \mathrm{~mm}$ working ports, and right flank $5 \mathrm{~mm}$ assistant port. Endoscopic devices including ultrasonic dissection, Endoclips and linear stapler used during procedure were introduced through the LLQ port. After mobilization and transection of the sigmoid colon, the specimen was extracted through a Pfannensteil incision. End to end colonic anastomosis done using endoscopic circular stapler with reinforced interrupted absorbable sutures. Postoperative course was unremarkable except for an abdominal wall hematoma due to anticoagulation. He was seen in clinic following discharge and pathology revealed negative lymph node involvement.

Discussion: Kartagener syndrome, includes the triad of situs inversus, bronchiectasis and sinusitis. According to our literature review, 13 cases were reported of laparoscopic colonic resection in patients with situs inversus alone. Taking into consideration that patients with Kartagener syndrome have associated comorbidities that can increase patient morbidity and mortality. Appropriate preoperative evaluation and multidisciplinary involvement, in addition to several technical intraoperative considerations can make laparoscopic sigmoid resection feasible. Points to consider include optimization of patient's comorbidities, preoperative CT angiography and colonoscopy with tumor tattooing. In addition, intraoperative port placement, surgeon assistant position and the presence of an experienced laparoscopic surgeon allow for a smooth operative course.

Conclusion: Laparoscopic sigmoid colectomy can be challenging in patients with Kartagener syndrome due to mirror imaged anatomy and associated comorbidities. Advances in laparoscopic methods now allow patients with rare anatomic variations to benefit from laparoscopic surgery and its associated advantages. 


\section{P319}

\section{Transanal Endoscopic Microsurgery in the Patient with Early Rectal Cancer: Role of the Sentinel Node Concept}

Vladimir Grubnik, Prof, MD, Viktor Grubnyk, Vadym Ilyashenko, PhD; Odessa National Medical University

Transanal endoscopic microsurgery (TEM) is effective procedure in selected group of patients' with early stage rectal cancer. Nevertheless, in the group of patients' with lymph nodes metastasis TEM is not acceptable. This group of patient needs total mesorectal excision (TME). The reported risk for lymph node metastasis (LNM) in early rectal cancer ranges from 10 to $20 \%$. Radical surgery can reduce the risk of metastasis. However, surgery has high morbidity and mortality as well as cost.

Aim of the Study: Was elaboration of indications for TEM based on sentinel lymph node (SLN) concept.

Methods: For last 2 years, TEM was performed on 28 patients' with early rectal cancer. There were 19 women and nine men, age 68 to 87 . Localization of tumors was $4-12 \mathrm{~cm}$ from anus. Mean size of tumors was $3.8 \mathrm{~cm}$. Full thickness excision was performed in all patient with suturing of mucosa. During last year in 7 patiens with early stage rectal cancer we used indocyanine green (ICG) with fluorescent imaging for mapping sentinel lymph node. ICG was injected in four quadrants to submucosa around the tumor. During the laparoscopy SLN was detected and removed with morphological examination. Results: Among seven patients in five patients, SLN was negative. TEM was performed in these patients with good results. After 6-10 months no recurrence or metastasis were detected in this patients. In 2 patients with positive SN laparoscopic TME was performed with low colorectal anastomosis. Anastomotic complication was occurred in one patient.

Conclusion: TEM procedure is highly effective in selected group of patients with early rectal cancer. Mapping and examination of SLN can clarify indication for the TEM in the patients with early rectal cancer.

\section{P320}

\section{Risks Associated with Anesthesia Services for Colonoscopy: A Meta-analysis}

Muna Lougheed, David Pace, Mark Borgaonkar, Aryan Modasi; Memorial University

Background: The present study aims to assess whether the use of anesthesia services during colonoscopy is associated with an increased risk of rare complications.

Methods: A search of Medline, PubMed and Cochrane databases identified studies that compared complication rates of colonoscopies performed with the use of anesthesia services versus endoscopist-administered sedation. The four outcome variables analyzed were aspiration pneumonia, bowel perforation, splenic injury, and overall complication rate. Random effects meta-analyses were performed using an odds ratios (OR). The ROBINS1 tool was used to assess the studies for risk of bias.

Results: Eight retrospective cohort studies were considered suitable for metaanalysis consisting of 7,752,580 colonoscopies. Use of anesthesia services during colonoscopy was associated with an increased risk of aspiration pneumonia (OR $1.79(1.34,2.38), \mathrm{P}<0.0001)$. There was no association found between the use of anesthesia services during colonoscopy and splenic injury rate $(\mathrm{OR} 0.90(0.61,1.31, \mathrm{P}=0.58)$, bowel perforation $(\mathrm{OR} 1.33(0.95,1.88)$, $\mathrm{P}=0.10)$, or overall complication rate $(\mathrm{OR} 1.33(0.99,1.79), \mathrm{P}=0.06)$.

Conclusions: An increased risk of aspiration pneumonia is associated with the use of anesthesia services during colonoscopy. This risk should be considered when anesthesia services are used.

\section{P321}

Colonoscopy-Assisted Percutaneous Sigmoidopexy: A Novel, Safe, and Efficient Treatment for Inoperable Cases with Sigmoid Volvulus

Tomonori Imakita, MD, Yutaka Suzuki, MDPhD, Hironori Ohdaira, PhD, Yasunobu Kobayashi, MD, Taigo Hata, MD, Mutsumi Kaji, MD, Mitsuyoshi Urashima, MDPhD; International University of Health and Welfare

Background: Sigmoid volvulus (SV) shows a high recurrence rate even after the correction of torsion using an intestinal endoscope. Intestinal resection is radical, but the most patients with SV are too old and poor physical condition to take operation under general anesthesia.

Objective: To assess whether percutaneous fixation of the sigmoid colon from the surface of the abdomen using an endoscope (endoscopically assisted percutaneous sigmoid fixation) can be performed safely and without adverse events, and whether it can prevent recurrence of sigmoid torsion.

Patients: SV patients with American Society of Anesthesiologists physical status classification $\geq 3$ or Barthel index $<30$ were included. Patients with intestinal necrosis, who were unable to reposition, whereas who could take intestinal resection were excluded. As a result, eight patients were treated with CAPS.

Interventions: First, preparation of the intestinal tract was done. Under X-ray observation, the intestinal endoscope was inserted to the fixation site, and the puncture site was identified by transmitted illumination and finger pressure. An exploratory puncture was made with a $23-\mathrm{G}$ needle under local anesthesia to decide the fixation site. A skin incision was made, and sigmoid colon fixation was performed using a two shot anchor. The procedure was repeated at 5-10 sites and complete.

Main Outcome Measurements: Perioperative adverse events; relapse within 12 months postoperative.

Results: Endoscopically-assisted percutaneous sigmoid fixation was carried out in eight cases. Median age was 72.5 years (25-75\%): 59-78.5 years). ASAPS classifications were class 2, 1 patient; class 3, 4 patients; and class 4, 3 patients. Median Barthel index was 10 (25-75\%: 0-20). Median procedure time was $72.5 \mathrm{~min}(25-75 \%$ : $56-80 \mathrm{~min})$, median time required for fixation was $16 \min (25-75 \%$ : $15-21.5 \mathrm{~min})$. Perioperative adverse events were only one case of subcutaneous emphysema. There were no cases of serious adverse events such as peritonitis, small intestine injury, intraabdominal abscess, or intestinal obstruction, and no cases of surgical wound infection. There were no cases of recurrence during the 1-year follow-up period. Three patients died, the result of aspiration pneumonia in two patients and senile decay in one patient. As these were all more than four months after the procedure, they were judged to be death from other illnesses having no direct relationship with the procedure.

Conclusion: CAPS was carried out safely and no recurrence was found over the long term. 


\section{P322}

Reducing Variability in Outcomes: Conversion Rate Analysis in Minimally Invasive Sigmoidectomy

Elizabeth R Raskin, $\mathrm{MD}^{1}$, Dongjing Guo ${ }^{2}$, Shilpa Mehendale, $\mathrm{MD}^{2}$; ${ }^{1}$ Loma Linda University, ${ }^{2}$ ISI

Introduction: Providing predictable outcomes after minimally invasive surgery (MIS) is an important priority for patients, hospitals and policy makers. The aim of this study was to examine the patterns and markers associated with conversion of MIS to open procedures in high volume hospitals and to understand the specific risk factors associated with high and lower conversion rates hospitals.

Methods and Procedures: Using the Premier health care database, we selected data from institutions who performed at least 100 laparoscopic-assisted (LA) or robotic-assisted (RA) sigmoidectomy cases during 2013 and 2015Q3. Data from non-elective cases or patients $<18$ years old were excluded. The median hospital level conversion rate was selected as the cutoff to categorize hospitals as "higher-conversion rate" or "lower-conversion rate" hospitals. Data from patients treated at the above categorized hospitals were compared. A step-wise backward model selection method was applied to identify the risk factors associated with conversion using logistic regression model for both cohorts. Unadjusted conversion rates and adjusted odds ratios for the different surgical approaches were reported.

Results: Data for 8821 patients from 75 hospitals were analyzed. The median conversion rate was $10.85 \%$. Hospitals with bed size $<300$ were more likely to have lower conversion rates.

Among patients from higher-conversion rate hospitals, the conversion rate for LA and RA approaches were $18.41 \%$ (95\%CI: 17.15-19.67\%) and $11.33 \%$ (95\%CI: 9.02-13.99\%), respectively (Figure A); After adjusting for patients' age, Charleson score, race and insurance type in the multivariable logistic regression model, LA approach still had 75\% (95\%CI: 36-127\%) higher risk for conversion than the RA approach (Figure B). Among patients from lower-conversion rate hospitals, the conversion rate for the LA and RA approach were 7.33\% (95\%CI: $6.49-8.16 \%$ ) and $2.89 \%$ (95\%CI: $1.70-4.09 \%$ ), respectively (Figure A). After adjusting for patients' age, Charleson score, and hospital bed size in the multivariable logistic regression model, patients underwent LA sigmoidectomy were 2.5 (95\% CI: 1.64.0) times more likely to be converted to open than patients who underwent RA sigmoidectomy (Figure C).
Subset analyses of patients with malignancy and benign disease showed similar results.

Conclusions: In high-volume hospitals, the RA approach demonstrated significantly lower risk for conversion than the LA approach in both higher-conversion rate and lower-conversion rate hospitals. This study demonstrates reduced variability in conversion rates and consistency with the robotic-assisted approach compared to laparoscopy.

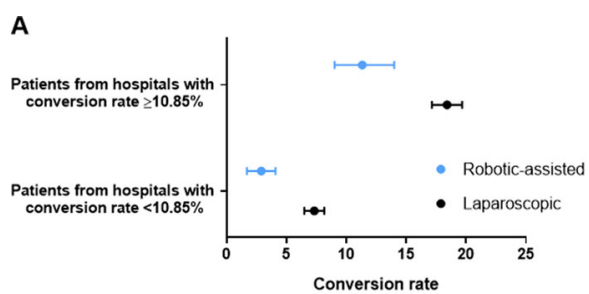

B

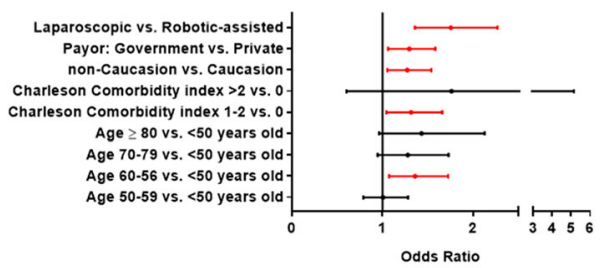

C

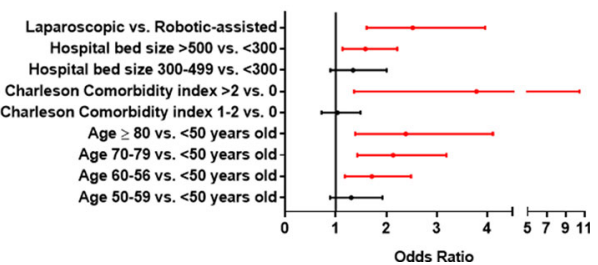


P323

\section{Narrative Operative Reports for Rectal Cancer - Now Insufficient?}

Alvaro Mendez, MD, Caren Nituda, BSN, RN, CEN, CPON, Raymond Yap, MD, BmedSci, MsurgEd, FRACS, Arman Erkan, MD, Justin Kelly, MD, FRCS, Bruce Orkin, MD, FACS, FASCRS, John Rt Monson, MD, FRCS, FACS, FASCRS; AdventHealth

Introduction: The National Accreditation Program for Rectal Cancer (NAPRC) has released guidelines for operative reports, that contains twenty mandatory elements (ASA score, case status, name of operation, modality, tumor location, distance from anal verge, mobilization of splenic flexure, level of ligation of IMV, level of ligation of IMA, distal margin, type of reconstruction, anastomotic testing method, creation of stoma, en-bloc resection, metastectomy, completeness of tumor resection, intraoperative complications, blood transfusion, TME photographed and short narrative). Although previous studies have highlighted deficiencies in narrative operative reports, there has been no comparison to the elements requested in the NAPRC guidelines. The aim of this study is to assess and compare current narrative operative reports to the new national standard.

Methods and Procedures: Data from consecutive rectal cancer operative reports from two tertiary institutions in 2016-17 was extracted and analyzed. Each note was independently graded on the 20 elements required by the NAPRC.

Results: A total of 100 operation notes were analyzed. Overall, adherence to the NAPRC standards was only $56.1 \%$. Four elements (case status, operation, modality and the short narrative) were present in $100 \%$ of reports. A further four elements (IMA and IMV level ligation, mobilization of splenic flexure and stoma) scored above $60 \%$. Four seemingly important elements (distal margin, completeness of the resection, distance from the anal verge range and tumor location) were only documented in $10-40 \%$ of cases. Not a single report included either ASA or documentation that the TME specimen had been properly photographed.

Conclusion: The evaluation of current operative reports shows that compliance with new NAPRC guidelines for operative reporting was low. A significant improvement in current documentation practice is required to achieve NAPRC accreditation standard. Synoptic reporting may provide a solution to this challenge.

\section{P324}

\section{The Risk Factors of Recurrence Stage II Colorectal Cancer}

Sigeki Yamaguti, Professor, Yasumitu Hirano, PhD, Toshimasa Ishii, $\mathrm{PhD}$, Hiroka Konndou, Kiyoka Hara, Asami Suzuki, Sinntarou Isikawa, Takuhisa Okada, Nao Obara, Sinyiti Sakuramoto, Kouzyun Okamoto, Isamu Koyama, Liming Wang; Saitama medical university international medical center

Background: The risk factors of recurrence in patient with stage II colorectal cancer have not been clarified in Japan, and the indications for adjuvant chemotherapy have also not been determined. In addition, it is not clear whether stage II colon and rectal cancer have the same risk factors for recurrence. The aim of the present study was to identify the risk factors of postoperative recurrence in stage II colorectal cancer.

Patients and Methods: We retrospectively reviewed a database of 990 patients who had undergone curative surgery for stage II colorectal cancer in our hospital between 2007 and 2018. There were 619 patients with colon cancer and 371 patients with rectal cancer. Patients characteristics and pathological features including age, gender, family history, BMI, tumor diameter, gross type of tumor, infiltration degree (T3/T4), tumor grade, perineural invasion, vascular invasion, lymphatic invasion, pathologic examination of lymph node number and pre-operative carcinoembryonic assay (CEA) level were compared in patients with recurrence and in patients without recurrence.

Results: The mean age of patients with colon cancer was 69.5 years and 66.4 years with rectal cancer. 508 cases $(82.1 \%)$ were treated laparoscopically for colon cancer, and 285 cases (76.8\%) for rectal cancer. The median follow-up period for colon cancer was 42.2 months and 41.8 months for rectal cancer. Forty-four recurrences occurred in colon cancer $(7.1 \%)$, and forty-four recurrences occurred in the rectal cancer $(11.9 \%)$. The pre-operative serum CEA level was significantly high in recurrent colorectal cancer patients. The proportion of $\mathrm{T} 4$ was also significantly high in recurrent patients. In addition, higher BMI, larger tumor diameter, poorly differentiated adenocarcinoma, and lympho-vascular invasion were also thought to be risk factors of recurrence in rectal cancer patients.

Conclusion: Our study showed that serum CEAlevel and infiltration degree (T4) could be used as prognostic factors that suggest a higher risk for recurrence in patients with colorectal cancer. 
P325

Selective Portal Vein Ligation and Liver Regeneration. An Experimental Model

I. Fedoriv, $\mathrm{MD}^{1}$, I. Garcia-Alonso, MD, $\mathrm{PHD}^{2}$, B. Herrero De La Parte, PHD $^{2}$, J. Guitierrez-Saenz ${ }^{2}$; ${ }^{1}$ Dpt. of Surgery. Ivano-Frankivsk national medical university. Ukraine., ${ }^{2}$ Laboratory of Experimental Surgery. Dpt. of Surgery. University of The Basque Country. Spain Introduction: Selective portal branch ligation is being proposed as a useful technique to increase remnant liver following liver metastases surgical removal. It would be useful to check drugs which can enhance liver regeneration in this scenario without increasing tumor growth. We have developed a murine model to carry out these kind of experiments.

Methods: Three months old male WAG/RijHsd rats were randomly allocated into three groups of 7 animals each. The first group was used as control to obtain normal values of liver lobes volume and weight. The other two groups were subjected to selective ligation of the portal branch irrigating the left lateral liver lobe, and the animals were sacrificed after 3 (group PVL-3D) or 7 days (group PVL-7D). When the animals were sacrificed blood samples were obtained and the whole liver was carefully removed. The volume and weight of the whole liver and of the left lateral lobe were assessed.

Results: The left lateral lobe volume and weight was $40 \%$ of the whole liver in the control group; while it dropped to $16 \%$ three days after portal ligation, and $12 \%$ when assessed a week later. The total liver weight remained constant following portal branch ligation: Control $8.7 \pm 1$, PVL-3D $7.3 \pm 1.1$, PVL-7D $7.4 \pm 0.9 ; \mathrm{p}=0.85$ ). Conclusion: Ligation of the portal branch irrigating the left lateral liver lobe, reduced the relative volume \& weight of the left lateral lobe of the liver, while not affecting total liver mass.

\section{P326}

Risk Reduction of Anastomotic Leakage After Intraoperative ICG Fluorescence Imaging in Patients with Left-sided Colon or Rectal Cancer

Takeshi Yanagita, MD, Masayasu Hara, Korehito Takasu, Yuzo Maeda, Nozomu Nakai, Takahisa Hirokawa, Kazuyoshi Shiga, Hiroki Takahashi, Yoichi Matsuo, Shuji Takiguchi; Nagoya City University

Introduction: Postoperative anastomotic leakage (AL) is still one of the most devastating complications after surgery. Not only causing severe postoperative general conditions, but also AL leads to oncological poorer prognosis. Previous investigations have already revealed several risk factors, among which, intestinal perfusion at anastomotic site is thought to be one of the most influential factors. ICG fluorescence imaging made it possible to visualize tissue perfusion more clearly. Although resent studies have shown a promising result, studies with relatively small number of patients are available so far, and no report have demonstrated its efficacy statistically. we evaluated its efficacy in terms of decreasing the incidence of $\mathrm{AL}$ in patients with left-sided colon or rectal cancer by comparing with those without ICG assessment.

Methods: A single-center, observational study was performed in consecutive 130 patients with left-sided colon or rectal cancer who underwent ICG fluorescence imaging during elective operations from March 2015 to December 2017 (group I). On the other hand, 130 patients who had similar diagnoses and procedures as ICG group from November 2013 to February 2015 were reviewed on charts as a historical control group (group C).

Data was analyzed using inverse probability of treatment weighting based on propensity scores to control for bias and the efficacy of ICG fluorescence imaging were assessed via a weighted Cox proportional hazards model in addition to number needed to treat (NNT).

Results: Patients' characteristics like age, gender, BMI etc. were comparable between both groups, except tumor location and preoperative chemoradiation therapy (CRT).

The number of cases with rectal cancer and CRT was statistically larger in group I than group $\mathrm{C}$, which led to the significant difference of anastomotic technique (double stapling technique was preferred in group I). Planned transactional line of colon was changed in thirteen patients after ICG assessment because of suspicious poor blood flow. Symptomatic AL occurred in 5 patients $(3.8 \%)$ in group I and in 11 patients $(8.5 \%)$ in group $\mathrm{C}$ with weighted $\mathrm{P}$ value of 0.073 and NNT was calculated to 17.9. It was evaluated that 315 cases in each group were necessary to show the significant difference from the weighted data.

Conclusion: In conclusion, this study demonstrates ICG assessment had tendency to decrease AL, and could be beneficial for at least one in every eighteen patients from the result of NNT. Additional 200 cases in each arm are necessary to prove statistical significance in terms of preventing AL. 


\section{P327}

\section{Surgical Outcome of Laparoscopic Surgery for Right Colon} Cancer: Single Institute Experience

Shinobu Ohnuma, Takeshi Naitoh, MD, Hideaki Karasawa, MD, Atsushi Kohyama, MD, Hideyuki Suzuki, MD, Kazuhiro Watanabe, MD, Hirofumi Imoto, MD, Akihiro Yamamura, MD, Takeshi Aoki, MD, Naoki Tanaka, MD, Hiroaki Musha, MD, Takanori Morikawa, MD, Fuyuhiko Motoi, MD, Takashi Kamei, MD, Takanori Ishida, MD, Michiaki Unno, MD; Tohoku University Hospital

Aim: The aim of this study is to investigate surgical outcome of the patients with laparoscopic surgery for right colon cancer in single institute.

Methods: One-hundred thirty-four patients ( $\mathrm{M}: \mathrm{F}=63: 71$, median age, 72 years) who had laparoscopic surgery for right colon cancer (Stage 0 - III) from October 2008 to July 2018 were retrospectively analyzed. The median follow-up period was 36 months.

Results: The pathological disease stage (pStage) was $0(\mathrm{n}=7)$, I $(\mathrm{n}=43)$, IIA $(\mathrm{n}=40)$, IIB $(\mathrm{n}=6)$, IIC $(\mathrm{n}=2)$, IIIA $(\mathrm{n}=7)$, IIIB $(n=25)$, and IIIC $(n=4)$, respectively (TNM7th). Operative time, blood loss, and postoperative hospital stay were $212 \min (93$ - 453), $42.5 \mathrm{~g}(1-695)$, and 10 days $(5-72)$, respectively (median). Postoperative complications (Clavien-Dindo above grade II) were observed in 16 patients $(11.9 \%)$. One patient $(0.7 \%)$ required conversion to a laparotomy. The number of patients with recurrence was $9(6.7 \%)$, whose pStages were IIA $(\mathrm{n}=1)$, IIB $(\mathrm{n}=2)$, IIIA $(\mathrm{n}=2)$, IIIB $(n=3)$, and IIIC $(n=1)$, respectively. Among them, initial recurrence was observed in liver $(n=4)$, para-aortic lymph node $(\mathrm{n}=2)$, peritoneum $(\mathrm{n}=1)$, brain $(\mathrm{n}=1)$, and port site $(\mathrm{n}=1)$. Those recurrences were diagnosed within 1 year $(n=4), 2$ years $(n=3)$, and 3 years $(n=2)$, respectively. The liver metastases of two patients and a port-site recurrence of one patient were surgically removed, and the brain metastasis in one patient were treated with Cyberknife. The remaining four patients with recurrence had chemotherapies, and two of those died of progressive disease. 5-years over-all survival rate and disease-free survival (DFS) rate of all patients were $98 \%$ and $91 \%$. 5-years DFS of Stage IIA, IIB, IIC, IIIA, IIIB, and IIIC were 96.7, 60.0, 100, 66.7, 84.7, and 66.7\%, respectively. Lymphatic invasion, vascular invasion, and lymph node metastasis were significantly correlated with recurrence $(p=0.0005$, 0.007 , and 0.008).

Conclusions: Surgical outcome of laparoscopic surgery for right colon cancer is safe and feasible.

\section{P328}

Is the Laparoscopic Total Mesorectal Excision with Selective Lateral Lymph Node Dissection for Locally Advanced Rectal Cancer After Preoperative Neoadjuvant Chemoradiotherapy Safe? A Single-center Study from China

Xuyang Yang, Chaoyang Gu, Lina Ye, Tao Hu, Liang Bi, Mingtian Wei, Xiangbing Deng, Wenjian Meng, Ziqiang Wang, Zongguang Zhou; Department of Gastrointestinal Surgery, West China Hospital, Sichuan University, No. 37 Guo Xue Alley, Chengdu 610041, Sichuan Province, CHINA

Background: To evaluate the technical feasibility, safety and oncological outcomes of laparoscopic total mesorectal excision (TME) with selective lateral lymph node dissection (sLLND) in patients with locally advanced rectal cancer (LARC) and clinically suspected swollen lateral lymph node (LLN) treated with preoperative neoadjuvant chemoradiotherapy (nCRT).

Methods: Consecutive patients with LARC and swollen LLN underwent laparoscopic TME and sLLND following preoperative nCRT between October 2012 and May 2018 were reviewed from a prospectively collected database at West China Hospital in China. Patient demographics, operation safety, perioperative complications, short-term oncological outcomes were analyzed.

Results: A total of 34 patients underwent laparoscopic TME and sLLND following preoperative nCRT were identified. All procedures were completed under laparoscopy without conversion to open surgery. The median operation time and blood loss was $310 \mathrm{~min}$ (ranged from 170 to 540) and $90 \mathrm{ml}$ (ranged from 30 to 450), respectively. The median number of LLN harvested was 10.5 (ranged from 1 to 29). LLN metastasis was found in eight patients $(23.5 \%)$. The major postoperative complications were found in three patients $(8.8 \%)$. The median hospital stay was 7 days (ranged from 4 to 27), and the median time to earlier resumption of fluid intake was $72 \mathrm{~h}$ (ranged form 36 to 120). The postoperative 30-day mortality rate was $0 \%$. With the median follow-up of 24 months, two anastomtic recurrence were found and no lateral pelvic recurrence had developed. For the whole patients, the 2-year OS, RFS were $90 \%$ and $95 \%$, respectively. Conclusion: With the advantage of a minimally invasive approach, laparoscopic TME and sLLND following preoperative nCRT in patients with LARC and swollen LLN was safe and feasible. This procedure obtain acceptable perioperative and short-term oncological outcomes.

Keywords: Laparoscopy; lateral lymph node dissection; lateral lymph node; neoadjuvant chemoradiotherapy; rectal cancer 
P329

\section{Appendiceal Diverticulum Presenting as Appendicitis: Case Series}

Indraneil Mukherjee, Zach Garcia, Weihua Song, Jocelyn Villanueva, Kokila Mody; Staten Island University

Introduction: Appendiceal diverticulitis, a rare but distinct clinical entity that was 1st described in 1983 by Kelynak. It can be both congenital or acquired, however the former is extremely rare with an incidence of $0.014 \%$ while acquired has an incidence of $1.9 \%$. Appendiceal diverticulitis may mimic acute appendicitis, however, it occurs in relatively older age group, with a fourfold increased risk of perforation and 30 times increased mortality as reported in the literature. They are also frequently associated with mucinous adenomas and carcinoid tumors.

Presenting Cases: We present two cases of congenital appendiceal diverticulitis encountered in our hospital in the last one year.

Case 1: He is a 30-year-old man, who presented to the ED with right lower abdomen pain with nausea and vomiting. The patient was afebrile with a WBC count of $13.1 \mathrm{~K} / \mathrm{mm}^{3}$. Acute appendicitis was suspected, and the patient underwent a Laparoscopic appendectomy. The histological diagnosis revealed an acute appendicitis and periappendicitis, in a $3.4 \mathrm{~cm}$ long tip of appendix and a separate $1.7 \mathrm{~cm}$ long congenital diverticulum.

Case 2: She was a 30-year-old lady, with right lower abdomen pain for 1 day. She presented with a WBC count of $8.9 \mathrm{~K} / \mathrm{mm}^{3}$. Post Laparoscopic appendectomy, her histological diagnosis revealed, a $5 \mathrm{~cm}$ appendix with acute appendicitis in the appendiceal diverticulum.

Both patients were discharged on post-op day 1 and recovered well. Discussion: Histologically acquired diverticuli are usually seen at the distal end of the appendix, ranging from $2-5 \mathrm{~mm}$ and described as "pseudo diverticuli". They have only the mucosa and submucosa herniate through a defect in the muscle layer. A herniation of all three layers is seen in the congenital form, which is located on the antimesenteric edge of the appendix.

Appendiceal diverticulitis has been classified in 4 sub-types. Type 1 occurs when a normal appendix is found with an acutely inflamed diverticulum. Type 2 implies an acutely inflamed diverticulum with surrounding appendicitis. Type 3 is a normal appendicitis with an incidental uninvolved diverticulum. Type 4 is an incidental appendiceal diverticulum with no evidence of appendicitis or diverticulitis. Both of our cases were Type 2.

Results: Appendiceal diverticulitis while rare should be considered in right lower quadrant pain. Due to its milder symptoms at presentation, they can often get missed and result in perforation. Therefore, we recommend prompt appendectomy on diagnosis.

\section{P330}

The Impact of Opioid Utilization on Quality Outcomes and Costs in Laparoscopic Colorectal Surgery

Thais Reif De Paula, MD, Ravi P Kiran, MD, Deborah S Keller, MS, $\underline{\mathrm{MD}}$; Columbia University Medical Center

Background: The use of laparoscopy is proven to improve patient outcomes and costs. Prior work showed that laparoscopy had higher daily opioid use than open colorectal surgery, with lower overall use from the shorter length of stay (LOS). With the opioid epidemic and need to optimize improve outcomes and costs in the shift towards value-based care, investigation into the link between opioid utilization and outcome measures is warranted. Our goal was to evaluate the relationship between opioid utilization and quality outcomes of LOS, readmissions, complications and costs in elective laparoscopic colorectal surgery.

Methods: The Premier Database was reviewed for inpatient laparoscopic colorectal procedures where the patients received opioids during the postoperative course from 1/1/2014-9/30/2015. Opioid use was stratified into 4 groups based on the distribution of total Morphine Milligram Equivalents (MME/stay) in the population $(<50$, $50-110,111-250,>250)$. Univariate analysis compared patient and provider demographics across the utilization groups. After controlling for patient and hospital characteristics, multivariate regression analyzed the relationship between opioid use, costs, and quality outcomes.

Results: During the study period, 18,395 patients met inclusion criteria. The overall mean MME/stay was 62.27 , and the mean days of opioid use was 3.61. For MME by group, 34\% used $<50 \mathrm{MME}, 24 \%$ each used 50-110 and 111-250, and $18 \%$ used $>250$. In the univariate analysis, total opioid MME was directly associated with overall complications, LOS, readmissions, and total costs (all $\mathrm{p}<0.001$ ) (Table 1). The multivariable analysis confirmed an independent effect of opioids on each of the quality outcome measures. Compared to low ( $<50 \mathrm{MME} /$ stay $)$ opioid use, higher utilization was associated with a 57\% longer LOS (OR 1.57, 95\% CI 1.54-1.60, $\mathrm{p}<.0001$ ), 63\% higher overall complications (OR 1.63, 95\% CI $1.47-1.82, \mathrm{p}<.0001), 74 \%$ higher readmission rate (OR 1.74, 95\% CI $1.48-2.05, \mathrm{p}<.0001)$, and $28 \%$ higher total costs of care (OR $1.28,95 \%$ CI $1.26-1.30, \mathrm{p}<.0001)$.

Conclusions: There is a direct relationship between postoperative opioid dose and common quality outcome measures after elective laparoscopic colorectal surgery. Higher opioid doses were independently associated with higher complication and readmission rates, longer LOS, and higher costs. Furthermore, incremental increases in daily opioid use by MME was associated with corresponding increases in complications, readmissions, LOS, and costs. Given these results, greater emphasis should be placed on reducing opioid use to improves patient and financial outcomes after elective laparoscopic colorectal surgery. With the transition to value-based care, opioid use could become an addition quality measure

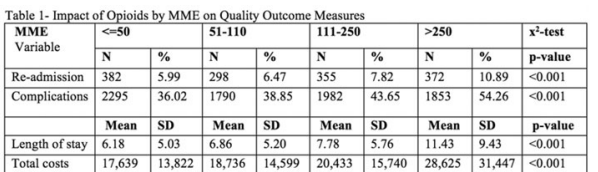




\section{P331}

LOCULA- A minimally invasive procedure for Pilonidal Sinus: Pilonidal disease management needs a paradigm shift from more to less

Pankaj Garg, MBBS, MS, FASCRS ${ }^{1}$, Sachi Singhal, MBBS ${ }^{2}$, Sachin B Jamma, MBBS, $\mathrm{MS}^{3}$, Suresh Vasistha, MBBS, $\mathrm{MS}^{4} ;{ }^{1}$ Garg Fistula Research Institute, Panchkula, India, India, ${ }^{2}$ Dayanand Medical College, Ludhiana, Punjab, India, ${ }^{3}$ Ashwini Rural Medical College, Kumbhari, Solapur, Maharashtra, India, ${ }^{4}$ Mangalam Hospital, Gurgaon, India

Purpose: Pilonidal sinus disease (PND)(including acute abscess) is very much similar to any subcutaneous abscess and should be treated like one. Logically also excision is unwarranted as PND is not a malignancy. Therefore, standard treatment of a routine abscess, simply laying open and curettage of sinus under local anesthesia(LOCULA), should suffice as adequate treatment for PND. We tested the efficacy of laying open and curettage of sinus under local anesthesia(LOCULA) procedure in all types of PND (simple and complicated).

Methods: All patients with chronic PND, recurrent disease and abscess were prospectively recruited between March 2011 and June 2018. Laying open with curettage of sinus cavity and its deroofing was done under local anesthesia on an outpatient basis. The wound was allowed to heal by secondary intention(Figure-1). The primary outcome measure was cure rate (recurrence). The secondary outcome measures were hospital stay, operating time, time taken to return to work, healing time and complication rate.

Results: Eighty-one(81) consecutive patients were prospectively recruited over a 7.5 years and were followed for 3-89 months(median- 26 months). One patient was lost to follow up. Mean age $23.4 \pm 4.7$ years and $\mathrm{M} / \mathrm{F}-65 / 15.24 / 80(30 \%)$ presented as pilonidal abscess and 56/80(70\%) as chronic pilonidal disease. 21/80(26\%) had recurrent disease. The operating time was $21.0 \pm 5.5 \mathrm{~min}$ and the hospital stay after the operation was $61.9 \pm 21.5 \mathrm{~min}$. The patients were able to resume their normal work in $3.8 \pm 3.1$ days and the healing time was $44.6 \pm 8.3$ days. $76 / 80(95 \%)$ patients had complete resolution of the disease and four $(5 \%)$ had a recurrence. All the recurrences happened in the patients who didn't adhere to the prescribed recommendations after the complete healing. Three patient with recurrence was operated again with the same procedure and got cured. The fourth patient was lost to follow up. Thus the overall cure rate was $98.75 \%$. Two patient had a minor bleeding within the first week after the operation. Both the patients were managed conservatively in the outpatient clinic and the wound got healed subsequently. Conclusions: LOCULA had cure rate \& complication rate of $95 \%$ (overall- $98.75 \%$ ) and $2.5 \%$. respectively. The procedure is also much less morbid(done under local anesthesia, no admission required, resumption of normal work within hours and much smaller wound). Laying open(deroofing) and curettage under local anesthesia (LOCULA) as an outpatient procedure should be the first line procedure for all types of Pilonidal disease and abscess.

\section{P332}

\section{Is Simultaneous Laparoscopic Multi-organ Resection Possible in Colorrectal Surgery?}

María Elena Peña, Mariana Matzner Perfumo, José Piatti, Maximiliano Enrique Bun, Nicolás Rotholtz; Hospital Alemán de Buenos Aires

Introduction: Laparoscopic approach is nowadays the treatment of choice in elective colorectal surgery since it provides faster recovery with less postoperative morbidity. In certain occasions, it is necessary to associate a second organ resection. Whether simultaneous multiorgan resection contraindicates the mini-invasive approach or modifies postoperative evolution is still a matter of debate. The aim of this study is to analyze the feasibility and safety of laparoscopic colorectal surgery combined with another abdominal organ resection.

Methods and Procedures: Patients operated due to colorectal disease from 2000 to 2018 were identified. Elective laparoscopic resections of the colon and rectum were included. The sample was divided into two groups: surgeries associated with another abdominal organ resection (G1) and those not associated with second resection (G2). We analyzed demographic factors, intraoperative variables and postoperative morbidity and mortality using Dindo-Clavien score.

Results: A total of 1585 surgeries met the inclusion criteria. Eightyeight patients belonged to G1 and 1497 to G2. Mean age was 60 years old ( $\mathrm{p}=\mathrm{NS})$ with a higher proportion of males in G2 (G1: $39(44 \%)$, $\mathrm{G} 2: 827(55 \%), \mathrm{p}<0.05)$. No differences were found when comparing smoking, body mass index, previous abdominal surgery or ASA score. Surgical colorectal procedures were: $824(52 \%)$ left colectomies, $382(24 \%)$ right colectomies, 57 (3.5\%) segmental colectomies, $144(9 \%)$ total colectomies, $156(10 \%)$ low anterior resections and proctectomy and $22(1.5 \%)$ abdominoperineal resections $(\mathrm{p}=\mathrm{NS})$. The most frequently associated organ resected were: $26(29.5 \%)$ hepatectomies, 18 (20\%) cholecystectomies, $14(16 \%)$ enterectomies and $11(12.5 \%)$ adnexectomies. Intraoperative complications (G1: $15(17 \%), \mathrm{G} 2: 108(7 \%), \mathrm{p}<0.05)$, conversion rate (G1: 25 (28\%), G2: $105(7 \%), \mathrm{p}<0.05)$ and the use of postoperative intensive care unit (G1: $28(31 \%), \mathrm{G} 2: 176(12 \%), \mathrm{p}<0.05)$ were more frequent in G1. Operative time and length of hospital stay were also significantly higher in G1 (G1: $227 \mathrm{~min}, \mathrm{G} 2: 173 \mathrm{~min}, \mathrm{p}<0.05$ and G1: 7.1 days, G2: 4.7 days, $p<0.05$ ). No differences were found in reoperation rate, rehospitalization, morbidity and mortality at 30 days.

Conclusions: Laparoscopic colorectal surgery associated with another abdominal organ resection is feasible and safe. In spite of being more complex procedures, they do not increase postoperative morbidity and mortality. 
P333

\section{Robotic Colostomy Reversal (RCR) at an Academic Medical} Center: A Review of Six Cases

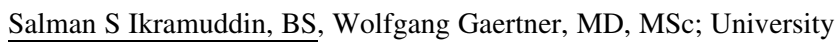
of Minnesota

Introduction: Hartmann's reversal is a demanding surgical procedure with considerable risk. Laparoscopic approaches have become the standard of care and have been shown to offer benefits compared to open surgery.1Recently, the use of robotics for colon and rectal procedures has grown. This is primarily driven by improved 3D visualization, more precise manipulation of tissues, and superior ergonomics.2Colostomy reversal remains an attractive target for robotic surgery although its outcomes remain unstudied. We report the initial outcomes of patients who have undergone robotic colostomy takedown in the setting of a Hartman's procedure.

Methods: Review was performed of 6 cases of robotic colostomy reversal at an academic medical center. Cases were limited to the previous 3 years with IRB approval.

Results: Six patients were identified with a mean age (range) of 55.6 yrs (35-74). Five patients were male. A total of 4 ports were used and the DaVinci Xi was used in all cases. There were no intraoperative conversions. Indications for colostomy (n) were diverticulitis (5) and one case of extra mammary Paget's disease. Mean (SD) estimated blood loss was $75 \mathrm{ml}( \pm 45)$, length of stay was 3.5 days $( \pm 1.2)$, return of bowel function 2.2 days $( \pm 1.1$ ). Average (range) time to reversal was 4.5 months $( \pm 1.5)$. There were no readmissions, wound infections, anastomotic leaks in the post-operative period. All patients are still in follow up with no complications to date.

Conclusions: Robotic Hartman's takedown is feasible. Comparison with laparoscopic and traditional approaches is warranted to ascertain the advantages to patient and surgeon alike following robotic colostomy takedown.

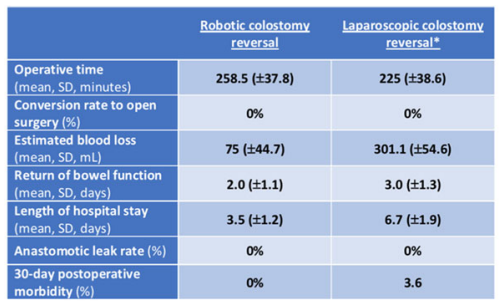

\section{P334}

Comparison of Self-expandable Metallic Stents and Transanal Decompression Tubes as a Bridge to Surgery for Treating Malignant Colorectal Obstruction

Masafumi Tomita, MD, Kouji Yasuda, Nozomi Kashu, Hiroyuki Yoshitake, Tomoya Takami, Koutaro Hatano, Naoki Kataoka, Tomoyuki Yamaguchi, Yoshiharu Shouno, Shinichiro Makimoto; Kishiwada Tokushukai Hospital

Background: Self-expandable metallic stents (SEMS) are widely accepted for treating malignant colorectal obstruction (MCO) in stenting procedures as a bridge to surgery (BTS). Transanal decompression tubes (TDT) for MCO are also used in East Asia, especially in Japan. This study aimed to retrospectively evaluate the safety and efficacy of SEMS and TDT for MCO.

Methods and Procedures: We identified 70 patients who underwent procedures using TDT or SEMS at the Kishiwada Tokushukai Hospital. From January 2009 to December 2011, 36 consecutive patients underwent TDT for MCO. After January 2012 (when the SEMS procedure became covered by the Japanese national health insurance system) to December 2014, 34 patients underwent SEMS for MCO. There was no significant difference in the TNM stages of the two groups. Procedural complications, postoperative complications, and 3-year disease-free survival rates were compared between the two groups. Technical success was defined as accurate SEMS or TDT placement on the first attempt with no adverse events. Clinical success of BTS was defined as decompression and relief of obstructive symptoms until surgery with no stent-related complications or need for endoscopic re-intervention or emergency surgery.

Results: The technical success rate was $91.7 \%$ (33/36) in the TDT group and $97.1 \%$ (33/34) in the SEMS group. The clinical success rate was $69.7 \%(23 / 33)$ in the TDT group and $97.0 \%(32 / 33)$ in the SEMS group. Procedure-related preoperative complications occurred in $5.6 \%$ of TDT and $8.8 \%$ in SEMS cases; perforation occurred in $2.8 \%$ of cases in the TDT group and $2.9 \%$ of cases in the SEMS group. Primary anastomosis was possible in $71.4 \%$ of TDT and $93.9 \%$ of SEMS cases, $12.0 \%$ and $6.5 \%$ of whom had anastomosis leakage, respectively. The overall stoma creation rate was $28.6 \%$ of TDT and $6.1 \%$ of SEMS cases. The postoperative complication rate was $20.0 \%$ and $6.1 \%$ and mortality rate was $5.6 \%$ and $0 \%$, respectively; the 3 -year disease-free survival rate was $64.0 \%$ and $63.4 \%$, respectively. Conclusion: SEMS for MCO as BTS was safer and more effective than the TDT procedure. Oncological outcomes were similar for SEMS and TDT. Thus, SEMS procedures may be useful and less burdensome for patients with MCO. 


\section{P336}

\section{Compliance with ERAS Protocol in Colorectal Surgery is Associated with Short-Term Outcomes and Long-Term Survival Benefit}

Natalia Gajewska, Grzegorz Torbicz, Magdalena Mizera, Jan Witowski, Magdalena Pisarska, MD, Mateusz Rubinkiewicz, MD $\mathrm{PhD}$, Piotr Major, MD, PhD, Michal Pedziwiatr, MD, PhD; 2nd Department of General Surgery, Jagiellonian University Medical College, Cracow, Poland

Introduction: Better adherence to ERAS is associated with improved short-term outcomes and increased long-term survival. Enhanced recovery after surgery (ERAS) pathways have proven to enhance postoperative recovery and reduce postoperative morbidity and length of hospital stay after colorectal cancer surgery. However, despite the benefits of the ERAS program in short-term results, little is known about its impact on long-term results.

Materials and methods: Between the years 2013 and 2015, 335 patients underwent laparoscopic colorectal cancer resection in 2nd Department of General Surgery Jagiellonian University Medical College. Patients were divided into two groups according to their degree of adherence to the ERAS interventions: group $1-<80 \%$ (103 patients) and group $2-\geq 80 \%$ (232 patients). The relation between the rate of compliance to the ERAS protocol and 3-year survival was analysed according to the Kaplan-Meier method with Log-rank test.

Results: The groups were similar in terms of demographics and surgical parameters. The median compliance to ERAS interventions was $85 \%$. Our analysis revealed, that adherence to the ERAS protocol in the group 2 with $\geq 80 \%$ adherence was associated with significantly shorter length of hospital stay ( 6 vs 4 days, $p<0.0001$ ), lower rate of postoperative complications $(44.7 \%$ vs. $23.3 \%, p<0.0001)$ and improved in functional recovery parameters: tolerance of oral diet $(53.4 \%$ vs. $81.5 \%, \mathrm{p}<0.0001)$ and mobilization $(77.7 \%$ vs. $96.1 \%$, $\mathrm{p}<0.0001)$ on the first postoperative day. Increased adherence to the ERAS protocols (group 2) was associated with significantly improved long-term overall survival compared with patients with lower adherence (group 1) ( $<<0.0007$ ). 3-year overall survival was $89 \%$ and $76 \%$ in group 1 and 2 , respectively.

Conclusion: High adherence to the ERAS protocol improve shortterm outcomes such as length of hospitalization, morbidity, recovery parameters, as well as long-term survival after laparoscopic colorectal cancer surgery.

\section{P337}

The Perinephric Fat Thickness Measured on Computerized Tomography Predicts the Technical Difficulty of Laparoscopic Colectomy

Tomoyuki Ueki, Hiromichi Sonoda, Toru Miyake, Tomoharu Shimizu, Masaji Tani; Shiga University of Medical Science

Background: It is considered that high visceral fat volume affects the technical difficulty in laparoscopic surgery. However, complex software image processing is required to calculate the visceral fat by using computerized tomography. To predict the amount of visceral fat more easily, we developed a practical technique by using "the perinephric fat thickness" measured on computerized tomography. In this study, we investigated the correlation between the perinephric fat thickness (PFT) and the conventional obesity parameters and the possibility that PFT could be a useful predictor of technical difficulty in laparoscopic colectomy.

Methods: We retrospectively reviewed 77 consecutive patients who received laparoscopic sigmoidectomy or high anterior resection for colorectal cancer between April 2014 and June 2018 in Shiga University of Medical Science hospital. We examined five obesity parameters as below, body mass index (BMI), subcutaneous fat area (SFA), visceral fat area (VFA), total fat area (TFA: SFA + VFA), and PFT. Such parameters were calculated by using CT images scanned preoperatively. We used "AZE Virtual Place" to measure SFA, VFA and TFA on one cross-sectional scan obtained at the level of left renal vein. We defined PFT as the distance from the renal capsule to the sidewall in parallel to the left renal vein.

Results: The median age was 70 years old (38-91). 35 patients were male, and the other 42 patients were female. The tumor locations were described as below (Sigmoid : Rectosigmoid $=49: 28$ ). The median maximum tumor diameters were $34 \mathrm{~mm}$ (10-90). The median BMI, SFA, VFA, TFA, and PFT were 21.7 (15.1-33.7), $82.4 \mathrm{~cm}^{2}$ (15.5236), $104 \mathrm{~cm}^{2}(6.2-367.1), 193.7 \mathrm{~cm}^{2}$ (23.5-603.2), and $12.6 \mathrm{~mm}$ $(1.77-29.4)$, respectively. The median operative time and the intraoperative blood loss were $201 \mathrm{~min}$ (134-343) and $9 \mathrm{~g}$ (0-450), respectively. Postoperative complications (Clavien-Dindo classification, $>$ Grade 1 ) were occurred in 12 patients $(15.6 \%)$. The median length of the postoperative hospital stay was 9 days (7-40). BMI, SFA, VFA, and TFA had significant correlations with PFT $(\mathrm{p}=0.0003,0.03,<0.0001,<0.0001)$. We divided patients into high and low groups in accordance with the median of each obesity parameters. The high groups of all obesity parameters: BMI, SFA, VFA, TFA and PFT required a significantly longer operative time than the low groups $(\mathrm{p}=0.014,0.014,0.023,0.0003,0.009)$. In twoway analysis of variance, only the high group of TFA tended to require a longer operative time $(\mathrm{p}=0.089)$.

Conclusion: PFT may be a simple and useful predictor comparable to VFA for assuming the technical difficulty of laparoscopic colectomy. 


\section{P338}

\section{Can Statistical Process Control (SPC) to Drive Improvement in Length of Stay After Colorectal Surgery?}

Thais Reif, MD, Deborah S Keller, MS, MD, Guanying Yu, MD, Haiqing Zhang, MD, Laura Hyde, MD, Ahmed Al-Mazrou, MD, Ravi P Kiran, MD; Columbia University Medical Center

Background: New methods of improving surgical quality are needed. A key quality outcome measure is lengthof stay(LOS), as it's linked to complications and costs. A novel method to reduce LOS is to apply Statistical Process Control(SPC) to identify process measures- specific institutional practices that lead to an outcome measure- related to outliers from the mean. SPC uses statistical methods to monitor and control a process, reducing variation and improving quality. Our goal was to determine if SPC can be used to identify outliers, patterns for outliers, and and their impact on quality outcome measures in elective colorectal surgery.

Methods: Review of a prospective divisional database was performedfor elective colorectal procedures using an abdominal approach from 1/1/13-5/1/ 2018. Univariate analysis was performed to identify outliers, and compare the demographic, operative, and outcome variables acrossoutliers and non-outliers. Control charts were used to display the data graphically and analyze the process performance of LOS over time. The upper and lower control limits were setat \pm 1 standard deviation(SD) from the mean.

Results: 1,097 consecutive patients were evaluated. The overall mean LOS was 6.6 days(SD 4.4), giving control limits of 11 and 2. There were 115 LOS outliers. The mean outlier LOS was 21.4(SD 12.6). The process of LOS was unstable, with large deviations from the mean. However, the rate of outliers annually and their spread around the mean was consistent across years. Outliers had comparable age, gender, BMI, albumin, primary diagnosis, and procedure performed to non-outliers, but significantly greater co-morbidities and higher rates of previous abdominal surgery(both $\mathrm{p}<0.05$ ). LOS outliers had significantly higher rates of intraoperative complications $(23.5 \%)$, post-operative complications $(81.7 \%)$, post-discharge complications(19.1\%), unplanned reoperations $(20.8 \%)$, readmissions $(26.0 \%)$, and use of post-discharge nursing facilities (21.7\%) than non-outliers(all $\mathrm{p}<0.05$ ).

Conclusions: Using SPC, outliers for LOS and the trends over time were easily identified. The number of outliers and spread of variation above the mean LOS remained stable over time. The outliers had a significant impact on quality outcome measures, with higher rates of complications, readmission, reoperation, and healthcare utilization. Being an outlier may be a marker of a sicker patient. Regardless, there are consistent patterns for these LOS outliers, and the current "one-size fits all approach" may not be the best fit for these complex patients. With this, further work can focus on developing models to identify the process measures predictive of a prolonged LOS outcome measure, and proactively developing cost-effective care practices

\section{P339}

\section{The Challenging Case of Non-cutaneous Kaposi's Sarcoma Presenting as Recurrent Rectal Abscesses}

Steven Schulberg, DO, Kevin Bain, DO, Josef Shehebar, MD; NYU Langone Hospital - Brooklyn

Introduction: Kaposi's sarcoma is a fatal disease that typically presents with cutaneous manifestations in immunocompromised individuals. There are a small number of documented cases where patients diagnosed with this disease present without cutaneous lesions.

Case Presentation: 35 year-old male presented with two weeks of left buttock pain. A CT scan confirmed a $1.8 \mathrm{~cm}$ perianal abscess with linear stranding in the left ischioanal fossa concerning for perianal fistula. The patient was taken to the OR for drainage. Flexible sigmoidoscopy revealed rectal ulcers, with biopsies taken. The patient was discharged home on antibiotics.

The patient returned with increasing pain. Physical exam revealed a new area of fluctuance, and the patient was taken back to the OR. A large abscess was encountered with a new perirectal tract. Copious pus was expressed and a seton was placed. Postoperatively the patient was treated with IV antibiotics. Increasing purulent drainage prompted re-exploration, and a new supragluteal abscess was discovered and drained.

This unusual recurrence prompted workup for an underlying immunocompromised state. HIV testing was positive, with a CD4 count of 14 . The patient was started on HAART therapy. Pathology from the rectal biopsies returned confirming Kaposi Sarcoma (KS). Metastatic workup revealed pulmonary and hepatic lesions. The patient adamantly refused chemotherapy and was subsequently lost to follow up.

Discussion: KS is an aggressive tumor that classically presents with cutaneous manifestations. Mild forms can present with involvement of lymph nodes, and lesions in skin and oropharyngeal mucosa. The multicentric nature of the tumor can result in a severe progression involving visceral organs, often including the pulmonary and gastrointestinal systems.

The prevalence of KS in AIDS patients was 20,000 times that of immunocompetent individuals in the 1980s. HAART therapy allowed the incidence of $\mathrm{KS}$ in AIDS patients to decrease from approximately $14 \%$ to around $2 \%$ by the early 2000s. AIDS-associated KS accounts for an estimated $94 \%$ of all reported cases. Low CD4 counts in immunocompromised individuals increases the likelihood of disease onset.

The ability of KS to metastasize proves to be fatal without prompt initiation of treatment. KS presenting without cutaneous lesions is an uncommon occurrence, and can prove problematic and delay diagnosis.

Conclusion: Prompt diagnosis of Kaposi's sarcoma and initiation of treatment is vital to decrease disease progression. A high index of suspicion should be present in immunocompromised patients, and clinicians must recognize atypical presentations in order to improve long term survival.

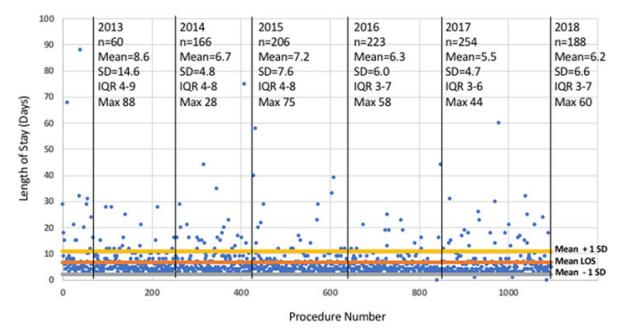




\section{P340}

\section{Robotic and Laparoscopic Colon Surgery: A Single Center} Experience

\section{Daisy Dan ${ }^{1}$, Ian Reight, $\mathrm{MD}^{2} ;{ }^{1}$ Colby College, ${ }^{2}$ Maine General} Medical Center

Introduction: Robotic-assisted minimally invasive colon surgery has been increasingly used for the treatment of various benign and neoplastic diseases in the past few years. Recent research has provided evidence that robotic assistance in surgeries offers more degrees of freedom, three-dimensional visualization, and possible better surgical outcome. However, there is limited data and research on colon surgeries to verify whether robotic-assisted colon surgeries would be a safe and alternative option. This study is designed to compare robotic-assisted and conventional laparoscopic colon surgeries through a retrospective chart review.

Methods and Procedures: The surgical techniques, the conversion rate from laparoscopic or robotic-assisted surgery to open, length of hospital stay, operative time and patient charges from patients that underwent colon surgeries with the $\mathrm{Da}$ Vinci Si robot (Intuitive Surgical) at Maine General Medical Center, Augusta, ME during 2015-2017 were collected analyzed $(n=338)$.

Results: There were significant differences in patient charges, length of stay, the operative time between either minimally invasive laparoscopic or robotic-assisted and open colon surgeries. Both patient charges and length of stay are comparable between laparoscopic and robotic-assisted colon surgeries. Robotic-assisted colon surgeries were found to have longer operative time in comparison to both laparoscopic and open colon surgeries. Decreasing trends in the conversion rate and length of stay when patients underwent roboticassisted colon surgeries were observed.

Conclusions: From the decreasing trend in clinical outcomes found in this study supported that robotic-assisted colon surgery can be a safe and feasible alternative to the conventional laparoscopic approach. Increased operative time is one of the disadvantages of robotic-assisted colon surgeries and can be attributed to mainly robot docking.

\section{P341}

Barriers to Adherence with a Post-Operative Enhanced Recovery Program in Colorectal Surgery

Richard Hu, Juan Mata, MD, Nicolo Pecorelli, MD, Pepa Kaneva, MSc, Lawrence Lee, MD, PhD, Barry L. Stein, MD, A. Sender Liberman, MD, Patrick Charlebois, MD, Julio Flavio Fiore Junior, $\mathrm{PhD}$, Liane Feldman, MD; McGill University

Introduction: Enhanced recovery programs (ERP) are perioperative evidence-based care bundles that improve morbidity and length of stay in colorectal surgery patients. However, adherence to ERP elements in the postoperative period remains low and may negatively affect outcomes. Much data have focused on process measures to improve adherence, but little is known regarding patient factors that may contribute to low adherence. The aim of this study was to identify patient-reported barriers to adherence to an ERP for elective colorectal surgery.

Methods: A secondary analysis was performed with data obtained from a randomized trial designed to study the effect of a mobile application device on adherence in 2016-17 at a single universityaffiliated specialist referral centre. Adult patients who underwent elective colorectal surgery were included. Target discharge day was postoperative day (POD) 3. Grounded theory methodology was used to identify and categorize patients' self-reported barriers to achieving ERP milestones on POD 1 and 2 for mobilization (time spent out of bed), ambulation (number of hallway laps walked in a day), gum chewing, breathing exercises and intake of liquids, nutritional drink and solid food. Patients were visited by an evaluator daily assessing adherence to each ERP element. If a goal was not reached, the patients were asked to provide explanations, which were then compiled.

Results: In the original study, 97 patients were randomized, with data from 93 patients available on POD1 and 62 available on POD2 (due to early discharges). Elements with the highest adherence across POD1 and 2 were breathing exercises (90\% and 90\%), solid food intake (83\% and $65 \%$ respectively) and liquid intake (81\% and $65 \%)$, while lower adherence was seen for mobilization (46\% and 35\%), ambulation (59\% and 50\%), nutritional drink intake (44\% and 35\%) and gum chewing $(63 \%$ and $47 \%)$. Barriers to mobilization included patient preference (e.g. "prefer the comfort of bed", "didn't feel like doing it"), pain, fatigue and nausea/vomiting. Barriers to adherence with nutritional drink were patient preference and nausea/vomiting, as well as non-patient dependent factors such as medical restrictions (i.e. NG insertion, medical order) and the nutritional drink not being supplied. Patient preference and nausea/vomiting were reported as the most common barriers for gum chewing.

Conclusion: Self-reported barriers to postoperative ERP elements were identified in colorectal surgery patients. "Patient preference" was a common reason for non- adherence. This suggests that strategies to maximize patient engagement may be helpful in improving postoperative adherence. 
P342

\section{Laparoscopic Repair of Post-operative Iatrogenic High} Rectovaginal Fistula

Kumar Parth, Dr, Gaurav Singh, Dr, Yashas H Ramegowda, Dr, Srikanth Gadiyaram, Dr; Sahasra Hospitals, Centre of Excellence for Gastroenterology, Bangalore, India

Background: Rectovaginal fistula(RVF) is a rare complication following hysterectomy. Surgical correction traditionally requires a laparotomy. We herein report our experience with laparoscopic repair of RVF

Mathods: Data of patients who presented with postoperative high rectovaginal fistula were analysed from a prospective database. The clinical details, evaluation, imaging findings, details of surgery, postoperative morbidity and follow-up were studied.

Results: Five patients, median age 36 years (range- 19- 47 years) were managed over a seven year period. Multidetector CT scan of the pelvis with rectal contrast defined the fistula in all patients. Laparoscopic repair was performed in all five. In four patients fistula was dismantled, rectal side was freshened and closed with interrupted absorbable sutures and repair buttressed with omentum. In one patient pedicled gracilis muscle with an intact neurovascular bundle was used as interposition graft after dismantling the fistula. All patients had a covering stoma. There median hospital stay was 3 (2-5) days.. One patient had recurrent fistula,which required a second repair by laparotomy, dismantling and interposition by rectus abdominus muscle flap. All patients are well with no recurrence at a median follow up was 36 (range 12-86) months.

Conclusion: Laparoscopic repair of iatrogenic high rectovaginal fistula is safe and feasible. It offers all advantages of minimal access surgery and a cure in the vast majority.

\section{P343}

A Comparative Effective Analysis of Perioperative Outcomes of Open, Laparoscopic and Robotic Abdominoperineal Resections for Rectal Cancer

Dakota T Thompson, MD, Paolo Goffredo, MD, Alan F Utria, MD, Jennifer Hrabe, MD, Muneera R Kapadia, MD, Irena GribovskajaRupp, MD, Sajida Ahad, MD, Imran Hassan, MD; University of Iowa

Introduction: Abdominoperineal resections (APR) using laparoscopic and robotic platforms are being increasingly utilized for surgically managing rectal cancer (RC). We hypothesized that laparoscopic and robotic APR would be associated with a shorter length of stay (LOS) and less morbidity compared to open APR. We analyzed demographics and perioperative outcomes of patients undergoing open, laparoscopic and robotic APR for RC using the American College of Surgeons National Surgical Quality Improvement Program (ACS-NSQIP) database Proctectomy Targeted Participant Use File (PUF).

Methods: Patients undergoing an elective APR for RC were identified using CPT codes 45110 and 45395. Patients with CPT 45395 were divided into laparoscopic or robotic APR based on NSQIP definitions. Patient demographics, disease and treatment characteristics, operative time, LOS, and perioperative clinical and oncologic outcomes between approaches were compared.

Results: A total of 787 patients were identified. Age, gender, BMI, neoadjuvant chemoradiotherapy, and preoperative diagnosis of locally advanced cancer were not significantly different between the three approaches (Table). Laparoscopic approach was associated with a lower incidence of positive circumferential radial margin (CRM) and a higher proportion of $\geq 12$ lymph node harvest compared to open APR. After adjusting for available confounders, laparoscopic $(\mathrm{OR}=0.58, \quad \mathrm{CI}=0.34-0.97, \quad \mathrm{p}=0.040)$ and robotic approach $(\mathrm{OR}=0.42, \mathrm{CI}=0.24-0.75, \mathrm{p}=0.003)$ was associated with a shorter LOS. However, operative time and Clavien-Dindo complications were not significantly different between approaches.

Conclusion: Laparoscopic and robotic APR for RC are associated with fewer in-hospital days; however, there were no significant differences in perioperative complications. Further investigation into oncologic outcomes is essential to validate the benefit of laparoscopic and robotic APR for RC.

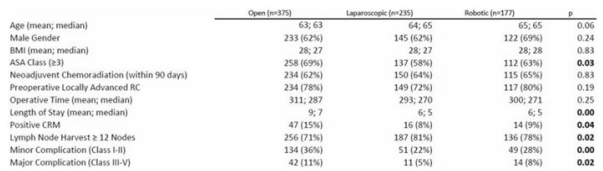




\section{P344}

Colon Surgery in Texas: A Comparison of Open, Laparoscopic and Robotic Surgeries with Regards to Cost and Utilization Using a Large Administration Database

Evan Liggett, MD, William Klingsporn, MD, Ira L Swinney, MS, Mallory Schenk, BS, Alan Tyroch, MD, Brian Davis, MD, Christopher Dodoo, MS; Texas Tech HSC Paul Foster School of Medicine

Introduction: Laparoscopic surgery has become the standard of care for the most common surgical procedures performed. However, laparoscopic techniques have not reached this same penetrance in colorectal surgery. We set out to determine the percentage laparoscopic colon (LC) surgeries constituted in Texas by querying an administrative claims based database. The secondary outcome that we studied was the cost of LC verus open and robotic techniques.

Methods: The Texas Inpatient Public Use Data File (PUDF) was queried using ICD-9-CM diagnostic and procedure codes to determine overall utilization of LC in Texas between 2013-14 for reporting facilities. We specifically looked at cost, length of stay, and complications of LC, open colectomy (OC) and robotic assisted colectomy (RAC). Quantitative variables were summarized using mean and standard deviation (SD). Categorical variables were described using frequencies and proportions. Differences in postoperative complications were assessed and reported using the generalized linear model and further reported as prevalence ratio (PR) with their $95 \%$ confidence interval (CI). Further, a cost analyses were carried out using a linear regression model on reported total charges. This was reported using the regression coefficients (RC) and CI. All analyses were carried out using STATA 15.

Results: In the state of Texas between 2013-14 there were 20,454 colectomies performed. Of these $12,328(60.3 \%)$ were OC, 7,536 $(36.8 \%)$ were LC, and 590 (3.9\%) were RAC. Average total cost per procedure was $\$ 117,113$ for OC, $\$ 75,741$ for $\mathrm{LC}$, and $\$ 81,996$ for RAC. Average length of stay for each technique was 10.6 days for OC, 6.1 days for LC, and 5.1 days for RAC. White, non-Hispanic patients were more likely to undergo LC (PR 1.14 and 1.16). The risk of a post-operative complication occurring was higher in the open procedure than a laparoscopic procedure.

Conclusions: LC accounted for $36.8 \%$ of all colectomies performed in Texas between 2013-14. OC had twice the cost as LC and increased the length of stay by nearly 4 days. LC significantly decreased the cost and length of stay for patients undergoing surgery while not significantly increasing perioperative complications.

\section{P345}

The Effect of Enhanced Recovery After Surgery (ERAS) Protocol on Colorectal Surgery Outcomes in a Metropolitan Community Hospital

Young Lee, MD, MSc, Greg Zielinski, MD, Jasmine Bhinder, BS, Victoria Agramonte, RN, MSN, Ritha Belizaire, MD, Pratibha Vemulapalli, MD, FACS; The Brooklyn Hospital Center

Introduction: The enhance recovery after surgery (ERAS) protocol was developed by incorporating evidence based medicine into patient care to improve post-operative outcomes and cost. ERAS programs envelop a set of recommendations focusing on patient education, optimal fluid management, minimal incision length, decreased used of tubes and drains, opioid sparing analgesia, and early oral intake and mobilization. Studies involving ERAS protocol in colorectal surgery have shown to decrease the hospital length of stay and overall morbidity. We report the one year outcome focusing on surgical site infections (SSI) and length of stay (LOS) after implementation of the ERAS protocol for patients undergoing elective colorectal surgery in our metropolitan community hospital.

Methods: The one year outcome of patients undergoing elective colorectal surgery under the ERAS protocol, launched in 2017, was compared to the patients who received conventional colorectal surgery perioperative care in 2016 at The Brooklyn Hospital Center. A multidisciplinary committee, including surgeons, anesthesiologist, nurses, social workers, pre-surgical testing and surgery office staff, was formed to develop a perioperative protocol based on core ERAS guidelines established by previous studies. This protocol was implemented with the goal of improving elective colorectal surgical outcomes. Specifically, the hospital length of stay and surgical site infection rates were assessed.

Results: A total of 134 patients underwent colorectal surgery from 2016 until present in 2018. Of these patients, seventy-one received conventional perioperative care and sixty-three underwent the ERAS protocol. The surgical site infection rate was $7.9 \%$ in 2016 before the start of ERAS protocol at our institution. With the implementation of the ERAS protocol in 2017, we saw a drastic reduction in surgical site infection rates to $3.4 \%$ and once we were able to demonstrate full bundle compliance, we saw a decrease to $1.6 \%$. In addition, when comparing hospital length of stay, we demonstrated a decrease from 11 days to 4 days in the conventional perioperative care and ERAS group, respectively, while our institution implemented ERAS and adopted laparoscopic techniques.

Conclusion: Our results demonstrate that the implementation of ERAS protocol, with an emphasis on laparoscopic colon techniques, in elective colorectal surgical patients at a metropolitan community hospital was effective in improving surgical site infection rates and reducing hospital length of stay dramatically. 
P346

Oncological and Clinical Safety of Single-Incision Laparoscopic Surgery for Colon Cancer with Previous Abdominal Surgery: A Propensity Score-Matched Comparison

Yasumitsu Hirano ${ }^{1}$, Chikashi Hiranuma, $\mathrm{MD}, \mathrm{PhD}^{2}$, Masakazu Hattori ${ }^{2}$, Kenji Douden ${ }^{2}$, Shigeki Yamaguchi ${ }^{1}$; ${ }^{1}$ Saitama Medical University International Medical Center, ${ }^{2}$ Fukui Prefectural Hospital

Introduction: Previous abdominal surgery (PAS) may affect the operative time and intra-operative bowel injury, and can also influence the postoperative complications including postoperative bowel obstruction and paralytic ileus. At the beginning of laparoscopic experience, PAS was even considered contraindications for this approach. However, the advances in laparoscopic surgery, the indications for laparoscopy was extended to patients with PAS without adverse consequences on outcome. In this study, we evaluated the outcome of SILC in locally advanced colon cancer patients with PAS with a propensity score matched comparison to assess its perioperative and long-term oncological outcomes.

Methods: 68 locally advanced colon cancer patients with PAS who electively underwent SILC were included in this study. 68 patients were then chosen out of a collective of 118 patients without PAS in a propensity score matched design. Short-term clinical outcomes in both groups were compared, and verified its long-term oncological outcome.

Results: In the patients with PAS, the mean skin incision length was $2.91 \mathrm{~cm}$, and the median estimated blood loss and median operating time were $27.9 \mathrm{~mL}$ and $180.0 \mathrm{~min}$, respectively. The mean number of harvested lymph nodes was 27.6. There were not significant differences in all these variables between two groups. There were no 30-days mortalities in both groups. Postoperative complications were occurred 7 cases $(10.3 \%)$ in each group, and the rate was not significantly different. There were also no significant differences in postoperative hospital stay between both two groups (15.1 vs 10.5 days; $\mathrm{p}=0.091)$. There were 10 occurrences of tumor recurrence or metastasis $(14.7 \%)$ in 68 patients with the median follow-up 55 months in patients with PAS. The 5-year relapse-free survival rates were $71.0 \%$ in patients with PAS and $76.0 \%$ in patients without PAS, respectively, and the 5-year overall survival rates were $75.4 \%$ with PAS and $88.9 \%$ without PAS, respectively. No significant differences were revealed between both groups (RFS; $p=0.69$, OS; $\mathrm{p}=0.117)$.

Conclusions: Our initial experiences may suggest that long-term oncological and clinical safety of SILC for colon cancer patients with previous abdominal surgery. However, further studies need to be undertaken to prove its superiority to conventional laparoscopic surgery in colon cancer patients with previous abdominal surgery.

\section{P347}

Laparoscopic Right Hemicolectomy with Intracorporeal Anastomosis and Pfannenstiel Extraction Facilitated by Intuitively-Controlled Articulating Device: A Novel Technique

Mercedes Pilkington, $\mathrm{MD}^{1}$, Christa N Grant, $\mathrm{MD}^{2}$, Jose Diaz-Miron, $\mathrm{MD}^{1}$, James D Geiger, MD ${ }^{1}$; ${ }^{1}$ University of Michigan, Pediatric Surgery Section, ${ }^{2}$ Penn State Hershey Children's Hospital

Introduction: A new technique for laparoscopic right hemicolectomy (LRH) was adopted at our pediatric institution that utilized an intuitively-controlled articulating device. This facilitated an intracorporeal anastomosis with a Pfannenstiel extraction site with the proposed advantage of a rapid return of bowel function and minimal cosmetic impact. We describe our technique and early intraoperative and short-term outcomes.

Methods and Procedures: A retrospective review was carried out of all patients who have undergone a LRH at our institution from January 2017 to September 2018. All cases were carried out with the described surgical technique.

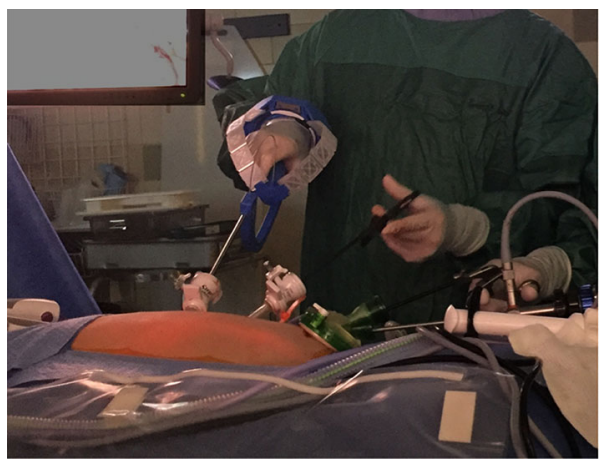

A Pfannenstiel incision with an advanced access platform and two to three additional ports were utilized. Medial-to-lateral dissection was carried out in a standard fashion and the side-to-side anastomosis was created intracorporeally with endostaplers. The common enterotomy was sewn using a novel intuitively-controlled articulating instrument. The specimen was extracted from the Pfannenstiel incision.

Results: A total of five laparoscopic right hemicolectomies have been completed with this technique since January 2017. The median age [IQR] was 14 years [11-16], median BMI 17.5 [17.3-21.2], and 2/5 patients were female $(40 \%)$. Four patients had an appendiceal neuroendocrine tumour and one had a mucinous neoplasm of the appendix; all were referred after incidental findings post-laparoscopic appendectomy and were discussed pre- and post-operatively at our institutional multi-disciplinary tumour board. All cases were completed with a Pfannenstiel incision for extraction with an intracorporeal anastomosis utilizing this novel tool; no case required conversion to open. A median of 2 [23] additional ports were used. Median operating time was $180 \mathrm{~min}$ [169-221]. All cases were carried out by fellows who had each performed fewer than five cases with this new tool. No patients had residual tumour in the specimen, 3 patients $(60 \%)$ had one positive lymph node (LN) and a median of 39 [17-45] LNs were retrieved. The median length of stay was 3 days [3-6]. There were no major complications to date with a median length of follow-up 291 days [271403].

Conclusions: The use of a novel articulating instrument facilitates the intracorporeal anastomosis and thus the use of a Pfannenstiel extraction rather than a larger umbilical incision. In early experience, this combination leads to a short hospital stay and improved cosmesis. 


\section{P348}

\section{Negative Pressure Wound Therapy is Beneficial in the Treatment} of Pilonidal Disease

Elisa Bianchi, MD, Titi Adegboyega, MD, Shirley Shih, MD, Cici Zhang, MD, David E Rivadeneira, MD, MBA; Huntington HospitalNorthwell Health

There are multiple surgical approaches to the treatment of Pilonidal Sinus Disease (PSD). All are associated with wound complications including surgical site infections (SSI), dehiscence, and recurrence. Negative Pressure Wound Therapy (NPWT) has been reported to decrease wound complications after surgery. We report our experience with NPWT after excision with primary closure of PSD.

Methods: We report 65 consecutive patients that underwent excision of pilonidal sinus disease with primary closure and placement of Negative Pressure Wound Therapy dressing. NPWT was places over the closed incision for 3-7 days at $-120 \mathrm{~mm}$ hg. We compare these results with 65 patients consecutive patients prior to using NPWT who underwent excision PSD with primary closure. Both groups studied where well matched and there was no difference in Age, sex, OR time, Blood loss, incisions size. Early period complications (wound site infection, wound dehiscence, abscess, hematoma and seroma formation) developing within 30 days of operation was recorded from the medical files. Data were analyzed using SPSS ver. 12.0 (SPSS Inc., Chicago, IL, USA). Comparison of groups was done with ANOVA

Results: Mean age of patients was $25.4 \pm 5.7$ years and the male: female ratio was 4:1. Operation time did not differ between groups. Significant decrease in early wound complications occurred in those patients treated with NPWT, $11 \%(6 / 65$,$) compared to those patients$ that did not have NPWT, 25\% (16/65) p, <0.001. Surgical site infection was significantly decreased in the NPWT group, $4.6 \%(3 / 65)$ compared to those who did not have NPWT 15\% (10/65), $\mathrm{p}<0.001$. Recurrence of pilonidal disease was significantly decreased in the NPWT patients 3\% (2/65) compared to non-NPWT $12 \%(8 / 65)$, $\mathrm{p}<0.0001$.

The use of Negative Pressure wound therapy is beneficial in patients undergoing excision and primary closure of pilonidal sinus disease. NPWT leads to a decrease in early wound complications including wound seroma, dehiscence and SSI. In addition NPWT also significantly resulted in a decrease in recurrence of disease.

We recommend the use of NPWT in the surgical management of pilonidal disease.

\section{P349}

To Compare the Outcome of Gum Chewing Versus No Gum Chewing on the Duration of Post Operative Ileus and Total Length of Hospital Stay in Cases of Re Laparotomy for Stoma Closure in a Lower Middle Income Country

Samiullah Bhatti, $\mathrm{Dr}^{1}$, Shabbar H Changezi, $\mathrm{Dr}^{1}$, Awais A Malik, $\mathrm{Dr}^{2}$, Usman I Butt, Dr ${ }^{1}$, Waris Farooka, Prof ${ }^{1}$, Mahmood Ayyaz, Prof ${ }^{1} ;{ }^{1}$ Services Hospital Lahore, ${ }^{2}$ Lahore General Hospital

Introduction: Postoperative ileus (POI) is generally referred to as the transient impairment of bowel motility after abdominal or other surgery. The potential complications of prolonged POI include increased postoperative pain, nausea and vomiting, delay in postoperative mobilization, prolonged hospitalization, and increased health-care costs. In view of these complications and economic burden a number of pharmacologic and non-pharmacologic strategies have been adopted all over the world to reduce the burden of postoperative ileus. The use of gum chewing has emerged as a new and simple modality for decreasing POI.

Materials and methods: 100 patients having ileostomies, fulfilling inclusion and exclusion criteria were recruited from outpatient department and divided into two groups. Group A patients were allowed to chew gums while Group B patients were not allowed to chew gums after the reversal of ileostomies. All patients were followed and monitored for the mean time to pass 1 st flatus and mean length of hospital stay.

Results: Mean age of the patients in group A was $26.12( \pm 7.1)$ years and in group B was $28.80( \pm 10.5)$ years. There were $25(50 \%)$ males and $25(50 \%)$ females in group A. In group B there were $29(58 \%)$ males and $21(42 \%)$ females.

Mean BMI for Group A was 23.5( \pm 5.3$)$ and Group B was $21.4( \pm 4.6)$.The mean time to pass flatus was noted to be significantly shorter $18.36( \pm 8.43$ ) hours in the chewing group (group A) while in no chewing gum group (group B) it was $41.16( \pm 6.14$ ) hours (p-value 0.000 ). The mean length of hospital stay was significantly short 84 $( \pm 8.3$ ) hours in the chewing group (group A) as compared to 107.04 $( \pm 6.4$ ) hours in no gum group (group B) (p-value 0.000).

Conclusion: It is concluded that post-operative chewing of gum after the reversal of ileostomies have a definitive role in reducing postoperative ileus and total length of hospital stay. 
P350

Prospective Multicenter Study of Reduced Port Surgery Combined with Transvaginal Specimen Extraction for Colorectal Cancer Resection

Hidekazu Takahashi, MD, $\mathrm{PhD}^{1}$, Atsushi Hamabe, $\mathrm{MD}, \mathrm{PhD}^{2}$, Tsuyoshi Hata, MD, PhD ${ }^{1}$, Yuji Nishizawa, $\mathrm{MD}, \mathrm{PhD}^{3}$, Atsushi Nishimura ${ }^{4}$, Masaaki Itoh ${ }^{3}$, Ichiro Takemasa, MD,

$\mathrm{PhD}^{2}$; ${ }^{1}$ Department of Gastroenterological Surgery, Osaka University Graduate School of Medicine, ${ }^{2}$ Department of Surgery, Surgical Oncology and Science, Sapporo Medical University, ${ }^{3}$ Department of Colorectal and Pelvic Surgery, National Cancer Center Hospital East, ${ }^{4}$ Department of Surgery, Institute of Gastroenterology, Nagaoka Chuo General Hospital

Background: For colorectal cancer treatment, conventional laparoscopic surgery combined with a transvaginal specimen extraction (TVSE) was less painful than laparoscopic surgery with a small laparotomy. However, the relevance of TVSE combined with reduced port surgery (RPS) remains unknown. This study investigated the feasibility of TVSE with RPS. We also evaluated the pain, aesthetic satisfaction, and quality of life of patients after TVSE with RPS.

Methods: This prospective multicenter study enrolled 10 patients with colorectal cancer that underwent RPS with TVSE at three institutions. This study is registered with UMIN-CTR, UMIN000016859. A pre-specified protocol specified the surgical approach, colorectal resection procedures, and the transvaginal approach. We administered questionnaires to assess pain (visual analogue scale [VAS]), subjective/objective wound healing aesthetics (photo series questionnaires [PSQ]), and quality of life (QOL).

Results: The median estimated blood loss was $32 \mathrm{ml}$ (range $<1$ to $154 \mathrm{ml}$ ). Resected specimen findings indicated that oncological clearance was secured in all cases. No operative complications occurred, except one urinary tract infection, which was promptly cured with antibiotics. On day 0, pain was rated $2.3 \pm 0.67$ at rest and $4.9 \pm 0.82$ during sneezing; these ratings gradually declined over time. The QOL scores showed that, compared to before surgery, after surgery, patients reported significant deterioration of physical functioning (96.67 \pm 1.49 vs. $87.33 \pm 2.71)$, emotional functioning $(93.33 \pm 2.72$ vs. $86.67 \pm 2.22), \quad$ fatigue $(7.78 \pm 3.72$ vs. $26.67 \pm 8.31)$, and pain $(6.67 \pm 3.69$ vs. $18.33 \pm 4.61)$. Nevertheless, no significant difference was observed on other scales. The PSQ showed that patient ratings of wound aesthetics after TVSE were not inferior to ratings from patients after conventional laparoscopy or single incision laparoscopic surgery, and they were significantly superior to patient ratings of wounds after laparotomy.

Conclusion: TVSE with RPS for colorectal cancer was feasible for the short-term surgical safety. Moreover, this approach might be less painful than other procedures for colorectal cancer.
P351

32-Year-Old Male, Hx of FG Syndrome, Presenting with Metastatic Colon Cancer with Metastases to Liver Status Post Combined Colon and Liver Resection

Taylor D Coleman, MD, Christine Tat, MD, Joaquin Estrada, MD, FACS, Jan Kaminski, MD, FACS; University of Illinois, Metropolitan Group Hospitals

Colorectal carcinoma (CRC) is the most common malignancy of the GI tract. Many molecular mechanisms underlying development remain unknown. Our case presents a rare genetic condition, FG Syndrome, with numerous genes on the $\mathrm{X}$ chromosome that have been found to be associated with increased risk for progression to CRC. Our patient, a 32-year-old male with FG Syndrome, intellectual disability, schizo-affective disorder, imperforate anus originally presented with constipation, abdominal pain, and intermittent rectal bleeding. Work-up revealed a fungating, obstructing mass at the sigmoid colon with biopsy consistent with adenocarcinoma. The patient underwent a combined laparoscopic left segmental colectomy with primary anastomosis as well as an open partial hepatectomy. Pathology was consistent with a pT3N2aM1a lesion.

Although CRC can be most commonly associated with a variety of genetic, environmental, and dietary factors, this report will focus on its association with FG Syndrome. Most mutations that lead to FG syndrome involve the MED12 gene, though it is important to note there are numerous genes associated with the syndrome's various presentations. These genes include Mecp2, CASK, and FLNA. In a patient with a genetically predisposed syndrome, who then develops $\mathrm{CRC}$ at an early age, it is reasonable to suspect that the explanation may be found at the genetic level. Variations in expression of these aforementioned genes have been shown to be, 1. Risk factors for the development of CRC, and 2. Prognostic factors after the diagnosis of CRC.

When evaluating our patient who has presented with newly diagnosed $\mathrm{CRC}$ at a young age, a work up is completed that includes testing for some of the more common causes of genetically associated predisposition to the development of this malignancy. Testing included Microsatellite Instability/HNPCC work up which showed microsatellite stable tumor. Another test screened for a pathogenic variant of RAS mutation including KRAS and NRAS. There was no pathogenic variant detected. These results leave us raising questions in need of further investigation, such as "do the mutations associated with FG Syndrome have a causative effect or prognostic function in the disease process?" "Which of these mutations could provide a possible screening test for these patients in the future?" "Would patients with FG syndrome benefit from early screening for CRC?" Although FG Syndrome is a rare condition, the ideas and questions discussed in this paper can be applied far past the narrow spectrum of this one disorder. 


\section{P352}

\section{Comparison of Robotic Versus Laparoscopic Sigmoid Colectomies: A Single Surgeon's Experience}

Elizabeth E Price, DO, Matthew Robinson, DO, Robert Carman, DO; UPMC Pinnacle Community Osteopathic Hospital

Introduction: Robotic surgery is becoming more prevalent in colorectal surgery. Some question if the cost associated with robotic training and its equipment is superior to its laparoscopic counterpart. There have been few studies comparing postoperative outcomes and pain control between the two approaches to identify if one approach is superior to the other. This study compares operative times and postoperative outcomes between laparoscopic and robotic approaches by a single fellowship-trained colorectal surgeon during his first two years of performing robotic colectomies.

Methods: The study is a retrospective single institution review of patients who underwent an elective sigmoid colectomy by a single surgeon. Sixteen patients had an elective laparoscopic sigmoid colectomy from August 2014 to December 2016 and twenty-one patients had an elective robotic sigmoid colectomy from November 2016 to July 2018. All resections were performed for diverticular disease, polyps unamenable to endoscopic resection or malignancy. Data was collected on anesthesia times, procedure times, length of stay, return of bowel function, days until on discharge diet and postoperative days requiring intravenous narcotics.

Results: Mean anesthesia time was $198 \mathrm{~min}$ and $215 \mathrm{~min}$ in the robotic and laparoscopic groups respectively. Mean procedure time was $166 \mathrm{~min}$ robotically compared to $171 \mathrm{~min}$ laparoscopically. Our data shows decreased operative and anesthesia times in the robotic group. Mean length of stay was 3.67 days for the robotic group versus 3.75 days for the laparoscopic group. Return of bowel function was 2.24 days for the robotic group versus 2.31 days for the laparoscopic group. Postoperative day to discharge diet was 3.24 days in the robotic group and 3.56 days in the laparoscopic group. Patients had less postoperative pain in the robotic group than the laparoscopic group. A majority of the robotic group patients did not require patient-controlled-analgesia and days until off intravenous narcotics was decreased compared to the laparoscopic group, 0.62 days versus 1.38 days.

Conclusion: A robotic approach for elective sigmoid colectomy appears to be an acceptable alternative to a laparoscopic approach. On average, anesthesia and operative times were decreased in the robotic group compared to the laparoscopic group. Length of stay, return of bowel function and day until discharge diet were similar between the two groups. However, postoperative pain was decreased in the robotic group as evident by reduced patient-controlled-analgesia and intravenous narcotic use. Further study is needed with larger sample sizes to define if a robotic approach is superior over a laparoscopic approach.

\section{P353}

\section{Colorectal Anastomotic Leak Rate: A Single Institution's Experience}

Anthony Dinallo, $\mathrm{MD}^{1}$, Danielle Craigg, $\mathrm{MD}^{1}$, Megan Navarro ${ }^{2}$, Abdelrahman Attili, MD ${ }^{3}$, Timothy Bourdeau ${ }^{1}$, Janelle Spence ${ }^{1}$, Haane Massarotti, MD ${ }^{1}$, Allen Chudzinski, MD ${ }^{1}$; ${ }^{1}$ Florida Hospital Tampa, ${ }^{2}$ University of South Florida, ${ }^{3}$ University of Central Florida

Purpose: An anastomotic leak is a devastating complication for both the patient and the surgeon, leading to an increased length of stay, higher local recurrence, greater cost and increased mortality. Leak rates in colorectal surgery range from $2.4 \%$ to $19 \%$. The purpose of this study is to compare a single institution's anastomotic leak rate with national averages provided by the American College of Surgeons National Surgical Quality Improvement Program (NSQIP).

Methods: Over a two year period (March 2016 - March 2018) with IRB approval, 163 patients were reviewed who underwent colorectal resections and primary anastomoses. Anastomotic leak was the primary outcome measured. Each complication was also evaluated including pneumonia, urinary tract infection, and venous thromboembolism. Data is expressed as mean in percentages and length of stay as median percentage (mean \pm SD) where appropriate.

Results: Of the 163 patients who underwent colorectal resections, three patients experienced an anastomotic dehiscence $(1.8 \%)$. Two of these patients had robotic assisted procedures: one total abdominal colectomy and one ileocecectomy. The other patient underwent an open right colectomy. This leak rate of $1.8 \%$ was less than the NSQIP rate of $2.7 \%$. Other outcomes such as length of stay, death, readmission within 30 days, and complications including: pneumonia, cardiac anomalies, UTI's, DVT's, surgical site infections, ileus, and renal complications, were evaluated and compared to NSQIP (table 1). The actual outcome for surgical site infection (SSI) of $2.4 \%$ was statistically different from NSQIP outcome of 7.4\% ( $\mathrm{p}=0.029)$. All other predicted and actual outcomes were not statistically different from NSQIP outcomes.

Conclusion: This study reveals a single institution's anastomotic leak rate to be less than the national average provided by NSQIP. Given the devastating impact of anastomotic leaks, it is important to be recognized as a marker of surgical quality when evaluating surgical performance.

\begin{tabular}{|l|ccc|}
\cline { 2 - 4 } \multicolumn{1}{c|}{} & \multicolumn{3}{c|}{ All Procedures } \\
\hline Outcome & $\mathrm{N}=163$ & $\mathrm{~N}=163$ \\
Serious Complication & $12.6 \%$ & $11.0 \%$ & $12.4 \%$ \\
Any Complication & $15.9 \%$ & $11.0 \%$ & $15.8 \%$ \\
Pneumonia & $1.6 \%$ & $1.2 \%$ & $1.5 \%$ \\
Cardiac Complication & $0.7 \%$ & $4.3 \%$ & $0.6 \%$ \\
SSI & $8.4 \%$ & $2.4 \%$ & $7.4 \%$ \\
UTI & $1.9 \%$ & $0.0 \%$ & $1.5 \%$ \\
Venous thromboembolism & $1.5 \%$ & $0.6 \%$ & $1.3 \%$ \\
Renal Failure & $1.0 \%$ & $0.0 \%$ & $0.8 \%$ \\
Ileus & $11.9 \%$ & $17.2 \%$ & $11.4 \%$ \\
Anastomotic Leak & $3.0 \%$ & $1.8 \%$ & $2.7 \%$ \\
Readmission & $9.6 \%$ & $4.2 \%$ & $8.9 \%$ \\
Length of Stay (LOS) & 5 days, $5 \pm 2$ & 5 days, & $1.7 \pm 6$ \\
Death & $1.2 \%$ & $1.8 \%$ \\
Discharge to Nursing or Rehab & $6.0 \%$ & $3.7 \%$ \\
\hline \multicolumn{3}{|c|}{$0.8 \%$} \\
\hline \multicolumn{1}{|c|}{ Surgical Approach } & Robotic & $4.7 \%$ \\
\hline \multicolumn{1}{|c|}{ Leak Rates } & $1.85 \%$ & Laparscopic & Open \\
\hline
\end{tabular}


P354

Does the Time Elapsed Between Cases Affect Operative Outcomes in the Early Experience of Transanal Total Mesorectal Excision?

Johnny K Chau, MD, Joshua Soloman, Hamzeh Naghawi, MD, Carmen Mueller, MD, MEd, Patrick Charlebois, MD, A. Sender Liberman, MD, Barry Stein, MD, Lawrence Lee, MD, PhD; McGill University

Introduction: A significant proportion of patients still experience poor resection quality for extraperitoneal rectal cancer. Trananal total mesorectal excision (TA-TME) may improve resection quality but is technically challenging. The learning curve of TA-TME may also be affected by the time elapsed between cases (operative interval). Therefore, the objective of this study was to determine the effect of operative interval on the early outcomes of TA-TME and to compare it to laparoscopic TME for mid/low rectal adenocarcinoma.

Methods: All consecutive cases undergoing curative intent sphincter-saving proctectomy for mid and low rectal adenocarcinoma at a single high-volume specialist referral centre from 2012-2018 were reviewed. All cases were performed by fellowship-trained colorectal surgeons. All TA-TME cases were performed by two-team approach including one surgeon who was specifically trained in TA-TME in every case from 2017/07 onwards. Operative interval was defined as the time elapsed since the last approach-specific case. Main outcome measure was poor-quality resection, defined as a composite of involved circumferential resection margin (CRM) or distal margin, or incomplete TME grade.

Results: A total of 128 patients were included(99 lap-TME, 29 TA-TME). All TA-TME cases were performed in 2016-2018 (4 in 2016-17, and 25 in 2017-2018). There were no differences in age, gender, and comorbid status. TATME patients were more likely to be obese $(19.4 \%$ vs. $3.0 \%, \mathrm{p}=0.003)$. Distance to anal verge (TATME $6.6 \mathrm{~cm}$ (SD1.6), lap $6.7 \mathrm{~cm}(\mathrm{SD} 1.9), \mathrm{p}=$ $0.854)$, pre-treatment threatened/involved CRM $(32.1 \%$ vs. $19.4 \%, \mathrm{p}=0.197)$ and neoadjuvant $($ chemo)radiotherapy $(67.9 \%$ vs. $67.7 \%, \mathrm{p}=0.986)$ were similar between groups. TATME patients were more likely to receive a handsewn coloanal anastomosis $(32.1 \%$ vs. $15.2 \%, \mathrm{p}=0.043)$ and a longer procedure time $(341 \mathrm{~min}(\mathrm{SD} 159)$ vs. $285 \mathrm{~min}(\mathrm{SD} 71), \mathrm{p}=0.007)$. There was no difference in the proportion of poor quality resections between TATME and lap-TME $(10.3 \%$ vs. $13.8 \%, p=0.575)$. Anastomotic complications, overall 30-day morbidity and length of stay were similar between groups. The TATME group had a longer median interval between procedures (4.6 weeks [IQR2.0$11.0)$ vs. 3.6 weeks $[\mathrm{IQR} 1.7-8.9, \mathrm{p}=0.003)$. Shorter operative interval was associated with lower risk of poor-quality resection for TA-TME (OR 0.70 per week, 95\% CI 0.49-0.99), but not for lap-TME (OR 1.03 per week, 95\% CI0.951.12).

Conclusions: In our early experience with TA-TME, there was no differences in surgical and clinical outcomes compared to lap-TME for patients undergoing curative sphincter-saving proctectomy for mid and low rectal cancer. Shorter interval between TA-TME cases may result in improved outcomes and should be factored into the learning curve and adoption process.

\section{P356}

Significantly Elevated Levels of Plasma Programmed cell Death Ligand 1 (PDL-1) in Wound Fluids After Colorectal Cancer Resection are a Likely Source of the Persistently Elevated Plasma Levels Noted Post-surgery

Hmc Shantha Kumara, $\mathrm{PhD}^{1}$, Neil Mitra, $\mathrm{MD}^{1}$, Dasuni Niyagama Gamage, MD ${ }^{1}$, Carl S Winkler, MD ${ }^{1}$, Jaspreet K Sandhu, MD ${ }^{2}$, Xiaohong Yan, $\mathrm{PhD}^{1}$, Vesna Cekic, $\mathrm{RN}^{1}$, Nipa D Gandhi, $\mathrm{MD}^{1}$, Richard L Whelan, MD ${ }^{1}$; ${ }^{1}$ Mount Sinai West Hospital, Department of Surgery, New York,USA, ${ }^{2}$ Brookdale University Hospital \& Medical Center, Department of Surgery,Brooklyn,New York,USA

Introduction: The protein B7 Homolog 1 (B7-H1), also named Programmed cell Death Ligand 1 (PDL-1), has proangiogenic and immunosupressive effects. PDL-1 is a ligand for PD-1 which is expressed on activated T cells; PDL-1 binding renders activated lymphocytes senescent. Recently, it has been shown that PD-L1 plasma levels are elevated for 4 weeks after colorectal cancer (CRC) resection. Added "free" PDL-1 in the blood may contribute to surgery-related immunosuppression by binding to PD-1 expressing circulating T-lymphocytes. The source of the plasma B7-H1 increase is unknown. It has earlier been shown that plasma levels of VEGF and 11 other proangiogenic proteins are elevated x 3-5 weeks after surgery; the healing wounds may be the protein source since wound fluid (WF) levels are 3-40 x higher than blood levels. This study's purpose is to determine perioperative PDL-1 levels in blood and WF taken after CRC resection.

Method: Consenting patients (pts) undergoing elective CRC enrolled in an IRB approved tissue/data bank in whom an intra-abdominal Jackson Pratt (JP) drain had been placed were studied. Demographic, Clinical and pathologic data were reviewed. Preoperative (preop) and post-operative (postop) blood and postop wound fluid (WF) samples were simultaneously collected at 1 or more postop time points, centrifuged and were stored at $-80^{\circ} \mathrm{C}$. Late samples (POD 7-20) were bundled into 7 day blocks and considered as single time points. PDL-1 levels were determined in duplicate via ELISA. The Wilcoxon and Mann and Whitney test were used for analysis (significance $\mathrm{p}<0.05$ ).

Results: Preop and 1 or more late plasma and WF samples from 23 CRC patients (colon 4, rectal 19; mean age $65.2 \pm 11.4$ years) were analyzed. The surgical methods were: laparoscopic, 13pts; hand assisted 7; open, 3. The mean incision length $(\mathrm{cm})$ for the MIS and Open pts was $8.4 \pm 4.2$ and $25.0 \pm 8.8$, respectively. The mean length of stay was $8.9 \pm 5.3$ days. Plasma PDL-1 levels on postop day (POD) 1, 3, and 7-13 were significantly higher than preop levels $(\mathrm{p}<0.001)$. Also, WF PDL-1 levels were significantly higher than the plasma levels at each postop time point $(\mathrm{p}=0.02-0.003)$.

Conclusion: PDL-1 WF levels were significantly higher than corresponding plasma levels (also elevated) for 3 weeks. The highest PDL-1 levels were noted POD7-13. A portion of the added PDL-1 in the plasma may originate in the healing wounds. Elevated plasma PDL-1 may suppress TIL's which may facilitate tumor growth. Larger perioperative PDL-1 studies and studies investigating plasma PD-1 levels are warranted.

Table 1: PDL-1 protein levels in preop and post op Plasma vs. postop Wound fluids
\begin{tabular}{|l|l|l|l|l|l}
\hline Time & Plasma ( pg/ml) & (n) & Wound fluids (pg/ml) & (n) & \multicolumn{2}{|c|}{$P$} \\
\hline PreOp & $52.24 \pm 22.47$ & 23 & N/A & N/A & N/A \\
POD1 & $71.12 \pm 34.75$ & 23 & $87.14 \pm 32.35$ & 22 & 0.02 \\
\hline POD3 & $68.75 \pm 27.34$ & 22 & $87.71 \pm 30.86$ & 19 & 0.02 \\
\hline POD 7-13 & $74.84 \pm 38.01$ & 13 & $197.1 \pm 212.0$ & 16 & 0.003 \\
\hline
\end{tabular}




\section{P357}

\section{Protective Ileostomy for Patients with T3 and T4 Rectal Cancer} Before Neoadjuvant Therapy

George Dejeu, Adrian Maghiar, Octavian Maghiar, Dan Ciurtin, Marius Sfirlea; Spital Pelican

We started using protective ileostomy for the patients that were diagnosed with $\mathrm{T} 3$ and $\mathrm{T} 4$ rectal cancer before neoadjuvant therapy in the past 24 months, looking at the effect this had on the readmision for intestinal occlusion of those patients during the course of the oncologic treatment before surgery. Taking into account two groups of patients, on a case match basis, we matched the last 30 patients we had diagnosed with $\mathrm{T} 3$ and T4 low rectal cancer patients, that had an ileostomy put before the start of neoadjuvant therapy to 30 patients of the same metrics besides the ileostomy from 3 years ago. The 2 groups were no different in female/male composition, age, tumor grade, comorbidities, chronic treatment, neoadjuvant treatment type. Our findings show that the number of patients that could not go through with the neoadjuvant therapy went down from $18 \%$ to $7,5 \%$. With the number of patients that did not finally get the surgical treatment (for different reasons, that were not discussed in this present study) rising from $78 \%$ to $85 \%$. Both statistically significant. Moreover, the most important finding in our opinion is the reduction in the need for readmission on a surgical ward from $32 \%$ to $12 \%$ of patients included in the study, and reduction of the readmission stay from a average of 4 days to 2 in the ileostomy group. We continue to study the effect of this on patients with $\mathrm{T} 3$ and $\mathrm{T} 4$ low rectal cancer patients, considering the need to apply this technique to all patients with low rectal cancer (we use a 100\% ileostomy technique for all patients with low rectal cancer resection with anastomosis in our clinic, using the transanal, laparoscopic or open technique for the surgery).

\section{P358}

Robotic Assisted Colorectal Surgery: Does BMI Affect Conversion Rate and Postoperative Complications?

Erin Worden, MD, Lala Hussain, MSc, Hamza Guend, MD; Good Samaritan Hospital

Background: Robotic-assisted surgery offers the advantages of instrumentation with added degrees of freedom, 3-dimensional surgeon controlled camera, and stable retraction. We hypothesize that such advantages may reduce the rate of conversion to open surgery in obese patients. The primary aim of our study was to determine if obesity increases the rate of conversion in patients undergoing robotic colorectal resection.

Methods: This study was a retrospective review of a prospectively collected database of 97 patients at a community-teaching hospital who underwent robotic colorectal resection between 2015-2018. The patients were divided into two groups, control (BMI $\left.<30 \mathrm{~kg} / \mathrm{m}^{2}\right)$ and obese (BMI $\geq 30 \mathrm{~kg} / \mathrm{m}^{2}$ ). Patient demographics, perioperative variables, histopathologic data, post-operative complications, and conversion rates were collected and compared. The primary outcome was conversion rate. Major complications were defined as leak, intraabdominal abscess, pneumonia, cardiac complications, re-operation, DVT, and compartment syndrome. Minor complications were defined as ileus, urinary tract infection, surgical site infection, and acute kidney injury.

Results: There were 47 patients in the obesity group (mean $35.6 \mathrm{~kg}$ / $\mathrm{m} 2$ ) and 50 patients in the control group (mean $25.1 \mathrm{~kg} / \mathrm{m}^{2}$ ). There was no significant difference in regards to age, sex, ASA class $(\mathrm{p}=0.353)$, or pathologic data. Leak rate was $10 \%$ in BMI $<30$ and $8.5 \%$ in BMI $>30$. The overall conversion rate was $6.2 \%$. The rate of conversion was not significantly different between the two groups $(10 \%$ vs $2.1 \% ; p=0.205)$. There was no significant difference in post-operative major complications $(\mathrm{p}=0.417)$ and minor complications $(p=0.805)$. There was a significant difference in radial margin $(\mathrm{p}=0.046)$, but no difference in distal margins $(\mathrm{p}=1)$.

Conclusion: Obesity does not impact conversion rate or post-operative complications in patients undergoing robotic-colorectal surgery, but there was a significant difference in circumferential margins.

\begin{tabular}{|c|c|c|c|}
\hline \multicolumn{4}{|c|}{ Table 1. Perioperative Outcomes } \\
\hline & $\begin{array}{c}\mathrm{BMT}<30 \\
\mathrm{n}=50\end{array}$ & $\begin{array}{c}\mathrm{BMI} \geq 30 \\
\mathrm{n}=47\end{array}$ & $\mathrm{p}$-value \\
\hline Age & $60.1( \pm 11.6)$ & $60.2( \pm 10.8)$ & 0.979 \\
\hline $\begin{array}{l}\text { Sex } \\
\text { Female }\end{array}$ & $17(34 \%)$ & $23(48.9 \%)$ & 0.135 \\
\hline Male & $33(66 \%)$ & $24(51.1 \%)$ & \\
\hline \begin{tabular}{|l|} 
Operative time \\
\end{tabular} & $486( \pm 160.3)$ & $496( \pm 151.1)$ & 0.75 \\
\hline Conversion rate & $5(10 \%)$ & $1(2.1 \%)$ & 0.205 \\
\hline Lymph node yield & $22.6( \pm 11.5)$ & $22.2( \pm 10.3)$ & 0.852 \\
\hline $\begin{array}{l}\text { Total Mesorectal Grade } \\
1 \\
2\end{array}$ & $\begin{array}{c}29(80.6 \%) \\
4(11.1 \%)\end{array}$ & $\begin{array}{l}25(78.1 \%) \\
7(21.9 \%)\end{array}$ & 0.143 \\
\hline 3 & $3(8.3 \%)$ & & \\
\hline Distal Margin & $4.0( \pm 2.44)$ & $4.39( \pm 3.89)$ & 0.574 \\
\hline Circumferential Margin & $1.67( \pm 1.73)$ & $2.54( \pm 2.11)$ & 0.046 \\
\hline Major complications & $10(20 \%)$ & $6(12.8 \%)$ & 0.417 \\
\hline Minor complications & $11(22 \%)$ & $9(19.1 \%)$ & 0.805 \\
\hline Leakrate & $5(10 \%)$ & $4(8.5 \%)$ & 1 \\
\hline Readmission ( $\leq 30$ days) & $8(16 \%)$ & $7(14.9 \%)$ & 1.0 \\
\hline
\end{tabular}


P359

Selective Use of Intraoperative Perfusion Assessment using Immunofluorescence Angiography in High Risk Colorectal Anastomoses Results in Important Changes in Management

Mohammed M Al-Abri, MD, Noura Alhassan, MD, Patrick Charlebois, MD, A. Sender Liberman, MD, Gerald Fried, MD, Berry L Stein, MD, Carmen Mueller, MD, Liane Feldman, MD, Pepa Kanava, Msc, Lawrence Lee, MD, PHD; McGill University Health Centre

Background: Immunofluorescence angiography (IFA) with indocyanine green (ICG) can be useful to assess anastomotic perfusion in high risk bowel surgery, and may help reduce the risk of leak. However, the indications for its use are unclear. Past studies have not demonstrated a high proportion of patients in which management was altered, however these studies described its use in unselected patients. Perfusion assessment with IFA may be most beneficial in high-risk patients. The objective of this study was to evaluate the effect of intraoperative perfusion assessment using IFA on operative decisionmaking and postoperative outcomes in patients with high-risk colorectal anastomoses.

Methods: A prospective cohort study was performed on patients undergoing intraoperative perfusion assessment using IFA during colorectal surgery at a single high-volume referral centre from $03 / 2018$ to $08 / 2018$. A high-risk anastomosis was defined as an extraperitoneal rectal anastomosis or emergency surgery. Use of IFA was at the surgeon's discretion. Perfusion assessment involved a $3 \mathrm{cc}$ injection of reconstituted $(25 \mathrm{mg} / 10 \mathrm{~mL})$ ICG with subsequent IFA. Changes from planned transection lines were recorded. Secondary outcome was anastomotic leak rates within 30-days after surgery.

Results: 21 patients were included in this study ( $80 \%$ male, mean age 63 years (range 50-86), body mass index $27 \mathrm{~kg} / \mathrm{m}^{2}$ (range 21-36). Most patients $(90 \% ; 19 / 21)$ underwent low anterior resection, including $43 \%$ transanal total mesorectal excisions. Another 2 patients underwent extended left hemicolectomy with a Deloyer's procedure (right colon derotation and colorectal anastomosis). Changes in management based on ICG-IFA occurred in 29\% (6/21) of patients. Four patients had revision of the proximal margin (range $2-10 \mathrm{~cm}$ revision) and in 2 patients ICG-IFA allowed for an anastomosis to be performed in the context of middle colic artery division for a Deloyer's prceodure. In the 6 patients where ICG-IFA altered management, only 1 patient experienced a complication (ileus). In the other 15 patients, only 1 patient experienced an anastomotic leak (7\%).

Conclusion: In our early experience, selective use of ICG-IFA in patients with high-risk colorectal anastomoses results in changes in management in an important proportion of patients with good results. This technology may be of greatest value in selected high sk patients.

\section{P360}

\section{Cranial First Approach in Laparoscopic Right-Sided Colon Cancer Surgery}

Toru Tonooka, Nobuhiro Takiguchi, Atsushi Ikeda, Hiroaki Soda, Isamu Hoshino, Hisashi Gunji, Arihito Yoshizumi, Yoshihiro Nabeya; Chiba Cancer Center

Aim: Laparoscopic surgery for right-sided colon carcinoma is difficult to standardize due to technical difficulty by the presence of adjacent organs and major vessels to be preserved. There are different approaches to overcome the hurdle, such as cranial first, median first and retroperitoneal first approach, although leading to difficulty in formulating the procedure standardization. The aim of this study is to verify the validity of the cranial first approach in laparoscopic rightsided colon cancer surgery.

Method: From 2013 to 2017, either laparoscopic ileocecal resection or laparoscopic right hemicolectomy was performed for 26 cases of right-sided colon cancer patients. We created the three dimensional CT angiography for preoperative simulation, and all cases were performed in cranial first approach procedure. We evaluated the clinicopathological findings and the accomplishment rate of the procedure.

Results: All 26 cases were accomplished by the laparoscopic surgery and there was no conversion to open surgery. The median operative time and blood loss were $205 \mathrm{~min}$ and $19 \mathrm{ml}$, respectively. The median number of harvested LNs was 30. There were 5 cases with positive LN metastasis. The median length of stay was eight days. There was no major complication and postoperative course was uneventful in all cases.

Conclusion: Laparoscopic right-sided colon cancer surgery was safely performed in cranial first approach. From the accomplishment rate and the quality of the surgical result, the validity of the cranial first approach was confirmed. 


\section{P362}

Are We Ready for Bundled Payments for Major Bowel Surgery?

Udai S Sibia, MD, MBA, Justin J Turcotte, MBA, John R Klune, MD, MBA, Glen R Gibson, MD, FACS; Anne Arundel Medical Center

Introduction: The Centers for Medicare \& Medicaid Services (CMS) recently announced new voluntary episode payment models for major bowel surgery that aim to align incentives across participating healthcare providers in an attempt to reduce healthcare costs and improve the overall quality of care. The purpose of this study was to examine the financial impact of bundled payments for major bowel surgery.

Methods: This was a case-series of all patients who underwent major bowel surgery from July 2016 through June 2018. Procedures were categorized using Medicare Severity Diagnosis Related Group (MSDRG) coding which classifies patients into groups based on pre-existing comorbidity: patients without comorbidity (WOC; $n=217$ ), with comorbidity ( $\mathrm{WC} ; \mathrm{n}=410$ ), and with major comorbidity (MC; $\mathrm{n}=171$ ). The primary endpoint of the study was hospital costs for the index admission, the rate of 30-day readmissions and the corresponding hospital costs for those readmissions. We assumed the median reimbursement in a fee-for-service model to be the bundled payment for the entire episode of care which included 30-day readmissions.

Results: A total of 700 patients underwent 798 procedures, with mean age 62.1 years and mean BMI $29.2 \mathrm{~kg} / \mathrm{m}^{2} .54 \%$ were female. Percentage of patients with an ASA score $>2$ were as follows: $30.0 \%$ for WOC, $53.4 \%$ for WC, and $75.4 \%$ for MC. Forty-seven $(6.7 \%)$ patients required a total of 53 reoperations during the initial index admission. The median length of stay, median hospital costs of the index admission, and 30-day readmissions rate were substantially lower for patients WOC ( 2.0 days; $\$ 10,766 ; 6.0 \%)$ compared to those WC (4.0 days; $\$ 14,370 ; 11.4 \%)$ and to those with MC (13.0 days; $\$ 28,162 ; 13.4 \%$ ). Overall, 76 patients were readmitted 103 times for a total cost of $\$ 395,362$. In our current fee-for-service reimbursement model, net hospital margins were $0.9 \%$ for WOC, $-5.2 \%$ for WC, and $-12.4 \%$ for MC. In a potential bundled payment reimbursement model where hospital readmissions would not be reimbursed, net hospital margins would decrease to $-2.9 \%$ for WOC, $-16.7 \%$ for WC, and $9.9 \%$ for $\mathrm{MC}$

Conclusions: Patients undergoing major bowel surgery are often a heterogeneous population with varied pre-existing comorbid conditions. These patients require a high level of complex care and hence greater hospital resources which can have a tremendous impact on hospital costs. As CMS continues to test alternative bundled payments, health systems must aim to minimize costs without compromising quality of care. This will continue to be a challenge as our population ages.

\section{P363}

Are Right-Sided Colectomies for Neoplastic Disease at Increased Risk of Primary Postoperative Ileus Compared to Left-Sided Colectomies? A Coarsened Exact Matched Analysis

Richard Garfinkle, MD, Faisal Al-Rashid, MD, Nancy Morin, MD, Carol-Ann Vasilevsky, MD, Gabriela Ghitulescu, MD, Julio Faria, MD, Marylise Boutros, MD; Jewish General Hospital, Montreal, QC, Canada

Introduction: Primary postoperative ileus (pPOI) is the most common gastrointestinal complication following colonic resection. The objective of this study was to determine whether right-sided colectomies (RC) were associated with a higher incidence of pPOI compared to left-sided colectomies (LC).

Methods and Procedures: Adult patients who underwent elective colectomy for neoplastic disease between 2012-2016 were retrospectively identified using the American College of Surgeons National Surgical Quality Improvement Program database. RC and LC were defined as having an ileocolic or colocolic/colorectal anastomosis, respectively. Coarsened Exact Matching (CEM) was used to balance the two groups (1:1) on the following nine a priori selected confounders: age, sex, body mass index, American Society of Anaesthesiologists score, preoperative albumin, bowel preparation, operative approach, operative time, and perioperative transfusions. The association between type of colectomy and pPOI, defined as POI in the absence of intra-abdominal sepsis, was then assessed in a multiple logistic regression analysis of the matched data, accounting for any residual imbalance. Secondary outcomes (NSQIP-defined major morbidity, length of stay, 30-day readmissions, and 30-day mortality) were assessed by multiple logistic and quantile regression, respectively.

Results: Of 40,636 patients who underwent a colectomy for neoplastic disease, 15,231 underwent a RC and 25,405 a LC. There were several important differences on univariate analysis of the unmatched data. After CEM, 12,949 matched patients remained in each group, and all important confounders were well balanced. The incidence of pPOI was higher in the RC group $(11.5 \%$ vs. $8.8 \%, \mathrm{p}<0.001)$. On multiple logistic regression, $\mathrm{RC}$ was associated with a $35 \%$ higher odds of developing pPOI compared to LC (OR 1.35, 95\% CI 1.251.47). RC was also associated with increased risk for NSQIP-defined major morbidity (OR 1.10, 95\% CI 1.01-1.20), 30-day readmission (OR 1.16, 95\% CI 1.06-1.27), and increased length of stay ( $\beta=0.16$ days, $95 \%$ CI $0.11-0.22)$, but not increased risk for 30-day mortality (OR 0.99 , 95\% CI $0.76-1.28$ ).

Conclusion(s): pPOI is more common after RC than LC. Given the high prevalence of RC for neoplastic disease, future research should aim at better understanding the pathophysiology behind this increased risk and identifying interventions to mitigate pPOI in this population. 


\section{P364}

Laparoscopic Procedure Combined with ICG Lymphatic Imaging for Splenic Flexure Advanced Colon Cancer

Kazuki Ueda, MD, Junichiro Kawamura, MD, Hokuto Ushijima, MD, Koji Daito, MD, Tadao Tokoro, MD, Yoshinori Yane, MD, Yasumasa Yoshioka, MD, Jin-Ichi Hida, MD, Kiyotaka Okuno, MD; Kindai University Faculty of Medicine

The laparoscopic procedure for splenic flexure advanced colon cancer is oncologically difficult to perform. The reasons are including anatomical surrounding the important organs and various variation of dominant vessels. Our indication for advanced colon cancer is almost all cases with the exception of the invasion to surrounding organs. Moreover, to perform adequate lymphadenectomy, we utilized indocyanine green (ICG) fluorescence imaging from October 2016 as a clinical trial (UMIN000025300).

We present the laparoscopic procedure for splenic flexure advanced colon cancer using ICG fluorescence lymphatic flow imaging.

A $0.3 \mathrm{ml}$ of ICG $(2.5 \mathrm{mg} / \mathrm{ml})$ was injected to the submucosal layer around the tumor at one point using 23-gauge localized injection needle under colonoscopy prior to the surgery (one or two days before). During the surgery, the lymphatic flow and lymph nodes (LNs) identified using ICG fluorescence imaging. The lymphadenectomy was performed the laparoscopic conventional fashion proceeding medial to lateral (LNs dissection and vessel ligation), lateral detachment, opening the bursa omentalis and transverse colon take-down.

From January 2005 to December 2017, we experienced 938 cases of colorectal cancers. Among these cases, splenic flexure colon cancer was 61 cases $(6.5 \%)$. ICG fluorescence imaging during surgery was performed in 8 cases. Except one case, all the case were advanced cancers. In the case with liver metastases, ICG positive (ICG(+)) LNs were failed to detect. The ICG (+) LNs were detectable in the other 7 cases. Of these seven cases, 4 cases were detected alongside the left colic artery and 3 cases were alongside middle colic artery (left branch). Some metastatic LNs were not detected as an ICG (+), but these LNs were included ICG $(+)$ area.

The splenic flexure advanced colon cancer was rare among colon cancer. The ICG fluorescence imaging is detectable lymphatic flow and LNs during laparoscopic surgery. It is helpful to perform adequate lymphadenectomy for colon cancer. In this presentation, we will show the laparoscopic procedure for splenic flexure advanced colon cancer and present the efficacy and the limitation of ICG fluorescence imaging.

\section{P365}

Meta-analysis of Clinical and Pathological Outcomes of Transanal Versus Laparoscopic Total Mesorectal Excision for Rectal Cancer

Magdalena Mizera, Grzegorz Torbicz, Natalia Gajewska, Jan Witowski, Mateusz Rubinkiewicz, MD, PhD, Agata Czerwinska, MD, Piotr Zarzycki, MD, Piotr Malczak, MD, Piotr Major, MD, PhD, Michal Pedziwiatr, MD, PhD; 2nd Department of General Surgery, Jagiellonian University Medical College, Cracow, Poland

Background: Transanal total mesorectal excision (TaTME) is designed particularly to overcome the limits of open and laparoscopic approach. The data comparing this new technique with laparoscopy is constantly expanding. This meta-analysis is designed to compare TaTME with standard laparoscopic TME (LaTME).

Methods: We searched Medline, Embase and Scopus databases covering a up to July 2018. Inclusion criteria were: (1) study comparing laparoscopic resection of rectal cancer vs. TaTME for rectal cancer, (2) reporting of overall morbidity, operative time or major complications, (3) full text papers. The outcome measures were: overall morbidity, intraoperative adverse events, operative time, anastomotic leakage, intra-abdominal abscess, reoperations, length of hospital stay (LOS), oncological outcomes such as completeness of mesorectal excision, R0 resection rate, lymph node yield, distal resection margin (DRM) and circumferential resection margin (CRM).

Results: Ten studies were eligible with total of 698 patients. We found statistically significant differences in regard to major complications in favor of TaTME $(6.03 \%$ vs. $12.24 \%$ : $\mathrm{RR}=0.52 ; 95 \% \mathrm{CI}$ $0.25-0.96 ; p=0.04)$. We did not find significant differences regarding overall complications, intraoperative adverse effects, operative time, anastomotic leakage, intra-abdominal abscess occurrence, surgical site infections, reoperations and length of stay, completeness of mesorectal excision, R0 resection rate, number of harvested lymph nodes, DRM and CRM.

Conclusion: This is an updated meta-analysis including all most recently published trials. It shows benefits of TaTME technique in terms of major postoperative complications. In other clinic-pathological features TaTME is not superior to LaTME. Nonetheless, the quality of the data is still limited. To properly evaluate the feasibility, safety and efficacy of TaTME the results of large randomized trials are required. 
P366

\section{Minimally Invasive Proctectomy for Rectal Cancer: The New Standard of Care?}

Azah Althumairi, MD, James P Taylor, MBBChir, MPH, Miloslawa Stem, MS, Susan L Gearhart, MD, Sandy Fang, MD, Chady Atallah, Bashar Safar, MD, Jonathan Efron, MD; Johns Hopkins

Introduction: Prior randomized trials showed comparable short-term outcomes between open and minimally invasive proctectomy (OP and MIP) for rectal cancer. We hypothesize that short-term outcomes for MIP have improved as surgeons have become more experienced with this technique over time.

Methods and Procedures: Retrospective cohort study of rectal cancer patients who underwent elective proctectomy using the proctectomy-targeted American College of Surgeons National Surgical Quality Improvement Program (ACS-NSQIP) 2016 database. Patients were stratified into three groups based on intent-to-treat protocol: OP, MIP, and hybrid proctectomy (HP). Multivariable logistic regression analysis was used to assess the impact of operative approach on 30-day overall morbidity. Subgroup analysis for laparoscopic and robotic proctectomy (LP and RP) patients was also performed.

Results: 1,781 procedures were performed (42.78\% OP, $34.59 \%$ MIP [58.77\% LP, 41.23\% RP], and 22.63\% HP). MIP and HP patients had less comorbidities and their overall morbidity rates were significantly lower for patients who underwent MIP and HP (36.88\% vs. $26.95 \%$ vs. $20.84 \%$, respectively; $\mathrm{p}<0.001$ ) (TABLE). Clavien-Dindo III-V complications, readmission, reoperation, and anastomotic leak rates were comparable between OP and MIP. OP patients had significantly longer hospital length of stay (LOS) (7 vs. 5 days for MIP and HP, $\mathrm{p}<0.001$ ). Interestingly, HP patients had significantly lower rates of overall morbidity, organ space SSI, anastomotic leak, Clavien-Dindo III-V complication, and shorter operative time when compared to the MIP patients. There was no significant difference in overall morbidity between RP and LP patients $(29.01 \%$ vs. $24.02 \%, \mathrm{p}=0.169)$. Adjusted analysis showed similar results with MIP and HP having significantly decreased odds of overall morbidity in comparison to OP (OR 0.68, 95\% CI 0.53-0.86; OR 0.48, 95\% CI 0.36-0.65; both $\mathrm{p}<0.001$ ), and RP being equivalent with LP in terms of overall morbidity (OR $0.80,95 \%$ CI $0.54-1.17, \mathrm{p}=0.243$ ).

Conclusions: Patients selected to undergo MIP had significantly better overall morbidity rates and shorter LOS when compared with patients who underwent OP. Appropriate selection of patients for MIP can result in better outcomes.

\begin{tabular}{|c|c|c|c|c|c|c|}
\hline \multicolumn{7}{|c|}{ TABLE. Unadjusted Rates of 30-Days Post-OPerative Outcomes Stratified by Operative Approach } \\
\hline & $\begin{array}{l}\text { Open } \\
76242788 \\
\end{array}$ & MII & & Hybrid & & \\
\hline $\begin{array}{l}\text { Outcomenen(s) } \\
\text { Overall morbidity }\end{array}$ & $\frac{762(42.78)}{281(36.88)}$ & $\frac{6166(34.59)}{166(26.95)}$ & $<0.001$ & $\begin{array}{l}403(22.63) \\
84(20.84)\end{array}$ & & \\
\hline Wound infection & $81(10.63)$ & 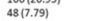 & & $25(6.64)$ & & \\
\hline Pneumonia & $16(2.10)$ & & 0.676 & $6(1.99)$ & 0.466 & 0.718 \\
\hline $\begin{array}{l}\text { Urinarytract infection } \\
\text { VTe }\end{array}$ & 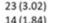 & 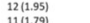 & 0.209 & $12(2.98)$ & 0.969 & 0.289 \\
\hline $\begin{array}{l}\text { VVE } \\
\text { Cardiac complication }\end{array}$ & 14(1.144) & $\begin{array}{l}11(1.79) \\
3(0.49)\end{array}$ & $\begin{array}{l}0.943 \\
0.078\end{array}$ & $\begin{array}{l}2(0.5) \\
2(1.50)\end{array}$ & $\begin{array}{l}0.061 \\
0.239\end{array}$ & 0.073 \\
\hline $\begin{array}{l}\text { Cardiac complication } \\
\text { Shockssepsis }\end{array}$ & 32(19.20) & $\begin{array}{l}36.049) \\
26.22)\end{array}$ & $\begin{array}{l}0.088 \\
0.984\end{array}$ & $\begin{array}{l}2(1.00) \\
11(2.73)\end{array}$ & $\begin{array}{l}0.239 \\
0.206\end{array}$ & $\begin{array}{l}0.999 \\
0.213\end{array}$ \\
\hline Unplanned intubation & $7(0.92)$ & $4(0.65)$ & 0.763 & $\begin{array}{l}210.50) \\
21050\end{array}$ & 0.727 & 0.999 \\
\hline Bieeding requiring transtusion & $159(20.87)$ & $52(8.44)$ & c0.001 & $28(6.95)$ & $<0.001$ & 0.386 \\
\hline $\begin{array}{l}\text { Renal compliketition } \\
\text { On ventiliter } 48 \text { hrs }\end{array}$ & $\begin{array}{l}14(1.84) \\
50.65)\end{array}$ & 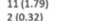 & 0.943 0.940 & $\begin{array}{l}7(1.74) \\
2(1.050)\end{array}$ & $\begin{array}{l}0.903 \\
0.999\end{array}$ & $\begin{array}{l}0.954 \\
0.650\end{array}$ \\
\hline Organ space SSI & $57(7.48)$ & $52(8.44)$ & 0.511 & $\begin{array}{l}210.050) \\
17(4.22)\end{array}$ & $\begin{array}{l}0.039 \\
0.030\end{array}$ & $\begin{array}{l}0.050 \\
0.009\end{array}$ \\
\hline Anastomotic leak & $23(3.02)$ & $21(3.41)$ & 0.682 & $4(0.99)$ & 0.029 & 0.015 \\
\hline Clavien-Dindo III-V & $126(16.54)$ & $91(14.77)$ & 0.372 & $\begin{array}{l}40(9.93) \\
06(1)\end{array}$ & 0.002 & 0.024 \\
\hline $\begin{array}{l}\text { Readimssision } \\
\text { Mortality }\end{array}$ & $\begin{array}{l}\begin{array}{l}131(17.19) \\
5(0.65)\end{array} \\
\end{array}$ & $\begin{array}{l}105(17.05) \\
2(0.32)\end{array}$ & $\begin{array}{l}0.943 \\
0.470\end{array}$ & 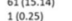 & $\begin{array}{l}0.368 \\
0.671\end{array}-120$ & 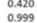 \\
\hline Reoperation & $\begin{array}{l}30.009 \\
46(6.04)\end{array}$ & $27(4.38)$ & 0.173 & $\begin{array}{l}19(4.71) \\
19.7 .7)\end{array}$ & 0.368 & 0.420 \\
\hline $\begin{array}{l}\text { Length th tsty, days, median (IIRR) } \\
\text { operative }\end{array}$ & $\begin{array}{l}7(5,10) \\
2855(209,394)\end{array}$ & $\begin{array}{l}5(4,7) \\
2092(225,5,392)\end{array}$ & $\begin{array}{c}c 0.001 \\
0.163\end{array}$ & $\begin{array}{l}544,8) \\
2732(20-350]\end{array}$ & $\begin{array}{l}0.001 \\
0.212\end{array}$ & $\begin{array}{l}0.552 \\
0.008\end{array}$ \\
\hline
\end{tabular}

\section{P367}

Robotic Surgery for Rectal Cancer: A Single Center Experience of 75 Consecutive Cases

Savulescu Florin, MD, PhD, Cirlan Cristian, MD, Blajut Cristian, MD, Budrugeac Iulia, MD, Iordache Mihai, MD, Iordache-Petrescu Madalina, MD; Dr. Carol Davila Central Military Emergency University Hospital

Background: Minimally invasive techniques have revolutionized the field of general surgery over the few last decades. The available literature on minimally invasive colorectal cancer surgery demonstrates that this approach is feasible and associated with better short term outcomes than open surgery while maintaining equivalent oncologic safety. Despite its advantages, in complex procedures such as rectal surgery, laparoscopy has not achieved a high penetration rate because of its steep learning curve, its relatively high conversion rate and technical challenges. Robotic-assisted surgery has been evolving now for over two decades and has finally matured into a technology that has caused a monumental shift in the way colorectal surgeries are performed.The aim of this study was to present a single center experience with robotic surgery for rectal cancer focusing mainly on early and mid-term postoperative outcome.

Methods: A series of 75 consecutive patients who underwent robotic rectal surgery between December 2014 and July 2018 in "Dr. Carol Davila" Central Military Emergency University Hospital, in Bucharest, Romania was analyzed retrospectively in terms of demographics, pathological data, surgical and oncological outcomes.

Results: Seventy-two patients underwent robotic sphincter-saving resection, and three patients underwent robotic abdominoperineal resection. There ware two conversions. The median operative time for sphincter-saving procedures was $210 \mathrm{~min}$. The median time for robotic abdominoperineal resection was $180 \mathrm{~min}$. The median number of retrieved lymph nodes was 22 . The median hospital stay was 6 days. In-hospital mortality was nil. The overall morbidity was $20 \%$. The median length of follow-up was 23 months.

Conclusion: Our preliminary results suggest that robotic-assisted surgery for colorectal cancer can be carried out safely and according to oncological principles. Robotic surgery is advantageous for both surgeons (in that it facilitates dissection in a narrow pelvis) and patients (in that it affords a very good quality of life via the preservation of sexual and urinary function in the vast majority of patients and it has low morbidity and good midterm oncological outcomes). In rectal cancer surgery, the robotic approach is a promising alternative and is expected to overcome the low penetration rate of laparoscopy in this field. 


\section{P368}

Fecal Incontinence After Surgical Treatment of Middle-Low Rectal Cancer. Laparoscopic Low Anterior Resection Versus Tatme

Cristina Mosquera, Eugenio Licardie, Dayana Bravo, Carla Madarro, Jesús Rosales, Jose Antonio Romero, Pablo Concheiro, Jose Francisco Noguera, PhD; Hospital Universitario A Coruña

Introduction: Worldwide, colorectal cancer(CRC) is the second most frequently diagnosed cancer in women and third one in men. Approximately 43,030new cases of rectal cancer are diagnosed annually in the United States. $\mathrm{CRC}$ is the most frequent cancer in Spain.

The surgical treatment of choice for middle-low rectal cancer is the low anterior resection; the main goal of this study is to compare the severity of faecal incontinence in two groups of patients, both diagnosed with middle-low rectal cancer, some of them underwent a Laparoscopic Low Anterior Resection(LADR) and the other by TaTME(Transanal total mesorectal excision).

Methods: Comparative cross-sectional study of two groups of patients, each one includes 12patients, classified based on the surgical technique.

\begin{tabular}{lcc}
\hline & TaTME(median) & LADR(median) \\
\hline Age(year) & 65 & 59.5 \\
Tumoral size(centimeters) & 4 & 4.7 \\
$\begin{array}{l}\text { Tumor distance from anal } \\
\quad \text { verge(centimeters) }\end{array}$ & 9.5 & 10 \\
$\begin{array}{l}\text { Height of the } \\
\text { anastomosis(centimeters) }\end{array}$ & 4 & 6 \\
\begin{tabular}{l} 
Post-surgical Wexner score \\
\hline
\end{tabular}
\end{tabular}

We used the magnetic resonance(MR) to study the tumor locally. For the analysis of results, we used the post-surgical Wexner score. We divided the results into 3 groups:Perfect continence(0points), Mild-Fecal Incontinence $(<9)$, Severe-Fecal Incontinence $(\geq 9)$. Several studies claims that the 9 point gravity cut-off is where the quality of life of patients was affected.
Results: To analyze the results we use the nonparametric Mann-Whitney Test. $\mathrm{P}$ value of $<0.05$ was considered significant.

\begin{tabular}{llllllll}
\hline & Age & BMI & $\begin{array}{l}\text { MR } \\
\text { size }\end{array}$ & $\begin{array}{l}\text { MR } \\
\text { height of } \\
\text { tumour }\end{array}$ & $\begin{array}{l}\text { Closed } \\
\text { ileostomy } \\
\text { time }\end{array}$ & $\begin{array}{l}\text { Height } \\
\text { anastomosis }\end{array}$ & $\begin{array}{l}\text { Wexner } \\
\text { Score }\end{array}$ \\
\hline Mann- & & & & & & Whitney &, 862 \\
& & & & & & U-test & \\
\hline 260 & 495 &, 475 &, 354 & $\mathbf{, 0 4 9}$ & $\mathbf{0 8 1}$ & & \\
\hline
\end{tabular}

Most of patients, regardless of the surgical technique used, presented some level of faecal incontinence. The comparison of obtained results between both groups was not statistically significant $(\mathrm{p}=0.081)$, but it is in the limit of significance.

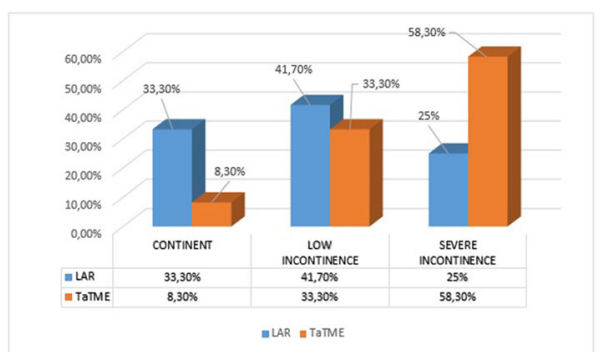

There is a statistically significant difference in the height of the anastomosis, being lower in the patients with TaTME.

Conclusion: TaTME versus conventional laparoscopic approach seeks to facilitate the surgery in complex pelvic regions. COLOR III study demonstrated the similarity between both techniques in oncological results.

Faecal incontinence is a frequent complication of rectal surgery, regardless of the technique used, requiring studies of larger sample size and longer-term follow-up to try to detect the possible differences between both techniques. 


\section{P369}

\section{Robotic Versus Laparoscopic Combined Resection for Rectal} Cancer and Liver Metastases

Jiyang Li, Yunhe Gao, Wenquan Liang, Kecheng Zhang, Jianxin Cui, Zhi Qiao, Bo Wei, Hongqing Xi, Lin Chen; Chinese PLA General Hospital

Background: Laparoscopic surgery is expanding in the treatment of liver metastases from gastrointestinal cancer due to its growing acceptance in safety, feasibility and efficacy recent years. Surgical robotic systems have recently supplemented and improved laparoscopic techniques. In this study, we compared the outcomes of robotic and laparoscopic combined resection for rectal cancer and liver metastases.

Methods: This retrospective study used data from a prospective database at the Chinese People's Liberation Army General Hospital. From January 2009 to December 2016, 133 rectal cancer patients with liver metastasis accepted laparoscopic or robotic rectal cancer resection, combined with simultaneous or staged hepatectomy or radiofrequency ablation (RFA) for liver metastases. The 133 patients enrolled in the study were divided into two groups: a robotic surgery group $(\mathrm{n}=18)$ and a laparoscopic group $(\mathrm{n}=115)$ which included three-dimensional laparoscopic $(\mathrm{n}=12)$ and two-dimensional laparoscopic $(\mathrm{n}=103)$. Primary endpoints were duration of surgery, intraoperative blood loss, conversions to an open procedure, resection margin status, in-hospital mortality and other postoperative recoveryrelated parameters.

Results: Forty-five patients underwent a simultaneous rectal and liver resection (mean operating time (robotic vs. laparoscopic): $426 \mathrm{~min}$ vs. $378 \mathrm{~min}, \mathrm{P}=0.029$ ), thirty patients had a simultaneous resection of primary rectal cancer and RFA of liver (mean operating time (robotic vs. laparoscopic): $382 \mathrm{~min}$ vs. $315 \mathrm{~min}, \mathrm{P}=0.046$ ), twenty-one patients underwent a staged rectal and liver resection (mean operating time (robotic vs. laparoscopic): $594 \mathrm{~min}$ vs. $530 \mathrm{~min}, \mathrm{P}=0.057$ ), and thirty-seven patients had a staged resection of primary rectal cancer and RFA of liver (mean operating time (robotic vs. laparoscopic): $521 \mathrm{~min}$ vs. $484 \mathrm{~min}, \mathrm{P}=0.142$ ). There were 9 conversions to an open procedure in the laparoscopic group. No in-hospital mortality occurred. An R0 resection margin was achieved in 98\%, with 3 positive cases in the laparoscopic group. Estimated blood loss, time to first flatus and postoperative complications, postoperative in-hospital days and 30-day readmission rates were similar in the two groups. On multivariate regression analysis, longer operation time and more conversion rates were not associated with the choice of minimal invasive techniques.

Conclusions: Robotic combined resection for rectal cancer and liver metastases was a safe and feasible minimal invasive approach for the management of rectal cancer with liver metastases. Although it has a longer operation time, its low mortality rate, conversion rate and comparable postoperative outcomes describes its bright prospect.

\section{P370}

\section{Trauma-Induced Cecal Bascule}

Robin Osofsky, MD, Heesung Park, BS, Sarah Popek, MD; University of New Mexico

Intro: Cecal bascule is a subtype of cecal volvulus characterized by anterior fold of the cecum into mid abdomen without any torsion, resulting in a closed loop obstruction. The infrequency with which it is encountered makes cecum bascule a challenging subject to study. Case Report: A 53-year-old male with past surgical history of childhood open appendectomy presented to the emergency department with acute onset of epigastric abdominal pain 3 days after a bicycle accident. He was diagnosed with multiple rib fractures. He was recovering at home and developed acute onset of severe abdominal pain and distention which worsened throughout the day, fever, and nausea without emesis.

Upon presentation to the emergency department, the patient's vital signs were stable, and lab values were within normal limits. His abdominal pain resolved with intravenous hydromorphone. The patient underwent $\mathrm{CT}$ of abdomen and pelvis with intravenous contrast to rule out delayed presentation of trauma-related injury. This revealed an abnormally rotated cecum in the upper mid abdomen with dilation of $12 \mathrm{~cm}$, concerning for cecal bascule. He was admitted for observation and was treated non-operatively. The following day, the patient had no abdominal pain, was tolerating a solid diet, passing flatus and stool, and he was discharged with outpatient follow-up for cecal bascule.

Colonoscopy was performed 2 weeks after discharge, which was normal aside from a large redundant cecum. The patient was offered options of non-operative management, cecectomy, and right hemicolectomy, and elected robotic-assisted right hemicolectomy with ileocolic anastomosis.

Four months after initial presentation, the patient underwent elective robotic assisted right hemicolectomy. Intraoperatively, the cecum was noted to be extremely mobile and redundant with no other gross abnormalities. The patient had no postoperative complications and was discharged on postoperative day one. Pathology of the resected colon and terminal ileum was unremarkable. At follow-up four weeks later, the patient reported having returned to work, resuming his activities of daily living, and with normal appetite and return of regular bowel habits.

Discussion: This case illustrates the potential for trauma inducing cecal bascule in a patient with a history of appendectomy. Although rare, cecal bascule should be considered as a differential diagnosis in trauma patients with cecal distention in order to provide appropriate counseling and treatment. 
P371

Ex Vivo Bovine Needle Puncture Bowel Wall Lift Histological Study: Lifts are Most Commonly Generated with a Sclerotherapy Catheter/Needle

Carl Winkler, $\mathrm{MD}^{1}$, Jaspreet Sandhu, $\mathrm{MD}^{2}$, Xiaohong Yan, $\mathrm{PhD}^{1}$, Neil Mitra, MD ${ }^{1}$, Dasuni Niyagama Gamage ${ }^{1}$, Vesna Cekic, RN ${ }^{1}$, Hmc Shantha Kumara, $\mathrm{PhD}^{1}$, Richard L Whelan, $\mathrm{MD}^{1}$; ${ }^{1}$ Mount Sinai West Hospital, ${ }^{2}$ Brookdale University Hospital Medical Center

Introduction: A mucosal "lift", generated by injecting fluid into the submucosal layer, needs to be established prior to endoscopic submucosal dissection (ESD) and endoscopic mucosal resection (EMR). Lifts are most often generated with a sclerotherapy catheter/needle. Although the muscularis propria or subersosal bowel wall layers may be inadvertently injected it is assumed that only submucosal injection can result in a "stable" lift. Recently, it was noted that stable "deep wall lifts" can result from injection into the muscularis propria or subserosal layers. This ex vivo bovine study was carried out to histologically assess needle injection generated bowel wall lifts to determine how often deep wall lifts occur.

Methods: Ex vivo bovine colorectal specimens were injected: 1) via colonoscope and sclerotherapy needle into an intact colon or 2) via a syringe and needle into the wall of an opened colon specimen. After visually grading each lift (superficial or deep) a full thickness piece of the bowel wall (including the lift) was harvested and placed in formalin. Days later, after dehydration in alcohol, the specimens were paraffin embedded and then cut into sections that were $\mathrm{H} \& \mathrm{E}$ stained. A pathologist assessed each slide to determine which layer(s) had actually expanded.

Results: A total of 5 bovine large bowel specimens were utilized and 83 stable lifts generated that were visually and histologically assessed (46 via scope and sclerotherapy needle into closed colon; 37 via direct syringe injection into opened colon). As per visual inspection, the location of the lifts was judged to be submucosal in 25 (30\%), deep in $16(19 \%)$, and mixed (submucosal \& intramuscular) in $42(51 \%)$. As per the histologic evaluation of "readable" slides (74/ $83)$, the lift location(s) were; submucosal in $12(16 \%)$, deep in 31 $(42 \%)$, and mixed in $31(42 \%)$. The deep lift location breakdown was muscularis propria in $25(34 \%)$ and subserosal in $6(18 \%)$.

Conclusions: A pure submucosal lift was obtained only $16 \%$ of the time with needle injection into ex vivo bovine colon; mixed (42\%) and deep lifts $(42 \%)$ where more common. Ex vivo models increase the odds of deep wall lifts because the tissue is non vital and easily damaged, however, deep wall lifts do occur in the clinical setting. Safe ESD is not possible with a deep lift. Lifts must be carefully assessed before starting ESD and a more superficial layer sought if a deep lift is suspected.

\section{P372}

Risk Factors for Additional Port Insertion in Single-port laparoscopic appendectomy

Yoo Shin Choi, Byung Kwan Park, MD; Chung-Ang University College of Medicine

Introduction: Recently, single-port laparoscopic appendectomy (SPLA) has been widely adopted to improve cosmetic outcomes and reduce surgical trauma. However, conversion to conventional laparoscopic appendectomy (CLA) might not lead to these advantages. We aimed to evaluate risk factors for conversion to CLA from SPLA in acute appendicitis.

Methods: Between August 2015 and December 2016, the characteristics, operative data, and postoperative surgical outcomes of patients who underwent intended SPLA at Chung-Ang University Hospital were retrospectively reviewed. Conversion to CLA was defined as any insertion of additional port, and complicated appendicitis was defined as gangrenous or perforated appendicitis with or without fluid collection, abscess, or peritonitis in preoperative CT. Univariable and multivariable analyses were performed to identify predictive factors for conversion to CLA.

Results: Of 409 patients, 65 (15.9\%) were treated with additional port insertion. On univariable analysis, age, sex, body mass index, complicated appendicitis, preoperative serum platelet count, and $\mathrm{C}$-reactive protein (CRP) level were associated with conversion to CLA. After multivariable analysis, old age $(\mathrm{OR}=1.031 ; 95 \% \mathrm{CI}$, $1.014-1.049 ; \mathrm{p}<0.001)$, male sex $(\mathrm{OR}=2.119 ; 95 \% \quad \mathrm{CI}$, $1.131-3.971 ; \mathrm{p}=0.019)$, increased serum CRP level $(\mathrm{OR}=2.944$; 95\% CI, 1.433-6.047; $\mathrm{p}=0.003)$, and complicated appendicitis $(\mathrm{OR}=3.330 ; 95 \% \mathrm{CI}, 1.304-8.503 ; \mathrm{p}=0.012)$ were significant risk factors for conversion to CLA. Overall complication rate was similar between patients who underwent SPLA and those who converted to CLA $(1.5 \%$ vs. $1.5 \%, \mathrm{p}>0.999)$.

Conclusion: The conversion rate to CLA was $15.9 \%$. Serum CRP level and complicated appendicitis were strong predictive factors for conversion to CLA from SPLA in acute appendicitis. 
S284

Surg Endosc (2019) 33:S130-S413

P373

Surgical Site Infections After Colorectal surgeries: A Risk that can be Decreased

Abdelrahman Attili, MD ${ }^{1}$, Danielle Craigg ${ }^{2}$, Anthony Dinallo ${ }^{2}$, Megan Navarro ${ }^{2}$, Mane Massarotti ${ }^{2}$, Allen Chudzinski ${ }^{2} ;{ }^{1}$ University of Central Florida, ${ }^{2}$ Florida Hospital Tampa

Introduction: Surgical site infections (SSI) are considered a major cause of morbidity and an independent predictor for mortality in surgical patients. It accounts for approximately a quarter of all nosocomial infections and it contributes to increase length of hospital stay along with increased cost of health care up to $\$ 15,000$ per case. Colorectal surgery is considered to be associated with higher rates of SSI compared to other surgeries. In this study we compared our SSI rate to the National Surgical Quality Improvement Program (NSQIP) predicted rate after using our adopted protocol for colorectal bundle.

Methods: With institutional review board approval, all patients undergoing colorectal resections in our institution between 2016-2018 were prospectively followed. We have adopted a specific protocol which was applied to all of our patients that includes, precise enhanced recovery after surgery, mechanical and antibiotic bowl preparation, perioperative antibiotics, specific intraoperative scrubping technique using alcohol and chlorhexidine, wound irrigation, the use of a wound protector, and the application of negative pressure wound vacuum followed by post operative wound monitoring. Patients demographic data, risk factors for SSI, and perioperative outcomes including SSI rate were collected and analyzed. Data is presented as median (mean $\pm \mathrm{SD}$ ).

Results: A total of 163 patients underwent colorectal resections within the study period. Fifty four percent had robotic approach, twenty two percent had laparoscopic approach, while twenty four percent had open approach. Age was $60.5(59.8 \pm 14.15), 54.6 \%$ were men, body mass index of $26.4(27.22 \pm 5.59) \mathrm{kg} / \mathrm{m}^{2}, 46 \%$ were smokers, and $12.4 \%$ were diabetic [Table 1]. Four patients had ISIs $(2.4 \%)$ which was statistically different from the predicted NSQIP outcome of $7.4 \%$ for these patients $(\mathrm{p}=0.029)$ [Table 2]. One patient had superficial wound infection that was treated with antibiotics, another three patients had deep wound infections manifested by anastomotic leak.

Conclusion: Our results suggests that using this specific protocol will help to substantially decrease the rate of SSI after colorectal surgeries compared to the predicted NSQIP SSI rate for these patients. This protocol is safe and simple, and may reduce the post operative monbidity after colorectal surgeries.

\begin{tabular}{|l|c|}
\hline Democraphics & Total Patients (N=163) \\
\hline Age (years) & $60.5(59.8+/-14.15)$ \\
\hline BMI kg /m ${ }^{2}$ & $26.4(27.217+/-5.59)$ \\
\hline Gender & \\
\hline Male & $54.60 \%$ \\
\hline Female & $45.40 \%$ \\
\hline Peri-operative Risk Factors & \\
\hline Smoking Hr & $46.30 \%$ \\
\hline Coronary Artery Disease & $5.50 \%$ \\
\hline Anemia & $12.30 \%$ \\
\hline CORD & $2.50 \%$ \\
\hline Diabetes & $12.35 \%$ \\
\hline Hypertension & $28.40 \%$ \\
\hline Transfusions (intraoperative) & $0.61 \%$ \\
\hline OR Time (ins) & $273(279.78+/-119.28)$ \\
\hline Surgical Approach & \\
\hline Robotic & $53.70 \%$ \\
\hline Laparscopic & $22.22 \%$ \\
\hline Open & $23.46 \%$ \\
\hline Robotic to Open & $0.62 \%$ \\
\hline
\end{tabular}

Table 1: Patients Demographics, perioperative risk factors for SSI and Surgical approach.

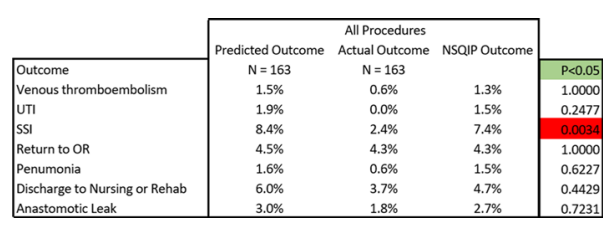

Table 2: Actual outcomes compared to NSQIP outcomes.

Springer 
P374

Results of Laparoscopic Intersphincteric Resection with Handsewn Coloanal Anastomosis for Rectal Low Cancer

Viet Trung Lam, MD, PhD; Cho Ray hospital, Ho Chi Minh City, Vietnam

Overview: Rectal cancer is one of popular cancers of digestive tract. With low rectal cancer which located $<5 \mathrm{~cm}$ from anal verge, the traditional method of surgery is abdominoperineal resection in which total anus and sphincter were resected and permanent colostomy. Recent studies have shown favorable outcomes of sphincter saving surgery with laparoscopic approach for low rectal cancer.

Aim: To evaluate the feasibility and results of laparoscopic intersphincteric resection with hand-sewn coloanal anastomosis for low rectal cancer. Method: Prospective study. From 4/2009 to 6/2017 we performed laparoscopic intersphincteric resection with hand-sewn coloanal anastomosis in 25 patients with low rectal cancer about $1-4 \mathrm{~cm}$ from dentate line with no sphincter involvement.

Results: Mean operation time was $270 \mathrm{~min}$. No intraoperative complications. Postoperative complications was $12 \%(3 / 25)$ included 2 cases had late anastomotic leak $(8 \%)$ which were re-operated on. There was no mortality. Anastomotic stenosis happened in 1 case (4\%) which was treated conservatively with anal dilatation. With the follow-up time 34.8 months (4-101 months), most of patients had stool $6-10$ times/day in the first 1-3 months which was gradually less frequently to $1-3$ times/day in the following 3 months. There was no local recurrence. One case with distal metastasis (liver and lung) treated with chemotherapy and targeted therapy is still in good health. One patient died at 20 months due to liver and peritoneal metastasis. Conclusion: Laparoscopic intersphincteric resection with hand-sewn coloanal anastomosis is feasible, safe and effective in functional and oncological results with selected patients. Less pain, early recovery, lower cost for not using staplers besides preserving sphincter function are those of its advantages. It is needed to have longer follow-up time to evaluate the long-term functional and oncologic outcomes.

Key words: Intersphincteric resection, coloanal anastomosis, low rectal cancer

\section{P375}

\section{Using Machine Learning to Predict Learning Curves}

Yuanyuan $\mathrm{Gao}^{1}$, Uwe Kruger ${ }^{1}$, Xavier Intes ${ }^{1}$, Steven Schwaitzberg ${ }^{2}$, Suvranu De ${ }^{1}$; ${ }^{1}$ ensselaer Polytechnic Institute, ${ }^{2}$ University at Buffalo

Background: Most surgical training programs depend on repetition of bimanual motor tasks to build muscle memory. However, such a time-limited process does not ensure attainment of proficiency due to inherent differences of initial skill levels and learning abilities. To better customize the training program for individual learners at the initial learning stage, we need to understand how to predict the final learning effect using initial learning stage information. Nonetheless, this specific approach has not been fully understood though several learning curve models exist.

Methods: In this study we use learning curve data from a metaanalysis of several studies performed on both real and virtual simulations of the Fundamentals of Laparoscopic Surgery (FLS) tasks. The learning curve features extracted from this dataset are used to train a supervised machine learning model, kernel partial least squares (KPLS) to develop a robust model for predicting learning curves. The performance of KPLS model is compared with the classic log-linear model. Based on the features, the trainees are also classified into learning groups using an unsupervised machine learning approach $(\mathrm{k}$ means).

Results: We show that the information present in the first ten trials of bimanual motor tasks can be utilized to predict the number of trials required to achieve proficiency $(\mathrm{R} 2=0.82)$ and the final performance level $(\mathrm{R} 2=0.99)$. In contrast, the classic log-linear model does not output accurate prediction in this scenario $(\mathrm{R} 2=-87.46$ and $\mathrm{R} 2=-$ 2.84 respectively). Furthermore, a single learning ability factor could be defined that could classify learners into groups with unique learning characteristics.

Conclusion: Using sophisticated machine learning models we show, for the first time, that the initial few trials of bimanual surgical motor tasks contain sufficient information to predict the learning curve and a single factor is able to capture complexities of learning behavior. Use of such models holds the potential for personalization of training regimens, leading to greater efficiency and lower costs. 
P376

Design and Development of A Virtual Endoluminal Surgical Simulator (VESS) for Endoscopic Submucosal Dissection

Zhaohui Xia, $\mathrm{PhD}^{1}$, Sudeep Hegde, $\mathrm{PhD}^{2}$, Nicholas Milef ${ }^{1}$, Suvranu $\mathrm{De}, \mathrm{PhD}^{1}$, Daniel Jones, $\mathrm{MD}^{3}$, Mandeep Sawhney, $\mathrm{MD}^{3}$, Cullen Jackson, $\mathrm{PhD}^{3}$; ${ }^{1}$ Center for Modeling, Simulation and Imaging in Medicine (CeMSIM), Rensselaer Polytechnic Institute, Troy, $\mathrm{NY},{ }^{2}$ Department of Industrial and Systems Engineering, University at Buffalo, Buffalo, NY, ${ }^{3}$ Beth Israel Deaconess Medical Center, Harvard Medical School, Boston, MA

Introduction: Endoscopic Submucosal Dissection (ESD) is a minimally-invasive technique that allows complete en-bloc resection and lower recurrence rates at long-term follow-ups compared to endoscopic submucosal resection (EMR). In ESD procedures, a fluid is injected underneath the lesion, and then the submucosal layer is dissected by a specialized knife below the lesion. However, performing ESD is technically demanding, and there is lack of sufficient number of expert instructors for ESD training in Western countries. One approach to reducing this gap is to design and develop an ESD surgical simulator as part of a training curriculum.

Objectives: The aim of the study is to design and develop a virtual endoluminal surgical simulator (VESS) with visual and haptic feedback along with assessment metrics based on a cognitive task analysis (CTA) approach, which can be used to train endoscopists in ESD and assess the trainees' performance. Methods and Procedures: To develop a virtual ESD simulator that will allow trainees to hone their ability to recognize and anticipate critical obstacles during the performance of ESD procedures, and enhance their decision-making skills, the results of a CTA are being integrated into the development of the simulator as learning objectives and associated metrics.

Results: From the CTA, we derived several key learning objectives that center on the cognitive skills required for ESD. Some of these include: (1) recognizing polyp boundaries to ensure accurate marking for complete en-bloc resection; (2) deciding on appropriate approach for initial incision (e.g., circumferential, pockets); (3) choosing appropriate coag and cutting current settings according to field dynamics; (4) adapting approach to account for tissue retraction and maximize field visualization; (5) maintaining the orientation of lesion boundaries and tissue layers during cutting to minimize tissue damage and perforations; (6) recognizing vessel type and choosing electrocautery settings accordingly to minimize bleeding in the field.

Conclusions: This study presents a novel CTA-based approach to developing a virtual ESD simulator that involves high-fidelity anatomical organ modeling, physics-based tool-tissue interactions, and performance metrics based on a cognitive task analysis.

\section{P377}

Bimanual Wrist-Squeezing Haptic Feedback Changes SpeedForce Tradeoff in Robotic Surgery Training

Eric $\mathrm{Cao}^{1}$, Sergio Machaca ${ }^{1}$, Timothy Bernard ${ }^{2}$, Brett Wolfinger ${ }^{1}$, Zachary Patterson ${ }^{3}$, Amy $\mathrm{Chi}^{1}$, Gina Adrales, $\mathrm{MD}^{4}$, Katherine J Kuchenbecker, $\mathrm{PhD}^{5}$, Jeremy D Brown, $\mathrm{PhD}^{1} ;{ }^{1}$ Johns Hopkins University, ${ }^{2}$ University of Maryland Baltimore County, ${ }^{3}$ Carnegie Mellon University, ${ }^{4}$ Johns Hopkins Medical Institute, ${ }^{5}$ Max Planck Institute for Intelligent Systems

Introduction: Most clinically approved robot-assisted surgical systems such as the Intuitive Surgical da Vinci provide no haptic feedback. Consequently, safely handling tissue with the robotic instruments can be challenging, especially for novice trainees. While most surgical training paradigms focus on maximizing speed and minimizing tissue interaction forces, task completion time is often the primary performance metric. We hypothesize that providing novices with tactile feedback of the force magnitude they apply while training will significantly reduce their root-mean-square (RMS) force magnitudes while increasing their completion time compared to trainees receiving no feedback. Methods: Novice participants with no prior da Vinci or clinical experience $(\mathrm{n}=8)$ performed a ring rollercoaster training task twelve times using a da Vinci S surgical robot. Participants were instructed to finish each trial as quickly as possible, to use both hands, and to avoid dropping or damaging the ring. Wrist-squeezing tactile actuators delivered bimanual haptic feedback in proportion to the magnitude of the 3D force vector applied to the track. Participants in the feedback group $(n=4)$ received haptic feedback for all twelve trials, while participants in the no-feedback group $(n=4)$ completed all trials without haptic feedback. Trial duration and RMS force were calculated for all trials

Results: Participant in both groups significantly reduced their trial duration between the beginning (trials 1-3) and end (trials 10-12) of the experiment ( $p<0.01$ for both groups). While participants in the feedback group were significantly slower than participants in the no-feedback group in the first three trials $(\mathrm{p}<0.01)$, this between-group difference was not significant by the last three trials of the experiment. Regarding the forces produced on the task materials, neither group significantly increased or decreased their RMS force between the beginning (trials 1-3) and end (trials 10-12) of the experiment. However, participants in the feedback group produced significantly less RMS force than participants in the no-feedback group overall for the 12 trials of the experiment $(\mathrm{p}<.001)$

Conclusions: Providing novice da Vinci users with tactile feedback of the forces they exerted did not affect final task completion time and significantly reduced the applied forces. These results highlight the potential efficacy of this approach to helping early novices understand and reduce the forces applied via a robot-assisted surgical system, potentially reducing tissue damage and suture breakage in the clinical setting later on.

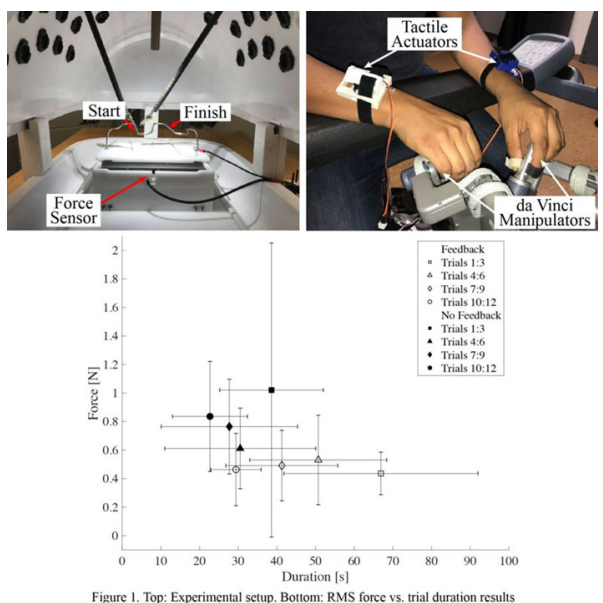




\section{P378}

Solutions for Patient Safety (SPS): How to Manage Risks Associated with the Use of Electrosurgery in the Operating Room ?

J Gugenheim, Jf Gravie, A Deleuze, F Borie, M Mathonnet, B Millat; Federation of Visceral and Digestive Surgery

In France, solutions for Patient Safety are established by expert's consensus under the guidance of the High Authority of Health (HAS) and based on an adverse events database established by HAS in 2006. The database collects informations on adverse events from all surgical specialties and allows analyze of the causes and barriers leading up to adverse events.

This study analysis 614 adverse events involving electrosurgery. Under the direction of the Federation of Visceral and Digestive Surgery (FCVD) the data analysis was performed by a multidisciplinary working group including physicians from 8 different specialties, pharmacists, biomedical engineers and operating room nurses. As a result several key points focussing on prevention, response, and risk mitigation of electrosurgical injuries were established:

\section{Prevent:}

These key points describe the different actions to be implemented by each actor in the operating room; control and adjustment of the equipment, patient positioning, safe use of the dispersive and active electrode, team communications, management of patient carrying implantable electronic devices such as a cardiac defibrillator.

2. Recover:

The key points specify the actions to be implemented in case of cutaneous or visceral burns and OR fires.

3. Mitigate:

This point describes the recommendations to be implemented regarding:

- The patient: inform, monitor, detect.

- The medical file: patient documentation, description of the incident and recovery measures

- The institutions (hospital, risk management organization, national agency...): report the event, analyze the causes, describe the implementation of corrective actions and measure their effectiveness.

In summary, SPS aims to prevent, recover, and mitigate adverse events related to the use of the electrosurgery. SPS intends to provide professionals in the operating room a safety tool in their daily practice with electrosurgical instruments.

\section{P379}

\section{Resource Optimization in Proficiency-Based Suturing Skills Training of Medical Students}

Madeline Lemke, Hillary Lia, Alexander Gabinet-Equihua, Guy Sheahan, Andrea Winthrop, Stephen Mann, Gabor Fichtinger, Boris Zevin; Queen's University

Background: Suturing is a fundamental skill in undergraduate medical education. It can be taught by faculty-led, peer-led, and holography augmented methods; however, the most educationally effective and cost-efficient method for proficiency-based teaching of suturing is yet to be determined.

Methods: We conducted a randomized controlled trial comparing faculty-led, peer-led, and holography augmented proficiency-based suturing training to preclerkship medical students. Holography augmented training provided holographic, voice-controlled instructional material. Technical skill was assessed using hand motion analysis every ten sutures and used to construct learning curves. Proficiency was defined by one standard deviation within average faculty surgeon performance. Intervention arms were compared using one-way ANOVA of the number of sutures placed, full-length sutures used, time to proficiency, incremental costs incurred. Participant preferences were surveyed. Results: Forty-four students were randomized to the faculty-led $(n=16)$, peer tutor-led $(n=14)$ and holography augmented $(n=14)$ intervention arms. At proficiency, there were no differences between groups in the number of sutures placed, full-length sutures used, and time to achieve proficiency (Table 1). The computer-augmented method was costlier than faculty-led and peer tutor-led instruction $(\$ 247.00 \pm \$ 12.05, \mathrm{p}<0.001)$ due to the high cost of the equipment. Faculty-led teaching was the most preferred method $(78.0 \%)$, while computer-augmented was the least preferred $(0 \%) .90 .6 \%$ of students reported high confidence in performing simple interrupted sutures, which did not differ between intervention arms (faculty-led $100.0 \%$, peer tutor-led $90.0 \%$, holography augmented $83.3 \%, \mathrm{p}=0.409) .93 .8 \%$ of students felt the program should be offered in the future.

Conclusion: Proficiency-based teaching of suturing using faculty-led and peer tutor-led instructional methods were superior to holography-augmented method with respect to costs and participants' preferences despite being educationally equivalent.

Table 1. Outcomes

\begin{tabular}{|c|c|c|c|c|}
\hline & $\begin{array}{l}\text { All } \\
(n=44)\end{array}$ & $\begin{array}{l}\text { Faculty- } \\
\text { led } \\
(n=16)\end{array}$ & $\begin{array}{l}\text { Peer tutor- } \\
\text { led } \\
(\mathrm{n}=14)\end{array}$ & $\begin{array}{l}\text { Holography } \\
\text { augmented } \\
(\mathrm{n}=14)\end{array}$ \\
\hline $\begin{array}{l}\text { Number of sutures } \\
\text { placed } P=0.376\end{array}$ & $\begin{array}{l}90.3 \\
\quad(55.6)\end{array}$ & $\begin{array}{l}80.0 \\
\quad(47.2)\end{array}$ & $\begin{array}{l}85.0 \\
\quad(58.6)\end{array}$ & $\begin{array}{r}107.4 \\
(61.3)\end{array}$ \\
\hline $\begin{array}{l}\text { Number of full-length } \\
\text { sutures used } \\
\qquad \mathrm{P}=0.735\end{array}$ & $\begin{array}{l}10.1 \\
\quad(6.0)\end{array}$ & $\begin{array}{l}9.3 \\
(5.3)\end{array}$ & $\begin{array}{l}10.3 \\
(7.0)\end{array}$ & $\begin{array}{l}11.0 \\
(6.0)\end{array}$ \\
\hline $\begin{array}{l}\text { Time to proficiency } \\
\text { (minutes) } \mathrm{P}=0.390\end{array}$ & $\begin{array}{l}174.3 \\
\quad(100.3)\end{array}$ & $\begin{array}{l}158.1 \\
\quad(89.2)\end{array}$ & $\begin{array}{l}162.2 \\
(98.9)\end{array}$ & $\begin{array}{l}205.0 \\
\quad(113.2)\end{array}$ \\
\hline $\begin{array}{l}\text { Incremental cost per } \\
\text { particpant }(\mathrm{CAD}) \\
\mathrm{P}<0.001^{*}\end{array}$ & $\begin{array}{l}\$ 209.28 \\
\quad(\$ 50.37)\end{array}$ & $\begin{array}{l}\$ 200.11 \\
\quad(\$ 60.19)\end{array}$ & $\begin{array}{l}\$ 182.05 \\
\quad(\$ 40.42)\end{array}$ & $\begin{array}{l}\$ 247.00 \\
\quad(\$ 12.05)\end{array}$ \\
\hline
\end{tabular}

All values are reported as mean(standard deviation); *P-values $<0.05$ is considered statistically significant. 


\section{P380}

Determining Endoscopic Competency by Contrasting Endoscopic Experience, GAGES and BEST Box Scores

Ilay Habaz, Bsc ${ }^{1}$, Allan Okrainec, $\mathrm{Dr}^{1}$, Ethan Weiss ${ }^{1}$, Silvana Perretta, Dr ${ }^{2}$, Else V Velden ${ }^{2}$, Ludovica Guerriero ${ }^{2}$, Fabio Longo ${ }^{2}$, Pietro Mascagni ${ }^{2}$, Louis Liu, $\mathrm{Dr}^{3}$, Timothy Jackson, $\mathrm{Dr}^{1}$, Lee L Swanstorm, Dr ${ }^{4}$, Eran Shlomovitz, Dr ${ }^{1} ;{ }^{1}$ University Health Network, Department of General Surgery, ${ }^{2} \mathrm{IHU}-$ Strasbourg, Strasbourg, France, ${ }^{3}$ University Health Network, Department of Gastroenterology, ${ }^{4}$ The Oregon Clinic, Division of GI/MIS, Portland, OR, USA

Introduction: To investigate the relationship between the Basic Endoscopic Skills Training (BEST) Box, Global Assessment of Gastrointestinal and Endoscopic Skills (GAGES) rating tool and number of endoscopic procedures performed to evaluate the relationship between number of endoscopic procedures performed and endoscopic competency.

Methods: A total of 55 participants were tested. Participants were scored on the BEST box by a trained proctor using an objective scoring formula which rewards speed and accuracy. GAGES scores were separately evaluated by a trained administrator. "Ability to keep clear view" was removed, as this is a skill that BEST box does not simulate, thus the passing score on GAGES was set at $15 / 20$. Prior endoscopic experience was assessed using a pre-test questioner to gauge number of endoscopic cases performed. For the analysis, participants were placed subgroups according to procedure numbers (Table 1). Due to a small sample size $(n=1)$, the '101-200 cases' group was excluded.

Results: A significant positive correlation was observed between user experience, BEST box score and GAGES score (Table 1). The $>200$ endoscopy group had the highest GAGES pass rate at $90 \%$, this is statistically significantly better as compared to users who performed $\leq 100$ procedures $(\mathrm{p}<.001)$. The ' $>200$ ' also had the highest BEST box score mean and pass rate $(M=83.5, \mathrm{p}<.001)$ compared to groups with $\leq 100$ procedures $(\mathrm{M}=60.43)$

Conclusion: Due to inconsistent sample sizes and variability within each group the ability to detect a linear progression of experience among groups was not possible. However, there is enough evidence to suggest that individuals with less than 100 endoscopy procedures have insufficient endoscopic skills. Additionally, the results suggest that BEST box may be an objective assessment tool to assess user competency. Further investigation is required to determine if a higher threshold should be recommended.

\begin{tabular}{|c|c|c|c|c|c|}
\hline \multirow{2}{*}{$\begin{array}{l}\text { Number of Endoscopic } \\
\text { procedures performed }\end{array}$} & \multirow[t]{2}{*}{$\mathrm{N}$} & \multicolumn{2}{|c|}{ GAGES } & \multicolumn{2}{|c|}{ BEST box } \\
\hline & & $\bar{x}$ & Pass rate (\%) & $\bar{x}$ & Pass rate (\%) \\
\hline None & 28 & 12.4 & \begin{tabular}{|l|l|}
17.9 \\
\end{tabular} & 59.2 & \begin{tabular}{|l|}
7.1 \\
\end{tabular} \\
\hline $1-10$ & 9 & 11.4 & 22.2 & 60.6 & 11.1 \\
\hline 11-50 & 4 & 12.0 & 0 & 62.4 & 0 \\
\hline $51-100$ & 3 & 13.0 & 33.3 & 65.8 & 0 \\
\hline $101-200$ & $1^{*}$ & 18.0 & 100 & 79.0 & 100 \\
\hline$>200$ & 10 & 17.9 & 90 & 83.5 & 70 \\
\hline
\end{tabular}

\section{P382}

Simulation Platforms to Assess Laparoscopic Suturing Skills: A Scoping Review

Elif Bilgic $^{1}$, Motaz Alyafi, MD ${ }^{1}$, Tara Landry, MLIS ${ }^{2}$, Melina C Vassiliou ${ }^{1}$; ${ }^{1}$ McGill University, ${ }^{2}$ Montreal General Hospital Medical Library

Background: Laparoscopic suturing (LS) has become a common technique used in a variety of advanced laparoscopic procedures. LS is, however, a challenging skill and trainees should be competent to perform LS at the end of their training. The purpose of this review is to identify simulation platforms available for assessment of LS skills, and to determine the characteristics of the platforms and the type of LS skills being assessed.

Methods: A scoping review was conducted between January 1997 and October 2017 for full-text articles. The search terms included "laparoscopic suturing" and "competence". Extracted data included characteristics of the simulation platforms, suturing type, and five sources of validity evidence: content (can the simulation tasks measure suturing skills), response process (can the scoring be done accurately), internal structure (are the scores consistent), relationship to other variables (do the task scores correlate with other assessments or differentiate between training levels), and consequences (what are the implications of incorporating the task for assessment into the training programs).

Results: Fifty-six studies were selected. Among them, 42 used a box trainer (36 inanimate, 5 ex vivo, 1 not specified(NS)), 10 augmented reality, 3 virtual reality, 0 in vivo methods, and 1 cadaver. The majority of the suturing was done using basic intracorporeal knottying(ICK) techniques (interrupted, 1 suture ICK), similar to the suturing done in the Fundamentals of Laparoscopic Surgery (FLS) ICK task. Most of the validity evidence was derived from internal structure (rater reliability) and relationship to other variables (compare training levels/case experience, compare various metrics). Consequences were not addressed in any of the studies.

Conclusion: We identified many types of simulation platforms used to assess LS skills, with most of them targeting basic skills. Platforms assessing the competence of trainees in advanced LS were limited in number and validity evidence. Therefore, future research should focus on assessment of advanced LS skills that better reflect the current practice environment. 


\section{P383}

\section{Deep Learning Analysis of Functional Brain Imaging Predicts Surgical Skill Level}

Yuanyuan Gao $^{1}$, Pingkun Yan $^{1}$, Uwe Kruger ${ }^{1}$, Lora Cavuoto ${ }^{2}$, Steven $\bar{D}^{\text {D Schwaitzberg }}{ }^{2}$, Xavier Intes ${ }^{1}$, Suvranu De ${ }^{1} ;{ }^{1}$ Rensselaer Polytechnic Institute, ${ }^{2}$ University at Buffalo

Background: Biofeedback/neurofeedback has been recently proposed to enhance motor skill execution. To implement this new paradigm to surgical applications, it is however imperative that surgical skill level assessment been achieved in real time. Though, the currently employed quantitative gold standard for skill assessment, i.e. the Fundamentals of Laparoscopic Surgery (FLS) score cannot be computed in a real-time and automatic fashion. In this study, we further investigate the potential of functional near-infrared spectroscopy (fNIRS) to impact surgical skill assessment methodologies by exploring the potential of fNIRS technology to predict surgical skill level in real-time.

Methods: In this study we acquired PFC region hemodynamic data via fNIRS technology from 13 medical students while they practiced Pattern Cutting task in both physical and virtual simulators. We developed a Deep Learning architecture called thereafter "Brain-Net" to utilize the features extracted from fNIRS data to predict the FLS score. The accuracy of the model is validated by 10 -fold cross validation scheme while the robustness is assessed by running multiple rounds of cross validation with randomly shuffled samples for each round. The performance of the model is also tested across physical/virtual simulators and skilled/unskilled learners.

Results: The mean and standard deviation of the 10-fold cross validation R2 values of 'Brain-NET' are 0.67 and 0.04 , respectively. The error distribution is not significantly different across physical/virtual simulators and skilled/unskilled learners.

Conclusion: The 'Brain-NET' 2D CNN enables predicting surgical skill level accurately and robustly using brain imaging data in real-time. The prediction performance is also stable across platforms and cohorts. This approach takes a step forward to a potential intraprocedural neurofeedback system to enhance surgical motor skill training.

\section{P384}

Do Laparoscopic Surgery Naïve Operators Prefer ThreeDimensional Images?

Kazuhiro Endo, MD, $\mathrm{PhD}^{1}$, Yoshinori Hosoya, $\mathrm{MD}, \mathrm{PhD}^{1}$, Takumi Saito, $\mathrm{MD}, \mathrm{PhD}^{2}$, Yasunaru Sakuma, MD, $\mathrm{PhD}^{1}$, Hisanaga Horie, $\mathrm{MD}, \mathrm{PhD}^{1}$, Joji Kitayama ${ }^{1}$, Alan Lefor, $\mathrm{MD}, \mathrm{PhD}^{1}$, Naohiro Sata, $\mathrm{MD}, \mathrm{PhD}^{1}$; ${ }^{1}$ Jichi Medical university, ${ }^{2}$ Saiseikai Iwaizumi Hospital

Background: Due to difficulties with stereoscopic vision or unpleasant symptoms, some surgeons prefer not to use this technology. We investigated whether the three-dimensional (3D) laparoscope was more effective than the two-dimensional (2D) laparoscope for naïve operators performing a simple task. We also conducted a survey regarding preferences, impressions and any symptoms when viewing images using a 3D laparoscope compared to a $2 \mathrm{D}$ laparoscope.

Methods: 118 fourth year medical students participated in this study. The peg transfer task of the SAGES FLS program was selected, and performed continuously for two minutes. Subjects were given a document and a verbal explanation. They then observed a demonstration before being randomly divided into two groups. The 2Dto3D group performed the task with a $2 \mathrm{D}$ laparoscope before performing it with a 3D laparoscope. The 3Dto2D group performed the task with a $3 \mathrm{D}$ laparoscope before performing it with a $2 \mathrm{D}$ laparoscope. The ENDOEYE 3D Flex and the ENDOEYE HD (Olympus Corporation, Tokyo, Japan) were used. The number of movements and drops were counted. We also conducted a survey regarding preferences of images, impressions regarding the endoscopic images and occurrence of any symptoms. Results (Table): There were no significant differences in the number of peg movements or drops between two groups. While many subjects in the 3Dto2D group tended to prefer the three-dimensional images, the number of subjects who preferred the three-dimensional images was below $50 \%$ in both groups.(47\% vs $41 \%$ ) Many subjects assessed the three-dimensional laparoscope as being superior in terms of image visibility $(69 \%)$ and depth perception (74\%). However, many subjects also found eye fatigue $(68 \%)$ and nausea $(46 \%)$ to be unpleasant.

\begin{tabular}{|c|c|c|c|}
\hline & $\begin{array}{l}\text { 3Dto2D } \\
\operatorname{group}(\mathrm{n}=59)\end{array}$ & $\begin{array}{l}2 \text { Dto3D } \\
\operatorname{group}(n=59)\end{array}$ & \\
\hline $\begin{array}{l}\text { First trial: Number of } \\
\text { movements }\end{array}$ & 8.41 & 8.34 & $p=0.88$ \\
\hline $\begin{array}{l}\text { Second trial: Number of } \\
\text { movements }\end{array}$ & 9.59 & 10.32 & $p=0.19$ \\
\hline First trial: Number of drops & 0.64 & 0.75 & $p=0.56$ \\
\hline $\begin{array}{l}\text { Second trial: Number of } \\
\text { drops }\end{array}$ & 0.67 & 0.58 & $p=0.48$ \\
\hline
\end{tabular}

Conclusions: 3D laparoscopy did not yield significantly different results for laparoscopy naïve operators performing the peg transfer task compared to $2 \mathrm{D}$ laparoscopy. 3D endoscopy was considered to have superior image visibility and depth perception. However, many subjects also experienced eye fatigue and nausea. 


\section{P385}

\section{Applicability of Competency Based Medical Education in Kuwait's General Surgery Residency Program}

\section{Yousef Almuhanna, MD; University of Ottawa}

Kuwait's general surgery residency program is one of the oldest and most challenging training programs in Kuwait, running for over 25 years. The program had went through several evolutionary modifications throughout the years to match the dynamic changes that occur globally in the field. The program managed to establish residents committee where residents are actively involved in decision making of the program through their chief resident. Another major change was creating a position of site chiefs, where they can monitor and follow residents progress in each hospital. They are also taking an active role in relocating residents in case adequate exposure becomes a challenge. The program started by accepting 4 residents per year, however capacity went up to 105 years ago. On the other hand, Kuwait has 1 school of medicine, 1 university hospital, and 5 other general hospitals. Plastic surgery, transplant surgery, paediatric surgery, and neurosurgery have their own centres.

Kuwait Institution for Medical Specialization, the body that runs the program, have recently been accredited by the Royal College of Surgeons of Canada. The program was also affiliated by the American College of Surgeons though Kuwait Chapter. We aspire, by our solid and strong affiliations, to achieve international standards of training, and eventually, creating opportunity of international training.

Our focus is on the recently established surgical foundations body, that is run by a distinguished North American graduate qualified in medical education. The aim is to incorporate Competency Based Medical Education and the designated Entrustable Professional Activity in our training. Our vision entails certain exposures in certain hospitals: trauma, acute care, bariatrics, abdominal wall reconstruction, and hepatobiliary surgeries. Feedback will be bidirectional, to ensure the progress of both trainees and and supervisors. A logbook would be provided to each resident, and what we found is quite similar to the Joint Surgical Colleges Fellowship Examination. We aspire to issue the resident's wellbeing, by applying mentorship. Each and every resident will have a mentor, and that would help them get through to reach their target. Annual review courses and exams in associated with The American College of Surgeons In-training examination and Canadian Associated of General Surgery. We will also incorporate Fundamentals of Endoscopic Surgery, Fundamentals of Laparoscopic Surgery, Basic Endovascular Skills for Trauma, Advanced Trauma Operative Management, and Advanced Surgical Skill for Exposure in Trauma courses into our rapidly growing program.

\section{P386}

\section{Virtual Pointer as a Telementoring Guidance Aid for Laparoscopic Surgical Training}

Hannah Mcgowan ${ }^{1}$, Yuanyuan Feng, MS ${ }^{1}$, Azin Semsar, MS $^{1}$, Hamid Zahiri, $\mathrm{DO}^{2}$, Adrian Park, $\mathrm{MD}^{2}$, Andrea Kleinsmith, $\mathrm{PhD}^{1}$, Helena Mentis, $\mathrm{PhD}^{1}$; ${ }^{1}$ University of Maryland Baltimore County, ${ }^{2}$ Anne Arundel Medical Center

Introduction: Effective means of telecommunication is pertinent for the surgical field, where transfer of knowledge is complex, i.e. involves knowledge of procedure, anatomy and use of surgical instruments. We previously showed a Virtual Pointer system (VP) can aid in laparoscopic instructional learning in an onsite training situation. Here, we examine the effectiveness of using the VP in a telementoring setting, where guidance is provided remotely. Successful telementoring guidance may be beneficial for creating more realistic OR training settings.

Methods and Procedures: We performed a counterbalanced, within subjects controlled experiment, comparing VP guidance in a colocated condition with a telementoring condition. Trainees were eight surgical residents. Trainees performed a simulated laparoscopic task on a physical model under the guidance of one trainer (attending surgeon). Trainee performance questionnaires (Global Rating Scale instrument) and trainer/trainee cognitive load questionnaires (PAAS scale) were distributed to trainer and trainees. Scores were rank transformed using ARTool in R (version 3.5.1). A two-way ANOVA test was run to analyze effect of condition and run order on performance and cognitive load scores. A one-way ANOVA test was run to examine score differences within runs.

The VP uses the Microsoft Kinect system and allows for real-time video telestration, enabling a trainer to point or draw free handedly over live laparoscopic video. In this study, we defined VP guidance in a colocated setting as the control and VP guidance in a telementoring condition, through use of a divider, as the experimental condition (Figure 1). Results: There is no effect of condition (co-located or telementoring) or an interaction effect of condition and run order on trainer's perception of trainee performance and trainee/trainer cognitive load.

A significant effect of run order; however, illustrates increased perception of trainee performance as assessed by the trainer $(p=0.002)$ (Figure 2) and significantly decreased trainee cognitive load over the runs $(\mathrm{p}=0.00018$ ) (Figure 3 ).

Conclusion: Overall, there is no significant difference of performance or cognitive load scores between the co-located and telementoring conditions. A significant effect of run order on performance and cognitive load scores suggests that learning is observed in both conditions over time. From these results, we can conclude that use of the VP in the telementoring condition was as effective as in the co-located setting, which has previously been shown to increase trainee performance and understanding. Further studies are needed to objectively measure trainee's performance and cognitive load in telementoring training conditions.

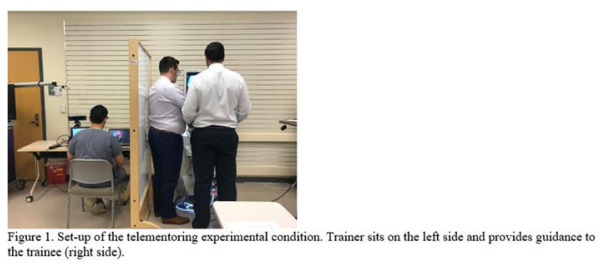

Mean of trainee performance scores as assessed by traine

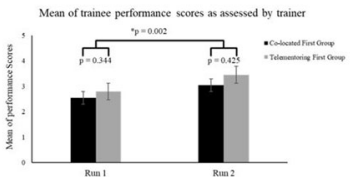

Figure 2. Comparison of effect of condition and run order on tramee performance as assessed by traine

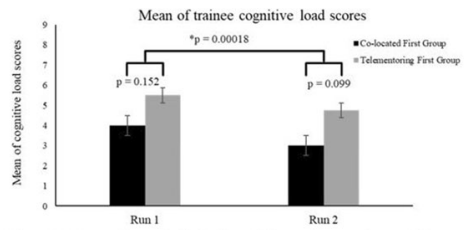

Figure 3. Comparison of effect of condition and run order on trainee cognitive load scores. 
P387

\section{Mobile App for Laparoscopic Surgery Training}

Juan Sanguinetti, $\mathrm{MD}^{1}$, Edgar Y Figueredo, $\mathrm{MD}^{2} ;{ }^{1}$ Maldonado Medical Asisstance, Cantegril Sanatorium, ${ }^{2}$ University of Washington

Purpose: To present a mobile App for tablets and mobile phones (Smart Phones) for training and teaching laparoscopic surgery in lowcost pelvitrainers.

For our simulator prototype we used 2 models, one of them a standard triangular prismatic prototype and a new model created by the research team which has only 2 boards (a base and a front or work board (Figures 1 and 2)); it was manufactured with MDF lacquered boards, in order to obtain a simulator by using less materials, making the simulator more economic, foldable and reproducible. Simulators are approximately $3 \mathrm{~kg}$, the angle between the front board and the base can vary from 90 to 70 degrees, the distance between ports and trocars varies from 14 to $24 \mathrm{~cm}$ and are adapted for simulating the distance of trocars in adult and pediatric surgery.

We intended to design a trainer accessible to the resident and the surgeon not only in the hospital or facility but also at home in order to extend training time. We should further improve the design and add elements in order to make different and varied exercises. The app accompanying this trainer is not only novel in our field but also there is no app on the website with the dynamics or features of the one developed by our work team, which could make it a fundamental tool created with a very low budget. These and other tools will surely change the training of residents in our environment, and the team is interested in these intervening in the training of colleagues from the region. We managed to make the simulators and the app in less than a year with an almost solo work, with no external financial support from private or public entities, only with the educational interest from the designer and contributors.

From the above it is concluded that with the necessity of ex vivo training, in order to reduce complications inherent to the learning curve of each surgeon, it is necessary to develop low-cost simulators for laparoscopic surgery in our environment. It was possible to develop a mobile app for laparoscopic surgery training. The next objective is to demonstrate, by a prospective, longitudinal and comparative study, the method's usefulness and applicability, in order to standardize its use both in Uruguay and Argentina.

\section{P388}

Laparoscopic Liver Surgery Training Course on Thiel Embalmed Bodies: Program Evaluation, Trainer's Long Term Feedback and Steps Forward

Nikdokht Rashidian, $\mathrm{MD}^{1}$, Mariano Giglio, $\mathrm{MD}^{2}$, Vincenzo Scuderi, $\mathrm{MD}^{1}$, Francesca Tozzi, MD ${ }^{1}$, Aude Vanlander, MD ${ }^{1}$, Katharina D'herde, $\mathrm{MD}, \mathrm{PhD}^{3}$, Adnan Alseidi ${ }^{4}$, Wouter Willaert, $\mathrm{MD}, \mathrm{PhD}^{5}$, Roberto Ivan Troisi, $\overline{\mathrm{MD}}, \mathrm{PhD}^{2}$; ${ }^{\mathrm{I}}$ Department of General, HepatoBiliary and Liver Transplantation Surgery, Ghent University Hospital Medical School, Belgium, ${ }^{2}$ Department of Clinical Medicine and Surgery Federico II University, Naples, Italy, ${ }^{3}$ Department of Anatomy, Embryology, Histology and Medical Physics, Ghent University Hospital Medical School, Belgium, ${ }^{4}$ Division of Pancreas, Liver and Biliary Surgery. Virginia Mason Medical Center, Seattle USA, ${ }^{5}$ Division of Gastrointestinal Surgery, Ghent University Hospital Medical School, Belgium

Background: The purpose of this study was to evaluate the feedback of participants upon hands-on laparoscopic liver surgery course on Thiel human bodies and define ways to improve this type of training. Moreover, we attempted to identify the obstacles to implement laparoscopic liver surgery.

Methods: From 2010 to 2017, 10 laparoscopic liver surgery masterclasses have been organized in a hepatobiliary department. During the first day, state of art lectures, technical tips and different topics in laparoscopic liver surgery were given and debated. Live streaming interventions were planned and discussed interactively in the boardroom. Additionally, a video session was carefully prepared to show different approaches to the liver for different indications. On the second day, the participants were able to develop their skills with a hands-on training on Thiel human bodies under the proctorship of international experts. A 23-question anonymous survey was electronically sent to 119 participants between November 2017 and January 2018, exploring their characteristics and asking for evaluation of the course.

Result: Sixty-four surgeons (53.8\%) responded to the survey; 42 $(65.6 \%)$ were employed at a university hospital; $39(60.9 \%)$ were in the first decade of their practice as a consultant surgeon. Forty-three $(67.2 \%)$ surgeons reported an increased percentage of LLS cases afterward. Training on Thiel cadavers was considered superior (49.2\%) to other training options including proctoring in operating room $(34.9 \%)$, simulators $(6.3 \%)$, video training $(4.8 \%)$ and training on pigs $(4.8 \%)$. Obstacles identified contained inadequate training, patient's referral pattern, financial issues, lack of dedicated surgical team and time constrains.

Discussion: This survey revealed that a structured short-time program incorporating interactive discussion, live operations and hands-on training on bodies under proctorship may enhance efficient training in laparoscopic liver surgery and offer an effective solution to implement the limits of the training, and eventually promotes its distribution. Practicing on Thiel human bodies seems to be a promising option amongst all available educational models and could be improved by using re-perfused Thiel human bodies. In a step forward for upcoming courses the importance of team building has to be addressed. 


\section{P389}

Evaluating Resident Performance and Training Curve in Laparoscopic Inguinal Hernia Totally Extraperitoneal Repair with a Procedure Specific Checklist

Christian Massier, William Walsh, DO, Jillian Sinopoli, DO;

Background: Laparoscopic inguinal hernia extraperitoneal repair is a difficult task to learn. The number of cases required to become proficient during surgical training is not known. This study aimed to assess the reliability of a procedure specific scoring systems, to compare resident performance along training years and construct personalized feedback of operative skill to trainee. Methods: Resident operative performance was recorded prospectively and analyzed in relationship to their prior experience in laparoscopic hernia repair and other complex laparoscopic procedures as well as anatomical difficulty of the patient. Performance (amount of attending assistance) and duration was recorded using procedure-specific checklist (access, cord dissection, hernia sac reduction, mesh placement).

Results: The study included 12 residents in a general surgery training program and 100 procedures performed over 18 months. A strong positive correlation was found between prior resident experience and each of the surgical steps. Mesh placement scores are stronger correlated with experience and difficulty than other procedural steps. Operative times are inverse correlated with experience. Performance correlates negatively with anatomical difficulty. Conclusion: Progress is quick only for port placement. Proficiency can be achieved after 10 cases for easy hernias but may take more then 30 cases for difficult hernias. Learning curve mirrors number of prior complex laparoscopic cases. Individual resident performance over time can be analyzed and personalized feedback constructed to guide further training needs.

\section{P390}

\section{Computer-Assisted Navigation for Laparoscopic HBP Surgery}

Masaharu Ishida, Takanori Morikawa, Tatsuo Hata, Masahiro Iseki, Tatsuyuki Takadate, Kyohei Ariake, Kei Kawaguchi, Kunihiro Masuda, Takeshi Aoki, Masamichi Mizuma, Ohtsuka Hideo, Hiroki Hayashi, Kei Nakagawa, Fuyuhiko Motoi, Takashi Kamei, Takeshi Naitoh, Michiaki Unno; Tohoku University

Introduction: Due to the absence of tactile sensation, it is difficult to recognize target structures that cannot be seen under laparoscopy. We are developing intraoperative navigation to obtain good orientation by applying preoperative images and real-time operation images. Here, we show our achievement of intraoperative navigation for laparoscopic hepatobiliary pancreatic surgery.

Methods: For preoperative simulation, an accurate 3D image is created from preoperative. In our department, in all cases of laparoscopic hepatectomy and pancreatectomy. For intraoperative navigation, the 3D image from preoperative $\mathrm{CT}$ is superposed on the actual surgical monitor of laparoscopic surgery in real time. The 3D images overlaid on the surgical image can be deformed according to the position of the camera and the movement of the targeted organ.

Results: By using the preoperative simulations, we can understand anatomical and positional relationships of each case preoperatively. Real-time intraoperative navigation can make it possible to recognize the positions of vessels and tumors difficult to observe from the surface.

Discussion: In laparoscopic surgery, by intervening in the monitor image, intraoperative navigation, that is difficult in open surgery, becomes possible. Because the positional error is not small, it is actually applied for surgical education for practitioners. As IT technology advances, intraoperative navigation will become a useful tool for improving endoscopic surgical techniques and surgical outcomes.

\section{P391}

Improving Operative Room Performance in Laparoscopic Surgery of Surgical Residents via Short Term Focused Training A Quality Improvement Study

Chaithra L, Virinder Kumar Bansal, Asuri Krishna, Om Prakash, H. K. Bhattacharjee, M. C. Misra; AIIMS, New Delhi

Introduction: Focused training outside OR has been considered an important tool for quality improvement of surgical resident performance. However whether it translates into a significant contributor is yet to be ascertained. There are presently limited randomized trials to objectively assess the effectiveness of focused training outside the OR in laparoscopy of surgical residents and their operative performance.

Materials and Methods: The study was a single blinded double armed randomized control study conducted between July 2016to March 2018. Eighteen fourth and fifth semester students of surgical residency were included in the study. The baseline performance was assessed in OR by performing laparoscopic cholecystectomy by validated scores i.e. GOALS, additional 5 criteria, task specific check list, error checklist, Visual analogue score, then the residents were randomized into Trained $(n=9)$ and Control group $(n=9)$.Training group received a 5 days short term focused training on Teubingen trainer with integrated porcine model and the improvement was reassessed in OR after 4 weeks.

Results: Demographic profile and baseline score were comparable. The training group showed statistical significant GOALS $(9.88 \pm 1.76$ to $12 \pm 0.66, \quad \mathrm{p}$ value- 0.05$)$,task specific checklist $(42.22 \pm 10.92$ to $53.33 \pm 14.14$, p-value 0.027 ), error checklist. The operating time significantly reduced $(\mathrm{p}$ value $=0.0025)$.VAS was suggestive of similar level of difficulty.

Conclusion: Our study concludes that the training on the Tuebingen Trainer with integrated porcine organs results in a statistically significant improvement in the operating room performance of surgical residents as compared to the non trained residents thereby indicating a transfer of skills from training to operative room. 
P392

\section{A Single-Blinded Randomized Control Study to Assess the Predictive Validity of Robotic Skills Simulation}

Daniel Cheng, MD, MPH, Christopher Mcnicoll, MD, MPH, Chad Cross, PhD, PStat, Heidi Ryan, MD, Shawn Tsuda, MD, Jennifer Baynosa, MD, Charles St. Hill, MD, MSc; University of Nevada, Las Vegas School of Medicine

Introduction: Current literature lacks a detailed assessment of robotic simulation in residency. We conducted a study to assess the effect of robotic simulation on resident performance.

Methods: A single-blinded randomized control study was conducted with 30 residents. All surgeries performed were robotic transabdominal pre-peritoneal inguinal hernia repairs. Subjects were timed and scored on key steps, errors, and complications. Subjects completed a baseline Surgery 1, and were randomized to Control $(n=4)$ and Advanced Training (AT) $(n=4)$ groups. The AT group trained using the simulator until proficiency. Subjects then completed Surgery 2 and Surgery 3. Performance was compared between the Control and AT groups using two-sample t-tests. Performance was compared within the groups, from Surgery 1 to Surgery 2, using paired samples t-tests. Owing to small sample size, we calculated exact p-values and bootstrapped confidence intervals.

Results: Control and AT groups had similar baseline training and performance in Surgery 1. AT group had improved performance after simulator training, taking less time and committing less errors. The difference in performance was largest in Surgery 2, and significant for suturing of the peritoneum $(\mathrm{p}=0.030)$. Exact p-values identified the AT group was significantly faster at mesh placement in Surgery $2(\mathrm{p}=0.031)$. From Surgery 1 to Surgery 2 , the AT group performed mesh placement faster $(p=0.025)$. Bootstrapped confidence intervals identified the AT group was significantly faster at mesh placement ($8.50,-3.25)$ and peritoneum suturing $(-22.00,-2.25)$ from Surgery 1 to Surgery 2. Errors committed and complications were not significantly different.

Conclusion: Although sample size makes it difficult to draw definitive conclusions, the data suggests robotic skills simulation accelerates proficiency. Given it has no additional risk to patients, simulation should be included in all curriculums that teach robotic surgery.
P393 A Novel Use of Multi-perspective Two-Dimensional Versus Single
Perspective Two-Dimensional View in Laparoscopy

Zachary Sanford, $\mathrm{MD}^{1}$, Robert Turner, $\mathrm{PhD}^{1}$, Ivan George ${ }^{1}$, Alex Addo, MD ${ }^{1}$, Helena Mentis, $\mathrm{PhD}^{2}$, Reza Zahiri, $\mathrm{DO}^{1}$, Adam S Weltz, $\mathrm{MD}^{1}$, Adrian Park ${ }^{1} ;{ }^{1}$ Anne Arundel Medical Center, ${ }^{2}$ University of Maryland, Baltimore County

Introduction: Traditional laparoscopy uses a single-camera to provide solitary 2D-perspectives of a 3D-operative space, limiting vital depth cues and spatial awareness while potentially increasing mental workload and operative error. We present a novel study to characterize the impact of multiple simultaneous 2D-visual-perspectives on surgeons' cognitive and physical workload in laparoscopy.

Methods: The effect of visualization on subjective visual comfort, mental workload, task proficiency, and ergonomic risk was prospectively investigated. Surgeons completed fundamental laparoscopic skills zip tie (ZT), pattern cut (PC), intracorporeal suture (IS) in single versus multi-perspective visualization. Baseline studies were conducted using configurations reflecting current laparoscopic displays while experimental design included three distinct and perpendicular 2D-visualizations (tip-of-the-scope, aerial, lateral perspectives). The NASA-TLX questionnaires was used to elicit subjective mental workload, visual comfort, and body pain/fatigue. Oculometry data were also recorded.

Results: Nine surgeons familiar with laparoscopy were recruited. No significant mental workload increase was noted from use of multiple versus single visual perspectives. Reductions in perceived mental and physical demand as well as effort and frustration were observed during the zip tie task $(\mathrm{p}<.05)$. Oculometry data suggest participants chiefly utilized tip-of-the-scope view (65\% ZT, 72\% PC, 74\% IS) while periodically scanning the aerial perspective (25\% ZT, $22 \%$ PC, $23 \%$ IS) and rarely incorporating the lateral perspective. Qualitative feedback indicates strong preference for the multi-perspective visual setup, especially for added aerial view.

Conclusions: Our findings demonstrate surgeons may benefit from multi-perspective visualization during laparoscopy. Additional work is required to explore potential benefits of multi-perspective visualization in optimizing movements and awareness in 3D-space.
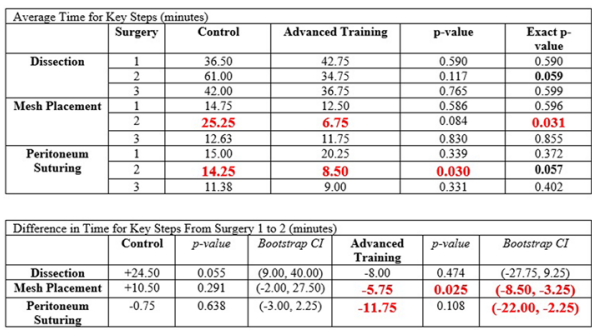

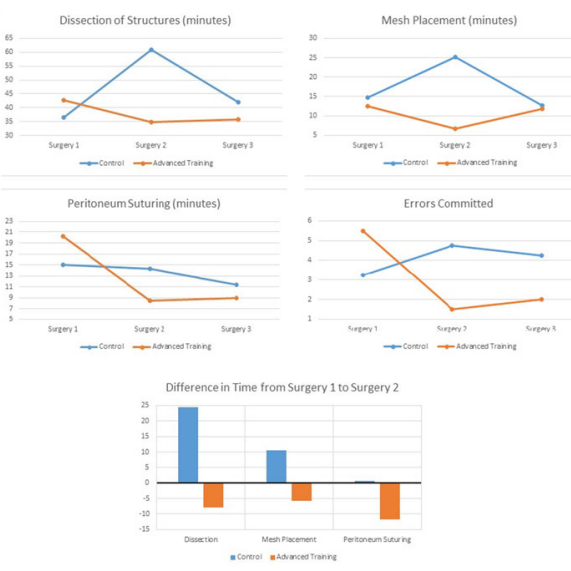




\section{P394}

\section{Identifying the Further Learning Objectives for the Safe and Effective Use of Ultrasonic Energy Devices: FUSE Certified Surgeons' Perspective}

Yuichi Nishihara, $\mathrm{MD}^{1}$, Yusuke Watanabe, $\mathrm{MD}^{2}$, Takahiro Homma ${ }^{3}$, Toshiro Tanioka, $\mathrm{MD}^{4}$, Yasuhito Tanase, $\mathrm{MD}^{5}$, Tomokazu Kishiki, $\mathrm{MD}^{6}$; ${ }^{1}$ Department of Surgery, National Hospital Organization Tokyo Medical Center, ${ }^{2}$ Department of Gastroenterological Surgery II, Hokkaido University Faculty of Medicine, ${ }^{3}$ Department of General Thoracic and Cardiovascular Surgery, University of Toyama, ${ }^{4}$ Department of Gastric Surgery, Tokyo Medical and Dental University, ${ }^{5}$ Department of Obstetrics and Gynecology, Nara Medical University, ${ }^{6}$ Department of Surgery, Kyorin University School of Medicine

Introduction: Ultrasonic transection and coagulation devices (USD) are commonly used in open and minimally invasive procedures after electrosurgical instruments. There may be latent contents for using USDs that are not taught in the current Fundamental Use of Surgical Energy (FUSE) program. The objective of this study was to identify the further learning objectives for the safe and effective use of USDs. Methods: A qualitative methodology using a focus group of FUSE certificated surgeons was used to gain an in-depth understanding of the safe and effective use of USDs. The focus group session was conducted under the condition that surgeons were able to use USDs with a piece of flank steak as hands-on. The session was recorded and transcribed. After comparing FUSE contents, learning objectives were generated from the recorded data of the focus group. Experimental and clinical scenarios based on findings identified were corrected.

Results: A total of 6 FUSE certificated surgeons were participated (4 GI surgeons, 1 thoracic surgeon, and $1 \mathrm{GYN}$ ). The following learning themes were generated from the data: the detailed explanation of cavitation, the tissue injury mechanisms that are different from cavitation, the difference in lateral thermal effect based on philosophy between USDs and vessel sealing devices, the characteristics of blade temperature among devices and temperature trends during activation, the difference in blade temperature based on different techniques (fine, piecemeal, bit by bit cutting technique increases blade temperature significantly and rapidly compared to full-length, chunk cutting), difference in vessel sealing between arteries and veins, and the unknown possibility of tumor cell dissemination. Some experimental and clinical scenarios regarding findings identified will be demonstrated.

Conclusions: Several specific matters for the safe and effective use of USD were identified. Some themes that emerged can be used to focus research in areas to advance in-depth understanding. The learning objectives identified can be used to enrich the existing FUSE program

\section{P396}

\section{Improving Voluntary Robotic Simulator Use Through Competition}

James D Roy, MD, John D Hunter, MD, Seth-Hill, BS, MS, Joseph E Stahl, BS, Paul F Rider, MD, William O Richards, MD; University of South Alabama

Introduction: The aim of this study is to examine if a robotic surgery simulation competition, incentivized by prizes, increases voluntary utilization of a robotic simulator. Virtual reality surgical simulation is a validated method for training residents and surgeons on robotic platforms. Additionally, robotic virtual reality simulation training has been shown to improve basic skillsets and lead to improved operative skills. A major hurdle to the use of simulation in resident training is voluntary utilization by trainees.

Methods and Procedures: This is a single center, IRB exempt, retrospective cohort study performed from 12/19/17 until 6/21/18. Open access to the da Vinci $\mathrm{Xi}^{\circledR}$ surgeon console and Mimic Simulation ${ }^{\circledR}$ $\mathrm{dV}$-Trainer ${ }^{\circledR}$ software was afforded to residents beginning on $12 / 19$ / 17. A robotics competition was announced on 4/20/2018 and held on 6/21/18.

Prizes for winners of each PGY class and an overall winner were announced, but specific prizes were not revealed until after the competition concluded. A total of 19 residents met inclusion criteria. The competition included three simulated tasks. Technical skill outcomes for each of the three tasks were quantified with an overall score. Technical proficiency was measured by time to complete, economy of motion, instrument collisions, excessive instrument force, instruments out of view, and workspace range.

Overall simulator use was compared for all residents before and after announcement of the competition using the Wilcoxon Sum Rank tests. Correlation of simulator use with overall score and technical proficiency was analyzed using Spearman's Rho. Comparison of specific technical proficiency measures between the top, middle and bottom scoring competition participants were analyzed using the Kruskal-Wallis tests. Statistical analyses were performed using IBM SPSS Statistics version 25.

Results: Following announcement of the competition and prizes, voluntary robotic simulator use increased by $132 \%(\mathrm{p}=0.008)$. There were 8 novel users following announcement of the competition. Total completed attempts correlated significantly with overall competition score $(\mathrm{p}<0.001)$. Total attempts in the study period also positively correlated with improved economy of motion $(\mathrm{p}=0.039)$, decreased time of excessive force ( $\mathrm{p}=0.038)$, and decreased instrument collisions $(\mathrm{p}=0.013)$. Among our grouped skill categories, average time to complete exercises $(\mathrm{p}=0.023)$, average economy of motion $(p=0.031)$, and number of instrument collisions $(p=0.002)$ significantly varied between the top, middle, and bottom performing groups.

Conclusions: Competition along with prizes significantly increases voluntary use of robotic simulators among residents. Additionally, increased utilization of robotic simulators leads to improved technical performance among surgical trainees. 


\section{P397}

\section{Training Effects of Visual Stroboscopic Impairment on Surgical} Performance: A Randomized-Controlled

Dmitry Zavlin, $\mathrm{MD}^{1,2}$, Vishwanath Chegireddy, $\mathrm{MD}^{1}$, John J Nguyen-Lee, $\mathrm{MD}^{3}$, Linden Shih, $\mathrm{BS}^{1}$, Anna M Nia, MS $\bar{S}^{4}$, Jeffrey D Friedman, MD, FACS ${ }^{1}$, Anthony Echo, MD ${ }^{1}$; Institute for Reconstructive Surgery, Houston Methodist Hospital, ${ }^{2}$ Department of Surgery, Easton Hospital, ${ }^{3}$ Department of Surgery, Houston Methodist Hospital, ${ }^{4}$ School of Medicine, University of Texas Medical Branch

Background: There have been numerous advances to accelerate and improve quality of surgical training to meet the growing US demand of graduating surgeons. In this randomized-controlled study, the authors investigated the learning effects of limited visual input with stroboscopic glasses on surgical proficiency in untrained novice surgeons.

Methods: Medical students were randomized into either the experimental group wearing stroboscopic eyewear $(n=11)$ or the reference group $(n=11)$. For five weeks, the subjects were scored during standardized surgical tasks from the ACS/APDS Resident Skills Curriculum: knot tying, simple interrupted sutures, and a running stitch. In addition, we preoperatively employed the State-Trait Anxiety Inventory and postoperatively, the NASA Task Load Index

Results: The demographic characteristics of our study participants were uniformly distributed: each group had 7 males and 4 females. Average ages were 23.6 and 24.2 years $(p=0.471)$. The anxiety was low during all five sessions and indifferent between both groups. At the end of the study, no changes were observed in the stroboscopic group for the knot tying task $(p=0.619)$. However, for the simple interrupted and the running stitch, the students with stroboscopic glasses performed significantly better $(p=0.001$ and $p=0.024$, respectively). The stroboscopic students also had significantly lower workload scores $(\mathrm{p}=0.001)$.

Conclusion: Regular training with stroboscopic glasses has a significant positive effect on the technical skills of novice surgical trainees with regards to more complex tasks such as multiple simple interrupted suturing or running suture. Intermittently impaired vision is beneficial in the early education of surgical students and residents.
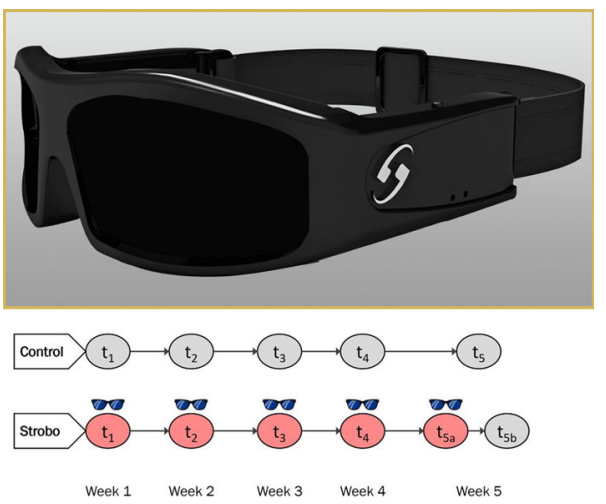

\section{P399}

Motion Analysis of General Surgery Residents Performing the Laparoscopic Peg Transfer Task and the Effect of a Secondary Task

Dustin Baker, MD ${ }^{1}$, Jacquelyn Pastewski, MD ${ }^{1}$, Amy Somerset, $\mathrm{MD}^{1}$, Kelsey Leonard ${ }^{2}$, Victoria Roach, $\mathrm{PhD}^{2}$, Kathryn Ziegler, $\mathrm{MD}^{1}$, Pavan Brahmamdam, MD ${ }^{1}$; ${ }^{1}$ Beaumont Health, Department of Surgery, Royal Oak, MI, USA, ${ }^{2}$ Oakland University William Beaumont School of Medicine, Rochester, MI, USA

Introduction: Using motion analysis, we aim to identify differences between junior and senior residents performing the laparoscopic peg transfer task, and the impact of a distracting secondary task on performance. Motion analysis is able to discriminate between expert and novice laparoscopic surgeons, but has not been used to illustrate motion patterns unique to surgical residents at different stages of training.

Methods: General Surgery residents at our institution were asked to perform the laparoscopic peg transfer task on a simulator equipped with a motion tracker. They were also asked to perform the simulated task while completing a secondary task (simple verbal arithmetic). The velocity and acceleration of movement in three degrees of freedom - pitch, yaw, and roll - were measured during task completion. The number of extreme events, defined as velocity or acceleration exceeding $1 \mathrm{SD}$ above or below the mean, were tabulated. The performance of junior residents (PGY 1-2, $\mathrm{n}=14$ ) was compared to senior residents (PGY 3-6, $\mathrm{n}=23$ ) using student's t-test. SPSS 25 (IBM) was used for all statistics.

Results: Average task completion time was equivalent between the groups (junior: 84.5 vs. senior: $71.1 \mathrm{~s}, \mathrm{p}=0.1$ ). With the addition of the secondary task, the average task completion time in the junior group significantly improved (84.5 vs. $75.2 \mathrm{~s}, \mathrm{p}=0.04)$ but was not significantly different from the senior group, $(75.2$ vs. $68.3 \mathrm{~s}, \mathrm{p}=0.31)$. The junior group answered more arithmetic questions $(57.90$ vs. $46.0, \mathrm{p}=.03)$ with greater accuracy $(57.6 \%$ vs. $45.8 \%, \mathrm{p}=.03)$ than the senior group.

In the motion analysis of peg transfer task alone, the senior group executed significantly fewer extreme motion events specific to acceleration in pitch, (16.63 vs. $20.69, \mathrm{p}=0.04$ ). The senior group was found to execute more extreme motion events specific to velocity in roll, $(16.14 \mathrm{vs.} 15.11, \mathrm{p}=0.038)$. In the secondary task condition, the senior group had fewer extreme acceleration events specific to pitch, $(14.69$ vs. $22.22, \mathrm{p}<.001)$. There were no differences within groups regarding number of extreme events for each motion parameter with addition of a secondary task.

Conclusion: Motion analysis identified that senior residents execute fewer extreme acceleration events specific to pitch, which corresponds to motion in the vertical plane. This was true even when a secondary task was added. This suggests that senior residents execute movements more smoothly during peg transfer, relative to junior residents, even when task completion time is equivalent. Motion analysis may prove useful for real-time feedback during laparoscopic skill acquisition. 
P400

Development of A Physics-based Interactive Virtual Endotracheal Intubation (ETI) Simulator

Zhaohui Xia, $\mathrm{PhD}^{1}$, Hong $\mathrm{Li}^{1}$, Nicholas Milef ${ }^{1}$, Adam Ryason ${ }^{1}$, Daniel Jones, $\mathrm{MD}^{2}$, Suvranu De, $\mathrm{PhD}^{1}$, Stephanie Jones, $\mathrm{MD}^{2}$; ${ }^{1}$ Center for Modeling, Simulation and Imaging in Medicine (CeMSIM), Rensselaer Polytechnic Institute, Troy, NY, ${ }^{2}$ Beth Israel Deaconess Medical Center, Harvard Medical School, Boston, MA

Introduction: Endotracheal intubation (ETI) is one of the most frequently performed procedures in operating rooms (OR), intensive care units (ICUs) and emergency departments (ED). Failure to intubate may result in severe morbidity and mortality. The traditional training methods, e.g., plastic mannequins or patient-based training, limit the opportunity for residents to deliberately practice this skill in varied patient anatomical and clinical conditions. Therefore, a virtual reality (VR)-based ETI trainer with visual and haptic feedback as well as skill assessment is highly desired.

Objectives: The goal of this study is to develop a high-fidelity physics-based anatomically accurate virtual airway skill trainer for ETI, which aims to provide trainees real time visual and haptic feedback, allowing them to attain competence in a controlled environment with no risk to patients.

Methods: There are four modules involved in the virtual ETI simulator: (1) real time rendering; (2) haptic interface; (3) physics-based simulation; and (4) performance recording and assessment metrics. Real time rendering allows the training tasks of ETI to be performed in a high-fidelity 3D virtual environment. Haptic feedback mechanisms provide the interaction forces, while physics-based simulation algorithms enable the complicated simulation for tissue deformation, collision detection and tool-tissue interactions. Meanwhile, the ETI simulator collects the trainees' performance data to provide feedback. Results: Four tasks including positioning the patient, insertion of the direct laryngoscopy blade into the oropharynx, achieving the optimal laryngeal view, and inserting the endotracheal tube are designed for the training of ETI procedures with two haptic tools (one for the laryngoscopy blade and the other for the endotracheal tube). The first task aims to tilt and elevate the patient's head for proper positioning. The second task involves inserting the laryngoscope into the mouth and interacting with the vallecula, avoiding collision with the teeth. The goal of the third task is obtaining a clear view of the vocal cords and epiglottis. The fourth task is to insert the endotracheal tube, while avoiding contact with the surrounding anatomy. Experiments on performing the ETI tasks show that the simulator is able to run at interactive rates.

Conclusions: A novel high-fidelity physics-based virtual ETI simulator has been developed. Our simulator can provide interactive training experience and collect useful performance information which can be further analyzed for evaluation purpose.

\section{P402}

Ontology as a Tool for Computerized Evaluation of Surgical Skills and Procedures in Endoscopic Surgery: A Feasibility Study

Kazuhiko Shinohara, MD, PhD; School of Health Sciences, Tokyo University of Technology

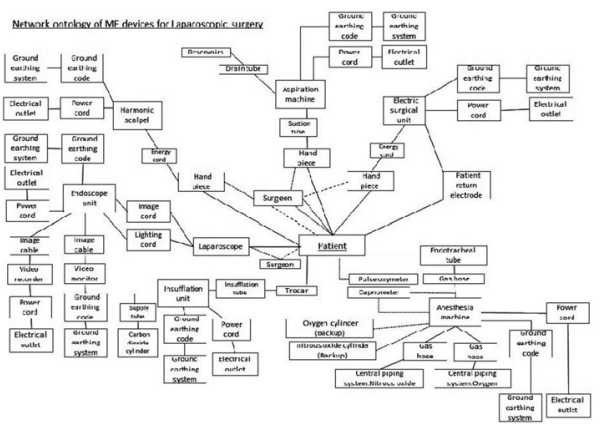

Background and Objectives: Artificial intelligence (AI) has recently been receiving increasing attention in the medical field. Ontology is defined as explicit formal specification of terms or concepts in a domain and relations among them. The successful introduction of AI to the medical field requires ontological and semantic descriptions of clinical procedures. This study suggests a method of ontological analysis for endoscopic surgery and investigates the feasibility and challenges of applying an ontological approach to surgery.

Materials and Methods: We analyzed the surgeon's technique during basic training in the box trainer, such as pegboard transfer, cutting pattern, and suturing, and described these as an ontological procedure. In addition, the network and connectivity between medical electronic (ME) devices, such as the electric surgical unit, endoscope unit, and anesthesia machine were ontologically investigated and described.

Results: Surgical maneuvers in the box trainer for endoscopic surgery were successfully classified and described with reference to the relevant ontological concepts. Ontological descriptions of the connection status of the patient, ME devices, and energy supply outlets were successfully designated.

Conclusion: Ontological descriptions of surgical procedures and the network of ME devices for endoscopic surgery are possible. Ontological descriptions of the procedures in endoscopic surgery can be applied in other areas, such as computerized evaluation of medical training and certification, navigation and automation systems for robotic surgery, and medical alert systems for patient safety. This study revealed the need for simple and practical methods of clinical ontology. 


\section{P403}

\section{Validation OF A Virtual Cricothyrodotomy Simulator VAST-} CCT

Di Qi, $\mathrm{PhD}^{1}$, Uwe Kruger, EngD ${ }^{1}$, Nicholas Milef, $\mathrm{BS}^{1}$, Mohamad R Abu-Nuwar, $\mathrm{MD}^{2}$, Mohamad I Haque, $\mathrm{MD}^{3}$, Robert Lim, $\mathrm{MD}^{4}$, Daniel B Jones, $\mathrm{MD}^{2}$, Melih Turkseven, $\mathrm{PhD}^{1}$, Emil Petrusa, $\mathrm{PhD}^{5}$, Doga Demirel, $\mathrm{MSc}^{6}$, Tansel Halic, $\mathrm{PhD}^{7}$, Suvranu De, $\mathrm{ScD}^{1}$, Noelle Saillant, MD ${ }^{5}$; ${ }^{1}$ Rensselaer Polytechnic Institute, ${ }^{2}$ Beth Israel

Deaconess Medical Center, ${ }^{3}$ Anderson Simulation Center, Madigan Army Medical Center, ${ }^{4}$ Tripler Army Medical

Center, ${ }^{5}$ Massachusetts General Hospital, ${ }^{6}$ University of Arkansas at Little Rock, ${ }^{7}$ University of Central Arkansas

Introduction: Cricothyroidotomy is a low-frequency yet high-risk emergency procedure conducted to secure a patient's airway. Traditional training methods offer limited practice opportunities and lack objective and automated assessment. Virtual reality simulation possesses great potential as an alternate training tool overcoming these limitations. A virtual airway skills trainer (VAST) for cricothyroidotomy (CCT), VAST-CCT, was developed to simulate the major steps of CCT. This study aims to assess the face and construct validity of the VAST-CCT simulator.

Methods and Procedures: 47 clinicians were recruited at the 2018 annual conference of the Society of American Gastrointestinal and Endoscopic Surgeons and assigned to one of two groups according to their levels of expertise in CCT: expert $(n=9)$ and novice $(n=38)$. The participants performed four critical steps in cricothyroidotomy on the simulator including 1) identifying landmarks, 2) making incision on the skin, 3) puncturing and dilating the cricothyroid membrane, and 4) intubation. Participants' performance was evaluated based on the overall completion time (seconds) as well as task completeness and accuracy quantified with an overall score, referred to as performance score.

All data (performance scores and completion time) was examined for normality using the Anderson-Darling test. The standard F test is utilized to assess the equality of variances between the novice and expert data. A 2-sample $\mathrm{T}$ test (with an equal variance assumption) was used to compare the performance scores between the expert and novice groups, as each sample was drawn from a normal distribution. Due to the non-normal distribution of the completion time, and the data distributions of the expert and novice groups had the same shape (examined using the Kolmogorov-Smirnov test), a Mann-Whitney U test was utilized to compare the completion time between these two groups.

Participants were also asked to comment on the realism of the simulator by answering 9 questions on a five-point Likert-type scale.
Results: Statistical analysis results showed that the experienced surgeons performed significantly better $(\mathrm{p}=0.014)$ and faster $(p=0.007)$ than the novice group. $75 \%$ of the participants reported that the simulator was useful (score of at least 4 out of 5) for training cricothyroidotomy surgical skills and hand-eye coordination ability. Conclusion: The VAST-CCT simulator showed face and construct validity and thus constitutes a promising tool for training in cricothyroidotomy. Virtual training of such rare and emergent procedure may help ensure competency in a critical skill when limited opportunities exist clinically.

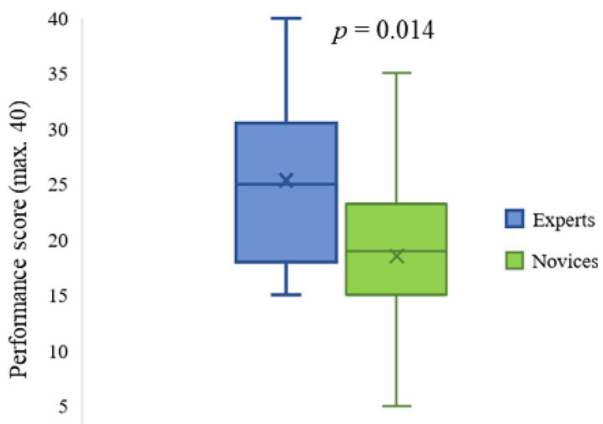

Fig.1: Experts vs. Novces - Performance score

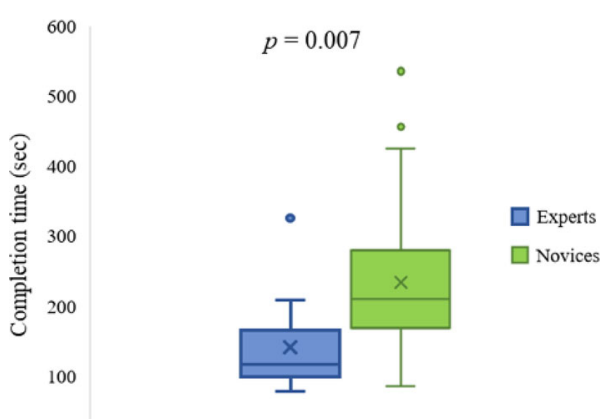

Fig.2: Experts vs. Novces - Completion time 


\section{P404}

Development of an Electric Fire for In-Situ Operating Room Fire Team Training: An Experimental Study

Yusuke Watanabe, $\mathrm{MD}^{1}$, Chisato Ichimaru, $\mathrm{MD}^{2}$, Kozue Satou, $\mathrm{RN}^{2}$, Yo Kurashima $^{1}$, Toshiaki Shichinohe, MD ${ }^{1}$, Satoshi Hirano, $\mathrm{MD}^{1}$; ${ }^{1}$ Department of Gastroenterological Surgery II, Hokkaido University Faculty of Medicine, ${ }^{2}$ Teine Keijinkai Medical Center

Introduction: Operating Room (OR) fire is a rare but critical adverse event. OR teams must be able to manage and protect the patient in the case of a fire. Current simulations for OR fire use a manikin model with smoke only or virtual reality. The purpose of this study was to examine the face validity of an electric fire that can be used for in situ OR fire interdisciplinary simulation training.

Methods: We developed a safe, easy to set-up electric fire which is designed to be used with a manikin model. This simulated electric fire does not create toxic smoke and there are no associated safety risks, such as carbon monoxide poisoning. After explaining the scenario of OR fire simulation, OR personnel from a teaching hospital were asked to observe an electric fire using a patient manikin model in the OR. Participants were asked about their agreement with the following statements for the electric fire, using a 10-point Likert scale: the reality of the electric fire, the usefulness of an electric fire for OR fire training, the additional value of an electric fire to simulation reality, and the need for simulation training in OR fire. Participants were also asked to complete a questionnaire including demographics and experience with OR fire. Data are expressed as $\mathrm{N}(\%)$ and median [IQR].

Results: A total of 15 OR personnel (12 OR nurses, 2 surgeons, and 1 anesthesiologist) participated (median years of experience in the OR 3 [2-8]; 53\% female). Of participants, 3 (20\%) experienced an OR fire in their practice. None of the participants have experienced any training regarding OR fire. On a scale of $1-10$, with 10 indicating strongly agree, participants rated reality of the electric fire (6[4-8]), the usefulness of an electric fire for OR fire training (10 [7$10])$, additional value of an electric fire to simulation reality (8 [8$10])$, and the need for simulation training in OR fire (10 [10-10]).

Conclusions: The face validity of an electric fire for OR fire team training was successfully established. In-situ OR fire team simulation with an electric fire may contribute to OR personnel's better preparedness for preventing and managing OR fires.

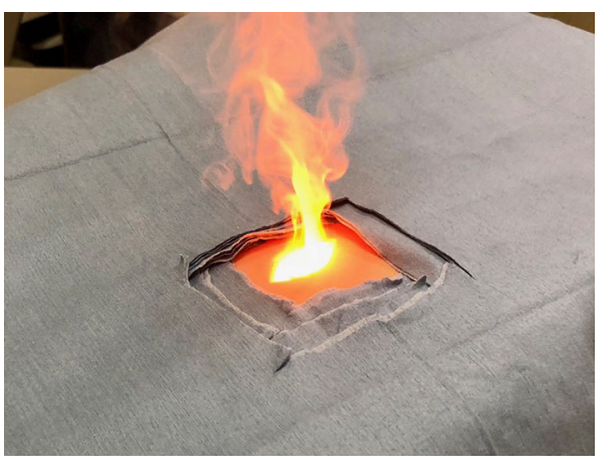

\section{P405}

Evaluating The Interrater Reliability of the Basic Endoscopic Skills Training (BEST) Box in Live And Delayed Video Settings

Ethan Weiss ${ }^{1}$, Silvana Perretta, $\mathrm{MD}^{2}$, Ilay Habaz, $\mathrm{BSc}^{1}$, Dennis Tchoudnovski ${ }^{1}$, Pietro Mascagni, $\mathrm{MD}^{2}$, Ludovica Guerriero, $\mathrm{MD}^{2}$, Else Van Der Velden ${ }^{2}$, Fabio Longo, MD $^{2}$, Lee L Swanstrom, MD², Allan Okrainec, $\mathrm{MD}^{1}$, Eran Shlomovitz, $\mathrm{MD}^{1}$; ${ }^{1}$ Division of General Surgery, University Health Network, Toronto, Canada, ${ }^{2}$ Institute for Image Guided Surgery IHU-Strasbourg, Strasbourg, France

Objective: The increasing use of flexible endoscopy as a minimally invasive tool has generated demand for a model which can easily train users on basic flexible endoscopic skills. The Basic Endoscopic Skills Training (BEST) box has been validated as a low-cost flexible endoscopy trainer. The BEST box consists of a conversion kit to the Fundamentals of Laparoscopic Surgery (FLS) box whereby 6 different flexible endoscopy skills are simulated. This pilot study aims to investigate the inter-rater reliability (IRR) as well as the consistency between live and delayed video scoring.

Methods: 40 participants were rated by several live proctors (L) and on video by two trained, remote proctors (V1 and V2). All proctors used the same previously validated BEST box scoring formula. To assess inter-rater reliability, interclass correlation coefficients (ICC) with absolute agreement and two-way mixed models were used. ICC values $>0.9$ and $>0.8$ suggest excellent and great reliability, respectively.

Results: Inter-rater reliability between the delayed video raters, as well as between the delayed video raters and the live raters is excellent (Table 1). ICCs remain consistently high when separately analysing the individual tasks including absolute time and errors.

Conclusion: The scoring system for the BEST box demonstrates high inter-rater reliability and remains consistent both when scored live as well as over video. The results of this study add further evidence to the validity of the BEST box as an objective tool for endoscopic evaluation. The ability for accurate video scoring adds future possibilities for remote scoring and telementoring.

Table 1. Interclass correlation coefficients for inter-rater reliability of BEST scores.

\begin{tabular}{ll}
\hline Two or three raters & ICC $(\mathbf{9 5 \%}$ CI $)$ \\
\hline V1 vs V2 $(n=39)$ & $0.96^{* * *}(0.93-0.98)$ \\
V1 vs L $(n=36)$ & $0.96^{* * *}(0.91-0.98)$ \\
V2 vs L $(n=37)$ & $0.93^{* * *}(0.86-0.96)$ \\
V1 vs V2 vs L $(n=36)$ & $0.97 * * *(0.94-0.98)$
\end{tabular}

Note: $* * * \mathrm{p}<.001$ 
P406

\section{Evaluation of Surgical Skills in New Developed VR Simulator for Laparoscopic Sigma Resection}

Koji Ikeda, Nobuyoshi Takeshita, Koichi Teramura, Masaaki Ito; National Cancer Center East

Background: While conventional VR simulators have been useful to learn the surgery process seeing surgical anatomy, dissection procedures performed in real surgery could not be reproduced in these VR systems. We developed a new model of VR simulator, Lap-PASS LP-100, in collaboration with a company. This includes the similar human plane structure in which laparoscopic trainings for dissection and exposure are possible

Objective: The objective of this study is to evaluate whether surgical performance tried in this VR can reflect actual surgical skills.

Methods: Fifty subjects including 44 surgeons and 6 medical non-professional were asked to undergo a surgical procedure in a certain step of laparoscopic sigma resection in VR and the procedures were scored. In the step, they dissected the meso-sigmoid colon three times to be scored. Two expert endoscopic surgeons having case experiences of more than 100 laparoscopic surgery, evaluated their surgical procedures performed in the VR simulator in a blind manner, using GOALS, global operative assessment of laparoscopic skills. This includes five evaluationitems as follows; 1 . Depth perception, 2. Bimanual dexterity, 3.Efficiency and 4. Tissue handling. Each item counts for 5 points, and so the total score is 20 points.

Results: Among fifty subjects, 11 were colorectal surgeons and 18 had surgical experiences with more than 100 laparoscopic surgeries. Total score evaluated in surgeons with more than 100 experiences of laparoscopic surgery was significantly superior to the others $(15.0 \pm 0.86$ vs $10.3 \pm 2.08, \mathrm{p}=0.032)$. Especially, two items of Bimanual dexterity $(\mathrm{p}=0.0022)$ and Efficiency $(\mathrm{p}=0.0045)$ in GOALS were found to be the great difference between two groups.

Conclusion: The current VR simulator succeeded in distinguishing between expert surgery and others. This can include real surgical plane anatomy in laparoscopic colectomy, which can be the effective tools leading to actual exercise for dissection skills in laparoscopic surgery.

\section{P407}

Competence in Laparoscopic Cholecystectomy, Implementation of an Assessment Tool in The fellowship Program

Mickael Chevallay, Emilie Liot, MD, Ian Fournier, MD, Monika Hagen, MD, Stefan Mönig, Professor, Philippe Morel, Professor, Christian Toso, Professor, Frederic Ris, Professor, Minoa Jung, PD, MD; Geneva university hospital

Introduction: To achieve proficiency in performing a surgical procedure is a milestone in the career of a trainee. Objective measures to assess the surgical skills are emphasized in the medical education. We suggest an objective assessment tool which was based on a competency assessment tool developed for colorectal surgery and adapted for the standardized procedure of laparoscopic cholecystectomy (LCAT)(Figure 1). We aimed to assess the feasibility of the implementation of the LCAT and to evaluate this tool by comparison of intraoperative assessors scores with scores submitted by independent experts after reviews of video records

Methods: Laparoscopic cholecystectomy was divided into 4 steps (exposition, calot dissection, cholangiography, resection). Steps were subdivided in 4 task including instrument use, tissue handling, execution and end result. Scoring system went from 1 to 4 with 4 representing the best performance. For general assessment, we added visual analog scale (VAS) and objective structured assessment of technical skills (OSATS) and an overall autonomy score. We included laparoscopic cholecystectomies which were performed by residents under the supervision of board-certified surgeons from 2013 to 2015 . Every procedure was assessed by means of the LCAT filled at the end of the procedure by the supervising surgeon (live). Video records of the same procedures were then anonymized and analyzed by two independent reviewers (reviewer A and B) which were blinded from the performing trainees and patients identity. Scores of assessments were compared between the supervising surgeons and the respective reviewers $\mathrm{A}$ and $\mathrm{B}$ and between the two reviewers $\mathrm{A}$ and $\mathrm{B}$.

Results: We identified 42 cholecystectomies performed by 21 trainees which were assessed by 15 surgeons. Scoring results were comparable between the live assessor and Reviewer A with a mean value for exposition of 3.21(SD $0.46) v s$ 3.23(0.43), Calot dissection:3.20(0.42)vs3.0(0.46), cholangiography $3.26(0.53) v s 3.23(0.52)$, gallbladder resection 3.18(0.49)vs3.19(0.45), OSATS3.77(0.65)vs3.75(0.73), VAS5.29(0.80)vs5.4(0.73), autonomy $5.17(1.17) v s 5.19(1.44)$. Scores between the live reviewer and reviewer B were comparable except for gallbladder resection $(3.18(0.49) \mathrm{vs} 2.89(0.63), \mathrm{p}=0.01)$ and VAS(5.29(0.8)vs4.64(1.19), $\mathrm{p}=0.01)$.

Comparison of scores of reviewer A and reviewer B showed significantly different means in gallbladder resection $(3.19(0.55) \mathrm{vs} 2.89(0.63), \mathrm{p}=0.004)$ and $\operatorname{VAS}(5.45(0.73) \mathrm{vs} 4.64(1.19), \mathrm{p}=0.004)$, whereas the other procedural steps as well as OSATS and overall autonomy scored comparably.

Conclusion: Implementation of a competency assessment tool for evaluation of surgical skills during laparoscopic cholecystectomy is feasible. Comparable scoring between live assessors and blinded reviewers may demonstrate the objectivity of this tool to measure trainee performance. General assessment showed significant interrater differences and might be less suitable for objective evaluation.

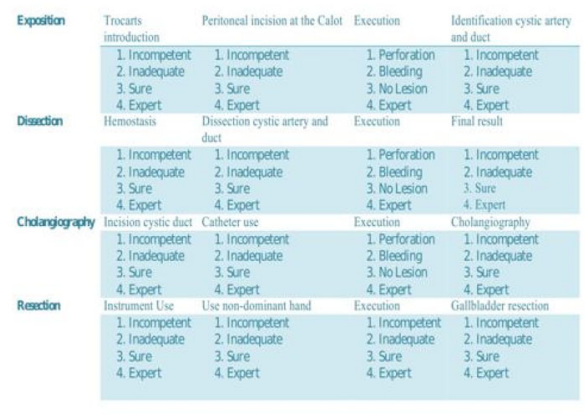

Figure 1. Laparoscopic competency assessment tool for laparosescopic cholecystectomy. 


\section{P408}

\section{The Effect of Laparoscopic Task Workshops on Prefrontal Cortex Activity in Medical Students: A Randomised Controlled Pilot Study Using Functional Near-Infrared Spectroscopy}

Sujith Wijerathne ${ }^{1}$, Howard Khoe ${ }^{2}$, Jun Wei Low ${ }^{2}$, Jackie $\mathrm{Ho}^{1}$, Raquel Maia ${ }^{1}$, Hrishikesh Salgaonkar ${ }^{1}$, Davide Lomanto ${ }^{1}$, Roger $\mathrm{Ho}^{1} ;{ }^{1}$ National University Hospital, Singapore, ${ }^{2}$ National University of Singapore

Introduction: Neurobiological feedback in surgical training could translate to better educational outcomes such as measures of learning curve. The variation in brain activation of medical students when performing laparoscopic tasks before and after a training workshop is not properly studied before and we planned to do this using functional near infrared spectroscopy (fNIRS). The fNIRS is a non-invasive optical brain imaging tool that measures cortical oxygenation change which is used as a marker of pre-frontal cortex activity (PFCA).

Methods and Procedures: This randomised controlled trial examined the PFC activity differences in two groups of novice medical students during the acquisition of 4 basic laparoscopic tasks. Both groups were shown an instructional video of the tasks and the "Trained-group" underwent an additional standardised one-to-one training. The PFCA was measured pre and post intervention using a portable fNIRS device. Primary outcome was the difference in the PFCA pre and post intervention. Secondary outcomes were the differences in PFCA between the 4 tasks and between the sexes.

Results: 16 trained and 16 untrained medical students with an equal sex distribution and a comparable age distribution were involved in the study. All students were right handed. Trained group had a significantly attenuated PFCA in the "Precision-cutting" $(p=0.011)$ and "Suture-insertion" $(p=0.025)$ tasks compared to the untrained group. Subgroup analysis based on sex revealed significant attenuation in PFCA in trained females compared to untrained females across 3 of the 4 laparoscopic tasks: "Pegs-transfer" $(\mathrm{p}=0.013)$, "Precision-cutting" $(\mathrm{p}=0.034)$, "Suture-insertion" $(\mathrm{p}=0.03)$. No significant PFCA attenuation was found in male students who underwent training compared to untrained males.

Conclusion: A standardised laparoscopic training workshop promoted greater PFCA attenuation in female medical students compared to males. This suggests that female and male students respond differently to the same instructional approach. These results may have implications for surgical training and education such as a greater focus on one to one surgical training for female students and use of PFCA attenuation as a form of neurobiological feedback as a measure of learning curve in surgical training.

\section{P409}

\section{Make Your Own Laparoscopic Simulator' Results from a Competition Held for Surgical Residents in Surgicon}

Samiullah Bhatti, Dr ${ }^{1}$, Awais A Malik, Dr ${ }^{2}$, Shabbar H Changezi, Dr ${ }^{1}$, Usman I Butt, Dr ${ }^{1}$, Waris Farooka, $\mathrm{PhD}^{1}$, Mahmood Ayyaz, Prof ${ }^{1}$; ${ }^{1}$ Services Hospital Lahore, ${ }^{2}$ Lahore General Hospital

Introduction: Laparoscopic surgery is the cornerstone of modern surgical practices. Young trainees, who are trained on simulators, can achieve basic laparoscopic skills that are comparable to those achieved after traditional training in the operating room. These simulators are very expensive and are not affordable by residents in a low economic country like Pakistan.

Methods and Procedures: A competition was held at a surgical conference to allow for residents to come up with ideas to design their own simulators. A team of consultants visited and tested each simulator for uniqueness, looks, effectiveness, cost and reproducibility.

Results: 97 residents from various hospitals participated in the competition. A total number of 11 simulators were made by all the residents. Simulator named "Galilleo" won the 1st prize, while simulator named "I-Simulator" and "periscope" won the second and third prize respectively.

Conclusion: Medical competitions are a healthy activity which can probe residents to think out of the way and come up with ideas which can benefit themselves as well as others.

\section{P410}

\section{Assessing the Educational Value of Online Pediatric Surgical Videos}

James M Prieto, $\mathrm{MD}^{1}$, Alicia Gaidry, $\mathrm{MD}^{1}$, Karen Kling, $\mathrm{MD}^{2}$, Ivan M Gutierrez, MD ${ }^{1}$, Donald J Lucas, MD, $\mathrm{MPH}^{1}$, Robert Ricca, $\mathrm{MD}^{3}$, Stephen Bickler, $\mathrm{MD}^{2}$, Romeo C Ignacio, $\mathrm{MD}^{1}$; ${ }^{1} \mathrm{Naval}$ Medical Center San Diego, ${ }^{2}$ Rady Children's Hospital San Diego, ${ }^{3}$ Naval Medical Center Portsmouth

Introduction: Recent studies indicate that surgical residents commonly use online videos when preparing for operative cases. Among the many resources available, YouTube is the most frequently used. The objective of this study was to evaluate the educational value of online videos of commonly performed pediatric surgical procedures.

Methods: A YouTube search was performed for "Laparoscopic Pyloromyotomy" and "Thoracoscopic Congenital Diaphragmatic Hernia (CDH) Repair". Videos were filtered in order of their number of views and screened to exclude any irrelevant content. The five with the highest number of views were selected for each operation. Videos were graded on their technical proficiency and educational value using the Global Operative Assessment of Laparoscopic Skills (GOALS) score, and the "Educational Value Score" (EVS). The EVS is operation specific, and was created using the key steps of each procedure as outlined by the SAGES Manual of Minimally Invasive Pediatric Surgery. Videos were rated by six pediatric surgeons and eight general surgery residents who have completed a pediatric surgery rotation as a senior resident. Statistical analysis was performed using the t-test and ANOVA.

Results: The mean educational value score of pyloromyotomy videos was significantly higher than that of thoracoscopic $\mathrm{CDH}$ repair videos (26 vs 21 , $\mathrm{p}<0.001)$. Technical proficiency was also rated significantly higher in the pyloromyotomy category $(16.5 \mathrm{vs} 13.2, \mathrm{p}=0.01)$. In both operations, the video with the highest number of views had a significantly higher educational value score as compared with the video with the least views ( 25.5 vs $16.5, p=0.004$; 23.6 vs $15.6, p=0.01)$. There was no statistically significant difference in the way residents and pediatric surgeons scored the videos. Commonly highlighted deficiencies included lack of any audio or text explanations $(50 \%)$, or failure to discuss any of the operation's common pitfalls $(70 \%)$.

Conclusion: There is a high degree of variability in the quality of online videos of pediatric surgical procedures. Based on our preliminary analysis, videos of more technically challenging procedures (i.e. thoracoscopic $\mathrm{CDH}$ repair), are likely to be of less quality and educational value. Videos with higher view counts may be more likely to be of higher educational value. A structured format to include audio narration, demonstration of key steps of each procedure, and an explanation of the common pitfalls could enhance the educational value of all surgical videos. A centralized online repository where residents could access videos of common pediatric surgical procedures would be a valuable teaching tool. 
P411

Skill Transference and Learning Curves Between Robotic and Laparoscopic Platforms: Does the Initial Learning Platform Impact Skills Acquisition?

Hannah Harris ${ }^{1}$, Kristen Quinn, $\mathrm{MD}^{2}$, Grace $\mathrm{Chen}^{1}$, Kayla Diaz ${ }^{1}$, Alan Harzman, $\mathrm{MD}^{1}$, Xiaodong (phoenix) Chen, $\mathrm{PhD}^{1}$, Syed Husain, $\mathrm{MD}^{1}$; ${ }^{1}$ Ohio State University, ${ }^{2}$ Medical University of South Carolina

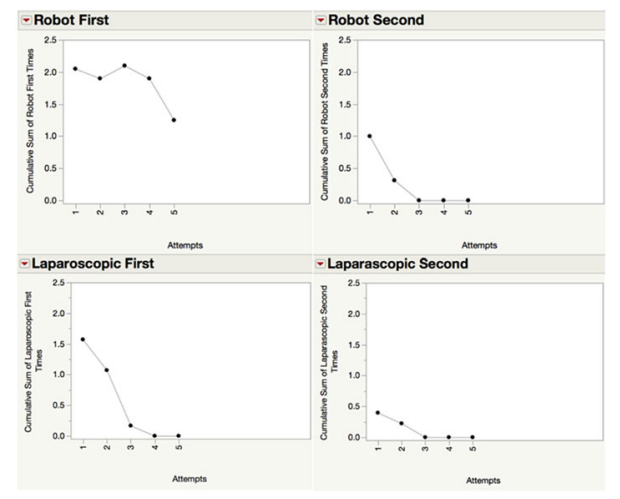

Introduction: This is the first phase of a project sponsored by a SAGES robotic surgery grant. While there has been increased adoption of the robotic platform in general surgery over the last few years, there is no consensus on how the introduction of this new modality impacts resident education. Our previous work has indicated that exposure to the robotic platform may interfere with laparoscopic skill acquisition in naïve operators (medical students)

We hypothesized that similar effects would be identified in surgical residents.

Methods and Procedures: Twelve general surgery interns were randomized into two groups. Group 1 performed five repetitions of the peg-transfer task using the robot first followed by performance of same tasks using the laparoscopic trainer. Group 2 performed the same tasks in reverse order. Participants were timed and errors were recorded. Transference of skill was measured by comparing the improvement in baseline performance levels between first and second attempt for each modality. Learning curves were calculated as the number of attempts required to reach best performance level (plateau) for each modality. Accuracy was calculated by the number of drops during the peg transfer tasks. A one-sided CUSUM analysis was used to evaluate the learning curves and Wilcoxon SignedRank Test was used to compare the times to task completion between the groups.

Results: Task completion was consistently faster $(\mathrm{p}<0.05)$ and more accurate $(13$ vs. 73 drops) with the robot compared to laparoscopy. There appeared to be transference of skill between both modalities. However, a more robust improvement was noted in the baseline skill level in the group that was exposed to laparoscopy first followed by robotic tasks (25.79\%) compared to the group with exposure to robotic tasks before laparoscopy $(16.37 \%)$.

Conclusions: These results are consistent with our previous research in that introduction to laparoscopic technology before robot improves acquisition of skill using the robotic platform. Introduction to robotic platform before exposure to laparoscopy might result in a decreased training efficiency of students' robotic training, as the robot-first group's learning curve did not reach a plateau. The phase 2 of this project investigating this effect in senior residents is currently under way and will be reported separately.

\section{P412}

Publication Patterns and the Impact of Self-citation Among General Surgery Residency Programs

Benjamin C Clark, MD ${ }^{1}$, Robert J Conrad, MD ${ }^{1}$, Dylan M Russell, $\mathrm{MD}^{1}$, Fadi M Balla, MD, $\mathrm{FACS}^{2}$, Robert B Lim, MD, FACS ${ }^{1}$, Dwight C Kellicut, MD, FACS ${ }^{1}$, Christopher G Yheulon, MD, FACS ${ }^{1} ;{ }^{1}$ Tripler Army Medical Center, ${ }^{2}$ Emory University MIS Fellowship

Introduction: For the few scientists who receive a Noble prize, the impact and relevance of their research is unquestionable. For the rest of us, how does one quantify the impact and relevance of an individual's research? The Hirsch Index (H-Index) is a widely utilized academic metric that measures both productivity and citation impact. In general, a higher $\mathrm{H}$-Index signifies more impactful publications. Both citation and self-citation affect H-Index. The purpose of this study is to define the publication patterns and the impact of selfcitation among professors in general surgery residency programs.

Methods: Through the Association of Program Directors in Surgery website, 65 surgical residency programs were randomly selected from the 259 listed. A list of Professors was obtained from these programs. Publication patterns of each professor were analyzed. Using the Scopus database, we calculated the number of publications, citations, self-citations, and $\mathrm{H}$-Index for each surgeon. We determined the number of faculty in which the H-Index changed due to self-citation and the magnitude of those changes.

Results: A total of 685 Professors of Surgery were identified. The mean number \pm SD of publications, citations, and H-Index for the cohort were 81.4 $\pm 93.5,2752 \pm 4935$, and $20.5 \pm 17.3$, respectively. The self-citation rate for the entire cohort was $4.06 \%$. Excluding self-citations reduces the mean number of citations to $2640 \pm 4604$ and H-Index to $20.1 \pm 17.0$. The H-Index remained unchanged for $72 \%$ (496/685) of surgeons. Only $2.9 \%$ (20/685) had a change in H-Index of greater than two integers.

Conclusion: Self-Citation is infrequent $(4.06 \%)$ and has a minimal impact (average $\mathrm{H}$-Index change of 0.3 ) on the academic profile of professors in general surgery residency programs.

\section{P413}

Examination of the VR + AR Training for Understanding of Regional Anatomy in the Laparoscopic Training of the Students

Takashi Iwata, $\mathrm{MD}, \mathrm{PhD}^{1}$, Kozo Yoshikawa, $\mathrm{MD}, \mathrm{PhD}^{2}$, Jun Higashijima, $\mathrm{MD}, \mathrm{PhD}^{2}$, Tomohiko Miyatani, $\mathrm{MD}, \mathrm{PhD}^{2}$, Takuya Tokunaga, $\mathrm{MD}, \mathrm{PhD}^{2}$, Masaaki $\mathrm{Nishi}^{2}$, Hideya Kashihara ${ }^{2}$, Chie Takasu ${ }^{2}$, Mitsuo Shimada ${ }^{2}$; ${ }^{1}$ Research Center for Education of Health Bioscience, Tokushima University Graduate School, ${ }^{2}$ Department of Digestive Surgery, Tokushima University Graduate School

Background: The qualitative evaluation for laparoscopic training of medical students was performed using Rubric evaluation, and weak points in conjunction with the lack of anatomical knowledge were derived. To conquer these weak points, virtual reality (VR) + augmented reality (AR) training for understanding of regional anatomy was investigated.

Materials and Methods: One hundred and six students in 5th grade of Tokushima Univ participated basic laparoscopic task training (gummy band ligation, beads transfer, delivery of beads, gauze excision) with training box and sham laparoscopic cholecystectomy with virtual simulator. Rubric evaluation, as qualitative evaluation, which includes the evaluation standards for each maneuver were performed before and after basic task training and sham operation. The group which self-evaluation was higher in a Rubric evaluation was investigated.

The 3D image of vessels and bile duct obtained from MDCT of real patient was projected in reality space with Microsoft HoloLens. Training of AR image using HoloLens was performed for understanding of regional anatomy. After training with HoloLens, rubric evaluation was performed in sham operation again.

Results: Rubric evaluation in basic task training showed no difference between self-evaluation and evaluation by tutor before and after practice. In sham laparoscopic cholecystectomy, several students showed higher score than tutor, especially in part of extension of operation field by elevation of the Gall bladder, exposure of triangle of Calot, and exposure of cystic duct.

After AR training, all students showed high score in questions related regional anatomy during operation. Especially, rubric evaluation of students who showed high self-evaluation in sham operation showed same score with tutor.

Conclusions: As Rubric evaluation showed weak points of detailed parts of maneuver, and $\mathrm{VR}+\mathrm{AR}$ was useful for understanding details of regional anatomy for laparoscopic training. 


\section{P414}

Advanced Endoscopic Polypectomy Skills Acquisition Via a Novel Bench-Top Training Program

Yohei Kono $^{1}$, Kota Momose ${ }^{1}$, Talal Alzghari ${ }^{1}$, Reem Alharbi ${ }^{2}$, Patrick Dolan ${ }^{1}$, Jackly Juprasert ${ }^{1}$, Russell Corwin ${ }^{1}$, Andrew Harvey ${ }^{1}$, Takahiro Hiratsuka ${ }^{3}$, Jeffrey Milsom ${ }^{1}$, Sam K Sharma ${ }^{1}$; ${ }^{1}$ Weill

Cornell Medicine, ${ }^{2}$ Princess Nourah bint Abdulrahman

University, ${ }^{3}$ Oita University

Introduction: Hypothesis - to determine whether a bench-top combination training model and program could lead to the acquisition of advanced ESD skills in a group of trainees of varying endoscopic experience.

Complex endoscopic skills needed for procedures such as ESD require stepwise training methods to build confidence and neuromuscular pathways ('muscle-memory'). The lack of suitable Western training models/methods may partly account for ESD's relatively low uptake. In Japan, ESD training follows a program of increased difficulty leading to operator competence. We aimed to simulate some of these core principles in our model and training program.

Methods: We constructed a 3-D printed abdomino-pelvic housing into which we attached silicone inserts to mimic the abdominal wall. Fresh porcine distal colon was mounted within the housing to give an accurate human anatomical representation. $3 \mathrm{~cm}$ diameter pseudopolyps were marked on the mucosal surface using electrocautery. 2 trainees of varying endoscopic experience but no ESD experience participated (Trainee A $>1000$ endoscopies, Trainee $\mathrm{B}=0$ endoscopies). Parameters measured were; total procedure time, negative horizontal margin rate and perforation rate. The training program consisted 30 rectal ESD's followed by 6 procedures at different proximal anatomical locations (descending $(n=6)$, transverse $(n=6)$ and $\operatorname{cecum}(n=6))$ to incrementally increase difficulty and build on previously acquired skills. All procedures were video recorded and feedback given to the trainee post-procedure. All procedures were performed using standard endoscopic equipment

Results: 2 trainees performed a total of 96 ESD procedures. All procedures were completed.

Trainee A $(\mathrm{n}=48)$ : Total mean procedure time was $30.0 \pm 10.1$ (mean $\pm \mathrm{SD}$ ) min. No perforations were observed. Regression analysis showed decreasing trend at each anatomical location (Rectum: $\mathrm{R}^{2}=0.42$, Descending colon: $\mathrm{R}^{2}=0.81$, Transverse colon: $\mathrm{R}^{2}=0.48$, Cecum: $\left.\mathrm{R}^{2}=0.49\right)$.

Trainee B $(n=48)$ : (no colonoscopy experience). Total mean procedure time was $28.8 \pm 12.7$ (mean $\pm \mathrm{SD}$ ) min. Perforation rate was $6.3 \%$ (3/48). Regression analysis showed decreasing trend over time at the rectum, transverse colon and cecum (Rectum: $R^{2}=0.46$, Transverse colon: $\mathrm{R}^{2}=0.96$, Cecum: $\mathrm{R}^{2}=0.89$ ).

Learning curve: Total procedure time decreased significantly from the first half of the training program to last (Trainee A: $p<0.001$, Trainee B: $p=0.003$ ).

Conclusions: The training model and program led to the acquisition of advanced endoscopic skills in both trainees. Previous endoscopic experience did not affect skill acquisition. Such a training program should be considered for all clinicians undertaking ESD as part of clinical practice.
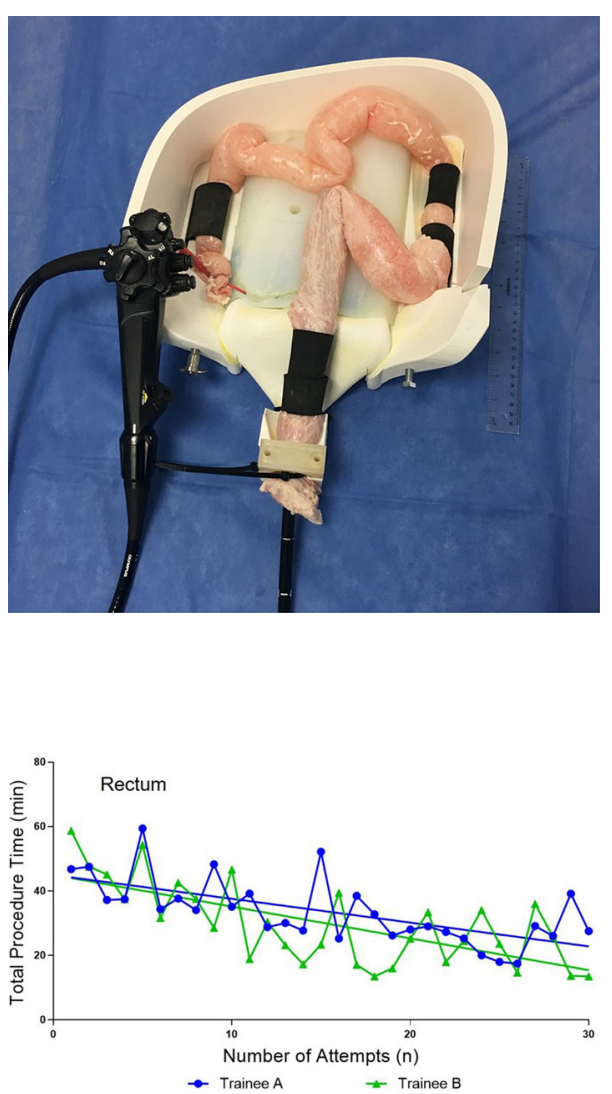

- Trainee A $A$ Trainee B

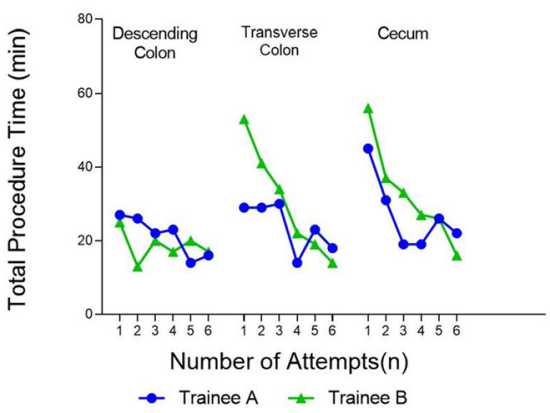




\section{P415}

Technique of Esophagojejunostomy Using OrVil After Laparoscopy Assisted Total Gastrectomy(LATG) for Gastric Cancer

S Sakuramoto, $\mathrm{PhD}, \mathrm{H}$ Sugita, $\mathrm{PhD}, \mathrm{K}$ Watanabe, MD, S Ishii, $\mathrm{PhD}, \mathrm{S}$ Itou, MD, N Fujiwara, PhD, Y Miyawaki, PhD, H Sato, PhD, S Yamaguchi, PhD, I Koyama, PhD; Saitama Medical University, International Medical Center

Introduction: During esophagojejunostomy using a circular stapler after LATG, placement of the anvil head via the transabdominal approach proved difficult. The authors report on a method modified for laparoscopy-assisted, esophagojejunostomy performed by placing the pretilted anvil head(OrVil) via the transoral approach.

Methods and Procedures: Between January 2013 and August 2018, esophagojejunostomy was performed using OrVil in 98 patients after LATG. The anesthesiologist introduced the anvil while observing its passage through the pharynx. During the anastomosis, we kept the jejunum fixed in position with a silicone band Lig-A-Loops, thereby preventing the intestine from slipping off the shaft of the stapler.

Results: Esophagojejunostomy using the OrVil was achieved successfully in all patients. No other complications, such as hypopharyngeal perforation and/or esophageal mucosal injury, occurred during passage. The postoperative complications of anastomosis were leakage in two patients and stenosis in 5 patients, in whom mild relief was achieved using a bougie.

Conclusion: Esophagojejunostomy using the OrVil is a simple and safe technique.

\section{P416}

The VITO (pn 20150100457, 2015) Novel Training Kit to Limit Down the Learning Curve of Upper GI Endoscopy: Initial Evaluation

Konstantinos S Mammas, Adamantia S Mamma; Program of Excellence-Siemens program 2014-16

Aim: To evaluate the VITO (pn 20150100457, 2015) training kit learning curve in terms of the so called "machine learning curve".

Materials and methods: The novel training kit was used in combination with the necessary set of devices for upper GI endoscopy i.e. Gastroscopy or ERCP (the central unit, the monitor and the flexible endoscope $=$ Gastroscope or Duodenoscope respectively to the procedure) by a beginner trainee in flexible endoscopy, but specialist surgeon who had never applied any type of upper GI endoscopy in the past in a period of six months. During the experimental period the trainee assisted in upper GI clinical endoscopy sessions on human subjects without having the right to perform any by him-self. Referring to VITO based Gastroscopy (initiation and retrieval of the Endoscope) training, the trainee's trials consisted of fourteen series $(\mathrm{N}=14)$ of ten $(\mathrm{n} 1=10)$ kit based Gastroscopies sets of ten trials $(\mathrm{n} 2=10)$ for each series in each session on several time points in a period of six months. The same took place for VITO based ERCP training with a Duodenoscope (initiation and retrieval of the Duodenoscope) in the same training session, counting eleven series $(\mathrm{N}=11)$ of ten $(\mathrm{n} 1=10)$ kit based ERCPs sets of ten trials $(\mathrm{n} 2=10)$ for each series in each session. The metric that used for learning was the mean time for finishing the task in terms of the analysis of the mean time of each series spent in each training kit based set: i.Diagnostic gastroscopy session (to enter, to retrieve and the total) and ii.Diagnostic ERCP session (to enter, to retrieve and the total), to compute the sequential training session after which the mean time spent for training was no statistically different than the previous training sessions (ANOVA, SPSS 17.0).

Results: I. Gastroscopy: Progressively, after the 8th series, which took place after a month of training no statistically significant difference was noticed among sessions $(20,7 \pm 1,8, p=0.663)$. The trainee managed to perform Gastroscopy on a patient after a month. II. ERCP: Progressively, after the 6th series, which took place also after a month of training no statistically significant difference was noticed among sessions $(17,7 \pm 1,5 \mathrm{~s}, \mathrm{p}<0,110)$. The trainee managed to perform a clinical diagnostic ERCP on a patient after six months.

Conclusions: The VITO training kit seems to be feasible and reliable for surgeons in training. Further evaluation combined with the design's modification for a self-centered or collaborative learning in Gastroscopy, ERCP and EUS, is being developed. 


\section{P417}

Novel Robotic Transanal Approach to Early Rectal Neoplasia: A Single Surgeon's Experience with the Medrobotics Flex*TM Colorectal Drive

Ada E Graham, MD, Natalie Pudalov, Vincent Obias, MD; George Washington University Hospital

Introduction: Treatment for early rectal cancer has evolved from the total mesorectal excision with data to support local excision. The nonlinear anatomy of the rectum and distal colon make surgical access and visualization of these regions challenging given the linear nature of most instrumentation used. We present our experience and results from the first 11 human subjects who have undergone surgery for early rectal neoplasia with a new robotics surgical system: the Medrobotics FlexTM Colorectal Drive, which uses a transanal approach with fully articulating instruments to address pathology in the distal colon and rectum. We aimed to assess the safety and feasibility of this new platform in this retrospective review.

Methods and Procedures: Preoperatively, patients were selected based on favorable polyp and lesion characteristics to demonstrate the feasibility and safety of this transanal approach. In this retrospective review of a single surgeon's database, 11 patients were deemed appropriate candidates. Patient age, sex, operative time, estimated blood loss, post-operative complications and margins were recorded.

Results: Polyps were removed at distances 2 to $17 \mathrm{~cm}$ from the anal verge, and as large as $8 \mathrm{~cm}$, without complications. One case involving a GIST tumor had a positive deep margin, which was resected with a deeper biopsy to achieve negative margins. A case concerning a single tumor at $12 \mathrm{~cm}$ was unable to be resected and required operative conversion, thought to be a combination of surgeon learning curve and sub-optimal patient positioning. Average operative time was $110 \pm 39.9 \mathrm{~min}$, average blood loss $9.1 \pm 13.6 \mathrm{cc}$. All patients were discharged on the day of surgery without post-operative complications.

Conclusions: This is the first data on a new transanal, endoluminal robotic platform that can maintain pneumorectum, the Medrobotics FlexTM Colorectal Drive, which was demonstrated to be safe and clinically feasible for the removal of early anorectal neoplasia.

\section{P418}

\section{The Endoluminal Treatment of Tumor Obstruction of UGI}

Yerlan Abdirashev, MD, Nurken Abdiyev, Sholpan Izmagambetova, MD, Amir Uzhakhov, MD; JSC "National Scientific center of surgery named after A.N. Syzganov's"

Aims: The severe and complicated malignant stenosis of UGI show up a difficult problem. The purpose is retrospective analysis of SEMS inserting to UGI cancer obstruction.

Methods: From 2014 to 2018 we analyzed of 69 cases of inoperable cancer esophagus and GOO. 53 cases (77.3\%) of stenosis caused by esophageal cancer (EC) and EJ, $16(22.7 \%)$ patients had malignant GOO. The AC of esophagus and gastric was diagnosed in $51(73.5 \%)$ cases, SCC of esophagus in $8(5.6 \%)$ patients, $1(0.7 \%)$ patient had metastatic lymph node compression of middle part of esophagus and $9(6.3 \%)$ cases of advanced tumors of HPB system. 3 $(2.7 \%)$ cases of EC were complicated by esophageal-bronchial fistula and esophageal-pleural fistula.

Results: To $69(100 \%)$ patients were implanted 77 SEMS. For all patients we used FCSEMS and PCSEMS. The 2 stents "into in" were deployed for 9 $(9.3 \%)$ patients and 1 stent to $60(86.8 \%)$ patients. For patients with leakage we installed FCSEMS. 10 (7\%) patients with esophageal obstruction had diameter less than $5 \mathrm{~mm}$ that appear difficulties to conduct delivery system into stenosis. To these patients before stenting the bougienage was done (33-39 Fr.). The technical success is $100 \%$ and clinical regress of obstruction $95.3 \%$. Early complications: bleeding in $3(2.7 \%)$ patients and migration in $4(3.5 \%)$ patients. Late complications: migration $5(7.5 \%)$, in growth $3(2.7 \%)$, stent disruption $1(0.7 \%)$, obstruction by food $1(0.7 \%) .6(5.2 \%)$ patients noted the heartburn. The stent migration was noted in patients with FCSEMS. All complications were solved by endoscopic (reposition) and drug therapy (PPI). Lethal outcome: $1(0.7 \%)$ patient died caused by profuse bleeding after 1 month of inserting SEMS.

Conclusions: FCSEMS is effective for fistula formation and prevent in growth tissue. PCSEMS prevent migration. In the case of severe stenosis the bougienage before stenting is necessary. However, it requires delicate performance. SEMS shows highly effective for palliation.

\section{P419}

\section{Salvage Therapy for Postoperative Residual Chest Pain Due to Esophageal Achalasia}

Chiaki Sato, MD, PhD, Yusuke Taniyama, MD, PhD, Tadashi Sakurai, MD, PhD, Makoto Hikage, MD, PhD, Kai Takaya, MD, $\mathrm{PhD}$, Takuro Konno, MD, PhD, Ken Koseki, MD, Ryohei Ando, MD, Takeshi Naitoh, MD, PhD, Michiaki Unno, MD, PhD, Takashi Kamei, MD, PhD; Dept of surgery, Tohoku Univ Hospital

Introduction: Chest pain is a common symptom of esophageal achalasia and markedly lowers patient's quality of life. However, some patients continue to experience chest pain after surgery, even when symptoms, such as dysphagia, have resolved. The mechanism behind chest pain caused by esophageal achalasia is poorly understood.

Case: We report the case of a 47-year-old female with residual chest pain after Heller-Dor operation whose dysphagia was resolved. High-resolution manometry (HRM) confirmed spastic simultaneous contractions in her middle esophagus. We diagnosed her as having type III achalasia, and assumed that the cause of her chest pain was incomplete myotomy of spastic simultaneous contractions in the middle esophagus. We performed the peroral endoscopic myotomy (POEM) procedure in the middle esophagus. Because she was type III achalasia, the length of the myotomy was adjusted to the length of the spastic segment in the esophagus, as observed on HRM. The POEM procedure improved her chest pain.

Conclusion: The cause of continued chest pain after surgical treatment for esophageal achalasia may be due to incomplete myotomy of the esophageal inner muscle, and POEM is effective for treating this pain. 
P420

Is It Time For General Surgeons To Place Wireless pH Probe at the Time of Initial Endoscopy for Patients with Gastroesophageal Reflux Symptoms?

Medhat Fanous, MD, FACS, Anja Jaehne, MD, Amanda Lambert, $\mathrm{RN}$, David Lorenson, RN; Aspirus Iron River Hospital

Introduction: Patients with symptoms of Gastroesophageal reflux disease (GERD) are often given a trial of proton pump inhibitors (PPIs). Despite low diagnostic yield, esophagogastroduodoscopy (EGD) is usually offered when empiric treatment fails. When a $\mathrm{pH}$ study is warranted to confirm diagnosis, it requires repeated endoscopy with potential procedural risks and additional cost. Gastroenterologists rather than general surgeons typically place $\mathrm{pH}$ probes.

Methods: A retrospective chart review was undertaken of patients presenting with GERD symptoms, to a general surgical clinic, between August 2015 and December 2017, who underwent EGD with concomitant placement of wireless $\mathrm{pH}$ probe. The probe was placed $6 \mathrm{~cm}$ proximal to the gastroesophageal junction. A DeMeester score $\geq 14.7$ was considered positive.

Results: 298 patients were evaluated (table 1). PPI usage was $11 \pm 9.1$ years. 197/298 (66.2\%) had previous EGDs. 281/298 (94.2\%) patients were tested off PPIs. 223/298 (74.8\%) had positive $\mathrm{pH}$ tests. Aspiration of $\mathrm{pH}$ probe occurred when one patient $(0.3 \%)$ violently coughed during placement. This patient was intubated and capsule was retrieved via bronchoscopy. Inconclusive studies were noted in 6/298 (2\%) patients. Four of them were due to misplacement (proximal or distal to intended location) and two had faulty probes.

Conclusion: Concomitant EGD and placement of $\mathrm{pH}$ probe by endoscopically trained surgeon is safe and confirms the diagnosis of GERD. Positive $\mathrm{pH}$ studies avoid repeating EGDs for the purpose of diagnosing GERD. Negative $\mathrm{pH}$ studies warrant a search for potential alternative diagnosis and can result in a decrease of inappropriate PPI utilization.

\begin{tabular}{|c|c|}
\hline Table One & \\
\hline Demographic & \\
\hline Female:Male & 203:95 \\
\hline Age & $16-79(57 \pm 14.7)$ \\
\hline BMI & $30.3 \pm 6.3$ \\
\hline Duration (years) & \\
\hline GERD symptoms & $14.7 \pm 11.3$ \\
\hline PPIs & $11 \pm 9.1$ \\
\hline Previous EGDs & \\
\hline None & $101 / 298(33.8 \%)$ \\
\hline Yes & $197 / 298(66.2 \%)$ \\
\hline Number of EGDs & $1-9(1.9)$ \\
\hline pH study & \\
\hline Off PPI & $281 / 298(94.2 \%)$ \\
\hline On PPI & $17 / 298(5.8 \%)$ \\
\hline Positive $\mathrm{pH}$ study & $223 / 298(74.8 \%)$ \\
\hline Positive $\mathrm{pH}$ study on PPI & $9 / 17(52.9 \%)$ \\
\hline Negative $\mathrm{pH}$ study & $69 / 298(23.1 \%)$ \\
\hline Inconclusive & $6 / 298(2 \%)$ \\
\hline DeMeester score & \\
\hline Composite & $28 \pm 26$ \\
\hline Day 1 & $25 \pm 27$ \\
\hline Day 2 & $30 \pm 29$ \\
\hline Complications & \\
\hline Aspiration of Bravo capsule & $1 / 298(0.03 \%)$ \\
\hline Misplacement of Bravo capsule & $4 / 298(0.01 \%)$ \\
\hline
\end{tabular}

\section{P421}

Imaging Through Blood Super-Resolution Based Flexible Microendoscope

Omer Wagner, $\mathrm{PhD}^{1}$, Asaf Shahmoon, $\mathrm{PhD}^{1}$, Zeev Zalevsky, Prof ${ }^{2} ;{ }^{1} \mathrm{Z}$ square, ${ }^{2}$ Bar Ilan University

Objective of the technology: The recent development of micro-endoscopic imaging tools opened a new door of medical modalities and opportunities of capturing images in a minimally invasive way. The main still remaining problem is that although the micro-endoscope can reach close to the desired location, the imaging resolution is still limited. This is due to the scattering of the blood and other residual liquids separating the endoscopic tip and the inspected object. Here we aim to show, for the first time, a wavelength-multiplexing based super-resolving concept that is integrated with the multi-core micro-endoscope (sub-mm diameter) to allow high resolution imaging through blood for liquid thickness of even more than $1 \mathrm{~cm}$.

Description of the technology: Our innovative solution involves usage of temporally-pulsed and spectrally wide-band laser. The laser should have short temporal pulses in the pico-sec regime and at its output we have special grating and a spatial 2-D transmission mask allowing to project wavelengths' dependent high-resolution spatial orthogonal encoding patterns (different spatial patterns for each wavelength). The short temporal pulses allow the high-resolution encoding pattern to reach the inspected object through the scattering blood medium (ballistic photons) without being spatially blurred. The light is intentionally collected via low resolution optics (small NA). The high-resolution reconstruction is obtained by passing the collected low-resolution data through a similar grating and 2-D mask. They multiply each wavelength dependent low-resolution image via its corresponding high resolution spatial decoding pattern. Thus, the grating and the 2-D spatial mask through which the light is collected do an all-optical decoding. Then we are summing all the images together to form the super-resolved reconstruction of the inspected object that was imaged through the highly scattering blood medium.

Preliminary results: We will present our novel conceptual design for the micro-endoscopy based super-resolving imaging module and then validate it via thorough numerical investigation as well as via experimental optical bench testing and validation. We will demonstrate the capability of performing high resolution imaging through blood medium of thickness of above $1 \mathrm{~cm}$ and characterize the performance envelope.

Conclusions: Here we present the first ever design of portable super-resolving micro-endoscopic imaging module providing high imaging resolution (HD pixels-wise resolution and high NA of the imaging lens) despite of its ultra-thin sub-mm external diameter. All this is obtainable even when the imaging is done via highly scattering medium as blood with thickness of above $1 \mathrm{~cm}$. 
$\mathbf{P 4 2 2}$

Anti-reflux Mucosectomy is Feasible and Effective After Failed Fundoplication

Meghana Vellanki, MD, Subhash Chandra, MBBS; Creighton University School in Medicine

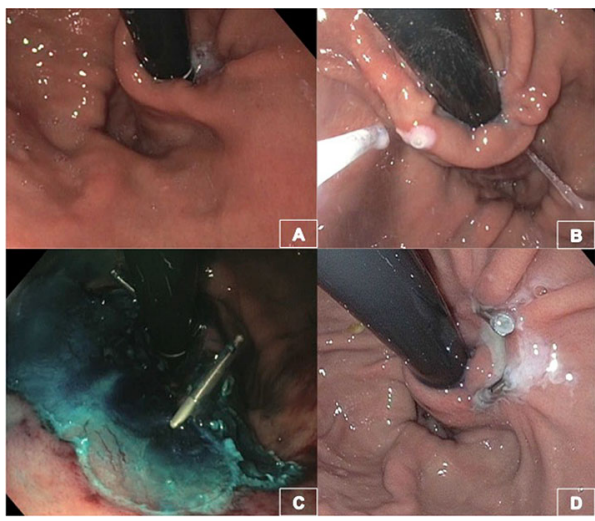

Introduction: Incompetent gastroesophageal junction (GEJ) is the cause for GERD in most patients. The lower esophageal sphincter (LES), crus of diaphragm, GEJ flap valve and angle of His maintain the competency of the GEJ. Anti-reflux interventions aim at reestablishing GEJ flap valve and gastropexy. Persistent high abdominal pressure leads to failure of the intervention overtime. Repeated surgical procedures are a high-risk surgery. Therefore, nonsurgical antireflux interventions as a primary or a savage modality to reestablish GEJ competency are needed. This is further important as adverse outcomes with long term proton pump inhibitor (PPI) use are being increasingly recognized. Haruhiro Inouea described mucosal resection of gastric cardia to re-establish GEJ flap valve with promising anti-reflux effect. Here, we report first anti-reflux mucosectomy (ARM) after failed fundoplication demonstrating its effectiveness and feasibility.

Case Description: A 65-year-old Caucasian female, with a BMI of 36 underwent Nissen fundoplication for GERD. This was taken down and a Toupet partial fundoplication was performed 3 years ago due to progressive dysphagia. She had good response for 1 year but symptoms of GERD recurred. She was back on twice daily PPIs. She also had dysphagia to solids and worsening regurgitation. Esophageal $\mathrm{pH}$ monitoring off PPIs had DeMeester score of 39 and symptoms of heartburn and regurgitation were related with acid reflux. Esophageal impedance manometry showed good deglutitive relaxation of LES with intact peristalsis but median distal contractile integral was 648 with $30 \%$ incomplete bolus clearance. Endoscopy showed loose gastroesophageal junction (Panel A). Piecemeal mucosal resection after submucosal lift was performed covering $2 / 3 \mathrm{rd}$ circumference in the gastric cardia along the lesser curvature, starting at the $\mathrm{z}$ line (panel $\mathrm{B}, \mathrm{C}$ ). This was performed in the endoscopy suite under general anesthesia. Patient was instructed to take twice a day PPIs for 1 week and then decrease to once a day. At three-week follow-up, patient was on once a day PPI, had no acid reflux but had persistent regurgitation while eating. Endoscopy showed tight GEJ flap valve and scar was still healing (panel D). Patient was instructed to continue once PPIs for 2 more weeks and eat slow which improved her regurgitation. She continued to do well four months later without symptoms of acid reflux.

Discussion: ARM is feasible and effective anti-reflux intervention even in presence of hiatal hernia and failed fundoplication. Durability of response is unknown.

\section{P423}

Endoscopic Management of Octopus Food Bolus Causing Esophageal Obstruction

Marc Ong, MBBS, MRCS, FRCS ${ }^{1}$, Wei-Wen Ang, MBBS, MRCS ${ }^{1}$, Vishal G Shelat, MBBS, MRCS, FRCS ${ }^{2} ;{ }^{1}$ Khoo Teck Puat Hospital, ${ }^{2}$ Tan Tock Seng Hospital

A 55-year-old man presented with acute onset of dysphagia following a meal which consisted of rice and octopus. He reported vomiting immediately after congestion of food/liquid thereafter. No other symptoms or signs were present. Laboratory tests did not reveal any abnormalities. A computed tomography of his neck and thorax was performed which revealed esophageal dilation with an apparent obstruction by a hyperdense mass (Figure A).

An esophagogastroduodenoscopy was promptly performed which showed an octopus lodged at the distal esophagus $5 \mathrm{~cm}$ from the cardio-esophageal junction (Figure B). Initial attempts at extraction of the food bolus proximally were unsuccessful as it was firmly impacted. Subsequently a gentle pressure with the tip of the endoscope was applied directly on the food bolus but to no avail. Finally, the scope was maneuvered carefully past the food bolus into the stomach, retroflexed and a toothed retrieval forceps was used to grasp the distal edge of the octopus head. The food bolus was then gently pulled into the stomach (Figure C). The patient was discharged well from the hospital 2 days later.

Foreign body ingestion and food bolus impaction are frequent problems encountered in endoscopic practice with majority occurring in the pediatric population. $80-90 \%$ of ingested foreign body/food bolus will pass spontaneously, while the remaining $10-20 \%$ of cases will require endoscopic management and less than $1 \%$ require surgery. There seems to be an association with dietary habits of different regions with food boluses encountered. In the United Stated, beef particularly and chicken are most common causes of food impaction, whereas in Asia and coastal countries, fish is most common. The "push technique" is the primary method recommended with high success rates, however applying excessive force can cause esophageal perforation. Failing which, retrieval either en bloc or piecemeal may be attempted using a variety of instruments including grasping forceps, snares, retrieval nets or endo baskets. Airway protection is pivotal if the latter method is adopted, necessitating endotracheal intubation or the use of overtubes for protection of the esophageal/pharyngeal mucosa from trauma especially during the retrieval of sharp objects. 


\section{P424}

Endoscopic Management of Ventriculoperitoneal Shunt Erosion into the Duodenum

Seyed A Arshad, MD, Andrew Christensen, MD, Edward Auyang, MD, MS, FACS, Jason Mckee, MD, FACS; University of New Mexcio

Ventriculoperitoneal (VP) shunt placement is a common procedure performed for the management of hydrocephalus. Of the complications associated with this procedure, bowel perforation constitutes around $0.1 \%$. [1] Colonic and gastric perforation is the most common, with small bowel perforation being exceedingly rare. [2,3] Standard management of hollow viscus perforation includes exploratory surgery. Described is the case of a rare duodenal perforation, managed only by upper endoscopy.

A 16-year-old female with past medical history significant for hydrocephalus, multiple previous VP shunt placements and manipulations, and multiple previous abdominal and pelvic surgeries presented to the emergency department with a two day history of generalized abdominal pain, however, no overt signs of peritonitis. A CT showed a previously abandoned VP shunt perforating the duodenum. Retrospective review of a CT scan performed one year prior also showed this shunt eroding into the duodenum.

Given that the erosion of the shunt into the duodenum had been longstanding, it was presumed that there likely was a well formed tract around the catheter. With the patient's history of multiple previous abdominal and pelvic operations, an attempt was made at endoscopic removal of the shunt.

Under general anesthesia, an upper endoscopy was preformed showing the catheter entering the third portion of the duodenum and extending distally. The catheter was able to be removed with use of an endoscopic grasper. Examination of the shunt ex vivo showed a cracked end, likely from prior attempts at shunt removal. Post-operative x-ray showed removal of the suspect shunt in its entirety.

Post-operatively, the patient was kept NPO with nasogastric tube decompression for a period of 5 days. At that time, an upper gastrointestinal contrast study was performed which showed no extravasation from the duodenum. The patient's diet was able to be advanced and the patient was subsequently discharged with no further sequela.

Although exceedingly rare, small bowel perforation by ventriculoperitoneal shunts is a possible complication of placement. Traditionally managed by exploratory surgery, endoscopic management of this complication is possible and should be considered as an adjunct to surgical intervention. Granted the use of endoscopy has been described for menagement of gastric and colonic perfortion, and for verification of me this is the first case describing the use of endoscopic-only management of VP shunts eroded into the small bowel. [4]
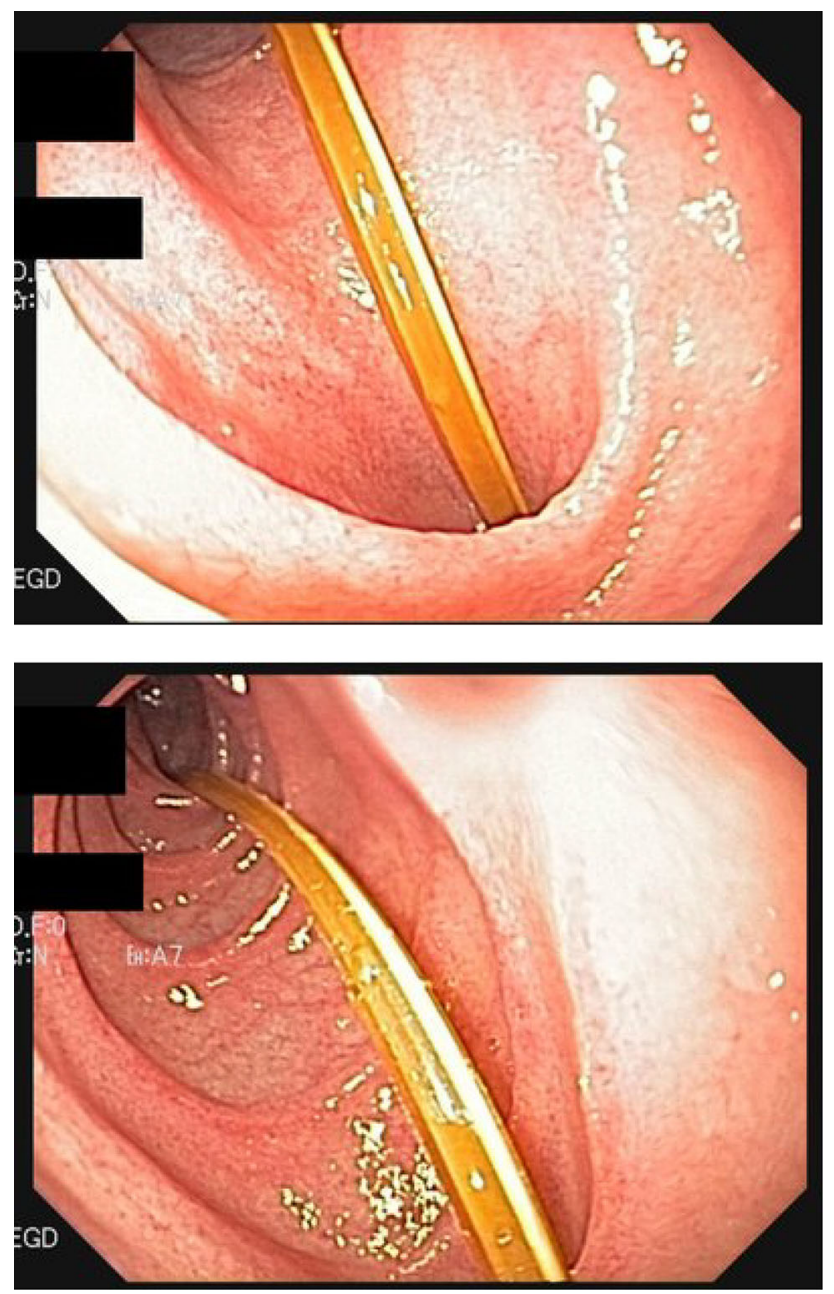
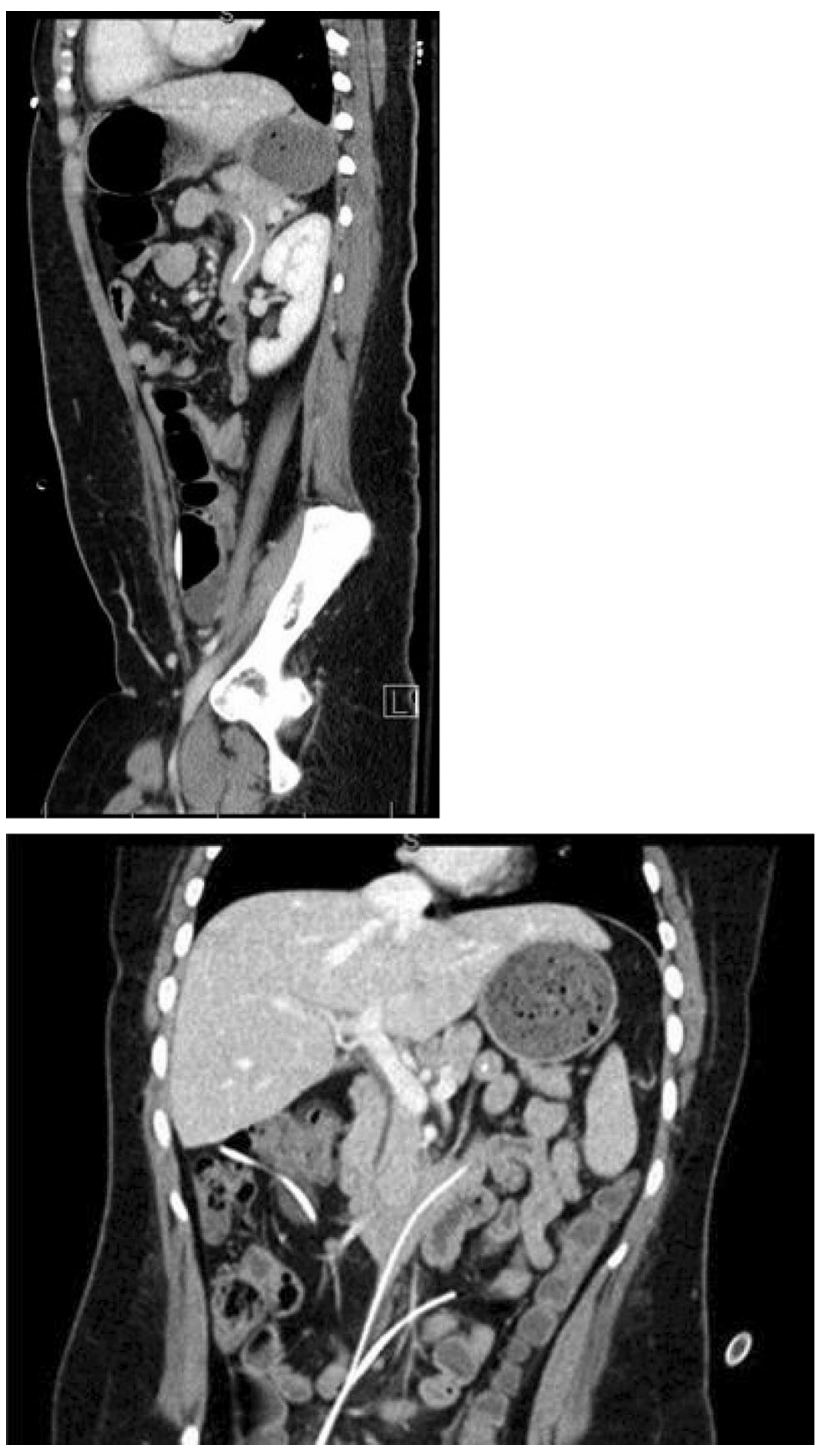

References

1. Sathyanarayana S, Wylen EL, Baskaya MK, Nanda A. Spontaneous bowel perforation after ventriculoperitoneal shunt surgery: case report and a review of 45 cases. Surg Neurol. 2000:54(5):388-96.

2. Bourm K, Pfeifer C, Zarchan A. Small bowel perforation: a rare complication of ventriculoperitoneal shunt placement. J Radiol Case Rep. 2016;10(6):30-35.

3. Odebode TO. Jejunal perforation and peroral extrusion of a peritoneal shunt catheter. $\mathrm{Br}$ Neurosurg, 2007:21(2):235-6.

4. Park CK, Wang KC, Seo JK, Cho BK. Transoral protrusion of a peritoneal catheter: a case report and literature review. Childs Nerv Syst. 2000;16(3):184-9. 


\section{$\mathbf{P 4 2 5}$}

\section{Drain Erosion: An Under-Reported Surgical Complication?}

Megan Sippey, MD, Jeffrey Hardacre, MD, FACS, Jeffrey Marks, MD, FACS, FASGE; Case Western Reserve University

Abdominal drains are frequently used as a surgeon's surveillance tool, with the benefit of serving a potential therapeutic role, for high risk anastomoses. Although rare, surgical drains can erode into nearby intraabdominal organs. We report two cases of abdominal surgical drain erosion into small bowel.

Case 1: A 17 year old male underwent pancreaticoduodenectomy for a near-complete transection of the pancreatic head and a perforation of the duodenum sustained in a motor vehicle collision. A flat Jackson-Pratt drain was placed anterior to the pancreaticojejunostomy. After 3 months, the drain continued to produce approximately $100 \mathrm{cc}$ daily. A fluoroscopic drain study confirmed filling of the jejunum (Figure 1). With suspicion for a pancreaticojejunostomy leak, the patient underwent enteroscopy with intention of endoscopically clipping or oversewing the suspected fistula. On endoscopic inspection, however, the drain was visualized intraluminally in close vicinity to an intact pancreatic anastomosis (Figure 2). The drain was removed and the tract healed spontaneously.

Case 2: A 62 year old female underwent Billroth II distal gastrectomy for a Stage IIIb gastric adenocarcinoma. On post-operative day 6, she developed peritonitis and was found to have a leak at the duodenal staple line on abdominal re-exploration. This was repaired with a Graham patch type repair using a mobilized piece of fat from the anterior aspect of the hepatic artery, and covered by a serosal patch using the efferent limb of the gastrojejunostomy. Two 3/16 inch round Jackson-Pratt drains were placed, one inferior and one superior to the repair. One drain had cessation of output and was removed. After 5 months, the second drain had persistent bilious output of 20-30 cc daily, despite being taken off of suction. A fluoroscopic drain study showed communication with small bowel in the left upper quadrant, and contrast passing through the gastrojejunostomy to fill the residual stomach (Figure 3). Interestingly, the area of communication was not at the site of the initial duodenal leak. The drain was slowly backed out over 6 weeks. She had intermittent drainage from the former tube site, which resolved spontaneously.

Drain erosion, with an otherwise intact surgical anastomosis, should be kept in mind in patients with persistent drainage despite a recuperating clinical picture. The advanced endoscopist is uniquely equipped to confirm this diagnosis and manage the residual fistula tract should spontaneous closure fail.

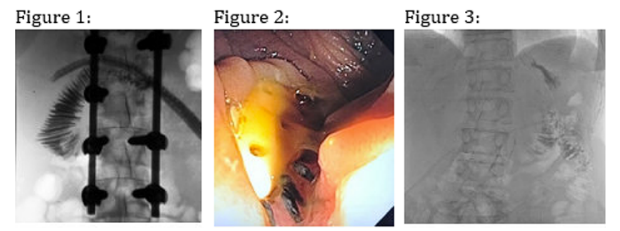

\section{P427}

Role of Weerda Diverticuloscope for Removal of Foreign Bodies Impacted in Upper Esophagus: Back to Rigid?

Carlo Galdino Riva, Stefano Siboni, Davide Ferrari, Marco Sozzi, Alberto Aiolfi, Luigi Bonavina; IRCCS Policlinico San Donato

Introduction: Foreign body removal is a common emergency procedure. The main location of impaction is the upper esophagus. In non-complicated cases, flexible endoscopy is the treatment of choice. Rigid endoscopy is not mentioned in most guidelines, but it provides good exposure and safe extraction of larger size or sharp objects.

Methods: A systematic review of the literature was conducted, including all the articles that matched the words "Rigid endoscopy AND flexible endoscopy AND foreign body". Heterogeneity was evaluated using I2index and Cochrane Qtest. Among 185 records screened, 7 studies were reviewed in full-text, of whom only 5 matched the inclusion criteria. Data collected from each work were: study characteristics, number of patients included in the series, localization of the foreign bodies, characteristics of the foreign bodies, endoscopic success rate, overall complication rate and perforation rate. Eventually, the results of the systematic review were summarized qualitatively into a frequentist meta-analysis.

Results: One thousand four hundred and two patients were included in the study. FE was performed in 736 patients and RE in 666. Overall, $101(7.2 \%)$ complications occurred. The most frequent complications were mucosal erosion (26.7\%), mucosal edema (18.8\%), and iatrogenic esophageal perforations (10.9\%). Compared to FE, the estimated RE pooled success OR was 1.00 (95\% CI $0.48-$ 2.06; $\mathrm{p}=1.00$ ). The pooled $\mathrm{OR}$ of iatrogenic perforation, other complications, and overall complications were 2.87 (95\% CI $0.96-$ 8.61; $\mathrm{p}=0.06), 1.09$ (95\% CI $0.38-3.18 ; \mathrm{p}=0.87)$, and $1.50(95 \%$ CI $0.53-4.25 ; \mathrm{p}=0.44$ ), respectively. There was no mortality. Conclusions: FE and RE are equally safe and effective for the removal of esophageal FB. To provide a tailored or crossover approach, patients should be managed in multidisciplinary centers where expertise in RE is also available. From a global health-care perspective, formal training and certification in RE should be reappraised. 
P428

The Impact of Bowel Diameter, Injection Method (Sclerotherapy Needle vs Punctureless High Pressure Injection) and Endoscope Type (Upper vs Low, and Different Diameter) on Endoscopic Bowel Wall Injections for Tattoo or Mucosal Lift

Neil Mitra, MD ${ }^{1}$, Dasuni Niyagama Gamage, $\mathrm{MD}^{1}$, Xiaohong Yan, $\mathrm{PhD}^{1}$, Jaspreet Sandhu, $\mathrm{MD}^{2}$, Carl Winkler, MD ${ }^{1}$, Vesna Cekic, $\mathrm{RN}^{1}$, Hmc Shantha Kumara, $\mathrm{PhD}^{1}$, Richard L Whelan, $\mathrm{MD}^{1}$; ${ }^{1}$ Mount Sinai West Hospital, ${ }^{2}$ Brookdale University Hospital Medical Center

Endoscopic bowel wall injections are most commonly performed in order to tattoo the location of a lesion or to generate a mucosal lift for ESD and EMR. The sclerotherapy needle is, by far, the most common injection method, however, needleless and punctureless high pressure injection methods are being used more often and offer advantages over needle puncture for lift establishment prior to and during ESD and EMR. The injection physics/mechanics for each method are quite different. The starting point for sclerotherapy needle injections is usually deep in the bowel wall because a firm push of the needle is needed to penetrate the mucosal surface. In contrast, the needleless system is more likely to generate a submucosal lift because there is no puncture; of note, this technique will not generate subserosal tattoos. As regards the sclerotherapy needle, when establishing a submucosal lift (ESD/EMR) or subserosal tattoo the angle of incidence between the needle and the bowel surface should be as tangential as possible so as to facilitate placement of the entire beveled needle tip into the desired layer (vs fully through the wall or in several layers). In contrast, a 90 degree angle of incidence is best when using a punctureless high pressure injection to create a submucosal lift; a tangential angle may result in mucosal injury or a poor lift. The ability to direct the needle or high pressure catheter at the desired angle is heavily influenced by the diameter of the bowel segment in question as well as the type of endoscope being used. Large diameter bowel facilitates right angle injections but makes tangential injection difficult. Likewise, the type of endoscope (upper vs lower, thin vs thick) also impacts the endoscopist's ability to make a tangential or right angle injection due the fact that the point at which the distal scope shaft bends in response to wheel deflection varies (closer or further from the tip) based on the scope's diameter. A colonoscope requires a larger bowel diameter in order to attain a 90 degree incidence and is better for making tangential injections. The opposite is the case for upper scopes. This poster will explain how the two bowel injection methods work, differ, and are best used. It will also demonstrate, via pictures and drawings, how the bowel wall diameter together with the endoscope design determine the injection angle options and limitations.

\section{P429}

Use of EUS-Directed Gastro-gastric Lumen-Apposing Metal Stents as a Viable Alternative for Gastric Remnant and Biliary Tree Access in Roux-en-Y Anatomy: A Tertiary Referral Center Experience

Alvaro F Galvez, MD ${ }^{1}$, Alexa Pesce ${ }^{2}$, Dane Scantling, DO, MPH ${ }^{1}$, Lynsey Daniels, MD ${ }^{1}$, Elizabeth Renza, $\mathrm{MD}^{1}$, Anand Kumar, MD, $\mathrm{MPH}^{1}$, Neal Patel, MD ${ }^{1}$, Erkanda Ikonomi ${ }^{1}$; ${ }^{1}$ Hahnemann University Hospital, ${ }^{2}$ Drexel University College of Medicine

Objectives: The anatomic challenges presented by Roux-en-Y gastric bypass (RYGB) are formidable. Access to the excluded gastric remnant or biliary tree is a common difficulty for surgeons and endoscopists after gastric reconstruction, especially in patients with multiple revisional surgeries and abundant abdominal adhesions. We present a case series in which gastric remnant access was achieved endoscopically, avoiding the need for surgery.

Methods: Three patients with a history of RYGB were enrolled for this case series. Each was considered high risk for a laparoscopic surgical intervention and each required access to the gastric remnant or biliopancreatic limb for different therapeutic purposes. Endoscopic ultrasound (EUS) was utilized to create a gastro-gastric fistula, maintained open with a lumen-apposing metal stent (LAMS). In one instance, this was done for decompression of the remnant. In the second case, for biliary stenting via ERCP; and in the third, it was utilized for percutaneous feeding access in a patient with failure to thrive.

Results: No patient suffered a leak or complication from stenting. Feeding access, gastric remnant decompression and biliary stenting were all accomplished successfully through the stented gastro-gastric fistula. The LAMS used for ERCP was removed electively 9 weeks after placement. The other 2 LAMS were not removed at follow up and were patent on the most recent imaging in all cases.

Conclusions: Endoscopic creation of a gastro-gastric fistula can be a safe and reliable means of accessing the excluded gastric remnant and biliary tree, avoiding the need for laparoscopic or open surgery. Additional patient enrollment is needed to ascertain long term outcomes.

Keywords: Roux-en-Y gastric bypass (RYGB), lumen-apposing metal stent (LAMS), gastric dilation, biliary leak 
P430

Simethicone Decreases Bloating and Improves Bowel Preparation Effectiveness: A Systematic Review and Meta-Analysis

Muhammad Moolla, BSc, Jerry Dang, MD, Ashley Shaw, MD, Thuc Nhi T Dang, MD, Chunhong Tian, PhD, Shahzeer Karmali, MD, MPH, Richard Sultanian, MD, MSc; University of Alberta

Introduction: We performed a systematic review and meta-analysis to determine if the addition of simethicone to bowel preparations prior to colonoscopy had an impact on bowel cleanliness and patient tolerability. Simethicone is an adjunct frequently used but there is currently no consensus on whether it should be recommended for routine use in combination with standard bowel preparations. Furthermore, mounting evidence suggests that simethicone adheres to the lining of the working channel on some of the endoscopes despite reprocessing, leading to the formation of biofilms. Given conflicting evidence regarding the effectiveness of simethicone as an adjunct to bowel preparation combined with recent data suggesting simethicone may contribute to biofilm formation, it is important to reassess the potential benefits of simethicone when added to bowel preparation regimes prior to colonoscopy.

Methods and Procedures: A comprehensive search was conducted for studies that compared colon cleansing of patients that received standard bowel preparation alone and in combination with simethicone prior to colonoscopy. Only randomized controlled trials were included. The primary outcomes were colon cleanliness and tolerability. Meta-analysis was performed to compare the number of successful bowel preparations amongst patients receiving polyethylene glycol (PEG) versus patients receiving PEG + simethicone. For tolerability, rates of nausea, vomiting, abdominal pain, and abdominal bloating were compared amongst patients receiving PEG versus patients receiving PEG + simethicone.

Results: Sixteen randomized controlled trials with 5630 patients were included in meta-analysis. Overall, PEG with simethicone improved colon cleansing compared with PEG alone (OR 1.48, CI 1.11 to $1.97, \mathrm{P}=.008$ ). This improvement was seen in patients receiving single dosing (OR 1.83 , CI 1.20 to $2.79, \mathrm{P}=.005$ ) but not in patients receiving split dosing (OR 1.32 , CI 0.72 to $2.43, \mathrm{P}=.38$ ). The rates of nausea (OR 0.96 , CI 0.75 to $1.24, \mathrm{P}=.75$ ), vomiting (OR $1.00, \mathrm{CI} 0.69$ to 1.44 , $\mathrm{P}=.99$ ), and abdominal pain (OR 0.69 , CI 0.40 to $1.18, \mathrm{P}=.17$ ) were not significantly different between PEG and PEG + simethicone cohorts. For abdominal bloating, the PEG cohort had greater odds of experiencing bloating than the PEG + simethicone cohort (OR 2.33, CI 1.70 to $3.20, \mathrm{P}<.00001)$.

Conclusion: Simethicone, when combined with PEG, resulted in superior colon cleanliness compared to PEG alone. However, this improvement was not seen in patients receiving split-dose PEG, which is now the standard of care. Furthermore, simethicone decreased abdominal bloating but has no effect on nausea, vomiting, and abdominal pain.

\section{P431}

\section{Acute Appendicitis Diagnosed During Screening Colonoscopy}

Lucia Collar Yagas, MD, Joaquin J Estrada, MD, FACS, FACRS, Jan P Kaminski, MD, MBA; Advocate Illinois Masonic Medical Center

Case: A 63-year-old male presented for his first screening colonoscopy. On the day of the procedure, he was asymptomatic and physical examination was unremarkable. He underwent an uncomplicated colonoscopy. A $6 \mathrm{~mm}$ pink-tan, sessile, polypoid lesion was found at the appendiceal orifice with thick yellowish-white oozing. After the colonoscopy, the patient developed right lower quadrant abdominal pain. On examination McBurney's sign was positive. A diagnostic laparoscopy was done revealing an inflamed appendix. An appendectomy was performed. Histopathologic examination of the specimen confirmed the diagnosis.

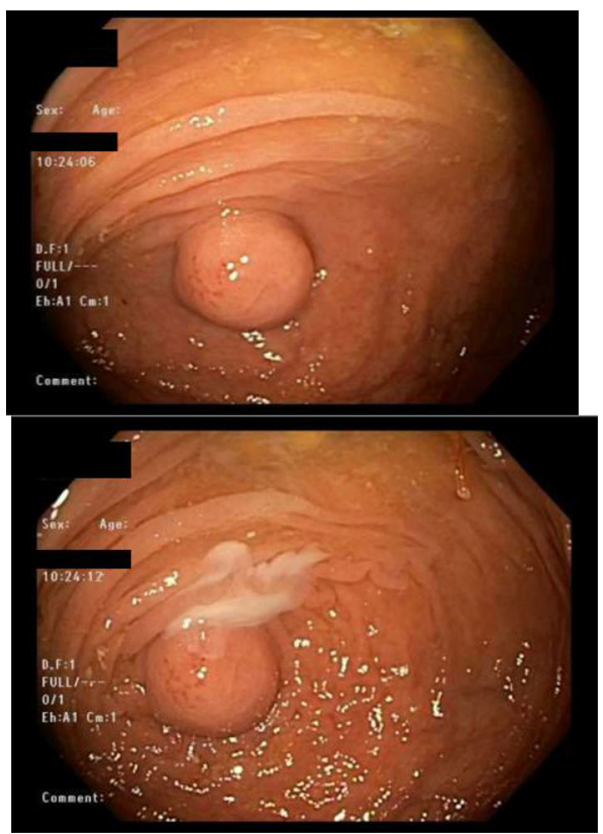

Discussion: In the few cases of appendicitis diagnosed by colonoscopy reported, the scope was performed because a diagnosis other than appendicitis was suggested by atypical clinical presentations or non-diagnostic imaging studies. Less commonly, this diagnosis was made during an endoscopy done in an asymptomatic patient such as in our case. Reported endoscopic findings were bulging near the appendiceal orifice, submucosal tumor-like protruding lesion, and spontaneous discharge of pus from the appendiceal orifice into the cecum.

It has been suggested that appendicitis can develop as a consequence of colonoscopy. Establishing causality is difficult, but there is an association between both entities. This relationship is most likely underreported, but it is estimated to be as high as 3.8 appendicitis cases per 10000 procedures.

The etiology of acute appendicitis is believed to be obstruction of the appendiceal lumen. Related to colonoscopy, the obstruction may occur during the preparation phase because of the aggressive catharsis or even during the procedure. It could be a result of air insufflation forcing fecal material into the appendiceal lumen, or from barotrauma. Other hypotheses are local edema caused by direct intubation of the appendiceal orifice, and mucosal inflammation from exposure to residual glutaraldehyde-type solution used in the cleaning of the endoscope. All these mechanisms could either initiate appendicitis or exacerbate a previously subclinical disease. Early stages of acute appendicitis might be symptom-free when the inflammation is only limited to the mucosa without serositis. It remains to be determined if in this case, appendicitis was caused by the colonoscopy or the patient had an early or subclinical disease when he presented for the procedure. It cannot be assured that colonoscopy did not have a role in the pathogenesis of the disease or the precipitation of symptoms.

Conclusion: Even though acute appendicitis during or after colonoscopy is an uncommon event, a high index of suspicion must be kept to facilitate early diagnosis and treatment. 
P432

Outcomes of Percutaneous Endoscopic Gastrostomy in Patients at a Tertiary Care Center in Northeast Brazil

Luiz Eduardo C Miranda, MD, phD ${ }^{1}$, Diego L Lima, $\mathrm{MD}^{2}$, Ana Clara G Miranda ${ }^{1}$, Mathews F Costa ${ }^{1}$, Marcel Roland ${ }^{1} ;{ }^{1}$ University of Pernambuco, ${ }^{2}$ State Servers Hospital

Background: Percutaneous endoscopic gastrostomy (PEG) is the main accepted method for long-term tube feeding, although it has been shown to be related to high short-term mortality. Recommending the procedure to the patient who naturally dies few days after it is a bad strategy. We investigated the risk factors associated with early mortality after PEG to improve the clinical indication.

Method: Retrospective short-term survival analysis was conducted on the medical records of 150 patients with PEG placement. The resultant data were analysed by the Kaplan-Meier method. Multivariable Cox proportional regression models were also built to test the effects of PEG on mortality.

Results: A total of 150 patients who submitted to PEG were studied (70 male). Patient mean age was $77.8 \pm 15.9$ years. Of the participants, $87(58.0 \%)$ had blood hypertension; 51 patients had diabetes $(34.0 \%) ; 6(4 \%)$ patients had chronic renal disease; and $6(4 \%)$ had malignancy. Chronic neurodegenerative diseases were the more common clinical indication for PEG. The 30-day and 60-day proportional mortality probability rates were $11.05 \%$ and $15.34 \%$ respectively. A multivariate Cox proportional regression model, haemoglobin (HR 4.39, 95\% CI 1.30-14.81, p 0.017) and pre-procedure UCI staying (HR 0.66, 95\% CI $0.50-0.87, \mathrm{p} 0.004$ ) were significant predictors of early mortality. A haemoglobin cut-off value of $10.05 \mathrm{~g} \mathrm{\%}$ was shown to have a sensibility of $82.6 \%(61.2 \%$ to $95 \% \mathrm{CI}$ ) and an acceptable sensitivity of 59.0 (50.6\% to $68.6 \% \mathrm{CI}$ ), and a likelihood ratio of 2.06 for eight weeks mortality.

Conclusion: In patients who had been subjected to the PEG procedure for long-term nutrition, low haemoglobin, pre-procedure UCI staying are associated with the risk of early mortality. PEG should not be indicated in such cases.

\section{P433}

Development of a Proficiency-Based Skills Program for FES ${ }^{\text {TM }}$ Competency: Determining Expert Performance Criteria on the Simbionix GI Endoscopy-Fundamental Skills Modules

Cheyenne C Sonntag, MD ${ }^{1}$, Katelin A Mirkin, MD ${ }^{1}$, John M Levenick, MD ${ }^{1}$, Jennifer L Maranki, MD ${ }^{1}$, Emmannuelle D Williams, $\mathrm{MD}^{1}$, Abraham Mathew, MD ${ }^{1}$, David B Stewart, $\mathrm{MD}^{2}$, Vamsi V Alli, $\mathrm{MD}^{1}$, Randy S Haluck, MD ${ }^{1}$, Eric M Pauli, MD ${ }^{1}$; ${ }^{1}$ Penn State Hershey Medical Center, ${ }^{2}$ University of Arizona

Introduction:Passing the Fundamentals of Endoscopic Surgery $\left(\mathrm{FES}^{\mathrm{TM}}\right)$ is a requirement for certification eligibility by the American Board of Surgery for all general surgery graduates. There is a need for evidence-based options for endoscopy training that will prepare trainees to pass FES $^{\mathrm{TM}}$ technical skills examination. The purpose of this study is to establish expert performance criteria on $\mathrm{FES}^{\mathrm{TM}}$ training modules to set minimum proficiency levels for trainees.

Methods: Expert performance criteria were established for the 9 currently available Simbionix GI Endoscopy-Fundamental Skills Modulesby expert endoscopists. Modules included: Basic Scope Manipulation; Endoscopic Navigation; Mucosal Evaluation (Basic, Advanced I, II); Retroflexion (Basic, Advanced); Task Targeting with Tool (Basic, Advanced) Demographic information and annual case experience was collected. Each expert demonstrated baseline passage of the FES ${ }^{\mathrm{TM}}$ exam skills portion. Experts performed five repetitions for each module and mean scores were calculated. Performance metrics included speed and precision as reported by the module software. Minimum standard of skill proficiency for trainees were then set at one standard deviation from the mean expert score for each module.

Results: Six expert endoscopists (4 male, mean age 40 years, 6 right handed) were recruited for study participation, with five experts completing each module five times. Experts reported on average 408 (range 100-800) upper and 251 (range 5-500) lower endoscopy procedures annually. Mean expert scores for each module and establishment of minimum standard of skill proficiency for trainees were calculated (Table 1).

Conclusion: There remains a clear need to define and validate a hands-on technical skills training program that promotes endoscopic skills competency and passage of the technical skills portion of the FES $^{\mathrm{TM}}$ exam. Establishing expert performance criteria on $\mathrm{FES}^{\mathrm{TM}}$ training modules is the first step in development of a proficiencybased skills program. Future work aims to assess construct validity of the program.

Table 1:

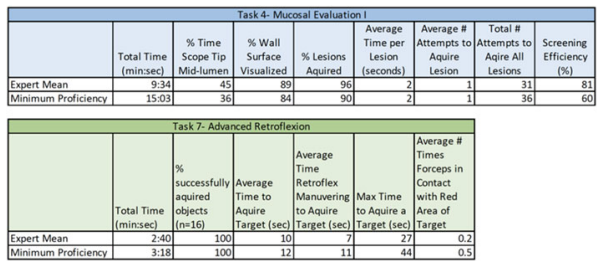




\section{P434}

Safety of Novel Submucosal Injection Agent to Perform Per-oral Pyloromyotomy: Single Center Pilot Study

Andrew T Strong, MD, Joshua P Landreneau, MD, MSc, Kevin ElHayek, MD, FACS; Section of Surgical Endoscopy, Department of General Surgery Cleveland Clinic

Introduction: Endoscopic submucosal tunneling techniques such as per-oral esophago-myotomy or per-oral pyloromytomy (POP) rely on gaining access to a submucosal plane in the gastrointestinal tract. A fluid lift technique is typically used to facilitate accessing this space. Admixtures of dye and saline are most often used, they are readily available and inexpensive. However, these mixtures must be mixed at the bedside, and rapidly diffuses into the tissue, necessitating frequent re-injection. A novel submucosal injection agent recently approved for endoscopic resection, (Eleview ${ }^{\mathrm{TM}}$, Aries Pharmaceuticals). This novel solution contains dye, medium chain triglycerides, soluble polymeric thickener and an emulsifier in a premixed single use ampule. This study evaluated in this pilot study for utility in performing POP procedures.

Methods: After approval by the Institutional Review Board, consecutive patients undergoing POP therapy for medically refractory gastroparesis between November 2017 and May 2018 by a single endoscopist (KE) were identified and retrospectively reviewed. All submucosal injections were performed with a novel submucosal injection agent (Image 1). Outcome variables included procedure time and safety.

Results: During the study period, there were 38 patients included. There were 33 female (86\%), with a mean ager of 46 years. The causes of gastroparesis were idiopathic in $50 \%$, diabetes related in $26 \%$, post surgical in $5.2 \%$ and multiple potential causes in $15.7 \%$. Among the patients included, $38 \%$ had undergone prior surgical or endoscopic intervention for gastroparesis. The POP procedure was technically successful in all cases with a mean operative time of $23.9 \mathrm{~min}$. There were no cases where re-injection was required to complete the POP. Visualization was noted to be temporarily compromised by bubbling of the injected solution in some cases (image 2). Most patients were discharged the same day $(60.5 \%)$. There were two patients readmitted within 30 days, one with suspicion for perforation that was found to be negative, and one for delayed post procedural bleeding.

Conclusion: The use of a novel submucosal injection agent during POP appears to be safe and effective, and should be considered for use in endoscopic tunneling procedures. The long lasting fluid cushion avoids the need for re-injection, and it pre-mixed packaging reduces the change for medication errors associated with bedside compounding. Comparative studies should be performed to evaluate this novel submucosal injection agent standard saline lift technique for tunneling procedures.

\section{P436}

Sodium Phosphate is Superior to Polyethylene Glycol in Constipated Patients Undergoing Colonoscopy: A Systematic Review and Meta-analysis

Jerry T Dang, MD, Muhammad Moolla, BSc, Thuc Nhi T Dang, MD, Ashley Shaw, MD, Chunhong Tian, PhD, Shahzeer Karmali, MD, MPH, Richard Sultanian, MD, MSc; University of Alberta

Introduction: We performed a systematic review and meta-analysis to determine the optimal bowel preparation in constipated patients undergoing colonoscopy. Constipation is an important and highly prevalent predictor of inadequate bowel preparation during colonoscopy. In North America, between $2 \%$ and $28 \%$ of the general population suffer from constipation. Despite the high prevalence of constipation, to our knowledge, no meta-analysis on optimal bowel preparations for constipated patients has been performed. Furthermore, while the literature recognizes constipation as a predictor for poor bowel preparation, specific recommendations regarding the preparation of these patients for colonoscopy remain scarce.

Methods and Procedures: A comprehensive search of electronic databases (MEDLINE, EMBASE, SCOPUS, and Web of Science) was performed. Studies were assessed using the following inclusion criteria: adult patients (age $\geq 18$ years), randomized controlled trial study design, studies comparing two or more different bowel preparations, studies that included constipated patients, and studies that assessed the effectiveness of the bowel preparation. The primary outcome of interest was the quality of the bowel preparation measured with either validated or non-validated scales. Studies varied in the criteria used to distinguish patients as constipated, therefore, for this review patients were considered constipated based on the method the study authors used to define constipation. Where possible, metaanalysis was performed to compare the percentage of successful bowel preparations amongst constipated patients receiving Polyethylene Glycol (PEG) versus constipated patients receiving Sodium Phosphate $(\mathrm{NaP})$.

Results: Preliminary database search yielded 1581 articles after duplicates were removed. After screening of the titles and abstracts using the exclusion criteria, 358 full-text articles were retained. Full text articles were reviewed and eight studies meeting the inclusion criteria were included for qualitative synthesis. Three randomized controlled trials identified a total of 1636 constipated patients, of whom 225 were eligible for meta-analysis. Of those, 107 (47.6\%) received $\mathrm{NaP}$ and $118(52.4 \%)$ received PEG. The weighted mean age was 57.6 years and weighted sex was $53.5 \%$ female. When compared to the PEG cohort, the (NaP) cohort had a higher likelihood of having a successful bowel preparation (odds ratio [OR] 1.87, CI 1.06 to 3.32, $\mathrm{P}=0.003$ ).

Conclusion: In chronically constipated patients undergoing colonoscopy, the use of $\mathrm{NaP}$ resulted in superior colonic cleanliness when compared to PEG. As such, in constipated patients who are otherwise healthy and do not have contraindications to $\mathrm{NaP}$, we recommend endoscopists choose NaP over PEG to improve the quality of bowel preparation. 
P437

Percutaneous Endoscopic Gastrostomy in Amyotrophic Lateral Sclerosis Patients: Outcomes of a Dedicated Surgery and Anesthesia Protocol

David J Morrell, MD ${ }^{1}$, Marvin H Chau, BS ${ }^{2}$, Vamsi V Alli, MD ${ }^{1}$, Elizabeth H Sinz, MD ${ }^{1}$, Sprague W Hazard, MD ${ }^{1}$, Zachary Simmons, $\mathrm{MD}^{1}$, Eric M Pauli, MD ${ }^{1}$; ${ }^{1}$ Penn State Health Milton S. Hershey Medical Center, ${ }^{2}$ The Pennsylvania State University College of Medicine

Introduction: Patients requiring percutaneous endoscopic gastrostomy (PEG) for amyotrophic lateral sclerosis (ALS) related dysphagia represent a clinical challenge. Diminished pulmonary function and aspiration risks can lead to anesthesia-related complications and gastric displacement from hemidiaphragm elevation may preclude safe gastric access. This study reports the efficacy and outcomes of a dedicated anesthesia/surgery management protocol for ALS patients undergoing PEG.

Methods: In 2013, a PEG protocol for ALS patients was developed by an ALS neurologist, two neuro- anesthesiologists, and one surgical-endoscopist. The protocol emphasized efficient pre-operative evaluation, rapidly metabolized anesthetic agents, reduced procedural times, and minimization of opioid use. Outcomes were analyzed following implementation of the protocol through retrospective review of the medical record. Preoperative weight loss, pulmonary function tests, total analgesia, procedural time, and 90-day morbidity and mortality were recorded.

Results: From 2013-2018, 39 ALS patients (mean age 64.7 years, $56.4 \%$ female, mean BMI $25.1 \mathrm{~kg} / \mathrm{m}^{2}, 82 \%$ outpatient) received a PEG under the protocol. PEGs were performed by one surgical-endoscopist using a standard Ponsky/pull method and safe-tract technique with anesthesia administered by one of two dedicated neuro-anesthesiologists. Mean percentage weight loss 6 months before PEG was $9.6 \%$ with $41 \%$ of patients meeting criteria for severe malnutrition. Mean forced expiratory volume was $55.4 \%$ predicted and mean forced vital capacity was $55.1 \%$ predicted. Mean anesthesia time (propofol induction to anesthesia emergence) was $32.2 \mathrm{~min}$ and mean operative time (endoscope insertion to dressing placement) was 14.8 min. Transversus abdominis plane block with liposomal bupivacaine was performed in $46.2 \%$. There were no anesthetic or operative complications and all attempts at PEG placement were successful. Mean perioperative opioid requirement was 4.58 morphine milligram equivalents (MME). With a mean follow-up of 6.3 months, all PEGs were functional and there were no surgical site complications. One patient was re-evaluated within 30 days for loosening of a painful PEG bumper. Thirty-day readmission rate was $5.1 \%$ ( 1 acute respiratory failure, 1 benign postoperative pneumoperitoneum) and 90-day mortality was $19.4 \%$ (all unrelated to PEG). Mean time from surgery to death was 9.3 months.

Conclusions: Protocols for optimizing PEG may help overcome challenges present in the ALS patient population. Despite patient comorbidities, protocol implementation and dedicated team members resulted in a high procedural success rate, low complication rate, and low MME requirement. Further study is warranted to optimize the timing of PEG placement in relation to ALS disease progression and determine the utility of regional anesthesia during PEG placement.

\section{P438}

Periampullary Duodenal Diverticulum ERCP Incidental Finding and Association with Difficult Biliary Cannulation. A Case Series

Carlos M Zavaleta, MD, Fernando A Quesada, MD; Intergastro

Introduction: A classification for Periampullary Duodenal Diverticulum (PAD) was proposed by Boix et al. in three types based on location. These anatomical variants may require more expertise from endoscopist in charge to achieve a successful procedure as it has been reported as a factor associated with difficult biliary cannulation.

Aim: To assess differences in difficult biliary cannulation and therapeutic failure between PAD types during ERCP.

Materials and Methods: A 5 year-period (2013-2017) from a Database of indicated/performed ERCPs was reviewed. Patients reported with findings of PAD on their first ERCP attempt were identified and included. ERCP Procedures had been performed by two endoscopic surgeons with more than 10 years of experience at the moment of the Study period.. Patients with PAD were distributed by the three Boix types. Cannulation difficulty was measured, defined as directed by the ESGE guidelines, and measured with Boix grading of 4 grades. Also therapeutic failure rate was measured. Inferencial Statistical analysis of comparison was done using Chi Square test considering $\mathrm{P}$ value $\leq 0.05$ significant for a difference.

Results: 234 patients were included, 157 Women and 77 men with a female/male ratio of 2.03:1, and a median age of 73 years old. Distribution of subjects by Type of PAD: Type I (Inside diverticulum): 69 (29.49\%), Type II (in the margin of the diverticulum): 123 (52.56\%), and Type III ( $<2 \mathrm{cms}$ near the diverticulum): 42 (17.95\%). A difference between difficult cannulation and PAD type was observed, with PAD type I having more frequence on the highest type of difficulty (grade 4) $(11.6 \%)$, and grade $3(21.73 \%) \mathrm{p}=0.000$. Therapeutic failure rate was higher for Type I: $26.08 \%$, beign statistically significant $\mathrm{P}=0.000$.

Conclusion: In our Study, PAD Type I has the highest rate of difficult biliary cannulation and therapeutic failure. 


\section{P439}

Intraoperative Endoscopy; A Safe and Valuable Tool for the Diagnosis and Treatment of Midgut Pathology

Amanda Fazzalari, $\mathrm{MD}^{1}$, Shruthi Srinivas, $\mathrm{BS}^{1}$, Natalie Pozzi, $\mathrm{MD}^{2}$, Christopher Schlieve, MD ${ }^{1}$, Jonathan Green, MD $^{1}$, Demetrius Litwin, MD, MBA, FACS ${ }^{1}$, David Cave, MD, $\mathrm{PhD}^{1}$, Mitchell Cahan, MD, MBA, FACS ${ }^{1}$; ${ }^{1}$ University of Massachusetts, ${ }^{2}$ Saint Mary's Hospital

Objective: Intraoperative endoscopy (IOE) was first described in the late 1960s and is regarded as the gold standard for complete evaluation of the small bowel. However, with the advent of deep endoscopy and video capsule endoscopy, IOE has been used less frequently. Recently we published a large series demonstrating that IOE is a valuable tool for the diagnosis and treatment of small intestinal bleeding (SIB) and non-adhesive obstructive small bowel disease (OSBD; Green et al., 2018). Existing literature lacks clear guidelines vis-a-vis IOE; therefore, we propose safe and effective methods to guide the surgeon's approach to IOE.

Methods and Procedures: As we recently described, IOE is indicated in patients with SIB that is visualized but cannot be treated via endoscopy. For OSBD, IOE is indicated when computed tomography and initial endoscopies are non-diagnostic and there is a suspicion for a resectable lesion (i.e. tumor or diverticulum). The procedure begins with standard diagnostic laparoscopy and complete evaluation of the small bowel. The evaluation includes visualizing a lesion or tattoos which may have been marked endoscopically beforehand. If no small bowel lesion is visualized, a six-centimeter supra-umbilical incision is made and an Alexis ${ }^{\circledR}$ wound protector is inserted for the purpose of IOE. The small bowel is eviscerated for complete visual inspection and manual palpation. If no lesion is palpated, IOE follows via an enterotomy made in proximity to the anticipated lesion or between proximally and distally marked ink tattoos. The enteroscope is secured with a purse string suture to prevent leakage of enteric contents. The small bowel mucosa is examined retrograde and anterograde, with the gastroenterologist controlling the enteroscope and the surgeon simultaneously advancing the scope and telescoping the bowel extracorporally over it. Carbon dioxide is ideally used for insufflation. Definitive treatment depends on the type of lesion identified, with small bowel resection being the most common procedure. Post-operative management is patient and provider dependent. Generally, nasogastric tubes and urinary indwelling catheters are removed on postoperative day one and diet is advanced as tolerated.

Conclusions: IOE is a safe, fast, and effective method for diagnosing and treating SIB and OSBD undiagnosed by conventional modalities. While the majority of the reports describing IOE have been published in Europe or Asia, we have proven that this technique is accurate and valuable in North America. Here we provide clear guidelines regarding the indications and appropriate technique by which to perform IOE in the United States.

\section{P440}

Does Routine EGD Prior to Eus Reveal Findings of Significance

Yuan Y Stevenson, MD, Nan Walsh, CRNP, Robert D Fanelli, MD, MHA, FACS, FASGE; Guthrie Robert Packer Hospital

Introduction: Side-viewing endoscopes used for endoscopic ultrasound (EUS) provide almost no view of the esophagus during insertion. Esophagogastroduodenoscopy (EGD) routinely is performed immediately prior to EUS to rule out obstructive lesions, stenoses, or to identify the location of mucosal and submucosal lesions that are the target of EUS. Few studies have looked at the impact the findings on routine EGD have on clinical management. This study reviews the significance of findings on routine EGD completed prior to EUS. Methods: A retrospective chart review was conducted at a 254-bed tertiary care teaching hospital. A total of 379 patients underwent 395 EGD/EUS during 13 months from June 2017 through June 2018. EGD findings were categorized into four significance categories: 1) normal/no reportable findings; 2) minimally significant findings/no intervention required; 3) significant findings/ minor intervention required; 4) significant findings/surgery or other major intervention required. The distribution of significance categories for EGD findings was calculated. Categorical variables were analyzed using a Chi square test of association and continuous variables were analyzed using a oneway ANOVA. Four patients did not have EGD reports in the electronic health record and therefore were excluded.

Results: The majority of EGD findings were category $2(50.38 \%)$, followed by category $1(39.24 \%)$, category $3(5.32 \%)$, and then category $4(5.06 \%)$. There was no significant difference in mean age of patients across significance levels. Conclusions: Routine EGD prior to EUS improves safety by ruling out obstructing lesions and stenoses, and localizes mucosal/submucosal lesions for study. Abnormal EGD findings are identified in $60.76 \%$ of patients, but intervention is required in only $10.92 \%$. 


\section{P441}

Endoscopic Management of Complete Bowel Obstruction Secondary to Ingestion of Water Absorbing Beads

Seyed A Arshad, MD, Andrew Christensen, MD, Edward Auyang, MD, MS, FACS, Jason Mckee, MD, FACS; University of New Mexico

Pediatric foreign body ingestion is a common issue with a reported 100,000 ingestions per year [1]. Most of these are accidental with a high majority being coins. A reported 50-90\% of foreign bodies pass spontaneously, with $10-20 \%$ requiring removal, and less than $1 \%$ requiring surgical intervention [2]. The ingestion of Orbeez, water absorbent polymer beads, has increased in the past years with a 110 patient case-series published describing no reports of intestinal obstruction as well as in vitro studies concluding low risk of obstruction [3,4]. There have however been two publications of intestinal obstruction secondary to these beads, each requiring operative intervention $[5,6]$. Described is the case of a rare complete bowel obstruction secondary to ingestion of these water absorbing beads, managed non-operatively with upper endoscopy.

An otherwise healthy 19-month-old female presented with a one day history of nausea, vomiting, and intoleration of oral intake. During a bout of emesis, the patient expelled a $1 \mathrm{~cm}$ fully saturated bead, concerning the parents for foreign body ingestion. The patient underwent a computed tomography (CT) scan, which was significant for a dilated second portion of the duodenum with nonpassage of oral contrast beyond the stomach. Given the patient's clinical and radiographic findings, there was concern for complete bowel obstruction at the level of the proximal duodenum.

Under general anesthesia, an upper endoscopy was performed. The scope was advanced to the duodenum where a mass of gelatinous material from the saturated beads was fully obstructing the lumen. Due to the location and impacted nature of the beads, attempts at intact bead removal were unsuccessful. Using a grasper, the beads were further broken down and suctioned through the scope piece-meal until the obstruction was resolved. The scope was then able to be advanced beyond the ligament of Treitz, assuring resolution of the obstruction. Post-operatively, the patient's diet was able to be advanced and was subsequently discharged with no long-term sequela.

Although common in the pediatric population, foreign body ingestion rarely requires operative intervention. There have now been two case reports of bowel obstruction secondary to these water-absorbing beads, but all previous cases have opted for operative intervention. We believe this is the first case describing endoscopic management of complete bowel obstruction secondary to these beads. Although one must be prepared to continue on to operative intervention, endoscopic evaluation and management is a safe and effective initial approach to this rare, but increasing cause for obstruction.

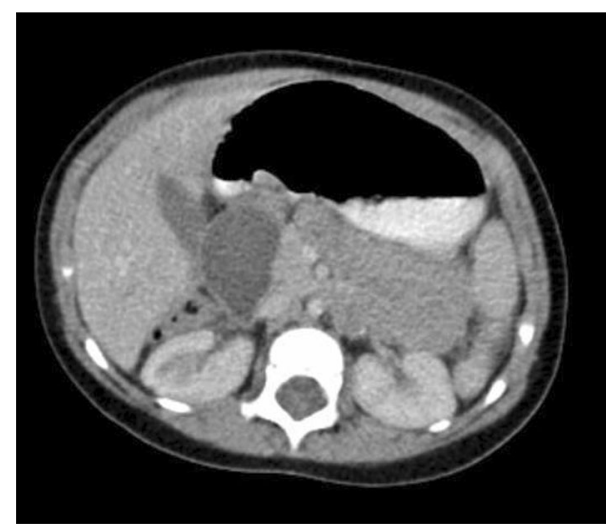

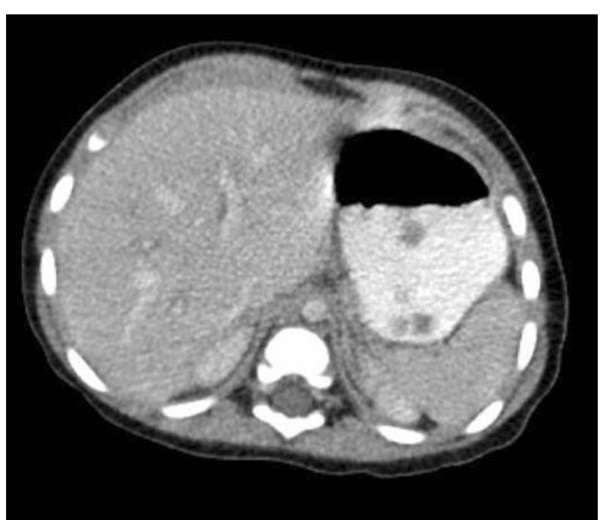

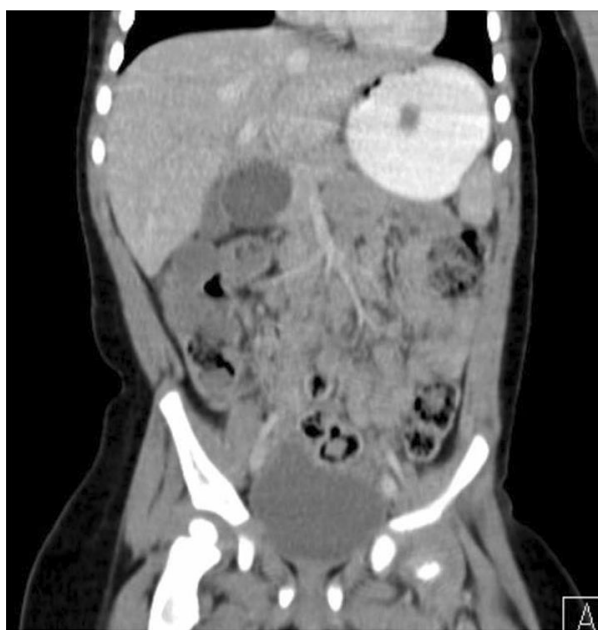

\footnotetext{
References

Kay M. Wyllie R. Pediatric foreign bodies and their management. Curr Gastroenterol Rep. 2005;7(3):212-8.

Wright CC, Closson FT. Updates in pediatric gastrointestinal foreign bodies. Pediatr Clin North Am. 2013;60(5):1221-39.

Forrester MB. Pediatric Orbeez Ingestions Reported to Texas Poison Centers. Pediatr

Emerg Care. 2017

列 absorbing bead--risk of pediatric bowel obstruction?. Pediatr Emerg Care. 2015;31(6):416-8.
Zamora IJ, Vu LT, Larimer EL, Olutoye OO. Water-absorbing balls: a "growing" problem. Pediatrics. 2012;130(4)::1011 caused by ingestion of water-storing gel beads. J Pediatr $S$ wro lobstruction in a child
} 


\section{P442}

Laparoscopic Para-esophageal Hernia Repair Including an Anterior 180 Degrees Fundoplication \& Mid-term Outcomes in a Single Center

Anne-Sophie S Studer, MD, Teodoros Thomopoulos, MD, Michele Podetta, MD, Henri Atlas, MD, Ronald Denis, MD, Pierre Garneau, MD, Radu Pescarus, MD; Hopital Sacré Coeur de Montréal

Introduction: Surgical management of large paraesophageal hernias (PEH) remains challenging and may be associated with high morbidity. As an alternative to Nissen fundoplication, an anterior 180 degrees wrap has been described in the treatment of hiatal and PEH. Materiel \& Methods: Retrospective analysis of patients operated for $\mathrm{PEH}$ that included an anterior 180 degrees fundoplication, between October 2014 and May 2018, was performed. Pre- and post-operative clinical and objective findings (EGD, UGI study, CT abdomen or gastric emptying test) were recorded. Following laparoscopic hernia reduction and hiatal closure, the anterior gastric fundus was sutured as an anterior valve to the top of the left crus and at various locations on the right crus.

Results: Overall, 26 patients (20 Female; 6 Male), with a mean age of 70 years, underwent PEH repair with an anterior fundoplication. $12 / 26(46 \%)$ patients were obese (BMI over $30 \mathrm{~kg} / \mathrm{m}^{2}$ ). Pre-operative dysphagia, heartburns, epigastric pain, and regurgitations and nausea were respectively present in $65,65,58$ and $35 \%$ of cases. All patients underwent primary laparoscopic PEH repair with partial anterior fundoplication, 17 patients $(65 \%)$ had biological mesh reinforcement and 1 patient underwent a Collis gastroplasty. Four patients $(15 \%)$ had an incarcerated PEH and underwent emergency surgery. Early post-operative morbidity rate and 30-day post-operative mortality rate were respectively $15,4 \%$ ( 1 post-operative confusion, 1 pneumonia, 1 angina pectoris and 1 myocardial infarction) and $0 \%$. Median length of stay was 2 days (1-9). Median follow-up was 13 months (1-32). Anatomic hiatal hernia recurrences were diagnosed in 7 cases $(27 \%)$, based on barium study (in 1 patient) or EGD (in 6 patients). Mean time of recurrence diagnosis occurred at 18,5 months. Only one patient was asymptomatic, three others complained bloating, 2 others regurgitations and nausea, and the last one, dysphagia and epigastric pain. There were 7 late complications with a mean time of occurrence of 20 months (4 oesophagitis, 2 gastroparesis, and 1 gastric ulcer). Four of those happened in a context of hiatal hernia recurrence. One patient with gastroparesis underwent a laparoscopic pyloroplasty 5 months post-op. No surgical revision was needed for the PEH recurrences.

Conclusion: Laparoscopic anterior partial fundoplication appears to be a safe option in the armamentarium of the PEH surgeon.

\section{P443}

\section{Pattern of Hiatal Hernia Repair: An Analysis of the National Inpatient Sample}

Zhamak Khorgami, MD ${ }^{1}$, Guido M Sclabas, MD ${ }^{1}$, Jamin G Graham, $\mathrm{MPH}^{2}$, C. Anthony Howard, MD ${ }^{1}$, Geoffrey S Chow,

$\mathrm{MD}^{1}$; ${ }^{1}$ University of Oklahoma, College of Medicine, Tulsa, USA, ${ }^{2}$ Trinity Medical Sciences University

Introduction: Patients with symptomatic hiatal hernias undergo surgical repair as a primary operation or in conjunction with other gastric and esophageal operations. The purpose of this study is to evaluate the pattern, cost, and outcomes after Hiatal Hernia Repair (HHR) in the United States.

Method: A retrospective analysis of the Nationwide Inpatient Sample (HCUP-NIS) was performed. Patients 18 years of age and older who underwent HHR were included for analysis. Patient demographic factors, surgical approach, concurrent operations, length of stay, mortality, and rate of HHR over the study period were evaluated. A multivariate regression analysis was performed to identify predictors of an open operation in patients undergoing HHR as the principal surgery.

Results: 46,590 patients (mean age $58.4 \pm 15.8$ years) were analyzed, and 30,893 (66.3\%) were female. A laparoscopic approach was utilized in $39,734(78.8 \%)$, with minimal change over the study period. A transthoracic approach was utilized in 486 patients (1\%). HHR was the primary operation in $40,788(87.5 \%)$ patients. The most common associated principal procedures were esophagomyotomy in 2,627(45.3\%), sleeve gastrectomy in 538(9.3\%), and Roux-en-Y gastric bypass in 241(4.2\%). In patients with HHR as the principal procedure, a gastrostomy tube was placed in 2,200(5.4\%) and partial gastrectomy performed in $177(0.4 \%)$ patients. The median (Interquartile range, IQR) length of stay was 2(2) days in laparoscopic cases, and 5(5) days in open cases $(\mathrm{p}<0.001)$. The median (IQR) total charge was $\$ 30,700(30,100)$ for laparoscopic repair, and $\$ 40,500(45,500)$ for open repair $(\mathrm{p}<0.001)$. Post-operative mortality was $0.5 \%$ (167) in laparoscopic cases, and $1.3 \%$ (129) in open cases $(\mathrm{p}<0.001)$. Age, gender, and race were similar in open and laparoscopic groups. Patients emergently admitted to a hospital had higher rates of open HHR than patients undergoing elective repair $(26.4 \%$ vs $18.8 \%, p<0.001)$. On multivariate analysis, independent patient factors predicting open HHR included alcohol abuse, chronic kidney disease, coagulopathy, fluid and electrolyte abnormality, paralysis, and weight loss.

Conclusion: The majority of HHR are performed with a laparoscopic approach, although open repair is performed in more than one-fifth of patients nationally. Length of stay, mortality, and total charges are lower in patients undergoing laparoscopic repair. Additional education during residency and fellowship programs, development of foregut specific service lines, along with orienting trainees and surgeons about referral to specialized centers may improve patient outcomes and decrease healthcare expenditure related to hiatal hernias. 


\section{P444}

\section{Gastroparesis Cardinal Symptom Index Scoring Does Not Predict} Delayed Gastric Emptying in Patients with GERD

Sarah Marowski, Yiwei Xu, Luke M Funk, Jacob A Greenberg, Anne O Lidor, Amber L Shada; University of Wisconsin School of Medicine and Public Health

Gastroparesis (GP) causes nausea, vomiting, and reflux. These symptoms are also associated with gastroesophageal reflux disease (GERD), and it is often difficult to distinguish between the two. Currently, gastric emptying scans (GES) and the Gastroparesis Cardinal Symptom Index (GCSI) are used to evaluate gastroparesis. The GCSI has been validated for use in diabetic gastroparesis patients but not in other patient populations, such as patients with GERD. This study aims to evaluate the usefulness of GCSI in identifying GP in patients with a primary diagnosis of GERD who presented to our clinic for anti-reflux surgery.

Retrospective review of an institutional foregut surgery database was performed to identify adult patients with GES. Diabetic or idiopathic gastroparesis patients with no history of foregut surgery were excluded. The rest were sorted by normal (GERD) or abnormal (GERDDGE) GES. GERD-Health Related Quality of Life (HQRL) and GCSI scores including subcategory scores in fullness, bloating and nausea/ vomiting were calculated and compared between groups. A Wilcoxon RankSum was used to compare scores between groups.

There were 137 total patients identified. Thirty-four of these with diabetic or idiopathic gastroparesis were excluded. The remaining patients included 67 with GERD and 36 with GERD-DGE. GERDDGE patients were more likely to be female and diabetic compared to GERD patients ( $p=0.03$ and 0.02 respectively). The GERD-DGE group had significantly higher gastric emptying halftime compared to the GERD group $(p=<0.001)$. Reflux was the primary reason for evaluation for both GERD and GERD-DGE groups. Mean HRQL score was 23 in the GERD group and 16 in the GERD-DGE ( $\mathrm{p}=\mathrm{NS})$. Mean GCSI was 24 for both groups $(p=N S)$. There was no difference between the GERD and GERD-DGE groups in any of the GCSI subcategory scores.

In terms of symptom scoring, patients with GERD and GERD-DGE do not have significantly different scores. The GCSI is not a good predictor of DGE in patients who present with GERD. A GES is still the best test to evaluate gastroparesis in this patient demographic. Our clinical practice is to perform GES on all patients who present with GERD and clinical concern for DGE.

\section{P445}

Comparison Between a New Gasless Abdominal Wall-Lifting Laparoscopic Device and Conventional CO2 Pneumoperitoneum in Elderly Gastric Cancer Patients: A Prospective Pilot Study

Jianxin Cui, Yunhe Gao, Hongqing Xi, Kecheng Zhang, Jiyang Li, Yingwen Cai, Xinxin Wang, Canrong Lu, Lin Chen; Chinese PLA General Hospital

Background: Elderly gastric cancer (GC) who underwent laparoscopic surgery often suffered cardiopulmonary overloading and complications due to carbon dioxide penumoperitoneum. The aim of this study was to validate a new gasless abdominal wall-lifting laparoscopic device (GAWLD) and comparison with conventional $\mathrm{CO} 2$ pneumoperitoneum (COP) approach.

Methods: In this pilot study, elderly GC patients (age $\geq 80$ ) cases underwent laparoscopic distal gastrectomy with newly-designed GAWLD $(n=20)$ or traditional COP $(n=20)$ were consecutively recorded. Peri-operative surgical parameters and intra-operative cardiopulmonary/anesthesia changes were repeatedly measured and analyzed.

Results: Patient baseline characteristics were comparable between the two groups. No conversion occurred in these two groups. During the procedure, the mean end-tidal $\mathrm{CO} 2($ ET-CO2) $(\mathrm{p}<0.05)$ and mean airway pressure was significantly elevated in COP group $(\mathrm{p}<0.01)$. The docking time was not significantly different (GAWLD vs COP, $12.4 \pm 5.6$ vs $10.2 \pm 4.9 \mathrm{~min}, \mathrm{p}=0.09$ ) and the operation time was also comparable $(175.6 \pm 67.7$ vs $198.0 \pm 52.1 \mathrm{~min}, \mathrm{p}=0.12$ ). The post-operative complication rate was similar $(\mathrm{p}=0.556)$ while one patient in the COP group has encountered $\mathrm{CO} 2$ penumoperitoneum relating complication. The return of bowl function was similar between these two groups while the post-operative hospital stay was shorter in the GAWLD group $(10.3 \pm 5.7$ vs $12.1 \pm 7.9$ day, $\mathrm{p}=0.207)$.

Conclusion: The GAWLD is safe and feasible in elderly gastric cancer patients. Moreover, it carries several advantages comparable to those of conventional $\mathrm{CO} 2$ pneumoperitoneum on perioperative parameters. Therefore, it could be regarded as an alternative for elder GC patients with high cardiopulmonary risk.
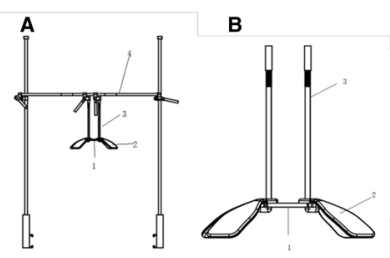

C

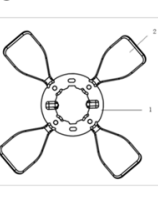

Figure 1 A schematic and photo of gasless abdominal wall-lifting laparoscopic device (GAWLD). (A: Overview of the GAWLD; B: Front view of the wall-lifting module; $C$ : cross sectional view of the wall-lifting module.) 


\section{P446}

Laparoscopic Distal Pancreatectomy for Giant Pancreatic Cystic Neoplasms -Is the Effort Worthwhile?

Srikanth Gadiyaram, Dr, Yashas H Ramegowda, Dr, Gaurav Singh, Dr; Sahasra Hospitals, Center of Excellence for Gastroenterology

Background: Laparoscopic distal pancreatectomy with (LDPS) or without splenectomy for cystic tumors in the body and tail has become the standard of care. We herein report three patients with giant pancreatic cystic neoplasms (GPCN) who underwent a distal pancreatectomy with splenectomy.

Methods: Records of patients who underwent LDPS for giant cystic tumors (Greater than $5 \mathrm{~cm}$ in diameter) by first author (SG) in the last three years were reviewed. All patients received pre-operative presplenectomy prophylaxis. LDPS was done using a five port technique. Resected specimens were extracted in an endobag. A tube drain was placed at the completion of the procedure adjacent to the transected border of pancreas.

Results: Three patients, one male (Case 1) and two female (Case2, Case 3) age 69, 25 and 64 years respectively were operated during the study period. The first two patients presented with pain abdomen which led to detection of the cystic tumors on Ultrasound (USG) imaging. Case 3 was asymptomatic and detected on USG during routine health check. MDCT was performed in all patients. The sizes of the tumors were 8,12 and $9 \mathrm{~cm}$ in maximum diameter respectively. All patients had the splenic artery stretched out around the cranial parts of the neoplasm. Splenic vein was stretched and displaced in the first two and thrombosed in the last patient. An endoscopic ultrasound (EUS) was performed in all patients and a preoperative diagnosis of serous cystic neoplasm was made in one and mucinous neoplasm in the other two. All patients underwent LDPS using a five port techique, the pancreas was transected using Endo GIA white cartridge and specimen extraction was done in an endobag by enlarging the umbilical port site, left subcostal incision by joining left midclavicular and anterior axillary ports and in one through a Pfannenstiel incision. All three patients made an uneventful postoperative recovery. Oral intake was started on first postoperative day and surgical drain was removed on postoperative day two in all. Hisopathology showed a serous cystadenoma, solid cystic pseudopapillary tumor and mucinous cystic neoplasm respectively. At a follow-up of 72, 48 and 42 months all patients are asymptomatic.

Conclusion: Laparoscopic distal pancreatectomy is feasible in patients with GPCN and offers the benefits of lesser postoperative pain and early recovery. Splenectomy was required in all patients because of technical reasons viz; gross distorsion of splenic vessels, thrombosis of splenic vein.

\section{P447}

\section{Our Stylized Procedure in Laparoscopic Nissen Fundoplication} for GERD Patients

Tatsushi Suwa, MD, PhD, Kenta Kitamura, MD, Tomonori Matsumura, MD, Ayato Obana, MD, Norio Mizugichi, MD, Mayuko Nakayama, MD, Kazuhiro Karikomi, MD, Motoi Koyama, MD, PhD, Yoshinobu Sato, MD, PhD, Ryuji Yoshida, MD, Hiroyuki Suzuki, MD, Shigeru Masamura, MD, PhD; Kashiwa Kousei General Hosp

Introduction: Laparoscopic techniques in anti-reflux surgery for GERD patients are still considered complicated by many surgeons. We have established our simple anti-reflux surgery procedure with less bleeding and less operative time.

\section{Surgical Procedure}

Setting: Our 5-trocar setting with patients in the reverse Trendelenburg's position is as follows: $12 \mathrm{~mm}$ trocar just below the navel (A), $5 \mathrm{~mm}$ trocar at the upper right abdomen for pulling up lateral segment of the liver, $5 \mathrm{~mm}$ trocar at the upper right abdomen, $12 \mathrm{~mm}$ trocar at the upper left abdomen (B), $5 \mathrm{~mm}$ trocar at the middle left abdomen (C).

\section{Step 1}

Under laparoscopic view, left part of the lesser omentum was cut with preserving the hepatic branch of vagus nerve. The right crus of the diaphragma has been dissected free from the soft tissue around the stomach and abdominal esophagus. In this step the fascia of the right crus should be preserved and the soft tissue should not been damaged to avoid unnecessary bleeding. After cutting the peritoneum just inside the right crus, the soft tissue was dissected bluntly to left side. Then the inside margin of the left crus of the diaphragma was recognized from the right side. In this part of the procedure, laparoscope uses trocar (A), the assistant uses trocar (B) to pull the stomach to left lower side and the operator's right hand uses trocar $(\mathrm{C})$.

Step 2

The branches of left gastroepiploic vessels and the short gastric vessels were divided with ultrasonic coagulation and dissection device. The left crus of the diaphragma was exposed and the window at the posterior side of the abdominal esophagus was widely opened. In this part of the procedure, laparoscope uses trocar (A) at the beginning of dividing left gastroepiploic vessels, trocar (B) when dividing short gastric vessels.

Step 3

The right and left crus are sutured with interrupted stitches to reduce the hiatus. From the right side, the fundus of the stomach is grasped through the widely opened window behind the abdominal esophagus. Then the fundus of the stomach is pulled to obtain a 360 degree "stomach-wrap" around the abdominal esophagus (fundoplication). Using 2-0 non-absorbable braided suture, stitches are placed between both gastric flaps.

Results: We have performed this procedure in 91 cases. A favorable outcome was assessed by radiograms performed on 4-5 postoperative day. The patients are mostly satisfied with the postoperative results. 
P448

\section{Circumference Heller Myotomy for Achalasia with Chest Pain}

Fumiaki Yano, MD, Nobuo Omura, Kazuto Tsuboi, Masato Hoshino, Se Ryung Yamamoto, Shunsuke Akimoto, Takahiro Masuda, Katsunori Nishikawa, Norio Mitsumori, Hideyuki Kashiwagi, Katsuhiko Yanaga; The Jikei University School of Medicine

Background/Aim: Although the typical complaint of patient with achalasia is dysphagia, nearly half of patients also experience chest pain, which in some cases may be intense enough to be mistaken for myocardial infarction. Further, while the main treatments for achalasia, Heller-Dor procedure (HD) and Per-oral endoscopic myotomy (POEM), have been shown to improve dysphagia, their effects on accompanying chest pain have not been satisfactory, which becomes a major issue in postoperative QOL of the patient. Here we report on the significance of the newly devised Circumference Heller myotomy (CHM) on the alleviation of chest pain.

Concept: One of the causes of chest pains in achalasia is thought to be strong contractions of the esophagus. Auerbach's plexus extends around the entire circumference of the esophagus between the inner circular muscle layer and outer longitudinal muscle layer in a weblike fashion, which stimulates the muscular layers. As the muscle layer incision in HD and POEM is placed only longitudinally, they fail to achieve complete division of the sprawling web of nerves. Therefore if, a partial lateral incision of CHM is made for the whole circumference in addition to the conventional longitudinal esophageal muscle layer incision, the top and bottom of the Auerbach's plexus can be fully severed, which may interrupt propagation of the esophageal contraction wave. In CHM, an incision is made in the whole circumferenceof the muscle layer, approximately $2 \mathrm{~cm}$ superior to the esophagogastric junction with a width of about $1 \mathrm{~cm}$, after the conventional longitudinal muscle layer incision for HD. With approval from the IRB, informed consent was obtained and the treatment outcomes were investigated in 10 patients who underwent CHM (7 female, average age 49.2 years, the preoperative morphologic type was St:Sg:aSg = 6:2:2, and grade of dilatation was I:II:III = 1:7:2) who were observed for 3 or more months post-surgery.

Results: The average operation time was $151 \mathrm{~min}$, the amount of blood loss was minimal in each case, and no intraoperative complications occurred. The median postoperative hospital stay was 4 days, which was equivalent to conventional HD and POEM. At 3 months after surgery, patients' dysphagia had improved in all cases. Chest pain also improved in all cases, of whom 5 cases $(50 \%)$ had complete disappearance of pain. The incidence of postoperative reflux esophagitis was $40 \%$ (4/10), which was slightly higher than HD, but were mild in all cases.

Conclusion: CHM may be effective for chest pain in patients with achalasia.

\section{P449}

Clinical Outcomes of Laparoscopic Versus Open Gastrectomy for Gastric Cancer: A Meta-Analysis

Caleb J Ba Mendoza ${ }^{1}$, Raúl Hernández Rubio ${ }^{1}$, Rafael Miret ${ }^{1}$, Madeline Jacobs ${ }^{1}$, Aliu Sanni, MD, FACS ${ }^{2}$; ${ }^{1}$ Philadelphia College of Osteopathic Medicine, ${ }^{2}$ Eastside Bariatric and General Surgery LLC

Introduction: According to the National Institute of Health and the National Cancer Institute, the yearly number of new cases and deaths from gastric cancer was 7.3/100,000 and 3.2/100,000, respectively. With a $30.6 \% 5$-year survival rate and an estimated 10,960 new cases in 2017, finding efficient ways of management is imperative. The safety of Laparoscopic Gastrectomy (LG) for benign lesions has been proven but not yet documented in cancerous lesion management. The aim of this study was to compare clinical outcomes of LG vs. Open Gastrectomy (OG) for gastric carcinoma.

Methods and Procedures: A systematic review was conducted through PubMed to identify relevant publications between 2008-2014 that compared LG and OG. Outcomes include postoperative morbidity and mortality, intraoperative bleeding and number of harvested lymph nodes. Results are expressed as standard difference in means with standard deviation. Statistical analysis was done using fixedeffects meta-analysis to compare the mean value of the separate groups (Comprehensive Meta-Analysis Version 3.3.070 software; Biostat Inc., Englewood, NJ).

Results: Five out of 411 articles were included and assessed in this meta analysis. Among the studies, 344 patients underwent LG and 288 underwent OG. Intraoperative bleeding $(0.727 \pm 0.065$, $\mathrm{p}=.001)$ was lower in the LG group. Morbidity $(\mathrm{OR}=1.189$, $\mathrm{p}=0.310)$, Mortality $(\mathrm{OR}=1.043, \mathrm{p}=0.937)$ and Lymph Node Harvesting $(0.079 \pm 0.064, p=0.214)$ were similar in both groups. Conclusion: Clinical outcomes using Laparoscopic Gastrectomy is as safe and possibly superior to Open Gastrectomy for gastric cancer surgery. 


\section{P450}

\section{Recurrent Jejunojejunostomy and Gastrojejunostomy Intussusception Complicated by Ischemic Bowel}

Sheena Say Hoon Phua ${ }^{1}$, Bo Chuan Tan ${ }^{2}$, Chun Hai Tan², Tzu-Jen $\mathrm{Tan}^{2} ;{ }^{\mathrm{I}}$ Khoo Teck Puat Hospital, National Healthcare Group, ${ }^{2}$ Khoo Teck Puat Hospital

Introduction: Gastrojejunostomy intussuception is a rare but serious complication of previous gastric surgery, in particular gastrojejunal bypass and Bilroth II gastrectomy. The incidence is less than $0.1 \%$. The incidence of double intussusception with a jejunoejunostomy intusscepting into a gastrojejunostomy, is even rarer, with only 3 similar cases in literature thus far.

Case Presentation: We present the case of a 55 year old gentleman with previous gastrojejunostomy bypass due to chronic duodenal ulcer, and jejunojejunostomy subsequently for efferent loop obstruction, who was referred to us for intestinal obstruction. CT abdomen and pelvis suggest intussuception of small bowel into the stomach. He underwent an emergency laparotomy with reduction of small bowel intussuception with good recovery after. He developed abdominal pain again 2 months later and CT investigation revealed possible ischemic bowel secondary to jejunojejunostomy and gastrojejunostomy intussusception. He underwent exploratory laparotomy, distal gastrectomy with en bloc resection of gastrojejunostomy and jejunojejunostomy roux-en-y reconstruction, and recovered uneventfully post operatively. We postulate this gentleman predisposition for double intussusception could be a result of the wide gastrojejunostomy and the proximity of the gastrojejunostomy to jejunojejunostomy.

Conclusion: Double intussusception with jejunojejunostomy into gastrojejunostomy is an extremely rare complication of gastrectomy and gastrojejunostomy. A high index of suspicion is needed to detect it and early surgical management is required. Surgical management with reduction alone may not be sufficient and interventions such as gastropexy or reconstruction should be performed. There is however no clear guideline currently on the best definitive management as this is a rare condition that is not frequently encountered.
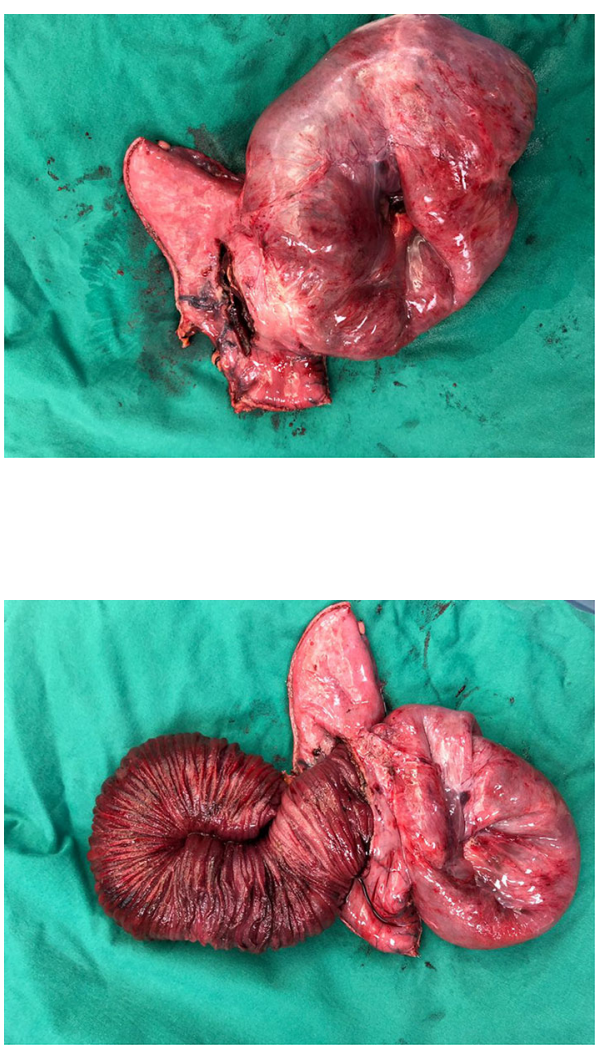

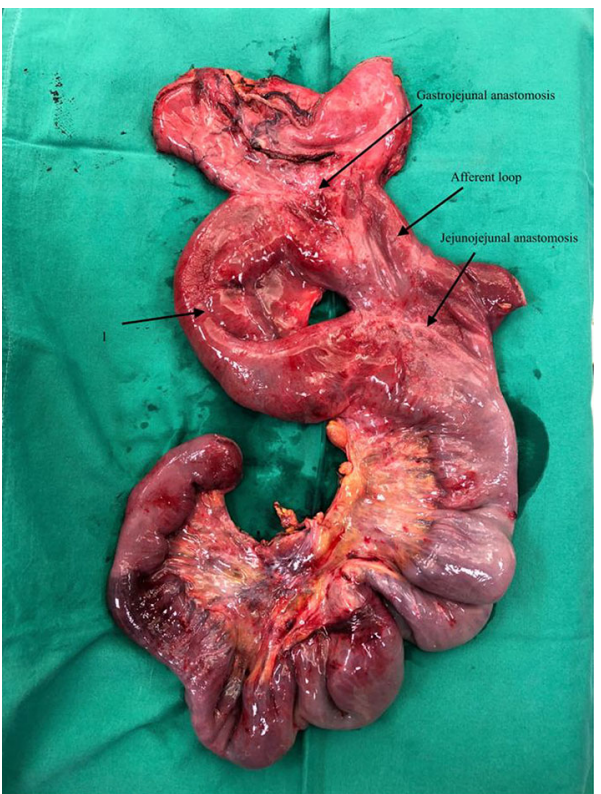


P451

Laparoscopic Splenectomy for Massive Splenomegaly by Anterior Approach - Tips \& Tricks

Sendhil Kumar, PhD, Piyush Patwa, V. Kandasamy; Gateway Clinics

Laparoscopic Splenectomy Anterior approach -The splenocolic ligament was dissected, followed by upward transection of the splenogastric ligament and short gastric using the harmonic scalpel. The splenic artery was identified along the upper margin of the pancreatic body and appropriately dissected. The arterial inflow was interrupted by applying two silk ties proximally and an endoclip to reduce spleen size and minimize blood loss during splenectomy. The splenic veins were dissected and securely ligated with silk ties and endoclips. The splenogastric ligament was transected starting from the upper pole, followed by transection of the splenocolic, splenorenal and splenophrenic ligaments. The secondary pedicular vessels were transected and ligated. Specimen retrieved out in toto through mini- Pfannenstiel incision. The presence or absence of accessory spleens should always be verified. A 22 years young unmarried female patient diagnosed to have Hereditary spherocytosis with Cholelithiasis who was on medical therapy for past 4 years. She was evaluated and referred for surgery by a haematologist.

The patient was vaccinated to decrease the risk of overwhelming postsplenectomy Infection (OPSI) by organisms such as Streptococcus pneumoniae, Haemophilus influenzae type B, and Neisseria meningitidis. All three vaccines were given to patient and she was also educated prior to discharge on the risk of OPSI \& her immunocompromised state.

\section{$\mathbf{P 4 5 2}$}

\section{Learning Curve from Laparoscopic to Robotic Heller Myotomy. A Myth?}

James Kurtz, Joseph Lim, Rene Borscheid, Edward Cho, Houssam Osman, Rohan Jeyarajah; Methodist Richardson Medical Center

Introduction: Heller myotomy is the standard surgical treatment for achalasia. Although robotic surgery is increasingly utilized in general surgery, the literature provides few guidelines on the learning curve for adoption. There have been no publications addressing the robotic learning curve for Heller myotomy. We sought to determine the learning curve for robotic Heller myotomy.

Methods: This was a retrospective review of all laparoscopic (group A) and robotic (group B) Heller myotomies performed by a single surgeon at one of two tertiary care hospitals from August, 2012 to April, 2018. The main outcome measures included estimated blood loss, operative time, conversion to open, length of hospital stay, and presence of leak. We also looked at these outcome measures as cumulative robotic experience increased.

Results: In total, 68 patients underwent laparoscopic or robotic Heller myotomy within the stated date range. Group A consisted of 14 patients that had a laparoscopic approach and group B consisted of 54 patients that had a robotic approach. There was no statistically significant difference in operative time, conversion to open, leak, or length of stay when comparing the two groups. As cumulative robotic experience increased, there was no statistically significant difference in estimated blood loss, operative times, conversion to open, leak, or length of stay. There was a trend toward shorter operative times and decreased EBL as robotic experience increased.

Conclusions: When a surgeon has performed the necessary case volume to master the laparoscopic approach to Heller myotomy, there is no learning curve to incorporate the robotic approach.

\begin{tabular}{lcc}
\hline & Group A $(\mathrm{n}=14)$ & Group B $(\mathrm{n}=54)$ \\
\hline median OR time & 92 & 93 \\
conversion to open & 0 & 0 \\
leak & 0 & 0 \\
median EBL & 30 & 40 \\
median LOS & 2 & 2 \\
\hline
\end{tabular}

\section{P453}

\section{Single Port Laparoscopic Management of Small Bowel} Obstruction with Intestinal Resection

Fernando Arias, MD, FACS ${ }^{1}$, Gabriel Herrera, MD ${ }^{1}$, Manuel Arrieta, $\mathrm{MD}^{2} ;{ }^{1}$ University Hospital Fundacion Santa Fe de Bogota -

University of the Andes, Bogota, Colombia, ${ }^{2}$ University Of Sabana, Colombia

Introduction: Laparoscopic surgery for small bowel obstruction have been accepted in guidelines. Reports indicates minimal invasive surgery results in less adhesion formation, less postoperative pain and shorter hospital stay. However, this approach has technical issues because small bowel dilatation can limitate operative space, which can lead to a higher risk of iatrogenic bowel injury. Althought, the role of single port laparoscopic surgery remains unclear, in this case report we managed closed loop bowel obstruction managed single port laparoscopic surgery and intestinal resection.

Methods: We present a 25 years old male with a history of annular pancreas with multiples procedures for correction in childhood and an open appendectomy 3 years ago. He presented to the emergency department with a 3 days history of general abdominal pain associated with nausea, vomiting and obstipation. Patient referred 2 similar episodes in the past. Abdominal CT showed a high grade obstruction with a transition point between small bowel segments. Informed consent was obtained and (patient) he was scheduled for surgery.

Results: We performed a single port laparoscopic founding a closed loop bowel obstruction with a necrotic segment due to a severe adhesions syndrome. Patient required complete adhesiolysis and resection of $40 \mathrm{~cm}$ of small bowel with a side by side anastomosis. The surgical time was 135 min and bleeding about $50 \mathrm{cc}$. On postoperative day 3 , the patient tolerated food and on day 5 was discharged. Follow up to 20 months with no new episodes of small bowel obstructions.

Conclusions: Minimal invasive management is still controversy for the management of small bowel obstruction, however, it can be used safely despite the number of adhesions founded, obtaining excellent outcomes in selected patients when is performed by an experienced surgeon. 


\section{P454}

The Participation of Surgeons In Diagnostic Workup of Gastroesophageal Reflux Disease Increases Utilization of Antireflux Surgery

Medhat Fanous, MD, FACS, Anja Jaehne, MD, David Lorenson, RN, Amanda Lambert, RN, Sarah Williams, RN; Aspirus Health System

Introduction: Patients presenting with putative symptoms of gastroesophageal reflux disease (GERD) are usually evaluated by gastroenterologists who perform the diagnostic workup and determine when to refer for surgical consideration. The multiple diagnostic studies can be overwhelming and leads to dropouts. In rural setting, without gastroenterology services, the surgeon can diagnose and perform surgical interventions. The aim of this study is to assess the completion of the required diagnostic studies and progression to surgical intervention.

Methods: This is a retrospective chart review of patients who presented with GERD symptoms between August 2015 and January 2018. Standardized workup included upper gastrointestinal study (UGI) and esophagogastroduodenoscopy (EGD) with concomitant wireless $\mathrm{pH}$ placement. High resolution impedance manometry was selectively offered to patients who reported dysphagia or had evidence of esophageal dysmotility on UGI.

Results: 429 patients were evaluated. Their data is listed in table 1. Proton pump inhibitors were used by $82.2 \%$ of patients. Required diagnostic workup was completed by $92.7 \%$ of all patients. Nearly $75 \%$ were suitable candidates for anti-reflux surgery. Approximately $2 / 3$ of these patients proceeded with anti-reflux surgery.

Conclusion: Surgical anti-reflux program with diagnostic and therapeutic capabilities results in high rate of completion of diagnostic workup and increase in utilization of anti-reflux surgery.

\section{Table 1}

Demographics

Female : Male

$283: 146$

Age (years)

BMI

$58.1 \pm 15$

Symptoms

Duration (years)

Classic

Extraesophageal

Both

Anti-secretory medicine

PPIs

Duration of PPIs (years)

Daily use of PPIs

BID use of PPIs

Combination with $\mathrm{H} 2$ blockers

GERD questionnaires (on PPIs)

GERD- health related quality of life (HRQL)

Heartburn score

Regurgitation score

Reflux Symptom index (RSI)

GERD symptom score (GERSS)

Diagnostic studies

Not completed

Completed

Surgical candidacy

Not a candidate

Candidate

proceed with anti-reflux surgery

continue medical therapy

$14.9 \pm 12.2$

$60 / 429(13.9 \%)$

$14 / 429(3.3 \%)$

$355 / 429(82.8 \%)$

$353 / 429(82.2 \%)$

$11 \pm 9$

$249 / 432(57.5 \%)$

$104 / 432(24.0 \%)$

$84 / 432(19.3 \%)$

$32.7 \pm 18.2$

$14 \pm 8.1$

$11.8 \pm 8.4$

$24.8 \pm 12.7$

$23.5 \pm 14.4$

$31 / 429(7.3 \%)$

$398 / 429(92.7 \%)$

$100 / 398(25.2 \%)$

298/398 (74.8\%)

$194 / 298(65.2 \%)$

$104 / 298(34.8 \%)$

\section{P455}

Lymphnode Dissection Along the Left Recurrent Laryngeal Nerve After Esophageal Stripping and Modified Circular Stapling for Safe Anastomotsis in VATS-E

Hiroshi Makino ${ }^{1}$, Hiroshi Yoshida, $\mathrm{PhD}^{2}$, Hiroshi Maruyama ${ }^{1}$, Tadashi Yokoyama ${ }^{1}$, Atsushi Hirakata ${ }^{1}$, Junji Ueda ${ }^{1}$, Hideyuki Takata $^{1}$, Yuta Kikuchi ${ }^{1}$, Takuma Iwai ${ }^{1}$, Masafumi Yoshioka ${ }^{1}$, Nobuyuki Sakurazawa ${ }^{3}$, Tsutomu Nomura ${ }^{2}$; ${ }^{1}$ Department of Surgery, Nippon Medical School, Tama-Nagayama Hospital, ${ }^{2}$ Department of Gastrointestinal and Hepato-Biliary-Pancreatic Surgery, Nippon Medical School, ${ }^{3}$ Department of Surgery, Nippon Medical School, Chiba-Hokusoh Hospital

Background: The working space in the upper mediastinum is limited and lymph-node(L.N.) dissection along the left recurrent laryngeal nerve is difficult despite of a clear operative view of the middle and lower mediastinum in prone position VATS-E(Video-Assisted Thoracoscopic Surgery of Esophagus). Anastomosis by a circular stapler in the narrow neck field is difficult. We report our technique of the L.N. dissection along the left recurrent laryngeal nerve and safe anastomosis. Methods

-Patients

One hundred thirty patients (27 in left lateral and 103 in prone position), with esophageal carcinomas underwent VATS-E, respectively.

-Methods

VATS-E in prone position At first the patients are fixed at semi-prone position and esophagectomy is performed in prone position that can be set by rotating and 5 ports are used at the intercostal space (ICS). Esophagectomy and the L.N. dissection are performed with pneumothorax by maintaining $\mathrm{CO} 2$ insufflation.

L.N. dissection around left recurrent laryngeal nerve Working space at the left upper mediastinal area for L.N. dissection around recurrent laryngeal nerve is limited in prone position. To obtain the space the residual esophagus is stripped in the reverse direction and retracted toward the neck after the stomach tube is removed through the nose.

Anastomosis At first the circular stapler is introduced into the gastric conduit and joined to an anvil, and close a little. And then a joined anvil is placed into the proximal esophagus and secured by means of a pursestring suture. The gastric conduit opening is closed by a linear stapler, and the anastomosis is completed.

Results

1. The rate of permanent and transient recurrent laryngeal nerve paralysis were $2.6 \%$ and $22 \%$, respectively.

2. The rate of anastomotic leak and postoperative pneumonia was $4.0 \%$ and $2.9 \%$,

Discussion

1. L.N. dissection along the left recurrent laryngeal nerve after esophageal stripping is available in prone position of VATS-E.

2. Our anastomotic technique is safe.

$104 / 298(34.8 \%)$


P456

\section{Systematic Review of Laparoscopic Partial Splenectomies in Adults}

Noeline Rajarajan, Julie Pepe, Paula Veldhuis, Sebastian De La Fuente; Florida Hospital Orlando

Introduction: Laparoscopic total splenectomy continues to be the operation of choice for lesions of the spleen. Total removal of the spleen, however, can result in severe early and late complications. Preservation of splenic parenchyma has been increasingly desired particularly when research has shown that only 20-30 percent is required to fulfill its immunological function and prevent the complications associated with asplenia. Laparoscopic partial splenectomy (LPS) continues to remain a marginally performed procedure and, despite its perceived benefits, the literature is still scare. In this study, we reviewed the current literature and discuss the feasibility of partial laparoscopic and robotic splenectomies.

Methods: A literature search was conducted on August 2017 for LPS using PubMed, Ovid, Clinical Key and Cochrane Library. The results were limited to material published from 2010-present, case reviews and studies which contained 2 or more patients, patients $>13$ years old. Demographic data, pathology of lesion, surgical technique, estimated blood loss, blood transfusion requirement, conversion rates and complications of surgery were extracted. Results: From the 28 publications that were retrieved after review, only 9 fulfilled the criteria for LPS. The total number of patients who underwent laparoscopic partial splenectomy were 85 ( 39 males and 46 females). The main pathologies encountered included cysts $(n=24)$ hemangiomas $(n=21)$, and trauma $(\mathrm{n}=21)$. The remaining etiologies comprised lymphangiomas, hamartomas, metastasis, metaplasia and an abscess. The mean operative time for a LPS was $144 \mathrm{~min}$ with documented times ranging from 80 to $226 \mathrm{~min}$. Average blood loss in the LPS group was $243 \mathrm{cc}$ (elective cases $274 \mathrm{cc}$ vs. emergency cases $174 \mathrm{cc}$ ). Despite this blood loss only $6 \%$ of elective cases required blood transfusion (4/60). Complications were seen in $16 \%$ of patients, $7 \%$ had fluid, $9 \%$ included atelectasis in 2 patients, one patient who developed a pulmonary embolism and one patient developed a venous thrombosis. Conversion to total laparoscopic splenectomy was $6 \%$ (5\% in the elective LPS group and $9.5 \%$ in the emergency group). There was only patient converted to an open approach

Conclusion: Minimally invasive partial splenectomy is a safe surgical procedure in both elective and emergency settings in selected patients and should be restricted to benign etiologies. LPS allows for preservation of the spleen with acceptable post-operative complications.

\section{P457}

\section{Laparoscopic Gastrectomy for Advanced Gastric Cancer}

Hung-Kuang Wei, MD; Koo Foundation Sun Yat-Sen Cancer Center, Taipei, Taiwan

From 2008 to 2016, we have 179 consecutive gastric cancer patients to recevie pure laparoscopic-appraoch gastrectomies. The mean age is 59 years-old and medium follow-up time are 30 months.

For the technical safety, the surgical complication rate is $13.4 \%(24 / 179)$ and the surgical mortality rate is $0.5 \%(1 / 179)$. For oncological outcome, the 5-year overall survival of this cohort is $87.1 \%$.

We devide these patients into two groups by surgical pathology result as early stage(AJCC 7TH stage 0 \& stage I) and advanced stage(stage II, III, IV). There are 101 and 78 patients in each groups, respectively. The total gastrectomy procedures are been perfomed more in advanced stage group $(\mathrm{n}=19$, $\mathrm{p}=0.0004)$. The surgical complication rate had no significant difference. $(11.85 \% \& .11 .54 \%, p=0.519)$ in two groups.

When we devide patients into different time-periods(early(2008 2012) and late(2012 2016)), there are obviously higher percentage of advanced stage surgeries $(54.5 \%)$ been perfomred in the late period than only $28.4 \%$ in the early period.

Laparoscopic-approach gastrectomy for gastric cancer patients seem not increase complication rates after accumulating adquate experiences.

\section{$\mathbf{P 4 5 8}$}

Complications and Readmission Following Same Day Discharge After Elective Laparoscopic Nissen Fundoplication

Tarik K Yuce, MD ${ }^{1}$, Ryan J Ellis, MD, MS ${ }^{1}$, Ryan P Merkow, MD, $\mathrm{MS}^{1}$, Nathaniel J Soper, $\mathrm{MD}^{2}$, Karl Y Bilimoria, MD, $\mathrm{MS}^{1}$, David D Odell, MD, MS ${ }^{1}$; ${ }^{1}$ Surgical Outcomes and Quality Improvement Center, ${ }^{2}$ Department of Surgery, Northwestern Memorial Hospital

Introduction: Traditionally, laparoscopic Nissen fundoplication (LNF) was associated with inpatient hospitalization. More recently, insurers and health systems have pushed for same day discharge. However, surgical outcomes in these patients are poorly understood. We describe the frequency of complications and readmission after same day discharge following elective Nissen fundoplication.

Methods and Procedures: The American College of Surgeons National Surgical Quality Improvement Program (ACS-NSQIP) database was used to identify all elective LNF from 2012 to 2016. Patients discharged on the day of surgery were compared to those discharged on postoperative day (POD) 1 or 2 . Outcomes studied included 30-day readmission and death or serious morbidity (DSM). Associations between same day discharge and both DSM and 30-day readmission were assessed using multivariable logistic regression. Readmission was further analyzed using Kaplan-Meier failure estimates and log-rank tests for equality.

Results: Of 7,734 patients who underwent elective LNF, 568 (7.3\%) were discharged on the day of surgery. Patients were less likely to be discharged on the day of surgery if they had an ASA class $>$ III $(26.8 \%$ vs $36.6 \%$, $\mathrm{p}<0.001)$, preoperative history of COPD $(2.3 \%$ vs $4.6 \%, \mathrm{p}=0.011)$, preoperative steroid use $(2.1 \%$ vs $4.8 \%, \mathrm{p}=0.004)$, or dyspnea $(4.9 \%$ vs $9.0 \%$, $\mathrm{p}=0.001)$. On multivariable analysis, there was no association between day of discharges and DSM (OR 0.91, 95\% CI 0.36-2.29) or 30-day readmission (OR $0.97,95 \%$ CI $0.62-1.52$ ). While patients discharged on the day of surgery had higher unadjusted rates of early readmission, Kaplan-Meier analysis showed no difference in rates of hospital readmission between groups at 30-days from discharge $(3.9 \% \mathrm{v} 4.1 \%, \mathrm{p}=0.325$, Figure 1$)$. The most common 30-day readmission diagnoses overall were nausea/vomiting $(11.5 \%)$, dysphagia (11.1\%), and abdominal pain $(5.7 \%)$.

Conclusions: Same day discharge of patients following Nissen fundoplication was not associated with an increase in perioperative complications or 30-day readmission. However, an increase in early readmission following same day discharge may be present. Furthermore, interventions focused on improvement of postoperative abdominal pain along with prevention of nausea and vomiting may provide an opportunity to improve readmission rates. Future work focused on identifying patients least likely to have early readmission after same day discharge is warranted.

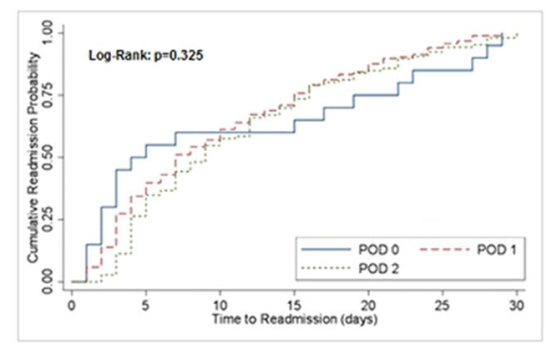

Figure 1. Time to Readmission following discharge based on Kaplan-Meier Method 


\section{P459}

\section{Gastric Volvulus in an Elderly Patient with Recurrent Hiatal Hernia Treated with Laparoscopic Surgery}

Maria K Bejar, MD, Adolfo Leyva, MD, Eduardo Gonzalez, MD, Mario Rodarte, MD; Tecnológico de Monterrey, Escuela de Medicina y Ciencias de la Salud - SSNL. Monterrey, N.L

Introduction: Gastric volvulus is an abnormal rotation of the stomach along its longitudinal or transverse axis. $80-90 \%$ occur in adults after the 5th decade of life and have a 30-50\% mortality.

Objective: case report of a patient with acute gastric volvulus treated with succesful laparoscopic surgery.

Summary: a 64 year old male with pathologic history of type II hiatal hernia $(\mathrm{HH})$ and Gastroesophageal Reflux Disease, treated with laparoscopic fundoplication and cruroplasty. He attends the hospital due to intense thoracoabdominal pain for the past $12 \mathrm{~h}$, nausea and dyspnea. He refers having ocassional morning cough, pyrosis and dyspnea on exertion for the last two months. He has a BMI of $26 \mathrm{~kg} /$ $\mathrm{m}^{2}$ and presents tachypnea, tachycardia, left lung base hypoventilation, epigastric abdominal pain, no peritoneal signs and diminished peristalsis. Laboratory tests were normal. The Chest X-ray (Fig 1a) showed an air-fluid level in the left hemithorax. In the abdomen Computed Tomography (CT) (Fig 1b) a type III HH was observed with total protrusion of the stomach through the diaphragm and rotation of the same in its longitudinal axis. Upper endoscopy was made, but the scope couldn't advance. A laparoscopic approach was performed, herniated stomach (Fig 2) was reduced and the previous fundoplication dismantled. During hernia reduction, a left tension pneumothorax was produced so a pneumo-kit was placed, resolving the problem. The greater curvature of the stomach was ischemic and it was incidentally perforated (Fig 3), having gastric material contaminating the abdominal cavity. A subtotal gastrectomy and an omega gastro-jejunal anastomosis were made (Fig 4). The hermeticity of the anastomosis was verified through an air test, with no leaks observed. The postoperative course was uneventful.

Discussion: Gastric volvulus is an uncommon condition; $70 \%$ are due to secondary causes. $60 \%$ are organo-axial volvulus. Borchadt's triad occurs in $70 \%$ of the acute cases. CT has a $100 \%$ sensitivity. Upper endoscopy should be done in the operating room to try to detorse and reduce the volvulus. The current surgical technique includes dissection and excision of the herniary sac, reposition of the gastroesophageal junction $2-3 \mathrm{~cm}$ below the diaphragm to diminish recurrence and cruroplasty. Mesh placement is recommended except when there is contamination.

Conclusions: the laparoscopic approach is the first choice of treatment for gastric volvulus, due to better results in terms of intrahospitalary stay, patient satisfaction and symptomatic resolution. A gastric resection is justified when there is ischemia, necrosis or perforation secondary to strangulation.

\section{$\mathbf{P 4 6 0}$}

Surgeon Management of PPIs Post Heller Myotomy

Andrew W White, MD, Taylor Wearda, Carl Westcott, MD; Wake Forest Baptist Hospital

Introduction: Most patients who present for achalasia surgery are already on PPI therapy. Achalasia surgery can cause reflux and the immediate cessation of PPIs can cause rebound hyperacidity. GI and family physicians often do not understand these nuisances as they relate to post-operative PPI need. These factors promote PPI overuse in post surgery patients. Presently, there is growing evidence that prolonged PPI therapy has significant and avoidable side effects. We examined a surgeon supervised PPI treatment course for post Heller myotomy patients.

Methods and Procedures: A retrospective chart review was performed for a single surgeon over the last four years. We measured PPI use pre and post-operatively where the surgeon actively managed PPI use. They were followed at 4 month intervals in the surgery clinic or by phone to determine the need for PPI and to wean as permitted. Those patients who remained on a PPI were again contacted and a weaning program initiated as symptoms allowed.

Results: A total of 54 patients were treated. The average age was 57.4 years with equal distribution between male and female. 53 of 54 underwent Dor fundoplication. $62.9 \%$ of patients were on PPIs preoperatively. At the initial post-operative visit, $51.9 \%$ of patients used PPIs and $20 \%$ of patients were using a PPI BID. 17 patients were lost after the initial post-operative visit and 11 of these patients remained on a PPI. The average follow up for all patients was 7.3 months. At their most recent visit, $37 \%$ of patients remained on a PPI with $14.8 \%$ of patients taking a PPI BID. 15 of the 20 patients who remained on PPIs after their most recent follow up were reached by phone to attempt weaning off of PPIs. 6 were weaned successfully leaving 14 patient or $25 \%$ of patients remaining on PPIs. These patients were given a weaning plan for discontinuing their PPIs and are being followed actively.

Conclusion: Long term follow up with a surgeon for patients undergoing Heller myotomy increases the opportunity to wean PPIs. We were able to wean PPI use in our patient population from $51.9 \%$ at the initial post-operative visit to $25.9 \%$ at most recent contact. We therefore believe that the patient's surgeon is often the best suited provider for PPI management post Heller myotomy. 


\section{P461}

Long-Term Dysphagia Resolution Following Poem Versus LHM in Patients with Achalasia

Grace E Shea, BA, Kevin D Davies, MS, Manasa Venkatesh, MA, MS, Sally Jolles, MA, Tyler M Prout, MD, Amber Shada, MD, Jacob A Greenberg, MD, EdM, Anne O Lidor, MD, MPH, Luke M Funk, MD, MPH; University of Wisconsin Hospital and Clinics, Madison, WI

Introduction: The objective of this study was to evaluate patient-reported outcomes for achalasia patients who underwent either peroral endoscopic myotomy (POEM) or laparoscopic Heller myotomy (LHM) and had at least one year of follow-up. LHM has historically been considered the gold standard treatment for achalasia. However, POEM is a less invasive procedure and offers a quicker recovery. Although some studies have compared short-term outcomes of LHM to POEM, the strongest predictors of long-term dysphagia resolution are unclear.

Methods and Procedures: Data from our single academic institutional foregut database were used to identify achalasia patients who underwent LHM or POEM over a 9-year period. Postoperative outcomes were assessed via telephone for patients with at least one year of follow-up using Eckardt dysphagia scores. Electronic health record data were reviewed to extract patient characteristics and operative data. Patient age, sex, type of operation, and length of follow-up were included in a multivariable linear regression model with Eckardt score as the outcome.

Results: Our cohort included 125 patients (93 LHM and 32 POEM). Sixty-four patients completed a phone survey at the one year or greater time interval (response rate $=51 \%$ ). Mean Eckardt scores were 2.95 and 1.85 at a median follow-up of 48 and 12 months for LHM and POEM patients, respectively (an Eckhardt score $\leq 3$ is considered successful). On multivariable analysis, operative approach was not associated with a statistically significant difference in dysphagia outcomes.

Conclusions: POEM and LHM were associated with similar rates of dysphagia resolution at a median of 3 years of follow-up. Both procedures should be considered durable treatment options for achalasia.

Table 1. Bivariate and Multivariate Predictors of Postoperative Dysphagia

\begin{tabular}{llll}
\hline Variable & & $\begin{array}{c}\text { Bivariate } \\
\text { Analysis* } \\
\beta \text {-coeff } \\
\text { p-value) }\end{array}$ & $\begin{array}{l}\text { Multivariate } \\
\text { Analysis* } \\
\text {-coeff } \\
\text { (p-value) }\end{array}$ \\
\hline Sex (N, \%) & & $0.14(0.81)$ & $-0.20(0.74)$ \\
Male & $36(56.3)$ & & \\
Female & $28(43.8)$ & & \\
Age (mean, SD) & $56.2(16.9)$ & $0.01(0.65)$ & $0.01(0.48)$ \\
Procedure Type (N, \%) & & $1.10(0.07)$ & $1.21(0.09)$ \\
POEM & $21(32.8)$ & & \\
LHM & $43(67.2)$ & & \\
Follow-up interval & 29.5 & $0.01(0.54)$ & $0.0001(0.99)$ \\
$\quad$ (months) & 12 & & \\
POEM & 48 & & \\
LHM & & & \\
\hline
\end{tabular}

*The reference group consisted of male sex and POEM as an operative approach.

\section{P462}

Is Barium Esophagram Enough to Guide Operative Technique for GERD Patients? Comparison of Esophageal Motility found on Barium Esophagram to High Resolution Manometry

Robert Roether, Amy Banks-Venegoni, MD, Brittany Kern, MD, David Scheeres, MD; Spectrum Health/Michigan State University

Background: Practice patterns amongst surgeons in the preoperative evaluation of Gastro-Esophageal Reflux Disease (GERD) and the type of anti-reflux fundoplication procedures performed vary widely. The Esophageal Diagnostic Advisory Panel recommends tailoring the type of anti-reflux fundoplication based on findings of the esophageal peristaltic wave and degree of esophageal dysmotility. High resolution manometry (HRM) is the gold standard for diagnosing esophageal dysmotility. Many surgeons, however, only use a barium esophagram (BE) to identify the presence of esophageal dysmotility and use its diagnostic findings to guide the type of fundoplication performed.

Aim: To determine if barium esophagram alone is sufficient to diagnose esophageal dysmotility when compared to high resolution manometry.

Methods: This is a retrospective review of patients that underwent a laparoscopic full or partial fundoplication by a single surgeon from 10/1/2015 to 7/1/ 2018. All HRM reports were blinded and read by an independent surgeon with an inter-rater reliability of $100 \%$.

Results: There were 65 patients total, 30 patients had both BE and HRM studies to make a comparison. None of the 30 patients $(0 \%)$ had dysmotility identified on both manometry and BE. Ten of $30(33.3 \%)$ had dysmotility identified on HRM without identification on BE. Five of $30(16.7 \%)$ had dysmotility on BE, but no dysmotility on HRM. Half of the patients had negative findings for dysmotility on HRM and BE. Utilizing HRM as the gold standard, BE had a Sensitivity of $0 \%$ (CI $0 \%-27.8 \%$ ), Specificity of $75 \%$ (CI $53.1 \%$ - $88.8 \%$ ), Positive Predictive Value of $0 \%$ (CI $0 \%-43.4 \%$ ), and Negative Predictive Value of $60 \%$ (CI $40.7 \%-76.6 \%$ ). The accuracy of BE was $50 \%$.

Conclusion: The data from this pilot study demonstrate that barium esophagram is not an effective diagnostic tool to determine if esophageal dysmotility is present. Implications of the results suggest that surgical decision making should not be based on barium esophagram alone, which has a PPV of $0 \%$ and accuracy of $50 \%$, and should be reinforced by acquisition of high resolution manometry. 


\section{P463}

The Tip of the Iceberg: An Unusual Case of Diaphragmatic Hernia Presenting as a Breast Abscess

Victoria Needham, MD, Alexandra Argiroff, MD, Diego Camacho, MD; Montefiore Medical Center

Introduction: While many diaphragmatic hernias (congenital or acquired) are asymptomatic, they may also present with subacute symptoms such as pain or dypsnea, or as a surgical emergency via organ strangulation. This case scenario illustrates an atypical presentation of a perforated viscus within the hernia sac leading to enterocutaneous fistula.

Case Report: An 83-year-old female with a surgical history inclusive of hiatal hernia repair and multiple ventral hernia repairs presented to the emergency room with purulence from her left breast, after drainage of a breast abscess earlier that day in clinic. Upon review of her recent mammogram noting bowel loops in the left chest, the patient was directed to the emergency room for further workup. CT scan was performed showing a colon-containing diaphragmatic and ventral hernia protruding between left-sided ribs, with an overlying left breast collection, concerning for enterocutaneous fistula (Picture 1). She had normal vitals, laboratory results, and a benign abdominal exam. Decision was made for operative exploration after preoperative medical clearance.
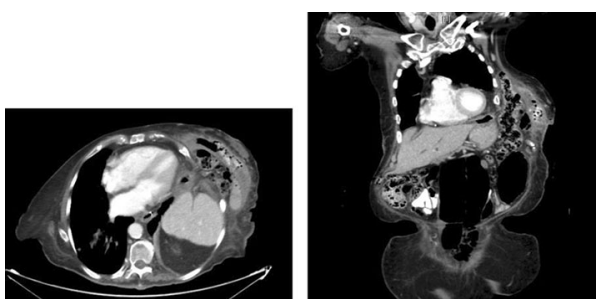

\section{Picture 1}

An open incision was made via an existing upper abdominal chevron scar, revealing a mesh directly under the subcutaneous tissue. The mesh was opened and extensive lysis of adhesions was performed in the left upper quadrant, revealing a colon-containing hernia sac through the diaphragm and through the left lateral ribs into the breast (Picture 2). The hernia sac was dissected down into the abdominal cavity, revealing extensive colonic diverticulosis and inflammation as well as a perforated diverticulum with surrounding abscess. The distal transverse colon and splenic flexure were completely mobilized, and a segmental colectomy was performed to include the area of involved pathology, with primary anastomosis. Remaining hernia sac was dissected to identify clean fascial edges, and the diaphragmatic defect was repaired primarily (Picture 3 ). The patient's postoperative course was uncomplicated.

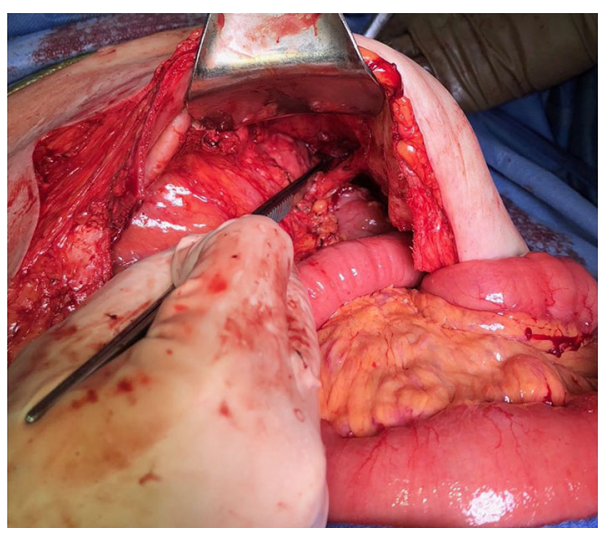

Picture 2

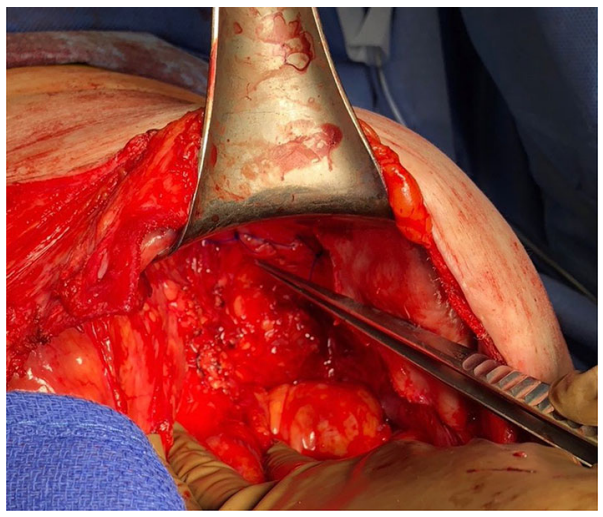

Picture 3

Conclusions: It is important to consider unusual presentations of diaphragmatic hernia, including pathologies arising from any of the organs contained in the hernia sac, such as illustrated in this case report. Upon operative exploration, the hernia sac and its contents should be dissected from the chest, the diaphragmatic defect cleared, and in the absence of contraindications (such as contamination in our case), a mesh should be placed to reinforce the repair. If technicaly feasible, a laparoscopic abdominal approach is preferred to an open approach. In certain cases, transthoracic approach may be necessary to release long-standing adhesions. 


\section{P464}

Intragastric Laparoscopic Resection of $8 \mathrm{~cm}$ Submucosal Gastric Mass to Preserve Total Gastric Volume and Avoid Partial Gastrectomy

Ryan Fairley, DO ${ }^{1}$, Danial Cottam, MD $^{2}$, Helmuth T Billy, $\mathrm{MD}^{1}$; ${ }^{1}$ Community Memorial Hospital, Ventura California, ${ }^{2}$ Bariatric Medical Institute, Salt Lake City, Utah

Large submucosal intragastric neoplasms can be difficult to resect endoscopically. Laparoscopic resection of gastric neoplasms often require partial gastrectomy with complex reconstruction. Complications including gastric paresis, gastric and bile reflux are often consequences of billroth I and II reconstructions. Preservation of total gastric volume when approaching submucsal lesions while obtaining complete resection with clear margins is a desireable outcome when approaching large intragastric lesions.

A 70 year old otherwise healthy man presented with a large, $6-8 \mathrm{~cm}$ gastric lesion, not amenable to endoscopic resection. presented for surgical and laparoscopic resection. The lesion was located along the lesser curve in the midbody of the stomach and extended from the distal cardia to the angularus incissura.

Laparoscopic resection was undertaken with the goal of preserving total gastric volume and avoiding partial gastrectomy necessitating reconstruction. Laparoscopic approach using a four trocar technique and internal liver retraction using a three hook trapeeze approach was perfromed. A left lateral subcostal $5 \mathrm{~mm}$ trocar, left mid abdominal $12 \mathrm{~mm}$ trocar, umbilical $12 \mathrm{~mm}$ trocar and right epigastric $5 \mathrm{~mm}$ trocar were employed. Intraoperative EGD confrimed the location of the mass in a submucosal location along the lesser curve.

Using a $3 \mathrm{~cm}$ gastrotomy on the anterior gastric wall between the angularus and the greater curve the gastric mass was successfully delevered through the gastrotomy. Using a $60 \mathrm{~mm}$ stapler the mass was successfully resected using two firings of the stapler across the mucosal base of the lesion. The lesion was successfully removed from the gastric wall without compromising lesser curve blood supply. The stomach was not resected and total gastric volume was preserved. The small gastrotomy was closed laparoscopically in two layers. Intraoperative completion endosco;y confirmed no intragastric bleeding along the line of resection and no leakage along the lesser curve or the site of the gastrotomy. Total operative time was $45 \mathrm{~min}$.

The pateint had an uneventful recovery and was tolerating clear liquids on post operative day one and was discharged on post operative day two. There were no complications and the patient had a full recovery. Final pathology was consistent with a benign large submucosal lipoma. Surgical margins were clear and the lipoma was completely resected

Resection of large submucosal gastric wall lesions can be resected without compromise of gastric wall volume

\section{P465}

Totally Laparoscopic Distal Esophagectomy with No Cervical or Thoracic Access: A Case Series

Erik A Green, MD, MPH, Kais Rona, MD, Christopher Ducoin, MD, MPH; Tulane University

Background: Esophagectomy remains a mainstay of treatment for esophageal malignancies as well as for refractory benign disease. Advances in laparoscopic and thoracosopic surgery have helped promote a variety of minimally invasive esophageal resection procedures. All of these, however, require a thoracic and/or neck incision. Here we describe a case series of patients treated with a totally laparoscopic esophagectomy technique.

Methods: A retrospective review of patients who underwent totally laparoscopic esophagectomy from April 2016 to April 2018 was conducted. Inclusion criteria comprised of benign stricture less than $5 \mathrm{~cm}$ from the GE junction, adenocarcinoma Siewart classification IIIII, and those with T1-T3 disease no evidence of nodal or metastatic disease.

Our standard approach consisted of four surgical ports placed in the upper abdomen. Intra-abdominal and mediastinal dissection was performed laparoscopically. Anastamosis in all 5 cases was achieved with an EEA stapler with the anvil passed trans-orally. Esophagoscopy was used as an adjunct, and a jejunal feeding tube was placed in all cases.

Results: Five patients underwent a totally laparoscopic distal esophagectomy procedure. Median age was 65 years (44-77 years) and the male to female ratio was $2: 3$. The indication for resection in 3 patients was benign distal stricture, refractory to more conservative treatments. Adenocarcinoma of the distal esophagus was the indication in the other 2 patients. In the 2 adenocarcinoma cases neoadjuvant chemoradiation was given, the initial staging was T2/ 3NOM0, and both were Siewert type II.

No cases required conversion to an open procedure. The mean operative time was $289 \mathrm{~min}$ (234-322 min). There was no 30-day mortality. Mean ICU stay was 12.8 days (2-32 days) and mean hospital stay 15.6 days (7-32 days). 3 of 5 patients had an uneventful post-operative course. 2 of 5 patients, including one case of benign stricture and one case of adenocarcinoma, experienced post-operative complications. One patient had respiratory failure with reintubation, and the other a contained leak, both necessitated a prolonged ICU stay. Neither of the two patients required reoperation or revision. R0 resection was achieved in both cases of adenocarcinoma.

Conclusion: We present a case series of 5 patients who underwent a totally laparoscopic transhiatal distal esophagectomy. Our preliminary results suggest it may be a safe alternative for distal benign strictures as well as for select patients with adenocarcinoma of the distal esophagus. Continued refinement of this technique may help decrease the morbidity and mortality of esophagectomy by avoiding a thoracic or neck incision. 


\section{P466}

Endoscopic and Fluoroscopy Guided EndoVive ${ }^{\circledR}$ Gastrojejunostomy Tube Placement for Patients with Esophageal Perforations

Jaideep Das Gupta, MD, James Verlanic, MD, Nida Bham, MD, Adam Delu, MD, Neelakantan Prakash, MD, Jess Schwartz, MD; University of New Mexico

Background: Esophageal perforation carries a high morbidity and mortality rate if not treated appropriately and aggressively. The treatment for esophageal perforation includes medical management, endotherapy or surgery. Yet, for all options, feeding access and decompression remains an issue. Gastrojejunostomy (GJ) tube is a practical option to allow post-pyloric feeds and gastric decompression. However, there is difficulty placing GJ-tubes in a short and timely fashion after management of esophageal perforations.

Methods: We present a hybrid approach of endoscopic and fluoroscopic guided GJ tube placement for patients with esophageal perforations. Our institution used the EndoVive ${ }^{\circledR}$ (Boston Scientific, Natick, MA) enteral feeding devices for our cohort of patients.

Results: We employed the technique of placing GJ-tubes within two days after the initial management of the esophageal perforation for six patients. All patients successfully tolerated the procedure and enteral feeds without any complications.

Conclusion: Hybrid method of endoscopic and fluoroscopy guided placement of EndoVive ${ }^{\circledR}$ (Boston Scientific, Natick, MA) GJ-tube is ideal for gastric decompression and post-pyloric feeding access shortly after the initial management of an esophageal perforation.

Key Words: esophageal perforation; gastrojejunostomy tube; enteral feeding access; EndoVive ${ }^{\circledR}$ (Boston Scientific, Natick, MA)

Abbreviations: GJ-tube - Gastrojejunostomy tube; G-tube - Gastrostomy tube; IR - Interventional Radiology; PEG - Percutaneous endoscopic gastrostomy; TTP - Through the PEG; J-tube - Jejunostomy tube; LOT Ligament of Treitz; GEJ - Gastroesophageal junction; NJ-tube - nasojejunal tubes.

\section{P467}

The Value of a Negative Preop Restech Test for Postop Results Gastrointestinal Function Testing Using the New Minimally Invasive Laryngopharyngeal PH Probe

Dolores T Mueller, Laura Knepper, Florian Gebauer, Wolfgang Schröder, Christiane J Bruns, Jessica M Leers, Hans F Fuchs; University of Cologne, Department of Surgery

Introduction: Laryngopharyngeal $\mathrm{pH}$-monitoring (Restech) is a relatively new reflux testing device that needs more validation. It was developed to detect both liquid and acidic gas vapor, and the pharyngeal probe placement may lead to more reliable results in patients with laryngopharyngeal symptoms.

A negative Restech result could be used as a screening instrument for gastroesophageal reflux and help decide whether patients should be included into a diagnostic pathway or benefit from a PPI therapy. Aim of this study is to examine the value of negative Restech test results using a large patient collective.

Methods and Procedures: In our esophageal center of excellence, more than 300 esophageal surgeries are performed annually. All patients undergoing minimally invasive or open upper gastrointestinal surgery are prospectively entered in our IRB approved database and undergo a routine check-up program with postoperative surveillance following surgery. All patients with benign disease received a complete diagnostic work-up for gastroesophageal reflux including symptom evaluation, endoscopy, 24-hour impedance $\mathrm{pH}$-metry, high resolution manometry and Restech. Only patients with a complete dataset and oropharyngeal reflux symptoms were offered inclusion into this study and evaluated using 24-h laryngopharyngeal and simultaneous esophageal impedance $\mathrm{pH}$-monitoring.

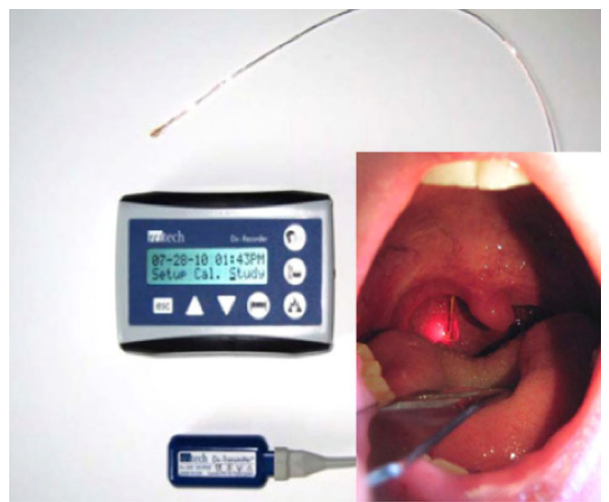

Results: A total of 155 [99 females] consecutive patients with suspected gastroesophageal reflux disease and oropharyngeal symptoms that were seen between 10/2013 and 08/2018 were included and underwent 24-h laryngopharyngeal with concomitant esophageal $\mathrm{pH}$-monitoring.

Restech evaluation was negative for reflux (= normal) in $55.5 \%(\mathrm{n}=86$, mean RYAN Score upright 2.5 [2.12-8.57], mean RYAN Score supine 2.2 [2.175.86]). In $45.3 \%$ of Patients with a normal Restech evaluation, 24-hour $\mathrm{pH}$ metry was pathologic $(n=39$, mean DeMeester Score 55.98 [14.8-254.9]). Nearly half of the patients with a normal RYAN and a normal DeMeester Score $(\mathrm{n}=20)$ did not complain about heart burn but only oropharyngeal symptoms. No patient with a normal RYAN Score and a normal DeMeester Score underwent antireflux surgery.

Conclusion: As shown in earlier research, Restech and 24-hour $\mathrm{pH}$ do not necessarily need to correspond. More women than men presented with oropharyngeal reflux related symptoms.

A negative Restech examination combined with a negative 24-hour $\mathrm{pH}$ metry may help to support the decision for or against antireflux surgery but is alone not suitable as a negative screening tool for GERD. 
P468

\section{A Novel Technique of Single-Port Thoracic-Assisted Totally Laparoscopic Esophagogastrectomy for Siewert Type 2 Adenocarcinoma of Esophagogastric Junction}

Wei Wang, Wenjun Xiong, Jin Wan, Xiaofeng Zhu; Guangdong Provincial Hospital of Chinese Medicine

Background: The incidence of Siewert type ? adenocarcinoma of esophagogastric junction (AEG) is increasing, especially in Asia, but laparoscopic surgery for type 2 AEG is not widely accepted due to the technical challenges and unknown prognosis. To address this problem, we developed a novel technique of single-port thoracic-assisted laparoscopic esophagogastrectomy.

Methods: From January 2017 to August 2018, 13 consecutive patients with advanced Siewert type 2 AEG underwent single-port thoracic-assisted laparoscopic radical esophagogastrectomy. After completly abdominal lymphadenectomy and mobilization of the stomach laparoscopically, the left diaphragm was incised 5 to $7 \mathrm{~cm}$, followed by a transthoracic assisted port was inserted into the thoracic cavity through the left axillary frontline at the sixth to eighth intercostal spaces. Then lower mediastinal lymphadenectomy and transection of the lower esophagus were completed with the transthoracic supplementary port. An intrathoracic Roux-en-Y esophagojejunostomy was performed by using the transthoracic assisted port, which was finally used for thoracic drainage.

Results: All procedures were completed successfully without surgical morbidity and mortality. The median operative time was 380.5 (255.0-480.0) $\mathrm{min}$. The time for reconstruction was 40 (29 to 52) $\mathrm{min}$ including 28 (20 to 35) $\mathrm{min}$ for intrathoracic esophagojejunostomy and 12 (10 to 17 ) min for extraperitoneal jejunojejunostomy. The median blood loss was $200(60.0-500.0) \mathrm{ml}$. The median tumor diameter was 4.2 (2.5 to 6.7 ) $\mathrm{cm}$ with a median esophagus invasion of $2.2(0.6$ to 3.5$) \mathrm{cm}$. The median number of lymph nodes harvest was 45 (37 to 60). The median numbers of lower mediastinal lymph nodes and metastatic mediastinal lymph nodes were 4(0-10)and 1(0-3, respectively. The rate of metastatic mediastinal lymph nodes was $24.4 \%$. Metastatic mediastinal lymph nodes were harvested from 6 patients $(6 / 12,50.0 \%)$. The median time of first flatus was 4 (2 to 7 ) days. The median hospital stay was 12 (10 to 17) days. One patient developed a thoracic infection and was cured with thoracic drainage and antibiotic treatment.

Conclusions: Our initial result suggested that this novel technique was safe and feasible for Siewert type 2 AEG, associated with the advantages of complete lower mediastinal lymphadenectomy and easy intrathoracic Roux-en-Y esophagojejunostomy by using the transthoracic assisted port.

\section{P469}

Control of Reflux Following Magnetic Sphincter Augmentation in Patients with Severe Esophagitis

Evan T Alicuben, MD, Jamil S Samaan, BS, Nikolai Bildzukewicz, MD, Kamran Samakar, MD, Caitlin C Houghton, MD, Adrian Dobrowolsky, MD, Kulmeet K Sandhu, MD, John C Lipham, MD; Keck Medicine of USC

Introduction: Magnetic sphincter augmentation offers an alternative to fundoplication for control of gastroesophageal reflux disease. The Food and Drug Administration has listed precautions for use of the device, but there is a growing literature demonstrating safety in those populations. To date, outcomes in patients with severe esophagitis have not been described. Our aim was to investigate the efficacy of the device in these patients with more severe reflux disease.

Methods: A retrospective review of all patients who underwent magnetic sphincter augmentation for gastroesophageal reflux disease was conducted. Demographics, pre-operative studies, operative characteristics and post-operative outcomes were studied. All patients underwent upper endoscopy as part of the pre-operative evaluation. Esophagitis was diagnosed based on direct mucosal evaluation and classified according to the Los Angeles system. Follow up was performed via symptom resolution, endoscopy, videoesophagram and $\mathrm{pH}$ testing.

Results: There were 24 patients, 10 males and 14 females with a median age 62 years (range 32-77 years) and median BMI 28.3 (range 23.3-44.3). 20 patients had Grade C esophagitis and 4 patients had Grade D esophagitis. Hiatal hernia repair was performed in 19 patients, 15 of which had a hernia size more than $3 \mathrm{~cm}$. The median length of stay was less than 1 day.

The median follow up was 1 year with 6 patients having follow up beyond 2 years. 16 patients had post-operative endoscopies performed, $12(75 \%)$ of which had resolution of their esophagitis. 3 patients had LA Grade B esophagitis and 1 patient had LA Grade C esophagitis.

15 patients (65\%) experienced post-operative dysphagia, 9 of which resolved and 6 patients having mild dysphagia at last follow up. 18 $(82 \%)$ patients were no longer using acid suppressing medications. 12 patients had post-operative $\mathrm{pH}$ studies completed. 6 patients had normalization of DeMeester score. There were no major complications. 2 patients had recurrent paraesophageal hernias and underwent repair.

Conclusions: Placement of the magnetic sphincter augmentation in patients with severe esophagitis was safe and effective. These findings add to the growing body of evidence demonstrating efficacy in patients with FDA precautions and should be used when counseling patients on treatment options. 


\section{P470}

1st Case Report :Total Laproscopic Transhiatal Esophagectomy in Esophageal Carcinoma

Danish Ali, Muhammad Adeel Kaiser, Anwar Zeb Khan, Muhammad Awais Amjad Malik, Muhammad Farooq Afzal; Lahore General hospital, Lahore

Objective: To share our experience of total laparoscopic transhiatal esophagectomy for esophageal carcinoma. Introduction Esophageal carcinoma is the eleventh most common carcinoma world wide.It has poor prognosis.Surgery remains the best choice for operable oesophageal carcinomas. Laproscopic transhiatal oesophagectomy has been reported as a safe alternative to open procedure in selected patients with esophageal carcinomas.

Case Report: In this case report we describe a case of $20 \mathrm{yrs}$ old female who presented with difficulty in swallowing and weight loss.Barrium swallow shows mucosal irregularity and a growth projecting into lumen from right lateral wall of middle $2 / 3$ of esophagus. Biopsy of lesion under endoscopy demonstrates a well differentiated squamous cell carcinoma of esophagus(Keratinizing type). We performed transhiatal laproscopic esophagectomy,esophagogastrotomy and pyloroplasty after neo adjuvant chemo-radio therapy. Histopathology analysis of tumor reveals histological characteristics typical of well differentiated squamous cell carcinoma of esophagus. 5 months after surgery endoscopic assessment reveals no evidence of recurrence of carcinoma. The patient is alive with no evidence of dysphagia and recurrence of tumor.

Conclusion: Comprehensive treatment of neoadjuvant chemo- radio therapy and transhiatal laproscopic esophagectomy appeared to be effective in this case. Laproscopic procedure.appears to be safe and minimally invasive which reduces the perioperative pain,,complication,and hospital stay with better outcome.It should be considered as a treatment of choice in esophageal carcinomas.

\section{P471}

Laryngopharyngeal pH Monitoring (Restech) May Predict a Successful Surgical Outcome for Regurgitation and Oropharyngeal Symptoms After Laparoscopic Antireflux Surgery

Dolores T Mueller, Laura Knepper, Wolfgang Schröder, Florian Gebauer, Christiane J Bruns, Jessica M Leers, Hans F Fuchs; University of Cologne, Department of Surgery

Introduction: Laryngopharyngeal $\mathrm{pH}$-monitoring (Restech) is a relatively new reflux testing device that needs more validation. It was developed to detect both liquid and acidic gas vapor, and the more consistent pharyngeal placement may lead to more reliable results, especially when laryngopharyngeal symptoms such as cough, hoarseness and globus sensation are present. Aim of this study is to determine if Restech can identify patients with a successful outcome for certain symptoms after antireflux surgery.

Methods and Procedures: In our esophageal center of excellence, more than 300 esophageal surgeries are performed annually. All patients undergoing minimally invasive or open upper gastrointestinal surgery are prospectively entered in our IRB approved database and undergo a routine check-up program with postoperative surveillance following surgery. All patients with benign disease received a complete diagnostic work-up for gastroesophageal reflux including symptom evaluation, endoscopy, 24-hour impedance pH-metry, high resolution manometry and Restech. Only patients with a complete dataset and oropharyngeal reflux symptoms were offered inclusion into this study and evaluated using 24-h laryngopharyngeal and simultaneous esophageal impedance $\mathrm{pH}$-monitoring.

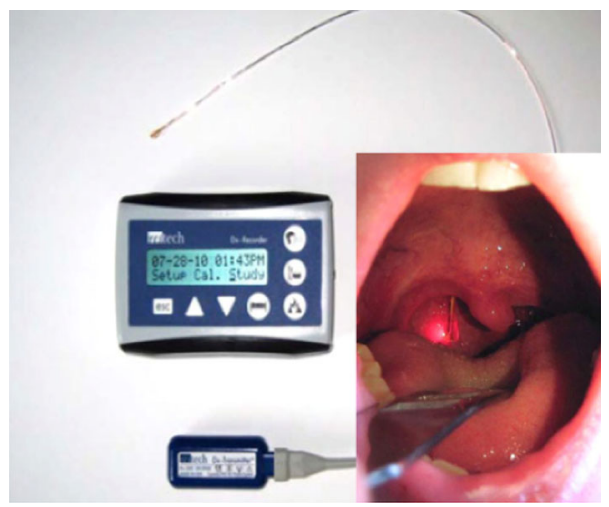

Results: A total of 155 [99 females] consecutive patients with suspected gastroesophageal reflux disease and oropharyngeal symptoms that were seen between 10/2013 and 08/2018 were included and underwent 24-h laryngopharyngeal with concomitant esophageal $\mathrm{pH}$-monitoring.

A total of 24 of these patients with laryngopharyngeal symptoms underwent laparoscopic antireflux surgery from 10/2013 - 02/2018 and had a complete follow up. Restech evaluation was abnormal in $62.5 \%(\mathrm{n}=15$, mean RYAN Score upright 121.73 [35.1-386.05], mean RYAN Score supine 5.74 [2.1750.62]). Two out of preoperatively 12 [16.7\%] patients with a pathologic Restech test complained about regurgitation after surgery versus 4 out of preoperatively 8 [50\%] patients with a normal Restech result. No patient with an abnormal Restech result out of preoperatively 11 complained about extraesophageal symptoms after the surgery versus 1 from preoperatively 4 [25\%] patients with a negative Restech.

Conclusion: An abnormal Restech result better identifies a successful outcome for regurgitation and extraesophageal symptoms after antireflux surgery. All patients had a complete resolution of extraesophageal symptoms after surgery. 


\section{P472}

\section{The Registry of Anti-reflux Surgery (ROARS) Results Of 1173 Patients at 1 Year}

Elisa Furay, $\mathrm{MD}^{1}$, SD Doggett, $\mathrm{PA}^{1}, \mathrm{~K}$ Freeman, $\mathrm{NP}^{2}$, Francis $\mathrm{P}$ Buckley, MD ${ }^{1}, \mathrm{R}$ Bell, MD ${ }^{2}$; ${ }^{1}$ Dell Medical School at The University of Texas at Austin 1, ${ }^{2}$ Institute for Esophageal and Reflux Surgery

Objective: Prospectively collected data from the Registry of Anti-Reflux Surgery (ROARS) were used to evaluate patient reported outcomes after laparoscopic magnetic sphincter augmentation (LMSA) and compare these to laparoscopic Nissen fundoplication (LNF), laparoscopic partial fundoplications (LPF), and all fundoplications (LNF + LPF).

Methods: The ROARS database is a prospectively collected multi-center quality registry. We prospectively evaluated 1173 patients undergoing either LMSA, LPF, or LNF. We compared each groups GERD-HRQL, regurgitation, dysphagia, and bloat scores preoperatively, at 6 months and at 1 year. Chronic acid suppression use at 6 months and 1 year postoperatively were compared. These variables were compared using a univariate analysis and statistical significance was determined by a p-value $<0.05$.

Results: Of a total of 1173 registry patients 638 underwent LMSA, 382 underwent LPF, and 153 underwent LNF. When comparing LMSA and LPF postoperative quality of life scores at 6 months and 1 year the LMSA had significantly improved 6 month and 1 year bloat score $(0.89 \pm 1.1$ versus $1.2 \pm 1.5 ; \mathrm{p}=.02,1.1 \pm 1.3$ versus $1.9 \pm 1.7 ; \mathrm{p}=.0001$ respectively) and 1 year GERD-HQRL score $(5.7 \pm 6.5$ versus $8.6 \pm 9.1 ; p=.002)$. Comparing LMSA to LNF, the patients who underwent LNF had improved 6 month dysphagia score $(1.8 \pm 2.2$ versus $.97 \pm 1.8 ; \mathrm{p}=.04)$. Comparing LMSA to all fundoplications, LMSA had significantly improved 6 month and 1 year HQRL scores $(5.2 \pm 6.7$ versus $6.5 \pm 7.9 ; \quad p=.47 ; 5.7 \pm 6.5$ versus $8.2 \pm 8.9 ; \mathrm{p}=.004$ respectively), as well as 6 month and 1 year bloat scores $(.89 \pm 1.1$ versus $1.2 \pm 1.4 \mathrm{p}=.02 ; 1.1 \pm 1.3$ versus $1.7 \pm 1.6 ; \mathrm{p}=.001$ respectively). There was no difference in the use of chronic acid suppression at 6 month or 1 year when comparing LMSA to any fundoplication group. Conclusion: Results from the ROARS database indicate that LMSA has improved or comparable quality of life metrics when compared to LPF, LNF and all fundoplications.

\section{P473}

Gastrectomy for Gastrointestinal Stromal Tumors: Does Robotic Assistance Affect Perioprative and Oncologic Outcomes?

Patrick J Sweigert, MD ${ }^{1}$, Emanuel Eguia, MD, MHA ${ }^{1}$, Marc H Nelson, MD ${ }^{1}$, Haroon Janjua, $\mathrm{MS}^{2}$, Gerard J Abood, MD, FACS ${ }^{1}$, Bipan Chand, MD, FACS, FASMBS, FASGE ${ }^{1}$, Paul C Kuo, MD, MS, MBA, FACS ${ }^{2}$, Marshall S Baker, MD, MS, MBA ${ }^{1}$; ${ }^{1}$ Loyola University Medical Center, ${ }^{2}$ University of South Florida

Introduction: Laparoscopic (LG) approaches to gastrectomy for gastrointestinal stromal tumors (GIST) have been shown to provide clinical outcomes similar to open (OG) approaches. The safety and oncologic efficacy of roboticassisted gastrectomy (RG) in the treatment of GIST has not been well studied. Methods: We queried the National Cancer Database to identify patients undergoing gastrectomy for GIST between 2010 and 2015. Patients with metastatic disease and tumor invasion into adjacent organs were excluded. Patients were stratified into OG, LG and RG cohorts in an intention-to-treat fashion. Multivariable logistic regression was used to compare margin status, 90-day mortality, 30-day readmission rate and length of stay (LOS) while controlling for patient age, sex, race, comorbidity score, tumor size and pathologic stage, cancer facility type and gastric resection type. Kaplan-Meier (KM) survival functions and multivariable cox proportional hazard models were used to compare rates of overall survival (OS).

Results: 22,665 (51.2\%) patients under went OG; $2,192(42.1 \%)$ LG, and 350 $(6.7 \%)$ RG. On univariate analysis, RG was more likely to be performed at Academic/Integrated Cancer Centers than either LG or OG $(61.7 \%$ vs $55.6 \%$, $\mathrm{p}=0.01 ;$ vs $52.4 \% \mathrm{p}<0.01)$. Patients undergoing RG and LG were more likely than those undergoing OG to have low grade/small tumors with $94.6 \%$ of RG, $89.8 \%$ of LG and $78.2 \%$ of OG cases done for stage I-II disease $(\mathrm{p}<0.01)$. The extent of resection was most often a partial gastrectomy ( $93.7 \%$ vs $98.0 \%$ vs $98.3 \%, \mathrm{p}<0.01)$. RG was associated with a lower rate of conversion to OG than LG $(5.1 \%$ vs $10.8 \%$, p < 0.01$)$. Unadjusted KM analysis (Figure 1) demonstrated a survival advantage for RG and LG relative to OG ( $p<0.01, p<0.01)$ but no difference in OS between RG and LG $(p=0.14)$. On adjusted analysis, patients undergoing RG had increased risk of having a margin positive resection than those undergoing either LG (OR 1.74; 95\% CI[1.07-2.82]), or OG (OR 1.69; 95\% CI[1.02-2.78]). RG (OR 0.32; 95\% $\mathrm{CI}[0.23-0.43], \mathrm{p}<0.01)$ and LG (OR 0.31; 95\% CI[0.26-0.36], p < 0.01) were associated with lower risk of prolonged LOS and LG with lower risk of readmission compared to OG (OR 0.69; 95\% CI[0.49-0.97], $\mathrm{p}<0.05$ ). There were no differences in adjusted OS.

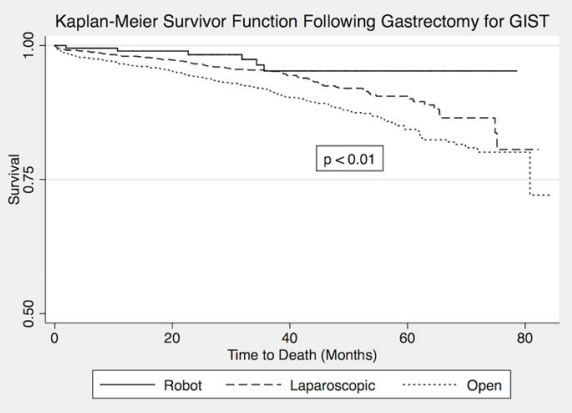

Conclusion: Compared to the OG, robotic-assisted partial gastrectomy for nonmetastatic GIST is associated with increased risk of margin positive resection that is independent of tumor size and grade but affords a lower risk of prolonged hospitalization and similar patterns of overall survival. 


\section{P474}

\section{Standardization of Surgical Technique in Laparoscopic Distal Pancreatectomy}

Yuji Morine, MD, Mitsuo Shimada, Satoru Imura, Tetsuya Ikemoto, Shuichi Iwahashi, Yu Saito, Shinichiro Yamada; Department of Surgery, Institute of Biomedical Sciences, Tokushima University

Background: Recent advances of diagnostic tool could contribute to the increasing detection of cystic or solid benign tumors, and even early malignancy. Laparoscopic distal pancreatectomy (Lap-DP) is being accepted as a feasible and safe surgical alternative to open distal pancreatectomy (DP) especially for such benign tumor located in the pancreatic tail and body. However, the expertise in Lap-DP is more difficult to acquire than that in other laparoscopic procedures, it is important to standardize the technique for performing Lap-DP. Hence, we standardized a technique of Lap-DP, and demonstrated the surgical outcomes in this study.

Standardization: Trocar placement: In supine position, after the insertion of $12 \mathrm{~mm}$ camera trocar on hypo-umbilical region, four other trocars are inserted into near bilateral costal arch $(5 \mathrm{~mm})$ and bilateral flank region $(10 \mathrm{~mm})$ in direct visualization.

Division of gastrocolic ligament: After retraction of liver left lobe, the omental bursa is opened, and splenocolic ligament is also dissected. Stomach is lifted and fixed to upper abdominal wall.

Individual division of splenic artery: after dissection of gastropancreatic fold, the splenic artery was identified and individually divided.

Dissection of inferior boarder of pancreas: The inferior border of pancreas is dissected from medial to left lateral, and retroperitoneum was dissected along lower level of the pancreas. The superior mesenteric vein (SMV) is exposed, and also the pancreas is mobilized over portal vein and SMV

Division of pancreas: Transection site of pancreas is covered by NEOVEIL sheet $^{\circledR}$. After 5 min clamping of transection site, pancreas was divided using autosuture device taking $5 \mathrm{~min}$, and then splenic vain $(\mathrm{SpV})$ is divided individually. (If tumor is located in pancreas tail, pancreas division including $\mathrm{SpV}$ is done at the left side of gastropancreatic fold.)

Pancreas and spleen mobilization: pancreas including tumor was dissected from retroperitoneum using anterior RAMPS (Radical antegrade modular pancreatectomy with splenectomy) method. (For malignant tumor, posterior PAMPS method is applied.)

Outcomes: Twenty-nine Lap-DP were performed for the following disease: $\operatorname{IPMN}(\mathrm{n}=7), \operatorname{MCN}(\mathrm{n}=3), \operatorname{SCN}(\mathrm{n}=5), \operatorname{SPT}(\mathrm{n}=1)$, neuroendcrine tumor $(\mathrm{n}=5)$, others $(\mathrm{n}=4)$ and pancreas cancer $(\mathrm{n}=4)$. The median blood loss and operative time were $74 \mathrm{~g}$ and $277 \mathrm{~min}$, respectively. Regarding short term surgical complication, grade $\mathrm{C}$ pancreatic fistula was observed in only one patients $(3.4 \%)$

Conclusion: Lap-DP is gaining acceptance as a standard approach for removal of various pancreatic diseases. Moreover, standardization of surgical procedure could establish Lap-DP as a safe and feasible procedure.

\section{P475}

The Role and the Feasibility of Single Incision Laparoscopic Surgery in the SBO Operation

Yozo Suzuki, MD, PhD, Masahisa Ohtsuka, MD, PhD, Manabu Mikamori, MD, PhD, Takuro Saito, MD, PhD, Kenta Furukawa, MD, $\mathrm{PhD}$, Mitsunobu Imasato, $\mathrm{MD}, \mathrm{PhD}$, Kentaro Kishi, $\mathrm{MD}, \mathrm{PhD}$, Masahiro Tanemura, MD, PhD, Hiroki Akamatsu, MD, PhD; Osaka Police Hospital

Background: Small bowel obstruction (SBO) has a variety of backgrounds and its operation procedure is often modified to meet the intraoperative requirements. Single incision laparoscopic surgery is a laparoscopic procedure which leaves a single small incision in navel, and has been reported to be less invasive than and as safe and efficient as the conventional multiport laparoscopic surgery. The role of SILS in the SBO operation has not been well studied.

Objective: The aim of this study was to examine the perioperative outcome and feasibility of single incision ileus operation (SIL-IO).

Methods: This is a retrospective study from a prospectively collected database. The investigation took place in a high-volume mutidiciplinary acute hospital in Japan. From July 2013 to June 2018. Patients who had operation for SBO caused by adhesion or strangulation were analyzed. Cancerous or perforated SBO, or intussusceptions were excluded.

Results: Out of consecutive 70 patients, 31 patients were started with SIL-IO. The background of the patients who had SIL-IO was as follows; median age was 69 (range; 20-94) year-old, 14 (45\%) were female, $26(84 \%)$ were American Society of Anesthesia Performance Status 0 or 1, Preoperative diagnoses were adhesion in $12(39 \%)$ and strangulation in $19(61 \%)$, the first SBO episode in $17(55 \%)$, the number of past abdominal operative history was 0 in $6(19 \%)$ and one in $18(58 \%)$. Severe adhesion was observed in $4(13 \%)$. Operation mode was as follows; small bowel resection in $13(42 \%)$, derotation in $5(16 \%)$, adhesiolysis in $10(32 \%)$, debanding in $3(10 \%)$. Perioperative outcome was as follows; the median operation time was 108 (38-390) min, bleeding Volume was $0(0-800 \mathrm{~mL})$, being completed by SILS in $23(74 \%)$ and converted to OS in $8(26 \%)$ (4; unable to detect responsible lesions, 2; difficult to perform procedure, 2; broad necrosis of intestine detected), intraoperative damage to organ occurred in 7 (serosal damage due to adhesiolysis). On univariate analysis, male $(\mathrm{P}=0.031)$, severe adhesion $(\mathrm{P}=0.016)$, and ASAPS $>l=3 \quad(\mathrm{P}=0.011)$ were independent risk factors of conversion. On multivariate analysis, male $(P=0.0097)$ and severe adhesion $(P=0.025)$ were independent risk factors of conversiion.

Conclusions: SILS in selected patients is a reasonable option in SBO. 
P476

\section{Laparoscopic Hiatus Hernia Repair: An Early Experience}

Ashish Prasad Rajbhandari, MS, Surgery; Nepal Medical College Teaching Hospital

Laparoscopic PHH repair (LPHHR) has emerged at the forefront of PHH treatment. Laparoscopic fundoplication now represents the gold standard in GERD therapy. Laparosocpic fundoplication has just been started in Nepal.A total of 10 cases were performed from January 2016 to june 2017 at Nepal Medical College Teaching Hospital. 2 patients presented with recurrent vomiting, 3 cases with the symptoms of GERD and dysphagia being the most common presenting symptom. All cases were female with age ranging from 12 -70 years. Endoscopic evaluation was done in all cases with grade III and Grdae II Hiatus hernia being the finding in 5 cases each.6 cases underwent Nissen fundopolication while 4 underwent Dor procedure. All cases were done through 5 ports and duration of surgery varied from $1-2 \mathrm{~h}$. None of the cases were of complicated type. Drains were put in all cases. All cases were started on liquid diet on first post operative day; drains were taken out on third post operative day and were discharged on fifth post operative day without any post operative complications. 2 cases required post operative dilatation on follow up with 1 cases requiring repeated dilatation for 3 months. In conclusion laparoscopic hernia fundoplication is a safe and relatively easy procedure and a good alternative to the open technique.

\section{P477}

\section{Laparoscopic Resection of Gastrointestinal Stromal Tumors Near Gastro-esophageal Junction}

Gaurav Singh, Dr, Yashas H Ramegowda, Dr, Srikanth Gadiyaram, Dr; Sahasra Hospitals, Centre of Excellence for Gastroenterology, Bangalore, India

Introduction: Organ preservation is a cornerstone in the surgical removal of gastro-intestinal stromal tumors (GISTs). Laparoscopic surgery and the current surgical technology have enabled the same in the management of GISTs near gastro-esophageal junction (GEJ), without the need of a total gastrectomy.

Methods and Procedures: This is a retrospective analysis from a prospective database of consecutive patients who were diagnosed to have GISTs located near GEJ (GEJ GIST) during last twelve years and underwent resection for the same by a single surgeon. GEJ GISTs were defined as those with an upper border within $5 \mathrm{~cm}$ of esophago-gastric line. Clinico-pathologic details, intraoperative course, short and long term outcomes were analyzed.

Results: A total of nine patients were planned for the laparoscopic resection of GEJ GISTs. All the procedures were completed laparoscopically without any intra-operative complications. The mean age of the patients was $54 \pm 17$ years with a male:female ratio of $8: 1$. The most common presentation was epigastric pain/discomfort (5), followed by an incidental imaging finding (2), malena (1) and dysphagia (1). The mean tumor size was $4.5 \pm 1.1 \mathrm{~cm}$. The mean distance between the upper border of the tumor and esophago-gastric line was $2.7 \pm 1.2 \mathrm{~cm}$. The most common procedure performed was wedge resection in six patients followed by eversion technique, intra-gastric approach (stapled resection) and proximal gastrectomy in one patient each. In two patients who underwent wedge resection, Toupet fundoplication was also performed. The median hospital stay was 3 days (range 1-5 days). The most common tumor risk stratification was low risk (4), followed by high risk (3) and intermediate risk (2). The median follow-up was 31 months (range 2-121 months). One of the patients presented with a solitary omental metastasis at one year four months follow-up which as resected following 3 months of imatinib therapy and showed myxoid degeneration. He is recurrence free at ten year follow-up. There was no evidence of recurrence in any other patient. The patient who underwent proximal gastrectomy had persistent reflux symptoms needing long term PPI therapy. Among the rest of the patients, only one patient who underwent wedge resection had mild reflux symptoms requiring intermittent PPI therapy.

Conclusion: Laparoscopic resection for GEJ GISTs is safe and feasible. The technique of resection utilized must be based on tumor factors and the surgeon's experience.

\section{P478}

Tumor Deposits Counted as Positive Lymph Nodes in Stage III Gastric Cancer: A Novel N Category in TNM Staging

Wenquan Liang, Kecheng Zhang, Hongqing Xi, Jianxin Cui, Aizhen Cai, Jiyang Li, Yunhe Gao, Xiaosong Wu, Xinxin Wang, Bo Wei, Lin Chen; Chinese PLA General Hospital

Background: Tumor deposits (TDs) are defined as satellite tumor nodules without histologic evidence of residual lymph node in peritumoral or regional lymph node areas. TD are markers of poor prognosis in patients with advanced gastric cancer. The accurate staging of gastric cancer is of utmost importance for treatment plan and prognosis. However, the role of perigastric TDs in gastric cancer TNM staging is poorly studied.

Methods: We established a novel N category strategy which counted tumor deposits as positive lymph nodes in TNM staging and evaluated its prognostic value for stage III gastric cancer. The positive lymph nodes in this novel $\mathrm{N}$ were calculated as the numbers of standard positive lymph nodes plus TDs. The database (2011-2014) was retrospectively reviewed for resectable stage III gastric cancer and stratified by presence of TDs. Univariate and multivariate analyses were performed to evaluate the overall survival (OS) for this novel $\mathrm{N}$ strategy.

Results: Out of 1987 patients, 285 (14.3\%) cases with TDs were identified. TDs were associated with pTNM-stage, N-stage, poor differentiation and vascular invasion. The median survival time of positive TD patients was significantly shorter as compared to negative TD patients 27 months vs. 41 months, $\mathrm{P}=0.02$ ). A significant positive correlation was observed between the number of TDs and tumor stage, and the number of TDs $>3$ was a powerful and independent predictor of prognosis. Further, the novel $\mathrm{N}$ strategy showed better differentiation ability in survival curves. However, cox regression multivariate analysis showed that both novel $\mathrm{N}$ and the standard $\mathrm{N}$ categories were independent prognostic factors and the performance of the novel $\mathrm{N}$ category did not reveal significant improvement compared to the standard one (c-index 0.611 vs. $0.623, \mathrm{p}=0.17$ ).

Conclusions: TDs are an independent predictive prognostic factor in stage III gastric cancer and may be appropriately considered as a form of positive lymph nodes. 


\section{P479}

Enhanced Recovery Protocol for Patients Receiving 3D Laparoscopy-Assisted Gastrectomy: High Compliance Rate Correlated with a Shortened Postoperative Stay

Kecheng Zhang, Canrong Lu, Jianxin Cui, Xiaohui Huang, Lin Chen; Chinese PLA General Hospital

Background: Chinese Society of Surgery and Chinese Society of Anesthesiology has jointly released the Chinese Expert Consensus and Clinical Practice Pathway for Enhanced Recovery After Surgery (ERAS) (2018 edition) in January 2018. We investigated the impact of enhanced recovery protocol on patients receiving 3D laparoscopyassisted gastrectomy in a single arm case series.

Methods: A prospectively maintained database was retrieved for 3D laparoscopy-assisted gastrectomy in ERAS settings. Patient demographics and perioperative outcomes were collected. ERAS protocol compliance rates were assessed. Patient nutritional status was evaluated by Nutritional Risk Screening 2002 (NRS-2002). Univariate and multivariate binary logistic regression analysis was performed to investigate influential factor for delayed discharge [postoperative hospital stay (PHS) $>7$ days].

Results: Between January and June 2018, a total of 84 patients receiving 3D laparoscopy-assisted gastrectomy in ERAS settings were included in the final analysis. The median age was 63.5 years [interquartile range, (IQR): 53.0-66.0] with 16 females (19.0\%) and 68 males $(81.0 \%)$. The median body mass index (BMI) was 24.0 (IQR: $21.0-26.0) \mathrm{kg} / \mathrm{m}^{2}$. Ten patients $(11.9 \%)$ had NRS-2002 scores $\geq 3$. The median operation time for 3D laparoscopy-assisted gastrectomy was 230.0 (IQR: 200.0-250.0) min and median blood loss was 100.0 (IQR: 100.0-100.0) ml. Four patients $(4.8 \%)$ was converted to open surgery. Eight patients $(9.5 \%)$ had postoperative complications $\geq$ grading II and mortality rate was $0 \%$. The median PHS was 7.0 (IQR: 5.0-9.0) days. The ERAS protocol compliance rate was 70.0\% (IQR: $50.0 \%-90.0 \%$ ). Univariate regression analysis revealed operation time, blood loss and ERAS protocol compliance were significantly associated with delayed discharge (all $\mathrm{P}<0.05$ ), while multivariate analysis showed only ERAS protocol compliance was statistically significant [odds ratio (OR): $0.65, \mathrm{P}=0.001$ ]. Correlation analysis has also demonstrated the PHS was negatively correlated with the ERAS compliance (Spearman $\mathrm{r}=-0.7, \mathrm{P}<0.001$ ). Patients with high compliance rates had significantly less hospital cost $(\mathrm{P}=0.031)$.

Conclusions: ERAS protocol is safe and feasible for patients receiving 3D laparoscopy-assisted gastrectomy. High ERAS compliance accelerates recovery, facilitates early discharge and saves cost.

\section{$\mathbf{P 4 8 0}$}

Study of Continuous Intraoperative Vagus Nerve Stimulation for Monitoring the Recurrent Laryngeal Nerve Function During Thoracoscopic Esophagectomy in a Prone Position for Esophageal Cancer

Soji Ozawa, Kazuo Koyanagi, Akihito Kazuno, Miho Yamamoto, Yamato Ninomiya, Kentarou Yatabe; Department of Gastroenterological Surgery, Tokai University School of Medicine, Japan

Introduction: Recurrent laryngeal nerve paralysis caused by lymph node dissection during thoracoscopic esophagectomy is one of the most important postoperative complications causing hoarseness, aspiration, pneumonia, or dysphagia. Continuous intraoperative vagus nerve stimulation for monitoring the recurrent laryngeal nerve function during thyroid or parathyroid surgery has been performed to avoid nerve injury. The aim of this study was to clarify whether such monitoring could be useful during thoracoscopic esophagectomy for esophageal cancer. To the best our knowledge, this is the first study of continuous nerve stimulation during thoracoscopic esophagectomy in a prone position for esophageal cancer.

Methods and Procedures: This study was a prospective, interventional, single arm, open-label study and was approved by the Institutional Review Board for Clinical Research, Tokai University. Twenty-eight patients with esophageal cancer were enrolled; all the patients were scheduled to undergo an elective thoracoscopic esophagectomy in a prone position with 3 -field lymph node dissection. The nerve function was continuously monitored using NIMResponse $^{\circledR} 3.0$ (Medtronic Japan) with an EMG endotracheal tube and an APS electrode attached to the vagus nerve. The endoscopic diagnosis of vocal cord movement and the clinical diagnosis of hoarseness were assessed before and after surgery.

Results: The median patient age was 68.5 years; 23 males and 5 females were examined. Twelve patients received neoadjuvant chemotherapy, and 2 patients received neoadjuvant chemoradiotherapy. pStage 0 , I, II, III, and IVa were observed in 4, 4, 12, 6, and 2 patients, respectively.Left recurrent laryngeal nerve paralysis occurred in 4 patients (14\%) whose EMG amplitude had decreased to below $50 \%$ of the initial value. The cause of left recurrent laryngeal nerve paralysis was presumed to be excessive traction during the thoracoscopic procedure in 1 patient and during the cervical procedure in 2 patients, and thermal injury caused by energy devices during the thoracoscopic procedure in 1 patient. All 3 patients, who were followed up for more than 3 months, recovered from the temporary left recurrent laryngeal nerve paralysis.

Conclusion: Continuous intraoperative vagus nerve stimulation for monitoring the recurrent laryngeal nerve function during thoracoscopic esophagectomy for esophageal cancer revealed that the potential causes of left recurrent laryngeal nerve paralysis included not only thoracoscopic lymph node dissection, but also cervical lymph node dissection. This monitoring method seems to be useful for identifying harmful procedures intraoperatively and for establishing safer procedures while performing lymph node dissection in the vicinity of the recurrent laryngeal nerve. 


\section{P481}

\section{Results of Colon By-Pass Surgery with Left Colon in Treatment of Esophageal Chemical Stricture}

Viet Trung Lam, MD, PhD, Dung Tien Tran Phung, MD, PhD; Cho Ray hospital, Ho Chi Minh City, Vietnam

Background: Esophagectomy to treat esophageal chemical stricture is often combined with high morbidity due to extended adhesion and cicatrisation around the affected esophagus. Aim: To evaluate the feasibility and results of colon by-pass surgery with left colon in treatment of esophageal chemical stricture.

Method: Prospective study. All cases with esophageal chemical stricture on the middle and lower third of esophagus with complete stricture were included. We use left colon with left colonic pedicle via substernal tunnel to perform an isoperistaltic bypass from cervical esophagus to stomach. Results: From 01/2015 to 01/2018, we performed 8 cases of colon by-pass surgery with left colon in treatment of esophageal chemical stricture at Choray hospital. Mean operation time was $4 \mathrm{~h}$. There was no postoperative anastomotic leak or stricture. Patients can eat well after operation without regurgitation. The controlled postoperative endoscopy and contrast study showed good anastomosis. With mean follow-up time of 22 months, there was no further complications noted. Conclusion: Colon by-pass surgery with left colon is feasible and effective in treatment of esophageal chemical stricture in selected patients. It improves patients quality of life without the need of esophagectomy. Longer follow-up time would be needed to further evaluate the role of this operation.

Keyword: colon by-pass surgery, esophageal chemical stricture.

\section{P482}

\section{Laparoscopic Gastrectomy for Gastric Cancer: A Single Center 12 Year Experience and Learning Curve}

Carlo Marino, MD, Cristián Jarry, MD, Vanessa Ahumada, Fernando Crovari, MD, Rodrigo Muñoz, Nicolás Quezada, Enrique Norero, Julián Varas, Pablo Achurra; Pontificia Universidad Católica de Chile

Background: Laparoscopic gastrectomy has shown to be a feasible approach for gastric cancer but involves long learning curves. As a learning curve progress, operating time, complications and conversion rates commonly diminish.

The aim of this study is to describe our experience and learning curve in laparoscopic gastrectomies in gastric cancer patients.

Methods: Retrospective study of our prospective database. Data from all gastric cancer associated gastrectomies between March 2005 and August 2017 performed in our center, were recorded. Surgical approach (open/laparoscopic), operative time (minutes), early complications, biopsy record and other perioperative variables were considered of interest. Our center's first laparoscopic gastrectomy for gastric cancer was done in 2005. Statistical analysis was performed, using non-parametric tests as needed.

Results: A total of 331 gastrectomies for gastric cancer were performed through the 12 years recorded. Patients mean age was 61 years. 38\% (128) of all gastrectomies were performed by a laparoscopic approach. During the first year (2005), only 4 out of 35 gastrectomies (11\%) were minimally invasive; this proportion showed a progressive growth, reaching 70\% (18/26) in 2017. As the number of cases increased, the learning curve showed improvement in terms of mean operative time (from 7 to $4 \mathrm{~h}$ ), early complications rate and conversion rate (from $10 \%$ to 5\%). Of note, most conversion cases at the beginning of the series were due to the size of the tumor and bleeding, while in the lasts, adherences and vascular involvement mainly explained conversion cases.

At the beginning of our experience, most laparoscopic cases where subtotal gastrectomies but in the final years the rate of laparoscopic approach in total and subtotal gastrectomies was similar (70\% and $60 \%$ respectively of all gastrectomies).

In terms of tumor size, first 50 laparoscopic cases were significantly smaller than the last 50 cases and also smaller than open approach cases. T0-2stage tumors represented $50 \%$ of the first 50 laparoscopic cases, $40 \%$ of the last 50 laparoscopic cases and only $25 \%$ of open cases (p: 0.001 ). Operative time, hospital stay and node count are also reported.

Conclusions: Laparoscopic surgery in gastric cancer has a long learning curve. Mean operative time, conversion rates and complications decrease as experience increases. In our experience, as the learning curve develops, more complex and larger tumors can be safely managed with a minimally invasive approach.

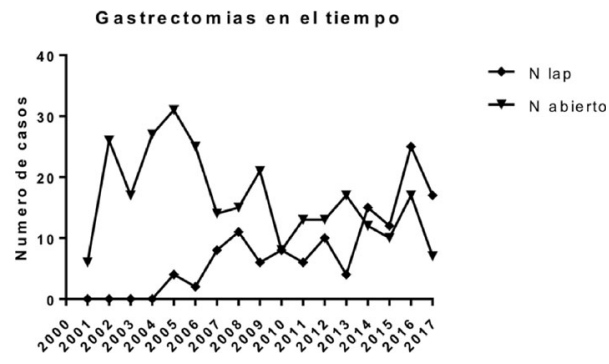

\section{P483} Heller Myotomy and Dor Fundoplication for Esophagogastric
Junction Outflow Obstruction: A Case Series

Timothy W Mansour, MD, William O Richards, MD; University of South Alabama

Esophagogastric Junction Outflow Obstruction (EGJOO) is a variant of achalasia that is characterized by increased lower esophageal sphincter (LES) tone in the setting of preserved peristalsis. It is a relatively new diagnosis since the advent of high resolution manometry (HRM) and its management has not been well studied. Heller Myotomy and Dor Fundoplication has been shown to be an excellent treatment for achalasia, but its use in EGJOO is uncertain. We present our experience with six patients who have received this operation for EGJOO. The primary presenting complaints of patients with manometric diagnosis of EGJOO are reflux (6/6 patients) and dysphagia (5/6). They all completed the Gastroesophageal Reflux Disease Health Related Quality of Life (GERD-HRQL) tool to quantify the severity of their symptoms both pre- and post-operatively. All of the patients who received the operation, had a marked improvement in reflux symptoms with a decrease from an average pre-operative GERD-HRQL score of 39 to an average post-operative score of 15.5. Four out of the five patients with dysphagia had complete resolution of dysphagia symptoms, with one patient reporting minimal change in dysphagia symptoms. Interestingly, the patient who reported minimal improvement had post-operative manometry that showed resolution of the EGJOO (normal peristalsis and IRP on the post-operative study). Four of the six patients studied reported complete satisfaction with the symptom improvement that the procedure provided. The other two patients (one neutral, one dissatisfied) both presented with very high pre-operative GERD-HRQL scores (average 47.5) and still had bothersome reflux symptoms (average GERD-HRQL 34) despite improvement in dysphagia. None of the patients in this series experienced post-operative complications. While EGJOO remains a poorly understood disease entity, Heller Myotomy and Dor Fundoplication seems to be an effective treatment for patients who present with dysphagia and reflux symptoms. 
P484

P486

\section{Novel Technique Using Intraoperative Impedance Planimetry for Magnetic Sphincter Augmentation Following Laparoscopic Sleeve Gastrectomy}

Keith J King, MD, Ragui Sadek, MD, FACS, Andrew M Wassef, BS; Rutgers Robert Wood Johnson Medical School

Background: Magnetic sphincter augmentation (MSA) using the LINX ${ }^{\circledR}$ Reflux Management System provides an alternative surgical option for patients experiencing medically refractory gastroesophageal reflux disease (GERD) following laparoscopic sleeve gastrectomy (LSG). A critical component in successful MSA, particularly in patients with a previous LSG, is appropriate size selection and placement of the LINX ${ }^{\circledR}$ device. We present a novel case of managing medically refractory GERD with laparoscopic MSA after LSG using intraoperative impedance planimetry $\left(\right.$ EndoFLIP $\left.^{\circledR}\right)$ to measure esophagogastric junction (EGJ) distensibility.

Methods: In September 2018, laparoscopic LINX ${ }^{\circledR}$ magnetic sphincter device placement with intraoperative impedance planimetry was performed in a 58 -year-old female with a DeMeester score of 51 who had undergone LSG 65 months prior. A small hiatal hernia was identified and repaired posteriorly in interrupted fashion. The sizing device was used to select a size 16 LINX $^{\circledR}$ device which was placed in proper anatomic alignment. Intraoperative impedance planimetry measurements were taken to confirm adequate distensibility and an adequate crural repair. An EGJ distensibility index of $2 \mathrm{~mm}^{2} / \mathrm{mmHg}$ was measured. Results: Laparoscopic MSA was well tolerated without complications and the patient was discharged home within $24 \mathrm{~h}$. She experienced some postoperative dysphagia which progressively improved during subsequent follow up. The severity and frequency of the patient's reflux, regurgitation, and epigastric pain were significantly improved postoperatively compared to preoperative evaluation with a reduction of her GERD score. Conclusions: Magnetic sphincter augmentation is a safe and effective surgical option for addressing medically refractory GERD in patients with a previous LSG. Using intraoperative impedance planimetry may provide benefit in appropriate device calibration and placement as well as potentially decreasing postoperative dysphagia.

\section{P485}

\section{Post-operative Dysphagia Following Magnetic Sphincter Augmentation for Gastroesophageal Reflux Disease}

\section{Catherine Tsai, Ulf Kessler, Rudolf Steffen, Joerg Zehetner; Clinic Beau-Site}

Background: Gastroesophageal reflux disease (GERD) is the most prevalent gastrointestinal disorder of the esophagus in the Western population. The magnetic lower esophageal sphincter augmentation (MSA) device is a novel treatment technique with promising results. As it becomes an increasingly common surgical option, understanding the temporary early side effects becomes important. Dysphagia is the most common temporary side effect after MSA, occurring in up to $83 \%$ of patients. While most cases selfresolve by three months, some cases persist and require further intervention. The aim of this study was to evaluate predictors of persistent post-operative dysphagia leading to intervention, as well as its impact on quality of life.

Methods: From August 2015 to September 2018 we reviewed the electronic medical records of all patients with GERD receiving a laparoscopic MSA (LINX reflux management system, Torax Medical) with posterior cruroplasty (PC). Demographic details, procedure details and outcome variables were recorded. Pre- and post-operative GERD-HRQL scores were obtained to assess symptom response. Patients with post-operative dysphagia requiring balloon dilation were identified, and variables were analyzed to asses for risk factors

Results: There were 118 patients $(M=59, F=59)$ with GERD treated with MSA-PC. Mean age was 50 years (R18-80) and mean duration of symptoms was 10.4 years (R1-40). One patient was converted to a Nissen fundoplication for persistent GERD. Based on GERD-HRQL scores ( $\mathrm{n}=73$ responses), $100 \%$ of patients had improved symptoms after MSA-PC. Post-operative dysphagia was present in 80 patients $(67.8 \%)$. Of them, 20 (16.9\%) required balloon dilation for persistent dysphagia. Patients received a median of 1 dilation at a mean of 5.6 months (R1-13) after MSA-PC. The MSA device was explanted in two patients, while dysphagia resolved in 15 patients. Two patients have persistent symptoms and the remaining patient has insufficient follow-up. Compared to patients who did not require dilation, dilated patients were more likely to have atypical GERD symptoms pre-operatively. However, $92.3 \%$ of non-explanted patients requiring dilation reported an improved quality of life.

Conclusion: Post-operative dysphagia after MSA-PC is a common temporary side effect and most commonly seen in patients with atypical GERD symptoms pre-operatively. Most cases of persistent dysphagia can be treated with a single balloon dilation. Despite requiring a dilation, the majority of patients still report an improved quality of life.
Laparoscopic-Assisted Percutaneous Endoscopic Gastrostomy, A Single Center Case-Series

Nir Hus Md., Phd., Daud Lodin, MD, Anupam K Gupta; FAU

Introduction: Gastroscopic assisted percutaneous endoscopic gastrostomy (PEG) is an easy means to provide enteral access in patient unable to intake nutrition via oral route. However, in many patients with unfavorable body habitus secondary to prior abdominal surgery, interpositioning of the colon between the stomach and anterior abdominal wall, laparoscopic means can allow this procedure to be performed safely. In this particular patient population, laparoscopic-assisted percutaneous endoscopic gastrostomy (LAPEG) can provide an alternative route for enteral access. Methods: This is a retrospective case-series of patients, who underwent LAPEG placement. Inclusion into our study involved patients who were unable to undergo Gastroscopic assisted PEG due to abnormal body habitus, previous abdominal procedures, or unfavorable anatomy. Patients were placed under general anesthesia and a $5 \mathrm{~mm}$ video port was inserted at the level of the umbilicus. Patients were placed in steep reverse Trendelenburg position to mobilize the colon away from the stomach. In some patients, we were required to place a single additional $5 \mathrm{~mm}$ port to complete lysis of adhesions and to facilitate a window for stomach opposition to abdominal wall. Intra-abdominal pneumoperitoneum pressures were reduced and a gastroscope was introduced. Stomach was insufflated via gastroscopy and brought close to the anterior abdominal wall. A needle was introduced into the stomach percutaneously, while ensuring no damage to the surrounding structures. The needle was visualized via gastroscope and a guidewire was introduced and brought out through the oral cavity. The gastrostomy tube was passed over the guidewire into the stomach using the Seldinger technique and the approximation was again confirmed via laparoscopic and gastroscopic views. The trocar ports were closed only with skin sutures. Their new gastrostomy tubes were immediately put to use post-operatively with a test administration of clear liquids and medications.

Results: 24 patients met our inclusion criteria. There were 18 males ( 75 percent) and 6 females ( 25 percent) with a mean age group of 75 and a mean body mass index of 22 . We were required to place an additional $5 \mathrm{~mm}$ port in 4 (17 percent) patients. We were able to successfully place gastrostomy tubes in all our patients and all were found patent and functional after immediate postoperative administration of liquids and medications. No postoperative complications resulted from this procedure.

Discussion: LAPEG is a safe and effective method to provide enteral access in patients, who are unable to receive gastroscopic assisted PEG.

\section{P487}

\section{Endoscopy-Assisted Laparoscopic Transgastric Resection of a Gastric Gastrointestinal Stromal Tumor (GIST) Located in the Cardia Near the Gastroesophageal Junction: First Institutional Experience}

Krista Angeli P Delos Santos-Belen, MD, Miguel C Mendoza, MD, Judy Carissa M Atazan, MD; Asian Hospital and Medical Center

Introduction: Cooperative laparoscopic and endoscopic surgery for the management of gastric gastrointestinal stromal tumors (GIST) have been developed in the past years, with the goal of integrating intraluminal and extraluminal approaches to identify precise lesion location, achieve negative surgical oncologic margins, and perform reconstruction with good functional outcome. Different variations of this hybrid approach have been conducted by institutions abroad with promising results. In the Philippines, minimally invasive surgical approach to gastric tumors have yet to be accepted by gastroenterologists and surgeons alike. This case report aims to provide a detailed description of our institution's first experience in performing an endoscopy-assisted laparoscopic transgastric resection of GIST near the GEJ. Methods: A 33 year old female, diagnosed with gastric GIST, underwent endoscopy-assisted laparoscopic transgastric resection. Preoperative evaluation consisted of endoscopy with ultrasonography and abdominal CT scan, which showed a $3.3 \times 1.9 \mathrm{~cm}$ submucosal mass located at the cardia near the GEJ, with no evidence of nodal or distant organ metastasis. Due to the proximity of the tumor to the GEJ, an endoscopy-assisted laparoscopic transgastric resection was performed. Key steps for this technique are as follows: 1) Liver retraction, 2) Mobilization of the greater curvature of the stomach, 3) Endoscopic localization of the tumor, 4) Laparoscopic gastrotomy in the anterior wall overlying the tumor, 5) Stapled submucosal resection with 1-cm margins, while the gastroscope is inserted to ensure patency of the GEJ, 6) Stapled closure of the gastrotomy, 7) Repeat endoscopy to check for completion of resection, patency of the GEJ, bleeding, and anastomotic leakage, 8) Extraction of tumor using a specimen bag to prevent tumor rupture or spillage. Results: The patient was able to tolerate regular diet on 1st postoperative day and was discharged on 3rd postoperative day. Short term outcomes evaluated immediately postop, on 1-week and on 1-month followup consults included: postoperative bleeding, anastomotic leakage, and surgical site infection, none of which were reported. Final histopathology report revealed a $3 \times 3 \times 2 \mathrm{~cm}$ mass consistent with GIST, with negative margins, and mitotic rate $15 / 50 \mathrm{hpf}$, for which adjuvant Imatinib therapy was recommended.

Conclusions: This technique is specifically advantageous for lesions located near the GEJ, where preserving the lumen is crucial, while adhering to standard oncologic principles. Its safety and efficacy depends on the expertise and coordination of the gastroenterologists and surgeons. 
P488

Efficacy of Magnetic Sphincter Augmentation for Gastroesophageal Reflux Disease in Patients with BMI 35 or Greater

Jocelyn F Burke, MD ${ }^{1}$, Evan T Alicuben, MD ${ }^{1}$, Reginald C Bell, $\mathrm{MD}^{2}$, John C Lipham, MD ${ }^{1}$; Department of Surgery, Keck Medical Center of the University of Southern California, ${ }^{2}$ Institute of Esophageal and Reflux Surgery

Background: Magnetic sphincter augmentation (MSA) was FDA approved as a surgical option for patients with gastroesophageal reflux disease (GERD) in 2012. However, for patients with a bodymass index $(\mathrm{BMI}) \geq 35$, use of MSA is considered precautionary by the FDA. Debate exists on the effectiveness of Nissen fundoplication in this group. Roux-en-Y gastric bypass is considered an alternative to Nissen fundoplication for patients with a high BMI but is a much more extensive surgery than either fundoplication or MSA. We sought to determine the effectiveness and safety of MSA for treatment of GERD in patients with BMI $\geq 35$.

Methods: We retrospectively analyzed data of patients undergoing magnetic sphincter augmentation (MSA) surgery between 2007 and 2018 from prospectively collected databases at two esophageal surgery centers. We compared clinical, diagnostic, intraoperative, and postoperative results for patients with $\mathrm{BMI} \geq 35$ to those with $\mathrm{BMI}<35$.

Results: A total of 623 patients underwent laparoscopic MSA, 43 with BMI $\geq 35$ ("obese") and 580 with BMI $<35$ ("normal weight"). The populations were similar in age, duration of GERD symptoms, and preoperative GERD-Health-related quality of life (GERD-HRQL) survey and $\mathrm{pH}$ study DeMeester scores. The obese patients had a higher percentage of females $(77 \%$ vs. $46 \%$, $\mathrm{p}=0.0001)$ and larger hiatal hernia sizes measured via EGD $(3.4 \mathrm{~cm}$ vs. $2.4 \mathrm{~cm}, \mathrm{p}=0.006)$. Operative times were longer in obese patients $(83 \mathrm{vs} .68 \mathrm{~min}, \mathrm{p}=0.006)$, but hospital length of stay was similar ( 14.7 vs $13.1 \mathrm{~h}, \mathrm{p}=0.56$ ). Most importantly, the postoperative courses were similar for both groups. At a median follow up of 11 months (range 1-94), postoperative complications were not significantly different $(3 \%$ in obese vs. $10 \%$ in normal, $\mathrm{p}=0.13$ ), though obese patients had lower rates of dysphagia $(53 \%$ vs. $68 \%, \mathrm{p}=0.05)$. Both populations had similar rates of GERD-HRQL symptom improvement or resolution at last follow up ( $84 \%$ vs. $85 \%, p=0.57)$, postoperative DeMeester score improvement (median score 7.2 vs. $7.4, p=0.67$ ), low levels of continued PPI use (daily or occasionally, $21 \%$ vs. $19 \%, p=0.8$ ), and low rates of recurrent hiatal hernia $(11 \%$ vs. $10 \%, \mathrm{p}=0.88)$.

Conclusions: MSA for the treatment of GERD achieves similar improvements in quality of life, reduction of reflux, and symptom control for patients with BMI $\geq 35$. MSA represents a safe and effective option for GERD control in these patients who are unable or unwilling to undergo a more invasive Roux-en-Y gastric bypass surgery.

\section{P489}

\section{How to Manage Efferent Loop Syndrome with Endoscopic} Techniques

Derek Lim, DO, Kevin Bain, DO, Prashant Sinha, MD; NYU Langone Hospital - Brooklyn

Introduction: Efferent loop syndrome (ELS) is defined as a mechanical obstruction of the efferent enteric jejunal limb. ELS can occur after a Whipple, Billroth II gastrectomy, or Roux-en-Y gastric bypass. Gastrojejunal anastomotic stenosis rates in these surgeries range from $0.2-1.7 \%$. However, these documented rates include afferent limb syndrome and roux limb obstruction.

Case Presentation: We present the case of a 60 year old female with gastric adenocarcinoma presenting for elective resection. The patient underwent a hemi-gastrectomy with Billroth II reconstruction. On postoperative day (POD) three, the patient was started on a clear liquid diet. On POD four through six, the patient continued to have bowel movements, however, was unable to tolerate oral intake. An UGI series was obtained, with findings suspicious for obstruction of the efferent limb. The patient was taken for esophagogastroduodenoscopy, and there was evidence of a stenosed Billroth II gastrojejunal anastomosis. The anastomosis was transversed, and a covered stent was placed under fluoroscopic guidance. Post procedurally a repeat UGI series confirmed a patent stent. The patient's diet was advanced and tolerated, and the patient was discharged home. Discussion: There are two types of "loop syndromes" which may occur after gastric surgery - afferent and efferent. Efferent loop syndrome is less common of the two. Both syndromes are characterized by a mechanical obstruction of the gastrojejunostomy, causing obstruction of gastric emptying. The gold standard to diagnosing ELS is an upper endoscopy; however, radiographic imaging can aid in the diagnosis. UGI series can demonstrate regurgitation of oral contrast into the afferent limb and gastric pouch. The mainstay treatment of ELS has historically been surgical intervention. However, with recent advancements in endoscopic procedures, there have been published techniques using metal stents, double pigtail stents and naso-jejunal tube stenting to bypass areas of stenosis.

In 2016, Chang et al. described the effectiveness of endoscopic stent therapy in the management of postoperative foregut surgery complications (anastomotic leak, lumen stenosis, fistula formation). They reported an $88.9 \%$ success rate in treating stenosis with the end point of being able to tolerate a diet. Double pigtail and naso-jejunal tube stenting have also been documented to have success rates as high as $95 \%$.

Conclusion: This case describes a rare post-gastrectomy complication which was successfully treated with modern endoscopic technique. With modern advancements in endoscopic techniques, patients suffering from efferent loop syndrome are able to avoid the morbidity associated with operative repairs.

\section{P490}

Laparoscopic Antireflux Procedures with Hepatic Shoulder Technique for the Surgical Management of Large Paraesophageal Hernias and Gastroesophageal Reflux Disease: A Follow-up Study

Philippe J Quilici, MD, Alexander Tovar, MD; Providence Saint Joseph Medical Center

Background: Numerous techniques have been historically proposed in the management of gastroesophageal reflux and para-esophageal hernias [PEH]. A follow-up study to a novel laparoscopic approach introduced in 2009 and performed in 49 patients is presented.

Methods: All procedures were performed via laparoscopy. Thirty-two patients underwent a Nissen Fundoplication, eleven a reduction of the PEH with a Nissen fundoplication, two without a fundoplication and four with a Collis-Nissen fundoplication. In all patients, the left hepatic lobe was freed, repositioned, and anchored under and inferior to the gastroesophageal junction, propping the gastroesophageal junction anteriorly. This maneuver entirely covers and closes the diaphragmatic defect.

Results: At the time of laparoscopy, a number of patients were found not to be suitable candidate for this procedure [morphology of the left hepatic love]. Forty-nine procedures were completed One patient was re-explored on POD 2 for a tight hiatus post-Collis Fundoplication. Postoperatively, all other patients did well without notable, unusual complaints. The average length of stay was 2.2 days. Although not statistically significant, 43 patients had no recurrence of symptoms with the longest follow-up at 10 years, two patients were lost to follow-up, one patient had a recurrence of the PEH and three patients stated they are experiencing some form of gastro-esophageal reflux requiring medical management

Conclusions: In selected patients, patients with an "at-risk" crural closure during a laparoscopic anti-reflux procedure or PEH can safely be managed via a laparoscopic antireflux procedure with the hepatic shoulder technique. This technique has shown good early postoperative results and could be used as an alternative to a laparoscopic Mesh reinforced fundoplication, in difficult crural closures or in the management of large para-esophageal hernias. 
P491 P493

\section{Robotic Transhiatal Esophagectomy: Initial Experience with a New Approach}

Sharona B Ross, MD, Danielle K Craigg, MD, Melissa Gonzalez, Janelle Spence, BA, Vladimir Faustin, MS, Timothy Bourdeau, BS, Iswanto Sucandy, MD, Alexander S Rosemurgy, MD; Digestive Health Institute Florida Hospital Tampa

Introduction: As minimally invasive operations become increasingly prevalent, the advantages and possible risks of new operative approaches should be further investigated, especially those involving a new platform. This study was undertaken to examine our results with robotic transhiatal esophagectomy (THE) and to compare our results with those reported through NSQIP and predicted by the NSQIP Risk Calculator. Methods: Patients undergoing THE are followed with IRB approval. We compared the operative data and hospital courses from our institution to NSQIP data for patients undergoing THE 2012-2018 and the NSQIP Risk Calculator. For illustrative purposes, data are expressed as median, mean $\pm \mathrm{SD}$, where appropriate. Significance was accepted at $95 \%$ probability.

Results: 45 patients underwent robotic THE, 62\% of which occurred from 2015-2018. Our patients were very similar to those in NSQIP, except our patients were more likely to be discharged to nursing or rehabilitation facility $(\mathrm{p}<0.05) .6$ (13\%) patients had conversions to 'open' operations, commonly due to failure to progress (often due to morbid obesity); $0 \%$ of the last 25 robotic operations were converted to 'open'. Robotic operations took 330, $355 \pm 107 \mathrm{~min}$; estimated blood loss was 200, $223 \pm 144 \mathrm{~mL}$. Our institution had no patients return to the O.R. which is superior to predicted and NSQIP outcomes (0\% vs $14 \%$ vs $13 \%, \mathrm{p}<0.05$ ). $1(2.2 \%)$ patient died within 30 days of the operation, due to cardiac arrest.

Conclusions: Predicted outcomes of our patients undergoing robotic THE are similar to those reported by NSQIP (Table). This approach was effective in reducing our conversions to 'open', and reoperation, and reducing the need for nursing home care after hospitalize while maintaining similar rates to the national average. Even though this is a new approach to a conventional esophagectomy, our data supports positive outcomes regarding minimally invasive approaches to to complex oncologic foregut surgery.

\begin{tabular}{|c|c|c|c|}
\hline & NSQIP Outcome & \begin{tabular}{|l|} 
Predicted Outcome \\
\end{tabular} & Actual Outcome \\
\hline Outcome & & $N=45$ & $N=45$ \\
\hline Serious Complication & $31 \%$ & $33 \%$ & $40 \%$ \\
\hline Any Complication & $34 \%$ & $33 \%$ & $40 \%$ \\
\hline Pneumonia & $12 \%$ & $13 \%$ & $18 \%$ \\
\hline Cardiac Complication & $2 \%$ & $4 \%$ & $4 \%$ \\
\hline SSI & $15 \%$ & $11 \%$ & $11 \%$ \\
\hline UTI & $2 \%$ & $3 \%$ & $2 \%$ \\
\hline DVT & $4 \%$ & $5 \%$ & $2 \%$ \\
\hline Renal Failure & $2 \%$ & $2 \%$ & $4 \%$ \\
\hline Readmission & $12 \%$ & $9 \%$ & $27 \% \wedge$ \\
\hline Return to O.R. & $13 \%$ & $14 \%$ & $0 \%{ }^{*} x$ \\
\hline Length of Stay (LOS) & N/A & $12,13 \pm 3$ days & $8,15 \pm 17$ days \\
\hline Death & $1 \%$ & $4 \%$ & $2 \%$ \\
\hline$D / C$ to Nursing or Rehab & $10 \%$ & $33 \%{ }^{\circ}$ & $24 \%$ \\
\hline
\end{tabular}

\section{P492}

\section{Laparoscopic Esophageal Diverticulectomy with Heller's Cardiomyotomy}

Venkatarami Reddy Vutukuru, Associate Professor; Sri Venkateswara Institute of Medical Sciences

A 68 yr lady, presented with complaints of retrosternal discomfort, dyspepsia and regurgitation of swallowed food of 6 months duration. Known hypothyroid on oral thyroxine supplements. On evaluation with barium study and endoscopy, there was a large diverticulum in the lower esophagus about $2 \mathrm{cms}$ proximal to GE junction. CT chest revealed a focal outpouching from right lateral aspect of the distal esophagus. Manometry showed abnormal relaxation of lower esophageal sphincter. Planned for surgery. Patient underwent laparoscopic transhiatal esophageal epiphrenic diverticulectomy using EndoGI stapler with cardiomyotomy and partial anterior (Dor's) fundoplication. Postoperative period was uneventful.
Gastric Plexiform Fibromyxoma Arising in the Cardia in an Adolescent Male. A Rare Tumor with Unusual Presentation

Awrad Nasralla, MD, Mufeed Alwabari, MD, Osama Alsaif, MD, Samir S Amr, MD; King Fahad Specialist Hospital -Dammam

Introduction: The objective is to report the sixth case of gastric plexiform fibromyxoma in the pediatric population with unusual location in the cardia. It is an exceedingly rare mesenchymal tumor, that usually affects adults. It has non-specific clinical manifestations. Treatment is surgical resection of the tumor; however, the type of surgery depends on the size, and location of the tumor. Preoperatively, this tumor is usually diagnosed as gastrointestinal stromal tumor (GIST), and the correct diagnosis is made on histopathological examination following surgical resection.

Case Presentation: A 16-year-old boy presented with a history of two episodes of hematemesis, without associated other gastrointestinal symptoms. Past medical, surgical, and family histories were unremarkable.

On physical examination, the patient was overweight, looking pale. His vital signs were within normal limits. His abdomen was soft, not tender, nor distended, with no palpable masses. Laboratory investigations revealed low hemoglobin value $(8.2 \mathrm{~g} / \mathrm{dL})$. CT scan revealed lobulated submucosal gastric mass at the gastric cardia measuring $4.3 \times 4 \times 4.7 \mathrm{~cm}$.

Upper gastrointestinal endoscopy showed normal esophagus, submucosal mass $(4 \mathrm{~cm})$, with deep ulcer at the cardia, the first and second parts of the duodenum were normal (Figure 1). In addition, EUS was done which demonstrated a submucosal mass at the cardia measuring $5 \times 3 \mathrm{~cm}$. It was oval in shape, heterogenous, echogenic, soft, with no appreciable adjacent lymph nodes.

The patient underwent laparotomy, with wedge resection of the mass and primary anastomosis. Postoperative course was uneventful. Pathological examination of the specimen revealed gastric plexiform fibromyxoma with the size of $4 \times 4.9 \times 4.5 \mathrm{~cm}$, margins were free from the tumor.

Discussion: Plexiform fibromyxoma is most commonly found in the gastric antrum $(88 \%)$. Other reported locations were duodenal bulb (22\%), gastric body $(2.6 \%)$, and gastric fundus $(1.3 \%)$. There is one report of plexiform fibromyxoma located in esophagus. Our case is the first one to report the tumor at the gastric cardia near the gastroesophageal junction in a child. In this case, gastric plexiform fibromyxoma was not expected due the age of the patient and the location of the tumor.

Conclusion: Plexiform fibromyxoma can be found in children and it can be located anywhere in the stomach. Images and endoscopy aid in assessing the location, and the size of the tumor, which helps to decide the best surgical technique. However, the diagnosis usually established after histopathogical examination of the tumor following surgery.

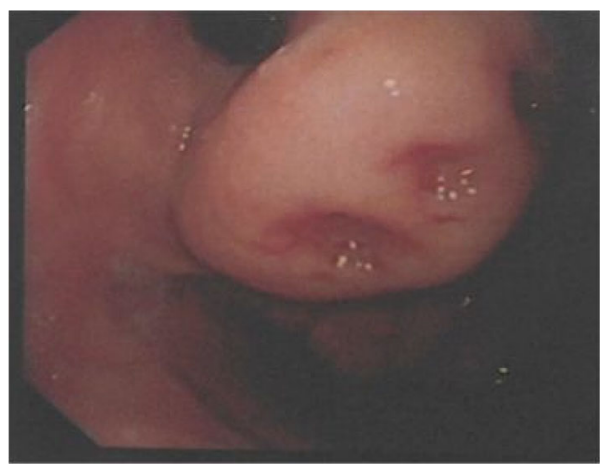

Figure 1: Endoscopic picture 
$\mathbf{P 4 9 5}$

\section{Robot Assisted Hiatal Hernia Repair Demonstrates Favorable Outcomes Compared to Laparoscopic Hiatal Hernia Repair}

Basem G Soliman, MD, Duc T Nguyen, MD, PhD, Edward Y Chan, $\mathrm{MD}$, Ray K Chihara, MD, PhD, Edward A Graviss, PhD, MPH, FIDSA, Leonora M Meisenbach, DNP, RN, ACNPBC, Min P Kim, MD, FACS; Houston Methodist Hospital

Introduction: We postulated that the use of robotics may significantly improve outcomes in hiatal hernia repair.

Methods and Procedures: We performed a retrospective analysis of prospectively collected STS database at a single institution of patients who underwent elective hiatal hernia repair from 2012 to 2017 using either laparoscopy or the da Vinci Xi robot. We compared patient characteristics and outcomes. Univariate and multivariate logistic regression modeling was performed to determine the factors associated with postoperative morbidity.

Results: There were 295 patients underwent elective hiatal hernia repair using either a laparoscopic $(\mathrm{n}=152)$ or robotic $(\mathrm{n}=143)$ technique. There was no significant difference in age, gender, BMI, smoking history, presence of co-morbidity nor hiatal hernia type. Seventy percent of the cases were a repair of either type III or type IV hiatal hernia. There were significantly higher ASA III and IV $(7.9 \%$ vs $4.9 \%, p=0.04)$ and lower redo-repair $(8 \%$ vs $21 \%, p<0.001)$ in the laparoscopic group compared to the robotic group. Hospital length of stay was significantly shorter $(1.3 \pm 1.8 \mathrm{vs}$ $1.8 \pm 1.5, \mathrm{p}<0.001)$ and there were significantly lower rates of complication $(6.3 \mathrm{vs.} 19.1 \%$, $\mathrm{p}=0.002)$ after robotic compared to laparoscopic hiatal hernia repair. There was no difference in readmission rate and mortality. Multiple logistic regression analysis showed that age and laparoscopic technique were associated with complications after surgery.

Conclusion: Use of the da Vinci Xi robot in our institution significantly improves outcomes compared to laparoscopic hiatal hernia repair despite higher incidence of re-operative cases in the robotic group. This may be due to the improved visualization and articulation of instruments that the robot provides over a laparoscope during a complex hiatal hernia repair.

Table 1. Pateint Characteristic:

\begin{tabular}{lrccc}
\hline & $\begin{array}{l}\text { Total } \\
\mathbf{n}=\mathbf{2 9 5}\end{array}$ & $\begin{array}{l}\text { Lap } \\
\mathbf{n = 1 5 2}\end{array}$ & $\begin{array}{l}\text { Robot } \\
\mathbf{n = 1 4 3}\end{array}$ & p-value \\
\hline ASA classification & & & & 0.04 \\
II & $115(39)$ & $49(32.2)$ & $66(46.2)$ & \\
III & $161(54.6)$ & $91(59.9)$ & $70(49)$ & \\
IV & $19(6.4)$ & $12(7.9)$ & $7(4.9)$ & \\
Hernia Type & & & & 0.14 \\
I & $87(29.5)$ & $37(24.3)$ & $50(35)$ & \\
III & $197(66.8)$ & $109(71.7)$ & $88(61.5)$ & \\
IV & $11(3.7)$ & $6(3.9)$ & $5(3.5)$ & \\
Reoperation & $42(14.2)$ & $12(7.9)$ & $30(21)$ & 0.001 \\
\hline
\end{tabular}

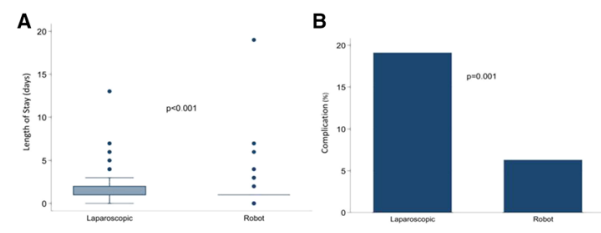

\section{P496}

Normal Johnson-DeMeester Scores Should not Preclude Patients from Anti-reflux Surgery

Alex Addo, MD, Zachary Sanford, MD, Adam S Weltz, MD, James Clemmens, Reza Zahiri, DO, Adrian Park, MD, FACS; Anne Arundel Medical Center

Introduction: The Johnson-DeMeester (JD) score is one of the main parameters derived from $\mathrm{pH}$ testing as a means to determine a patient's acid exposure and is often used as an objective data point to determine potential need for anti-reflux surgery. The purpose of this study was to determine if a normal JD score alone is sufficient to preclude patients from consideration for laparoscopic anti-reflux surgery (LARS).

Methods: A retrospective study was conducted to review quality of life (QOL) outcomes after LARS in patients with a normal $(<14.7)$ versus abnormal $(>14.7)$ JD score at a high volume foregut disorder center between October 2012 and April 2018. [AW1] Patient QOL was assessed using four validated surveys: Reflux Symptoms Index (RSI), GERD Health Related Quality of Life Score (GERD-HRQL), Laryngopharyngeal Reflux-Health Related Quality of Life Instrumen (LPR-HRQL), and Swallowing Quality of Life (SWAL). Additional study outcomes included 30-day wound and non-wound related complications, readmission and reoperation rates.

Results: 144 patients with mean follow up of 1.8 years ( 1 month -5 years) were selected for thi study, $38(26.4 \%)$ with normal and $100(73.6 \%)$ with abnormal JD scores. The two groups had significantly different mean JD scores $(7.40$ vs $57.64, \mathrm{p}<0.05)$. Among the normal and abnorma cohorts thirty day wound and non-wound related complications were $0 \%$. All patients derived significant QOL benefits from operative intervention regardless of JD scores with improvements in mean RSI (65\%), GERD-HRQL (79\%), LPR-HRQOL (77\%), and SWAL (19\%) surveys through five years [AW2] in greatest follow up $(\mathrm{p}<0.01)$. A comparison between the groups did not show any differences in post-operative outcomes and [AW3] percent improvement in QOL outcomes. No patients required reoperation. Patient satisfaction was strong at long term follow up, with both groups reporting either "satisfied" or "neutral" in $94 \%$ of cases.

Conclusion: Our findings confirm that the JD score alone does not correlate with QOL outcomes after laparoscopic anti-reflux surgery. Patients with objective diagnosis of GERD despite a normal JD score should not be precluded from consideration for anti-reflux surgery.

\section{P497}

Robotic Distal Gastrectomy with Indocyanine Green Fluorescence-Guided Lymphadenectomy and Anastomotic Perfusion Assessment: Preliminary Experience

Wanda Petz, MD, Emilio Bertani, MD, Simona Borin, MD, Alessandra Piccioli, MD, Uberto Fumagalli Romario, MD, Giuseppe Spinoglio, MD, FACS; European Institute of Oncology

Background: Robotic gastrectomy is a feasible alternative to laparoscopic gastrectomy, providing similar short-term clinical benefits over open gastrectomy for cancer.

Robotic surgery, conceived to overcome technical limitations intrinsic to the laparoscopic approach, may be of particular interest in complex surgical procedures as gastrectomy with D2 lymphadenectomy.

We present a preliminary experience of robotic distal gastrectomy with the use of Indocyanine Green (ICG) fluorescence to visualize the lymphatic draining area and to assess stumps perfusion before anastomosis.

Methods: The day before surgery, in patients scheduled for robotic distal gastrectomy a gastroscopy is performed and an injection of $2.5 \mathrm{mg}$ of ICG sis realized adjacent to the tumor in the submucosa.

Robotic distal gastrectomy is performed with the Da Vinci Xi System inserting four robotic trocars along a transverse umbilical line

Site of primary tumor and lymphatic draining basin are visualized with the Firefly camera modality, thus guiding D2 lymphadenectomy, which is systematically performed.

After intravenous ICG injection $(0.05 \mathrm{mg} / \mathrm{kg}$ of ICG solution at a dilution of $2.5 \mathrm{mg} / \mathrm{ml})$, perfusion of gastric and jejunal stump is assessed, and an intracorporeal gastro-jejunostomy with robotic linear stapler and manual closure of inserting holes is performed.

The specimen is extracted through a Pfannenstiel incision.

Results: From July 2016 to August 2018, 14 patients (8 males, 6 females; median age 70 years, median BMI $24 \mathrm{~kg} / \mathrm{m}^{2}$ ) with antral adenocarcinoma received a robotic distal gastrectomy with D2 lymphadenectomy

Preoperative staging identified three nodal-positive patients, who received neoadjuvant chemotherapy, according to Institutional policy.

No intraoperative complications nor conversions to open surgery occurred; ICG fluorescence allowed the visualization of the site of primary tumor, of lymphatic draining basin and of gastric and jejunal stumps perfusion before anastomosis in all patients.

Median number of harvested lymph nodes was 37 (range 19-53); median time to first bowel movements 3 days (2-5), median time to soft oral diet 4 days (3-7), median hospital stay 7 days (5-18). Anastomotic or duodenal stump leak did not occurr in any patient, while two patients (14\%) required a percutaneous drainage of peripancreatic and hematic pelvic fluid collection.

Conclusions: In this preliminary experience, robotic distal gastrectomy confirmed to be feasible and safe, providing good clinical results.

Moreover, the use of ICG fluorescence allowed to safely perform an extended lymphadenectomy, and the intraoperative assessment of good perfusion of gastric and jejunal stumps before anastomosis led to the absence of anastomotic complications. 


\section{P498}

\section{Minimally Invasive Options for Gastrointestinal Stromal Tumors} of the Stomach

Laura Mazer, MD, Patrick Worth, MD, Brendan Visser, MD, FACS; Stanford University

Introduction: Gastrointestinal stromal tumors (GIST) are rare mesenchymal tumors, most commonly in the stomach. Surgical resection remains the mainstay of cure. Selecting a resection approach depends on tumor size and location.

Methods: Retrospective review of all patients undergoing surgery at one academic center between 2000-2018. Comparisons and descriptive statistics using Chi square and student's t test.

Results: 77 patients underwent resection, $69 \%$ laparoscopic. Patients undergoing open operations had significantly larger tumors ( $8.1 \mathrm{vs} 4.3 \mathrm{~cm}, \mathrm{p}=<0.001)$ Operative time was not significantly longer for laparoscopic resection, (122 vs $105 \mathrm{~min}, \mathrm{p} 0.264$ ), length of stay was significantly shorter ( 4 vs 7 days, $\mathrm{p}<0.001)$. Complication rate was higher in open operations $(41 \%$ vs $17 \%$, $p<0.001)$. There were four types of resection: stapled wedge resection, resection of a full thickness 'disk' of stomach around the tumor with a primary closure, partial gastrectomy with reconstruction, and laparoscopic trans-gastric (endoluminal) resection.

\begin{tabular}{lllll}
\hline & $\begin{array}{l}\text { Stapled } \\
\text { wedge } \\
(\mathrm{n}=47)\end{array}$ & $\begin{array}{l}\text { Disk } \\
\text { resection } \\
(\mathrm{n}=9)\end{array}$ & $\begin{array}{l}\text { Partial } \\
\text { gastrectomy } \\
(\mathrm{n}=16)\end{array}$ & $\begin{array}{l}\text { Endoluminal } \\
\text { excision } \\
(\mathrm{n}=5)\end{array}$ \\
\hline $\begin{array}{l}\text { Laparoscopic (\%) } \\
\text { Tumor size (mean, cm) }\end{array}$ & 79 & 67 & 31 & 100 \\
Location (\%) Cardia & 4.8 & 5.8 & 7.7 & 4.2 \\
Lesser curve & 33 & 22 & 6 & 100 \\
Body & 25 & 33 & 13 & 0 \\
Greater curve & 17 & 22 & 31 & 0 \\
Antrum & 21 & 11 & 19 & 0 \\
Tumor spillage (n) & 4 & 11 & 31 & 0 \\
Endoscopy (\%) & $2(4 \%)$ & $0(0 \%)$ & $1(6 \%)$ & $0(0 \%)$ \\
Operative time (mean, min) & 28 & 11 & 0 & 100 \\
Length of stay & 101 & 142 & 146 & 175 \\
\hline
\end{tabular}

Conclusions: Wedge resection is most feasible for tumors on the greater curve or body, far enough from the pylorus and gastroesophageal junction to avoid narrowing inflow or outflow. A partial gastrectomy is sometimes required for large tumors or those close to the esophagus or pylorus. For small intraluminal tumors, a laparoscopic transgastric approach is ideal. A review of the technical details of each resection can aid in operative planning for difficult tumors.

\section{P499}

\section{Laparoscopic Management of Parasitic Liver Cysts:}

\section{A Retrospective, Comparative Study}

Ibrahim A Salama, MDPhD; Department of Surgery,National Liver Institutee, Menoufia University,Egypyt

Introduction: Parasitic liver cysts are common in many areas of the world. In our country, hydatid disease is the commonest to cause cystic lesions. We reviewed our data to compare and analyze the outcome of laparoscopic management of these patients. This paper assesses the feasibility and safety of laparoscopic management of hepatic hydatid disease in a tertiary center in Egypt.

Materials and Methods: We retrospectively reviewed our operative and inpatient data at the National liver institute, Menoufia university, Egypt for clinical and operative, postoperative details patients with preoperative diagnoses of a hepatic hydatid disease starting from June 2012 to June 2015. Results: 47 patients had operative management for hepatic hydatid cystic lesions. Twenty seven patients had laparoscopic management and 20 patients were managed through open procedures. Operative procedure included endocystectomy in all laparoscopic cases and while three of the open group had liver resection in addition. Hospital stay was at a mean of 3 days for lap cases and 6 cases for open group. Complications included one cases of bile leak and minor wound infections in the open group. While there were no complications in in the lap group. Follow up showed occurrence of recurrence in two of the open group only.

Conclusions: With conventional laparoscopic instruments, the laparoscopic approach in the management of hepatic hydatid cysts is safe and feasible even. It showed shorter operative time and hospital stay with relatively decreased postoperative complication rate.

\section{P500}

\section{A Spade Shaped Anastomosis Following Proximal Gastrectomy Using Double Suture Fixing Posterior Wall of Esophagus to Anterior Wall of the Stomach (SPADE Operation)}

Wonho Han, Bang Wool Eom, Hong Man Yoon, Junsun Ryu, YoungWoo Kim; National Cancer Center

Objective: Aim of this study was to show early clinical outcome compared with previous proximal gastrectomy (PG) cases.

Background: PG has been performed as function-preserving surgery in proximally located early gastric cancer. As gastroesophageal reflux is a major pitfall of this operation, we devised a modified esophagogastrostomy (EG) anastomosis to fix the distal part of the posterior esophageal wall and the proximal part of the anterior stomach wall to produce an anti-reflux mechanism and named it as SPADE operation.

Methods: A total of 56 patients who underwent PG from January 2012 to March 2018 were retrospectively reviewed. Thirty patients underwent conventional esophagogastrostomy (CEG) anastomosis using circular stapler. Twenty-six patients underwent the SPADE operation. Early postoperative clinical outcomes related reflux symptoms, endoscopic findings and postoperative complications were compared as a case-control study.

Results: Follow-up endoscopy showed more frequent reflux esophagitis in the CEG group than in the SPADE group $(30 \%$ vs $15.3 \% \mathrm{p}=0.19)$. Bile reflux was higher in the CEG group $(26.7 \%$ vs $7.7 \% \mathrm{p}=0.08)$ and residual food was also higher in the CEG group in endoscopic finding with statistical significance $(\mathrm{p}=0.01)$.

In the CEG group, 13 patients (43.3\%) had mild reflux symptoms, while three patients had severe symptoms. In the SPADE group, three patients had mild reflux symptoms (11.5\%), while one had severe symptoms without statistical significance (absolute difference $31.8 \%, 95 \% \mathrm{Cl} 1.11-29.64$, $\mathrm{p}=0.01)$.

Conclusion: A novel modified EG called SPADE operation showed potential in reducing gastroesophageal reflux in $\mathrm{PG}$

\section{P501}

Umbilical Tape Provides Better Correlation with Final EndoFLIP Distensibility Index Compared to LINX Sizing Device

Rodrigo Pedraza, MD, Edward Y Chan, MD, Ray K Chihara, MD, PhD, Leonora M Meisenbach, MSN, APRN, ACNPBC, Min P Kim, MD; Houston Methodist Hospital

Background and Aim: The use of a magnetic sphincter augmentation device (LINX) has been shown to be safe and efficacious for the management of gastroesophageal reflux disease (GERD). The endoluminal functional lumen-imaging probe (EndoFLIP) distensibility index (DI) has been correlated with significant dysphagia after surgery. We want to determine if umbilical tape can better predict the final EndoFLIP distensibility index compared to the LINX sizer.

Methods: From 5/2017 to 2/2018, all patients who underwent LINX placement with EndoFLIP analysis were evaluated. Our technique included the placement of an EndoFLIP to assess EGJ distensibility. An umbilical tape is marked at different sites according to the LINX available sizes. We also used the LINX sizer to measure the EndoFLIP distensibility index (Figure 1). The umbilical tape is placed around the EGJ and is tightened on each size mark while evaluating the EGJ distensibility using the EndoFLIP ${ }^{\circledR}$. The LINX size is selected based on distensibility values (Figure 1).

Results: A total of 30 patients were evaluated. The patient demographics included a mean age of 54 $83 \%$ females, a median ASA of 2, and an average BMI of 30.5. On esophagram, $43 \%$ had type III hiatal hernia and 57\% had type I hiatal hernia. On manometry, peristalsis was normal on $>70 \%$ of swallowing episodes for all patients. The mean preoperative DeMeester score was 34 . All procedures were performed robotically. The median LINX size was 16 (14-17). Intraoperatively, there were no complications and the average operating time was $3 \mathrm{~h}$. Thirteen patients had an EndoFLIP assessment using the sizing tool provided by the manufacturer and there was no correlation between the sizing too EndoFLIP value and the final value after LINX placement. Seventeen patients had umbilical tape used for sizing and there was perfect correlation in $94 \%$ of the patients. The mean LOS was 1 day (0-3) and there were no postoperative complications, readmissions, or reoperations. The mean pre-intervention EGJ distensibility index was 2.9 as compared with postoperative $1.5, \mathrm{p}<0.0001$. The mean preoperative GERD QOL was 24 as compared with postoperative 5.5, $\mathrm{p}<0.0001$

Conclusions: Our analysis revealed that umbilical tape provides a better predictive final EndoFLIP DI after LINX placement compared to the LINX sizer. Further research is needed to assess whether this approach may potentially avoid placement of an improper size of LINX device. 
P502

A Novel Laparoscopic Approach for Management of an Incarcerated Gastric Herniation Through a Percutaneous Endoscopic Gastrostomy Site

Alexander Gonzalez-Jacobo, DO, Faith Buchanan, Hubby Buehler, Jacob Eisdorfer, DO, Javier Andrade, MD; St. John's Episcopal Hospital

Introduction: Percutaneous endoscopic gastrostomy (PEG) is a common procedure frequently performed to provide nutrition via enteral access for patients that are unable to feed orally. PEG tube insertion has fallen into favor due to its minimally invasive technique in addition to its costeffectiveness compared to open gastrostomy. Although it boasts an impressive safety profile and is a reasonably straightforward procedure, PEG tube placement does entail risks. We present a rare case of an elderly female patient who experienced prolapse of the gastric wall from the PEG site and a novel laparoscopic/endoscopic approach to the subsequent repair.

Case Description: A 90-year-old female, with extensive comorbidities was admitted to our institution for hypotension and electrolyte abnormalities. During her workup, it was discovered that the patient had developed gastric prolapse and herniation surrounding the PEG tube due to increased abdominal pressure caused by pulmonary fibrosis and chronic ventilator support. The PEG tube was removed and a gastrograffin study was performed which showed no extravasation. Attempts were made to externally reduce the prolapse but were unsuccessful. Due to concerns of ischemia to the prolapsed gastric wall the decision was made to attempt the reduction surgically. The abdomen was accessed laparoscopically and the prolapsed stomach was identified. Adhesions were lysed and small incisions were made on either side of the PEG tract to mobilize the stomach through the abdominal wall. A partial gastrectomy was then performed from the exterior with an Endo-GIA stapler. The stomach was returned into the abdomen and the integrity of the repair was confirmed via endoscopy. Due to the continued requirement for enteral feeding, a laparoscopic-assisted endoscopic gastrostomy tube was then placed along with multiple T-fasteners to secure the anterior gastric wall to the abdominal wall preventing future gastric prolapse. The patient experienced an uneventful post-operative course and was discharged.

Discussion: Exploratory laparotomy has been previously described for repair of gastric prolapse, however a review of current literature has yielded no reports of laparoscopic-endoscopic assisted repair of the aforementioned condition in the adult population. Studies have shown PEG has a major complication rate of up to $11 \%$. Due to the infrequency of our patients' complication, no standard has been identified in adults for repair of gastric prolapse through gastrostomy. The advancements of laparoscopic technique as well as the decreased recovery time accompanying laparoscopic repairs, suggest laparoscopic-endoscopic assisted repair of gastric prolapse is a superior method of repair in clinically stable patients.

\section{P503}

\section{Optimal Surgical Management of Medically Refractory Gastroesophageal Reflux Disease and Concurrent Esophagogastric Junction Outlet Obstruction}

Michael A Antiporda, MD ${ }^{1}$, Christy M Dunst, MD², Kelly R Haisley, $\mathrm{MD}^{1}$, Walaa Abdelmoaty, $\mathrm{MD}^{1}$, Kevin M Reavis, $\mathrm{MD}^{2}$, Daniel Davila Bradley, $\mathrm{MD}^{2}$, Steven R Demeester, MD², Lee L Swanstrom, $\mathrm{MD}^{3}$; ${ }^{1}$ Providence Portland Medical Center, ${ }^{2}$ The Oregon Clinic, ${ }^{3}$ Institute for Image Guided Surgery (IHU-Strasbourg)

Introduction: The significance of manometric esophagogastric junction outlet obstruction (EGJOO) in the setting of objective GERD is unknown. Some hypothesize that adding myotomy at the time of fundoplication is beneficial in these patients, particularly those with significant dysphagia. Others worry that myotomy compromises reflux control. The aim of this study is to explore post-operative outcomes in patients treated for GERD plus EGJOO.

Methods: At our institution, the decision to add Heller myotomy for EGJOO in the setting of GERD is per surgeon preference. We retrospectively reviewed all patients who underwent surgery for GERD with concurrent EGJOO (defined as IRP $>15$ ). Objective GERD was defined by abnormal $\mathrm{pH}$ testing or endoscopic evidence of erosive esophagitis, or Barrett esophagus. Patients with named hypercontractility disorder, paraesophageal hernia, or prior foregut operation were excluded. Pre-operative objective testing included HRM, EGD, pH study, and UGI. Pre- and postoperative symptoms were assessed via validated questionnaire. Peri-operative variables including operative approach, length of stay, and complications were recorded.

Results: Twenty-seven patients underwent anti-reflux surgery in the setting of elevated IRP from 2013 to 2018 (mean age 57, 37\% male, 33\% BMI > 30). Pre-op HRM revealed mean IRP 19.3 and ineffective esophageal motility in 19\%. Median pre-op DeMeester score was 22.2. Pre-op EGD revealed erosive esophagitis or Barrett esophagus in 11 patients. Commonest symptomatic indication was regurgitation (93\%) followed by heartburn (82\%) and dysphagia to solids (64\%). Operative treatment included Heller myotomy in 33\%. Complete fundoplication was performed in 52\% and partial fundoplication in the remainder. Median length of stay was two days. There were eight complications including two sub-clinical leaks that resolved without additional procedural intervention (one patient had undergone concurrent pyloroplasty and the other underwent concurrent endoscopic cricomyotomy). Surgery resulted in overall improved rates of heartburn, regurgitation, and dysphagia. When stratified by performance or omission of myotomy, there was a trend in the short term towards improved rates of dysphagia (22\% vs 33\%) and regurgitation (0\% vs $11 \%)$ but increased persistent pyrosis $(22 \%$ vs $11 \%$ ) in the myotomy group, but these were not statistically significant. Conclusions: Ideal treatment of GERD and EGJOO is unknown. The addition of myotomy may be helpful in patients with primary complaint of dysphagia, and does not itself appear to increase risks of surgery. Tailoring of the anti-reflux operation to address the most troublesome complaint of the patient with GERD and EGJOO may lead to the best overall symptomatic outcome.

\section{P504}

Increased Morbidity After Paraesophageal Hernia Repair is Associated with Collis Gastroplasty and Open Approach

Sarah Samreen, MD, Bhavani Pokala, MD, Laura E Flores, Priscila R Armijo, MD, Dmitry Oleynikov, MD; University of Nebraska Medical Center

Introduction: Paraesophageal hernia repair (PEH) requires an adequate length of intra-abdominal esophagus. For the management of short esophagus, a collis gastroplasty can be performed to lengthen the functional distal esophagus. A fundoplication can then be performed without tension. Our aim was to examine the frequency and outcomes of PEH with and without Collis gastroplasty. Methods: The 2014-2016 National Surgical Quality Improvement Program (NSQIP) database was analyzed for adults who underwent elective PEH performed by general or thoracic surgery. Emergent cases and those unable to be followed for 30 days were excluded. Cases with and without Collis gastroplasty were compared. Statistical analysis was performed using IBM SPSS 25.0, $\alpha=0.05$

Results: 12,900 patients were included in this study $(\mathrm{C}: 241 ; \mathrm{NC}: 12,599)$. Patient demographics and symptoms were similar with the exception of a higher rate of dyspnea ( $36 \%$ vs $16.5 \% ; \mathrm{p}=.017$ ) and older mean age ( $64 \pm 12.7$ vs $61 \pm 14.3$ years) in the Collis group. Majority of cases were performed laparoscopically (C:84.2\%, NC:92.6\%) and by general surgeons (C:79.8\%, NC:95.6\%). $10.75 \%$ of PEH cases performed by thoracic surgeons included Collis gastroplasty vs $2.21 \%$ performed by general surgery $(\mathrm{p}=<.001)$. In the Collis group, there was significantly higher rates of organ space infection $(2.6 \%$ vs $0.8 \% ; \mathrm{p}=.002)$, superficial site infection $(12 \%$ vs $3.5 \%$; $\mathrm{p}=.002)$, and sepsis ( $2.6 \%$ vs $0.7 \% ; \mathrm{p}=.001)$. Mean length of stay was also longer in the Collis group (3.89 vs $2.90 ; \mathrm{p}=.001)$. Logistic regression revealed that open approach and Collis gastroplasty were independently associated with higher risk of superficial site infection (OR:3.378 and OR:6.611), organ space infection (OR:3.263 and OR:2.303), and sepsis (OR:3.681 and OR:3.492), all $\mathrm{p}<.05$.

Conclusions: Collis gastroplasty is associated with increased morbidity. Short esophagus can often be elongated safely with extended mediastinal dissection to provide adequate intra-abdomina esophageal length and avoid the need for a Collis gastroplasty.

\section{P505}

Regression of Ultra-Short Segment Barrett's Esophagus and Intestinal Metaplasia of the Gastric Cardia After Anti-reflux Surgery: A Single Center Experience

Ashwin Kurian, MD, Loren Estoesta; SurgOne, P.C. - Denver Esophageal and Stomach Center

Introduction: Barrett's esophagus (BE) is a precancerous condition that results from gastroesophageal reflux disease. Recent studies have demonstrated that Cardiac Intestinal Metaplasia (CIM) progresses to endoscopically confirmed Barrett's over time. The regression rates of ultrashort segment BE and CIM following anti-reflux surgery has not been clearly elucidated.

Methods: Ultra-short segment BE or CIM was defined by intestinal metaplasia seen on standard endoscopic forceps biopsy of an irregular squamo-columnar junction. Patients who underwent antireflux surgery between November 2013 to November 2017, with a preoperative diagnosis of either ultra-short segment BE or CIM were retrospectively reviewed. Patients underwent surgery primarily for symptomatic reflux disease. Index endoscopy, anti-reflux surgery and surveillance endoscopy were all performed by the same surgeon. Standard 4-quadrant forceps biopsy of the squamo-columnar junction were performed both preoperatively and 1 year post fundoplication. Results: Twenty-seven patients with a diagnosis of ultra-short segment BE or CIM, underwent laparoscopic anti-reflux surgery (9:male; 18 :female). Mean age was $57+14$ years and mean BMI was $28.6+5.6$. Nine patients were lost to follow-up. The mean length of time for follow-up was $12.3+0.76$ months. The median length of stay following the laparoscopic fundoplication was $2+2.1$ days. Nine patients $(50 \%)$ had regression of intestinal metaplasia on their postoperative surveillance endoscopy.

Conclusion: One year after anti-reflux, 50\% of patients showed regression of short-segment BE and CIM. The regression of ultra-short segment BE and CIM can be an added benefit of anti-reflux surgery in addition to the resolution of reflux symptoms. 
P506

Laparoscopic Synchronous Left Hepatectomy and Distal Gastrectomy with Lymphnodal Dissection for Carcinoid Tumor of the Gastric Antrum with Left Lobe Liver Metastasis

Srikanth Gadiyaram, Dr, Gaurav Singh, Dr, Yashas H Ramegowda, Dr; Sahasra Hospitals, Center of Excellence for Gastroenterology

Background: Surgery remains the mainstay of treatment for neuroendocrine tumors of stomach with resectable liver metastasis. Distal gastrectomy with lymphnodal dissection is increasingly being performed laparoscopically by several centres across the world. Also, laparoscopic left hepatectomy is being performed laparoscopically in some centres routinely. We herein present an operative video of laparoscopic synchronous resection, viz; laparoscopic distal gastrectomy with lymphnode dissection and left hepatectomy.

Case Report: 65 year old lady evaluated with upper gastroscopy for evaluation of upper abdominal pain had a antral tumor which was confirmed to be a carcinoid tumor on histopathology and immunohistochemistry. A DOTANOC-PETCT showed an increased uptake in gastric antral lesion, hepato-duodenal enlarged lymphnodes and uptake in lesions in Segment 2, 3 and 4 of live and no uptake elsewhere. The patient was planned for the aforementioned operative procedure which was performed in the following steps

Step 1-Five port laparoscopic procedure. Lesser sac was opened, right gastroepiploic vessels dissected and divided. First part of duodenum looped and staple transected

Step 2-Hepatoduodenal lymphnode clearance done

Step 3-Left hepatic artery looped, ligated and divided. Left portal vein looped and ligated in continuity

Step 4 -Line of demarcation marked out. Parenchymal transection done using harmonic for superficial transection, CUSA for deeper parenchymal transection, and structures $3 \mathrm{~mm}$ or more clipped and divided. The left portal pedicle was staple (endo GIA vascular load) transected, and left hepatic vein encountered during deeper dissection, was dissected and secured with an endo GIA vascular load. The left triangular ligament was divided.

Step 5-Proximal gastric marging for transection defined, and staple divided

Step 6-Specimen retrieval, viz; distal stomach, lymphnodes and left liver placed in endobag and removed through $5 \mathrm{~cm}$ left subcostal incision.

Step 7-Gastrojejunostomy completed through the wound of specimen retrieval

Step 8-Hemostasis checked, lavage done and drain placed along transected liver and excited from right anterior axillary line port.

Conclusions: Synchronous laparoscopic gastrectomy and major hepatectomy is safe and feasible. This has to be viewed keeping in mind the potential need for repeat surgeries in these patients in the long term for any recurrent disease.

\section{P507}

\section{Magnetic Sphincter Augmentation (MSA) is an Effective Treatment in Patients with Severe Reflux Disease}

Shahin Ayazi, MD, Ping Zheng, MD, Kirsten Newhams, MD, Fahim Habib, MD, Jonathan Levy, MD, Tameka Scott, DO, Kristy

Chovanec, BS, Philip Jackson, BS, Yoshihiro Komatsu, MD, Toshitaka Hoppo, MD, Ali H Zaidi, MD, Blair A Jobe, MD; The Esophageal and Lung Institute, Allegheny Health Network

Introduction: The performance and durability of the various types of fundoplication is variable when stratified by disease severity. For example, partial fundoplication has been demonstrated to be an inadequate surgical treatment in patients with a very high DeMeester score (DS). To date, magnetic sphincter augmentation (MSA) has not been evaluated in this context and the majority of severe disease patients are referred for Nissen fundoplication. We hypothesize that MSA is an effective treatment in patients with severe reflux.

Methods and Procedures: Baseline clinical and objective data were prospectively collected in patients who underwent MSA between June 2013 and July 2017. Outcomes were assessed using disease-specific questionnaires, upper endoscopy, $\mathrm{pH}$ monitoring. PPI use, and need for device removal. Based on previous studies, a DS $\geq 50$ was used as a cutoff to define severe disease. The study population was divided using this cutoff and outcomes were compared with those with less severe disease (DS $<50$ ).

Results: A total of 334 patients underwent MSA. The mean (SD) age was 52.7 (14.1) and 60\% were female. There was no difference between groups in regard to age, BMI or duration of follow up ( $p>0.05)$. Patients with severe reflux had a higher mean preoperative DS compared to those with less severe disease [78.2 (11.1) vs. $22.8(1.3),(\mathrm{p}<0.0001)]$. Patients with severe disease had a higher preoperative prevalence and severity grade of esophagitis [prevalence: $67 \%$ vs. $44 \%$, $\mathrm{p}=0.0016$, and combined LA grade C and D: $18 \%$ vs. $7 \%, \mathrm{p}=0.0076)]$.

At a mean postoperative follow up of 13.6 (10.4) months, there was no difference between the GERD-HRQL total score in patients with severe disease compared to those with less severe disease [10.8 (12.9) vs. $9.8(10.9), \mathrm{p}=0.53]$. This was also true for GERD-HRQL heartburn score [3.2 (6.0) vs. 3.2 (5.3), $\mathrm{p}=0.98]$ and RSI total score [8.1 (8.7) vs. $9.4(8.8), \mathrm{p}=0.32]$. Postoperative DS was not different between groups [17.3 (23.0) vs. 14.1 (33.9), $\mathrm{p}=0.71]$, and there was no difference in the prevalence or grade of esophagitis [4 $(20.0 \%)$ vs. $17(15.0 \%), \mathrm{p}=0.52]$, or PPI use [6 $(11.5 \%)$ vs. $19(8.2 \%), p=0.42$ ]. There were similar rates of postoperative dysphagia $(10 \% \mathrm{vs}$. $14 \%, \mathrm{p}=0.42)$ and need for device removal $(3 \%$ vs. $5 \%, \mathrm{p}=0.63)$.

Conclusion: MSA is an effective treatment in patients with severe reflux disease and provides the same degree of clinical and objective improvement across the spectrum of disease severity.

\section{P508}

Outcomes of Magnetic Sphincter Augmentation in Patients with Compromised Esophageal Motility

$\underline{\text { C Daniel Smith }}{ }^{1}, \mathrm{~J}$ Patrick Waring ${ }^{2} ;{ }^{1}$ Esophageal Institute of Atlanta, ${ }^{2}$ Digestive Healthcare of Atlanta

Introduction: Magnetic sphincter augmentation (MSA) with the Linx Device (Johnson \& Johnson, Cincinnati, $\mathrm{OH}$ ) was approved by the FDA in 2012 for use in patients with $\mathrm{pH}$ proven gastroesophageal reflux disease. It is recommended that patients undergo objective testing regarding esophageal motor function prior to Linx implantation to ensure effective esophageal motility to overcome the augmentation effect of the device. Patients with poor esophageal motility should be at risk for post-op dysphagia. Current recommendations are that MSA be limited to patients with esophageal body pressure $\geq 35 \mathrm{mmHg}$ and no less than $70 \%$ peristalsis. Here is described an experience and outcomes using MSA in patients with compromised esophageal motility.

Methods: A prospectively maintained database of 278 patients undergoing Linx implantation over a 7-year period was analyzed. All patients underwent esophageal motility testing as part of their preoperative work-up. Patients who did not undergo esophageal motility testing were not offered MSA. All patients are informed about their esophageal motility status prior to MSA. Patients who underwent MSA and did not meet the above esophageal motility criteria were identified and their clinical course and outcomes analyzed and reported.

Results: Sixteen patients were identified who did not meet the above esophageal motility criteria for MSA. All 16 patients had been informed preoperatively of the risk of post-op dysphagia, yet were highly motivated to proceed with MSA. Outcomes are summarized below.

Six of sixteen patients that did not meet recommended preoperative esophageal motility criteria for MSA experienced persistent developed dysphagia and required removal of the device.

\begin{tabular}{|l|c|c|c|c|}
\hline \multicolumn{5}{|c|}{ MSA Outcome With Impaired Esophageal Motility } \\
\hline & & $\begin{array}{c}\text { Linx } \\
\text { Remowal } \\
(\mathrm{n})\end{array}$ & $\begin{array}{c}\text { Linx } \\
\text { Removal } \\
(\%)\end{array}$ & $\begin{array}{c}\text { Removal Time } \\
(\mathrm{d} \text { postop) }\end{array}$ \\
\hline Total No. Subjects & 16 & 6 & $37.5 \%$ & \\
\hline Esophageal Body Pressure Abnormal & 10 & 2 & $20.0 \%$ & $>365$ \\
\hline Peristalsis Abnormal & 4 & 2 & $50.0 \%$ & $90-270$ \\
\hline Both Abnormal & 2 & 2 & $100.0 \%$ & $<30$ \\
\hline
\end{tabular}

Conclusion: Some patients with impaired esophageal motility can be successfully managed with MSA. Abnormal peristalsis and esophageal body pressure predicts the worst outcome and early Linx removal. Abnormal peristalsis appeared to be the best predictor of persistent post-op dysphagia. 
P509

\section{Experience with U-Shaped and Keyhole-Shaped Configuration of Urinary Bladder Extracellular Surgical Device in Repair of Paraesophageal and Recurrent Hiatal Hernia}

Lauren Rabach, MD, Sarah Keville, BS, Adham Saad, MD, Vic Velanovich, MD; University of South Florida

Background: Paraesophageal hernia (PEH) and recurrent hiatal hernia (RHH) with mesh utilization is performed for patients with large symptomatic hiatal hernias and/or reflux. The urinary bladder matrix surgical device (UBMSD) for PEH/RHH repair has shown promising results. U-shaped and keyhole are the two most commonly used configurations; however, there is little data comparing them. We report here our experience with these two configurations of this mesh.

Methods: A review of PEH repairs between 2013 and 2018 was performed from a single institution. Patients with PEH/RHH that required mesh placement were identified and analyzed for demographic information, perioperative/intraoperative details, and postoperative outcomes. Results: Of the patients undergoing PEH/RHH repair, 110 were repaired with UBMSD. Of these, 88 were repaired with the U-shaped configuration, while 22 the keyhole configuration. Of the U-shaped configuration patients, $70 \%$ were for PEH, $28 \%$ for RHH, $1 \%$ for a type I HH; while of the keyhole-shaped patients, $73 \%$ were for PEH and $27 \%$ for RHH. The median follow-up time was 8 weeks. There was no difference in in-hospital complication rates, $20 \%$ vs $18 \%$, respectively. However, $24 \%$ of patients with the U-shaped configuration recurred, compared to $9 \%$ of patients with the keyhole configuration. The average time for recurrence in our patient cohort was around 14 months postoperatively. Postoperative bloating and dysphagia was seen in $6 \%$ of our patients, however all of them had the U-shaped mesh configuration. $42 \%$ (8 of 19) of the U-shaped patients underwent repair of the recurrence. Of note, the recurrences were found anterior and to the left of the esophagus at an area uncovered by the mesh. Both of the patients with recurrences after the keyhole configuration had repairs.

Conclusion: In conclusion, there was no difference in complication rates between the configuration types. The keyhole configuration may carry lower risk of recurrent hernia. Postoperative dysphagia and bloating may also be lower in the keyhole configuration. Site of recurrence of the U-shaped configuration appeared to be in the region of the hiatus not covered by the mesh.

\section{P510}

Short- and Long-Term Outcomes of Laparoscopic ParenchymaSparing Resections for Pancreatic Neuroendocrine Neoplasms

Renchao Zhang, Yiping Mou, Qicong Zhu; Department of Gastrointestinal \& Pancreatic Surgery, Zhejiang provincial people's Hospital

Background: Pancreatic neuroendocrine neoplasms (PNENs) are rare neoplasm with long life expectancy. In this setting, patients may benefit from laparoscopic parenchyma-sparing resections. There are few reports of laparoscopic parenchyma-sparing resections for PNENs. The aim of this study was to evaluate the Short- and long-term outcomes of laparoscopic parenchyma-sparing resections for PNENs.

Methods: A retrospective study was performed for patients with PNENs who underwent laparoscopic parenchyma-sparing pancreatectomy between March 2005 and May 2018. The patients' demographic data, operative results, pathological reports, hospital courses, morbidity and mortality, and follow-up data (including pancreatic function, till May 2018) was analyzed.

Results: Thirty-five patients were identified. There were 9 male and 26 female patients, with median age of 46 years (range, 25-75 years). The mean BMI was $24.6 \pm 3.3 \mathrm{~kg} / \mathrm{m}^{2}$. Nine patients received laparoscopic enucleation, 20 received laparoscopic spleen-preserving distal pancreatectomy, and 6 received laparoscopic central pancreatectomy. The operative time, intraoperative blood loss, transfusion rate, postoperative hospital stay were $186.4 \pm 60.2 \mathrm{~min}, 165 \pm 73.0 \mathrm{ml}, 0,9$ day (range, 5-23 days), respectively. The morbidity rate, $=$ Grade $?$ complication rate and the incidence of pancreatic fistula formation were $34.2 \%, 11.4 \%, 28.6 \%$, respectively, with no mortality. The rate of follow-up was $92.8 \%$, and the median follow-up time was 55 months (range, 3-158 months). One patients had diabetes after laparoscopic spleen-preserving distal pancreatectomy, no patien had symptom of pancreatic exocrine insufficiency. One patient developed recurrence 3 years after laparoscopic enucleation and was managed with surgical resection. The other patients survived without metastases or recurrence during the follow-up.

Conclusions: Laparoscopic parenchyma-sparing resections for PNENs is safe and feasible, and have favorable short- and long-term outcome.

Key words: Parenchyma-sparing; pancreatic neuroendocrine neoplasms; Laparoscopy; Pancreatectomy.

\section{P511}

\section{Management of Gastrointestinal Stromal Tumors; Our Experience}

Samiullah Bhatti, Dr ${ }^{1}$, Shabbar H Changezi, $\mathrm{Dr}^{1}$, Awais A Malik, $\mathrm{Dr}^{2}$, Usman I Butt, $\mathrm{Dr}^{1}$, Waris Farooka, Prof ${ }^{1}$, Mahmood Ayyaz, Prof ${ }^{1} ;{ }^{1}$ Services Hospital Lahore, ${ }^{2}$ Lahore General Hospital

Introduction: Gastrointestinal stromal tumors (GISTs) are the most common mesenchymal tumors of the gastrointestinal tract. Surgical (open/laparoscopic) treatment is the only chance of cure for patients with a primary localized GIST.

Materials and Methods: We present our experience of managing gastrointestinal stromal tumors from June 2015 till June 2018 .

Results: we treated 6 cases of gastrointestinal stromal tumors from June 2015 till June 2018. 5 $(83 \%)$ of them were male and $1(16 \%)$ was female. Mean age of the patients was 44.1 Year (range 17-64 years). All of them were located in stomach. Mean size of the tumor was $10.65 \mathrm{~cm}$ (range $6.5-14.2 \mathrm{~cm}$ ). one of them was with $>5$ mitosis $/ 50 \mathrm{HPF}$ all others were having $<5 / 50 \mathrm{HPF} .4 \mathrm{of}$ them underwent open resection of the tumor while 2 of them underwent laparoscopic resection. No complication occurred per operative or post operatively. All of the patients were given imatinib postoperatively. All of them underwent resection with $2 \mathrm{~cm}$ resection margin.

Conclusion: Surgical resection remains the treatment of choice and offers the only chance of cure from GIST. The main operative principle is resection of the tumor with negative microscopic margins. Wide resection of the tumor (e.g. $2 \mathrm{~cm}$ margin) has not been shown to improve outcomes.
Minimally Invasive Surgery for Siewert Type II Adenocarcinoma
Based on the Distribution of Lymph Node Metastasis or Recurrence

Kazuo Koyanagi, MD, $\mathrm{PhD}^{1}$, Soji Ozawa, $\mathrm{MD}, \mathrm{PhD}^{1}$, Kentaro Yatabe, MD ${ }^{1}$, Yamato Ninomiya, $\mathrm{MD}^{1}$, Miho Yamamoto, $\mathrm{MD}^{1}$, Akihito Yamamoto, $\mathrm{MD}^{1}$, Yuji Tachimori ${ }^{2}$; ${ }^{1}$ Department of Gastroenterological Surgery, Tokai University School of Medicine, ${ }^{2}$ Cancer Care Center, Kawasaki Saiwai Hospital

Aim: Based on the distribution of lymph node (LN) metastasis and LN recurrence of Siewert type II adenocarcinoma, appropriate surgical procedure was investigated, and also minimally invasive surgery for Siewert type II adenocarcinoma was introduced.

Methods: One hundred and sixty-eight patients with Siewert type II adenocarcinoma who underwent surgery without any neoadjuvant therapy between 2001 and 2016 were enrolled. Metastatic stations and recurrent LN sites were classified into five zones; cervical, upper/middle/ lower, mediastinal, and abdominal zones. After investigating the correlation between LN metastasis or recurrence and esophageal invasion length (EIL) of the tumor, we have introduced a laparoscopic and thoracoscopic surgery for Siewert type II adenocarcinoma since 2018.

Results: Overall LN metastasis or recurrence in the cervical, upper/middle/lower, mediastinal LN zones were detected in $4,8,11$, and 25 patients, respectively. Overall LN metastasis or recurrence in the abdominal LN zone was detected in 87 patients, however, metastasis or recurrence in No. 4 LN (LN along the short and left gastroepiploic artery) station was detected in only one patient. Siewert type II patients with an EIL of more than $25 \mathrm{~mm}$ had a higher incidence of more advanced stage $(\mathrm{P}<0.001)$ and more overall metastasis or recurrence rate in the upper $(\mathrm{P}=0.01)$, middle $(\mathrm{P}<0.001)$, and lower $(\mathrm{P}=0.002)$ mediastinal zones than those with an EIL of less than or equal to $25 \mathrm{~mm}$. Based on these retrospective results, a laparoscopic and thoracoscopic surgery including the mediastinal LN dissection and intrathoracic reconstruction using gastric conduit have been introduced for Siewert type II tumors with invasion to the thoracic cavity. Prone position and overlap anastomosis for reconstruction were used during thoracoscopic procedure. We have conducted this procedure in three patients. Mean clinical EIL was $26 \mathrm{~mm}$, mean operative time was $587 \mathrm{~min}$, blood loss was $268 \mathrm{~mL}$, and there were no intraoperative complications. Pathologically, mean length of proximal margin from the tumor was $63 \mathrm{~mm}$, and abdominal $\mathrm{LN}$ metastasis was detected in two patients, but no mediastinal LN metastasis were detected. Despite short follow-up period, no recurrence including $\mathrm{LN}$ recurrence were detected.

Conclusion: Appropriate area of $\mathrm{LN}$ dissection and reconstruction methods were investigated based on the analyses of distribution of LN metastasis once the tumor has invaded more than $25 \mathrm{~mm}$ to the esophageal wall in Siewert type II adenocarcinoma. Laparoscopic and thoracoscopic surgery should be less invasive and could be conducted safely. 


\section{P513}

PET Positive Esophageal Leiomyoma: A Case Series and Literature Review

James Kurtz, Rene Borscheid, Edward Cho, Houssam Osman, Rohan Jeyarajah; Methodist Richardson Medical Center

Introduction: Esophageal leiomyomas are rare, benign tumors that can be adequately treated with limited resection or enucleation. Distinguishing a leiomyoma from a more aggressive entity can present a diagnostic challenge. It has been suggested that positron emission tomography (PET) scanning can help determine the likelihood of an invasive cancer.

Case series: We present a series of two patients with large esophageal leiomyomas that had increased uptake of $18 \mathrm{~F}$-fluorodeoxyglucose on PET scan. Both patients underwent transhiatal esophagectomy.

Patient A had a pre-operative endoscopic ultrasound biopsy that showed spindle-cell neoplasm in the esophageal mass. He also had Barrett's esophagus with high grade dysplasia. Intra-operative frozen section also showed spindle cells and was labeled as probable leiomyoma but cannot rule out sarcoma. After discussion with operative team, pathologist, and radiologist that read the PET/CT scan the decision was made to perform Transhiatal esophagectomy. The final pathology showed an $8.8 \mathrm{~cm}$ leiomyoma as well as evidence of Barrett's esophagus with high grade dysplasia. The postoperative course was uneventful and the patient was discharged on post-operative day number nine. Patient B had a history of achalasia treated with prior thoracic esophagomyotomy who was referred for reflux refractory to balloon dilations and medications. Workup including CT scan, upper GI contrast study, endoscopic ultrasound, PET/CT, and manometry revealed a distal esophageal mass that was PET avid. His endoscopic biopsy showed no malignant cells. Intra-operative frozen section was described as leiomyoma versus gastrointestinal stromal tumor. Final pathology was consistent with $8.5 \mathrm{~cm}$ leiomyoma that stained positive for smooth muscle and desmin. The postoperative course was uneventful and the patient was discharged home on post-operative day number eight.

Conclusions: The patients in this series had large, symptomatic esophageal leiomyomas with a positive PET scan and underwent an operation fit for an invasive cancer. Only on final pathology could we fully determine that a limited resection would have been acceptable. This underscores the diagnostic uncertainty that comes with large esophageal leiomyomas and also calls into question the utility of PET scan to aid in characterization of tumor aggressiveness. These cases and updated literature review help to expand the awareness of the PET/CT limitations in determining aggressiveness of esophageal submucosal tumors.

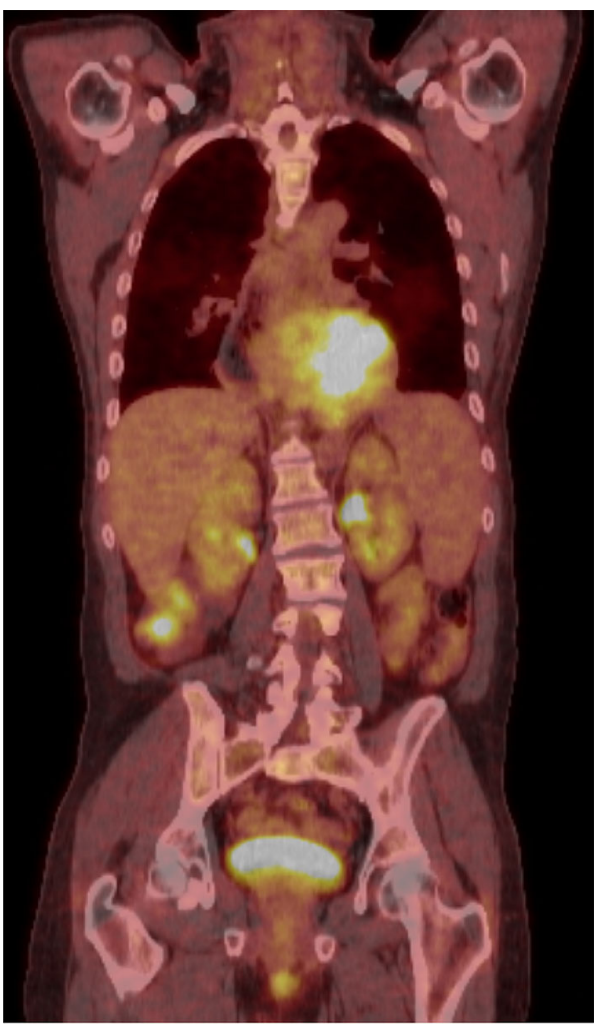

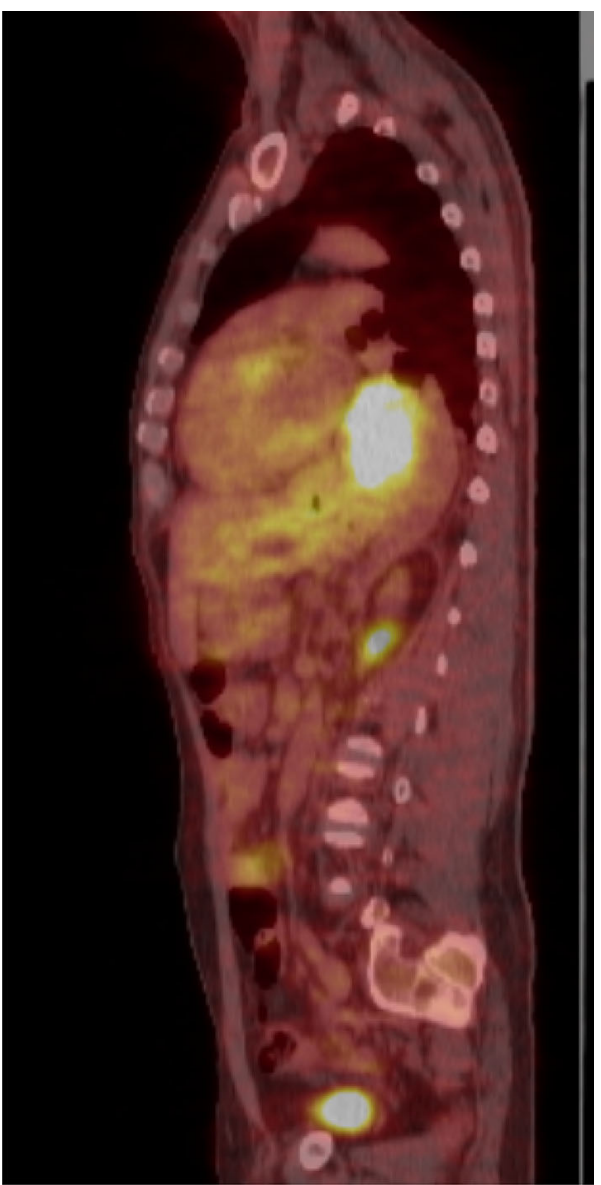

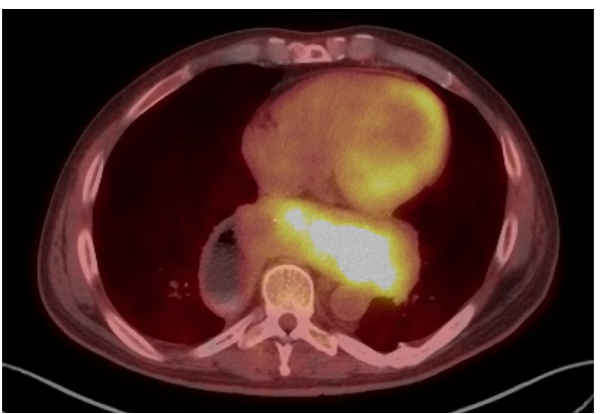


P514

\section{Laparoscopic Transgastric Resection of Gastric Submucosal Tumors Located Near the Gastroesophageal Junction}

\author{
Benjamin White, MD, Chitharanjan Pullattrana, MD; Medical \\ University of South Carolina
}

Background: Gastric submucosal tumors (SMT) near the gastroesophageal (GE) junction typically cannot be excised endoscopically, and historically have required potentially morbid proximal gastric resection and reconstruction. Laparoscopic-assisted transgastric resection (LTR) has been described in recent years as a novel and minimally invasive solution with good oncologic outcomes. Here we present the largest single institution series to date, describe our operative technique, and affirm that this approach is safe and efficacious. Methods: We performed retrospective review of consecutive patients undergoing LTR over a three-year period between 2015 and 2018 at our institution. Results: 15 patients, 7 male (46.7\%) and with a mean age of 56.5 years (range 32-82) with gastric SMT located at the GE junction underwent LTR. Mean BMI was 32.9 (22.7-42). No conversions to open or traditional laparoscopic wedge resection occurred. There were no intraoperative complications. Mean operative time was $93.7 \mathrm{~min}$ (59-157), and mean EBL was $32 \mathrm{~mL}$ (5-200). Intraoperative endoscopy was performed in all cases. One patient required transfusion of one unit of PRBCs. Mean length of stay was 1.8 days (1-6), with 8 patients (53\%) being discharged on postoperative day 1. Mean tumor size was $3.6 \mathrm{~cm}$ (2.2-7), and R0 resection was achieved in all cases. There was no tumor capsular rupture. Leiomyoma (6) and GIST (5) were the most common final pathologic findings. There were no mortalities or major 30-day morbidities, no ICU admissions, no readmissions, and no reoperations. One patient had dysphagia at 4 months postoperatively, which was managed endoscopically. Conclusions: Laparoscopic transgastric resection is a safe, replicable, well tolerated, and oncologically sound technique for resection of gastric submucosal tumors located near the GE junction.

Keywords: Gastroesophageal junction, submucosal tumor, GIST, leiomyoma, laparoscopic transgastric resection

\section{P515}

\section{Case Report: Minimally Invasive Approach to SMA Syndrome with a Review of Diagnostic Criteria and Treatment Options}

\section{Lindsay Tse, DO; Houston Methodist Hospital}

Superior Mesenteric Artery Syndrome is a rare and potentially life threatening entity that has a reported incidence in medical literature of 0.1 to $0.3 \%$. SMA syndrome occurs when the third portion of the duodenum is compressed by a narrowed angle ranging from six and twenty -five degrees between the abdominal aorta and the superior mesenteric artery. The decreased angle causes a mechanical compression, which is often secondary to a lack of retroperitoneal and visceral fat. Symptoms are often nonspecific, and a definitive diagnosis is often difficult to obtain. This case report describes a 20 year old female diagnosed with superior mesenteric artery syndrome who presented for surgical consultation after years of failing medical interventions. She described an extensive history of nausea and vague post prandial abdominal pain unrelieved by numerous medical interventions including long term PPI use, diet modification, and treatment for irritable bowel syndrome. Her BMI was consistently 18 or under throughout her life, and she reported being unable to gain weight due to lack of caloric intake. Physical exam and laboratory testing were unremarkable. EGD revealed a single gastric polyp which was removed by cold snare, but was otherwise unremarkable. Upper GI demonstrated a functional stenosis at the third portion of the duodenum as it transverses the spine, and CTA abdomen/pelvis showed a discrete transition in caliber from the proximal to distal duodenum with a decreased aortomesenteric angle. Treatment options included forced increased caloric intake, jejunostomy tube placement, duodenojejunostomy, and/or ligation of the ligament of Treitz. The patient underwent a laparoscopic duodenojejunostomy using two $3 \mathrm{~mm}$ ports and one $12 \mathrm{~mm}$ port. At the time of surgery, the superior mesenteric artery was easily visualized, and the duodenum was nearly two times the diameter of the small bowel immediately distal to the artery. A hand sewn, two layer anastomosis was created to bypass the area of obstruction. Post operatively, the patient recovered uneventfully, and is currently tolerating a soft diet with improvement in symptoms. Minimally invasive laparoscopic duodenojejunostomy is a safe and effective treatment option for select patients with superior mesenteric artery syndrome.

\section{P516}

\section{Does Preoperative Splenic Embolization Facilitate Laparoscopic Splenectomy in Massive Splenomegaly?}

Hamda Al Zarooni, MD, Ajda Altinoz, MD, Shadi Al Bahri, Guido Mannaerts, MD, PhD; Tawam Hospital Johns Hopkins International

Introduction: Massive splenomegaly is defined as a palpable spleen more than $8 \mathrm{~cm}$ below the costal margin, $>20 \mathrm{~cm}$ in diameter, or weighs $>1000$ gm. We present a case of massive splenomegaly who underwent splenic artery embolization to facilitate laparoscopic splenectomy.

Case Presentation: A 22 year old male with a history of beta thalassemia major undergoing regular blood transfusions presented with generalized weakness and abdominal distension. On examination, his spleen was palpable to the left lower abdomen. Workup showed pancytopenia, CT abdomen revealed a splenic diameter of $27 \mathrm{~cm}$, splenic and portal vein dilation up to $18 \mathrm{~mm}$. Distal splenic artery embolization was performed by interventional radiology using Gelfoam. Day 3 post-embolization, patient underwent Laparoscopic splenectomy. He was positioned supine. Ports placed according to splenic borders. A $12 \mathrm{~mm}$ right hypochondrial camera port, $5 \mathrm{~mm}$ epigastric, $12 \mathrm{~mm}$ infraumbilical and a $5 \mathrm{~mm}$ left paraumbilical port. Spleen was retracted through the left paraumbilical port using a fan retractor. An electrothermal bipolar sealing device introduced through the infraumbilical port was used for dissection of the splenic ligaments and hilum. Splenic artery control was stapled using a white $(2.8 \mathrm{~mm})$ cartridge. Specimen was retrieved through a pfannenstiel incision in three portions.. No remnants left in the abdomen. Spleen measured $27 \mathrm{~cm}$ in size. Drain was placed in the splenic bed. Intraoperative blood loss was $100 \mathrm{ml}$. The patient was discharged on postoperative day 3 without complications. Drain was negative for amylase. Postsplenectomy vaccination was administered.

Discussion: Splenectomy for massive splenomegaly was conventionally done via midline laparotomy; however with the emergence of minimally invasive surgery and interventional radiologic techniques, laparoscopic splenectomy in massive splenomegaly is now feasible. Supine position as opposed to the conventional right lateral decubitus allowed for easier port placement and ergonomics, as the spleen was occupying the left side of the abdomen. Removal of the specimen via pfannenstiel incision saves time, carries low morbidity, is cosmetically acceptable and provides adequate histology. According to our literature review, expected benefits from splenic artery embolization included decreased intraoperative bleeding and reduction in splenic size. Despite the spleen not decreasing in size in our case, potential intraoperative hemorrhage was significantly reduced.

Conclusion: Combining laparoscopic splenectomy with preoperative splenic artery embolization in massive splenomegaly may reduce intraoperative blood loss, morbidity, conversion rates and hospital stay. Further studies are required to study the best interval between embolization and surgery to maximize potential benefits. 
P517

\section{Elective Versus Non-elective Laparoscopic Paraesophageal} Hernia Repairs: Comparison Using the NSQIP Database

William Sherrill, MD, Brent D Matthews, MD, Caroline E Reinke, MD, MSHP; Carolinas Medical Center

Background: Utilization of minimal invasive surgery (MIS) is on the rise, and laparoscopic paraesophageal hernia (lapPEH) repairs are an increasingly common procedure. Most lapPEH repairs are performed electively, but a subset are performed during an unplanned admission. We aimed to investigate the outcomes of patients who underwent non-elective lapPEH repair compared to elective lapPEH repair. We hypothesized that patients undergoing non-elective lapPEH repair would have similar 30-day outcomes to elective patients.

Methods: This retrospective study utilized 2012-2016 NSQIP PUFs. We identified patients by CPT codes $(43281,43282)$. Case status was classified as elective, emergency, or urgent (non-elective, non-emergency). We compared preoperative patient characteristics, operative characteristics, and 30-day postoperative complication rates by case status. Logistic regression was used to examine the impact of case status on death or serious morbidity (DSM) and 30-day mortality.

Results: We identified 16,647 lapPEH repairs. 91\% were performed electively, $7 \%$ urgent and $2 \%$ emergencies. Elective patients were younger, had a higher BMI, were more likely to be functionally independent, and were more likely to have dyspnea with moderate exertion $(\mathrm{p}<0.01)$. Non-elective cases had higher rates of preoperative sepsis $(\mathrm{p}<0.01)$. Mortality rates were higher in the non-elective cases $(0.5 \%$ elective, $3 \%$ urgent, $2 \%$ emergency, $\mathrm{p}<0.01)$. Undergoing non-elective lapPEH repair is associated with increased odds of DSM, even after controlling for patient characteristics (Table 1). While both urgent and emergency cases were associated with an increased risk of death in univariate analysis, this association was no longer significant in emergent cases after adjustment.

Conclusions: For lapPEH repairs, patient characteristics are significantly different in the non-elective cases and mortality is higher in the urgent and emergent population. When the skill set is available, utilization of MIS techniques in the non-elective lapPEH repairs almost certainly benefits the patient, but is not enough to create outcomes equivalent to those seen in the elective procedures.

Table 1: Multivariable Analysis comparing case status

\begin{tabular}{lllll}
\hline DSM & OR & CI & p-value & \\
\hline Elective & Ref & Ref & Ref & \\
Urgent & 2.11 & $1.7-2.6$ & $<0.01$ & \\
Emergency & 2.49 & $1.8-3.5$ & $<0.01$ & \\
\hline Mortality & OR & CI & p-value \\
\hline Elective & Ref & Ref & Ref \\
Urgent & 2.18 & $1.3-3.7$ & $<0.01$ \\
Emergency & 0.75 & $0.23-2.48$ & $<0.01$ \\
\hline
\end{tabular}

\section{P518}

\section{Intraoperative EGD During Heller Myotomy: Updating Guidelines}

Elizabeth H Bruenderman, MD, Robert C Martin, MD, PhD, Matthew P Fox, MD, Victor H Van Berkel, MD, PhD, Stacy B Block, ARNP, Farid J Kehdy, MD; University of Louisville

Background: Heller myotomy remains the optimal treatment for achalasia. SAGES guidelines, last updated in 2011, recommend the routine use of intraoperative esophagogastroduodenoscopy (EGD) during Heller myotomy to assess for mucosal perforation and for adequacy of the myotomy. An examination of recent literature reveals little regarding the true utility of EGD in preventing postoperative leaks and/or myotomy failure. This study aimed to evaluate the effect of intraoperative EGD on surgical outcomes after Heller myotomy.

Methods: Patients with achalasia who underwent Heller myotomy between 2013 and 2018 in a single center were retrospectively identified and data extracted via an IRB-approved protocol. Patient cohorts were divided into those who underwent intraoperative EGD and those who did not. Intraoperative EGD evaluated myotomy adequacy by assessing distensibility of the lower esophageal sphincter (LES) with air insufflation and the effort required to push the endoscope through, both before and after the myotomy. To evaluate for perforation, EGD also included visual assessment of the mucosa and air leak test after the myotomy. Patient characteristics and surgical outcomes were compared between groups.

Results: Sixty-one patients were reviewed, 46 (75.4\%) underwent intraoperative EGD, $15(24.6 \%)$ did not. There were no differences in age, gender, BMI, operative times, or lengths of stay. A total of $5(8.2 \%)$ mucosal perforations occurred, 2 (4.3\% of cohort) in the EGD group and 3 (20\% of cohort) in the non-EGD group $(\mathrm{p}=0.09)$. All perforations, regardless of EGD use, were recognized laparoscopically. Of the entire cohort, the non-EGD group was more likely to undergo postoperative esophagogram $(15,100 \%)$ than those who underwent EGD $(17,37.0 \%)(\mathrm{p}<0.01)$. Postoperative leak rate was $0 \%$ for both groups. Failed myotomy, defined as recurrent symptoms post myotomy with objective evidence of constriction at the LES, occurred in $5(11.1 \%)$ who underwent EGD and $1(6.7 \%)$ who did not $(\mathrm{p}=1)$.

Conclusions: In patients undergoing Heller myotomy for achalasia, SAGES guidelines advocate routine intraoperative EGD to assess the integrity of the gastroesophageal mucosa and adequacy of the myotomy. In this study, EGD did not detect mucosal perforations that were not detected laparoscopically, nor did EGD alter postoperative leak rates or myotomy failure rates. Performing an EGD with air leak test does, however, provide an immediate and reliable indicator of an intact repair after perforation. We recommend modifying SAGES guidelines to emphasize the use of EGD in the setting of known or suspected perforation, rather than routine use in every Heller myotomy. 
P519

\section{Our Gastro-Esophageal Reflux Test Deciding Surgical Indication and Results of Laparoscopic Nissen Fundoplication for GERD Patients}

Tatsushi Suwa, MD, PhD, Kenta Kitamura, MD, Ayato Obana, MD, Norio Mizuguchi, MD, Mayuko Nakayama, MD, Kazuhiro Karikomi, MD, Motoi Koyama, MD, PhD, Yoshinobu Sato, MD, PhD, Ryuji Yoshida, MD, Hiroyuki Suzuki, MD, Shigeru Masamura, MD, PhD; Kashiwa Kousei General Hospital

Introductions: The indication of laparoscopic anti-reflux surgery for GERD patients is difficult to be judged fairly. We have established "Reflux Test" as the tool for the decision in the surgical indication for GERD patients.

\section{SURGICAL INDICATION}

Reflux Test: At the standing position a patient swallows $300 \mathrm{ml}$ barium solution. After total solution goes into stomach, a patient lies down at the flat position.

Then a patient changes the position to left lateral decubitus position, flat position, right lateral decubitus position and flat position again every $10 \mathrm{~s}$ in the order.

During this procedure, gastro-esophageal reflux was evaluated and assigned to severe, moderate and slight category. If the reflux was observed slightly up to cervical esophagus, the case was assigned to moderate category. If the reflux was observed intensely up to cervical esophagus, the position was returned to head high position for the safety and the case was assigned to severe category. The anti-reflux surgery was considered in the moderate and severe categories. Results: We have performed laparoscopic Nissen procedure in 91 cases. The outcome was assessed by Reflux Test performed on 4-5 postoperative day, and the results showed the reflux was disappeared in every cases.

Median follow-up period of this study was 55 months (3-106 months). In 11 cases $(12.1 \%)$ PPI was restarted before 6 months after the anti-reflux surgery. In 23 cases $(25.3 \%)$ PPI was restarted after the anti-reflux surgery during the whole follow-up period of this study.

The BMI of the patients had no relationship to the needed restart of PPI.

To evaluate the degree of esophagitis objectively before and after the antireflux surgery we designed "the esophagitis score". In this scoring method, a number from $0-5$ was assigned according to the degree of esophagitis along with the LA classification. The results of the study have shown that the reflux esophagitis was improved obviously after the anti-reflux surgery even in the PPI restarted group $(\mathrm{p}<0.001)$.

Discussion: The number of GERD patients who needed anti-reflux surgery seems to be so high. To extract the patients who needed it remarkably is important. The anti-reflux surgery is most effective for the patients who really have the obvious reflux. Reflux Test is feasible because of its convenience and visual effects for the patients. The results of the laparoscopic Nissen fundoplication were good and satisfied by the patients mostly.

\section{P520}

\section{Dynamic Evaluation of Luminal Esophageal Pressures During Anti-reflux Procedures: A Pilot Study}

Dessislava I Stefanova, MD, Timothy M Ullmann, MD, Adham Elmously, MD, Katherine D Gray, MD, Jessica N Limberg, MD, Thomas J Fahey III, MD, Felice H Schnoll-Sussman, MD, Philip O Katz, MD, Rasa Zarnegar, MD; NYP-Weill Cornell Medical Center

Introduction: Anti-reflux surgery involves augmentation of the lower esophageal sphincter (LES), however to date little is known about intraoperative changes in LES pressures. Moreover, there is lack of data on the effects of mesh on LES pressure. As such, it is critical for surgeons to maintain a balance between too much and too little augmentation of the LES in order to resolve reflux without causing dysphagia. We hypothesized that dynamic intraoperative LES monitoring may assist in determining the effects of essential steps in anti-reflux surgery.

Methods: Patients with type II/III hiatal hernias undergoing an anti-reflux procedure were enrolled in a prospective database. All operations were performed using robotic-assisted laparoscopic technique with biosynthetic mesh reinforcement of the hiatus using a keyhole technique. Repair was determined on a per-patient basis based on preoperative manometric and clinical characteristics. Intraoperative LES measurements were collected using EndoFlip, which utilizes planimetry impedance, to measure cross-sectional area, pressure, and distensibility of the LES. Correct positioning was ensured by intraoperative visualization and wave narrowing on the EndoFlip display. Data were collected at four crucial timepoints: pre-repair, post hiatal hernia closure, post mesh placement, and post-fundoplication or magnetic augmentation.

Results: A total of 35 patients were included; seventeen patients (48.6\%) were female with a mean age of 55.5 years (range $20-86$ years). The median preoperative DeMeester score was 32.9 (IQR 19-60). The cohort underwent Nissen $(45.7 \%)$, Toupet $(42.9 \%)$, or LINX $(11.4 \%)$ procedures. Five enrolled patients $(14.2 \%)$ had previous anti-reflux surgery.

Cross-sectional area (CSA) of the LES decreased significantly following closure of the hiatus (50 [IQR 37-66 $\mathrm{mm}^{2}$ ] vs. 31 [IQR 24-42.5 $\left.\mathrm{mm}^{2}\right], \mathrm{p}=0.0003$ ) and remained relatively unchanged thereafter, while pressure increased incrementally from induction to augmentation $(25.7[\mathrm{IQR} 21.5-33.2 \mathrm{mmHg}$ ] vs 40[IQR 35.6-44.1 mmHg], p < 0.0001) (See Figure). Distensibility of the LES significantly decreased from pre- to post-fundoplication/augmentation (1.9 $\mathrm{mm}^{2} / \mathrm{mmHg}$ [IQR $1.3-2.5 \mathrm{~mm}^{2} / \mathrm{mmHg}$ ] vs. $0.8 \mathrm{~mm}^{2} / \mathrm{mmHg}$ [IQR $0.56-1 \mathrm{~mm}^{2} /$ $\mathrm{mmHg}$ ], $\mathrm{p}<0.0001)$.

Mesh placement did not significantly alter cross-sectional area (31[IQR $24-42.5 \mathrm{~mm}^{2}$ ] vs 29 [IQR 23-42 $\mathrm{mm}^{2}$ ] $\mathrm{p}=0.32$ ), or pressure (33[IQR 29-36.7 $\mathrm{mmHg}$ ] vs 35.8[IQR 29-38.7 $\mathrm{mmHg}$ ], $\mathrm{p}=0.20)$.

Discussion: Dynamic intraoperative LES monitoring demonstrated an increase in pressure and a decrease in cross-sectional area and distensibility following fundoplication. Closure of the hiatus produced the most dramatic decrease in LES cross-sectional area, possibly indicating that this plays the most critical component in restoring LES competence. Mesh placement did not significantly change LES parameters. Correlation of these changes with postoperative clinical outcomes is warranted.

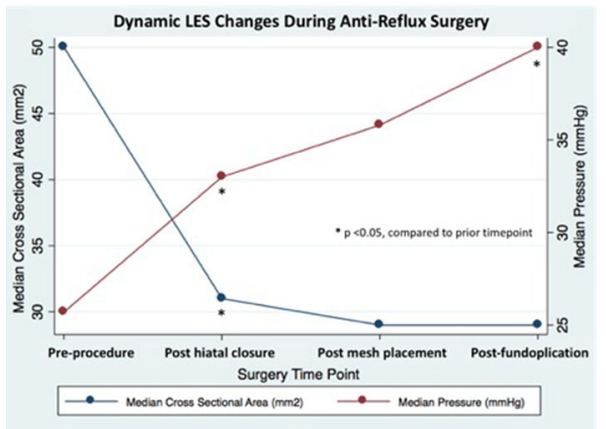


P521

\section{An Unusual Case of an Esophageal Polyp}

Ernest G Chan, MD, MPH, Patrick G Chan, MD, MPH, Omar Awais, DO, Rajeev Dhupar, MD, James D Luketich, MD, Matthew J Schuchert, MD; UPMC

Introduction: Polyps can develop at any portion of the gastrointestinal tract and are most common in the colon. In the esophagus, an overwhelming majority of these polyps are benign lesions originating from the cervical esophagus. While polyps more commonly become neoplastic in the colon, a rare percentage have the ability to develop into a malignancy in the esophagus. We present the diagnostic work-up, operative management, and postoperative course of an unusual case of an esophageal polyp.

Case Description: A 68-year-old male presented to the gastroenterology department with significant signs of anemia including a sudden drop in hemoglobin. Upon upper endoscopy, he was noted to have a $4 \mathrm{~cm} \mathrm{X} 7 \mathrm{~cm}$ mass in the proximal thoracic esophagus with a well demarcated stalk which began at $20 \mathrm{~cm}$ from the incisors. The mass extended to $27 \mathrm{~cm}$ from the incisors. A barium swallow was obtained for additional work-up which showed a filling defect in the proximal esophagus outlining a lengthy stalk arising from the esophagus at the level of the cricopharyngeus. The patient was brought to the operating room for endoscopic evaluation and attempt at endoluminal excision. However, upon inspection of the mass, there were several areas of nodularity suspicious for malignancy. Multiple attempts at snaring the lesion was unsuccessful give the sheer girth of the polyp. Next, attempts were directed to cauterize the stalk to detach the lesion from the mucosa. However, due to the fear of thermal injury to the esophagus and the close vicinity to the cricopharengeous, the operation was again aborted. Therefore, a final attempt via a left neck exploration with creation of an esophagotomy was planned to achieve the complete removal of the esophageal polyp. The esophagus was circumferentially dissected and a $4 \mathrm{~cm}$ longitudinal esophagotomy was created along the lines of the longitudinal fibers. The stalk of the pedunculated mass was divided, over-sewn with an absorbable suture, and cauterized. Final pathology was positive for squamous cell carcinoma with an associated malignant spindle cell stromal component.

Discussion: Esophageal polyps are defined in the literature as macroscopic, well-demarcated projections of epithelial tissue above the mucosa with a fibrovascular core. While majority of these polyps are indeed benign, there is potential for malignancy. Extensive work up including computed tomography and barium esophagram can provide prudent information regarding structural characteristics of the lesion. We recommend further observation and follow up to ensure there is no residual disease.

\section{P522}

Minimally Invasive Approach to Paraesophageal Hernia Repair is Superior to Open, Even in the Emergent Setting: A Large National Database Analysis

Salim Hosein, MD, Laura Flores, Priscila R Armijo, MD, Tyson Carlson, Dmitry Oleynikov, MD; University of Nebraska Medical Center

Introduction: We aimed to examine the utilization of various operative approaches to hiatal hernia repair (HHR) in elective and emergent/urgent (U) settings and to compare outcomes.

Methods: Vizient database was queried from 2015 to 2017 for adult patients with HHR using ICD codes. Patients were grouped into open (OHHR), laparoscopic (LHHR), or robot-assisted (RHHR) groups, and further stratified by elective or $\mathrm{U}$ status and severity of illness at admission. Surgical outcomes and costs were compared across all groups. Statistical analysis was done using SPSS v.23.0.

Results: 9,171 adults underwent HHR during this period (OHHR: $\mathrm{N}=1,534$; LHHR: $N=6,796$; RHHR: $N=841$ ). Overall, LHHR was the most frequently utilized approach $(74.1 \%)$, followed by OHRR $(16.7 \%)$ and then RHHR $(9.2 \%)$. Table 1 demonstrates the utilization of each approach in both elective and $\mathrm{U}$ settings. OHHR approach was employed three times as frequently in $\mathrm{U}$ settings, compared to elective and $9.6 \%$ of elective, minor illness severity patients had OHHR. Overall analysis revealed that OHHR had longer average length of stay (LOS, 9.41 vs. $<4$ days, $\mathrm{p}<0.05$ ) and higher post-operative complication rates $(8.8 \%$ vs $<3.8 \%, \mathrm{p}<0.05)$, mortality $(2.7 \%$ vs $<0.5 \%)$ and mean direct costs $(\$ 27,842$ vs $<\$ 10,407)$, when compared to both LHHR and RHHR. Further analysis of mild to severely ill elective cases demonstrated LHHR and RHHR to be better than OHHR in terms of complications $(p<0.05)$, cost $(p<0.001)$ and LOS $(p<0.013)$; there were insufficient extremely ill elective patients for meaningful analysis. In the U setting, minimally invasive approaches predominate, overtaken by OHHR only for the extremely ill. Despite the U setting, for mild-moderately ill patients, OHHR was statistically inferior to both LHHR and RHHR in terms of LOS ( $=0.002$, $\mathrm{p}<0.0001)$ and cost $(\mathrm{p}=0.0133, \mathrm{p}<0.001)$. In severe-extremely ill patients, despite being proportionately more utilized, OHHR was not superior to LHHR; in fact, complication, cost and mortality trends (all p > 0.05) favored LHHR. Conclusions: Our analysis demonstrated LHHR to currently be the most employed approach overall. LHHR and RHHR were associated with lower cost, decreased LOS, complications and mortality compared to OHHR, in all but the sickest of patients. Patients should be offered minimally invasive HHR, even in urgent/emergent settings, if technically feasible.

\begin{tabular}{|c|c|c|c|}
\hline Table 1. Outcomes between app & $\begin{array}{l}\text { aches, all cases } \\
\text { Laparoscopic } \\
\mathrm{N}=6,796(74.1 \%)\end{array}$ & $\begin{array}{c}\text { Robotic } \\
\mathrm{N}=841(9.2 \%)\end{array}$ & $\begin{array}{c}\text { Open } \\
\mathrm{N}=1,334(16.7 \%)\end{array}$ \\
\hline \multicolumn{4}{|l|}{ Elective } \\
\hline Minor $(\mathrm{N})$ & 4,551 & 603 & 553 \\
\hline Moderate $(\mathrm{N})$ & 476 & 72 & 128 \\
\hline Severe $(M)$ & 222 & 24 & 83 \\
\hline Extreme $(\mathrm{N})$ & 8 & 5 & 5 \\
\hline \multicolumn{4}{|l|}{ Emergent } \\
\hline Minor $(\mathrm{N})$ & 1,021 & 108 & 313 \\
\hline Moderate $(\mathrm{N})$ & 293 & 16 & 183 \\
\hline Severe $(\mathrm{N})$ & 141 & 5 & 113 \\
\hline Extreme $(\mathrm{N})$ & 62 & 2 & 110 \\
\hline Overall Complications (N) & $253(3.7 \%)^{*+}$ & $17(2.0 \%)^{x,+}$ & $135(8.8 \%) * *$ \\
\hline Mortality (N) & $30(0.4 \%)^{-}$ & $1(0.1 \%)^{*}$ & $42(2.7 \%) \cdot *$ \\
\hline LOS (days) & $3.9(5.22)$ & $3.44(5.01)$ & $9.41(10.21)$ \\
\hline Mean Direct Cost (\$) & $8,596(9,002)$ & $10,406(9,142)$ & $27,842(16,163)$ \\
\hline
\end{tabular}


P523

\section{Three-Dimensional (3D) Visualization Provides Better Outcome} in Single-Port Laparoscopic Distal Gastrectomy

So Hyun Kang, Yo-Seok Cho, Sa-Hong Min, Young Suk Park, SangHoon Ahn, Do Joong Park, Hyung-Ho Kim; Seoul National University Bundang Hospital

Introduction: The use of a three-dimensional (3D) camera for laparoscopic surgery has been reported in literature. However, there are only few comparative studies demonstrating its benefits, and no reports on the application of 3D vision to single-incision laparoscopic surgery. This study aims to compare 3D vision to the previous two-dimensional (2D) system in solo single-incision laparoscopic distal gastrectomy (SIDG).

Methods: Medical charts of 179 gastric cancer patients who underwent solo SIDG from February 2014 to December 2017 were retrospectively reviewed. Patients were grouped into either 2D group or 3D group depending on the type of camera used. All the operations were performed by a single surgeon using a flexible camera (Olympus, Japan), fixed onto a passive scope holder without the use of a scopist or an assistant. Operative data, postoperative outcome, and early complication were analyzed.

Results: Ninety had their operations under 2D vision and 89 used the 3D scope. In both groups, there was no difference in age, body mass index, staging, and other demographic or histopathologic criteria. Operative time was significantly faster in the 3D group $(115.6 \pm 34.0$ vs. $129.4 \pm 38.5$ min., $p=0.012)$ and EBL was also less $(20.7 \pm 30.0$ vs. $35.1 \pm 56.0 \mathrm{ml}, \mathrm{p}=0.034)$. Patients in the 3D group started small fluid diet faster $(2.5 \pm 0.9$ vs. $3.0 \pm 1.1$ postoperative days, $\mathrm{p}=0.006)$, and were discharged faster $(4.4 \pm 1.7$ vs. $5.2 \pm 3.1$ postoperative days, $p=0.024$ ). Early complication was also less in the 3D group $(2.2 \%$ vs. $6.7 \%)$ but there was no statistical significance $(\mathrm{p}=0.140)$.

Conclusion: The use of the 3D camera improves operative outcome and hospital stay in patients undergoing solo SIDG.

\section{P524}

\author{
Male Sex Is an Independent Predictor of 30-Day Mortality \\ in PEH Repair
}

Alexis Graham, MD, Miloslawa Stem, MS, Alisa Coker, MD, Gina Adrales, MD; Johns Hopkins Hospital

Introduction: Increased age, higher American Society of Anesthesiologists (ASA) score, and frailty are known risk factors for morbidity and mortality following paraesophageal hernia (PEH) repair. Understanding the effect of sex on outcomes may impact the approach to repair.

Methods: A retrospective cohort study of ACS-NSQIP patients who underwent primary laparoscopic PEH repair between 2012-2016 was performed. Those undergoing concurrent bariatric and esophagectomy procedures were excluded. Primary outcomes included 30-day morbidity, mortality, readmission, and reoperation with patients stratified by sex (Table). Multivariable logistic regression analysis was used to identify risk factors for emergent repair and to assess the impact of sex on outcomes. Separate analyses were performed for elective and emergent cases.

Results: Of the 15,596 patients who met study criteria, $71.3 \%$ were female $(\mathrm{F})$ and $28.7 \%$ were male $(\mathrm{M})$. Of these, $97.8 \%(\mathrm{n}=15,429)$ underwent elective repair $(97.9 \% \mathrm{~F})$ and $2.2 \%(\mathrm{n}=347)$ underwent emergent repair $(67.4 \% \mathrm{~F})$. Within the elective repair group, men were younger (age $>65$ years; 39.9 vs $50.3 \%$ ), thinner (BMI $>30$; 37.2 vs $51.1 \%$ ), and less healthy (ASA $>3 ; 2.7 \%$ vs $1.9 \%$ ) with an increased incidence of bleeding diathesis (2.3 vs $1.7 \%$ ), longer operating times (min; $155 \pm 78$ vs $142 \pm 69$ ), and increased mortality ( 0.82 vs $0.48 \%)$, when compared to females, respectively. Significant risk factors for mortality included male sex (OR 1.86; 95\%CI (1.20-2.89), $\mathrm{p}=0.006)$, COPD (OR 1.99 (1.11-3.57), $\mathrm{p}=0.021)$, bleeding diathesis (OR $3.34(1.67-6.68) ; \mathrm{p}=0.001)$, age $>65 y$ (3.6 (1.97-6.56), p < 0.001), and ASA $>3$ (6.48 (3.05-13.78), $\mathrm{p}<0.001)$. There was no difference between sexes in morbidity, readmission, or reoperation.

Among the emergent group, baseline characteristics between sexes where comparable except for longer operative time in males $(181 \pm 74 \mathrm{~min})$ vs females $(157 \pm 72 \mathrm{~min})$. There was no significant difference in outcomes between sexes in the emergent group. Significant risk factors for emergent repair included age $\geq 75$ (OR 1.48, (1.02-2.15), p = 0.039), ASA III and IV-V (OR 1.95, (1.48-2.56); OR 8.90, (5.85-13.55); respectively; both $\mathrm{p}<0.001)$, and bleeding diathesis (OR 2.85, (1.83-4.42), $\mathrm{p}<0.001)$.

Those with BMI $>30$ had a decreased mortality risk in the elective setting (OR $0.59(0.36-0.96), p=0.034)$ and a decreased risk of needing emergent repair (OR $0.60(0.44-0.81), \mathrm{p}=0.001)$.

Conclusion: Male sex was a significant predictor of 30-day mortality in PEH repair independent of poor health. While higher BMI appears to be protective, it may correlate with female sex. Differences in fat distribution between sexes may contribute to longer operative times and subsequently greater morbidity in males.

\begin{tabular}{|c|c|c|c|c|c|c|}
\hline & & ive & & Eme & gent & \\
\hline & Male & Female & & Male & Female & \\
\hline Outcome (\%) & 4366 & 10,883 & & 113 & 234 & \\
\hline & (28.63) & (97.90) & $p$ & $(32.56)$ & $(67.44)$ & $p$ \\
\hline Overall morbidity & $245(5.61)$ & $688(6.32)$ & 0.098 & $19(16.81)$ & $53(22.65)$ & 0.209 \\
\hline Serious morbidityb & $101(2.31)$ & $242(2.22)$ & 0.736 & $9(7.96)$ & $27(11.54)$ & 0.306 \\
\hline Mortality & $36(0.82)$ & $52(0.48)$ & 0.011 & $1(0.88)$ & $5(2.14)$ & 0.668 \\
\hline Readmission & $252(5.77)$ & $669(6.15)$ & 0.379 & $10(8.85)$ & $24(10.26)$ & 0.680 \\
\hline Reoperation & $105(2.40)$ & $269(2.47)$ & 0.810 & $6(5.31)$ & $20(8.55)$ & 0.283 \\
\hline
\end{tabular}




\section{P525}

\section{Magnetic Sphincter Augmentation: Poor Consensus Among Experts Regarding Key Technical Aspects of LINX Implantation}

\section{Steven Leeds, MD, Marc Ward, MD; Baylor University Medical} Center at Dallas

Introduction: Magnetic sphincter augmentation (MSA) of the lower esophageal sphincter (LES) by LINX implantation is a highly effective GERD treatment. Reports describe significant improvements in GERD-HRQL, normalization of esophageal acid exposure, and freedom from PPIs for more than $85 \%$ of carefully-selected GERD patients at 5 years after MSA. However, many key technical aspects of LINX implantation are not standardized. The purpose of this study was to determine how often surgical experts agree on technical aspects of the MSA procedure.

Methods and Procedures: We created a survey form comprising 12 questions on various technical aspects of the MSA procedure including patient selection criteria, hiatal dissection and repair procedures, criteria for selecting the proper site for placement, and criteria for the ideal appearance of the MSA implant after placement. We obtained a list of MSA procedure preceptor surgeons from the LINX manufacturer (Ethicon, Somerville, NJ), and sent the survey to those surgical experts. Consensus on individual questions was defined as $\geq 70 \%$ agreement among the responding surgeons.

Results: We sent the survey to 37 surgical experts, and 24 responded. The mean number of MSA procedures performed by the responders was 210 (range $10-600$ ). There was consensus on 4 questions: 1) hiatal hernia size is not a contraindication to MSA implantation, 2) tension at the crural closure does not influence the decision to implant the device, 3) LES muscle has no characteristic laparoscopic appearance, and 4) the posterior vagus nerve is one important landmark for localizing the LES. Consensus was not achieved for the other 8 questions that included criteria for the ideal location for LINX placement, criteria for ideal appearance of the LINX with regard to the LES, the need for circumferential mobilization of the esophagus, criteria for determining the adequacy of mediastinal dissection, and the critical anatomical landmarks needed to ensure proper device placement. Notably, for the question "What do you consider the ideal location for LINX placement with respect to the LES?", $38 \%$ responded middle, $29 \%$ proximal, $25 \%$ distal, $4 \%$ immediately proximal and $4 \%$ immediately distal.

Conclusions: There is poor consensus among surgical experts on key technical aspects of MSA including what is the ideal anatomic location for LINX implantation. It is not clear how this variability in technical aspects of the procedure might influence its outcomes. Our results highlight the need for an expert consensus conference to standardize key technical aspects of the operation.

\section{P526}

\section{Outcomes of Lung Transplantation in Patients with Absent Esophageal Body Motility}

Takahiro Masuda, MD, Sumeet K Mittal, MD, Balazs Kovacs, MD, Michael A Smith, MD, Rajat Walia, MD, Jasmine Huang, MD, Ross M Bremner; Norton Thoracic Institute; St. Joseph's Hospital and Medical Cener

Introduction: Impaired esophageal motility results in poor esophageal clearance, and can exacerbate gastroesophageal reflux which is a potential risk for allograft dysfunction in lung transplant (LTx) recipients. However, the clinical course after LTx in patients with absent esophageal body motility has not been well described. The aim of this study is to explore the outcomes of LTx in patients with absent contractility.

Methods: We retrospectively reviewed the records of all patients who underwent lung transplantation at our institution between 2015 and 2016. Patients who underwent high-resolution esophageal manometry both before and after LTx were identified. We compared patients who diagnosed with absent esophageal body motility before LTx and patients who diagnosed with normal esophageal motility both pre- and post-LTx. Manometric diagnoses were performed based on Chicago classification v3.0.

Results: Of the 204 patients who underwent LTx during the study period, preLTx absent esophageal body contractility was seen in 10 patients including 2 patients diagnosed with achalasia type I, and 36 patients were diagnosed with normal motility both pre- and post-LTx. In patients with absent motility, there were 2 patients with chronic obstructive pulmonary disease, 7 patients with idiopathic pulmonary fibrosis, and 1 patient with scleroderma. Lung allocation score was higher in patients with absent esophageal body contractility than those with normal motility ( 40.3 vs $35.1, \mathrm{p}=0.033$ ). The $1-, 2-$, and 3-year post-LTx survival for patients with absent motility were 80,80 , and $37.5 \%$, respectively, and was lower than normal motility cohort (100, 93.5, 84.6\%, respectively; log-rank test, $\mathrm{p}=0.002$ ). Based on 24-hour $\mathrm{pH}$ monitoring, significantly greater \%time $\mathrm{pH}<4$ and DeMeester score were noted in patients with absent motility than those with normal motility before LTx $(8.9 \%$ vs $2.5 \%, \mathrm{p}=0.014 ; 30.3$ vs $10.5, \mathrm{p}=0.016)$, and the same trend was seen after LTx although it did not reach statistical significance $(8.2 \%$ vs $2.9 \%, p=0.176$; 27.6 vs $17.8, p=0.176)$. Importantly, esophageal body motility was improved after LTx in six out of 10 patients with absent motility. Two of 6 patients with improved esophageal motility showed normal esophageal body motility.

Conclusions: We have demonstrated that esophageal motility does improve in some patients after LTx even in patients with absent esophageal motility. The 2-year outcome of LTx in patients with absent motility seems to be reasonable, although the 3-year outcome requires improvement. Fundoplication for patients with improved esophageal motility may help better outcomes.

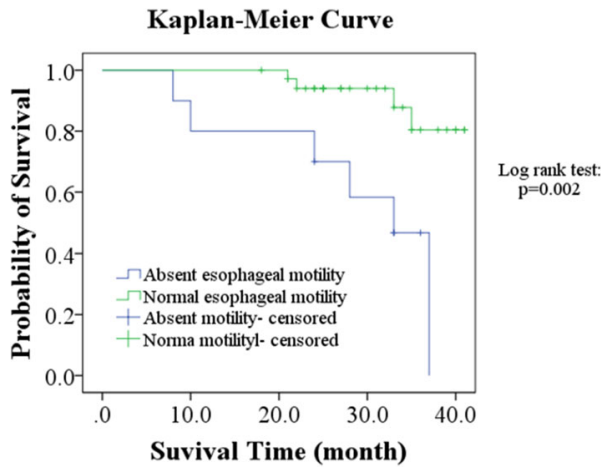


P527

Laparoscopic and Endoscopic Cooperative Surgery for Gastrointestinal Stromal Tumor of the Stomach

Norimasa Koide, Kazuyoshi Suda; Niigatakensaiseikai Sanjyo Hospital

Introduction: Gastrointestinal stromal tumors (GISTs) are the most common types of mesenchymal tumors of the gastrointestinal tract, and these tumors occur most frequently in the stomach. Complete resection is the curative treatment for limited gastric GIST. Many reports showed laparoscopic resection was useful procedure for gastric GIST. Recently several reports shows laparoscopic and endoscopic cooperative surgery (LECS) appears to be a preferable choice. The aim of this study is to investigate the LECS for gastric GIST in our institution.

Methods and Procedures: From April 2017 and September 2018, three patients with gastric GIST underwent LECS at our institution. The surgical outcomes were analyzed retrospectively.

Results: The patients consisted of one man and two women with a mean age of 69 years (ranging from 53-80). Mean tumor size was $5.7 \mathrm{~cm}$ (ranging from 3.2100 ), with the following anatomic distribution: fornix in two and body in one respectively. Mean operating time was 269 min (ranging from 104-352), and mean blood loss was $128 \mathrm{ml}$ (ranging from 5-360). There were no conversions to open surgery and no major intraoperative complications. The time to resumption of food intake was 2 days (ranging 1-3) and average length of postoperative hospital stay was 16 days (ranging from 5-33). Postoperative complications were neurogenic bladder dysfunction. There were no recurrences and deaths because of gastric GIST.

Conclusion: LECS for gastric GIST is a safe and feasible procedure except for longer operative time because there were no major surgical complications during perioperative period.

\section{P528}

Minimally Invasive vs. Open Pancreatectomy After Neoadjuvant Therapy for Pancreatic Adenocarcinoma: Short- and Long-Term Outcomes

Susanna W De Geus, MD, PhD, Jacob Nudel, Sing Chau Ng, Tara S Kent, David Mcaneny, Jennifer F Tseng, Teviah E Sachs; Boston Medical Center

Background: Neoadjuvant therapy is increasingly used for the treatment of (borderline) resectable pancreatic adenocarcinoma. Previous studies have shown that neoadjuvant chemotherapy with or without radiotherapy induces significant metabolic and inflammatory changes in remnant pancreatic parenchyma and the peripancreatic tissue. However, it remains unknown how these changes impact the outcomes of minimally invasive versus open pancreatic surgery.

Methods: Patients that underwent neoadjuvant chemotherapy with or without radiotherapy followed by surgery for pancreatic adenocarcinoma between 2010 and 2015 were identified from the National Cancer Data Base. Propensity score models were creating predicting the odds of undergoing minimally invasive surgery. Patients were matched based on propensity score. Short- and longterm outcomes were compared after matching. Survival analysis was performed using the Kaplan-Meier method.

Results: 1,640 patients were identified. $1,319(80.4 \%)$ and $321(19.6 \%)$ patients underwent open and minimally invasive pancreatic surgery, respectively. $71(22.1 \%)$ procedures that started minimally invasive were converted to open. After matching, length of stay was significantly shorter (median length of stay: 7 vs. 8 days; $p=0.0008$ ) after minimally invasive surgery compared to open surgery. However, positive resection margins $(16.8 \%$ vs. $14.3 \%$; $\mathrm{p}=0.3839)$ and readmission rates $(8.1 \%$ vs. $3.4 \% ; \mathrm{p}=0.0111)$, were significantly higher after minimally invasive surgery. Survival (median survival: 26.8 vs. 25.0 months; log-rank $\mathrm{p}=0.062$ ) was comparable between both groups. Conclusion: The use of neoadjuvant therapy and minimally invasive surgical techniques have both soared in the past decade. Although further studies are warranted, the results of this study suggest that neoadjuvant therapy and minimally invasive surgery may safely be combined for pancreatic cancer treatment.

\section{P529}

Endoluminal Functional Luminal-Imaging Probe (ENDOFLIP ${ }^{\circledR}$ ) Can Help Prevent Significant Dysphagia After Magnetic Sphincter Augmentation Device (LINX ${ }^{\circledR}$ ) Placement

Rodrigo Pedraza, MD, Edward Y Chan, Ray K Chihara, MD, PhD, Leonora M Meisenbach, MSN, APRN, ACNPBC, Min P Kim; Houston Methodist Hospital

Background: Magnetic sphincter augmentation device (LINX) placement has been demonstrated to provide optimal mid-term outcomes for the management of gastroesophageal reflux disease (GERD). However, dysphagia following LINX placement has been reported as frequently as $83 \%$. The endoluminal functional luminal-imaging probe $\left(\right.$ EndoFLIP $\left.^{\circledR}\right)$ provides an objective functional distensibility assessment of the GEJ. We aimed to investigate clinical outcomes following LINX placement while using intraoperative EndoFLIP ${ }^{\circledR}$. Methods: The data were obtained from a prospectively maintained database. From $5 / 2017$ to $2 / 2018$, all patients who underwent LINX placement with the use of intraoperative EndoFLIP were included in our study. During the procedure, the EndoFLIP is placed and inflated after circumferential esophageal mobilization. The EndoFLIP values at baseline, following crural closure, and after LINX placement were recorded and compared. Modification was performed during the operation so that the final EndoFLIP value is $>0.5$ with $30 \mathrm{cc}$ of fluid in the balloon.

Results: A total of 30 patients were evaluated. The average age of patients was 54 with $83 \%$ females, a median ASA of 2 and an average BMI of 30.5. There were $43 \%$ of patients with type III hiatal hernia and $57 \%$ had type I hiatal hernia and $23 \%$ underwent redo-hiatal hernia repair. The peristalsis was $>$ $70 \%$ for all patients on manometry and the mean preoperative DeMeester score was 34. All patients underwent robot-assisted hiatal hernia repair with LINX and the median LINX size was 16 (14-17). There was no intraoperative and immediate postoperative complications or 30-day readmissions, and the median LOS was 1 (0-3). The pre-intervention EGJ distensibility index on EndoFLIP was 2.9 as compared to postoperative $1.5, \mathrm{p}<0.0001$. The mean preoperative GERD QOL was 24 as compared with postoperative 5.5, $\mathrm{p}<0.0001$. There was no significant difference of average dysphagia score (15 scale) between prior to surgery $(2.2 \pm 0.33)$ and after the surgery $(1.4 \pm 0.26, \mathrm{p}=0.058)$. One patient had a dysphagia score of 5 after surgery but further work-up showed an esophageal spasm as the cause and the symptoms resolved with muscle relaxant administration. None of the patients needed steroids for treatment of post LINX dysphagia.

Conclusions: Our study showed that the use of EndoFLIP for intraoperative EGJ distensibility assessment during LINX placement could help prevent significant dysphagia after surgery. The use of EndoFLIP may provide an objective metric to predict successful functional GEJ following LINX placement. 
P530

\section{An Assessment of Bio-Absorbable Mesh in High-Risk Patients Undergoing Complex Abdominal Wall Reconstruction}

Omar Toubat, BA, Evan T Alicuben, MD, Kamran Samakar, MD, Namir Katkhouda, MD, Caitlin C Houghton, MD, John C Lipham, MD, Kulmeet Sandhu, MD, Adrian Dobrowolsky, MD, Nikolai A Bildzukewicz, MD; Department of Surgery, Keck School of Medicine of USC

Introduction: Abdominal wall reconstruction (AWR) with mesh placement is commonly performed in patients with complex ventral hernias. Recently, bio-absorbable mesh has emerged as an alternative to synthetic and biologic mesh types, however few studies have evaluated its efficacy in AWR. The aim of this study was to describe our institutional experience with bio-absorbable mesh in a high-risk patient cohort.

Methods and Procedures: Patients who underwent incisional hernia repair with component separation and bio-absorbable mesh placement (Gore Bio-A or Bard Phasix) between 2013 and 2018 were identified. Demographic and surgical characteristics and relevant outcome data were retrospectively collected from patient records.

Results: In total, 50 patients ( 32 males, $64.0 \%$ ) were included, with a mean age of 58.7 years and mean BMI of 30.7 (range 21.0-44.9). 9 (18.0\%) patients had a modified Charlson-Deyo (CD) comorbidity score of 1 and $33(66.0 \%)$ patients had a CD score of greater than $2.9(18.0 \%)$ patients had a history of wound infection. Operative characteristics are summarized in Table 1. The median LOS following surgery was 4 days (range 1-20). The mean follow-up period after discharge was 8.9 months (range 0.4-39.4). Postoperatively, $11(22.0 \%)$ patients experienced at least one seroma, $14(28.0 \%)$ had a surgical site infection, and $5(10.0 \%)$ experienced surgical site bleeding/hematoma formation. Of the six patients that required surgical reintervention, 3 underwent surgical debridement, 2 underwent surgical repair for hernia recurrence, and 1 underwent both debridemen and repair for recurrence. Overall, $7(14.0 \%)$ patients experienced a recurrence, with a mean time to recurrence of 1.3 years.

Conclusion: Bio-absorbable mesh is an emerging alternative to synthetic and biologic mesh in incisional hernia repair. The use of bio-absorbable mesh in conjunction with component separation is a durable approach for complex AWR.

\begin{tabular}{lc}
\hline Table 1 & Number \\
\hline Characteristic & \\
\hline Surgical Status & 48 \\
Elective & 2 \\
Emergent & \\
Repair Type & 27 \\
Initial & 23 \\
Recurrent & \\
Mesh Placement & 19 \\
Onlay & 24 \\
Underlay/Retrorectus & 7 \\
Onlay and Underlay/Retrorectus & 18 \\
Component Separation & 17 \\
Bilateral Posterior & 11 \\
Bilateral Anterior & 4 \\
TAR & \\
Bilateral Posterior and Anterior & \\
\hline
\end{tabular}

\section{P531}

\section{Pre-peritoneal Complex Ventral Hernia Repair}

Shyanie J Kumar, MD, Zachary Warriner, MD, Wesley Edmunds, John S Roth, MD; University of Kentucky

This is a technique video for totally extraperitoneal approach (TEP) with component separation for complex ventral hernia repair (VHR). TEP involves hernia sac identification and preservation. Hernia sac is dissected circumferentially until edges of intact anterior fascia identified. Posterio component separation performed as required for fascial closure. Hernia sac is then imbricated within the preperitoneal space or posterior rectus sheath in the midline. Mesh is placed as retromuscular sublay and linea alba restored ventral to mesh. TEP involves hernia sac identification and preservation. Hernia sac is dissected circumferentially until edges of intact anterior fascia identified. Posterior component separation performed as required for fascial closure. Hernia sac is then imbricated within the preperitoneal space or posterior rectus sheath in the midline. By leaving the hernia sac unopened we save operative time by avoiding lysis of adhesions and decrease our enterotomy rate. Mesh is placed as retromuscular sublay and linea alba restored ventral to mesh. We have described five years' experience of totally extraperitoneal approach with favorable outcomes.

\section{P532}

\section{Risk Factors for Postoperative Urinary Retention After Laparoscopic Inguinal Hernia Repair: A 3-Year Single Institution Analysis}

Courtney L Devin, MD, Guillaume S Chevrollier, MD, Richard Zheng, MD, Samantha L Savitch, BA, Michael J Pucci, MD, Karen A Chojnacki, MD, Ernest L Rosato, MD, Adam C Berger, MD, Francesco Palazzo, MD; Thomas Jefferson University

Introduction: Urinary retention (UR) is common after laparoscopic inguinal hernia repair (LIHR), with reported incidence ranging from $2-30 \%$. We sought to determine the incidence of postoperative UR at our institution and identify risk factors for its development.

Methods and Procedures: A retrospective chart review of all patients undergoing LIHR from March 2014 to March 2017 was performed. Postoperative UR was defined as the inability to void requiring urethral catheterization prior to discharge or return to the emergency department requiring catheterization. Demographics and perioperative factors were compared between patients with and without UR using Fisher's exact tests and student's t-tests. Univariate analysis was conducted by logistic regression analysis and odds ratios (OR) were calculated to identify risk factors for UR. All statistical analysis was performed with Stata v13.0 and significance was set at $\mathrm{p}<0.05$.

Results: Of the 354 patients who underwent laparoscopic inguinal hernia repair, 23 patients (6.7\%) experienced postoperative urinary retention. Patients who developed urinary retention were sig nificantly older $(53$ vs $65, \mathrm{p}<0.001)$. Other comparisons between groups are presented in the Table. On univariate analysis, we found the following factors to be significant: age $>65$ years $(\mathrm{OR}-1.1, \mathrm{p}=0.001)$, securing mesh with tacker $(\mathrm{OR}-4.8, \mathrm{p}=0.001)$, peritoneal violation (OR-3.5, $p=0.008$ ), amount of intraoperative intravenous fluids (OR-1.0, $p=0.009$ ), history of benign prostatic hyperplasia $(\mathrm{OR}-5.3, \mathrm{p}=0.002)$, diabetes $(\mathrm{OR}-5.2, \mathrm{p}=0.014)$, and prior IHR (OR-3.5, $\mathrm{p}=0.008)$. Midazolam $(\mathrm{OR}-4.3, \mathrm{p}=0.005)$ and Propofol $(\mathrm{OR}-5.2, \mathrm{p}=0.009)$ use were also significant factors for UR.

Conclusions: Postoperative urinary retention after LIHR is likely multifactorial. In this study, patient characteristics, surgical technique, perioperative medications, and fluids administered appeared to be linked with higher rates of postoperative UR. Larger studies are needed to assess these as independent risk factors for UR after LIHR. These results can inform the preoperative counseling and the development of strategies to mitigate UR after LIHR.

\begin{tabular}{llll}
\hline & No Retention & Retention & p-value \\
\hline BPH & 30 & 5 & 0.001 \\
DM2 & 17 & 3 & 0.007 \\
Prior IHR & 67 & 7 & 0.005 \\
\hline
\end{tabular}


P533

Evaluation of a U-Shaped Device Derived from Urinary Bladder Matrix in a Laparoscopic Hiatal Hernia Model

Adam Young, PhD ${ }^{1}$, Collin E. M Brathwaite, MD, FACS, FASMBS ${ }^{2}$, Thomas W Gilbert, $\mathrm{PhD}^{1} ;{ }^{1}$ ACell Inc, ${ }^{2} \mathrm{NYU}$ Winthrop Hospital

Mesh reinforcement of hiatal hernia repairs has been previously shown to reduce recurrence rates compared to primary repair alone, however, the risk of esophageal erosion and stricture limit the utility of many materials for this application. Recent publications have described early clinical successes in using urinary bladder matrix (UBM) for reinforcement of hiatal and ventral hernia repairs. In this study, a porcine model of laparoscopic hiatal hernia repair was utilized to compare the tissue responses between non-reinforced primary repairs to repairs reinforced with a "U-shape" surgical graft composed entirely of UBM.

Hiatal hernias were simulated in pigs by laparoscopically dissecting the phrenoesophageal ligament and separating the left and right crus. The defect was then repaired by suturing the crura with an EndoStitch device. Five pigs were then closed with only this primary suture repair, while another five pigs received reinforcement of the primary repair with a UBM surgical graft that was prefashioned into a "U-shape" configuration (Fig 1a,b). All animals were survived for two months, at which point the animals were evaluated endoscopically for esophageal stricture or erosion. The repair site was then harvested for histological evaluation.

All animals recovered from surgery and gained weight with no signs of dysphagia. At two months post-operatively, endoscopic evaluation showed slight stricture at the GE junction in both reinforced and non-reinforced animals as a result of the procedure, but no evidence of erosion. Adhesions to the underlying viscera were minimal and similar in both groups as well. Histologic evaluation found only minimal evidence of the UBM device remaining, with the rest of the device having remodeled into tissue that was largely indistinguishable from the surrounding diaphragmatic tissue (Fig 1c). An increase in granulation tissue was noted in the reinforced animals compared to the non-reinforced controls.

This study highlights the safety of a UBM-derived device in the laparoscopic reinforcement of hiatal hernia repairs. The device was completely remodeled into site appropriate tissue that minimized the risk of stricture or erosion of the esophagus. Although this is an early time point, the deposition of new granulation tissue and absence of complications provides histologic evidence for the successful clinical outcomes that have been previously published for UBM devices in the reinforcement of hiatal hernia repairs.

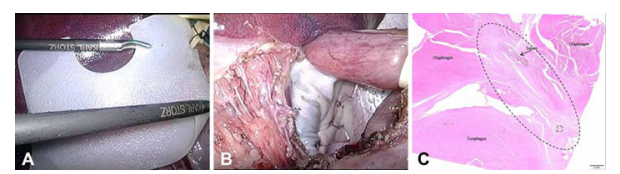

Figure 1: U-shaped UBM device was inserted laparoscopically without damage (A) and tacked to the diaphragm (B). At 2 months, histology (C) showed UBM (oval) had remodeled without damaging surrounding anatomy.

\section{P534}

\section{Epidemiological Profile and Pre and Post-natal Care Assessment in Patients with Congenital Diaphragmatic Hernia: A Descriptive Study}

Melina F Pires, Medical Student ${ }^{1}$, Rodrigo M Gallindo, MD, phD ${ }^{1}$, Raquel N Cordeiro, Medical Student ${ }^{1}$, Diego L Lima, $\mathrm{MD}^{2} ;{ }^{1}$ Pernambuco Health College, ${ }^{2}$ State Servers Hospital

Objective: To assess epidemiology, pre and post-natal care of patients born with congenital diaphragmatic hernia $(\mathrm{CDH})$ in a tertiary hospital in the northeast of Brazil.

Methods: A retrospective, descriptive, cross-sectional study was performed with the analysis of the medical records of patients diagnosed with CDH and treated between January 2008 and December 2017. We studied the aspects related to prenatal, to postpartum and to the postnatal periods, observing survival rates, characteristics of the hernia and the treatment of the disease. The study was developed upon the completion of forms according to the data collected from the medical records of these patients and their mothers.

Results: The study analyzed 54 patients: $64,8 \%$ were diagnosed before birth. $38 \%$ showed the defect on the left side of diaphragma and $50 \%$ of the patients had intrathoracic liver. $63 \%$ of the patients had surgery to repair the defect. $53,7 \%$ of the children with congenital diaphragmatic hernia died.

Conclusion: Due to the high mortality rate especially in those patients who had other abnormalities besides the $\mathrm{CDH}$, it is necessary to investigate these malformations during the prenatal care and postnatal period.

\section{P535}

\section{Longstanding Post-Traumatic Diaphragmatic Hernia with Massive Herniation: Management Strategies}

John P Skendelas, MD, Jody Kaban, MD, FACS, Ajay Chopra, MD; Albert Einstein College of Medicine/Jacobi Medical Center

Introduction: Post-traumatic diaphragmatic hernias with delayed presentation are an insidious clinical entity with variable presentation following blunt thoracoabdominal trauma. While the majority of these injuries are identified early and amenable to laparoscopic repair, symptoms may develop years or even decades following injury. The true incidence of these injuries remains elusive and only a constellation of case reports exist highlighting their complexity.

Case Presentation: Here we report the case of a 33 year old male with history of blunt thoracic injury 20 years prior who presented with dyspnea on exertion and chest $x$-ray findings consisten with left diaphragmatic hernia containing the stomach, pancreas, small bowel, and colon. The patient underwent comprehensive preoperative evaluation and consented to procedural intervention. The surgery began with a laparoscopic approach, allowing for complete reduction of the foregut contents; however, the limited insufflated space was quickly obliterated. To proceed, a left thoracotomy was made for further visualization and attempted takedown of dense colonic adhesive attachments. This too posed challenging and required midline laparotomy for definitive adhesiolysis, colonic hernia reduction, and esophageal hiatus reconstruction with an overlay $9 \mathrm{~cm}$ partiallyabsorable lightweight mesh. Due to difficult ventilation and the risk of abdominal compartment syndrome, the patient remained intubated post-operatively. The abdomen was partially closed with a Wittman's patch.

The patient returned to the operating room on POD\#1 for persistent air leak, left lower lobe wedge resection, and reduction of recurrent diaphragmatic hernia. Primary abdominal closure occurred on POD\#5. Post-operative recovery was prolonged by left lower lobe MSSA pneumonia with discharge on POD\#31. The patient was followed closely in the outpatient setting until recognition of left lung empyema on POD\#56, which required chest tube drainage. At this point, the role of decortication was proposed to the patient, who refused. Instead, intrapleural irrigation using piperacillin/tazobactam was administered for 14 days with resolution of the patient's underlying symptoms, leukocytosis, and radiologic evidence of empyema. The patient remained well on last follow up appointment 10.5 months after the index operation.

Conclusion: Management of longstanding diaphragmatic hernias pose a complex clinical problem whose solutions are highly dependent on the chronicity and magnitude of thoracic herniation Anticipation of intra- and post-operative complications is integral to recognize the need for a thoracic surgeon or open thoracoabdominal operative approach to address limitations of laparoscopy, for the takedown of dense thoracic adhesions or loss of abdominal domain, as evident in this case of massive herniation.

\section{P536}

Antireflux Surgery in Patients with Laryngopharyngeal Reflux Without Large Size of Hiatal Hernia

Mykola Paranyak, Vladimir Grubnik, Professor, MD, Viktor Grubnyk; Odessa National Medical University

Background: Laryngopharyngeal reflux (LPR) is described as the retrograde flow of stomach content to the larynx and pharynx. In such case most commonly manifested symptoms are cough, sore throat, hoarseness, dysphonia, globus and only $40 \%$ patients with LPR have typical GERD symptoms. Also PPI therapy are less effective in patients with LPR in comparison with patients who have typical features of GERD.

Purpose: To compare the outcomes between surgical treatment and conservative therapy in patients with laryngopharyngeal reflux.

Materials and Methods: For the period March 2014 to October 2016 in Odessa Regional Hospital we performed a study, where selected patients reported of having chronic cough and globus pharyngeus, with LPR which was diagnosed according to the 24-hour impedance-pH monitoring. Excluded criteria for our study were a diagnosis of hiatal hernia (HH) type II or III. We offered patients two ways of treating the disease: surgical method and PPIs therapy (where we emphasized that conservative therapy could be for life). After 36 patients (28- HH type I, 8- without features of $\mathrm{HH})$ underwent laparoscopic Nissen fundoplication (LNF) without cruroraphy. PPIs therapy group included 16 patients (6- without HH, 10 - HH type I). In both groups we assessed 24-h impedance$\mathrm{pH}$ monitoring, and quality of life (GERD-HRQL) questionnaires.

Results: Baseline characteristics were similar between groups. After a median follow-up of 26 months the prevalence of cough $(14.3 \%$ vs $48.4 \% ; \mathrm{p}<0.05)$, globus pharyngeus $1(9.9 \%$ vs $39.7 \%$; $<0.05$ ), was significantly lower in patients who underwent LNF. RSI improved from $22.8 \pm 5.4$ to $11.83 \pm 4.8(\mathrm{p}<0.001)$ in the first group and from $17,8 \pm 4,6$ to $10,4 \pm 3,4$ $(?<0,05)$ in the conservative therapy group. Based on the questionnaire GERD-HRQL results, in the first group the quality of life improved from $16,4 \pm 5,3$ to $6.2 \pm 0.6(\mathrm{P}<0.001)$, in the second group from $15.9 \pm 6.6$ to $11.4 \pm 1.5(\mathrm{P}<0.05)$.

Conclusion: LNF had higher efficacy in long-term eliminating of LFR symptoms in comparison with PPIs in patients without large sizes of HH. Also LNF showed better results in quality of life improvements in patients with LFR than PPIs. 
P537

\section{Management of Re-Re Recurrent Inguinal Hernia with Enterocutaneous Fistula After TEP Repair: A Rare Case Report}

Manish Kumar Gupta, Associate Professor, Sarrah Idrees, Dr, Rathindra Sarangi, Dr; Sir Ganga Ram Hospital, New Delhi
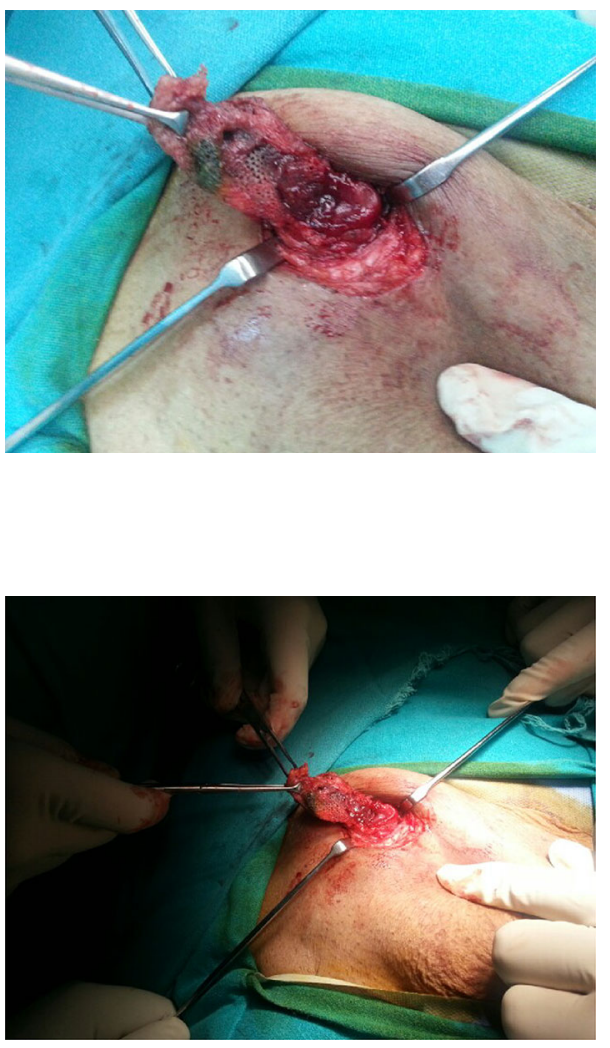

Introduction: Introduction of synthetic mesh was a landmark step forward in the management of hernia repair and has significantly reduced recurrence rates. But in addition to the benefits, some more problems have come in picture like 'mesh infection and mesh erosion in bowel'. This phenomenon especially after laparoscopic surgeries gives rise to chronic discharging sinus at the port site, abscess formation around mesh and even sepsis. It appears that laparoscopic hernia repair is a promising method but having chances of mesh infection owing to difficult approach and lack of uniformity in sterilization.

Case Report: A 64 years normotensive, non-diabetic gentleman presented to us in the out-patient department with the complaints of pus discharging opening in the right groin region for 8 months. He underwent open herniorraphy for right inguinal hernia two and half years back. He developed recurrence of hernia after 1 years of herniorraphy for which he underwent totally extraperitoneal (TEP) repair of inguinal hernia. He remained asymptomatic for few months and subsequently developed pus discharging opening in the right groin with reducible swelling at the same site for which he presented in our OPD.

On local examination, there was a sinus opening in the right inguinal region just above the midpoint of the inguinal ligament with active pus discharge. A visible cough impulse or recurrent right inguinal hernia was also noted.

MRI revealed an organized area of inflammation beneath the anterior abdominal wall in the right iliac fossa of $1.9 \times 2.2 \mathrm{~cm}$ with adjoining ileal loops. Decision for exploration of sinus with explantation of mesh and need for diagnostic laparoscopy was taken. A $3 \mathrm{~cm}$ tract was extending up to the abscess cavity which was present over the mesh. The sinus tract was exposed and abscess cavity was drained. The infected mesh was also removed and enteric content was noted coming out through an opening at the base of the mesh. On diagnostic laparoscopy, there was an ileal loop which was densely adherent to the right inguinal region. The fistulous opening was freshened and enterotomy was closed intracorporeally with vicryl 3-0 in two layers. The muscle defect was repaired with vicryl 2-0 and the right inguinal wound was left open for healing by secondary intention. After 6 months, he underwent hernioplasty by " 555 Manish Technique" using all $5 \mathrm{~mm}$ ports. On 1st and 6th month of follow up visits, patient was asymptomatic and showed no recurrent

\section{P538}

Laparoscopic Treatment of a Coexisting Femoral and Obturator Hernias: Case Report and Review of the Literature

Diego L Lima, $\mathrm{MD}^{1}$, Lucas M Aires Camara, $\mathrm{MD}^{1}$, Lucas M Vasconcelos De Albuquerque, $\mathrm{MD}^{1}$, Raquel N Cordeiro ${ }^{2}$, Roberto $\mathrm{P}$ Ramos, MD ${ }^{1}$, Flavio Malcher ${ }^{3}$, Marconi R De Lemos Meira ${ }^{1}{ }^{1}$ State Servers Hospital, ${ }^{2}$ Faculdade Pernambucana de Saúde, ${ }^{3}$ Montefiore Hospital, NYC

Case Report: A 68 years-old, female patient, with history of abdominal pain in the left iliac foss for 6 months, associated to a bulge in the left inguinal area, reducible to manual maneuvers. Ultrasound show a left inguinal hernia with tear of $1 \mathrm{~cm}$. Besides, an umbilical hernia was also noted in this exam. Patient was submitted to a laparoscopic treatment (TAPP technique). A suprambilical incision with the insertion of a 11-mm trocar was performed. The umbilical hernia was treated, and its herniary sac removed. The pneumoperitoneum was created by the open technique. A 5-mm trocar was positioned in the paraumbilical at the right e another in the left. During the procedure, a left femoral hernia, an obturator hernia (with pre-peritoneal fat content), and a lipoma in the left inguinal canal were found. The peritoneum was dissected with scissors, and, afterwards, the lipoma and the femoral herniary sac were reduced. Then, the peritoneum was disected to the pelvic floor. The herniary content of the obturator hernia was reduced. A polipropilen $15 \times 15 \mathrm{~cm}$ mesh was positioned in the pre-peritoneal region with a laparoscopic stapler. The parietal peritoneum was repositioned with a barbed suture, and some tears in the peritoneum were sutured. The umbilical tear was sutured with vicryl 2-0. The total surgery time was $90 \mathrm{~min}$. The patient had no further complications, with no pain in the post-operatory, and was discharged in the following day.

Discussion: Femoral and obturator hernias are difficult to diagnose. CT scan is, usually, the only way to have a proper identification of the obturator hernia. Anatomically, the femoral and obturator canal are close, what can difficult the identification of the hernias, looking just like a big inguinal hernia. In the current report, the ultrasound didn't differentiate the hernias in their right position, mentioning an inguinal hernia. An ipsilateral femoral and obturator hernias should be considered in cases of bowel obstruction with swelling in the inguinal region. The treatment of obturator hernia is always surgery. Laparotomy is the most common approach; however, many studies demonstrated the feasibility of laparoscopic techniques.

Conclusion: The laparoscopic treatment of femoral and obturator hernias is doable, and the TAPP technique can change the previous diagnosis, with great benefits for the patients.

\section{P539}

\section{Comparisons of Abdominal Wall Reconstruction for Ventral Hernia Repairs, Open versus Robotic}

Barbara Nguyen, $\mathrm{MD}^{1}$, Bryan David, $\mathrm{MD}^{1}$, Kensey Gosch, $\mathrm{MS}^{2}$, George B Sorensen, $\mathrm{MD}^{2} ;{ }^{1}$ University of Missouri-Kansas City General Surgery, ${ }^{2}$ St. Luke's Hospital

Background: An increasingly multifaceted surgical population necessitates effective, safe, ventra hernia repairs. Approaches to complex hernias include advances in abdominal wall reconstruction, such as transverse abdominis release (TAR), and diverse operative technology to achieve this While robotic assisted laparoscopic repair provides a minimally invasive approach to a maximally invasive surgery, the cost of utilizing this tool—and longer operative times-have argued against its use. As robotic surgery emerges as a major surgical instrument, an analysis of its outcomes, particularly examining perioperative, postoperative, and follow-up periods, can better clarify its role.

Methods: A retrospective review was performed from September 2016 to February 2017 of patients undergoing TAR and sublay mesh placement. Various components of surgery, hospitalization, and outcomes were compared between patients receiving standard open repair (SOR) and those receiving robotic-assisted repair (RAR) using Student's t-test or Wilcoxon Signed Rank test for continuous variables and Pearson's X2 test for categorical variables.

Results: Of the 43 patients reviewed, 16 were SOR, versus 27 RAR. Patients were comparable in age, gender, BMI, and number of diabetic patients. The average size of defect was similar between SOR and RAR groups $\left(242 \mathrm{~cm}^{2}\right.$ vs. $216 \mathrm{~cm}^{2}$ respectively, $\left.\mathrm{p}=0.40\right)$. Perioperatively, RAR patients had longer operative times than OAR patients, at $272 \mathrm{~min}$ compared to $206 \mathrm{~min}(\mathrm{p}<0.001)$ Estimated blood less (EBL) was less in the RAR group $(43 \mathrm{~mL})$ than OAR $(147 \mathrm{~mL}, \mathrm{p}<0.001)$. Postoperatively, OAR patients averaged 9.6 hospital days, while RAR patients averaged 3.0 days $(\mathrm{p}<0.001)$. Half of the OAR patients and one RAR patient required critical care management during hospitalization. More RAR patients had seromas; none required surgical intervention. Both groups had four patients present to the emergency department within 30 days, with two in the OAR group readmitted.

Conclusions: Compared to SOR patients, RAR had a significantly shorter length of stay and less EBL. However, they also had longer operative times, which may be reflective of learning curves with robotic training and ongoing development of reconstruction techniques. More postoperative complications requiring critical care management and readmission were noted in the SOR group. While the robotic approach may require more operative time, shorter hospital length of stay with less postoperative complications would suggest its ultimate benefit in abdominal wall reconstruction, as it has less hospital costs, and provides better outcomes for patients. 


\section{P541}

Laparoscopic Diagnosis and Treatment of a Complex Incisional Inguinal Hernia

Jonah White, MD, Jeffrey Chiu, MD, Bernadette Profeta, MD, Francisco Couto, MD, Alexandros Coutsoumpos, MD, Rachael Gentry, PAC, Scott Bloom, MD, Steve Eubanks, MD; Florida Hospital

A 59-year-old male had previously undergone percutaneous coronary artery revascularization with access through the right femoral artery. He subsequently required a right femoral endarterectomy with right femoral to popliteal bypass due to occlusive disease and chronic right femoral artery dissection as a complication of femoral access from his coronary artery catheterization. The patient developed graft occlusion 3 years later and required revision and replacement of the femoral to popliteal bypass graft. The patient had a moderate sized, soft, reducible, right groin hernia on physical exam. CT scan demonstrated a right femoral hernia containing several loops of small bowel without any evidence of bowel obstruction. The patient was subsequently offered laparoscopic right femoral hernia repair with mesh with posterior approach due to multiple open procedures in the anterior groin. Intraoperatively, he was found to have a right inguinal hernia lateral to the inferior epigastric vessels secondary to dilation of the indirect space from his previous femoral artery procedures instead of a true femoral hernia. The hernia defect was repaired with the laparoscopic intraabdominal posterior approach using prosthetic mesh without complication. This case demonstrates the usefulness of laparoscopic posterior approach in the repair of a complex groin hernia as an alternative to anterior approach, which would have been complicated by the assumption of a true femoral hernia on this patient's CT scan.
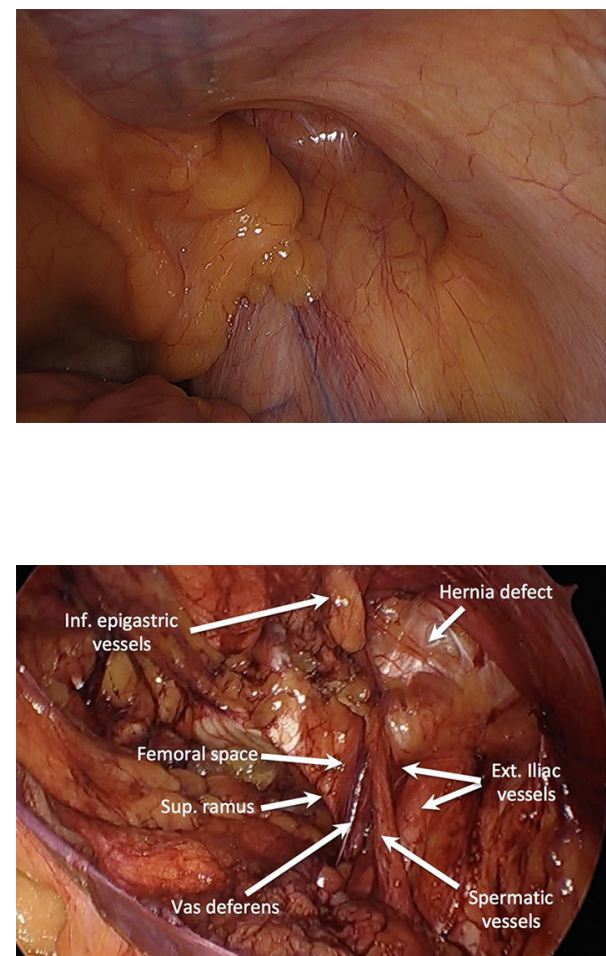
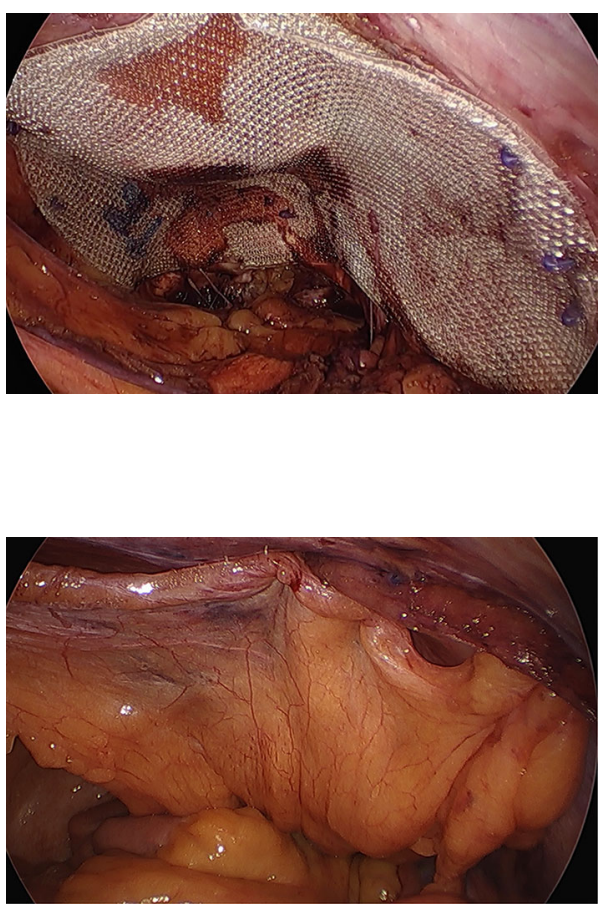

\section{P542}

Transabdominal Extraperitoneal Repair of Spigelian Hernia: Care Report and Literature Review

Christrian Massier, MD, William T Walsh; South Pointe Hospital

Introduction: We report a recent case of an emergent repair of an incarcerated Spigelian hernia via laparoscopic transabdominal extraperitoneal (TAP) approach with a polypropylene mesh.

Presentation of Case: A 50-year-old female presented to ED with vague abdominal pain and obstructive symptoms. Physical exam was non-specific without peritoneal signs. A contrast enhanced compute tomography was obtained showing left sided incarcerated Spigelian hernia and ventral hernia. On initial induction of pneumoperitoneum, the incarcerated hernia spontaneously reduced and there was an incidental contralateral Spigelian hernia. Patient underwent a left TAP repair and primary repair of right Spigelian and ventral hernia.

Discussion: These hernias are clinically difficult to diagnosis due to their interstitial location. There should be a high index of suspicion for the correct diagnosis to be made via CT scan of the abdomen. There is little literature to state the best approach for repairing these hernias. There have been advocates for open transabdominal, extraperitoneal, and primary closure. The laparoscopic repair is preferred because the outer external oblique remain intact with these hernias and allows for complete inspection of the abdomen for other fascial defects.

Conclusion: The use of a TAP repair provides the opportunity to close a Spigelian hernia defect and place a mesh without violating the anterior muscle layer and prevent direct contact between mesh and bowel. 
P543

P545

\section{A Novel Technique for Ventral Hernia Repair: Less Postoperative Pain with Lower Recurrence Rates Using a Combined Laparoscopic and Open Repair}

Natasha Denicola, MD ${ }^{1}$, Claire M Eden, BA ${ }^{2}$, Fred Kimmelstiel, $\mathrm{MD}^{1} ;{ }^{1}$ Icahn School of Medicine at Mount Sinai St. Luke's Roosevelt Hospital, ${ }^{2}$ Icahn School of Medicine at Mount Sinai

Background: Open ventral hernia repair may be limited by the ability to adequately visualize the underlying peritoneum and ensure complete reduction of the hernia sac for flat placement of mesh. Although laparoscopic repair permits better visualization, long term outcomes are unknown and many patients experience significant postoperative pain, especially with larger mesh use and more fixation materials.

Methods: We retrospectively analyzed all patients who underwent ventral hernia repair by a single surgeon who performed a hybrid open and laparoscopic technique from 2010-2018. An open incision was made over the hernia to reduce the sac and clear off the fascia circumferentially. 2 $5 \mathrm{~mm}$ trocars were placed in the bilateral upper quadrants or suprapubic regions. The sac was fully reduced under pneumoperitoneum to ensure circumferential clearance around the defect. A traditional open composite mesh was placed through the fascial defect, manipulated laparoscopically and secured with fascial sutures. Tacks were rarely used, usually for mesh grafts $>8 \mathrm{~cm}$. We evaluated perioperative outcomes to determine the feasibility and effectiveness of this technique. Results: 181 patients with ventral hernias underwent hybrid repair over an 8-year period. Median age was 50 years and $62 \%$ were male. Median BMI was 28 (IQR 25-32). $9 \%$ were smokers and $4 \%$ used steroids perioperatively. $50 \%$ had prior abdominal surgery and $12 \%$ had a prior ventral hernia repair. The most common indication for repair was pain $(91 \%)$ from an incarcerated hernia (59\%). At the time of surgery, median hernia defect size was $2.0 \mathrm{~cm}$ (IQR $1.9-2.5 \mathrm{~cm}$ ), and median mesh diameter was $6.4 \mathrm{~cm}$ (IQR 6.4-8.0 cm). 1 patient required conversion to open repair. Median EBL was $5 \mathrm{cc}$ (IQR $5-10 \mathrm{cc}$ ). Postoperatively, only $3 \%$ of patients experienced pain after 2 weeks. There were 11 (6\%) 30-day perioperative complications; 6 seromas managed conservatively, 4 superficial surgical site infections managed with antibiotics and 1 small bowel obstruction requiring reoperation. There were no mesh infections. At a median follow-up time of 4 months (IQR 2-12 months), 7 (4\%) patients experienced hernia recurrence at a median time of 19 months (IQR 13-44 months); 2 were managed with re-operation (both laparoscopic).

Conclusions: Our experience using a hybrid technique for ventral hernia repair suggests that it is safe and effective with low rates of postoperative complications and recurrence. Comparison to open and laparoscopic long-term outcomes will better define its utility.
Safety and Efficacy of Robotic-Assisted Repair of Inguinal Hernia

Marissa C Maas, Evan T Alicuben, MD, Caitlin C Houghton, MD, Kamran Samakar, MD, Kulmeet K Sandhu, MD, Adrian Dobrowolsky, MD, Nikolai Bildzukewicz, MD; Keck School of Medicine of USC

Introduction: Robotic technology provides another tool in the arsenal of the minimally-invasive surgeon with emerging uses for general surgical procedures. Given the limited working space in the pelvis, the increased dexterity offered by the robot may prove beneficial in the repair of inguinal hernias. Currently, there are only a few studies examining the use of robotic technology for inguina hernia repair. Our aim was to detail our experience with robotic inguinal hernia repair at an academic institution.

Methods and Procedures: We performed a retrospective chart review on all patients who had undergone robotic inguinal hernia repair from March 2015 to April 2018. Preoperative demographics, operative characteristics, and postoperative outcomes were analyzed using RStudio software. The primary outcome was hernia recurrence.

Results: There were 43 patients, 40 of which were male. The patients had a mean age of 56 years (range 18-85 years). Thirteen of the patients had bilateral hernias. The mean patient BMI was 26.4 (range 17.5-42.3). All operations were performed transabdominally and all but one included fixation of synthetic polypropylene mesh. Regarding mesh attachment, 23 patients had suture only, 14 had tacks only, 1 had a combination of suture and tacks, 2 had suture and glue, 2 had tacks and glue. The mean patient in-room time was $4.0 \mathrm{~h}$, mean operative time was $2.9 \mathrm{~h}$, and the mean robot docked time was $2.0 \mathrm{~h}$. Thirty-two patients were discharged on the day of surgery. One patient was kept overnight for treatment of urinary retention. The other 10 patients were kept for one or two nights for observation. There was one bladder injury discovered intraoperatively, which was primarily repaired. Post-operatively, none of the patients had wound infections, 11 developed seromas, and one patient had a groin hematoma. At a median follow up of 37.5 days, there was one recurrence. This was discovered incidentally during a urological procedure and was repaired. Conclusions: Robotic surgery in inguinal repair is safe and effective and should be considered a viable alternative to laparoscopic and open repairs. Longer term studies will further define the role of this technology.

\section{P544}

\section{Complications of Minimally Invasive Abdominal Wall Reconstruction: A Single Institution Experience}

Richard Lu, MD ${ }^{1}$, Alex Addo, $\mathrm{MD}^{1}$, Allison Estep, $\mathrm{MD}^{2}$, Reza Zahiri, DO ${ }^{1}$, Igor Belyansky, MD, FACS ${ }^{1} ;{ }^{1}$ Anne Arundel Medical Center, ${ }^{2}$ MedStar Health Baltimore

Introduction: Minimally invasive retromuscular abdominal wall reconstruction is a relatively novel approach. As this technique becomes more widespread, it is crucial to consider its complications, particularly those that are unique to these operations. We present our institution's complications with this approach.

Methods: A review of our center's prospectively maintained database of hernia patients was conducted. This identified 435 patient who had undergone laparoscopic or robotic Rives-Stoppa and transversus abdominis release reconstructive procedures between August 2015 to August 2018 . Results: Mean follow-up was 7.9 months (range, 1-31 months). The overall complication rate was $9 \%(\mathrm{~N}=41)$ with the most common being postoperative seromas $(3 \%, \mathrm{~N}=15)$. Four of these were retromuscular, the remaining were subcutaneous. The majority of these required interventions with either aspiration in clinic or placement of a percutaneous drain for retromuscular seromas. Three subfascial seromas became infected which were explored and drained endoscopically. Subfascial hematomas were seen in $1 \%$ of patients $(\mathrm{N}=3)$ with one requiring endoscopic drainage. A unique but devastating complication of retrorectus hernia repairs is the failure of the posterior rectus sheath which required urgent reoperation due to exposure of bowel to mesh. This occurrence was seen in 3 patients. Two patients had injuries to the linea alba which were not identified at the time of the original surgery. This resulted in destabilization of the abdominal wall and early recurrences requiring emergent intervention. The overall recurrence rate was $1 \%(N=5)$. Postoperative ileus and small bowel obstruction were observed in $2 \%$ of patients $(\mathrm{N}=6)$ and managed nonoperatively. Venous thromboembolism disease (VTE) and cardiopulmonary events were observed in $1 \%$ of patients $(\mathrm{N}=6)$. There was one mortality in the early perioperative period due to a fatal pulmonary embolus.

Conclusions: In the recent past several novel MIS reconstructive abdominal techniques have been described by our group. As other surgeons begin to adopt some of these more complex approaches, it is critical to consider the unique complications and be prepared to implement potential management strategies. While the overall complication rates are low in our high-volume center, it is not clear if these results are reproducible by other hernia centers. Furthermore, additional efforts should be made to determine overall complication rates in low-volume centers.

\section{P546}

\section{Readmission After Laparoscopic Treatment of Inguinal Hernia}

Adrian Maghiar, George Dejeu, Marius Sfirlea, Dan Ciurtin, Codruta Macovei, Florin Fasie, Paula Badea; Spital Pelican

We use the laparoscopic treatment of inguinal hernia in our clinic, Pelican Hospital in Oradea, Romania, for 10 years. With a percentage of about $60 \%$ of cases being operated laparoscopically. In this study we looked at the readmission rates for inguinal hernia patients operated laparoscopically or using the Lichtenstein technique.

Of 100 patients included in the study, that started in january 2018, 62\% were operated laparoscopically (52 using TEP technique and 10 using TAPP technique), the rest were operated with Lichtenstein technique. We had 17 readmisions for various reasons, 12 for patients from the Lichtenstein group and 5 from the lap group. 4 patients form the Lichtenstein group for surgery unrelated conditions and 1 from the lap group. The rest were a mixture of acute postoperative pain, haematoma and seroma. No patients spent more than 2 days in hospital. 7 patients from the Lichtenstein group needed some form of surgical drainage for the haematoma/seroma, and 1 patient from the TEP group. No patients presented with signs or symptoms of surgical site infection, and no patients needed antibiotic treatment. Looking at the data we conclude that using a lap technique we reduce the number of patients with complications in the first 30 days after hernia surgery that need readmission and surgical treatment, lowering the economic impact of such surgery and accelerating the return to normal life for patients. We continue to research the impact of lap surgery in addition to newer pain control techniques on hernia patients. 
P547

HandX, a Hand-Held Smart Motorized Laparoscopic Device as an Alternative to Robotic Suturing in Laparoscopic Hernia Repair: First Human Cases

\section{Amir Szold, MD; Assia Medical Group, Assuta Medical Center}

Introduction: Suturing the abdominal wall and peritoneum is becoming a current trend in laparoscopic hernia repair. As laparoscopic suturing is technically demanding, it has justified the use of expensive robotic systems to perform this part of the operation. In order to try and make the procedure more cost-effective we have used a novel, hand-held motorized device, HandX, for suturing during laparoscopic hernia repair

Materials and methods: HandX is a hand-held motorized and computerized device that has all necessary degrees of freedom to perform complex motions in minimally invasive surgery. It is controlled by a sophisticated human interface unit that requires a short learning curve.

We have used the device for laparoscopic suturing and tissue manipulation in various laparoscopic procedure as part of a clinical trial, including in hernia repair, and recorded adverse events and device performance. After surgery, the surgeons filled a validated usability questionnaire to asses the device performance. In addition all cases were recorded for further analysis.

Results: HandX was used in 21 human cases, including 10 hernias. These included 2 TAPP procedures where the device was used to suture the peritoneum, and 8 cases in which it was used for primary repair of the abdominal wall defect prior to mesh placement. Using the device facilitated suturing in difficult angles and was performed following a short learning curve. The usability score of the device for most relevant questions was very good to excellent.

Conclusions: Although limited by the early experience and small number of patients HandX seems to enhance the surgeons capabilities and allow for more cost effective suturing in laparoscopic hernia repair. It demonstrarted the capacity of a robot with the advantages of laparoscopy in an affordable way

\section{P548}

The Role of CT-Based Volumetric Analysis of Abdominal Wall Anatomy in Predicting Tension-Free Midline Approximation in Abdominal Wall Reconstruction with Components Separation. A Pilot Study

Mazen Al-Mansour, MD, Greg Gagnon, MS, Alexander B Knee, MS, Sheina Theodore, MD, Jacqueline J Wu, MD, John Romanelli, MD, Neal E Seymour, MD; Baystatea Medical Center-University of Massachusetts Medical School

Objective: To evaluate the ability of abdominal wall volume measurements based on 3D-reconstruction of abdominal CT-scans to predict tension-free midline approximation (TFMA) in patients undergoing abdominal wall reconstruction with components separation (AWRCS).We hypothesized that CT-based volume measurements can better predict TFMA compared to linear measurements (e.g. defect width)

Methods: In this pilot study, we retrospectively reviewed the charts of 18 patients who underwent AWRCS (Nov 2008-Aug 2017). We collected demographic, historical, hernia-specific and operative data. Linear measurements were obtained from CT images and used to calculate the hernia sac volume (HSV) and abdominal cavity volume (ACV). 3D-reconstruction was performed to measure the HSV and ACV (Figure 1). Logistic regression analysis was used to determine how well clinical and CT parameters discriminated failure of TFMA. Pearson correlation was used to evaluate the agreement between calculated and measured parameters.

Results: Mean age was 55.9(10.9) years, 6 patients were female. Tables 1 and 2 and Figure 2 summarize the results.

Conclusions: Among the variables assessed in this pilot study, measured HSV/ACV ratio using 3DCT reconstruction was more predictive of TFMA than calculated volumes and linear measures (e $\mathrm{g}$. defect width). There was good agreement between measured and calculated values of HSV, ACV and HSV/ $\mathrm{ACV}$. The findings of this pilot study need to be further investigated with a larger sample size.
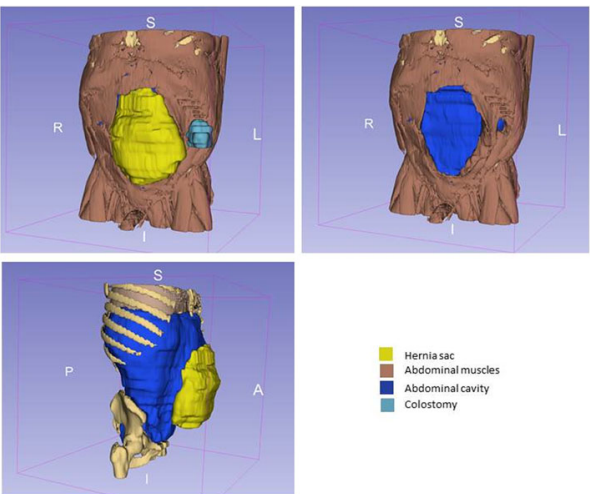

\begin{tabular}{|c|c|c|c|c|}
\hline & $\begin{array}{l}\text { TFMA } \\
(\mathrm{N}=15)\end{array}$ & $\begin{array}{l}\text { Failure of TFMA } \\
(\mathrm{N}=3)\end{array}$ & $\begin{array}{l}\text { Total } \\
(\mathrm{N}=18)\end{array}$ & AUC \\
\hline BMI $\left(\mathrm{kg} / \mathrm{cm}^{2}\right)^{\mathrm{a}}$ & $35.6(9.4)$ & $36.4(3.6)$ & $35.8(8.6)$ & 0.58 \\
\hline Recurrent hernia & $8(53.3 \%)$ & $2(66.7 \%)$ & $10(55.6 \%)$ & 0.57 \\
\hline Stoma present & $1(6.7 \%)$ & $1(33.3 \%)$ & $2(11.1 \%)$ & 0.63 \\
\hline Defect width $(\mathrm{cm})^{\mathrm{a}}$ & $13.6(90.6)$ & $13.4(4.0)$ & $13.5(4.1)$ & 0.53 \\
\hline Calculated HSV/ACV ratio ${ }^{a}$ & $0.07(0.06)$ & $0.09(0.07)$ & $0.08(0.06)$ & 0.60 \\
\hline Measured HSV/ACV ratio ${ }^{a}$ & $0.05(0.04)$ & $0.10(0.07)$ & $0.06(0.05)$ & 0.76 \\
\hline
\end{tabular}

${ }^{\mathrm{a}}$ Mean (SD)

AUC area under the curve

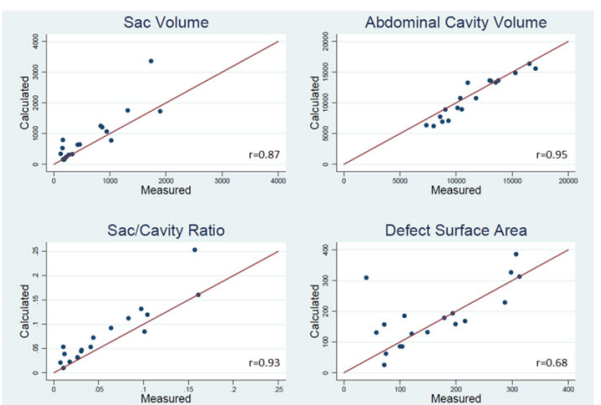




\section{P549}

Ventral Hernia Repair in Obese Patients: Tailored Preoperative Weight Loss and Results in an Asian Cohort

Raquel A Maia, MD, Lee Leng Loh, RN, Hrishikesh Salgaonkar, MS, Sujith Wijerathne, FRCS, Asim Shabir, MBBS, MMed, FRCS, Davide Lomanto, MD, PhD, FAMS; National University Singapore

With the growing incidence of abdominal hernias due to the high volume of laparotomies performed annually and the exponential global growth of obesity in the last decades both, general surgeons and specialists in bariatric surgery, have a new dilemma: when and how to operate an obese patient with ventral hernia.

In our experience at National University Hospital in Singapore, 25 patients with $\mathrm{BMI} \geq 30$, underwent preoperative weight management by a specialized bariatric team either for 4 weeks with a regime of 1400 calories or a very lowcalorie diet (VLCD) of 800 calories under hospitalization care based on patient compliance and weight loss. We compared the results between patients underwent weight loss management with a VLCD, patients with a diet of 1400 per day and patients without any type of weight management on the preoperative period and their results after surgery, from 2016 to June 2018. We evaluate 28 consecutive patients with mean BMI 29,5 and max BMI 41,2, for elective ventral hernia repair. Fifteen patients underwent laparoscopic ventral hernia repair with prosthetic mesh in an intra peritoneal approach with defect closure (IPOM- plus); 9 patients had a robotic ventral hernia mesh repair with a preperitoneal approach (PPOM) and 4 patients a synchronous ventral hernia repair and bariatric procedure with LSG and IPOM plus approach for the hernia repair. In our mean follow up of 18 months no recurrence was seen, 1 patient was converted to open due to strong adhesions, no mesh or wound infection was noted.

\section{P550}

\section{Minimally-Invasive or Less Open Sublay (MILOS) Repair for Incisional Hernias: The Creighton Experience}

Dustin J Tubre, MD, Alexander Schroeder, MD, Robert J Fitzgibbons, MD, FACS; Creighton University School of Medicine

Introduction: Retrorectus mesh placement is widely accepted as the gold standard for the repair of incisional hernias. The open procedure requires an extensive abdominal wall dissection but has the advantage of extraperitoneal mesh placement. Laparoscopic intraperitoneal onlay mesh (IPOM) repair avoids the extensive dissection and is associated with less surgical site infections, however the intraperitoneal mesh placement can result in a variety of adverse outcomes. Recently a hybrid operation, termed the MILOS (mini/less open sublay) technique has been described by Dr. W. Reinpold (Hamburg, Germany). We present preliminary data from an ongoing study at Creighton University Medical Center (CUMC) comparing MILOS repair to open retrorectus repair

Methods: Dr. Reinpold personally demonstrated the first two MILOS procedures at our institution. Subsequent procedures were performed independently. Patient data and outcomes from MILOS repairs and open retrorectus repairs are collected prospectively. An enhanced recovery after surgery (ERAS) protocol is utilized to reduce opioid use. Follow-up is carried out at 2 weeks, 6 months, and 12 months.

Results: Eight MILOS repairs and 11 open retrorectus repairs have been performed since April 2018. The two groups were comparable in terms of patient characteristics and included mostly midline incisional hernias. Hernia size (mean, $45.1 \mathrm{~cm}^{2}$ vs. $333.8 \mathrm{~cm}^{2}, \mathrm{p}=0.004$ ) was larger in the open retrorectus group, while mesh sizes were similar (mean, $348.9 \mathrm{~cm}^{2}$ vs. $406 \mathrm{~cm}^{2}, \mathrm{p}=0.40$ ). Hospital stay was shorter in the MILOS group (mean, 2.5 days vs 5.6 days, $\mathrm{p}=0.01$ ). There was a trend towards a lower morphine equivalent dose in the MILOS group (mean, $3.0 \mathrm{mg}$ vs. $15.2 \mathrm{mg}, \mathrm{p}=0.26$ ). There were no immediate complications or recurrences.

Conclusion: Our initial experience with the new MILOS technique indicates its reproducibility and safety in large incisional hernias. This is an ongoing study and results will be updated as the sample size increases.

\section{P552}

\section{Evaluation of Intraperitoneal Tissue Attachments to Biologically} Derived Surgical Meshes in a Rabbit Model

Hannah B Baker, PhD, Adam Young, PhD, Thomas Gilbert, PhD; ACell Inc

The utility of biologically derived surgical meshes relies on their capacity to integrate with host tissue and remodel over time. However, development of mesh attachment to viscera after intraperitoneal placement must be minimized to limit risk of bowel obstruction and intestinal fistula. Herein, the utility of surgical grafts derived from urinary bladder matrix (UBM) and small intestine submucosa (SIS) to minimize tissue attachments was investigated in a rabbit model of intraperitoneal tissue attachment.

The rabbit model entailed excision of the peritoneum and abrasion of the cecum to induce the formation of tissue attachment between the bowel and peritoneum ( $n=6$ per treatment and time point). Commercially available UBM and SIS grafts (3 variable thickness UBM grafts and 1 SIS graft) were placed intraperitoneally and sutured to the peritoneum to reinforce the midline repair. Animals with non-reinforced repairs were used as controls. At 14 and 90 days, the number and severity of tissue attachments between the device and the surrounding viscera were assessed and tissues were processed for histology.

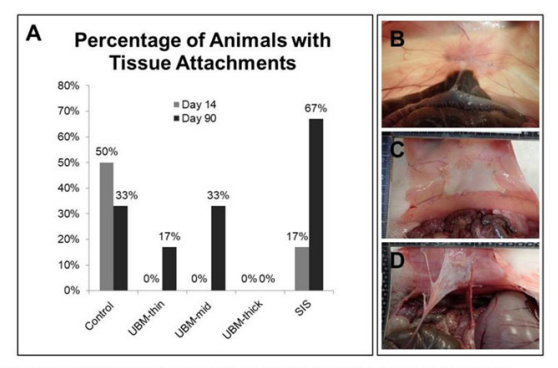

Fig. 1. Tissue Attachment Characterization. Percentage of animals with device-tissue
attachments (A), 90 day necropsy images: Control (B), UBM-middile thickness (C), and SIS (D)

At both time points, all three UBM groups had fewer animals with tissue attachments (Fig. 1) and lower average severity scores than the SIS group. When compared to controls, the UBM groups had fewer and/or smaller tissue attachments (single vs multifocal) and lower attachment severity scores at both time points. At 14 days, all devices remained largely non-resorbed while at 90 days moderate device degradation was observed across all groups, with the thinnest UBM devices appearing substantially degraded. Histologic evaluation revealed closure of the midline defect and granulation tissue deposition for all groups, with appreciable differences in semi-quantitative histological assessments for certain cell types. At 14 days, all surgical meshes showed increased numbers of macrophages compared to control animals. Lymphocytes were also more readily apparent in UBM compared to SIS devices. At 90 days there were increased numbers of multinucleated giant cells for SIS compared to UBM. This study evaluated the ability of biologically-derived surgical meshes to minimize tissue attachments in a preclinical model in which adhesion formation would normally be expected. UBM devices all showed performance that was similar to or better than SIS devices with respect to limiting tissue attachment formation when the devices are placed intraperitoneally. The results of this study support the use of UBM-based devices for intraperitoneal repair reinforcement as well as laparoscopic deployment of biologically derived surgical meshes. 
P553

Prospective Evaluation of Poly-4-hydroxybutyrate Mesh in CDC Class I, High-Risk Ventral and Incisional Hernia Repair: 3-Year Follow-up

John S Roth, Professor of Surgery ${ }^{1}$, G J Anthone ${ }^{2}$, D J Selzer ${ }^{3}$, Ben K Poulose $^{4}$, J G Bittner ${ }^{5}$, William W Hope ${ }^{6}$, R M Dunn ${ }^{7}$, Robert J Martindale $^{8}$, Matthew J Goldblatt ${ }^{9}$, David Earle ${ }^{10}$, John Romanell ${ }^{11}$, Greg Mancini $^{12}$, J A Greenbert ${ }^{13}$, J G Linn ${ }^{14}$, E Parra-Davilla ${ }^{15}$, Brian J Sandler ${ }^{16}$, G R Voeller ${ }^{12}$; ${ }^{1}$ University of Kentucky, ${ }^{2}$ Nebraska Methodist Hospital, ${ }^{3}$ Indiana University, ${ }^{4}$ Ohio State University, ${ }^{5}$ Virginia Commonwealth University, ${ }^{6} \mathrm{New}$ Hanover Regional Medical Center, ${ }^{7}$ University of Massachusetts, ${ }^{8}$ Oregon Health Sciences University, ${ }^{9}$ Medical College of Wisconsin, ${ }^{10}$ Lowell General Hospital, ${ }^{11}$ Baystate Medical Center, ${ }^{12}$ University of Tennessee, ${ }^{13}$ University of Wisconsin, ${ }^{14}$ Evanston, IL, ${ }^{15}$ Celebration, ${ }^{16}$ University of California, San Diego

Introduction: Long-term resorbable mesh represents a promising technology for complex ventral and incisional hernia repair (VIHR). Preclinical studies indicate that Poly-4-hydroxybutyrate (P4HB) bioresorbable mesh supports strength restoration of the abdominal wall. The objective of this study is to evaluate outcomes of CDC Class I/High-Risk patients undergoing VIHR with P4HB mesh. Methods: This is a prospective multi-institutional study of patients undergoing retrorectus or onlay VIHR. Inclusion criteria are: CDC Class I, defect size $10-350 \mathrm{~cm}^{2}, \leq 3$ prior repairs, and $\geq 1$ highrisk criteria (i.e. obesity, active smoker, COPD, diabetes, immunosuppression, coronary disease, steroid use, hypoalbuminemia, advanced age and renal insufficiency). Physical exam and/or quality of life surveys are performed at regular intervals through 5 years ( 60 months).

Results: 121 patients $(46 \mathrm{M}, 75 \mathrm{~F})$ with a mean age of $54.7 \pm 12.0$ years and BMI of $32.2 \pm 4.5 \mathrm{~kg}$ / $\mathrm{m}^{2}$ underwent VIHR. Comorbid conditions include: obesity (78.5\%), hypertension $(59.5 \%)$, COPD $(28.1 \%)$, cardiovascular disease $(34.7 \%)$, diabetes $(33.1 \%)$, active smoker $(23.1 \%)$, malignancy (24.8\%), immunosuppression (8.3\%), chronic corticosteroid use (5.0\%), hypoalbuminemia (2.5\%), advanced age $(5.0 \%)$, and renal insufficiency $(0.8 \%)$. Hernia types include: primary ventral hernia $(14.0 \%)$, primary incisional hernia $(44.6 \%)$, recurrent ventral hernia $(12.4 \%)$, and recurrent incisional hernia $(28.9 \%)$. Mean defect and mesh size are: $115.7 \pm 80.6 \mathrm{~cm}^{2}$ and $459.38 \pm 172.3 \mathrm{~cm}^{2}$, respectively. Repair types include: retrorectus $(43,35.5 \%)$, retrorectus with component separation $(45,37.2 \%)$, onlay $(24,19.8 \%)$ and onlay with component separation $(8,6.6 \%)$, and other $(1,0.8 \%)$. 82 patients $(67.8 \%)$ have completed at least 36 months follow-up to date. Postoperative wound infection, seroma requiring intervention, and hernia recurrence occurred in $11(9.1 \%), 8(6.6 \%)$ and 19 (15.7\%) Among the recurrences, nine occurred in the onlay group and 10 in the retrorectus group. Conclusion: Intermediate term outcomes following VIHR with P4HB mesh are favorable and demonstrate low recurrence rates at the 3-year (36-month) postoperative time frame. 5-year (60-month) follow-up is ongoing.

\section{P554}

\section{Laparoscopic and Percutaneous Short Stitch Technique Repair for Ventral Hernia (A Novel Technique)}

Abdullah Aldohayan, Mohanad Albalawi, Dr, Rasha Basha, Maram Alaqel, Hassan Arishi, Dr; King Saud University Hospital

Background: Ventral hernia repair (VHR) is a common procedure. Despite the frequency of VHR, the optimum repair method has not been established yet. The aim of this study is to analyze outcomes of VHR using a combined open (small bite short stitch technique (SBSST)) and laparoscopic technique.

Method: A retrospective review was conducted for 46 patients who received a combined laparoscopic and open ventral hernia repair at the medical city of King Saud University in Riyadh, Saudi Arabia. Main outcome measures included postoperative complications and recurrence.

Surgical Technique: Mesh size is determined by adding $5 \mathrm{~cm}$ longer than hernia defect size. The mesh is mounted with five sutures to the edges and center of the mesh number as per the principles set by the author of patent number US92049SSB. After ports are established and reduction of hernia sac and content is carried out, a small incision is made over the neck of sac where the sac is incised with sac excision, the mesh is introduced through the defect of the wound in aseptic technique which then followed by primary repaired using (SBSST). The sutures are retrieved through the abdominal wall and mesh is tacked to the abdominal wall using capture. Transfacial suture through abdominal wall keeps the mesh in place.

Results: The study started from May 2016 to May 2018, there are 46 patients who underwent ventral hernia repair using this technique were included. Majority of patients were female $(\mathrm{N}=28)$. The mean age and body mass index (BMI) was 52.2 years and $32.36\left(\mathrm{~kg} / \mathrm{m}^{2}\right)$ respectively. The mean defect size was $33.9 \mathrm{~cm} 2$. Postoperative complications included 2 seroma $(4.3 \%)$ occurrences, 1 hematoma (2.1\%) and 1 superficial infection $(2.1 \%)$. At a mean follow up of 17.1 months (range 6 to 28), there was no evidence of hernia recurrence or chronic pain.

Conclusion: We demonstrate that a combined laparoscopic and open (SBSST) hernia repair approach yielded favorable results especially in terms of hernia recurrence rate reduction and longterm patient satisfaction.

\section{P555}

Flank Incisional Hernia After Lateral Approach Spine Operations: Presentation and Outcomes After Repair

Negin Fadaee ${ }^{1}$, Erfan Zarrinkhoo, BS ${ }^{2}$, Khadij Assani, BS, $\mathrm{BA}^{3}$, Shirin Towfigh, $\mathrm{MD}^{4} ;{ }^{1}$ University of California, Los Angeles, ${ }^{2}$ Wake Forest School of Medicine, ${ }^{3}$ AT Still University School of Osteopathic Medicine, ${ }^{4}$ Beverly Hills Hernia Center

Introduction: The lateral flank approach to lumbar spine surgery is considered a new minimally invasive alternative to the anterior abdominal approach. Flank incisional hernia is one complication. Our aim is to review our experience with repair of these hernias and try to decipher technical reasons for their occurrence so that these can be prevented, with the goal of improving overall outcomes.

Methods: Retrospective review of all patients referred for evaluation of lateral bulging of the abdominal wall after Lateral Interbody Fusion (LIF), from August 2013 to August 2017.

Results: In 4 years, 14 patients were evaluated: 12 were diagnosed with incisional hernias at the LIF access site and 2 with denervation injury without hernia defect.

\begin{tabular}{|c|c|}
\hline Hernia Characteristics: & $\mathrm{N}=12$ \\
\hline $\begin{array}{l}\text { Demographics } \\
\text { Age, years, average (range) } \\
\text { Sex, M (\%) } \\
\text { BMI, kg/m, average (range) }\end{array}$ & $\begin{array}{l}67(50-78) \\
1(8 \%) \\
28(21-38)\end{array}$ \\
\hline Size of defect, cm; average (range) & $20(4-44)$ \\
\hline $\begin{array}{l}\text { Type of Fascial Closure } \\
\text { Single layer, External oblique only, slowly absorbable suture } \\
\text { Two layer, rapidly absorbable suture } \\
\text { Not specified }\end{array}$ & $\begin{array}{l}5(42 \%) \\
1(8 \%) \\
6(50 \%)\end{array}$ \\
\hline $\begin{array}{l}\text { Type of Defect } \\
\text { Partial thickness, External oblique preserved } \\
\text { Full thickness }\end{array}$ & $\begin{array}{l}7(58 \%) \\
5(42 \%)\end{array}$ \\
\hline $\begin{array}{l}\text { Presentation } \\
\text { Notice of Initial Bulge, months, average (range) } \\
\text { median, months } \\
\text { Time to Diagnosis, months, average (range) }\end{array}$ & $\begin{array}{l}11(1 \text { day }-7 \text { years }) \\
216(1-50)\end{array}$ \\
\hline \multicolumn{2}{|l|}{ Hernia Repair: } \\
\hline $\begin{array}{l}\text { Technique } \\
\text { Robotic } \\
\text { Laparoscopic } \\
\text { Open onlay }\end{array}$ & $\begin{array}{l}6(50 \%) \\
4(33 \%) \\
2(17 \%)\end{array}$ \\
\hline $\begin{array}{l}\text { Outcomes } \\
\text { Pre-op pain score, average (range) } \\
\text { Early post-op pain score, average (range) } \\
\text { Chronic pain } \\
\text { Recurrence }\end{array}$ & $\begin{array}{l}6(0-10) \\
4(0-8) \\
4 / 7(57 \%) \\
4 / 12(33 \%)\end{array}$ \\
\hline
\end{tabular}

Conclusions: This is the largest study addressing incisional flank hernias after LIF. This complication is under-represented in the spinal literature $(0.17 \%$ prevalence). We show that the patients present early postoperatively with their incisional hernia (median 2 months), and yet most hernias are not diagnosed for 16 months. The hernia is typically partial thickness, as the technique for access site closure is single layer closure of the external oblique with rapidly absorbable suture. To help reduce risk of hernia, we recommend closure in multiple layers with slowly absorbable suture; this should include closure of all fascial layers, not just the external oblique.

Although LIF is considered a minimally invasive spinal procedure, the morbidity from hernia complications cannot be overlooked. These hernias are difficult to repair with suboptimal outcomes: ? recurrence and over half with chronic pain. We prefer robotic approach with primary closure of the defect and extraperitoneal sublay mesh. 
P556

\section{Autologous Augmentation of Hiatal Hernia Repair with Platelet Concentrate Improves Results in Patients with Large Hiatal Hernias}

Vladimir Grubnik, MD, Professor, Viktor Grubnyk, Vadim Ilyashenko, PhD, Mykola Paranyak; Odessa National Medical University

Introduction. Following our hypothesis we believe that the use of a mesh implant with autologous PRP can be effective to prevent the large number of recurrence rate in patients with large $\mathrm{HH}$. The Purpose of Our Study is to evaluate whether the addition of PRP to self-fixated ProgripTM mesh would improve HH repair.

Methods. The study was performed on 104 patients with gastroesophageal reflux with large hiatal hernias (the size of hernias 10-20 cm2), who underwent Nissen fundoplication and crura repair. Al patients were divided into two groups: I group (54 patients) - we used non-absorbable self-fixating ProgripTM mesh for reinforcement of crura repair, II group (50 patients) - crura repair was done without mesh. In the patients of I group pieces of mesh were infiltrated by $2-4 \mathrm{ml}$ of autologous PRP. We determined type-I, and type-III collagens in patients of both groups. PRP was prepared from $20 \mathrm{ml}$ blood of the patient. The groups were evaluated according to the following criteria: dysphagia, patient's symptomatic outcome judgment according to the GERD-HRQL questionnaire and patient's satisfaction, hiatal hernia recurrence according to upper endoscopy and barium contrast swallow study. Followup was completed in $100(96 \%)$ patients with a mean follow-up duration of 29 months (range 12-48 months)

Results. There were no serious complications in the patients of both groups. Mean operative time was not significantly different in both groups. The quantity of total, type-I, and type-III collagens was significantly lower by about $55 \%$ in patients of both groups. In the patients of I group there were no problem with fixation of the mesh. The surgeons can identify very strong fixation of the ProgripTMmesh infiltrated by autologous PRP to the crura after pressure on it for 1-2 min. During the 48 months of follow-up, no recurrences occurred in the mesh group and 6 recurrences appeared in the non-mesh group $(\mathrm{p}<0.05)$. Patient satisfaction was significantly higher in the mesh group $(\mathrm{p}<0.05)$.

Conclusion. Our study has shown that the collagen content is reduced in patients with large HH. Thus, in such patients it is advisable to use a mesh for crural reinforcement. Use of autologous PRP can increase strength of fixation of ProgripTM mesh. Mesh reinforcement of crura repair improves results of laparoscopic $\mathrm{HH}$ repair in the patients with large $\mathrm{HH}$.

\section{P557}

\section{Operative Outcomes of Elective Giant Para-esophageal Hernia Repair at High Volume Centers - A Systematic Revie}

Rafik Sorial, Sofia Valanci, Pepa Kaneva, Julio F. Fiore Jr, Gerald M Fried, Liane S Feldman, Lorenzo E Ferri, Lawrence Lee, Carmen L Mueller; McGill University

Introduction: Laparoscopic repair of giant paraesophageal hernia (LPEHR) has historically been associated with significant morbidity and mortality, such that elective repair is only recommended for symptomatic patients. Since LPEHR was initially reported in the 1990s, technological improvements, specialization in surgical training and regionalization of care have occurred such that recent reports suggest modern era outcomes of LPEHR have improved. Elective repair may now be safer than previously thought, yet historical data continue to inform patient selection for surgery and informed consent discussions.

The objective of this study is to systematically review modern era surgical outcomes for elective laparoscopic giant paraesophageal hernia repair (LPEHR) performed at high volume center.

Methods: A systematic review was conducted according to PRISMA guidelines to identify all studies published between 01/2013-08/2018 reporting outcomes after elective repair of giant ( $>50 \%$ herniated stomach) paraesophageal hernia. High volume centers were defined as those reporting $>15$ cases/year over the study period. Review articles, those including $>10 \%$ Type I PEH or revision surgeries, any co-surgeries other than cholecystectomy, pediatric series and those not reporting primary data were excluded. The MINORS instrument was used to assess quality of observational studies. Data heterogeneity precluded meta-analysis.

Results: Eleven identified articles were included, of which ten were observational studies and one was an RCT. The mean MINORS score for observational studies was 14.0 (SD: 3.6; range: 9-18). A total of 1,926 patients underwent primary repair of type II-IV PEHs (63-78\% female; mean age range 57-71 years). PEHs were classified by type in 8 studies (1146 patients) and were mostly Type IIIs (mean incidence MI $=88 \%$ ). Procedures were begun laparoscopically in all studies except one (61\% open approach) with conversion to open reported in $7 / 1707$ cases $(\mathrm{MI}=0.2 \%)$. Collis gastroplasty was reported in 5 studies $(\mathrm{MI}=2.7 \%)$ and crural mesh reinforcement in 8 studies $(\mathrm{MI}=64 \%$ ). Median length of stay ranged from 2-4 days. Complications within 30 days of surgery were reported in 10 studies $(\mathrm{MI}=11 \%$ ), with $30 / 1809$ cases severe (Clavien-Dindo grade $\geq 3$; $\mathrm{MI}=2.5 \%)$. Peri-operative death was reported in 1 case $(\mathrm{MI}=0.1 \%)$. Nine studies reported anatomical recurrences $(\mathrm{MI}=21 \%$ ) of which revision was performed in 30 cases $(\mathrm{MI}=4.4 \%)$. Post-operative quality of life scores were reported in $6(54.5 \%)$ studies, with all reporting statistically significant improvement over pre-operative values.

Conclusion: Modern-era morbidity and mortality after elective primary LPEHR in high volume centers is low. Patient selection for surgery and pre-operative consent discussions should reflect modern and institutional-specific outcomes data.

\section{P558}

\section{Lateral (Port Site) Hernias Following Laparoscopic Ventral Hernia Repair}

Karla Bernardi, MD, Oscar A Olaverria, MD, Nicole B Lyons, BS, Puja Shah, Alexis N Milton, BS, Shinil K Shah, MD, Lillian S Kao, MD, MS, Tien C Ko, MD, Mike K Liang, MD; McGovern Medical School at UTHealth

Introduction: Lateral hernias due to port placement occur at a rate of 5\% $(0-30 \%)$ and are an under-reported complication following laparoscopic ventral hernia repair (LVHR). The majority of lateral hernias occur at previous $10-12 \mathrm{~mm}$ port incisions, which are often needed to safely introduce large pieces of mesh. One way to avoid using such a complication is to place the 10-12 $\mathrm{mm}$ port through the ventral hernia defect. However, there is theoretical concern for increased risk of surgical site infection (SSI) with this technique. We hypothesized that introducing mesh through a 10-12 mm port placed through the defect will decrease the rate of lateral hernias with no increased risk of SSI.

Methods: This is a prospective cohort study of patients who underwent LVHR at a single academic institution from 2014-2017. All patients had mesh introduced through a $10-12 \mathrm{~mm}$ port placed through the ventral hernia defect. Observed outcomes were compared to expected outcomes based upon data from published systematic reviews. The primary outcome was prevalence of latera hernias from port placement. Diagnosed by clinical exams and verified by radiographic imaging. Secondary outcomes included hernia recurrence, overall post-operative hernia occurrence, surgical site occurrences (SSO), and SSI. Binomial tests were performed and Bonferroni penalties applied ( $\mathrm{p}<0.01$ was considered significant).

Results: A total of 317 patients underwent LVHR repair. Within this cohort, patients had the following comorbidities: obesity $(66.9 \%)$, recent tobacco use $(8.8 \%)$, and diabetes $(18.9 \%)$. Most patients had a ventral incisional hernia $(61.2 \%)$ and $19.2 \%$ were recurrent. Hernias were on average $4.7 \pm 3.3 \mathrm{~cm}$ in width on preoperative imaging.

At a median follow-up of 21 (range 11-41) months, there was 1 (0.3\%) lateral hernia and $13(4.1 \%)$ hernia recurrences for an overall post-operative hernia occurrence of $14(4.4 \%)$. Compared to expected rates in the literature, there were significantly fewer observed lateral hernias and hernia recurrences. There was no difference in the observed versus expected rates of SSI and SSO (Table). Conclusion: During LVHR, introduction of the mesh through a $10-12 \mathrm{~mm}$ port placed through the defect may decrease the prevalence of lateral hernias without increasing the risk of developing a SSI or recurrence. While further studies are needed to confirm these results, lateral 10-12 mm ports can be safely avoided during LVHR.

Table

\begin{tabular}{|l|l|l|l}
\hline & Expected & $\begin{array}{l}\text { Observed } \\
\mathrm{N}=317\end{array}$ & P-Value \\
\hline $\begin{array}{l}\text { Postoperative Hernia } \\
\text { Hernia Recurrence } \\
\text { Lateral }\end{array}$ & $\begin{array}{l}15 \% \\
10 \%\end{array}$ & $\begin{array}{l}4.4 \%(14) \\
4.1 \%(13) \\
0.3 \%(1)\end{array}$ & $\begin{array}{l}0.020 \\
0.008 \\
0.001\end{array}$ \\
\hline Surgical Site Occurrence & $10 \%$ & $11.0 \%(36)$ & 0.817 \\
\hline Surgical Site Infection & $1 \%$ & $0.6 \%(2)$ & 0.385 \\
\hline
\end{tabular}


P559

\section{Operative Outcomes of Ventral Hernia Repairs in Heart Transplant Patients: A Single Center Experience}

Kambiz Kamrani, Alexis Okoh, Adam Kopelan; Newark Beth Israel

Background: There are scant data in the literature regarding the incidence and outcomes of ventral hernia repairs (VHR) after orthotopic heart transplantation (OHT). This study aims to describe a single centers experience with VHRs in OHT patients.

Methods: Data on all patients who underwent an OHT at a single center over a 13-year period was retrospectively reviewed. The incidence of post-transplant ventral hernia that required repair by a single surgeon was calculated. Baseline characteristics including risk factors for developing a ventral hernia and operative outcomes post-VHR were investigated.

Results: Between 2003 and 2016, a total of 603 OHT procedures were performed. Of these $21(3.5 \%)$ had a ventral hernia that required repair which was performed in a median duration of 2 (min:0.08 max:11) years after OHT. The study cohort consisted of $18(85 \%)$ males with mean \pm SD age and body mass index of $59 \pm 10$ years, $32.7 \pm 5.6 \mathrm{~kg} / \mathrm{m} 2$ respectively. Six $(29 \%)$ patients had a prior history of smoking, 5 (24\%) were insulin dependent diabetics, 13 $(62 \%)$ had prior abdominal surgery and $11(52 \%)$ were bridged to OHT with a durable ventricular assist device. VHRs were performed via an open approach in $16(76 \%)$ of which 7 needed component separations. Size of defects repaired was $\leq 100 \mathrm{~cm} 2$ in $14(67 \%)$. In-hospital mortality and re-admission within 30 -days were $0 \%$. Mean postoperative length of stay was 2 days. After a median follow-up of 4-years, recurrence requiring repair occurred in one patient after a year.

Conclusion: In a contemporary series of OHT patients, the incidence of ventral hernias post-transplant that required repair is reportedly low. Ventral hernias of various sizes in OHT patients can be repaired safely with favorable outcomes.

\section{P560}

\section{Laparoscopic Repair of Right Diaphragmatic Hernia with Liver Herniation}

Venkatarami Reddy Vutukuru, Associate Professor; Sri Venkateswara Institute of Medical Sciences

A $45 \mathrm{yr}$ lady, presented with pain in right lower chest and upper abdomen since 1 year. Increase in severityof symptoms since 10 days. Patient also has exertional breathlessness since 10 days. No history of trauma. On evaluation with chest radiograph and chest $\mathrm{CT}$ scan, there was a right diaphragmatic hernia with liver, small and large bowel in the right pleural cavity. Patient underwent surgery. Laparoscopic reduction of hernial contents with anatomical repair of hernia. Postoperative period was uneventful.

\section{P561}

Results of Robotic Ventral Hernia Repair with Phasix Bioabsorbable Mesh: A Fellowship Council Accredited Program's experience

Stefanie J Haynes, DO, Frederick Sabido, MD; Richmond University Medical Center

Introduction: The use of the robotic platform for ventral hernia repair has been proven to result in less surgical site infections (SSIs) and surgical site occurrences (SSOs) in multiple studies. We report our experience which further demonstrates the impact that robotics has had on reducing recurrence rates and post-operative complications specifically when fully resorbable mesh is utilized.

Methods: The American Hernia Society Quality Collaborative database was used to examine patients undergoing robotic ventral hernia repair from March 2017 - August 2018 by a Program Director and Fellow. The primary outcome measured was recurrence at 4 weeks, 20 weeks and 17 months. Secondary outcomes included SSIs, SSOs, readmission and chronic pain.

Patient demographics and intraoperative data were collected. All repairs utilized the Intuitive daVinci Si or Xi robotic platform. Primary closure of the hernia defect was completed with absorbable unidirectional barbed suture (Quil) and uniquely a fully resorbable Phasix mesh $(8,11$, or 15$)$ was positioned as an intraperitoneal onlay (IPOM).

Results: A total of 48 patients undergoing ventral hernia repair were examined- 22 female and 26 male. The mean age was 54 and BMI 33.8. The types of ventral hernias repaired were umbilical $(40 \%)$, incisional $(33 \%)$, epigastric $(21 \%)$, and Spigelian $(0.06 \%)$. The mean ASA class was 1.9 and total operative time was 0-59 min. All patients were discharged on the same day post operatively. Recurrence at 4 weeks was $0 \%, 20$ weeks $0 \%$ and 17 months $4 \%$ (2/ 48). There were no SSIs, SSOs, readmissions or mesh infections reported. The average pain score post operatively was moderate and current pain was $0 / 10$ on the pain scale.

Conclusions: This preliminary data demonstrates that fully resorbable mesh combined with the benefits of robotic technique for ventral hernia repair can reduce or eliminate the incidence of recurrence and post-operative complications at 17 months which has not been shown to date. 
P562

Wound Complications After Inguinal Hernia Repair, Has Robotic Surgery Improved Outcomes?

Carla N Holcomb, MD, MSPH ${ }^{1}$, Li-Ching Huang ${ }^{2}$, Savannah Renshaw $^{1}$, Susan Maurer, MD ${ }^{1}$, Noah Switzer, MD ${ }^{1}$, Michael Meara, $\mathrm{MD}^{1}$, Courtney Collins, $\mathrm{MD}^{1}$, Vimal Narula, $\mathrm{MD}^{1}$, Benjamin Poulose, $\mathrm{MD}^{1}$; ${ }^{1}$ Ohio State University Medical Center, ${ }^{2}$ University of Vanderbilt

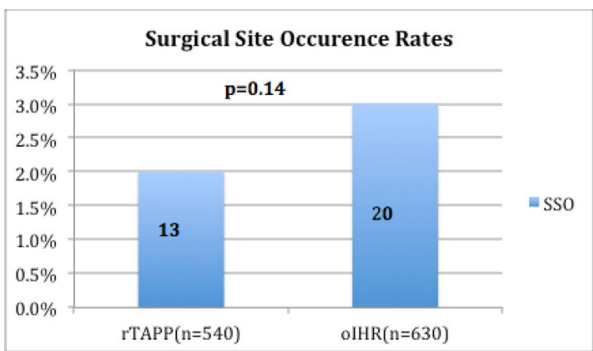

Figure 1. Surgical site occurrence rates for robotic transabdominal preperitoneal nguinal hernia repair (CTAPP versus open inguinal hernia repair (glHB) in 1170

Introduction: Minimally invasive inguinal hernia surgery has been associated with lower surgical site infections compared to open surgery. However, there is speculation that the preperitoneal approach may lead to higher rates of seroma formation. Robotic assisted inguinal surgery has become increasingly popular yet it is unclear if this approach is an improvement on the standard open Lichtenstein repair in terms of surgical site complications. The purpose of this study was to compare surgical site complication rates after robotic assisted transabdominal preperitoneal repair(rTAPP) of inguinal hernias to open Lichtenstein hernia repair(oIHR).

Methods: The study consisted of multi-institutional data from patients undergoing elective, nonrecurrent inguinal hernia repair with either rTAPP or oIHR in the Americas Hernia Society Quality Collaborative from 2013-2018. Outcomes of interest included surgical site occurrence (SSO), which is a composite variable of surgical site infection, skin or soft tissue ischemia, wound drainage, seroma, or hematoma. Wilcoxon rank-sum test was used for continuous variables and Pearson Chi square analysis was used for categorical variables.

Results: The study population consisted of 1170 inguinal hernia repairs including 540 rTAPP $(46 \%)$ and $630 \mathrm{oIHR}(54 \%)$. Patients that underwent oIHR were more likely to be older (65 years [55-73 years] vs 60 years[48-70 years], $\mathrm{p}=0.01)$ and have higher rates of diabetes mellitus $(11 \%$ vs $8 \%), p=0.06$ ) compared to rTAPP. There was no significant difference between groups regarding hernia size (rTAPP $2.0 \mathrm{~cm}[1.0-5.0 \mathrm{~cm}]$ vs oIHR $2.0 \mathrm{~cm}[1.6-3.0 \mathrm{~cm}], \mathrm{p}=0.84)$. Overall SSO rates were low at $2.8 \%(\mathrm{n}=33)$ and did not significantly differ by $\operatorname{rTAPP}(2 \%)$ vs oIHR $(3 \%)$, $\mathrm{p}=0.14$. The most common SSO in both groups was seroma but this was not significantly different in either group $(1.6 \%$ for rTAPP versus $1.4 \%$ for oIHR $p=0.17)$. There was no significant difference in any postoperative complication ( $5 \%$ vs $5 \%$ ), hernia recurrence $(0.2 \%$ vs $0.0 \%$ ) or 30 day readmission( $1 \%$ vs $1 \%$ ) for rTAPP vs oIHR, respectively.

Conclusions: In this study, the rate of any surgical site complication after open or robotic inguinal hernia repair was $2.8 \%$ and this did not differ by robotic or open approach. Both approaches to inguinal hernia repair appear to have low surgical site complication rates and equivalent outcomes.

\section{P563}

Evaluating Abdominal Wall Function Post Ventral Hernia Repair: A Comparison of Open Versus Robotic-Assisted Retromuscular Techniques. An Americas Hernia Society Quality Collaborative Study

Noah Switzer, Savannah Renshaw, Carla Holcomb, Susan Maurer, Courtney Collins, Michael Meara, Vimal Narula, David Renton, Benjamin Poulose; The Ohio State University

Introduction: To examine abdominal wall function specific quality of life metrics comparing open to robotic-assisted retromuscular hernia repair

Retromuscular ventral hernia repairs are a common surgical technique for the management of incisional hernias. The placement of mesh in a well vascularized plane, free from abdominal viscera, with wide overlap of the hernia defect, have led to lower recurrence rates and durability that make it arguably the gold standard for small to moderate sized hernias.

Robotic-assisted retromuscular repairs have been shown to have similar objective outcomes to the open approach. However, a comparison between the two groups in terms of abdominal wall function. The Hernia Related Quality of Life Survey (HerQLes) was developed to address this, as a patientcentered method for the evaluation of abdominal wall function.

Methods and Procedures: This is a retrospective review of prospectively collected data using the Americas Hernia Society Quality Collaborative data between 2013 - 2018. Included subjects were $>18$ years old, had an open or robotic-assisted retromuscular ventral hernia repair performed with HerQLes summary scores available. The HerQLes score is scored out of 100, where a higher number indicates a better quality of life. Wilcoxon rank sum test and Pearson Chi square test were used. A 3:1 propensity-matched analysis was performed with the groups matched on baseline HerQLes scores, age, sex, BMI, smoking status, ASA Class, diabetes and hernia size.

Results: The propensity-matched analysis included 120 patients, stratified 3:1 into open $(\mathrm{N}=90)$ and robotic $(\mathrm{N}=30)$ cohorts. The median age was 61 [52-68] and 58 [51-63] years for open and robotic, respectively. Sixty-nine percent of the open population were female compared to $73 \%$ in the robotic arm. The median hernia width was $6 \mathrm{~cm}$ and $7 \mathrm{~cm}$ for the open and robotic groups, respectively. Post-operative complications rates were similar in both groups $(\mathrm{p}=0.29)$. One-year recurrence rates were not statistically different(19\% vs. $23 \%)$ in the open and robotic groups, respectively $(\mathrm{p}=0.6)$

The median HerQLes summary scores at 30 -days( 48 vs 48 ) and I year( 81 vs. 82 ) were not significantly different between the open and robotic groups, respectively $(\mathrm{p}=0.54, \mathrm{p}=0.86)$. This resulted in median change from baseline to 1 year of 21.7 for both $(\mathrm{p}=0.6)$.

Conclusion: Both open and robotic-assisted retromuscular ventral hernia repairs are associated with improved patient reported quality of life outcomes, with no significant outcome differences between the techniques. Larger study populations are still needed to further evaluate this topic.

\section{P564}

\section{Comparison of Pure Polypropylene Mesh vs. Covered Mesh in Laparoscopic Incisional and Ventral Hernia Repair: A Non Randomised Cohort Study}

Om Prakash, Shardool Vikram Gupta, Virinder Kumar Bansal, Subodh Kumar, Asuri Krishna, M. C. Misra; AIIMS, New Delhi

Introduction: The literature continues to be ambiguous on the type of mesh to be used in laparoscopic incisional/ventral hernia repair. This study presents non randomised comparison of pure propylene and covered mesh in laparoscopic incisional and ventral hernia repair.

Materials and methods: Patients undergoing laparoscopic incisional or ventral hernia repair in a single surgical unit from 2004 to 2015 were studied. The data was collected from a prospectively maintained database and analysed retrospectively.These patients were divided into two group depending on the type of mesh used i.e. pure polypropylene (PP group) mesh or covered mesh (CM group).Laparoscopic repair of incisionaland ventral hernia was done following a standard protocol.A mesh of appropriate size was used to overlap all the defects with a margin of at least $4-5 \mathrm{~cm}$ in all directions. Mesh fixation was done with a $5 \mathrm{~mm}$ non absorbable or absorbable fixation device in double crown fashion along with 4 corners transfascial sutures.Patients were followed up in the follow up clinic at 1 week, 6 weeks, 3 months, 6 months and yearly thereafter. Results: Eight hundred eighty patients underwent incisional and ventral hernia repair during this period. Laparoscopic repair was performed in 500 patients. Conversion to open repair was done in 30 patients because of dense adhesions. Pure polypropylene mesh was used to repair the hernia in 174 patients ( $\mathrm{PP}$ group) and covered mesh was used in 326 patients (CM group).Commonest index surgery of incisional hernia was lower section Caesarean section. Seroma formation difference was not statistically significant between the two groups. Wound infection was comparable in both groups (4.6\% in PP and 5.2\% in CM). Post operative pain scores were similar in both groups. Three patients in both groups required relaparoscopy for unsettled abdomen. Six patients (3.4\%) in PP group and 8 patients (2.5\%) in CM group had prolonged ileus. Sixteen patients $(9.2 \%)$ in PP group while 28 patients $(8.6 \%)$ in $\mathrm{CM}$ group required readmission for various reasons.Subacute intestinal obstruction was seen in 12 patients(6.9\%) in PP group while in 18 patients $(5.5 \%)$ in $\mathrm{CM}$ group There were 28 recurrences, $11(5.9 \%)$ in pure polypropylene group and $17(4.9 \%)$ in covered mesh group.The median duration of recurrence was 2 years with a range of 16 to 48 months. Conclusion: Outcomes with pure polypropylene mesh are comparable to covered mesh for laparoscopic ventral and incisional hernia repair with regards to postoperative pain, complications and recurrence rates. 
P565

Laparoscopic versus open ventral hernia repair in the elderly: a propensity score matched analysis

Susanna W De Geus, Alaina D Geary, Nkiruka Arinze, Sing Chau Ng, Cullen O Carter, Donald T Hess, Jennifer F Tseng, Luise I Pernar; Boston Medical Center

Background: Ventral hernia repair is common in the expanding aging population, but remains challenging due to their frequent comorbidities. The purpose of this study is to compare the surgical outcomes of open vs. laparoscopic ventral hernia repair in elderly patients.

Methods: Patients ${ }^{3} 65$ years of age that underwent elective open or laparoscopic ventral hernia repair were identified from the American College of Surgeons National Surgical Quality Improvement Project (NSQIP) database. To reduce potential selection bias, propensity scores were created for the likelihood of undergoing laparoscopic surgery based on patients' age, sex, race, BMI, ASA class, functional status, inpatient/outpatient surgery, diabetes mellitus, disseminated cancer, dialysis, hypertension, COPD, congestive heart failure, bleeding disorder, and steroid use. Patients were matched based on the logit of the propensity scores. Surgical outcomes were compared after matching using the WilcoxonMann-Whitney test and logistic regression.

Results: $18,820(76.5 \%)$ and 5,795 $(23.5 \%)$ patients underwent open and laparoscopic ventral hernia repair, respectively. Open surgery was associated with age $>70$ years $(54.6 \%$ vs. $52.7 \% ; \mathrm{p}=0.0103)$, non-white race $(8.6 \%$ vs. $6.8 \% ; \mathrm{p}<0.0001)$, ASA class III $(60.3 \%$ vs. $57.3 \% ; \mathrm{p}<0.0001)$, BMI $£ 29$ ( $46.7 \%$ vs. $41.8 \% ; \mathrm{p}<0.0001)$, inpatient procedure $(42.5 \%$ vs. $34.9 \% ; \mathrm{p}<0.0001)$, COPD $(7.5 \%$ vs. $6.6 \% ; \mathrm{p}=0.0225)$, steroid use $(4.4 \%$ vs. $3.7 \% ; \mathrm{p}=0.0156)$, history of congestive heart failure $(0.6 \%$ vs. $0.4 \%$; $\mathrm{p}=0.0316)$, and bleeding disorders ( $3.8 \%$ vs. $3.1 \% ; \mathrm{p}=0.0099)$. After matching, baseline characteristics were equally distributed across treatment groups, with 5,795 patients in each group. Open surgery was associated with significantly shorter operating time (63 vs. $84 \mathrm{~min} ; \mathrm{p}<0.0001)$. Patients who underwent open surgery were more likely to develop surgical site infections ( $3.4 \%$ vs. $1.6 \%$; Odds Ratio [OR], 2.146; $\mathrm{p}<0.0001)$, postoperative hemorrhage $(0.7 \%$ vs. $0.3 \%$; OR, $2.343 ; \mathrm{p}=0.0019)$, and sepsis $(0.6 \%$ vs. $0.3 \%$; OR, $1.847 ; \mathrm{p}=0.0291)$ compared to laparoscopic ventral hernia repairs. The incidence of myocardial infarction, pulmonary complications, venous thromboembolism, urinary tract infection, postoperative septic shock, length of stay, reoperation, and readmission were not statistically significant between groups.

Conclusion: Although open surgery remains the most prevalent in the elderly population, the results of this study suggest that laparoscopic surgery is safe and associated with lower risk of sepsis, bleeding, and surgical site infections.

P566

\section{Robotic Paraesophageal Hernia Repair: A Single-Center Experience in a Third World Country}

Ruben Luna, MD, Carlos Luna, MD, Daniel Gomez, Luis F Cabrera, MD, Laura Castro; Bosque University

Introduction: Robotictechnology is an emerging technology that has been developed in order to overcome some limitations of the standard laparoscopic approach, offering a stereoscopic three-dimensional visualization of the surgical field, increased maneuverability of the surgical tools with consequent increased movement accuracy and precision and improved ergonomics.Laparoscopic surgery is considered the standard approach for the treatment of paraesophageal hernias. Despite its advantages, this approach is technically demanding with a significant learning curve. There is no consensus on the optimal technique for hiatal hernia repair, and considerable recurrence rates are reported.

Objectives: Describe the feasibility and safety of roboticassistance for the treatment of paraesophageal hernias in a third world country.

Materials and Methods: Between August 2016 and June 2018. Patients who underwent elective or urgent Robotically assisted paraesophageal hernia repair were included in a prospectively collected database. Demographic data, American Society of Anesthesiologists classification, preoperative testing, operative time, length of hospital stay, conversion rate, morbidity, recurrence and mortality were recorded and reviewed retrospectively. Results: 4 underwent elective and 1 patient underwnt urgent Robotically assisted paraesophageal hernia repair, the type of the hernia, was type IV $100 \%$. Operative time averaged $196 \mathrm{~min}$, including robot setup time. There were no conversions. The average blood loss was $23 \mathrm{~mL}$. Perioperative complications, including intraoperative and 30-day complications, were $0 \%$.The mean length of hospitalization was 5 days. No recurrence. There were no deaths.

Conclusions: Robotically assisted paraesophageal hernia repair has proved to be feasible and safe with a learning curve comparable to the standard laparoscopic approach in a third world country

\section{P567}

Feasibility of Single Incision Laparoscopic TEP Hernia Repair Under Local Anesthesia for Elderly Patients with Inguinal Hernia

Norihito Wada, MD, PhD, FACS, Toshiharu Furukawa, MBA, MD, PhD, Yuko Kitagawa, MD, PhD, FACS; Department of Surgery, keio University School of Medicine

Introduction: Inguinal hernia is a benign disease. The indication for surgical treatment is relative. For the elderly patients with inguinal hernia, the lifestyle is not so active and the life expectancy is limited. If the symptom of hernia is minimal, the risk may exceed the benefit of surgery. We have developed a novel minimally invasive technique of single-port in the lower abdomen laparoscopic TEP inguinal hernioplasty under local anesthesia which have the potential to expand the indication of laparoscopic surgery for inguinal hernia. Materials \& Methods: From January 2012 to June 2018, a consecutive group of 169 patients with bilateral inguinal hernia was included. The clinical data of the patients aged 80 or older (group $\mathrm{E}, \mathrm{n}=23$ ) were compared with those of the patients aged less than 80 (group $\mathrm{Y}, \mathrm{n}=146$ ). Obese patients, patients with giant hernia or irreducible hernia were excluded. We used $0.5 \%$ lidocaine with epinephrine as local anesthesia. An incision of $30 \mathrm{~mm}$ in the lower abdomen was made and a wound protector with sealing silicon cap was installed. We used three 5-mm trocars and a 5-mm flexible laparoscope. A flat self-fixating mesh with resorbable microgrip was placed over the myopectineal orifice. No tacking devices were used.

Results: The mean \pm SD [range] age of group $\mathrm{E}$ and $\mathrm{Y}$ were $84.0 \pm 4.0$ [80-96] and $65.6 \pm 9.9$ [27-79], respectively. Female sex of group $\mathrm{E}$ and $\mathrm{Y}$ were $17.4 \%$ and $11.0 \%$ respectively $(\mathrm{P}=0.59)$. The mean $\pm \mathrm{SD}$ [range] operating time of group $\mathrm{E}$ and $\mathrm{Y}$ were $162 \pm 38$ [76-300] and $164 \pm 42$ [100-254] minutes, respectively. Surgical complications were not observed except for 7 cases of minor seromas (4.1\%). Pneumoperitoneum due to peritoneal injury was occurred in 18 cases $(10.7 \%)$ and managed by closing the defect. During median follow-up of 45 months, we observed 1 hernia recurrence $(0.59 \%)$ in group Y. No 30-day mortality was observed.

Conclusions: The mid-term outcomes of elderly patients were comparable to those of younger patients. Surgical invasiveness of this technique was minimal because the area of dissection in the preperitoneal space is smaller than that of umbilical TEP. Postoperative recovery was rapid and patients can walk soon after surgery. This novel procedure may be feasible in elderly patients. The indication of TEP may be expanded to the elderly patients at high risk of general anesthesia. 


\section{P568}

The Use of Biologic Mesh for Repair of Ventral and Incisional Hernia: A Systematic Review

Alejandro Rodriguez, MD, Jennifer Minneman, MD, Rebecca P Petersen, MD, Andrew S Wright, MD; University of Washington

Background: Biologic grafts are widely used as an alternative to synthetic materials in ventral hernia repair. In the United States, there are currently at least a dozen commercially available biologic grafts. However, the indications for use and individual outcomes are unclear.

Methods: A systematic search was performed using the EMBASE and PubMed platforms. Additional abstracts were identified through other sources. Inclusion criteria were studies describing ventral or incisional hernias, studies with $\mathrm{N} \geq 20$, and reporting of basic demographic information and key outcomes (recurrence and wound complications). The PRISMA checklist for systematic reviews was followed.

Results: The search strategy yielded 773 abstracts, with 5 articles being identified through other sources. Seventy-two abstracts were assessed for eligibility. All 30 included studies were retrospective. Outcomes for 2,423 patients were analyzed. Porcine acellular dermal matrix was the most commonly mesh studied followed by human acellular dermal matrix. Recurrence rates ranged from 0 to $35 \%$. Pooled results by mesh type are included in table 1.

Conclusion: The ideal choice of biologic mesh for ventral hernia repair remains unclear. The available literature is characterized by heterogeneity in patient selection and outcomes reporting. Standardized reporting of patient characteristics and clinical outcomes will be crucial in order to guide clinical decision-making.

\begin{tabular}{lllll}
\hline Summarized Results & & & & \\
\hline Mesh & $\begin{array}{l}\text { Studies } \\
(\mathrm{n})\end{array}$ & $\begin{array}{l}\text { Patients } \\
\text { (n) }\end{array}$ & $\begin{array}{l}\text { Recurrence } \\
\text { (median) }\end{array}$ & $\begin{array}{l}\text { SSO } \\
\text { (median) }\end{array}$ \\
\hline $\begin{array}{l}\text { Porcine Acellular } \\
\text { Dermal Matrix (non- } \\
\text { crosslinked) }\end{array}$ & 12 & 683 & $8.2 \%$ & $28.5 \%$ \\
$\begin{array}{l}\text { Porcine Acellular } \\
\text { Dermal Matrix } \\
\quad \text { crosslinked) }\end{array}$ & 4 & 927 & $11.3 \%$ & $28.7 \%$ \\
$\begin{array}{l}\text { Human Acellular } \\
\quad \text { Dermal Matrix }\end{array}$ & 10 & 675 & $18.5 \%$ & $46.9 \%$ \\
$\begin{array}{l}\text { Bovine Pericardium } \\
\text { Bovine Acellular Dermal } \\
\text { Matrix }\end{array}$ & 2 & 64 & $9.6 \%$ & $3.2 \%$ \\
\hline
\end{tabular}

\section{P569}

\section{Inflamed Adipose Tissue and Fibro/Adipo Proliferative} Fibroblasts in the Pathogenesis of Incisional Hernia

Finosh G Thankam, Gunasekar Palanikumar, Nicholas K Larsen, Robert J Fitzgibbons, Devendra K Agrawal; Creighton University

Incisional hernia $(\mathrm{IH})$ is a major complication of laparotomy and is common after laparoscopic procedures where specimen extraction mini laparotomy is required. The information regarding the basic biology of IH formation, progression, and healing responses are limited in the literature. In this study we are focusing on the association of adipocyte mediated inflammatory responses in IH pathology. In order to examine our hypothesis, we collected adipose tissue samples adjacent to the hernia defect from patients undergoing repair of their IH. The tissues were evaluated for histomorphological alterations by H\&E, the gene expression of cytokines, and macrophage markers (CCR7, CD206, CD68) by real time PCR. The protein expression of those markers was determined by immunofluroscence analysis. The H\&E showed many small disorganized adipose tissues where as the control tissue has more organized and large adipose tissue. Inflammatory markers IL6, IFN- $\gamma$, and IL1B were observed to be more in the adipose tissue of IH patient than from the control patients. The expression of M1 macrophage marker (CCR7) predominated in the adipose tissue of IH patient when compared to the control patients. The expression of M2 macrophage marker (CD206) were similar in all the tissue samples. We established that adipose tissue cells in patients with IH produce inflammatory adipokines which could damage adjacent structures such as fascia and muscle because of collagen degradation from production of collagen. This could interfere with the healing process and can cause recurrent hernia. We also looked into fibroblasts in the hernia sacs and borders. We hypothesized that a switch in fibroblast phenotype results in altered ECM and pathological fibroblast phenotypes could also contribute to the underlying pathophysiology of IH. Initial H\&E staining revealed ECM damage, increased cellularity, and collagen disorganization. The protein expression of biomarkers (CD34, CD90, CDH11, PDPN, PDGFR- $\alpha$ and FSP1) was examined by immunofluorescence analysis. Immunopositivity was shown for all markers suggesting the presence of fibro/adipo-proliferative fibroblasts. The isolated fibroblast cells from hernia tissue also revealed the presence of these markers in the in vitro culture. The high density of fibro/adipo-proliferative fibroblasts in the IH tissues suggests a strong association of these cells in the development of $\mathrm{IH}$ following a laparotomy. The understanding of the underlying molecular mechanism associated with the fibro/adipobiology of IH pathology could serve to develop promising therapeutic strategies to prevent $\mathrm{IH}$. 
P570

\section{Pneumomediastinum: An Unusual Complication After Totally Extraperitoneal Inguinal Hernia Repair}

Derek Lim, DO, Shinban Liu, DO, Claudia Kim, DO, Michael Timoney, MD; NYU Langone Medical Center

Case Description: A 30-year-old female with no past medical or surgical history presents with a symptomatic right inguinal hernia for 1 month prior to evaluation. She underwent a laparoscopic totally extraperitoneal (TEP) inguinal hernia repair. Three infraumbilical ports were used and the pre-peritoneal space was insufflated to $12 \mathrm{mmHg}$. Appropriate structures were visualized and the hernia was repaired with mesh in the standard fashion after extensive dissection. She was discharged the same day with no complaints.

She returned to the emergency room that night complaining of sharp pleuritic chest pain which radiated to the back and neck. She denied any fevers, dysphagia, cough or shortness of breath. Physical exam was negative for chest wall or neck crepitus. CT chest demonstrated evidence of pneumomediastinum and pneumoperitoneum. She was admitted and observed overnight with improvement of symptoms the following morning. She was discharged after tolerating a diet and without requiring any further interventions.

Discussion: Laparoscopic totally extraperitoneal (TEP) inguinal hernia repair is a growing technique amongst minimally invasive surgeons. This approach allows for the repair of all three inguinal spaces (direct, indirect and femoral) as well as bilateral inguinal hernias with only three incisions. Pneumomediastinum is a rare complication after a TEP procedure, with a reported incidence of only $0.1-0.3 \%$. Insufflated carbon dioxide can enter into the thoracic cavity through three fascial planes; subcutaneous fascia, retroperitoneum (myopectineal dissection) or transdiaphragmatic (congenital diaphragmatic hernia or aortic/esophageal hiatus). Each route may present differently ranging from chest wall crepitus to a pneumothorax. Risk factors include high working $\mathrm{CO} 2$ insufflation pressures $(>10 \mathrm{mmHg}$ ), extensive dissection, and prolonged duration of surgery. $\mathrm{CO} 2$ diffuses through tissue very rapidly and often patients can be observed for $24 \mathrm{~h}$ and managed conservatively. There is no role for repeat imaging as long as the patient remains asymptomatic and stable.

Although uncommon, early recognition of pneumomediastinum is important to prevent further complications such as respiratory distress or cardiac compromise. Anesthesiology must be cognizant intraoperatively as a pneumothorax secondary to progressive pneumomediastinum may complicate airway management.

\section{P571}

\section{Atypical Mycobacteria Associated Mesh Infections Following Laparoscopic Inguinal Hernia Repair - An Underreported Clinical Concern}

Sunay N Bhat, MS, Santhosh Koppal, DNB, Gokul K Shanker, MSDNBMBA; VGM Gastro centre, Coimbatore

Introduction: The incidence of atypical mycobacteria associated mesh infections (AMAMI) is underreported. These infections are often treated as surgical site infections which do not respond to regular antibiotics. The present study is aimed at diagnosing AMAMI and its treatment options. Methods: Twelve cases of AMAMI after laparoscopic inguinal hernia repair presented between 2012 to 2017. All cases were started on antimicrobial therapy initially but the wounds never healed. All cases presented with erythematous, indurated,painful swellings at the working port site. Four cases had persistent discharging sinus, 3 cases had pain around the site of mesh placement and low grade fever. Wound swab was sterile. Ultrasonogram and CECT whole abdomen showed abscess cavity in the abdominal wall extending to the pre-peritoneal space without signs of peritonitis. After failure to respond to broad spectrum antibiotics, atypical mycobacteria infection was strongly suspected. Pus sent for PCR and found positive for mycobacterium fortuitum in 5 of these cases. A clinical diagnosis of AMAMI was made. All the cases were treated conservatively initially with oral Clarithromycin and oral Ciprofloxacin.Four cases needed mesh explantation. A drain was left in situ for 1-2 weeks. There have been no such infections at our institute after the use of plasma sterilizer.

Results: After two weeks of therapy, the amount of discharge from the wound reduced and indurations subsided. In 8 cases, the symptoms improved considerably after treatment for 6 to 8 weeks. Oral Clarithromycin and Ciprofloxacin were continued for 12 weeks to prevent relapse. Four cases underwent mesh explantation after 6 to 8 weeks of no significant clinical improvement with conservative treatment. Nine cases were asymptomatic after 1 year of completion of treatment. Three cases were lost for follow-up.

Conclusion: Conservative management with second line of anti-tubercular drugs for a period of 12 weeks may be successful in controlling AMAMI in most cases and the morbidity of mesh explantation can be avoided. Many pus samples failed to confirm the presence of atypical mycobacteria but since the presentation in all the cases were similar, the response with oral Clarithromycin and oral Ciprofloxacin has been satisfactory in our series. Whenever a tacker is reused, it should be sterilized by ethylene oxide or plasma sterilization. Where ethylene oxide sterilization facility is not available, tacker reuse may be a point of concern in preventing atypical mycobacterial infection and 1.0 polypropylene suture for fixing the mesh may be used.

\section{P572}

\section{Robotic Extended Totally Extraperitoneal (eTEP) Approach: Evolution of Robotic Ventral Hernia Repair}

Jeremy A Warren, $\mathrm{MD}^{1}$, Marcia Alayon-Rosario, $\mathrm{MD}^{2}$, Joseph A Ewing, $\mathrm{PhD}^{2}$, Alfredo $\mathrm{M}$ Carbonell, $\mathrm{DO}^{1} ;{ }^{1}$ University of South Carolina School of Medicine Greenville, ${ }^{2}$ Greenville Health System

Robotic technology facilitates reproduction of complex abdominal wall reconstruction with retromuscular placement of prosthesis in a minimally invasive fashion. A transabdominal approach is typically used, often using additional myofascial release in the form of transversus abdominis release (TAR). However, this approach may unnecessarily employ TAR for smaller hernia defects that would not require TAR in an open repair. Use of an extended totally extraperitoneal (eTEP) approach mitigates the overutilization of TAR.

Methods: Prospective, surgeon entered data in the Americas Hernia Society Quality Collaborative (AHSQC) was used to identify all robotic retromuscular ventral hernia repairs (rRMVHR) performed at our institution between October 2013 and September 2018. Patients repaired with the standard transabdominal technique were compared to eTEP. Demographic, operative and outcomes data were compared. Primary endpoints were hospital length of stay (LOS)and use of TAR. Analysis was performed with Chi square test using R Statistical Software.

Results: Of 195 total rRMVHR were performed, of which 51 were eTEP and 144 transabdominal. Mean age was higher in the eTEP group ( 60.8 vs 55.7 years; $p<0.001)$, but other comorbidities were no different between groups. Hernia width was smaller for eTEP (median $6 \mathrm{~cm}$ vs $8 \mathrm{~cm}$; $\mathrm{p}=0.042$ ). There was no difference in surgical site infection ( 0 vs $2.1 \%$ ), surgical site occurrence (25.5 vs $39.6 \%$ ), or those requiring procedural intervention (3.9 vs $4.2 \%$ ) between eTEP and transabdominal rRMVHR. LOS was significantly shorter with eTEP (median 0 vs 1 day; $\mathrm{p}<0.001)$. Use of TAR was significantly reduced with eTEP $(13.7$ vs $67.4 \% ; \mathrm{p}<0.001)$. Conclusion: Robotic eTEP repair represents an evolution of rRMVHR that more closely approximates open repair and use of TAR. The eTEP also reduces LOS, with most patients discharged on the day of surgery.

\section{P574}

\section{Subsequent Hernia Repair After Inguinal Herniorrhaphy}

Igor Wanko Mboumi, MD, Yiwei Xu, MHS, Jacob Greenberg, MD, Luke Funk, MD, Amber Shada, MD, Anne Lidor, MD; University of Wisconsin

Background: Inguinal hernia repair is one of the most frequently performed procedures in the United States. The optimal surgical approach, whether laparoscopic or open, remains a subject of debate. While certain clinical scenarios may favor laparoscopic herniorrhaphy, such as bilateral or recurrent cases, the majority of inguinal hernia repairs in this country are performed open. This study endeavoured to determine the rate of subsequent hernia surgery after initial inguinal hernia repair and to identify risk factors that predict the need for reoperation.

Methods: Data from the Truven Health MarketScan ${ }^{\circledR}$ Research Databases were analyzed. Adult patients with a CPT procedure code for outpatient inguinal hernia repair from 2012-2014 were included. Only patients with continuous enrollment for 6 months prior to, and following, surgery were included. Performance of another inguinal hernia repair within at least 6 months of the initial surgery was captured using the same procedure codes. Multivariable logistic regression was used to identify risk factors for reoperation.

Results: 88,331 cases were identified ( $91.4 \%$ male), with mean age of 55 . Most patients underwen open herniorrhaphy, and men underwent laparoscopic and bilateral repair more frequently than women $(32.1 \%$ vs. $27.2 \%$ and $13.4 \%$ vs. $7.1 \%)$. The overall rate of reoperation for both men and women was similar $(8.3 \%$ vs. $7.9 \%$, respectively). On adjusted analysis, surgery for recurrent inguinal hernia resulted in significantly increased odds for another hernia repair $(1.16, \mathrm{CI}=$ $1.06,1.26)$, and patients who underwent bilateral repair had a significantly lower odds of reoperation $(\mathrm{OR}=0.48, \mathrm{CI}=0.44,0.53)$.

Conclusion: The rate of subsequent hernia repair after inguinal herniorraphy is significant. Most notable is the nearly $50 \%$ reduction in the odds of subsequent inguinal herniorrhaphy in patients who undergo bilateral inguinal hernia repair initially. This may reflect an advantage of laparoscopy in affording a means to identify and treat a subclinical hernia on the contralateral side.

\begin{tabular}{|c|c|c|c|c|c|}
\hline \multirow{2}{*}{\multicolumn{2}{|c|}{ Characteristics }} & \multicolumn{4}{|c|}{ Odds ratios (OR) } \\
\hline & & Univariable & $95 \% \mathrm{Cl}$ & Multivariable & $95 \% \mathrm{Cl}$ \\
\hline \multirow[t]{3}{*}{ Age group } & 18-44 & Ref. & - & Ref. & - \\
\hline & $45-64$ & 1.40 & $1.31,1.49$ & 1.38 & $1.29,1.47$ \\
\hline & $\geq 65$ & 1.39 & $1.30,1.50$ & 1.28 & $1.19,1.39$ \\
\hline \multirow[t]{3}{*}{ Charlson index } & 0 & Ref. & - & Ref. & - \\
\hline & 1 & 1.27 & $1.06,1.20$ & 1.08 & $1.01,1.16$ \\
\hline & $\geq 2$ & 1.31 & $1.20,1.42$ & 1.24 & $1.14,1.36$ \\
\hline \multicolumn{2}{|l|}{ Female } & 0.95 & $0.87,1.04$ & 0.96 & $0.88,1.04$ \\
\hline \multirow[t]{3}{*}{ Surgery type } & Open & Ref. & - & Ref. & - \\
\hline & Lap & 0.92 & $0.88,0.97$ & 1.07 & $1.01,1.13$ \\
\hline & Converted & 0.44 & $0.34,0.57$ & 0.51 & $0.39,0.66$ \\
\hline \multicolumn{2}{|l|}{ Bilateral } & 0.48 & 0.44 .0 .53 & 0.48 & $0.44,0.53$ \\
\hline Recurrent & & 1.22 & $1.12,1.33$ & 1.16 & $1.06,1.26$ \\
\hline
\end{tabular}




\section{P575}

\section{Paediatric Laparoscopic Inguinal Hernia Repair}

Bindey Kumar, MCh, Prem Kumar, MD; All India Institute Of Medical Sciences, Patna

Objective: To present our experience of reducing risk of recurrences in paediatric laparoscopic inguinal hernia repair.

Material and Procedure: The retrospective review includes all case of paediatric laparoscopic inguinal hernia repair(PLIHR) between 2015-2018 at our centre The surgical technique in which we use to cut the peritoneal cuff proximal to deep inguinal ring(DIR) and then suturing the cut margin led to recurrences of hernia.We modified the technique and after dissection of the sac in the inguinal canal distal to DIR, sac was cut in the inguinal canal and suturing was done.

The cure rate between two procedures was compared by using student t-test for two proportions. Results: 97 paediatric patients underwent laparoscopic inguinal hernia repair.First technique of cutting the peritoneal cuff proximal to DIR and suturing in first 50 patients had $92 \%$ success rate.The modified technique in which sac was cut distal to DIR and suturing had $100 \%$ success rate. Associated anomalies identified in this study were hypospadias,undescended testis,umbilical hernia and hydrocele.

There was no associated injury to vas,adjacent structures or testis.

Conclusion: PLIHR done with cutting the hernial sac distal to DIR and suturing is easy method and had no recurrence.

\section{P576}

\section{The Laparoscopic Approach for Rectus- Adductor} Tendonopathy- Sport's Hernia

\section{David S Edelman; Doctor's Hospital, Coral Gables}

Introduction: Athletes with osteitis pubis and core muscle injuries have many options available for treatment. MRI can show pubic bone edema, rectus muscle insertion atrophy and/or adductor tendonopathy. I have surgically repaired 165 athletes using laparoscopically placed biologic mesh for a "sport's hernia" with excellent results. This presentation will review the injury and repair involving the rectus-adductor tendon injury onto the pubic bone.

Methods: A 28 year old professional athlete presented 2 weeks after an open core muscle injury repair. MRI showed disruption of the adductor tendon from the pubis and persistent opposing side rectus abdominis muscle insertion atrophy. A 3 port, pre-peritoneal, posterior, laparoscopic approach with biologic mesh was performed.

Results: A $3 \mathrm{~cm}$ left groin incision was made to evacuate a hematoma and reattach the left adductor tendon to the pubis with a 0 ethibond suture along with a $4 \times 7 \mathrm{~cm}$ biologic mesh reinforcement. This was followed by a laparoscopic pre-peritoneal bilateral rectus and adductor reinforcement with two pieces of $7 \times 10 \mathrm{~cm}$ biologic mesh, absorbable tacks and fibrin sealant. Rehabilition was started one week later with full activity at 1 month. A post-op MRI at 1 month showed complete healing with hypertrophy of the repaired muscles. A similar case in a 45 year old Olympic weight lifter who set a world record in his age class 4 months post op. Other similar cases will be discussed.

Conclusion: The laparoscopic, posterior, pre-peritoneal repair of the Rectus-Adductor tendon with biologic mesh is safe and effective in treating athletes with core muscle injuries.

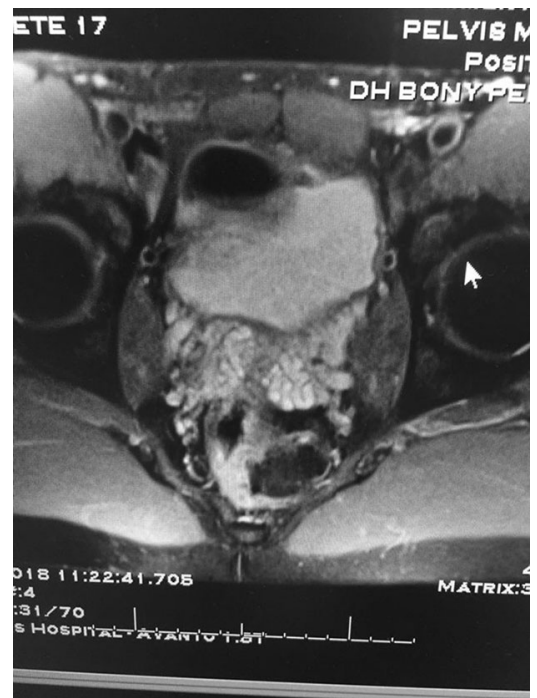

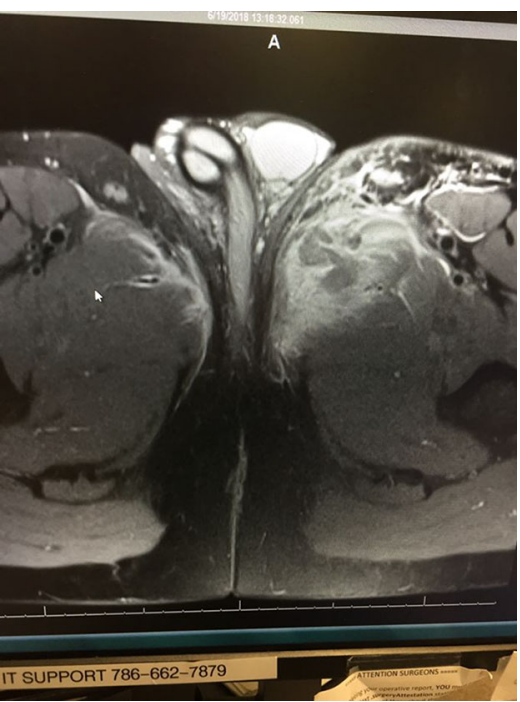
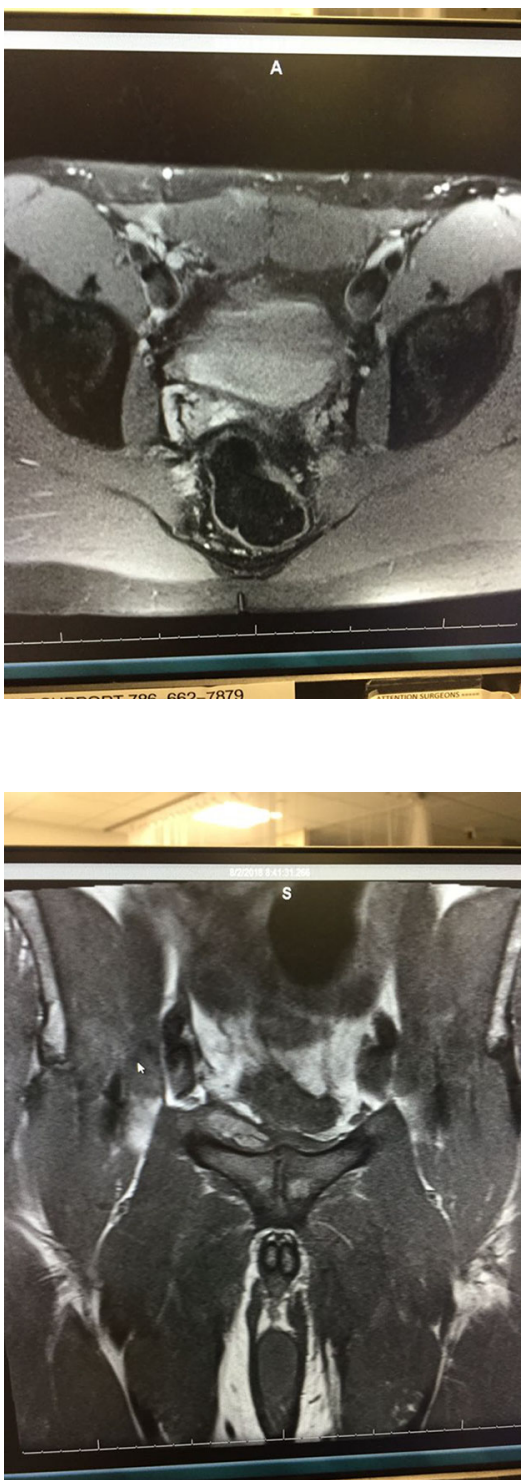
P577

\section{Repeat Transabdominal Preperitoneal (TAPP) Mesh Repair after previous anterior and posterior repair for Re-recurrent Inguinal Hernia}

Krista Angeli P Delos Santos-Belen, MD, Miguel C Mendoza, MD, Judy Carissa M Atazan, MD; Asian Hospital and Medical Center

Introduction: Current guidelines of the international hernia societies recommend a posterior laparoscopic approach with either TAPP or TEP for a recurrent inguinal hernia following a previous anterior open repair, having the advantage of operating through virgin tissue and giving improved mechanical strength using mesh. Likewise, the anterior approach is recommended following a posterior repair. However, in cases of re-recurrence, there is still paucity of data on the role of a repeat posterior laparoscopic approach. This case report aims to show the critical steps in performing a repeat TAPP after TAPP for a re-recurrent inguinal hernia.

Methods: A 58 year old male underwent open anterior tissue repair for right inguinal hernia at age 19 , followed by TAPP for recurrence at age 55 . Re-recurrence was noted 3 years after TAPP, presenting with a reducible inguinal mass, with no evidence of obstruction, incarceration, or strangulation.

Results: Standard position and port placement for TAPP was done. Creation of the peritoneal flaps was carefully performed due to postoperative tissue fibrosis and the presence of the previous mesh. Further dissection revealed a re-recurrent direct inguinal hernia on the right $(3.5 \mathrm{~cm}$ and $2.0 \mathrm{~cm})$. Hernia sac was isolated with preservation of the spermatic cord structures. We placed one $10 \mathrm{x}$ $15 \mathrm{~cm}$ with additional half $10 \times 15 \mathrm{~cm}$ mesh, ensuring sufficient overlap, followed by fixation with tackers. Closure of the peritoneal flaps was completed using absorbable barbed continuous sutures. Short term outcomes evaluated were postoperative bleeding, surgical site infection, and inadequate pain control, none of which were reported.

Conclusions: The safety and feasibility of a repeat TAPP for re-recurrence primarily depends on the expertise of the surgeon. Future studies should aim to determine factors that affect short and long term outcomes, including the rates of perioperative complications and re-recurrence.
P579

Post Operative Pain Following Mesh Fixation of Ventral Hernia Repair: Comparison of Transfascial with Intracorporal Suture Fixation

Manoj K Choudhury, MS, FMAS, Kaushik Baruah, MS; Nemcare Superspeciality Hospital

Objective: Fixation of mesh is the most important step of ventral hernia repair. Transfascial mesh fixation has been the most accepted of all the methods, but the significant pain during early post operative period following fixation is still a cause of concern. In search of reducing the pos operative pain, the intracorporal suture fixation has been carried out in our centre. So the study is undertaken to determine the post operative pain following intracorporal suture fixation of mesh in ventral hernia repair and to compare the severity and duration of pain with transfascial fixation method.

Method \& Procedures: 81 cases are taken up for study during the period from august,2015 to july, 2018.

Cases were admitted after clinical diagnosis and complete evaluation. Ultrasonography was the routine diagnostic investigation specially to measure the defect. CT scan was done in selected cases of complex hernias. Transfascial and intracorporal suture for mesh fixation were done in 33 and 47 cases respectively for the comparative study.

One $10 \mathrm{~mm}$ and two $5 \mathrm{~mm}$ trocars were introduced after $\mathrm{CO} 2$ insufflation. Abdominal cavity was examined and adhesiolysis was done to carry out the release of hernia content. Defect/s were cleared and prepared for mesh fixation with or without closure of the defect. Size of the composite mesh was determined by the size of the defect/s. 2-0 polypropylene was used to centralize the mesh with the centre of the hernia defect. Mesh was aseptically introduced through the $10 \mathrm{~mm}$ trocar and fixed with the centralizing suture. Fixation of the mesh was done either by transfascial sutures or by intracorporal sutures to fix the mesh with the abdominal wall. Ports were closed after completion of mesh fixation.

Post operative pain was recorded as per visual analog score 0 - 10 (0- no pain, 10- most severe pain) and compared the severity on day $0,2,7,14,30$ and 60 .

Results: Average age of the patients was between 35 and 65 years and was mostly female $(83.75 \%)$. Comparative study of severity and duration of pain were found to be significantly less with intracorporal method in comparison to transfascial method.. Day 0 (t-value 5.201, $\mathrm{p}<0.01$ ) Day 2 (t-value 7.693, p $<0.01$ ), Day 7 (t-value-9.738, p $<0.01$ ), Day 14 (t-value 9.571, p $<0.01$ ) Conclusion: Intracorporal mesh fixation is a technically demanding technique in comparison to transfascial method. But the study has shown significant reduction of post operative pain with intracorporal technique in comparison to transfascial.

\section{P580}

Endoscopic Assisted Linea Alba Reconstruction (ELAR) : Our Experience

Ajay Bhandarwar, MS, FMAS, FIAGES, FAIS, FICS, FBMS ${ }^{1}$, Girish D Bakhshi, MS, MRCS ${ }^{2}$, Shivang D Shukla, $\mathrm{MBBS}^{1}$, Amol N $\overline{\mathrm{Wagh}}$, MS, FMAS, FIAGES, FAIS, FICS, FBMS ${ }^{1}$, Shekhar A Jadhav, MS, FMAS $^{1}$, Amarjeet E Tandur, MS ${ }^{1}$, Jalbaji More, $\mathrm{MS}^{1}$, Nidhisha Sadhwani, MBBS ${ }^{1}$, Priyanka Saha, MBBS ${ }^{1}$, Khushboo Kadakia, MBBS $^{1} ;{ }^{1}$ Grant Government Medical College \& Sir J.J. Group of Hospitals, Mumbai, India, ${ }^{2}$ Grant Govt. Medical College \& Sir J. J. Group of Hospitals, Byculla,,Maharashtra, India

Introduction: Diastasis of recti is separation of two sides of rectus abdominis muscle. It has more prevalence in newborns and postpartum women.

It usually occurs due to stretching of criss cross fibres of line alba due to stretching of abdomen under combined with effects of relaxin in postpartum women.

Materials and methods: In our tertiary care set up, we operated 5 cases of diastasis of recti through endoscopic plication with or without onlay mesh.

Diagnosis was made by BEER classification by placing ultrasound probe on xiphoid sternum, 2 cms above it and $3 \mathrm{cms}$ below.

One $10 \mathrm{~mm}$ port was inserted above pubis symphysis and two $5 \mathrm{~mm}$ working ports were placed $5 \mathrm{cms}$ on either side. Meticulous dissection was performed up to $3 \mathrm{~cm}$ laterally from mid line so as to avoid skin necrosis. Plication of recti was done from pubic symphysis up to xiphoid sternum using non absorbable sutures in a continuous fashion. Umbilicus was hitched to fascia for cosmesis An onlay mesh was placed in 5 patients

A subcutaneous drain was inserted so as to avoid seroma formation. Skin was closed using non absorbable sutures.

Technique details and outcomes will be discussed.

Results:

Average post operative time was - $80 \mathrm{~min}$

Average time for resumption of diet : evening of surgery.

Average post operative stay : 3 days

Average time for removal of drain $<20 \mathrm{cc}: 1.5$ days

Patients were followed up after 3 months.

Pain was assessed on visual analogue scale. (0-10)

Recurrence was objectified on using using beer classification. 
P581

\section{Long Acting Local Anesthetic Nerve Blocks in Laparoscopic Ventral Hernia Repair}

Michael Lew, MD, Rachel Lewis, MD, Miles Landry, MD, Brandie Forman, Bruce Ramshaw, MD; University of Tennessee Knoxville

Background: Laparoscopic ventral hernia repair is a commonly performed procedure. Metrics important to both patients and surgeons include pain control and length of stay. Length of stay is associated to increased costs and inversely associated with value. Decreasing opioid usage is part of a national health care initiative.

Methods: In an attempt to measure and improve outcomes, we implemented a clinical quality improvement (CQI) program for ventral hernia patients. From 2012 to 2018, consecutive laparoscopic ventral hernia repair patients from a single hernia program were evaluated. One initiative during this time period was the use of a long acting local anesthetic nerve block.

Results: A total of 112 patients underwent laparoscopic ventral hernia repair. The no block group comprised of 47 patients: $57 \%$ were female, mean age was 63 , mean BMI was 35.6 , and $60 \%$ had a recurrent hernia. The long acting local anesthetic block group comprised of 65 patients: $54 \%$ were female, mean age was 58 , mean BMI was 35 , and $51 \%$ had a recurrent hernia. The no block group had a larger hernia size (98 to $\left.73 \mathrm{~cm}^{2}\right)$ and mesh size $\left(526\right.$ to $\left.477 \mathrm{~cm}^{2}\right)$. The no block group had a longer post anesthesia care unit time (172 to $110 \mathrm{~min}$ ), and length of stay (4.4 to 2.5 days). Opioid usage was higher in the no block group in the post anesthesia care unit (12 to $7 \mathrm{mg}$ morphine equivalents) and overall (116 to $53 \mathrm{mg}$ morphine equivalents).

Conclusion: Long acting local anesthetic abdominal nerve blocks during laparoscopic ventral hernia repair were associated with decreased post anesthesia care unit time and length of stay. They were also associated with decreased opioid usage overall and in the post anesthesia care unit. Long acting local anesthetic abdominal nerve blocks were used as part of a CQI program to improve outcomes. As with any CQI analysis, other factors may have contributed to these outcomes and these results may be different in another local environment.

\section{P582}

\section{Fatty Inguinal Hernia (Sacless Inguinal Hernia)}

Abdullah Aldohayan, FRCS, Glas, Mohanad Albalawi, Rasha Bassas, Maram Alaqel, Hassan Arishi; King Saud University Hospital

Background: Fatty Inguinal Hernia $[\mathrm{FIH}]$ is inguinal defect filled with Extraperitoneal fat and no sac. Laparoscopic repair of an inguinal hernia requires the presence of a SAC, either direct or indirect inguinal hernia. Many surgeons aborted the laparoscopic surgery in absence of the sac. The preoperative examination is important to diagnose the FIH.

Aim: In presence of suspicion of FIH, laparoscopic examination of deep inguinal \& Hesselbach's triangle is necessary to diagnose and manage.

Materials and methods of FIH: The study started from January 2017 to June 2018. All the patients were either examined clinically or by ultrasound of the groan.

Results: There were 50 patients who were diagnosed with an inguinal hernia, 8 fatty indirect inguinal hernias, and 3 fatty direct inguinal hernias are encountered.

Conclusion: For a long time, patients who had FIH were managed improperly, hereby we illuminate the case of FIH which surgeon can miss if we're not aware of FIH.

\section{P583}

Comparison of Peri-operative Outcomes Between Low Pressure and Standard Pressure Insufflation Systems During Laparoscopic Ventral Hernia Repair

Sung G Lee, MD, Rachel Lewis, MD, Miles Landry, MBBS, Thomas Kennedy, MD, Sujata Datta, MD, Michael Lew, MD, Brandie Forman, Hernia Clinician, Bruce Ramshaw, MD, FACS; University of Tennessee at Knoxville

Introduction: Most laparoscopic procedures utilize high flow insufflators at an intra-abdominal pressure setting of $15 \mathrm{mmHg}$. This standard pressure insufflation may alter physiology intraoperatively by reducing femoral venous flow, respiratory compliance, cardiac output, and portal venous flow. Also, low pressure pneumoperitoneum may help to decrease shoulder and abdominal wall visceral pain that can occur in patients who undergo laparoscopy at standard insufflation pressures.

Methods and Procedures: This project is a part of Clinical Quality Improvement (CQI) effort for patients with ventral/incisional hernias implemented by a multi-disciplinary hernia team. This patient group underwent a LVHR between April 2012 and July 2018. The initial 79 patients had standard laparoscopy at a pressure setting of $15 \mathrm{mmHg}$. A low pressure pneumoperitoneum system was introduced as a potential process improvement after a literature search was completed. This attempted process improvement was initiated, in part, after a patient experienced a $\mathrm{CO} 2$ embolus with standard insufflation. Since introduction of the low pressure system, 34 patients have undergone LVHR. A variety of patient factors, treatment factors and outcome measures are collected and analyzed by the hernia team in an attempt to improve outcomes.

Results:

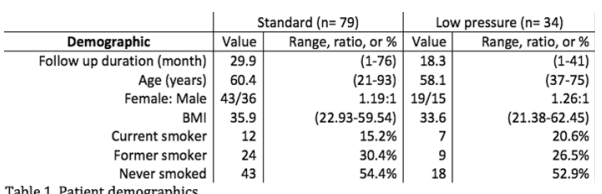

No major difference in demographics between two groups. Other than follow up for low pressure group was shorter compare to standard pressure group.

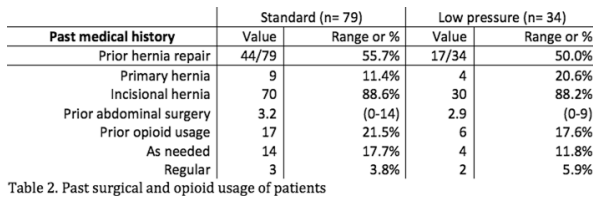

Both groups had similar prior surgery experience and opioid usage.

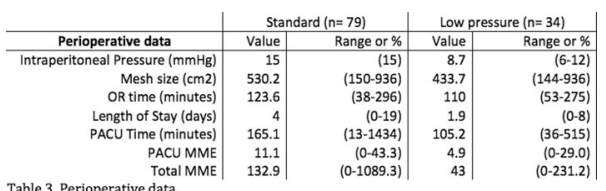

The average intraperitoneal pressure setting utilized during LVHR was $8.7 \mathrm{mmHg}$ for the low pressure group and $15 \mathrm{mmHg}$ for the standard pressure group. There was a decrease in length of stay by 2.1 days, a decrease of PACU Morphine Milligram Equivalents (MME) of 6.2 MME and a decrease of total hospital stay MME of 89.9 MME for the low pressure group compared with the standard pressure group.

Conclusions: This attempt at process improvement demonstrated beneficial effects for patients who underwent LVHR with a low pressure pneumoperitoneum system. As with any CQI analysis, other factors may have contributed to these outcomes and these results may be different in another local environment. 
P584

\section{Management of Eventration of the Diaphragm with Laparoscopic Plication \& Dual Mesh Repair - Long Term Results in 3 Cases}

\author{
K. Sendhil Kumar, MD, Piyush Patwa, MD; Gateway Clinics \& \\ Hospitals
}

Eventration of the diaphragm is an abnormal elevation of the dome of diaphragm. It is a condition in which all or part of the diaphragm is largely composed of fibrous tissue with only a few or no interspersed muscle fibers. It can be complete or partial.

Patients presented with recurrent Dyspnea on exertion and respiratory infections for last few years gradually increasing in its trend. Treatment is individualized. Most healthy asymptomatic adults with isolated diaphragmatic paralysis or suffer only from mild breathlessness on exertion can be managed conservatively. Patients are often observed for a year or so after the diagnosis is made, and are offered surgery only if the symptoms worsen. Diaphragmatic eventration could cause compression of lung by abdominal organs and reinforcement of diaphragm through plication might increase the diaphragm strength and diminish the clinical symptoms.The most commonly used treatment for this condition is diaphragmatic plication which can be done open, thoracoscopic or Laparoscopic surgery. Multiple sutures are placed on the paralyzed diaphragm to pleat it. In our series, A $15 \mathrm{x} 15 \mathrm{cms}$ size dual mesh was fixed over plicated Diaphragm in all the three cases. This makes the lax diaphragm taut and moves it to a lower position which is appreciated at the end of procedure. Lung expands and functions better.

\section{P585}

\section{Dynamic MRI to Assess Complex Abdominal Wall Hernias}

Nicole Cherng, MD, Kashayar Rafatzand, MD, Gabriela SantosNunez, MD, James Carroll, MD, Philip Cohen, MD, Richard Perugini, MD; University of Massachusetts Medical School

Introduction: Multiple techniques and variations have been described for complex abdominal wall hernia repair, and include rationale for types of synthetic mesh, location of mesh, and the performance of and type of lateral release. Decisions to utilize these techniques stem from surgeon preference and severity of the defect. Commonly accepted grading systems rely on CT in the relaxed state, and thus are limited to defect location and dimension. Such systems are inadequate in differentiating severity of physiologic impact. We developed a protocol utilizing dynamic MRI (dMRI) to image the abdominal wall in relaxed state and in Valsalva, which enables quantification of abdominal wall muscle dysfunction, improving the assessment of subjects with complex abdominal wall hernia and impacting surgeon decision-making.

Methods: A registry was created under IRB approved protocol of patients presenting for repair of abdominal wall hernias with a preoperative dMRI. From relaxed state images, the hernia was categorized using the European Hernia Society (EHS) scoring system. Valsalva images were compared with the relaxed images to assess degree of rectus dysfunction $(0=$ no hernia, $1=$ diastasis only, 2 $-5=$ hernia with increasing degree of rectus dysfunction). We compared our system of grading abdominal wall dysfunction to the EHS system.

Results: We evaluated 21 subjects, with a male to female ratio of 11:10, a median age of 60 years (range 34-74), and a median BMI of 30 (range 25.643.1). Five hernia were classified as functional class 2 (minimal rectus dysfunction). For these subjects, the recti contracted and hernia defect was stable to decreasing with Valsalva. Three underwent open Rives Stoppa (RS) repair with retro-rectus mesh, one underwent laparoscopic repair with sublay mesh, and one underwent external oblique release (EOR) with onlay mesh. Seven hernia were classified as most severe physiologic dysfunction (class 5). The recti were atrophic and elongated with Valsalva. Four underwent RS repair with retro-rectus mesh, and three underwent EOR with onlay mesh. Eleven hernia were EHS W2 (width 4-10 cm). In these subjects, dMRI differentiated between normal rectus function and severe rectus dysfunction.

Conclusions: We present our initial experience with dMRI in the evaluation of complicated abdominal wall hernia. dMRI offers an improved assessment of physiologic dysfunction of the abdominal wall. We identified variation in surgeon technique in subjects with similar severity of hernia in this small series, and plan to utilize dMRI for both preoperative stratification and for postoperative assessment of physiologic outcomes.

\section{P586}

\section{Does Laparoscopic Inguinal Hernia Surgery Affects the Vascularity of Testes}

Mahendra Lodha, Dr; AIIMS Jodhpur, India

Purpose: The effect of laparoscopic TEP repair on testicular perfusion is unclear. The procedure entails dissection of testicular blood vessels off the hernial sac and incorporation of a prosthetic mesh. This carries at minimum, a theoretical risk of compromise in testicular blood supply, which in turn may affect fertility. Our study aims to establish if any alteration in testicular perfusion occurs in very early ( $24 \mathrm{~h}$ ), early (1 week) or late postoperative period (3 months) after laparoscopic TEP repair in the Indian population.

Methods: In our prospective trial, 20 patients underwent unilateral and 8 underwent bilateral laparoscopic TEP hernia repairs using standard technique by experienced surgeons. Flow parameters of testicular, capsular and intratesticular artery were noted using color Doppler ultrasound preoperatively and postoperatively and the postoperative resistive indexes of operated side $(n=36)$ were compared with preoperative values. Additionally, for unilateral repairs, flow parameters on operated side were compared with the non-operated side.

Results: No statistically significant difference was noticed in the resistive index of the arteries upon comparing these postoperative with preoperative values. For unilateral repairs, the flow parameters of the operated side were comparable with that of non-operated side (i.e. $\mathrm{p}[0.05$ ).

Conclusion: Laparoscopic TEP performed by experienced surgeons does not alter testicular flow dynamics in early or late postoperative period.

\section{P587}

\section{Clinical Intra Operative Tool to Determine the Adequacy of Laparoscopic Inguinal Hernia Repair in Pediatrics}

Muhammad Armughan ${ }^{1}$, Malik Muhammad Makki ${ }^{2}$, Muhammad Tariq ${ }^{1}$, Javed Iqbal ${ }^{1}$, Samia Yunas ${ }^{3} ;{ }^{1}$ Bahawal Victoria Hospital, Bahawalpur, ${ }^{2}$ Sheikh Khalifa Medical City Ajman, UAE, ${ }^{3}$ Emirates Specialty Hospital, Dubai

Introduction: Laparoscopic Inguinal hernia repair in pediatric is a safe and feasible procedure. There are two main approaches which has been successfully practiced are extra peritoneal and intraperitoneal. Recurrence in case of laparoscopic repair consider more than open repair. We develop a intraoperative clinical tool or method which could reassure adequacy of repair.

Methods and Procedure: It is a common practice to evacuate air or fluid from the scrotum or labia before tying a suture around internal opening and ligating the hernia. We used scrotal air to check air tight closure of internal ring. We first encircle and tie internal ring in our laparoscopic technique. Later assistant hold the scrotum and push than push scrotal inside abdomen. Operating surgeon should check air air leak at level of internal opening. We assumed if internal ring is air tight close than chances of recurrence inguinal hernia me be minimum.

We used all male pediatric patients who can accumulate enough air in their scrotum during pneumoperitoneum. We followed patient first 06 months than 1 year for recurrence.

Result: There were fifteen (15) male child patient who were analyzed intraoperative and followed at 06 month and 1 -year. There was one case with intraoperative air leak at internal opening. This patient was tied again securely without air leak. Same patient got recurrence at at 10 months. Three patients missed their one year follow-up. We did not notice recurrence in remaining patients.

Conclusion: This was a small study based on the fact that air tight internal ring might have less chance of recurrence. Which were observed in majority of our patients. Only one patient developed recurrence even with air tight closure of internal ring. We checked retrospectively and found thin peritoneal layers at internal ring. We concluded religating the internal opening might have weakened the internal ring which cause recurrence. 
P588

\section{Outcomes of Laparoscopic Totally Extraperitoneal Inguinal Hernia Repair (LAP TEP) with Fixation Device (PROTACK ${ }^{\text {TM }}$ ) and Self Fixating Mesh (PROGRIP ${ }^{\mathrm{TM}}$ )}

Peeravudh Chantana, MD, Ratchanon Bongsuwan, Setthasiri Pantanakul, MD, Panot Yimcharoen, MD; Bhumibol Adulyadej hospital

Background: Inguinal hernia is a commonly encountered surgical condition. Laparoscopic techniques have become more popular in the recent years. Laparoscopic approach provides considerable advantage in shortening post-operative recovery time. Fixation of mesh is a challenge point in laparoscopic hernia repair. This study will compare outcome between mesh fixation by fixation device and self-fixating mesh, done by laparoscopic TEP.

Objective: To compare post-operative outcome using parameters of pain, length of hospital stay, operative time, time of recovery to effective life activities, short term recurrence and complications, in patients underwent LAP TEP with the use of mesh with fixation device and self-fixating mesh

Materials and Methods: Data was collected from 2015 to 2018 and reviewed. Patients undergoing LAP TEP with use of fixation device (ProTack ${ }^{\mathrm{TM}}$ ) and self-fixating mesh (ProGrip ${ }^{\mathrm{TM}}$ ). The procedures were performed by expert in laparoscopic surgery. One-hundred-and-thirty-one (131) patients with complete medical records met the inclusion criteria.

Results: In total, 131 Lap TEP surgery was performed, fixation devices were used in 112 cases $(85 \%)$ while in 19 cases (15\%) self-fixating mesh was independently placed. Male patients were the most common visitor with the condition (97\%). The mean age was 66.45 (22-91) years old. Most of the participants had bilateral disease $(56 \%)$ and indirect inguinal hernia was the most commonly found Operative time, estimate blood loss, length of hospital stay, time of recovery to effective life activities within a week were not different between two groups. Three-month post-operative pain with mild severity were found in 3 cases with placement of fixation device. Mean score of the first day post-operative pain was 1.6 in self-fixation mesh and 2.3 in fixation device. Six-month recurrence was found in 3 cases in fixation device group. Complications were found in 10 cases with placement of fixation device, comprised of 3 cases of seroma, 6 cases of hematoma and 1 case of wound infection.

Conclusion: This study revealed the outcome of fixation technique. Self-fixating mesh was found superior in pain outcome both first day and at 3 month. Complications and recurrences were found only in fixation device group. Long term and larger study may be needed to confirm the effectiveness of self-fixating mesh.
P590

\section{Nissen Fundoplication Supplemented by a New Wrap Fixation Technique}

Mykola Paranyak, Vladimir Grubnik, Professor, MD, Viktor Grubnyk; Odessa National Medical University

Background: Nearly $10 \%$ of patients who undergo laparoscopic anti-reflux surgery at long-term follow-up have low degree of satisfaction and need surgical reintervention mostly because of hiata hernia $(\mathrm{HH})$ recurrence, wrap migration or disruption. Some surgeons after determining such complications perform a fixation of fundoplication wrap to the esophagus, but such a technique increases the risk of esophageal injury.

Purpose: The aim of our prospective study was to evaluate and compare several technics of wrap fixation and determine whether modified Nissen fundoplication (MNF) reduces failure rate in the long term follow up.

Materials and Methods: From November 2012 to October 2014 one hundred and thirty-eight patients who underwent anti-reflux surgery were selected and divided into two groups. In the first group which included 87 patients we performed the following manipulations: NF was supplemented with suturing a wrap to the diaphragmatic crura (52 patients) on each side using two nonabsorbable stitches. This technique allows us to create a more symmetrical wrap. In the cases of weak conditions of the crura ( 35 patients) fundoplication wrap was sutured to the body of the stomach using two non-absorbable stitches on each side. The control group (51) underwent classic Nissen fundoplication (NF) without wrap fixation. All patients were assessed before and afte surgery using validated symptoms and quality of life (GERD-HRQL) questionnaires, 24-h impedance-pH monitoring and barium-swallow, also we used visual analog scale to quantify pain intensity in the first month after surgery.

Results: Baseline characteristics were similar between groups. There were no conversion to open procedure or mortality. At 41,6 months (range 18-57) of a follow-up, the overall rate of complications after MNF was 1,14\% (1 HH reoccurrence) and NF 7,84\% (3 HH reoccurrence, 1slipped wrap). Patients in MNF group show more significant improvement in GERD-HRQL score $(\mathrm{p}<0.01)$ and DeMeester index compared to the NF group. There were no significant differences in pain intensity after surgery between two groups (p ? 0.05)

Conclusions: According to our study MNF is a safe technique and can make a positive impact on reducing the failure rate of laparoscopic anti-reflux surgery. Also it minimized the risk of a slipped wrap and intrathoracic migration of the wrap.
P589

Postoperative Management of Patients with Refractory Ascites Following Hernia Repair: Does a Temporary Peritoneal Dialysis Catheter Placement Improve Outcomes?

Amro Wafi, $\mathrm{MD}^{1}$, Archana Ramaswamy, MD, MBA ${ }^{2} ;{ }^{1}$ University of Minnesota, Department of Surgery, ${ }^{2}$ Minneapolis VA Health Care System

Introduction: Surgery in the setting of decompensated liver disease carries significant morbidity and mortality. Abdominal wall hernia is a common problem in this population, especially in patients with refractory ascites. Frequently, hernia repair is performed on an emergent basis, which carries an even higher risk of morbidity and mortality. It's generally accepted that preoperative management of ascites reduces risk of recurrence as well as morbidity and mortality. However, there is no standardized consensus on how to manage these patients post operatively. This study examines the utility of placing a temporary peritoneal dialysis catheter for postoperative management of ascites in improving outcomes.

Methods: A retrospective chart review of patients who underwent abdominal wall hernia repair at the Minneapolis VA between 1998 and 2015. Primary outcome measures included: hernia recurrence, wound complications, and 30-day mortality. Secondary outcome measures included: hospital length of stay and 3 year mortality. Standard statistical analysis was utilized using Chi square tests and $t$ test. $P$ values $<0.05$ were considered statistically significant.

Results: A total of 44 patients were identified with one female patient. 31 patients had a peritoneal dialysis catheter placed intraoperatively, and 13 patients underwent hernia repair without drain placement. Patients in the peritoneal dialysis catheter group were noted to have a higher MELD score $(\mathrm{p}=0.04)$ and a higher Child-Pugh score $(\mathrm{p}=0.007)$. Hernia repair was performed emergently in $64 \%$ of patients in the peritoneal dialysis catheter as opposed to $61 \%$ in the second group $(\mathrm{p}=0.85)$. There was no statistical difference between the two groups in terms of wound complications $(\mathrm{p}=0.06)$, hernia recurrence $(\mathrm{p}=0.08)$, and death at 30 days $(\mathrm{p}=0.83)$. The hospital length of stay was longer for patients in the peritoneal dialysis catheter group $(p=0.04)$.

Conclusion: Patients with higher MELD or Child-Pugh score were more likely to have a peritoneal dialysis catheter placed at the time of the hernia repair. However, we did not find a statistical significant between the primary outcomes in the two groups. More work is needed to come up with a standardized way to treat patients with ascites following abdominal wall hernia repair.

\section{P591}

\section{An Experience of Over 1500 Cases of Laparoscopic Groin Hernia Surgery at a Tertiary Care Centre}

Virinder Kumar Bansal, Subodh Kumar, Asuri Krishna, Om Prakash, W. F. Khan, M. C. Misra; AIIMS, New Delhi

Background: Hernia surgery is one of the most common procedures worldwide. Laparoscopic mesh repair is the standard of care in the management of inguinal hernia. TEP and TAPP are standard techniques for laparoscopic repair of groin hernia. We present our experience of more than 1500 cases of laparoscopic groin hernia repair.

Methods: This study is a retrospective analysis of prospectively maintained database of all patients who underwent laparoscopic groin hernia repair in a single surgical unit from January 2004 to July 2018. Patient's demographic profile, hernia characteristics were noted. Clinical outcomes included were operation time, intra-operative and postoperative complications, length of postoperative hospital stay, hernia recurrence, chronic pain (defined as pain that persisted for more than 3 months), recurrence, seroma and wound infections. Patients were followed up in the outpatient clinic by the attending surgeons during the postoperative course

Results: Over ten years duration, TEP repair was performed in 915 patients and TAPP on 635 Mean age of patients was 50.7 years (range 17-86 years). Both the techniques were comparable in terms of operative time, intraoperative complications and post operative outcomes. However there was a significantly higher pain scores ( $\mathrm{p}$ value $<0.05$ ) at $24 \mathrm{~h}$ in the TAPP group. Chronic pain( $>3$ months) score was not significantly different between the groups. The incidence of seroma was higher in TEP and scrotal edema was more common after TAPP repair. Recurrence was noted only in 7 cases. 5 patients presented with contralateral hernia and 2 patients had Sub acute interstinal obstruction in the post-operative period and one patient had mesh infection and mesh had to be removed. In 7 patients with recurrence, 3 were operated and TAPP was done. Cause of recurrence was mesh protrusion in 2 patients(light weight mesh was used), migration of mesh and medial recurrence (non fixation). 4 patients didn't come for follow up.

Conclusion: TEP and TAPP repair are comparable and they should be considered as complementary procedures. 
P592

\section{A Novel, No Cost, Simple Paper Model Technique for Placement Of IPOM}

Sachin B Jamma, MS, MNAMS, FMAS, DipALS, Balasaheb G Shitole, MS; Ashwini Rural Medical College,Solapur

Objective: Correct placement of the mesh around the defect is always a cause of anxiety in Surgeon's mind, especially for beginners. Various methods are described to place IPOM accurately however they come with a cost. Purpose of the study was to find out a simple, no cost method to place IPOM accurately around the defect in ventral hernias especially for countries with limited resources.

Methods and Procedures: Basic principles of IPOM surgery observed in study were the overlap of mesh has to be $5 \mathrm{~cm}$ all around the defect, mesh should never touch the bare skin and perfect asepsis. The study was performed on sixteen randomly selected patients posted for IPOM or IPOM plus after thorough clinical examination and investigations. Size of defect was judged earlier however intra operatively size of the defect was measured by using spinal needle technique in all sixteen cases. The defect was marked externally on skin with marker pen. The size of IPOM was selected so as to get an overlap of $5 \mathrm{~cm}$ all around the defect. Once the size of mesh was decided, after changing the gloves, mesh was delivered from sterile foil to a separate sterile instrument trolley. While changing the gloves the sterile paper glove cover/glove bag was preserved. The mesh edges were drawn on this sterile paper glove cover with marker pen. Just outside the marked edges of mesh, points of transfixation sutures ( 2 or 4-as per the plan of surgery) were plotted. Then this paper glove cover with markings was placed on anterior abdominal wall after deflating pneumo so that the defect will be in the centre of the sketch with a $5 \mathrm{~cm}$ overlap. The points of transfixation were now marked on abdominal wall puncturing the points marked on paper with a marker pen so that skin puncture later will be at these points for transfixation sutures. Rest of the surgical procedure was carried out as planned.

Result: With this technique, we observed precise and perfect placement of the mesh in all sixteen cases with an overlap of $5 \mathrm{~cm}$ all around the defect. Inraoperative Revision was not needed in any case.

Conclusion: This simple, cheap technique using a paper glove cover will definitely ease Surgeon's life on table avoiding contact of mesh to skin \& achieving a good $5 \mathrm{~cm}$ overlap of mesh to the edge of defect.

\section{P593}

Emergency Surgery for Complicated Large Midline Incisional Hernias in Elderly: Is Nihilistic Attitude Justified?

Nitin Vashistha, MS, FACS ${ }^{1}$, Dinesh Singhal, MS, FACS, DNB, Surgical, Gastroenterology ${ }^{1}$, Sunil Choudhary, MS, FRCS $^{2}$, Raj Tobin, $\mathrm{MD}^{3}$; ${ }^{1}$ Department of Surgical Gastroenterology, Max Super Speciality Hospital, ${ }^{2}$ Department of Plastic \& Reconstructive Surgery, Max Super Speciality Hospital, ${ }^{3}$ Department of Anesthesiology, Max Super Speciality Hospital

Background: There is reasonable consensus that laparoscopic repair (LR) is the preferred modality for management of ventral hernias. However LR is technically difficult in patients with large incarcerated hernia, small defects with large hernia sacs, loss of domain and obesity. The scenario becomes particularly demanding when elderly patients with multiple co-morbidities present with such complex hernias. In view of high risk of adverse events, surgical intervention is often deferred by patients \&/or surgeons. Many of these patients present as emergency with hernia associated complications such as obstruction \&/or strangulation and pose even more formidable challenge for management.

We present our experience in management of complicated large incisional hernias.

Patients \& Methods: From January 2013 to July 2018, consecutive patients with complicated large midline incisional hernia with defect $\geq 10 \mathrm{~cm}$ were retrospectively analysed.

Following initial optimisation in surgical intensive care unit emergency surgery was performed by same team of GI surgeons. For giant ventral hernia $(\geq 15 \mathrm{~cm})$ abdominal wall reconstruction was done by plastic surgery team. In the postoperative period patients were managed in SICU and were shifted out once they were hemodynamically stable and oral feeding had commenced. Demographic data, operative details, postoperative outcomes and follow up was recorded from institutional electronic health record system.

Results: Study group comprised of 12 patients ( 1 male and 11 females) with a median age of 72 (range $38-80$ ) years. The body mass index for the cohort was 27 (range $21-35.1$ ). An overview is presented in Table 1

At surgery, 04 patients were found to have strangulation of which 02 (one aged 80 years) died in early postoperative period. Of these 04 patients vacuum assisted closure was done in 2 while one each had component dissection \& repair and onlay mesh repair due to large size $(24 \mathrm{~cm})$ of defect.

Of the 3 patients with wound related complications, one had necrosis of the skin while 2 developed seroma. At a median follow up of 12 (range 2 -52) months, 2 patients (16.6) developed recurrent hernia. All surviving patients have expressed satisfaction with outcomes with improvements in body image and health related quality of life.

Conclusions: Emergency repair for complicated large midline incisional hernias in elderly should preferably be performed at tertiary care centres with experienced teams. In such scenario, acceptable perioperative and medium term outcome can be attained in this high risk group of patients. Image 1
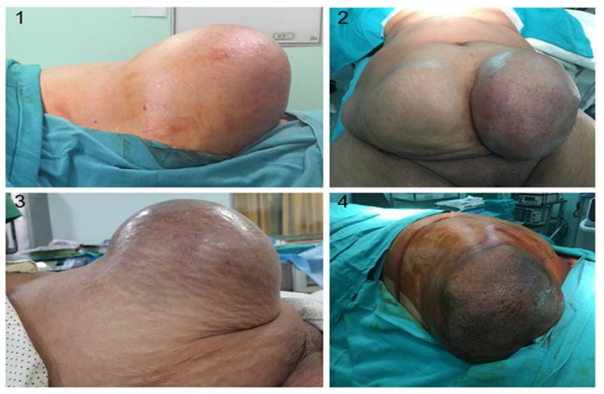

\begin{tabular}{|l|l|}
\hline Table 1. Results at a glance & \\
\hline Parameter & Value \\
\hline Median Age (range) years & $72(38-80)$ \\
\hline Sex (M:F) & $1: 11$ \\
\hline Median size of the defect (range) cm & $12(10-24)$ \\
\hline Presentation - Obstruction: Strangulation & $8: 4$ \\
\hline Repair with: without mesh & $9: 3$ \\
\hline Placement of mesh & Onlay -3 \\
& Sublay -2 \\
\hline Median length of stay (range) days & $8(3-15)$ \\
\hline Wound complications $-n$ (\%) & $3(25)$ \\
\hline Mortality $-n(\%)$ & $2(16.6)$ \\
\hline Recurrence $-n(\%)$ & $2(16.6)$ \\
\hline
\end{tabular}




\section{P594}

\section{Umbilical Hernia Repair in Women of Childbearing Age}

Susan Maurer $^{1}$, Savannah Renshaw ${ }^{1}$, Li-Ching Huang ${ }^{2}$, Carla Holcomb $^{1}$, Noah Switzer ${ }^{1}$, Courtney Collins ${ }^{1}$, Vimal Narula ${ }^{1}$, Benjamin Poulose ${ }^{1} ;{ }^{1}$ The Ohio State University, ${ }^{2}$ Vanderbilt University

Introduction: Women of childbearing age represent a unique challenge to hernia surgery, particularly for umbilical hernias secondary to physiologic changes associated with pregnancy. Thus far, no consensus has been developed for the most appropriate way to approach umbilical hernia repair in women aged 18-45. This study looks to try to understand the current management of this patient population and outcomes associated with these management decisions.

Methods: A retrospective review of prospectively collected data was conducted in women aged 18-45 with umbilical hernia repair using the Americas Hernia Society Quality Collaborative. Recurrence rate and abdominal wall function, based on the HerQLes assessment tool, at both 30 days and 1 year were determined for open repair versus laparoscopic repair. The impact of mesh use on recurrence was also evaluated in the open repair group.

Results: A total of 510 women were identified, with 479 (93.9\%) undergoing open repair (OUHR) and 71 undergoing laparoscopic repair (LUHR). The LUHR group included patients with higher BMI, ASA class, hernia size and hernia grade. Within the open hernia repair group, 247 patients were repaired primarily and 192 were repaired with mesh. Recurrence rate at 30 days in the OUHR group was $0.004 \%$, while recurrence was $3 \%$ in the LUHR group $(\mathrm{p}=0.036)$. Fifty-one patients had completed 1 year follow-up, $16 \%$ of those patients in the OUHR group were found to have a recurrence, while $0 \%$ of the LUHR group were found to have a recurrence $(\mathrm{p}=0.3)$. Within the OUHR group, $1 \%$ of the primary repairs and $0 \%$ of the mesh repairs had recurred at 30 days $(\mathrm{p}=0.21)$. At 1 year $(\mathrm{n}=45)$, this was found to be $17 \%$ and $12 \%$, respectively $(\mathrm{p}=0.67)$. At 1 year, 32 patients had completed the HerQLes assessment tool. Those in the LUHR group trended toward a higher summary score $(\mathrm{p}=0.15)$, and therefore higher quality of life after their UHR.

Conclusions: While there are many patient factors that are considered when determining which type of umbilical hernia repair, the ideal repair of women of childbearing age has not yet been elucidated. The AHSQC database shows that most surgeons elect to perform an open primary repair and with 30 day recurrence rates this seems to be the appropriate choice; however, at 1 year, the recurrence rate is less in the laparoscopic repair group.
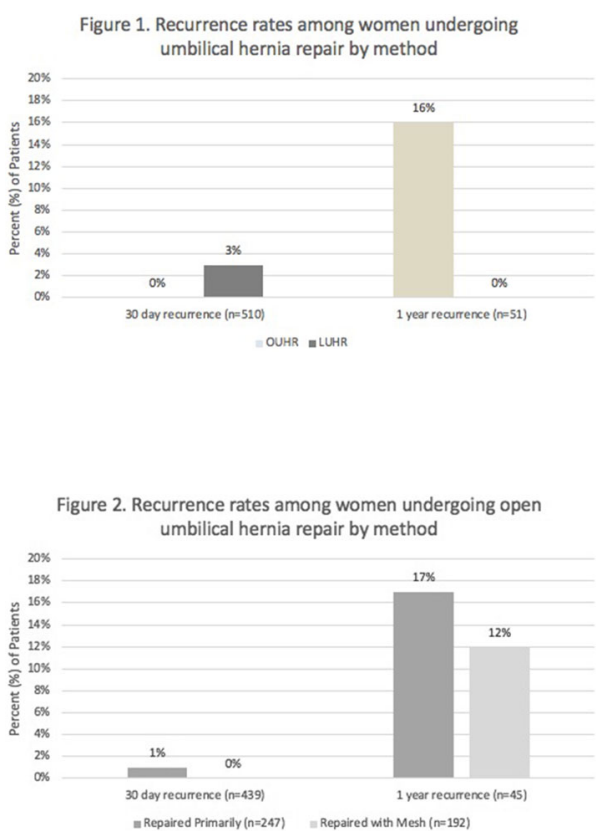

\section{P595}

\section{6-Month Post-operative Quality of Life and Pain Comparisons Between Robotic and Laparoscopic Inguinal Hernia Repair}

Noah Switzer, Savannah Renshaw, Carla Holcomb, Susan Maurer, Courtney Collins, Michael Meara, Vimal Narula, David Renton, Benjamin Poulose; The Ohio State University

Introduction: To examine the European Hernia Society Quality of Life (EuraHS QoL) scores at 6 months post-inguinal hernia repair comparing laparoscopic versus robotic techniques.

Minimally invasive inguinal hernia repairs have been shown to be associated with decreased post-operative pain and length of stay when compared to the open approach. While laparoscopy is more common and less expensive, robotic surgery is being increasingly utilized due to its 3Dimensional visualization and wristed motion.

Few studies exist comparing robotic and laparoscopic inguinal hernia repairs, with no consensus on superiority. The importance of focusing on patient's quality of life is being increasingly recognized. The European Hernia Society developed a validated and effective hernia specific quality of life score (EuraHS QoL) that scores patients in three domains: pain, restriction of activities and esthetical discomfort.

Methods and Procedures: This is a retrospective review of prospectively collected data using the Americas Hernia Society Quality Collaborative data between the years 2013 and 2018. Included subjects were $>18$ years old, had a laparoscopic or robotic unilateral inguinal hernia repair performed electively with 6 month EuraHS information available. The EuraHS score is 9 questions in 3 domains, where a higher score from 0-10 is considered a worse state. The Wilcoxon rank sum test and Pearson Chi square test were used.

Results: This study included 148 patients, separated into laparo$\operatorname{scopic}(\mathrm{N}=115)$ and $\operatorname{robotic}(\mathrm{N}=33)$ cohorts. The two groups had similar basic demographics with respect to age, race, smoking status, ASA class, immunosuppressant use, chronic obstructive pulmonary disease, and diabetes status.

Post-operative complication rates were also comparable, with $10(9 \%)$ patients in the laparoscopic arm and 5(15\%) patients in the robotic arm $(\mathrm{p}=0.28)$. No hernia recurrences or mesh infections were reported, with 1 re-admission in the robotic group.

The overall median EuraHS scores at baseline were 24 and 33 for the laparoscopic and robotic cohorts, respectively $(\mathrm{p}=0.16)$. This was separated into the pain, restriction and cosmetic domains and at baseline the median values were $6,9,5$ and 10,17, and 6 for the laparoscopic and robotic groups respectively. At 6 months, the overall change from baseline was similar, -20.0 and -23.0 , respectively $(\mathrm{p}=0.75)$. At 6 months, the median scores for pain, restriction and cosmetic domains were 0 for both groups.

Conclusion: Both laparoscopic and robotic inguinal hernia repairs are associated with improved patient reported quality of life outcomes at six months post-operatively, with no significant outcome differences between the techniques. 
P596

Laparoscopic Management of Congenital Diaphragmatic Hernia in Adults

Ajay H Bhandarwar, MS, FMAS, FIAGES, FAIS, FICS, FBMS ${ }^{1}$, Khushboo N Kadakia, MBBS ${ }^{1}$, Amol N Wagh, MS, FMAS, FIAGES, FAIS, FICS, FBMS ${ }^{1}$, Shekhar A Jadhav, MS, FMAS ${ }^{1}$, Amarjeet E Tandur, MS ${ }^{1}$, Priyanka Saha, MBBS ${ }^{1}$, Soumya Chatnalkar, MBBS ${ }^{1}$, Jai Rathore, MBBS ${ }^{1}$, Shivang Shukla, MBBS ${ }^{1}$, Sunita R Gajbhiye, MBBS, MD Associate Professor ${ }^{2} ;{ }^{1}$ Grant Government Medical College \& Sir J.J. Group of Hospitals, Mumbai, India, ${ }^{2}$ Govt. Medical College, Nagpur,Maharashtra,India

Introduction: Congenital diaphragmatic hernia typically presents in childhood but its presentation in adults is an extremely rare entity. Surgery is indicated for all types of diaphragmatic hernia. In recent years, there has been a shift from open surgical repair to laparoscopic approach. With our study, we aim to establish that diaphragmatic hernia repair by laparoscopic approach is safe, feasible and effective method with minimal intra operative and post-operative complications and decreased post-operative hospital stay .

Materials and Methods: We did a retrospective study of 8 patients with diaphragmatic hernia, operated between March 2010 to October 2016. All patients with age 15 and above were considered as adults and included in the study. One patient had Morgagni hernia and the remaining patients had Bochdalek hernia. Patients presented most commonly with symptoms of abdominal pain, nausea, vomiting, respiratory distress. Patients were diagnosed with diaphragmatic hernia based on CT imaging. All patients were managed laparoscopically with no conversion to open. The contents of the hernia were reduced with harmonic scalpel, defect closed with polypropelene continues sutures and a composite mesh was placed over the sutured defect and fixed with tackers. All patients had an uneventful recovery and were asymptomatic at year of follow up.

Conclusion: Congenital diaphragmatic hernia is an uncommon presentation in adults. Thus laparoscopic approach is safe, feasible and effective method for diaphragmatic hernia repair in adults with minimal morbidity and mortality.

\section{P597}

\section{Laparoscopic Repair of Incarcerated Bochdalek Hernia in Elderly. A Rare Emergency Easily Overlooked}

Sendhil Kumar, PhD, Piyush Patwa, V. Kandasamy; Gateway Clinics

Congenital posterolateral diaphragmatic defects, such as Bochdalek hernias (BHs), usually present during the neonatal period with respiratory symptoms and are associated with significant mortally. However, a subset of patients with BHs may remain asymptomatic during childhood, and the condition may present as a surgical emergency in adulthood. Surgical repair of the defect is the recommended therapy for of diaphragmatic defects has been performed via laparotomy or thoracotomy, but the use of laparoscopic has challenged the use of these traditional procedures. However the laparoscopic management of incarcerated BH is rarely reported. In the present paper, we report the case of a patient who presented gastric volvulus that was caused by an incarcerated stomach through a Bochdalek defect and treated using a laparoscopic approach.

\section{P598}

\section{Is Robotic Surgery Safe and Feasible in a County Hospital}

Oscar A Olavarria, MD, Karla Bernardi, MD, Aghogho Idiaghe, RN, Todd D Wilson, MD, Mike K Liang, MD, Curtis J Wray, MD, Tien C Ko, MD, Lillian S Kao, MD, MS, Shinil K Shah, DO; McGovern Medical School at UTHealth

Introduction: Safety net hospitals, such as county hospitals, have been demonstrated to be outliers for surgical complications on multiple national databases. Reasons include low socioeconomic status patients with poorly controlled comorbid conditions, system based issues, and limited hospital resources. Adoption of complex technologies, such as robotic surgery, may not result in the same outcomes as those achieved in other facilities. The aim of this study was to determine the safety and feasibility of the adoption of robotic surgery in a county hospital.

Methods: This was a review of the first 95 patients who underwent robotic assisted laparoscopic procedures at a single county hospital. Primary outcome was major complications defined as Clavien-Dindo class 3-5 complications within 30 days of surgery. Secondary outcomes included conversions from robotic to open procedures, blood transfusions, readmissions, reoperations, operative time, minor complications (Clavien-Dindo 1-2) including wound complications, and 30 day hospital length of stay. Multivariable linear regression was performed to identify variables associated with longer operative time.

Results: A total of 95 patients underwent robotic assisted laparoscopic surgery between January-September 2018. Of these, 30 (31.6\%) were overweight and $51(53.7 \%)$ were obese, $19(20 \%)$ had diabetes mellitus, $68(71.5 \%)$ had a previous abdominal surgery and $14(14.7 \%)$ had a previous ventral hernia repair (VHR). In our series: $69(72.6 \%)$ patients had a VHR, $14(14.7 \%)$ had an inguinal hernia repair of which $8(8.4 \%)$ were bilateral, and $10(10.5 \%)$ had foregut surgery (hiatal hernia repair, Heller myotomy or other gastroesophageal surgery). There was 1 major complication, a pulmonary embolism following a robotic hiatal hernia repair.

There were no conversions to open procedures, blood transfusions, procedure related readmissions or reoperations. The mean $\pm \mathrm{SD}$ operative time was $133 \pm 62 \mathrm{~min}$. There were 3 surgical site occurrences ( 3 seromas) and no surgical site infections. The 30 day hospital length of stay, median (IQR), was 0 (0). On multivariable analysis, procedure type (ventral, unilateral-inguinal, bilateral-inguinal and foregut), previous abdominal surgery, and first fifty cases were independently associated with longer operative time (Table).

Conclusion: Robotic assisted laparoscopic surgery is safe and feasible at a county hospital. Given the high risk patient population and high complication rate based upon national data, county hospitals may see substantial benefit by developing robotics programs. Further studies are needed to assess the effectiveness and value (outcomes/costs) of robotic platforms in general surgery and other specialties at safety net hospitals.

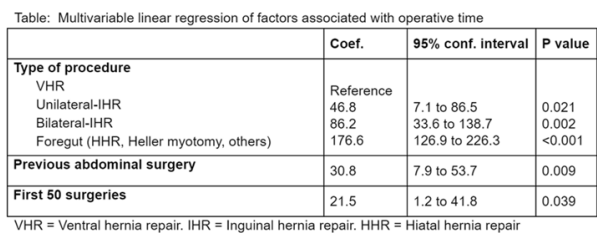


P599

Effective Inguinal Ring Closure Methods to Prevent Recurrence After Laparoscopic Inguinal Hernia Repair for Indirect Inguinal Hernias with Large Inguinal Rings

Fujio Ito, PhD, MD, Junichi Miura, PhD, MD, Takao Tsuchiya, PhD, MD, Satoshi Otani, PhD, MD, Takahiro Saito, PhD, MD, Atsushi Nishimagi, MD; Iwase General Hospital

Introduction: In laparoscopic inguinal hernia repair, tack fixation to the iliopubic tract, vascular triangle (triangle of doom) and nerve triangle (triangle of pain) cannot be performed, which consequently leads to concerns regarding the recurrence of indirect inguinal hernias with large inguinal rings due to postoperative eversion or displacement of the lower part of the mesh. To avoid migration or displacement of the mesh after the repair procedure until it becomes adherent to the surrounding tissue through scarring, we perform suture closure of the enlarged inguinal ring or mesh suturing to the hernia orifice and find these methods to be useful in preventing recurrence of the hernia. Here, we report our findings.

Materials and Methods: Thirty patients who underwent laparoscopic hernia repair (transabdominal preperitoneal/totally extraperitoneal) for indirect inguinal hernia with $>3 \mathrm{~cm}$ hernia orifices were investigated. After examining the peritoneal dissection area, inguinal ring closure was performed using one of the following three methods:

Method 1. Suture closure using the preperitoneal fascia

When the inguinal ring is about $3-4 \mathrm{~cm}$ and sufficient preperitoneal fascia remains after dissection of the peritoneum, or when the iliopubic tract cannot be exposed easily, the preperitoneal fascia is approximated with purse-string sutures or continuous sutures to close the hernia orifice.

Method 2. Suture closure of the transverse aponeurotic arch and iliopubic tract

This method is used when the hernia orifice exceeds $4 \mathrm{~cm}$ or the lateral triangle is enlarged, and when approximation of the transverse aponeurotic arch and iliopubic tract is feasible. Without getting close to the course of the genitofemoral nerve or lateral femoral cutaneous nerve, the iliopubic tract is exposed and visualized at the center of the inguinal ring and 2-3 sites are sutured to reduce the size of the inguinal ring. Importantly, the iliopubic tract is superficially sutured. Method 3. Mesh suturing to the transverse aponeurotic arch and iliopubic tract

When the hernia orifice exceeds $4 \mathrm{~cm}$ but approximation of the transverse aponeurotic arch and iliopubic tract is structurally not feasible, the mesh is sutured to the hernia orifice with low tension sutures.

Results: Using the above three methods, inguinal ring closure was achieved in all cases with enlarged inguinal rings; the patients are recovering well without chronic pain or recurrence. Discussion: By only suturing sites that can be safely visualized without performing tack fixation, it was feasible to safely close large inguinal rings.

\section{P600}

Mesh or No Mesh for Laparoscopic Hiatal Hernioplasty: Prevention of Recurrence vs Avoiding Esophagic Erosion, Facing a Large Surgical Dilem

Daniel Gomez, MD, FACS ${ }^{1}$, Luis F Cabrera, General Surgeon ${ }^{2}$, Andrez Mendoza, General Surgeon ${ }^{2}$, Mauricio Pedraza ${ }^{2}$, Jean Pulido $^{2}$, Eric E Vinck ${ }^{2}$, Sebastian Sanchez ${ }^{2} ;{ }^{1} \mathrm{CPO},{ }^{2}$ Bosque University

Introduction: The use of mesh in the laparoscopic repair of paraesophageal hernias has been shown to reduce recurrence, only a few studies have shown a prevention of it as reported by Frantzides et al. The incidence of complications such as erosion of the esophagus is $10.1 \%$ as reported by Sathasivam and collaborators, using polypropylene and polytetrafluoroethylene meshes, however in the market the polyester mesh covered with collagen is offered as an option to avoid this type of complications.

Objective: To show our experience in the laparoscopic repair of giant hiatal hernia using this type of specific mesh in order to establish if there are complications associated with it.

Materials and Methods: Retrospective, descriptive observational study, including symptomatic patients with giant hiatal hernia grade III and IV, taken to laparoscopic hiatal hernioplasty with polyester mesh covered with collagen (Parietex Composite HiatalR), in our institution, from November 2013 to February 2017. Evaluating the following variables: age, sex, type of hernia for esophageal, surgical time, bleeding, recurrence, esophageal mesh incorporation, complications, conversion and mortality.

Results: 24 patients were taken to laparoscopic hiatal hernioplasty with polyester mesh covered with collagen (Parietex Composite Hiatal ${ }^{\circledR}$, Medtronic Inc., Minneapolis, USA) with 0\% conversion rate. The average size of hiatal hernias was $4.3 \mathrm{~cm}$ and the most common type of hernia was III in $86 \%$. With an average surgical time of $92.6 \mathrm{~min}$ and an average hospital stay of $26.3 \mathrm{~h}$. However, at 36 months, esophageal erosion occurred due to the incorporation of collagencoated polyester mesh (Parietex Composite Hiatal ${ }^{\circledR}$ ), with an incidence of this complication of $4.1 \%$ and a recurrence incidence rate of $4.1 \%$ at 24 months. Dysphagia incidence of $8.3 \%$.

Conclusions: The use of the mesh in the laparoscopic repair of para esophageal hernias should be routine, in order to minimize hernia recurrence, however, as it can be seen in our study, the ideal mesh has not been designed yet for the esophageal hiatus, since, despite using a mesh technology covered with the latest technology such as collagen-coated polyester mesh (Parietex Composite Hiatal $^{\circledR}$, Medtronic Inc, Minneapolis, USA), a case of esophageal erosion was presented.

\section{P601}

\section{Outcomes of Totally Extraperitoneal and Trans-Abdominal Preperitoneal Laparoscopic Spigelian Hernia Repair}

Kara Donovan, BS, Merritt Denham, BS, Kristine Kuchta, MS, Joann Carbray, BS, Michael Ujiki, MD, John Linn, MD, Woody Denham, MD, Stephen Haggerty, MD; NorthShore University HealthSystem

Introduction: Spigelian hernias (SH) are rare intraparietal hernias occurring just medial to the semilunar line. They often involve older patients, are difficult to diagnose, and have a high risk for incarceration and strangulation. Historically repaired using open surgery, several small series have reported laparoscopic repair over the last two decades. Both totally extraperitoneal (TEP) and transabdominal preperitoneal (TAPP) approaches have been described. However, there is limited outcome data including both of these approaches. We present the largest series to date of laparoscopic Spigelian hernia repair including both popular approaches.

Methods and Procedures: Consecutive patients $(n=73)$ undergoing laparoscopic spigelian hernia repair from 2009-2018 were identified from a prospectively managed quality database. All procedures were performed at a single institution by one of four general surgeons. Patients were divided based on laparoscopic approach used, totally extraperitoneal (TEP) group $(n=33)$ and a transabdominal preperitoneal (TAPP) group $(n=40)$. A descriptive analysis of patient demographics, surgical characteristics, and post-operative complications was performed on both groups. Results: In the TAPP cohort, $70 \%$ of patients were female and mean age was $65( \pm 14)$ years. Median hernia size was $4.9 \mathrm{~cm}^{2}$ and mean operative time was $77( \pm 44)$ minutes. $95 \%$ used tacks, with a mean of $9( \pm 1)$ tacks. Median length of stay (LOS) was $25 \mathrm{~h}$. Five $(12.5 \%)$ patients had an ED visit within 30 days of repair. One (2.5\%) patient had a readmission, one had a reoperation, and one experienced a surgical site infection (SSI). One patient recurred, with a median follow-up of 957 days.

In the TEP cohort, $61 \%$ were male and mean age was $71( \pm 12)$ years. Median hernia size was 3.1 $\mathrm{cm}^{2}$ and mean operative time was $48( \pm 22)$ minutes. $79 \%$ used tacks, with a mean of $7( \pm 3)$ tacks. Median LOS was $7 \mathrm{~h}$. One (3.0\%) patient had an ED visit within 30 days of repair. There were no readmissions, reoperations, SSI, or recurrence, with a median follow-up of 259 days.

Conclusion: Our large series indicates both the TEP and TAPP approach to laparoscopic spigelian hernia repair are safe and effective, can be performed on an outpatient basis and should be favored over open surgery. The laparoscopic approach used should be based on surgeon experience and preference.

\section{P602}

\section{Laparoscopic vs Open Ventral Hernia Repair}

Mohannad S Eledreesi, MD, Turki Howiti, MD, Hussam Adi, MD, MMAS, FACS, FASMBS; North West Armed Forces Hospital,Tabuk,Saudi Arabia

Background: Ventral hernia ( $\mathrm{VH})$ remains a signficant problem that follow previuos laparotomy. Although an increase evidence is available for the benefit of laparoscopic repair (LR), open repai $(\mathrm{OR})$ is still the first choice for many surgeons.

Objective: Our aim is to compare LR vs OR hernia repair with regard to operative results, perioperative and postoperative complications, and recurrence rate.

Methods: We reviewed the medical records of patients who underwent $L R$ and OR ventral hernia repair in King Salman Armed Forces Hospital, Saudi Arabia from 1/1/2012 till 31/7/2015 with minimum of 12 months follow up.

Results: A total of 121 patients underwent VH repair at King Salman Armed Forces Hospital from 1/1/2012 till 31/7/2015. Of these, 78 were male $(64.4 \%)$. The etiologies of the $\mathrm{VH}$ were as follows: umbilical (17/121), paraumbilical (87/121), epigastric (2/121), incisional (15/121). The mean age was 43.5 year. The mean fascial defect size was $14.1 \pm 2 \mathrm{~cm}^{2}$. Comorbidities included diabetes $2.5 \%$ and hypertension (7.4\%). Mean Body Mass Index (BMI) was $27.2 \mathrm{~kg} / \mathrm{m}^{2}$. eighty four patients (69.4\%) underwent OR. None of LR had conversion. Mean operative time was $76 \mathrm{~min}$ (77 $\mathrm{min}$ and $75 \mathrm{~min}$ for OR and LR, respectively). Five cases (4.13\%) had recurrence (4 after OR and one after LR with rate of $3.3 \%$ and $0.8 \%$, respectively). Recurrence rate was $1.6 \%(\mathrm{n}=2)$ in $\mathrm{BMI}<30 \mathrm{~kg}$ / $\mathrm{m}^{2}$ and $2.4 \%(\mathrm{n}=3)$ in $\mathrm{BMI} \geq 30 \mathrm{~kg} / \mathrm{m}^{2}$. Seven cases $(5.7 \%)$ developed seroma $(5$ cases in OR and 2 cases in LR with rate of $4.1 \%$ and $1.6 \%$, respectively). Five patients had wound infection, 4 in OR and one in LR. Only one case of hematoma rate complicated OR.

Conclusion: LR of VH has better short -term results than OR. However, a larger sample and long term follow up are required. 
P603

\section{Pre-operative Administration of Tamsulosin for Prevention of Post-operative Urinary Retention in Males Undergoing Elective Inguinal Hernia Repair}

Anthony Tabatabai, DO, John Vance, DO, Christopher Ash, DO, Mark Vance, DO, Andrea Goethals, DO, Kimberly Barber, PhD; Genesys Regional Medical Center

Introduction: The current standard of care does not include prophylactic doses of an alphaadrenergic blocker in preventing post-operative urinary retention among patients undergoing hernia repair. This study aimed to test the efficacy of tamsulosin for preventing post-operative urinary retention among males undergoing elective inguinal hernia repair. Avoiding post-operative urinary retention may decrease medical costs, hospital admissions, extended recovery observation, and return to emergency room for inability to urinate

Methods and Procedures: This was a double blind, randomized controlled prospective trial evaluating two preoperative doses of tamsulosin an alpha-adrenergic blocker in preventing postoperative urinary retention (POUR). Patients were enrolled from January 2017 to September 2018 in a community hospital. Tamsulosin versus placebo medication in males over the age of 30 who have no history of benign prostatic hypertrophy nor who are taking any alpha-adrenergic blocker or 5 alpha reductase blocker for issues of urinary retention were selected. Patients were undergoing elective laparoscopic, open, or robotic assisted unilateral or bilateral inguinal hernia repair. They received two doses of $0.4 \mathrm{mg}$ (tamsulosin vs placebo) preoperatively, the night before surgery and the morning of surgery. POUR was defined as the inability to spontaneously urinate following surgery requiring straight catheterization or placement of a Foley catheter for urinary retention within $72 \mathrm{~h}$ of surgery. Post-operative catheterization rates were compared for significance at $\mathrm{p}<0.05$ with Chi square analysis.

Results: A total of 210 patients were enrolled into the study. Of them 193 (92\%) completed taking the drug as directed and having an inguinal hernia repair. Patients were randomly assigned to either the tamsulosin group (110 or $57 \%$ ) or the placebo group ( 83 or $43 \%$ ). A total of $16(8.3 \%)$ patients had urinary retention. The tamsulosin group had $4(4.8 \%)$ retentions and the placebo group had 12 $(11 \%)$. This was a $42 \%$ relative decrease for the treatment group $(\mathrm{p}=0.10)$. Procedures were further subdivided by technique, $105(54 \%)$ Robotic assisted, 64 (33\%) open, and $24(12 \%)$ laparoscopic. Among 105 robotic procedures, there was a relative difference of $52 \%$ in retention rates between groups [3 (6.7\%) tamsulosin, $8(13.3 \%)$ placebo, $\mathrm{p}=0.13$ ].

Conclusion: Though it did not reach statistical significance, a clinically meaningful difference was observed in the rates of retention between the two groups. This large difference was supported among those with robotic assisted procedures and suggests that prophylaxis with a selective alpha 1-A adrenergic receptor antagonist may prevent urinary retention following hernia surgery.

\section{P604}

\section{Revealing Nationwide Readmission and Cost Patterns After Liver Transplant}

Alvaro Castillo, $\mathrm{MD}^{1}$, Allison D Rice, $\mathrm{MD}^{1}$, Kristian C Enestvedt, $\mathrm{MD}^{2}$, Joshua Parreco, $\mathrm{MD}^{1}$, Susan L Orloff, $\mathrm{MD}^{2} ;{ }^{1}$ University of Miami, ${ }^{2}$ Oregon Health and Science University

Introduction: Hospital readmissions are becoming an important quality metric in liver transplantation. However, limited data exist examining the relationship between center experience and readmission rates. The purpose of this study was to evaluate the risk factors for readmission after liver transplant to determine performance and costs stratified by transplant center volume. Methods: The Nationwide Readmissions Database for 2010-2014 was queried for all adult patients undergoing liver transplant in the U.S. The outcomes of interest were non-elective readmission to index and non-index hospitals within 180 days. High volume centers were identified as performing the highest quartile of liver transplants each year. Univariable analysis was performed for the outcomes of interest and the significant variables were used for multivariable logistic regression. Costs were calculated using the cost to charge ratio and results were weighted for national estimates.

Results: There were 33,287 patients who underwent liver transplant during the study period. The highest quartile centers performed between 80 and 177 procedures per year (unweighted). The readmission rate within 180 days was $36.4 \%$, of which $7.6 \%$ were readmitted to a non-index hospital. The median initial admission cost was $\$ 94,215(\$ 67,183-\$ 145,487)$ and median readmission cost was $\$ 17,341(\$ 7,249-\$ 40,181)$. High volume centers performed $52.1 \%$ of procedures and were associated with a decreased median initial admission cost $(\$ 85,091$ [\$63,274-\$131,852] vs $\$ 103,727$ [\$73,838-\$161,228] $\mathrm{p}<0.01)$ and decreased median readmission cost $(\$ 16,579$ $[\$ 6,929-\$ 39,299]$ vs $\$ 18,001[\$ 7,799-\$ 40,476] \mathrm{p}<0.01)$. High volume hospitals were also associated with a decreased risk for readmission $(\mathrm{OR} 0.71[0.68-0.75] \mathrm{p}<0.01)$ however, they were associated with an increased risk for readmission to a different hospital (OR 1.83 [1.56-2.14] $\mathrm{p}<0.01$ ). The most common primary diagnosis code on readmission was "complications of transplanted liver" $(25.0 \%)$ and on readmission to a non-index hospital was "acute kidney failure" $(7.8 \%)$

Conclusions: This study represents the first nationwide evaluation of readmission after liver transplant including readmissions to hospitals outside the liver transplant network. High volume liver transplant centers were associated with decreased cost and readmission rates. However, these centers have an increased risk for readmission to a non-index hospital. These findings have implications for quality metrics, benchmarking, regional transfer patterns, and readmission reduction strategies.

\section{P605}

\section{Laparoscopic Splenectomy for Nodular and Cystic Lesions in the Spleen}

Hironori Shiozaki, Takeshi Gocho, Keigo Nakashima, Rui Marukuchi, Yoshihiro Shirai, Jungo Yasuda, Kenei Furukawa, Shinji Onda, Hiroaki Shiba, Yuichi Ishida, Katsuhiko Yanaga; The Jikei University School of Medicine

Background: Laparoscopic splenectomy is becoming the standard procedure for benign splenic disorders including hematologic diseases and hypersplenism. However, the feasibility and safety of laparoscopic splenectomy for nodular and cystic splenic lesions are yet to be elucidated.

Methods: Twelve patients with splenic nodular or cystic lesions who underwent laparoscopic splenectomy between April 2003 and June 2018 were retrospectively reviewed, in which patien factors (age, sex), lesion factors (diagnosis, size, number) and surgical factors (procedures, operation time, blood loss, postoperative complication, postoperative hospital stay) were assessed. Results: The median age was 44.5 ( 21 to 72 ) years, and 6 patients $(50 \%)$ were male. Splenectomy was performed by either pure laparoscopy $(\mathrm{n}=3)$, hand - assisted laparoscopic surgery (HALS) $(n=5)$ or single incision laparoscopic surgery (SILS) $(n=4)$, for solid lesions in 8 and cystic lesions in 4 patients. Eleven patients had a single lesion, of which maximum diameter was 50.5 (range, 28 - 100) $\mathrm{mm}$. The operation time was 178.5 (range, 90 - 254) $\mathrm{min}$ and intraoperative blood loss was 12.5 (range, 0 - 622) g. Splenectomies were performed without intraoperative or postoperative complications of Grade ?b or more by Clavien-Dindo classification except for 2 cases with postoperative pancreatic fistulas which were Grade A by International study group of postoperative pancreatic fistula (ISGPF). All laparoscopic splenectomies were performed withou tumor rupture during the procedure. Final diagnosis was confirmed pathologically as hemangiomas in 3 , SANT in 3, inflammatory pseudotumor in 2 , malignant lymphoma in one, lymphangioma in one, simple cyst in one and pseudocyst in one patient

Conclusions: Laparoscopic splenectomy for the nodular and cystic lesion is safe and feasible.

\section{P606}

Feasibility of Laparoscopic Splenectomy in Large Pyogenic Splenic Abscess: An Initial Experience from Tertiary Hospital of India

Rushil Jain, Vimal Jain, Shalit Jolly; Mata Chanan Devi Hospital New Delhi

Objective of the Study: To assess the feasibility of laparoscopic splenectomy in cases of large pyogenic abscess (although the incidence of pyogenic abscess across the globe is very less) in a tertiary care referral hospital of new delhi india.

Methods and Procedure: Five patients during the duration of two years (2014-2016) of fever of unknown origin and abdominal pain reporting to hospital undergone computed tomography and detected splenic abscess were included in the study.

Patients were subjected to pre anaesthetic check up and were planned for laparoscopic splenectomy, though consented for open splenectomy also.

This is a short case series because incidence of pyogenic abscess is less than one percent across the globe.

All patients undergone laparoscopic splenectomy by standard accepted technique.

Results: All five patients sucessfully underwent laparoscopic splenectomy out of which three were females with age ranging from 16-34 years where as male were two with age between 18-36. Average time in operative procedure was $2.4 \mathrm{~h}$ with average intraoperative blood loss of $130 \mathrm{ml}$ All patients recovered well in post operative period with no mortality. All patients were vaccinated as per the guildlines and schedule. Two patients had platelet counts of ten lakhs for which prophylactic aspirin was started.

Conclusion: Laparoscopic splenectomy is a safe and acceptable procedure in large splenic abscess with relatively little long learning curve in such cases. 
P607

\author{
Laparoscopic Liver Cyst Aspiration in a Twin Pregnancy: Case \\ Report
}

Emeka Ray-Offor, MBBS, DMAS, FWACS, FMAS, FACS ${ }^{1}$, Rex F Ijah, MBBS, MPH, MSMAS, FMAS ${ }^{1}$, Ogheneochuko D Ray-Offor, MBBS, FWACS ${ }^{2}$, Onochie Nweze, MBBS, FWACS ${ }^{3}$, Job Otokwala, MBBS, FWACS ${ }^{4} ;{ }^{1}$ Oak Endoscopy Centre \& Department of Surgery University of Port Harcourt Teaching Hospital Port Harcourt Rivers State Nigeria, ${ }^{2}$ Oak endoscopy Centre Port Harcourt Rivers State \& Department of Radiology University of Port Harcourt Rivers State Nigeria, ${ }^{3}$ Federal Medical Centre Bayelsa State Nigeria, ${ }^{4}$ Department of Anaesthesiology University of Port Harcourt Teaching Hospital Port Harcourt Rivers State

Background: Laparoscopy offers accurate visualization with minimal uterine manipulation in nonobstetric surgery during pregnancy.

Aim: To highlight the role of laparoscopy in non-obstetric surgery during pregnancy

Case Report: A 27-year-old Gravida 2 para $1+0$ was referred to our centre for laparoscopy with a month history of epigastric pain insidious in onset, sharp, intermittent not related to meals and relieved by analgesics. There was no nausea vomiting, jaundice, change in bowel habit or previous abdominal surgery. Physical examination revealed no pallor nor jaundice. Vital signs were stable: Temperature 360C; Pulse rate 90 beats/cycle; respiratory rate 20cycles/min but had hepatomegaly and a fundal height of 18 weeks.

Haemoglobin count was $10.5 \mathrm{~g} / \mathrm{dl}$. All other relevant investigations results were within normal range except low total protein $5.2 \mathrm{~g}$, and globulin $1.3 \mathrm{~g} / \mathrm{dl}$. Abdominal ultrasound scan showed enlarged liver harbouring a cyst $16.09 \mathrm{~cm} \times 12.02 \mathrm{~cm}$ in the right lobe at segments V and VI and a twin gestation of live males with estimated gestational age of 18 weeks 3 days. An elective laparoscopic procedure was scheduled. General anaesthesia was administered in left lateral supine position with prophylactic antibiotic given and deep vein thrombosis prophylaxis by compression stockings. Access was via a $10 \mathrm{~mm}$ midline epigastric port by open technique then laparoscope inserted. Capnoperitoneum was maintained at $14 \mathrm{mmHg}$. A $5 \mathrm{~mm}$ working port was inserted under direct vision in right subcostal region mid clavicular line. Cyst was visualized and completely aspirated without spillage yielding $2.5 \mathrm{~L}$ of purulent fluid (culture of aspirate yielded no organism). The operating time was forty minutes. She was admitted overnight for observation, oral intake commenced about $12 \mathrm{~h}$ later then discharged home after an uneventful hospital stay. Foetal heart monitoring of twin gestation was routinely done and satisfactory. The follow-up visit at 7th day post op was unremarkable.

Conclusion: Laparoscopic surgery is not contraindicated in sub-acute, non-obsteric conditions in pregnancy.

\section{P608}

\section{Pulling the Trigger: Laparoscopic Posterior Neurectomy in Patients with Abdominal Cutaneous Nerve Entrapment Syndrome, A Case Series}

Brian Bassiri-Tehrani, MD, Donna Bahroloomi, MD, A. Douglas Heymann, MD, FACS; Lenox Hill Hospital, Zucker School of Medicine at Hofstra/Northwell

Background: Anterior Cutaneous Nerve Entrapment Syndrome (ACNES) is an elusive cause of chronic abdominal pain that is secondary to the branches of the intercostal nerves becoming impinged as it pierces the anterior abdominal wall. Causes can include trauma, previous surgery, or pregnancy. The diagnosis is made on physical exam with a positive Carnett sign, and, usually with a therapeutic injection of local anesthetic at the focal area of pain and tenderness. These symptoms, however, are only temporarily treated by local anesthetic injections. Occasionally, patients require surgery to cut down to and resect a segment of the nerve branches known as an anterior neurectomy. This is effective in $75 \%$ of patients. For the remaining one-fourth of patients suffering from pain refractory to these interventions, pain is often described as "lateralizing" or migrating toward the lateral abdominal wall. Posterior neurectomy is a novel surgical approach that may provide sustained relief for patients with ACNES. This unique laparoscopic approach allows the surgeon to perform extensive lateral dissection of the intercostal nerves with minimal morbidity, and affords potential for removal of a longer segment of the lower thoracoabdominal intercostal nerves, thus eliminating the "lateralization" pain that some patients experience after an anterior release. This study is the first to describe the results of a laparoscopic posterior neurectomy in patients suffering from ACNES.

Methods: A database of ACNES patients' refractory to lidocaine injections who underwent a laparoscopic posterior neurectomy between the years of 2016 and 2018 in the Northwell Health System were retrospectively evaluated. Pain was recorded prior to treatment and after 6 weeks using a pain intensity numeric rating scale $(0-10)$. Success was defined as a $\geq 50 \%$ pain score reduction or $\geq 2$ point verbal rating scale reduction.

Results: Three adult ACNES patients who had lidocaine injections with temporary relief were included in this study. One patient had a laparoscopic posterior neurectomy with pain relief, however had a recurrence of ACNES in 12 months and underwent a repeat laparoscopic posterior and open anterior neurectomy with good relief. The other two patients had a laparoscopic posterior neurectomy with sustained mitigation of symptoms ( $\geq 50 \%$ PI-NRS reduction) postoperatively. Conclusions: Laparoscopic posterior neurectomy is a novel, minimally invasive surgical technique to treat ACNES that allows surgeons to resect a longer segment of nerves and its branches. This may potentially alleviate lateralization pain, although further studies with larger sample sizes need to be conducted in order to corroborate these results.

\section{P609}

The Clinical Use of Indocyanine Green as a Near-Infrared Fluorescent Contrast Agent for Image-Guided Laparoscopic Surgery

Konstantinos G Tsallis, Pr, Nikolaos Antoniou, Dr, Orestis Ioannidis, Ioannis Mantzoros, Savvas Simeonidis, Stathis Kotidis, Manousos-G Pramateutakis, Lydia Loutzidou, Dimitris Konstantaras, Stamatis Angelopoulos; Aristotle University

Background: Advances in laparoscopic imaging the past decade has brought to light new achievements as major contributions to intraoperative decision during laparoscopic procedures. Among these are the use of high definition and 3D imaging systems, and more recently indocyanine green (ICG) near infrared (NIR) fluorescence guided laparoscopy. The aim of this study was to present our experience with different laparoscopic procedures using ICG fluorescence imaging Patients and Methods: 52 ICG guided laparoscopic procedures were performed: 42 cholecystectomies ( 36 ellective and 6 urgent) 1 adrenalectomy, 2 segmental hepatectomies, 4 colectomies, 1 spleenctomy, 1 lymph node biopsy and 1 accesory spleen laparoscopic rescection mimicking pancreatic tail cancer. Visualization of structures was provided by a high resolution camera and a light source emitting both visible and near infra-red (NIR) light.

Results: After injection of ICG, vessels,organs and biliary structures were clearly identified. No injection-related adverse effects were reported. ICG-fluorescent imaging identified both of the microscopically confirmed HCC and CRC metastases in surgical specimens. Also provide valuable information to conduct a safe laparoscopic cholecystectomy spleenectomy, adrenalectomy and ensure adequate vascular supply for colectomy, or find lymph nodes. There were no bile duct injuries or anastomotic leaks.

Conclusions: The use of (ICG), a non-radioactive, inexpensive fluorescent dye, in laparoscopic operations permits visualizing anatomic structures, perfusion and perfusion defects as well as the biliary and lymphatic system. In liver surgery, the system is used to visualize liver metastases or primary tumors of the liver. Due to its versatile cross-discipline applications, in our opinion we consider this fluorescence technology as a future standard imaging technique that will be found in every OR.

\section{P610}

Percutaneous Assited Transprosthetic Endoscopic Therapy (PATENT) for Necrotizing Pancreatitis

Claudio Navarrete, $\mathrm{MD}^{1}$, Cecilia Castillo, $\mathrm{MD}^{2}$, Eduardo Valdivieso, $\mathrm{MD}^{3}$, Oscar Barrera, $\mathrm{MD}^{3}$, Manuel Arrieta, $\mathrm{MD}^{4} ;{ }^{1}$ Chief Department of Surgery Clínica Santa María and Latin American Gastrointestinal Endoscopy Training Center, Santiago, Chile., ${ }^{2}$ Digestive Disease Department, Clínica Alemana, Santiago, Chile, Latin American Gastrointestinal Endoscopy Training Center, ${ }^{3}$ Associate professor Latin American Gastrointestinal Endoscopy Training Center, ${ }^{4}$ University Of Sabana, Colombia

Introduction: The paradigm of open surgical management in patients with pancreatitis complicated by necrosis and infected collections has recently changed. The use of minimally invasive surgery (MIS) techniques has shown promising results and reduces the morbidity and mortality of traditional surgery. One of the different MIS approaches is the Percutaneous Assisted Transprosthetic endoscopic therapy (PATENT), a hybrid technique that combines the best of the endoscopic and retroperitoneal approaches.

Methods: Retrospective, descriptive study of patients who were admitted to our hospital with acute pancreatitis complicated by necrosis or infected collections between June 2010 and July 2017. In our series we performed a step-up approach management, where the necrotized pancreas or retroperitoneal infected collection was initially drained percutaneously through a CT-guided catheter. If the procedure failed, patients underwent the combined endoscopic and retroperitoneal debridement PATENT. In the procedure a biliary guidewire is inserted through the drainage catheter to reach the collection and then the catheter is removed. Later, the tract between abdominal wall and the collection is dilated and the distance is measured to choose the length of a prothesis to be inserted, in our series a fully covered self-expandable metal stent was used. Afterwards, the endoscope is advanced through the expanded stent toward the collection and debridement is performed. Finally, a collector system is left, allowing endoscopic re-exploration to be performed if necessary. We collected demographic and outcome data on patients who underwent the procedure including: gender, age, etiology of pancreatitis, collection size, number of interventions, complications and mortality.

Results: A total 19 patients underwent PATENT: eleven women (57.8\%) and eight men (42.2\%) with a median age of 34 years (Range: 24-46). The average size of the drained collections was $17 \mathrm{~cm}$ and the success rate was $93.85 \% .3$ patients $(15.7 \%)$ had complications and one patient died $(5.26 \%)$. None of the complications needed open surgery and no cases of 30 - day - mortality after discharged were identified. Conclusions: Our preliminary experience using PATENT for patients with complications of severe pancreatitis demonstrated the technique to be safe and reproducible, with an excellent success rate and low morbidity and mortality. We consider this technique to be an important option to be considered along different MIS methods for this catastrophic pathology. 
P611

\section{The Importance of Integrating Minimally Invasive Surgery in Developing Nations}

Charlotte Horne, MD, Godwin Onyema, MD, Afam Onyema, Kevin El-Hayek, MD; Cleveland Clinic

Laparoscopic surgery has routinely become the standard approach and standard of care for numerous surgical procedures in the developed world. Currently, over 3.5 million laparoscopic procedures are performed annually in the United States ${ }^{1}$. However, many developing nations are still routinely performing these surgeries via open techniques due to lack of infrastructure or lack of training. Laparoscopic surgery has been shown to decrease both wound morbidity and length of stay. It also allows for faster return to work ${ }^{2,3,4}$, which can significantly benefit populations where work is imperative for those to meet their basic needs.

To address these issues, we conducted a mission trip to Awka, Nigeria under the GEANCO Foundation to establish infrastructure, teach the surgeons and operating room personal the techniques, appropriate instrument handling, basic troubleshooting and equipment sterilization procedures. Nigeria presents an excellent location for this, as it is the largest country in Africa, is a regional power in Africa and has one of the top twenty largest economies in the world ${ }^{5}$. Despite this, the human development index ranks 152 in the world, 70 million lack access to safe drinking water and 110 million people are lacking appropriate sanitation $^{6}$. Due to these public health concerns, minimally invasive surgery has the potential to significantly decrease wound morbidity.

To establish the infrastructure, donated products were obtained from device and medical redistribution companies. Lectures were conducted daily and included basic laparoscopic principles as well as procedure specific techniques. We chose patients who required a cholecystectomy, appendectomy or inguinal herniorrhaphy as these procedures would commonly need to be performed locally and are appropriate to establish a basic laparoscopic skill set. Working directly with one surgeon and his senior registrar, techniques for abdominal entry, basic procedural related steps, equipment care and troubleshooting were taught.

Over 7 days, a total of 11 laparoscopic cases were performed. Conversion to open was required in two patients. All 11 patients were discharged home at the completion of the mission without inpatient complication. Overall length of stay after all procedures was approximately 1.5 days, mostly due to patient discomfort in being discharged home earlier. There is a significant need in developing countries for improved access to what is considered standard of care in the United States. We advocate for establishing the infrastructure and teaching laparoscopic techniques to greatly decrease post-operative morbidity.

\section{P613}

Transversus Abdominis Plane Block Versus Port Site Infiltration for Post-Operative Analgesia After Laparoscopic Cholecystectomy - A Prospective Randomized Triple Blinded Study

Anubhav Vindal, MS, DNB, MRCSEd, FRCSGlas, FACS, FAIS, Hitesh Sarda, MBBS, Pawanindra Lal, MS, DNB, FRCSEd, FRCSGlas, FACS; Division of Minimal Access Surgery, Department of Surgery, Maulana Azad Medical College, New Delhi, India

Introduction: Transversus abdominis plane (TAP) block is a regional anesthetic technique that provides analgesia to the parietal peritoneum, skin and muscles of the anterior abdominal wall innervated by somatic nerves from T6 to L1 spinal segments. It is usually administered either blindly (double pop technique) or under ultrasound guidance. A less commonly used and recently described technique is laparoscopic guided TAP block which can be administered by the surgeon at the time of surgery without the need for any additional equipment. We present the results of our prospective randomized triple blind study comparing laparoscopically guided 4-quadrant TAP block with the widely used port site local anesthetic infiltration after laparoscopic cholecystectomy.

Methods and Procedures: Hundred patients with symptomatic gallstones planned for laparoscopic cholecystectomy were included in the study and randomized into two groups of TAP block and port site infiltration respectively. All patients received a total of $40 \mathrm{ml}$ of $0.25 \%$ bupivacaine as the local anesthetic agent

Patients in group A received TAP block with bupivacaine in 4 abdominal quadrants: $10 \mathrm{ml}$ each at bilateral subcostal and lumbar regions using a $18 \mathrm{G}$ intravenous cannula guided laparoscopically. Correct plane of infiltration was confirmed by observing Doyle's bulge through the laparoscope. Port site infiltration was done with $40 \mathrm{ml}$ of $0.9 \%$ saline $(10 \mathrm{ml}$ at each of the 4 port sites) to blind the surgeon.

Patients in group B received infiltration with bupivacaine, $10 \mathrm{ml}$ at each of the 4 port sites and TAP infiltration with $40 \mathrm{ml}$ of $0.9 \%$ saline (as described above).

Post-operative pain (at 1, 3, 6, 12, $24 \mathrm{~h}$ and 1 week) (using VAS), time of return to activities of daily living and patient satisfaction (using Capuzzo score) were recorded by an observer who was also blinded.

Results: The mean VAS at 1, 3, 6, 12, $24 \mathrm{~h}$ and 1 week was less in Group A compared to Group B ( $\mathrm{p}=0.831,0.819,0.082,<0.05,<0.05$ and $<0.01$ respectively) as was the mean time to return to activities $(\mathrm{p}<0.01)$. The mean Capuzzo score (measuring patient satisfaction) was found to be higher in Group A compared to Group B $(\mathrm{p}<0.001)$. Conclusion: TAP block is a safe and easy to use technique that reduces severity of postoperative pain and analgesia requirement after laparoscopic cholecystectomy thereby aiding enhanced recovery, early discharge and improved patient satisfaction. It does not require any special or additional equipment and can be administered by the surgeon himself.

\section{P614}

\section{Laparoscopic Cysto Gastrostomy in Pseudocyst Pancreas : Handsewn \& Stapled}

Ajay H Bhandarwar, MS, FMAS, FIAGES, FAIS, FICS, FBMS, Priyanka Saha, MBBS, Khushboo Kadakia, MBBS, Jai Rathore, MBBS, Amol N Wagh, MS, FMAS, FIAGES, FAIS, FICS, FBMS, Shekhar A Jadhav, MS, FMAS, Amarjeet E Tandur, MS, Soumya Chatnalkar, MBBS, Jalbaji P More, MS, Ruchira Bhattacharya, MBBS; Grant Government Medical College \& Sir J.J. Group of Hospitals, Mumbai, India

Introdution: Management of pseudo cyst of pancreas ranges from conservative to endoscopic/open/laparoscopic surgery.

Drainage procedures includes: cystogastrostomy, cystojejunostomy and cystoduodenostomy. Laparoscopic drainage procedure can be attempted using endo-stapler or with intra corporeal suturing.

Materials and methods: In a retrospective study of 63 patients including both male and female with age 18-65 years underwent laparoscopic cystogastrostomy from may 2013 to 2017.

Of these 39 patients underwent stapler cystogastrostomy and 34 underwent handsewn anastomosis. These patients were followed up for a period of 1 year.

Patients were assessed in terms of duration of surgery,cost, length of hospital stay, and complications.

Results: Laparoscopic cysto- gastrostomy is a safe and effective technique for management of pseudocyst of pancreas.

Stapler cystogastrostomy was found to be more costly, less time consuming with lesser rates of anastomotic leak and better haemostasis of suture line and easier to perform. Sutured cystogastrostomy has a steep learning curve as it requies meticulous intra corporeal suturing. 


\section{P615}

\section{Health Literacy, A Management Dilema in Carcinoma}

Samia Yunas ${ }^{1}$, Malik Muhammad Makki ${ }^{2}$, Saba Yunas ${ }^{3} ;{ }^{1}$ Emirates Specialty Hospital, Dubai, ${ }^{2}$ Sheikh Khalifa Medical City Ajman, UAE, ${ }^{3}$ Dublin

Introduction: American Cancer society indicates 100\% Breast Cancer survival rate for early disease. Despite improvement in screening and treatment of Breast Carcinoma, SEER (Surveillance, Epidemiology \& End Result Program) data from 2009-2013 shows new Breast cancer case were 12.5 per 100,000 women/year and the deaths were 21.5 per 1000 women/year. The estimated cost was calculated as 180 billion dollars.

Literacy has a profound impact on understanding disease process and outcome. Being educated, does not mean health literate. In 2003 NAAL, studied that only 12 percent of adult demonstrate Proficient health literacy in United States. An individual's health literacy may be worse than his general literacy.

Low health literacy leads to lack of screening, advanced stage at diagnosis, decreased acceptance and poor compliance with treatment. It ends with worse mortality and morbidity outcome. Material \& Methods: Our cross sectional study enrolled 100 histopathologically proven breast carcinoma patients. Different variables included patient's education level, duration of symptoms, clinical stage on first clinical visit and TNM classification.

Result: Total of $22 \%$ patients presented in stage- 1 while $12 \%$ in advance metastasis. Patient's first healthcare advice was at 1-5 months while Speciality clinic visit was within one year. $18 \%$ of the patients finished high school and 33\% were illiterature.

Conclusion: People with low literacy seek late medical advice and present at advance stage. Their knowledge about disease process and disease outcome was not optimal. Social, cultural and family factors were big contributors. Improved health literacy is the key to improve disease outcome \& lower health cost burden.

\section{P617}

Sentinel Lymph Node and Function-Preserving Gastrectomy in the Patients with Early Gastric Cancer

Vladimir Grubnik, MD, Professor, Vadim Ilyashenko, PhD, Viktor Grubnyk, Resident, Raisa Romak, PhD, Yurii Grubnik, MD, Professor; Odessa National Medical University

Laporoscopy-assisted distal gastrectomy or laporoscopy-assisted total gastrectomy with D2 lymph node dissection is standard for treatment of gastric cancer. However, patients' quality of life maybe seriously affected by late-phase complications, including dumping syndrome, weight loss, nutritional deficit. Partial gastrectomy, segmental gastrectomy, proximal gastrectomy with limited lymph node dissection can improve postoperative late-phase function.

Sentinel lymph node (SLN) concept theoretically can improve results of surgical treatment of early gastric cancer.

Aim of the Study: was analyze the possibility of function preserving gastrectomy based on the sentinel node concept.

Methods: During last 5 years in our clinics we used mapping procedures in the patients with gastric cancer. In our clinics patients with clinical T1,T2 tumors primary lesions less than $5 \mathrm{~cm}$ in diameter, and clinical N0 gastric cancer undergo SN mapping and biopsy. Blue dye was injected into 4 quadrants of the submucosal layer of the primary side with endoscopic puncture needle and the beginning of surgery in 16 patients. Blue lymphatic vessels and blue-stained lymph nodes can be indentified by laparoscopy within $15 \mathrm{~min}$ of the blue dye injection. After dissection and examination of SLN the patients were operated. Laparoscopic distal gastrectomy with D2 lymph node dissection was performed in all 16 patients. New technology indocyanine green (ICG) fluorescent imaging was used for sentinel node mapping in 7 patients with gastric cancer.

Results: Among16 patients sentinel lymph nodes were negative in 7 patients. No metastasis were detected in other lymph nodes, which were removed during D2 lymph node dissection,. In 9 patients with positive SN metastasis were detected in 8 patients. Among 7 patients in whom we used ICG fluorescent mapping positive SN was detected in 2 patients. Laparoscopic-assisted dista gastrectomy with $\mathrm{D} 2$ lymph dissection was performed in these patients. In 5 patients with negative SN partial wedge resection was performed in 2 patients, segmental pylorus preserving gastrectomy was performed in 3 patients. During follow-up period from 3 to 18 months no recurrences or metastasis were detected in these group of patients. QoL in this group of patients was much better than in the patients with conventional distal gastrectomy.

Conclusion: ICG fluorescent method is highly effective for detection of sentinel nodes. In the patients with early stages gastric cancer function preserving gastrectomy based on SN navigation may be promising strategy to achieve better results.

\section{P618}

Efficacy of Laparoscopy in Diagnosis and Treatment of Chronic Abdominal Pain of Unknown Origin

Jamil S Samaan, BS, Emily S Chang, BA, Nadav J Hart, BA, Evan T Alicuben, MD, Allan Ghlandian, MD, Kamran Samakar, MD; University of Southern California

Introduction: Chronic abdominal pain of unknown etiology is a difficult condition to treat. Patients often undergo exhaustive medical testing without a definitive diagnosis. Diagnostic laparoscopy (DL) has the potential to diagnose and treat these patients although there is limited evidence of its efficacy. We aim to evaluate the role of laparoscopy in both diagnostic and therapeutic management of chronic abdominal pain of unknown etiology. Additionally we aim to explore patient satisfaction and efficacy of laparoscopy.

Methods and Procedures: This was a retrospective review of twenty-nine patients who underwent diagnostic laparoscopy for chronic abdominal pain of unknown origin from July 2009 to November 2017. Preoperative information was collected through chart review and patients were contacted by phone and given a follow-up survey to determine recurrence of pain and their post-operative satisfaction.

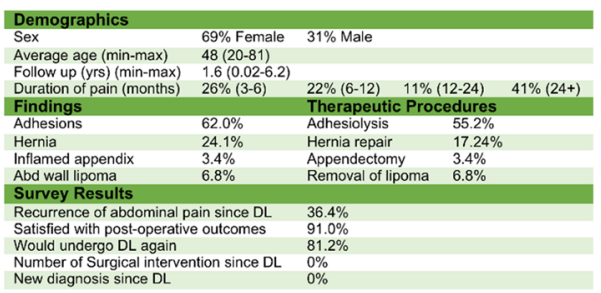

Conclusion: $86.2 \%$ of laparoscopies performed resulted in a therapeutic procedure demonstrating the efficacy of laparoscopy as a diagnostic and therapeutic method. Patient satisfaction and willingness to undergo the procedure again were very high. Laparoscopy appears to be an effective modality for the diagnosis and treatment of chronic abdominal pain with high patient satisfaction. Diagnostic laparoscopy should be considered early in the evaluation of patients with chronic abdominal pain of uknown etiology. 
P619

Strategy for the Treatment of Elderly Patients with Siewert Type II Esophagogastric Junction Cancer

Masazumi Sakaguchi, MD, PhD, Norihiro Shimoike, MD, Shin Akagawa, MD, PhD, Seiichiro Kanaya, MD, PhD; Osaka Red Cross Hospital

Background: There is a lack of clarity on the optimal surgical procedure for Siewert type II esophagogastric junction cancer (type II EGJ cancer). We proposed laparoscopic proximal gastrectomy (LPG) with esophagogastric tube reconstruction as the treatment of choice for early-stage type II EGJ cancer and/or for high-risk patients who required tumor resection. In the present study, we evaluated the shortterm outcomes of patients with type II EGJ cancer undergoing LPG. Method: We performed a retrospective descriptive study of patients with type II EGJ cancer who underwent LPG at our hospital between April 2011 and April 2018.

Results: We identified 29 consecutive patients with type II EGJ cancer who were planned to undergo LPG with transhiatal lower paraesophageal lymphadenectomy. The median follow-up was 591 days (interquartile range, IQR: 1042-329). The median age was 75 years (IQR: $80-68$ ). The number of patients with ASA-PS 2 or 3 was $21(72.4 \%)$. The number of patients with ECOG-PS 2 or 3 was 14 $(48.1 \%)$. The clinical stage (AJCC 7th) was IA:IB:IIA:IIB:IIIA:IIIB:IIIC:IV = 9:4:1:2:4:1:7:1. All tumors were found to be an adenocarcinoma. Only 3 patients received neoadjuvant chemotherapy. Twenty-eight patients underwent LPG with perigastric, suprapancreatic, and transhiatal lower paraesophageal lymphadenectomy, and one patient converted to transthoracic esophagectomy to guarantee safety of the proximal margin. The Clavian - Dindo classification grade 2 and higher complication rate was $48.3 \%$. The median postoperative stay was 16 days (IQR: 30-13). The pathological stage was IA:IB:IIA:IIB:IIIA:IIIB:IIIC:IV = 6:4:4:1:3:2:5:4. The 1-year overall survival rate was found to be $72.4 \%$.

Conclusion: LPG can be a successful treatment for elderly patients.

\section{P620}

\section{Beneath the Bandages: Conceptualizing Surgery in Patient Narrative}

Magdalene Lugowski, MD; Memorial University of Newfoundland

Introduction: Patients often turn to narrative to grapple with the meaning of illness. Technology such as social media encourages such activity on a large scale. However, narratives of surgery are complex to construct and commonly incorporate information from multiple sources. It is worth considering the ways in which patients reconstruct their surgical experiences to understand what information patients seek out and how surgeons can be of help.

Methods: This analysis offers a close reading and considers the narrative techniques used in two accounts of surgery written by patients two hundred years apart. Frances Burney's account of her mastectomy, completed without anaesthesia, was composed as a letter to her sister Esther Burney. The second text, Brain on Fire, was originally written as an article for the New York Post by Susannah Cahalan, and later expanded into a book, which has, in turn, inspired a movie by the same title. She too recounts her surgery, but twice removed from her lived experience, by both madness and modern anaesthesia.

Results: Though their circumstances differ greatly, Burney and Cahalan deploy some of the same techniques to narrate their surgical experience. Subverting genre expectations regarding the directness of their chosen methods of communication - letter, memoir - the authors create carefully crafted texts, in which they assume the medical gaze for their own use, mingling this with their own voices to reclaim the experience. The effect differs however, as Burney's account contains the vivid recollection of truly living through surgery unanaesthetized, while Cahalan must fall back on her professional persona, and rely on her medical record and the voices of others, as her own memories (if they exist) are largely inaccessible. In adopting the medical gaze, both authors engage to an extent with the experience of their surgeons. The construction of each narrative and the various literary techniques employed by each author raise questions about the extent to which anyone can narrate their own surgery coherently and invite readers to contend with the complexity of trying to capture such an experience. Conclusion: The texts considered here were written by women who were writers before they became patients, and they employ their preexisting narrative skills to produce these accounts, using similar techniques. Exploring the ways in which patients narrate surgical experience can allow clinicians to develop effective partnerships with patients that support this process of seeking meaning in illness. 


\section{P621}

\section{Management of a Migrated Jejunostomy Tube}

Brandon C Dessecker, MD, Safi Haq, MD, D James Smith, MD; Northside Medical Center/Western Reserve Health Education

Introduction: Enteral feeding accesses are some of the most morbid surgical procedures. Misplacement, dislodgement, clogging, and wound complications are among the most common of these complications. We present a case of a patient who had underwent placement of a feeding jejunostomy secondary to esophageal cancer where the tube migrated after a recent replacement.

Case Report: 59 year old male with a history of esophageal cancer status post esophagectomy and gastric pull through as well as a feeding jejunostomy placement presented to the emergency room after he thought that his feeding catheter had fallen out overnight. He was unable to find the catheter within his home. He recently was seen at another institution after he had his feeding catheter fall out as well. A Foley catheter was placed at the institution in order to allow for feeding access. He underwent abdominal radiography, which demonstrated the catheter in his abdomen (Figure 1), and further underwent computerized tomography (CT) in order to further evaluate the location of the catheter. CT demonstrated the catheter within his terminal ileum and the Foley catheter balloon inflated.

He was admitted with plans to monitor radiography in hopes that the tube would pass into the colon. The following day there was noted to be no movement of the catheter on radiography. Given this, an attempt was made to was made to attempt to deflate the balloon using a percutaneous, CT guided needle, which was unsuccessful. The following day he proceeded to surgery for small bowel enteroscopy, through his jejunostomy, in order to attempt to remove the catheter from proximally. This was also unsuccessful, and required a laparotomy with enterotomy to remove the catheter (Figures 2). In image 2, by the surgeon's right hand hemorrhage was noted at the small bowel from the attempted CT deflation of the catheter.

Conclusion: While this is a rare complication of a jejunostomy tube, there are several lessons that can be learned. We believe that the Foley catheter balloon was overinflated, which allowed peristalsis to carry it through the small bowel before lodging at the ileocecal valve. While a Foley catheter can be used for maintaining an enteral feeding tract, true feeding tubes have an external bumper, which allows apposition against the abdominal wall and prevent tube migration.
Figure 1

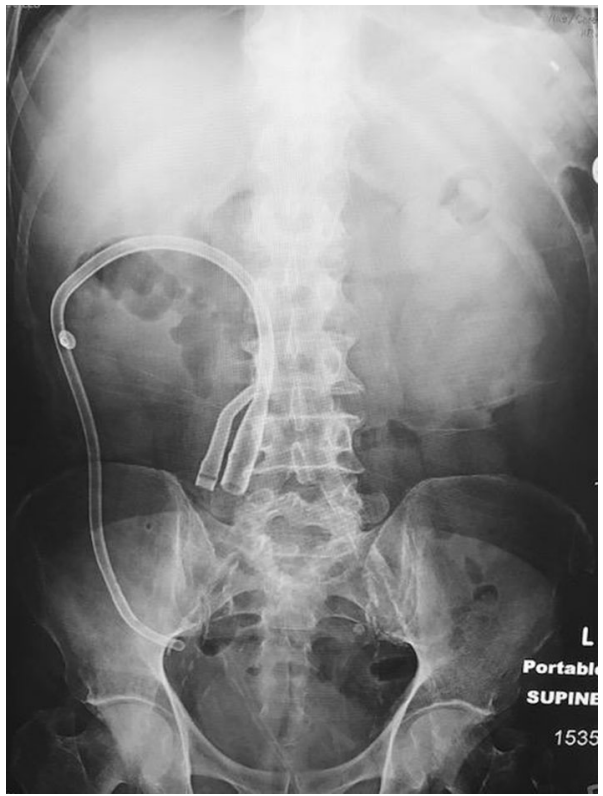

Figure 2

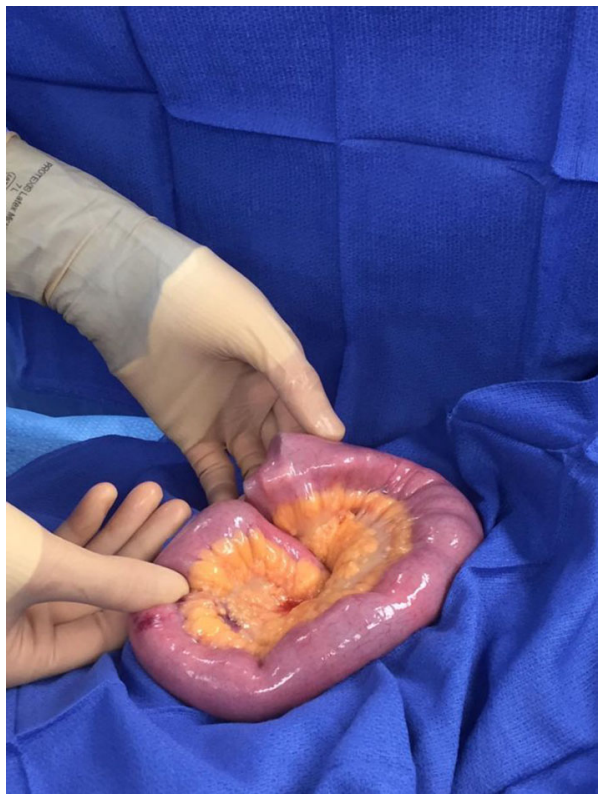


P622

The Development and Future of Digest Tract Reconstruction After Distal Gastrectomy: A Systemic Review and Meta-analysis

Xijie Chen ${ }^{1}$, Yunzhi Chen ${ }^{1}$, Shi Chen ${ }^{2}$, Junsheng Peng ${ }^{2} ;{ }^{1} \mathrm{MD},{ }^{2} \mathrm{Ph} . \mathrm{D}$

Abstract: Background: Billroth I, Billroth II, Roux-en-Y, and Un-cut Roux-en-Y are common reconstruction techniques of distal gastrectomy. Which of these techniques is better has yet to be established. We performed an indirect comparison to evaluate which technique was optimal for preventing reflux symptoms.

Methods: The PubMed, Cochrane Collaboration, Embase, ClinicalTrials.gov and Web of Science databases were searched to identify clinical trials that compared at least two of the reconstruction skills among Billroth I, Billroth II, Roux-en-Y, and Un-cut Roux-en-Y. Data on reflux gastritis, intraoperative blood loss, bile reflux and postoperative hospital stays were extracted from the included clinical trials for meta-analysis using a random-effects model.

Results: Twenty-four articles that included 5419 individuals were assessed as eligible for metaanalysis. The indirect comparison suggested that Roux-en-Y reconstruction significantly reduces reflux gastritis, and it tended to rank first and had the highest probability of preventing bile reflux. No significant differences were found in intraoperative blood loss and postoperative hospital stays. Conclusion: This indirect comparison suggested some superiority of Roux-en-Y reconstruction after distal gastrectomy. Further perspective clinical trials are required to provide evidence for the optimal reconstruction skil

\section{P624}

Short-Term Outcomes of Laparoscopic Spleen-Preserving Distal Pancreatectomy: A Comparative Analysis with Laparoscopic Distal Pancreatectomy with Splenectomy

Takanori Morikawa, MD, $\mathrm{PhD}$, Masahiro Iseki, MD, PhD, Masaharu Ishida, MD, PhD, Tatsuyuki Takadate, MD, PhD, Kyouhei Ariake, $\mathrm{MD}, \mathrm{PhD}$, Kei Kawaguchi, MD, PhD, Kunihiro Masuda, MD, PhD, Takeshi Aoki, MD, PhD, Hideo Ohtsuka, MD, PhD, Masamichi Mizuma, MD, PhD, Shinobu Ohnuma, MD, PhD, Hiroki Hayashi, $\mathrm{MD}, \mathrm{PhD}$, Kei Nakagawa, MD, PhD, Fuyuhiko Motoi, MD, PhD, Takashi Kamei, MD, PhD, Takeshi Naitoh, MD, Ph, FACS, Michiaki Unno, MD, PhD; Tohoku University

Introduction: Laparoscopic distal pancreatectomy (LDP) is widely accepted as a standard treatment for the tumors located in the pancreas body or tail, and spleen-preserving distal pancreatectomy was established in benign or low-grade malignancies of the distal pancreas which do not need lymphatic clearance. However, it is still controversial whether or not to preserve spleen in case of LDP for benign or low-grade malignant tumor, because laparoscopic spleen-preserving distal pancreatectomy (LSPDP) has some technical difficulties. In our institution, we have performed LSPDP only for the patients with small neuroendocrine neoplasms based on preoperative imaging for the reason of its low malignancy. The aim of this study is to evaluate the feasibility and safety of LSPDP compared with LDP with splenectomy (LDPS)

Methods and Procedures: From July 2009 to August 2018, we performed 76 LDPs (LSPDP: $\mathrm{n}=14$ LDPS: $n=62$ ) in our institution. We reviewed clinical records of these patients, and clinicopathological findings, surgical outcomes, and diagnostic accuracy were retrospectively analyzed.

Results: In this cohort, there were 54 females, and the median age was 55.5 years old. Two patients who received LDPS were converted to open surgery due to severe pancreatitis and adhesion around the pancreas. On the other hand, one patient in the LSPDP group was converted to hand-assisted LDPS because of strong inflammation between the pancreas and the splenic vessels. There was no significant difference in their characteristics such as age, sex, body mass index and ASA score, however LSPDP was significantly associated with small-sized tumor and nodular tumor. The operation time of LSPDP was significantly longer than LDPS ( $p=0.02)$, meanwhile blood loss of each group was similar $(p=0.39)$. In addition, the length of skin incision in the LSPDP group was significantly smaller than the LDPS group. The rate of complication including pancreatic fistula and postoperative courses had no significant difference between groups.

Conclusion: In selected patients, LSPDP is safe and feasible procedure with smaller incision and similar postoperative outcomes compared with LDPS.

\section{P625}

Endoscopic Thyroidectomy Via Bilateral Axillo-Breast Approach With or Without Intraoperative Nerve Monitoring in Patients with Papillary Thyroid

\section{A Very Low-Cost Negative Pressure Wound Therapy as an Alternative to Periotoniostomy: Case Report}

Matheus D Gomes Gonçalves, MD ${ }^{1}$, Diego L Lima, MD ${ }^{2}$, Raquel N Cordeiro, Medical Student ${ }^{3}$, Ítalo C Moreira, MD ${ }^{1}$, Fabio Eduardo R Rabelo Ferreira, $\mathrm{MD}^{1}$, Carlos Esdras A Moraes, MD ${ }^{1}$, Balduíno G Nóbrega Junior, $\mathrm{MD}^{1}$, Henrique $\mathrm{G}$ Araújo, $\mathrm{MD}^{1}$, Adalberto $\mathrm{G}$ Araújo ${ }^{1}$, Márcio Rogério C Carvalho, $\mathrm{MD}^{1}$; ${ }^{1}$ Getulio Vargas Hospital, ${ }^{2}$ State Servers Hospital, ${ }^{3}$ Pernambuco Health College

Case Report: A 48 years-old male with an ileostomy secondary to a previous firearm wound presents with a parastomal hernia and intestinal semi-occlusive obstruction. Patient was submitted to surgical repair with the resection of the distal ileum, with a latero-latero ileum-ascending colon anastomosis. Furthermore, a loop colostomy with the transverse colon was performed. In the 5th postoperatory day, there was anastomotic dehiscence. The patient was submitted to a new laparotomy where an enteric resection, an ileostomy, and a mucous fistula were performed. The patient had two more surgical procedures due to small bowel perforation, and a peritoneostomy was performed with the Bogota bag, due to the impracticable aspect of the aponeurosis. Three days later, due to great accumulation of intra-abdominal secretion and clinically worse, the Bogota bag was removed, and the surgical team opted for a negative pressure wound therapy after a cavity toilet. The patient changed 10 times the dressings and the therapy had total time of 38 days. Since it's a public hospital with limited access to this type of therapy, the department of surgery developed a low-cost therapy using dressings, suction drains, sterile videosurgical plastic bags, and a transparent polyurethane film connected to the hospital vacuum system with a negative pressure of $125 \mathrm{mmHg}$. Every time they changed the dressings and other materials, it had a cost of USD 18 Conclusion: The low-cost negative pressure wound therapy was safe and effective for the patient who was discharged after 38 days in good clinical conditions. This therapy was affordable for the department which can't afford other dressings available in the market. Methods: The prospective cohort consisted of 50 patients undergoing endoscopic thyroidectomy

\section{Jiyang Li, Chen Liu, Hongqing Xi, Bo Wei, Lin Chen, Zhi Qiao;} Chinese PLA General Hospital

Brackground: Bilateral axillo-breast approach has the cosmetic superiority of various techniques for endoscopic thyroidectomy. Nevertheless, inadequate exposure of surgical field may increase the risk of recurrent laryngeal nerve injury. Vocal cord palsy occurs in $12 \%$ of patients with papillary thyroid carcinoma after endoscopic thyroidectomy. In recent years, intraoperative nerve monitoring (IONM) has been introduced into thyroidectomy procedures. This study investigated the risk of postoperative vocal cord palsy with or without the use of intermittent IONM in endoscopic thyroidectomy. for papillary microcarcinoma from January 2015 to December 2017. IONM was used in 26 operations $(52 \%)$, which is named IONM group; the other 24 patients not using IONM were included in the NO-IONM group. Transient hoarseness was diagnosed within 6 weeks after surgery. Permanent palsy of the recurrent laryngeal nerve was defined as that persisting after 6 months. The latest follow-up date was June 30, 2018. Patient demographics, clinicopathologic characteristics and surgical-related parameters were analyzed. Univariable and multivariable logistic regression analyses were used to examine risk factors for vocal cord palsy.

Results: No significant differences in demographic or clinicopathologic characteristics were noted between the groups. The number of retrieved ipsilateral lymph nodes was larger in the IONM group compared with the NO-IONM group $(4.3$ vs $2.1 \mathrm{P}=0.030)$. Mean operation time was shorter for the IONM group compared with the NO-IONM group (118 vs. $149 \mathrm{~min}$, respectively), but this did not reach statistical significance $(\mathrm{P}=0.125)$. Estimated blood loss, drain maintenance, drain amount and hospitalization length were similar between the two groups. Transient hoarseness occurred in 3 patients $(6 \%)$ of the NO-IONM group and 1 patient $(2 \%)$ of the IONM group. Permanent vocal cord palsy occurred in 2 patient $(4 \%)$ of the NO-IONM group. Other postoperative complications included 2 cases of postoperative bleeding, 1 case of skin bruising on the chest wall, and 5 cases of transient hypocalcemia. In the multivariable analysis, the use of IONM was associated with a decreased risk of transient hoarseness ( $95 \%$ OR $0.91,0.64$ to 1.47 ), but decreased risk of permanent vocal cord palsy ( $95 \%$ OR: $0.59,0.21$ to 0.98$)$.

Conclusion: With satisfactory cosmetic results, endoscopic thyroidectomy via bilateral axillobreast approach with IONM reduced the risk of permanent vocal cord palsy in patients with papillary thyroid carcinoma. This technical combination might gain wider acceptance in the near future. 
P626

Laparoscopic Management of a Rare Intra-abdominal Undescended Testicular Malignancy in an Adult

Ajay Bhandarwar, MS, FMAS, FIAGES, FAIS, FICS, FBMS, FIAGES, FAI ${ }^{1}$, Raj N Gajbhiye, Professor \& Head, Surgery,Govt. Medical College, Nagpur ${ }^{2}$, Nidhisha Sadhwani, MBBS ${ }^{1}$, Amol Wagh, MS, FMAS, FIAGES, FAIS, FICS, FBMS ${ }^{1}$, Eham Arora, MS, DNB ${ }^{1}$, Shekhar Jadhav ${ }^{1}$, Ameya Tibude, MS ${ }^{1}$, Shivang Shukla ${ }^{1}$; ${ }^{1}$ grant Government Medical College \& Sir J.J. Group of Hospitals, Mumbai, India, ${ }^{2}$ Govt. Medical College, Nagpur,Maharashtra,India

Introduction: Intra-abdominal undescended testes in adults is extremely rare, so is the development of a malignancy in it.

On our extensive search in literature, we could not find any documented case of an intra-abdominal undescended testicular neoplasm in adults managed laparoscopically. To the best of our knowledge, this is the first ever reported case.

Clinical Case: A 52 yr old male patient with an intra-abdominal undescended testicular malignancy was operated laparoscopically while confirming with the oncosurgical principles. Histopathology was suggestive of a seminoma with tumour free margins. The patient developed no local recurrence in a follow up period of 6 months.

Conclusion: There is currently no universally accepted consensus of the role of minimally invasive surgery in the treatment of an intraabdominal undescended testicular cancer.

Based on our experience, for an operable undescended intra-abdominal testicular malignancy, laparoscopic resection is an easy and a viable option.

\section{P627}

Plan-do-check-act (PDCA) Approach to Improve Quality of Care for Surgical Patient: A Randomized Controlled Trial

Hemanga Bhattacharjee, Pritam Mandal, Virinder Bansal, Anu Sachdeva, Mamta Sood, Ashok Deorari; All India Institute of Medical Sciences

Introduction: Adequate and relevant preoperative information is pertinent for patient satisfaction, thus for quality of care. The spectrum of information patients desire may vary according to their socioeconomic or cultural background. Hence, patients' participation in the development of a preoperative information module is important in quality improvement initiative.

Plan-Do- Check-Act (PDCA) cycles and Focussed Group Discussion (FGD) are important aspects of Quality Improvement (QI) initiative. Herein, we have described the development of a module for preoperative information and a randomised controlled trial to evaluate the effectiveness of this module.

Materials and Methods: The study was conducted in a public funded teaching hospital catering to a population of middle and low socio economic strata. Institute's ethics committee approved the study protocol. The study was conducted in two phases i.e. Phase 1: Development of the QI module and Phase 2: Evaluation of the module by a randomized study. Patients undergoing laparoscopic cholecystectomy and laparoscopic groin hernia surgery were enrolled.

Phase 1: Researchers did the root cause analysis, interviewed thirty patients and prepared the first draft of the QI module as a leaflet. Module was then implemented in 30 patients. Patients' satisfaction score (PSS) was recorded on a standardized validated proforma. Six randomly selected patients from this group were then called for FGD. QI module was then improvised based on patients' feedback during FGD. PDCA cycles were repeated and additional information was added to the QI module based on FGD feedback. Post 3rd PDCA, PSS reached more than $90 \%$ and the QI module, comprising scientific, social and logistical information on the disease and the treatment process, was standardized.

Phase 2: Sixty patients were randomized. Control group $(\mathrm{n}=30)$ received spoken information, as done in conventional protocol; study group $(n=30)$ received spoken and written information in the form of QI module (leaflet) in addition to conventional protocol. WHOQOL BREF questionnaire was used for assessment of quality of life. Patients' satisfaction was analyzed using PSS.

Results: The demographic profile and preoperativeWHOQOL BREF scores were identical between the two groups.In post-operative period, all domains of WHOQOL BREF showed improvement, however, the improvement in psychological health and overall domain was statistically significant in study group as compared to control group ( $p=0.002$ and 0.01 respectively). The overall quality of life, satisfaction with health and Patients Satisfaction Score (PSS) were significantly more in study group $(\mathrm{p}=0.02,0.005$ and 0.001 respectively).

Conclusion: Patients' satisfaction and perception of care can be improved by preoperative QI module. 


\section{P628}

'Double Volvulus' of Small Intestine: A Rare Cause of Mechanical Obstruction in Chronic Intestinal Pseudo Obstruction

Nitin Vashistha, MS, FACS ${ }^{1}$, Dinesh Singhal, MS, FACS, DNB, Surgical, Gastroenterology ${ }^{1}$, Shanti Swaroop Dhar, MD, DM², Bharat Aggarwal, MD ${ }^{3}$, Anurag Krishna, MS, $\mathrm{Mch}^{4} ;{ }^{1}$ Department of Surgical Gastroenterology, Max Super Speciality Hospital, Delhi, India, ${ }^{2}$ Department of Gastroenterology, Max Super Speciality Hospital, Delhi, India, ${ }^{3}$ Department of Radiology, Max Super Speciality Hospital, Delhi, India, ${ }^{4}$ Department of Pediatric Surgery, Max Super Speciality Hospital, Delhi, India

Background: Chronic intestinal pseudo obstruction (CIPO) is a rare syndrome of intestinal dysmotility caused by disorder of enteric nerve or muscles. The characteristic feature is recurrent intestinal obstruction in absence of mechanical lesion. It usually progresses to debilitating intestinal failure with potentially life threatening complications.

Case report: We managed 2 teenaged real brothers presenting with CIPO. The younger brother had multiple surgeries and died due to intestinal failure. The elder brother became symptomatic for the disease at the age of 15 years and had repeated hospitalizations for recurrent intestinal obstructions which were managed conservatively (Figure 1). At the age of 21 years he was readmitted with acute intestinal obstruction retention of urine. Following initial failed conservative management, abdominal computed tomography was performed which was suggestive of small intestine volvulus (Figure 2). At laparotomy there was volvulus at proximal ileum with 360 degrees rotation (Figure 3). On further exploration there was another volvulus in distal ileum with 3 turns of 360 degrees each (Figure 4). There was narrowing at terminal ileum approx $5 \mathrm{~cm}$ proximal to IC junction. Derotation of both volvulus was performed. Postoperative recovery was slow but progressed to initiation of enteral feeding.

Discussion: A majority of CIPO patients undergo a mean of 2.96 useless and potentially dangerous surgeries (Stanghellini 2005). Hence it is extremely important to make all efforts to avoid a non therapeutic laparotomy. The most important clue to diagnosis of CIPO is recurrent symptoms and signs of bowel obstruction with radiologically dilated bowel in the absence of demonstrable obstructing luminal lesion. A judicious use of abdominal computed tomography (CT) scan can be an invaluable adjunct for the purpose. Our patient was taken up for surgery only after a definitive diagnosis of small bowel volvulus was made. Few case reports have previously reported small bowel volvulus in the setting of CIPO (de Betue CT 2011). However to the best of our knowledge volvulus at two different locations causing obstruction (Double Volvulus) in one patient at the same time has not been reported to date.

Conclusions: Initial management for CIPO patient presenting with AIO is essentially conservative. Patients not responding to expectant management should be investigated with abdominal CT scan to rule out mechanical cause of AIO. Patients with mechanical AIO should be managed by appropriate surgery. All endeavors should be made to avoid non therapeutic laparotomy.

\section{Figure 1}

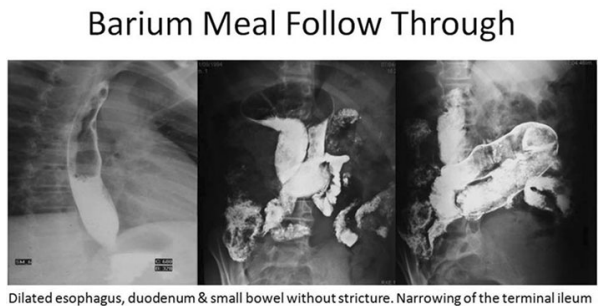

Figure 2

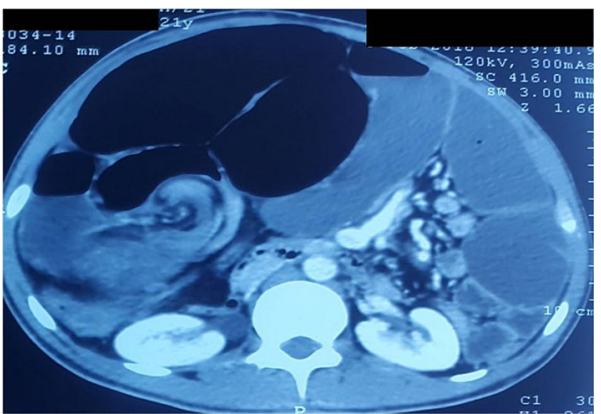

Figure 3

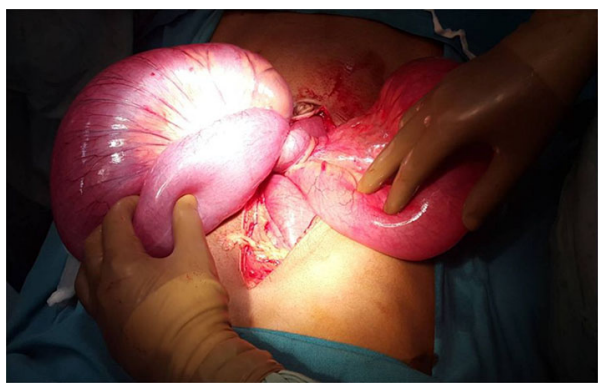

Figure 4

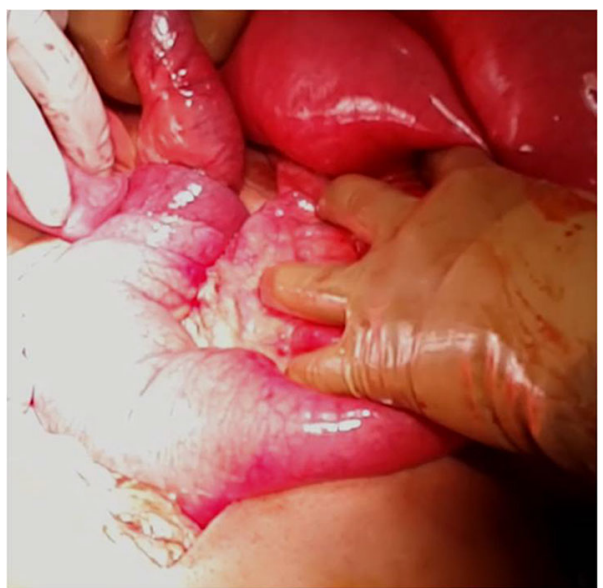




\section{P629}

\section{Pancreatoduodenectomy Damage Control with Gastrointestinal Reconstruction Two Times: Strategy Against Severe Trauma Pancreaticoduodenal. Multicenter Study}

Luis F Cabrera, General Surgery ${ }^{1}$, Mauricio Pedraza ${ }^{1}$, Sebastian Sanchez $^{1}$, Juliana Ordonez, MD, FACS, General Surgeon ${ }^{2}$, Paula Lopez $^{1}$, Felipe Bernal ${ }^{1}$, Patricia Parra ${ }^{1}$, Carlos Lopez ${ }^{1}$, Jean A Pulido ${ }^{1}$, Daniel Gomez, MD, FACS ${ }^{2}$; ${ }^{1}$ Bosque University, ${ }^{2} \mathrm{New}$ Granada Military University

Introduction: An emergency pancreatoduodenectomy is a surgery that is currently used for the management of severe pancreatoduodenal trauma after damage control surgery.

Objectives: To show the experience of pancreatoduodenal trauma management with emergency pancreatoduodenectomy and damage control.

Materials and Methods: We carried a retrospective observational study of a series of cases with severe pancreatoduodenal trauma and reviewed articles published in journals indexed in PubMed and ScienceDirect databases in the last 10 years. We completed the search with the review of classic articles on the subject and compared our experience with those reported in the world medical literature.

Results: In a period of 6 years, 4 patients with severe pancreatoduodenal trauma with injuries due to firearm projectile were obtained, of which $75 \%$ were men, with an average age of 17.5 years, with an average ISS score of 39.5 points and associated vascular trauma in $100 \%$ of cases. In the 4 cases, damage control and emergency pancreatoduodenectomy were performed and in a second surgical time the gastrointestinal tract reconstruction was carried out. A $75 \%$ incidence of surgical site infection were reported, $25 \%$ health care associated pneumonia and $50 \%$ postoperative pancreatic fistula. With an average stay in the intensive care unit of 12.25 days, average hospital stay of 29.5 days and no mortality. Conclusions: We evidenced that the resection after the damage control surgery in pancreatoduodenal trauma is effective for the management of this entity, although its accomplishment is a surgical challenge, it is a very useful procedure in the trauma surgery. Keywords: pancreas; duodenum; abdominal trauma; penetrating injuries; pancreaticoduodenectomy.

\section{P631}

Opioid Use Reduction After Subcutaneous Infiltration with Local Anesthesia During Surgery

Adrian Maghiar, George Dejeu, Mihai Botea, Dan Ciurtin, Marius Sfirlea, Octavian Maghiar, Cipri Puscas; Spitalul Pelican Oradea

We present the preliminary findings of a study we started in our institution, Pelican Hospital in Oradea, Romania. In the past 12 months we started a study to quantify the reduction of opioid pain medication need reduction in patients with major abdominal surgery that get wound margins infiltration of local anesthesia during closure.

During this period we measured the pain score as decided by the patients (using a 10 degrees pain score meter) at $1 \mathrm{~h}, 3 \mathrm{~h}, 6 \mathrm{~h}, 12 \mathrm{~h}$ and $24 \mathrm{~h}$ after surgery. We divided the patients in 3 groups. First group, the control, we used our usual protocol of pain management using opioids, the second group we used a revised protocol using paracetamol and metamizole, and the third group we used our revised protocol and the infiltration of the surgical wounds at the time of closure with local anesthesia.

The results show a clear reduction in pain score at 1,3 and $6 \mathrm{~h}$ after surgery for the group using the local anesthesia, with a statistical significance. The difference between the standard and revised protocols of pain management (with no local anesthesia) was not statistically significant, with a very slight advantage for the opioid group. However the results at 12 and $24 \mathrm{~h}$ were more interesting, with pain scores much lower for the local anesthesia group, and also the revised protocol group, with a overall reduction in the need for opioid treatment of more than $80 \%$ when compared with a similar group from 12 months before the study begun. We continue to research the implications of the use of the revised protocol, that now includes the infiltration of surgery wounds with local anesthesia at the time of closure. And are looking at the economic impact on the surgery ward budget the reduction in opioid use brings. One interesting finding is the reduction in hospital stay for patients with less than 2 doses of opioid needed for pain control, of as much as 3 days on average, an effect that we are currently studying more closely with a different study.

\section{P630}

\section{Long-Term Follow-up Outcomes of Fundoplication in the Obese Population}

Hana Fayazzadeh, MD ${ }^{1}$, Christopher R Daigle, $\mathrm{MD}^{2}$, Raul J Rosenthal, MD ${ }^{3}$, Stacy A Brethauer, MD ${ }^{1}$, Philip R Schauer, MD ${ }^{1}$, Kevin M El-Hayek, MD ${ }^{1}$, Matthew Kroh, $\mathrm{MD}^{4}$; ${ }^{1}$ Cleveland Clinic, ${ }^{2}$ Cleveland Clinic Akron General, ${ }^{3}$ Cleveland Clinic Weston Florida, ${ }^{4}$ Cleveland Clinic Abu Dhabi

Objective: Gastro-esophageal reflux disease (GERD) occurs in $20 \%$ of the adult population in the United States. Its incidence is increased in patients with higher Body Mass Index (BMI). The gold-standard anti-reflux option is laparoscopic fundoplication, however its effectiveness is reportedly less in patients with high BMI. We aim to describe long-term outcomes of primary fundoplication in obese patients at our institution.

Methods: All patients with $\mathrm{BMI} \geq 30 \mathrm{~kg} / \mathrm{m}^{2}$ who underwent primary fundoplication between 2005 and 2015 were retrospectively studied. Pre-operative findings, surgical details, and long-term outcomes (follow-up time $\geq 6$ months) are reported.

Results: A total of 125 patients with medically refractory GERD who underwent primary Nissen $(\mathrm{N}=120)$ or Toupet $(\mathrm{N}=5)$ fundoplication were included. The mean age and BMI were $54.8 \pm 13.8$ years and $33.3 \pm 3.4 \mathrm{~kg} / \mathrm{m}^{2}$, respectively. Fundoplication was performed via laparoscopy in $86 \%(\mathrm{~N}=108)$. Mean operative time was $150.1 \pm 96 \mathrm{~min}$, estimated blood loss was $42.4 \pm 49.8 \mathrm{ml}$, and duration of stay was $3.3 \pm 2.7$ days. Mesh reinforcement was used in $24 \%$ of patients $(\mathrm{N}=30)$. During the post-operative period with mean follow up of $67.9 \pm 37.6$ months, patients with BMIs $30-35 \mathrm{~kg} / \mathrm{m}^{2}, 35-40 \mathrm{~kg} / \mathrm{m} 2$, and $\geq 40 \mathrm{~kg} / \mathrm{m}^{2}$ reported recurrent symptoms $29 \%(\mathrm{~N}=26), 70 \%(\mathrm{~N}=19)$, and $50 \%$ $(\mathrm{N}=4)$ of the times, respectively. Follow up radiographic or endoscopic evaluations confirmed anatomical recurrence in $21 \%(\mathrm{~N}=19), 55 \%(\mathrm{~N}=15)$, and $38 \%(\mathrm{~N}=3)$ of the aforementioned BMI categories, respectively.

Conclusions: Primary fundoplication in obese population may not result in the satisfactory outcomes due to high BMI and other comorbid conditions. Weight loss interventions may improve the benefits in this specific population.

\section{P632}

\section{Dissection Around Trachea and Bronchus in Salvage Thoracoscopic Esophagectomy}

Yusuke Taniyama, Tadashi Sakurai, Makoto Hikage, Chiaki Sato, Hiroshi Okamoto, Kai Takaya, Takeshi Naitoh, Michiaki Unno, Takashi Kamei; Tohoku University, Department of Surgery

Background: Salvage thoracoscopic esophagectomy after chemoradiation therapy is extremely difficult because of fibrotic and edematous change in mediastinal tissue. Especially when the tumor is adjacent to trachea or main bronchus, dissection would be much harder. Furthermore, tracheobronchial necrosis might happen in salvage esophagectomy if the surgeon did not pay much attention to preserve tracheobronchial blood supply. Thus, we present the twists and methods of dissecting around trachea and bronchus in salvage thoracoscopic esophagectomy, especially focused on the preservation of blood flow of trachea and bronchus.

Surgical Procedure: Prone position with single-lumen intubation tube are employed for thoracoscopic esophagectomy in our institution. Preservation of bronchial artery is important to prevent tracheobronchial necrosis which can be fatal complication. To preserve this artery, restructuring of bronchial artery by 3D-CT help to understand the location of this artery. We also avoid injuring the longitudinal anastomosis of inferior thyroid artery and bronchial artery which locate along both side of the tracheal wall. Dissection of carinal lymph node is not recommended, except the case which have metastasis in those lymph nodes. Magnified view by thoracoscopy is necessary during the dissection of trachea and bronchus in salvage esophagectomy. Intraoperative bronchoscopy would help to dissect the strong fibrotic adhesion between tumor and tracheobronchial membrane. Transmitted light at the tracheobronchial membrane from thoracoscopic view, or traction of the membrane from bronchoscopic view is the signal of thinning tracheobronchial membrane. Singlelumen intubation make this procedure possible.

Result: 108 cases of salvage esophagectomy was performed in our institution from 2001 to 2017. Among them, tracheobronchial necrosis had happened in 4 cases. All those cases were operated before 2008 when we started to preserve tracheobronchial blood supply. Although 12 cases had direct invasion to tracheobronchial and resulted in R2 resection, none of cases showed tracheobronchial injury during operation.

Conclusion: It is important to preserve the tracheobronchial blood supply in salvage esophagectomy. Magnified view by thoracoscopy and observation by bronchoscopy helps dissecting the fibrous tissue between tracheobronchial membrane and tumor. 
P633

\section{A Case of Tuberculous Peritonitis and the Role of Laparoscopy}

Fernando Arias, MD, FACS, Alma Burbano, Natalia Cortes M, Camilo Cetares, MD, Sergio Caceres, MD, Jose Daniel Guerra, MD; Fundacion Santa Fe de Bogota

We present the case of a previously healthy 23 year old female with abdominal pain, distention, nausea, vomit, diarrhea and fever of acute onset. Six months prior to this event she refers pleuritic chest pain and occasional fever. A cavitated pulmonary lesion on chest CT was found. Complete blood count evidenced lymphopenia, elevated erythrocyte sedimentation rate and an elevated CA 125. A bronchoscopy was perform and the lavage was negative. She developed ascites that was analyzed via paracentesis with a positive ADA. A CT scan revealed peritoneal thickening and a omental cake. Peritoneal TB was suspected and also a malignant mesothelioma was consider in the differential diagnosis. A single site laparoscopy (LESS) approach was performed for definitive diagnosis. Findings were a severe peritonitis with adhesions of the viscera with a friable serosa, the greater omentum was attached to the anterior abdominal wall, with fibrinoid membranes and loculated ascites. Multiple biopsies were performed, which were positive for Mycobacteria. A test for infection with the human immunodeficiency virus was negative. The sensitivity of asciticfluid culture for M. tuberculosis is low, and the diagnosis can require peritoneal biopsy. The patient was started on TB standard treatment with a favorable outcome.

\section{P635}

Clutter-Less (C-Less) Triangulation: Laparoscopy in Children by Replacing Conventional Ports

Minu Bajpai, MD, PhD, Kanika Sharma, MCh, Prabudh Goel, MCh; All India Institute of Medical Sciences, New Delhi, India

Objective: To facilitate laparoscopy in children by replacing ports with percutaneously placed stay sutures (Port-Less Triangulation) during laparoscopy.

Materials and Methods: Laparoscopic procedures in children from the period, December 2012 to July 2018 were included in this study. The effectiveness of replacing port in the small peritoneal cavity of children using percutaneously placed stay sutures ('pick \& fix') instead of conventional ports, was studied. The ease of placement of sutures, clarity of target, reduced operating time and per-operative complications were all noted. Procedure related morbidity and eventual patient/care-giver satisfaction with cosmetic outcome were also noted. Results: 90 children underwent laparoscopic procedures during the aforementioned duration and included cholecystectomy(64), appendicectomy(18) \& colectomy (8). Age ranged between 18 months to 16 years. Clarity of target, by removing the clutter of multiple ports (Clutter-Less) \& instruments and ease of dissection, by decreasing the total number of ports across the variety of procedures to which it was applied, was observed. Cosmetic results were excellent Conclusion: The technique underscores the minimally invasive virtue of laparoscopy \& could be applied to the wide range of endoscopy procedures. It, thus, acts as a substitute for conventional ports, the ultimate result being good cosmetic outcomes, ease of dissection with reduced overall operating time and patient satisfaction.

Keywords: C-LESS; Paediatric laparoscopy; Percutaneous 'Pick \& Fix' stitch; Cholecystectomy; Appendicectomy; Colectomy

\section{P636}

\section{Advantage of Laparoscopy in Diagnosing Tuberculous Peritonitis: A Case Report}

Leo Yamada ${ }^{1}$, Shinji Ohki ${ }^{1}$, Daisuke Ujiie ${ }^{1}$, Takahiro Sato ${ }^{1}$, Takkeshi Tada $^{1}$, Hiroyuki Hanayama ${ }^{1}$, Kenji Gonda ${ }^{1}$, Zenichiro Saze ${ }^{1}$, Tomoyuki Momma ${ }^{1}$, Hirokazu Okayama ${ }^{1}$, Wataru Sakamoto ${ }^{1}$, Hisahito Endo ${ }^{1}$, Shotaro Fujita ${ }^{1}$, Tomohiro Kikuchi ${ }^{1}$, Azuma Nirei ${ }^{1}$, Motonobu Saito $^{1}$, Koki Kuwabara ${ }^{2}$, Eisei Endo ${ }^{1}$, Koji

Kono ${ }^{1} ;{ }^{1}$ Department of Gastrointestinal Tract Surgery, School of Medicine Fukushima Medical University, ${ }^{2}$ Shirakawa Hospital

Background: In Japan, compared with other asian countries, the number of tuberculosis patients is under control (morbidity incidence rate 14.4) and relatively it's difficult to find tuberculous peritonitis(0.04-0.5\% in all the tuberculosis patients).

But recent global process of people passing could make it happen to import the unaccustomed disease. Like our case tuberculosis peritonitis is no exception.

Here we present a case report of tuberculous peritonitis and using diagnostic laparoscopy, we could make a diagnosis with minimally invasive procedure and could make it possible to treat immediately.

Case Presentation: A 30-year-old man from Myanmar with past medical history of pulmonary tuberculosis at the age of three. He admitted to our institution with right lower abdominal pain, fatigue and slight fever lasting for several weeks. Blood test and abdominal ultrasound revealed the increase of inflammatory response (WBC7200, CRP13), high score of CA125 and ascites. With additional CT scan, not only ascites, panniculitis with peritoneal nodules and the thicken omentum were detected.

Considering the possibility of tuberculous peritonitis and carcinomatous peritonitis, we performed a diagnostic laparoscopy in negative pressure room for preventing from perioperative contamination.

The operative findings, ascites with slightly cloudy, thickened omentum, a few millimeters of white nodules around the peritoneal and omentum were found.

Pathological diagnosis with the omental tissue during operation suggested a suspicion of tuberculous peritonitis due to the caseating granuloma, we could start the treatment with 4 kinds of antitubercular agents (INH + REP + PZA + EB)

Sensitive Mycobacterium tuberculosis was detected from the omental tissue culture after few weeks although both ascites culture and PCR were negative and ADA was under the reference value.

Conclusion: The complication of active pulmonary tuberculosis is about $10 \%$ and only pulmonary and pharyngeal tuberculosis are said to be highly contagious, but the report of tuberculosis peritonitis is also rare in the aspect of infectious prevention.

To determine the diagnosis, the detection of Mycobacterium tuberculosis is required from ascites culture or omental biopsy.

The diagnostic laparoscopy might be one of the effective method, because the former sensitivity is only $10-34 \%$, on the contrary the later has precise sensitivity $(93 \%)$.

In addition, the intraperitoneal features of tuberculosis peritonitis such as "cheese cake sign" are very characteristic.

We report our case of tuberculosis peritonitis, along with some literature review. 
P637

\section{Advanced Laparoscopic CAPD Catheter Insertion: Review of Results of 200 Cases}

Venkatarami Reddy Vutukuru, Associate Professor, Ram R, Professor; Sri Venkateswara Institute of Medical Sciences

Background: Peritoneal dialysis (PD) is increasingly being used as a primary mode of renal replacement therapy. Access is provided with a PD catheter. Various techniques of catheter insertion both laparoscopic and open are described in literature. Major problem is catheter dysfunction due to adhesions, catheter tip migration, omental wrapping. Advanced laparoscopic technique involves adhesiolysis, suture fixation of catheter, and Omentopexy. We present our results of CAPD catheter insertion using advanced laparoscopic technique. Materials \& Methods: All patients who underwent advanced laparoscopic PD catheter insertion and reinsertion between December 2013 to March 2018 were included. All patients underwent adhesiolysis if present, omentopexy in the left hypochondrium, catheter fixation with transabdominal suture. Post operatively followed up for complications such as bleeding, leak, catheter dysfunction, infection and the need for catheter removal. (Video of procedure will be shown)

Results: 200 cases of advanced laparoscopic CAPD catheter insertions were done. Of which, 172 were primary and 28 were reinsertions or correction of catheter dysfunction following open insertion earlier. Mean age is 53.48 years. Males constituted $72 \%$. Average duration was 28 (21-55) min. Adhesiolysis was done in 34\% of patients. 164 patients had omentopexy done. Others had parietal adhesions precluding the need for omentopexy. In 28 patients of catheter reinsertion, dysfunction was due to omental and bowel wrapping with catheter migration in 27 and 1 patient had catheter transected. Postoperatively, six patients had pericatheter leak which subsided. No infections noted. 4 (2\%) patients had catheter dysfunction which required redo surgery over a period of minimum 3 months follow up. Mean hospital stay was 2 days.

Conclusion: Advanced laparoscopic PD catheter insertion or reinsertion using adhesiolysis, catheter fixation and omentopexy has the lowest reported rate of catheter dysfunction even in patients with prior abdominal surgery.

\section{P639}

\section{Evaluation of Laparoscopic Hepatectomy for Hepatocellular Carcinoma}

Junji Ueda ${ }^{1}$, Hiroshi Makino ${ }^{1}$, Tadashi Yokoyama $^{1}$, Hiroshi $\overline{\text { Maruyama }}^{1}$, Atsushi Hirakata ${ }^{1}$, Hideyuki Takata ${ }^{1}$, Natsuki Seki ${ }^{1}$, Yuta Kikuchi ${ }^{1}$, Takuma Iwai ${ }^{1}$, Masafumi Yoshioka ${ }^{1}$, Mikihiro Okusa $^{1}$, Manpei Kawashima ${ }^{1}$, Toshiyuki Irie ${ }^{1}$, Atsushi Inukai ${ }^{1}$, Mikito Suzuki ${ }^{1}$, Takahiro Haruna ${ }^{1}$, Hiroshi Yoshida ${ }^{2} ;{ }^{1}$ Nippon Medical School Tamanagayama Hosipital, ${ }^{2}$ Nippon Medical School

Introdruction: Laparoscopic hepatectomy has spread worldwide with the development of various medical devices. We analyzed the use of laparoscopic hepatectomy for hepatocellular carcinoma at our institution.

Materials and Procedures: We analyzed 104 patients who underwent surgery for hepatocellular carcinoma from 2010 to 2018 at our institution. We divided these patients into two groups: the open hepatectomy group $(\mathrm{OH})$ and the laparoscopic hepatectomy group (LH). We evaluated the clinicopathological findings, overall survival, and recurrence-free survival. In the LH group, there were more early-stage patients than in the $\mathrm{OH}$ group. We further analyzed the two groups by limiting groups to patients with Stage I and II cancer. Results: In our study, 27 patients underwent laparoscopic hepatectomy (23.1\%). The operation time, intraoperative bleeding, duration of hospital stay after surgery, and tumor diameter were significantly less in the LH group. Between the two groups, mortality rate, morbidity rate, overall survival rate, and recurrence-free rate did not significantly differ. We limited the patients to those in stages I and II. There were 56 patients in stages I and II, and 23 patients were in the LH group. Intraoperative bleeding, duration of hospital stay after surgery, tumor diameter, and morbidity rate were significantly less in the LH group. Between the two groups, mortality rate, overall survival rate, and recurrence-free rate did not significantly differ.

Conclusions: Laparoscopic hepatectomy is a safe and feasible surgical method that results in less intraoperative bleeding and shorter hospital stay than open hepatectomy for both advanced and early-stage hepatocellular carcinoma.

\section{P638}

\section{Utility of the Incentive Spirometer}

Nicholas Morin, DO, George Blessen, RN, Erika King, MS, George Ferzli, MD; NYU Langone Brooklyn

Introduction: There is uncertainty as to whether the incentive spirometer (IS) confers a clinical benefit or reduces inpatient pulmonary complications when correlated with the most common hospital diagnoses. Does the patient know how to use one correctly, is it prescribed for the appropriate diagnosis, and is it at the bedside? if so, is it within reach? Our study aims to determine the patterns of use of the incentive spirometer.

Methods: From September 2016 to August 2017, patients admitted for more than $24 \mathrm{~h}$ were included.192 surgical and 206 medical patients were surveyed for usage and observed for receipt of the IS device. Questions asked where: Have you ever used the IS? "Have you been instructed on how to use the incentive spirometer? Have you used it today? How many times in the last hour have you used the IS? What is the number one reason for you to not effectively use the IS? Other questions: Did the patient receive the IS device? Did the patient have the capacity to use the IS? Was the IS within reach? Was the package open? Did the patient demonstrate correct usage of the IS?" We correlated our literature search with the top 10 most common admitting diagnosis and procedures at our institution. Results: Of the 192 surgical patients observed, 147 received the device. $72 \%$ of these patients demonstrated correct usage. $60 \%$ used the device that day, but only $15.6 \%$ for the prescribed 10 times/hour. It was in reach of $80.3 \%$. Average use per hour was 2.79 times. $3 \%$ of surgical patients did not have capacity. The IS was out of reach for $9 \% .12 \%$ reported that they did not know how to use the device. The most frequently reported reason among surgery and medical patients for not using the IS appropriately was pain $(60 \%$ and $67 \%$, respectively). Of the 206 observed medical patients, only $22 \%$ (45 patients) have received the device with only half admitting to using it on the day of questioning. $22 \%$ of these patients didn't know how to use the IS, and it was out of reach in $7 \% .80 \%$ had been educated. $57.8 \%$ showed correct usage. $100 \%$ of medical patients had capacity to use it. Medical patients use averaged 1.47 times per hour.

Conclusion: Our data show that the IS device has poor availability, improper application, and poor compliance among inpatient medical and surgical patients.

\section{P640}

\section{A Prospective Randomized Trial to Compare Time to Complete Laparoscopic Skill Test Between 3D Imaging System and Ultra- high Definition (4 K) Laparoscopic System}

Komol Chaivanijchaya, MD, Sikarin Sornphiphatphong, MD, Worawit Kattipatanapong, MD, Ittiphon Viratanapanu, MD, Suthep Udomsawaengsup, MD; Chulalongkorn hospital

Background: 3D (Three dimensional) imaging system provides depth perception of surgical field that may make laparoscopic surgery move precise and safe. There have many studies showed that can improve inanimate laparoscopic skill compared with 2D system. Development of $4 \mathrm{~K}$ (Ultra high definition) imaging system, the advantage include more detailed, color-correct images and greater depth perception. As well this study aims to compared the effect of $4 \mathrm{~K}$ imaging system to time for complete laparoscopic skill test over 3D imaging system

Methods: Sixth year medical student and first year resident include into this study. There were assign in two groups ( $3 \mathrm{D}$ and $4 \mathrm{~K}$ ) to perform 3 laparoscopic skill test (ring transfer, pattern cutting, suture/knotting) then they were switch to the other arm. Time to complete tasks were measured and total time to complete all test were also recorded and number of mistake were note in both groups. All participants completed questionnaires to report the inconveniences that occurred during the skill test.

Results: Time to complete all tasks in 3D imaging system was shorter than $4 \mathrm{~K}$ imaging system $(661 \mathrm{~s}$ vs. $746.88 \mathrm{~s}, \mathrm{p}<0.001)$. If consider time to complete each task the result showed shorter time in 3D imaging system than $4 \mathrm{~K}$ in all task, ring transfer.

Conclusions: The 3D vision systems significantly improve speed and accuracy when compared to the $4 \mathrm{~K}$ vision system based on shorter performance time in non experience trainees. 


\section{P641}

VATS Versus Fibrinolytics for Treatment of Primary Pediatric Empyema in Otherwise Healthy Children

Joseph Balaban ${ }^{1}$, Lorrie Burkhalter ${ }^{2}$, Diana L Diesen ${ }^{3} ;{ }^{1}$ UT

Southwestern, Dallas, Tx, ${ }^{2}$ Children's Health Dallas, ${ }^{3}$ UT

Southwestern, Department of Surgery; Children's Health Dallas

Introduction: Traditionally, Video Assisted Thorascopic Surgery (VATS) decortication has been the gold standard treatment for pediatric empyema. Recent studies have shown that fibrinolytics via tube thoracostomy produced similar outcomes while being less invasive and more cost-effective. Clinical practice started to change though difference in efficacy, hospital stay, cost, and failure rate are still debated.

Methods: We performed an IRB approved retrospective review of 161 otherwise healthy patients seen at a pediatric hospital from $2007-2017$ with a primary diagnosis of empyema treated with either fibrinolytics via chest tube or VATS decortication. Children were excluded if they had complex medical comorbidities or secondary empyema. This time period included our transition from first-line VATS treatment to fibrinolytic treatment.

Patient demographics, clinical and outcomes data points were collected. Age, size of effusion, necrotizing pneumonia, loculations, and mediastinal shift were specifically examined to determine if these subgroups had improved outcome with either VATS or fibrinolytics.

Results: There were 161 otherwise healthy pediatric patients treated for empyema between 2007-2017 with 95 patients treated with fibrinolytics and 66 treated with VATS. There were no differences seen in age, gender, race, ethnicity, weight, days of symptoms, asthma history, previous pneumonia, or WBC. Those patients in the fibrinolytic group did have more oxygen requirement on presentation and the fibrinolytic group were more likely to have loculated effusions $(\mathrm{p}<0.05)$.

The two groups (fibrinolytics vs VATS) had similar outcomes when it came to days of oxygen support, days of antibiotics, days of narcotic use, days until fever resolved, number of chest tubes, days intubated, LOS, and readmission rate The fibrinolytic group has slightly longer ICU days ( 3 vs 1.4 ) and received higher number of US but the same numbers of CT scans $(0.5$ vs 0.4$) \mathrm{p}<0.05$.

There was an $5.3 \%(5 / 95)$ failure rate in fibrinolytic patients and $7.6 \%$ in the VATS group $(5 / 66)$ defined as needing repeat procedure (CT placement).

In the subgroup of children $>5$, patients treated with VATS had shorter hospital LOS, ICU LOS, days of narcotics, and no treatment failures. Neither size of effusion, presence of necrotizing pneumonia, loculated effusion, nor mediastinal shift subgrouping correlated with improved outcome with one type of treatment.

Conclusion: Both VATS and fibrinolytic treat pediatric empyema in otherwise healthy children with fibrinolytic therapy offering a less invasive alternative. Further research is needed to determine if there are benefits of one approach over another for subpopulations of patients.

\section{P642}

Safety, Feasibility, and Effectiveness of Delta-Shaped Anastomosis: Outcomes of 358 Consecutive Cases

Masazumi Sakaguchi, MD, PhD, Norihiro Shimoike, MD, Shin Akagawa, MD, PhD, Seiichiro Kanaya, MD, PhD; Osaka Red Cross Hospital

Background: Since its development, delta-shaped anastomosis (DA) using only endoscopic liner staplers has been widely used during laparoscopic gastroduodenostomy. Herein, we report our DA technique, and short- and mid-term outcomes of 358 consecutive DA cases.

Technique: DA applies functional-end-to-end anastomosis in gastroduodenostomy. By using only liner staplers, a good visual field can be achieved during the procedure, thus ensuring safety. First, a V-shaped anastomosis was created between the remnant stomach and duodenal cut end by firing a $45-\mathrm{mm}$ endoscopic liner stapler. The resultant entry hole was closed by firing either one $60-\mathrm{mm}$ or two $45-\mathrm{mm}$ liner staplers. It is extremely crucial that the operator, assistant, and camera operator work in concert during this procedure.

Methods: We retrospectively identified 358 consecutive patients with gastric cancer who underwent DA from April 2011 to March 2018. We extracted data pertaining to the patient ages, BMIs, postoperative diet start dates, and complications associated with DA.

Results: The median follow-up period was 862 [interquartile range (IQR): 1380-408] days. The median patient age and BMI were 69 (IQR: 62-76) years and 22.6 (IQR: 24.8-20.7), respectively. The median postoperative diet start date was postoperative day 4 (IQR: 4-5 days). Complications of Clavien-Dindo grade 2 or greater included six cases of leakage $(1.68 \%)$, three of delayed gastric emptying $(0.84 \%)$, and one of bleeding $(0.28 \%)$. No patients experienced anastomotic stenosis.

Conclusion: DA is a safe, feasible, and effective procedure.

\section{P643}

Is Polyglycolic Acid (PGA) Nonwoven Fabric Suitable as an Anastomotic Portion Reinforcement Material of Automatic Suturing Device? Comparison with Early Bioresorbable Materials

Mitsuo Miyazawa, MD, FACS ${ }^{1}$, Katsuya Okada, MD $^{2}$, Masayasu Aikawa ${ }^{2}$, Shunsuke Ohnishi ${ }^{3}$, Yoshito Ikada ${ }^{4}$ ' $T$ Teikyo University Mizonokuchi Hospital, ${ }^{2}$ Saitama Medical University International Medical Center, ${ }^{3}$ Hokkaido University, ${ }^{4}$ Kyoto University

Background: Currently, in laparoscopic surgery, a sheet of bioabsorbable material is often used as a stumped closure reinforcement to reinforce the closure of the gastrointestinal tract and pancreatic stump with an automatic suturing device. We compared the bioabsorbable material which decomposes earlier than PGA nonwoven fabric and PGA nonwoven fabric, and evaluated the material of decomposition rate which is more effective as an anastomotic part reinforcement material.

Method: Split 2/3 lap of small intestine of pig and suture with 4 needles of suture. A PGA nonwoven fabric and a bioabsorbable polymer sheet (BAPS) (lactic acid: caprolactone 50: 50 copolymer, absorbed in vivo in about 6 to 8 weeks) which can be hydrolyzed at an early stage are stitched around the sewn portion I wore it. After 1, 3 weeks of suture reopening, the strength of the anastomotic part was examined by the tensile strength test, and the site was compared histologically. (Each $\mathrm{n}=3$ ).

Results: After 1, 3 weeks, no anastomotic site showed suture failure. Compared with PGA, the surrounding anastomotic part of BAPS was harder and thicker palpated. In the tensile strength test, BAPS had the highest tensile strength at 1 week compared to PGA (PGA and BAPS are equivalent at 3 weeks).

Conclusion and Discussion: As early as the anastomotic portion reinforcement material, it was shown that BAPS reinforces the anastomotic site highly in comparison with PGA. As a stump reinforcement material, it is thought that material that absorbs earlier than PGA is suitable. 


\section{P644}

\section{Type IIIb Congenital Intestinal Atresia: A Case Report}

Brian Bassiri-Tehrani, MD ${ }^{1}$, Alex Wang ${ }^{2}$, William Harris ${ }^{2}$, Edmund Kessler, MD ${ }^{1}$; ${ }^{1}$ Lenox Hill Hospital, Zucker School of Medicine at Hofstra/Northwell, ${ }^{2}$ SUNY Downstate Medical Center

Intestinal atresia is a congenital malformation that occurs in 1 out of 5,000 live births, with equal incidence in males and females. One out of three infants with intestinal atresia are premature. The authors present a case of a 1-day-old female born at 38-weeks gestation via normal spontaneous vaginal delivery with bilious vomiting on the first day of life.

Antecedent pregnancy was normal and routine ultrasound screenings showed no evidence of any underlying pathology. Birth weight was 3360 grams, height/length was 83rd percentile, head circumference 54th percentile. Apgar score was 9 at $1 \mathrm{~min}$ and 10 at $5 \mathrm{~min}$. Birth physical exam was unremarkable without evidence of dysmorphic signs or features.

On day of life 1 the baby was tolerating her first breast feeds, however overnight the baby had bilious emesis and had yet to pass meconium. The patient's abdomen was slightly more distended and tympanic. Plain film showed distended bowel. A replogle tube was placed. Vital signs were within the normal range and labs were unremarkable. Subsequently, an upper GI series was performed and showed a proximal small bowel obstruction.

A contrast enema was done and showed a patent rectum without evidence of a microcolon, Hirchsprung's disease or malposition. The baby was then taken to the operating room on day of life 2 for an exploratory laparotomy.

Upon entry into the peritoneal cavity, congenital adhesions were encountered and lysed. A midgut type IIIb intestinal atresia was found with an internal hernia through a mesenteric defect. A $15 \mathrm{~cm}$ segmental bowel resection was performed with a single layer hand sewn anastomosis was performed. Postoperatively, the baby was extubated and placed on nasal cannula with the replogle in place.

The baby required parental nutrition for weeks after surgery due to inability to consistently tolerate a PO diet despite repeated upper GI films ruling out a mechanical obstruction. Therefore, on post op day 20 , the baby started a 5 day course of erythromycin to promote GI motility. After the start of erythromycin, the baby was started on feeds and was gradually advanced with good tolerance. On post op day 37, the baby continue to tolerate a PO diet and was reintroduced to expressed breast milk. The baby was reliably tolerating a home breast feeding schedule by post op day 39 . On post op day 41 the baby was discharged home.
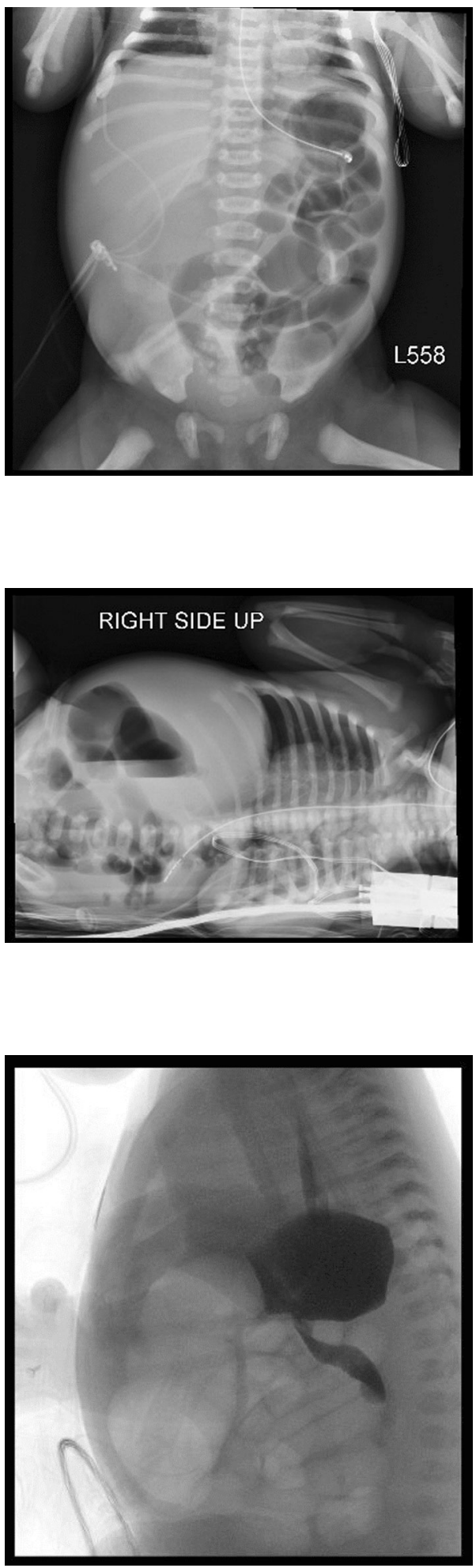

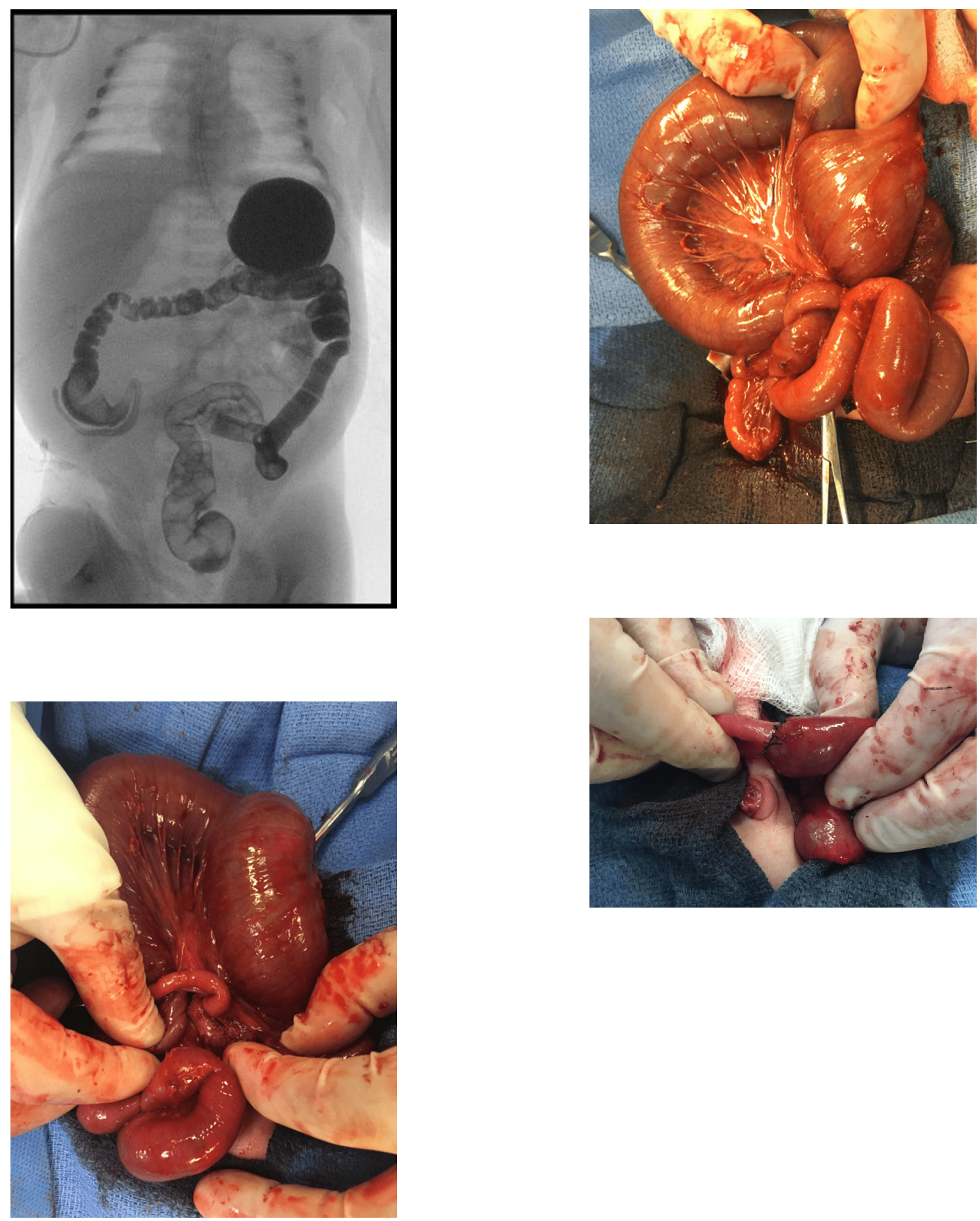


\section{P645}

\section{Effect of Skin Organization Glue Compared with Traditional Suture on the Abdominal Type II Incision Closure}

Xinquan Lu, MD, Xiaojiang Yi, MD, Hongming Li, MD, Dechang Diao, MD, Jin Wan, MD; Department of Gastrointestinal (Tumor) Surgery, Guangdong Province Hospital of Chinese Medicine, the Second Affiliated Hospital of Guangzhou University of Chinese Medicine

Objective: To o evaluate the advantages, disadvantages, safety and feasibility of skin organization glue and intradermal suture on the abdominal type II incision closure. Methods: A total of 298 patients with gastric cancer and colorectal cancer received aparoscopic radical resection between January 2015 and May 2018.The patients were divided into the skin organization glue group(n:168) and the traditional suture group $(\mathrm{n}: 130)$.For two groups of patients, the incision closure time, incision complication (incision infection, fat liquefaction, wound dehiscence, etc.), incision healing, hospitalization days, incision closure cost and total hospitalization cost were observed and evaluated. Results: the closure time of the skin glue group was $8 \min (10 \pm 3.5)$, and the suture group was $12 \min (12 \pm 3.2)$, the difference was statistically significant $(\mathrm{P}<0.05)$; the incision complication in the skin glue group was 3 cases and the suture group 5 cases, there were no statistically significant difference $(P>0.05)$; the healing rate of the first grade was 166 in the skin glue group and 126 in the suture group, and there was no significant difference between the two groups. The postoperative hospital days were compared, the average of the hospital days was $5(5 \pm 2)$ days, and the suture group was $7(7 \pm 3)$ days, there were statistically significant difference $(\mathrm{P}<0.05)$; the skin glue group of the incision closure cost was significantly higher than the suture group, but it did not increase the overall hospitalization cost of the patients.

Conclusion: the abdominal type II incision with skin organization glue is simple, timesaving, safe and feasible, and it can improve the patient's comfort. At the same time, it does not increase the total hospitalization expenses of the patients, and the patients are willing to accept it.

Keywords:organization glue;traditional suture;abdominal operation;type II incision

\section{P647}

\section{Cost and Outcomes of Laparoscopic Appendectomy and Cholecystectomy Across a Large, Integrated Health System}

Mike Mallah, MD, Michael Barringer, MD, Michael Thomason, MD, Elizabeth Ross, MSN, Brent Matthews, MD, Caroline Reinke, MD; Carolinas Medical Center

Introduction: Within our health system of 47 hospitals there is substantial variation in operative supply cost for low-risk minimally invasive surgeries. We hypothesized that after controlling for patient factors, there would be no association between cost and outcome. We also hypothesized that utilization of an energy device would be associated with a shorter operative time.

Methods: We examined operative room (OR) supply cost for all laparoscopic appendectomies and cholecystectomies captured in NSQIP from 1/2015-12/2017 and stratified them by supply cost quartile as well as presence or absence of an energy device. Each quartile was then compared by preoperative risk factors, operative time, and 30-day outcomes. Logistic regression was utilized to examine factors related to a composite outcome and linear regression was utilized to examine factors associated with operative time.

Results: 5,192 NSQIP cases with matched supply cost were identified. Mean supply cost for laparoscopic appendectomy was \$927 (IQR \$725-\$1,063) and for laparoscopic cholecystectomy was $\$ 584$ (IQR $\$ 427-\$ 653$ ). In both groups, significant differences were observed in ASA class, elective vs. urgent vs. emergent status, and wound classification between cost quartiles. Use of an energy device and operative time were also significantly different between the cost quartiles. Rates of postoperative bleeding, organ space SSI, and the composite outcome were differed significantly between cost quartiles for laparoscopic appendectomy, but not laparoscopic cholecystectomy. Increase in supply cost was associated with increased risk of the composite outcome, even after controlling for patient and disease factors (OR 1.06, $\mathrm{p}=0.04)$. Mean cost of case was greater and mean operative time was shorter for cases in which an energy device was used (\$535 more/6 min shorter for cholecystectomy; $\$ 259$ more $/ 6$ min shorter for appendectomy; $\mathrm{p}<0.01$ in both). The six minute decreased operative time was evident even after controlling for patient and case characteristics $(\mathrm{p}<0.01)$

Conclusion: Increased supply cost is associated with an increased rate of 30-day postoperative complications, even after controlling for patient and disease characteristics. Use of an energy device increases supply cost, with only a modest decrease in operative time. Standardizing supply cost on patient outcomes across a large group of hospitals and surgeons has the potential to increase value of care for patients.

\section{P648}

\section{Using Local Anesthesia at Time of Closure for Routine Lap Surgery}

George Dejeu, Adrian M Maghiar, Octavian Maghiar, Dan Ciurtin, Marius Sfirlea, Codruta Macovei, Mihai Botea, Cipri Puscas, Calina Tarau, Erika Bimbo-Szuhai, Rodica Gilau, Paula Badea, Florin Fasie; Spital Pelican

In our institution, Surgery Clinic, Pelican Hospital in Oradea, Romania, we started studying the effect of using local anesthesia at the trocar sites at time of closure for basic, routine lap surgery. We started a prospective study including all patients with laparoscopic cholecystectomy and appendectomy in the past 3 months. We compared this group of patients with a matched group for age, sex, diagnosis, comorbidities and previous chronic medication from 12 months ago. We used a standardised surgical approach and postop management with the only difference being the addition of local anesthesia infiltrations of trocar sites at time of closure. To compare the effect on pain management we compared the pain score cards our patients routinely complete at 3,6,12 and $24 \mathrm{~h}$ after this type of surgery. The extra pain medication needed was also noted, as was the use of opioids for pain management. We found the reduction of pain score at 3 and $6 \mathrm{~h}$ (statistically significant) and reduction of opioid use (by $80 \%$ ). The pain score at 12 and $24 \mathrm{~h}$ show a reduction in pain for the local anesthesia gorup, but not statistically significant. We also noted the reduction of hospital stay from 4 to 3 days for the local anesthesia group, but this aspect was not in our end points for the study. We are currently implementing into our standard approach to all surgery the use of local anesthesia at the surgery site at time of closure, and are researching the use of nerve block techniques and use of continuous intravenous local anesthesia for pain management for our major surgery patients.

\section{P649}

\section{Port Site Tuberculosis after Laparoscopy}

Mohammad Masud Karim, MBBS, Gold Medalist, FCPS, Surgery ${ }^{1}$, Moshammat Zebunnesa, MBBS, FCPS, MS ${ }^{2}$, Satyajit Dhar, MBBS, FCPS, MD ${ }^{2}$, Mamun Mustafa, MBBS ${ }^{2} ;{ }^{1}$ Noakhali Abdul Malek Ukil Medical College,Bangladesh, ${ }^{2}$ Chittagong Medical College, Chittagong

Background: After first laparoscopic cholecystectomy by Phillip Mauret in 1987 there has been rapid explosion in the field of minimal access surgery (MAS). With increasing use of laparoscopy for various surgical procedure, the occurrence of port site tuberculosis is seen more often a postoperative complication. Most of the cases present as non-healing port site wounds. The infection has been attributed to improper sterilization of laparoscopy instruments. There is concern about the effectiveness of the sterilizing reusable laparoscopic instruments by immersion in $2 \%$ gluteraldehyde (GTA). This article describes the various features of biopsy proven port site tuberculosis. However lack of awareness of this entity leads to prolonged morbidity and may need repeat surgical interventions. In case of nonhealing sinus following laparoscopic surgery keeping this possibility in mind will lead to early diagnosis and treatment.

Objective: To see the occurrence of port site tuberculosis after laparoscopic surgeries.

Materials and Methods: This is a retrospective, cross-sectional, observational study carried out in one of the private hospital in Chittagong during the period of October 2005 to March 2016. Study populations were all the patients underwent various laparoscopic procedures by our team during this period. It includes laparoscopic cholecystectomy, laparoscopic appendicectomy, diagnostic laparoscopy and laparoscopic ovarian cystectomies. Surgeries were performed Storz standard laparoscopy set. Port and hand instruments were reusable one made by same brand and all were immersed in 2\% GTA for 15-20 min before each use. $2 \%$ GTA has been changed after each 12 to 15 case intervals. More than one operation has been performed in the same afternoon in most occasions. Results: Among all the patients of laparoscopic surgeries (3720), seventeen cases were diagnosed as port site tuberculosis which is about $.45 \%$. Fifteen of them were female $(88.23 \%)$. Most of them were within 30-40 years of age groups. None of them had known tuberculosis prior to surgery. All were presented with non-healing port site discharge/sinus except one which was presented as abscess. All cases confirmed by biopsy. All the patients responded to four drugs anti-tubercular therapy for one year. Nine cases developed umbilical port incisional hernia later (52.94\%).

Conclusion: Port site tuberculosis is not so common but troublesome for the patients and surgeons. It's a burden to our health economy. Proper sterilization of instruments is the key. Use of $2 \%$ GTA is not cent percent effective against tuberculosis. Key words: Laparoscopy, Tuberculosis, Port site, Sterilization. 
P650

Impact of Laparoscopic Simultaneous Hepatectomoy in Treatment of Colorectal Cancer with Synchronous Liver Metastasis

Takeaki Ishizawa, MD, PhD, FACS ${ }^{1}$, Shoji Kawakatsu ${ }^{2}$, Yoshiya Fujimoto $^{2}$, Takafumi Sato ${ }^{2}$, Yoshihiro $\mathrm{Ono}^{2}$, Yoshihiro Mise ${ }^{2}$, Yosuke Inoue $^{2}$, Hiromichi Ito $^{2}, \mathrm{Yu} \mathrm{Takahashi}^{2}$, Masashi Ueno ${ }^{2}$, Akio Saiura ${ }^{2} ;{ }^{1}$ Cancer Institute Hospital/The University of Tokyo, ${ }^{2}$ Cancer Institute Hospital, Japanese Foundation for Cancer Research

Background: Although laparoscopic approach has been indicated widely in treatment of colorectal cancer with synchronous liver metastasis, safety and efficacy of laparoscopic simultaneous hepatectomy remains unclear.

Methods: Subject consisted of 258 patients who had undergone curative resection of colorectal cancer with simultaneous hepatectomy for synchronous liver metastasis. Surgical outcomes were compared between patients in the early period $(2006-2012, \mathrm{n}=117)$ and those in the latter period (2013-2017, $\mathrm{n}=141$ ). In the latter period, short-term outcomes were also compared between the open hepatectomy group (OH; open or laparoscopic colorectal resection with open hepatectomy) and the laparoscopic hepatectomy group (LH; laparoscopic colorectal resection with laparoscopic hepatectomy). Laparoscopic hepatectomy has been indicated as a standard care since 2013, basically for liver metastasis resected by 4 or less wedge resections and/or left lateral sectionectomy. Results: Indication of laparoscopic surgery had been extended over time; proportion of open hepatectomy and colorectal resection, open hepatectomy and laparoscopic colorectal resection, and laparoscopic hepatectomy and colorectal resection was 50, 45, and 5\% in the early period and 21 , 53 , and $26 \%$ in the latter period, respectively. In the latter period, length of postoperative hospital was shorter than the early period (median [range], 15 [6-71] days vs. 17 [7-84)] days; $\mathrm{P}=0.041$ ), while total operation time tended to be prolonged (519 [248-998] min vs. 425 [173-1116] min $\mathrm{P}<0.001)$. In the latter period, surgical outcomes in the LH group $(\mathrm{n}=37)$ were more favorable than those in the $\mathrm{OH}$ group $(\mathrm{n}=104)$ in terms of total operation time $(474$ [311-905] min vs. 548 [248-998] min; $\mathrm{P}=0.025)$, estimated blood loss amount $(200$ [5-700] $\mathrm{mL}$ vs. $580(0-1675) \mathrm{mL}$, $\mathrm{P}<0.001$ ), postoperative hospital stay $(13[8-42]$ days vs. 15 [8-71] days; $\mathrm{P}=0.004)$, and $\mathrm{R} 0$ rate for liver metastasis ( $100 \%$ vs. $89 \%$; $=0.008$ ), although hepatic transection speed in the $\mathrm{LH}$ group was slower $\left(0.61[0.14-1.18] \mathrm{cm}^{2} / \mathrm{s}\right.$ vs. $\left.1.36[0.34-4.7] \mathrm{cm}^{2} / \mathrm{s}\right)$. Morbidity and mortality were $5.4 \%$ and $0 \%$ in the LH group and $9.6 \%$ and $0 \%$ in the $\mathrm{OH}$ group, respectively.

Conclusion: Laparoscopic simultaneous hepatectomy with colorectal resection can be applied safely and may enhance patients' postoperative recovery, if liver metastasis can be removed by simple wedge resections and/or left lateral sectionectomy. Further improvement in hepatic transection techniques is needed to extend indication of totally laparoscopic approach to colorectal cancer with synchronous metastasis requiring complicated hepatectomy.

\section{P651}

\section{Mucosal Perforation During Laparoscopic Surgery for Achalasia}

Vladimir Grubnik, MD, Professor, Viktor Grubnik, Vadim Ilyashenko, PhD; Odessa National Medical University

The aim of this study: was to evaluate whether preoperative pneumatic balloon dilation (PBD) represents a risk factor for surgical complicated of laparoscopic Heller myotomy.

Methods: A retrospective analysis was done for surgical treatment of 97 patients with achalasia. From 1996 to 2016 years 97 patients with esophageal achalasia underwent laparoscopic Heller myotomy: 32 patients with preoperative PBD and 65 patients without PBD. Patients demographics: age, gender, body mass index, duration of symptoms; operative findings: operative time, blood loss, intraoperative complications, postoperative curse: course: complications, clinical symptoms, postoperative treatment; esophageal functional tests were assessed.

Results: No significant differences were observed in the patient's demographics. Operative finding were similar between two groups; however the incidence of mucosal perforation was significant by higher in the PBD group $(\mathrm{n}-6 ; 18.7 \%)$ compared to the non-PBD group $(\mathrm{n}-3,4.6 \%)(\mathrm{p}<0.01)$. In all patients with perforation. The Dor fundoplication was done in the $45 \%$ patients without perforation. In $40 \%$ of cases Heller myotomy was performed without Dor fundoplication. Lower esophageal sphincter pressure was effectively reduced in both groups, no differences were observed in 24-h pH monitoring profile between the two groups.

Conclusion. Preoperative PBD in the factor significantly associated with intraoperative mucosal perforation. Additional caution is recommended in identifying intraoperative mucosal perforation in patients with preoperative PBD during performing laparoscopic Heller myotomy.

\section{P652}

The Technique of Up-Steer Method of Laparoscopic Ultrasound Guidance for Radio-Frequency Ablation of Tumors Located in Hepatic Segment VII and Segment VIII

Wenbo Tang, Rong Liu, Minggen Hu, Chenggang Li, Kedi Zhang; Chinese PLA General hospital

Aims: to evaluate the effectiveness of a novel up-steer method for laparoscopic ultrasound guidance of radiofrequency ablation for tumors located in hepatic segment VII and VIII.

Methods: all the cases of tumors located in hepatic segment VII and VIII which were treated using laparoscopic ultrasound guided radiofrequency in our department by a single surgeon from February 2015 to August 2017 were retrospectively reviewed. Whether the procedure was performed using an up-steered method, the first puncture duration, the puncture failure time, the complication rate, the ablation area, and the local recurrence rate in one year were all compared. Results: a total of 64 tumors were enrolled in the present study, including 28 tumors ablated using a novel up-steered method. The overall puncture failure time was 8 times in the up-steered group, and the puncture failure time was 22 times in the conventional puncture group, with a puncture success rate of $77.8 \% \mathrm{Vs} 62.1 \%$, $(\mathrm{p}=0.086)$. The average puncture duration was $115.5 \pm 114.7 \mathrm{~min}$ in the up-steered group, and $151.4 \pm 159.9 \mathrm{~min}$ in the conventional group. There was no significant difference between the complication rate and the ablation area of the two group, and there was no local recurrence in one year after ablation.

Conclusion: the up steered method was helpful when performing the laparoscopic ultrasound guided radiofrequency for tumors located in hepatic segment VII and VIII.

\section{P653}

\section{Robotic Ventral Hernia Repair: A Canadian Perspective}

Moska Hamidi, MD, MPH, FRCSC ${ }^{1}$, Jensen Tan, MD, MSc, FRCSC $^{2}$, Lazar Klein, MD, MSc, FRCSC ${ }^{2}$, Steven Maclellan, MD, FRCSC ${ }^{2}$; ${ }^{1}$ Department of Surgery, St. Michael's Hospital, ${ }^{2}$ Department of Surgery, Humber River Hospital

Background:Minimally invasive ventral hernia repair is increasingly prevalent in Canada;however, the uptake of robotic assisted ventral hernia repair (RAVHR) has been slow compared to other countries.The open approach continues to be widely used for complex hernia repairs requiring components separation,despite a lower incidence of SSI and comparable recurrence rates with a minimally invasive repair.Given the reported success of RAVHR as a minimally invasive approach to these hernias, we sought to determine the feasibility of its routine use in a community hospital setting. There is currently no other Canadian experience reported on RAVHR.This report describes our initial series. Methods:We included all RAVHR performed at our institution, between Nov 2017-July 2018.We reviewed 30-day complications, mortality, LOS, opiates prescribed and operating time. A two-way scatter plot was used to describe the relationship between hernia size,number of cases performed and operating times.

Results:Eighteen patients from our institution performed by two robotic surgeons were analyzed.Mean follow-up period was 57 days.The mean age of our patients was 56 years (31-79),with the majority being male $(\mathrm{n}=12 ; 67 \%)$.All patients had an attempted RAVHR, with one patient $(6 \%)$ requiring a conventional laparoscopic repair.The average BMI was 30.13 (22.9-37). The mean defect size was $5.4 \mathrm{~cm}$ in maximal transverse diameter $(2.5-15 \mathrm{~cm})$ and all hernia defects were closed primarily.Fourteen patients $(78 \%)$ received a retrorectus mesh repair and 3 patients $(16.7 \%)$ received a component separation. The mean length of stay (LOS) was 1.28 days. The mean number of opiates prescribed at discharge was 16 tablets of Morphine $5 \mathrm{mg}$ equivalents. There was one bowel injury $(6 \%)$,and one paralytic ileus $(6 \%)$ in the same patient.There were no readmissions or mortalities. One patient developed an abscess at the umbilicus.The mean operating time was $223 \mathrm{~min}$ (118-516 $\mathrm{min}$ ); there was no significant change in operating time with increasing experience,as the hernia size varied, and sample size is limited. Pearson coefficient demonstrate the relationship between the hernia size and operating time, with a correlation of 0.65 (Figure 1).

Conclusion: The outcomes from our first series of RAVHR are consistent with previously published studies with respect to LOS and complication rates.Outcomes are also encouraging for using RAVHR for larger hernia requiring components separation.We have demonstrated the safety of this approach in a Community hospital setting in Canada.Our future study will assess the safety and cost-effectiveness of this approach in complex abdominal wall reconstruction with component separation as an alternative to open surgery in select populations.

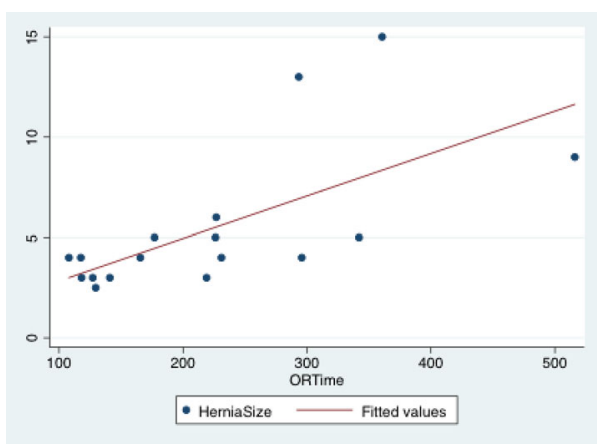

Figure 1-Pearson Correlation Hernia Size vs. OR time 


\section{P654}

First Robotic Spleen Preserving Distal Pancreatectomy for Trauma

Elijah Kim, MD, MPH, FACS, FCCP, Patrick Collier, MD, Corydon Siffring, MD, FACS, Erin Hagerman, MSN, AGACNP, Tina Molohon, TPC, RN, TNS, Douglas C Maibenco, MD, PhD, FACS; HSHS Medical Group Trauma/Acute Care Surgery, St. John's Hospital, Springfield, Illinois

Introduction: Robotic distal pancreatectomy for trauma has never been reported. We present a case of blunt abdominal trauma resulting in a Grade III injury of the pancreatic body with complete transection at the confluence of the inferior mesenteric and splenic veins. The patient was taken emergently to the operating room where a robotic assisted spleen preserving distal pancreatectomy was performed. We describe the patient selection, technical details, and postoperative course, as well as indications, contraindications, and implementation of robotic assisted surgery for trauma to the pancreas.

Methods and Procedures: The patient is an 18-year-old male who sustained a football injury to the left upper quadrant and presented hemodynamically stable. Computed tomography demonstrated a Grade III pancreatic injury. With the patient in right lateral decubitus position, one $12 \mathrm{~mm}$ and three $8 \mathrm{~mm}$ robotic trocars were used to gain entry. Inspection verified the pancreatic body to be transected. Venous bleeding was controlled and no active arterial bleeding was noted. The splenic vessels and the inferior mesenteric vein were identified and preserved. The proximal pancreatic body was closed using $2.5 \mathrm{~mm}$ staples, fibrin glue, hemostatic agent, and an omental buttress. The distal pancreas was morselized and retrieved via the $12 \mathrm{~mm}$ trocar site resulting in the loss of approximately $40 \%$ of pancreatic volume. Estimated blood loss was $100 \mathrm{~mL}$. Total operative time was $346 \mathrm{~min}$.

Results: Postoperatively, the patient was treated with total parenteral nutrition and octreotide. A left pleural effusion was drained under ultrasound guidance on day six. Enteral feeds were started on day six via a nasojejunal feeding tube. Abdominal drain output diminished significantly by day eleven. He was started on an oral diet and discharged home. Total length of stay was 13 days.

Conclusion: Although initially developed with remote trauma surgery in mind, robotic assisted surgery is currently utilized nearly exclusively for elective surgery. Search in the current literature demonstrates a noticeable absence of robotically assisted surgery for trauma. With appropriate patient selection, experienced trauma surgeons comfortable with robotic and open techniques, and hospitals prepared and equipped with sufficient capacity, regional trauma centers can safely provide minimally invasive robotic surgery for trauma and take full advantage of the modality including potentially minimizing pain, large incisions, blood loss, conversion rates to open, splenectomy rates, and length of stay. Ongoing progress in the adoption of robotic assisted trauma surgery is helping to realize some of the intentions that inspired the original developers of the technology.

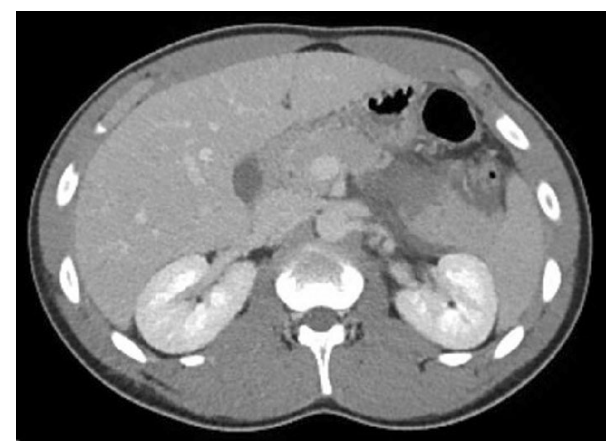

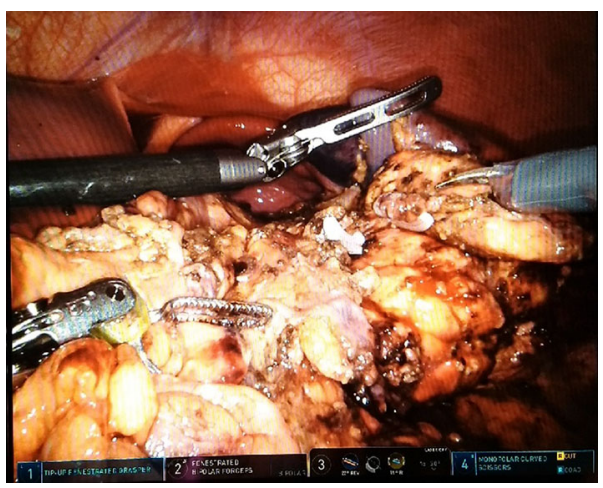
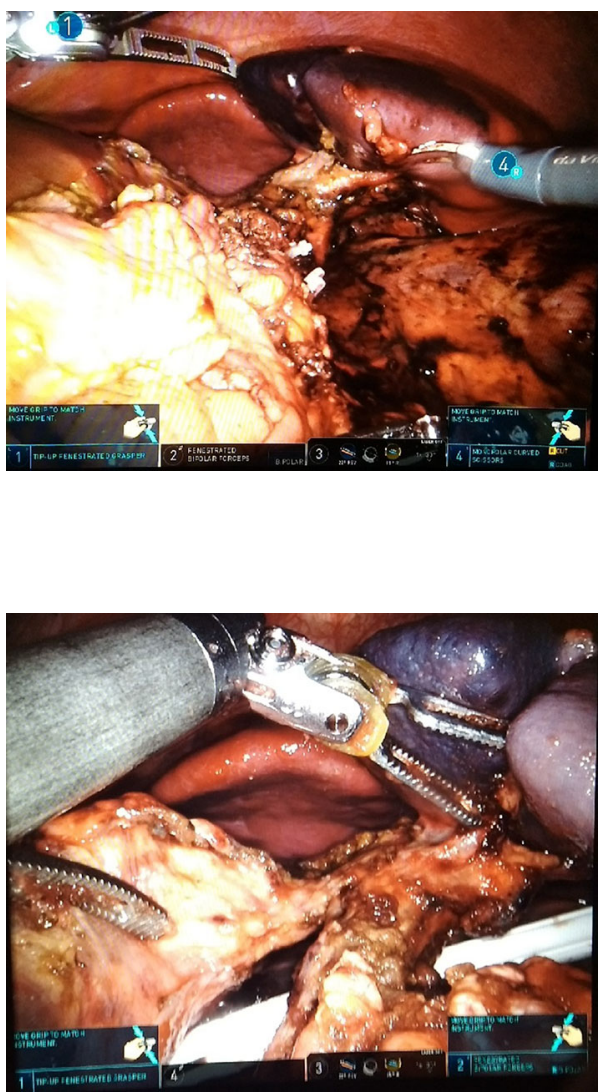
P656

\section{Robotic Removal of A Retroperitoneal Mucinous Cystic Neoplasm}

Cole P Rodman, MS, Sophia Roberts, Michael P Meara, MD, MBA, FACS; The Ohio State University Wexner Medical Center

Introduction: This is the case of a 24-year-old woman who presented with a large retroperitoneal mass which was found to be a primary retroperitoneal mucinous neoplasm. Such tumors are rare and less then 20 cases have been reported in the literature. Consequently, their histopathology is not well understood and preoperative diagnosis can often prove challenging. In this case the mass was initially diagnosed as a benign enteric duplication cyst. To our knowledge this is the only documented robotic removal of a retroperitoneal mucinous neoplasm.

Case Presentation: This is a twenty-four-year-old woman with an uncomplicated medical history complaining of right upper quadrant fullness and discomfort. After delivery of her second child in 2017, she subsequently noted a palpable mass in the right abdomen which became progressively more bothersome. Abdominal and pelvic CT scan obtained in January, 2018 showed a cystic mass in the right colic gutter suspicious for enteric duplication cyst. After four months of worsening symptoms, she decided to undergo resection in April, 2018.

The abdomen was accessed laparoscopically via the left upper quadrant and the cystic mass was identified. Electrocautery and blunt dissection were used to define the margins of the cyst and to free it from the surrounding retroperitoneum. The cyst was drained of all fluid with a laparoscopic aspiration needle prior to removal of the sac with an occlusive bag. After removal of the mass, all ports were removed and port sites were closed. The patient's postoperative course was uneventful and she was discharged on the day of surgery in stable condition.

The pathology report indicated that the sample from the mass was consistent with a mucinous cystic neoplasm $(\mathrm{MCN})$ with ovarian-type stroma. At follow-up one month after her surgery, the patient was well and only complained of occasional constipation.

Discussion: In addition to the rarity of extra-ovarian or extra-pancreatic, ovarian-type MCNs, as of this writing we are unaware of any case presentations of such tumors being managed robotically. The primary challenge of the case presented here was the radiologic and clinicopathologic similarity of this MCN and an enteric duplication cyst. In this case, the mass appeared as smooth and thin-walled with a single loculation. Ultimately, the diagnosis of MCN is postoperative, however we hope that the presentation of this case will serve as a reminder that care should be taken in the surgical approach to seemingly benign intrabdominal masses.

\section{P659}

The Impact of the Airseal ${ }^{\circledR}$ Valve-Less TROCAR System in Robotic Colorectal Surgery: A Single Surgeon Retrospective Review

Jessie O Paull, MD ${ }^{1}$, Ada Graham, $\mathrm{MD}^{2}$, Natalie Pudalov ${ }^{3}$, Vincent Obias, MD ${ }^{2}$, Abdullah Al Slami ${ }^{2} ;{ }^{1}$ Walter Reed National Military Medical Center, ${ }^{2}$ George Washington University Hospital, ${ }^{3}$ George Washington University School of Medicine and Health Sciences

Introduction: Traditional trocar systems suffer from several innate flaws due to their silicone sea design. The AirSeal ${ }^{\circledR}$ is a valve-less trocar system that overcomes these flaws by utilizing a system of laminar flow and $\mathrm{CO} 2$ recirculation. The purpose of this paper is to examine the effect of the AirSeal ${ }^{\circledR}$ versus a traditional trocar system in operative time, estimated blood loss and postoperative complications. To the best of our knowledge, this is the first analysis of this system in robotic colorectal surgery.

Methods: A single surgeon's database from 2015 and 2017 was reviewed and all robotic colonic resections with 2 or more cases per year were included for analysis, which included LAR and righ hemicolectomies. These dates were chosen as the surgeon began incorporating the valveless trocar system in routine surgical practice in early 2016, thus ensuring complete use of the traditional system in 2015 and complete use of the valveless system in 2017. Patient demographic information was evaluated and primary outcomes examined were operative time, estimated blood loss, postoperative complications and hospital length of stay.

Results: 42 patients were identified in the 2015 cohort (23 LAR and 19 right hemicolectomies) and 32 patients in the 2017 cohort (27 LAR and 8 right hemicolectomies). There was no statistical difference in sex, BMI or number of comorbidities per patient between the two years. Mean operative time for LAR was $276.8 \pm 69.8 \mathrm{~min}$ in 2015 and $214.5 \pm 54.9 \mathrm{~min}$ in $2017(\mathrm{p}=0.001)$ however this significant difference was not seen between right hemicolectomies. Mean estimated blood loss for LAR was $257.6 \pm 228 \mathrm{cc}$ in 2015 and $106.9 \pm 75 \mathrm{cc}$ in $2017(\mathrm{p}=0.005)$; again this significant difference was not appreciated for right hemicolectomies. There was no statistically significant difference in hospital length of stay, post-operative pneumonia, DVT/PE or 30-day readmissions between 2015 and 2017; however there were 3 prolonged intubations ( 2 in righ hemicolectomies, 1 in LAR) and 3 wound infections ( 1 in right hemicolectomies and 2 in LAR) in 2015 as opposed to no prolonged intubations and 1 wound infection in an LAR in 2017.

Conclusions: In patients undergoing low anterior resections, the AirSeal ${ }^{\circledR}$ trocar system was superior to the traditional trocar system in both operative time and estimated blood loss. There was also a trend towards decreased post-operative complication with AirSeal ${ }^{\circledR}$ use. The AirSeal ${ }^{\circledR}$ trocar system should be considered for use in distal colonic and rectal procedures.

\section{Development of a General Surgery Robotic Program: Is Progress for Everyone?}

Christina M Sharon, MD, Luca Milone, MD, Gonzalo Ausqui, MD; The Brooklyn Hospital Center

The DaVinci Platform for General Surgery is now used worldwide with excellent results throughout and although the future is bright in robotic surgery, some surgeons and institutions are still reluctant to invest time and resources into opening a robotic program. We report on our experience of building a General Surgery Robotic Program in a small community metropolitan hospital. The Robotic Program at The Brooklyn Hospital Center was launched in January of 2017 and by August of 2017, the General Surgery Department had commenced utilizing the DaVinci platform under the guidance of a Robotic fellowship trained surgeon. In a short twelve months, a single surgeon performed a total of 103 cases. These cases included 32 inguinal herniorrhaphy, 36 cholecystectomies, 24 ventral herniorrhaphy, of which 3 were transversus abdominus muscle releases, 8 colectomies, 1 adrenalectomy, 1 paraesophageal hernia, and 1 rectal resection. Of the 103 cases, we had 1 readmission within 30 days $(0.9 \%), 5$ surgical complications $(4.8 \%)$, and 1 mortality $(0.9 \%)$

Residents in the Surgical Program were actively involved in the Robotic Surgery program. From the beginning of the program, all residents in the General Surgery training program were required to complete online modules and one dry robotic lab prior to scrubbing into any robotic cases. Interns were limited to bedside robotic assistance while PGY 2 and above where allowed to enhance their skills on the console after performing a minimum of 15 cases at the bedside. At the completion of the year, Chief residents were able to perform uncomplicated herniorrhaphies and cholecystectomies independently. In addition to resident training, all operating room staff members that were part of the robotic team were trained individually, including weekly meetings that were held in order to allow for continued education.

In conclusion, our goal with this report is to shed light on the promising possibilities of piloting a robotic program in a small community metropolitan hospital and to encourage others to do the same. Success is measured by outcomes, and through our experience, we learned to achieve success starting with less complex procedures and to create a team environment that was conducive to minimizing complications. Resident training also plays an important role in creating enthusiasm around the robotic platform. 
P660

\section{Assessment of Current Robotic Surgical Training Curricula}

Jorge G Zarate Rodriguez, MD, William D Gerull, Michael M Awad, MD, PhD; Washington University in St Louis School of Medicine

Introduction: With the proliferation of robotic-assisted laparoscopic surgery (RALS), training programs have had to adapt to incorporate RALS-specific training. Without standardized curricula for RALS, we hypothesized the curricula on PubMed would vary in terms of their application of curriculum development pedagogy and in their applicability to the clinical realm.

Methods: Pubmed was queried using relevant MeSH terms and the terms "robotic surgery" and "curricul*." The results were reviewed for relevance, and scored by 2 independent reviewers for evidence of a modified Kern's sixstep model for curriculum development (Table 1), and were assigned a level based on Kirkpatrick's model of educational outcomes (Table 2).

Results: 228 of the 306 results returned by the query were discarded based on exclusion criteria. The remaining 78 were pared down to 44 based on relevance. All of the curricula accomplished at least 2 of the 7 modified Kern steps, most $(47.7 \%)$ accomplished 4 of 7 , and only one accomplished all 7 steps. Most articles $(70.5 \%)$ showed data that exhibited learner acquisition of knowledge and skills (Kirkpatrick level 2b). Two (4.6\%) articles presented evidence of organizational changes or patient benefits, achieving the highest Kirkpatrick levels, and two did not include any outcomes data.

Conclusion: Surgical training programs will need to adapt to ensure trainees develop the necessary skills to perform RALS. There is significant variation amongst published curricula in terms of the robustness of the educational experiences. Additionally, very few programs evaluate the effects of their curricula in a clinically-relevant manner.

Table 1

\begin{tabular}{ll}
\hline Kern step & Description \\
\hline 1 & Generalized needs assessment \\
2 & Targeted needs assessment \\
3 & Goals and objectives \\
4 & Educational strategies \\
5 & Implementation \\
$6 \mathrm{a}$ & Learner evaluation and feedback \\
$6 \mathrm{~b}$ & Curriculum evaluation and feedback \\
\hline
\end{tabular}

Table 2

\begin{tabular}{ll}
\hline Kirkpatrick level & Description \\
\hline 1 & Learner feedback \\
$2 \mathrm{a}$ & Changes in attitudes/perceptions \\
$2 \mathrm{~b}$ & Acquisition of knowledge/skills \\
3 & Changes in behavior \\
$4 \mathrm{a}$ & Changes in organizational practice \\
$4 \mathrm{~b}$ & Benefits to patient \\
\hline
\end{tabular}

\section{P661}

Robotic Repairs of Type IV Giant/Paraesophageal Hernias and Failed Anti-reflux Fundoplications

Sharona B Ross, MD, Alexander S Rosemurgy, MD, Danielle K Craigg, MD, Miguel Castro, Melissa Gonzalez, Iswanto Sucandy, MD; Digestive Health Institute Florida Hospital Tampa

Introduction: The repairs of a type IV giant/paraesophageal hernias and failed antireflux fundoplications are difficult, often exacerbated by operating through a reoperative field. With an experience of over 2,500 anti-reflux operations and with increasing penetrance of robotic surgery into our practice, we sought to document our outcomes with robotic redo hiatal hernia repairs/fundoplications and robotic repairs of type IV giant/paraesophageal hernias to evaluate the robotic approach. We hypothesize that the robotic approach is a safe and effective method to utilize in challenging fundoplications.

Methods: With IRB approval, 56 patients who had type IV giant/paraesophageal hernias $(\mathrm{N}=24)$ or failed antireflux fundoplications $(\mathrm{N}=32)$ were prospectively followed. Outcomes, including length of stay (LOS), estimated blood loss (EBL), operative time, intraoperative and postoperative complications, and conversions to 'open' operations were evaluated. Data are presented as median (mean + SD). Significance was accepted with $95 \%$ probability. Results: The 56 patients had a BMI of $26(25 \pm 3) \mathrm{kg} / \mathrm{m}^{2}$ and age of 67 $(66 \pm 11)$ years. With their operations, EBL was $25(53 \pm 75) \mathrm{mL}$, median operative time was $194 \mathrm{~min}(203 \pm 78)$, and LOS was $1(2 \pm 2)$ day; operative duration decreased progressively with experience $(p=0.023$, Figure). 4 patients had intraoperative complications (4 gastrostomies) and only four patients had postoperative complications ( 2 capnothorax [Clavian Grade 2b], 2 respiratory decompensation due to smoking/COPD [Clavien Grade 2a]). No patients were converted to an "open" operation. There were no differences between patients undergoing repairs of type IV giant/paraesophageal hernias or failed antireflux fundoplications, except all gastrostomies occurred (without consequence, Grade 2 Delphi classification) in those undergoing robotic repairs of failed antireflux fundoplications.

Conclusions: The robotic approach to repairing type IV giant/paraesophageal hernias and failed antireflux fundoplications seems easier than a laparoscopic approach; the redo operations are inherently more difficult. EBL, LOS, and frequency of notable complications were commendable and operative time was permissible and significantly decreased with experience. For these difficult operations a robotic approach will be our choice.

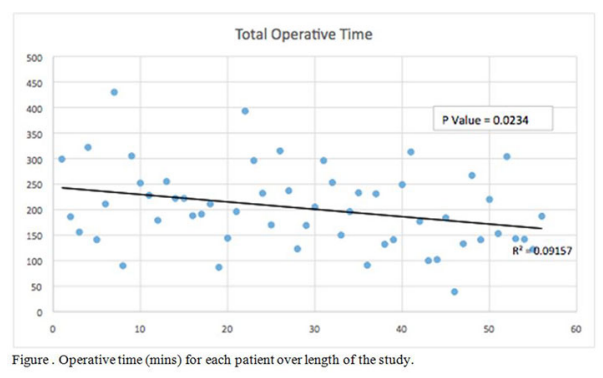




\section{P662}

Robotic-Assisted Laparoscopic Sigmoid Resection with Repair of Vaginal Defect and Diverting Loop Ileostomy for Treatment of Colovaginal Fistulas: A Case Series

Keitaro Nakamoto, Paul Bown; Marshall University Joan C. Edwards School of Medicine

Introduction: The objective of this study was to review our institution's experience on robotic-assisted laparoscopic sigmoid resection with repair of vaginal defect and diverting loop ileostomy as a primary treatment modality for colovaginal fistula.

Methods and Procedures: Patients with colovaginal fistulas treated by two surgeons (one general surgeon and one OB/GYN) between January 2018 and September 2018 were identified. A retrospective chart review was performed to determine outcomes including length of stay, operative times and complications.

Results: There were 4 patients identified. Median age was 72.5. All patient had a history of hysterectomy. All patients underwent cystoscopy with ureteral stent placement, robotic-assisted laparoscopic sigmoid resection with primary anastomosis, repair of vaginal defect, and diverting loop ileostomy. Average operative time was $223 \mathrm{~min}$. Median length of stay for initial procedure was 2.5 days. All patients underwent ileostomy reversal. Median time to ileostomy reversal was 35.5 days. Median length of stay after ileostomy reversal was 2.9 days. Median total length of stay overall was 5.4 days. There was one readmission due to high ileostomy output, otherwise there were no complications.

Conclusions: Robotic-assisted laparoscopic sigmoid resection, with vaginal defect repair and diverting ileostomy is a safe and effective way of managing colovaginal fistulas. This is an ongoing case series with more data expected to be added.

\section{P663}

Comparison of Intraoperative Outcomes Between Single-Incision Robotic Cholecystectomy and Multi-incision Robotic Cholecystectomy

\section{Philip Schertz, DO, Subhasis Misra; Brandon Regional Hospital}

Aim: The aim of this study is to evaluate the differences in key surgical factors for single-incision robotic cholecystectomy and multi-incision robotic cholecystectomy.

Method: A retrospective data review from August 2013 to April 2018 consisting of 104 single-incision and 105 multi-incision robotic cholecystectomies was done looking at factors including patient gender, age, operating time (skin incision to skin closure), robotic console time (docking to undocking), the preoperative diagnosis for surgery, any complications in surgery, length of stay (LOS), and estimated blood loss (EBL). Procedures with conversion away from original robotic cholecystectomy approach were excluded. Chi square analysis using p value of 0.05 as statistical significance were run between the two data sets.

Results: A total of 209 robotic cholecystectomy cases were reviewed since 2013. We found significantly less time with single-incision compared to multiincision $($ Single incision $=94.0 \mathrm{~min}$, multi-incision $=99.9 \mathrm{~min}, \mathrm{p}=0.016$ ) and estimated blood loss (single-incision $=11.52 \mathrm{~mL}$, multi-incision $=17.17 \mathrm{~mL}, \mathrm{p}=.004)$. There was no significant difference in age or robotic console time. The most common indication was symptomatic cholelithiasis overall, with equal cases of dyskinesia in single-incision approach, although there was no significant difference in indication between the two approaches. Intraoperatively there was a marginally significant use of irrigation in multi-incision (multi-incision 45 (42.9\%), single-incision 31 $(29.8 \%), p=0.0499$ ) and no difference in Firefly, perforation, or intraoperative cholangiogram use. Length of stay showed significant decreased stay in singleincision approach (single-incision 84 outpatient $(80.8 \%)$, multi-incision 75 $(71.4 \%) \mathrm{p}=0.0379)$.

Conclusion: In a single center, retrospective study, single-incision and multiincision robotic cholecystectomy showed single-incision is associated with less total operative time, blood loss, irrigation use, and shorter length of stay.

\section{P664}

\section{Cost Comparison of Single Site Robotic and Conventional Laparoscopic Cholecystectomy at a Single Institution}

Mary M Lee, DO, Kevin K Seeras, DO, John J Lim, MD; Henry Ford Macomb Hospital

Introduction: Single site robotic (SSR) cholecystectomy has become a popular technique at our institution with similar if not improved patient satisfaction compared to conventional laparoscopic (CL) cholecystectomy. Prior studies have suggested that these favorable patient outcomes are attenuated by the high cost of the robot. This study was designed to compare direct hospital costs between these two techniques on a case by case basis, excluding the capital purchase cost of the robot.

Methods: This is a retrospective, single site, single surgeon study comparing the cost of outpatient cholecystectomies using either single site robotic (SSR) or conventional four-port laparoscopic (CL) techniques. Patient demographic data, case duration, and hospital financial data were obtained from electronic medical records. We excluded those cholecystectomies requiring intraoperative cholangiograms, umbilical hernia repairs or other concomitant procedures such as liver biopsy. We analyzed the total cost of disposable equipment on a caseby-case basis and excluded amortization costs of the robot. Charges unique to the SSR group included robot-specific drapes, single site port, seals, hooks, hem-o-lock clips, and crocodile graspers while charges for the CL group included disposable trocars and endoclips. Continuous variables were described using the mean and SD or median (IQR) and categorical variables were described with the frequency and percentage. Student t test or Wilcoxon rank test was used for continuous variables, Chi square test or Fisher's exact test was used to categorical variables, as appropriate.

Results: A total of 125 patients were included in the analysis. These patients had either undergone conventional laparoscopy $(n=44)$ or single site robotic cholecystectomy $(\mathrm{n}=81)$ for an indicated diagnosis of biliary dyskinesia $(n=40)$ or symptomatic cholelithiasis $(n=85)$. There was no significant difference between groups in age, sex or ASA classification however, BMI was significantly higher in the CL group $(33.5 \pm 7.7$ vs $28.9 \pm 5.7, \mathrm{p}=0.002)$. Anesthesia time [119 (110-160) vs $134(122-170)$ minutes, $p=0.008]$ and skin-to skin operative time $(70.1 \pm 24.7$ vs $82.7 \pm 22.7 \mathrm{~min}, \mathrm{p}=0.005)$ were higher in the SSR group. After exclusion of the capital cost of the robot and operating room costs, there was no significant difference between median hospital cost for CL and SSR groups (\$488.9 (428.5-562.3) vs \$472.3 (293.5563), $\mathrm{p}=0.22$ ) per case.

Conclusions: Our data suggests that in the setting of outpatient cholecystectomy there is no difference in cost between SSR and CL cholecystectomy when anesthesia time and capital cost of the robot are excluded. 


\section{P665}

Use of Indocyanine Green Augmented Near Infrared Fluorescence Imaging in Identification of Biliary Anatomy During Robotic Radical Resection of Gallbladder Adenocarcinomas

Ali Ahmad, MD, FACS; University of Kansas School of MedicineWichita

Introduction: Gallbladder cancer remains a rare cancer with a poor prognosis. National guidelines recommend radical resection in the absence of metastatic disease. This often requires extensive dissection around the extrahepatic bile ducts. We report our experience of real-time near-infrared fluorescence (NIRF) imaging using indocyanine green (ICG) during robotic radical resection of gallbladder adenocarcinomas.

Methods: Six patients with gallbladder adenocarcinoma underwent robotic radical resection entailing central hepatectomy (segments IV-B and V) with regional lymphadenectomy. Real time NIRF imaging was performed using the da Vinci ${ }^{\circledR}$ Firefly system after administration of $5 \mathrm{mg}$ of ICG 30 to $60 \mathrm{~min}$ preoperatively. Primary objective was to determine safety of this technique. Results: Procedure was successfully completed in all patients. Four patients had incidentally discovered gallbladder cancer after laparoscopic cholecystectomy and had preoperatively known positive margins. Mean operative time was $220 \mathrm{~min}$. Mean intraoperative blood loss was $50 \mathrm{~mL}$ (30-250 mL). Median number of lymph nodes retrieved was 5 (4-7). High ligation of cystic duct was performed close to the common bile duct (CBD) junction with the assist of NIRF and negative margins were achieved in all patients. No procedural complications or mortality was seen at 30 days post-op.

Conclusion: Results from our limited experience demonstrate procedural safety and beneficial use of NIFC using ICG during robotic radical resection of gallbladder adenocarcinomas. It may assist in attainment of negative cystic duct margin and lymphatic clearance around the biliary tree especially in complex re-explorative biliary surgery.

\section{Table 1}

Patient Age Sex Lymph nodes Final Pathology Negative (Yrs) (Positive/Total) Margins

\begin{tabular}{llllll}
\hline 1 & 69 & F & $2 / 5$ & T2N1 & Yes \\
2 & 84 & M & $5 / 6$ & T3N2 & Yes \\
3 & 74 & F & $0 / 5$ & T2N0 & Yes \\
4 & 60 & F & $3 / 7$ & T3N2 & Yes \\
5 & 67 & F & $1 / 4$ & T3N1 & Yes \\
6 & 71 & M & $0 / 6$ & T2N0 & Yes \\
\hline
\end{tabular}
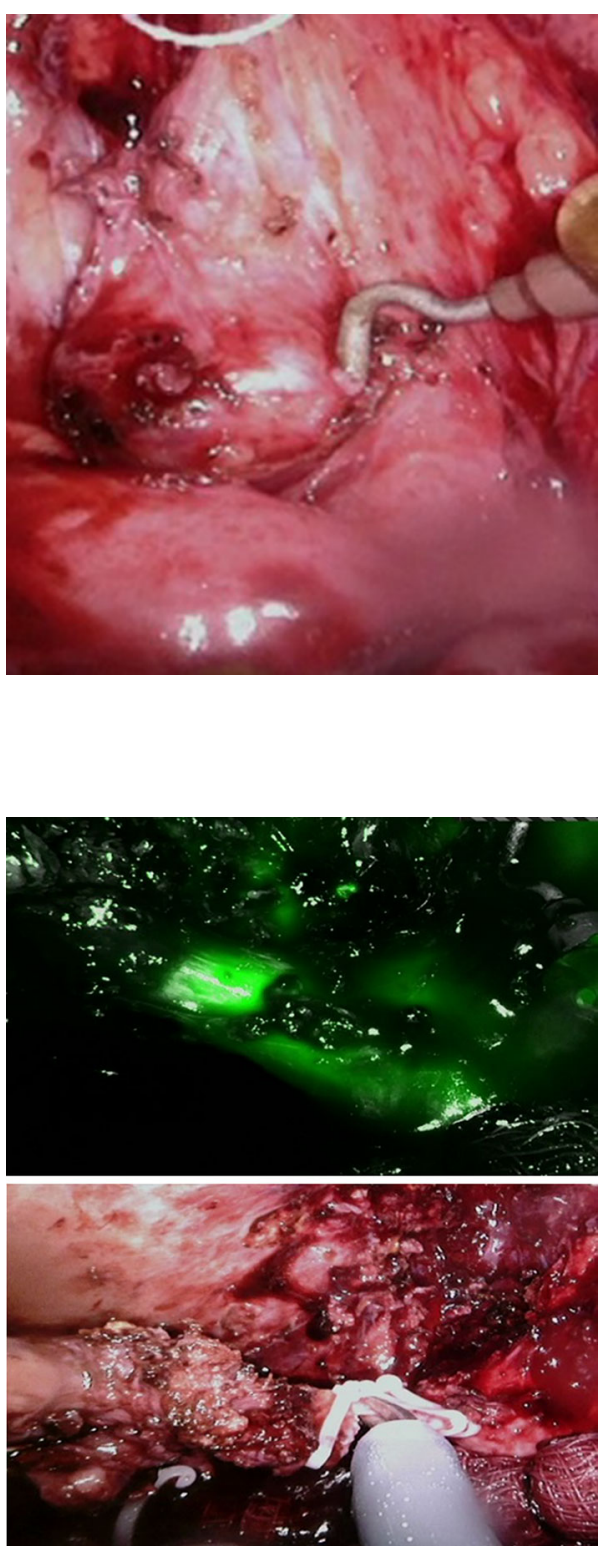
P666

\section{The Usefulness of Our Step-wise Training System for Robotic Assisted Gastrectomy}

Kozo Yoshikawa, Mitsuo Shimada, Jun Higashijima, Takuya Yoshimoto; University of Tokushima

Background/Aims: The purpose of this study is to examine the usefulness of the step-wise training system for introducing robotic assisted gastrectomy.

Step-wise training system: Our step-wise training consists of 5 parts (basic training, advanced training, clinical experience, self-assessment and feedback and brash-up in wet lab). This system is based on PDCA cycle of training. 1st step is training by simulator, 2nd step is training by a wet training using pigs and ultra-realistic practice models, 3rd step is checking the operation time in individual section in operation. 4th step is self-assessment and feedback by all member of our department. The last step is re-training in wet lab using pigs after recognition of the problems. Method: Initially robotic gastrectomy was performed without step-wise training(Group 1)(n=7 TG:2, DG:5)(Dec/2011-Oct/2012), Now robotic gastrectomy was performed with step-wise training(Group 2)(n=10 TG:1, DG:9)(May/2018-), and recent consecutive laparoscopic distal gastrectomy patients was examined (Group 3) $(\mathrm{n}=30)$. We examine the operation time and intraoperative bleeding and incidence of all surgical morbidities.

Result: Operative time was significantly shorter in group 2 than group $1(377 \mathrm{~min}$ vs $517 \mathrm{~min}$, $\mathrm{p}=0.01$ ). But group 1 took a significantly longer time compared to group 3 (377 $\mathrm{min}$ vs $270 \mathrm{~min}$, $p=0.01)$. When we focused on patients after technically stable in group $2(n=6)$, there was no significant difference compared with group $3(318 \mathrm{~min}$ vs $270 \mathrm{~min}, \mathrm{p}=0.08)$. In intra-operative bleeding, there was significant difference between group 1 and group $2(137 \mathrm{ml} \mathrm{vs} 15 \mathrm{~min}, \mathrm{p}=0.01$ ). On the other hand, there was no significant difference between group 2 and group $3(15 \mathrm{ml}$ vs $18 \mathrm{~min}$, $\mathrm{p}=0.6$ ). There was no surgical morbidity ( $>$ grade $3 \mathrm{CD}$ criteria) in all three groups

Conclusion: Robotic surgery was successfully introduced and safely performed, furthermore, our step-wise training system is useful to perform the robotic surgery. Tokunaga, Masaaki Nishi, Hideya Kashihara, Chie Takasu, Toshiaki

\section{P668}

Robotic vs. Standard Laparoscopic Cholecystectomy: Clinical Outcomes

P M Norwick, BS ${ }^{1}, \mathrm{~S}$ Shaheen, $\mathrm{MD}^{1}$, J Blebea, MD, MBA ${ }^{1}, \mathrm{~N}$ Conti $^{2}$, R Heckburn ${ }^{1}$, M Zayout ${ }^{3}$, J Clements, $\mathrm{PhD}^{1}, \mathrm{M}$ Ghanem, $\mathrm{MD}^{1}$; ${ }^{1}$ Central Michigan University College of Medicine, ${ }^{2}$ Case Western University/University Hospitals Medical Center, ${ }^{3}$ Royal College of Surgeons, Ireland, Dublin

Introduction: This pilot study was aimed to identify where a patient may or may not benefit from robotic assisted laparoscopic cholecystectomy, versus conventional laparoscopic cholecystectomy to uncover the overall utility of robotic assisted procedures for this common surgical operation. Methods and Procedures: A total of 244 patients were included. Subjects included all patients operated upon by a single surgeon between 2013-June 2018 who met the following inclusion criteria: 1) over age 18,2$)$ laparoscopic and robotic cholecystectomies performed by single surgeon. We compared clinical outcomes including, OR time, length of stay (LOS), readmission to the hospital, post-op ED visit, and post-op pain between non-robotic assisted single incision (Group A) and robotic assited laparoscopic (Group B) cholecystectomies. A Chi square analysis and t-test were used to compare these variables between the two groups. Propensity score matching (PSM) was used to predict the probability of having robotic surgery using gender, age, and BMI as variables.

Results: 144 patients were included in Group A and 100 in Group B. Compared with Group A, Group B was associated with shorter post-operative length of stay (mean, 0.8 vs. $1.61 ; \mathrm{p}=0.002$ ). There was no significant difference in OR time (mean, 64.82 vs. $65.04 ; \mathrm{p}=0.945$ ), readmissions $(4.0 \%$ vs. $3.5 \% ; p=0.830)$, post-operative $\mathrm{ED}$ visits $(7.0 \%$ vs. $7.6 \% ; \mathrm{p}=0.851)$ or post-operative pain $(13.0 \%$ vs. $21.3 \% ; p=0.137)$. Robotic cholecystectomy patients were younger (mean, 46.3 vs. $51.79 ; \mathrm{p}=0.023$ ) and had lower BMIs (mean, 30.6 vs. $32.7 ; \mathrm{p}=0.038$ ). There was no significant difference in gender between the two groups. Because of these differences, we conducted PSM which showed no difference between Group A and Group B regarding age, BMI, or gender and the statistical significance of LOS was maintained (mean, 0.93 vs. $1.9 ; p=0.009$ ).

Conclusion: These data demonstrate that robotic assisted cholecystectomies can reduce LOS for patients undergoing laparoscopic cholecystectomy, without increasing OR time. This enables surgeons to maintain OR fluency by not increasing the time it takes physicians to perform procedures. The reduced LOS would benefit both patient and hospital by decreasing risk of infection, while also reducing hopsital resource usage and cost.

\section{P669}

\section{Robotic Versus Laparoscopic Radical Cholecystectomy for Gallbladder Cancer at a High-Volume Minimally Invasive Center}

Ryan C Pickens, $\mathrm{MD}^{1}$, Jesse K Sulzer, MD, $\mathrm{PhD}^{1}$, Erin E Isenberg, $\mathrm{BSPH}^{2}$, Keith J Murphy, $\mathrm{MSPH}^{3}$, Lee Ocuin, $\mathrm{MD}^{1}$, Erin Baker, $\mathrm{MD}^{1}$, John B Martinie, MD, FACS ${ }^{1}$, David A Iannitti, MD, FACS ${ }^{1}$, Dionisios Vrochides, MD, PhD, FACS, FRCSC ${ }^{1}$; ${ }^{1}$ Department of Surgery, Atrium Health, ${ }^{2}$ University of North Carolina at Chapel Hill, ${ }^{3}$ Carolinas Center for Surgical Outcomes Science, Atrium Health

Introduction: This study aims to demonstrate that robotic-assisted radical cholecystectomy is safe and effective. Minimally-invasive radical cholecystectomy has gradually gained approval as a safe and oncologically acceptable modality in the treatment of resectable gallbladder cancer. While roboticassisted hepatopancreatobiliary surgery has been increasingly described with comparable results to laparoscopic approaches such as for primary liver and pancreatic tail malignancies, current literature for resection of gallbladder cancer has almost exclusively reported laparoscopic experience. We present a comparison of robotic-assisted and laparoscopic radical cholecystectomies at a high-volume center.

Methods and Procedures: A retrospective review was performed on all cases of robotic radical cholecystectomy (RRC) and laparoscopic radical cholecystectomy (LRC) at our institution from January 2013 to August 2018. Patients undergoing either radical cholecystectomy for gallbladder cancer detected preoperatively (cholecystectomy with $4 \mathrm{~b} / 5$ hepatectomy and portal lymphadenectomy) or a completion operation for malignancy identified after routine cholecystectomy ( $4 \mathrm{~b} / 5$ hepatectomy, cystic duct resection, and/or portal lymphadenectomy only) were both included. Any case converted to open was excluded from comparison. Operative details, tumor pathology, clinical and oncologic outcomes were analyzed.

Results: Twenty-seven patients underwent minimally invasive radical cholecystectomy for resectable gallbladder cancer (20 robotic, 7 laparoscopic). Fifteen patients (55.5\%) underwent a completion operation for cancer detected after cholecystectomy. No conversions to open occurred in the robotic cohort, however 1 patient was converted from laparoscopic to open due to safety concerns during adhesiolysis. Three patients in the RRC $(15 \%)$ did not undergo hepatic resection as the original tumor was on the gallbladder free edge and no residual tumor was identified on ultrasound. Median age, ASA score, estimated blood loss, and operative time were similar between groups. R0 resection was achieved in $66.7 \%$ of laparoscopic cases (4/6) and $83.3 \%$ of robotic cases (15/18). Median lymph node harvest was greater for the RRC $(p=0.043)$. Postoperative outcomes for median 30-day Clavien $3+$ complications, readmissions, and postoperative length of stay as well as final pathology for local invasion, nodal and metastatic spread were similar between cohorts. During a mean follow-up of 21 months (SD 27.18), 6 patients in the RRC (30\%) and 2 patients in the LRC (33.3\%) developed recurrence.

Conclusions: At a high volume minimally invasive center, robotic-assisted radical cholecystectomy is safe with comparable oncologic benefit when a minimally-invasive approach is possible for select patients. Increased utilization of minimally invasive surgery for gallbladder cancer may demonstrate additional technical benefits of robotic-assistance compared to laparoscopy for patients previously only offered open resection.

\section{Robotic Duodenal Sleeve Resection}

Jesse K Sulzer, MD, $\mathrm{PhD}^{1}$, Adeel Khan, $\mathrm{MD}, \mathrm{MPH}^{2}$, David Iannitti, $\mathrm{MD}^{1}$, Dionisios Vrochides, $\mathrm{MD}, \mathrm{PhD}^{1}$, John B Martinie, $\mathrm{MD}^{1} ;{ }^{1}$ Atrium Health, ${ }^{2}$ Washington University

Introduction: We have previously presented methods for successful robotic duodenal surgery including sleeve resections. After demonstrating technical feasibility we sought to investigate the safety profile of this new option for conditions of the duodenum that are not amenable to endo-

Methods: Retrospective review of all patients undergoing robotic duodenal sleeve resection at a tertiary care center from 2010-2018. Indications, procedural details and short and long term outcomes were reviewed

Results: Nine patients unresent sleeve (segmental) resection. Median age was $50 \mathrm{yrs}$, 67\% were male with a median BMI was 29.2. Sixty-seven percent were symptomatic with GI bleeding being the most common presentation. Adenoma was the most common diagnosis (56\%) followed by low grade neuroendocrine tumor. Median tumor size was $35 \mathrm{~mm}$ and all resections achieved negative margins. Median op-time was $222 \mathrm{~min}$ and EBL was $50 \mathrm{cc}$. Median postop length of stay was 5 days (range 4-12 days). Four patients had complications including one bleed requiring laparoscopic reoperation, two leaks managed non-operatively and a retroperitoneal hematoma requiring drainage for persistent pain. There were two 90-day readmissions and no 90-day mortalities. Recurrent disease or strictures were not seen in any patient after a mean follow-up of 3 months (range 1-15 months).

Conclusion: Robotic duodenal sleeve resection is a viable alternate to open or laparoscopic duodenal resection for lesions not amenable to endoscopic intervention. scopic intervention and can avoid morbidity related to an open procedure. 


\section{P670}

Achieving Cost Effectiveness in Robotic Rectal Cancer Surgery Single Tertiary Hospital Experience in the Indian Subcontinent

Venkatesh Munikrishnan, FRCS, Ajay C Vallabhaneni, MD, Sudeepta K Swain, MS, DNB; Apollo Hospitals, Chennai

Introduction and Aim: The use of robotic technique for rectal cancer surgery is slowly gaining popularity and acceptance but cost remains a main factor for more widespread uptake. The aim of this study was to find effective ways to control the costs without compromising the oncological principles of rectal cancer surgery.

Materials and Methods: This is a retrospective analysis of 108 patients with rectal cancer operated by an experienced colorectal surgeon using DaVinci Surgical Robot between August 2015 to January 2018 at a tertairy care hospital in India

Results: On analysis the average cost for robotic rectal cancer surgery was INR 504037.30 (\$ 6990.32). If the cost of first 54 cases(INR 536132.8/\$7423.80) is compared to the second 54 cases(INR 472536.20/\$6553.44), there is a drop in cost of about INR 63,596.80 ( $\$ 880.50)$. The cost savings can be attributed to high volume of rectal cancer surgery performed and the experience gained by the operating surgeon and the assisting team. We have a two-team approach, and member of each team has a set assigned duties which help to reduce time and overall costs. For Robotic Rectal Cancer surgery we use 3 arms only (a camera and 2 working arms) with single docking technique and reusable ports. Conclusion: Robotic Rectal Surgery can be cost effective without compromising on oncological principles by high volume surgeons using a trained two team approach using 3 arm technique with single docking and reusable ports.

\section{P671}

Usefulness of the Teleflex Mini-grasper in 160 Laparoscopic Cases

\section{Hugo Bonatti; Meritus Health}

Background: The Teleflex minigrasper is a $2.4 \mathrm{~mm}$ instrument, which is inserted into the abdominal cavity without use of a Trocar. Only limited experience in general laparoscopic surgery is available. We herein report a large case series, where the minigrasper replaced a trocar based instrument. Patients and Methods: From June 2015 to September 2018, the Teleflex minigrasper was used in 160 laparoscopic procedures including 73 cholecystectomies (LC), 51 appendectomies (LA), 25 inguinal hernia repairs (TAPP), 5 ventral hernia repairs $(\mathrm{LVH})$ and 6 exploratory laparoscopies with tissue biopsy and/or lysis of adhesions.

Results: In the vast majority of cases a two port plus minigrasper technique was used including all appendectomies. In subsets of cholecystectomies ( $\mathrm{n}=7$ i.e. $9.5 \%)$ and bilateral inguinal hernia repairs $(n=4$, i.e. $16 \%) 3$ trocars plus the minigrasper were inserted. For LC, the minigrasper was placed between an umbilical and a LUQ port and a modified dome down technique was used. For the majority of appendectomies the minigrasper was placed in the suprapubic area with $5 \mathrm{~mm}$ ports in the umbilicus and LUQ and appendix and mesoappendix were secured using an endoloop. For inguinal hernia repair a modified transperitoneal technique was developed creating the peritoneal flaps from a subumbilical vertical incision. The minigrasper replaced the Maryland grasper during intracorporeal suturing. There were no conversions to open surgery in this series and no complications related to the minigrasper were encountered. Cosmetic results were excellent and patient satisfaction was high.

Conclusions: The minigrasper can completely replace a $5 \mathrm{~mm}$ instrument in many commonly performed laparoscopic procedures including cholecystectomy, appendectomy and hernia repair, however, some technical modifications need to be made. Obesity does limit its usefulness.

\section{P673}

Pressurized Intra-Peritoneal Aerosol Chemotherapy (PIPAC) for Carcinomatosis Patients: A Laparoscopically Administered Palliative Treatment that is Well Tolerated, Repeatable, and Safer Than Systemic Chemotherapy

Carl Winkler, $\mathrm{MD}^{1}$, Jaspreet Sandhu, $\mathrm{MD}^{2}$, Dasuni Niyagama Gamage, MD ${ }^{1}$, Neil Mitra, MD ${ }^{1}$, Xiaohong Yan, $\mathrm{PhD}^{1}$, Hmc Shantha Kumara, $\mathrm{PhD}^{1}$, Nipa Gandhi, MD ${ }^{1}$, Richard Whelan, $\mathrm{MD}^{1}$; ${ }^{1}$ Mount Sinai West Hospital, ${ }^{2}$ Brookdale University Hospital Medical Center

Introduction: Most carcinomatosis patients (pts) with ovarian, colorectal, gastric, or appendiceal primary tumors are not candidates for cytoreductive surgery and HIPEC; most are treated with systemic (IV) chemotherapy (CT) which has notable morbidity and marginal responses. Because peritoneal implants are poorly vascularized, intra-lesion CT levels from IV CT are low vs. blood levels. PIPAC is an alternative palliative laparoscopic treatment approach that delivers an aerosol of CT directly into the peritoneal cavity under pressure $(12 \mathrm{mmHg})$. This results in minimal blood elevations and a low rate of systemic complications. No cytoreduction is done. In ex vivo animal studies aerosolized CT has been shown to penetrate the peritoneum more deeply (100-200 um) than liquid CT. PIPAC is being used in Europe but not in the US. This poster will introduce this method and give a brief summary of results to date.

Method: A $12 \mathrm{~mm}$ Hassan port is placed via cut down; after insufflation a $5 \mathrm{~mm}$ port is placed. MIS exploration (PCI scoring) and implant biopsy are followed by administration of an aerosol of 1-2 CT agents via high pressure injector and aerosolizing device via the $12 \mathrm{~mm}$ port. Once infused, a $12 \mathrm{mmHg}$ pneumo is maintained for $30 \mathrm{~min}$ after which the gas/aerosol is evacuated via filters into a canister and the ports removed. Discharge home is usually on postop day 1. PIPAC can be repeated (6 week intervals).

Results: As per the literature (16 series $>10$ pts) PIPAC was successfully given to $627 / 709(88.4 \%)$ who underwent a total of 1,408 PIPAC treatments for a variety of tumors (mean, 2.2 PIPAC/patient). The mean rate of grade 3 CTCAE (adverse events) was $6.5 \%$ per PIPAC (91/1408) and the 30 day mortality was $2 \%$. The objective response rate as judged by serial biopsy, PCI score, or RECIST criteria was $45 \%$ overall; for the 145 pts who got ${ }^{3} 2$ PIPAC the response rate was $74 \%$. The mean survival for this mixed group was 12.9 months. Of note, quality of life scores were maintained with PIPAC vs standard CT results. There have been no staff safety issues/problems.

Conclusion: PIPAC has been well tolerated with respectable response rates and low morbidity (vs IV CT). PIPAC allows pts to avoid IV CT and its higher morbidity. Prospective studies are underway to establish survival rates for each tumor type. A phase 1 FDA approved PIPAC trial will shortly begin in the US. 
P674

Post-Nisen Recurrent GERD and Severe Dismotility

of Esophagus. 1st Case Worldwide Treated

with Electrostimulation. 3rs Follow Up

David Aguirre-Mar, MD, MMHB, MBA, MEdu, MBA, MEdu;

Instituto Tecnologico de Estudios Superiores Monterrey

Introduction: Recurrent GERD is common clinical problem affecting Quality of Life worldwide. Redo surgery helps but deteriorated distal esophageal peristalsis(DEP) reduces best options. Electrical Stimulators (NES) at LES has reported good results controlling GERD in primary cases, with no previous published experience in redo cases. Prospective randomized protocol started to evaluate NES in recurrent GERD.

Abstract: Presenting 1stcase worldwide using NES for recurrent GERD. A 36 years-old male with twenty years with GERD. Symptoms: heartburn, regurgitation and chest pain. IBP's gained symptoms control. Upper Gastrointestinal Endoscopy (UGIE) revealed Grade D Esophagitis before and Non-Erosive disease after treatment. Barret's Esophagus was documented. Reported DeMeester Score 21, Acid exposure $<4$ at $5.8 \%$, LES pressure at $9 \mathrm{mmHg}$ with normal DEP. Floppy Nissen Fundoplication (NF) and small Hiatal Hernia (HH) repair was performed. No residual PO Medication, 4yrs symptoms control was accomplished. Recurrent disease found small recurrent $\mathrm{HH}$, DeMeester at 20, LES at $7.7 \mathrm{mmHg}$ and $80 \%$ of normal DEP were found. A redo operation was done and GER symptoms control, no residual POMed and normal endoscopic findings were achieved for 9 years. At recurrence, minimum $\mathrm{HH}$ at Barium x-Rays, Grade B Esophagitis at UGIE, DeMester Score at 10 and normal LES and DEP test failed $100 \%$ (Absent of Peristalsis). BMI was normal. Patient refused radical surgery options or to live under chronic POMed requesting surgical alternatives. Patient went to $\mathrm{HH}$ closure, Tupet reconstruction and NES placement under prospective protocol. Successfully done, three years later patient are doing with no dysphagia, light heartburn after heavy meals, using $150 \mathrm{Mg}$ PO Ranitidine at night, UGIE has reveled short segment Barret's Esophagus without dysplasia. QoLQ-GERD and Visyck's Score reported excellent satisfaction.

Discussion: NES was turned off to evaluate placebo effect. Symptoms recurred over a week and it was turned on again. Probably when NES is quiet, distal esophageal clearance at night may be compromised, prolonging acid exposure of the esophageal mucosa, causing intermittent esophagitis. We started Ranitidine PO Med every night to reduce the risk.

Conclusion: Initial experience is now 7 patients. Follow up data analysis is coming. At present, the procedure is feasible, secure, with $0 \%$ mortality and morbidity related to NES operation or its placement. Pacemaker needs be turned off to enter NMR due to risk of permanent magnetic damage and that could represents a discomfort in chronic elder or high sport patient groups. More studies and patient population are needed to reach any conclusion

\section{P675}

Near Real-Time Diagnosis of Omental Metastases in Patients with Primary Colorectal Cancer Using Rapid Evaporative Ionisation Mass Spectrometry (REIMS)

Eftychios Manoli ${ }^{1}$, Afeez Adebesin ${ }^{1}$, Sam Mason ${ }^{1}$, Zsolt Bodai ${ }^{1}$, Julia Balog ${ }^{2}$, Hiromi Kudo ${ }^{1}$, Steven Pringle ${ }^{2}$, Robert Goldin ${ }^{1}$, Ara Darzi ${ }^{1}$, Jamie Murphy ${ }^{1}$, Zoltan Takats ${ }^{1}$, James Kinross ${ }^{1}$; ${ }^{1}$ Imperial College London, London, United Kingdom, ${ }^{2}$ Waters Research Center, Budapest, Hungary

Introduction: The presence of peritoneal and omental metastatic disease from primary colorectal cancer alters a patient's optimal treatment strategy. Indeterminate lesions found at staging laparoscopy or at the time of resection can undergo subjective visual assessment and postoperative histological analysis an approach that is time-consuming and costly. There is an unmet need for novel tools that provide real-time tissue phenotyping. Rapid Evaporative Ionisation Mass Spectrometry (REIMS) performs real-time chemical analysis of biological tissues utilising electrosurgery-generated aerosols. This study aimed to integrate REIMS with the Harmonic ${ }^{\circledR}$ scalpel, assessing its efficacy in diagnosing omental metastases.

Methods: Patients undergoing surgical resection for colorectal cancer with suspected omental metastasis were recruited at St Marys Hospital, UK, as part of a prospective cohort study. Fresh frozen normal and tumour-infiltrated omentum samples were analysed ex vivo using a Harmonic $\mathrm{ACE}^{\circledR}+7$ fitted with a tapered-tip blade (Ethicon Endo-Surgery, JnJ). Sampling was performed at $5 \mathrm{~W}$, with aerosols aspirated into a Xevo G2-XS QToF mass spectrometer (Waters Corporation). The relative abundance of cellular metabolites was subjected to multivariate statistical analyses including principle components analysis and orthogonal partial least squares discriminant analysis in SIMCA (Umetrics). The ability of REIMS to predict the presence of tumour infiltration was assessed using leave-one-seventh-patient-out cross-validation.

Results: 14 patients (10 females, median age 58, range $25-82$ ) with primary colorectal cancer were included. 88 normal and 51 tumour-infiltrated omentum samples were analysed generating 139 spectra. REIMS was able to accurately distinguish normal from tumour-infiltrated omentum with an accuracy of $99.3 \%$, sensitivity of $98.8 \%$, specificity of $100 \%$, PPV of $100 \%$ and NPV of $98.8 \%$. The relative abundance of metabolites in the mass-to-charge range of 600-1000 Da were responsible for differentiating the normal and tumour groups; corresponding to a variety of cellular lipid species. This platform has been deployed in three human studies as a proof of concept, for providing realtime chemical staging of advanced colorectal cancer.

Conclusions: REIMS can be coupled to the Harmonic surgical device to accurately distinguish tumour-infiltrated and normal omentum in patients with colorectal cancer. This may provide the surgeon with real-time tissue feedback of indeterminant lesions, allowing intra-operative decision making to improve clinical outcomes. 


\section{P676}

\section{Laparoscopic Surgery with Minilap}

Quintin Gonzalez, FACS, MD, Monica De Jesus, MD, Jesus Bahena, MD, Guillermo Arana, MD, Sari Mejia, MD; Hospital Humanitas Medical Group Coyoacan
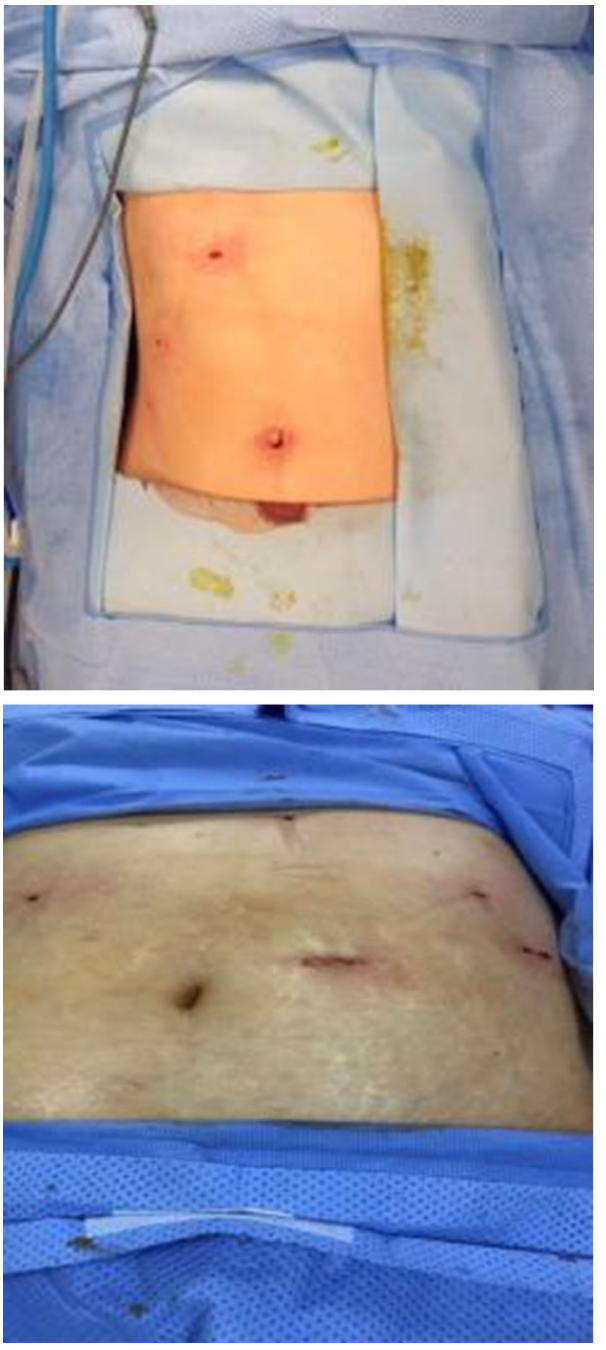

Background: In recent years laparoscopic techniques have evolved trying to improve surgical results and the mini laparoscopic approach has shown its advantages in terms of morbidity, less incisional pain, greater comfort and better cosmetic results, therefore, shorter hospital stay and return to daily activities early.

Objective: To prospectively analyze a sample of 30 patients, underwent to a minimally invasive laparoscopic surgery using minilap percutaneous tweezers (teleflex) determining its advantages.

Material and Method: A prospective study of 30 patients with a diagnosis of vesicular lithiasis, vesicular dyskinesia and GERD, with cholecystectomy and nissen fundoplication by laparoscopy, respectively, minilap tweezers, TeleflexR, percutaneous clamp type and minigresper clamp, both of $2.3 \mathrm{~mm}$ of diameter, which are introduced percutaneously into the abdominal wall without requiring the use of trocars.

Results: The MiniLap percutaneous tweezers were used in the 30 patients, 3 percutaneous forceps and 2 trocars were used in the fundoplication, in the cholecystectomy 2 percutaneous tweezers in the right flank, a subxifoid $5 \mathrm{~mm}$ and a $10 \mathrm{~mm}$ umbilical trocar (image 1 and 2), the first atraumatic clamp was used by the assistant to stabilize the gallbladder and raise it to expose the operative field, the second minilap clamp was used in the left hand of the surgeon to expose the calot triangle and facilitate the dissection with cautery hook in cases of Nissen fundoplication, the first clamp was used to pull the liver and expose the operative field and the second to facilitate the dissection of short vessels and esophageal pillars, closure by planes was carried out and steri-strips were placed in the access sites of the Percutaneous tweezers, both procedures were carried out without complications. Postoperative analgesia was achieved with intravenous paracetamol every $8 \mathrm{~h}$ obtaining adequate control and less pain, as well as $2.3 \mathrm{~mm}$ scars with a satisfactory aesthetic result (image 3 and 4). All patients were discharged the next day and there were no complications during ambulatory follow-up 7 days after surgery.

Conclusion: This study demonstrates that percutaneous retraction of the liver and vesicle with the percutaneous system of TeleflexR MiniLap is safe and effective in this series of patients. The complication rate implied by this technique is low and this new method provides less trauma, better cosmetic results than the conventional technique, as well as less postoperative pain and decreased hospital stay
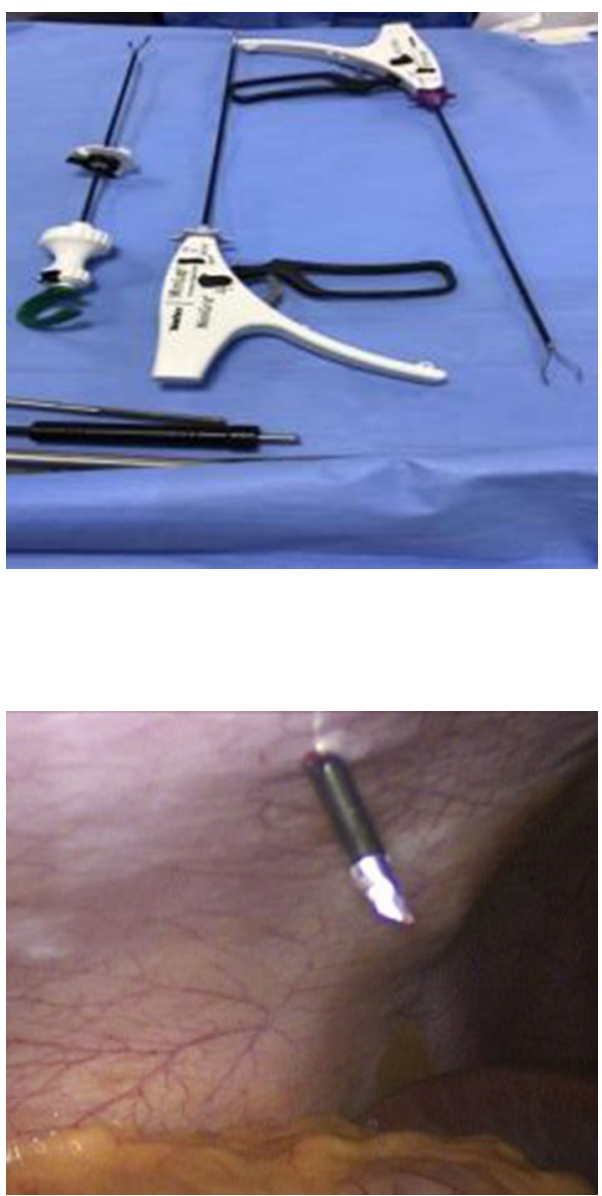
P677

\section{The First Robotic Pancreaticoduodenectomy in a Third World Country}

Ruben Luna, MD, Carlos Luna, MD, Luis F Cabrera, Daniel Gomez; Bosque University

Introduction: Pancreatoduodenectomy is the procedure of choice with curative option for the management of solid tumors of the head of the pancreas, however, despite advances in minimally invasive surgery in laparoscopy and robotic surgery of the last two decades, the vast majority of These procedures continue to be carried out openly, since, regardless of the development of the technology, complications vary from $25 \%$ to $65 \%$.

Objectives: Show our experience in the realization of the first robotic pancreatoduodenectomy in a third world country.

Materials and Methods: Patient underwent electiverobotic pancreatoduodenectomy was performed with the DaVinci XI robotic system. Demographic data, American Society of Anesthesiologists classification, preoperative testing, operative time, length of hospital stay, conversion, morbidity and mortality were recorded.

Results: The first robotic pancreatoduodenectomy was performed in a third world country, a surgical time of $480 \mathrm{~min}$, with acceptable intraoperative bleeding, no complications, adequate control of postoperative pain, minimal stay in the ICU, no pancreatic fistula and no mortality. Conclusions: Robotic pancreatoduodenectomy is a complex procedure, which should be performed by experienced surgeons, in reference centers and requires a long learning curve, however, the precise movement of the robotic arm and the high-definition, three-dimensional image allows a better dissection of the tissues.

\section{P679}

Robotic Assisted Resection for Duodenal Stromal Tumors: A Single Center Experience

Zhiming Zhao, Rong Liu, Yuanxing Gao, Wenbo Tang, Yang Wang, Zhuzeng Yin, Yong Xu; Chinese PLA General hospital

Aims: to discuss the value of robotic assisted resection in the treatment of duodenal stromal tumors. Methods: all the cases of pathology proved duodenal stromal tumors during and september 2014 and March 2018 were retrospectively reviewed, and all the cases who were performed robotic assisted resection on were enrolled in this study. The location of the tumors, the exact surgical procedure, the diameter of the tumor, the intraoperative duration, the intraoperative blood loss, the postoperative hospital stay, and the complication rate were all analyzed.

Results: a total of 17 cases were enrolled in the present study, including 8 males and 9 females, with an average age of 56.4 ( 34 to 71). The tumor were located in descending part in 12 cases, and in horizontal part in the other 5 cases. The average maximal diameter was $5.53 \mathrm{~cm}(1.5 \mathrm{~cm}$ to $10 \mathrm{~cm}$ ). Robotic pancreatoduodenectomy were performed in 8 cases, and local resection of tumors were performed in other 9 cases. The average intraoperative duration was $156 \mathrm{~min}$, the average intraoperative blood loss was $118 \mathrm{ml}$, and the avereage postoperative hospital stay was 9 days. There was one case of post operative panceratic fistula, and one case of postoperative infection. No patients died in 6 months.

Conclusion: Robotic assisted resection was safe for duodenal stromal tumors and had the advantages because of its minimally invasiveness. However, the compare of prognosis still needs to be proved in further studies.

\section{P678}

\section{Do Attending Physicians and Trainees Agree About What Happens in the Operating Room During Robot-Assisted Laparoscopic Hysterectomies?}

Alexandra J Berges ${ }^{1}$, S. Swaroop Vedula, MBBS, $\mathrm{PhD}^{2}$, Edward J Tanner, $\mathrm{MD}^{3}$, Amanda N Fader, $\mathrm{MD}^{3}$, Stacey Scheib, $\mathrm{MD}^{3}$, Betty Chou, $\mathrm{MD}^{3}$, Gregory D Hager, $\mathrm{PhD}^{2}$, C. C. Grace Chen, MD, MHS ${ }^{3}$, Anand Malpani, $\mathrm{PhD}^{2} ;{ }^{1}$ Johns Hopkins University School of Medicine, ${ }^{2}$ Malone Center for Engineering in Healthcare, ${ }^{3}$ Johns Hopkins Medicine Department of Gynecology and Obstetrics

Introduction: The objective in this study was to determine agreement between attending and trainee surgeons on important aspects of what happened in the operating room during robot-assisted laparoscopic hysterectomy (RALH) procedures, which are essential for teaching and assessment, e.g., case participation for accurate case logs, intraoperative skill assessment for performance evaluation and feedback.

Methods and Procedures: Attending and trainee surgeons performing 56 RALH procedures responded to questionnaires postoperatively concerning: 1 ) who performed each of 11 steps (L/R excise infundibulopelvic ligament, excise utero-ovarian ligament, skeletonize uterine vessel, electrodessicate uterine vessels, dissect bladder, colpotomy or amputate uterus, close vaginal cuff or cervical stump), 2) vaginal cuff closure technique (VCCT) and suture device (SD) used, 3) trainees' self-assessed skill and attending assessment of trainees' skill using GEARS, and difficulty of surgery. For bladder dissection, colpotomy, and vaginal cuff closure, we asked what percentage $(0,25,50,75$, or $100 \%)$ was performed by the trainee. We analyzed agreement between attending and trainee surgeons using Cohen's kappa for categorical variables and Krippendorff's alpha (KA) for ordinal variables, as well as Spearman's correlation (rho).

Results: Kappa was greater than 0.6 for 8 surgical steps (kappa $=0.71,0.62,0.82,0.85,0.71,0.78$, $0.86,0.86$ respectively), suggesting strong agreement between attending and trainee surgeons. We observed kappa values of 0.79 and 0.79 for VCCT and SD, indicating strong agreement.

There was weak agreement for depth perception and bimanual dexterity criteria of the GEARS scale (rho $=0.21,0.29$ and $\mathrm{KA}=0.158,0.007$, respectively). Skill assessments by attending and trainee surgeons on the domains of efficiency, force sensitivity, autonomy, and $\mathrm{r}$

obotic control exhibited moderate correlation and agreement $(r h o=0.468,0.534,0.440,0.495$; $\mathrm{KA}=0.473,0.506,0.412,0.484$, respectively)

There was a moderate level of agreement $(\mathrm{kappa}=0.484)$ on estimating difficulty of surgical case Conclusions: Our findings show that there is imperfect agreement between attending and trainee surgeons even with who performed parts of the procedure, which intuitively, should be unambiguous. They also disagreed on case difficulty, and technical skill. This incongruency on critical aspects of what happened in the operating room poses a significant challenge to teach and learn hysterectomy. 


\section{P680}

\section{A Web Platform for Image Segmentation and 3D Model Reconstruction}

Atul Kumar, $\mathrm{MD}, \mathrm{PhD}^{1}$, Wayne Shei-Wei Huang, $\mathrm{MD}^{2}$, Ching-Chun Huang, $\mathrm{PhD}^{3}$, Quentin Courbon, $\mathrm{BS}^{4}$, Yen-Yu Wang, $\mathrm{MS}^{5}$, Kai-Che Liu, PhD ${ }^{1}$, Lih-Shun Chen, MS ${ }^{1}$; ${ }^{1}$ IRCAD-AITS, Chang Bing Show Chwan Memorial Hospital, ${ }^{2}$ Show Chwan Memorial Hospital, ${ }^{3}$ National Chung Cheng University, Taiwan, ${ }^{4}$ University of Strasbourg, France, ${ }^{5}$ National Changhua University of Education, Taiwan

Introduction: Conventionally diagnosis in a medical image recorded as a text report and a pointer to the lesion in the image. Segmentation of the lesion area in the image is required to reconstruct its 3D model. A 3D model of lesion provides a better understanding of its extent. Moreover, such segmentation provides useful information for developing predictive models of the lesion. There are several tools available for image segmentation, however, to our knowledge, no web-based platform is available for medical image segmentation and 3D modeling. A web-based application is useful for creating a cloudbased knowledge system for research and development. Although one study [1] presented a framework for the similar application, our application uses different technology. In this work, we present the preliminary form of the web application which can be utilized for manual segmentation of medical images and further reconstruct a 3D virtual model from the segmented regions.

Methods: The application utilized HTML and Javascript as client side and Python as the server side programming language. A user can upload the image sequence files using the interface. The interface displays one of the images, and the user selects a region in the area of interest. The system records the pixel location of the selected regions, and a binary image mask for the region is created. The pixel coordinates of the binary mask are retrieved and scaled by the factor of image resolution to bring it into the real-world measurement. The edge coordinates are connected to neighboring points using Delaunay's triangulation.

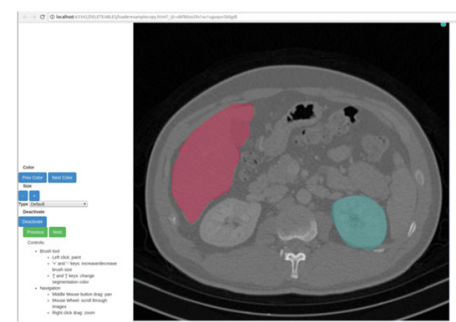

Results: The system was applied to a CT image dataset to find the region of interests (Figure 1). The 3D model reconstruction from the segmented region will be accomplished in the next step. The system is easy to use and does not require additional training. The accuracy of the segmentation would be user dependent.

Conclusion: The current work presents the preliminary results of an image segmentation web application which will be further used for 3D reconstruction of organs. Such a system would be useful for understanding the extent of lesion in three dimensions and creating a knowledge database for medical image diagnosis.

Acknowledgments:

We thank Chang Bing Show Chwan for supporting this study.

References:

[1] Jacinto, Hector, et al. "A web interface for 3D visualization and interactive segmentation of medical images." Proceedings of the 17th International Conference on 3D Web Technology. ACM, 2012

\section{P681}

\section{Are Small Community Hospitals Ready for Robotic Surgery Residency Training?}

Allyne Topaz, MD, Lauren Poliakin, MD, Luca Milone, MD; The Brooklyn Hospital

Background: Previous studies have shown positive outcomes involving dedicated resident training in robotic general surgery cases in small community hospitals. The aim of this study is to provide more evidence regarding a robotics curriculum in a general surgery residency program at a small community hospital with one DaVinci Xi. Our current robotic training education program begins in the use of surgical simulators in a risk-free environment ("dry-lab") to begin the development of basic robotic skills and bedside assistant training. The resident will advance to assisting at bedside in the operating room and eventually perform the procedures under the supervision of the robotic trained general surgeon.

Methods: 15 general surgery residents (PGY 1-5) without any exposure to robotic surgery were introduced to robotic training. All the residents were required to complete the online modules and dry robotic lab before scrubbing in robotic cases.

The dry-lab sessions were performed on Saturdays when the robot was readily available. The training involved the following exercises: peg transfer, right and left hand suturing, camera and clutching with ring tower transfer.

Interns were only allowed to learn bedside robotic assistance while PGY 2 and above were allowed to sit at the console once at least 15 cases at the bedside were performed.

Results: From January 2017 to September 2018, 103 robotic general surgery cases involving general surgery residents under the supervision of a single surgeon in a 297-bed community hospital. The cases included: 32 inguinal hernia repairs, 36 cholecystectomies, 24 ventral hernia repairs, 8 colectomies, 1 adrenalectomy, 1 paraesophageal hernia repair, 1 abdominoperineal resection. Each resident assisted bedside in about 12-15 cases. Each PGY3 and above resident performed 5 to 6 cases under the supervision of the surgeon. By the end, of the year chief residents were able to perform uncomplicated hernia repairs and cholecystectomy independently. $103(100 \%)$ were completed robotically. There was 1 readmission within 30 days $(0.9 \%)$ and 5 patients with complications $(4.8 \%)$, including 1 ventral hernia recurrence, 1 seroma, and 3 groin hematomas. There was 1 mortality $(0.9 \%)$; after an uncomplicated outpatient cholecystectomy, the patient was readmitted overnight after cardiac arrest at home.

Conclusion: There is an increase in demand for robotic general surgery cases, which prompts residency programs to train their general surgery residents in basic robotic skills. General surgery residency programs should incorporate a formal robotics curriculum with simulation training and early robotic surgery exposure. 


\section{P682}

Retroperitoneoscopic Versus Laparoscopic Enucleation for Distal Pancreatic Insulinomas: A Retrospective Study

Guodong Zhao, Yuyao Song, Rong Liu, Zhiming Zhao, Yuanxing Gao, Zhuzeng Yin, Yong Xu; Chinese PLA General hospital

Background: Retroperitoneoscopic pancreatic surgery has been reported to be safe and advantageous. However, studies on the comparison of retroperitoneoscopic and laparoscopic pancreatic surgery are still lacking. In this study, we sought to compare the efficacy and safety of retroperitoneoscopic enucleation (RE) with laparoscopic enucleation (LE) in the treatment of distal pancreatic insulinomas.

Study Design: This was a retrospective comparative study of the clinical data of 18 and 24 patients who underwent RE and LE, respectively, between January 2010 and February 2014. The operation time, intraoperative blood loss, and complications were in comparison.

Results: The results showed significantly shorter operation time $(89.23 \pm 17.18$ vs. $128.90 \pm 56.16 \mathrm{~min} ; \mathrm{P}=0.018)$ and less intraoperative blood loss $(34.23 \pm 27.07$ vs. $117.37 \pm 123.24 \mathrm{ml} ; \mathrm{P}=0.044)$ in $\mathrm{RE}$ group than in LE group. However, no significant difference was noted between the two groups in the time to oral intake, hospitalization, and incidence of pancreatic fistula.The incidence of postoperative hemorrhage was only one case in the LE group and none in the RE group.

Conclusion: RE is an alternative surgical approach for the management of selected distal pancreatic insulinomas, and appears to be more beneficial than LE in terms of direct access to the pancreas, shorter operation time, less blood loss, and probably less severe postoperative complications.

Keywords: Pancreas; insulinomas; laparoscopy; retroperitoneoscopy.

\section{P683}

Novel Fluorescent IV Dye for Real-Time Laparoscopic Identification of Ureters in an Inflammatory Swine Model

Lung W Lau, MD ${ }^{1}$, Michael Luciano, $\mathrm{PhD}^{2}$, Martin Schnermann, $\mathrm{PhD}^{2}$, Jaepyeong Cha, $\mathrm{PhD}^{1}$; ${ }^{1}$ Children's National Medical Center, ${ }^{2}$ National Cancer Institute

Introduction: Ureteral identification during laparoscopic surgery can be difficult, with iatrogenic ureteral injuries reported as high as 7.6 percent during colorectal and gynecologic surgeries. Current methods of identification, such as ureteral stents or watching for peristalsis, are not always effective. In settings of inflammation and distorted anatomy, ureteral identification is even harder, with greater risk for injury. The use of fluorescent dye can improve intraoperative ureteral identification without the need for any additional, invasive procedures. While no ureter-specific fluorescent dye exists for clinical use, our team has previously described the development of a pre-clinical ureter-specific dye, UL-766. Here, we present the use of the fluorescent dye during laparoscopy to assist in ureteral identification in an inflammatory swine model.

Method: With approval from IACUC, two $20-25 \mathrm{~kg}$ swine underwent laparoscopic abdominal surgery with use of an FDA-approved fluorescent laparoscopic system. Inflammation was created by sharp and blunt dissection of right ureter from surrounding tissues. Tissue surfaces were further irritated by rubbing with surgical gauze. The animals were allowed to recover and returned to the operating room after seven days. Images of the inflamed right retroperitoneum were taken before and after intravenous injection of the novel fluorescent dye. The dye was dosed at $120 \mathrm{ug} / \mathrm{kg}$, injected via the ear vein. The time to fluorescent visualization of the ureters was measured. Fluorescent signal was measured for up to four hours from initial dye injection. Ureter was dissected from inflamed tissue under fluorescence laparoscopy, and injury was replicated via partial transection. Urine was collected and analyzed by high-performance liquid chromatography (HPLC) for analysis of metabolic changes of the dye.

Results: Despite surrounding tissue erythema and edema, ureteral visualization under fluorescent laparoscopy was achieved within 5-10 min after dye injection. The fluorescent signal was visible for at least $4 \mathrm{~h}$ after injection. The fluorescent dye showed ureteral injury that would not have been seen under standard laparoscopy (Figure 1). Urine analysis show chemically unaltered dye after excretion.

Conclusion: UL-766 is a novel fluorescent dye useful for intraoperative identifications of ureters in an inflamed abdomen and visualization of ureteral injuries. Excretion of the dye is minimally metabolized indicating a likely linear excretion profile. With more preclinical data, this novel dye can become a valuable tool to prevent iatrogenic ureteral injuries during laparoscopic abdominal and pelvic surgeries.
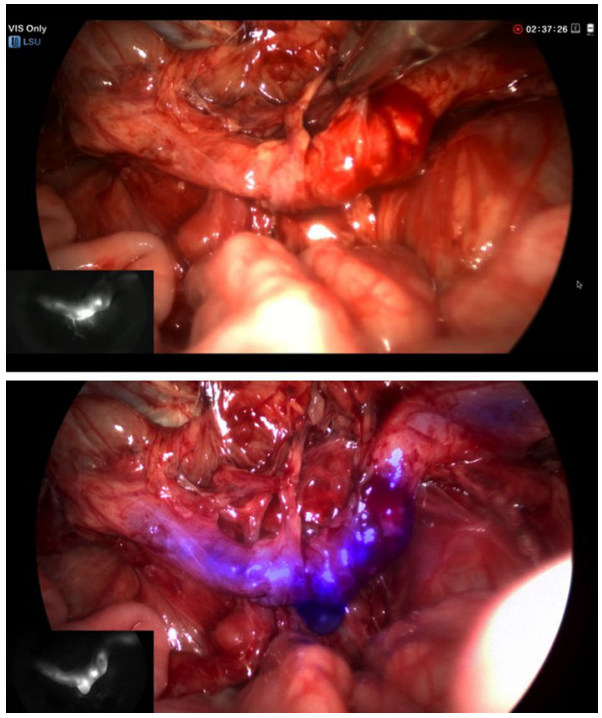

Figure 1: Fluorescent visualization of ureteral injury with effluent of urine from the ureter, visualized with and without fluorescence laparoscopy (blue). 


\section{P684}

The Surgical Outcomes of a Mesocolon Right Hole Approachversus that of the Traditional Approachfor Laparoscopic Enucleation of Pancreatic Head and Uncinateneuroendocrine Tumors: A Comparative Study of a Single Center

Yuyao Song, Guodong Zhao, Rong Liu, Zhiming Zhao, Yuanxing Gao, Zhuzeng Yin, Yong Xu; Chinese PLA General hospital

Background: Laparoscopic surgery for proximal pancreatic disease remains controversial due to the challenging anatomic pathways involving the gastrocolic ligament. In this study, we present a novel laparoscopic approach through an operation of the stoma of the right side of the mesenteric vessels,R-Hole for short,to enucleate proximal pancreatic lesions. We discuss the safety and efficacy of this approach to expand indications for laparoscopic pancreatic surgery.

Methods: A retrospective study was designed to compare the surgical outcomes of 15 cases who underwent a laparoscopic enucleation of proximal pancreatic neuroendocrine tumours via the R-hole approach (RHA)and 15 cases who underwent a laparoscopic enucleation of proximal pancreatic tumours via the gastrocolic ligament approach (GLA). All cases underwent surgery for proximal neuroendocrine pancreatic lesions $(\leq 2.5 \mathrm{~cm}$ diameter) from August 2010 to November 2017[Office1].The novel laparoscopic approach was designed to go through the transverse mesocolon to obtain direct access and easy manipulation.

Results: Compared with the GLA group,the RHA group exhibited a significantly shorteroperative time (mean 82.00 vs. $153.67 \mathrm{~min}$ ), shorter hospital stay (mean 10.00vs. 20.27 days), and comparable blood loss (mean $81.33 \mathrm{vs} .94 .00 \mathrm{ml}$ ). No significant difference was observed between the two groups in terms of open conversion rate, complication rate, and 90-daymortality rate.No relapse was detected in the follow-up period of $34.47 \pm 14.73$ months.

Conclusions: Our initialr esults indicate that laparoscopic enucleation via the mesocolon R-Hole approach would be a safer and more feasible alternative approach for selected proximal pancreatic lesions,with the potential advantages of easier exposure, shorter operative time, more simple manipulation, and shorter postoperative recovery than the conventional approach via the gastrocolic ligament.

Keywords: Anatomical pathway; pancreas; enucleation; mesocolon; laparoscopy; minimally invasive surgery

\section{P686}

\section{Laparoscopic Management of Paraganglioma of Urinary Bladder: A Case Report}

Lalit Aggarwal, MS, Megha Priyadarshi; Lady Harding medical college, New Delhi, India

Introduction: Paraganglioma are tumours arising from the neural crest cells of the sympathetic and parasympathetic paraganglia. Functional Paraganglioma presents with the symptoms of catecholamine excess that includes hypertension, flushing, diaphoresis etc. non -functional paraganglioma are usually found incidentally during imaging studies. Early diagnosis of functional paragangliomas are important because their removal is curative.

Bladder Paragangliomas are extremely rare tumours accounting for less than $0.05 \%$ of all bladder tumours and less than $1 \%$ of all pheochromocytomas. Functional tumours secrete catecholamines causing paroxysmal hypertension, palpitation and micturition syncope. In the genitourinary tract, the urinary bladder is the most common site for paragangliomas (79.2\%) followed by urethra, pelvis and ureter.

Case Presentation: 44 year old gentleman presented with palpitations and headache after mic turition for the past 10 years with increasing frequency of symptoms in last 8 months. He is a known case of hypertension for the past 6 years on Telmisartan $(20 \mathrm{mg})$.

General physical and systemic examination was normal.

Laboratory studies revealed an elevated $24 \mathrm{~h}$ Urinary Nor-epinephrine $322.4 \mathrm{mcg} / 24 \mathrm{~h}$ (normal range: $23-105$ ) and $24 \mathrm{~h}$ urinary VMA- $14.28 \mathrm{mg} / 24 \mathrm{~h}$ (normal range 1.6-7.3).

On CECT Abdomen a well defined intensely enhancing lesion (approx. $2.7 \times 2.4 \mathrm{~cm}$ ) was seen in relation to the base of the bladder.

Patient was started on Tab. Prazosin and Tab. Propanolol. He subsequently underwent LAPAROSCOPIC PARTIAL CYSTECTOMY WITH EXCISION OF TUMOUR.

Histopathology of the tumour revealed on gross examination Grey brown to yellow encapsulated soft tissue mass measuring $3 \times 2 \times 1.8 \mathrm{~cm}$ surface. S100 marker was POSITIVE on Immunohistochemistry.

In the postoperative period patient remained normotensive even after stopping all anti -hypertensive medications and urinary VMA came back to normal values.

Discussion: More than 180 cases of bladder paraganglioma have been reported in the literature, but only few managed laparoscopically. Our case, probably to the best of our knowledge is 12th such case managed successfully laparoscopically.

Conclusion: In summary, paragangliomas of the urinary bladder tend to be functional. Initia presentation is extremely varied, necessitating a low threshold of suspicion in the face of symptoms like hypertension, hematuria, headache and palpitations. We present this case to demonstrate the feasibility of a minimally invasive approach for these tumors over open approach. Laparoscopic Partial Cystectomy with excision of tumour is difficult but probably best possible method of surgical removal.

\section{P685}

Automatic Pixelwise Segmentation and Workflow Recognition in Laparoscopic Sigmoidectomy Using Convolutional Neural Networks

Nobuyoshi Takeshita, Daichi Kitaguchi, Masaaki Ito; National Cancer Center Hospital East

Introduction: Tracking and analyzing surgical tool movements, anatomical structures and bleeding in laparoscopic-surgical views are beneficial for training and safety. Furthermore, automatic recognition of surgical workflow is useful for operating room management and prediction of adverse event during surgery. In this work, we made use of the video database of laparoscopic sigmoidectomy and introduced semantic segmentation and workflow recognition using convolutional neural networks (CNN)

Methods: We used a CNN architecture to perform automatic segmentation of surgical tools, anatomical structures and bleeding: U-NET which was designed for biomedical image segmentation. For the semantic segmentation purpose, we manually labeled surgical tools, inferio mesenteric artery (IMA) and bleeding in the videos of laparoscopic sigmoidectomy from 300 cases of the database. We pixelwisely annotated spatial bounds of the objects. As for surgical tools, we used 1326 annotated frames for training data and 80 frames for test data in machine learning. Finally, we evaluated precision rate, recall rate and F-measure. The datasets for semantic segmentation of IMA and bleeding were created in the same way and then used as training and test data for machine learning. For the workflow recognition purpose, we labeled the surgical phases (Preparation, Recto-rectal space, Median to lateral approach 1, Inferior mesenteric artery transection, Median to lateral approach 2, lateral approach, Rectal mobilization, Mesorectal dissection, Transection and anastomosis, Inferior mesenteric vein transection, Closing) for every frame in the surgical videos. We used 63 videos for training data and 8 videos for test data in machine learning. Results: F-measures of the tip of grasper, linear dissector and Maryland were $0.873,0.763$ and 0.686 , respectively. The results of automatic semantic segmentation of IMA and bleeding were also acceptable from clinical perspective. F-measures of automatic recognition of surgical workflow were around 0.900 .

Conclusions: The results of semantic segmentation of surgical tools, IMA and bleeding seemed promising. Furthermore, the results of workflow recognition were acceptable for contribution in clinical fields. CNN approach must be feasible for automatic image recognition in laparoscopic surgery. 


\section{P687}

\section{Minilap Laparoscopic Cholecystectomy Vs Single Port}

Quintin Gonzalez, FACS, MD, Monica De Jesus, MD, Jesus Bahena, MD, Victoria Hernandez, MD; Humanitas Medical Group Coyoacan

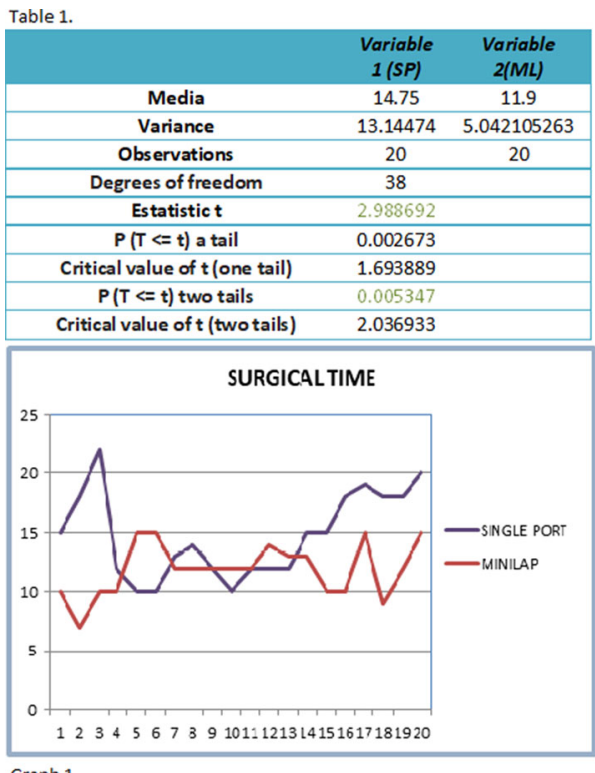

Graph 1

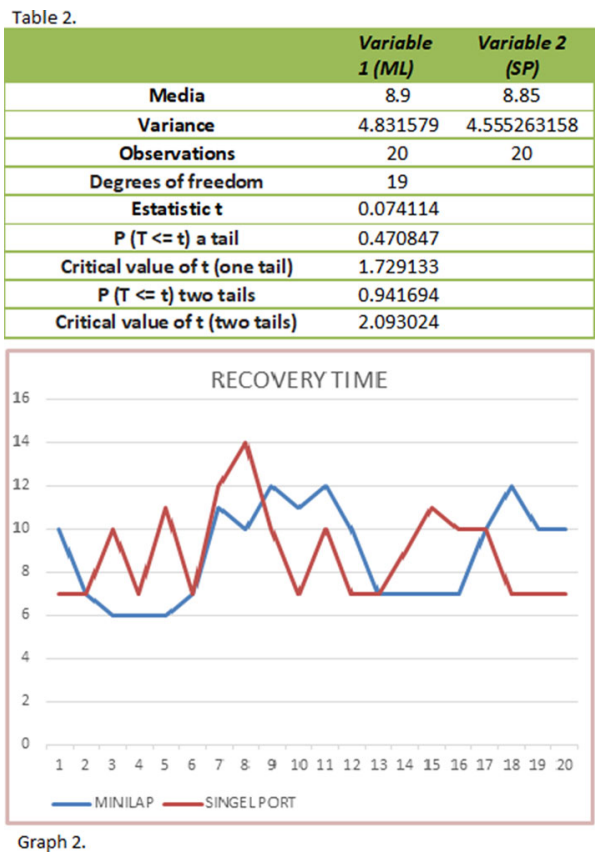

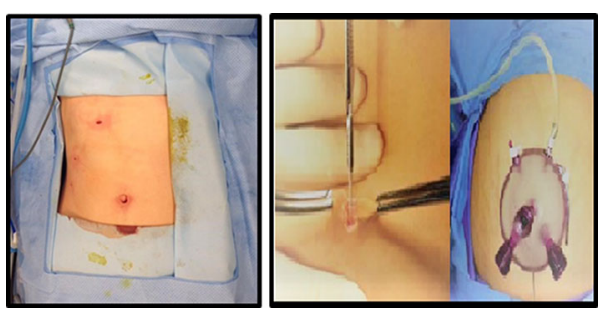

Background: In recent years there have been many attempts to improve the laparoscopic technique in order to minimize tissue trauma and invasion by reducing the size and number, so it has begun the surgical technique of minimally invasive obtaining technological advantages and the minilaparoscopy and single-port laparoscopy better cosmetic results and postoperative pain.

Materials and methods: A retrospective study June 2015 a total of 40 patients divided into two groups was performed July 2018, group A was operated using percutaneous clamps MiniLap ${ }^{R}$ and the B group intervened with point gel port single platform in from Mexico City, variables such as surgical time, days of hospital stay, postoperative pain, surgical incision and recovery time were noted.

Results: A total of 40 patients, divided into two groups, group A operated using percutaneous clamps MiniLap $^{\mathrm{R}}$ and the $\mathrm{B}$ group intervened with Gel point only port platform included surgery indication was benign diseases such as chronic cholecystitis symptomatic, dyskinesia and vesicular polyp. $72.5 \%$ were women with an average age of 40.6 years. Surgical time in group A (Minilap) was $12.1 \mathrm{~min}$ vs 14.7 single port, showing a statistically significant difference with a value of $\mathrm{p}=0.005$ using the Student t-test (table and graph No. 1), $98 \%$ of patients remained in the hospital $24 \mathrm{~h}$ withdrawing the next day, a patient was discharged after $72 \mathrm{~h}$ for postoperative ileus the surgical incisions in the group A few times percutaneous Minilap forceps, both of $2.3 \mathrm{~mm}$ previous infiltration with naropin and two $10 \mathrm{~mm}$ trocars and 5 respectively (image 1), the percutaneous wounds were confronted with steri-strips, the surgical incision in group B was $3 \mathrm{~cm}$ infraumbilical, using a gel platform with 4 trocars, placed in the shape of a rhombus (image 2) and faced with subdermal sutures, immediate postoperative pain is evaluated with EVA. (Table and graph 2)

Conclusions: This study shows that both groups have acceptable postoperative pain, short hospital stay, however in those patients treated with percutaneous MiniLap tweezers show a better satisfaction in terms of cosmetic results and for those patients managed with single port the surgical time is longer and for the surgeon, the position of the trocars is not very ergonomic since the operative field becomes very parallel, making difficult an adequate exposure of the anatomical structures which could impact the incidence of injuries. 


\section{P688}

Robotic Single-Site Cholecystectomy is as Cost Effective and Safer than Traditional Four Port Laparoscopic Cholecystectomy: A Retrospective Randomized Experience of a New York City General Surgical Group

Thomas J Shaknovsky, DO ${ }^{1}$, Purvee M Patel, BSA ${ }^{2}$; ${ }^{1}$ Southeast Alabama Medical Center, ${ }^{2}$ The Univeristy of Texas at Austin

Introduction: Since March 2014 our group has performed 200 Robotic SingleSite Cholecystectomies (RSS) with intra-operative fluorescent cholangiography (Fire-Fly). We analyzed our morbidity rates and procedure cost in comparison to traditional four port laparoscopic cholecystectomy (LC).

Methods: From March 2014 to December 2015, all RSS cases performed by our surgical group were retrospectively reviewed. All patients were randomized to RSS, regardless of BMI, diagnosis, co-morbidities, age or ASA (American Society of Anesthesiologist) class. Age, ASA, diagnosis, BMI, total operative time (docking and console time) and complications were retrospectively analyzed. Total cost of procedure was calculated at $\$ 1068$, excluding lifetime amortization of the robotic platform (Table 1).

Results: A total of 200 RSS were performed over a 21 month period. Mean age was 53 (21-83), mean BMI was 29 (19-53) and mean ASA was 2 (1-4). Total operative time was $68 \mathrm{~min}(25-130 \mathrm{~min})$. The patient cohort diagnosis breakdown was $29(14.5 \%)$ acute cholecystitis, $129(64.5 \%)$ with chronic cholecystitis, $34(17 \%)$ with cholelithiasis, $5(2.5 \%)$ with gallbladder polyps and $3(1.5 \%)$ with biliary dyskinesia. Diagnosis was confirmed by post operative pathology report. Three patients $(1.5 \%)$ developed an umbilical incisional hernia and none $(0 \%)$ required conversion to a open procedure. There were no deaths, re-admissions, bleeding complications, wound infections, cystic duct leaks $\left(0 \%\right.$ vs. $\left.0.38 \%^{1}\right)$ or common bile duct injuries $\left(0 \%\right.$ vs. $\left.0.50 \%{ }^{1}\right)$.

Conclusions: Robotic Single-Site Cholecystectomy (RSS) with intra-operative fluorescent cholangiography (Fire-Fly) is safe to perform on any patient regardless of diagnosis, BMI, age and ASA at an urban teaching community medical center. This procedure is as cost effective ( $\left.\$ 1068 \mathrm{vs.} \$ 1224^{2}\right)$ and more importantly associated with lower morbidity rates than traditional laparoscopic cholecystectomy (LC).

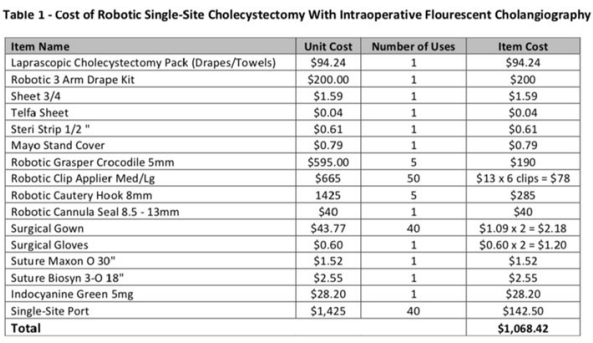

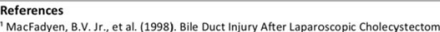
'MacFadyen, B.V. Ir, et al. (1998). B.
Surgicol Endoscopy, 12(4), 315-321. "IKramuddin, s., et al. Equipment Variation in Laparoscopic Cholecrstectomy. SAGES Annual Meeting: 2014 Apr 2-5; Salt Lake City, UT.

\section{P689}

The Use of Ureteral Stents with Indocyanine Green (ICG) in Robotic Colon Surgery

Matthew J Pergamo, MD, Michael A Granieri, MD, Aaron Weinberg, MD, Lee Zhao, MD, Mitchell Bernstein, MD, Alexis Grucela, MD; NYU School of Medicine

Introduction: Ureteral injury during colon resection (reported range 0.28$7.6 \%$.) can have devastating consequences for patients including increased morbidity, mortality, and cost. Ureteral stenting is commonly used to aid in ureteral identification and recognize intraoperative injury, especially in patients with diverticulitis, Crohn's Disease, reoperative pelvic surgery, or with history of radiation therapy. In patient's undergoing laparoscopic or robotic surgery, lighted stents have been used; however, at a higher cost. We report a novel technique for ureteral visualization by injecting standard ureteral catheters with indocyanine green (ICG) during robotic colon resection. We aim to show the benefits including low cost, excellent intraoperative visualization of the ureters, and low morbidity.

Methods and Procedures: An IRB approved review of our prospectively maintained database was conducted of all robotic colon resections performed at our institution. In select cases, cystoscopy with open ended ureteral catheter placement and ICG injection were performed by three experienced urologists. Once placed, $5 \mathrm{cc}$ of ICG was injected into the stent, which were then connected to a separate drainage bag. Surgeons were then able to identify the ureter and stent via firefly mode on the $\mathrm{Da}$ Vinci $\mathrm{Xi}^{\circledR}$ platform. Inclusion criteria were ureteral stent placement (Unilateral, Bilateral), ICG injection and colon resection. Surgeries were performed between 4/2017-8/2018. Data gathered included demographics, type and indication of resection, intraoperative and postoperative complications, and readmissions.

Results: 30 patients were identified who underwent robotic colon resection with ICG ureteral stent placement. The most common procedure performed was low anterior resection $40 \%(12 / 30)$ and the most common indication for surgery was diverticulitis $46.7 \%(14 / 30)$. There were no ureteral injuries $0 \%(0 /$ $30)$ and no major morbidities or mortalities $(0 \%)$. The ureters were identified using firefly mode in 100\%(30/30) of patients (Image A/B). Most patients underwent bilateral stent placement $66 \%(20 / 30)$, with remainder being unilateral. There was $1(3.3 \%)$ minor postoperative urologic complication, AKI from under-resuscitation, resolved with hydration. There were no $(0 \%)$ urinary tract infections. There were no 30-day readmissions. One $(3.3 \%)$ patient was seen in the emergency room within 30-days for a port site hernia not requiring operation. At our institution, lighted stents cost approximately $\$ 1500 /$ stent, while standard stents are \$100/stent and ICG \$508/vial resulting in \$254/ injection.

Conclusion: The use of ICG injected ureteral stents is a novel technique for intraoperative ureteral identification that is safe, effective, with time and cost saving benefits. This technique should be studied further in prospective randomized controlled trials to further quantify time and cost-benefit.

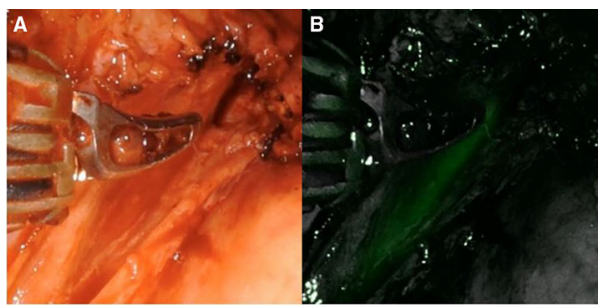


P690

\section{Minimally Invasive Robotic Liver Resections for Benign and Malignant Tumors. Institutional Outcomes of the Modern Technique}

Iswanto Sucandy, MD, Janelle D Spence, BA, Sydni Schlosser, Sharona B Ross, MD, Alexander S Rosemurgy, MD; Florida Hospital Tampa

Introduction: The nascent robotic approach for hepatic resections for benign and malignant liver tumors is gaining momentum because of perceived efficacy and utility with reduced morbidity. Herein, in considering this, we document our outcomes with robotic liver resections. Methods: With IRB approval, all patients undergoing robotic liver resections are prospectively followed. Patient demographics, operative outcomes, such as operative duration and estimated blood loss, length of stay (LOS), complications, and 30-day readmissions were collected and analyzed. Data are presented as median (mean $\pm \mathrm{SD}$ ). Results: 77 patients underwent robotic liver resections. Patients age was $63(62.1 \pm 13.9)$ years, $57 \%$ were women, BMI was $28(29.7 \pm 9.6) \mathrm{kg} / \mathrm{m}^{2}$. ASA Class was $3(2.5 \pm 0.55)$, and MELD score was $7(8.0 \pm 2.9) .58(75 \%)$ of the liver resections were undertaken for malignancy [hepatocellular carcinoma $(36 \%)$, metastatic colorectal cancer $(31 \%)$, intrahepatic cholangiocarcinoma $(10 \%)$, gallbladder cancer $(7 \%)$, and other metastatic tumors $(16 \%)], 7(9 \%)$ were undertaken for premalignant lesions, and $12(16 \%)$ were undertaken for benign lesions. $35 \%$ of patients underwent non-anatomical partial right hepatectomy, $21 \%$ non-anatomical partial left hepatectomy, $10 \%$ formal right hepatectomy, $13 \%$ formal left hepatectomy, and $12 \%$ central hepatectomy. $9 \%$ of patients underwent extended hepatectomy. Operative duration was $228(264.5 \pm 109.6)$ minutes and estimated blood loss was $150(254.9 \pm 300.4) \mathrm{ml}$. Conversion to an 'open' approach was necessary in two patients because of difficulty in assessing tumor margins and inability to reach a tumor located in segment 8, respectively. Length of hospital stay was 3 (4.9 \pm 4.6$)$ days. While there were no intraoperative complications, nine patients experienced postoperative complications (enterocutaneous fistula, respiratory failure, bile leak $(n=2)$, fluid collection $(\mathrm{n}=2)$, pleural effusion, wound infection, and intra-abdominal abscess). Eight patients required readmission to the hospital within 30 days of discharge due to wound infection, bile leak, fluid collection, pneumonia, jaundice, and intractable vomiting.

Conclusion: Our data support that robotic liver resection is a safe and feasible technique in liver surgery with favorable short-term outcomes. Robotic surgical system enhances application of minimally invasive surgery for complex abdominal operation such as liver resection.

\section{P691}

\section{Robotic-Assisted Simultaneous Repair of a Morgagni Hernia and Ventral Hernia in an Adult: Case Report}

David L Rhoiney, DO, Zachary Laucis, DO, Kevin Jamil, MD, Ray Laird, DO; Beaumont Health System-Trenton

Congenital diaphragmatic hernias (CDH) are rare malformations which are believed to occur in about 1 per 2000-5000 births. Morgagni hernias (MH) are a rare variation of CDH and are uncommon in adults. Laparoscopic repairs of these hernias have been well described in the literature, however there are few reports of repair via a robotic-assisted approach. We present our successful repair of a MH and ventral hernia in a 49 -year-old male utilizing the da Vinci XI surgical robot (Intuitive). We aim to add to the current literature by discussing our technique and approach in repair these two concurrent hernias. Conclusion: We believe robotic-assisted laparoscopic repair of $\mathrm{MH}$ are a technically feasible and safe option which provides a great outcome in adults presenting with this hernia type, while providing the ability to perform other repairs during the same operation.

\section{P692}

\section{Laparoscopic Testing of Port Bleeding}

Abdullah Aldohayan, FRCS, Glas, Mohanad Albalawi, Rasha Bassas, Maram Alaqel, Hassan Arishi; King Saud University Hospital

Background: Laparoscopic port bleeding is complication which cripple the meritus of laparoscopic surgery. The port bleeding can be arterial bleeding or venous bleeding. We advocate the probing the port pathway under the low pneumoperitoneum pressure $(5 \mathrm{mmHg})$.

Materials and methods: All patients are undergoing laparoscopic surgery in year 2017 are involved in this examination. After finishing the procedure, the pneumoperitoneum is lowered to $5 \mathrm{MMHg}$, the port size is examined and any plastic tube Nelaton catheter $14 \mathrm{Fr}$. is introduced for probing and clarification any bleeding which is controlled of its noted.

Results: Five cases are encountered among 120 patients, the bleeding is venous and controlled by diathermy in 4 and by suture in one.

Conclusion: Port bleeding can cumbersome that can delay healing and decrease patient satisfaction, by this technique probing the pathway of the trocar can clarify the venous bleeding and managing it.

\section{P693}

Sentinel Lymph Node Biopsy Using Indocyanine Green in Lung Cancer: A Systematic Review and Meta-Analysis

Warren Sun, MD ${ }^{1}$, Jerry T Dang, MD ${ }^{1}$, Aryan Modasi, MD, FRCSC ${ }^{1}$, Awrad Nasralla, MD ${ }^{1}$, Daniel Skubleny, MD ${ }^{1}$, Noah J Switzer, MD, MPH, FRCSC ${ }^{2}$, Daniel Birch, MD, MSc, FRCSC ${ }^{1}$, Simon Turner, MD, FRCSC ${ }^{1}$, Shahzeer Karmali, MD, MPH, FRCSC ${ }^{1}$; University of Alberta, ${ }^{2}$ Ohio State University Wexner Medical Center

Introduction: We aim to systematically review the literature and perform a meta-analysis on the diagnostic utility of ICG for SNNS in lung cancer. Lung cancer is the leading cause of cancer death worldwide. The use of sentinel node navigation surgery (SNNS) is well established in the literature to reduce operative mortality in cancers. Indocyanine green (ICG) has also been reported as an emerging technology in SNNS.

Methods and Procedures: A comprehensive search of MEDLINE, EMBASE, SCOPUS, Web of Science, and the Cochrane Library using search terms "lung/pulmonary" AND "tumor/carcinoma/cancer/neoplasm/adenocarcinoma/malignancy/squamous/carcinoid"

AND "indocyanine green" was completed in June 2018. Studies were limited to humans and English. 347 titles or abstracts were screened. Articles were selected by two independent reviewers based on the following inclusion criteria: (1) diagnostic accuracy study design; (2) ICG injected at the tumor site; (3) lymphadenectomy or systematic lymph node sampling was performed as gold standard.

Results: Nine primary studies were included with a total of 382 patients. $43.0 \%$ of patients were females, and the mean tumor size was $2.3 \mathrm{~cm}$. Additionally, $33.9 \%$ of tumors were located in the RUL, $5.3 \%$ in the RML, $17.2 \%$ in the RLL, $26.0 \%$ in the LUL, and $17.6 \%$ in the LLL. Pathologic analysis revealed $67.5 \%$ of tumors were adenocarcinomas, $21.3 \%$ were squamous cell carcinomas, $10.6 \%$ were other malignancies, and $0.6 \%$ were other benign tumors. Furthermore, $6.9 \%$ of patients received wedge resections, $48.0 \%$ received segmentectomies, $39.3 \%$ received lobectomies, and $5.8 \%$ received pneumonectomies. Sentinel node biopsies were successful in 298 patients, yielding a pooled identification rate of 0.75 ( 0.56 to $0.90)$. A meta-analysis of seven studies computed a diagnostic odds ratio, sensitivity, an specificity of 177.61 (45.64 to 691.14 ), 0.85 ( 0.71 to 0.94 ), and 1.00 (0.98 to 1.00 ), respectively. The summary receiver operator characteristic (SROC) demonstrated an AUC of 0.963 $(\mathrm{SE}=0.038)$ and a $\mathrm{Q}^{*}$ of $0.909(\mathrm{SE}=0.057)$. Only $59.5 \%$ of patients with histologically positive sentinel nodes had metastatic disease exclusively in the sentinel nodes.

Conclusion: Our review found suboptimal results for the use of ICG in SNNS for lung cancer. The diagnostic odds ratio, sensitivity, and SROC AUC were promising, but needs improvement before consideration as a potential treatment option for lung cancer. This was due to mediocre identification rate, and a poor representation of all metastatic nodes. Further research is required to develop a robust protocol for the use of ICG in SNNS for lung cancer. 


\section{P694}

\section{Percutaneous Surgery Procedures Under Stereotactic Guidance, Beyond Ablative Therapies}

Mariano E Gimenez, $\mathrm{MD}, \mathrm{PhD}^{1}$, Edgardo Serra, $\mathrm{MD}^{2}$, Mariano Palermo, $\mathrm{MD}^{2}$, Alain Garcia, MD ${ }^{1}$, Amilcar Alzaga, $\mathrm{MD}^{3}$, Eduardo Houghton, $\mathrm{MD}^{2}$, Pablo Acquafresca, $\mathrm{MD}^{2}$, Bernard Dallemagne, $\mathrm{MD}^{4}$, Jacques Marescaux, $\mathrm{MD}^{4} ;{ }^{1} \mathrm{IHU}$ Strasbourg, Institute of ImageGuided Surgery Strasbourg France. IRCAD, Research Institute against Cancer of the Digestive System Strasbourg France,Docencia Asistencia Investigación en Cirugía Invasiva Mínima (DAICIM) Foundation, Buenos Aires, Argentina., ${ }^{2}$ Docencia Asistencia Investigación en Cirugía Invasiva Mínima (DAICIM) Foundation, Buenos Aires, Argentina., ${ }^{3}$ Siemens Healthineers, Advanced Therapies, Surgery., ${ }^{4}$ IHU Strasbourg, Institute of Image-Guided Surgery Strasbourg France. IRCAD, Research Institute against Cancer of the Digestive System Strasbourg France

Introduction: The purpose of this study was to evaluate the feasibility and safety of stereotactic navigation ( $\mathrm{SN}$ ) for percutaneous abdominal procedures beyond tumor ablation.

Methods and Procedures: This experimental study, approved by the French National Ethics Committee, included four pigs. All procedures were performed under general anesthesia in an experimental hybrid operating room. Images of the targeted organs/structures were acquired with intraoperative cross-sectional imaging (CT or CBCT, Somatom Confidence, and ARTIS Zeego, Siemens Healthcare, Forchheim, Germany) and loaded in the optical stereotactic navigation system (CAScination, Bern,Switzerland) or worked directly in the workstation from the robotic angiography system. All animals were euthanized at the end of the experiments.

Procedures:

1. Percutaneous drainage of anatomical complex abdominal collections (two animals) of artificial intraabdominal collections, which were created in the subphrenic, peripancreatic and deep pelvic anatomical spaces.

2. Percutaneous cholecystic-gastrostomy (one animal). Dilatation of the biliary system was provoked by a laparoscopic partial ligation of the common bile duct 3 days before the procedure. Percutaneous cholecystostomy was first performed. Then, under SN a needle was inserted through the gallbladder towards the gastric lumen and a transhepatic biliary stent was deployed between the gallbladder and the stomach.

3. Percutaneous Selective Portal vein occlusion before hepatectomy (one animal). SN was used to guide the occlusion of the right portal vein with a percutaneous occluding device (Amsel Medical Cambridge, Mass.). The efficacy of the procedure was assessedby US Doppler and a laparoscopic exploration with intravenous injection of indocyanine green (ICG).

Results:

All percutaneous procedures were successful without the need for repositioning. 1. All intraabdominal artificial collections were drained successfully. There were no visceral injuries, no bleeding, no thoracic complications (subphrenic collections).

2. An efficient cholecysto-gastrotomy was obtained in 1 animal. Radiological control with contrast confirmed the patency of the anastomosis in the animal. There were no complications.

3. US Doppler and laparoscopic exploration with ICG confirmed the occlusion of one right Glissonian pedicle in the animal liver. There were no complications and laparoscopic right lobectomy was guided by the ICG boundaries.

Conclusion: This preliminary experimental study has shown that complex percutaneous procedures can be performed safely under stereotactic navigation guidance. This justifies further studies with the aim of reduction of the exposure of the patients and operators to X-rays during percutaneous procedures which require high precision.

\section{P695}

\section{Hernia Surgery and Robotics: A Comparative Study of Laparoscopic and Robotic Repairs}

\author{
Katie Marrero ${ }^{1}$, Sharique Nazir, MD ${ }^{2} ;{ }^{1}$ SGU, ${ }^{2}$ NYU Langone
}

Surgical innovation has been at the forefront of surgery over the last decade, fine tuning many surgical procedures. This innovation occurs with the desire to increase efficiency while decreased morbidity and mortality. Laparoscopic surgery has, with this refinement, become the mainstay for many operations including hernia repairs. This study looks to analyze the upcoming field of robotic surgery to its laparoscopic counterpart. With this analysis, novel contributions to the field can be added in regard to robotic hernia repair and its ability to change the way minimally invasive surgery is done.

Analysis was done of eighty-eight patients with hernia surgery who underwent either laparoscopic or robotic hernia repairs between 2016 and 2018. Several outcome parameters were noted, which include the duration of the operation postoperative complications, length of hospital day, and readmission rate.

The average OR time for the robotic group was approximately $151 \mathrm{~min}$ compared to $139 \mathrm{~min}$ for the laparoscopic group. Overall, the robotic surgery time became more efficient showing the possible initial learning curve physician have when transitioning to robotic repair and the gain in effectiveness they achieve with continued practice. The average length of stay for the robotic group was approximately $9 \mathrm{~h}$, $55 \mathrm{~min}$ compared to $11 \mathrm{~h}, 52 \mathrm{~min}$ in the laparoscopic group.

The difference in postoperative complications between the two groups were noted. In regard to hematomas, one laparoscopic patient complained of this while none experience this in the robotic group. For seroma complications, eight patients from the laparoscopic group were seen with this post-op compared to three patients in the robotic group. Of note, one patient experienced a surgical site infection from the robotic group while no one in the laparoscopic had this complication. For the patient experiencing an SSI, CT confirmed an abscess that was subsequently treated with antibiotics and resolved. Lastly, recurrences of hernia since surgery occurred in one patient, who was in the laparoscopic group.

This retrospective study was conducted to assess if the new robotic hernia surgery could be equal if not better than the previously accepted gold standard of laparoscopic repair. Interpreting this data shows that robotic hernia repairs are a suitable alternative to laparoscopic repairs. Robotic repairs were superior went assessing the length of hospital stay and post-operative complications. Thus, if these are major concerns for patients, robotic repair can be considered as a valuable alternative to the laparoscopic methods.

\section{P696}

\section{The Application of Robotic Surgery for Hepato-Pancreatico-} Biliary Disease in a Single Center: Experience of 2534 Cases

Rong Liu, Minggen Hu, Zhiming Zhao, Chenggang Li, Xianglong Tan, Yuanxing Gao; Chinese PLA General hospital

Aims: To evaluate the value of da Vinci robotic surgery system in the treatment of hepatopancreaticobiliary diseases.

Methods: the clinical data of a consecutive of 2534 patients who underwent roboticassisted laparoscopic surgery during 2011 and 2018 were retrospectively reviewed. The demographic characteristics, type of surgery, intraoperative blood loss, operative duration, post-operative hospital stay, conversion rate as well as the technical advantages and disadvantages of da Vinci robotic surgery system were all analyzed. Results: the clinical data of 2534 patients in total were reviewed in this study. The type of surgery included 1624 cases of robotic assisted pancreatectomies (including 623 cases of robotic assisted pancreaticoduodenectomies), 710 cases of robotic assisted liver resection and 200 cases of other resections. All the resections were successfully performed using 5 ports method (except for cases of combined resection involving multiple organs), with the techniques such as $\mathrm{L}$ and $\mathrm{R}$ pathway, " $1+2$ " pancreaticojejunostomy, En-bloc lymphadenectomy, indocyanine green-fluorescence imaging applied. The overall convertion rate was $5.05 \%$, and the 30 days mortality was $0.6 \%$.

Conclusion: da Vinci robotic surgery system is feasible and safe for resection of hepatopancreaticobiliary diseases, which is especially suitable for complexed operations with lots of suture or knotting, with a low conversion rate and low 30 days mortality. 
P697

Robotic Median Arcuate Ligament Release is a Safe and Effective Approach in the Treatment of Median Arcuate Ligament Syndrome

Michael Pryor ${ }^{1}$, Colton J Fernstrum ${ }^{1}$, Andrea Wolf, MD $^{2}$, Paul Wright, $\mathrm{MD}^{2} ;{ }^{1}$ Michigan State University College of Human Medicine ${ }^{2}$ Spectrum Health

Introduction: Median arcuate ligament syndrome (MALS) results from compression of the proximal celiac artery and celiac ganglion by the median arcuate ligament of the diaphragmatic crura. Minimal reports on treatment outcomes are available in the current published literature. We sought to examine outcomes in a consecutive cohort of patients with MALS treated by robotic median arcuate ligament release to evaluate the safety and efficacy of this approach.

Methods and Procedures: A retrospective chart review was performed to include consecutive patients with MALS that underwent robotic median arcuate ligament release from August 2012 to April 2018. Standard patient workup included history and physical examination, CT scan, and mesenteric Doppler ultrasound. Select patients underwent diagnostic angiography. Demographics, diagnostics, intra- and post-operative data were collected for each patient. The primary outcome measure was improvement/resolution of pain at first follow-up visit. Secondary outcomes included operative duration, length of stay, postoperative complications, and liberation from narcotics.

Results: Twenty-seven patients were found to meet the inclusion criteria and were included for study. Mean age of the cohort was $49 \pm 15.5$ years and two-thirds were female. The most common pre-operative symptoms were post-prandial abdominal pain $(25 / 27,93 \%)$, persistent/constant abdominal pain $(16 / 27,59 \%)$, weight loss $(15 / 27,56 \%)$, and nausea $(14 / 27,52 \%)$. Duration of symptoms was < 6 months in 14 (52\%), 6-12 months in $2(7 \%)$, and $>12$ months in $11(41 \%)$. Sixteen patients $(59 \%)$ were using narcotic medications for their pain at the time of surgical evaluation. CT was performed in $24(89 \%)$, mesenteric Doppler ultrasound in $19(70 \%)$, and angiogram in $6(22 \%)$. Celiac stenosis $>70 \%$ was observed in $24(89 \%)$ while median peak velocity was $311 \mathrm{~cm} / \mathrm{s}$ (range 184-562). Median operative duration was 95 min (range 53-358) and estimated blood loss was $<100 \mathrm{~mL}$ in $24 / 27$ (89\%) cases. Length of stay was one day in $22 / 27$ $(81 \%)$. Two cases required conversion to open, the first related to adhesive disease and the second due to disruption of the celiac trunk requiring ligation to control hemorrhage. This was the only major complication and this patient was discharged home on postoperative day 6 . Twenty patients (74\%) had complete and three (11\%) partial symptom resolution at initial follow-up for an $85 \%$ treatment response rate. Narcotic liberation was achieved in $9 / 16(56 \%)$

Conclusions: Robotic MAL release offers an attractive option for treatment of MALS with high response rates to treatment, short hospital stay, and opportunity for narcotic liberation.

\section{P698}

\section{Precision Surgery and Radiomics for Laparoscopic Liver Cancer Resection with Intraoperative Holographic 3DCT}

Maki Sugimoto, MD, $\mathrm{PhD}^{1}$, Mitsuo Shimada ${ }^{2}$, Gio Wakabayashi ${ }^{3}$; ${ }^{1}$ The University of Tokyo Research Center for Advanced Science and Technology, ${ }^{2}$ Tokushima University, ${ }^{3}$ Ageo Central General Hospital

The aim of precisionliver surgery is a balance of maximizing the removal of the target lesion, while maximizing the functional liver remnant and minimizing surgical invasiveness. We evaluated the radiomics application of precision approach using intraoperative holographic CTin laparoscopic complex liver surgery.

In 3 laparoscopic liver cancer surgeries, we reconstructed the 3D polygon models of the liver segments, planned surgical margins, tumor and vessels from patient individual preoperative CT. During surgery we performed 3D-CT cholangiography directly through a cystic duct. We could watch these holographic polygons above the operative field usinf our original mixed reality application to present 3D organ models with the wearable holographic google (HoloLens). This was presented as a hologram in which the organ floats in the direction of 360 degrees around the surgeon and the space on the field of sterilized surgical field before, after, and during surgery. As a result, in the space outside the endoscopic monitor, biliary anatomy models were corrected to the movement of the operator's eyes and hand gesture, and the stereoscopic relationship between each organ and the surgical margin, tumor position, artery, vein, bile duct, uterus, and myomas, was constantly observed from any directions.

As the spatial awarenesswas improved, the clinical usefulness was confirmed by avoiding misunderstanding in all cases. The immersive and presence feeling alleviate the stress of the surgeon and improve the accuracy, bleeding volume, and the operation time. It improved the sense of equilibrium and strengthened the space recognition ability.

\section{P699}

\section{Robotic Salvage Surgery for the Treatment of Recurrent Rectal} Cancer

Hye-Jin Kim, Gyu-Seog Choi, Jun Seok Park, Soo Yeun Park, Sung Min Lee; Kyungpook National University Chilgok Hospital

Background: Robotic approach can be a treatment option for patients with pelvic recurrence after primary resection for rectal cancer. However, data regarding patient selection, complication rates, and oncologic outcomes are rarely reported. We aimed to present initial experience and to evaluate feasibility, safety, and oncologic outcomes of robotic salvage surgery for recurrent rectal cancer. Methods: Eight patients who underwent robotic salvage surgery for local recurrence at the anastomotic site, lateral pelvic side-wall, or lateral pelvic lymph nodes (LPNs) were retrospectively evaluated from a prospectively maintained database.

Results: Two patients underwent pelvic mass excision with en bloc resection of anastomosis an redo-anastomosis, and six patients underwent lateral pelvic lymph node dissection (LPND) for LPN metastasis; one of these six patient underwent additional en bloc resection of anastomosis. Al patients achieved R0 resection. The median operation time was $165 \mathrm{~min}$ and the median estimated blood loss was $50 \mathrm{ml}$. There were no conversions. As for intraoperative complications, one patien experienced ureter injury during LPND because the metastatic LPN was closely abutting to the ureter. The median hospital stay was 7 days. In six patients who underwent LPND, the median number of harvested lymph nodes was 7 (range 2-13) and the median number of metastatic lymph nodes was 1 (range 0-2). With median follow-up 26 months, one patient developed lung and pelvic recurrence at 36 months after salvage operation and seven patients remained in disease-free state at the last follow-up.

Conclusion: Initial experience of robotic salvage surgery for pelvic recurrence in rectal cancer indicated that it is safe and feasible. Therefore, the robotic approach can be considered as a treatment option for the treatment of local recurrence in highly selected patients.

\section{Assessment of Content and Quality of Internet Information Regarding Robot-Assisted General Surgery}

Loren J Smith, Saeed Arefanian, MD, Jeffrey Blatnik, MD; Washington University School of Medicine in St. Louis

Introduction: Robot-assisted general surgery (RAGS) procedures have been growing in use and demand. Internet information about robotic surgery has similarly expanded, enhancing consumer access to information. Increasingly patients are taking an active role in their healthcare decisionmaking often consulting the internet for information upon which to base their decisions. The goal of this study is to analyze data from a survey of online information on RAGS to assess the type and quality of information available. Understanding the preconceptions created by readily-available internet resources can help physicians bridge the gap between patient perceptions of best medical care and effective treatment for specific concerns.

Methods: Nine search terms encompassing four categories of general surgery with the larges increase in robotics were entered into the three most-frequented search engines. Sites meeting the inclusion criteria were screened using a 114 dichotomous question internet screening rubric (ISR) The ISR evaluated sites based on 12 categories of comprehensiveness and 17 categories of conten thoroughness. Secondary financial gain was uncovered using The Open Payments Search Tool. Descriptive and statistical analyses include median, interquartile-range, Chi square, and the MannWhitney U Test.

Results: Sites providing RAGS or selling the da Vinci account for $85 \%$ of informational results. Many RAGS providers $(68 \%)$ receive secondary financial gain. The median comprehensiveness score is 4 of $12(\mathrm{IQR}=2)$. Discussion of risk, disadvantage, complication, and financial consideration are often absent (medians $=0$ ). In contrast, benefits and advantages predominate $(63 \%$ cited $3+$ surgical benefits ( $0-6)$ and $72 \%$ cited $4+$ patient benefits (0-8). $25 \%$ of sites specify RAGS as the sole surgical option. There is no statistical significance in disclosure parameters between hospitals and private practice, however, there is a slight credibility advantage to private practices $(\mathrm{p}=0.03)$. Only $50 \%$ of sites describe the robot as an instrument used in surgical procedures with $49 \%$ describing how it works. $35 \%$ neglect to state that the surgeon controls the robot and $60 \%$ fail to contextualize RAGS as a type of MIS. $84 \%$ of sites omit financial discussion completely.

Conclusions: The results underscore the promotional nature of information regarding RAGS, with the majority of informational sites directly or secondarily benefiting. The lack of depth and comprehensiveness demonstrates a trend toward superficial information over depth of content, often at the expense of a balanced assessment of the risks inherent in surgery. As a result, patients who investigate RAGS on the internet may conclude it is associated with non-supported benefits and outcomes. 


\section{P701}

Initiation of a Robotic Program in a Community Affiliate Hospital Setting-Safely and Cost-Effectively. It's Not Just for University Teaching Hospitals Anymore!

\section{Marian P Mcdonald, MD, MEd, FACS; St. Luke's University Health} Network

Introduction: Robotic surgery has resurged in recent years in Urology, Gynecology/ Oncology, and General Surgery. Technology and training have improved whereas most major teaching hospitals have at least one "robot" in use. Community hospitals have developed an interest in robotic programs for many of the same reasons that robotics is becoming favored in teaching hospitals-advanced laparoscopic techniques resulting in improved surgical outcomes.

Methods: We describe a community affiliate hospital program, initiating a Robotic program within nine months, with training of their Bariatric surgeons, General Surgeons, and aiding in recruiting Urologists and Gyn/Onc surgeons to the institution, increasing the OR utilization by these surgeons. All involved surgeons adopted an aggressive approach to campaigning for the robot and demonstrated cost savings to the hospital, so much so that the Intuitive robot was upgraded within ten months of acquiring the Si Robot.

Results: In the initial quarter of the acquisition of the Robot, with just Gyn/Onc and Urology only using the Robot, only 16 cases in a quarter were using the Robot. Within nine months, the number of cases jumped to 88 per quarter, the vast majority of them were Bariatrics and General Surgery. Interestingly, the number of Gyn/Onc and Urology cases also increased during that time when General Surgery came on board.

For General Surgery, a significant cost savings was determined for inguinal hernias and laparoscopic cholecystectomies. For one surgeon, the cost savings for ventral hernia repair robotically was $\$ 75,744$ (per year) and for inguinal hernias, the savings was $\$ 97,836$, for a total of $\$ 176,211$ for these two cases alone, using the robotic techniques. (This was not including the service agreements, which decreased significantly per case the more the robot was utilized.) In robotic inguinal hernias, there was demonstrated improvement in pain control postoperatively, with decreased opioid use.

Conclusions: Robotic surgery is not just for advanced teaching hospitals any more. The benefits of a robotic program can be demonstrated in common surgical procedures performed by community surgeons. A dedicated team approach is necessary to make the program safe and cost-effective. We describe how a community hospital can indeed develop a robotic program with the support of administration, a dedicated OR nursing team, and the collaboration of diverse surgeons interested in improving cost and patient outcomes.

\section{P702}

End-to-end Pancreatic Anastomosis Could be an Ideal Approach for Central Pancreatectomy

Zizheng Wang, Rong Liu, Zhiming Zhao, Yuanxing Gao, Zhuzeng Yin, Yong Xu; Chinese PLA General hospital

Objective: Central pancreatectomy with pancreaticienterostomy is one of the standard procedures of pancreatectomy. However, along with the resection of neoplasms, it brings additional injury, changes of anatomy and physiology, and risk of digestive tract fistula. In this study, we innovatively used the one-stage robotic end-to-end pancreatic anastomosis to replace conventional pancreaticoenterostomy in central pancreatectomy, and evaluated its safety and feasibility.

Methods: Prospectively collected clinical data of 11 consecutive patients underwent robotic central pancreatectomy with end-to-end pancreatic anastomosis between August 2017 and December 2017 were analyzed retrospectively.

Results: All operations completed successfully without conversion. The mean defect of main pancreatic duct was $4.3 \pm 1.0 \mathrm{~cm}$. The median operative time was 121 (range, 105 to 199) $\mathrm{min}$. The median estimated blood loss was 50 (range, 20 to 100) $\mathrm{ml}$. Seven patients had Grade B pancreatic fistula and no Grade C pancreatic fistula occurred. Nine patients had benign tumor, 1 had mass-forming chronic pancreatitis and 1 had pancreatic metastasis from renal cancer. MRCP at postoperative 6 months showed no stricture of main pancreatic duct but 3 patients had asymptomatic pancreatic pseudocyst.

Conclusion: Our series indicates that robotic central pancreatectomy with end-toend anastomosis is safe and feasible. It restores normal anatomy of pancreas and further reduces the inherent injury of central pancreatectomy. With its good repeatability and short-and long-term outcome, it could be an ideal approach to replace pancreaticoenterostomy in central pancreatectomy.

\section{P704}

Minimally Invasive Bariatric Surgery Leads to Significant Back Strain of Surgeons

Sara Monfared, $\mathrm{MD}^{1}$, Hamed Asadi, $\mathrm{MS}^{2}$, Denny $\mathrm{Yu}, \mathrm{PhD}^{2}$, Dimitrios Stefanidis, $\mathrm{PhD}, \mathrm{MD}^{1}$; ${ }^{1}$ Indiana University, ${ }^{2}$ Purdue University

Introduction: Work-related musculoskeletal injuries among surgeons are alarmingly high. Minimally invasive surgery, while popular with patients, is technically demanding and places increased ergonomic strain on the surgeon. The purpose of this study was to examine surgeon ergonomic risk during the performance of minimally invasive bariatric surgery operations.

Methods: An IRB-approved prospective study quantifying the intraoperative ergonomic risk factors during bariatric procedures was conducted. Ergonomic data from 5 bariatric surgery attendings and 2 fellows performing laparoscopic or robotic gastric bypass were collected. Surgeon ergonomic data, including workload, were recorded before, during, and after the procedures using subjective (Musculoskeletal Symptoms Response questionnaire and the National Aeronautics and Space Administration Task Load Index) and objective tools (electromyography to detect excessive muscle activity in the trapezius and deltoid muscles and inertial measurement units for motion tracking and body angulation). To determine the frequency of poor biomechanical postures during surgery, the relative frequency of static and demanding postures was calculated and compared to existing norms.

Results: Thirty surgeon participant observations were performed during 19 operations (13 laparoscopic and 6 robotic). All participants were right handed. Back stiffness was reported by $43 \%$ of surgeons before surgery and by $80 \%$ after surgery $(p=0.004)$. Surgeons who experienced back stiffness had significantly higher right deltoid muscle activity during the procedures compared with those who did not $(5.8 \pm 2.8$ vs. $4.5 \pm 2.2$, respectively; $p=0.03)$. The right deltoid and the right trapezius had the highest average muscle activity $(8.5$ and 5.7 respectively). At least $50 \%$ of operations were performed in static positions that require sustained muscle efforts. Neck postures were observed to be in demanding postures for $41 \%$ of the case that results in dangerously high joint loads (Table 1).

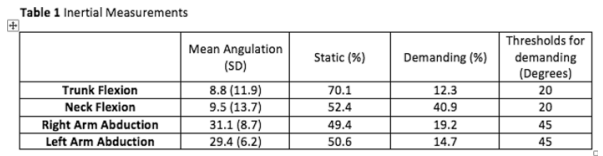

Conclusion: Minimally invasive bariatric surgery demands high static positioning with less weight shifting and significant strain on the back, specifically the right side. By determining direct body stresses during bariatric surgery, new technological advancements that can help reduce them can be tested or developed. 


\section{P705}

Detection of Extrahepatic and Intrahepatic Biliary Anatomy: Laparoscopic Intraoperative Cholangiography v. Robotic NearInfrared Fluorescence Cholangiography

Priti Lalchandani, MD, Jennifer A Smith, MD, Beverley A Petrie, MD; Harbor-UCLA Medical Center

Introduction: The inability to identify biliary anatomy has been identified as the cause of bile duct injury (BDI) in 71-97\% of laparoscopic cholecystectomies. Indocynanine green (ICG) has emerged as a safe and effective modality to visualize biliary anatomy. Near-infrared laser beams excite ICG causing emission of light and resultant imaging of the biliary tree. ICG is given prior to robotic near-infrared fluorescence cholangiography (NIFC) visualization of the biliary ducts. In this report, we compared the extrahepatic and intrahepatic biliary anatomy of a patient utilizing both laparoscopic intraoperative cholangiography (IOC) and robotic NIFC.

Method/Procedure: A 28-year-old presented with 5-years of post-prandial right upper quadrant pain and was found to have cholelithiasis and normal common bile duct on ultrasound. Robotic NIFC performed for academic purposes detected an aberrant cystic duct. Given the finding of a double cystic duct, the case was converted to a laparoscopic approach in order to perform a comparative evaluation of the biliary anatomy utilizimg IOC.

Results: Laparoscopic IOC did not reveal the aberrant double cystic duct seen on robotic NIFC (Figure 1,2). However, an incidental anomalous intrahepatic right posterior hepatic duct was noted on laparoscopic IOC (Figure 3). Interestingly, this intrahepatic abnormality was not seen on robotic NIFC. Ultimately the cholecystectomy was completed without BDI.

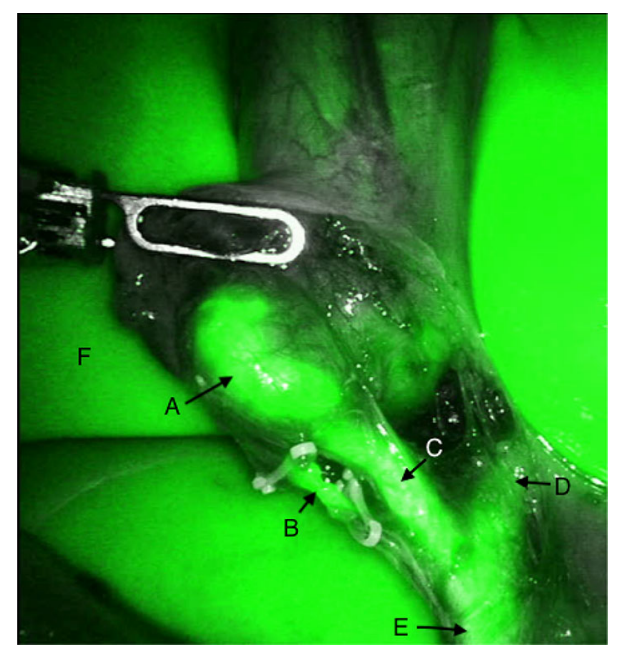

Figure 1. Robotic near-infrared fluorescence cholangiography. A Gallbladder infundibulum, B Anterior aberrant double cystic duct, C Cystic duct, D Common hepatic duct, E Common bile duct, F Liver.

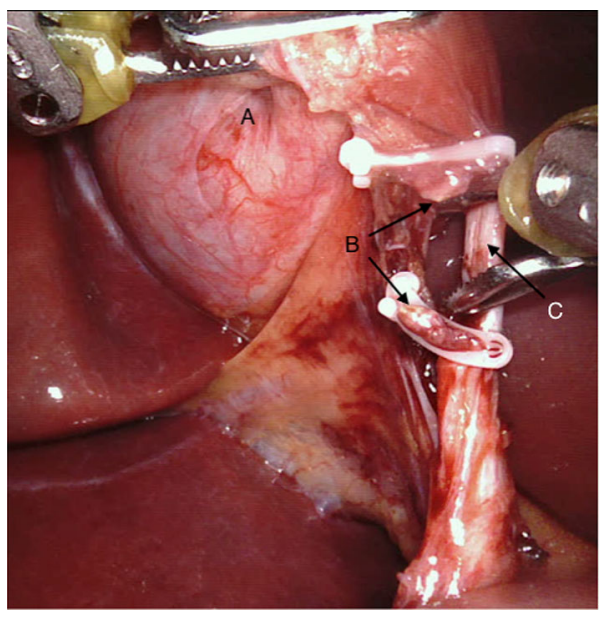

Figure 2. Robotic image of gallbladder with double cystic duct. A Gallbladder body, B Aberrant anterior double cystic duct (clipped and transected), C Cystic duct.

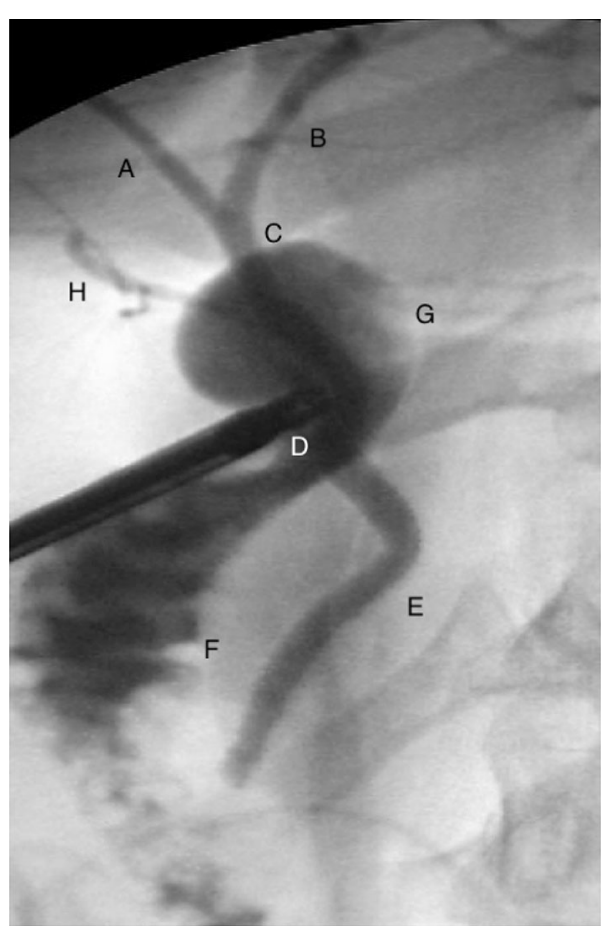

Figure 3. Laparoscopic intraoperative cholangiography.

A Right anterior hepatic duct, B Left hepatic duct, C Common hepatic duct, D Cystic duct, E Common bile duct, $\mathbf{F}$ Duodenum, G Contrast extravasation, H Right posterior hepatic duct.

Conclusion: Laparoscopic IOC has been well studied and has been shown to accurately detect extrahepatic and intrahepatic biliary anatomy. In recent studies, robotic NIFC has been reported to reveal extrahepatic biliary anatomy with noteworthy accuracy. Remarkably, robotic NIFC may actually outperform laparoscopic IOC in detection of extrahepatic biliary anatomy due to its four times standard view of the ICG image. Therefore, NIFC performed during robotic cholecystectomy is an effective procedure that helps real-time visualization of the extrahepatic biliary anatomy. Yet its ability to elucidate intrahepatic biliary anatomy and whether that information has a salient affect on minimally invasive cholecystectomy outcomes remain unclear. 


\section{P706}

\section{Feasibility of Robotic Assisted Heller Myotomy for Achalasia} in Pediatric Patients

William J Hammond, MSc, MD, Katherine D Gray, MD, Nicole J Croteau, MD, Thomas Ciecierega, MD, Demetri J Merianos, MD, Shaun A Steigman, MD, Rasa Zarnegar, MD; New York Presbyterian Hospital

Purpose: Surgical management of achalasia utilizing minimally invasive robotic-assisted technique in pediatric patients is controversial. We aimed to determine the feasibility of robotic Heller myotomy in the pediatric population in comparison to adults

Methods: We reviewed the records of pediatric patients $\leq 21$ years diagnosed with achalasia who underwent robotic-assisted Heller myotomy between 2012 and 2017. Comparison was made to adults $>21$ years old with achalasia who underwent robotic-assisted Heller myotomy during the same time period. Reoperative cases were excluded. Mannometry was completed to define preoperative esophageal motility by the Chicago Classification. Eckardt scores were calculated pre- and post-operatively. Clinical presentation, treatment, and outcomes were evaluated.

Results: 8 pediatric ( 5 male; median age 16.5 years) and 32 adult (14 male; median age 53.7 years) patients were identified with a median follow-up of 1.1 years (IQR: 0.6-1.6 years) and 0.3 years (IQR: 0.1-0.9 years), respectively. All pediatric patients had type II achalasia, and none had received medical or endoscopic therapy prior to Heller myotomy. Results comparing pediatric and adult patients are summarized in Table 1.

Conclusions: Surgery remains the cornerstone therapy for achalasia, and robotic-assisted minimally invasive approach in children appears to have comparable outcomes to adults. In our cohort of patients, robotic Heller myotomy was found to be a safe and effective therapy for pediatric patients with achalasia.

Table 1. Characteristics and treatment details

$\begin{array}{lll}\begin{array}{l}\text { Pediatric } \\ (\mathrm{n}=8)\end{array} & \begin{array}{l}\text { Adult } \\ (\mathrm{n}=32)\end{array} & \begin{array}{l}\text { P- } \\ \text { Value }\end{array}\end{array}$

Variable

Body Mass Index $-\mathrm{kg} / \mathrm{m}^{2}$

Median(IQR)

21.4

$(18.1-25.5) \quad(23.0-27.4)$

Pre-operative Eckardt Score

Median(IQR)

$4(3-7)$

$5(3-7)$

Chicago Classification

Type I

Type II

Type III

Type IV

Operative Time minutes

Median(IQR)

Length of Stay if

Admitted - days

Median(IQR)

30-day Post-operative

Complications

Post-operative Eckardt Score $<1$
0.16

$\begin{array}{lll}1.0(1.0-1.3) & 1.0(0.3-1.0) & \\ 0 & 3(9 \%) & 0.37 \\ 7(88 \%) & 26(81 \%) & 0.68\end{array}$

\section{P707}

\section{Nissen Fundoplication is Technically Feasible with the Senhance ${ }^{\circledR}$ Surgical System}

Robin Schmitz, $\mathrm{MD}^{1}$, Dietmar Stephan, $\mathrm{MD}^{2}$, Justin Barr, $\mathrm{MD}, \mathrm{PhD}^{1}$, Heike Saelzer, $\mathrm{MD}^{2}$, Michael Scheidt ${ }^{2}$, Joachim Labenz, $\mathrm{MD}^{2}$, Sabino Zani, MD ${ }^{1}$, Frank Willeke, $\mathrm{MD}^{2}$; ${ }^{1}$ Duke University Medical Center, Durham, NC, ${ }^{2}$ St. Marienkrankenhaus, Siegen, Germany

Purpose: Potential advantages of robotic assisted surgery are expected for intracorporal suturing, given its long learning curve in conventional laparoscopy. The success of laparoscopic fundoplication relies on precise suturing of the fundal wrap at the hiatus. Therefore robotic assistance could be a useful tool in this particular procedure.

Since February 2015 patients undergo anti-reflux surgery at our institution in an interdisciplinary setting. In March 2017, the Senhance ${ }^{\circledR}$ Surgical System (Transenterix Inc) was introduced in our clinical practiceand utilized in upper GI surgeries.

Methods: After team training and establishment of standard operating procedures in hernia repair, robotic assisted surgery at the gastro-esophageal junction was initiated. All patients gave informed consent for robotic assistance with prospective data acquisition and analysis. An independent investigator not involved in direct patient care performed the data analysis.

Results: Between March 2017 and January 2018, we performed a total of 22 antireflux surgeries with the Senhance ${ }^{\circledR}$ System. Ten patients underwent robotic assisted Nissen fundoplication. In two patients conversion to laparoscopic fundoplication was necessary. The cockpit time ranged from 62 to $172 \mathrm{~min}$. The docking time (positioning of the robotic arms after initiation of the pneumoperitoneum) ranged between 4 and $14 \mathrm{~min}$. We performed one relaparoscopy on the day of surgery due to pain without any significant intraoperative findings and one laparoscopic revision to Toupet fundoplication after 7 months due to dysphagia.

Conclusion: This is the first reported experience with the Senhance ${ }^{\circledR}$ Surgical System in upper GI surgery. Robotic assisted Nissen fundoplication is technically feasible utilising this new robotic platform. Larger case series and randomised trials are needed to verify the value for upper GI-surgery.

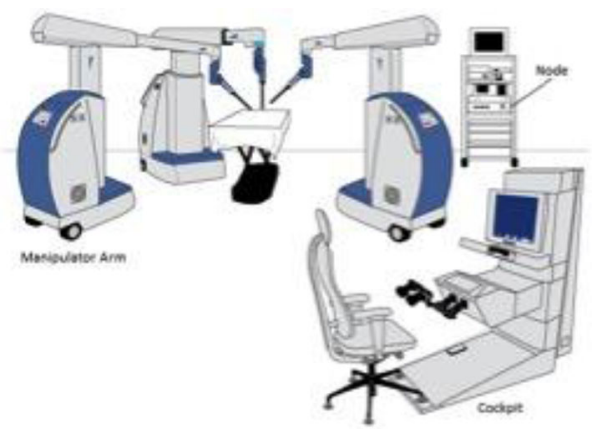




\section{P708}

Robotic Left-Sided Colorectal Resection with Natural Orifice Intracorporeal Anastomosis and Transrectal Extraction of Specimen: A Comparative Analysis

Ramon O Minjares-Granillo, MD ${ }^{1,2}$, Bertha A Dimas, MD $^{1,2}$, Samuel ${\mathrm{W} \text { Carson, } \mathrm{MD}^{1,3} \text {, Bobby L Johnson, MD }}^{1,3}$, Jean-Paul J Lefave, $\mathrm{MD}^{1,2,3}$, Eric M Haas, $\mathrm{MD}^{1,2,3}$; ${ }^{1}$ Department of Surgery, The University of Texas Medical School at Houston, Houston,

Texas, ${ }^{2}$ Southeast Clinical Research Associates, Inc., Houston,

Texas, ${ }^{3}$ Division of Colon and Rectal Surgery, Houston Methodist

Hospital, Houston, Texas

Introduction: We present the first known comparative analysis of robotic left-sided colorectal resection using natural orifice-assisted intracorporeal anastomosis (ICA) and removal of specimen. Although there has been a surge of enthusiasm and reports of outcome benefits following robotic ICA for right-sided colectomy, there are few reports investigating the feasibility, utility, benefits and limitations of robotic left-sided colectomy in the worldwide literature. We recently developed a stepwise approach designed for reproducibility and widespread adoption of this technique which we refer to as the Robotic NICE procedure: Natural orifice IntraCorporeal anastomosis with Extraction.

Methods: Thirty-five consecutive patients who underwent the NICE procedure over a four-month period were matched to a cohort of patients undergoing conventional robotic left-sided colectomy based on 4 matching criteria: surgeon, institution, disease, and procedure.
Patients who underwent neoadjuvant therapy were excluded. Demographic data, intraoperative parameters, costs, and short-term outcomes were analyzed in an IRB database.

Results: There was no significant difference in demographics between the groups including age, gender, BMI, and ASA. Diverticulitis was the most common diagnosis $(63 \%)$ followed by colorectal cancer (29\%). Overall mean operative time was similar between the NICE and conventional group ( 212 vs. $228 \mathrm{~min}, \mathrm{p}=0.298$ ). When comparing the NICE vs. conventional group, there was a significant difference in return of bowel function (1 vs. 2 days, $p=0.001$ ), length of stay ( 50 vs. $75 \mathrm{~h}, \mathrm{p}=0.001)$ and direct costs $(\$ 19,497 \mathrm{vs}$. $\$ 22,336, p=0.026)$. Significantly greater number of patients were discharged by POD 2 in the NICE group (74\%) compared to the conventional group (46\%) and all but 2 patients in the NICE group were discharged by POD $3(\mathrm{p}=0.025)$. Overall opioid use was lower in the NICE group but did not reach statistical significance (14 vs. $22 \mathrm{mg}$ morphine equivalent, $\mathrm{p}=0.13$ ). No significant difference was found in complication, readmission or reoperation rates. There was one surgical site infection in each group. No anastomotic leaks or mortalities were encountered.

Conclusion: The robotic NICE procedure is a promising approach that utilizes the rectum as a natural orifice for ICA and specimen extraction. It thereby eliminates the need for an abdominal incision other than the port sites. In this initial analysis there was a significant reduction in length of stay, earlier return of bowel function and decreased costs. Additional outcomes including reduced opioid requirements will be addressed in larger multicenter studies. 